| 071

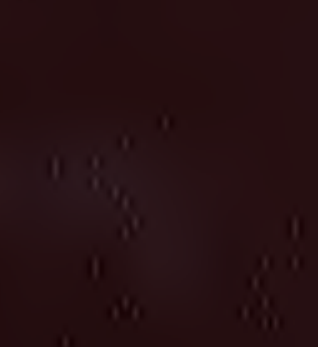

in.

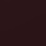




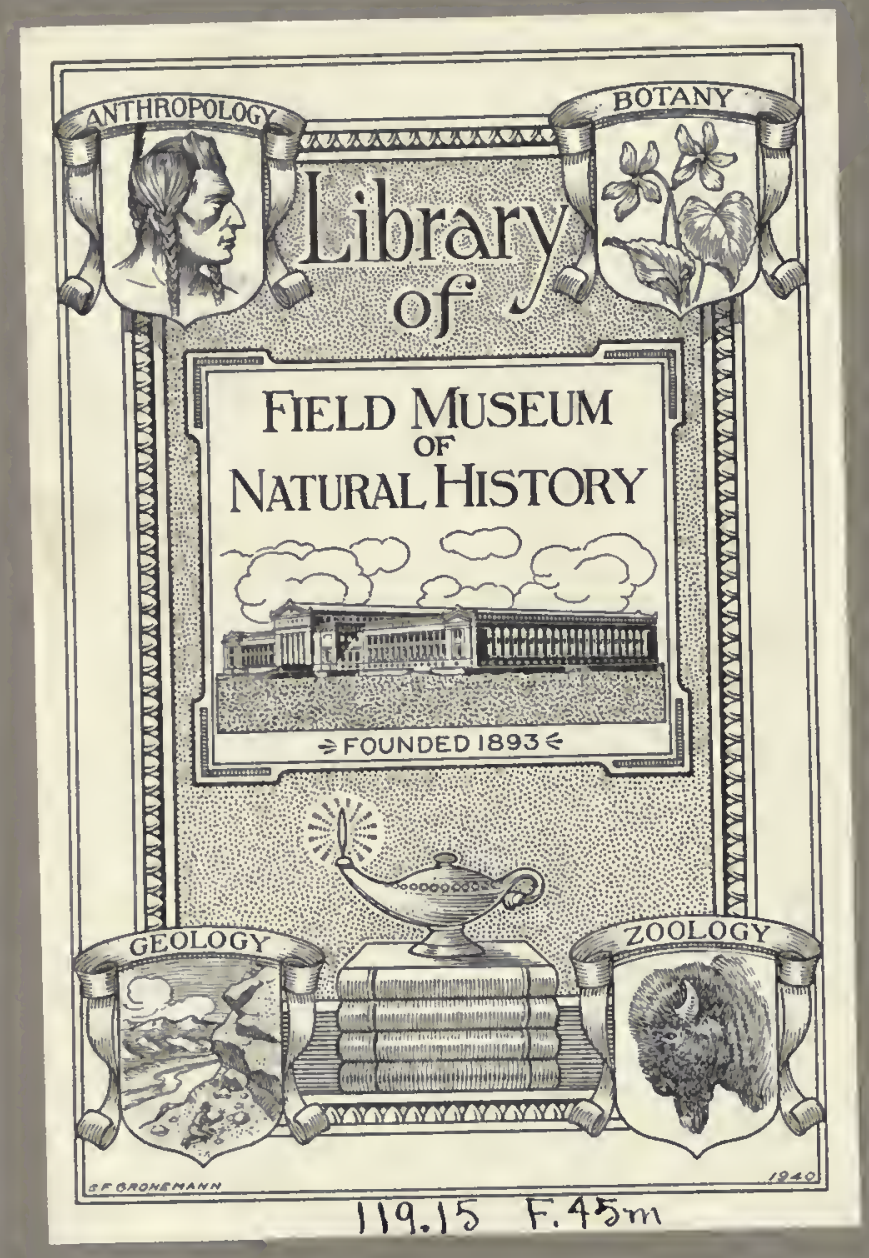





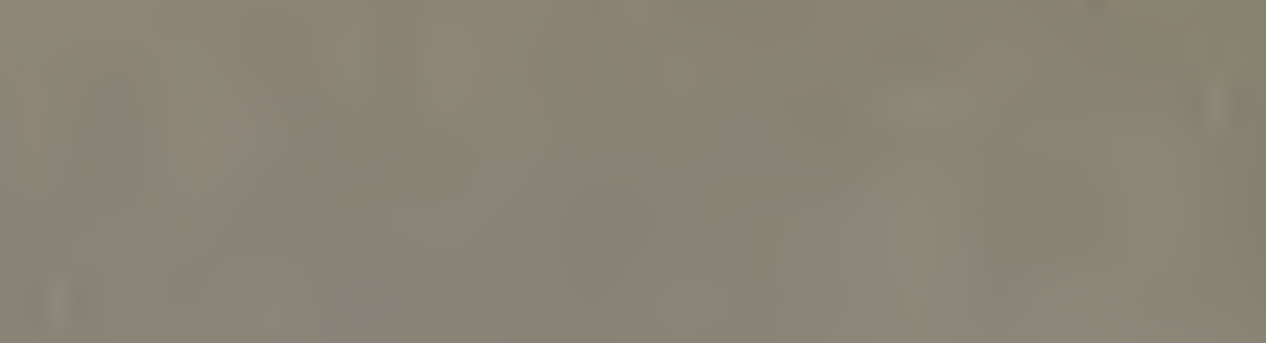

18.

4 


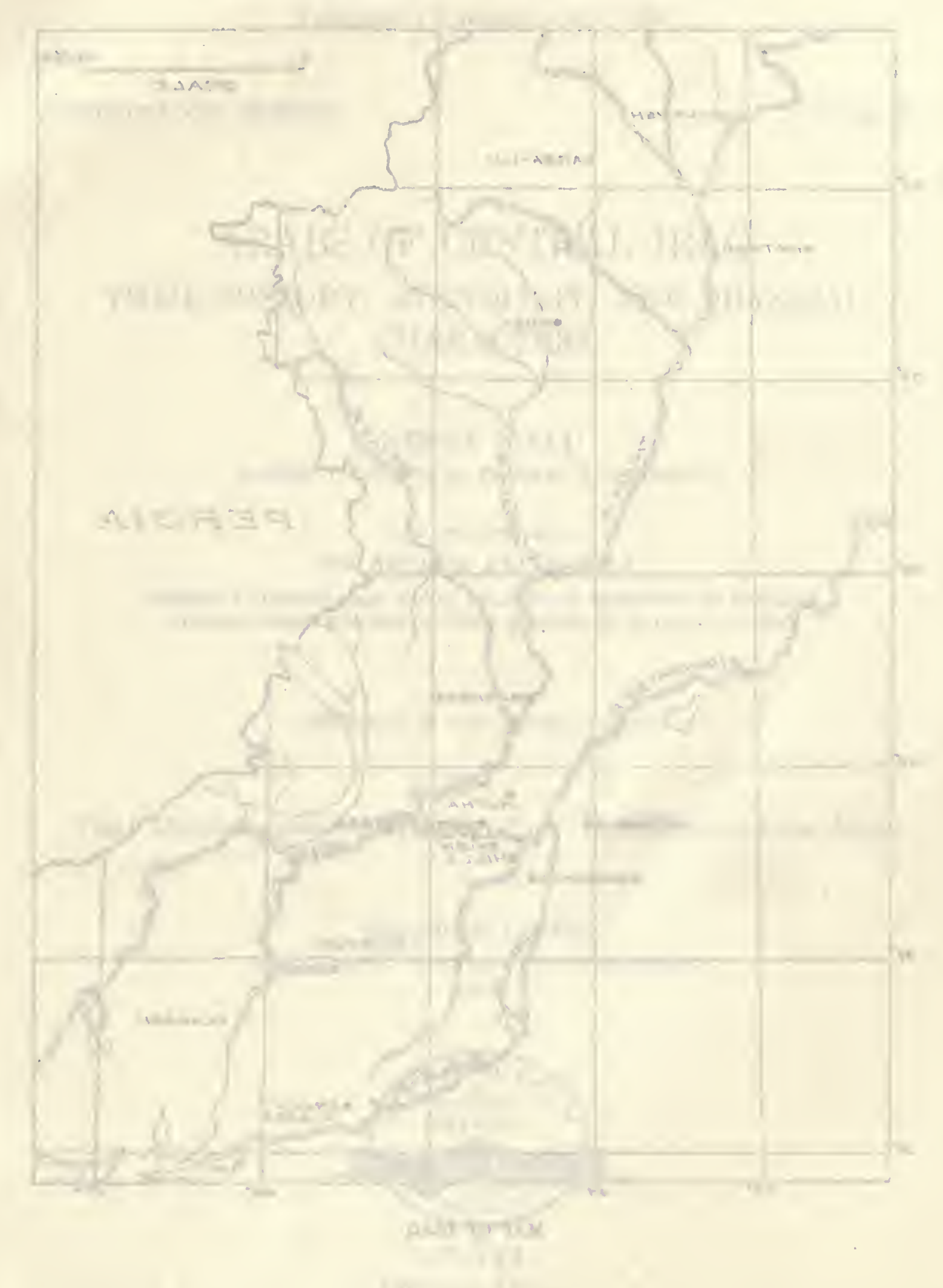




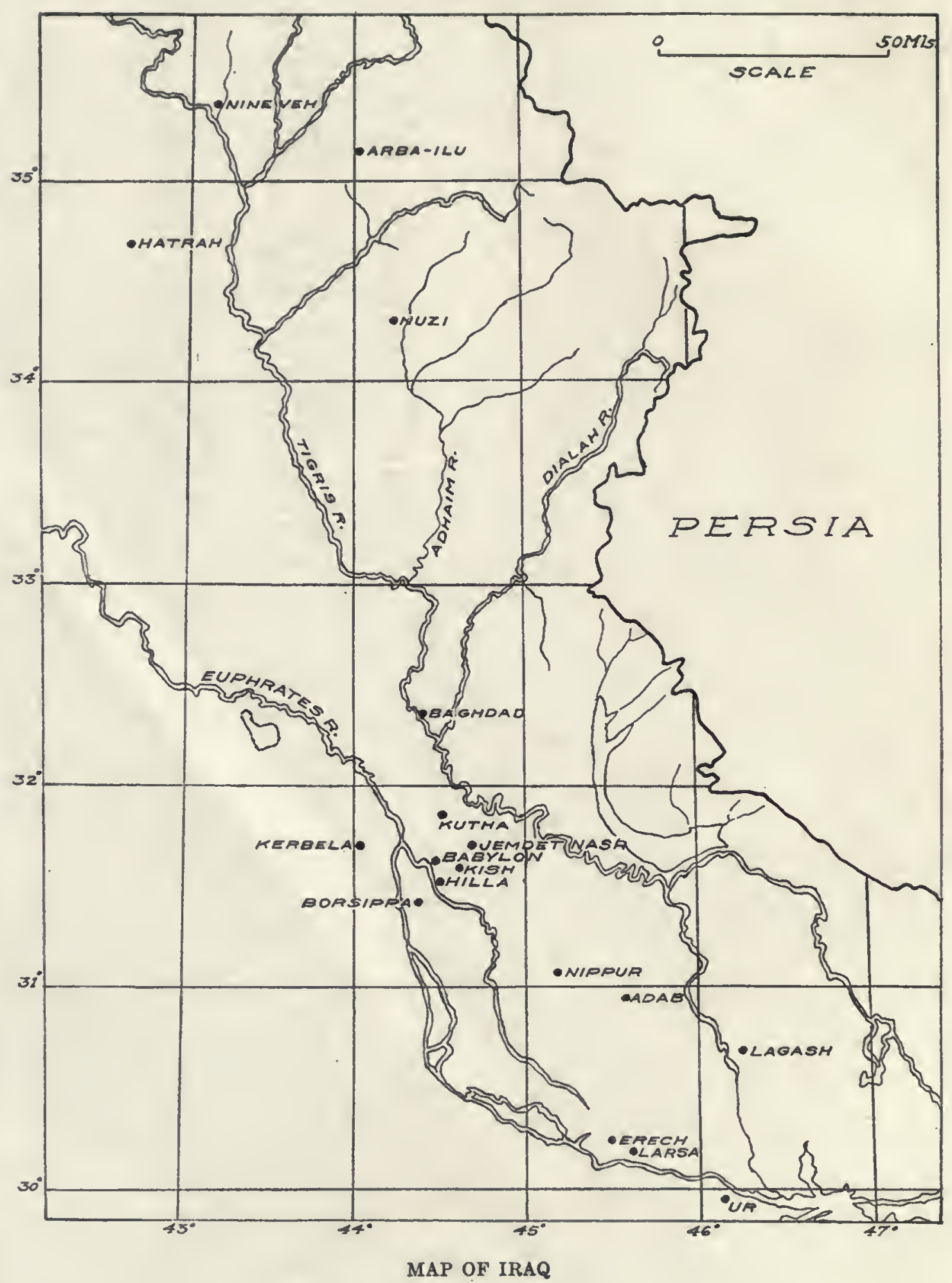


Field Museum of Natural History

Founded by Marsiall Field, 1893

ANTHROPOLOGY, MEMOIRS

Volume IV

\title{
ARABS OF CENTRAL IRAQ \\ THEIR HISTORY, ETHNOLOGY, AND PHYSICAL CHARACTERS
}

\author{
BY \\ HENRY FIELD \\ ASSISTANT CURATOR OF PHYSICAL ANTHROPOLOGY \\ WITH INTRODUCTION BY \\ SIR ARTHUR KEITH, F.R.S. \\ FORMERLY CONSERVATOR, ROYAL COLLEGE OF SURGEONS OF ENGLAND \\ CORRESPONDING MEMBER OF FIELD MUSEUM OF NATURAL HISTORY
}

156 Plates, 48 'Text Figures, 3 Maps

Field Museum-Oxford University Joint Expedition to Kish, IraQ

BERTHOLD LAUFER

LATE CURATOR, DEPARTMENT OF ANTHROPOLOGY

EDITOR

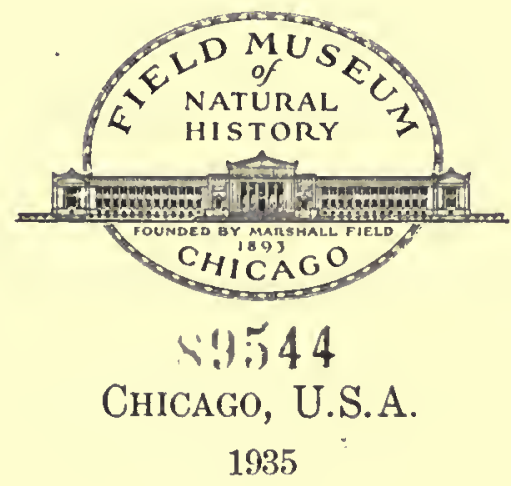




$$
\begin{aligned}
& \text { GIN } \\
& 2 \\
& .1354 \\
& =4 \mathrm{~m} \\
& \because .4
\end{aligned}
$$




\section{CONTENTS}

List of Illustrations . . . . . . . . . . . . 5

Preface . . . . . . . . . . . . . . 9

Introduction by Sir Arthur Keith . . . . . . . . . . . . 11

I. Arabs of the Kish Area . . . . . . . . . . . . . . . . . . 77

Geographical Location . . . . . . . . . . . . 77

Evidence of Prehistoric Culture . . . . . . . . . . . . . 77

History of Kish by Stephen Langdon . . . . . . . . . . 79

Social Life and Customs . . . . . . . . . . . . . . . 82

II. Anthropometric Data . . . . . . . . . . . . . . . 98

Measurements and Observations . . . . . . . . . . 98

Arabs of the Kish Area . . . . . . . . . . . . . 101

Soldiers of the Iraq Army . . . . . . . . . . . . . . . . 305

Ba'ij Beduins . . . . . . . . . . . . 410

III. Statistical Summaries . . . . . . . . . . . . . . . . 430

Vital Statistics . . . . . . . . . . . . 430

Qaraqosh .............. . . . 432

Arabs of the Kish Area . . . . . . . . . . . . . . . 434

Soldiers of the Iraq Army . . . . . . . . . . . . . 444

Ba'ij Beduins . . . . . . . . . . . . . . . . . . 448

Tattooing . . . . . . . . . . . . 455

Summary of Anthropometric Data from Southwestern Asia . . 456

Appendix: Field Museum Anthropological Expedition to the Near

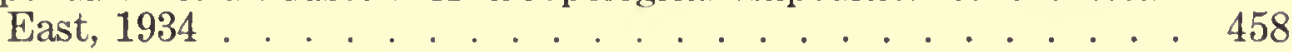

Bibliography . . . . . . . . . . . . 464

Glossary . . . . . . . . . . . . . . 467

Index . . . . . . . . . . . . . . . 469 


\section{.}




\section{LIST OF ILLUSTRATIONS}

I-LI. Arabs, Kish Area.

\section{PLATES}

LII-CVI. Iraq Soldiers, Hilla Camp.

CVII. Figs. 1-4. Iraq Soldiers, Hilla Camp. Figs. 5-8. Arabs, Kish Area.

CVIII-CXVII. Ba'ij Beduins, near Kish.

CXVIII. Ba'ij Beduins, near Kish. Figs. 1, 2. Herdsmen. Fig. 3. Black tent.

CXIX. Ba‘ij Beduins, near Kish. Fig. 1. Woman. Fig. 2. Woman with child. Fig. 3. Woman with spindle. Fig. 4. Woman weaving.

CXX-CXXII. Arab Children, Kish Area.

CXXIII. Building Mud House, Kish Camp.

CXXIV. Arab Dancing Girl and Musicians, Kish.

CXXV. Building Reed-mat House, Kish Excavations.

CXXVI. Arabs, Kish Area. Figs. 1, 2. Making coffee. Fig. 3. Beside the hearth. Fig. 4. Author, taking measurements.

CXXVII. Arabs, Kish Area. Fig. 1. Celebrating end of Ramadan. Fig. 2. Horsemen. Fig. 3. Workmen outside camp, Kish.

CXXVIII-CXXIX. Arab (No. 26), Kish Area.

CXXX-CXXXI. Arab (No. 2), Kish Area.

CXXXII-CXXXIII. Arab (No. 10), Kish Area.

CXXXIV-CXXXV. Arab (No. 74), Kish Area.

CXXXVI. Arabs, Kish Area.

CXXXVII. Arabs, Kish Area. Fig. 1. Native orchestra. Fig. 2. Woman. Fig. 3. Husband and wife.

CXXXVIII. Scenes at Mahewil and Karbala. Fig. 1. Gubbah, Mahewil near Kish. Fig. 2. Karbala. Fig. 3. Central arch of gubbah, Mahewil. Fig. 4. Palm groves, near Karbala.

CXXXIX-CXLIV. Chaldean Women and Girls, Qaraqosh.

CXLV-CXLVI. Types, near Altün Köprü.

CXLVII-CLIII. Chaldeans, Qaraqosh.

CLIV. Types, near Altün Köprü.

CLV. Chaldeans, Qaraqosh.

CLVI. Scenes near Qaraqosh and Kirkuk. Fig. 1. Girl in brickyard. Fig. 2. Street scene in Qaraqosh. Fig. 3. The winnower. Fig. 4. View of Qaraqosh village. Fig. 5. Threshing. Fig. 6. Wooden threshing sledge.

\section{TEXT FIGURES}

PAGE

1. Chart I. Head length, breadth, and capacity of thirty-eight Ba ij Beduins . . . 21

2. Chart II. Head length, breadth, and capacity of thirty-eight Ba'ij Beduins, thirty-five South Arabs, and six Somalis . . . . . . . . . . . . . . . 23

3. Chart III. Head length, breadth, and capacity of fifty Iraq soldiers (Nos. 500$550)$. . . . . . . . 2 24

4. Chart IV. Head length, breadth, and capacity of fifty Egyptians of the Khargha Oasis . . . . . . . . . 2 26

5. Chart V. Head length, breadth, and capacity of fifty men of Chatri caste, United Provinces. . . . . . . . . . . . . . . .

6. Chart VI. Head length, breadth, and capacity of fifty men of Pathan caste, Punjab . . . . . . . . . . . . . . 
7. Chart VII. Head length, breadth, and capacity of fifty Iraq soldiers (between

8. Chart VIII. Head length, breadth, and capacity of fifty Iraq soldiers .

PAGE

9. Chart IX. Head length, breadth, and capacity of fifty Kish Arabs from camp of Sheikh Miniehil (Nos. 1-50). . . . . . . . . . . . . . .

10. Chart X. Head length, breadth, and capacity of fifty Kish Arabs from camp of Sheikh Miniehil (Nos. 100-149) . . . . . . . . . . . . .

11. Chart XI. Head length, breadth, and capacity of fifty Kish Arabs from camps of Sheikh Swadi and Sayyid Ruda (Nos. 200-249) . . . . . . . . . . . .

12. Chart XII. Head length, breadth, and capacity of fifty Kish Arabs from camps of Sheikh Hunta and Sheikh Aziz .

13. Chart XIII. Minimal frontal width and maximal head width in three groups of

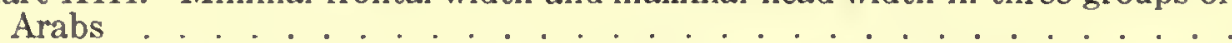

14. Chart XIV. Minimal frontal width and maximal head width in fifty men of Chatri caste, United Provinces, and fifty men of Pathan caste, Punjab . . .

15. Chart XV. Nasal width and length of fifty Egyptians of the Khargha Oasis . .

16. Chart XVI. Nasal length and width of fifty Kish Arabs (Nos. 1-50) . . . .

17. Chart XVII. Nasal length and width of thirty-eight Ba'ij Beduins . . . . . .

18. Chart XVIII. Nasal length and width of fifty Kish Arabs (Nos. 101-150). . .

19. Chart XIX. Nasal length and width of fifty Iraq soldiers (Nos. 500-549) . . .

20. Chart XX. Nasal length and width of fifty men of Chatri caste, United Provinces ...................

21. Chart XXI. Nasal length and width of fifty men of Pathan caste, Punjab . . .

22. Chart XXII. Upper face length and total face length of thirty-eight Ba'ij Beduins . . . . . . . . . . . . . . .

23. Chart XXIII. Upper face length and total face length of fifty Kish Arabs

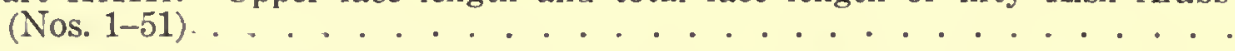

24. Chart XXIV. Upper face length and total face length of fifty Kish Arabs

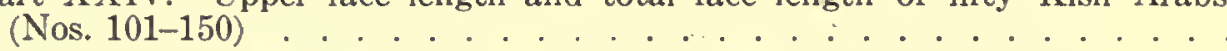

25. Chart XXV. Upper face length and total face length of fifty Kish Arabs (Nos. 200-251) . . . . . . . . . . . . . .

26. Chart XXVI. Upper face length and total face length of fifty Iraq soldiers (Nos. 500-551) . . . . . . . . . . .

27. Chart XXVII. Total face length and total face width of thirty-eight $\mathrm{Ba}^{\circ} \mathrm{ij}$

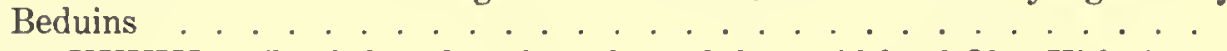

28. Chart XXVIII. Total face length and total face width of fifty Kish Arabs (Nos. 1-50) . . . . . . . . . . . . . . .

29. Chart XXIX. Total face length and total face width of fifty Egyptians of the Khargha Oasis.

30. Chart XXX. Total face length and total face width of fifty Kish Arabs (Nos. 100-150) . . . . . . . . . . . . .

31. Chart XXXI. Total face length and total face width of fifty Kish Arabs (Nos. 201-250)

32. Chart XXXII. Stature and sitting height of thirty-eight Ba'ij Beduins . . .

33. Chart XXXIII. Stature and sitting height of fifty Egyptians of the Khargha

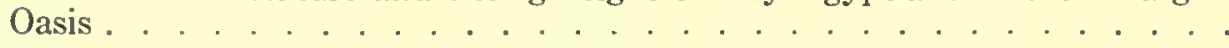

34. Chart XXXIV. Stature and sitting height of fifty Kish Arabs (Nos. 1-50) . .

35. Chart XXXV. Stature and sitting height of fifty Kish Arabs (Nos. 101-150) . .

36. Household Utensils . . . . . . . . . . . . . . . . . . . 91

37. Weapons and Implements . . . . . . . . . . . . . . . .

38. Graph I. Standing heights. Continuous curve, 376 Kish Arabs; dash curve, 228 Iraq soldiers; dotted curve, thirty-eight Ba'ij Beduins; dot-dash curve, composite of Kish Arabs and soldiers . 
39. Graph II. Index of relative sitting heights. Continuous curve, 376 Kish Arabs; dash curve, 228 Iraq soldiers; dotted curve, thirty-eight Ba'ij Beduins; dot-dash curve, composite of Kish Arabs and soldiers .

PAGE

40. Graph III. Cephalic indices. Continuous curve, $396 \mathrm{Kish}$ Arabs; dash curve, 227 Iraq soldiers; dotted curve, thirty-eight Ba'ij Beduins; dot-dash curve, composite of Kish Arabs and soldiers.

41. Graph IV. Cumulative percentages of cephalic indices. Continuous curve, 396 Kish Arabs; dash curve, 227 Iraq soldiers; dot-dash curve, thirty-eight Ba'ij Beduins .

42. Graph V. Fronto-parietal indices. Continuous curve, 395 Kish Arabs; dash curve, 227 Iraq soldiers; dotted curve, thirty-eight Ba'ij Beduins; dot-dash curve, composite of Kish Arabs and soldiers . . . . . . . . . . . . . . .

43. Graph VI. Total facial indices. Continuous curve, $385 \mathrm{Kish}$ Arabs; dash curve, 227 Iraq soldiers; dotted curve, thirty-eight Ba'ij Beduins; dot-dash curve, composite of Kish Arabs and soldiers . . . . . . . . . . . . . . . .

44. Graph VII. Upper facial indices. Continuous curve, $391 \mathrm{Kish}$ Arabs; dash curve, 226 Iraq soldiers; dotted curve, thirty-eight $\mathrm{Ba}^{6} \mathrm{ij}$ Beduins; dot-dash curve, composite of Kish Arabs and soldiers . . . . . . . . . . . . . . .

45. Graph VIII. Nasal indices. Continuous curve, 389 Kish Arabs; dash curve, 226 Iraq soldiers; dotted curve, thirty-eight Ba'ij Beduins; dot-dash curve, composite of Kish Arabs and soldiers . . . . . . . . . . . . . . . .

46. Graph IX. Ear indices. Continuous curve, 390 Kish Arabs; dash curve, 226 Iraq soldiers; dotted curve, thirty-eight Ba'ij Beduins; dot-dash curve, composite of Kish Arabs and soldiers . . . . . . . . . . . . . . . . . .

47. Graph X. Jugomandibular indices. Continuous curve, 393 Kish Arabs; dash curve, 227 Iraq soldiers; dotted curve, thirty-eight Ba'ij Beduins; dot-dash curve, composite of Kish Arabs and soldiers . . . . . . . . . . . . . . .

48. Graph XI. Jugofrontal indices. Continuous curve, $395 \mathrm{Kish}$ Arabs; dash curve, 227 Iraq soldiers; dotted curve, thirty-eight Ba'ij Beduins; dot-dash curve, composite of Kish Arabs and soldiers.

\section{MAPS}

Iraq . . . . . . . . . . . . . . . . . . facing page 1

Kish Area . . . . . . . . . . . . . . . . . . . . . . . . . . . . . . . . . 83

Trip from Kish to Tigris River . . . . . . . . . . . . . . . . . . 84 



\section{PREFACE}

This publication deals with the physical characters and anthropometric data collected in 1928 on Arabs and Beduins living near Kish, Iraq. During the winter season, from December, 1927, to March, 1928, I was attached as physical anthropologist to the staff of the Field Museum-Oxford University Joint Expedition, at Kish. The continual discovery of human skeletal remains prevented the collection of anthropometric data on the living Arabs until the close of the archaeological season.

Mr. Louis C. Watelin, field director of the expedition, then decided to work for two weeks at Jemdet Nasr, eighteen miles northeast of Kish. I accompanied him there, and, with the assistance of Mr. Eric Schroeder, who was attached to the expedition during that season, I began studies on a small group of the native workmen. Mr. S. Y. Showket, of the Mesopotamia Studio in Baghdad, was engaged to take front and profile photographs of each individual measured and studied.

Early in the month of June, Mr. Showket and an assistant photographer returned with me to Kish in order to obtain anthropometric data on five hundred individuals of that region.

I wish to express my gratitude to the late Dr. Berthold Laufer, who encouraged and aided my research work by his interest in racial and cultural problems of the Near East.

Before leaving the United States I had discussed various Arab racial problems with Professor E. A. Hooton, of Harvard University, who gave me valuable advice and suggestions. I am also indebted to Mr. Henry Balfour, Dr. R. R. Marett, and Dr. L. H. Dudley Buxton of the University of Oxford, under whose tuition no student of anthropology could fail to receive inspiration. Dr. Buxton, moreover, accompanied me to Kish for a period of three weeks during December, 1925, and January, 1926, and under his direction I learned the technique employed in recording anthropometric data.

Professor Stephen Langdon, who directed the excavations at Kish during twelve seasons, has been most generous with his assistance upon all occasions.

I am grateful to the various local sheikhs of the Kish area, particularly to Sheikh Hadji Miniehil and to the late Sheikh Hadji Hunta, who allowed me to make observations and measurements on themselves as well as on their tribesmen. Khalef-el-Jebbar, who was my assistant during the entire season of excavation, rapidly learned the methods employed in the preservation of skeletal material. He also assisted my anthropometric work in the Kish area and accompanied me to each Arab encampment. His chief duties were to further cordial relationships and to collect hair samples, which was no easy task.

I must add a few words of thanks to Mr. Showket and his assistant, who exposed over one thousand negatives, many hundreds of which were developed under adverse conditions. I wish also to express my thanks to the Mutasarrif 
of the Hilla Liwa and to the officer commanding the Hilla army camp, who granted me special permission to measure and photograph a number of the troops in his charge.

In Baghdad, Mr. A. Kerim, owner of the Mesopotamia Studio, was always generous in his assistance with the photographic work.

Upon my return to Chicago it was necessary to calculate the statistics, and in this task I was assisted by Professor F. E. Wood and Mr. G. E. Arnold, to whom I am deeply indebted.

Sir Arthur Keith, formerly Conservator of the Royal College of Surgeons of England, has written an introduction which places the Arab in a new light and shows his relationship to the main racial divisions of Asia, Africa, and Europe from Pleistocene times. I am most grateful to Sir Arthur, who has given me many valuable suggestions and who has devoted so much time and energy to this problem.

My thanks are due to Dr. William M. Krogman for his assistance with certain statistical additions, which were necessary for Sir Arthur Keith's summary of the anthropometric data.

I also gratefully acknowledge the aid of Mr. Carl F. Gronemann, Staff Illustrator of Field Museum, and Mr. Richard A. Martin in the preparation of drawings, maps, and graphs, of Miss Lillian A. Ross in seeing the manuscript through the press, and of Miss Alice Wilkinson and Miss Elizabeth Reniff, who assisted with the preparation of this Report.

The spelling of place names conforms to the system adopted by the British Permanent Committee on Geographical Names, published in London by the Royal Geographical Society.

As a result of the personal interest of King Ghazi and the generous cooperation of the government officials of Iraq, various problems connected with the study of the physical anthropology of the peoples of Iraq are now approaching solution. 


\section{ARABS OF CENTRAL IRAQ \\ INTRODUCTION}

It has given me great pleasure to write this introduction to Mr. Henry Field's Report for several reasons. The first, a minor reason, was this: When in 1926 I was investigating the human skulls and bones which Sir Leonard Woolley (Excavations at Al 'Ubaid, 1927) had discovered in the ancient graves of Ur, I found myself hampered by the almost complete lack of data concerning the racial characters of the modern inhabitants of Iraq. Dr. L. H. Dudley Buxton, when he was reporting on the ancient human remains discovered at Kish by the Field Museum-Oxford University Joint Expedition, was equally embarrassed (Excavations at Kish, by S. Langdon, I, 1924). He could not determine, any more than I could, to what extent the human types who lived in the ancient cities of the plain were represented among the inhabitants of modern Iraq. At Ur I found men and women who were long-headed and big-headed; at Kish Dr. Buxton found round-headed as well as long-headed people. We had no data to guide us as to whether or not these ancient long-headed and round-headed people were still represented among the Northern Arabs and to what extent. Herein lies part of the value of the investigations made by Mr. Field during his sojourn in Iraq during the winter season (1927-28). His Report begins to bridge a hiatus in modern anthropology.

A knowledge of the Northern Arabs was needed not only for such minor purposes as Dr. Buxton and I had in view, but for a much more important purpose, that of obtaining light on the evolution of eastern humanity. The origin of the Arab is the central enigma of this great problem. As I hope to show, the data collected and published in this Report provide us with the means of putting forward an explanation or theory of the evolutionary origin of the Arab and other types of the East.

Let me first indicate the scope covered by this publication. The people whose measurements and characters are recorded here were met in the Kish area, between the rivers. Only men were examined. Records were made of 667 individuals. These fall into the three following groups:

Group A.-Arabs living in the region of Kish in 1927-28. They are numbered in two series: Nos. 1-288 and 350-471-altogether 398 men.

Group B.-Iraq soldiers measured in 1928 in the army camp at Hilla on the Euphrates, 231 in number. In the series they are Nos. 500-732.

Group C.-Thirty-eight men of the Ba ij Beduin tribe, living fourteen miles northeast of Kish in the direction of Jemdet Nasr. This small but most valuable addition to the series, comprising Nos. 300-337, was also made in 1928.

The various measurements made of each individual and the racial features noted will be seen at a glance by consulting the schedule reproduced on page 101 (Arabs of the Kish Area, No. 1). Of each individual fourteen measurements were taken, three relating to stature, two to dimensions of head, 
seven to face, and two to external ear. Nine indices were calculated. Twentythree other features were noted and their development determined by eyemeasurement. A record was made of name, age, birthplace, and other items. More than fifty items were recorded concerning each individual examined, and, as the number included in this survey amounts to 667 individuals, the total observations recorded in Mr. Field's schedules are more than 33,000 in number. Thus, thanks to his labors, our knowledge of the modern Arabs of central Iraq has passed suddenly from a state of poverty to one of affluence.

Of all the records and measurements, those which were registered by the camera are for myself of the highest value. Altogether 581 individuals were photographed, and, as in almost every instance profile and full face of the subject measured were taken, the total number of photographs amounts to more than 1,150 records. Of the subjects photographed thirty-eight were Ba'ij Beduins, representatives of what may be spoken of as the essential Arab; 223 were men enrolled in the Iraq army, all natives of Iraq; 320 were Arabs living or working in the neighborhood of Kish. I do not underestimate for a moment the value of exact instrumental measurements. A science of anthropology cannot come into existence unless we use exact instruments and precise arithmetical records. Our general impressions are of no value to those who come after us; only definite measurements are of use to them. They can incorporate our labors with their own only if we leave behind us precise figures of what we have observed and measured. Nevertheless, we have a very imperfect knowledge of a people if we seek to realize their characteristics merely from the examination of tables of measurements, however elaborate these tables may be. We know a people only when we have seen them in the flesh with our own eyes. But there is one substitute - good photographic records.

We are apt to think of anthropology as a new science; in truth it is a very old art, perhaps the oldest. For since that remote time when man made conscious observations on the faces and habits of the members of his own family and of his own tribe, he has been an anthropologist. How soon we learn to discriminate by the cast of an eye the distinctive races of mankind! A Negro or a Chinese, a Hindu or an American Indian meets us in the street, and, before he has passed, we have assessed his racial characters and made our diagnosis. We can never hope to obtain, by the use of instruments, indices, and angles, the delicacy and precision of racial diagnosis reached by the tutored and experienced eye. Travelers and explorers may become so skilled that in a cosmopolitan crowd in an eastern bazar they will recognize the most delicate shades of race. They scarcely note the shape of head or even of face; a hundred minor points which they have never submitted to conscious analysis guide them to a true diagnosis.

If we could cultivate those natural gifts and apply them in such a way that the observations made by one observer could be so formulated that those made by another observer could be added to them, what an engine of progress would be at our disposal for the proper discrimination of the breeds and races of mankind! A series of photographs such as were collected by Mr. Field among the 
Arabs of Kish provides me with an excellent opportunity to test my theory in a practical way. In his series of photographs which reproduce the facial features is one of 223 men enrolled in the Iraq army. I prefer to begin with this group for two reasons: the men are in uniform, and their heads are cropped. An Arab in national dress presents us with a racial diagnosis already made; to ascertain how far we can distinguish Arabs by their outward features from men of other races, all should be dressed and barbered alike. The Iraq soldiers present us with our racial problem in an ideal form. The soldier series also presents the problem free from another great difficulty, the changes due to age. Presently, in discussing the photographs of the Ba'ij Beduins, I shall have to draw attention to the changes which transform the youthful Beduin into the venerable old sheikh, but among the soldiers we are not confronted with age changes; almost the entire series falls between the ages of seventeen and thirty.

In order that the degree of racial distinction of the Arab from surrounding nationalities and races should be tested properly there ought to be, dressed in the Iraq uniform, an equal number of photographs of Egyptian soldiers, of soldiers from the various parts of India, from Afghanistan, from Iran, from the Turkish army, from the Italian, French, and Spanish armies, and, more especially, photographs of Algerian soldiers. If in such a large, mixed series of photographs I could recognize every native of Arabia, then that would be proof that the Arab is a completely differentiated race-a Pan-diacritic race-a hundred-per-cent race (Sir Arthur Keith, Ethnos, 1931). Or if it happened, as I know would be the case, that I should succeed in recognizing only a portion of the Arab soldiers, I should then wish to know the proportion of my successes because that would be an indication of the extent to which Arabs are differentiated from surrounding peoples. Especially should I want to know the peoples that I was most likely to confuse with Arabs, for the extent of my mistakes would be an index of the relationship of the Arab to these other peoples.

Not having at my disposal materials for comparison from other countries I made a close examination of the series of Arab soldiers and noted those whom I believed I could recognize as Arabs from their external appearances. Of 223 men there were 122 (54 pel cent) whom I believe I could have picked out as Arabs from a cosmopolitan crowd. More especially is it important to note the peoples with whom the Arabs might be confused owing to high degrees of resemblance. The dominating resemblances I detected in the Iraq soldier series were to men of the Mediterranean type. Two examples of Arabs of the Mediterranean type are reproduced here to illustrate the kind of Arab I have in mind (Nos. 713 or 714 , and 500 or 504). In the series of 223 Arabs there were fifty-four whom I thought might be confused with Greeks or South Italians, giving a South European relationship of more than 24 per cent. The next group of aberrant Arabs had resemblances which linked them with peoples of Asia Minor and with Iranis. There were sixteen Arabs in this group, rather upward of 7 per cent. Certain minor relationships of these Arabs with the peoples of Africa and India I shall touch on presently. 
My evidence, as far as it goes, points to the Arab as a branch of the stock which occupies southwestern Asia and southern Europe; he is an imperfectly differentiated member-imperfect in a physical sense-of the great Caucasian stock. In my system of nomenclature, the Arab is a mesodiacritic race, a race differentiated to the extent that from 40 to 60 per cent of its members can be recognized at sight in any cosmopolitan crowd. It was not with the Egyptian or Indian soldier I was most likely to confuse the Arab soldier. The Arab is akin to the South European; among the Iraq soldiers there were 24 per cent who might have passed as Mediterraneans. The Arab also has affinities with certain Asiatic races ( 7 per cent).

Now this result is somewhat surprising in several respects. One would have expected a considerable number of Arabs to have Hamitic features, such as would lead us to confuse them with Egyptians or even with Somalis. In my list only nine Arabs are noted as showing Egyptian resemblances. Recently I had an opportunity to scrutinize Egyptian soldiers drawn up along the streets of Cairo on the occasion of the arrival of a foreign monarch. Most of them were recruits from provinces in the Delta. I came to the conclusion that the nationality of at least 85 per cent of these soldiers could have been recognized at sight. The Egyptian is more sharply differentiated as a racial group than even the Arab, but that is because he is more distantly related to the Caucasian stock than the Arab. Less than 10 per cent of Egyptians could pass undetected were they to enroll in a regiment recruited from a South European (Mediterranean) population. In reality the Arab and the Egyptian are sharply contrasted physical types. We shall return to the nature of the differences when discussing head form and stature.

One soldier, No. 522, springs at once to the eye as a Negro. No. 699 is more than half Negro; although his hair is cropped its tufted arrangement is clearly discernible. No. 572 plainly has Negro blood in him also. The Arab cannot be confused with the pure Negro, and yet even in the most representative specimens there are certain Negroid features. Sometimes these are very evident, as in the soldiers numbered $581,584,595,599,602,670,694,715,719,722,729$. Among these Negroid features which may be counted normal in Arabs are the full, rather everted lips, shortness and width of nose, certain blanks in the bearded areas of the face between the lower lip and chin and on the cheeks; large, luscious, gazelle-like eyes, a dark brown complexion, and a tendency for the hair to grow in ringlets. Often the features of the more Negroid Arabs are derivatives of Dravidian India rather than inheritances of Hamitic Africa. Although the Arab of today is sharply differentiated from the Negro of Africa, yet there must have been a time when both were represented by a single ancestral stock; in no other way can the prevalence of certain Negroid features be accounted for in the natives of Arabia.

Since the Arabs occupy a geographical place which lies between the center of the evolution of the Negro type in Africa and the center of differentiation of the Mongol type in Asia, it might be expected that we should find among them Mongolian as well as Negroid traits. This is not the case. Among the 223 
soldiers in the camp at Hilla only three show traits which could confuse them with the least differentiated Mongolian races. These are Nos. 556, 654, 682. The observer in making his record mentions the Mongolian appearance of No. 682 . There is the same absence of Mongolian features in the Kish series of photographs, representing 320 individuals. There is in this series a man (No. 29) whom I should have unhesitatingly recognized as a Southern Chinese, and yet his history points to a pure Arab descent. No. 2 might pass as an American Indian, also No. 380. No. 469 has some Chinese features. No. 28 might come from the neighborhood of Lake Baikal. Thus the affinities of the Arabs lie in the direction of Africa rather than Mongolia.

Let me now proceed to discuss another set of features which I noted in my survey of the photographs. They are features which I think most anthropologists would name Armenoid. Among such features I include a peculiarly shaped head, one in which the occiput is flattened, rising steeply from the nape of the neck. The post-auricular length of the head is thus curtailed; the vault of the head appears lofty and pushed forward. Now this shape of head seems to have been evolved in southwest Asia. It is found in its purest form among peoples who occupy a mountainous tract stretching from the Pamir and the western flanks of the Himalayas to the mountains of Anatolia and along the western shores of the Adriatic. There is good reason for believing that the Armenoid shape of head passes from one generation to the next according to Mendel's law. The Arab belongs to a long-headed race; we may presume, when we find the Armenoid skull in an Arab community, that it represents an importation from the north.

Another feature which I have reckoned among Armenoid characters - perhaps with less reason - is convexity of nose. In the Armenoid nose this organ assumes great prominence and there is great development of the nasal bones. An Armenoid or Semitic characterization of the nose is a mark of a certain branch of the Caucasian stock; it is confined to the Armenoid and Semitic stocks. Like the Armenoid head the Armenoid nose was also evolved in southwestern Asia, but the characteristic nose and head do not often occur in the same individual of any race. I cannot draw any reliable distinction which will serve to discriminate the Armenoid nose from the pronounced Semitic nose. Now a pronounced development of nose is very common among Arabs. It is not an imported feature like the Armenoid head, but an original trait shared with many other racial strains of southwest Asia. There never was a race in which all its individuals had pronounced noses any more than there has been one in which all the heads were molded in the Armenoid manner. A race is characterized by the number of its individuals who exhibit such features. After this digression I propose to touch briefly on resemblances to Armenians detected among the individuals photographed by Mr. Field.

Among the 223 soldiers I noted sixteen with Armenoid traits; among the 320 Arabs of the Kish area, twenty-eight; about 8 per cent of Arabs show Armenoid traits. Soldier No. 603 reproduces the Armenoid head, but his features are frankly Arab, and with the Negroid strain more marked than usual. The 
Armenoid head form is also recognizable in Nos. 553, 555, 568, 575, 603, 626, 676 , 686. Convexity of nose and certain other Armenian traits may be seen in Nos. $510,529,540,555,568,607,612,620,653,692,698,704,715$.

Among the Arabs of Kish (320 in number) it would be possible to cite excellent examples of the Armenian type, such as Nos. 26, 134, 142, 144, 243, $245,273,393$. As to the prevalence of the Armenoid head among the Kish Arabs we shall have a better opportunity of discussion when we come to deal with actual measurements; in the meantime we are dealing only with estimates made from photographic appearances.

There is one point that was elicited in the comparison of the soldier series with the Kish Arab series of photographs, which may be most suitably mentioned here. Among the soldiers, 223 in number, I noted only twelve with pronounced nasal development-prominent, long, convex noses-a little over 5 per cent, whereas among the Kish Arabs (320) I noted sixty cases, i.e. more than 18 per cent. This difference is largely attributable to the fact that while the soldiers were less than thirty years of age, many of the Kish Arabs were more than fifty years of age. The nose grows in size relatively and absolutely after middle age. After middle age there are apt to be a loss of teeth and a reduction in the size of the jaws, the nose being thus rendered more prominent. But with the nose, and it is the same with the external ear, growth goes on after middle life. The nose alone is sufficient to give the Arab his place in the Caucasian stock in spite of a brown or dark brown skin. Between the Arab and the Jewish nose it is hard to discriminate in many cases. In the Arab, as in the Jew, the nose is not usually compressed from side to side in the middle of its length; in both there is usually a fullness at the upper part of the bony aperture.

Perhaps the most valuable of the photographic records are those taken of the $\mathrm{Ba}$ 'ij Beduins, thirty-eight in number. Nearly all are in their national costumes so that an unprejudiced recognition of race is not possible. We cannot help noting the frequency with which prominent, convex, elongated noses occur among them.

One other valuable service that is rendered by the Beduin series is to illustrate the changes which the typical Arab undergoes from youth to old age. No. 302, M'hssein ibn Hashash, is twenty years of age - an excellent type of the young Arab. His hair is frizzly and reddish brown in color; his eyes are brown. There is a thin mustache on his upper lip, and thin hairs peer out from the point of his chin. The mouth is wide, the lips are quite full and rather thick. The forehead is wide, the face long and oval, the nose long, aquiline, and thick from side to side in the middle of its length; the eyes are full. A still younger stage is seen in Nos. 309 and 323, both being youths eighteen years old. I regard them as typical Arab youths. Then for a later stage of the same representative type I would cite No. 306, a man aged thirty. Note the usual Arab distribution of beard-bare patches on the lower lip between mouth and chin and thin areas on the cheeks. The nose has become more prominent and longel. A later stage is seen in No. 316, an Arab with a full beard and straight nose, a man with Euro- 
pean-like features. Then next to him comes Luti ibn M'Sara, No. 317, reputed to be ninety years of age. One has difficulty in believing that a youth could be like No. 323 at eighteen, then at fifty resemble No. 324, and finally assume the features seen in No. 317. In estimating racial affinities we have to take these age changes into account. I am of the opinion that as the Arab ages he turns more Europeanoid in features. Indeed I find it much harder to discriminate Arab, Egyptian, Hindu, and South European from one another in childhood than in manhood.

In discussing age changes I have selected the long-faced, aquiline-nosed Arab as a type. There are thirty-one of the thirty-eight Beduins who may be assigned to this class, the aquiline-nosed, long-faced type. But there is another type in which the features are irregular; especially is the bridge of the nose lacking in high development, leaving a notch at the junction of nose and forehead. The forehead in this group is often expanded and high, the face short. Seven of the thirtyeight Beduins are of this short-faced type. Among the Kish Arabs and especially the Iraq soldiers the short-faced type becomes relatively much more numerous. Among the Ba'ij Beduins the short-faced type occurs to the longfaced in a ratio of $26: 100$; among the Kish Arabs the ratio is $60: 100$; among the soldiers the ratio of short face to long face is $280: 100$. The aristocratic visage predominates among the Beduins; it is still common in the Kish Arab group but is overwhelmed by a more plebeian visage among the Iraq soldiers, unless the effects of shaving, barbering, and military dress have somehow deceived my eye.

Let me cite among the Kish population and Iraq soldiers examples of the two types just mentioned. Among the Beduins I have already cited, while discussing age changes, examples of the long-faced, aquiline-nosed type. I may now mention examples of the contrasted type among the Beduins-Nos. 307, 310, 315, 319, 322, 327. Among the Kish Arabs, as illustrative of the long face I would cite as examples Nos. $3,11,12,13,18,25,27,31,38,39,48,50,62,70,72,74,92,94$, 101, 103, and others. As examples of the short-faced, irregular-featured type, Nos. $1,4,9,23,28,44,45,46,69,87,90,93,95,96,102,104,168$. Among the Iraq soldiers I would cite as Type 1 Nos. 501, 503, 505, 506, 508, 510, 513, 515, 516, $517,522,523,527,528,529,536,553,565$, and others; as Type 2 Nos. 502, 507, $509,514,520,526,530,535,539,543,556,558,563,578,584,590,596,603,663$, 670,718 . It is among the short-faced, irregular-featured Arabs that one meets with examples reminiscent of the Dravidian of India on the one hand and of the Hamitic types of Africa on the other. Presently I shall have to discuss the origin of such types. It is possible that they represent survivals from an ancient or modern Negroid population of Arabia.

In the foregoing pages the photographic records have been submitted to an empirical method of anthropological analysis. I have been seeking to ascertain to what degree the modern people of Iraq have become differentiated from all surrounding peoples, and also with what peoples we are most likely to confuse them. The result of this analysis has been to prove that the races with 
which the Arab is most likely to be confused are those of the Caucasian stock. We must regard the Arab as a member of this stock. Although distinguishable by external appearances from Hamitic and other African races, the Arab is marked by numerous minor Negroid traits. While I have cited many examples of both the long-faced and the short-faced type of Arab, I have not as yet cited examples of the individuals who if regimented with other nationalities, such as Iranian, Greek, or South Italian, might pass as true members of these groups. Let me cite, first, a few examples of Europeanoids from the Beduin series. There is, first, the handsome Arab No. 320; if in European dress his color and the distribution of beard might reveal his race to the expert eye, but I doubt it. Nos. $306,307,310,312,316$, and several others, could, if put into uniform, pass as Europeans. Among the Iraq soldiers I should cite Nos. 706, 708, 709, 710, 711, 712, $713,714,716$ as types which might pass as Europeans. I diagnosed my racial types without referring to birthplace. On reading Mr. Field's notes I found that this string of European-like types came from Baghdad and still farther to the north-Mosul, Kirkuk, and Sulaimaniya. A further analysis revealed the fact that the men in whom I recognized European-like features came predominantly from the northeastern side of the plain-from the north bank of the Tigris and beyond. Fol example, Amara, Ba'quba, and Basra provided a higher proportion of European-like individuals than those coming from the towns on the Arabian side of the plain, the Euphrates side. Altogether I counted that 54 soldiers, out of a total of 223 (more than 24 per cent), might pass as South Europeans. Examples of these types are Nos. 500, 504, 515, 533, 537, 538, $615,623,628,648,661,667,684$.

As said before, the experienced traveler takes unconscious notice of a multitude of points in coming to a conclusion as to the race and place of any stranger he may chance to meet. There are two characters which weigh with him as of especial importance: the degree to which skin, hair, and eyes are pigmented; also the hair texture. Many Arabs, whose features might be those of a European, cannot pass as Europeans because of their degree of pigmentation. Now Mr. Field has recorded the color of hair and eyes of the various groups of Arabs measured by him. Unfortunately no standard set of colors was used to measure the degrees of pigmentation. There are certainly many Arabs who are not more deeply pigmented than many South Italians. The hair was dark brown or black in 86 per cent of the Beduins of the Kish area and in 98.5 per cent of the Iraq soldiers. There were four of thirty-two Beduins whose hair was light brown or reddish; among the Kish Arabs this group reached 3 per cent, but it formed one and a half per cent of the soldiers. Thus, as a whole, the Arabs are more deeply pigmented than any group of South Europeans. In this feature (pigmentation) they are nearer to the Dravidians of India and the Hamites of Africa than to any European group.

As regards eye color there is a considerable difference among the three groups of Arabs included in the anthropological census made by $\mathrm{Mr}$. Field. Those in which the eye (iris) seemed black or dark brown made up 23.7 per cent of the 
Beduins observed, 77.2 per cent of the Kish Arabs, and 86.6 per cent of the Iraq soldiers. Here again the soldiers appear as the most pigmented of the Arabs examined. In eyes reckoned as greenish brown the Beduins had 18.4 per cent, the Kish Arabs 12 per cent, and the soldiel's 7.3 per cent. The Beduins with blue brown eyes numbered 58 per cent, the Kish Arabs 8.3 per cent, the soldiers 5.4 per cent. As regards gray brown eyes, none was observed among Beduins, 2.5 per cent among the Kish Arabs and 0.7 per cent among the soldiers. Thus as regards coloring the Beduins in their prevalence of black hair (38 per cent) combined with eyes which were of a blue brown shade (58 per cent) differ from all the other Arabs observed. Blackness of hair and blueness of eyes are characteristics met in the darker (Celtic) peoples of western Europe.

Whether the Beduin type has been evolved in Arabia by isolation and selective breeding or whether it represents a type evolved to the north of Arabia that has mingled with native blood since its arrival, are matters open to debate. My opinion is that the Arab is a type which has been evolved from the general population of Arabia by selective breeding continued over many generations. But as will be mentioned below, there probably was, before this process of evolution set in, a Caucasian influx from the north.

Before proceeding to discuss the anthropological data collected by Mr. Field it is important that we should note the position occupied by Iraq, where the data were obtained, in an ethnological map of the Old World such as that charted by Ratzel. According to this author, Iraq lies on or near an ethnological frontier. To the north, in a mountainous region, lies the home of the Iranians and kindred peoples. In the Iranian group we may include the peoples of Asia Minor (Ottomans, as Ratzel has named them). The whole of Arabia, from the Red Sea to the Persian Gulf and from the Levant to the Indian Ocean, is indicated by Ratzel as the home of the Semites. The Semites are represented as spreading across northern Africa, occupying the southern shores of the Mediterranean. Adjoining Arabia on the west and south, but separated by the Red Sea, is the home of the Hamitic peoples. They occupy a tract of Africa, east of the Nile, which extends from Cairo in the north almost to Zanzibar in the south. Still farther away in the east is India, occupied by a dark-skinned people-Dravidians in the south, Aryo-Dravidians in the north. Now, our object is to discover the relationship of the Arab to these other peoples-to the Hamites, the Dravidians, the Aryo-Dravidians, the Iranians, other Semites, and South Europeans - in short, to all peoples whose skins are brown or even black in color and whose faces are Caucasian in type. Whatever theory we may advance to account for the origin of the Arab it must be one which will explain at the same time the distribution and the types of people found between India in the east and Egypt in the west.

We come now to the examination of the physical measurements made by Mr. Field of the Arabs living in the neighborhood of Kish. Our purpose is to ascertain what light these measurements throw on the physical constitution of the Arabian people and on the relationship of the Arab to races both past and 
present. I propose to begin with a consideration of the two measurements made of the head, its length and breadth. We shall consider the three groups taken in this order-Ba'ij Beduins (38), Kish Arabs (398), and Iraq soldiers (231).

The Beduins form a very small but a very valuable group, as they are representative of a separate class - the tribal Arab-in contradistinction to the town Arab. The average head length of this group of men of various ages is $191.37 \mathrm{~mm}$, which if we allow $8 \mathrm{~mm}$ for flesh would give a skull length of approximately $183 \mathrm{~mm}$ - a short skull. The coefficients of variation for each measurement are given in the table (p. 455). The average head width of the Beduin group is $140.24 \mathrm{~mm}$, which with flesh removed and skull dried would give a cranial width of about $130 \mathrm{~mm}$. Thus we must note that the head dimensions are small. The relationship of width to length (cephalic index) of the living head is 73 per cent. If we allow for flesh the index on the skull would be about two points less; namely, 71. Thus the Beduin is both small- and long-headed (dolichocephalic).

I am in full agreement with accepted anthropological practice-that averages of measurements with coefficients of variations must be obtained and recorded for the complete group of individuals examined, and if the groups are large enough, as in the case of the Kish Arabs (398) and Iraq soldiers (231), curves must be drawn to represent the distribution of each item of measurement. In averages and in distributional curves we lose sight of the individuals; very different kinds of men have their individuality obscured when included as items in the statements of averages and as units in distributional curves. Hence I have been in the habit, these twenty years past, of using an old-fashioned form of chart for the finer analysis of small racial groups. This form of chart is illustrated here (Chart I). The vertical lines of this chart represent the head length, having a range from 160 to $220 \mathrm{~mm}$, one of $60 \mathrm{~mm}$. The horizontal lines represent head width, beginning at $120 \mathrm{~mm}$ and ending at $170 \mathrm{~mm}$, a range of $50 \mathrm{~mm}$. Each individual is entered on the chart at the point where his measurement of head width crosses that of his head length. The " $O$ 's" on the chart represent the distribution of the thirty-eight Beduins. Four diagonal lines cross the chart from top left corner to bottom right; they are marked 70,75, 80, 85. A head falling on the 70-diagonal is one whose width is 70 per cent of its length, and so on with the other lines. The heads which fall between the 75 and 80 lines I name mesocephalic. In most human races this becomes the most crowded compartment of the chart. Heads falling on the 75-line and below the 70-line I name dolichocephalic; if on the 70-line or above it, they form an ultra-dolichocephalic group. Heads which fall on the 80 -line and above the 85 -line I name brachycephalic, and if on the 85-line or below it I name ultra-brachycephalic. Now, when we look at the cephalic chart of the Beduins, we see that eight of them are ultra-dolichocephalic (with a cephalic index of 70 or less), twenty-one of them are in the dolichocephalic group (70-75), while only seven lie in the mesocephalic group. Two fall in the brachycephalic group (80-85), but none are ultra-brachycephalic. The mass of the tribesmen fall between the 70 and 75 lines, but the individuals 
who stray away from the main mass are, for students of race, as important as the main mass itself.

A long experience in dealing with racial characters has convinced me that the actual dimensions of length and width are more important for the determination of the status of a race than a statement of the cephalic index - the relation of width dimension to length dimension. I have sought to bring out the value of actual dimensions by drawing diagonally in Chart I two lines at right angles

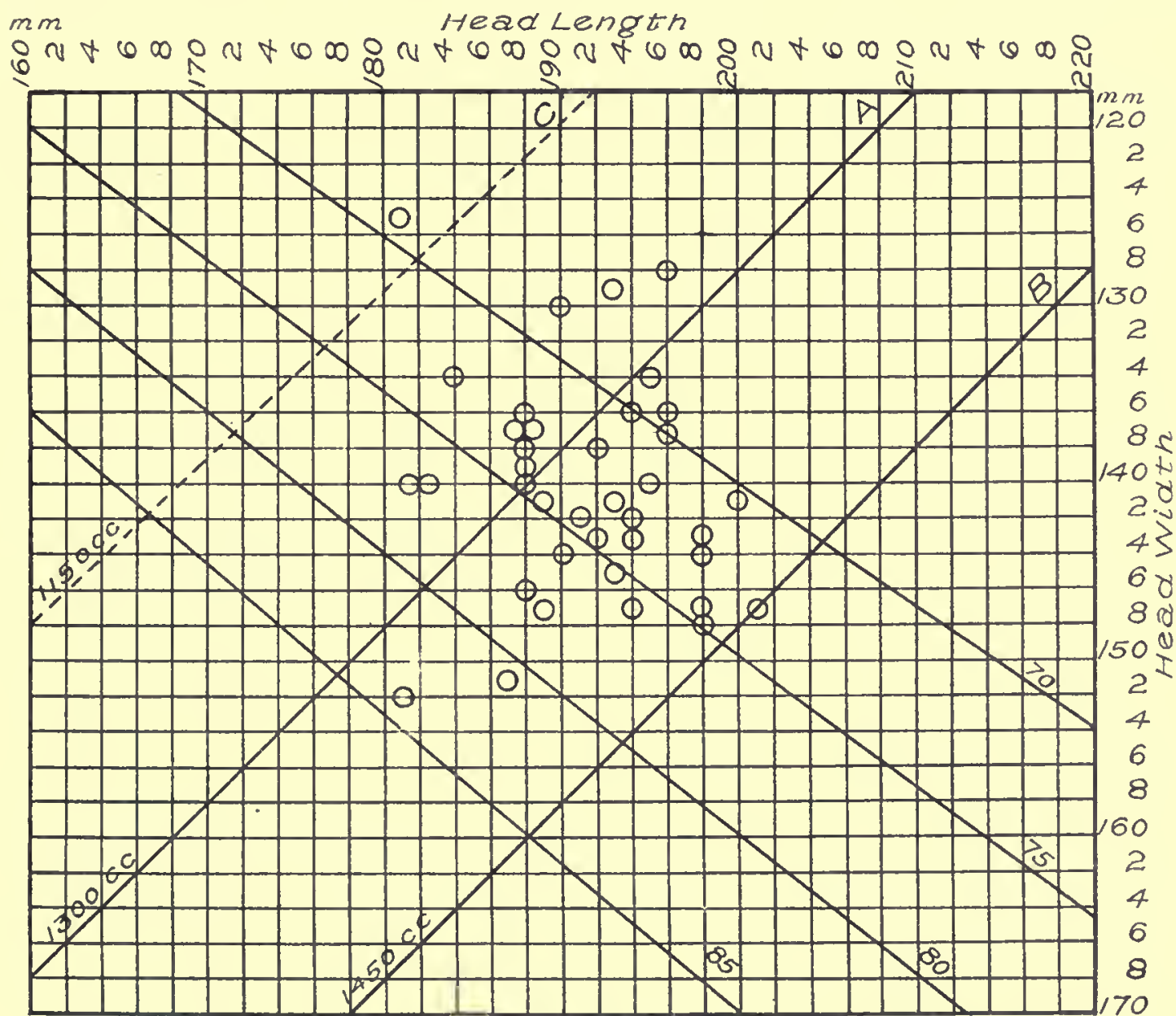

FIG. 1. Chart I. Head length, breadth, and capacity of thirty-eight Ba'ij Beduins. Each circle indicates an individual. Vertical lines indicate length of head; horizontal ones, width of head. Each individual is entered on chart at point where his length and breadth measurements meet.

to the cephalic index lines. One of these (line A) is drawn through the point where the vertical line 188 is crossed by the 140 horizontal. A head falling on this point (188 x 140) would have a skull length of $180 \mathrm{~mm}$ and a width of 130 mm - a small head with a capacity of about 1300 cc. All heads falling on this line or above and to the left of it, I count small heads. A curved line, with convexity downward and bending to the spectator's left, would give a more truthful result, but such a conventional line as $\mathrm{A}$ will serve our present purpose. The second diagonal (B) is drawn through the point which represents a head 198 $\mathrm{mm}$ long and $150 \mathrm{~mm}$ wide - one which gives a skull 190 by $140 \mathrm{~mm}$-with a 
brain capacity of about $1450 \mathrm{cc}$. This is not really a large head; the cranial capacity of the average Englishman is 1480 cc. All heads which fall on line $\mathrm{B}$ or below it (i.e. to the right of it as seen by the spectator) I count large heads; those which fall between the lines A and B I count heads of intermediate size. When we look at Chart I we see that the Beduins are distributed as follows: large head one; medium, twenty-four; small, thirteen. The Beduins are, compared to Europeans, small-headed and small-brained. We shall see presently how they compare with other groups of Arabs and other races of mankind.

If we pursue our analysis of the Beduins according to size and shape of head, they fall into the following groups:

\begin{tabular}{|c|c|c|c|c|c|c|}
\hline \multirow{2}{*}{ Heads } & \multicolumn{6}{|c|}{ Groups According to Cephalic IndeX } \\
\hline & $-70 /$ & $-75 /$ & - & $/ 80+$ & $185+$ & Tot: \\
\hline Small $(1300$ ce or less)... & 4 & 7 & 2 & 0 & 0 & 13 \\
\hline Medium . & 4 & 13 & 5 & 2 & 0 & 24 \\
\hline Large (1450 ce or more) & 0 & 1 & 0 & 0 & 0 & 1 \\
\hline Totals & 8 & 21 & 7 & 2 & 0 & 38 \\
\hline
\end{tabular}

Now the eccentric individuals revealed in Chart I are of as much interest as the average Beduin. We must keep an eye open for exceptionally smallheaded individuals. In Chart I a single very narrow-headed individual falls below a third line I have introduced-one which marks heads about $1150 \mathrm{cc}$ in size. In such a group of men it is usual to find two or three rising into the bigheaded group; here there is only one, and he reaches only the boundary line. The two round-headed individuals falling between the 80 and 85 lines in the medium-headed group are also of interest. There is in the Beduin only a slight tendency to produce individuals with round heads.

About the time Mr. Field was measuring the Arabs of central Iraq, Captain Bertram Thomas spent some months among the Arabs of the south coast, preparatory to his daring journey across the southern desert. In Chart II I have plotted observations made by Captain Thomas on thirty-five South Arabs, so as to make clear how markedly the South Arab differs from the North Arab in shape of head. The South Arab is intensely round-headed, while the North Arab is just as intensely long-headed. The facial type, so common among the Beduins of the north, does not occur in the south. But the North and South Arabs are alike in this: both are small-headed, as the following table shows.

\begin{tabular}{|c|c|c|c|c|}
\hline & $\begin{array}{l}\text { Small } \\
\text { heads }\end{array}$ & $\begin{array}{c}\text { Medium } \\
\text { heads }\end{array}$ & $\begin{array}{l}\text { Large } \\
\text { heads }\end{array}$ & Totals \\
\hline & 33 & 2 & 0 & 35 \\
\hline Arabs (38). & 13 & 24 & 1 & 38 \\
\hline
\end{tabular}

The South Arabs are smaller-headed than those of the north. Even allowing for the circumstance that the straight line A gives an unfair index of size of brachycephalic heads, the fact still remains that the South Arab is characterized not only by roundness of head, but also by smallness of head. A glance 
at Chart II will reveal the fact that, as Dr. Krogman was the first to observe, it is in length not in width of head that the North Arab exceeds his cousin of the south. One suspects that the South Arab has come by his roundness of head not by a swelling in its width, but by a contraction in its length.

Chart II brings out very well the extent to which the South Arab, as regards head form, differs from the Arab of the north. Only two of the Beduins cross the 80-line to fall in the brachycephalic group; only four of the South Arabs

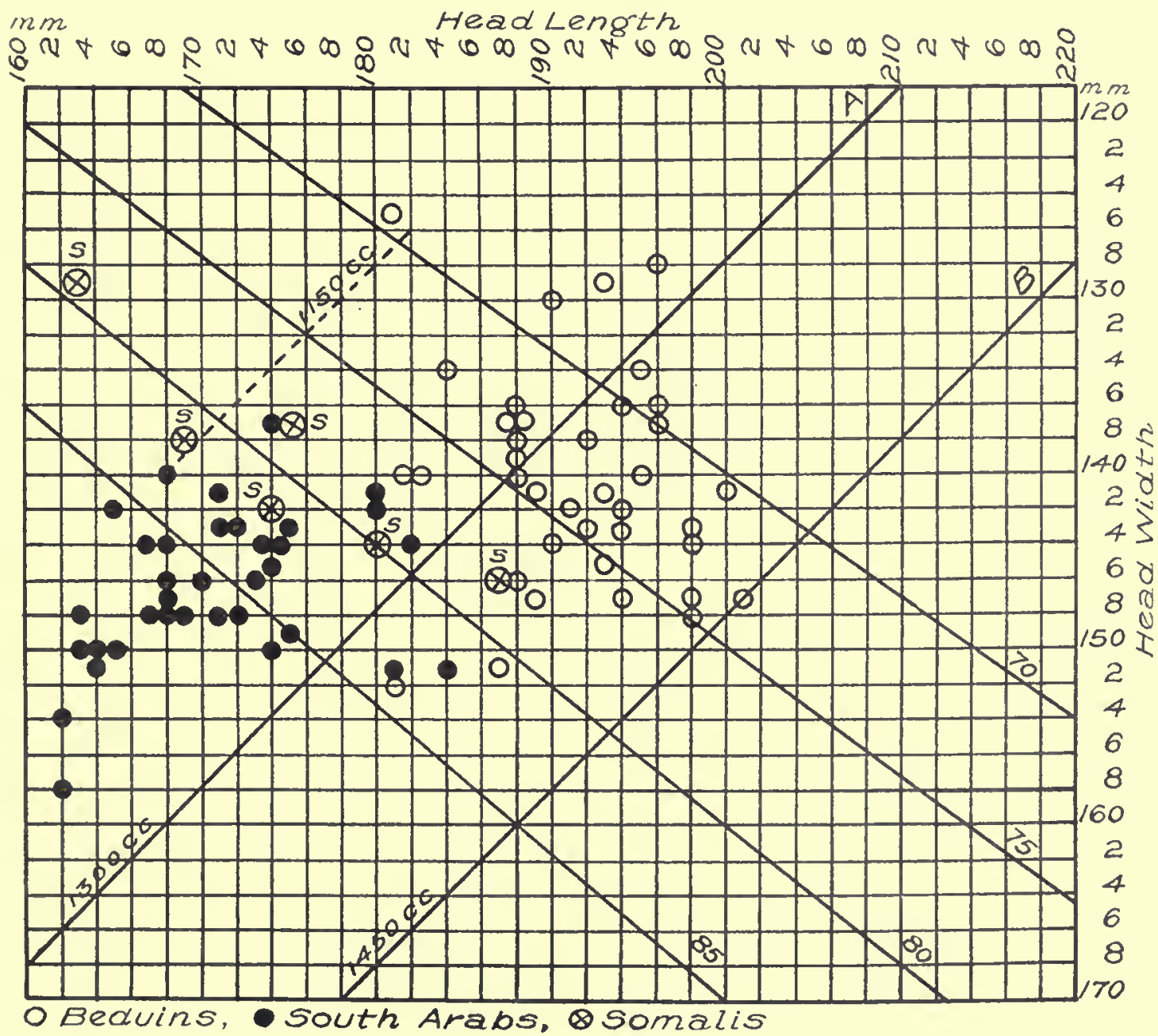

FIG. 2. Chart II. Head length, breadth, and capacity of thirty-eight Ba'ij Beduins, thirty-five South Arabs, and six Somalis.

cross the same line to enter the mesocephalic group. Captain Thomas included in his list six Somalis whom he found living in southern Arabia, and I introduce these representatives of a Hamitic people; they fall on either side of the 80-line, and five of them lie in the small-headed group. When tabulated, the difference in head form between the South Arab and the Beduin of the north is as follows:

\begin{tabular}{|c|c|c|c|c|c|c|}
\hline & \multicolumn{6}{|c|}{ Groups ACCording to Cephalic IndeX } \\
\hline & $-70 /$ & $-75 /$ & - & $/ 80+$ & $/ 85+$ & Totals \\
\hline & 8 & 22 & 6 & 2 & 0 & 38 \\
\hline South Arabs (35) & 0 & 0 & 4 & 11 & 20 & 35 \\
\hline
\end{tabular}


When we come to discuss the origin and affinities of the people of Arabia, we shall have to keep in mind the unexpected degree of brachycephaly which prevails in southern Arabia.

$m+n$

Heaolenoth

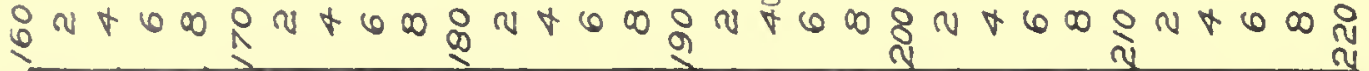

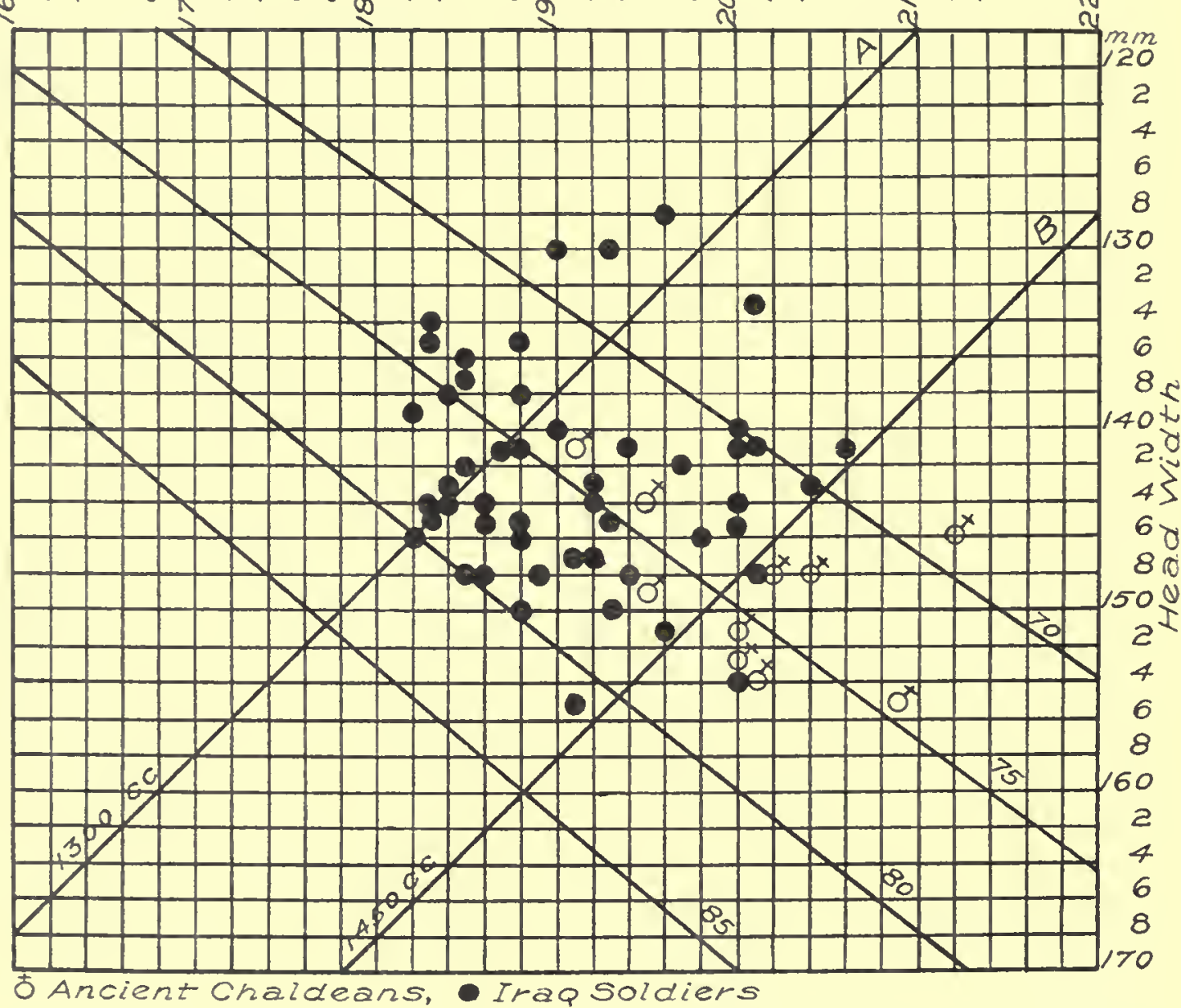

FIG. 3. Chart III. Head length, breadth, and capacity of fifty Iraq soldiers (Nos. 500-550) born near Kish, at Hilla, Nasiriya, and other towns on Euphrates side of plain, compared with ten ancient Chaldeans (males) whose skulls were found by Leonard Woolley near Ur in graves of fourth millennium B.C. The ancient skulls have had $8 \mathrm{~mm}$ added to their length and $10 \mathrm{~mm}$ to their width, to represent their original fleshy coverings and to make them comparable with heads of modern natives. The ancient skulls were described by Sir Arthur Keith (Excavations at Al 'Ubaid by Hall and Woolley, 1926). In some series of numbers, such as the present-Nos. 500-550, where 51 men are represented - one or more men of ten had to be omitted from the series because of lack of one measurement. Apparent discrepancies are due to such omissions. In all cases the group diagrammed contains fifty individuals.

In Chart III are plotted the head dimensions of fifty Iraq soldiers (I chose the opening series), Nos. 500-550. Most of the men were natives of villages in the southern part of the plain along the south bank of the Euphrates. I chose men from this district for three reasons: I wanted to compare them (1) with the Beduin group; (2) with a group chosen from the northern side of the plain; (3) with the men who lived in almost the same locality in the fourth millennium B.C. Let me first classify this group of Arab soldiers according to form and size of head: 
IRAQ SOLDIERS (NOS. 500-550)

\begin{tabular}{|c|c|c|c|c|c|c|}
\hline \multirow[b]{2}{*}{ Heads } & \multicolumn{6}{|c|}{ Groups According to CEPHALIC INDEX } \\
\hline & $-70 /$ & $-75 /$ & - & $/ 80+$ & $/ 85+$ & Totals \\
\hline Small. & 3 & 7 & 8 & 0 & 0 & 18 \\
\hline Medium... & 5 & 11 & 12 & 2 & 0 & 30 \\
\hline Large. ... . & 0 & 1 & 1 & 0 & 0 & 2 \\
\hline Totals........ & 8 & 19 & 21 & 2 & 0 & 50 \\
\hline
\end{tabular}

As regards head size, this group of soldiers is in close agreement with the Beduins; rather a higher proportion of soldiers falls in the small-headed group. Nor is there any marked difference in head form; there is a movement of the soldiers away from the dolichocephalic toward the mesocephalic group; there is a greater tendency toward the brachycephalic line among the soldiers than among the Beduins. In both groups there is the same representation of small, narrow-headed individuals; there is, too, as in the Beduins, a representation of brachycephaly; there are two individuals who fall into the round-headed group $(/ 80+)$.

To save description it is convenient to adopt a method to indicate classes grouped according to measurement; /80-/85 indicates individuals with a cephalic index of 80 or more, but under $85 ; / 180 \mathrm{~mm}-/ 185 \mathrm{~mm}$ indicates a group of people with head length of 180 or more, but under $185 \mathrm{~mm}$; and so on.

The position taken by the ten ancient inhabitants of Ur is very striking. Their skulls have been clothed in flesh to make them comparable with modern natives of the plain, $8 \mathrm{~mm}$ being added to length and 10 to width. Now as regards proportion of breadth of head to length of head, ancient and modern heads agree; they fall very evenly on each side of the 75-diagonal. But as regards size, seven of the ten ancients rise into the large-headed class, while only two of the series of fifty modern natives have equal preeminence. Three of the ancient heads fall in the medium-headed group, which contains thirty of the fifty soldiers. The modern Arab has the same form of head as the ancient inhabitants of Ur, but his head is less by at least $100 \mathrm{cc}$ of brain capacity. I am proceeding on the assumption that the modern Arab represents the same race as lived in the cities of the lower plain but that decay of the old civilization has resulted in the survival of a smaller-brained type. Or was it the big-headed people who died out and thus civilization decayed?

Before proceeding to examine the head form of various other groups of Arabs measured by Mr. Field, it may be well at this point to ascertain how the Arab compares, as regards size and shape of head, with other neighboring races. And here a strange fact emerges: while I see in so many external points resemblances of the Arab to the South European, it is not with Europeans but with nonEuropean races that confusion is likely to arise in the comparison of dimensions and shape of head. The Arab lies in the great brown belt which extends from Egypt to the farther shores of India. Let us see, first, how the Arab compares with the Egyptian in the points now under consideration. 
If the Egyptian chart (IV) is compared with that of the Beduins (I) or with that of the Iraq soldiers (III), a very considerable degree of resemblance is observed. The Egyptians fall into the following groups:

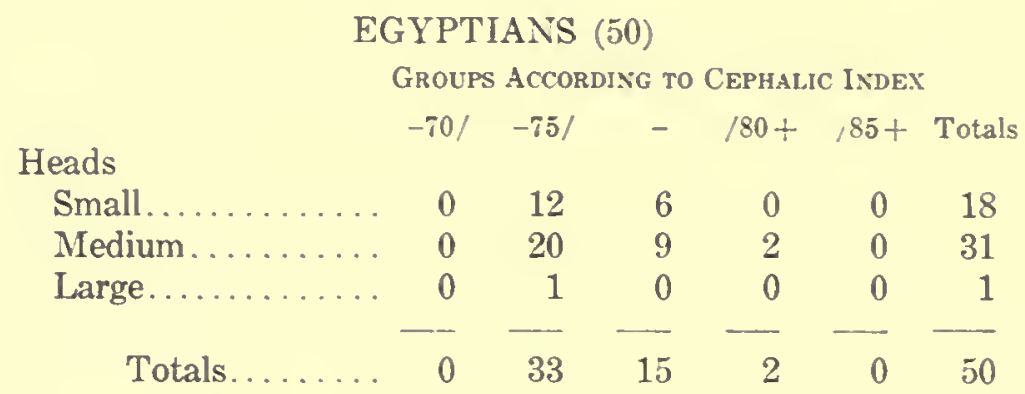

mm Head Length

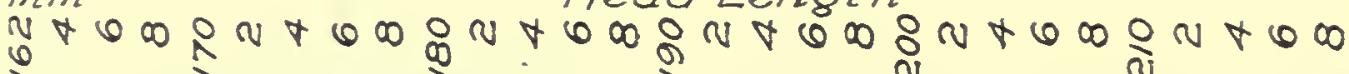

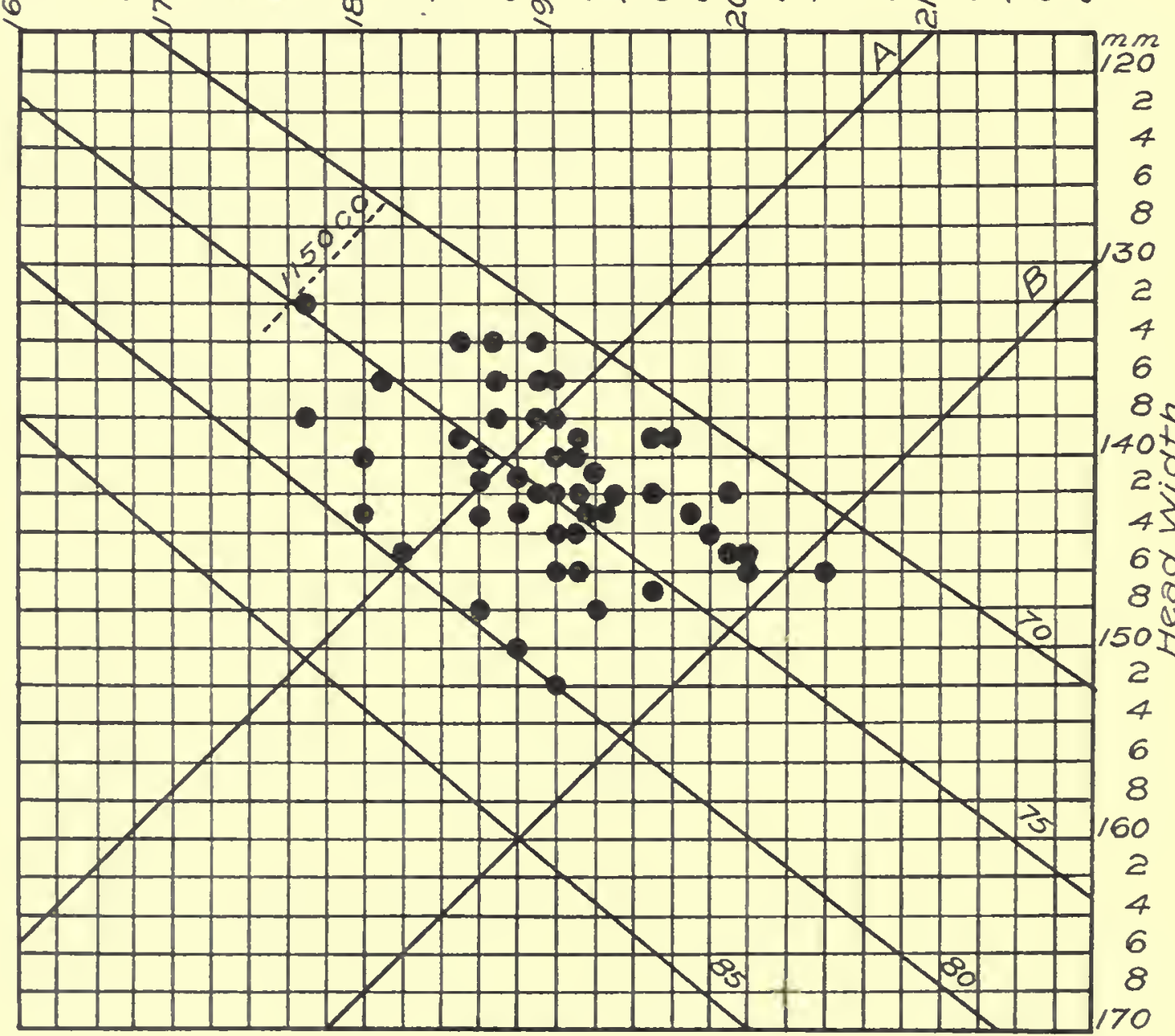

FIG. 4. Chart IV. Head length, breadth, and capacity of fifty Egyptians. Measurements taken and recorded by A. Hrdilicka (Natives of the Khargha Oasis, 1912). For this purpose I used the first fifty men of his series.

Now as regards distribution of head size, the Egyptians and the Iraq soldiers are almost identical: 


\begin{tabular}{|c|c|c|c|c|}
\hline & $\begin{array}{l}\text { Small } \\
\text { heads }\end{array}$ & $\begin{array}{l}\text { Medium } \\
\text { heads }\end{array}$ & $\begin{array}{l}\text { Large } \\
\text { heads }\end{array}$ & Totals \\
\hline Egyptians....... & 18 & 31 & 1 & 50 \\
\hline Iraq soldiers...... & 18 & 30 & 2 & 50 \\
\hline Beduins......... & 13 & 24 & 1 & 38 \\
\hline
\end{tabular}

The prominent feature of the Egyptian array of heads (Chart IV) is its compactness. Egyptian heads are grouped along each side of the 75-diagonal, none passing beyond the 70-line on the one side or the 80-line on the other. There is none of the Arab small-headed ultra-narrow-headed individuals; the Arab brachycephalic sports are also missing. There are, in the Egyptian series, one individual who almost sinks into the ultra-small-headed group and one who rises into the large-headed. It may be held that the uniformity of the Khargha Egyptian group is due to inbreeding and that the "outliers" of the Arab group are a result of outbreeding. But it is quite as probable that we have here an exhibition of real inherent traits of both peoples, the Arab having a tendency to vary, to produce sports, the Egyptian to breed true to type. In combining the Beduin and Iraq soldier charts we obtain a distribution of Arab head form and size which is very similar to that shown in the Egyptian chart. The real difference lies in the Arab tendency to produce eccentric forms.

Let us now turn to India and compare the head form and size of two peoples of that country with Mr. Field's Arabs. I shall take fifty men of the Chatri caste of the United Provinces to represent the Dravidian element. The fifty examples chosen are from tables in "The Tribes and Castes of Bengal" (II, 1891) by $\mathrm{H}$. H. Risley. In the following table the distribution is shown according to size and shape:

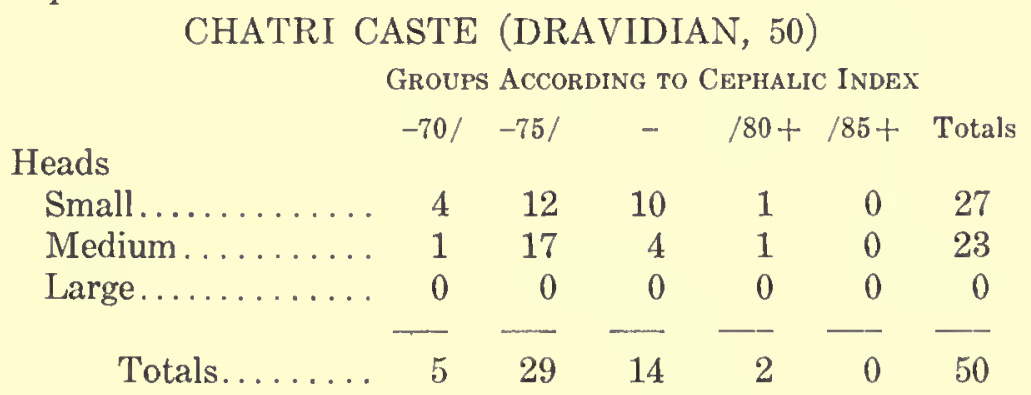

Now, the outstanding feature of the Dravidian head is its smallness; in this group of fifty Chatri twenty-seven fall among the small heads; only twenty-three rise into the medium group; not one reaches the upper limit, while three individuals fall on or below the 1150 cc-line. Yet, if we except the degree of smallness, the distribution of head form among the Chatri is not unlike that among the Beduins (Chart I). There are five ultra-dolichocephalic Chatri and two brachycephalic ones. There are reasons, besides the resemblances in head form, which induce us to suppose that Dravidian and Arab have in them an inheritance from a common stock - an inheritance which has been retained more completely by the natives of India than by the people of Arabia.

Turning to a group of Aryo-Dravidians, such as is represented in Chart VI (taken from Risley's measurements), we see that the Pathan distribution 
departs from that shown in Chart $\mathrm{V}$ of Dravidians and approaches that shown in Chart III of the Iraq soldiers. The fifty men included in Chart VI were members of the Pathan caste, Punjab. Their average stature is $1687 \mathrm{~mm}$ (5 feet 6.4 inches)-rather taller than the Beduins ( $1683 \mathrm{~mm}$ or 5 feet 6.3 inches), the Kish Arabs (1677 $\mathrm{mm}$ or 5 feet 6 inches), and the Egyptians of the Khargha Oasis ( 5 feet 5.5 inches), but shorter than the average of the soldiers of the Iraq army measured by Mr. Field. The average stature of the soldiers is $\mathbf{1 7 2 5 . 6}$

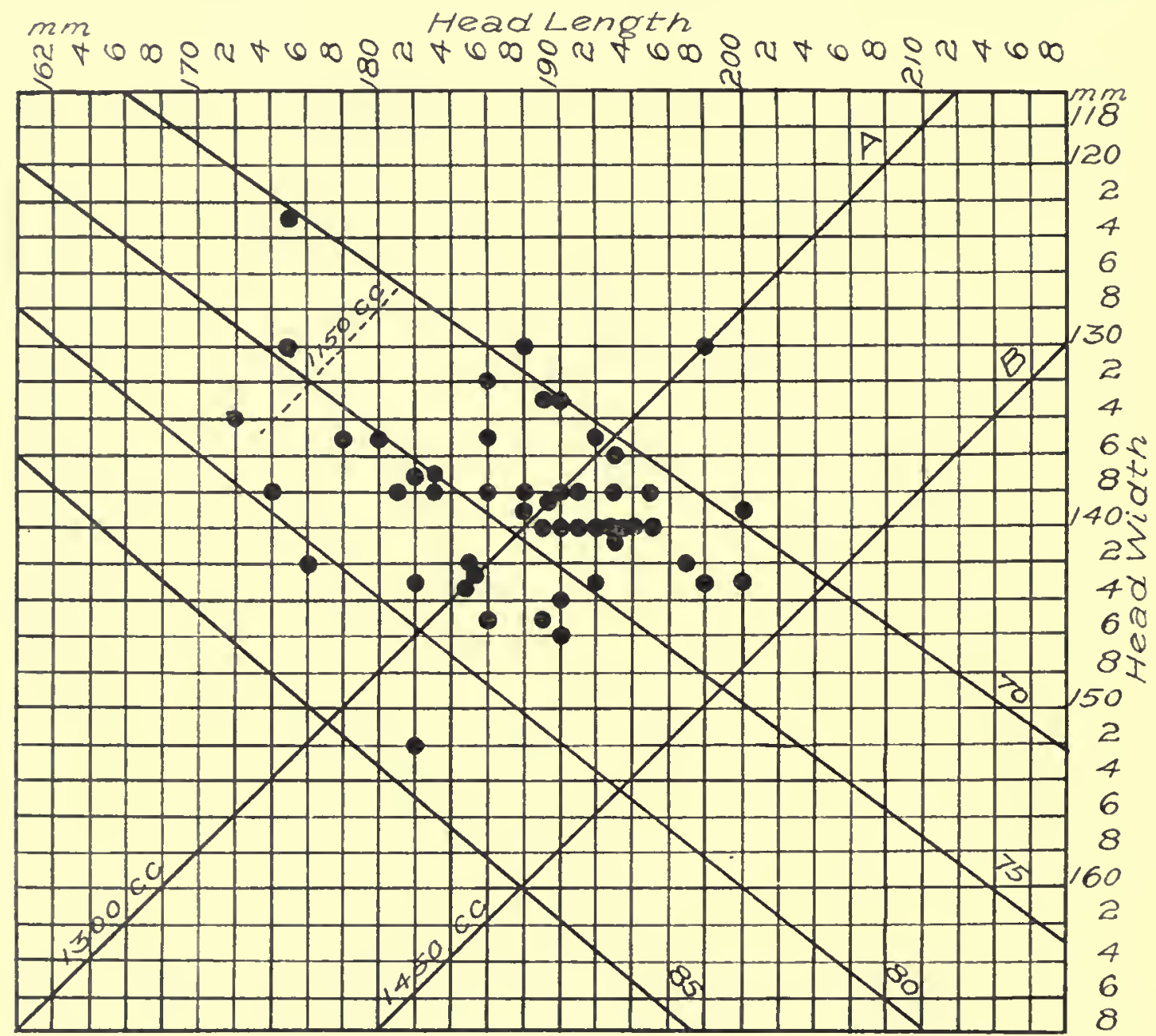

FIG. 5. Chart V. Head length, breadth, and capacity of fifty men of Chatri caste, United Provinces (after Risley).

$\mathrm{mm}$ ( 5 feet 8 inches). The Pathan is taller than the Chatri (Dravidian) by one inch (26 mm, to be exact), and it might be thought that the increase of head dimensions was directly correlated with increase of stature. When, however, we take into consideration the fact that the soldier Arab is two inches taller than either the Beduin or the Kish Arab and that the heads of these two latter groups are larger than those of the taller soldiers, we must hesitate in ascribing any very close correlation between stature and size of head. Mr. Field regards the greater height of the Arab soldier as being due to physical drill, but probably there has also been a selection of recruits. In proceeding we shall find that the soldier Arab 
departs in many respects from the Kish Arab. Whatever be the right explanation, the fact remains that a native people of the Punjab approximates in size and shape of head the Arab population of Iraq, and this must be given due weight when we come to work out the affinities of the Arab people.

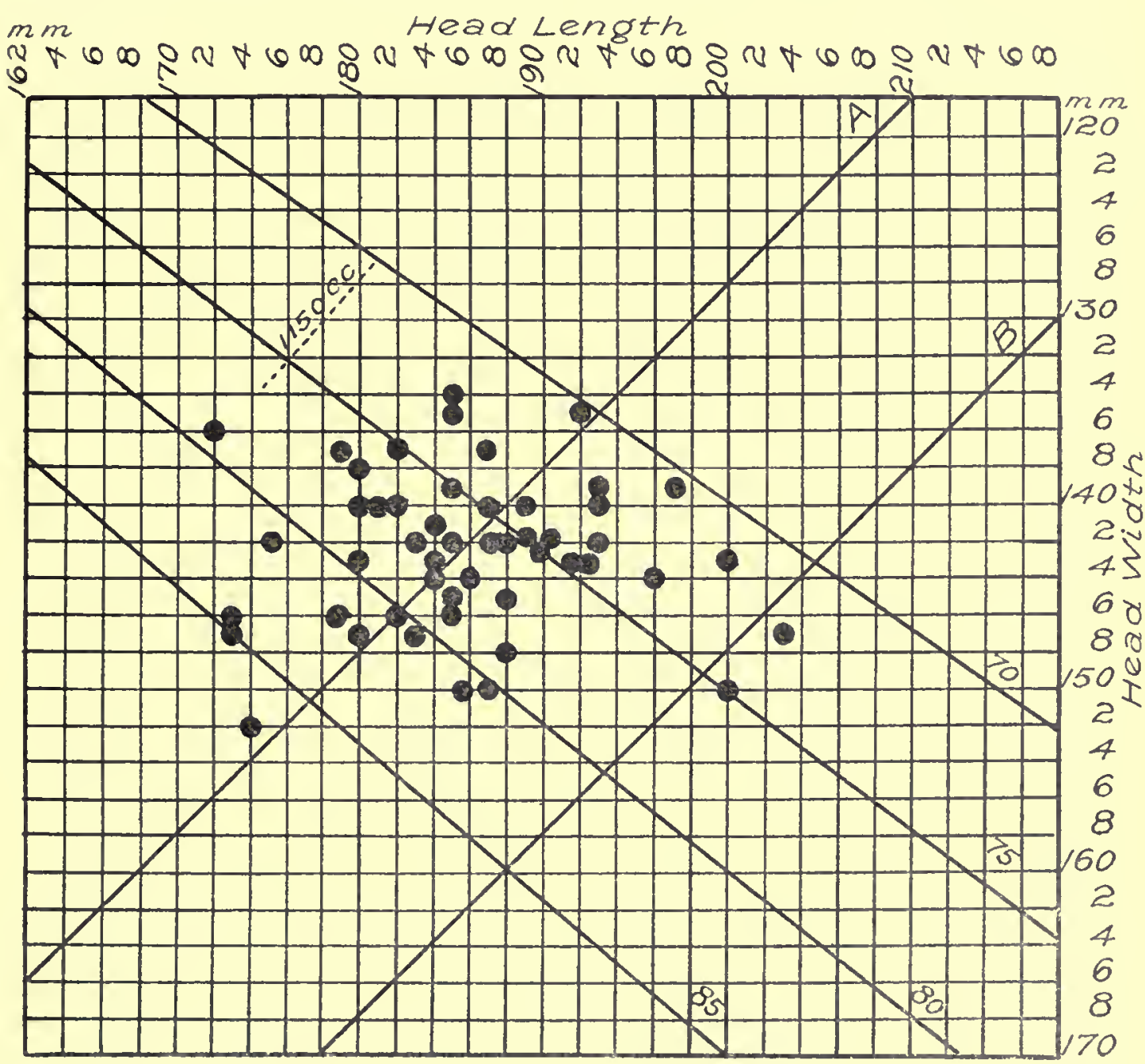

FIG. 6. Chart VI. Head length, breadth, and capacity of fifty men of Pathan caste, Punjab (after Risley).

In the following table the fifty Pathans, represented in Chart VI, are placed in groups according to size and shape of head:

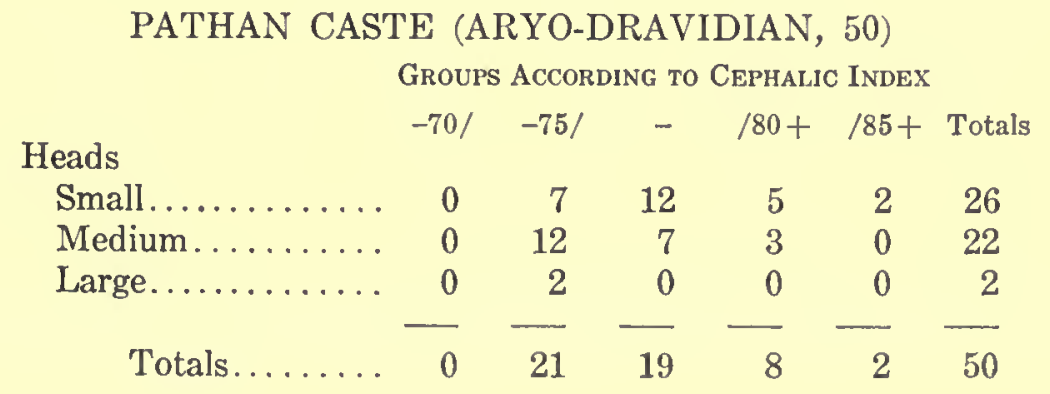


Now, among the Pathans as among the Dravidians there is a large representation of small heads; twenty-six of the fifty Pathans fall into the small-headed group, whereas among Arabs we expect only about one-third of that number to fall into the lower group. Two Pathans rise into the highest group, and one man falls almost on the 1150 cc-line. Most of the heads fall along either side of the 75-diagonal; there are no ultra-dolichocephals as among the Arab groups. On the other hand, there is a much stronger tendency among the Pathans than

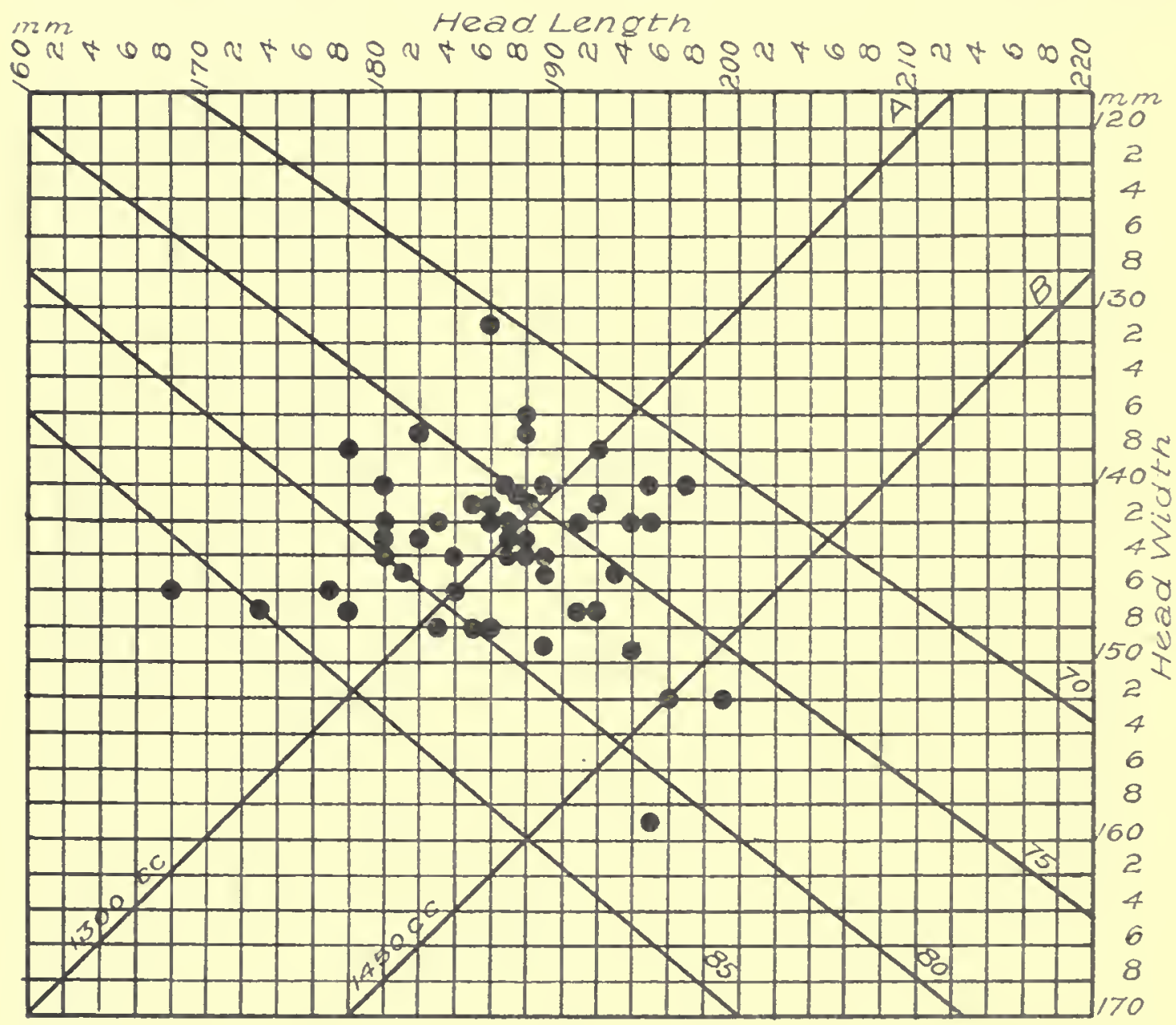

FIG. 7. Chart VII. Head length, breadth, and capacity of fifty Iraq soldiers (between Nos. 553-671), born in Nasiriya or Hilla. For comparison with Chart III.

among the Kish Arabs to brachycephaly. Indeed we see in Chart VI a group of short, round heads (seven in number) which fall into the compartments occupied by the South Arabs in Chart II. Thus, among the Pathans there is a greater tendency toward brachycephaly than in the North Arabs, but much less than among the South Arabs. Further, like the South Arabs, the Pathans and Dravidians are preponderatingly small-headed. The North Arab has more than a half ( 50 per cent) representation in the group of medium-sized heads.

Having made a cursory comparison of the Arab of central Iraq with the Arab of the south, the Somali, the Egyptian, the Dravidian, and the Aryo-Dravidian, 
I now return to the examination of the men measured by $\mathrm{Mr}$. Field in the neighborhood of Kish. The fifty soldiers whose head dimensions are reproduced in Chart III came from the south bank of the Euphrates, from Hilla, Nasiriya, and other places, but in this group (Nos. 500-550) there were also a number from villages farther afield. So I took another group of soldiers, all natives of Hilla or of Nasiriya (between Nos. 553-671) to see what degree of uniformity in head form there is among the Arabs of the south side of the plain. When the fifty men included in Chart VII are classified according to head dimensions the result is as follows:

\begin{tabular}{|c|c|c|c|c|c|c|}
\hline \multicolumn{7}{|c|}{ HALIC INDEX } \\
\hline Heads & $-70 /$ & -75 & - & $180+$ & $185+$ & Totals \\
\hline Small..... & 0 & 8 & 14 & 4 & 2 & 28 \\
\hline Medium.......... & 0 & 7 & 10 & 2 & 0 & 19 \\
\hline Large .............. . & 0 & 0 & 2 & 1 & 0 & 3 \\
\hline & 0 & 15 & 26 & 7 & 2 & 50 \\
\hline
\end{tabular}

These two groups of Arabs from the southern area (Charts III, VII) differ in important respects: in the first group the small heads number eighteen, whereas in the second they number twenty-eight; nevertheless there are three big heads in the second group, and two in the first. The chief difference, however, is a prevalence of ultra-dolichocephaly in the first group (eight individuals), while in the second there is none of this class; but there are nine individuals who are brachycephalic or ultra-brachycephalic. The distribution in this second group is strikingly similar to that given by the Pathans (Chart VI).

When passing in review the facial features revealed in these photographic records of Arabs I remarked on the predominance of the European visage among men from Baghdad, Kirkuk, Mosul, and Sulaimaniya. So I took the head dimensions of these men, thirty-one in number. I made up a group of fifty by adding nineteen natives from Kut al Imara, which is situated on the lower Tigris. When the head forms of these fifty men are plotted as in Chart VIII, the following is the result:

IRAQ SOLDIERS (50)

Groups According to Cephalic Index

\begin{tabular}{|c|c|c|c|c|c|c|}
\hline \multirow[b]{2}{*}{ Teads } & \multicolumn{6}{|c|}{ Groups According to Cephalic Index } \\
\hline & $-70 /$ & $-75 /$ & - & $180+$ & $/ 85+$ & Totals \\
\hline Small. & 1 & 3 & 9 & 8 & 0 & 21 \\
\hline Medium.... & 0 & 4 & 16 & 3 & 2 & 25 \\
\hline Large.......... & 0 & 0 & 3 & 1 & 0 & 4 \\
\hline Total & 1 & 7 & 28 & 12 & 2 & 50 \\
\hline
\end{tabular}

The first point to be noted in this group is the prevalence of brachycephaly; fourteen of the fifty men fall on or below the 80 (brachycephalic) line. There is in this group a considerable representation of the small brachycephal so 
prevalent among the South Arabs. The second point is the number of men falling in the large-headed group - four in number (all from Kut al Imara)-but were the divisional lines marking head size drawn more justly, two of the ultrabrachycephals might also be added to the large-headed group. Twenty-one men of this Baghdad group fall in the small-headed class, against twenty-eight in the Hilla group just examined. We note, too, in the Mosul-Baghdad group a single representative of the narrow-headed, small-headed type so common in

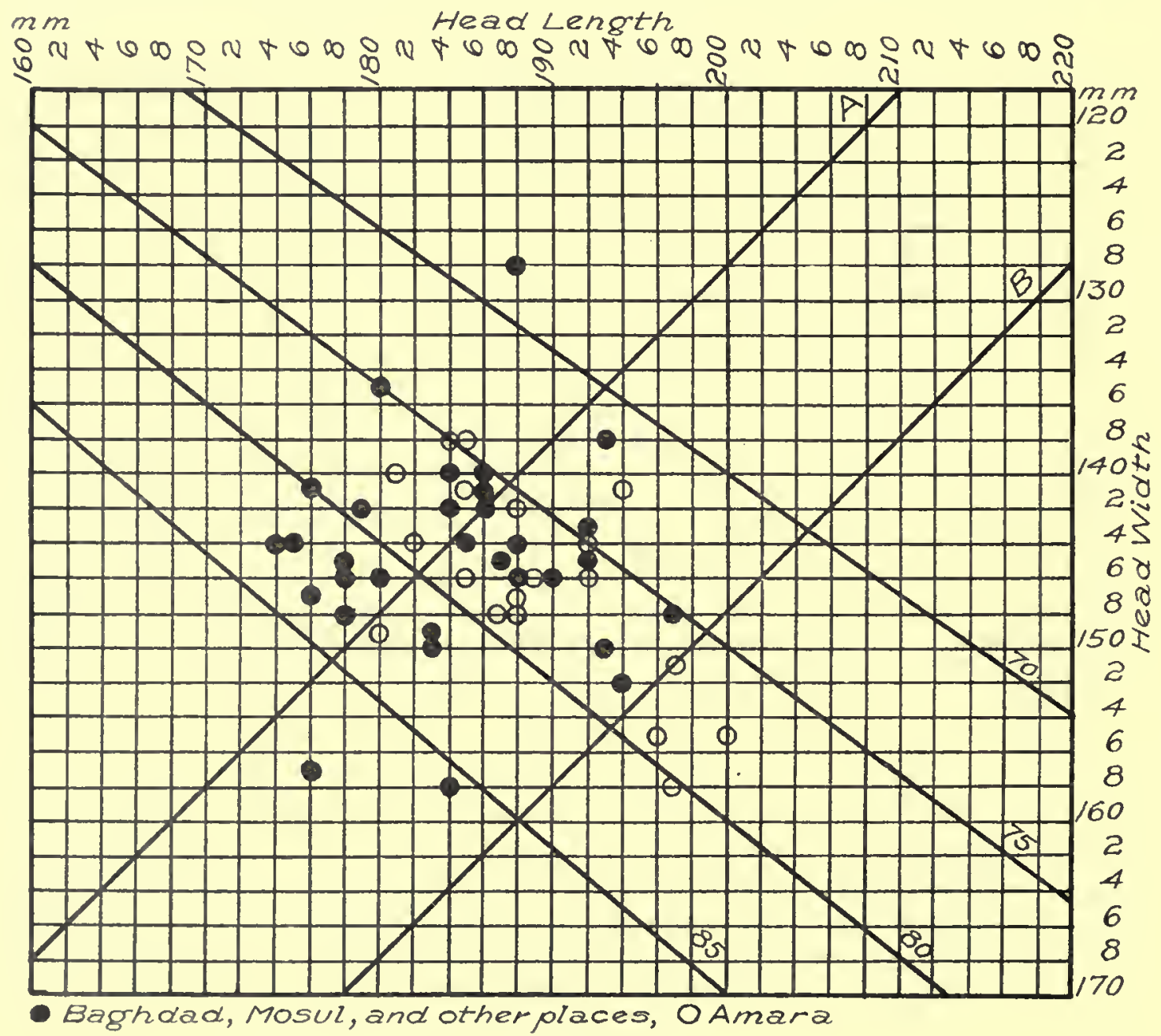

FIG. 8. Chart VIII. Head length, breadth, and capacity of fifty Iraq soldiers, thirty-one from Baghdad, Kirkuk, Mosul, and Sulaimaniya, nineteen from Kut al Imara.

the Beduin group. From the north comes a tendency to round-headedness and large-headedness.

As soldiers were recruited from various districts and represent selected samples of the population, I turned to the Arabs who lived in camps in the neighborhood of Kish to see if there was any trace to be found of the prevalence of local types. I took four groups of fifty from the $398 \mathrm{Kish}$ Arabs. The first group lived in the camp of Sheikh Miniehil (Nos. 1-50). When classified according to head dimensions these fifty men fall into the following classes: 
KISH ARABS (NoS. 1-50)

Groups ACCORDing to CEPHALIC INDEX

\begin{tabular}{|c|c|c|c|c|c|c|}
\hline \multicolumn{7}{|c|}{ /85+ Totals } \\
\hline \multicolumn{7}{|l|}{$\begin{array}{l}\text { Heads } \\
\text { Small }\end{array}$} \\
\hline Medium.......... & 0 & 12 & 8 & 1 & 0 & 21 \\
\hline Large... & 0 & 0 & 1 & 0 & 0 & 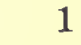 \\
\hline Totals & 0 & 20 & 24 & 4 & 2 & 50 \\
\hline
\end{tabular}
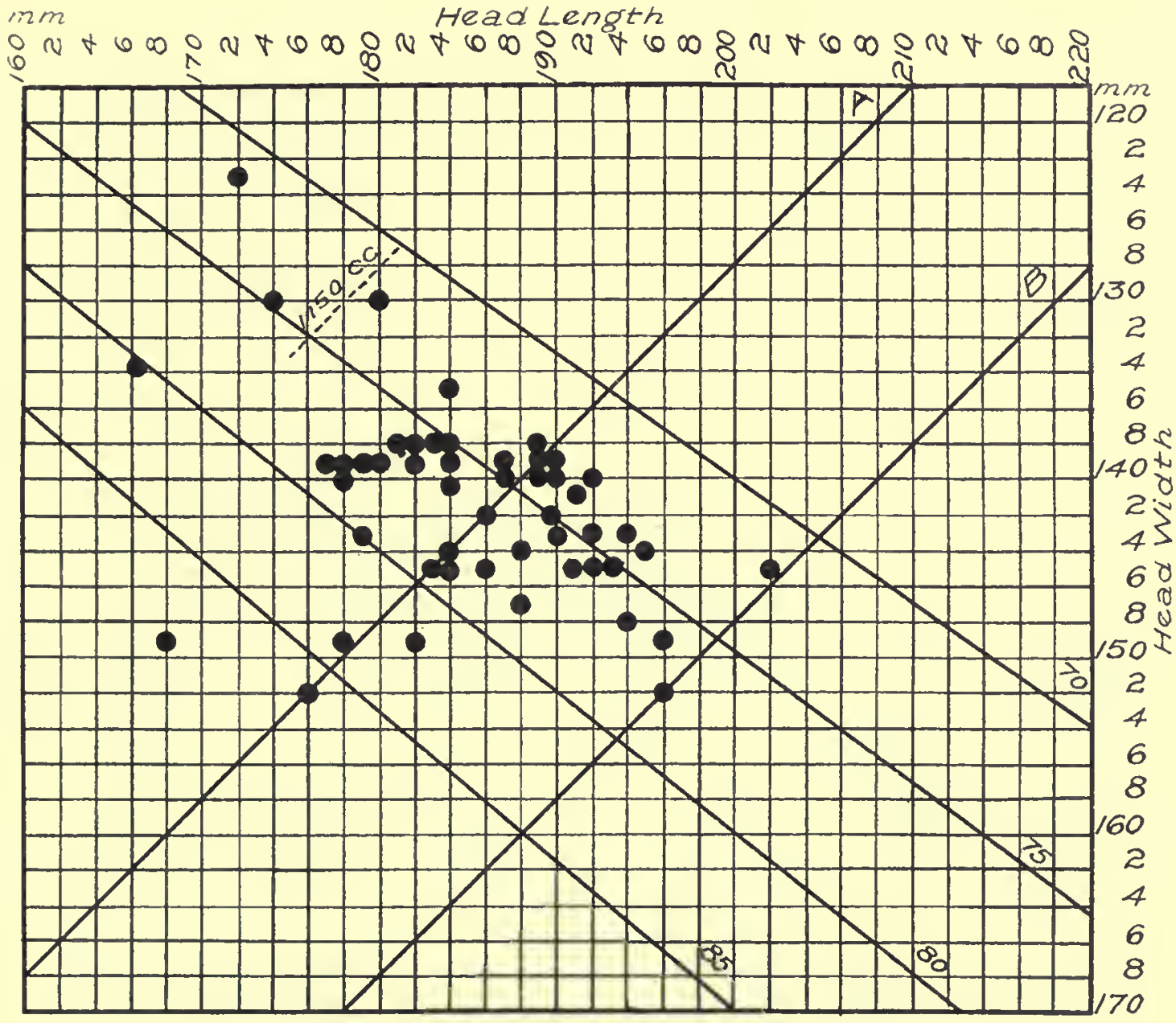

FIG. 9. Chart IX. Head length, breadth, and capacity of fifty Kish Arabs (Nos. 1-50) from camp of Sheikh Miniehil.

We are struck, first, by the number of very small heads; three fall below the 1150 cc-line. Only one reaches the 1450 cc-limit. There are no ultra-dolichocephals; there are six on or beyond the 80 -line; most fall on either side of the 75-line.

The next group also comes from the camp of Sheikh Miniehil (Nos. 100-149). A somewhat more compact group than the last; there is only one very small head; not one reaches the highest standard; there are two small-headed ultradolichocephals; and none of the five brachycephals reaches the 85-line. 
When classified the men in this group fall into the following classes:

$$
\begin{aligned}
& \text { KISH ARABS (Nos. 100-149) } \\
& -70 /-75 / \quad-\quad / 80+/ 85+\text { Totals }
\end{aligned}
$$

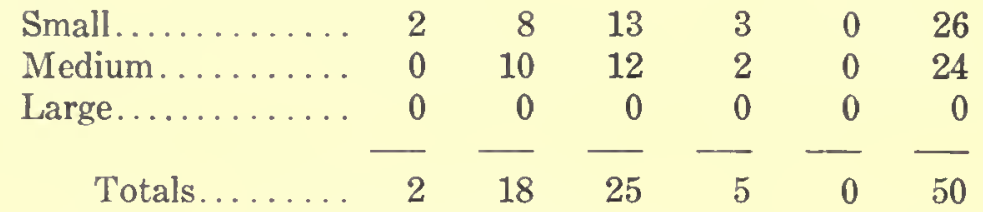

min Head Length

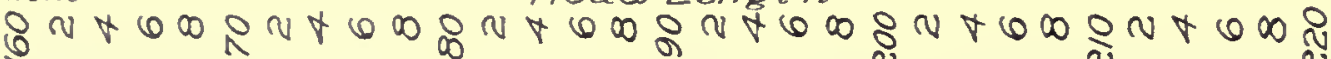

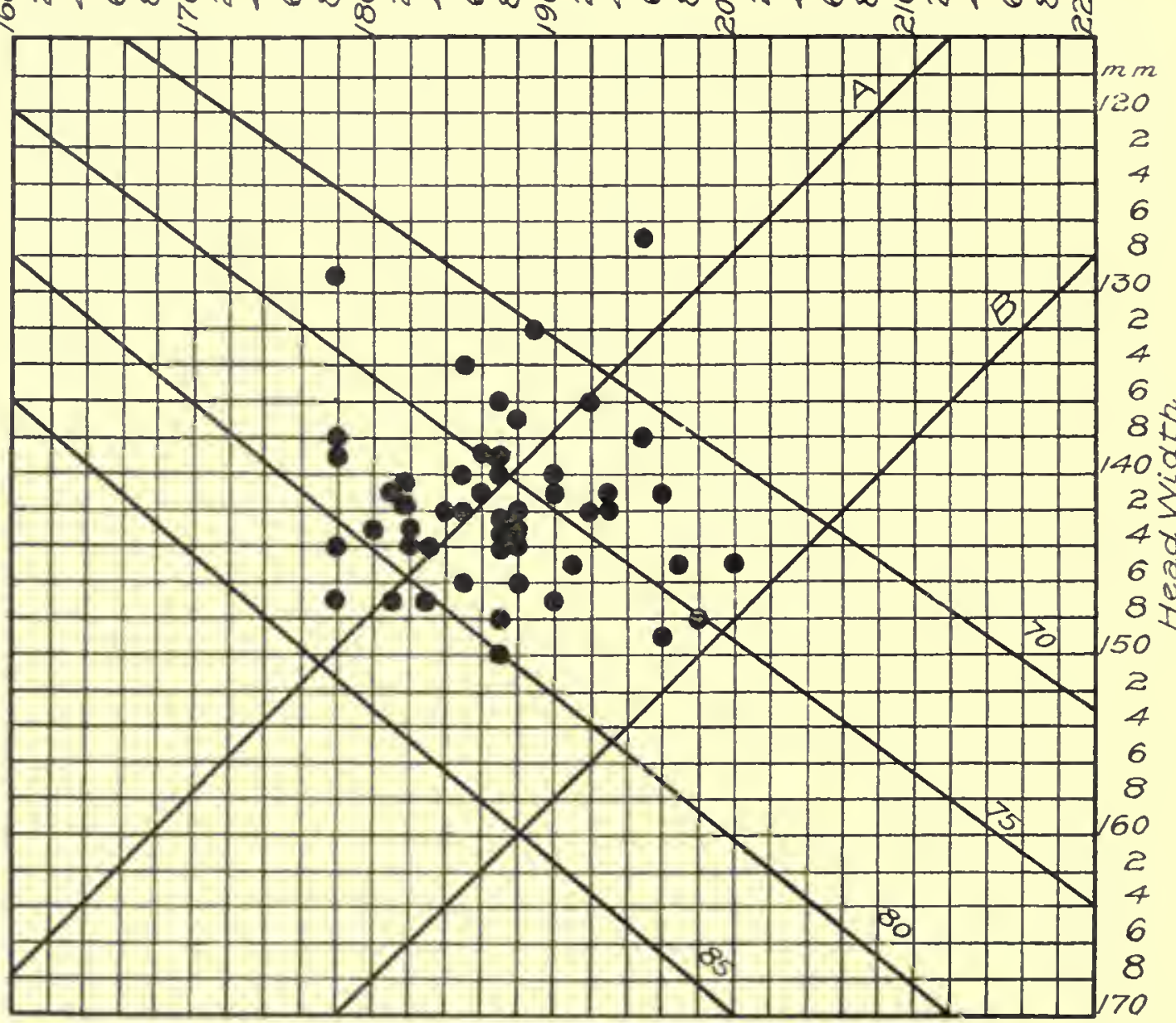

FIG. 10. Chart X. Head length, breadth, and capacity of fifty Kish Arabs (Nos. 100-149) from camp of Sheikh Miniehil.

The next group of Arabs (Nos. 200-249) is from other camps, that of Sheikh Swadi and that of Sayyid Ruda. They are larger-headed men than those of the two last groups; there is no very small head; four rise into the large-headed group. There are three ultra-dolichocephals and four brachycephals, but the latter fall just over the 80-line. This I look on as the chart which is most representative of the head of the Arab as regards size and shape. When classified the following is the result: 
KISH ARABS (Nos. 200-249)

Groups According to Cephalic Index

\begin{tabular}{|c|c|c|c|c|c|c|}
\hline Head: & $-70 /$ & $-75 /$ & 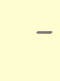 & $180+$ & $/ 85$ & Totals \\
\hline Small. & 2 & 8 & 8 & 1 & 0 & 19 \\
\hline Medium. & 1 & 11 & 13 & 2 & 0 & 27 \\
\hline Large & 0 & 1 & 2 & 1 & 0 & 4 \\
\hline Totals & 3 & 20 & 23 & 4 & 0 & 50 \\
\hline
\end{tabular}

Head Length

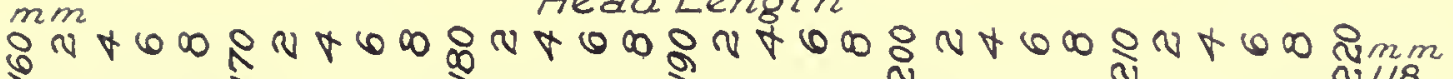

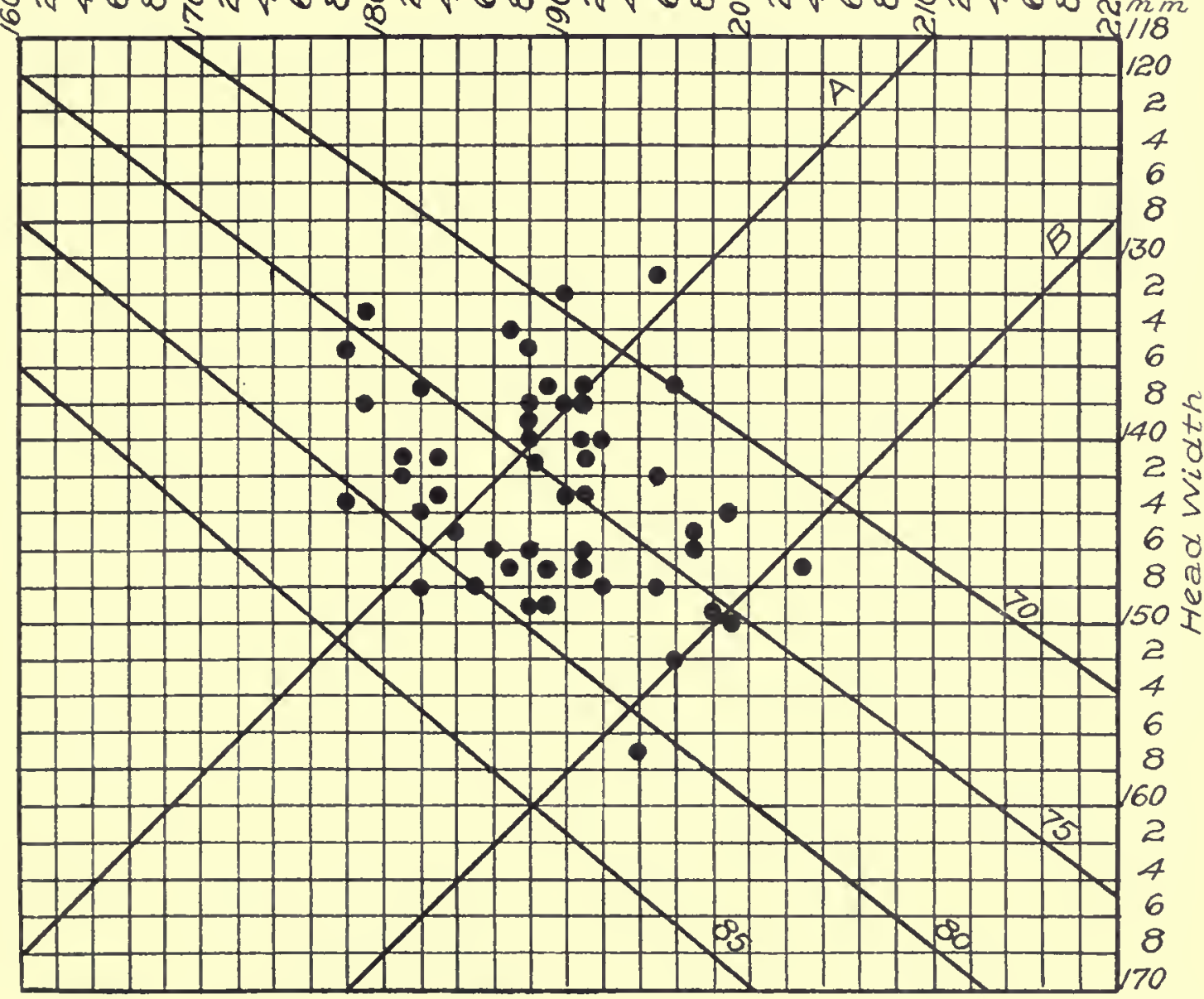

FIG. 11. Chart XI. Head length, breadth, and capacity of fifty Kish Arabs (Nos. 200-249) from camps of Sheikh Swadi and Sayyid Ruda.

The last group of Kish Arabs I included in my review is from the camp of Sheikh Hadji Hunta on the Tigris (seventeen men) and from the camp of Sheikh Aziz (thirty-three men).

We are struck with the dispersion of the individuals of this group. Three rise into the highest group, twenty-seven fall into the medium-headed group, and seven fall toward the bottom of the small-headed group, one being definitely below 1150 cc. There is a strong representation of ultra-dolichocephals (seven), and there is no ultra-brachycephal. 


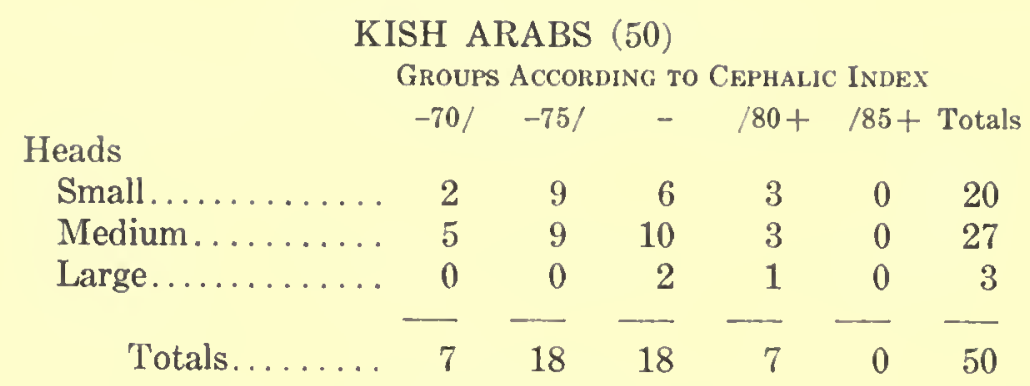

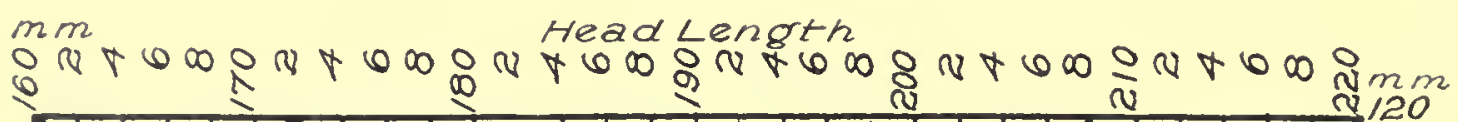

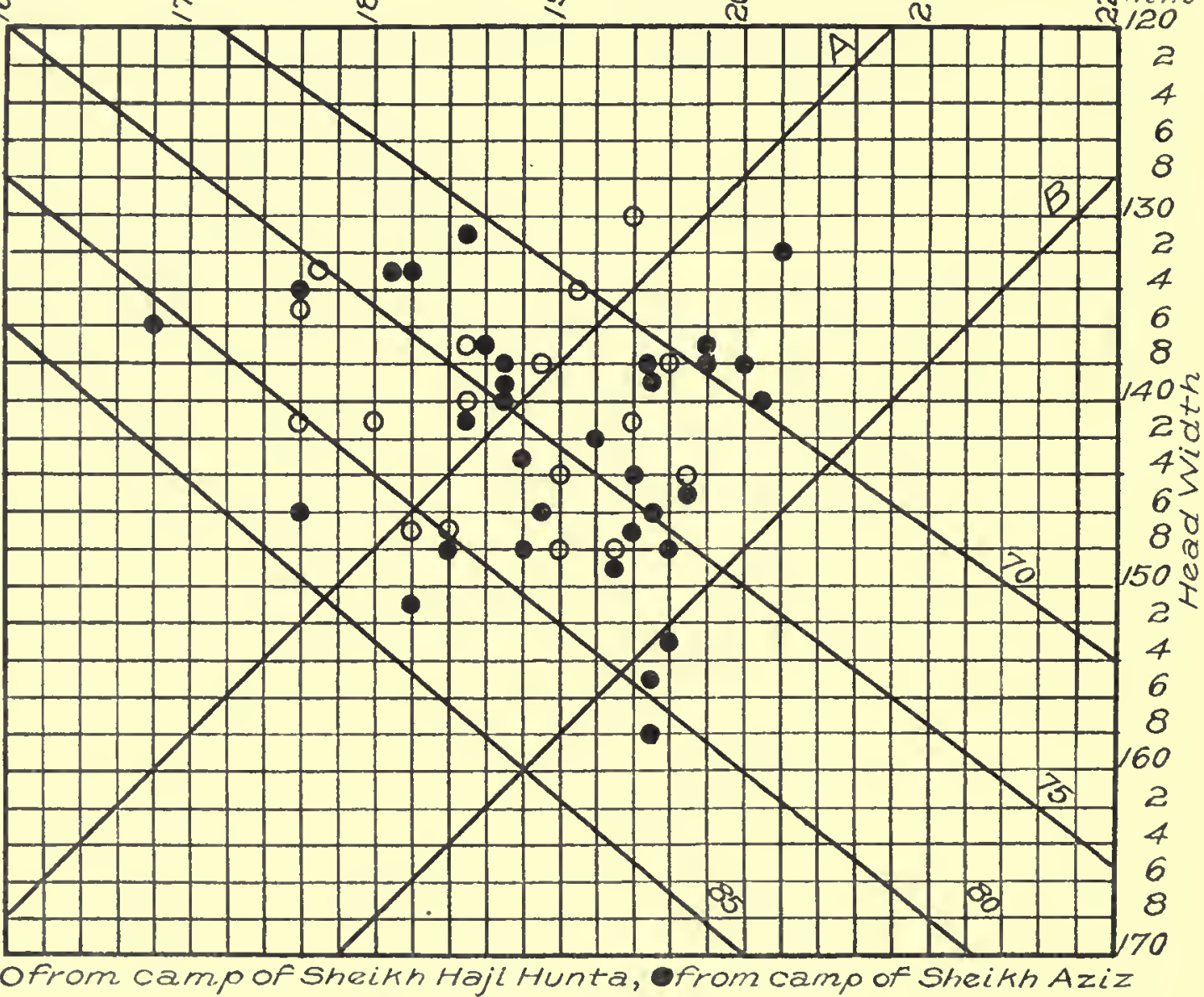

FIG. 12. Chart XII. Head length, breadth, and capacity of fifty Kish Arabs, seventeen from camp of Sheikh Hunta on the Tigris River, thirty-three from camp of Sheikh Aziz.

I have purposely broken up the masses of men measured by Mr. Field into small groups in order to see if in the army or among the Kish Arabs we should meet development of local types, but more especially to see if we should find a group which gave a chart with approximately the same distribution of classes as that given by the Ba'ij Beduins. We have encountered Arabs, both in the army and Kish groups, who have the same head dimensions as the Beduins, but no group gives approximately the same distribution. The Beduin, even in head form and size, has his points of differentiation. 'The soldiers from Mosul and Baghdad also differ from other groups in the proportions of their classes. 
Let me now take the two hundred Arabs depicted in Charts IX to XII and add the results together to obtain a general conspectus of the distribution of head classes among the people of the region of Kish:

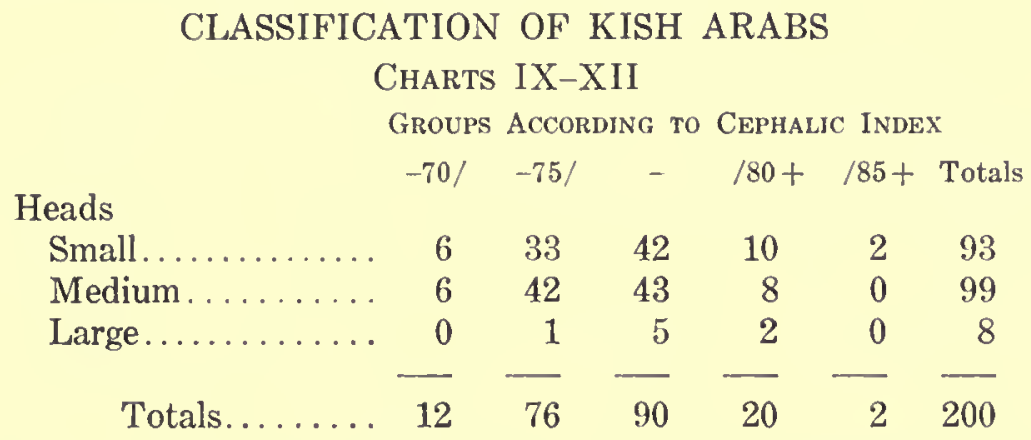

Thus, of the two hundred Arabs 4 per cent rise into the large-headed group, almost half (49.5 per cent) fall in the medium-headed group, while 46.5 per cent are small-headed. Of the small heads five (2.5 per cent) fall below $1150 \mathrm{cc}$; of the ultra-dolichocephalic small heads there are six (3 per cent), whereas among thirty-eight Beduins there are four examples (more than 10 per cent). Of the ultra-brachycephals there are only two examples ( 1 per cent). There are twelve ( 6 per cent) of ultra-dolichocephals. Most fall above the 70-line and below the 80-diagonal; 166 (83 per cent) fall within these limits; twenty (10 per cent) fall on or above the 80 -line and below the 85 -line and are brachycephalic. Thus the Arabs are predominantly dolichocephalic, but the tendency toward brachycephaly is not negligible, there being twenty-two men (11 per cent) of the two hundred men who fall into the brachycephalic classes.

Before passing on to consider measurements other than those relative to the chief dimensions of the head, it will be well to note again the averages and distributional curves which have been worked out from Mr. Field's data. The average measurements of the head in the various groups are as follows:

$\begin{array}{lccc} & \begin{array}{c}\text { Head length } \\ \mathrm{mm}\end{array} & \begin{array}{c}\text { Head width } \\ \mathrm{mm}\end{array} & \begin{array}{c}\text { Cephalic } \\ \text { index }\end{array} \\ \text { Ba'ij Beduins (38) } \ldots \ldots \ldots & 191.3 & 140.2 & 73.4 \\ \text { Kish Arabs }(398) \ldots \ldots \ldots & 188.4 & 141.7 & 75.3 \\ \text { Iraq army }(231) \ldots \ldots \ldots & 187.4 & 143.6 & 76.7\end{array}$

It will be noticed that in dimensions of length and of breadth of head the Kish Arabs hold a position intermediate between the other two groups. The shorter, wider head of the average army Arab must be due to the presence of the wider-headed men from the north. The head width of the Beduin has a higher coefficient of variation (4.32, against 3.74 for the width of head of the soldier and 4.01 for that of the Kish Arab). Nevertheless the peculiarity of the head diameters of the Beduins is due to the high proportion of ultra-dolichocephalic individuals.

In curves of distribution of head indices (Fig. 40) we lose the individual forms which are made patent in the charted groups. The curve of the cephalic 
index for the Iraq soldiers rises rather steeply to gain a single apex at 75-76 and thereafter sinks much more slowly than it rose. In the curve of the Kish Arabs there are two chief peaks, at 75 and 77 , and three minor peaks at 70, 73, and 80 . In our separate charted Kish groups we found no evidence of any differentiation among the individuals to help us in explaining the occurrence of such peaks. I suspect that such peaks have no racial or evolutionary significance. Whether the brachycephalic individuals represent a foreign ol imported element, or whether they are but the extreme of a natural series I will not attempt to decide. It is, however, most probable that Arabia has been invaded at diverse times from the round-headed zone which crosses Asia from the Pamir to Asia Minor.

The Arab impresses the onlooker as having a wide forehead. The average minimal frontal width in the group of Ba'ij Beduins is $110.8 \mathrm{~mm}$, which, if $10 \mathrm{~mm}$ is allowed for flesh, gives a cranial dimension of $100.8 \mathrm{~mm}$ - a high mean-width of forehead. The average for the Kish Arabs is $111.13 \mathrm{~mm}$, almost the same, but among the soldiers the average width of forehead is much greater (113.8 $\mathrm{mm}), 3 \mathrm{~mm}$ more than in the Beduins. The coefficient of variation was almost the same in the soldiers as in the Beduins, but rather higher for the frontal diameter of the Kish Arabs. This difference interested me, and so I prepared Chart XIII to serve a double purpose: (1) to give the distribution of width of forehead in various groups; and (2) to bring out the degree of correlation between width of forehead and greatest width of head. The vertical lines (Chart XIII) which represent width of forehead begin at $94 \mathrm{~mm}$ and end at $130 \mathrm{~mm}$, corresponding to a range on the skull of $84 \mathrm{~mm}$ to $120 \mathrm{~mm}$. I separate the individuals into classes in the following way: (1) very narrow foreheads, under $100 \mathrm{~mm}$, represented thus, $-/ 100$; (2) narrow foreheads, $100 \mathrm{~mm}$ and more but under $110 \mathrm{~mm}$, lepresented thus, $/ 100-/ 110$; (3) wide foreheads, represented thus, $/ 110-/ 120$; (4) very wide foreheads, more than $120 \mathrm{~mm}$, represented thus, $/ 120+$. The horizontal lines (Chart XIII) represent greatest width of head; the series begins at $120 \mathrm{~mm}$ (110 on the skull) and ends at $160 \mathrm{~mm}$ (150 on the skull). The individuals fall into four classes as regards width: (1) very narrow, represented thus, $/ 120-/ 130$; (2) narrow, $/ 130-/ 140$; (3) wide, $/ 140-$ 150 ; (4) very wide, $/ 150+$. In this classification individuals fall into sixteen sections. In Chart XIII three groups are represented: Beduins (38); Kish Arabs (50), Nos. 1-50; Arab soldiers (50), Nos. 500-549.

Let us first arrange the three groups of Arabs into classes according to width of forehead:

\begin{tabular}{|c|c|c|c|c|c|}
\hline & \multicolumn{4}{|c|}{ Minimal Frontal Width } & \\
\hline & $\begin{array}{c}\text { Very narrow } \\
-/ 100\end{array}$ & $\begin{array}{l}\text { Narrow } \\
/ 100-/ 110\end{array}$ & $\begin{array}{c}\text { Wide } \\
/ 110-/ 120\end{array}$ & $\begin{array}{l}\text { Very wide } \\
/ 120+\end{array}$ & Totals \\
\hline Ba ${ }^{\prime} \mathrm{ij}$ Beduins.... . & 0 & 19 & 18 & 1 & 38 \\
\hline Kish Arabs....... & 3 & 31 & 15 & 1 & 50 \\
\hline Arab soldiers..... & 0 & 2 & 36 & 12 & 50 \\
\hline
\end{tabular}

The Beduins are almost evenly divided between the narrow and wide classes of forehead; there are no very narrow and there is only one very wide. Among the Kish Arabs three are in the lowest class, and one is in the highest, 
but there are more than twice as many with narrow foreheads as with wide foreheads. The Kish Arab has a wider head than the Beduin (141.7:140.2), but far more Beduins have wide foreheads. Among the soldiers we meet an

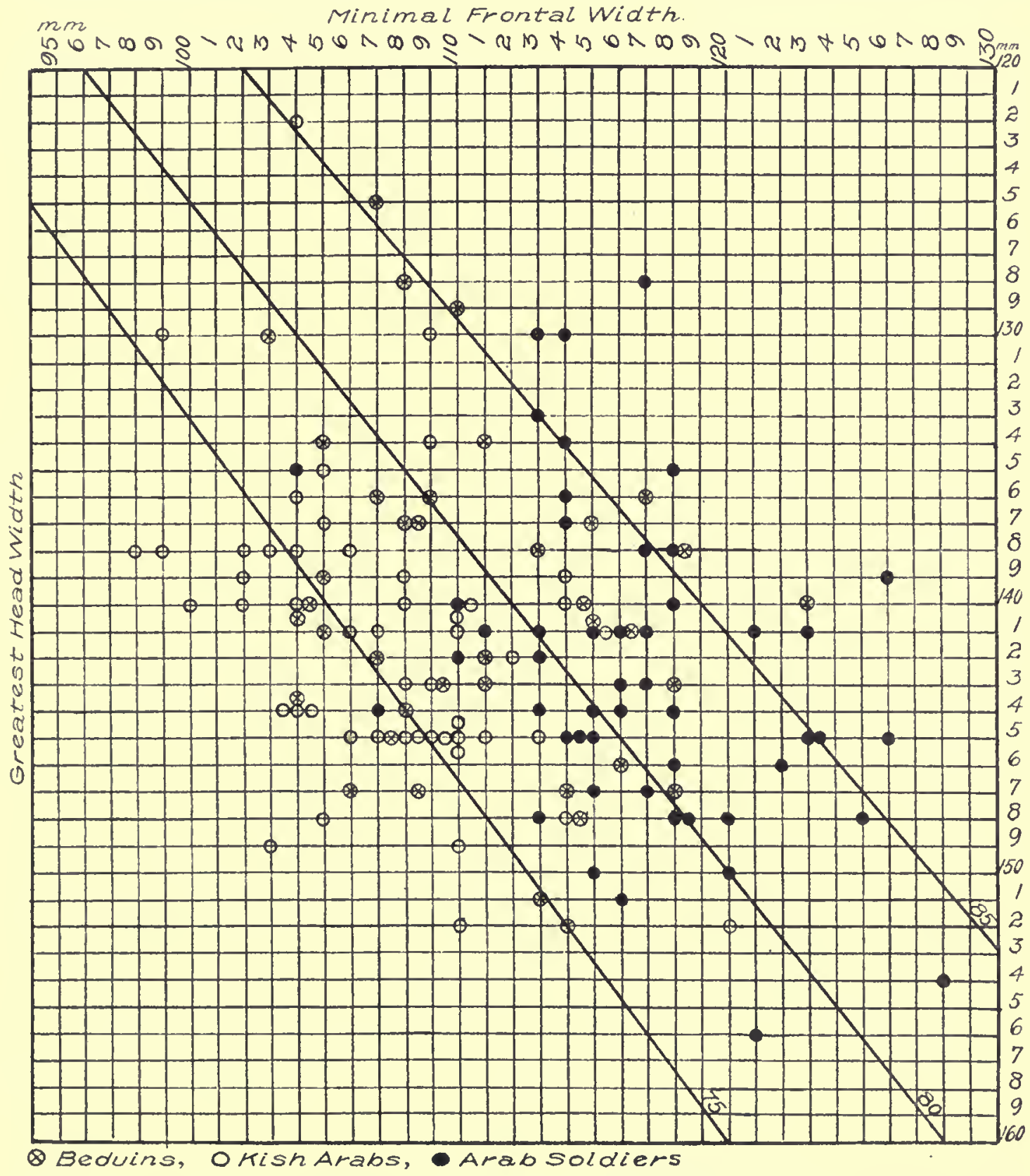

Fig. 13. Chart XIII. Minimal frontal width and maximal head width in three groups of Arabs: thirty-eight Beduins, fifty Kish Arabs (Nos. 1-50) and fifty Iraq soldiers (Nos. 500-549). Three diagonal lines divide the individuals plotted into four groups: (1) where the forehead is less than 75 per cent of width of head; (2) where less than 80 per cent, but 75 per cent or more, and so on.

unexpected predominance of width of forehead. Not one forehead falls into the lowest group, twelve rise into the highest; only two soldiers have "narrow" foreheads, while thirty-six have wide ones. I think it unlikely that there can be this difference between the soldier and other groups. The soldiers have their hair 
cropped and their foreheads exposed. I suspect that surface markings then appear different in the soldiers. Yet, as I shall have to remark later, the soldier class is differentiated from the other Arab groups by many features besides great width of forehead.

Now, one may justifiably suppose that there are races with heads relatively wide in front and relatively narrow farther back; or the opposite may occur, narrow foreheads and wide hinder heads. Let us see how the Beduins stand in this respect. The following table shows distribution of forehead width:

\begin{tabular}{|c|c|c|c|c|c|}
\hline \multirow{6}{*}{$\begin{array}{l}\text { Greatest head width } \\
\qquad \begin{array}{c}/ 120-/ 130 \ldots \ldots \\
/ 130-/ 140 \ldots \ldots \\
/ 140-/ 150 \ldots \ldots \\
/ 150+\ldots \ldots\end{array}\end{array}$} & & \multicolumn{2}{|c|}{ BEDUINS (38) } & & \\
\hline & $-/ 100$ & $/ 100-/ 110$ & $/ 110-/ 120$ & $/ 120+$ & Totals \\
\hline & 0 & 2 & 1 & 0 & 3 \\
\hline & 0 & 7 & 5 & 0 & 12 \\
\hline & 0 & 10 & 10 & 1 & 21 \\
\hline & 0 & 0 & 2 & 0 & 2 \\
\hline TTatos & - & -- & -10 & - & 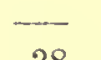 \\
\hline Totals..... & 0 & 19 & 18 & 1 & 38 \\
\hline
\end{tabular}

Noте: $120-130=$ head widths $120 \mathrm{~mm}$ in width or more but less than $130 \mathrm{~mm} ; 130-140=$ head widths $130 \mathrm{~mm}$ or more but less than $140 \mathrm{~mm}$; and so on.

There is a correlation between width of forehead and greatest width of head, capable, no doubt, of exact mathematical expression; but it serves my present purpose sufficiently well to point out that of the fifteen Beduins with a head width less than $140 \mathrm{~mm}$ nine had narrow foreheads, six had wide; of the twentythree with head widths more than $140 \mathrm{~mm}$ ten had narrow foreheads and thirteen had wide (one very wide).

Let us now tabulate fifty Kish Arabs in the same way.

\begin{tabular}{cccccc}
\multicolumn{7}{c}{ KISH } & $\begin{array}{c}\text { ARABS (Nos. 1-50) } \\
\text { Minimal Frontal W10th }\end{array}$ \\
Greatest head width & $-/ 100$ & $/ 100-/ 110$ & $/ 110-/ 120$ & $/ 120+$ & Totals \\
$/ 120-/ 130 \ldots \ldots \ldots$ & 0 & 1 & 0 & 0 & 1 \\
$/ 130-/ 140 \ldots \ldots \ldots$ & 3 & 11 & 1 & 0 & 15 \\
$/ 140-/ 150 \ldots \ldots \ldots$ & 0 & 19 & 13 & 0 & 32 \\
$150+\ldots \ldots \ldots$ & 0 & 0 & 1 & 1 & 2 \\
Totals..... & 3 & 31 & 15 & 1 & 50
\end{tabular}

Of the sixteen men with width of head less than $140 \mathrm{~mm}$ fifteen have nalrow or very narrow foreheads - a result very different from that found in the Beduins. Of the thirty-four men with heads $140 \mathrm{~mm}$ or more in width, nineteen have narrow foreheads, fifteen have wide foreheads, in one case very wide. Here again the result is not the same as yielded by the Beduins. Although there are relatively more wide heads among the Kish Arabs, a narrow forehead is much more common among them. The degree of difference is almost masked in the fronto-parietal index, the frontal width being 78.57 per cent of the parietal in the Kish Arabs 
and 79.29 per cent in the Beduins, a difference of 0.72 per cent. In the Iraq soldiers the forehead measures 79.28 per cent of the greatest width, 0.01 per cent less than in the Beduins.

The classes among the Iraq soldiers are as follows (see also Chart XIII):

\begin{tabular}{|c|c|c|c|c|c|}
\hline \multirow{6}{*}{$\begin{array}{c}\text { Greatest head width } \\
\text { /120-/130..... } \\
/ 130-/ 140 \ldots \ldots \\
/ 140-/ 150 \ldots \ldots \\
/ 150+\ldots \ldots \ldots\end{array}$} & \multicolumn{4}{|c|}{ Minimal Frontal Width } & \multirow[b]{2}{*}{ Totals } \\
\hline & $-/ 100$ & $/ 100-/ 110$ & $/ 110-/ 120$ & $/ 120+$ & \\
\hline & 0 & 0 & 1 & 0 & 1 \\
\hline & 0 & 1 & 9 & 1 & 11 \\
\hline & 0 & 1 & 24 & 8 & 33 \\
\hline & 0 & 0 & 2 & 3 & 5 \\
\hline Totals..... & 0 & 2 & 36 & 12 & 50 \\
\hline
\end{tabular}

Taking the twelve soldiers with a head width less than $140 \mathrm{~mm}$, only one has a narrow forehead as compared with fifteen of sixteen Kish Arabs. One of the narrow heads has a wide forehead. Then of the thirty-eight men with head widths of $140 \mathrm{~mm}$ or more only one has a narrow forehead. Twenty-six have wide foreheads and eleven have very wide; among the thirty-four Kish wider heads, only one has a very wide forehead and fourteen have wide foreheads. On the data as they stand the Kish Arabs have a relatively and absolutely narrow forehead, the opposite state prevailing among the soldiers and rather to a lesser degree among the Beduins than among the soldiers.

The difference in width of forehead is brought out in Chart XIII by three diagonal lines, which divide the individuals represented into four groups: those in whom the forehead is (1) less than 75 per cent of the width of head; (2) /75-/80; (3) $/ 80-/ 85$; (4) $/ 85+$. Into the lowest group fall nineteen Kish Arabs, but only one soldier. In the highest group $(/ 85+)$ there is one Kish Arab, but there are ten soldiers. The Beduins extend into both classes, ten in the lowest and five in the highest.

Width of forehead and its relationship to total head width are features which will prove of selvice in identifying racial relationships. I will confine myself to the comparison of our Arab measurement to two Indian peoples noted already in the discussion of length and breadth of head (see Charts V, VI) - the Chatri caste (Dravidian) of the United Provinces and the Pathan caste of the Punjab.

CHATRI CASTE (DRAVIDIAN, 50)

Minimal Frontal Width

$\begin{array}{cccccc}\text { Greatest head width } & -/ 100 & / 100-/ 110 & / 110-/ 120 & / 120+ & \text { Totals } \\ / 120-/ 130 \ldots \ldots & 2 & 0 & 0 & 0 & 2 \\ / 130-/ 140 \ldots \ldots & 8 & 18 & 0 & 0 & 26 \\ 1140-/ 150 \ldots \ldots & 2 & 17 & 2 & 0 & 21 \\ 1150+\ldots \ldots \ldots & 0 & 1 & 0 & 0 & 1 \\ \text { Totals.... } & 12 & 36 & 2 & 0 & 50\end{array}$


How different is the width of forehead in a Dravidian people as compared with that of Arabs is seen from the table given above. Forty-eight of the fifty Dravidians have narrow or very narrow foreheads. Among the Kish Arabs only three had very narrow foreheads, while sixteen had wide foreheads, one a very

Frontal Width

แ 6 -

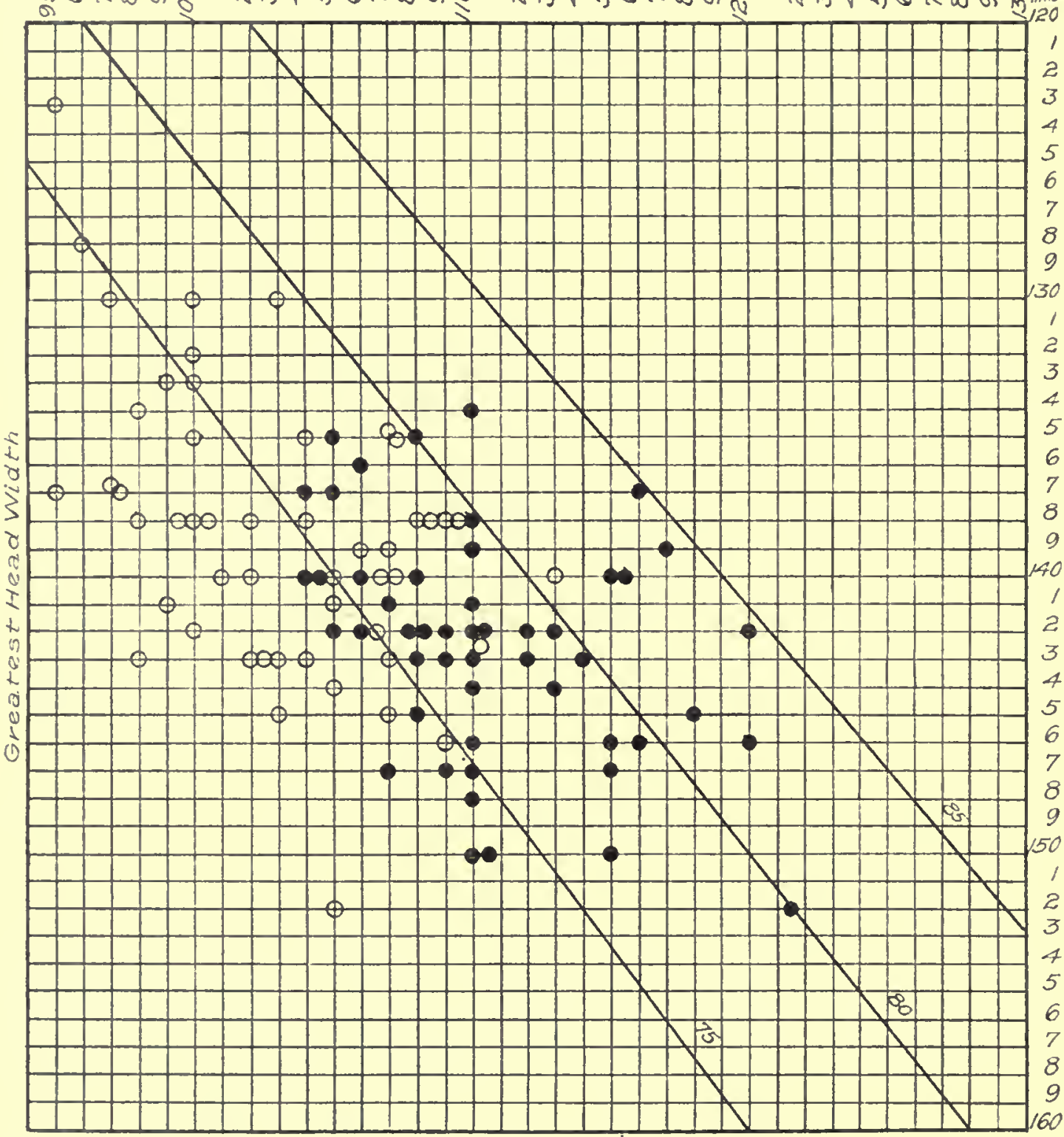

Ochatri, Pathan

FIG. 14. Chart XIV. Minimal frontal width (vertical lines) and maximal head width (horizontal lines) in fifty men of Chatri caste, United Provinces, and fifty men of Pathan caste, Punjab.

wide forehead. The correlation of width of forehead with maximal width of head is very different in these two races.

In Chart XIV are reproduced the frontal and maximal head widths of fifty men of the Pathan caste (Aryo-Dravidian) as well as fifty of a Dravidian people (Chatri). 'The classification of the Pathans is as follows: 


\begin{tabular}{cccccc}
\multicolumn{5}{c}{ PATHAN CASTE (ARYO-DRAVIDIAN, 50) } \\
Minimal Frontal Width \\
Greatest head width & $-/ 100$ & $/ 100-/ 110$ & $/ 110-/ 120$ & $/ 120+$ & Totals \\
$/ 120-/ 130 \ldots \ldots$ & 0 & 0 & 0 & 0 & 0 \\
$/ 130-/ 140 \ldots \ldots$ & 0 & 5 & 5 & 0 & 10 \\
$1140-/ 150 \ldots \ldots$ & 0 & 15 & 19 & 2 & 36 \\
$150+\ldots \ldots \ldots$ & 0 & 0 & 3 & 1 & 4 \\
Totals..... & 0 & 20 & 27 & $-\frac{1}{3}$ & 50
\end{tabular}

How markedly the Pathan and Chatri differ in width of forehead is seen from the fact that only two of the fifty Chatri have a wide forehead, while thirty of the Pathans have wide foreheads, three being very wide. The difference is also brought out in Chart XIV by the numbers falling below the 75-diagonal. They represent individuals with foreheads which are less than 75 per cent of the total width of head. Thirty-two Chatri of the fifty fall in this low class, while only eleven of the fifty Pathans keep them company. On the other hand nine Pathans rise above the 80-diagonal line, but only one Chatri. Among the Kish Arabs (see p. 40; Chart XIII) sixteen had wide foreheads. Hele as in the diameters of the head, there is a resemblance between the Arabs of central Iraq and the Aryo-Dravidian peoples of India. The prevalence of narrowness of forehead in the Kish Arabs is probably due to the survival among them of Dravidian influences.

The racial traits with which we have been dealing relate to the cranial or brain-containing part of the head. In our judgment of races we are influenced by facial traits far more than by shape and size of head. And of all the facial traits perhaps the shape and size and other characteristics of the nose claim our chief attention. To introduce Mr. Field's data relating to the nose, its length and width (alar width) as measured in the living face, I propose to refer first to a race in which the nose is wonderfully uniform in size and shape, the Egyptian. In Chart XV are set out the length and width of the noses of fifty Egyptians of the Khargha Oasis measured by A. Hrdlicka. In this chart the nose is divided into four classes as regards width: (1) noses less than $30 \mathrm{~mm}$ in width (represented by $-/ 30$ ), which are very narrow; (2) noses $30 \mathrm{~mm}$ in width but less than $36 \mathrm{~mm}$ (/30-/36), which may be named the medium narrow class; (3) noses $36 \mathrm{~mm}$ in width, but less than $42 \mathrm{~mm}$ (/36-/42), medium wide noses; (4) wide noses $42 \mathrm{~mm}$ in width and more $(/ 42+)$. As regards length, those less than $50 \mathrm{~mm}$ are counted short; $50 \mathrm{~mm}$ but less than $60 \mathrm{~mm}$ in length are counted of medium length, while those which are $60 \mathrm{~mm}$ and more form the long group.

When the fifty Egyptian noses are classified, we obtain the following groups:

\begin{tabular}{|c|c|c|c|c|c|}
\hline \multirow[b]{2}{*}{ Nasal length } & \multicolumn{3}{|c|}{ EGYPTIANS (50) } & \multirow[b]{2}{*}{$/ 42+$} & \multirow[b]{2}{*}{ Totals } \\
\hline & $-/ 30$ & $/ 30-/ 36$ & $/ 36-/ 42$ & & \\
\hline Short, $-/ 50$ & 0 & 8 & 17 & 3 & 28 \\
\hline Medium, $/ 50-/ 60$ & 1 & 3 & 16 & 2 & 22 \\
\hline Long, $/ 60+\ldots$ & 0 & 0 & 0 & 0 & 0 \\
\hline Totals....... & 1 & $\overline{11}$ & 33 & 5 & 50 \\
\hline
\end{tabular}


Of this series of fifty Egyptians twenty-eight have short noses, twentytwo have noses of medium length; none has a long nose. As regards width, one is narrow, eleven are moderately narrow, thirty-three moderately wide, and five very wide. Thirty-three individuals fall into two classes, the moderately wide and short (seventeen), and the moderately wide and moderately long

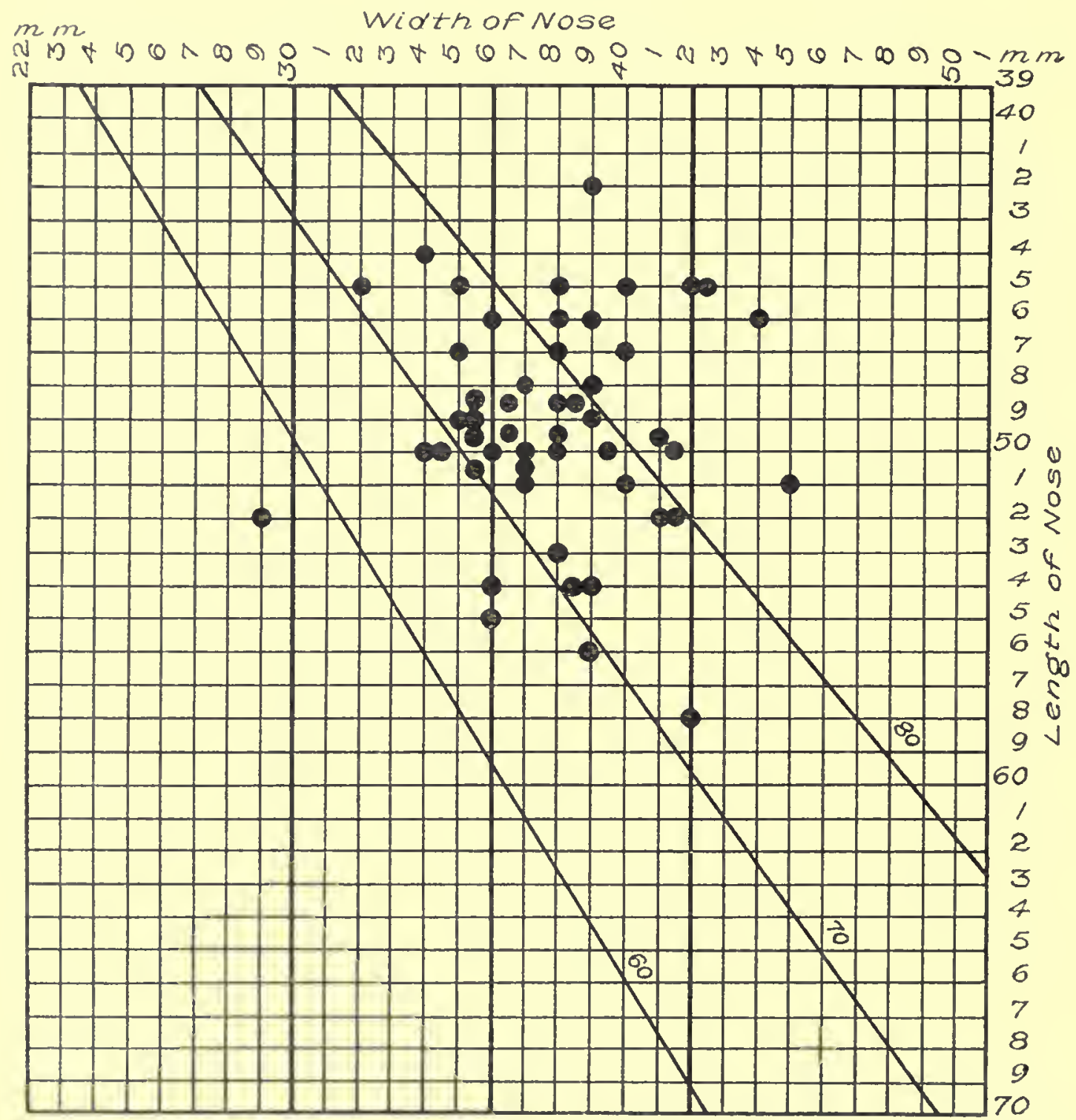

FiG. 15. Chart XV. Nasal width (vertical lines) and length (horizontal lines) of fifty Egyptians of Khargha Oasis, measured by A. Hrdlicka. Diagonal lines indicate percentage of nasal width to nasal length.

(sixteen). Only one individual has a nose in which the width is less than 60 per cent of the length; fourteen have noses with an alar width of 80 per cent of the length or more (see Chart XV). The Egyptian nose we may look upon as characteristic of a Hamitic people. The mean length of the nose of the Khargha Egyptian was $49 \mathrm{~mm}$, its mean width $37.3 \mathrm{~mm}$, the width being 76.3 per cent of the length. 
Let us now turn to the Arab nose, taking first a group of Kish Arabs (Nos. 1-50). How different the dimensions of the Arab nose are from those of the Egyptian will be seen at once if Chart XV is compared with Chart XVI. When classified according to dimensions of nose the fifty Kish Arabs fall into the following groups:

\begin{tabular}{|c|c|c|c|c|c|}
\hline \multirow[b]{2}{*}{ Nasal length } & \multicolumn{3}{|c|}{ (Nos. 1-50) } & \multirow[b]{2}{*}{$142+$} & \multirow[b]{2}{*}{ Totals } \\
\hline & $-/ 30$ & $/ 30-/ 36$ & $/ 36-/ 42$ & & \\
\hline Short, $-/ 50$ & 1 & 3 & 0 & 0 & 4 \\
\hline Medium, $/ 50-/ 60 \ldots \ldots$ & 4 & 16 & 10 & 0 & 30 \\
\hline Long, $/ 60+\ldots \ldots \ldots$ & 1 & 10 & 5 & 0 & 16 \\
\hline Totals. & 6 & 29 & 15 & 0 & 50 \\
\hline
\end{tabular}

Among the fifty Arabs there are six with very narrow noses (less than $30 \mathrm{~mm}$ in width), whereas among the Egyptians there is but one. Among the Arabs there is none with a very wide nose (42 $\mathrm{mm}$ and more); among the Egyptians there are five. Among the Arabs twenty-nine have moderately narrow noses, fifteen moderately wide ones; these proportions are reversed in the Egyptians. Only six Egyptians fall below the 70-diagonal (Chart XV), while below this mark fall forty-six of the Arabs. Six of the Arabs have noses with an index of 50 or less; fourteen of the Egyptians have an index of 80 or more. As regards length of nose, the difference is even more marked. Among the fifty Arabs there are sixteen with long noses (60 mm or more); among the Egyptians there is not one. Twenty-eight Egyptians have short noses, while only four Arabs have them. Nasal development is an Arab character.

Let us now turn to the thirty-eight Beduins. Their nasal chart is given below (Chart XVII). Compare this chart with that of the Egyptian nose (Chart XV), or with that of the Kish Arabs (Chart XVI). Among the Beduins there are no very narrow noses, whereas there are six in the Kish Arab group. There is one Beduin with a nose surprisingly long and wide, the only very wide nose in the Beduin group. Among the Beduins there is not a single short nose; there are four short noses in the Kish group. The prominent feature of the Beduin nose is its length; twenty-two fall into the long group, sixteen into the intermediate group. The proportions are reversed among the Kish Arabs. Among the Beduins are three with an index less than 50 and two with an index of 70 or more.

The average nasal dimensions in Mr. Field's three groups of Arabs are:

\begin{tabular}{|c|c|c|}
\hline & $\begin{array}{l}\text { External nasal } \\
\text { length }\end{array}$ & $\begin{array}{c}\text { Nasal (alar) } \\
\text { width }\end{array}$ \\
\hline Beduins & 60.45 & 35.16 \\
\hline $\mathrm{Ki}$ & 58.33 & 35.32 \\
\hline Iraq soldiers .... & 56.85 & 34.53 \\
\hline
\end{tabular}

The average length of nose is greatest in the Beduins, least in the Iraq soldiers; the width of nose is least among the soldiers, greatest among the Kish 
Arabs. We have previously had reason to suspect the retention of a Dravidian element among the Kish Arabs, which is here indicated by individuals with noses of more than average width.

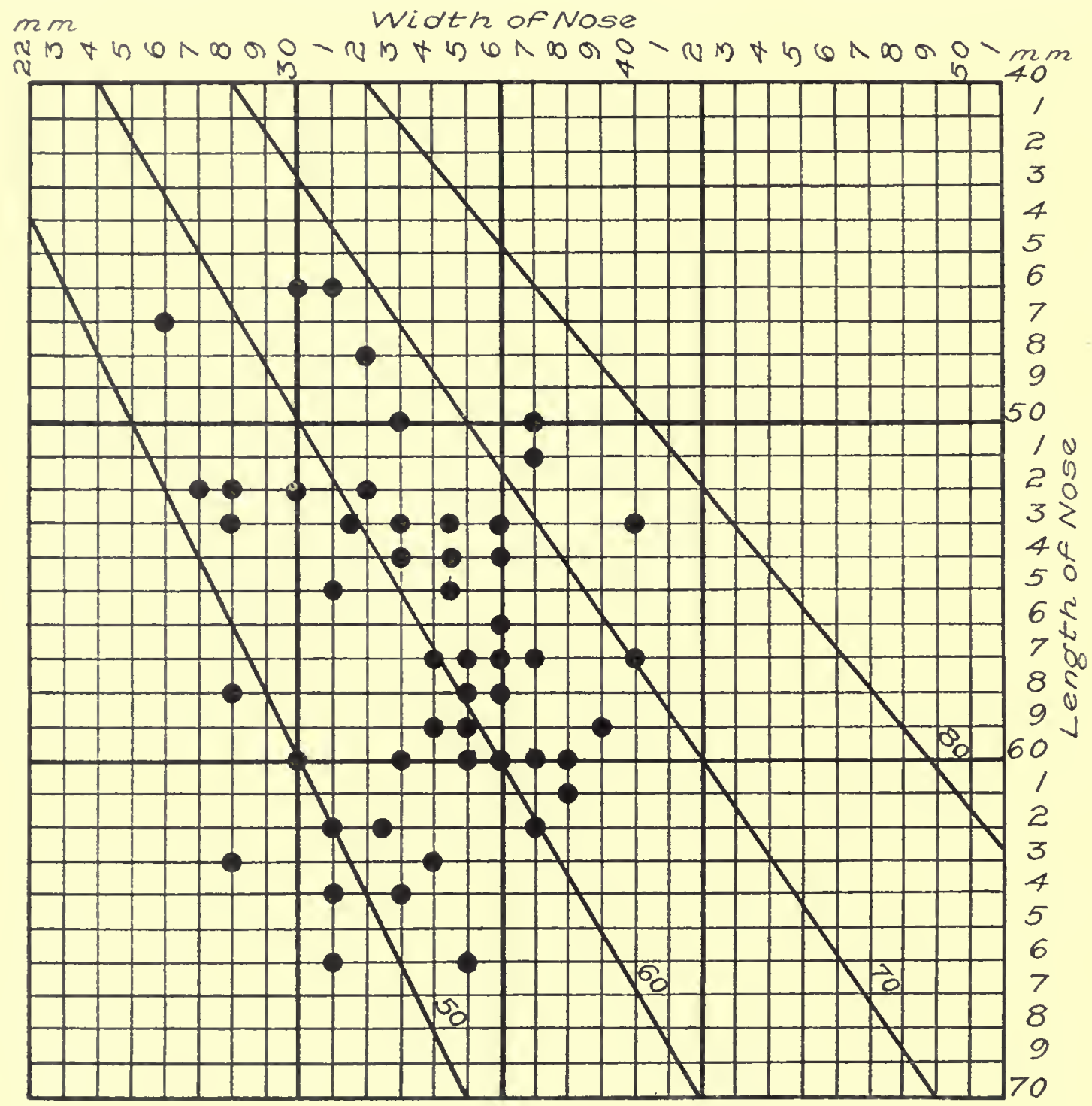

Fig. 16. Chart XVI. Nasal length (horizontal lines) and width (vertical lines) of fifty Kish Arabs (Nos. 1-50).

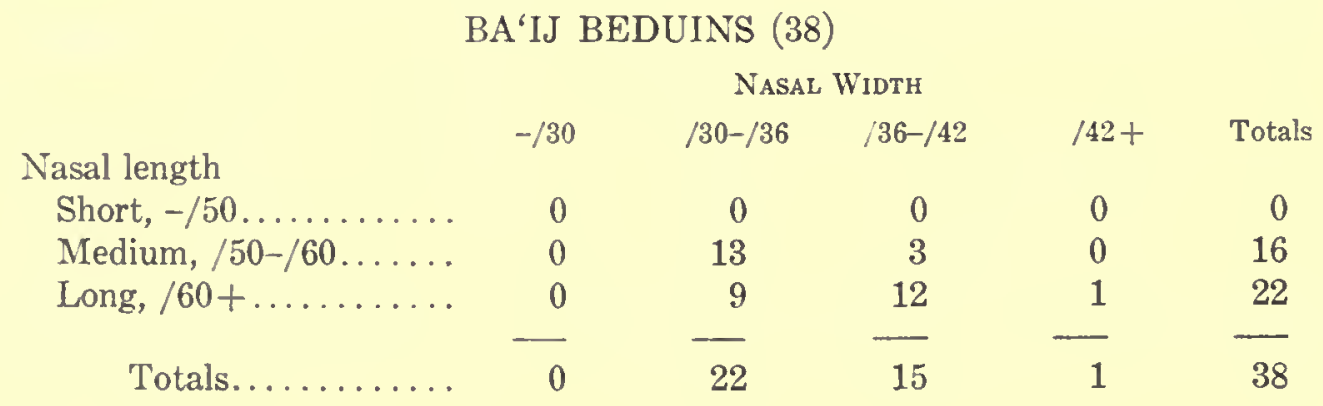


As the group of Kish Arabs charted above (Chart XVI, Nos. 1-50) was found to differ somewhat from other groups in head dimensions, I charted another $m m$ Width of Nose

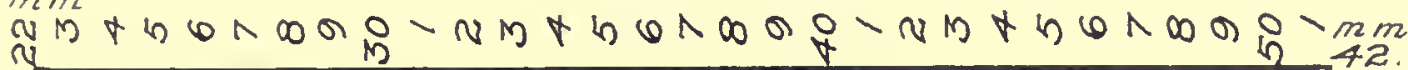

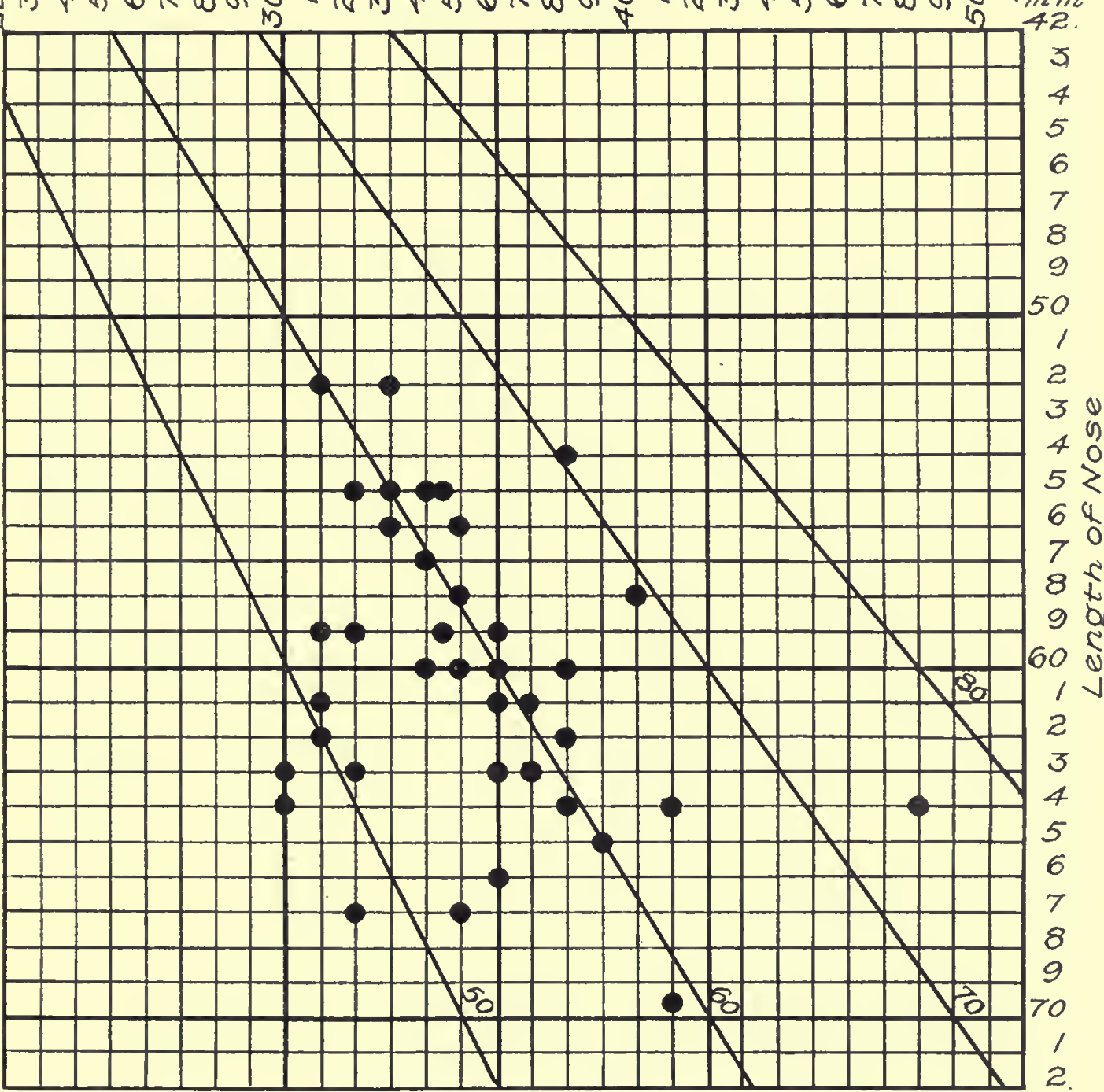

FIG. 17. Chart XVII. Nasal length (horizontal lines) and width (vertical lines) of thirty-eight Ba ${ }^{\text {ij }}$ Beduins.

group of Kish Arabs, Nos. 101-150 (Chart XVIII). When the second series of Kish Arabs is classified according to nasal dimensions we obtain these groups:

\begin{tabular}{|c|c|c|c|c|c|}
\hline \multirow[b]{2}{*}{ Nasal length } & \multicolumn{4}{|c|}{$\begin{array}{l}\text { (Nos. 101-150) } \\
\text { NASAL WIDTH }\end{array}$} & \multirow[b]{2}{*}{ Totals } \\
\hline & $-/ 30$ & $/ 30-/ 36$ & $/ 36-/ 42$ & $142+$ & \\
\hline Short, $-/ 50$ & 0 & 0 & 0 & 0 & 0 \\
\hline Medium, /50-/60. & 0 & 10 & 6 & 0 & 16 \\
\hline Long, $/ 60+\ldots . . . .$. & 0 & 15 & 16 & 3 & 34 \\
\hline Totals. & 0 & 25 & 22 & 3 & 50 \\
\hline
\end{tabular}


The very short-nosed and very narrow-nosed individual, which occurred in Group I, is here absent. Indeed, the nasal chart of the second group is almost the same as the chart of the Beduin group. There is a greater dispersion in the first group of Kish Arabs; in it six individuals had an index of 50 or less, four of

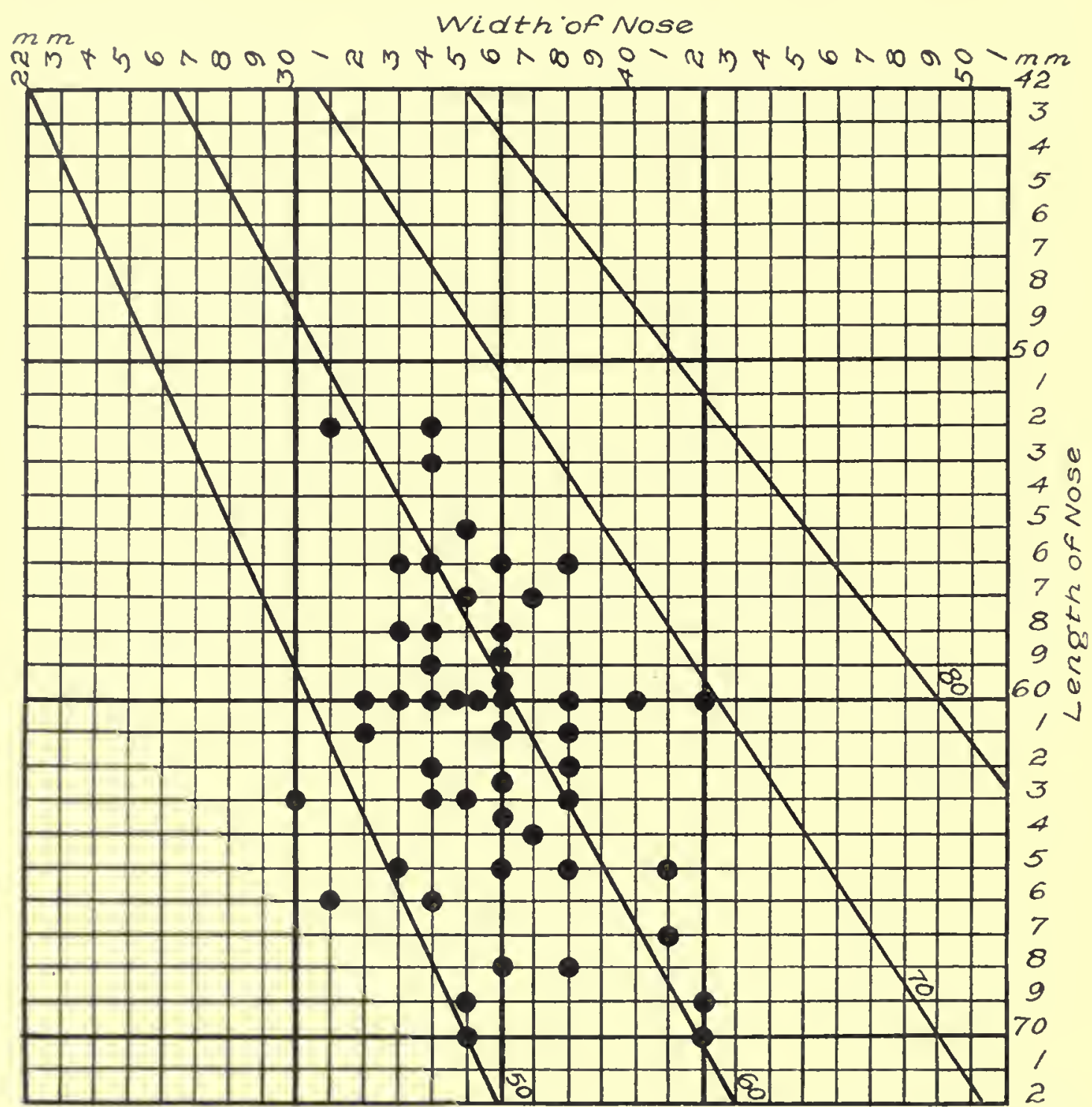

FIG. 18. Chart XVIII. Nasal length (horizontal lines) and width (vertical lines) of fifty Kish Arabs (Nos. 101-150).

70 or more; in the second group the corresponding numbers of such individuals are two and one.

The Iraq soldier surprised us by his width of forehead; there was also in him a greater tendency to brachycephaly than among the Kish Arabs. We will now see that the average length of the soldier's nose is less and the width less than in other Arab groups. In Chart XIX the nasal dimensions of fifty men (Nos. 500-549) are set out. When classified according to these dimensions, the following is the result: 
IRAQ SOLDIERS (Nos. 500-549)

\begin{tabular}{lccccc} 
& \multicolumn{5}{c}{ NASAL Width } \\
Nasal length & $-/ 30$ & $/ 30-/ 36$ & $/ 36-/ 42$ & $/ 42+$ & Totals \\
Short, $-/ 50 \ldots \ldots \ldots \ldots$ & 0 & 0 & 0 & 0 & 0 \\
Medium, $/ 50-/ 60 \ldots \ldots \ldots$ & 0 & 21 & 19 & 1 & 41 \\
Long, $/ 60+\ldots \ldots \ldots \ldots$ & 0 & 5 & 4 & 0 & 9 \\
Totals........... & 0 & 26 & 23 & -1 & 50
\end{tabular}

$\mathrm{mm}$

Width of Nose

NH+U

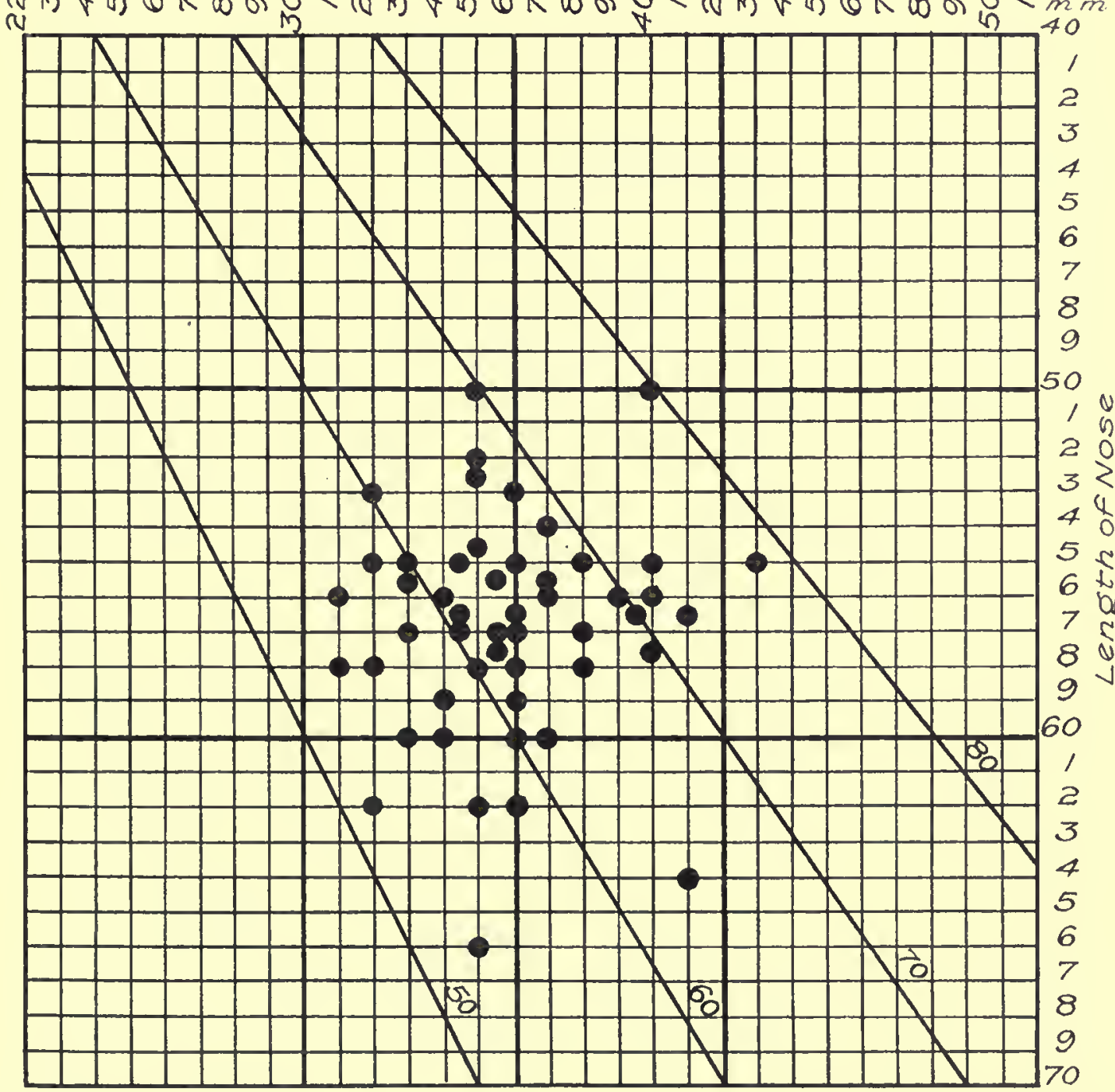

FIG. 19. Chart XIX. Nasal length (horizontal lines) and width (vertical lines) of fifty Iraq soldiers (Nos. 500-549).

The noses of the soldiers are predominantly medium in length; there are no short noses. There are forty-one of intermediate length and nine long noses; in the other Arab groups long noses tend to predominate. Nor are there among the soldiers any very narrow noses and there is only one very wide one. Not a soldier has a nose under the 50-diagonal, but there are seven on the 70-diagonal 
or above it. The rise in nasal index among the soldiers is not due to an increase of breadth but a decrease of length. The striking feature of Chart XIX is the compactness of the array, so different from other Arab groups. The difference is such that one is tempted to suppose that the soldiers are not recruited from the Arabs represented in the camps around Kish or in Beduin tents. The population
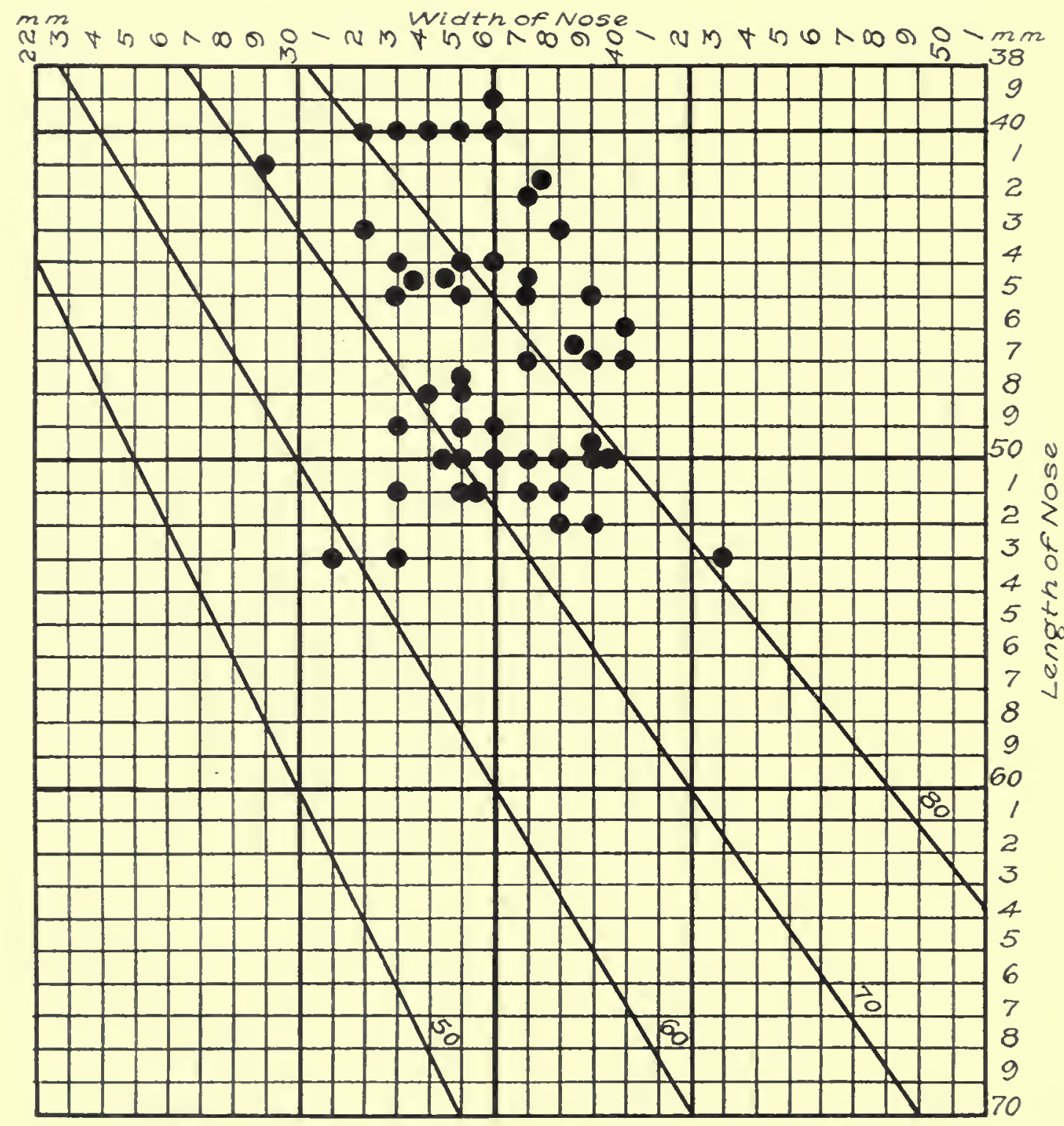

FIG. 20. Chart XX. Nasal length (horizontal lines) and width (vertical lines) of fifty men of Chatri caste, United Provinces (after Risley).

of Baghdad and of the lower towns on the north bank of the Euphrates has been fed, for a long time, by artisans drawn from populations other than the natives of Iraq. If soldiers were drawn mostly from town populations, especially those of the northeast, one could understand their wider foreheads and shorter, narrower noses.

As one has reason to suspect an evolutionary kinship of the Arab with the native races of India, we shall compare very briefly the Arab nose with that of 
groups of Indian people chosen to represent the Dravidian type (fifty men of the Chatri caste, United Provinces, and the Aryo-Dravidian type (fifty men of the Pathan caste, Punjab). Chart XX represents the Chatri (Dravidian) nose. When classified we get the following groups:

\begin{tabular}{rrrrrr}
\multicolumn{6}{c}{ CHATRI CASTE (DRAVIdiAN, 50) } \\
NASAL Width \\
Nasal length & $-/ 30$ & $/ 30-/ 36$ & $/ 36-/ 42$ & $/ 42+$ & Totals \\
Short, $-/ 50 \ldots \ldots$ & 1 & 16 & 16 & 0 & 33 \\
Medium, $/ 50-/ 60$ & 0 & 7 & 9 & 1 & 17 \\
Long, $/ 60+\ldots$. & 0 & 0 & 0 & 0 & 0 \\
Totals..... & 1 & 23 & 25 & 1 & 50
\end{tabular}

Among the Chatri there are no long noses; we have had to heighten the scale of Chart XX to bring in the noses which fall below $40 \mathrm{~mm}$ in length. There are thirty-three short noses and seventeen of medium length. The grouping is much the same as among the Egyptians save as regards three points: there are more noses of moderate length and of more than moderate width among the Egyptians; among the Egyptians there are five men with extremely wide noses (42 $\mathrm{mm}$ or more), whereas among the Chatri there is only one; eighteen of the Chatri have a nasal index of 80 or more, only one having an index below 60 . The Arab did not get his long nose from either Egypt or India.

In Chart XXI are set out the nasal dimensions of the Pathans (fifty men), and, when classified according to these dimensions, we obtain the following groups:

\begin{tabular}{|c|c|c|c|c|c|}
\hline \multirow[b]{2}{*}{ Nasal length } & \multicolumn{4}{|c|}{$\begin{array}{l}\text { NASAL WIDTH } \\
\text { NASA-DRAVIL }\end{array}$} & \multirow[b]{2}{*}{ Totals } \\
\hline & $-/ 30$ & $/ 30-/ 36$ & $/ 36-/ 42$ & $142+$ & \\
\hline Short, $-/ 50$. & 0 & 13 & 6 & 0 & 19 \\
\hline Medium, /50-/60. & 0 & 27 & 4 & 0 & 31 \\
\hline Long, $/ 60+\ldots$. & 0 & 0 & 0 & 0 & 0 \\
\hline Totals..... & 0 & 40 & 10 & 0 & 50 \\
\hline
\end{tabular}

The Pathans are regarded as Aryo-Dravidians, Aryan blood leading to lengthening and narrowing of the nose. Only one Pathan has a nasal index of 80 or more, and only one of 60 or less. The Pathan array is very compact. Neither in this group nor in the last were there noses both narrow and long; among the Pathans there is no very wide nose. The Pathans differ from the Chatri in having thirty-one noses of moderate length and only nineteen short, whereas the Chatri reversed the proportions, thirty-three short and seventeen of medium length. As regards width, among the Chatri moderately narrow and moderately wide noses were almost equally common, whereas among the Pathans the moderately narrow nose predominates. The Pathan nose is a step nearer to the Arab nose than is the Dravidian or Egyptian, but we have to 
go to the Northwest Frontier Provinces of India to find people who begin to rival the Arab in development of nose. The great nose is a Caucasian feature, and in spite of Negroid traits, pigmented skin, sparse development of beard, thick lips, and other features, the Arab has this Caucasian feature developed to its

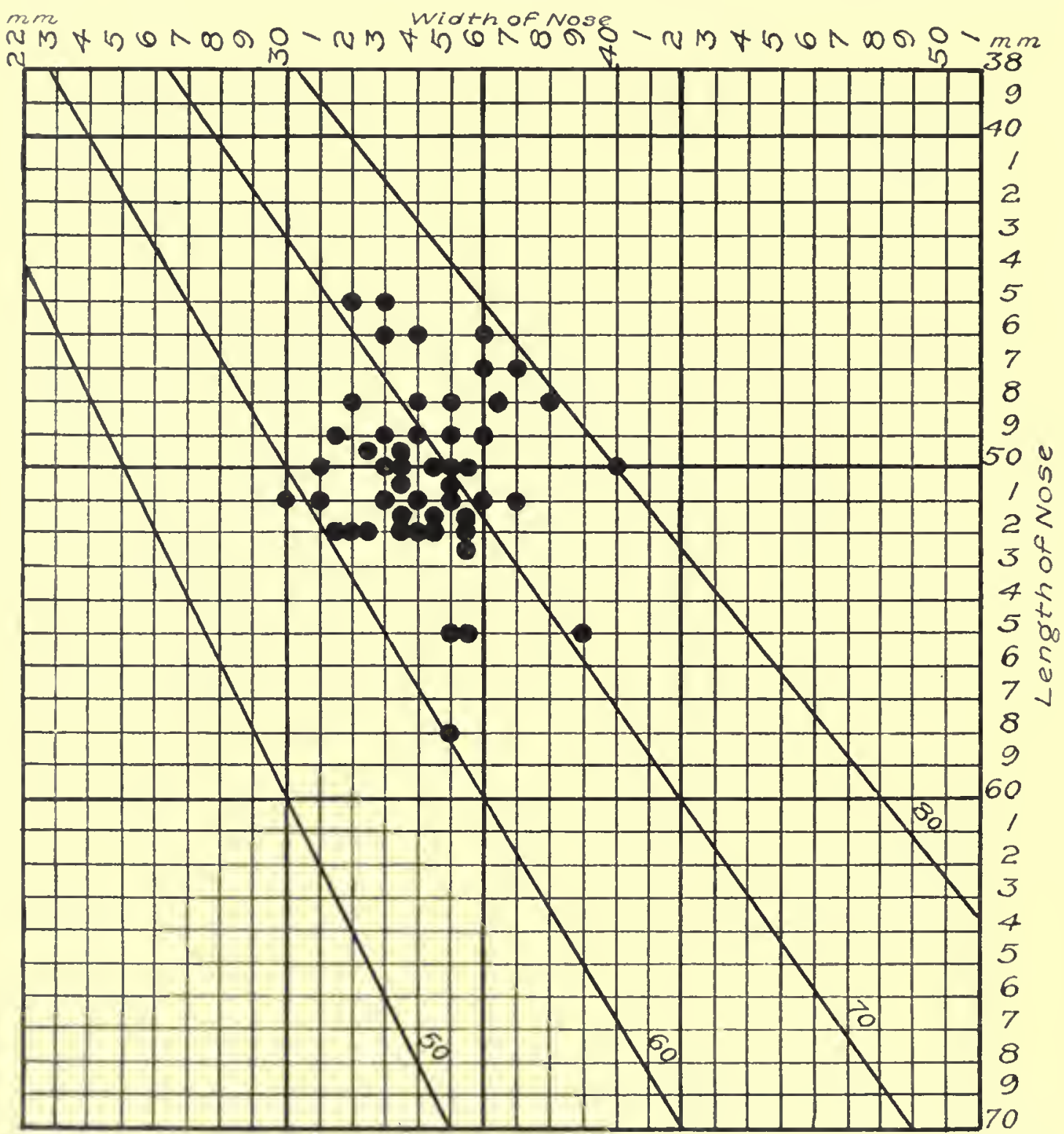

FIG. 21. Chart XXI. Nasal length (horizontal lines) and width (vertical lines) of fifty men of Pathan caste, Punjab (after Risley).

highest extent. One has reason to suppose that great nasal development is often associated with marked individuality of character.

Among eastern peoples distributed in the southwestern part of Asia from the Pamir to Asia Minor, there occurs a type of face which seizes upon the attention of the student of human races. People with this type of countenance are sometimes described as "ram-faced"; the upper face carrying the nose is 
long, while the mandibular part of the face is short. There are "ram-faced" Arabs, and it will be of interest to ascertain how often this type occurs in Mr. Field's various groups. We shall come by the prevalence of the type by comparing the upper face height or length with the total (naso-mandibular) length

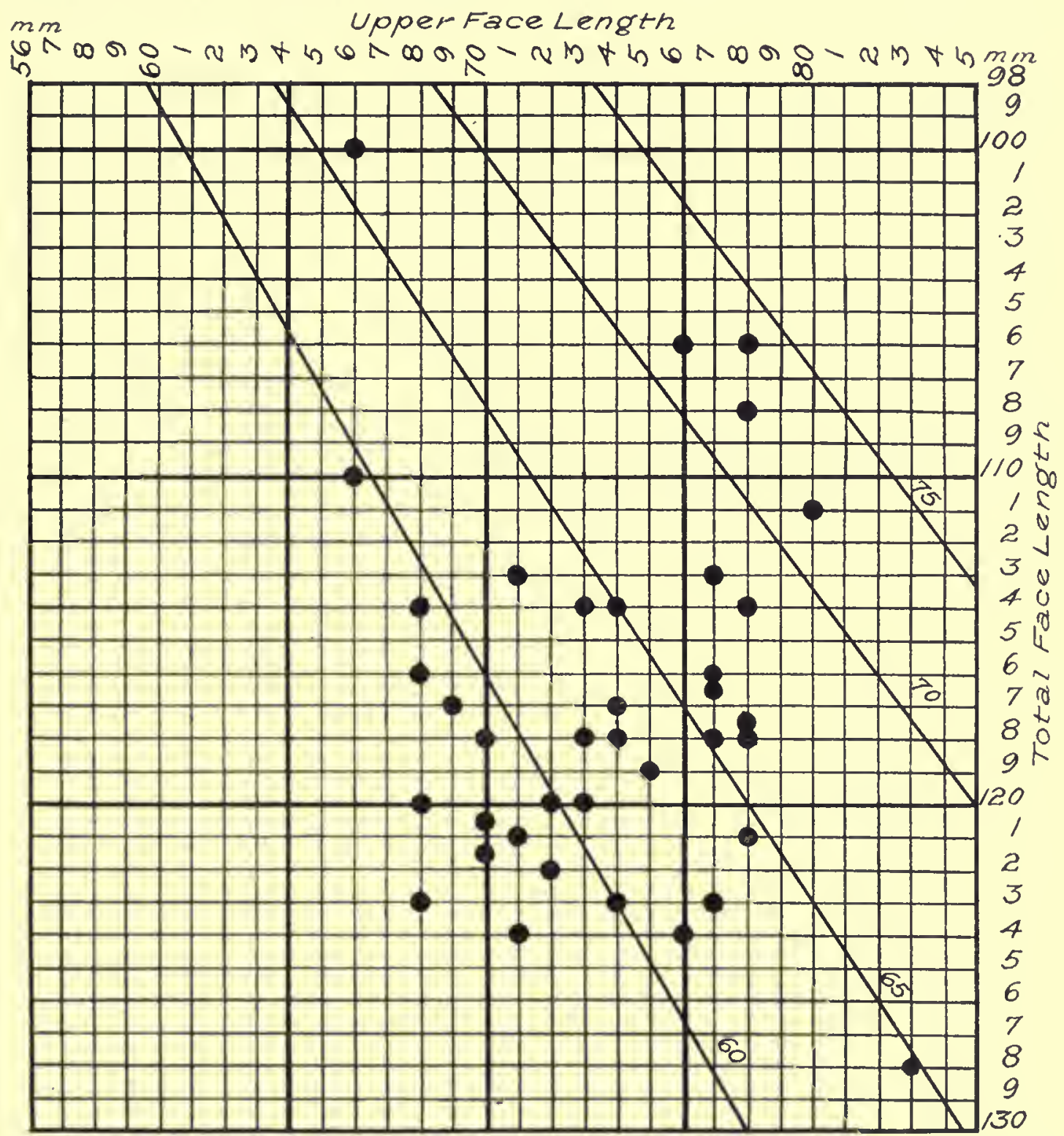

Fig. 22. Chart XXII. Upper face length (vertical lines) and total face length (horizontal lines) of thirty-eight Ba'ij Beduins. The diagonal lines indicate proportion of upper face length to total face length.

or height of the face. That the "ram-face" occurs more frequently in some of Mr. Field's groups than in others is evident when we examine his averages.

\begin{tabular}{|c|c|c|c|c|c|}
\hline & $\begin{array}{l}\text { Upper face } \\
\text { length } \\
\mathrm{mm}\end{array}$ & $\begin{array}{l}\text { Total face } \\
\text { length } \\
\mathrm{mm}\end{array}$ & $\begin{array}{l}\text { Relative face } \\
\text { index }\end{array}$ & $\begin{array}{l}\text { Total face } \\
\text { width } \\
\text { mm }\end{array}$ & $\begin{array}{l}\text { Total face } \\
\text { index }\end{array}$ \\
\hline & 73.63 & 117.2 & 62.82 & 128.5 & 91.4 \\
\hline & 72.97 & 119.8 & 60.91 & 129.5 & 92.73 \\
\hline q soldiers. & 73.88 & 120.92 & 61.10 & 133.85 & 90.5 \\
\hline
\end{tabular}


The Beduins have the shortest faces, the soldiers the longest; nevertheless, the upper face of the Beduin is very little shorter than that of the soldier. Therefore we should expect the "ram-face" to occur more frequently among Beduins than among the Arab soldiers. In fact, the soldiers are much bigger-faced than either Beduin or Kish Arab; the soldier's face on the average is $5.4 \mathrm{~mm}$ wider than the Beduin face. The total length of the face of the Kish Arab is greater than that of the Beduin, but as regards the upper (or nasal) part of the face, the Beduin has the higher measurement. In the averages the individual types are obliterated. We can elicit the occurrence of various types only by a process of graphic analysis such as I have been employing in connection with other features. We shall find that each group of Arabs has its prevalent types; the occurrence of group types is evidence, I think, of local evolution.

As the Beduins promise to provide us with the most characteristic Arab face form, let us study this group first. In Chart XXII each has been plotted according to the upper length and total length of face. As regards upper face length I use four groups: short, less than $64 \mathrm{~mm}(-/ 64)$; moderately short, $64 \mathrm{~mm}$ and more but less than $70 \mathrm{~mm}(/ 64-/ 70)$; moderately long $(/ 70-/ 76)$; and long $(/ 76+)$. As regards total length of face I also use four divisions: short faces, less than $110 \mathrm{~mm}(-/ 110)$; faces moderately short, $110 \mathrm{~mm}$ and mole but less than $120 \mathrm{~mm}(/ 110-/ 120)$; moderately long faces $(/ 120-/ 130)$; long faces $(/ 130+)$. When grouped by this system, the Beduins fall into the following classes:

\begin{tabular}{|c|c|c|c|c|c|}
\hline \multirow[b]{2}{*}{ Total face length } & \multicolumn{3}{|c|}{ BA‘IJ BEDUINS (38) } & & \\
\hline & $-/ 64$ & $/ 64-/ 70$ & $/ 70-/ 76$ & $/ 76+$ & Totals \\
\hline$-/ 110 \ldots \ldots \ldots \ldots$ & 0 & 1 & 0 & 3 & 4 \\
\hline$/ 110-/ 120 \ldots \ldots$ & 0 & 4 & 8 & 8 & 20 \\
\hline$/ 120-/ 130 \ldots \ldots$ & 0 & 2 & 8 & 4 & 14 \\
\hline $1130+\ldots$ & 0 & 0 & 0 & 0 & 0 \\
\hline Totals.... & 0 & 7 & 16 & 15 & 38 \\
\hline
\end{tabular}

Here we have twenty-four men who are short-faced (less than $120 \mathrm{~mm}$ ), yet nineteen have long upper faces, i.e. tend toward the "ram" type of visage. Or" we may state the matter in another way; fourteen of the thirty-eight have uppel" faces which are 60 per cent or less of the total face length, while four have faces in which the index is 70 or mole. The latter represent the "ram-faced" type. Among the Beduins there is not a single man who has a short upper face, but there are four with short total faces. Very remarkable is the number with very long upper faces (fifteen). Among the Beduins there occur sports - a very shortfaced man (upper and total) and a very long-faced man (upper and total); see Chart XXII. The eccentric individual is perhaps more interesting than the average man.

In Chart XXIII the facial dimensions of a group of Kish Arabs are displayed. When compared with Chart XXII, there is seen to be a greater degree of dispersion or scattering, as is apparent in the following table: 
KISH ARABS (Nos. 1-51)

$\begin{array}{rccccc}\text { Total face length } & -/ 64 & / 64-/ 70 & / 70-/ 76 & / 76+ & \text { Totals } \\ -/ 110 \ldots \ldots \ldots & 0 & 1 & 1 & 1 & 3 \\ / 110-120 \ldots \ldots & 2 & 9 & 5 & 1 & 17 \\ / 120-/ 130 \ldots \ldots & 0 & 4 & 12 & 7 & 23 \\ / 130+\ldots \ldots & 0 & 0 & 3 & 4 & 7 \\ \text { Totals.... } & 2 & - & - & - & -\end{array}$

mm Upper Face Length

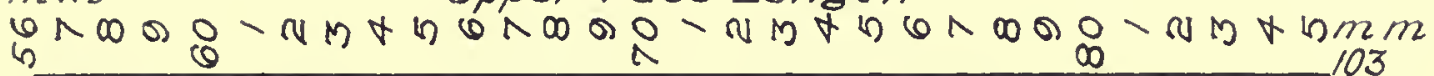

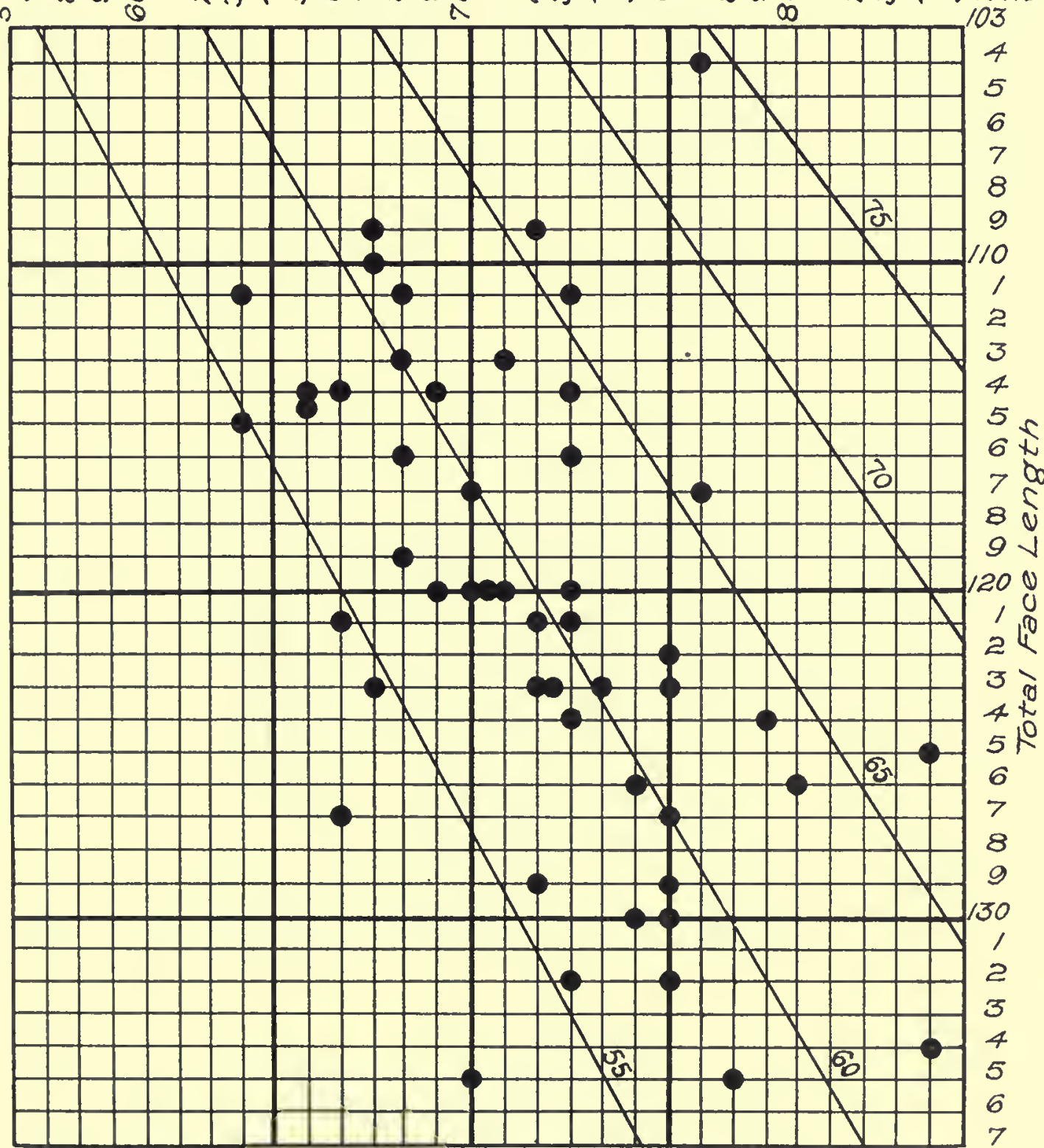

FIG. 23. Chart XXIII. Upper face length (vertical lines) and total face length (horizontal lines) of fifty Kish Arabs (Nos. I-51). 
Here extremes are represented more freely than among the Beduins; there are two men with very short upper face lengths and three other men with very short total face lengths. Whereas among the Beduins there were twenty-four

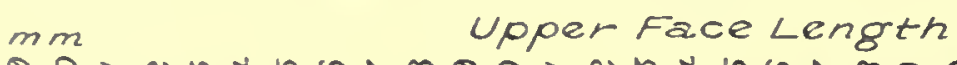
is - NM+

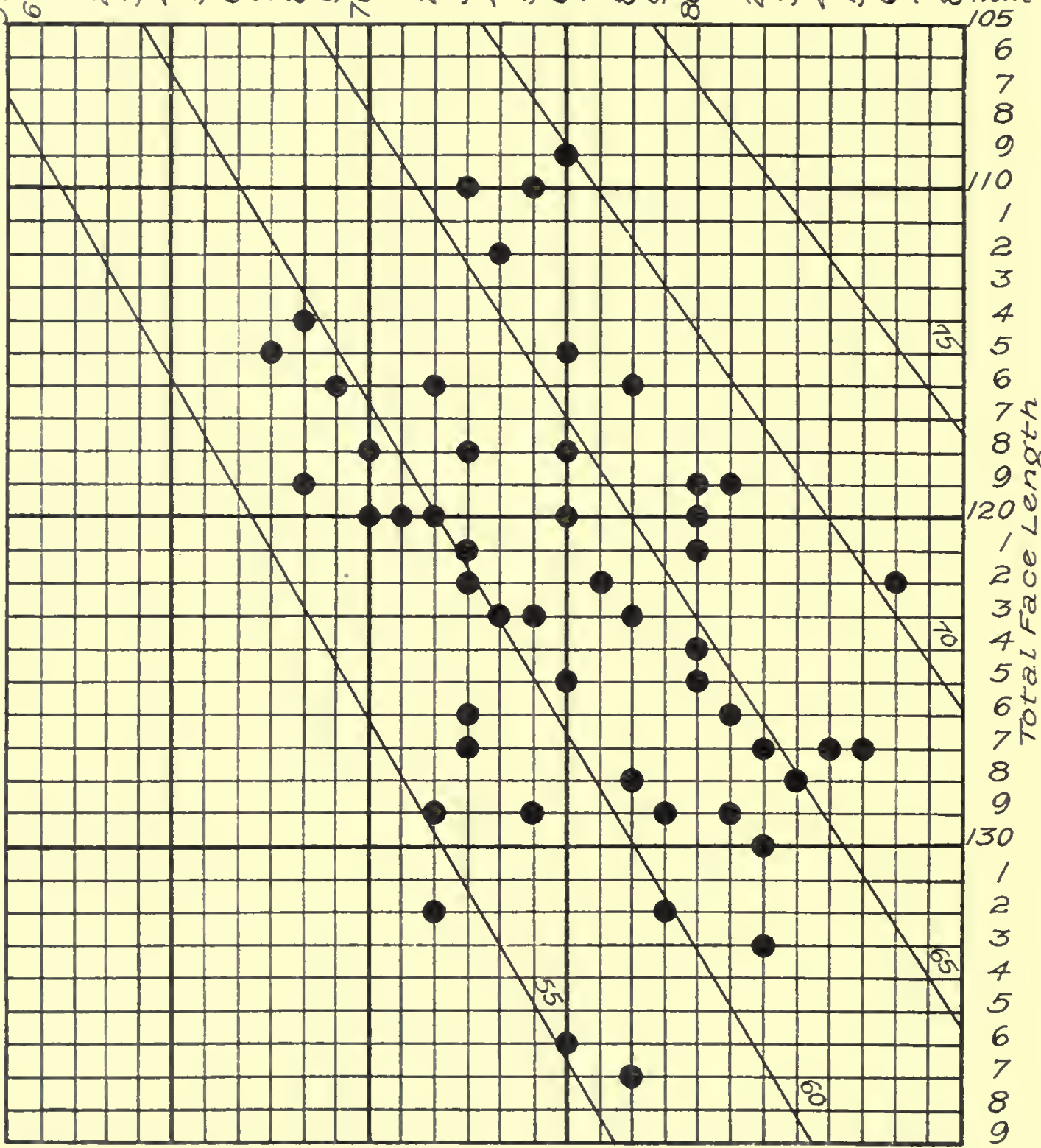

FIg. 24. Chart XXIV. Upper face length (vertical lines) and total face length (horizontal lines) of fifty Kish Arahs (Nos. 101-150).

men the length of whose faces fell below the $120 \mathrm{~mm}$ mark, yet nineteen of them had long upper faces (70 $\mathrm{mm}$ or more). In this group of Kish Arabs, out of the twenty short-faced men there are only eight who have long upper faces. The "ram-faced" type is much less common than among the Beduins. In seven of this group of Arabs the face was very long (more than $130 \mathrm{~mm}$ ); not one Beduin entered this class. Thirty-one of the thirty-eight Beduin Arabs had 
long upper faces (70 $\mathrm{mm}$ or more); thirty-four of this group of fifty Kish Arabs had faces $70 \mathrm{~mm}$ in length or more. The most remarkable feature of this group is seen in Chart XXIII; twenty-nine of the fifty had upper faces which were 60 per cent of the total face, or less; only one man rose above the 70 mark. We shall meet no other group of Arabs showing such strange faces and noses.

A second group of Kish Arabs, set out in Chart XXIV, has much the same dispersion as the last group, save that there are fewer individuals with such exceptional facial characteristics. A remarkable difference between the two samples lies in the grouping as regards the diagonal divisions (cf. Charts XXIII, XXIV). Twenty-nine men of the first group fell on or below the 60-diagonal; here there are seventeen. When the second group is classified, we see how the various compartments are filled:

KISH ARABS (Nos. 101-150)

\begin{tabular}{cccccc}
\multicolumn{7}{c}{ KISH ARABS (NOS. 101-150) } \\
UPPER FACE LENGTH \\
Total face length & $-/ 64$ & $/ 64-/ 70$ & $/ 70-/ 76$ & $/ 76+$ & Totals \\
$-/ 110 \ldots \ldots \ldots$ & 0 & 0 & 0 & 1 & 1 \\
$/ 110-/ 120 \ldots \ldots$ & 0 & 4 & 6 & 5 & 15 \\
$/ 120-/ 130 \ldots \ldots$ & 0 & 0 & 11 & 17 & 28 \\
$/ 130+\ldots \ldots \ldots$ & 0 & 0 & 1 & 5 & 6 \\
Totals..... & 0 & - & -18 & 28 & 50
\end{tabular}

In this group there is not a single very short upper face length and only one very short total face length. Of sixteen men who have a short total face length (less than $120 \mathrm{~mm}$ ) twelve have long upper faces. Indeed in this group the "ramface" is very prevalent. Twenty-eight men have an upper face length of $76 \mathrm{~mm}$ or more, and yet of the fifty only six have a long total face, $130 \mathrm{~mm}$ or more.

I have charted a third group of Kish Arabs to ascertain the degree of variation of type of face from group to group. The result, as regards this third group, is shown in Chart XXV, and in the following table:

KISH ARABS (Nos. 200-251)

\begin{tabular}{ccrccc} 
& \multicolumn{5}{c}{ UPPER FACE LENGTH } \\
Total face length & $-/ 64$ & $/ 64-/ 70$ & $/ 70-/ 76$ & $/ 76+$ & Totals \\
$-/ 110 \ldots \ldots \ldots$ & 0 & 1 & 1 & 0 & 2 \\
$/ 110-1120 \ldots \ldots$ & 2 & 13 & 11 & 0 & 26 \\
$1120-/ 130 \ldots \ldots$ & 0 & 6 & 9 & 5 & 20 \\
$/ 130+\ldots \ldots$ & 0 & 0 & 0 & 2 & 2 \\
Totals..... & 2 & -20 & -21 & -7 & 50
\end{tabular}

In this third group we obtain a dispersion in the Chart (XXV) very similar to that of the first group (Chart XXIII). There is the same preponderance of individuals on and below the 60-diagonal, the same absence of the relatively Iong upper face; not an individual in this third group reaches the 70-diagonal. 
There are, in the third group, the same number of eccentric individuals, two men with very short upper faces and two other men with shortness of the total face. As in the first group, too, the individuals with moderately long upper faces (/70-/76 $\mathrm{mm})$ are much more numerous than those possessing very long upper

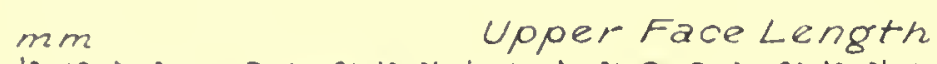

FIG. 25. Chart XXV. Upper face length (vertical lines) and total face length (horizontal lines) of fifty Kish Arabs (Nos. 200-251).

faces $(/ 76+)$, whereas in the second group of Kish Arabs long upper faces were in preponderance. Among the Beduins moderately long and long upper faces occurred in almost equal numbers. In this third group, of twenty-eight men whose total face lengths were less than $120 \mathrm{~mm}$, only twelve had a long upper face (more than $70 \mathrm{~mm}$ ). In this group there can be few of the "ram-faced" type. 
We have already found that the Iraq soldier departs in many points from the standard of the Kish Arab. We have seen that in his upper face length, his mm Upper Face Length

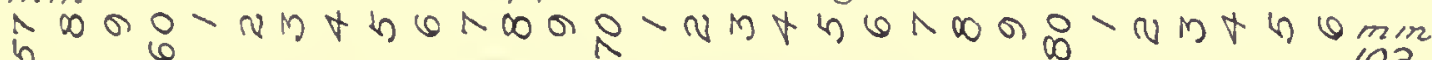

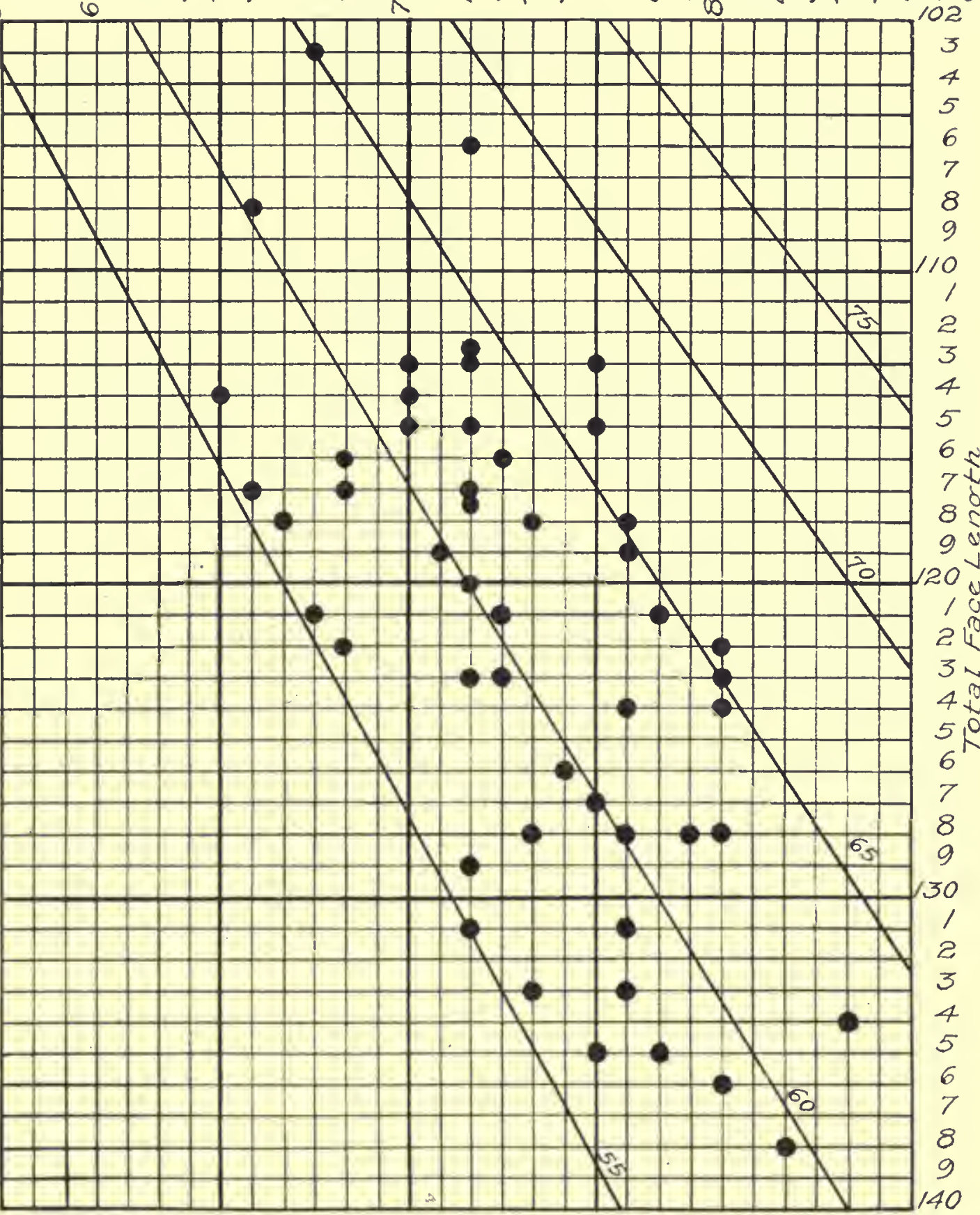

FIG. 26. Chart XXVI. Upper face length (vertical lines) and total face length (horizontal lines) of fifty Iraq soldiers (Nos. 500-551).

total face length and his total face width (bizygomatic) the soldier has greater averages than the Beduin and the Kish groups of Arabs. The Iraq soldier is biggerfaced than the other examples measured by Mr. Field. The fifty soldiers repre- 
sented in Chart XXVI have been examined already as regards diameters of head; their frontal width is greater, their noses shorter and narrower than those of the Beduins and Kish Arabs. The massiveness of face is brought out in the following classification:

\begin{tabular}{cccccc}
\multicolumn{7}{c}{ IRAQ SOLDIERS (NoS. 500-551) } \\
Total face length & $-/ 64$ & $/ 64-/ 70$ & $/ 70-/ 76$ & $/ 76+$ & Totals \\
$-/ 110 \ldots \ldots \ldots$ & 0 & 2 & 1 & 0 & 3 \\
$/ 110-/ 120 \ldots \ldots$ & 0 & 5 & 11 & 4 & 20 \\
$1120-/ 130 \ldots \ldots$ & 0 & 2 & 7 & 9 & 18 \\
$/ 130+\ldots \ldots \ldots$ & 0 & 0 & 2 & 7 & 9 \\
Totals..... & 0 & 9 & 21 & 20 & 50
\end{tabular}

The dispersion of the fifty soldiers is not unlike the second Kish Arab group shown in Chart XXIV, but is unlike the Beduin group (Chart XXII) and the first and third groups of Kish Arabs (Charts XXIII, XXV). In the first and third Arab groups a considerable number fell below the 55-diagonal; among the soldiers there is not one of this class. On the other hand none of the soldiers rises to or above the 70-group. We find, as was to be expected from the average face measurements of the soldier class, a large representation of long faces. Of the fifty men nine have faces $130 \mathrm{~mm}$ in length or more, whereas among the Beduins none reached this long class, and in the third group of Kish Arabs only two. On the other hand, long upper faces are rare. Only four of the fifty have a total face length less than $120 \mathrm{~mm}$, and at the same time reach or cross the $76 \mathrm{~mm}$ standard, whereas among the Beduins eleven of the thirty-eight had upper faces $76 \mathrm{~mm}$ long or more combined with a total face length less than $120 \mathrm{~mm}$. The most marked character of the soldier's face is the more harmonious increase of both parts of the face, the upper as well as the mandibular face. The "ramfaced" type is much less common among soldiers than among the general Arab population. The preponderance of the upper face in the Arab is a character which leads us to seek a relationship among upland peoples of southwest Asia. It is common among the people of Iran and among those who inhabit countries to the north and east of Iran.

My introduction to Mr. Field's monograph has led me to greater lengths than I intended, and in what I have to say regarding the shape of the Arab facethe proportion which its length (naso-mandibular) holds to its width measured between the most distant points of the zygomatic arches-I must be brief. Let us take the faces of the Beduin group first; the Beduin is the essential Arab. In Chart XXVII I have plotted the total face length and total face width of thirty-eight of the Ba'ij Beduins and have set out the results in a table on page 62 .

I recognize for the purposes of the present analysis three groups as regards length of face. I have made the following classes: short, less than $115 \mathrm{~mm}(-/ 115)$; medium, $115 \mathrm{~mm}$ and more but less than $125 \mathrm{~mm}$ (/115-/125); long, $125 \mathrm{~mm}$ or 
more $(/ 125+)$. As regards widths three classes were distinguished: narrow $(-/ 125 \mathrm{~mm})$; medium $(/ 125-/ 135)$; wide $(/ 135+)$. There is only one long-faced Beduin according to this scale; eleven have short faces; twenty-six have faces of medium length. Only eight of the thirty-eight have wide faces; nineteen have faces of medium width; eleven have narrow faces. In only one man does face

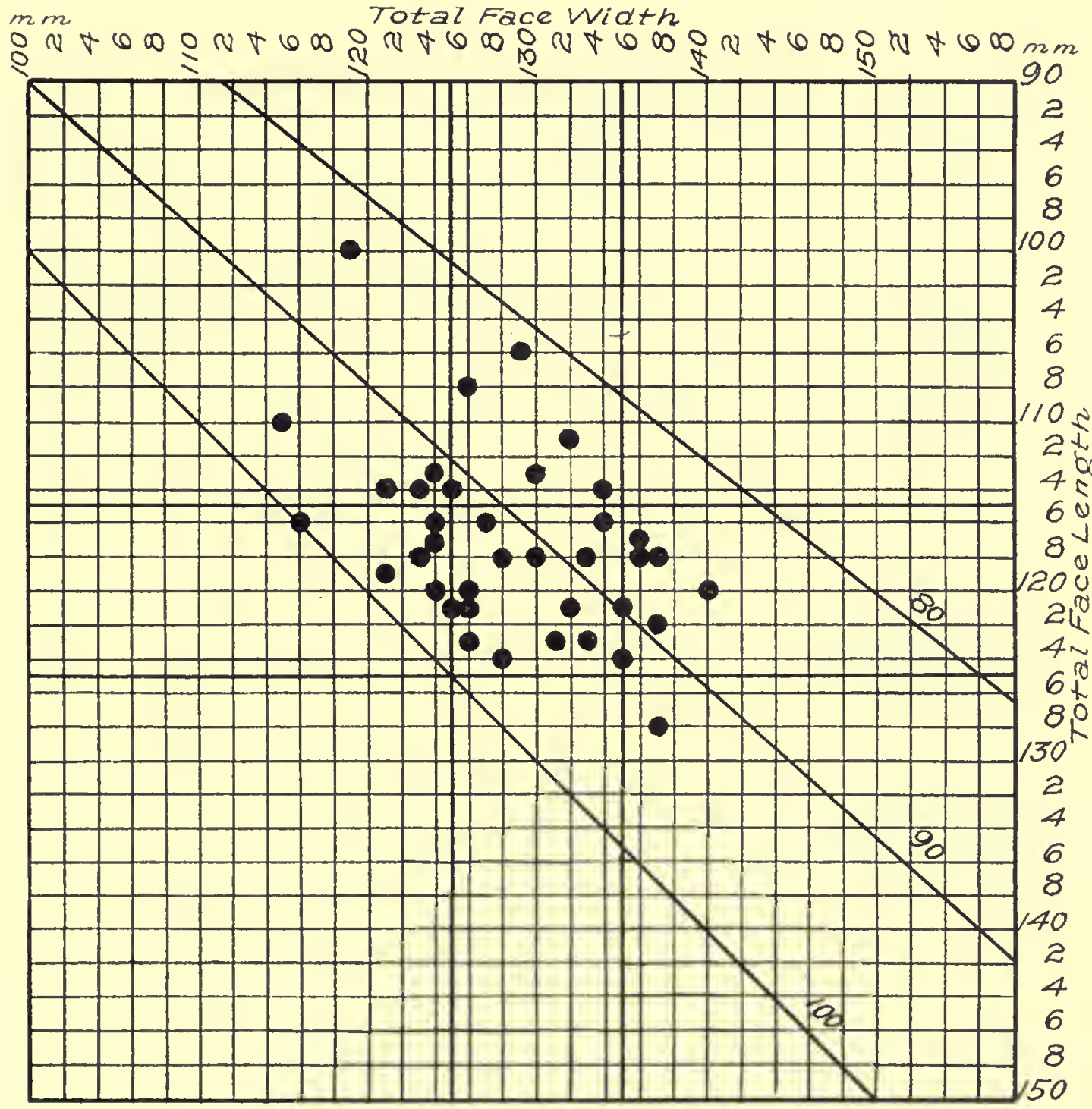

FIg. 27. Chart XXVII. Total face length (naso-mandibular) and total face width (bizygomatic) of thirty-eight Ba'ij Beduins.

length equal face width, and there is not one approaching the 80-diagonal at which the length of face equals 80 per cent of its breadth. The Arab face tends to moderate length and less than moderate width. Thirty of the thirty-eight have faces which are short or of medium length, and are narrow or of medium width. Long and wide faces such as occur often among the Arab soldiers are rare in this small Beduin group. 


\begin{tabular}{|c|c|c|c|c|}
\hline \multirow[b]{2}{*}{ Total face length } & \multicolumn{3}{|c|}{$\begin{array}{l}\text { BA'IJ BEDUINS (38) } \\
\text { TOTAL FACE WIDTH }\end{array}$} & \multirow[b]{2}{*}{ Totals } \\
\hline & $\begin{array}{l}\text { Narrow } \\
-/ 125\end{array}$ & $\begin{array}{l}\text { Medium } \\
/ 125-/ 135\end{array}$ & $\begin{array}{l}\text { Wide } \\
/ 135+\end{array}$ & \\
\hline Short, $-/ 115$ & 5 & 6 & 0 & 11 \\
\hline Medium, /115-/125. & 6 & 13 & 7 & 26 \\
\hline Long, $/ 125+\ldots \ldots$ & 0 & 0 & 1 & 1 \\
\hline Totals. . & $\overline{11}$ & 19 & 8 & 38 \\
\hline
\end{tabular}

Chart XXVIII shows how very different is the distribution of the face measurements in the first group of Kish Arabs (Nos. 1-50). 'There is a very much greater variety in the combinations of length and width than in the Beduins. There is not a single long, narrow face among the Beduins; in this group there are five individuals with such a face. In seventeen individuals the length of the face is $115-125 \mathrm{~mm}$ and the width is less than $125 \mathrm{~mm}$ (see Chart XXVIII). Only six Beduins are in this group. Among the Beduins, faces of medium width (nineteen of thirty-eight) are common; here there are but twelve, whereas there are thirty-two narrow faces. In this group of Kish Arabs the face tends to be longer and narrower, as will be seen in the following classification into groups:

\begin{tabular}{|c|c|c|c|c|}
\hline \multirow[b]{2}{*}{ Total face length } & \multicolumn{3}{|c|}{$\begin{array}{l}\text { BS (NOS. } 1-50) \\
\text { TOTAL FACE WIDTH }\end{array}$} & \\
\hline & $\begin{array}{c}\text { Narrow } \\
-/ 125\end{array}$ & $\begin{array}{l}\text { Medium } \\
/ 125-/ 135\end{array}$ & $\begin{array}{l}\text { Wide } \\
/ 135+\end{array}$ & Totals \\
\hline Short, $-/ 115$ & 10 & 3 & 1 & 14 \\
\hline Medium, $/ 115-/ 125$. & 17 & 2 & 3 & 22 \\
\hline Long, $/ 125+$ & 5 & 7 & 2 & 14 \\
\hline Totals. . & 32 & 12 & 6 & 50 \\
\hline
\end{tabular}

Among the Beduins there were thirteen men with faces which were medium in length and width; in the corresponding class of this group of Arabs there are only two such individuals. The largest class in the Kish Arabs is that to which are assigned faces which are narrow but of medium length.

Before analyzing the facial characters of other groups of Arabs it will be well at this point to refer to the facial dimensions of the typical Egyptian, as seen in the inhabitants of the Khargha Oasis. I have already referred to the data collected by A. Hrdlicka from the men of this oasis. In Chart XXIX the facial dimensions of Egyptian males are represented. When classified according to facial dimensions these fifty Egyptians fall into the following groups:

\begin{tabular}{|c|c|c|c|c|}
\hline \multirow[b]{2}{*}{ Total face length } & $\begin{array}{r}\text { GYPTI } \\
T\end{array}$ & $\begin{array}{l}\text { NS }(50) \\
\text { AL FACE W }\end{array}$ & & \multirow[b]{2}{*}{ Totals } \\
\hline & $\begin{array}{c}\text { Narrow } \\
-/ 125\end{array}$ & $\begin{array}{c}\text { Medium } \\
/ 125-/ 135\end{array}$ & $\begin{array}{l}\text { Wide } \\
/ 135+\end{array}$ & \\
\hline Short,- $/ 115 \ldots \ldots$. & 2 & 15 & 4 & 21 \\
\hline Medium, /115-/125. . & 0 & 19 & 7 & 26 \\
\hline Long, $/ 125+\ldots \ldots$ & 0 & 3 & 0 & 3 \\
\hline Totals. . & 2 & 37 & $\overline{11}$ & 50 \\
\hline
\end{tabular}


The Egyptian faces, like the Egyptian heads, when charted (XXIX) form a wonderfully uniform group - the dispersion being quite different from that met with in all Arab peoples. There are only two eccentric individuals in the fifty Egyptians, one with a short and narrow face, the other with a short and wide face. In not one is the face length equal to the face width; there are

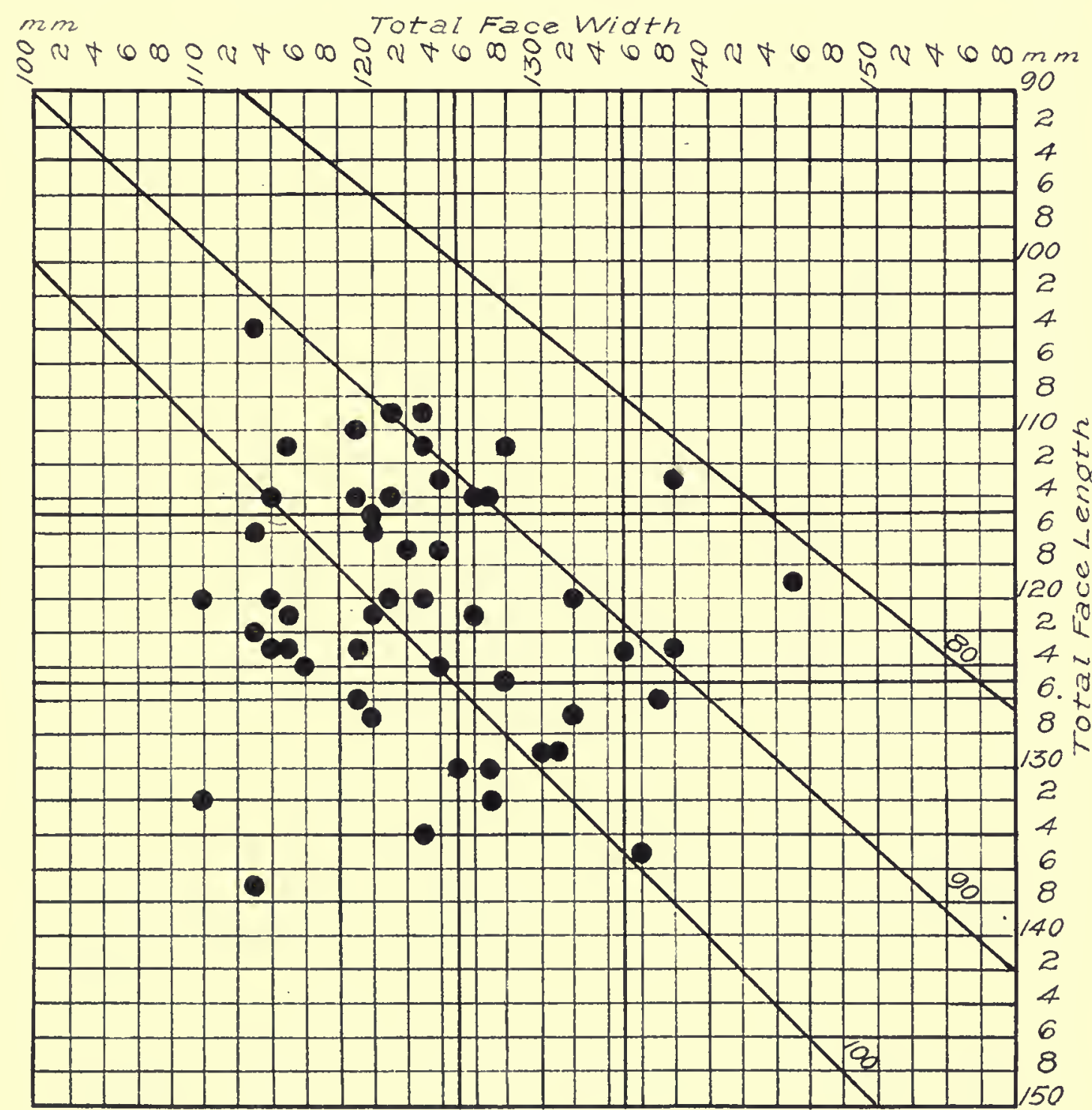

FIG. 28. Chart XXVIII. Total face length (naso-mandibular) and total face width (bizygomatic) of fity Kish Arabs (Nos. 1-50).

four in whom the face length is less than 80 per cent of the face width-Negro faces. In the Egyptian series there are only two narrow faces and both are short. Thirty-four of the fifty men fall into two groups: short faces of medium width, and faces which are medium in width as well as length. There are only three long faces and these are of medium width. There is no massive faceboth long and wide. Of the fifty Egyptians recorded by A. Hrdlicka thirty-seven have a face of medium width, twenty-six of medium length. Short faces are much 
more common among Egyptians than among Arabs; twenty-one of the fifty Egyptians noted here had short faces. The Egyptian face tends to be of medium width, but rather less than medium length. As compared with the Arab its tendency to uniformity is the prominent feature; the Arab face tends to vary widely in all its dimensions. Degrees of variability are a characteristic of race, and the Arab has a tendency to variability in a marked degree.
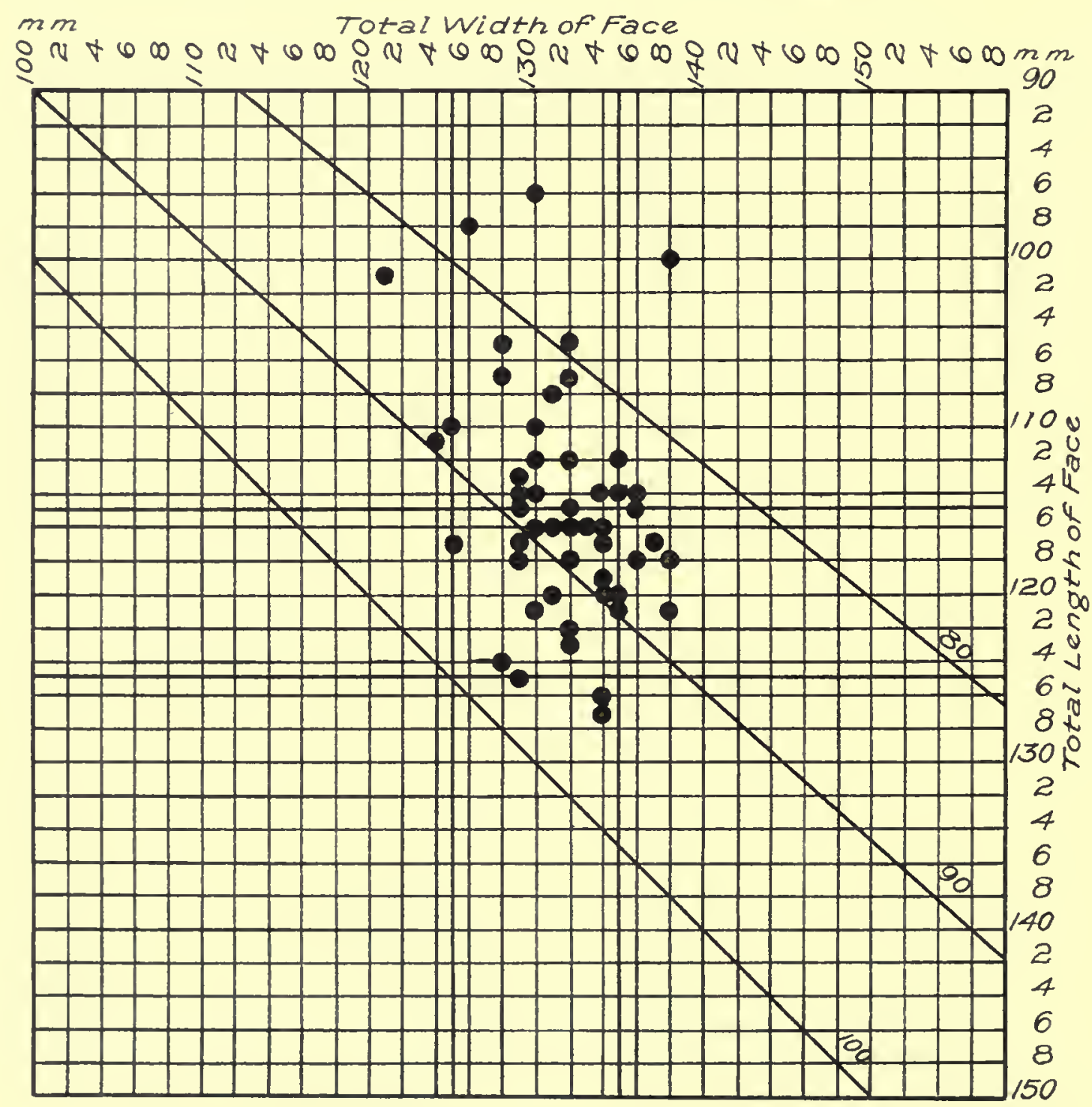

FIG. 29. Chart XXIX. Total face length (naso-mandibular) and total face width (bizygomatic) of fifty Egyptians of the Khargha Oasis (Hrdlicka's measurements).

We now return to the faces of the Kish Arabs. In Chart XXX is depicted the distribution of fifty faces (Nos. 100-150) according to length and breadth.

How different this group of Arabs is as regards face shape and size from the group depicted in Chart XXVIII is very apparent, and is at once brought out in the proportion of narrow-faced individuals. In the group containing Nos. 1-50 there were thirty-two Arabs with narrow faces, here there are but three. 
In the first group of Kish Arabs the people with faces of medium width numbered twelve; in this second group they amount to thirty-five. There were six widefaced people in the first group; in this there are twice as many, twelve. In the second group the long faces number twenty, in the first they numbered fourteen. In this second group we have, as among the soldiers, a tendency to
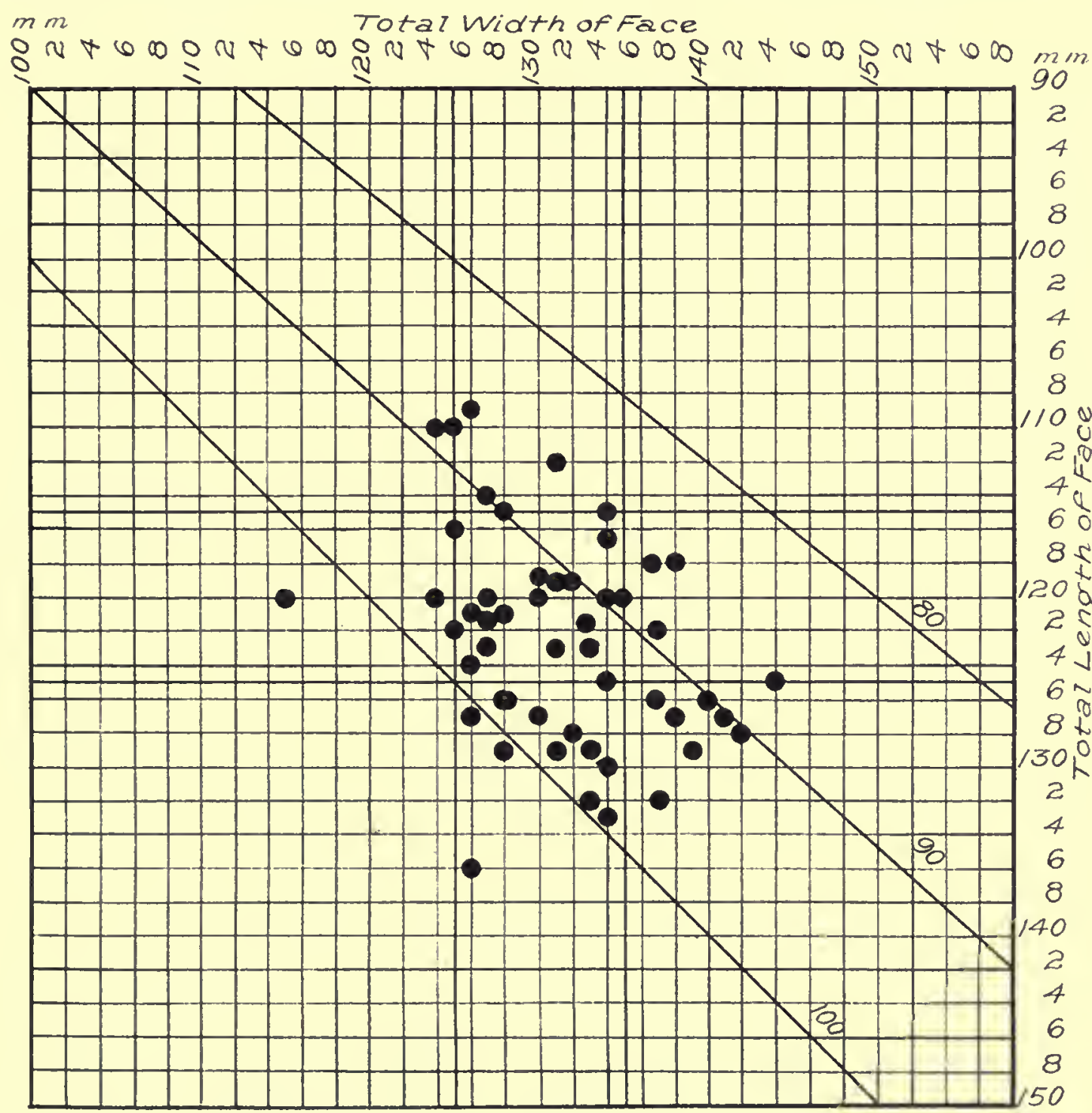

FIG. 30. Chart XXX. Total face length (naso-mandibular) and total face width (bizygomatic) of fifty Kish Arabs (Nos. 100-150).

massiveness of face - a face both long and wide. In the first group there were seventeen in whom the face length was equal to the face width or more; in this, the second group, there are but four. To find so great a local or group difference seems to me an evolutionary fact of great importance. This group differs as much from the Beduin group as from the first group of Kish Arabs. In the second group the spread is great, but in the first it was toward narrowness and shortness, here toward width and length of face, as in the Iraq soldiers. The table gives the following classification of the faces: 


\begin{tabular}{|c|c|c|c|c|}
\hline \multirow[b]{2}{*}{ Total face length } & RABS & TOTAL FACE WIDTH & & \multirow[b]{2}{*}{ Totals } \\
\hline & $-/ 125$ & $/ 125-/ 135$ & $/ 135+$ & \\
\hline Short, $-1115 \ldots$ & 1 & 4 & 0 & 5 \\
\hline Medium, /115-/125. . & 2 & 19 & 4 & 25 \\
\hline Long, $/ 125+\ldots \ldots$. & 0 & 12 & 8 & 20 \\
\hline Totals. & 3 & 35 & 12 & 50 \\
\hline
\end{tabular}

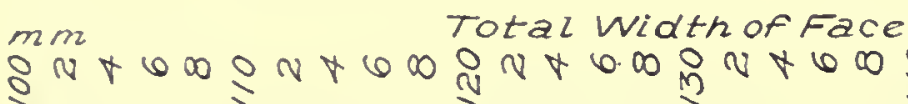

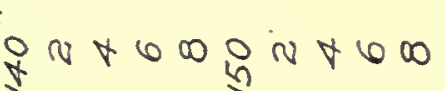

$m m$

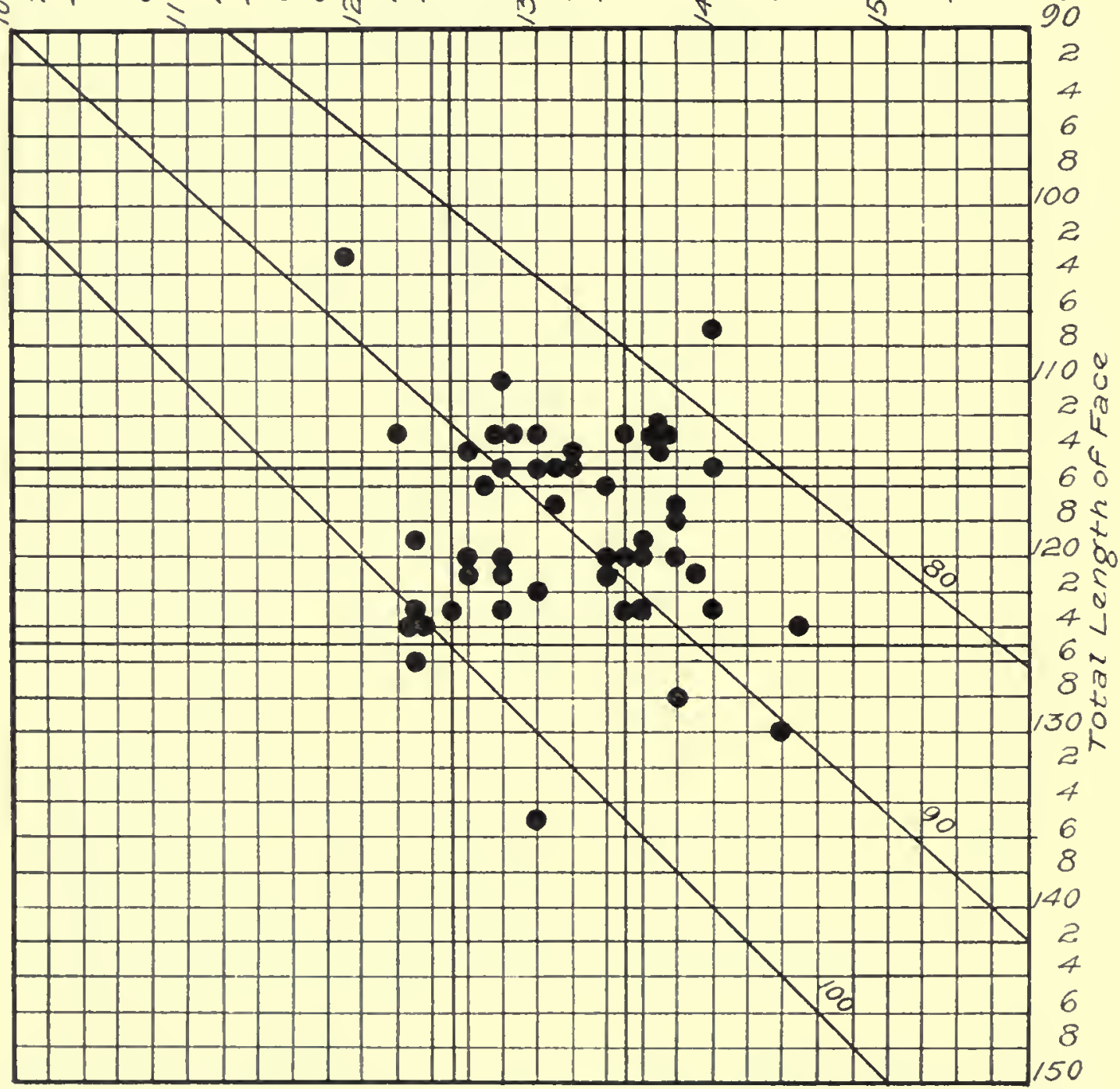

FIG. 31. Chart XXX1. Total face length (naso-mandibular) and total face width (bizygomatic) of fifty Kish Arabs (Nos. 201-250).

As the second group of Kish Arabs (Nos. 100-150) gave a variety of face forms so different from that encountered in the first group of Kish Arabs (Nos. 1-50), I took a third group (Nos. 201-250). The distribution of the facial forms of this third group is shown in Chart XXXI and its classification is as follows: 


\begin{tabular}{|c|c|c|c|c|}
\hline $\mathrm{KISH}$ & ARAB & $\begin{array}{l}\text { Nos. } 201 \\
\text { AL FACE }\end{array}$ & & \\
\hline Total face length & $-125 /$ & $/ 125-/ 135$ & $/ 135+$ & Totals \\
\hline Short, $-/ 115 \ldots \ldots \ldots$ & 2 & 6 & 6 & 14 \\
\hline Medium, /115-/125. . & 4 & 16 & 12 & 32 \\
\hline Long, $/ 125+\ldots . .$. & 1 & 1 & 2 & 4 \\
\hline Totals. & 7 & 23 & 20 & 50 \\
\hline
\end{tabular}

In the second group (Nos. 100-150) there were twenty Arabs with long faces and only twelve who had wide faces. Here the proportions are reversed; there are only four individuals with long faces, while twenty people are in the wide-faced classes. In group one (Nos. 1-50) there were thirty-two Arabs with narrow faces; here there are only seven. The present group (Chart XXXI) is in several respects intermediate to the groups shown in Charts XXVII (Beduins), XXVIII (Kish Arabs), and XXX (Kish Arabs). To me the occurrence of such groups, varying as regards dimensions of face, is of high interest. I do not think they are the result of random sampling, but represent local evolutionary specialization. The one point which is demonstrated is the extreme variability of the Arab as regards relative and absolute dimensions of face. And yet, in spite of this variability, expert travelers are able to recognize the Arab at sight.

The interpretation of the distributional curves which have been constructed from this data I must leave to those who have the necessary mathematical equipment. For my part I never like to lose sight of the individuals during the process of analysis; hence my adoption of graphic forms such as the charts shown above. I am sure that an examination of Figure 43, which shows the relationship of length to breadth diameters of the face, will repay examination. The curves rise steeply to an apex, and then fall slowly toward the right. I suspect this skew form is due to the tendency to narrowing of the face among most groups of Arabs and also to the peculiar and characteristic development of the upper face in so many.

Another matter into which Mr. Field inquired is of great interest. It refers to the manner in which the upper and lower incisor teeth meet. In the young of all races, when the incisors have just erupted, the lower incisors ascend behind the upper in mastication. In primitive races, among people who live much on uncooked food and use the incisor teeth for which civilized people employ knives, the incisor teeth in biting and chewing meet in the edge-to-edge position. In the more civilized races the overlap of childhood is retained; the natural edge-to-edge apposition of the incisors is seen only in a very small percentage of civilized individuals. In twenty-nine Ba'ij Beduins, Mr. Field found a marked incisor overlap in six (20.7 per cent), a slight overlap in twentythree; none of the Beduins had the edge-to-edge bite. Among 139 soldiers 0.7 per cent had under bite, 1.4 per cent had edge-to-edge, 90 per cent had slight overlap, while in 7.9 per cent there was marked overlap. In this point the Arab does not differ greatly from Europeans. Of the soldiers 99 per cent had sound dentitions, wherein they differ very much from soldiers of the west. 
I have already had occasion to call attention to the average stature of the various groups of men examined. The soldiers were, on an average, two inches taller than the other Arabs. Mr. Field is inclined to attribute this difference to physical training, but as we have found that the soldiers possess many other physical peculiarities besides excess of stature, I am inclined to think that the majority of the soldiers are not drawn from the same classes as the Beduins and Kish Arabs. They are selected individuals but even selection does not account for their wide foreheads, noses shorter and narrower than those of Beduin and Kishite, and the width, length, and massiveness of their faces.

I am now to make a brief analysis of the stature of the Arabs, taking first the Beduin group (thirty-eight). My method of analysis will be understood from an examination of Chart XXXII. We want to ascertain not only the frequency with which the various degrees of stature occur among the Arabs but also how far the stature of each individual depends on length of trunk or on length of leg. As regards stature I use four classes: the short, under $1600 \mathrm{~mm}(-/ 1600)$; the moderate or medium in stature, under $1700 \mathrm{~mm}$ but $1600 \mathrm{~mm}$ and over $(1700 /-$ $1600 /)$; the tall, under $1800 \mathrm{~mm}$ but $1700 \mathrm{~mm}$ and over $(1800 /-1700 /)$; the very tall $(/ 1800+)$. As regards length of trunk we have to make a finer division: very short, trunks under $750 \mathrm{~mm}(750 /-)$; short $(800 /-750 /)$; medium length $(850 /-800 /)$; long trunks $(900 /-850 /)$; and very long $(+900 /)$. When the sitting height stature of each of the thirty-eight Beduins is plotted we are again struck by the variability of the Arabs; this is brought out when they are classified according to their places in the chart.

\begin{tabular}{|c|c|c|c|c|c|c|}
\hline \multirow[b]{2}{*}{ Standing height } & \multicolumn{5}{|c|}{$\begin{array}{l}\text { BA'IJ BEDUINS ( } 38 \text { ) } \\
\text { Sitting Height (TRunk Length) }\end{array}$} & \multirow[b]{2}{*}{ Totals } \\
\hline & $\begin{array}{l}\text { Very long } \\
+900 /\end{array}$ & $\begin{array}{l}\text { Long } \\
900 /-850 /\end{array}$ & $\underset{850 /-800 /}{\text { Medium }}$ & $\begin{array}{c}\text { Short } \\
800 /-750 /\end{array}$ & $\begin{array}{l}\text { Very short } \\
750 /-\end{array}$ & \\
\hline Very tall, $/ 1800+\ldots$ & 0 & 0 & 0 & 0 & 0 & 0 \\
\hline Tall, $1800 /-1700 / \ldots$. & 1 & 7 & 3 & 1 & 0 & 12 \\
\hline Mediun, $1700 /-1600 /$. & 1 & 3 & 15 & 4 & 0 & 23 \\
\hline Short, $-/ 1600 \ldots . .$. & 0 & 0 & 2 & 1 & 0 & 3 \\
\hline Totals. . & 2 & 10 & 20 & 6 & 0 & 38 \\
\hline
\end{tabular}

Of the thirty-eight Beduins none rises to the $1800 \mathrm{~mm}$ limit ( 5 feet 10.8 inches), twelve are tall ( 5 feet 7 inches or more), twenty-three are of medium stature (more than 5 feet 3 inches), and three are short (below 5 feet 3 inches). Of the thirty-eight, two have really long trunks, six have short trunks. Foul men who rise into the tall group as regards stature have trunks which are moderate or short in length. These individuals are tall because of length of leg. On the other hand one longest-trunked man falls into the group of medium stature; another man, in the tall group, is there because of length of trunk. Three individuals fall into the short group (below 5 feet 3 inches); one is in this group because of shortness of trunk, the other two because of shortness of legs. Into Charts XXXII-XXXV I have introduced diagonals which separate the long- 
legged individual on the one side and the long-trunked individual on the other. The middle diagonal (A) is drawn so that it marks the line on which individuals whose trunks contribute 50 per cent to their stature would fall. The diagonal $\mathrm{B}$ marks the line on which the trunk contributes 47 per cent and the
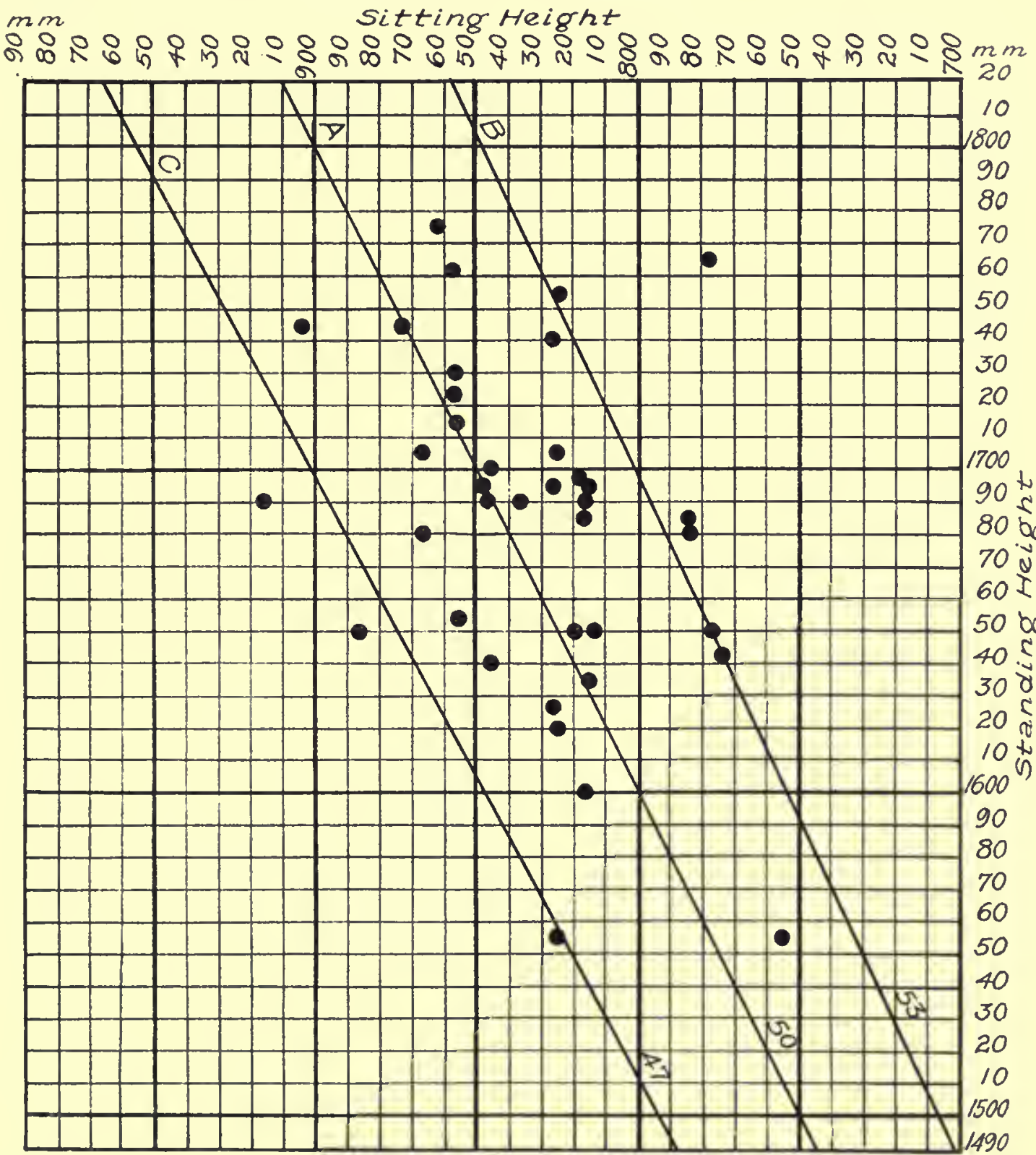

FIG. 32. Chart XXXII. Stature (horizontal lines) and sitting height (vertical lines) of thirty-eight $\mathrm{Ba}$ "ij Bcduins. Individuals falling on the middle diagonal (A) have a sitting height which is half their standing height. Diagonal $\mathrm{B}$ marks the line on which fall individuals whose limbs form 53 per cent of their standing height, diagonal $\mathrm{C}$ those in whom the limbs form 47 per cent of the stature.

limbs 53 per cent. The diagonal $\mathrm{C}$ indicates trunk 53 per cent and limbs 47 per cent. Thus the individuals falling outside or to the right of $B$ have a standing height in which the limbs make up 53 per cent or more of the stature; those falling on or outside $\mathrm{C}$ have trunks which contribute 53 per cent or more. In three Beduins the lower limbs make up 47 per cent or less of the stature; in 
five the limbs make up 53 per cent or more of the stature. It is sufficient for my present purpose to show how variable are the Arabs in stature, in trunk length, and in the combination of the two.

On every previous occasion when we had to contrast Egyptian with Arab, we have found the Egyptian the more stable in characters of body. It is so as l'egards standing height and sitting height. In Chart XXXIII are set out measurements made by A. Hrdlicka of the Egyptians of the Khargha Oasis and in the accompanying table the various combinations of stature and trunk length are classified:

\begin{tabular}{|c|c|c|c|c|c|c|}
\hline \multirow{2}{*}{ Standing height } & \multicolumn{5}{|c|}{$\begin{array}{l}\text { EGYPTIANS }(50) \\
\text { SITTING HEIGHT (Trunk }\end{array}$} & \multirow[b]{2}{*}{ Totals } \\
\hline & $+900 /$ & $900 /-850 /$ & $850 /-800 /$ & $800 /-750 /$ & $750 /-$ & \\
\hline $1800+\ldots \ldots$ & 0 & 0 & 0 & 0 & 0 & 0 \\
\hline $1800 /-1700 / \ldots$ & 0 & 5 & 1 & 0 & 0 & 6 \\
\hline $1700 /-1600 / \ldots \ldots \ldots$ & 0 & 17 & 19 & 0 & 0 & 36 \\
\hline$-/ 1600 \ldots \ldots \ldots \ldots$ & 0 & 0 & 7 & 1 & 0 & 8 \\
\hline Totals... & 0 & 22 & 27 & 1 & 0 & 50 \\
\hline
\end{tabular}

The Egyptian statures when plotted out (Chart XXXIII) form a compact array, very different from the Beduins in Chart XXXII. In increase of stature, trunk and limbs are involved to a nearly equal extent. But whereas well over half of the Beduins gained stature from length of leg the opposite is the case among the Egyptians; in only three do the legs take a larger part than the trunk in making the stature. In seven Egyptians the trunk forms 53 per cent of stature, or more. There were two Beduins with very long trunks $(900+)$; no Egyptian falls in this class. Thirty-six Egyptians fall into two neighboring compartments; in the same two compartments there are only eighteen Beduins. There are twelve Beduins more than $1700 \mathrm{~mm}$ in stature but only six of the fifty Egyptians are in this class. There are three short Beduins and eight short Egyptians. On the other hand there are twenty-two Egyptians who have long trunks $(900 /-850 /)$; only ten of the Beduins fall in this group. The Egyptian and Arab differ in the contributions made to their stature by trunk and by limb; the Egyptian is relatively long-trunked; the Arab long-legged.

We return to the consideration of stature and trunk length of the Arabs. The following is the classification of fifty of the Kish Arabs (Nos. 1-50):

\begin{tabular}{|c|c|c|c|c|c|c|}
\hline \multirow[b]{2}{*}{ Standing height } & \multicolumn{4}{|c|}{ KISH ARABS (Nos. 1-50) } & \multirow[b]{2}{*}{$750 /-$} & \multirow[b]{2}{*}{ Totals } \\
\hline & $+900 /$ & $900 /-850 /$ & $850 /-800 /$ & $800 /-750 /$ & & \\
\hline$/ 1800+\ldots \ldots \ldots$ & 0 & 0 & 0 & 0 & 0 & 0 \\
\hline $1800 /-1700 / \ldots \ldots$ & 0 & 8 & 10 & 0 & 0 & 18 \\
\hline $1700 /-1600 / \ldots \ldots$ & 1 & 3 & 14 & 6 & 0 & 24 \\
\hline$-/ 1600$. & 0 & 0 & 0 & 3 & 5 & 8 \\
\hline Totals... & 1 & 11 & 24 & 9 & 5 & 50 \\
\hline
\end{tabular}


The grouping of these Arabs is not as scattered as that of the Beduins, but still much more than that of the Egyptians. There is a great variation in stature; eighteen individuals are in the tall class, twenty-four have a medium stature, and eight men are short.

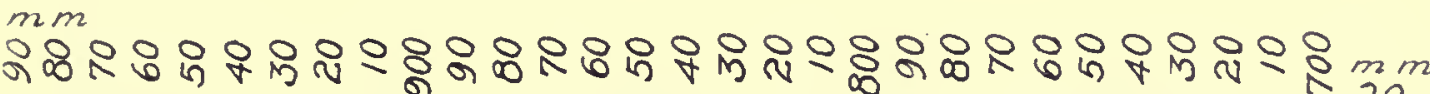

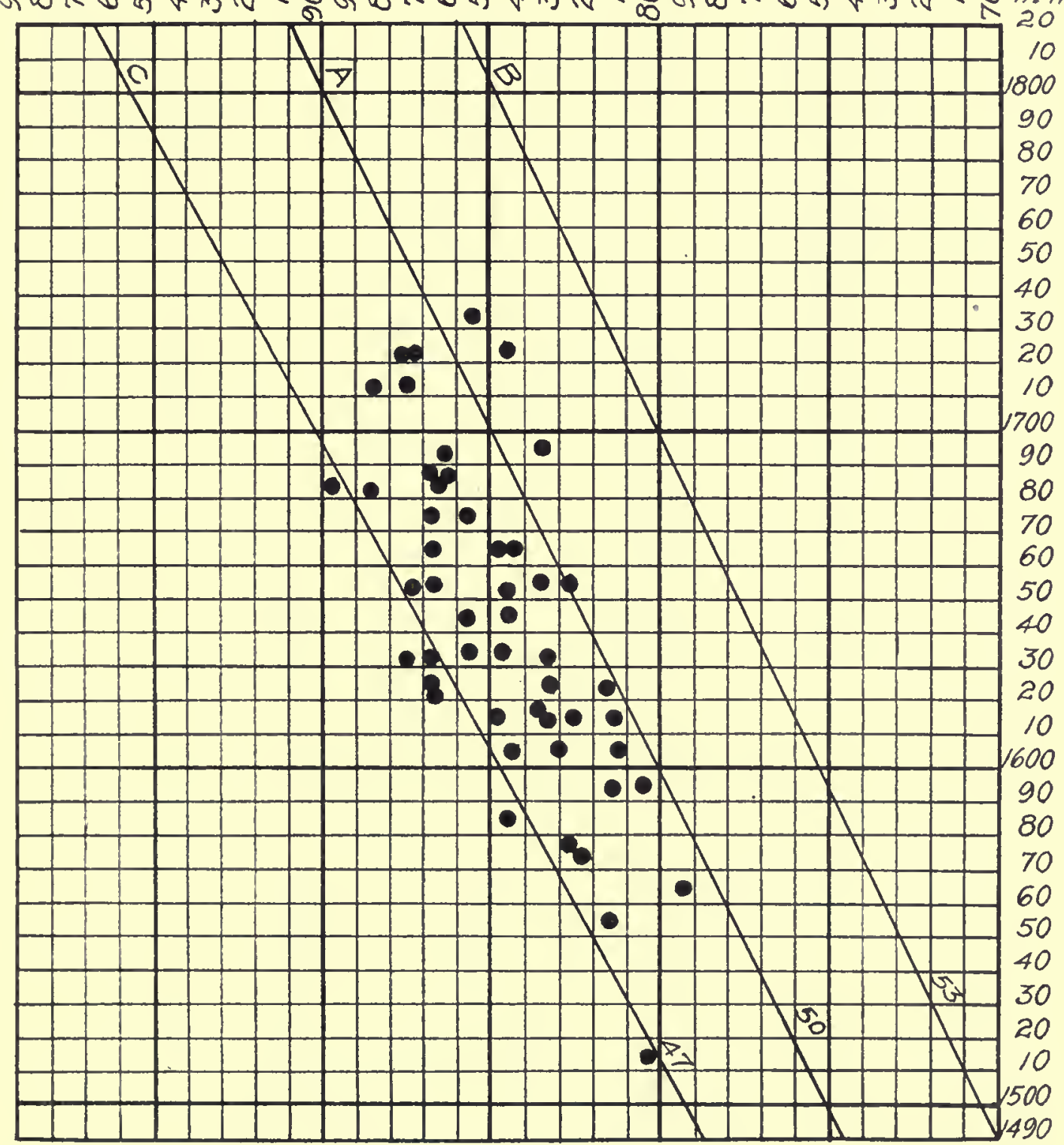

FIG. 33. Chart XXXIII. Stature (horizontal lines) and sitting height (vertical lines) of fifty Egyptians measured by A. Hrdlicka in the Khargha Oasis. Diagonal lines as in Chart XXXII.

As regards length of trunk the variation is also very great: twelve fall in the long-trunked groups; fourteen in the short-trunked groups. In six the lower limbs are 53 per cent or more of the stature; in only one man does the trunk form more than 53 per cent of the stature. Of the short-trunked individuals six are of moderate stature, eight are of short stature. The man with the longest 
trunk falls into the medium stature group. The distribution is not unlike that of the Beduins (Chart XXXII) except that among the latter there are fewer short people, especially people who are short because of reduction of both trunk

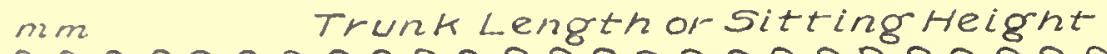

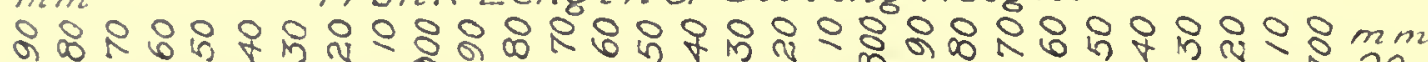

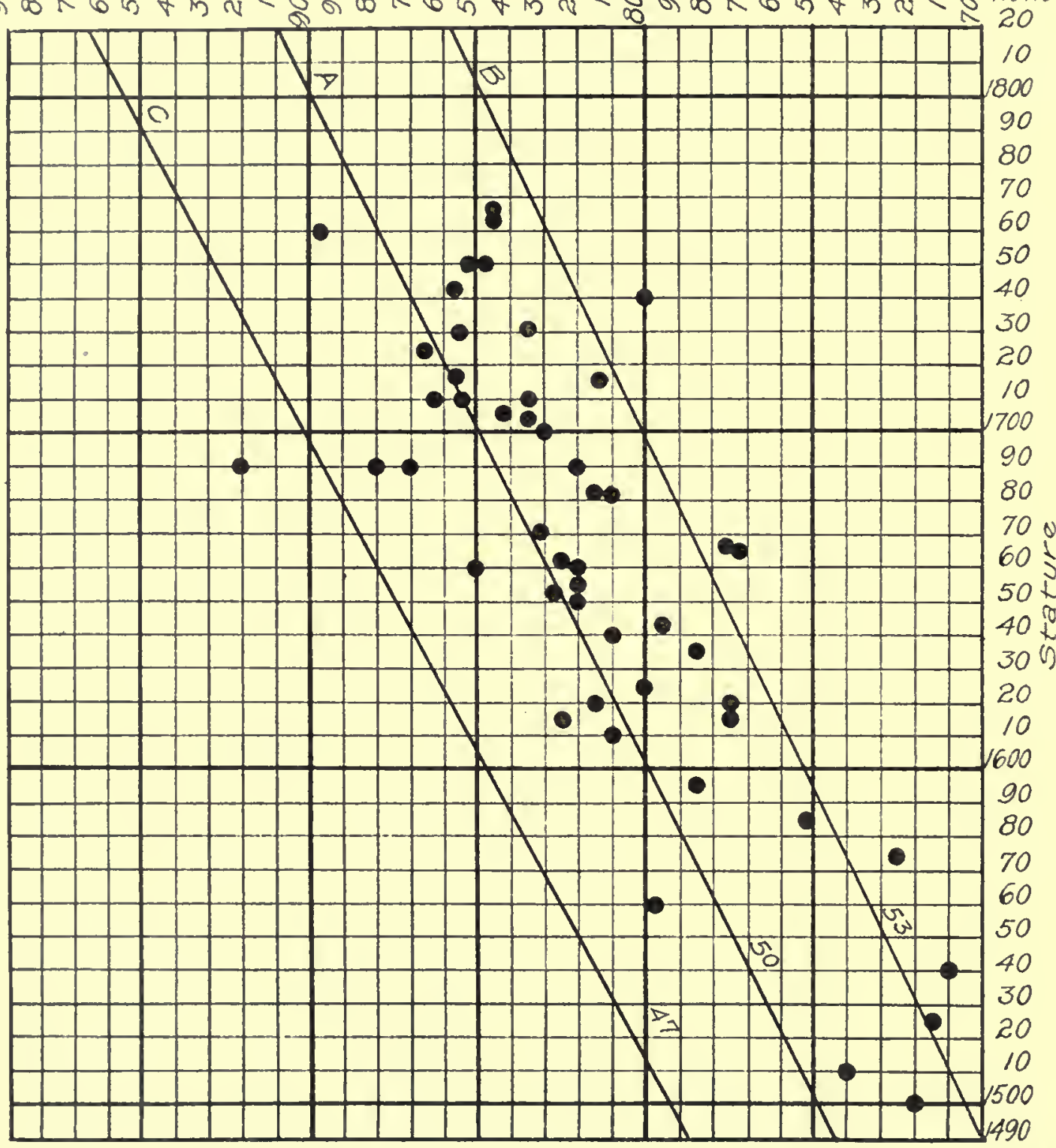

FIG. 34. Chart XXXIV. Stature (horizontal lines) and sitting height (vertical lines) of fifty Kish Arabs (Nos. 1-50). Diagonal lines as in Chart XXXII.

and limbs. There are twice as many long-legged Arabs in this group as in the Beduin group.

In order to ascertain the amount to which one group of Kish Arabs may differ from another group, I plotted the stature and sitting height of another section (Nos. 101-150). The results are shown in Chart XXXV and in the following table: 
KISH ARABS (Nos. 101-150)

SitTing Height (TRUNK Length)

\begin{tabular}{|c|c|c|c|c|c|c|}
\hline Standing height & $+900 /$ & $900 /-850 /$ & $850 /-800 /$ & $800,-750 /$ & $750 /-$ & Totals \\
\hline $1800+\ldots$ & 0 & 1 & 0 & 0 & 0 & 1 \\
\hline $1800 /-1700 / \ldots \ldots \ldots$ & 0 & 4 & 10 & 4 & 0 & 18 \\
\hline $1700 /-1600 / \ldots \ldots \ldots$ & 0 & 1 & 11 & 13 & 1 & 26 \\
\hline$-/ 1600$. & 0 & 0 & 2 & 1 & 2 & 5 \\
\hline Totals. & 0 & 6 & 23 & 18 & 3 & 50 \\
\hline
\end{tabular}

The distribution of the second group (Chart XXXV), although it corresponds in many respects to that shown in Chart XXXIV, differs in some respects. If we arrange the men in stature classes, these are almost identical in both lots of Arabs. But if we group the trunk lengths, we see that in the second series of Arabs there are eighteen in the short-trunked class, whereas in the first there are but nine. The first series has more long-trunked individuals. I have brought this difference out by the use of the diagonal lines. In the first series, six individuals are long-legged on this scale, i.e. have limbs which contribute 53 per cent or more to the stature; in the second series there are nineteen such long-legged individuals. The Arab stature is highly variable both in its absolute amount and in the proportions which trunk and limbs contribute to it.

The following conclusions may be presented. Considering the part that Arabs have played in the history of the world, as pioneers of civilization, as early cultivators of knowledge, and especially as the initiators in the development of religions, we have neglected them sadly in an anthropological and racial sense. No doubt our lack of information concerning the physical make-up of the Arab is due to Arab aloofness rather than to negligence on the part of anthropologists. Mr. Field has overcome all obstacles and in this monograph makes a royal gift to his fellow workers. He has made the Arab known to us in the mass. Where there was dearth of knowledge there are now facts in plenty. Altogether he has made records of 667 individuals; his photographic records are of inestimable value.

After working through these data, what are we to say as to the prominent features of this ancient people? I think the most conspicuous character is their degree of variability in every one of the physical characters tested. The second is the prevailing smallness of head, a characteristic we meet with among the Egyptians and more especially among the peoples of India. Nevertheless in every group there are a few big heads. I lay stress on these exceptional individuals because, although they may not represent the actual leaders of their community, they do represent Galton's exceptional individuals - thrown up in every animal community to play the part of leaders. Arabs are neither tall nor heavy men; their stature ranges from 5 feet 4 inches to 5 feet 8 inches; a very few tall peoplemore than 5 feet 11 inches in stature-occur; diminutive men are also very common.

It was fortunate that Captain Bertram Thomas should have placed the data he collected among the South Arabs at my disposal when I was in the midst of 
preparing this Report. No doubt the difference in physique between the South Arab and the North Arab is remarkable. The South Arab is predominantly round-headed and small-headed; the North Arab is also small-headed but longheaded. Among the North Arabs round heads do occur, but never-if we except $m m$ Sitting Height

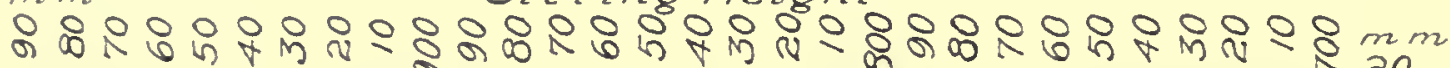

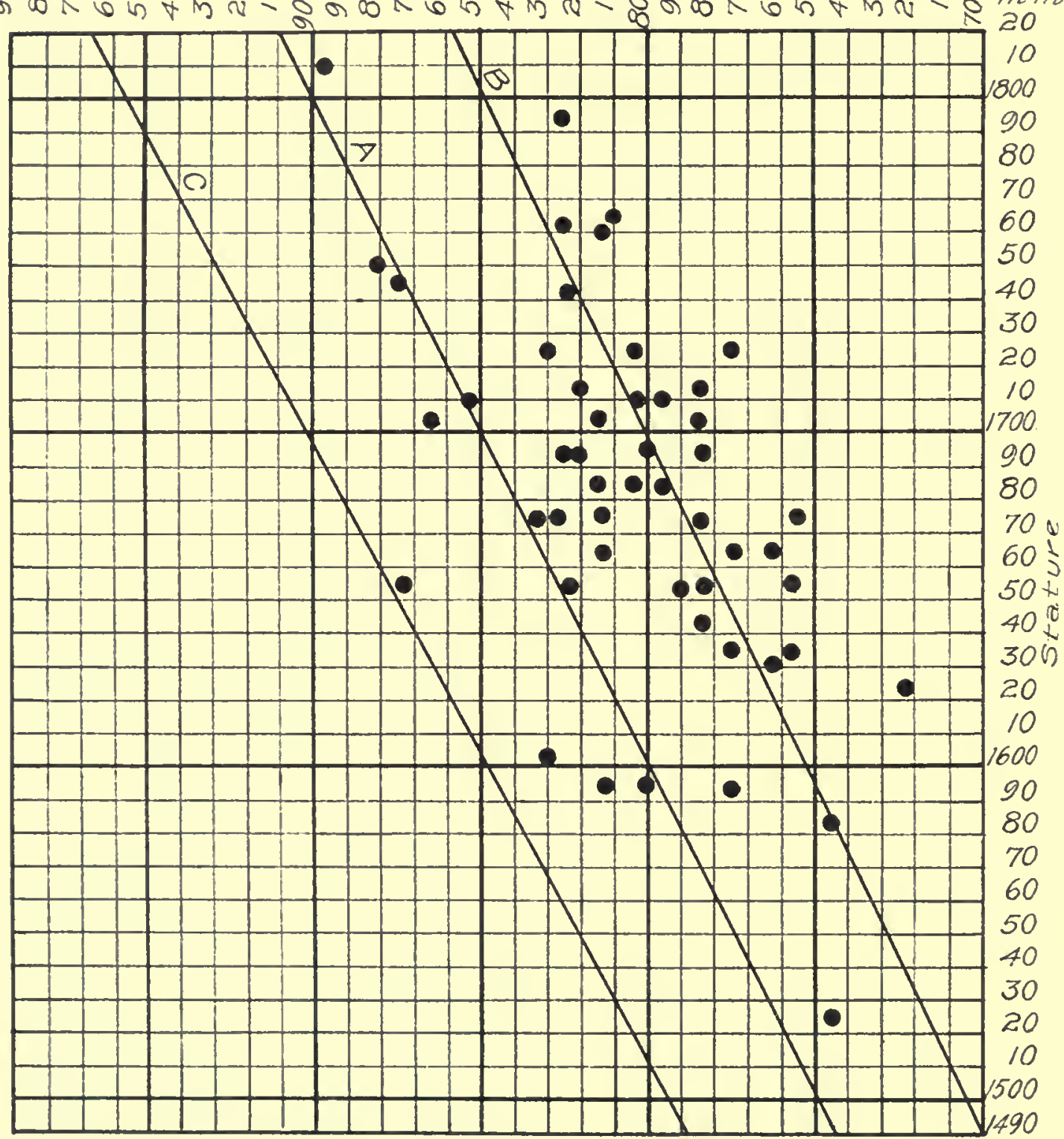

FIG. 35. Chart $\mathrm{XXXV}$. Stature (horizontal lines) and sitting height (vertical lines) of fifty hish Arabs (Nos. 101-150). Diagonal lines as in Chart XXXII.

northern communities - among more than 10 per cent of the general population. It is possible that the round-headedness of Arabs is due to racial contamination by peoples from the northern brachycephalic belt, but it may be that the brachycephalic individuals merely represent the normal extreme of a pure mesocephalic or dolichocephalic people. Certainly the brachycephaly of the 
South Arab cannot be explained in this way; the right explanation is not yet apparent. Among the South Arabs, too, fuzzy hair and other Hamitic traits, which link them to peoples of northeast Africa, occur much more frequently than among the North Arabs. In the south one never sees the clear-cut features of the Beduin of the north. And yet a comparison of North and South Arabs has led me to the conclusion that these two peoples must be counted members of the same race. In the South Arab as in the North we see, peering through a dark brown skin, the facial and bodily features which link the Arab race to the Caucasian stock and separate it from the equally dark-skinned peoples of Africa and India.

How does the Arab stand with regard to other races of mankind? On entering into this inquiry we must note the relationship of Arabia to adjacent racial frontiers. The Red Sea separates the great Arabian peninsula from the Hamitic peoples of Africa, many of which, to be sure, have received Arab infusion. Arabia is separated from the mainland of Asia by the Persian Gulf and the Gulf of Oman. This inlet of the Indian Ocean is also a racial frontier separating the Arab from a people not remotely akin to him, people of the Indo-Afghan type. Also, in the north the base of the peninsula abuts on another racial frontier, the southern frontier of the main or purer Caucasian stock. Then away in the east are the peoples of India, who have many other resemblances to the Arab besides a dark brown skin and dark brown or black hair. If we presume that the modern stocks of mankind have been evolved in or near the regions which they now occupy then we ought to find that the Arab has an evolutionary relationship to all surrounding peoples. That is what we have found in the course of our analysis. The Arab shares traits with Hamitic peoples of Africa, with the Dravidian and Indo-Aryan peoples of India, and with the peoples which extend from the gates of India to the Levant. The Arab's facial features are often so Caucasoid in appearance that we may mistake him for a South European but his pigmentation is usually deepel than that seen in South Europeans. Undoubtedly in his composition we recognize many Negroid traits, and traits which link him with Dravidian and with Hamite.

Now, how are we to account for Arabia's being occupied by people who are mainly Caucasian in their physical make-up and yet possess so many features in common with dark-skinned neighboring races? In seeking to explain these facts there are other circumstances and relationships which have to be considered. Even today a belt of pigmented human races crosses the Old World. At one extreme we have the Negro of Africa, at the other extreme the Negro of the Pacific. India lies midway in this pigmented belt, one which we suspect extended continuously in Pleistocene times from one extremity of the Old World to the other. On this theory the original inhabitants of Arabia were deeply pigmented and akin to the Hamites of Africa on the one hand and to the Dravidians of India on the other. To the north of the black belt there were two other evolutionary centers: the Mongolian, north of the Himalayas, and the Caucasian, north of the upland mountainous plateau which extends westward from the Himalayas across Iran to Asia Minor. That there was an early break-through from the Mongolian center at the eastern end of the 
Himalayas is manifest; the Mongol stock at different times broke into the black belt and spread out in the Pacific. There was a Caucasian southward migration at the western end of the Himalayas. In Pleistocene times the great Arabian peninsula was a land to tempt adventurous hunters. The peoples of Arabia might thus represent a mixture of darker-skinned Dravidians into which invaders from the southern or Semitic fringe of the Caucasian center had infused their blood. Such a theory explains many of the facts relating to the racial composition and affinities of the inhabitants of Arabia. Or did the evolutionary center of the Caucasian type actually extend into Arabia? In the latter case the Arab would be a pure evolutionary race.

Our interest in the ancient inhabitants of Arabia, particularly of the northern plain, has been stimulated by the expectation that we shall yet be able to prove that our modern way of living-our modern civilization-was initiated by a people or peoples living on or near the frontier of northern Arabia. Were the pioneers of civilization really Arabs (Semites)? Or were they of the less deeply pigmented Caucasian stock farther to the north? We have little evidence to sway us either way, but the only real difference I can perceive between the ancient Mesopotamians of Kish (fourth millennium B.C.) and the modern Arabs of central Iraq relates to size of skull and brain. The average cranial capacity of the ancient Mesopotamian or Arab exceeded that of the average modern inhabitant of central Iraq. I expect that it will yet be proved that the Arab of today is the descendant of the men who built the ancient cities and early civilization along the Euphrates and Tigris rivers. 


\section{ARABS OF THE KISH AREA}

\section{GEOGRAPHICAL LOCATION}

Ancient Mesopotamia, now called Iraq, was on the alluvial plain between the Euphrates and Tigris rivers. This location is of considerable importance, as it was undoubtedly one of the main factors which contributed to the political domination of this region in the beginning of the historical period.

Let us examine the geographical position of Iraq. To the west lie the North Arabian and Syrian deserts, which were fertile and well watered when man in various paleolithic periods inhabited this area. The general contour of this region shows a gradual rise from water level at the Euphrates to about 2,700 feet near landing ground $P$, and then a slight downward slope until the Harrat-ar-Rajil is reached. Farther to the west lie Syria, Palestine, and TransJordan. To the north of Iraq are Anatolia and the inhospitable mountain regions of Lake Van. To the east lies Iran, and to the south the Persian Gulf, with a long sea passage to India. The southwestern borderland of Iraq joins the great wilderness and deserts of Arabia.

There are, however, two gorges through which the Mesopotamian plain can be reached. These passes in the eastern mountains, which separate the plain from Iran, are located near Rowandiz and Sulaimaniya, where archaeological research should be conducted. Thus, from a geographical point of view, the hypothetical "Garden of Eden" was located in a position destined to be the center of cultural development as well as the natural battlefield for various peoples. It is therefore small wonder that the ancient city of Kish was founded on the banks of the Euphrates, since the site of this city is in the central southern portion of Iraq. Historical evidence shows that Kish was the "first city founded aftel the Flood," and from a geographical point of view this was not altogether unexpected. Kish lies sixty-five miles south of Baghdad and eight miles due east of the ruins of Babylon. The region in which these anthropometric studies have been made is in the vicinity of Kish, where the Field Museum-Oxford University Joint Expedition conducted excavations from 1922 to 1933.

\section{EVIDENCE OF PREHISTORIC CULTURE}

It is desirable to trace in outline the main features which dominated the history of Kish from a late neolithic phase of culture down to the time of the modern Arabs, who form the main study of this publication. At Kish no evidence for the existence of paleolithic man has yet come to light, although in a limited area virgin soil has been reached. At the time when man in the various paleolithic stages of culture inhabited northern Iraq and the North Arabian Desert and adjacent territory, I believe that the entire alluvial plain, at least south of Baghdad, was under water. This large lake connected with the Persian Gulf to the south and was replenished by the Tigris and the Euphrates. These two 
rivers at that time carried an enlarged volume of water owing to the increased rainfall over this entire area. Geological and climatological evidence lends support to this view, although it is impossible in the light of existing knowledge to correlate periods of time with the Pleistocene of western Europe or with the pluvial periods of British East Africa.

From archaeological and geological evidence obtained during the Field Museum North Arabian Desert Expeditions of 1927-28 it may be stated that man in various paleolithic phases of culture lived in the North Arabian Desert between Rutba and the Hedjaz Railway. Flint implements were collected from several hundred surface sites, and at Bayir Wells hand-axes, upper Chellean in type, were unearthed from a gravel bed at a depth of 11 feet 6 inches. Hundreds of crude paleoliths were collected, as well as a number of neolithic tools and rejects. The most recent implements, from a typological point of view, apart from those flakes made by modern Beduins, suggest an extremely late neolithic technique.

The earliest archaeological evidence from Kish, which is derived from a stratum between present water level and virgin soil, suggests a late neolithic phase of culture. Flint implements associated with a black incised ware as well as the entire absence of copper indicate that the earliest inhabitants at Kish were in an advanced neolithic cultural stage. From this archaeological evidence, combined with a comparative study of the flint-working technique employed, as well as from the geological and climatological data now available, I am suggesting the following hypothesis.

The earliest inhabitants of the North Arabian Desert, the Proto-Mediterraneans, lived in a lower paleolithic phase of culture, and there is abundant evidence of their wide distribution over this area. During the long period of time in which their flint-chipping technique evolved from crude hand-axes to late neolithic tools, the climate slowly changed and that once fertile and well-watered region gradually became less able to support the population. Finally a climax was reached about six or seven thousand years ago when a semi-nomadic existence became untenable.

This climatic change was the dominant factor in forming the civilizations of Mesopotamia and Egypt, to which our own civilization can be traced. There was an alternative for the early dwellers in North Arabia: they could remain as nomads in this hostile and barren wilderness, or they could trek to the east, west, or southwest. The former mode of life was chosen by the ancestors of the modern Beduins, who with their flocks and herds wander from well to well across the wilderness, which only becomes green after the spring rains. During the remainder of the year this region is swept by wind and sandstorms; in the summer a blazing heat beats down upon it. The Beduins must often long to see water in the dry stream beds of the countless wadis, which are the evidence of a former fertility and which are now waterless except for a few hours after heavy rains. The aridity of this region has definitely increased since the Roman occupation; for example, at Qasr-el-Burqu', the easternmost outpost of the Roman Empire, the legionaries constructed a reservoir about five hundred meters long 
and one hundred meters wide. When we visited this ruin in 1928, there was no water in the reservoir; according to oul Beduin guides, water could be obtained only from deep local wells, which had been dry for several years except during the rainy season.

It may be suggested that various groups of the prehistoric population wandered from this now inhospitable region to more fertile and well-watered lands. To the north and south lay desert or wilderness; they could travel westward into Palestine and eventually to the Nile, in a southwesterly direction into Abyssinia, or to the east until they found the pleasant lands watered by the Tigris and Euphrates. These wanderers brought with them a late neolithic culture which included or developed the making of heavy, black, incised pottery.

From among advantageous sites, Kish was chosen by these stragglers, who founded the first community at the spot, which was afterwards to be designated as "the first city founded after the Flood." From a physical standpoint, these settlers were part of the Proto-Mediterranean group whose descendants are the modeln Beduins of North Arabia. They were relatively tall in stature and dolichocephalic. About 4200 B.c. the Sumerians, who were probably brachycephalic and racially different in several respects, swooped down upon the settlers at Kish and conquered them in a decisive victory.

The Sumerians imposed their sway upon the conquered Proto-Mediterranean group and ruled them for several centuries, until they themselves were subjugated. It seems probable that the dawn of the fourth millennium before our era witnessed the arrival of Semites at Kish. This would account for the apparently mixed population discovered by means of skeletal remains from the lower levels. Undel' a second Sumerian domination, however, which began five centuries later, Kish grew to be the first capital of the civilized world. Stephen Langdon, Director of the Field Museum-Oxford University Joint Expedition, and Professor of Assyriology at the University of Oxford, has kindly contributed the following brief summary of the history of Kish.

\section{HISTORY OF KISH \\ STEPHEN LANGDON}

According to the consensus of traditions, Kish was the first capital of Sumer after the Flood, a date impossible to fix with certainty, but on our records clearly in the neolithic age and before 5000 B.C. Chronological traditions place this date about 34,000 B.C., but that is obviously impossible. The first dynasty at Kish had twenty-three kings, among whom was the famous Etana, the shepherd who ascended to heaven on the back of an eagle in quest of the plant of birth, that his wife might bear a son to inherit the kingdom. This legend was known throughout Sumer, Babylonia, and the entire ancient world. Seals of every Sumerian city have representations of this ascension of Etana. A fine Accadian poem based on an older Sumerian epic has been preserved in the myth of Etana.

About 4000 B.C., Kish, having been the capital of the entire land from the Persian Gulf to the Armenian plateau for centuries, lost the hegemony of the 
Sumerians. It is possible that the Semites then began to arrive, and a civilization of advanced type, marked by the invention of writing and the production of painted pottery, appeared all over the Kish area, and in fact all over Sumer. Kish, although not now the capital, thrived greatly under the dynasty of Erech and later under the dynasty at Ur. A sterile period followed-the great but brief period of painted pottery, which was in my opinion entirely a Sumerian culture. Soon after, about 3500 B.C., the first great buildings at Kish appeared, and the sterile period caused by the invasion of Elamitic conquerors of Awan ceased. Kish again became the capital, and at that time the first palace, which was excavated by Mr. E. Mackay, was built.

Later arose a line of kings whose tombs were found in a stratum eighteen feet thick, marking a long civilization characterized by great buildings and the savage rite of slaying servants to accompany their masters and mistresses into the future life. Copper and stone work, carving of precious stones, and gold and silver work then attained a high standard. These kings were undoubtedly the "pigtail people" of the palace carvings.

After the great tomb period, Kish lost the hegemony of Sumer for centuries, until about 3000 B.c., when the first famous woman in history, Kug-Bau, a wine merchant, appeared. She rallied the whole Sumerian people and enabled them to shake off the foreign rule of the northern Semites at Maer. She became queen of the whole land, and is said to have ruled one hundred years! She founded a dynasty of seven kings, her son Gimil-Sin succeeding her on the throne.

Then came the great Sargon, a priest in the temple of the war god of Kish, now the ruins Tell-el-Uhaimir, situated in western Kish. He was a pure Semite. Uniting the Semites against the Sumerians, he conquered in a few years the whole of Mesopotamia, Elam, Syria, Anatolia, and the islands of the Levant, and subdued Palestine and Phoenicia. He forsook Kish and founded his capital at Agade. This city has never been found, although it existed as a great city down to the days of Nebuchadnezzar. From records of this dynasty at Kish, Agade cannot be far away.

The Sargonic dynasty did little at Kish, but numerous inscriptions of that period have been unearthed. The great Sumerian dynasties at Kish, just before Sargon, had carried out colossal building operations - the entire temple area was reconstructed, the huge stage towers were rebuilt, and the palace was repaired. In such splendid condition did Sargon find Kish. But that famous son of Kish, and his equally famous sons and descendants, Rimush, Manistusu, Naram-Sin, and Shargalisharri, devoted their attention to Agade, where they created schools of sculpture, literature, and art.

As splendid and rapid as was the rise of the fortunes of Agade and its mighty empire, so sudden and humiliating was its fall. A few feeble kings ended its rule, and the barbarians of Gutium invaded the land. The whole of Sumer, including Kish, sank into political and cultural mediocrity, save at Lagash in the extreme south. After 125 years of barbaric decay, the Sumerians united under the leadership of Erech and Ur, and the Guteans were expelled. 
In 2416 B.C. arose the mighty kingdom of Ur, ruling the whole of western Asia for 108 years, succeeded by the contemporary rule of Isin and Ellasar, until the rise of the Semitic dynasty of Babylon, in 2169 B.C. Nothing is yet known of the history of Kish during the turmoil of the Gutean age, nor have we found anything of importance yet under the great kingdoms of Ur, Isin, and Ellasar. Only a few tablets dated during these latter kingdoms have come to light.

But the memory of former glory remained strong at Kish. The city asserted its ancient rights to become the capital and three independent kingdoms sprang up there suddenly to oppose the growing and aggressive kingdom of Babylon. From that period tablets dated by the names of these kings have been found. They failed, and Kish was soon received into the empire of Babylonia, with its capital at Babylon. Henceforth Kish remained, until the days of Nebuchadnezzar and Nabunidus, a thriving metropolitan suburb of Babylon, retaining the memories of its past and the privilege of being the center of the cult of the mighty earth goddess and of the god of war.

During the long history of Babylonia, 2169-538 B.C., when Cyrus put an end to the political kingdom of Sumer and Accad, little is known of the local history of Kish. We have found there nothing dating from the Cassite period (nearly five hundred years). Temples were repaired by the kings of Babylon at Kish. Their scribes wrote grammars, lexicons, and poems and edited ancient texts. A the end of the period Nebuchadnezzar rebuilt the temple of the earth goddess on a magnificent scale, unequaled anywhere in his kingdom.

There followed the Persian, Greek, and Parthian periods, extending to the third century of our era. Kish remained a thriving city and the center of a flourishing agricultural section of Babylonia during all these critical periods of ancient history. The commercial cuneiform records are especially prolific under the Persians of the dynasty of Cyrus, as well as under the Greeks; tablets from the period of Alexander the Great have been unearthed. Numerous Parthian tombs have also been found.

The Sasanian-Neo-Persian rule of Mesopotamia held sway from A.D. 226 to 628 . The Persians chose Kish as their western capital, at least from the fourth century onward, and two fine palaces (probably one near the Manichaean Church) have been found just east of the temple of the earth goddess.

Then followed the magnificent Arab period of the caliphs of Baghdad and the advent of Jewish influence at Kish. The caliphs had a palace at Abu Sudaira, three miles east of Kish, and their coins are found in many mounds of the city itself. Ruins of the canals of the caliphate period break the surface of the plain at and around Kish in every direction.

It is clear that the Kish area remained a thriving center of agriculture and the city a great metropolis as late as A.D. 1000. From that time its history can no longer be traced, until European travelers visited Kish in the nineteenth century and found it a desert with only one tribe of Arabs living in the district. Moslem and Turkish rule had put an end to the oldest consecutive city history of western Asia. It is probable that in A.D. 1000 families still lived in Kish who 
were related to the earliest Semitic people, of 3500 B.C. What was the Sumerian admixture of blood in them cannot be determined. Nor can the exact relationship of the modern Arab tribesmen to their predecessors be traced.

\section{SOCIAL LIFE AND CUSTOMS}

The Arabs who are the subject of these studies live within a small radius of Kish. They comprise three main groups: Arab workmen employed at the excavations and their relatives living near Tell-el-Uhaimir; members of the Iraq army stationed at Hilla camp; and a small group of Ba'ij Beduins.

It is desirable to study in some detail the physical characters and the life and customs of the modern inhabitants of the Kish area. These Arabs appear to belong to the same racial group and, from the result of preliminary studies on the excavated skeletal material, appear to be direct descendants of the people who were inhabitants of Kish in the early part of the fourth millennium before our era. In the modern population the predominant shape of the head is long and narrow (dolichocephalic). This also appears to have been the basic element in head form at the dawn of civilization in the "Fertile Crescent." There is also a small percentage of round-headed peoples or brachycephals among the modern Arabs. This latter element appears to have been superimposed upon the original dolichocephalic population. It is probable that the earliest inhabitants of Kish were conquered by brachycephalic invaders at an early date. A comparison of the anthropometric data obtained from the skeletal material and that recorded from the modern inhabitants will be of interest.

The purpose of this Report is to record the data obtained on modern peoples of the Kish area, accompanied by some notes on their life and customs observed during 1927 and 1928, when I was attached to the expedition.

From a cultural point of view the Arabs are entirely separate from the Beduins of Iraq, who wander at will in the desert (jazirah) between the Tigris and Euphrates. The Arabs and Beduins undoubtedly form part of the same racial group, but the centuries of agricultural life on the one hand and nomadism on the other have tended to separate them in many cultural adaptations. I shall record details of Beduin tribal life and customs in a subsequent publication.

Within a radius of several miles from Tell-el-Uhaimir, the deep red temple tower (zikkurat) dominating western Kish, there is a group of small Arab encampments, each of which is ruled over by a sheikh. The power of each sheikh depends largely upon the size of his camp and thus upon the number of men and their families who respect him as a leader and a dispenser of justice. These sheikhs are insignificant in power and wealth compared with the great Beduin sheikhs of the North Arabian and Syrian deserts. The location of the main villages in the Kish area, and more particularly of those encampments where the following anthropometric data were obtained, is shown upon the map (p. 83).

The majority of workmen at the Kish excavations were drawn from the village of Sheikh Hadji Miniehil, which lies about three miles west of Tell-el-Uhaimir. 


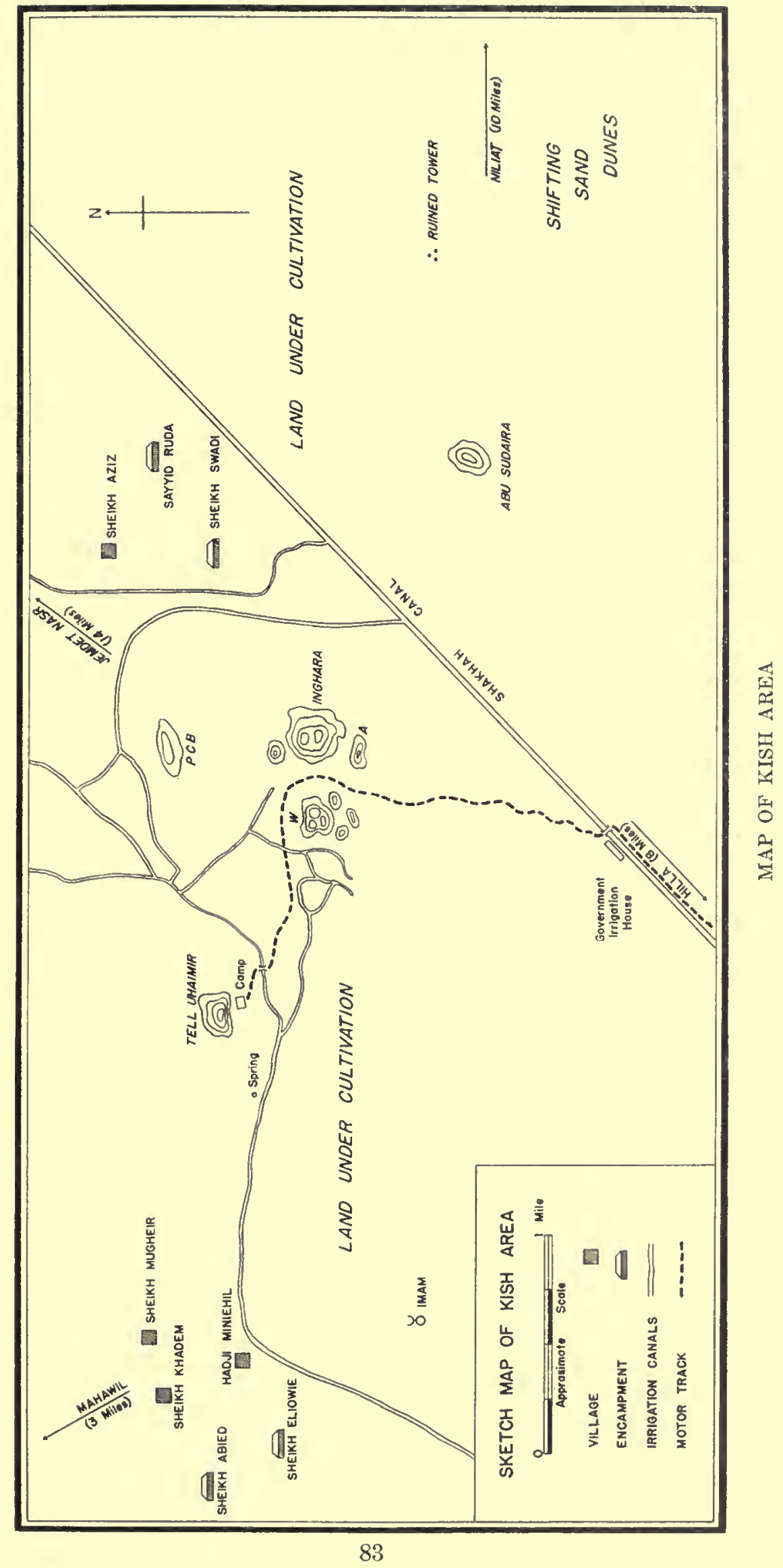


I was therefore able to examine the people of this large village closely, as the sheikh and his two sons, Ralli and Razi, were favorably disposed toward the members of the expedition. Before discussing the villages in detail it will be necessary to include a brief description of the country surrounding Kish.

With the exception of the two temple towers, Tell-el-Uhaimir and Tell Inghara, which dominate the western and eastern sections of Kish respectively, some low mounds covered with broken pottery sherds-relics of former habitation - and large dry Abbassid canals, the entire region between Hilla, on the

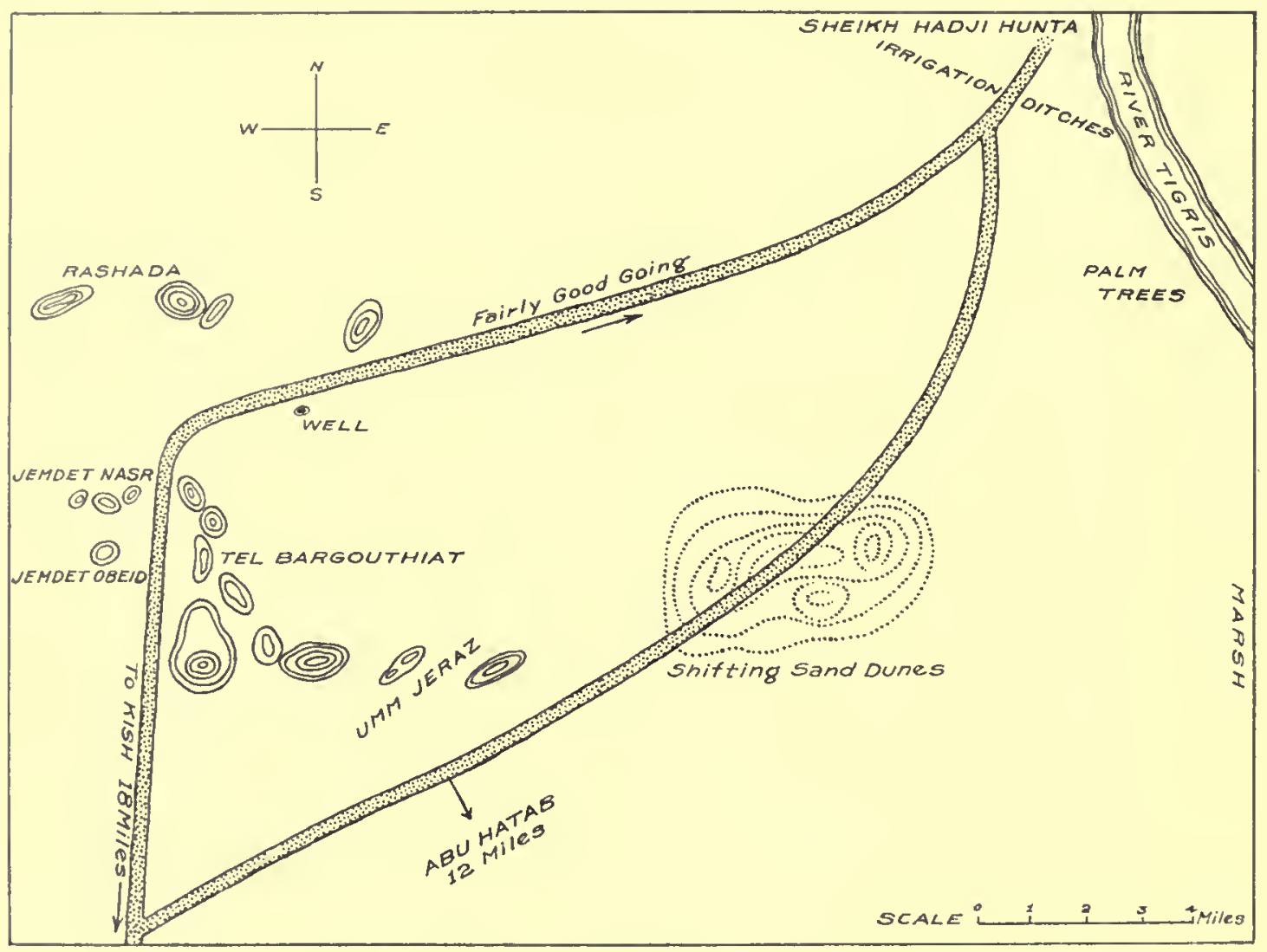

MAP OF TRIP FROM KISH TO TIGRIS RIVER

Euphrates, and the Tigris, is a flat, alluvial plain. The rivers have changed their courses many times during the past six thousand years. Even today the Euphrates, which flows past Babylon, is denuding its western bank and gradually moving away from the great complex of mounds once ruled over by King Nebuchadnezzar.

The Shatt-en-Nil is a small canal about twenty feet in width, which flows in an easterly direction from the Euphrates River to the eastern boundary of Kish near Abu Sudaira, and, turning northward, irrigates the country nearly one-third of the way toward Jemdet Nasr. There are numerous small canals which branch off from the Shatt-en-Nil. This network of canals partially irrigates the land, which is naturally fertile but for the most part lacks an adequate water supply. There are no palm trees except around several villages (Mahewil and others) 
between Kish and Tell Ibrahim (ancient Cutha). There is considerable growth of camel's thorn (hatab) and rank grass surrounding two large lakes south of the Shatt-en-Nil and about two miles from Tell-el-Uhaimir. During the winter these lakes are the abode of wild fowl, including ducks, geese, and flamingos.

The larger villages, such as that of Sheikh Hadji Miniehil, are composed of mud huts of various shapes and sizes. Around each village is a wall of mud brick, supplemented by a thick camel's-thorn fence. As an additional precaution against surprise visits of strangers there are numerous mongrel dogs which bark savagely and continually during the approach of a person either mounted or on foot. The Arabs apparently do not object to the almost incessant clamor of their dogs, as they make little or no effort to prevent it. Far into the night these dogs howl to their mates or in answer to the challenge of a fox, hyena, or jackal, as he prowls around in search of prey.

Each village has a large mud-walled hut roofed with beams of palm covered with reed mats overlaid with mud. This house belongs to the sheikh, and is the central meeting place for the men of the village. About this house are grouped the smaller mud huts of the inhabitants of the village. Around each entrance is a camel's-thorn fence with a narrow opening directly opposite the door of the hut. The mud huts are constructed in the following manner. A foreman or master mason is engaged to direct the work of building the side walls and roof. The floor of the proposed building is drawn on the ground by means of a pointed stick, four large stones being left at the respective corners as markers. Eight men are generally employed to assist the master mason. The division of labor is such that two men bring water in buckets to moisten the clay during its preparation for use as a building material. One man digs clay from the edges of a small hole in the ground, while two men, adding water and occasional handfuls of straw, knead the clay with their feet into the correct consistency.

In this connection it is interesting to note the punishment Pharaoh inflicted on the Israelites because Moses and Aaron begged (Exodus $\mathrm{v}: 1-22$ ) that the Israelites might pray and sacrifice to Jehovah three days' journey into the wilderness so that they might be delivered from pestilence or death by the sword. Pharaoh refused to grant this request and ordered the imposition of additional tasks upon the Israelites to keep them occupied. Pharaoh said, "Ye shall no more give the people straw to make brick, as heretofore; let them go and gather straw for themselves. And the number of the bricks which they did make heretofore, ye shall lay upon them; ye shall not diminish aught thereof: for they are idle; therefore they cry, saying, 'Let us go and sacrifice to our God.' ... So the people were scattered abroad throughout all the land of Egypt to gather stubble for straw."

The duty of the builder's chief assistant is to knead the clay which is taken from the damp pit into balls about twice the size of a baseball. These balls are then carried by two other assistants, one ball in each hand held at eye level with the palm upward, and thrown in turn to the master mason. The mason places the balls of clay one on top of another until the wall is about three feet 
high, when operations are interrupted for a day to allow the clay to dry out. Then another few feet are built on top of the first and allowed to dry. This program is followed until the walls are about ten feet high. As a plummet a stone attached to a string gives the more or less correct upright angle for the wall. The never-ending chain of men carrying water, stamping the clay, kneading it into balls, and carrying it to the mason, enables rapid building of the side walls.

The roof is a more complex matter. In the case of the central house of the sheikh, split palm trunks, purchased for one and one-half or two rupees (fortyeight to sixty-four cents) apiece in Hilla, are placed at intervals on top of the side walls of the hut. Reed mats, which are also purchased at Hilla for about eight annas (sixteen cents), are laid across the beams and tied down with twisted palm fiber. When this has been completed, the mud ball system is again commenced, and the mason stands upon the roof to catch the mud balls, placing them upon the top of the reed mats to form a continuous layer of mud (Plate CXXIII). A bucket of water is always beside the mason so that he can keep the clay at the desired consistency. In this way the mats are covered with a layer of mud, which is allowed to dry for several days. The same process is then repeated, and the hut finally becomes habitable.

Windows are made by cutting a hole in the wall and setting bottles or a pane of glass in the space, the intervening holes being filled with mud. The approximate total cost of building a hut of this kind with dimensions 18 feet long, 10 feet wide, and 10 feet high would be about $\$ 40.00$, and one week would be necessary for its construction. It is probable that houses of this type have been built in the Kish area for the past six thousand years.

A bridge ( $j$ isr) spans each irrigation canal, in order to allow the passage of wheeled traffic as well as to facilitate the portage of supplies from Hilla. Each bridge is constructed in the following manner: Large split palm trunks, similar to those used to support the roofs of mud houses, are laid across the stream at a convenient point. The spaces between the planks are filled with camel's thorn, and a layer is spread over the bridge. The entire surface is then covered with the arenaceous earth and stamped into place with the bare feet or pounded with a wooden beam. The result is eminently satisfactory for the passage of horses and donkeys, but always precarious for wheeled traffic.

The bridges which connect the various parts of the track between Hilla and Kish are kept in constant repair by Arab workmen, who use long-handled shovels to scoop the earth upon the surface of the bridge.

There are horses, donkeys, sheep, and chickens within the village walls. A few camels are often seen browsing upon tufts of camel's thorn. There is no community ownership; there is much individual pride of possession. Every Arab desires to own beautiful horses and he is a good judge of a horse from the standards of speed and endurance. Several times during the winter season of excavation every horse owner in the village of Sheikh Hadji Miniehil galloped across the hard, flat plain to the camp at Kish. Upon their arrival they raced each other around amid wild screams of excitement. The horses are small and 
stocky and poorly groomed, with the exception of those belonging to the sheikh or to his rich sons. The bridle consists of a rope tied as a haltel' over the head and passing around the nose through a slip knot. The loose end is held in the right hand and is used as the sole means of guiding the animal. For those accustomed to riding with a double-reined bridle and bit and perhaps even a standing martingale, the Arab's method of guiding the horse seems almost impossible. However, after a little practice the secret between horse and rider becomes established.

There are three kinds of saddles. The most common type used by the Arabs is a narrow-seated leather saddle with sharp angles in front and behind. There is often a silver knob or pommel on the front of the saddle to which the less skilful riders cling when the horse goes out of a walk. A thin, flat leather saddle which closely resembles the type used in racing in America and Europe is also used. Lastly, second-hand British military saddles are seen occasionally, but the price (twenty rupees, about $\$ 6.40$ ) prohibits their general use. The stirrups are very small metal loops; the leathers are short and often consist of thin leather or even string. Safety devices are unknown. The Arabs ride short with a forward seat, while little or no weight is placed on the stirrups. The saddle girths are narrow canvas bands attached on both sides beneath the saddle flap. Since the horses are fed only on grass and are usually turned out when not being ridden, their stomachs are dilated, and it is impossible to keep the girths tight. Consequently it is a common occurrence for the saddle to slip around beneath the horse.

Trappings of various kinds are worn behind the saddle. Saddlebags (khurj) of camel's hair or wool, with two large, wide pockets and decorated with colored tassels, hang on both sides. In Spain, the saddlebag (al-Forja) is carried in front of the rider. It has a loop which fits over the back portion of the saddle and which theoretically keeps it in place. The rich Arabs place a colored saddlecloth (gamis) from the back of the saddle to the crupper. Each rider generally carries a rifle slung over his back or he rests the butt of the firearm upon his right knee with the muzzle pointing in the air.

Donkeys and camels are used as beasts of burden. All necessities of life are brought from Hilla market on these overladen animals. Cattle and pigs are not kept, since they are deemed unclean according to Mohammedan law.

Each Arab wears a long cotton garment (dishdashi or thob) resembling a nightgown in appearance, which is fastened at the throat with several buttons. It has long, wide sleeves terminating in points. Over the dishdashi is worn a coarse brown cloak ('aba) which is made of camel's hair and resembles the standard type of university gown. On the head is worn a white or a black-and-white speckled cloth (kaffiyah), held in place by a brown or black camel's-hair head-dress ('agal).

The feet are often bare, but coarse leather sandals or shoes are sometimes worn. Each village has a shoemaker who knits the uppers from coarse string. The leather soles are purchased in Hilla. These shoes (kalhe) are also worn by Kurdish shepherds and by Beduin tribesmen of northern Iraq.

The sheikh or his son wears a white kaffyah with a gold or silver thread 'agal and a tight-fitting colored garment (zibun) over the dishdashi. The sons of a sheikh often wear European shoes. 
Each woman wears a dark blue dishdashi with a correspondingly dark blue kaffiyah, the ends of which are wound around the head. 'The feet are always bare. Each little boy and girl wears some form of white or blue dishdashi, and the children run about barefoot. Dark blue outer garments are generally worn by women and girls.

The men wear no ornaments or jewelry, but they are extremely vain concerning their personal appearance. Each young man carries a small folding mirror and frequently a brush and a small jar of kohl, which is applied above and below the eyes. The women also are fond of this form of cosmetic. In the graves excavated in Cemetery $\mathrm{A}$ and also in the graves in Y-trench at Tell Inghara, small shells (Pecten sp.) containing traces of kohl were found. 'These shells and the small copper toilet sets belonging to a period anterior to 3000 B.C. prove that the same types of cosmetics and depilatories have been in vogue at Kish for about five thousand years. The men and women apply henna to their hands to stain them a reddish brown, which is considered beautiful. Some of the women stain their feet with henna.

The majority of the men bear tattooed marks, while the women and girls of marriageable age are tattooed with blue spots or more complex designs. As a rule, the faces of these Arab women are not as heavily tattooed as those of the Beduins, who desire a tattooed upper lip and chin.

The women are not veiled, although young maidens always cover the lower portion of the face when a man approaches. The small groups have to a great extent intermarried, but they realize the necessity for fresh blood. The greatest possible care is taken to secure a suitable match. The method generally employed is as follows: A young man may fall in love with a girl whom he has known from childhood or who has taken his fancy when he visited some neighboring village. The boy's father calls on the girl's parents, who at once state that several other young men have requested their daughter's hand in marriage. The primary question to be solved is the amount of the girl's dowry. The girl is then brought into the family circle and admired, particularly by her parents. After considerable discussion over cups of coffee and glasses of tea, the girl's parents state the amount of money or property the young man must possess before he can marry her. When an agreement is reached, the girl and boy are told that their engagement has been arranged. The amount required varies from two hundred to five hundred rupees $(\$ 64-\$ 160)$. The young man has to collect this amount, which may be in animals or money.

When the money or its equivalent has been raised, the boy's father announces this to the parents of the girl. A date is set for the wedding, which is duly pelformed by the sheikh in the presence only of members of both families. In the stricter Mohammedan groups the bridegroom does not see the face of his bride until after they are married. Among the richest members of the community the marriage is celebrated with preliminary dances by the sisters and female cousins of the bride. They dance to the rhythmic beat of drums and wind instruments from dawn until far into the night. In the meantime the bride is not allowed out of her house until the wedding ceremony takes place. 
The Arabs, though fatalists, are superstitious, and this feeling of dread of the supernatural appears to be more deeply rooted in the women than in the men. The men, however, sometimes wear a stone on a string around the neck to remove a pain in the throat or to heal some disease which is gradually becoming chronic. Many of the women wear charms or amulets consisting of beads or cylinder seals which are supposed to keep away the evil eye. All young children wear blue beads of some kind around either the neck or the wrist, or sewed on their garments. The evil eye is credited with supernatural powers; the Arabs believe that it may be the will of Allah to allow an individual to be tormented by the Evil One, who, however, dreads the color blue and therefore cannot harm a person wearing blue beads. Unless there is sickness in the family the men ridicule the women for their superstitions. These same men, however, have blue beads sewn on the harnesses of their horses, camels, and donkeys. I have not seen any harness that does not include this simple protection against the evil eye. The beads are manufactured by the thousands at Birmingham, England, and cost of protection is thus negligible, even for the poorest Arabs.

\section{FOOD}

The main articles of food include unleavened cakes (chupatties), rice (timmin), sour milk (laban), and dried dates (tamr). The food is stored in earthenware containers. There are no special storehouses in these small villages. Meat is rarely eaten, owing to its high cost, but on festive occasions a lamb or kid is killed. All meat is eaten fresh, except that of the gazelle, which is allowed to hang for several days. Gazelles, which are to be found only in the jazirah, are rarely killed. I believe that there is prejudice against the killing of this animal, which may result from a former primitive clan totemism.

The unleavened bread (khubz) is baked in a crude oven ( furn) outside the hut. The oven, consisting of a hole in the ground, is lined with clay and partially filled with camel's thorn, which is fired. Coffee (gahwah) is prepared by roasting the beans in a long-handled iron dish (sage, in Kurdish). After being roasted, the beans are pounded with a heavy metal pestle in a large brass mortar (Plate CXXVI, Figs. 1, 2; Fig. 36, $k, l)$. The coffee grounds are boiled in an iron vessel, in which the sediment is allowed to accumulate. Wheat (huntah) and barley (sha'ir) are grown in this district. Charred grains of both these cereals have been found during the excavations (cf. my article, Amer. Anthr., XXXIV, pp. 303-309).

Pottery of various kinds is purchased in the market at Hilla, but I did not see any glassware in the various encampments visited, except small thick glasses for tea.

The water supply is obtained from a local spring, or is carried in large earthenware jars from the nearest irrigation canal.

Since Mohammed strictly forbade alcoholic liquor in any form, there is no drinking of any kind in these communities. In the town of Hilla, however, the general principles of Mohammed are not so religiously and strictly obeyed, and a local alcoholic drink called 'araq is imbibed. This beverage contains a high percentage of alcohol and is therefore very potent. The mention of araq, the only stimulant of which they have heard, is forbidden among the Kish Arabs. 
According to the "Encyclopaedia of Islam" $(1924$, pp. 1, 111) there is a description of the preparation of this intoxicating drink from the time of the Mughal Emperor Akbar, who lived in the second half of the sixteenth century. In Persia, however, according to Herbert (p. 261), "arrack and aquavitae [first edition: "arack or usquebagh distilled from dates or rice"] they also drink."

The lighting of the houses or tents is done by means of oil lanterns and hurricane lamps, which are purchased at Hilla.

\section{MUSIC}

The Arabs enjoy music (musiga), and often chanted some refrain during the work of excavation. In the evenings they sit around the fire (nar) and sing to the monotonous wail of the rababah, the single-stringed instrument of the Beduins.

An end-flute (mutlug) with two tubes of the same length held together by bitumen is also used by the Kish Arabs. This musical instrument is composed of cylindrical tubes open at both ends and is sounded by blowing across the upper end. A series of six open stops along each tube enables a variety of notes to be produced, since, by opening or closing the stops, the vibratile length of the column of air in the tube can be varied. There are two small reeds connected by a black thread, which can be raised or lowered to alter the pitch. This instrument is called mutlug by the Arabs, and duzahle by the Kurds, who call the reed mamzah. The strolling minstrel players use the rababah and several kinds of drums, with which they beat time for the dancing girls.

\section{WEAPONS}

An Arab is always armed with a rifle, a dagger, or a club, and after nightfall no man is anxious to travel without a rifle. There is no great danger, but at any moment in the darkness of the night the challenge Menu hadha? ("Who goes there?") may ring out. An unarmed man walking alone across the plain would be robbed by a stranger or by anyone who did not expect to be recognized. Apart from this danger, there are hyenas and foxes which may cross the path.

The Arabs are inexpert marksmen mainly because of the high price of cartridges, which practically forbids target practice. Throughout this region cartridges represent a form of wealth; consequently, few bullets are wasted. The cartridges are worn in native leather belts or in crossed bandoleers. The old Turkish rifle is the most common weapon, but occasionally an out-ofdate rifle of German pattern may be found among the Arabs. The ammunition is Turkish, and many cartridge cases bear a ten-year-old date of manufacture.

The dagger (khanjar) is a short, curved knife with a wooden or bone handle made from the horn of an ox or a water buffalo. The blade, which is made of good steel, is not decorated or marked in any way, although the bone handle may have silver bosses attached. The silver knobs are fashioned from hammered Turkish buttons, and they are in some degree decorative. The handle is fastened to the blade by means of two nails. The scabbard may be of leather, and in this case is undecorated. On the other hand, the sheath may consist 

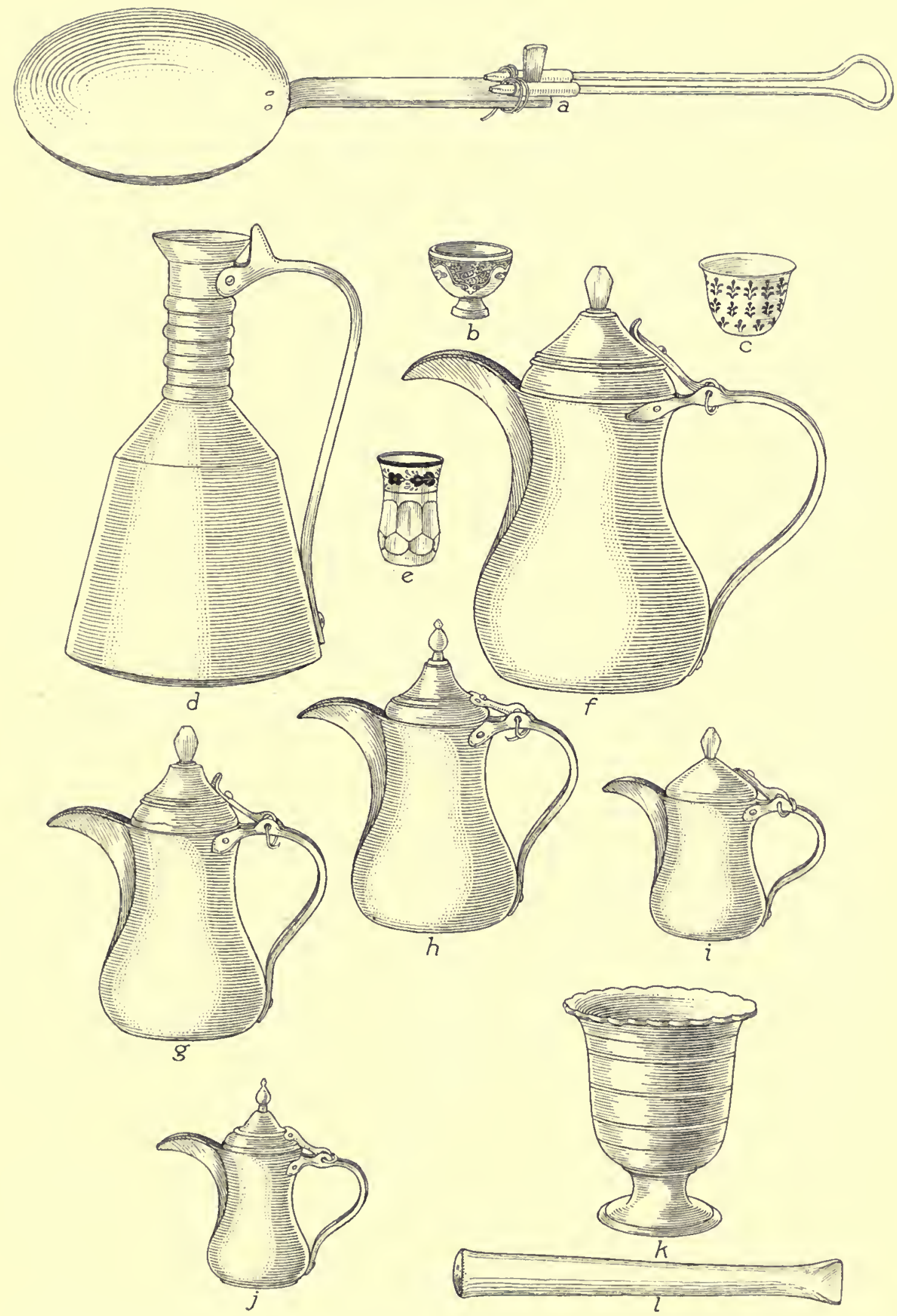

FIG. 36. Household Utensils. $a$, coffee roaster (mahmus); $b$, metal coffee cup holder (finjan); $c$, china coffee cup (finjan); $d$, metal water jar (bugwa); $e$, tea glass (istikan); $f$, large water pot (gumgum); $g, h$, coffee pots (telgamat); $i, j$, small copper coffee pots (dilal); $k$, brass mortar (hawan); $l$, pestle (id al-hawan). 
of two curved strips of wood fastened together by means of some resinous material and covered with hammered silver or gold. The metal is obtained by melting local currency, and thus an approximate estimate of the value of the dagger can be obtained. The metal work, although not done by the famed Amara silversmiths, is very fine. The upper portion of the scabbard may be ornamented with parallel lines of dots and more rarely with a semicircle of small turquois beads on the outer side. Each scabbard appears to have its individual motif of ornamental design, which is reproduced on both sides. The lower portion of the scabbard tapers to a curved point, and is decorated with a heavier band of metal ornamented with lines or dots. The point is broad and is decorated with five lines. On one silver scabbard fish and birds have been engraved (Fig. 37, $k$ ). The former are an emblem of happiness; the latter, a symbol of good news.

The khanjar is worn on the right side, and is held in position by a camel'shair girdle, which also serves to keep the dishdashi in place. The khanjar is a serviceable weapon; its curved blade inflicts a terrible blow. The excavators found these daggers most useful in removing the compact alluvial soil from around a buried object. The value of a khanjar varies from eight to ten rupees $(\$ 2.50$ $\$ 3.50)$ for an undecorated handle and scabbard, to forty rupees $(\$ 13.00)$ for a silver case, and up to five hundred rupees $(\$ 160)$ for a magnificent gold scabbard.

The striking club (Arabic, magwar; Kurd, mazrak) has a round wooden handle which varies from two to three feet in length. The head of the club is made of wood, bitumen, or stone (Fig. 37, $a-c$ ). In the last-named case a Sumerian ceremonial macehead is occasionally used, a nail being driven through the pierced stone into a wooden handle. The magwar is carried constantly by these Arabs as a means of protection.

\section{GAMES AND PASTIMES}

The Arabs drink large quantities of black coffee (gahwah) and sweetened tea (chai). They smoke long, native cigarettes, or suck the narghile. The cigarettes, which are very mild, are about twice the length of American cigarettes. Virginia or Turkish tobacco appears strong to the Arabs, and cigars are never smoked since they cause nausea. Coffee is drunk from china cups which bear red or blue designs (Fig. 36,c). These cups are often supported by brass holders, each of which is called a finjan. One finjan in Karbala bore the Arabic inscription, $f i$ Al-'ajalah al-nadamah ("Haste makes waste"). Tea is sipped from a thick glass container (istikan) which is made in Warsaw and imported through Iran.

During these pleasures they gossip continually and allow their innate inquisitiveness a free hand. Horses are their chief joy and pride. According to an old Arab proverb heaven on earth can be found only on the back of a horse or in the arms of a woman.

I have seen but two games enjoyed by these people. One of these is played as follows: A small hole is made in the ground, and a dagger is held in the right hand about six inches above the mark. The palm of the left hand is placed directly above the hole. As the dagger is plunged into the hole, 


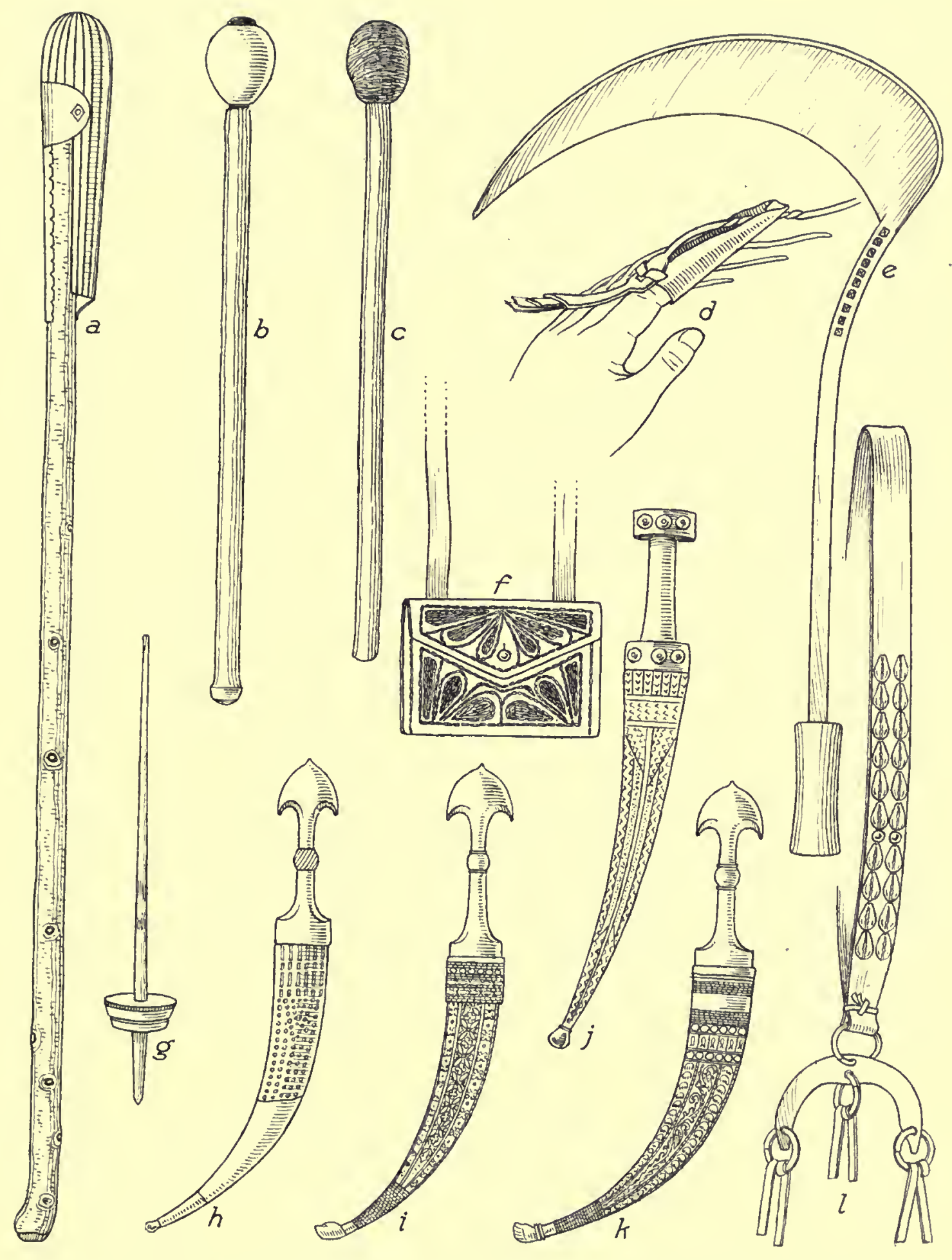

FIG. 37. Weapons and Implements. $a$, club (chmagh) with wooden head; $b$, club (sakhariya) with Sumerian macehead; $c$, club (magwar) with bitumen head; $d$, finger guard (chinagh); $e$, sickle (minjal); $f$, leather pouch (chis); $g$, spindle (maghzal); $h-k$, daggers (khanajir); l, donkey halter (rashmah). 
the left hand must be moved to the side and then replaced over the hole as the dagger is drawn upward into position. Skill is shown by the rapidity with which the dagger can be plunged into the hole as the hand is drawn aside. The left hand can be moved either toward or away from the person playing the game. Several of the Kish Arabs plunge and retract the dagger as fast as the eye can follow the rapid movements. The winner is merely the contestant who gains the most approval from the onlookers.

The other game is a variety of astragals, which is so called from the fact that it is played with the astragalus or knucklebone of a sheep or some other animal. This pastime is of great antiquity, since one of the British Museum Tanagra terra-cottas, which date from the eighth century before our era, represents two girls playing this game. In Horace's "Satires" (Book II) we read, "Ever since I saw you, Aulus, carrying your knucklebones and nuts in the loose fold [of your toga], giving them away and playing with them, but you, Tiberius, counting them and solemnly hiding them in holes, I have feared that different kinds of madness may seize you and that you will follow Nomentanus and you Cicula." (Nomentanus is used by Horace as a type of a prodigal and spendthrift; Cicula is a very cautious money-lender.)

The Kish Arabs draw a circle on the ground and place an astragal in the center. The object of the game appears to be to knock the astragal outside the perimeter of the circle by flicking another astragal against it with the thumb and forefinger. This game is akin to marbles, and is generally played only by children, although I have seen young men passing the time in this manner. (For further information see E. Lovett, "The Ancient and Modern Game of Astragals," Trans. Folklore Soc., XII, No. 3, pp. 280-293.)

In Hilla the Arabs play a game with three brass cups and a thimble. Each cup is turned upside down, and the thimble is artfully hidden beneath one of them. The game consists in deciding under which cup the thimble reposes. This is similar to the three-card trick, which pays the traveling expenses of many professional gamblers. The game of mancala is also played in the market at Hilla. I have never observed any ball games among these Arabs.

The Arabs lead a quiet existence provided that they do not draw police or government attention. The latter can be drawn through unwillingness to pay the annual taxation.

\section{TAXES}

The local government taxes agricultural Arabs by making them work a certain number of hours a year. The government pays a fixed wage of one rupee (thirty-two cents) daily. Each sheikh must supply the requisite number of men each day for two weeks out of every year. These men are employed either on irrigation and agricultural projects, or on road repairs. The government standard of wages fixes the salary of the workmen employed at the Kish excavations, since if lower wages were given they would prefer government service whenever opportunity offered. The Kish Arabs seemed to enjoy the governmental tasks mainly because of the additional revenue received thereby, although 
they in turn have to pay part of their salaries to the sheikh, who has given them this means of revenue. The objects of making money among these Arabs are to purchase live stock or a wife or wives.

\section{RELIGION}

At noon the older Arabs cease work in order to pray. Each man faces toward Mecca, places his ' $a b a$ on the ground at his feet, and, muttering several verses from the Koran, kneels on the 'aba with lips moving in prayer. He touches the ground with his forehead three times. Finally he arises to his feet, shakes the dust from his ' $a b a$, throws it around his shoulders, and continues with the affairs of the day.

Each holy man (imam) is buried in a sacred, domed building (gubbah). Dressed in flowing robes, I was allowed to enter the gubbah of Mahewil (Plate CXXXVIII, Figs. 1,3) between Kish and Tell Ibrahim. I took off my shoes and came into the holy place with a guide. The tomb was under the central portion of the small dome and consisted of a block of hewn stone supported by two flanking stone blocks. A high, wooden, openwork screen surrounded the tomb. At each of the four corners and on the central portion of each side and end of the screen were carved wooden rosettes. As we moved slowly around the tomb, my guide bent forward to press his lips against each rosette, murmuring prayers for help from the saint. This scene recalled the numberless pilgrims who with their kisses have partially worn away the bronze toes of St. Peter at Rome. Near the village of Sheikh Hadji Miniehil stands the domed Imam Sa'id. This tomb was considered so sacred that I was not allowed to enter.

Islam, which means submission to the divine will, is the name given by all Mohammedans to their religion. Every man must worship God five times each day: namely, at dawn, at noon, before and after sunset, and at the close of day. Before beginning his prayers, the worshiper turns toward the holy city of Mecca. While on a journey or during warfare or in special circumstances such as sickness, a man does not have to conform to the general rules of worship. During each week Friday is observed as a day of prayer, and in accordance with local custom the excavations at Kish were closed on that day, while work was continued during the Christian Sabbath. According to the Mohammedan doctrine, strict fasting is part of religion. In Islam the period of fasting falls in the month of Ramadan, during which period the Koran was revealed to the Prophet. The date of Ramadan, like the Christian Easter, varies each year. It begins with the rise of the new moon in March or April and lasts until the appearance of the next new moon. The fast commences daily when a white thread can be distinguished from a black one and continues until nightfall. During these long hours no particle of food or drink may pass the lips unless special dispensation is claimed.

The workmen employed at the Kish excavations occasionally had to work through the month of Ramadan. The older men adhered rigidly to the letter of the Moslem law, and found the continual work with a pick-ax almost too hard to endure. The younger men and basket boys made little attempt to abstain from food and water, since their youth obtains a special dispensation for them. 
Each evening at sunset the men gathered outside the village for the close of the day's fasting period. During Ramadan sounds of feasting reached the expedition camp at Tell-el-Uhaimir far into the night.

As the month of Ramadan draws to a close, great excitement prevails among the Arabs. There is considerable speculation on the probability of seeing the rise of the new moon. In many places, however, this spectacle is invisible because of clouds or mist. Therefore, when the new moon is visible in any portion of Iraq the information is telegraphed to every outpost, so that news of the termination of Ramadan can be relayed far and wide. This is followed by frenzied rejoicing among all Arabs, and there is singing, dancing, and revelry.

In a discussion regarding the local belief in life beyond the grave, the workmen stated that they believed implicitly in a future life. I asked them for their conception of heaven and of the intermediate step between life on the earth and the celestial state. One of the men replied that he visualized the transition as follows: Each person must cross a narrow bridge over a yawning chasm to the promised land. Each sinner, who has made little or no attempt to appease the Creator by ritualistic prayers, will be pushed off the bridge into the seething abyss to await purification before a further attempt can be made. There are two bridges, one for men and the other for women, who, however, are less harshly treated owing to the fact that they are seldom allowed to make their own decisions under Mohammedan law. Arabs do not believe that animals have a future life.

\section{EDUCATION}

With the exception of a few of the older men, whose religious zeal has inspired them to learn to read the words of Mohammed, the population is illiterate. The children attend no school, but are taught in the family. They devote their early childhood to play but are soon forced to assist with the work around the home, whether this be mud hut or black camel's-hair tent. From the moment when a child becomes helpful there is little respite allowed for play from sunrise to sunset. The children are proportionately more alert and intelligent than at the age of maturity. They are eager, quick, and responsive, but become slow and dullwitted in their late twenties.

The lazy or mischievous child is punished and often beaten by the mother; but the father, particularly if the child is a boy, will not touch one hair of his head. Paternal pride for the young boy who will carry on the family traditions is almost unbounded. A girl child is a disadvantage unless, when she reaches the marriageable age, she becomes the wife of a strong, healthy, and prominent young member of the group.

The young men and maidens talk and think of little but marriage and the family hearth. During excavation work, agricultural labor, or while riding across the plains, the Arabs chant romantic love songs.

Education, according to our concept, is unknown. There are no books or papers of any kind with the exception of copies of the Koran. These Arabs have seen no books, and it is often difficult for them to recognize the significance of a 
photograph. Many an Arab was unable to recognize his own photograph or those of his friends. He would turn the photograph on its side or upside down, and often was unable to see the resemblance even when the original was pointed out. However, there was a small percentage of men to whom the above would not apply. They were naturally of the more intelligent type, who were chosen as head men (rais) of the gangs (jogat) employed at the excavations.

The Kish workmen had little mechanical sense and seemed particularly slow in grasping even the most rudimentary mechanical principles. Achmed Hamadi (No. 34) was the most efficient mechanic, although his general intelligence did not appear to be above the average.

The Arabs are very inquisitive and have no hesitation in asking any question which may occur to them. Upon the occasion of my first visit to Kish, in the winter of 1925 , I was asked where the objects were shipped at the conclusion of each season of excavation. I replied that the most important archaeological treasures remained in the new Iraq Museum at Baghdad, while the other portions were sent to Chicago and Oxford. I was immediately questioned as to whether Oxford was near London, and I was impressed with their geographical knowledge until I discovered that London, Berlin, and Constantinople constitute all their knowledge of Europe. Not one of the 150 workmen had heard of the Americas. One of the foremen asked the distance and number of camel journeys from Kish to Chicago, but when I described the intervening ocean I was not believed by any member of the group. Repeatedly they would ask me to explain why we could not bring part of the great ocean to them, so that they might irrigate their properties.

These Arabs, from an early age, are interested in the moon and stars. The former controls the fast at Ramadan, and the latter guide them by night. As a result they know many of the stars by name. The evening star and the Dipper (mizan) are immediately recognized by young and old alike. It is interesting to note that when Babylon was the capital of central Mesopotamia, Kish had degenerated into a mere outpost and then was the center of astronomical and mathematical research. 


\section{ANTHROPOMETRIC DATA}

\section{MEASUREMENTS AND OBSERVATIONS}

The anthropometric statistics in this chapter were obtained from three groups of Arabs living at that time in the Kish area. The largest group includes 398 Arabs, who live in various encampments in the region of Tell-el-Uhaimir. The second group comprises 231 members of the Iraq army, located at Hilla in 1928. These Arabs were recruited from all over Iraq, and thus form a non-homogeneous group. The third series of anthropometric data was obtained from thirty-eight Beduins belonging to the $\mathrm{Ba}$ ij tribe, which wanders north and south between the Tigris and the Euphrates.

An anthropometer was used for measuring the stature, height from acromion to sole, and sitting height. This instrument is a graduated metal rod which is in sections, fixed to a metal base and provided with a sliding horizontal branch. In order to obtain the stature the sections of the anthropometer were screwed together and inserted in the metal base. The instrument was then placed against the main tent pole and tied with a loose string to hold it upright. Each subject was placed with his back to the tent pole and the sliding horizontal bar was moved down until it rested on the top of his head. With two Arab assistants it was possible to obtain this measurement, although the standing height figures of the Kish Arabs show considerable variation, since it was impossible to make them stand uniformly straight. The Iraq soldiers, however, were accustomed to drill and therefore stood erect.

The acromion to sole measurement was obtained on the standing individual by placing the horizontal bar of the anthropometer on the left superior and external border of the acromion process. This measurement immediately followed the standing height, since it involved little change of position.

The sitting height was obtained by seating the subject on a four-gallon gasoline can about $34 \mathrm{~cm}$ in height. The knees were flexed, the dorsal aspect of the trunk made contact with the anthropometric rod in the sacral region and between the shoulder blades. The axis of vision remained horizontal. The height of the vertex from the ground was measured and later the height of the seat subtracted to give the correct sitting height. It was found advisable to carry a gasoline can as a seat for this measurement, since the various native wooden stools varied in height, and it was easier to subtract a constant figure for the height of the seat from the ground.

The measurements obtained on the head were as follows: greatest occipital length, greatest breadth, minimum frontal diameter, bizygomatic breadth, bigonial breadth, total facial height, upper facial height, nasal height, nasal breadth, and left ear length and breadth.

Since there are several systems used in the taking of these anthropometric measurements, it will be necessary to state the methods employed. The spreading calipers were held with a branch reposing on the palm of each hand. Each 
forefinger guided the metal end of the calipers to the desired point, and when they were satisfactorily adjusted the reading was made. The elbows were held parallel to the ground and in some cases the butt or joint of the instrument rested against the chest in order to give support, while the scale was being read. The facial measurements were obtained with the same instrument, which was firmly held by the hands when the body could not be used as the support.

In the majority of cases the landmarks used for determining the exact point on the head upon which the extremities of the sliding calipers should be placed followed the international agreement for the unification of anthropometric measurements made on living subjects, contained in the report of the commission appointed by the Fourteenth International Congress of Prehistoric Anthropology and Archaeology at Geneva (1912), to supplement the work commenced by the Thirteenth Congress at Monaco (1906). This report was translated by W. L. H. Duckworth, and is quoted in A. Hrdlicka's "Anthropometry" (pp. 25-31).

The measurements on the head were taken in the following manner. The head length (glabella to opisthocranium) was measured with the spreading calipers from the most prominent point of the glabella to the most prominent point on the occiput, as shown by the maximum determinable spread of the branches of the compass. The two landmarks were taken in the median line and a slight pressure was used on the ends of the calipers.

The head breadth (euryon to euryon) was measured with the spreading calipers from the widest points above the supramastoid and zygomatic crest. The ends of the instrument were pressed lightly to the sides of the head and moved in an anterior to posterior line until the maximum reading was obtained.

The head height could not be measured, since the Arabs would not allow any instrument to penetrate the auditory meati.

The minimum frontal diameter was obtained by measuring the shortest horizontal diameter between the two temporal crests on the frontal bone. The ends of the spreading calipers were guided by each forefinger to the deepest part in the curve of each temporal line above the lateral angular processes of the frontal bone, and the reading thus obtained.

The bizygomatic breadth (zygion to zygion) was measured by placing the spreading calipers on the most widely separated points on the external surface of the zygomatic arches.

The bigonial breadth was obtained with the spreading calipers by placing the ends of the instrument on the most prominent external points of the angles of the lower jaw (gonion).

The total facial height was measured with the spreading calipers from the lowest point in the middle of the bony chin (menton) to the point in the median sagittal line where the nasal bones join the frontal bone (nasion). This measurement was obtained by holding the calipers in a vertical position, while the scale was read from the side.

The upper facial height was measured from the nasion to the point between the two front incisor teeth where they enter the gum of the upper jaw. 
The nasal height was measured with the spreading calipers from the nasion to that point where the septum of the nose joins the upper lip (subnasale).

The nasal breadth (alare to alare) was obtained with the spreading calipers by measurement of the maximum normal external breadth of the nasal alae, determined without the exertion of any pressure.

The sliding compass was used for the measurements on the ear. The left ear was measured wherever circumstances permitted. The ear length was measured from the highest point on the border of the helix to the lowest point on the lobe. The rod of the sliding compass was held parallel to the long axis of the ear.

The ear breadth was obtained with the sliding compass by measuring the distance between two lines parallel to the long axis of the ear, one of these lines being tangent to the anterior, the other to the posterior border of the helix.

In recording observations the following subdivisions were used: hair, eye, eyebrow, nose, teeth, ear, scapulae, chest, musculature, and health. The form of the hair was noted as straight, with low or deep waves, curly, frizzly, or woolly; the texture was recorded as coarse, medium, or fine; the quantity on the head, beard, and body was noted; the color was recorded as black, dark brown, reddish brown, light brown, golden, red, white, or gray. The color of the eye was noted as black, dark brown, blue brown, gray brown, green brown, blue, or gray; the sclera as clear, speckled, yellow, or bloodshot; the iris as homogeneous, rayed, or zoned. The thickness, concurrency, and lateral extension of the eyebrows were noted. Median or continuous brow-ridges, and the prominence of the glabella were observed. The nasion depression, nasal root, and thickness of the nasal tip and its elevation or depression were recorded. The nasal wings were compressed, medium, or flaring, and the nasal septum was straight or convex with an upward or downward inclination. The prominence of the malars was mentioned whenever conspicuous. The eruption of the teeth was recorded as complete or unerupted. The wear on the teeth, occurrence of caries, number lost, and whether the bite was under, edge-to-edge, slight over, or marked over were noted. With regard to the ears the development of the lobe, whether it was attached or free, the degree of roll of the helix and the presence of Darwin's point were recorded. The vertebral borders of the scapulae were straight, convex, or concave. The chest development, general musculature, and state of health were recorded. Any pathological characters were observed, particularly the occurrence of smallpox, "Baghdad boils," or attacks of fever. The presence or absence of tattooing was observed and a general discussion of body-marking in southwestern Asia, together with a detailed report on tattooing in Iraq, will be published later.

Since the anthropometric data already published on Arabs are limited, it seemed desirable to publish in full the figures and other data obtained. The statistics have been tabulated in Chapter III. It must be noted, however, that Dr. L. H. Dudley Buxton, with myself as the recorder, made anthropometric measurements on 164 adult Arab males at Kish during January, 1926. The results are included in the article by Buxton and Rice, entitled "Report on the Human Remains found at Kish" (J.R.A.I., LXI, 1931, pp. 57-119). The 
figures are quoted here, since these Arabs belong to the groups studied and included in this Report. Each individual was asked if he had been measured before, and if so was omitted, so that little duplication could have resulted.

In the following group 164 persons were measured in every case, except for the stature, when only 159 individuals were recorded.

MEAN MEASUREMENTS OF LIVING ADULT MALES FROM KISH

(After Buxton and Field. Season, 1925-26)

\begin{tabular}{|c|c|c|c|}
\hline Measurements & Mean & $\begin{array}{l}\text { Standard } \\
\text { deviation }\end{array}$ & $\begin{array}{l}\text { Coefficient } \\
\text { of variation }\end{array}$ \\
\hline Stature. & $169.56 \pm 0.33$ & $6.11 \pm 0.23$ & $3.61 \pm 0.14$ \\
\hline Glabella-occipital length & $189.94 \pm 0.33$ & $6.21 \pm 0.23$ & $3.27 \pm 0.12$ \\
\hline Greatest breadth. & $143.48 \pm 0.26$ & $4.88 \pm 0.18$ & $3.40 \pm 0.13$ \\
\hline Minimum frontal diameter. & $107.39 \pm 0.21$ & $3.99 \pm 0.15$ & $3.71 \pm 0.14$ \\
\hline Bizygomatic breadth...... & $133.32 \pm 0.26$ & $4.81 \pm 0.18$ & $3.61 \pm 0.13$ \\
\hline Bigonial breadth... & $104.83 \pm 0.29$ & $5.35 \pm 0.20$ & $5.10 \pm 0.19$ \\
\hline Nasal height. & $46.98 \pm 0.23$ & $4.27 \pm 0.16$ & $9.08 \pm 0.34$ \\
\hline Nasal breadth. & $33.54 \pm 0.15$ & $2.86 \pm 0.11$ & $8.43 \pm 0.32$ \\
\hline Upper facial height. & $66.51 \pm 0.23$ & $4.35 \pm 0.16$ & $6.54 \pm 0.24$ \\
\hline Total facial height. . & $114.53 \pm 0.37$ & $6.71 \pm 0.25$ & $5.86 \pm 0.22$ \\
\hline \multicolumn{4}{|l|}{ Indices } \\
\hline Cephalic index & $76.61 \pm 0.18$ & $3.43 \pm 0.13$ & $4.54 \pm 0.17$ \\
\hline Nasal in & $72.80 \pm 0.46$ & $8.64 \pm 0.32$ & $11.87 \pm 0.45$ \\
\hline Upper facial inde & $49.56 \pm 0.18$ & $3.45 \pm 0.12$ & $6.96 \pm 0.26$ \\
\hline Total facial index. & $85.14 \pm 0.29$ & $5.42 \pm 0.20$ & $6.37 \pm 0.24$ \\
\hline
\end{tabular}

\section{ARABS OF THE KISH AREA}

\section{No. 1. Plate I, Figs. 1, 2}

Locality: Jemdet Nasr. Date: March 12, 1928.

Birthplace: camp of Sheikh Miniehil. Age: 24; unmarried.

Brothers: 2 living, 3 dead. Sisters: 1 living.

Tattoo marks: horse on right arm.

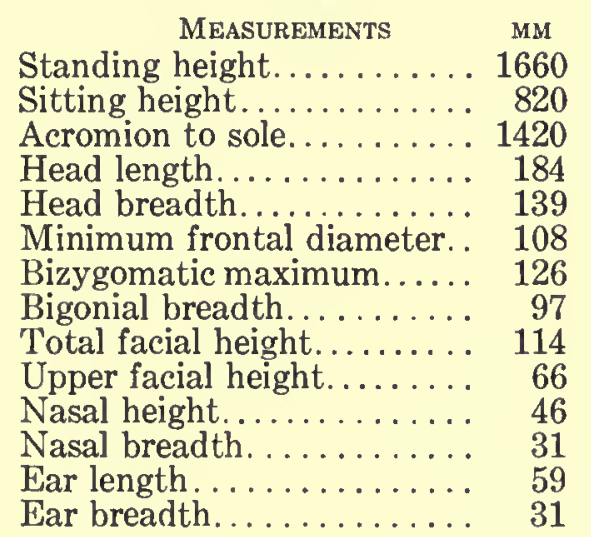

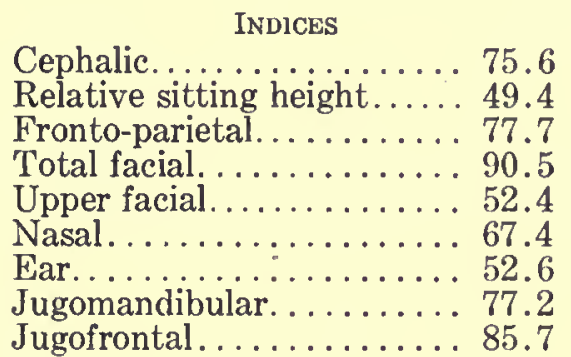

Jugomandibular.......... 85.7

HAlR.-Form: straight. Texture: coarse. Color: reddish brown. Quantity: head, plus; beard, medium; body, medium.

EYE.-Color: dark brown. Sclera: clear. Iris: homogeneous.

EyeBrow.-Thickness: plus. Concurrency: plus. Lateral extent: plus. Brow-ridges: median. Glabella: plus.

Nose.-Profile: straight. Tip: thickness plus, depressed. Wings: compressed.

TEETH.-Wear: none. Caries: none. Lost: none. 
EAR.-Lobe: attached. Size: plus. Helix: plus. Darwin's Point: plus. SCAPULAE.-Vertebral borders: convex.

CHEst.-Development: average plus.

Musculature.-Good.

HEALTH.-Good.

\section{No. 2. Plates I, Figs. 3, 4; CXXX; CXXXI}

Locality: Jemdet Nasr. Date: March 12, 1928.

Birthplace: camp of Sheikh Miniehil. Age: 25; unmarried.

Brothers: 2 living, 3 dead. Sisters: 1 living.

Tattoo marks: gazelle on right hand (identification mark); on right arm.

\begin{tabular}{|c|c|c|}
\hline MEASUREMENTS & MM & INDICES \\
\hline Standing height. . & 1700 & Cephalic. \\
\hline Sitting height. & 835 & Relative sitting height.....49.1 \\
\hline Acromion to sole. & 1435 & Fronto-parietal.......... 80.4 \\
\hline Head length. . & 192 & Total facial. \\
\hline Head breadth. & 143 & Upper facial. \\
\hline Minimum frontal diameter. . & 115 & Nasal. \\
\hline Bizygomatic maximum..... & 130 & . 52.4 \\
\hline Bigonial breadth.......... & 108 & Jugomandibular. . . . . . \\
\hline Total facial height......... & 129 & Jugofrontal. . . . . . . . 88.4 \\
\hline
\end{tabular}

Upper facial height. ........ 76

Nasal height............ 54

Nasal breadth............. 33

Ear length............ 63

Ear breadth............ 33

HAIR.-Form: straight. Texture: coarse. Color: dark brown.

EYE.-Color: dark brown. Sclera: speckled. Iris: homogeneous.

EYEBRow.-Brow-ridges: median.

Nose.-Profile: straight. Wings: compressed.

TEETH.-Wear: slight. Caries: none. Lost: none.

EAR.-Helix: plus. Darwin's Point: plus.

SCAPULAE.-Vertebral borders: convex.

CHEST.-Development: average.

Musculature.-Excellent.

HEALTH.-Good.

Brother of No. 1.

No. 3. Plate I, Figs. 5, 6

Locality: Jemdet Nasr. Date: March 12, 1928.

Birthplace: near Imam Sa'id. Age: 20 ; married 18 months. No children.

Brothers: 1 living. Sisters: 2 living.

Tattoo marks: none.

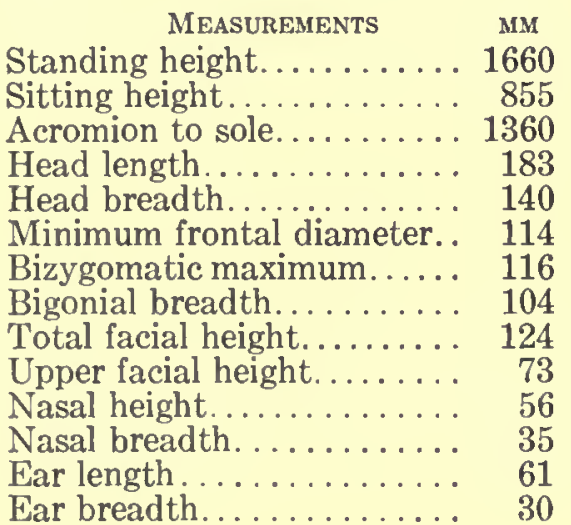

INDICES

Cephalic.............. 76.5

Relative sitting height...... 51.5

Fronto-parietal.......... 81.4

Total facial. ...............108.7

Upper facial............6 63.5

Nasal ................. 62.5

Ear................ 49.2

Jugomandibular. .......... 89.6

Jugofrontal........... 98.2 
HAIR.-Form: curly frizzly. Texture: medium-fine. Color: dark brown. Quantity: head, plus; beard, minus; body, medium.

EYE.-Color: dark brown. Sclera: speckled and yellow. Iris: zoned.

EYEBRow.-Brow-ridges: median.

Nose.-Profile: concave. Wings: medium.

TEETH.-Lost: none.

EAR.-Lobe: free.

SCAPULAE.--Vertebral borders: straight.

Chest.-Development: average.

Musculature.-Excellent.

HEALTH.-Excellent.

No. 4. Plate I, Figs. 7, 8

Locality: Jemdet Nasr. Date: March 12, 1928.

Birthplace: near Bagala on Euphrates. Age: 30 ; married 12 years. Sons: 1 living, 1 dead. Daughters: 2 living, 2 dead.

Birthplace of father and mother: Bughaila.

Brothers: 1 living. Sisters: 1 dead.

Tattoo marks: none.

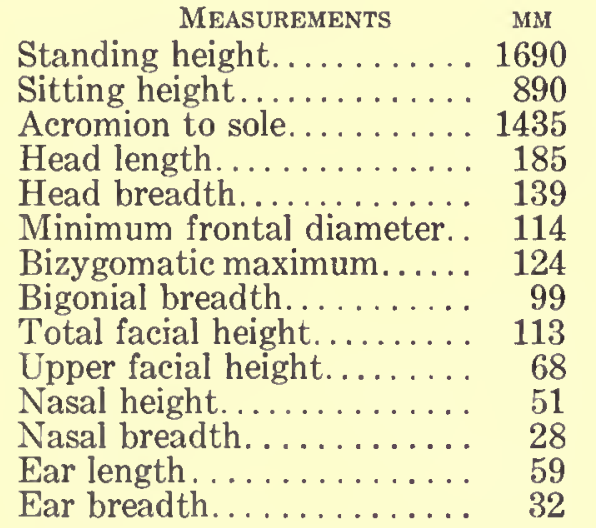

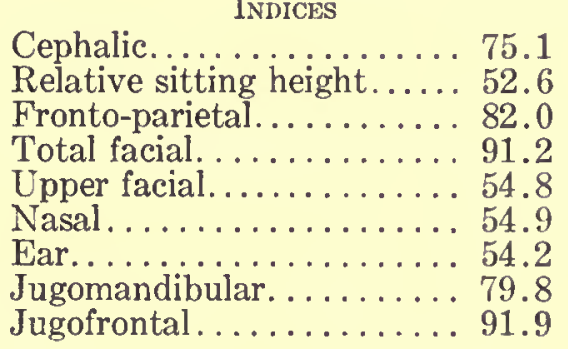

HaIr.-Form: low waves. Texture: coarse. Color: dark brown. Quantity: head, plus; beard, plus; body, plus.

EYE.-Color: dark brown. Sclera: speckled. Iris: rayed.

EyeBrow.-Thickness: plus. Concurrency: plus. Lateral extent: average. Brow-ridges: median. Glabella: average.

Nose.-Profile: straight. Tip: depressed.

TEETH.-Wear: slight. Lost: none.

EAR.-Lobe: attached. Size: plus. Helix: plus. Darwin's Point: double plus.

SCAPULAE.-Vertebral borders: straight.

CHEsT.-Development: average plus.

Musculature.-Excellent.

HEALTH.-Good.

\section{No. 5}

Locality: Jemdet Nasr. Date: March 12, 1928.

Birthplace: camp of Sheikh Miniehil. Age: 18; unmarried.

Brothers: 1 living, 2 dead. Sisters: 1 living.

Tattoo marks: none.

MEASUREMENTS MM

Standing height......... 1545

Sitting height............ 710

Acromion to sole........ 1273
INDICES

Cephalic............. 75.2

Relative sitting height.....45.9

Fronto-parietal......... 76.1 


\begin{tabular}{|c|c|}
\hline MEASUREMENTS & \\
\hline $\begin{array}{l}\text { Head length............ } \\
\text { Head breadth. . . . }\end{array}$ & \\
\hline Minimum frontal diameter. & \\
\hline Bizygomatic maximum... & \\
\hline Bigonial breadth. & \\
\hline Total facial height. & \\
\hline Upper facial height. & \\
\hline Nasal breadt & \\
\hline or length & \\
\hline breadth. & \\
\hline
\end{tabular}

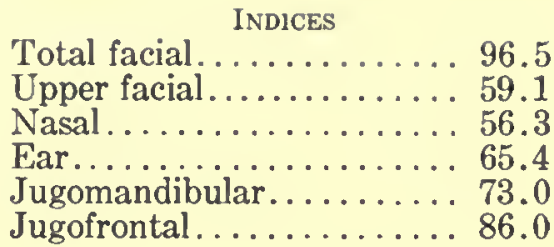

HaIn.-Form: straight. Texture: coarse. Color: dark brown. Quantity: head, double plus. EyE.-Color: dark brown. Sclera: clear. Iris: homogeneous.

Nose.-Profile: concave. Tip: depressed. Wings: compressed.

TEETH.-Wear: none. Caries: none. Lost: none.

EAR.-Lobe: attached. Helix: plus.

SCAPULAE.-Vertebral borders: straight.

CHEST.-Development: poor.

Musculature.-Fair.

HEALTH.-Good.

No. 6

Locality: Jemdet Nasr. Date: March 12, 1928.

Birthplace: camp of Sheikh Mugheir. Age: 16; unmarried.

Brothers: 3 living. Sisters: none.

Tattoo marks: none.

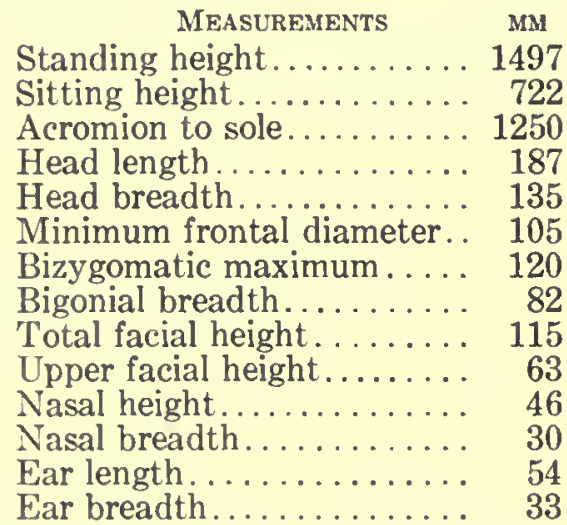

HAIR.-Texture: medium. Color: dark brown. Quantity: head, medium; beard, medium; body, medium.

EYE.-Color: dark brown. Sclera: clear. Iris: zoned.

EYEBROW.-Thickness: minus. Brow-ridges: median.

Nose.-Profile: straight. Tip: depressed. Wings: medium.

TEETH.-Caries: plus. Lost: none.

SCAPULAE.-Vertebral borders: straight.

Chest.-Development: average.

Musculature.-Good.

HeALTH.-Good.

No. 7. Plate II, Figs. 1, 2

Locality: Jemdet Nasr. Date: March 13, 1928.

Birthplace: camp of Sheikh Atiyeh. Age: 25; married for 7 years. No children.

Brothers: 1 living, 1 dead. Sisters: 3 living.

Tattoo marks: on glabella. 


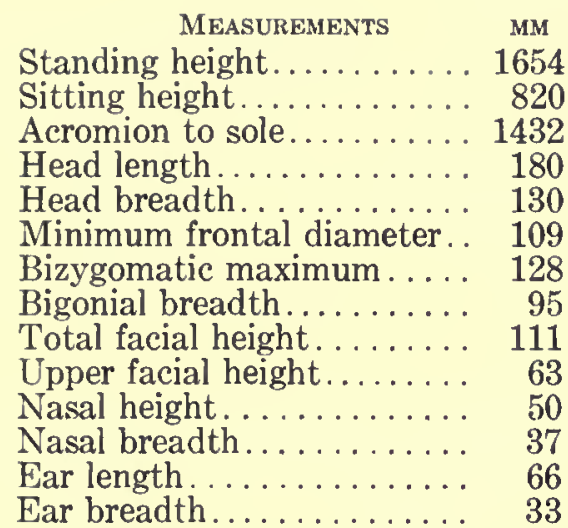

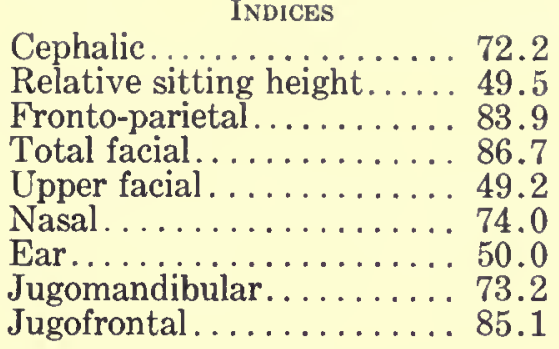

HAIR.-Form: curly frizzly. Texture: medium. Color: dark brown black. Quantity: head, double plus; beard, plus; body, plus.

EYE.-Color: dark brown. Sclera: speckled. Iris: homogeneous.

EYEBROW.-Thickness: plus. Brow-ridges: median.

Nose.-Profile: straight. Tip: thickness plus. Wings: medium-flaring.

TEETH.-Wear: slight. Caries: none. Lost: none.

EAR.-Lobe: free. Size: plus.

SCAPULAE.-Vertebral borders: convex.

CHEST.-Development: average.

Musculature.-Fair.

Health.-Good.

No. 8

Locality: Jemdet Nasr. Date: March 13, 1928.

Birthplace: the desert near Jemdet Nasr. Age: 25; unmarried.

Birthplace of father and mother: camp of Sheikh Miniehil.

Brothers: 2 living. Sisters: 2 living.

Tattoo marks: gazelle, as "identification mark."

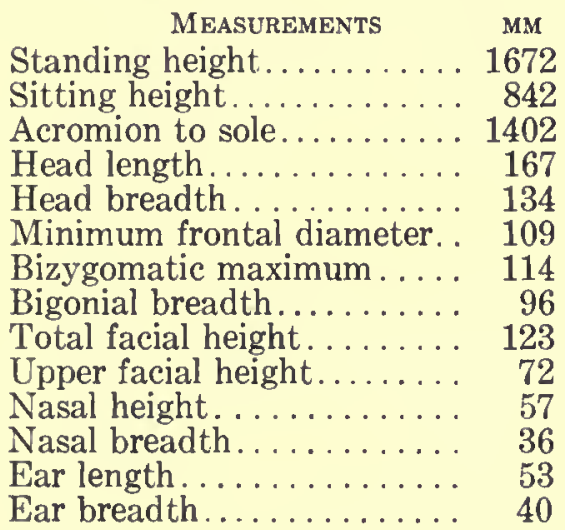

INDICES

Cephalic............. 80.2

Relative sitting height..... 50.3

Fronto-parietal......... 81.4

Total facial. . . . . . . . . . 92.8

Upper facial. ..........6. 63.1

Nasal. . . . . . . . . . . . 63.1

Ear................ 75.5

Jugomandibular ......... 84.2

Jugofrontal . . . . . . . . . . . 95.6

HAIR. -Form: low waves. Texture: medium. Color: dark brown. Quantity: head, plus; beard, plus; body, plus.

EYE.-Color: dark brown. Sclera: speckled. Iris: homogeneous.

EyeBrow.-Thickness: plus. Lateral extent: plus. Brow-ridges: median. Glabella: average. Nose.-Profile: concave. Tip: depressed. Wings: medium.

TEETH.-Wear: none. Caries: none. Lost: none.

EAR.-Lobe: attached. Size: minus. Helix: plus. Darwin's Point: plus.

SCAPULAE.--Vertebral borders: convex.

Chest.-Development: average. 
Musculature.-Good.

HEalth.-Excellent.

\section{No. 9. Plate II, Figs. 3, 4}

Locality: Jemdet Nasr. Date: March 13, 1928.

Birthplace: between Hilla and Baghdad. Age: 20; unmarried. Birthplace of father and mother: camp of Sheikh Miniehil. Brothers: 1 living, 1 dead. Sisters: 1 living, 1 dead.

Tattoo marks: gazelle on left forearm.

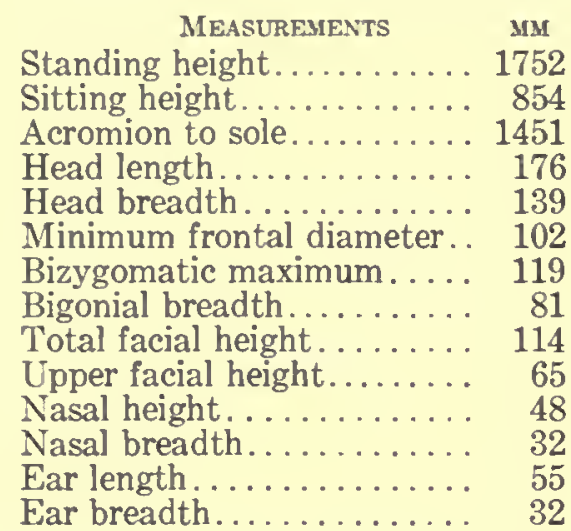

INDICES

Cephalic.............. 78.9

Relative sitting height..... 48.7

Fronto-parietal......... 73.4

Total facial. . . . . . . . . 95.8

Upper facial. .......... 54.6

Nasal. ..............66.6 66

Ear.................. 58.2

Jugomandibular. . . . . . 68.0

Jugofrontal.......... 85.7

HaIR.-Form: low waves. Texture: coarse. Color: dark brown. Quantity: head, double plus; beard, plus; body, plus.

EYE. -Color: dark brown. Sclera: clear. Iris: homogeneous.

Nose.-Profile: straight. Tip: thickness plus, elevated. Wings: compressed.

TeETH.-Wear: slight. Caries: none. Lost: none.

EAR.-Lobe: attached. Size: plus. Helix: plus. Darwin's Point: average.

SCAPULAE.-Vertebral borders: straight.

Musculature.-Fair.

HEALTH.-Good.

\section{No. 10. Plates II, Figs. 5, 6; CXXXII; CXXXIII}

Locality: Jemdet Nasr. Date: March 13, 1928.

Birthplace: camp of Sheikh Miniehil. Age: 19; unmarried.

Brothers: 3 living. Sisters: 2 living, 1 dead (infant).

Tattoo marks: gazelle on right forearm.

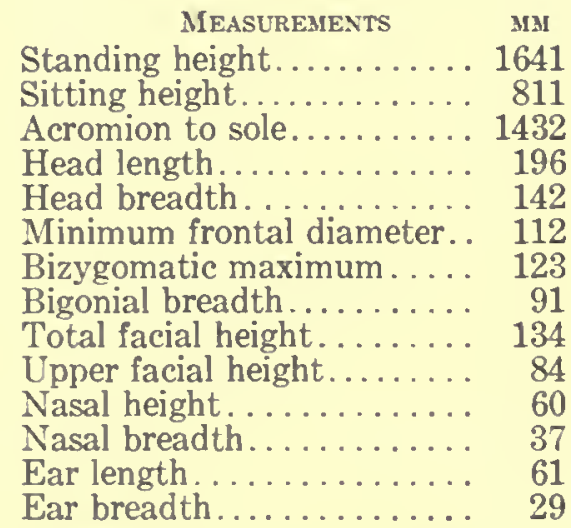

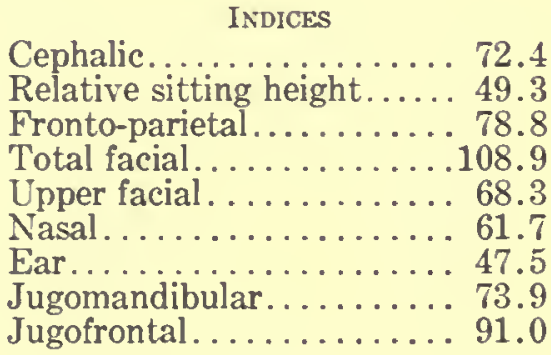

HaIR.-Form: low waves. Texture: coarse. Color: dark brown. Quantity: head, double plus; beard, average; body, average.

EYE.-Color: dark brown. Sclera: clear. Iris: homogeneous. 
EyeBRow.-Thickness: plus. Concurrency: plus. Lateral extent: plus. Brow-ridges: median. Glabella: plus.

Nose.-Profile: straight. Wings: flaring.

TEETH.-Wear: none. Caries: none. Lost: none. Condition: excellent.

EAR.-Lobe: free. Helix: minus. Darwin's Point: plus.

CHEST.-Development: good.

Musculature.-Good.

HEALTH.-Excellent.

\section{No. 11. Plate II, Figs. 7, 8}

Locality: Jemdet Nasr. Date: March 13, 1928.

Birthplace: camp of Sheikh Miniehil. Age: 25; married for 5 months.

Brothers: 2 living, 2 dead. Sisters: 1 living, 1 dead.

Tattoo marks: spot on end of nose.

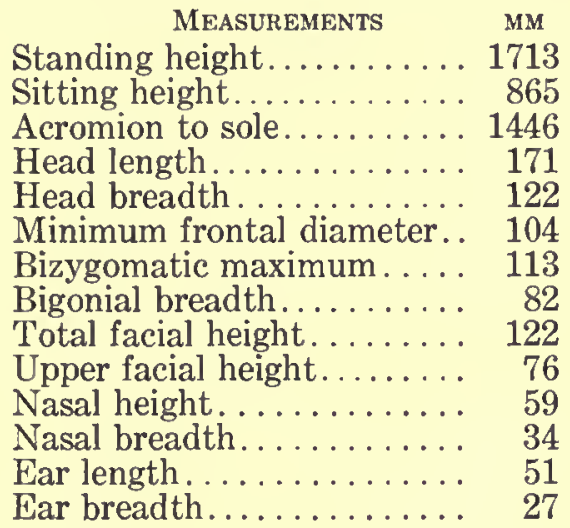

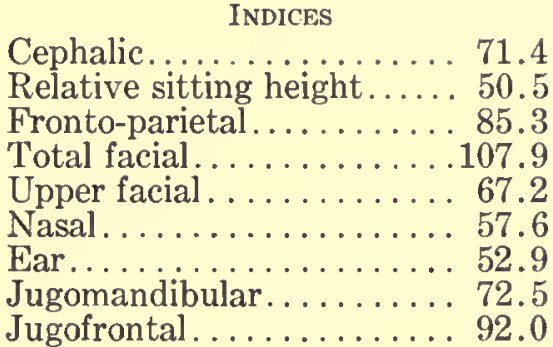

Cephalic.............. 71.4

Relative sitting height..... 50.5

85.3

Upper facial . . . . . . . 67.2

Nasal................ 57.6

Jugomandibular......... 72.5

Jugofrontal. . . . . . . . . 92.0

HAIR.-Form: low waves. Texture: coarse. Color: dark brown. Quantity: head, double plus; beard, medium; body, medium.

Eye.-Color: dark brown. Sclera: clear. Iris: homogeneous.

NosE.-Profile: concave. Wings: medium.

TEETH.-Wear: none. Caries: none. Lost: none.

EAR.-Lobe: attached. Size: average. Helix: average. Darwin's Point: plus.

SCAPULAE.-Vertebral borders: straight.

Chest.-Development: average.

MUSCUlature.-Good.

HEALTH.-Excellent.

No. 12

Locality: Jemdet Nasr. Date: March 13, 1928.

Birthplace: camp of Sheikh Mugheir. Age: 50; married for 30 years, 3 wives. Divorced one wife, one left him, and one with him now. Sons: 4, 0, 0, dead. Daughters: 3, 0, 0, living; $4,0,0$, dead.

Brothers: 1 living, 3 dead. Sisters: 2 living.

Tattoo marks: 2 spots on inside of right forearm.

\begin{tabular}{|c|c|c|}
\hline MEASUREMENTS & мM & INDICES \\
\hline Standing height.. & 1726 & Cephalic. \\
\hline Sitting height. . & 867 & Relative sitting height. \\
\hline Acromion to sole. & 1456 & Fronto-parietal. \\
\hline Head length. & 196 & Total facial. \\
\hline Head breadth. & 149 & Upper facial \\
\hline Minimum frontal diameter.. & 103 & Nasal. \\
\hline Bizygomatic maximum..... & 122 & Ear. \\
\hline Bigonial breadth. & 99 & Jugomandibular \\
\hline tal facial height. & 117 & Jugofrontal \\
\hline
\end{tabular}




\begin{tabular}{|c|c|}
\hline MEASUREMENTS & \\
\hline Nasal height. & $5 ?$ \\
\hline Nasal breadth & 3 \\
\hline length. . & \\
\hline
\end{tabular}

HaIR.-Form: low waves. Texture: coarse. Color: dark brown. Quantity: head, minus; beard, double plus; body, plus.

EYE.-Color: dark brown. Sclera: speckled and bloodshot. Iris: zoned.

EYEBRow.-Lateral extent: plus. Brow-ridges: median. Glabella: plus.

Nose.-Profile: concave. Wings: medium.

TeEth.-Wear: none. Caries: none. Lost: none. Condition: poor.

EAR.-Lobe: attached. Size: average. Helix: average. Darwin's Point: plus.

SCAPULAE.-Vertebral borders: straight.

CHEST.-Development: average.

Musculature.-Fair.

HEALTH.-Poor.

No. 13. Plate III, Figs. 1, 2

Locality: Jemdet Nasr. Date: March 13, 1928.

Birthplace: camp of Sheikh Miniehil. Age: 20; unmarried.

Brothers: 2 living. Sisters: 2 living.

Tattoo marks: band with two spots on right wrist; three spots on right and left frontals.

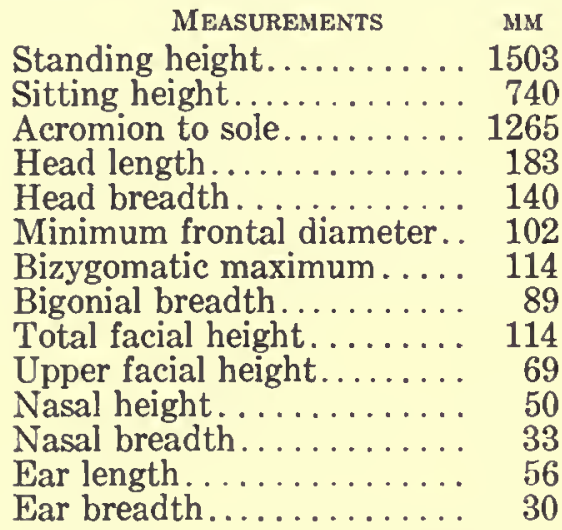

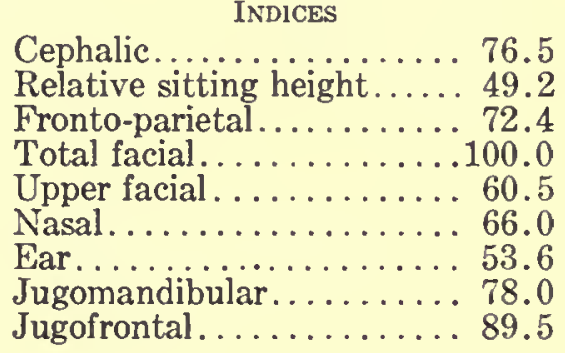

Cephalic ............... 76.5

Relative sitting height..... 49.2

Fronto-parietal.......... 72.4

Total facial.............100.0

Upper facial............ 60.5

Ear.......... 53.6

Jugofrontal. . . . . . . . 89.5

HAIR.-Color: dark brown. Quantity: head, plus; beard, double minus; body, double minus. EYE.-Color: dark brown. Sclera: speckled. Iris: homogeneous.

NosE.-Profile: straight. Tip: depressed. Wings: compressed.

TEETH.-Wear: none. Caries: none. Lost: none.

EAR. - Lobe: attached. Size: average. Helix: plus. Darwin's Point: average.

SCAPULAE.-Vertebral borders: straight.

CHEsT.-Development: poor.

Musculature.-Poor.

HEALTH.-Poor.

\section{No. 14. Plate III, Figs. 3, 6}

Locality: Jemdet Nasr. Date: March 13, 1928.

Birthplace: camp of Sheikh Miniehil. Age: 17; unmarried.

Brothers: 1 living, 3 dead. Sisters: 1 living.

Tattoo marks: spot on tip of nose and on right wrist.

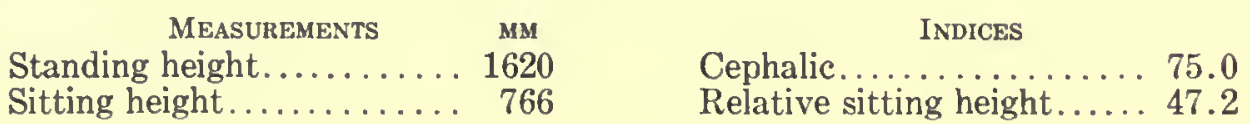




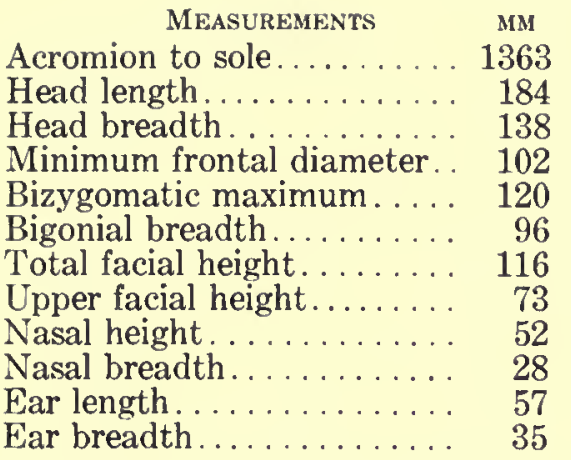

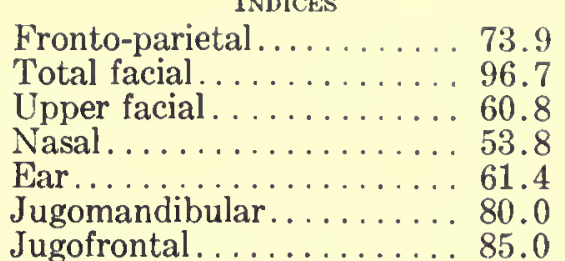

HaIR.-Form: low waves. Texture: coarse. Color: dark brown. Quantity: head, triple plus; beard, minus; body, minus.

EYE.-Color: dark brown. Sclera: clear. Iris: homogeneous.

EYEBrow.-Thickness: plus. Concurrency: plus. Lateral extent: average. Brow-ridges: continuous. Glabella: plus.

NosE.-Profile: straight.

TеEтн.-Wear: slight. Caries: none. Lost: none.

EAR.-Lobe: attached. Size: average. Helix: plus.

SCAPULAE.-Vertebral borders: straight.

CHEsT.-Development: average.

Musculature.-Fair.

HeALTH.-Excellent.

Locality: Jemdet Nasr. Date: March 13, 1928.

Birthplace: camp of Sheikh Miniehil. Age: 40 ; married for 22 years, 2 wives (both living, the second the aunt of No. 13). Sons: 1, 1, living. Daughters: 2,2 , living.

Birthplace of father: camp of Sheikh Miniehil. Birthplace of mother: camp of Sheikh Hadji Hunta on Tigris.

Brothers: none. Sisters: 1 living.

Tattoo marks: on right wrist.

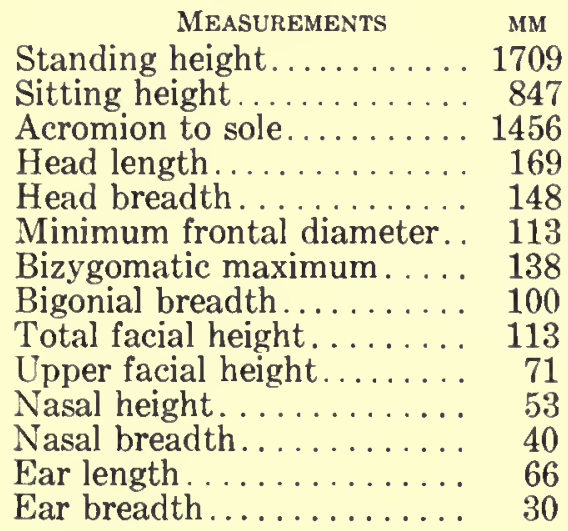

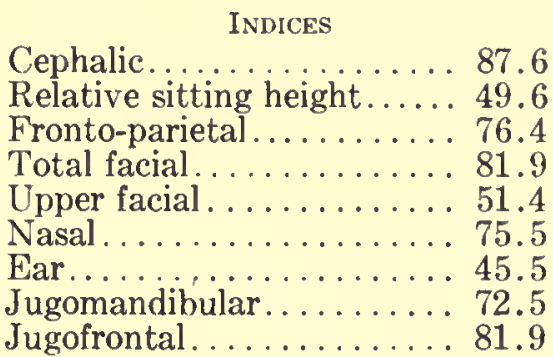

HaIR.-Form: low waves. Color: dark brown. Quantity: head, minus; beard, triple plus; body, triple plus.

EYE.-Color: dark brown. Sclera: clear. Iris: homogeneous.

EyeBrow.-Thickness: average. Concurrency: plus. Lateral extent: plus. Brow-ridges: median. Glabella: plus.

NosE.-Profile: concave. Tip: thickness plus, horizontal. Wings: medium.

TеEтн.-Wear: triple plus. Caries: triple plus. Lost: on right side 1 upper, 1 lower premolar, 2 lower molars. Condition: very poor.

EAR.-Helix: plus. Darwin's Point: plus. 
Chest.-Development: good.

Musculature.-Fair.

HEALTH.-Often fevered and has headaches.

No. 16

Locality: Jemdet Nasr. Date: March 13, 1928.

Birthplace: Hilla. Age: 28; married for 12 years. Sons: 2 living, 1 dead. Daughters: 1 living, 1 dead.

Tattoo marks: none.

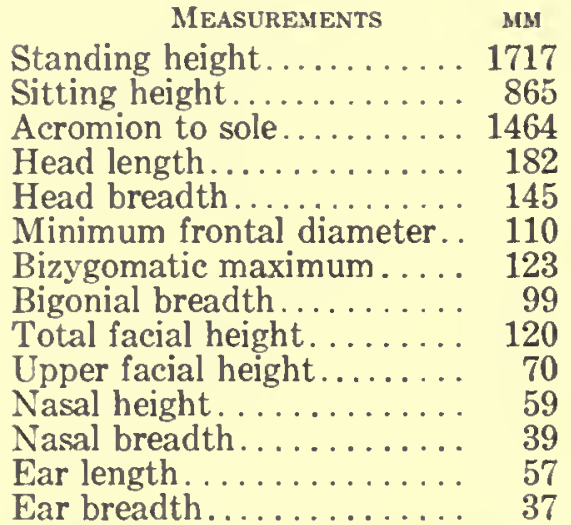

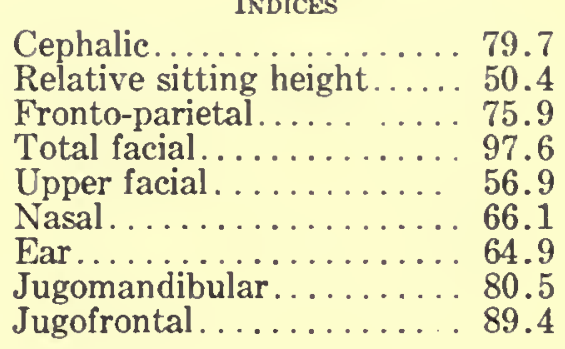

HAIR.-Form: low waves. Texture: fine. Color: dark brown. Quantity: head, double plus; beard, plus; body, plus.

EYE.-Color: dark brown. Sclera: speckled. Iris: zoned.

EYEBROW.-Thickness: minus.

NosE.-Profile: straight. Tip: thickness plus, horizontal. Wings: medium.

TEeTH.-Wear: double plus. Caries: plus. Lost: 2 right lower molars.

EAR.-Lobe: attached. Size: plus. Helix: minus. Darwin's Point: minus.

SCAPULAE.-Vertebral borders: straight.

CHEsT.-Development: good.

Musculature.-Good.

Health.-Fair.

\section{No. 17. Plate III, Figs. 4, 5}

Locality: Jemdet Nasr. Date: March 14, 1928.

Birthplace: camp of Sheikh Adi at Shomali. Age: 20; unmarried.

Birthplace of father: camp of Sheikh Miniehil. Birthplace of mother: camp of Sheikh Mutlukh at Butafia.

Brothers: 2 living. Sisters: none.

Tattoo marks: none.

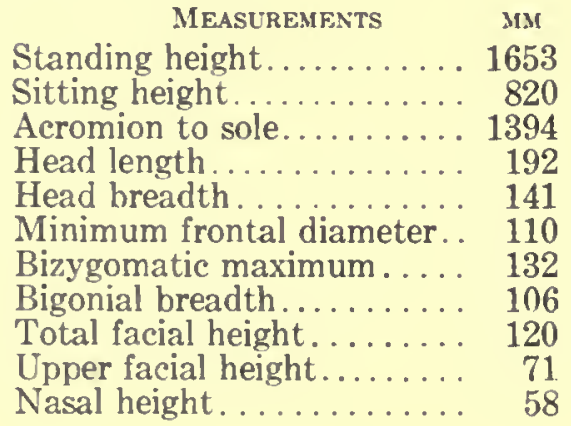




$\begin{array}{cr}\text { MEasurements } & \text { MM } \\ \text { Nasal breadth........... } & 35 \\ \text { Ear length } \ldots \ldots \ldots \ldots \ldots \ldots & 54 \\ \text { Ear breadth } \ldots \ldots \ldots \ldots \ldots & 36\end{array}$

HaIR.-Form: low waves. Color: dark brown. Quantity: head, double plus; beard, double plus; body, plus.

EYE.-Color: dark brown. Sclera: speckled. Iris: homogeneous.

Nose.-Profile: concave. Tip: depressed. Wings: compressed.

TEETH.-Wear: slight. Caries: none. Lost: none.

EAR.-Lobe: free. Size: plus. Helix: plus. Darwin's Point: plus.

SCAPULAE.-Vertebral borders: straight.

CHEsT.-Development: average.

Musculature.--Fair.

HEALTH.-Good.

No. 18. Plate IV, Figs. 1,2

Locality: Jemdet Nasr. Date: March 14, 1928.

Birthplace: camp of Sheikh Miniehil. Age: 24; married for 9 years. Sons: 2 living, 1 dead Daughters: 1 living.

Brothers: 4 living, 2 dead. Sisters: 3 living.

Tattoo marks: 2 spots on left hand.

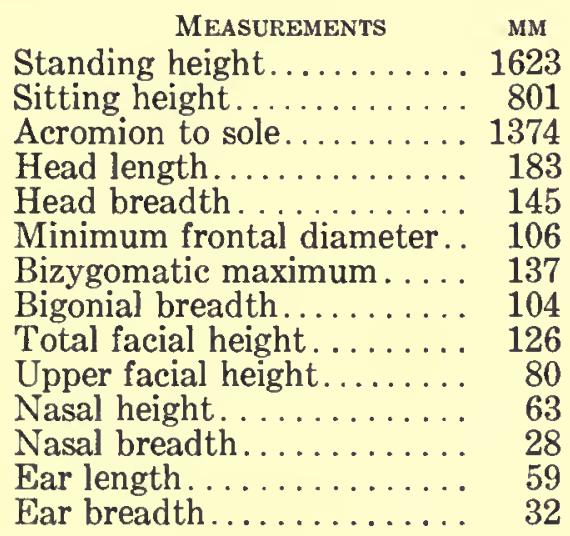

Cephalic............... 79.2

Relative sitting height..... 49.3

Fronto-parietal.......... 73.1

Total facial. ............. 91.9

Upper facial ............ 58.4

Nasal. ................ 44.4

Ear................ 54.2

Jugomandibular. . . . . . . 75.9

Jugofrontal. . . . . . . . . 77.3

HAIR.-Form: straight. Color: dark brown. Quantity: head, plus; beard, double plus; body, plus.

EyE.-Color: gray brown. Sclera: speckled. Iris: homogeneous.

EYeBrow.-Thickness: average. Concurrency: plus. Lateral extent: plus. Brow-ridges: median. Glabella: average.

Nose.-Profile: straight. Tip: thickness average, depressed. Wings: compressed.

TEETH.-Wear: slight. Caries: none. Lost: none.

EAR.-Lobe: attached. Size: average. Helix: plus. Darwin's Point: plus.

Chest.-Development: average.

Musculature.-Good.

HFALTH.-Always good.

No. 19

Locality: Jemdet Nasr. Date: March 14, 1928.

Birthplace: camp of Sheikh Miniehil. Age: 19; unmarried.

Brothers: 1 living, 4 dead. Sisters: 2 dead.

Tattoo marks: dog, gazelle, horse, etc., on right arm; design on forehead over glabella.

MEASUREMENTS MM

Standing height......... 1764

Sitting height........... 837

Acromion to sole.......... 1473
INDICES

Cephalic..............7 71.9

Relative sitting height..... 47.4

Fronto-parietal......... 81.6 


\begin{tabular}{|c|c|}
\hline MEASUREMENTS & MM \\
\hline Head length. & 196 \\
\hline Head breadth. & 141 \\
\hline Minimum frontal diameter. & 115 \\
\hline Bizygomatic maximum.... & 132 \\
\hline Bigonial breadth........... & 110 \\
\hline Total facial height. & 127 \\
\hline Upper facial height. & 76 \\
\hline Nasal height....... & 62 \\
\hline Nasal breadth & 37 \\
\hline Ear length. & 64 \\
\hline Ear breadth & 37 \\
\hline
\end{tabular}

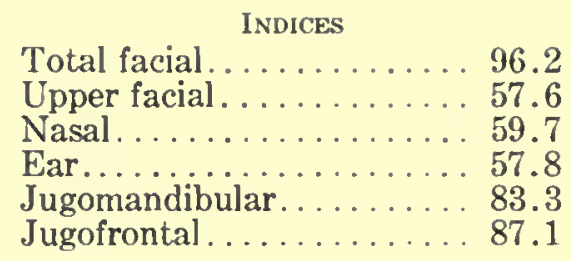

HAIR.-Form: straight. Texture: coarse. Color: dark brown. Quantity: head, plus; beard, minus; body, average.

EYE.-Color: gray brown. Sclera: clear. Iris: homogeneous.

EYeBrow.-Thickness: minus. Brow-ridges: median.

NosE.-Profile: straight. Tip: thickness, double plus; depressed. Wings: compressed.

TEETH.-Wear: slight. Caries: none. Lost: 1 right lower molar.

EAR.-Lobe: attached. Size: plus. Helix: plus. Darwin's Point: average.

SCAPULAE.-Vertebral borders: straight.

CHEST,-Development: average.

Musculature.-Good.

HEALTH.-Always excellent.

No. 20

Locality: Jemdet Nasr. Date: March 14, 1928.

Birthplace: near Jemdet Nasr. Age: 22; unmarried.

Birthplace of father: near Jemdet Nasr. Birthplace of mother: camp of Sheikh Miniehil. Brothers: 1 living, 2 dead. Sisters: 4 living, 1 dead.

Tattoo marks: snake design and gazelle on right arm.

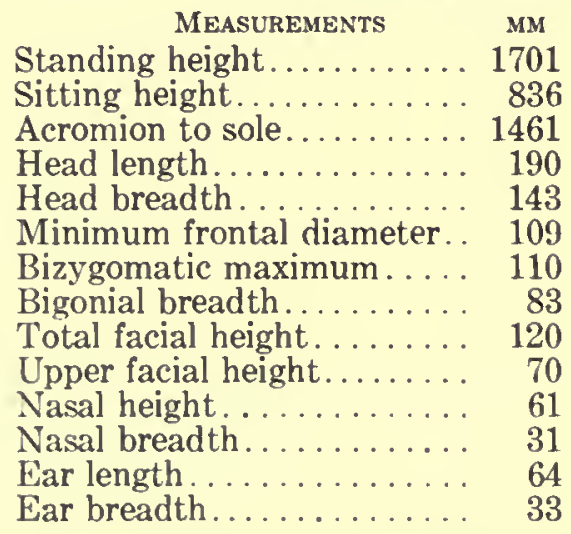

HAIR.-Form: straight. Texture: medium. Color: dark brown. Quantity: head, plus; beard, double plus; body, double plus.

EYE.-Color: gray brown. Sclera: clear. Iris: rayed.

Eyerrow,-Thickness: double plus. Concurrency: plus. Lateral extent: plus. Brow-ridges: median. Glabella: plus.

NosE.-Profile: straight. Tip: thickness plus, depressed. Wings: medium-flaring.

TeEth.-Wear: slight. Caries: none. Lost: none.

EAR.-Lobe: free. Size: plus. Helix: average. Darwin's Point: plus.

SCAPULAE.-Vertebral borders: straight.

Chrst.-Development: good.

Musculature.-Good.

HEALTH.-Always excellent. 
EYF.-Color: gray brown. Sclera: speckled. Iris: homogeneous.

Eyebrow.-Thickness: plus. Concurrency: average. Lateral extent: average. Brow-ridges: median. Glabella: average.

Nose.-Profile: concave. Tip: thickness plus. Wings: compressed.

TEETH.-Wear: none. Caries: none. Lost: none.

EAR.-Lobe: free. Size: average. Helix: plus. Darwin's Point: average.

SCAPULAE.-Vertebral borders: straight.

Chest.-Development: plus.

Musculature.-Good.

Health.-Good.

\section{No. 23. Plate IV, Figs. 3, 4}

Locality: Jemdet Nasr. Date: March 14, 1928.

Birthplace: Hindiya. Age: 24; married for 6 years. Sons: 2 living. Daughters: none. Brothers: 3 living, 1 dead. Sisters: 2 living, 2 dead.

Tattoo marks: on right hand.

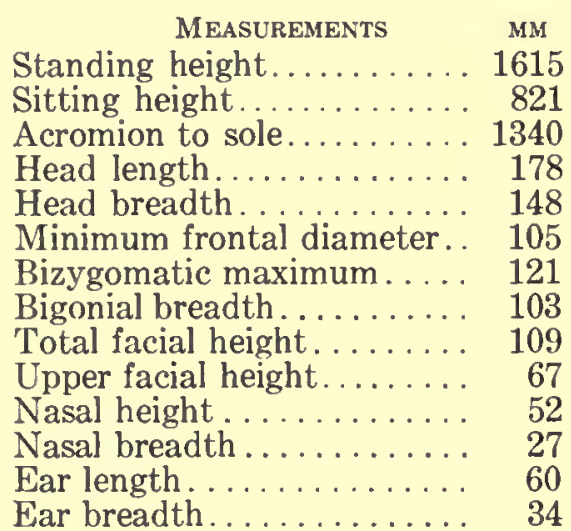

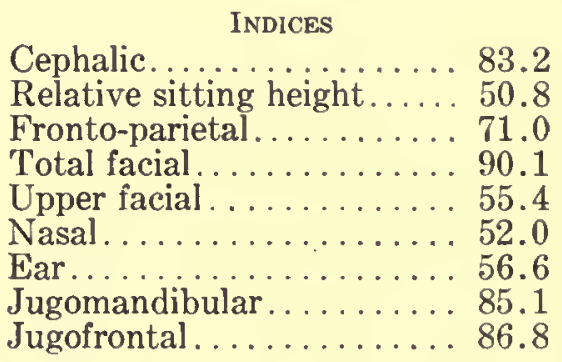

HAIR.-Texture: medium. Color: dark brown. Quantity: head, average; beard, triple plus; body, plus.

Eye.-Color: dark brown. Sclera: speckled. Iris: zoned.

EyeBrow.-Thickness: average. Concurrency: plus. Lateral extent: plus.

Nose.-Profile: concave. Tip: thickness, average; depressed. Wings: medium.

TеEтн.-Wear: average. Caries: average. Lost: none.

EAR.-Lobe: attached. Size: average. Helix: average. Darwin's Point: average.

SCAPULAE.-Vertebral borders: straight.

Chest.-Development: good.

Musculature.-Good.

HEALTH.-Generally good; sometimes fevered.

\section{No. 24}

Locality: Jemdet Nasr. Date: March 16, 1928.

Birthplace: desert near Kish. Age: 18; unmarried.

Birthplace of father and mother: camp of Sheikh M'Mahil.

Brothers: 1 living (half-brother). Sisters: none.

Tattoo marks: gazelle and dots on forearm, 3 dots and right angle on hand and wrist.

\begin{tabular}{|c|c|}
\hline MEASUREMENTS & MM \\
\hline Standing height. & 1575 \\
\hline Sitting height. & 724 \\
\hline Acromion to sole. & 1297 \\
\hline Head length. & 188 \\
\hline Head breadth. & 141 \\
\hline Minimum frontal diameter. . & 107 \\
\hline
\end{tabular}

Cephalic............ 75.0

Relative sitting height..... 46.0

Fronto-parietal.......... 75.9

Total facial..............105.2

Upper facial.............. 60.6

Nasal.............. 62.3 


\begin{tabular}{|c|c|}
\hline \multirow{4}{*}{\multicolumn{2}{|c|}{ MEASUREMENTS }} \\
\hline Bizygomatic maximum . & 11 \\
\hline & \\
\hline & \\
\hline & \\
\hline & \\
\hline & \\
\hline & \\
\hline & \\
\hline & \\
\hline & \\
\hline & \\
\hline & \\
\hline & \\
\hline & \\
\hline & \\
\hline & \\
\hline & \\
\hline & \\
\hline Bigonial breadth. & 9 \\
\hline Total facial height. & 120 \\
\hline Upper facial height. . . . . . . & b. \\
\hline Nasal height. & \\
\hline al breadth & \\
\hline ar length. & \\
\hline
\end{tabular}

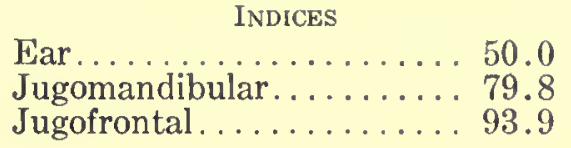

HAIR.-Form: deep waves. Texture: fine. Color: dark brown. Quantity: head, double plus; beard, minus; body, minus.

EyE.-Color: dark brown. Sclera: bloodshot. Iris: zoned.

EyenRow.-Thickness: average. Concurrency: average. Lateral extent: average.

Nose.-Profile: concave. Tip: depressed. Wings: flaring.

TEETH.-Wear: average. Caries: average. Lost: first lower premolar.

EAR.-Lobe: attached. Size: average. Protrusion: double plus.

SCAPULAE.-Vertebral borders: straight.

CHest.-Development: good.

Musculature.-Good.

HFALTH.-Excellent.

No. 25. Plate IV, Figs. 5, 6

Locality: Jemdet Nasr. Date: March 15, 1928.

Birthplace: camp of Sheikh Miniehil. Age: 25; unmarried.

Brothers: 3 dead. Sisters: 5 dead.

Tattoo marks: none.

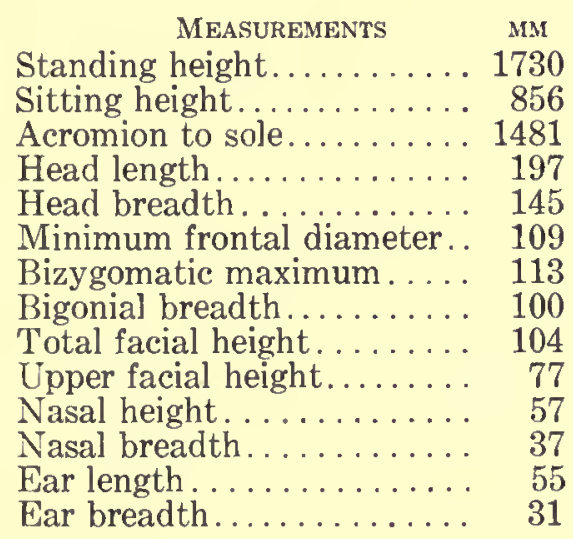

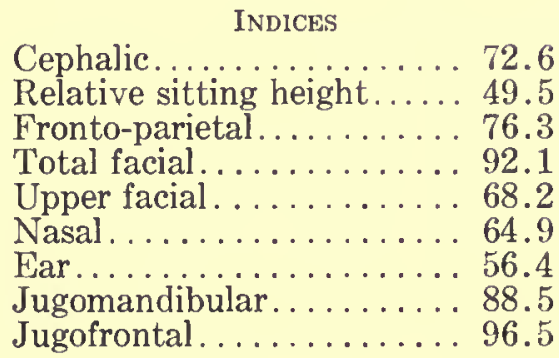

HaIR.-Form: low waves. Texture: medium. Color: dark brown. Quantity: head, plus; beard, average.

EYE.-Color: dark brown. Sclera: speckled and bloodshot. Iris: zoned.

EyEBRow.-Thickness: plus. Concurrency: plus. Lateral extent: plus. Brow-ridges: median. Glabella: average.

Nose.-Profile: straight. Tip: elevated. Wings: medium.

TEETH.-Wear: plus. Caries: plus. Lost: right lower, 2 premolars, 2 molars.

SCAPULAE.-Vertebral borders: straight.

CHEST.-Development: good.

Musculature.-Fair.

HEALTH.-Excellent.

No. 26. Plates IV, Figs. 7, 8; CXXVIII; CXXIX

Lncality: Jemdet Nasr. Date: March 15, 1928.

Birthplace: near arch of Ctesiphon. Age: 28; married for 10 years. Sons: 3 living, 1 dead. Daughters: none. 
Birthplace of father: Baghdad. Birthplace of mother: camp of Sheikh Miniehil. Brothers: 2 living, 1 dead. Sisters: 2 living.

Tattoo marks: on left hand.

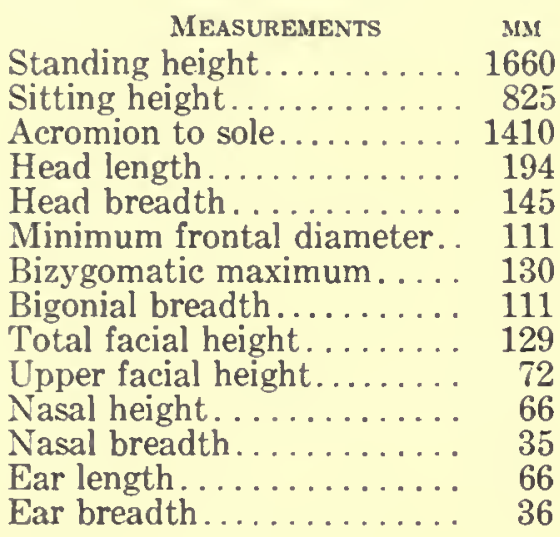

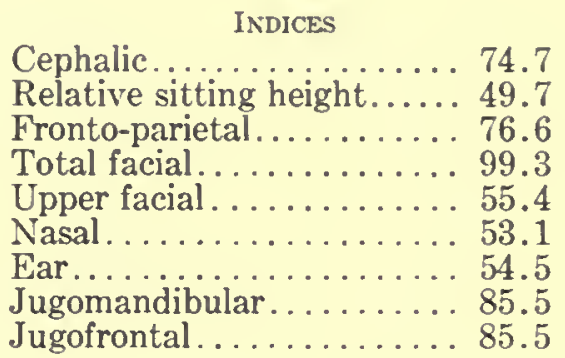

HAIR.-Form: low waves. Texture: medium. Color: dark brown. Quantity: head, plus; beard, plus; body, plus.

EYE.-Color: dark brown. Sclera: clear. Iris: homogeneous.

EYEBRow.-Thickness: plus. Brow-ridges: median. Glabella: average.

Nose.-Profile: concave. Wings: medium.

TEeTH.-Wear: plus. Caries: average. Lost: none.

EAR.-Lobe: free. Size: plus.

SCAPULAE.-Vertebral borders: straight.

CHEST.-Derelopment: good.

Musculature.-Good.

HEALTH.-Good.

Head and face closely resemble features portrayed in Sumerian art.

External eve folds: double plus.

Had fever and vomiting fits during cholera outbreak in 1927 and 1928. Also had jaundice.

No. 27. Plate V, Figs. 1, 2

Locality: Jemdet Nasr. Date: March 15, 1928.

Birthplace: camp of Sheikh Miniehil. Age: 18; unmarried.

Brothers: 2 living. Sisters: 1 living.

Tattoo marks: gazelle on right forearm.

\begin{tabular}{|c|c|c|}
\hline MEAsurements & MMI & INDICES \\
\hline Standing height....... & 1614 & Cephalic. \\
\hline Sitting height. . & 775 & Relative sitting height..... 48.0 \\
\hline Acromion to sole. & 1344 & Fronto-parietal........ \\
\hline Head length. & 186 & Total facial.... \\
\hline Head breadth. & 145 & Upper facial \\
\hline nimum frontal diameter. & 108 & Nasal. \\
\hline gomatic maximum. ... & 120 & Ear. \\
\hline nial breadth......... & 100 & Jugomandibular......... 83 \\
\hline al facial height. . . . . . . & 12 & Jugofrontal. ........... \\
\hline
\end{tabular}

Upper facial height........ 66

Nasal height........... 58

Nasal breadth........... 36

Ear length............ 62

Ear breadth.............. 33

Hair.-Form: low waves. Texture: fine. Color: dark brown.

EyE.-Color: dark brown. Sclera: clear. Iris: homogeneous.

Eyebrow.-Brow-ridges: median. Glabella: average. 
Nose.-Profile: concave. Tip: depressed. Wings: medium.

TEETH.-Lost: none.

EAR.-Lobe: free. Size: average. Helix: average. Darwin's Point: average.

SCAPULAE.-Vertebral borders: straight.

Chest.-Development: average.

Musculature.-Fair.

Health.-Good.

No. 28. Plate V, Figs. 3, 4

Locality: Jemdet Nasr. Date: March 15, 1928.

Birthplace: Hindiya. Age: 20; unmarried.

Brothers: none. Sisters: none.

Tattoo marks: none.

\begin{tabular}{|c|c|}
\hline MEASUREMENTS & MM \\
\hline Standing height. & 1632 \\
\hline Sitting height. & 786 \\
\hline Acromion to sole. & 1423 \\
\hline Head length.... & 187 \\
\hline Head breadth. & 138 \\
\hline Minimum frontal diameter. & 103 \\
\hline Bizygomatic maximum. . . . & 126 \\
\hline Bigonial breadth. & 113 \\
\hline Total facial height. . & 114 \\
\hline Upper facial height...... & 73 \\
\hline Nasal height. & 57 \\
\hline Nasal breadth & 36 \\
\hline Ear len & 63 \\
\hline & . \\
\hline
\end{tabular}

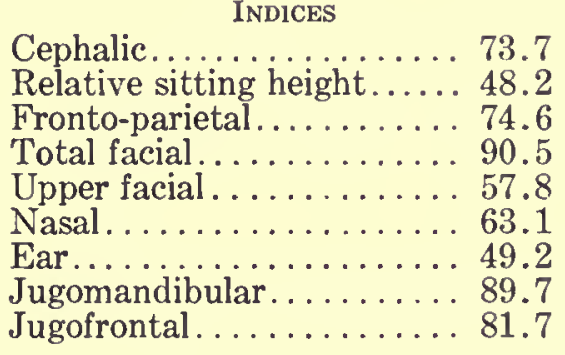

Halr.-Form: low waves. Texture: medium. Color: dark brown. Quantity: head, average; beard, plus; body, plus.

EYE.-Color: dark brown. Sclera: speckled and bloodshot.

EYEBrow.-Thickness: plus. Brow-ridges: median. Glabella: average.

Nose.-Profile: concave. Wings: flaring.

TeEth.-Wear: double plus. Caries: plus. Lost: none.

EAR.-Lobe: attached. Size: plus. Helix: plus. Darwin's Point: average.

SCAPULAE.-Vertebral borders: straight.

CHEST.-Development: double minus.

Musculature.-Poor.

Health.-Poor; sick every year with fever.

\section{No. 29. Plate V, Figs. 5, 6}

Locality: Jemdet Nasr. Date: March 15, 1928.

Birthplace: Seara on Euphrates. Age: 17; unmarried.

Birthplace of father and mother: camp of Sheikh Miniehil.

Brothers: 3 living. Sisters: 1 living, 1 dead.

Tattoo marks: none.

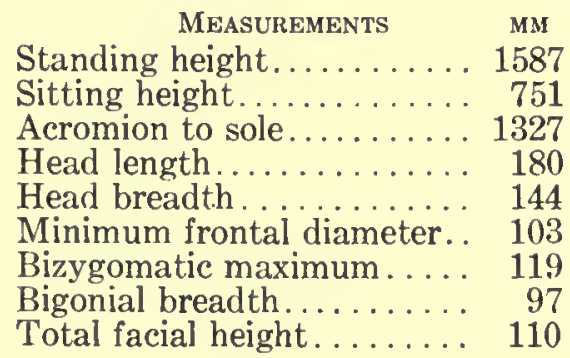

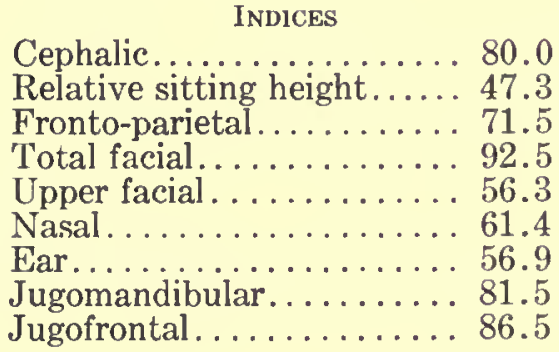




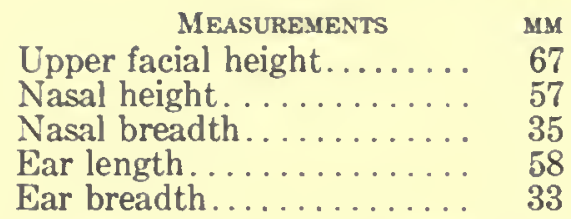

HaIR.-Form: low waves. Texture: medium fine. Color: dark brown. Quantity: head, plus; beard, double minus; body, plus.

EYE.-Color: dark brown. Sclera: clear. Iris: zoned.

EYEBROW.-Thickness: plus. Concurrency: average. Lateral extent: average. Bronc-ridges: median.

Nose.-Profile: concave. Wings: flaring.

TEETH.-Wear: none. Caries: plus. Lost: none.

EAR.-Lobe: free. Size: plus. Helix: average. Darwin's Point: average.

SCAPULAE.-Vertebral borders: straight.

CHEST.-Development: average.

Musculature.-Fair.

HEALTH.-Poor, often fevered, especially during 1927.

No. 30

Locality: Jemdet Nasr. Date: March 15, 1928.

Birthplace: near Bughaila. Age: 23; unmarried.

Brothers: 1 living, 3 dead. Sisters: 1 living.

Tattoo marks: on right wrist; spot on left cheek; spot above glabella.

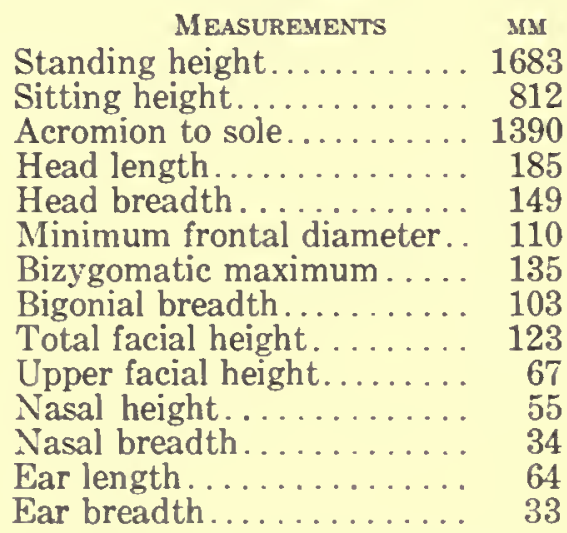

HAIR.-Form: straight, low waves at ends. Texture: medium. Color: dark brown. Quantity: head, double plus; beard, plus; body, plus.

EYE.-Color: dark brown. Sclera: clear. Iris: zoned.

EYEBRow.-Brow-ridges: median. Glabella: average.

Nose.-Profile: convex. Tip: elevated. Wings: medium.

TEETH.-Wear: plus. Caries: plus. Lost: none.

EAR.-Lobe: free. Size: double plus. Helix: average. Darwin's Point: average.

SCAPULAE.-Vertebral borders: straight.

CHEST.-Development: good.

Musculature.-Good.

HEALTH.-Always excellent.

Thickness of lips double plus, suggesting Negro blood.

\section{No. 31. Plate V, Figs. 7, 8}

Locality: Jemdet Nasr. Date: March 15, 1928.

Birthplace: near Jemdet Nasr. Age: 20; unmarried. 
Birthplace of father and mother: camp of Sheikh Miniehil.

Brothers: 1 living, 3 dead. Sisters: 3 living, 1 dead.

Tattoo marks: none.

\begin{tabular}{|c|c|}
\hline MEASUREMENTS & $\operatorname{MM}$ \\
\hline Standing height. . & 1751 \\
\hline Sitting height. & 850 \\
\hline Acromion to sole. & 1506 \\
\hline Head length. & 195 \\
\hline Head breadth & 14 \\
\hline Minimum frontal diameter. . & 108 \\
\hline Bizygomatic maximum . . . . . & 12 \\
\hline Bigonial breadth.... . . . . . & 100 \\
\hline Total facial height......... & 124 \\
\hline Upper facial height..... & 79 \\
\hline Nasal height. & 60 \\
\hline Nasal breadth & \\
\hline & 6 \\
\hline & \\
\hline
\end{tabular}

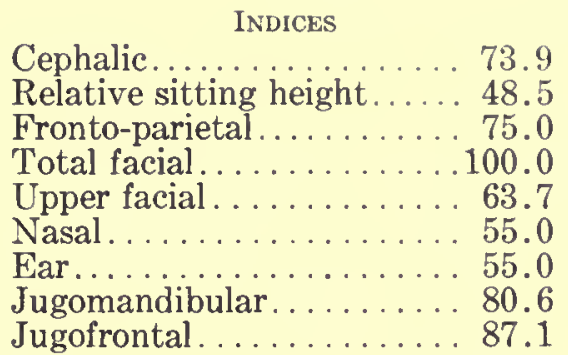

HAIR.-Form: low waves. Texture: medium. Color: dark brown. Quantity: head, plus; beard, average; body, average.

EYE.-Color: dark brown. Sclera: bloodshot. Iris: zoned.

EyeBrow.-Thickness: plus. Concurrency: plus. Lateral extent: plus. Brow-ridges: median. Glabella: average.

Nose.-Profile: concave. Tip: depressed. Wings: medium.

TeETH.-Wear: plus.

EAR.-Lobe: free. Size: plus. Helix: average. Darwin's Point: average.

SCAPULAE.-Vertebral borders: straight.

CHEST.-Development: poor.

Musculature.-Fair.

HEALTH.-Always good.

No. 32

Locality: Jemdet Nasr. Date: March 15, 1928.

Birthplace: Alhai on Tigris east of Jemdet Nasr. Age: 18; unmarried.

Brothers: 4 living. Sisters: 2 living.

Tattoo marks: 3 spots in line on right hand.

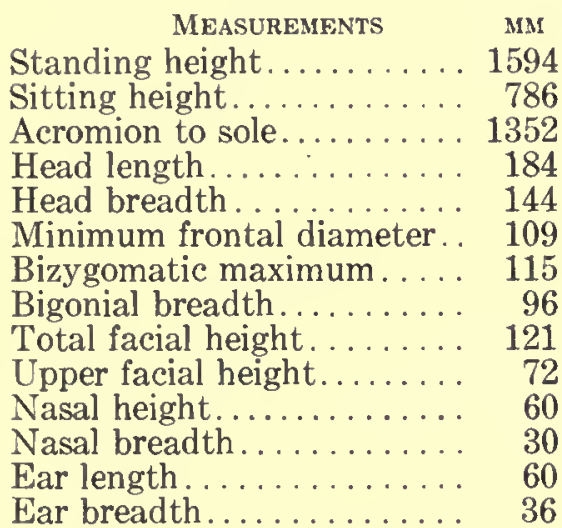

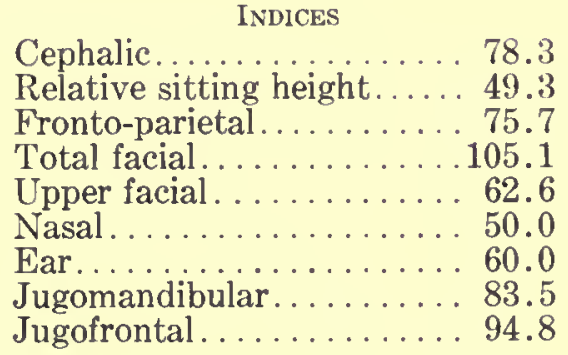

HAIR.-Form: straight. Texture: medium. Color: dark brown. Quantity: head, plus; beard, minus; body, average.

Eye.-Color: dark brown. Sclera: clear. Iris: zoned.

EyeBrow.-Thickness: plus. Concurrency: plus. Lateral extent: plus. Brow-ridges: median. Nose.-Profile: concavo-convex. Tip: elevated. Wings: medium.

TEETH.-Wear: average. 
EAR.-Lobe: free. Size: double plus. Helix: plus. Darwin's Point: minus.

SCAPULAE.-Vertebral borders: convex.

CHEST.-Development: average.

Musculature.-Fair.

HEALTH.-Good.

No. 33

Locality: Jemdet Nasr. Date: March 15, 1928.

Birthplace: camp of Sheikh Miniehil. Age: 30; married for 2 years. No children.

Brothers: 1 living, 1 dead. Sisters: 3 living, 1 dead.

Tattoo marks: gazelle on right forearm; spots on wrist.

\begin{tabular}{|c|c|c|}
\hline MEASUREMENTS & MM & INDICES \\
\hline Standing height....... & 1708 & Cephalic. \\
\hline Sitting height. . & 840 & Relative sitting height.. \\
\hline Acromion to sole. & 1456 & Fronto-parietal...... \\
\hline Head length. & 187 & Total facial. \\
\hline d breadth. & 145 & Upper facial \\
\hline Minimum frontal diameter. & 113 & Nasal. \\
\hline atic maximum.... & 115 & 60.0 \\
\hline iial breadth....... & 98 & Jugomandibular. . \\
\hline otal facial height. & 123 & Jugofrontal. \\
\hline
\end{tabular}

Upper facial height........ 74

Nasal height............ 54

Nasal breadth........... 34

Ear length............ 60

Ear breadth............. 36

HAIR.-Texture: medium fine. Color: dark brown. Quantity: head, average; beard, double plus; body, triple plus.

EYE.-Color: dark brown. Sclera: clear. Iris: zoned.

EYeBrow.-Thickness: double plus. Concurrency: plus. Lateral extent: plus. Brout-ridges: continuous. Glabella: p]us.

TEETH.-Wear: plus. Lost: 1 lower right molar.

EAR.-Lobe: free. Size: plus. Helix: average. Darwin's Point: average.

SCAPULAE.-Vertebral borders: straight.

ChEST.-Development: double plus.

Musculature.-Excellent.

HEALTH._Excellent since marriage; fevered often before.

\section{No. 34. Plate CVII, Figs. 7, 8}

Locality: Jemdet Nasr. Date: March 15, 1928.

Birthplace: Ramadi. Age: 25; married for 5 years. Sons: none. Daughters: 1 living. Birthplace of father: camp of Sheikh Miniehil. Birthplace of mother: Musaiyib near Hindiya. Brothers: 3 living. Sisters: 3 living.

Tattoo marks: on right hand, "to give strength."

\begin{tabular}{|c|c|c|}
\hline MEASUREMENTS & MM & INDICES \\
\hline Standing height....... & 1761 & $\ldots \ldots \ldots \ldots 72.9$ \\
\hline Sitting height. & 847 & Relative sitting height..... 48.1 \\
\hline Acromion to sole. & 1481 & Fronto-parietal......... 76.6 \\
\hline Head length.. & 188 & Total facial...... \\
\hline Head breadth. & 137 & Upper facial. \\
\hline Minimum frontal diameter. . & 105 & Nasal. \\
\hline Bizygomatic maximum. . . . . & 120 & . 53.9 \\
\hline Bigonial breadth...... & 109 & Jugomandibular. . . . . . . 90.8 \\
\hline Total facial height. . . . . . . . & 127 & Jugofrontal . . . . . . . . . . . 87.5 \\
\hline
\end{tabular}

Upper facial height. ...........

Nasal height

Nasal breadth............ 28

Ear length............... 65

Ear breadth........... 35 
HaIR.-Form: low waves. Texture: medium. Color: dark brown.

EYE.-Color: dark brown. Sclera: speckled and bloodshot. Iris: zoned.

EYEBRow.-Brow-ridges: median.

Nose.-Profile: convex. Tip: depressed. Wings: medium.

TEETH.-Wear: slight. Lost: 1 lower premolar on each side.

EAR.-Lobe: attached.

SCAPULAE.-Vertebral borders: straight.

Chest.-Development: plus.

Musculature.--Good.

HEALTH.-Excellent except for occasional fever attacks.

No. 35

Locality: Jemdet Nasr. Date: March 16, 1928.

Birthplace: camp of Sheikh Miniehil. Age: 16; unmarried.

Brothers: 2 living. Sisters: 3 living.

Tattoo marks: gazelle on right arm.

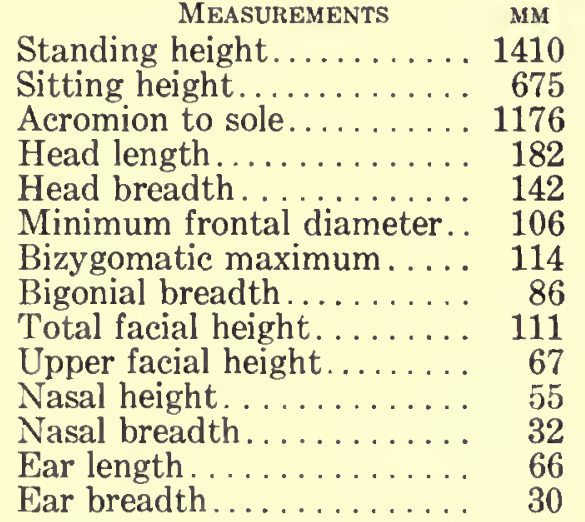

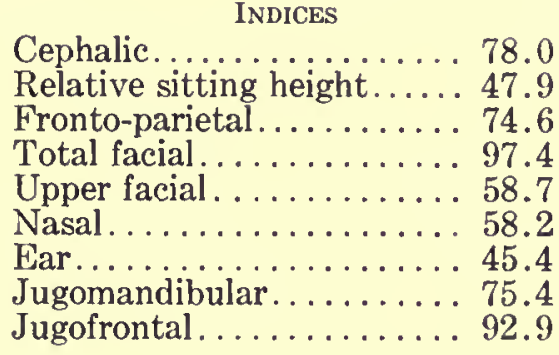

Cephalic...............78.0

Relative sitting height..... 47.9

ronto-parietal .........74.6

Upper facial........

Nasal. .............. 58.2

.

Jugofrontal. . . . . . . . . . 92.9

HaIR.-Form: low waves. Texture: fine. Color: dark brown. Quantity: head, plus; beard, minus; body, minus.

Eye.-Color: dark brown. Sclera: clear. Iris: zoned.

EyeBrow.-Thickness: minus. Concurrency: minus. Lateral extent: minus.

Nose.-Profile: convex. Tip: depressed. Wings: medium-flaring.

EAR.-Lobe: free. Size: plus.

SCAPULAE.-Vertebral borders: straight.

CHEST.-Development: average.

Musculature.-Good.

HEALTH.-Excellent.

Not included in statistical summaries.

No. 36

Locality: Jemdet Nasr. Date: March 17, 1928.

Birthplace: camp of Sheikh Mugheir. Age: 18; unmarried.

Brothers: 3 living. Sisters: none.

Tattoo marks: none.

MEASUREMENTS MM

Standing height ........ 1681

Sitting height........... 812

Acromion to sole......... 1411

Head breadth........... 136

Minimum frontal diameter.. 104

Bizygomatic maximum ..... 121
INDICES

Relative sitting height..... 48.3

Fronto-parietal......... 76.5

Total facial............. 94.3

Upper facial. . . . . . . . . . 53.7

Nasal. . . . . . . . . . . . 70.6

Ear............... 55.2 


\begin{tabular}{|c|c|c|}
\hline MEASURFmentS & MM & INDICES \\
\hline Bigonial breadth....... & 99 & Jugomandibular.... . \\
\hline Total facial height. & 114 & Jugofrontal . \\
\hline Upper facial height. & 65 & \\
\hline Nasal height. . . . . . . . . & 51 & \\
\hline Nasal breadth. & 37 & \\
\hline $\begin{array}{l}\text { Ear length } \\
\text { Ear breadth }\end{array}$ & 58 & \\
\hline breadth & & \\
\hline
\end{tabular}

HaIr.-Form: low waves. Texture: fine. Color: dark brown. Quantity: head, plus; beard, minus; body, minus.

EYE.-Color: dark brown. Sclera: clear. Iris: zoned.

EyeBrow.-Thickness: plus. Concurrency: plus. Lateral extent: plus. Brow-ridges: median.

NosE.-Tip: depressed. Wings: medium-flaring.

TEETH. - Wear: average.

EAR.-Lobe: attached.

SCAPULAE.-Vertebral borders: straight.

Chest.-Development: plus.

Musculature.-Good.

HEALTH.-Excellent except for occasional fever attacks.

No. 37

Locality: Jemdet Nasr. Date: March 17, 1928.

Birthplace: camp of Sheikh Miniehil. Age: 17; unmarried.

Brothers: 1 living, 2 dead. Sisters: 1 dead.

Tattoo marks: spot on center of back of right hand.

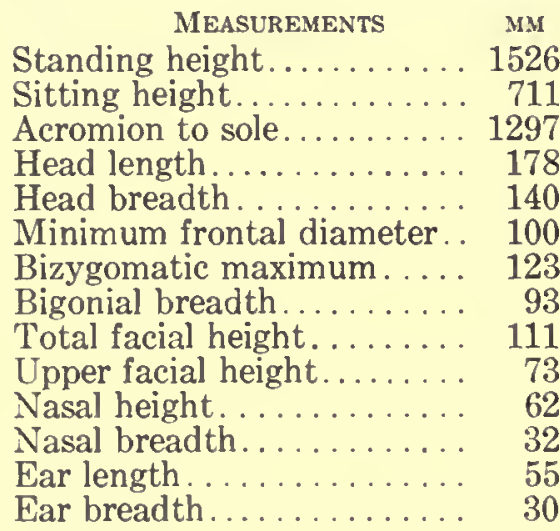

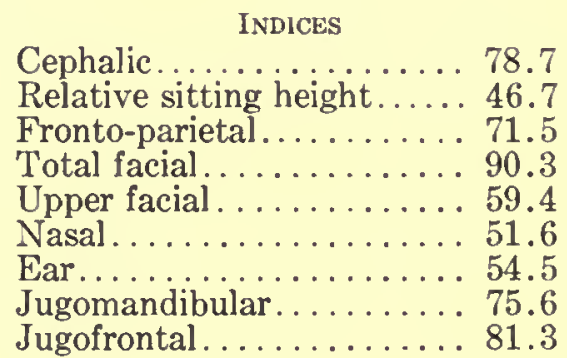

Jugofrontal ............ 81.3

HAIR.-Form: low waves. Texture: medium. Color: dark brown. Quantity: head, plus; beard, double minus; body, minus.

EYE.-Color: dark brown. Sclera: clear. Iris: rayed.

EYEBRow.-Brow-ridges: median.

Nose.-Profile: convex. Tip: depressed. Wings: medium.

EAR.-Lobe: free. Size: plus. Helix: average. Darwin's Point: plus.

SCAPULAE.-Vertebral borders: straight.

Musculature.-Good.

HEALTH.-Excellent.

No. 38. Plate VI, Figs. 1, 2

Locality: Jemdet Nasr. Date: March 16, 1928.

Birthplace: camp of Sheikh Mugheir. Age: 25; married for 3 years. Sons: none. Daughters: 1 dead.

Brothers: 4 living, 3 dead. Sisters: 1 dead.

Tattoo marks: gazelle on right forearm; 3 spots on both temples. 


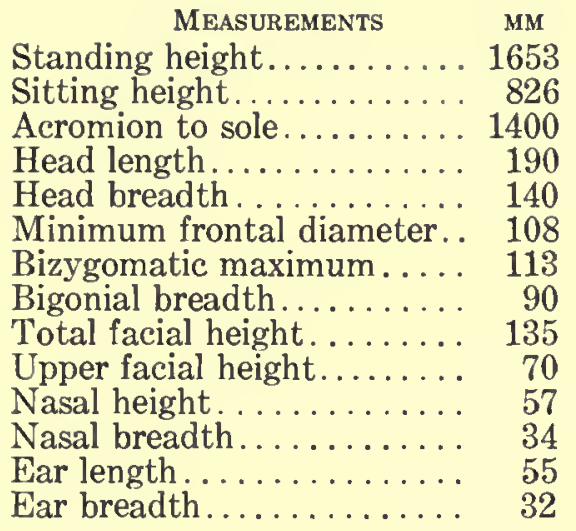

Cephalic................. 73.7

Relative sitting height..... 50.0

Fronto-parietal.......... 77.1

Total facial............. 119.5

Upper facial. . . . . . . . . 6 61.4

Nasal. ............... 59.6

Ear....................... 58.2

Jugomandibular......... 79.6

Jugofrontal. ............ 95.6

HaIR. - Form: low waves. Texture: fine. Color: dark brown. Quantity: head, double plus; beard, plus; body, plus.

EYE.-Color: dark brown. Sclera: speckled. Iris: rayed.

Eyebrow.-Thickness: double plus. Concurrency: plus. Lateral extent: plus. Brow-ridges: median.

NoSE.-Profile: straight. Wings: medium.

EAR.-Lobe: attached. Size: plus.

SCAPULAE.-Vertebral borders: straight.

Chest.-Development: plus.

Musculature.-Good.

HEALTH.-Excellent.

An excellent pickman. Brother of No. 40.

\section{No. 39. Plate VI, Figs. 3, 4}

Locality: Jemdet Nasr. Date: March 17, 1928.

Birthplace: Daghghara. Age: 20; unmarried.

Brothers: none. Sisters: 2 living.

Tattoo marks: on right wrist; single spot on both temples.

\begin{tabular}{|c|c|c|}
\hline Measurements & MM & INDICES \\
\hline Standing height. & 1739 & Cephalic. \\
\hline Sitting height. & 804 & Relative sitting height..... 46.3 \\
\hline Acromion to sole & 1472 & Fronto-parietal......... 73.5 \\
\hline Head length. & 188 & Total facial...... \\
\hline Head breadth. & 147 & Upper facial \\
\hline Minimum frontal diameter. & 108 & Nasal. \\
\hline atic maximum... & 119 & 58.2 \\
\hline breadth. & 97 & Jugomandibular... \\
\hline Total facial height........ & 126 & Jugofrontal \\
\hline
\end{tabular}

Upper facial height......... 75

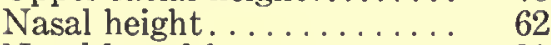

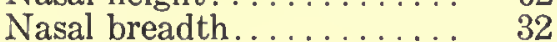

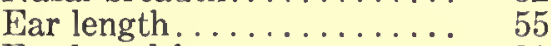

Ear breadth............ 32

HAIR.-Form: low waves. Color: dark brown. Quantity: head, plus; beard, plus; body, plus. EYE.-Color: green brown. Sclera: bloodshot. Iris: zoned.

Eyebrow.-Thickness: plus. Concurrency: plus. Lateral extent: plus. Brow-ridges: median. Nose.-Profile: straight. Tip: horizontal. Wings: compressed.

TEETH.-Wear: slight.

EAR.-Lobe: attached. Size: plus.

SCAPULAE.--Vertebral borders: straight.

Chest.-Development: plus. 
Musculature.-Good.

Health. - Excellent.

\section{No. 40. Plate VI, Figs. 5, 6}

Locality: Jemdet Nasr. Date: March 17, 1928.

Birthplace: camp of Sheikh Mugheir. Age: 25; unmarried.

Brothers: 4 living, 3 dead. Sisters: 1 dead.

Tattoo marks: gazelle on right hand.

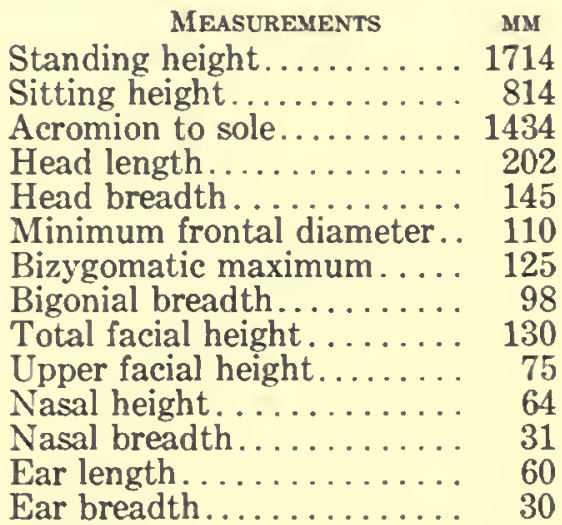

INDICES

Cephalic..............71.1

Relative sitting height..... 47.5

Fronto-parietal......... 75.9

Total facial ............... 104.0

Upper facial..........6 60.0

Nasal. ............... 48.4

Ear............... 50.0

Jugomandibular.......... 78.4

Jugofrontal............ 88.8

HalR.-Form: low waves. Texture: medium. Color: dark brown. Quantity: head, plus; beard, plus; body, plus.

EYE.-Sclera: clear. Iris: zoned.

EYEBRow.-Thickness: double plus. Concurrency: double plus. Lateral extent: plus. Browridges: median.

Nose.-Profile: convex. Tip: depressed. Wings: medium.

TEETH.-Wear: average. Caries: minus. Lost: 1 lower right molar.

EAR.-Lobe: attached. Size: average.

CHEST.-Derelopment: double plus.

Musculature.-Excellent.

HEALTH.-Excellent except for occasional attacks of fever which last from three to four days. Brother of No. 38.

No. 41

Locality: Jemdet Nasr. Date: March 17, 1928.

Birthplace: camp of Sheikh Miniehil. Age: 18; unmarried.

Brothers: 2 living, 3 dead. Sisters: 1 living, 1 dead.

Tattoo marks: none.

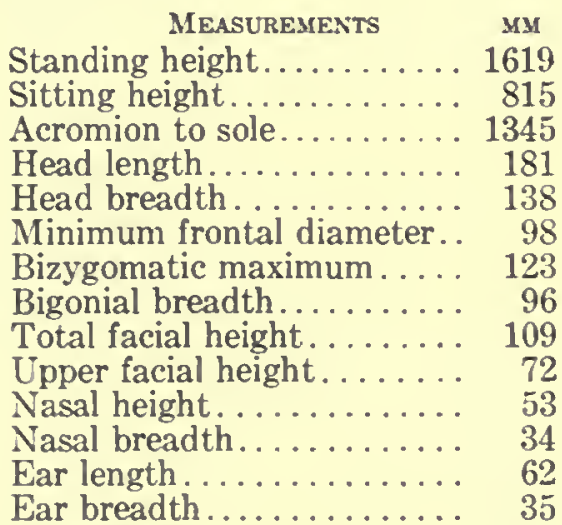

INDICES

Cephalic...............76.2

Relative sitting height...... 50.3

Fronto-parietal.......... 71.0

Total facial............... 88.6

Upper facial............ 58.5

Nasal. ..............6.6 64.2

Ear................... 56.5

Jugomandibular......... 78.0

Jugofrontal. ........... 79.7

HaIR.-Form: low waves. Texture: medium. Color: dark brown. Quantity: head, plus; beard, minus; body, minus. 
EYE.-Color: dark brown. Sclera: speckled. Iris: zoned.

Nose.-Profile: convex. Tip: depressed. Wings: flaring.

EAR.-Lobe: attached. Size: minus. Darwin's Point: double plus.

SCAPULAE.-Vertebral borders: straight.

Chest.-Development: average.

Musculature.-Good.

HEALTH.-Good.

No. 42

Locality: Jemdet Nasr. Date: March 17, 1928.

Birthplace: camp of Sheikh Miniehil. Age: 28; married for 8 years. Sons: 3 living. Daughters: none.

Brothers: none. Sisters: 3 dead.

Tattoo marks: none.

\begin{tabular}{|c|c|}
\hline MEASUREMENTS & MM \\
\hline Standing height....... & 1560 \\
\hline Sitting height. & 797 \\
\hline Acromion to sole. & 1345 \\
\hline Head length. & 179 \\
\hline Head breadth. & 138 \\
\hline Minimum frontal diameter. & 99 \\
\hline Bizygomatic maximum. ... . & 113 \\
\hline Bigonial breadth........... & 96 \\
\hline Total facial height......... & 116 \\
\hline Upper facial height......... & 68 \\
\hline Nasal height & 5 \\
\hline Nasal breadth & \\
\hline Ear length & 6 \\
\hline & \\
\hline
\end{tabular}

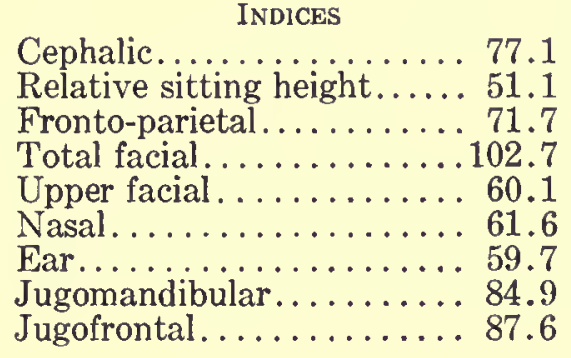

Halk.-Form: low waves. Texture: fine. Color: dark brown. Quantity: head, plus; beard, double plus; body, double plus.

Eye.-Color: dark brown. Sclera: clear. Iris: rayed.

EYEBROW.-Concurrency: plus. Lateral extent: plus.

Nose.-Profile: straight. Tip: depressed.

TEeTH.-Wear: plus. Caries: plus. Lost: 1 upper left molar.

EAR.-Lobe: attached. Size: plus. Helix: average. Darwin's Point: minus.

SCAPULAE.-Vertebral borders: straight.

Chest.-Development: average.

Musculature.-Fair.

HEalth.-Good except for slight fever attacks of four or five days' duration.

\section{No. 43. Plate VI, Figs. 7, 8}

Locality: Jemdet Nasr. Date: March 15, 1928.

Birthplace: camp of Sheikh Mugheir. Age: 20; unmarried.

Brothers: 2 living, 3 dead. Sisters: 1 living.

Tattoo marks: 3 dots on right hand.

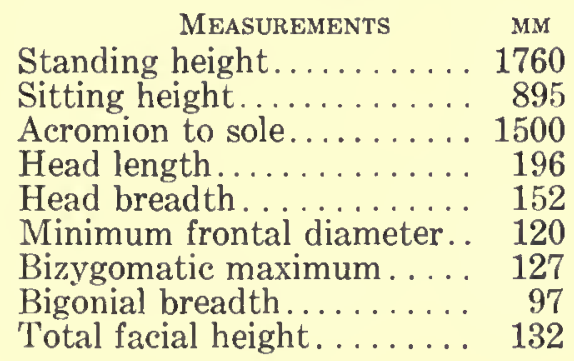




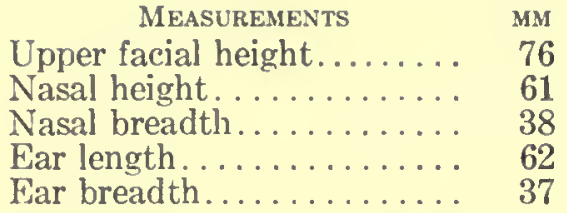

HAlR.-Form: deep waves. Texture: medium. Color: dark brown. Quantity: head, double plus; beard, average; body, average.

EYE.-Color: dark brown. Iris: homogeneous.

EYEBROW.-Thickness: plus.

Nose.-Profile: convex. Wings: medium.

EAR.-Lobe: attached. Size: plus.

SCAPULAE.-Vertebral borders: straight.

CHEsT.-Development: plus.

Musculature.-Excellent.

Health.-Excellent except for occasional fever attacks.

No. 44. Plate VII, Figs. 1, 2

Locality: Jemdet Nasr. Date: March 16, 1928.

Birthplace: Nasiriya. Age: 20; unmarried.

Birthplace of father and mother: camp of Sheikh Miniehil.

Brothers: 3 living, 1 dead. Sisters: 2 living.

Tattoo marks: on right wrist.

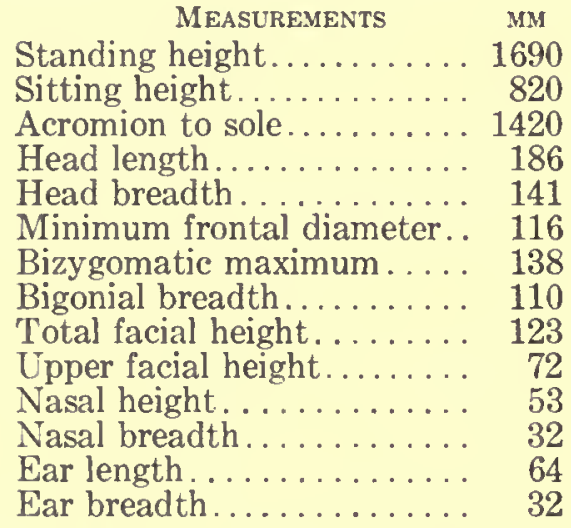

Cephalic............ 75.8

Relative sitting height..... 48.5

Fronto-parietal......... 82.3

Total facial........... 89.1

Upper facial. . . . . . . . . . . 52.2

Nasal. .............6. 60.4

Ear................. 50.0

Jugomandibular......... 79.7

Jugofrontal. . . . . . . . . 84.1

HaIr.-Form: low waves. Texture: medium. Color: dark brown. Quantity: head, plus; beard, minus; body, minus.

EYE.-Color: dark brown. Sclera: speckled. Iris: homogeneous.

EYeBRow.-Thickness: average. Concurrency: plus. Lateral extent: average. Brow-ridges: median.

Nose.-Profile: straight. Tip: horizontal. Wings: medium.

EAR.-Lobe: attached.

SCAPULAE.--Vertebral borders: straight.

Chest.-Derelopment: average.

Musculature.-Excellent.

HEALTH.-Excellent except for slight attacks of fever.

No. 45. Plate VII, Figs. 3,4

Locality: Jemdet Nasr. Date: March 17, 1928.

Birthplace: camp of Sheikh Miniehil. Age: 19; unmarried.

Brothers: 3 living, 1 dead. Sisters: 3 living, 1 dead.

Tattoo marks: on right wrist. 


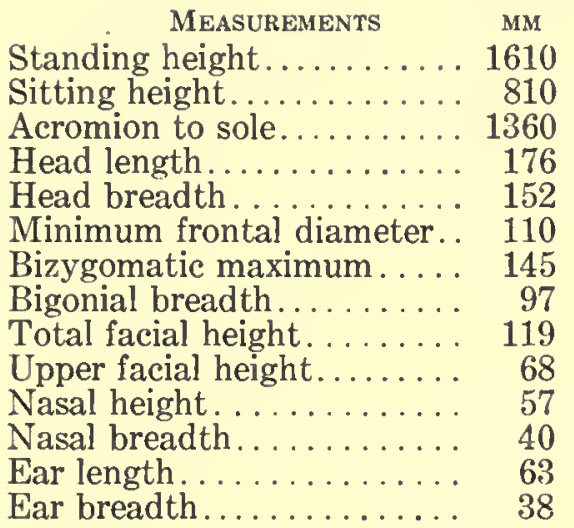

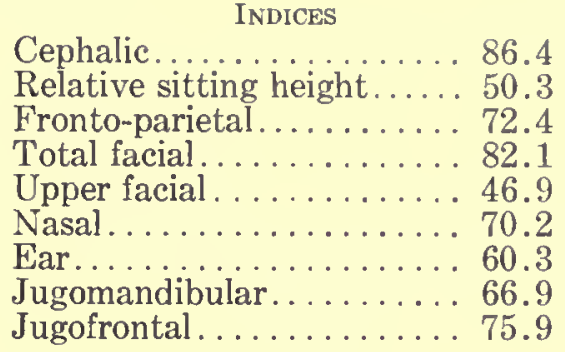

HaIR.-Form: low waves. Texture: fine. Color: dark brown. Quantity: head, plus; beard, minus; body, average.

Eye.-Color: dark brown. Sclera: speckled. Iris: homogeneous.

EyeBRow.-Thickness: minus. Concurrency: minus. Lateral extent: average. Brow-ridges: median.

TEeth.-Wear: none. Caries: plus. Lost: first lower right molar.

EAR.-Lobe: free. Size: plus. Helix: average. Darwin's Point: average.

SCAPULAE.-Vertebral borders: straight.

CHEST.--Development: plus.

MusculaAture.-Good.

HEALTH.-Excellent except for occasional fever attacks.

\section{No. 46}

Locality: Jemdet Nasr. Date: March 16, 1928.

Birthplace: camp of Sheikh Miniehil. Age: about 40; married for 7 years. Sons: 1 living, 1 dead. Daughters: none.

Brothers: none. Sisters: none.

Tattoo marks: on right wrist and forearm.

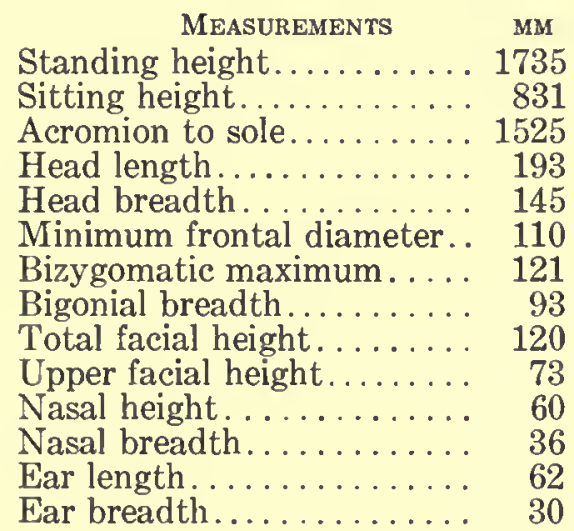

Cephalic............... 75.1

Relative sitting height..... 47.9

Fronto-parietal.......... 75.8

Total facial. ............ 99.2

Upper facial. . . . . . . 60.3

Nasal. ............... 60.0

Ear................ 48.4

Jugomandibular. . . . . . 76.9

Jugofrontal . . . . . . . 90.9

HaIr.-Form: low waves. Texture: medium. Color: dark brown and gray. Quantity: head, average; beard, average; body, plus.

EYE.-Color: dark brown. Sclera: speckled. Iris: homogeneous.

EyeBrow.-Brow-ridges: median.

Nose.-Profile: straight. Tip: depressed. Wings: medium.

TеEтн.-Wear: plus. Caries: plus. Lost: 1 upper right molar, 1 upper left molar.

EAR.-Lobe: free. Size: plus. Helix: plus. Darwin's Point: plus.

SCAPULAE.--Vertebral borders: straight.

CHEST.-Development: average. 
Musculature.-Good.

HEALTH.-Good. Fever attacks of five days' duration.

No. 47. Plate VII, Fig. 5

Locality: Jemdet Nasr. Date: March 17, 1928.

Birthplace: camp of Sheikh Miniehil. Age: 24; unmarried.

Brothers: 2 dead. Sisters: 3 living.

Tattoo marks: on right wrist.

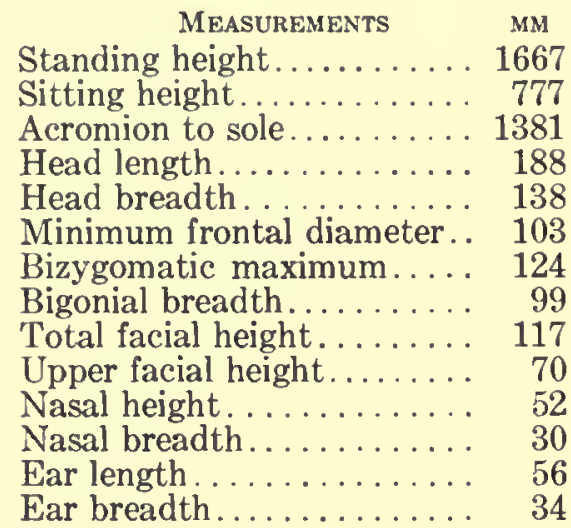

INDICES

Cephalic.............. 73.4

Relative sitting height.... 46.6

Fronto-parietal .......... 74.6

Total facial........... 94.4

Upper facial . . . . . . . . . . 56.4

Nasal. ............... 57.6

Ear...................6. 60.7

Jugomandibular. . . . . . 79.8

Jugofrontal. ........... 83.1

HaIR.-Form: low waves. Texture: fine. Color: dark brown. Quantity: head, double plus. EYE.-Color: dark brown. Sclera: clear. Iris: zoned.

Nose.-Profile: straight. Tip: depressed. Wings: medium.

TEETH.-Lost: 1 lower right molar.

EAR.-Lobe: attached. Helix: average. Darwin's Point: average.

CHEST.-Development: plus.

Musculature.-Good.

HEALTH.-Good.

\section{No. 48. Plate VII, Figs. 6,7}

Locality: Jemdet Nasr. Date: March 17, 1928.

Birthplace: camp of Sheikh Mugheir. Age: 25; married for 7 years. Sons: 1 living. Daughters: 1 living.

Brothers: 5 living. Sisters: none.

Tattoo marks: on right wrist; gazelle on arm.

\begin{tabular}{|c|c|}
\hline MEASUREMENTS & MM \\
\hline Standing height. & 1640 \\
\hline Sitting height. & 795 \\
\hline Acromion to sole. & 1393 \\
\hline Head length. & 188 \\
\hline Head breadth & 144 \\
\hline Minimum frontal diameter. . & 11 \\
\hline Bizygomatic maximum ..... & 12 \\
\hline Bigonial breadth. & 90 \\
\hline Total facial height. & 125 \\
\hline facial height......... & 84 \\
\hline Nasal height. & 66 \\
\hline Nasal breadth. & 31 \\
\hline Ear length. & 67 \\
\hline Ear breadt & a \\
\hline
\end{tabular}

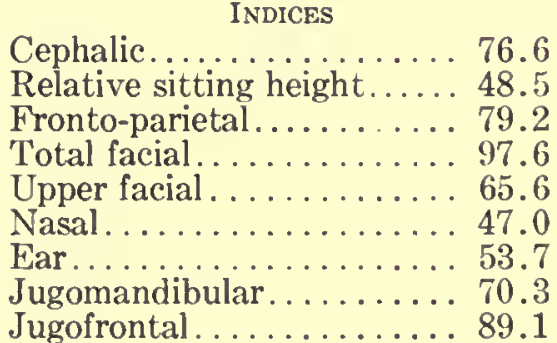

Jugofrontal . ......... 89.1

HAIR.-Texture: fine. Color: dark brown. Quantity: head, plus; beard, average; body, plus. EYE.-Color: dark brown. Sclera: speckled. Iris: homogeneous.

EYEBRow.-Thickness: plus. Lateral extent: plus. Brow-ridges: median. 
NosE.-Profile: straight. Tip: depressed. Wings: medium.

TEETH.-Wear: plus. Caries: average.

EAR.-Lobe: attached.

SCAPULAE.-Vertebral borders: straight.

Chest.-Development: double plus.

Musculature.-Excellent.

HEALTh.-Excellent.

No. 49

Locality: Jemdet Nasr. Date: March 16, 1928.

Birthplace: below Kut al Imara. Age: 30 ; married for 7 years. No children.

Birthplace of father: below Kut al Imara. Birthplace of mother: camp of Sheikh Swadi. Brothers: none. Sisters: none.

Tattoo marks: none.

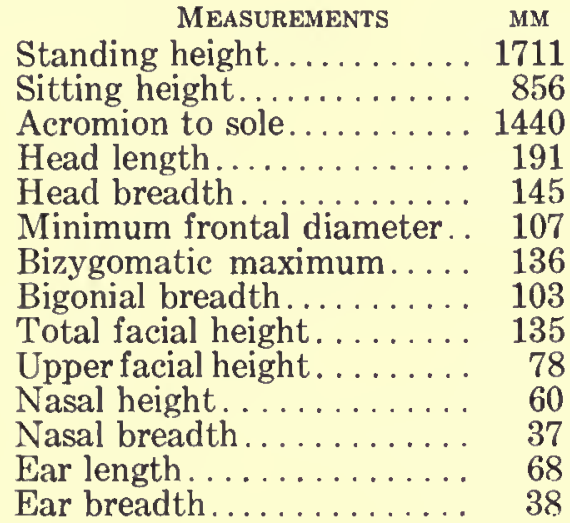

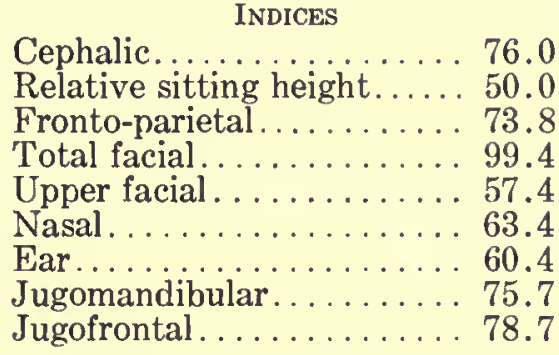

Ear breadth................ 38

HAIR.-Form: low waves. Texture: medium. Color: dark brown. Quantity: head, plus; beard, double plus.

EYE.-Color: dark brown. Sclera: speckled. Iris: homogeneous.

EyeBrow.-Thickness: double plus. Concurrency: average. Lateral extent: plus. Browridges: median.

Nose.-Profile: convex. Tip: depressed.

EAR.-Lobe: free. Size: plus. Helix: average. Darwin's Point: plus.

CHEST.-Development: plus.

Musculature.-Good.

HEALTH.-Good.

\section{No. 50. Plate VIII, Figs. 1, 4}

Locality: Jemdet Nasr. Date: March 15, 1928.

Birthplace: Hilla. Age: 23; unmarried.

Brothers: 1 living. Sisters: 2 living.

Tattoo marks: none.

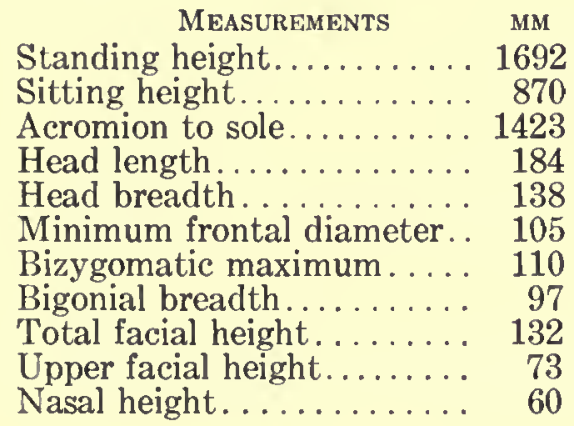

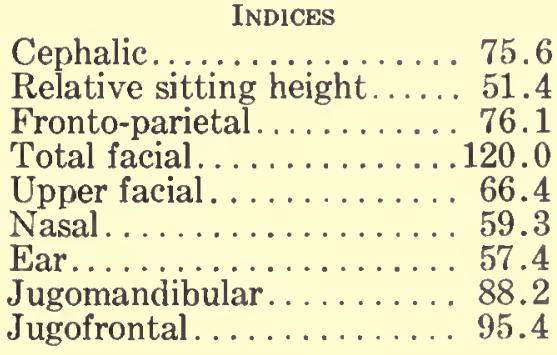




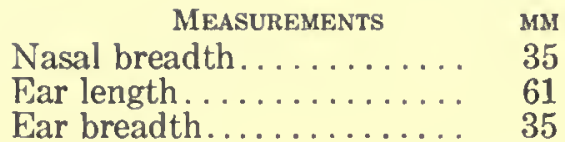

HAIR.-Texture: medium. Color: dark brown. Quantity: head, average; beard, average; body, minus.

Eye.-Color: dark brown. Sclera: clear. Iris: homogeneous.

EYEBRow.-Brow-ridges: median.

NosE.-Profile: straight. Wings: medium.

TeEth. - Wear: average. Caries: double plus. Lost: 1 lower right molar, 1 lower left premolar, and 1 lower left molar.

EAR.-Lobe: attached. Helix: average. Darwin's Point: average.

CHEsT.-Development: plus.

Musculature.-Fair.

HEALTH.-Good except for fever attacks lasting four or five days.

No. 51

Locality: Jemdet Nasr. Date: March 17, 1928.

Birthplace: camp of Sheikh Swadi. Age: 20; unmarried.

Brothers: 1 dead. Sisters: none.

Tattoo marks: none.

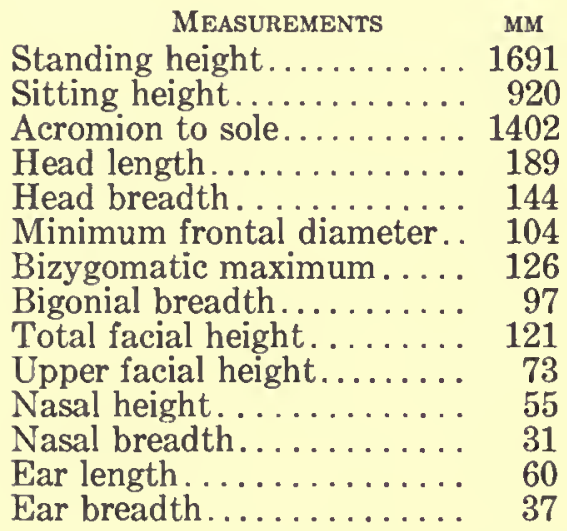

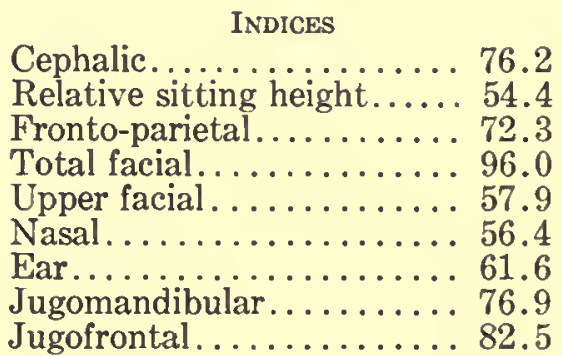

HAIR.-Texture: medium. Color: dark brown.

EYE.-Color: dark brown. Sclera: speckled. Iris: homogeneous.

EYEBRow.-Thickness: plus. Brow-ridges: median.

Nose.-Profile: straight. Wings: medium.

TEETH.-Wear: average. Caries: none. Lost: none.

EAR.-Lobe: free. Size: average.

SCAPULAE.-Vertebral borders: straight.

CHEst.-Development: plus.

Musculature.-Good.

HEALTH.-Good.

No. 52

Locality: Jemdet Nasr. Date: March 17, 1928.

Birthplace: camp of Sheikh Mugheir. Age: 25; unmarried.

Brothers: 2 dead. Sisters: 1 dead.

Tattoo marks: on right wrist.

MEASUREMENTS MM

Standing height.......... 1662

Sitting height......... 847
INDICES

Cephalic............. 73.0

Relative sitting height..... 50.9 


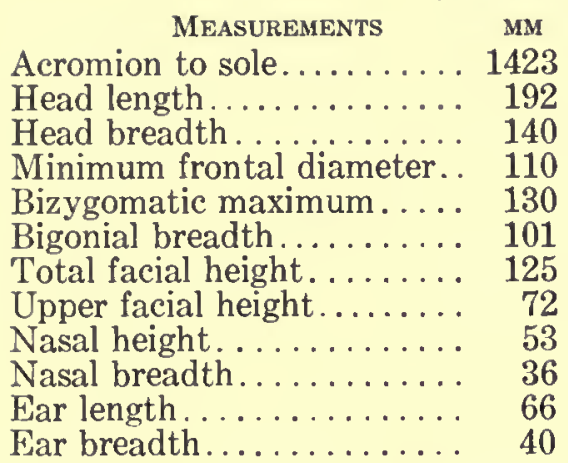

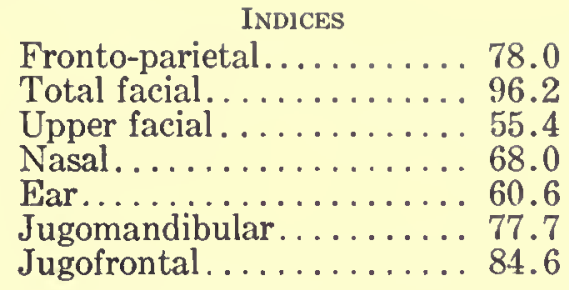

HAlR.-Form: low waves. Texture: fine. Color: dark brown. Quantity: head, plus; beard, plus; body, plus.

EYE.-Color: dark brown. Sclera: speckled. Iris: zoned.

EyeBrow.-Thickness: plus. Concurrency: average. Lateral extent: average. Brow-ridges: median.

Nosf.-Profile: straight. Tip: depressed. Wings: medium.

TEETH.-Wear: plus. Caries: none. Lost: none.

EAR.-Lobe: attached. Size: average. Helix: average. Darwin's Point: average.

SCAPULAE.-Vertebral borders: straight.

CHEST.-Development: plus.

Musculature.-Good.

HEALTH.-Good except that he is often fevered.

\section{No. 53}

Locality: Jemdet Nasr. Date: March 17, 1928.

Birthplace: camp of Sheikh Swadi. Age: 20. Married twice: first time 6 years ago, wife died 3 years after marriage. No children. Second time 3 years ago. Sons: 1 living. Daughters: none.

Brothers: 2 living. Sisters: none.

Tattoo marks: none.

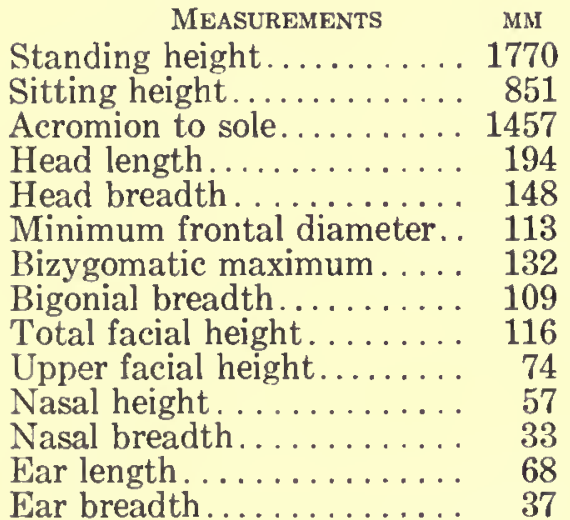

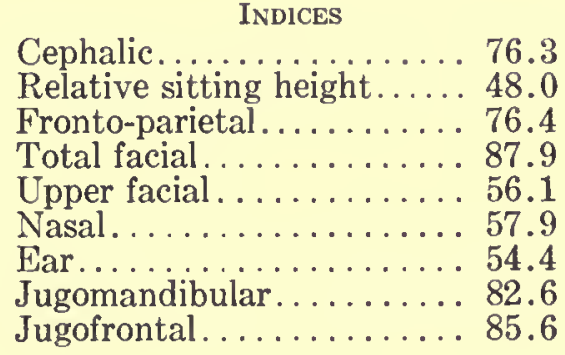

HAIR.-Form: low waves. Texture: medium. Color: dark brown. Quantity: head, average; beard, plus; body, plus.

EYE.-Color: dark brown. Sclera: clear. Iris: homogeneous.

EYEBRow.-Thickness: minus. Concurrency: average. Lateral extent: minus. Brow-ridges: median.

Nose.-Profile: straight. Wings: compressed.

TEETH.-Wear: average. Caries: none. Lost: none.

EAR.-Lobe: attached. Size: average. Helix: average. Darwin's Point: plus. 
SCAPULAE.-Vertebral borders: straight.

Chest.-Development: plus.

Musculature.-Good.

HEALTH.-Good.

Locality: Jemdet Nasr. Date: March 17, 1928.

Birthplace: camp of Sheikh Swadi. Age: 18; unmarried.

Brothers: 1 living, 1 dead. Sisters: 1 living.

Tattoo marks: on right wrist.

\begin{tabular}{|c|c|c|}
\hline MEASUREMENTS & MM & INDICES \\
\hline Standing height........... & 1610 & Cephalic............ \\
\hline Sitting height............ & 777 & Relative sitting height..... 48.3 \\
\hline Acromion to sole.. & 1292 & Fronto-parietal.......... 77.6 \\
\hline Head length..... & 181 & Total facial ............ 100.0 \\
\hline Head breadth & 138 & Upper facial.... \\
\hline Minimum frontal diameter. & 107 & Nasal...... \\
\hline Bizygomatic maximum.... & 120 & Ear. . \\
\hline Bigonial breadth.......... & 90 & Jugomandibular....... \\
\hline Total facial height..... & 120 & Jugofrontal . . . . . . . . . . 89.1 \\
\hline Upper facial height........ & 77 & \\
\hline Nasal height. . . . . . . . . . . & 59 & \\
\hline Nasal breadth & 37 & \\
\hline Ear length... & 65 & \\
\hline Ear breadth & 34 & \\
\hline
\end{tabular}

HaIR.-Texture: fine. Color: dark brown. Quantity: head, plus; beard, minus; body, plus. EYE.-Color: dark brown. Sclera: clear. Iris: homogeneous.

EyeBrow.-Thickness: plus. Concurrency: plus. Lateral extent: plus. Brow-ridges: median. NosE.-Profile: convex. Wings: flaring.

TеEтн.-Wear: average. Caries: none. Lost: none.

EAR.-Lobe: free. Size: average.

SCAPULAE.-Vertebral borders: straight.

CHEST.-Development: plus.

Musculature.-Fair.

HEALTH.-Good except for occasional fever of three or four days' duration.

Four plaits of hair. Thick lips suggestive of Negro blood.

No. 55

I ocality: Jemdet Nasr. Date: March 16, 1928.

Birthplace: near border of Iran. Age: 30-35; married for 10 years. Sons: 3 living, 1 dead. Daughters: none.

Brothers: 3 living, 1 dead. Sisters: 2 living.

Tattoo marks: on right wrist.

\begin{tabular}{|c|c|c|}
\hline MEASUREMENTS & MM & INDICES \\
\hline Standing height........ & 1656 & Cephalic. \\
\hline Sitting height. . & 881 & Relative sitting height.... 53.2 \\
\hline Acromion to sole.... & 1416 & Fronto-parietal......... 75 \\
\hline Head length. & 194 & Total facial. \\
\hline Head breadth. & 143 & Upper facial \\
\hline Minimum frontal diameter. & 108 & Nasal. \\
\hline Bizygomatic maximum . . . . & 124 & .. 57. \\
\hline Bigonial breadth......... & 106 & Jugomandibular. . . . . . . . 85. \\
\hline Total facial height. ....... & 123 & Jugofrontal \\
\hline
\end{tabular}

Upper facial height........ 68

Nasal height........... 60

Nasal breadth.......... 38 


$\begin{array}{cr}\text { Measurements } & \text { MM } \\ \text { Ear length .............. } & 63 \\ \text { Ear breadth............ } & 36\end{array}$

HAIR.-Texture: fine. Color: dark brown. Quantity: head, plus; beard, double plus; body, plus.

EYF.-Color: dark brown. Sclera: speckled. Iris: zoned.

EyEBRow.-Brow-ridges: median.

Nose.--Profile: concave. Tip: depressed. Wings: medium.

TeEth.-Wear: plus. Caries: none. Lost: none.

EAR.-Lobe: free. Helix: plus. Darwin's Point: plus.

CHEST.-Development: plus.

Musculature.--Excellent.

HFalth.-Excellent except for some attacks of fever.

No. 56

Locality: Jemdet Nasr. Date: March 17, 1928.

Birthplace: near Kut al Imara. Age: 20; unmarried.

Brothers: 1 living. Sisters: 1 living.

Tattoo marks: spots on forehead.

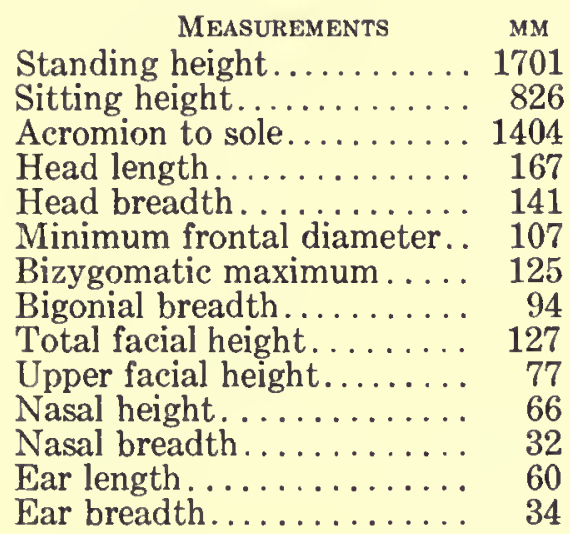

INDICES

Cephalic............. 84.4

Relative sitting height..... 48.6

Fronto-parietal......... 75.9

Total facial. . . . . . . . . . . 101.5

Upper facial. . . . . . . 61.6

Nasal............... 48.5

Far................ 56.7

Jugomandibular........ 75.2

Jugofrontal . . . . . . . . . . 85.6

HaIR. - Form: low waves. Texture: fine. Color: dark brown. Quantity: head, plus; beard, minus; body, average.

EYE.-Color: dark brown. Sclera: bloodshot. Iris: homogeneous.

EYEBRow.-Brow-ridges: median.

NosE.-Profile: concave. Tip: depressed. Wings: medium.

TеEтH.-Wear: plus. Caries: plus. Lost: none.

Ear.-Lobe: attached. Size: plus. Helix: plus. Darwin's Point: double plus.

CHEST.--Development: plus.

Musculature.-Good.

Health.-Good.

No. 57

Locality: Jemdet Nasr. Date: March 16, 1928.

Birthplace: near Kut al Imara. Age: 17.

Birthplace of father and mother: Kish near Abu Biaraq.

Brothers: 1 dead. Sisters: 1 dead.

Tattoo marks: none.

\begin{tabular}{|c|c|}
\hline MEASUREMENTS & MM \\
\hline Standing height. & 1677 \\
\hline $\begin{array}{l}\text { Sitting height.... } \\
\text { Acromion to sole }\end{array}$ & $\begin{array}{r}834 \\
1450\end{array}$ \\
\hline
\end{tabular}

Cephalic.... INDICES $\ldots . . .81 .9$

Relative sitting height...... 49.7

Fronto-parietal......... 71.8 


\begin{tabular}{|c|c|c|}
\hline MEASUREMENTS & MM & INDICES \\
\hline Head length. & 182 & Total facial.............102.5 \\
\hline Head breadth. & 149 & Upper facial. ......... 60.0 \\
\hline Minimum frontal diameter. . & 107 & Nasal. \\
\hline Bizygomatic maximum.... & 120 & 49.2 \\
\hline Bigonial breadth...... & 97 & Jugomandibular. \\
\hline Total facial height & 123 & Jugofrontal..... \\
\hline
\end{tabular}

Upper facial height. ......... 72

Nasal height. ........... 59

Nasal breadth.......... 32

Ear length.................. 61

Far breadth........... 30

HAIR.-Texture: medium. Color: dark brown. Quantity: head, plus; beard, average; body, plus.

EYE.-Color: dark brown. Sclera: clear. Iris: homogeneous.

EYEBRow.-Thickness: plus. Concurrency: plus. Lateral extent: plus. Brow-ridges: median.

NosE.-Profile: straight. Tip: depressed. Wings: medium.

TeETH.-Wear: none. Caries: none. Lost: none.

Far. - Lobe: free. Size: plus. Helix: plus. Darwin's Point: plus.

SCAPUlaE.-Vertebral borders: straight.

Chest.-Development: plus.

Musculature.-Good.

HEalth.-Excellent.

\section{No. 58}

Locality: Jemdet Nasr. Date: March 17, 1928.

Birthplace: Daghghara. Age: 18; unmarried.

Birthplace of father and mother: camp of Sheikh Swadi.

Brothers: none. Sisters: 2 dead.

Tattoo marks: on back of right hand.

\begin{tabular}{|c|c|}
\hline MEASUREMENTS & MM \\
\hline Standing height....... & .1583 \\
\hline Sitting height. & 776 \\
\hline Acromion to sole & 1344 \\
\hline Head length. & 178 \\
\hline Head breadth. & 145 \\
\hline Minimum frontal diameter. & 105 \\
\hline Bizygomatic maximum . . . . & 115 \\
\hline Bigonial breadth. & 94 \\
\hline Total facial height. & 121 \\
\hline Upper facial height. . & 76 \\
\hline Nasal height. & 56 \\
\hline Nasal breadth & 31 \\
\hline Ear len & 57 \\
\hline$D \quad$ b & 30 \\
\hline
\end{tabular}

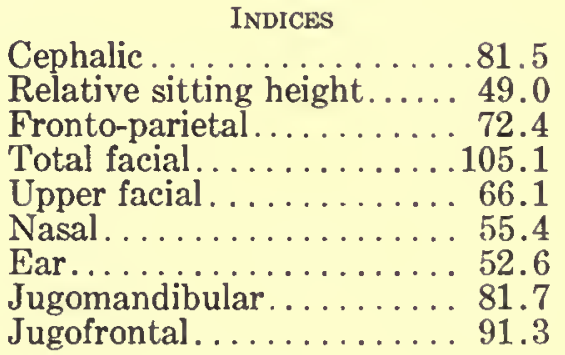

HaIR.-Form: low waves. Texture: fine. Color: dark brown. Quantity: head, plus; beard, minus; body, minus.

EYE.-Color: dark brown. Sclera: clear. Iris: homogeneous.

EYEBRow.--Thickness: minus. Concurrency: minus. Lateral extent: minus. Brow-ridges: median.

Nose.-Profile: straight. Tip: depressed. Wings: medium.

TeETH.-Wear: none. Caries: none. Lost: none.

EAR.-Lobe: attached. Size: minus.

SCAPULAE.-Vertebral borders: straight.

CHEST.-Development: average.

Musculature.-Fair.

HEALTH.-Excellent. 
No. 59

Locality: Jemdet Nasr. Date: March 16, 1928.

Birthplace: near Muftiya. Age: 20; unmarried.

Birthplace of father: near Muftiya. Birthplace of mother: camp of Sheikh Atiyeh. Brothers: 3 living. Sisters: 7 living, 1 dead.

Tattoo marks: none.

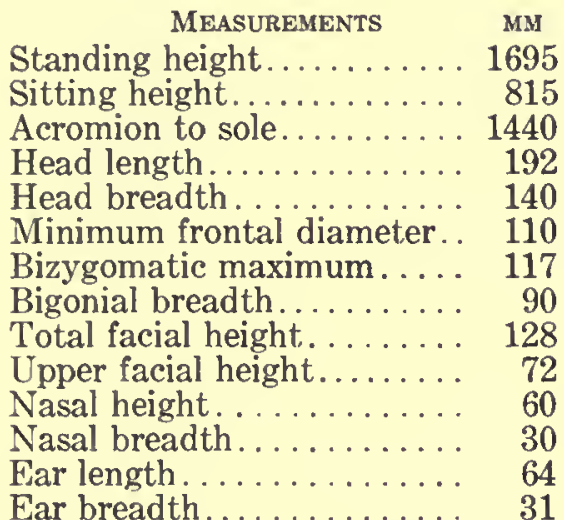

INDICES

Cephalic.............. 72.9

Relative sitting height..... 48.1

Fronto-parietal.......... 78.6

Total facial................. 109.4

Upper facial. ..........6 61.5

Nasal. ............. 50.0

Ear............... 48.4

Jugomandibular. . . . . . 76.9

Jugofrontal. ........... 94.0

HaIR.-Form: low waves. Texture: medium. Color: dark brown. Quantity: head, minus; beard, plus; body, plus.

EYE.-Color: dark brown. Sclera: clear. Iris: homogeneous.

Nose.-Profile: straight. Tip: depressed. Wings: medium.

TEETH.-Wear: plus. Caries: minus.

EAR.-Lobe: attached. Size: plus.

SCAPULAE.-Vertebral borders: straight.

CHEST.-Development: average.

Musculature.-Fair.

HEALTH.-Good.

Skin very dark in color, suggesting Negro admixture. Marked wrinkling on forehead.

No. 60

Locality: Jemdet Nasr. Date: March 18, 1928.

Birthplace: camp of Sheikh Mugheir. Age: 20; unmarried.

Brothers: 4 living. Sisters: 3 living.

Tattoo marks: none.

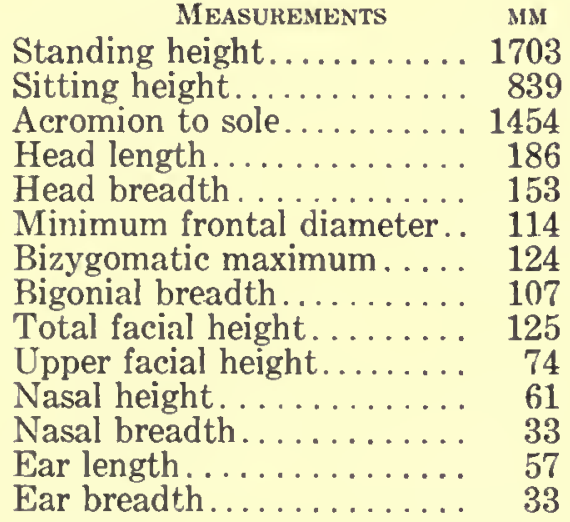

Cephalic............82.2

Relative sitting height..... 49.3

Fronto-parietal......... 74.5

Total facial.............. 100.8

Upper facial . . . . . . . . . . . 59.6

Nasal. .............. 54.1

Ear........................ 57.9

Jugomandibular......... 86.3

Jugofrontal . . . . . . . . 91.9

HAIr.-Texture: medium. Color: dark brown. Quantity: head, plus; beard, minus; body, average. 
EYE.-Color: dark brown. Sclera: clear. Iris: zoned.

Nose.-Tip: depressed. Wings: medium.

TEETH.-Wear: slight.

EAR.-Lobe: attached. Size: plus. Helix: average. Darwin's Point: plus.

SCAPULAE.-Vertebral borders: straight.

CHEST.-Development: plus.

Musculature.-Good.

HEAlTH.--Excellent except for occasional fever attacks.

No. 61

Locality: Jemdet Nasr. Date: March 18, 1928.

Birthplace: near Jemdet Nasr. Age: 20; unmarried.

Birthplace of father and mother: camp of Sheikh Mugheir.

Brothers: 2 living, 1 dead. Sisters: 2 living.

Tattoo marks: on right wrist.

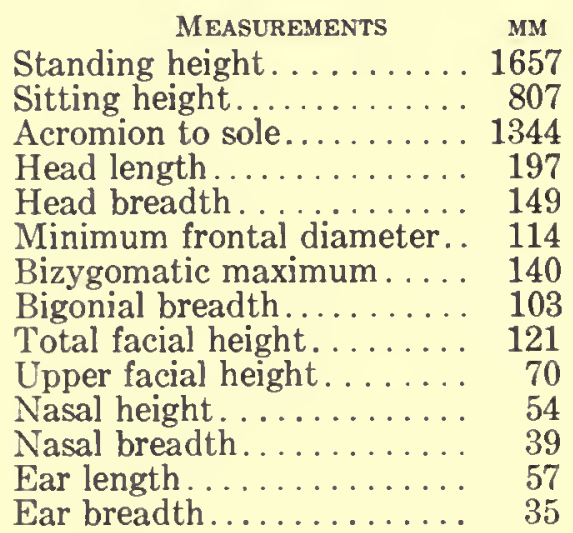

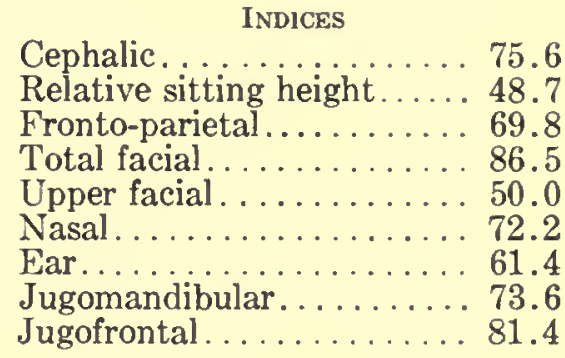

HAIR.-Form: low waves. Texture: medium. Color: dark brown. Quantity: head, average; beard, average; body, average.

EYE.-Color: dark brown. Sclera: clear. Iris: homogeneous.

EYEBRow.-Brow-ridges: median.

Nose.-Profile: straight. Tip: depressed. Wings: medium.

TEETH.-Caries: none. Lost: none.

EAR.-Lobe: attached.

SCAPULAE.-Vertebral borders: straight.

Chest.-Development: plus.

Musculature.-Good.

HEALTH.-Good except for frequent fever attacks, especially in January.

No. 62. Plate VIII, Figs. 2,3

Locality: Jemdet Nasr. Date: March 17, 1928.

Birthplace: camp of Sheikh Miniehil. Age: 38; unmarried.

Brothers: 2 living, 1 dead. Sisters: 1 living, 1 dead.

Tattoo marks: on right hand.

\begin{tabular}{|c|c|}
\hline MEASUREMENTS & MM \\
\hline Standing height. & 1586 \\
\hline Sitting height. & 806 \\
\hline Acromion to sole & 1351 \\
\hline Head length. & 184 \\
\hline Head breadth............ & 142 \\
\hline Minimum frontal di & 106 \\
\hline
\end{tabular}




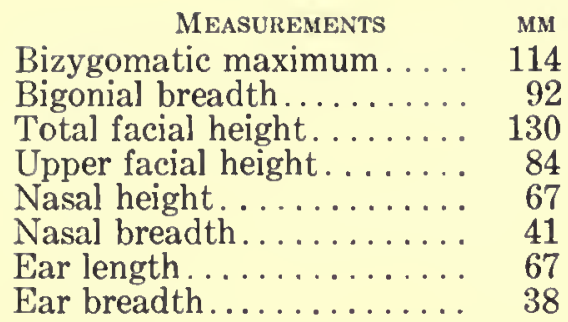

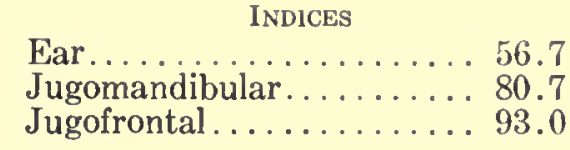

INDICES

14

(1)

67

38

HAIR.-Form: low waves. Texture: medium. Color: dark brown. Quantity: head, average; beard, plus; body, double plus.

EYE.-Sclera: speckled and bloodshot. Iris: rayed.

EyEBRow.-Thickness: double plus. Concurrency: plus. Lateral extent: plus. Brow-ridges: median. Glabella: plus.

Nose.-Profile: straight. Tip: depressed. Wings: medium-flaring.

TEeTH.-Wear: plus. Caries: plus. Lost: none.

EAR.-Lobe: attached. Helix: plus. Darwin's Point: double plus.

SCAPULAE.-Vertebral borders: straight.

CHEST.-Development: average.

Musculature.-Fair.

HEALTH.-Good but often fevered.

\section{No. 63. Plate VIII, Figs. 5, 6}

Locality: Jemdet Nasr. Date: March 18, 1928.

Birthplace: camp of Sheikh Hadji Hunta on Tigris. Age: 48 ; married for 6 years. No children. Birthplace of father and mother: camp of Sheikh Atiyeh.

Brothers: 3 dead. Sisters: 1 living.

Tattoo marks: on right wrist and gazelle on forearm.

HAIR.-Texture: medium. Color: dark brown. Quantity: head, plus; beard, double plus; body, double plus.

ExE.-Color: dark brown. Sclera: clear. Iris: homogeneous.

Eyebrow.-Concurrency: plus. Brow-ridges: median.

Nose.-Profile: convex. Tip: depressed. Wings: medium.

TeETH.-Wear: plus. Caries: plus. Lost: 2 right upper molars, 1 right lower molar.

EAR.-Lobe: free. Helix: plus. Darwin's Point: average.

SCAPULAE.-Vertebral borders: straight.

CHEST.-Development: plus.

Musculature.-Good.

HEALTH.-Excellent.

No measurements obtained.

Lips thick. Prominent in malar region. Marked wrinkling on forehead.

Hard worker, employed as sakka (water-carrier).

\section{No. 64}

Locality: Jemdet Nasr. Date: March 16, 1928.

Birthplace: camp of Sheikh Miniehil. Age: 27; married for 6 years. Sons: none. Daughters: 1 living.

Brothers: 2 living, 2 dead. Sisters: 1 living.

Tattoo marks: spot on end of nose.

\begin{tabular}{|c|c|c|}
\hline MEASUREMENTS & MM & INDICES \\
\hline Standing height. . . . . . & 1648 & Cephalic............ \\
\hline Sitting height... & 817 & Relative sitting height..... 49.6 \\
\hline Acromion to sole..... & 1404 & Fronto-parietal........ 80.2 \\
\hline
\end{tabular}




\begin{tabular}{|c|c|c|}
\hline MEASUREMENTS & MM & INDICES \\
\hline Head length. & 188 & Total facial.......... \\
\hline Head breadth. & 136 & Upper facial. \\
\hline Minimum frontal diameter. . & 109 & Nasal...... \\
\hline Bizygomatic maximum .... & 120 & .56 .6 \\
\hline Bigonial breadth......... & 104 & Jugomandibular........ 86.7 \\
\hline al facial height. . . . . . & 134 & Jugofrontal........... \\
\hline
\end{tabular}

Upper facial height. ...... 73

Nasal height........... 58

Nasal breadth........... 35

Ear length ............... 60

Ear breadth........... 34

HAIR.-Form: curly frizzly. Texture: coarse. Color: dark brown. Quantity: head, double plus; beard, plus; body, double plus.

EYE.-Color: dark brown. Sclera: clear. Iris: zoned.

EYEBRow.-Thickness: plus. Brow-ridges: median.

Nose.-Profile: concave. Tip: depressed. Wings: medium.

TEETH.-Lost: 1 lower left premolar.

EAR.-Lobe: attached.

SCAPULAE._-Vertebral borders: straight.

Chest.-Development: plus.

Musculature.-Excellent.

HEalth.-Excellent.

No. 65

Locality: Jemdet Nasr. Date: March 17, 1928.

Birthplace: camp of Sheikh Miniehil. Age: 19; unmarried.

Brothers: 1 living. Sisters: 2 dead.

Tattoo marks: on right wrist and two spots on frontal.

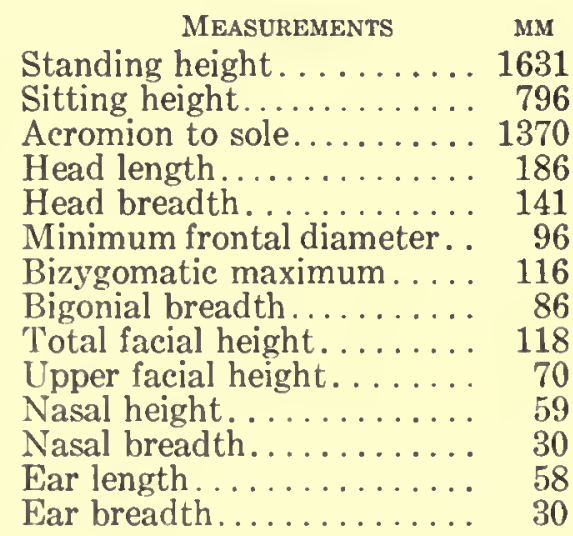

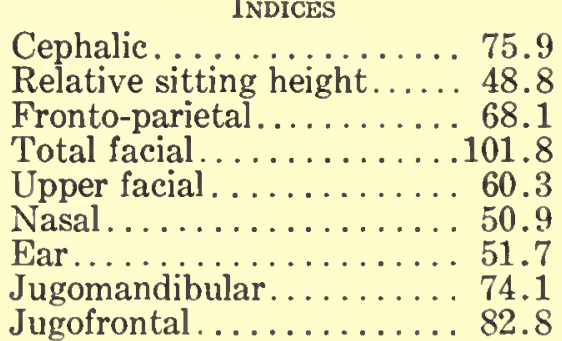

Cephalic............ 75.9

Relative sitting height..... 48.8

Total facial.............101.8

Upper facial . . . . . . . . . . 60.3

Nasal................ 50.9

Jugomandibular......... 74.1

Jugofrontal............8.8 82.8

Halr.-Form: low waves. Texture: medium. Color: dark brown. Quantity: head, plus; beard, minus; body, average.

EyE.-Color: dark brown. Sclera: clear. Iris: homogeneous.

EYeBrow.-Brow-ridges: median.

Nose.-Profile: concave. Tip: depressed.

Теетн.-Wear: triple plus. Caries: triple plus. Lost: none. Condition: bad.

EAR. - Lobe: free. Size: average. Helix: average. Darwin's Point: plus.

SCAPULAE.-Vertebral borders: straight.

Chest.--Development: plus.

Musculature.-Good.

Health.-Good. 
No. 66

Locality: Jemdet Nasr. Date: March 17, 1928.

Birthplace: Hindiya. Age: 17; married for a few months.

Brothers: 1 living. Sisters: none.

Tattoo marks: on right wrist.

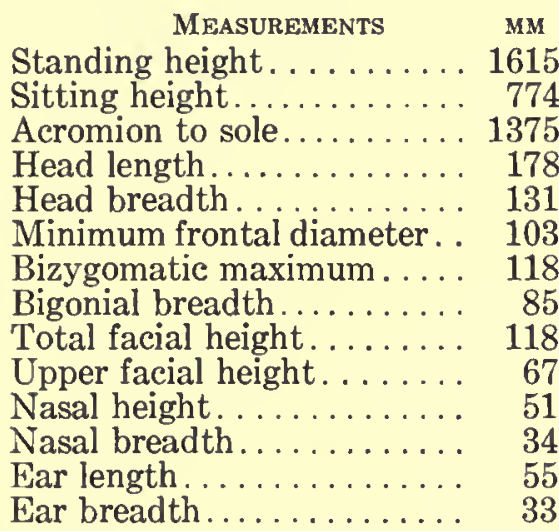

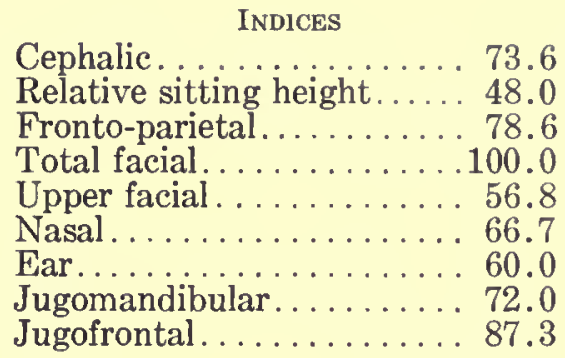

HAIR.—Texture: medium. Color: dark brown. Quantity: head, plus; beard, plus; body, plus. EYE.-Color: dark brown. Sclera: clear. Iris: zoned.

EyeBrow.-Thickness: plus. Concurrency: plus. Lateral extent: plus. Brow-ridges: median. Nose.-Profile: straight. Tip: depressed. Wings: medium.

TEETH.-Lost: none.

EAR.-Lobe: free.

CHEST.-Development: plus.

Musculature.-Good.

HEALTH.-Good except for fever attacks which last six days.

No. 67. Plate XXIII, Figs. 3, 4

Locality: Jemdet Nasr. Date: March 17, 1928.

Birthplace: camp of Sheikh Miniehil. Age: 20; unmarried.

Brothers: 1 dead. Sisters: 1 living.

Tattoo marks: three spots on right wrist.

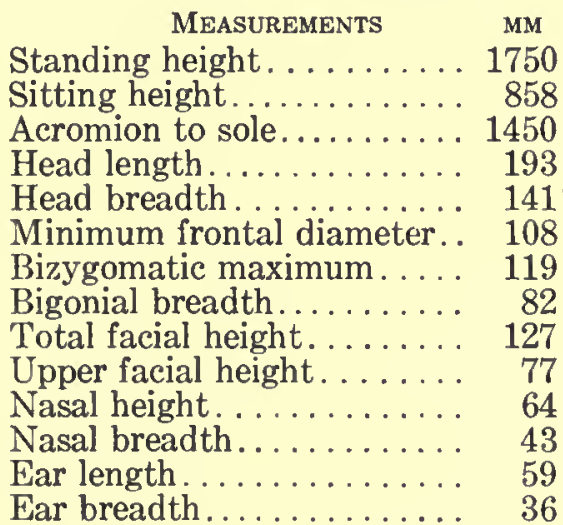

HAIR.-Texture: medium. Color: dark brown. Quantity: head, plus; beard, minus; body, plus.

EyE.-Color: dark brown. Sclera: clear. Iris: zoned.

EYEBRow.-Brow-ridges: median.

Nose.-Profile: convex. Tip: elevated. Wings: medium-flaring. 
TEETH.-Caries: average. Lost: 2 upper molars on each side.

EAR.-Lobe: free. Size: plus. Darwin's Point: plus.

CHEST.-Development: plus.

Musculature.-Excellent.

HEALTH.-Good.

No. 68

Locality: Jemdet Nasr. Date: March 16, 1928.

Birthplace: camp of Sheikh Mugheir. Age: 27; married for 4 years. Sons: 1 living. Daughters: none.

Brothers: 5 living. Sisters: none.

Tattoo marks: none.

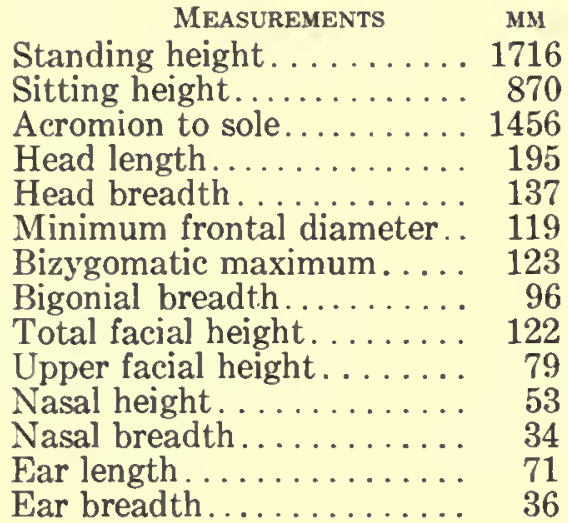

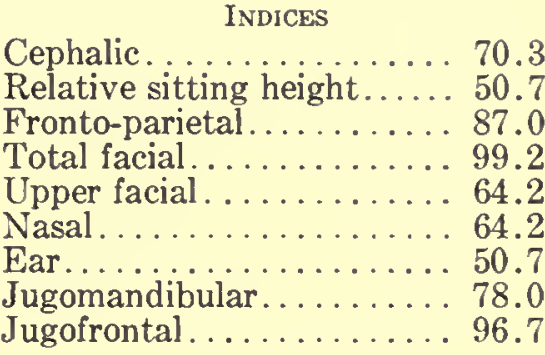

HAIR. - Texture: medium. Color: dark brown. Quantity: head, plus; beard, plus; body, plus. EYE.-Color: dark brown. Sclera: speckled and bloodshot. Condition: bad.

EYEBROw.-Concurrency: plus. Brow-ridges: median.

Nose.-Profile: convex. Tip: depressed. Wings: medium.

TEeTH.-Wear: plus. Caries: plus. Lost: none.

SCAPULAE.-Vertebral borders: straight.

CHEsT.-Development: plus.

Musculature.-Fair.

HEALTH.-Fair; often fevered and suffers from headaches.

No. 69

Locality: Jemdet Nasr. Date: March 16, 1928.

Birthplace: camp of Sheikh Miniehil. Age: 23; unmarried.

Brothers: 5 living, 1 dead. Sisters: 1 living, 1 dead.

Tattoo marks: spot on end of nose and on right wrist; 3 spots on each temple.

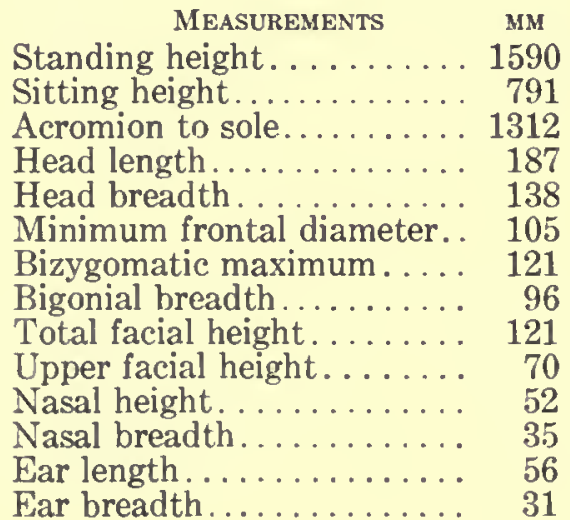


HAIr.-Texture: medium. Color: dark brown. Quantity: head, double plus; beard, plus; body, plus.

EYe.-Color: dark brown. Sclera: clear. Iris: zoned.

Eyebrow.-Thickness: double plus. Concurrency: plus. Lateral extent: plus. Brow-ridges: median.

Nose.--Profile: straight. Tip: depressed. Wings: medium.

TeEth.-Wear: plus. Caries: plus. Lost: none.

EAR.-Lobe: attached. Size: minus.

SCAPULAE.-Vertebral borders: straight.

CHEST.-Development: plus.

MusculaAture.-Good.

HEALTH.-Good except for occasional attacks of fever.

No. 70

Locality: Jemdet Nasr. Date: March 16, 1928.

Birthplace: camp of Sheikh Miniehil. Age: 17; unmarried.

Brothers: 3 living, 1 dead. Sisters: 1 living.

Tattoo marks: 3 spots on right wrist.

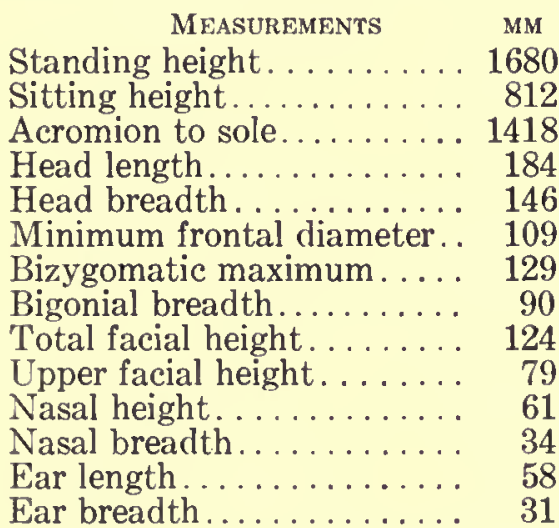

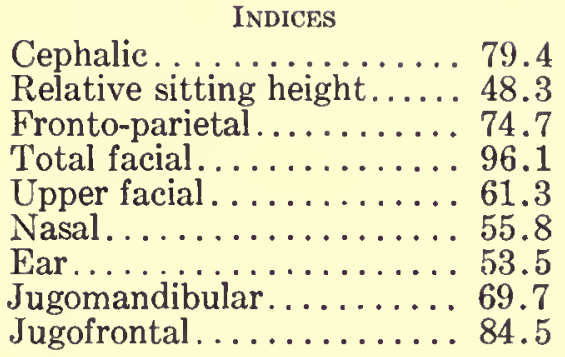

HAIR.-Texture: medium. Color: dark brown. Quantity: head, plus; beard, minus; body, average.

EYF.-Color: dark brown. Sclera: clear. Iris: rayed.

EYeBrow.-Thickness: plus. Concurrency: double plus. Lateral extent: plus. Brow-ridges: median.

Nose.-Profile: straight. Tip: depressed. Wings: medium.

TEeTH.-Caries: none. Lost: none.

EAR.-Lobe: attached.

SCAPULAE.-Vertebral borders: straight.

ChEsT.-Development: plus.

Musculature.-Good.

HEALTH.-Good.

Locality: Jemdet Nasr. Date: March 16, 1928.

Birthplace: camp of Sheikh Atiyeh. Age: 20; unmarried.

Brothers: 3 living, 1 dead. Sisters: 2 living.

Tattoo marks: gazelle on right forearm; series of spots on both temples.

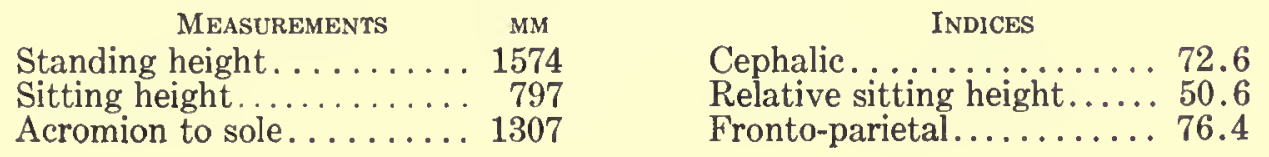




\begin{tabular}{|c|c|c|}
\hline $\begin{array}{l}\text { MEASUREMENTS } \\
\text { MeAs }\end{array}$ & мM & INDICES \\
\hline Head length............. & 186 & Total facial........ \\
\hline Head breadth. . . . . . . . & 135 & Upper facial. \\
\hline Minimum frontal diameter. & 103 & Nasal. \\
\hline Bizygomatic maximum.... & 107 & Ear. \\
\hline Bigonial breadth.. & 84 & Jugomandibula \\
\hline Total facial height. & 127 & Jugofrontal. \\
\hline Upper facial height. & 61 & \\
\hline Nasal height. & 52 & \\
\hline Nasal breadth & 33 & \\
\hline $\begin{array}{l}\text { Ear length. } \\
\text { Ear breadti }\end{array}$ & 6 & \\
\hline Ear breadth & & \\
\hline
\end{tabular}

HAIR.-Texture: medium. Color: dark brown. Quantity: head, average; beard, minus; body, minus.

EYE.-Color: dark brown. Sclera: clear. Iris: zoned.

EYEBRow.-Thickness: double plus. Concurrency: average. Lateral extent: plus. Browridges: median.

Nose.-Profile: concave. Tip: depressed. Wings: medium.

TEETH.-Wear: plus. Caries: plus. Lost: none.

EAR.-Lobe: attached. Size: average. Helix: double plus. Darwin's Point: average.

SCAPULAE. - Vertebral borders: straight.

CHEST.-Development: plus.

MusCulatuRe.-Fair.

HEALTH.-Excellent.

No. 72

Locality: Jemdet Nasr. Date: March 17, 1928.

Birthplace: Daghghara. Age: 18; unmarried.

Birthplace of father and mother: camp of Sheikh Swadi.

Brothers: 1 living. Sisters: 2 living.

Tattoo marks: on right wrist and gazelle on right forearm.

\begin{tabular}{|c|c|c|}
\hline MEASUREMENTS & MM & INDICES \\
\hline Standing height. . . . . . . & 1654 & $\begin{array}{l}\text { Cephalic. } \\
\text { Relative sitting height. }\end{array}$ \\
\hline Sitting height. & 808 & Relative sitting height. \\
\hline Acromion to sole. & 1400 & Fronto-parietal...... \\
\hline Head length. & 178 & Total facial. \\
\hline Head breadth. & 144 & Upper facial \\
\hline Minimum frontal diameter. . & 110 & Nasal. \\
\hline Bizygomatic maximum . & 126 & Ear ...... \\
\hline Bigonial breadth. & 96 & Jugomandibular. \\
\hline Total facial height. & 113 & Jugofrontal....... \\
\hline Upper facial height. & 67 & \\
\hline Nasal height.. & 54 & \\
\hline $\begin{array}{l}\text { Nasal breadth } \\
\text { Ear length... }\end{array}$ & 30 & \\
\hline ar bread & 3 & \\
\hline
\end{tabular}

HAIR.-Texture: medium. Color: dark brown. Quantity: head, average; beard, minus; body, minus.

EYE.-Color: gray brown. Sclera: clear. Iris: rayed.

EyeBrow.-Thickness: average. Concurrency: plus. Lateral extent: average. Browridges: median.

NoSE.-Profile: straight. Tip: depressed. Wings: medium.

TeETH.-Wear: average. Caries: average. Lost: none.

EAR.-Lobe: free. Size: plus. Darwin's Point: plus.

SCAPULAE.-Vertebral borders: straight.

CHEST.-Development: average. 
Musculature.-Good.

HeALTh.-Good.

No. 73

Locality: Jemdet Nasr. Date: March 16, 1928.

Birthplace: camp of Sheikh Swadi. Age: 30 ; unmarried.

Brothers: 3 dead. Sisters: 2 dead.

Tattoo marks: none.

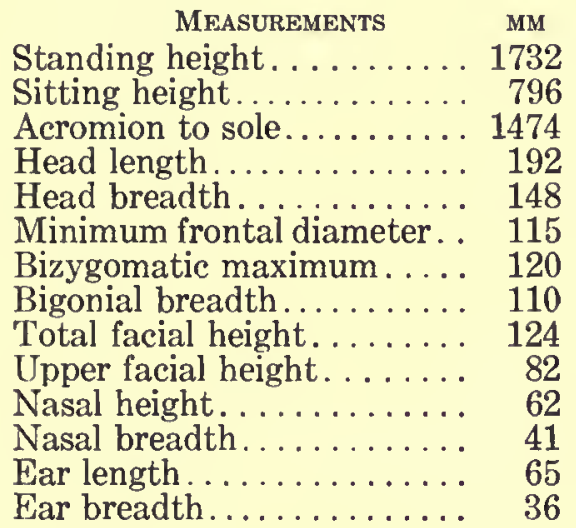

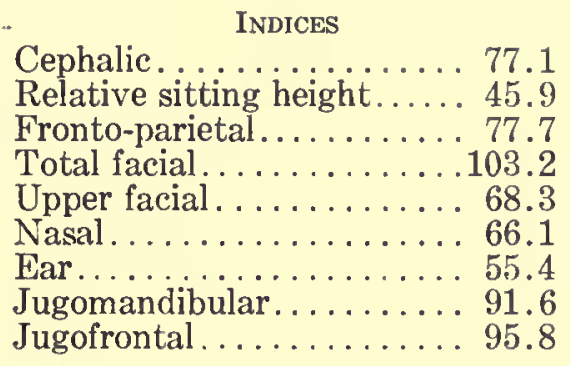

HAIR.-Texture: fine. Color: dark brown and gray. Quantity: head, double plus; beard, plus; body, double plus.

EYE.-Color: dark brown. Sclera: bloodshot. Iris: rayed.

Nose.-Profile: convex. Tip: depressed. Wings: medium.

TEETH.-Wear: plus. Caries: plus. Lost: none.

EAR.-Lobe: attached. Size: plus.

SCAPULAE.-Vertebral borders: straight.

Chest,-Development: plus.

Musculature.-Good.

HEALTH.-Good.

\section{No. 74. Plates IX, Figs. 1, 2; CXXXIV; CXXXV}

Locality: Jemdet Nasr. Date: March 16, 1928.

Birthplace: camp of Sheikh Miniehil. Age: 25; unmarried.

Brothers: 3 living. Sisters: 3 living, 1 dead.

Tattoo marks: none.

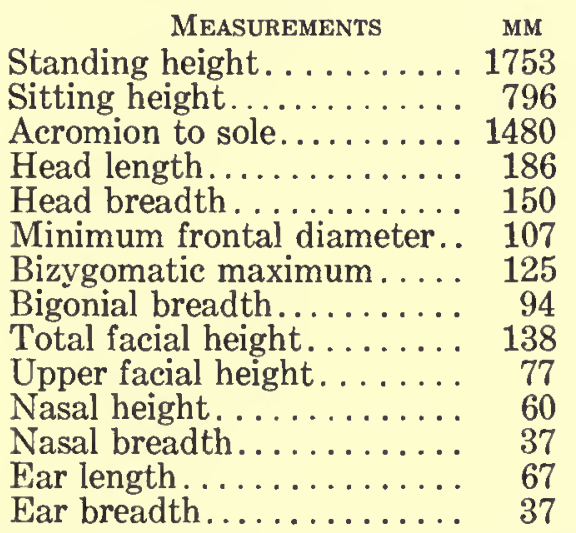

INDICES

Cephalic............ 80.7

Relative sitting height..... 45.3

Fronto-parietal.......... 71.4

Total facial. . . . . . . . . 110.4

Upper facial . . . . . . . . . 61.6

Nasal. ...............6 61.7

Ear................... 55.3

Jugomandibular.......... 75.1

Jugofrontal........... 85.6

HAIR.-Texture: medium. Color: dark brown. Quantity: head, double plus; beard, plus; body, plus. 
EYE.-Color: dark brown. Sclera: speckled and bloodshot. Iris: rayed.

EyeBrow.-Thickness: double plus. Concurrency: plus. Lateral extent: average.

NosE.-Profile: straight. Wings: medium.

TEETH.-Lost: none.

EAR.-Lobe: attached. Size: average.

SCAPULAE.-Vertebral borders: straight.

CHEST.-Development: plus.

Musculature.-Good.

HEALTH.-Good.

No. 75

Locality: Jemdet Nasr. Date: March 17, 1928.

Birthplace: near Kut al Imara. Age: 17; unmarried.

Brothers: 1 living. Sisters: 1 living.

Tattoo marks: on right wrist.

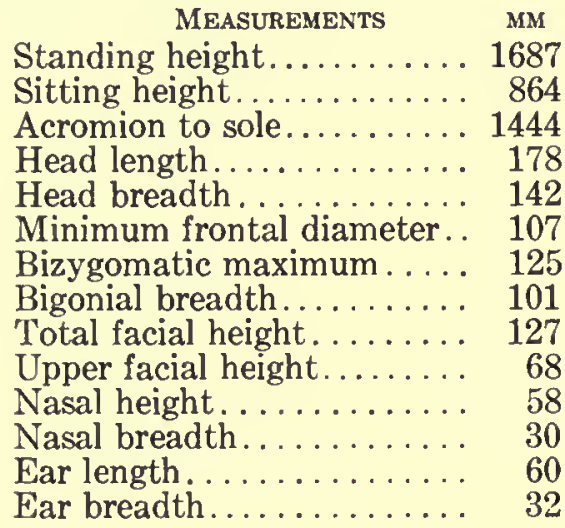

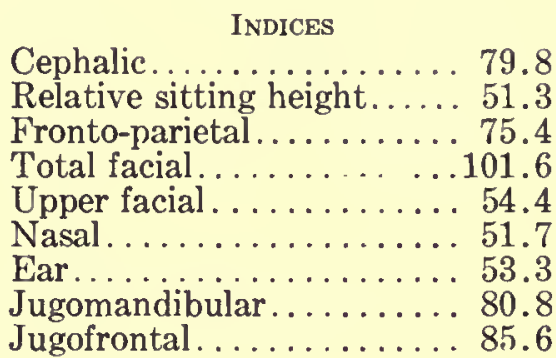

Cephalic............. 79.8

Relative sitting height..... 51.3

Upper facial. . . ........... 54.4

Nasal. ................. 51.7

Jugomandibular........ 80.8

Jugofrontal ........... 85.6

No. 76

Locality: Jemdet Nasr. Date: March 16, 1928.

Birthplace: Kut al Imara. Age: 60 ; married for 30 years. Sons: 1 living. Daughters: 2 living. Brothers: none. Sisters: 1 living.

Tattoo marks: on right wrist.

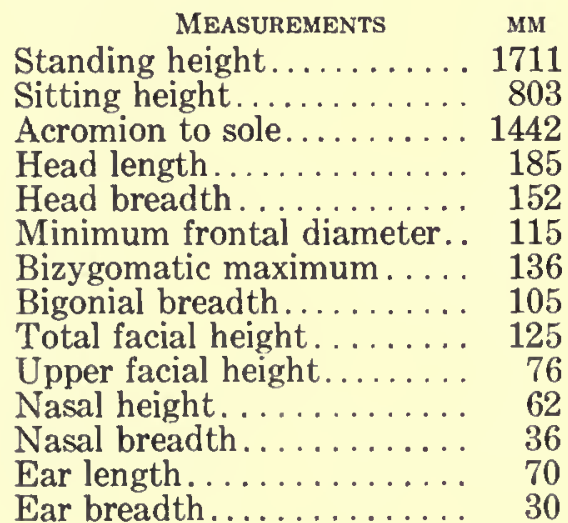

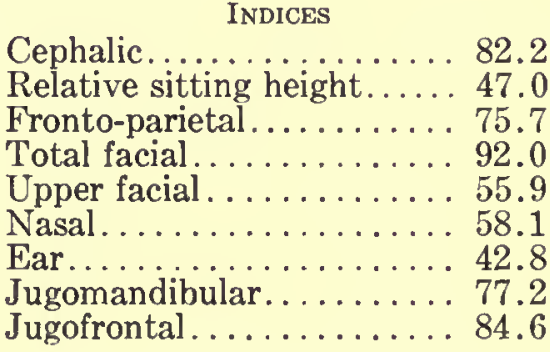

No. 77

Locality: Jemdet Nasr. Date: March 16, 1928.

Birthplace: camp of Sheikh Swadi. Age: 40. Married twice: first time 10 years ago, wife died; second time 7 years ago. Sons: 2 living. Daughters: 1 living, 1 dead. 
Brothers: none. Sisters: 1 living.

Tattoo marks: none.

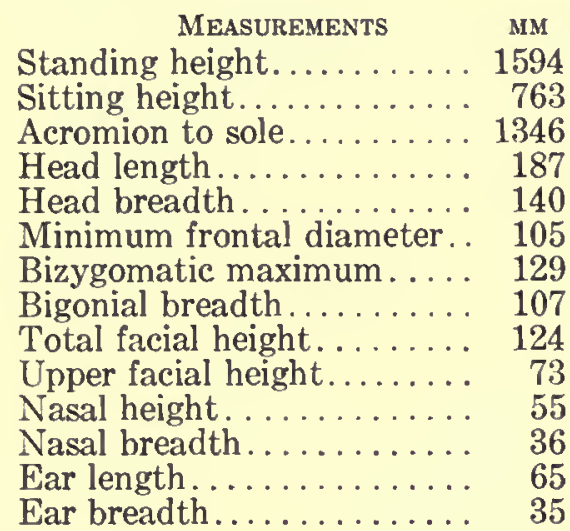

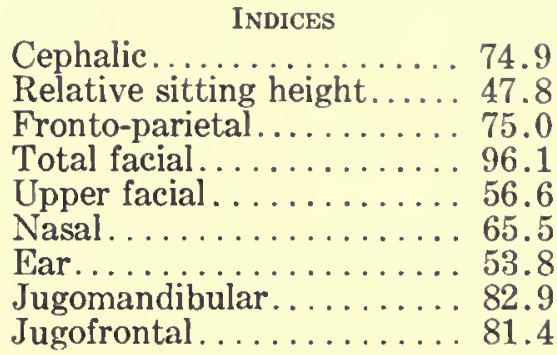

No. 79

Locality: Jemdet Nasr. Date: March 18, 1928.

Birthplace: camp of Sheikh Miniehil. Age: 15; unmarried.

Brothers: 1 living, 2 dead. Sisters: 1 living, 2 dead.

Tattoo marks: gazelle on right forearm.

\begin{tabular}{|c|c|c|}
\hline MEASUREMENTS & мM & INDICES \\
\hline Standing height....... & 1491 & Cephalic. \\
\hline Sitting height.. & 711 & Relative sitting height..... 47.7 \\
\hline Acromion to sole. & 1263 & Fronto-parietal......... 75.0 \\
\hline Head length. & 180 & Total facial. \\
\hline Head breadth. & 144 & Upper facial. . . . . . . . 60.5 \\
\hline Minimum frontal diameter. & 108 & Nasal.... \\
\hline Bizygomatic maximum . . . . & 119 & Ear. \\
\hline Bigonial breadth......... & 80 & Jugomandibular. . \\
\hline Total facial height. . . . . . . & 115 & Jugofrontal. . . . . . . . . 90. \\
\hline Upper facial height........ & 72 & \\
\hline Nasal height. . . . . . . . . . & 31 & \\
\hline Nasal breadth........... & 28 & \\
\hline Ear length. ........... & 62 & \\
\hline eadth.... . . . . . & 36 & \\
\hline
\end{tabular}

No. 80

Locality: Jemdet Nasr. Date: March 18, 1928.

Birthplace: camp of Sheikh Hadji Hunta. Age: 15; unmarried.

Birthplace of father: camp of Sheikh Atiyeh. Birthplace of mother: camp of Sheikh Hadji Hunta.

Brothers: 2 living, 1 dead. Sisters: 3 living.

Tattoo marks: on right wrist and gazelle on inside of right forearm.

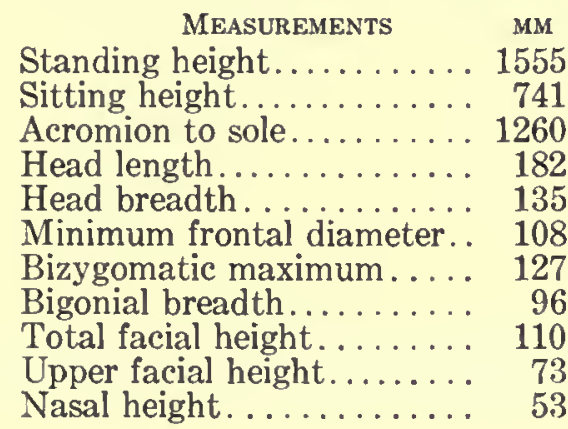

Cephalic............... 74.2

Relative sitting height...... 47.6

Fronto-parietal..........8 80.0

Total facial.............. 86.7

Upper facial. ........... 57.5

Nasal. .................. 60.4

Ear.................... 65.4

Jugomandibular............. 75.6

Jugofrontal. .......... 85.0 


\begin{tabular}{|c|c|}
\hline MEASUREMENTS & MS \\
\hline Nasal breadth.... . & 32 \\
\hline $\begin{array}{l}\text { Ear length.... } \\
\text { Ear breadth... }\end{array}$ & $\begin{array}{l}55 \\
3\end{array}$ \\
\hline
\end{tabular}

No. 81

Locality: Jemdet Nasr. Date: March 18, 1928.

Birthplace: Karbala. Age: 18; unmarried.

Birthplace of father and mother: Hindiya.

Brothers: 3 living, 1 dead. Sisters: 2 living, 1 dead.

Tattoo marks: on right wrist.

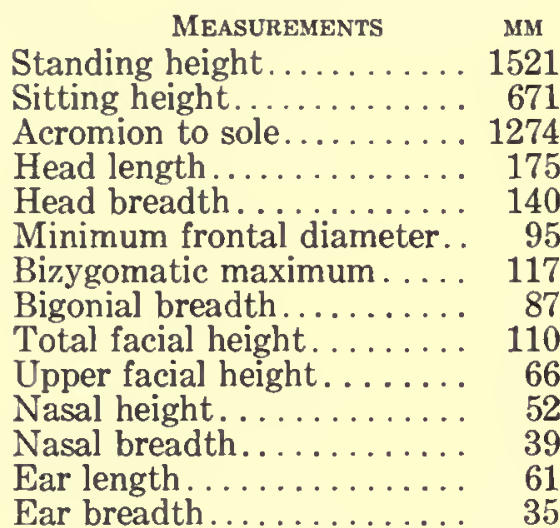

INDICES

Cephalic............880.0

Relative sitting height..... 44.1

Fronto-parietal.........6. 67.8

Total facial............. 94.1

Upper facial. ........... 56.4

Nasal. ................ 75.0

Ear............... 57.4

Jugomandibular........ 74.4

Jugofrontal. . . . . . . 81.2

No. 82

Locality: Jemdet Nasr. Date: March 18, 1928.

Birthplace: Hindiya. Age: 15; unmarried.

Brothers: 3 living, 1 dead. Sisters: 2 living, 1 dead.

Tattoo marks: on right wrist.

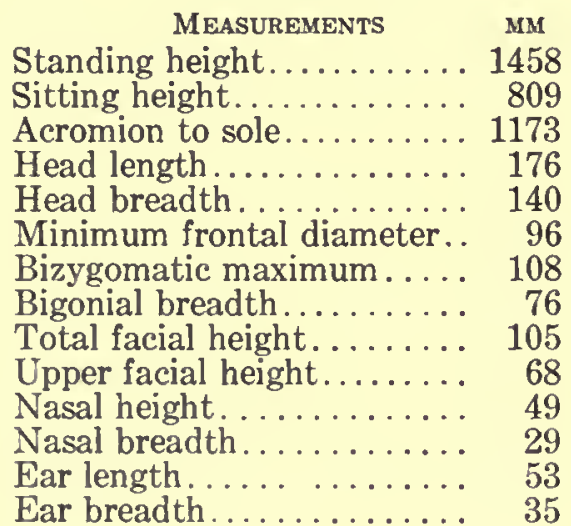

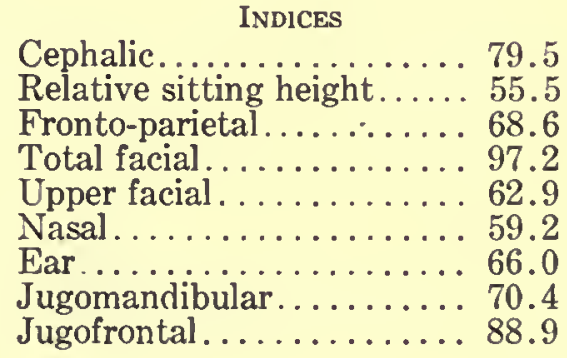

No. 83

Locality: Jemdet Nasr. Date: March 18, 1928.

Birthplace: camp of Sheikh Swadi. Age: 16; unmarried.

Brothers: none. Sisters: none.

Tattoo marks: none.

MEASUREMENTS MM

Standing height......... 1504

Sitting height.......... 716

Acromion to sole......... 1263
Cephalic............ 79.8

Relative sitting height.....47.6

Fronto-parietal......... 73.2 
Brothers: 2 living. Sisters: none.

Tattoo marks: none.

\begin{tabular}{|c|c|}
\hline MEASUREMENTS & MM \\
\hline Standing height. & 1666 \\
\hline Sitting height. & 769 \\
\hline Acromion to sole. & 1413 \\
\hline Head length... & 188 \\
\hline Head breadth. & 143 \\
\hline Minimum frontal diameter. & 100 \\
\hline Bizygomatic maximum. ... & 117 \\
\hline Bigonial breadth.......... & 95 \\
\hline Total facial height. . & 121 \\
\hline Upper facial height.... & 85 \\
\hline Nasal height. . . . . . . . . . . . & \\
\hline Nasal breadth. & \\
\hline Ear length ............. & \\
\hline Wor hro & \\
\hline
\end{tabular}

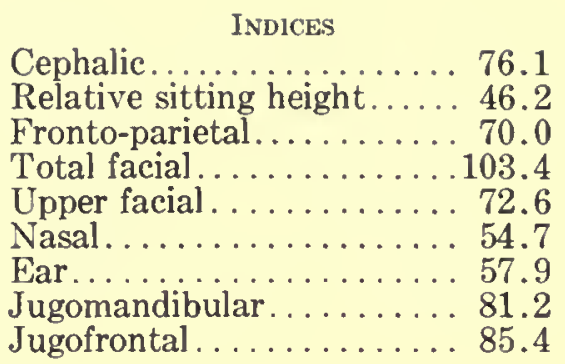

No. 87

Locality: Jemdet Nasr. Date: March 18, 1928.

Birthplace: Hindiya. Age: 17; unmarried.

Brothers: 2 living, 3 dead. Sisters: 1 living, 1 dead.

Tattoo marks: on inside of elbow.

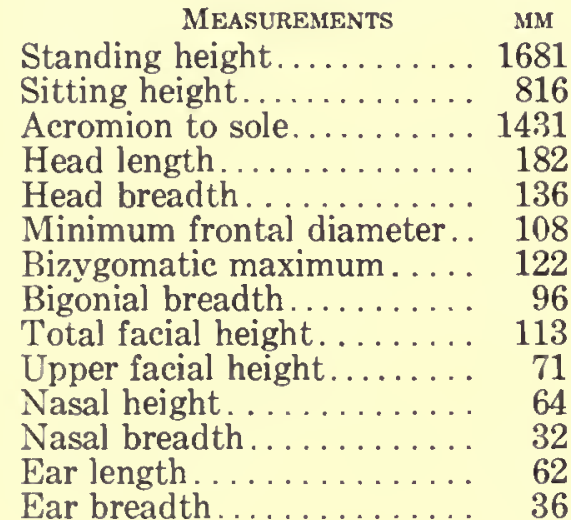

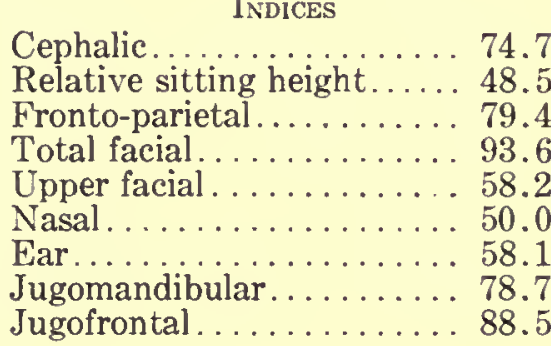

No. 88

Locality: Jemdet Nasr. Date: March 18, 1928.

Birthplace: camp of Sheikh Miniehil. Age: 17; unmarried.

Brothers: 2 living. Sisters: 1 living.

Tattoo marks: spot on end of nose.

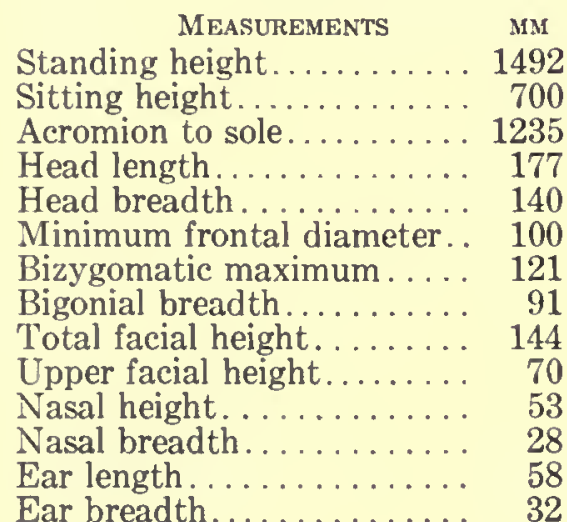

Cephalic..............79.1

Relative sitting height..... 46.9

Fronto-parietal......... 71.5

Total facial............. 119.0

Upper facial. . . . . . . . . 57.8

Nasal............... 52.8

Ear................. 55.2

Jugomandibular. . . . . . . . 75.2

Jugofrontal. . . . . . . . . 82.7 
No. 89

Locality: Jemdet Nasr. Date: March 18, 1928.

Birthplace: camp of Sheikh Swadi. Age: 90 . Married twice: first time 50 years ago, wife died after 2 years; second time 5 years ago.

Sons: 1 living. Daughters: none.

Brothers: none. Sisters: none.

Tattoo marks: none.

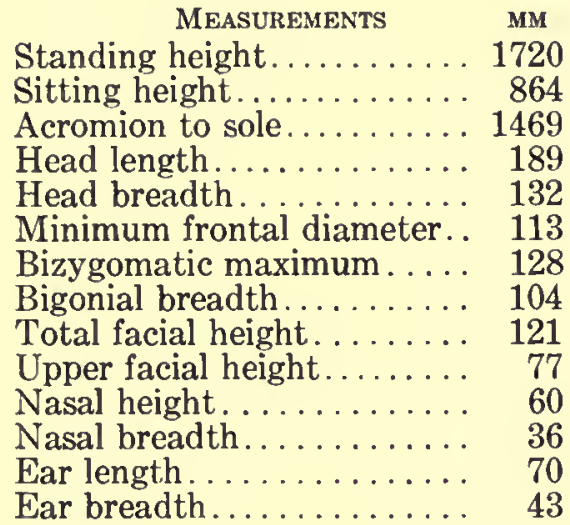

\section{INDICES}

Cephalic.............. 69.8

Relative sitting height..... 50.2

Fronto-parietal.......... 85.6

Total facial. ............ 94.6

Upper facial . . . . . . . . 60.2

Nasal. ..............6. 60.0

Ear................. 61.4

Jugomandibular. . . . . . . . . 8 81.3

Jugofrontal. ........... 88.2

\section{No. 90. Plate IX, Figs. 5, 6}

Locality: Jemdet Nasr. Date: March 18, 1928.

Birthplace: camp of Sheikh Swadi. Age: 50; married for 4 years. Sons: 1 living. Daughters: 2 dead.

Brothers: 2 dead. Sisters: 1 dead.

Tattoo marks: on right forearm.

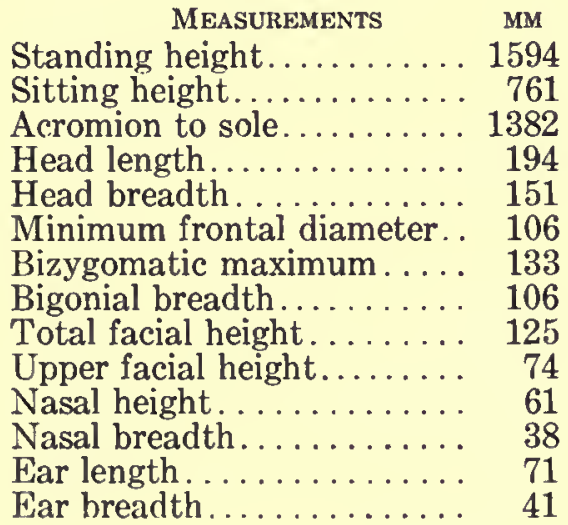

\section{INDICES}

Cephalic.............. 77.8

Relative sitting height..... 47.7

Fronto-parietal.......... 70.2

Total facial........... 94.0

Upper facial. . . . . . . . . . 55.6

Nasal................6. 62.3

Ear............... 57.7

Jugomandibular........... 79.7

Jugofrontal. . . . . . . . . 79.7

\section{No. 91. Plate IX, Figs. 7, 8}

Locality: Jemdet Nasr. Date: March 18, 1928.

Birthplace: Hindiya. Age: 25; married for 6 years. Sons: 2 living. Daughters: none. Brothers: 1 living. Sisters: 1 living.

Tattoo marks: none.

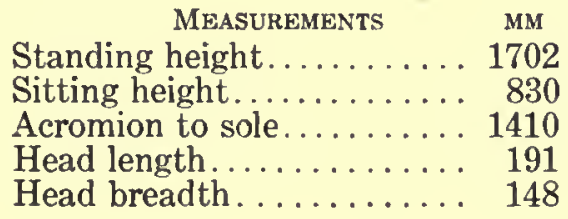

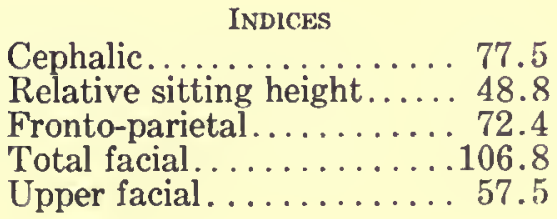




\begin{tabular}{|c|c|c|}
\hline MEASUREMENTS & мM & INDICES \\
\hline Minimum frontal diameter. & 107 & $\ldots \ldots \ldots$ \\
\hline Bizygomatic maximum.... & 120 & Ear. \\
\hline Bigonial breadth...... & 90 & Jugomandibular. \\
\hline Total facial height........ & 128 & Jugofrontal..... \\
\hline Upper facial height. & 69 & \\
\hline Nasal height......... & 56 & \\
\hline $\begin{array}{l}\text { Nasal breadth } \\
\text { Ear length. }\end{array}$ & 35 & \\
\hline Ear length.. & 60 & \\
\hline Ear breadth & 40 & \\
\hline
\end{tabular}

\section{No. 92. Plate X, Figs. 5, 6}

Locality: Jemdet Nasr. Date: March 18, 1928.

Birthplace: Bismaya. Age: 50; married for 30 years. Sons: 3 living. Daughters: 1 living, 1 dead.

Brothers: 3 dead. Sisters: none.

Tattoo marks: none.

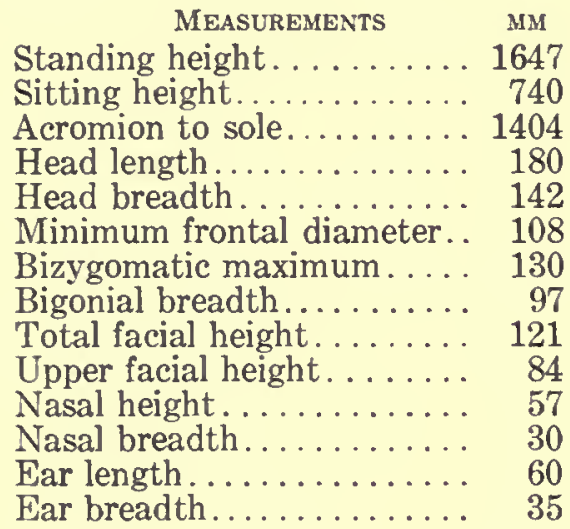

\section{INDICES}

Cephalic............. 78.9

Relative sitting height..... 45.0

Fronto-parietal.......... 76.1

Total facial . . . . . . . . . 93.0

Upper facial . . . . . . . . . . 6. 64.6

Nasal. . . . . . . . . . . . 52.7

Ear................ 58.4

Jugomandibular. . . . . . 74.6

Jugofrontal. ............ 83.1

No. 93

Locality: Jemdet Nasr. Date: March 18, 1928.

Birthplace: Hindiya. Age: 25; married for 6 years. No children.

Brothers: 2 living. Sisters: none.

Tattoo marks: none.

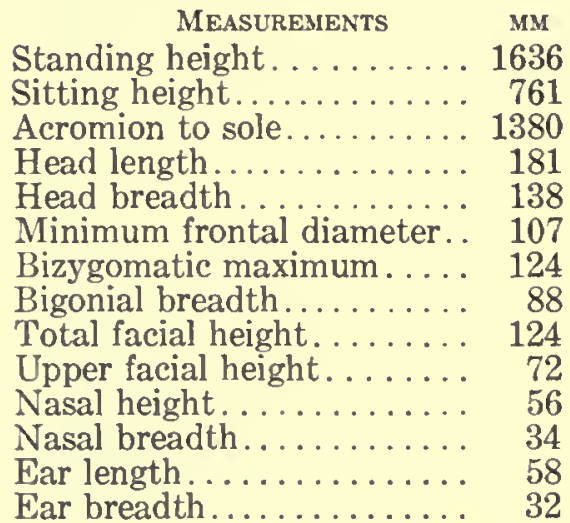

\section{INDICES}

Cephalic............... 76.3

Relative sitting height..... 46.5

Fronto-parietal......... 77.6

Total facial.............. 100.0

Upper facial. ........... 58.1

Nasal. .............. 60.7

Ear.................... 55.2

Jugomandibular........... 71.0

Jugofrontal............ 86.3

No. 94

Locality: Jemdet Nasr. Date: March 18, 1928.

Birthplace: camp of Sheikh Swadi. Age: 20; unmarried.

Brothers: none. Sisters: none.

Tattoo marks: gazelle on right forearm. 


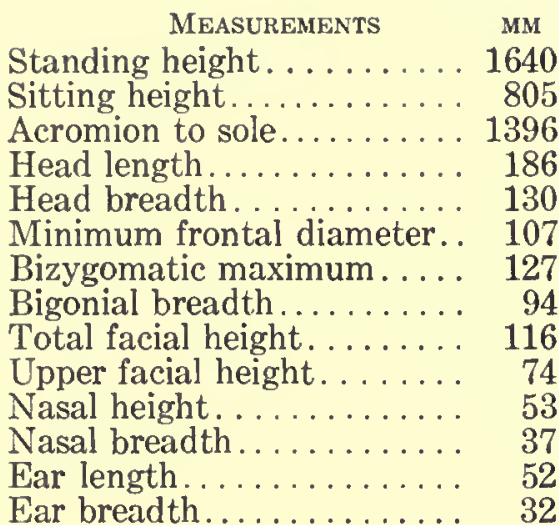

INDICES

Cephalic............ 69.9 Relative sitting height.....49.1

Fronto-parietal........ 82.4

Total facial.............. 91.4

Upper facial. . . . . . . . 58.3

Nasal. . . . . . . . . . . . 69.8

Ear...............6. 61.5

Jugomandibular . . . . . . . 74.0

Jugofrontal . . . . . . . . . . 84.2

\section{No. 95. Plate X, Figs. 7, 8}

Locality: Jemdet Nasr. Date: March 18, 1928.

Birthplace: camp of Sheikh Miniehil. Age: 19; unmarried.

Brothers: 3 living. Sisters: 2 living.

Tattoo marks: none.

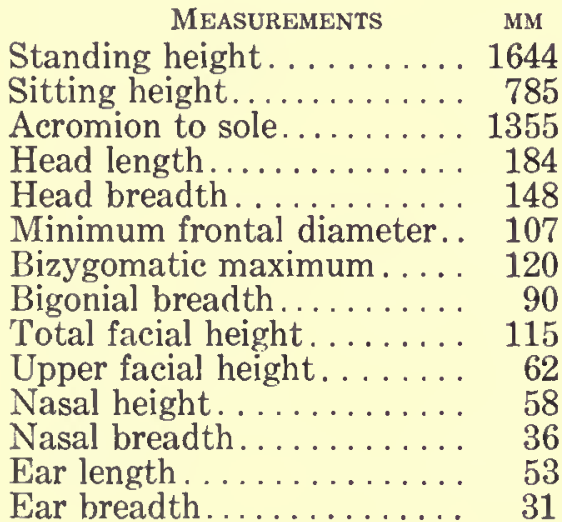

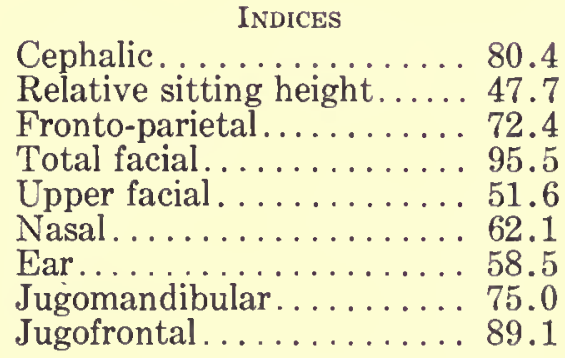

HAIR.-Form: deep waves. Texture: medium. Color: dark brown. Quantity: head, plus. EYE.-Color: dark brown.

NosE.--Profile: concavo-convex. Wings: flaring.

\section{No. 96. Plate XI, Figs. 1, 2}

Locality: Jemdet Nasr. Date: March 15, 1928.

Birthplace: camp of Sheikh Miniehil. Age: 40; married for 15 years. Sons: 2 living. Daughters: 1 living.

Brothers: 1 living. Sisters: 4 living.

Tattoo marks: 3 spots on right hand.

MEASUREMENTS MM

Standing height. . . . . . . 1545

Sitting height........... 764

Acromion to sole........... 1310

Head length............ 186

Head breadth............ 144

Minimum frontal diameter.. 105

Bizygomatic maximum..... 115

Bigonial breadth.......... 85

Total facial height ......... 117

Upper facial height. . . . . . . 74

Nasal height. ........... 61
INDICES

Cephalic............77.5

Relative sitting height..... 49.5

Fronto-parietal......... 72.9

Total facial.............111.5

Upper facial. . . . . . . . . 64.4

Nasal. ............... 52.5

Ear.................6. 62.1

Jugomandibular........ 73.9

Jugofrontal ... . . . . . . . . 91.3 


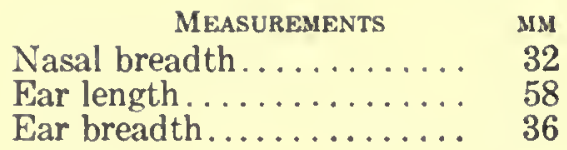

HAIR.-Texture: coarse. Color: dark brown. Quantity: head, average; beard, double plus; body, double plus.

EYE.-Color: green brown. Sclera: clear. Iris: zoned.

EyeBrow.-Thickness: double plus. Concurrency: double plus. Lateral extent: plus. Browridges: median.

Nose.-Profile: concave. Tip: depressed. Wings: flaring.

TEETH.-Wear: plus. Caries: plus. Lost: 3.

EAR.-Lobe: free. Helix: plus. Darwin's Point: plus.

SCAPULAE.-Vertebral borders: convex.

CHEST.-Development: poor.

Musculature.-Poor.

HEALTH.-Poor, often sick.

No. 97

Locality: Jemdet Nasr. Date: March 15, 1928.

Birthplace: Jebel Hamrin. Age: 55; married for 20 years. Sons: 1 living, 1 dead. Daughters: none.

Brothers: 1 dead. Sisters: 1 dead.

Tattoo marks: none.

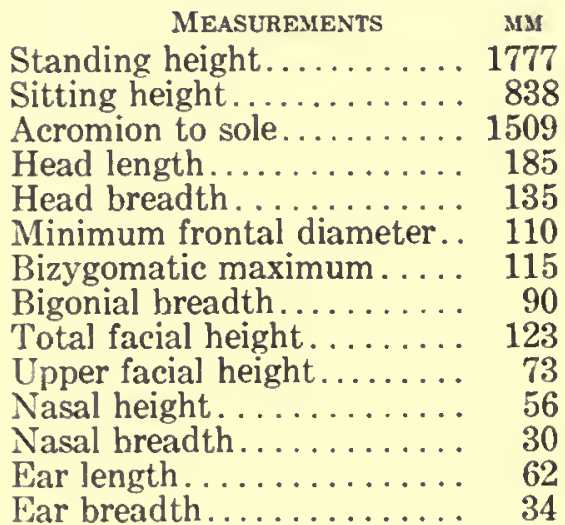

INDICES

Cephalic...............73.0

Relative sitting height......47.1

Fronto-parietal......... 81.5

Total facial. ............. 107.0

Upper facial . . . . . . . 63.5

Nasal. . . . . . . . . . . . 53.6

Ear.............. 54.8

Jugomandibular . . . . . . . . 78.3

Jugofrontal . . . . . . . . . . 95.6

No. 100

Residence: camp of Sheikh Miniehil. Date: June 4, 1928.

Age: 25 ; married for 1 year. No children.

Brothers: 2 living, 1 dead. Sisters: none.

Tattoo marks: on left wrist.

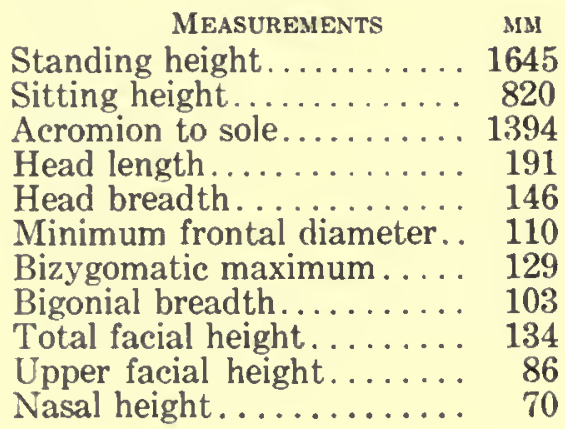

Cephalic............76.5

Relative sitting height...... 49.8

Fronto-parietal......... 75.4

Total facial. . . . . . . . . 103.9

Upper facial. . . . . . . . . 66.6

Nasal. . . . . . . . . . . 57.2

Ear............... 59.7

Jugomandibular. . . . . . . . 79.8

Jugofrontal. . . . . . . . 85.3 


\begin{tabular}{|c|c|}
\hline MEASUREMENTS & MM \\
\hline Tasal breadth & 4 \\
\hline 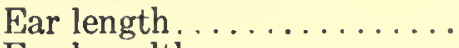 & 67 \\
\hline Ear breadth ............. & 40 \\
\hline
\end{tabular}

HAIR.-Form: low waves. Color: dark brown. Quantity: head, plus; beard, plus; body, plus. EYE.-Color: dark brown.

Nose.-Profile: convex. Tip: depressed.

TeETH.-Wear: plus. Caries: none. Lost: 1 left premolar.

EAR.-Lobe: free. Helix: average. Darwin's Point: plus.

Musculature.-Excellent.

HEALTH.-Excellent.

Circular shaved area on back of head.

No. 101. Plate XI, Figs. 3, 4

Residence: camp of Sheikh Miniehil. Date: June 4, 1928.

Age: 30 ; unmarried.

Brothers: 3 living. Sisters: 1 living.

Tattoo marks: on right wrist.

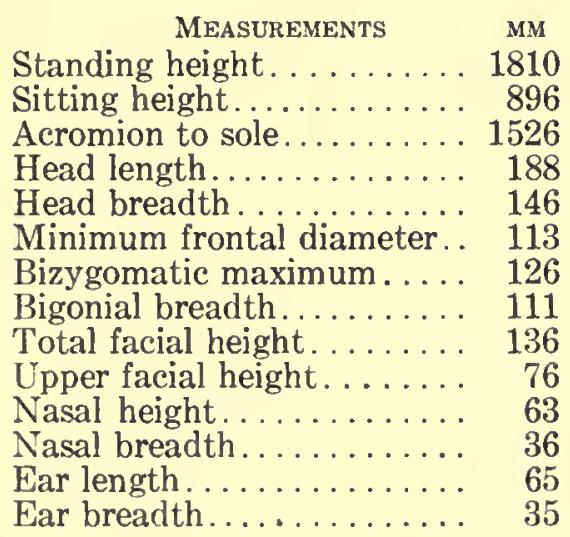

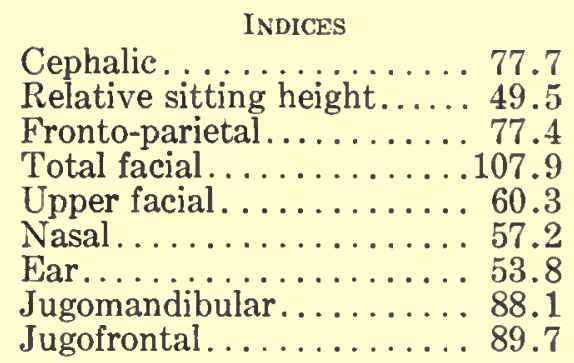

HAIR.-Form: deep waves. Texture: coarse. Color: dark brown. Quantiy: head, double plus; beard, plus; body, double plus.

EYE.-Color: dark brown. Sclera: clear. Iris: rayed.

EYEBRow.-Thickness: minus. Concurrency: minus. Latcral extent: average.

Nosf.-Profile: convex. Tip: depressed.

TeEth.-Wear: plus. Caries: none. Lost: none.

EAR.-Lobe: free. Size: average. Helix: plus. Darwin's Point: average.

Musculature.-Good.

HEALTH.-Excellent.

\section{No. 102. Plate XI, Figs. 5, 6}

Residence: camp of Sheikh Miniehil. Date: June 4, 1928.

Age: 20; unmarried.

Brothers: 4 living. Sisters: 2 living.

Tattoo marks: on right wrist.

\begin{tabular}{|c|c|}
\hline MEASUREMENTS & MM \\
\hline Standing height. & 1625 \\
\hline Sitting height. & 757 \\
\hline Acromion to sole. & 1375 \\
\hline Head length.......... & 181 \\
\hline Head hreadth & 141 \\
\hline
\end{tabular}




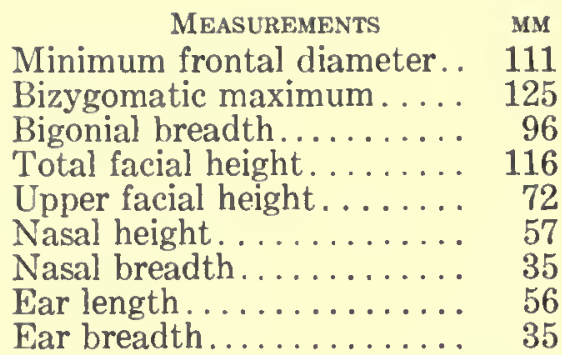

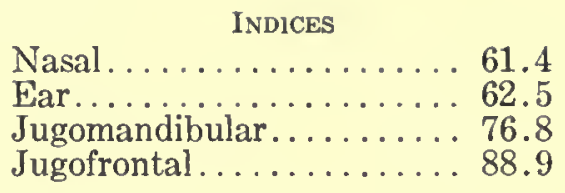

HAIR.-Form: low waves. Texture: medium. Color: dark brown. Quantity: head, double plus; beard, minus; body, plus.

EYE.-Color: dark brown. Sclera: yellow. Iris: zoned.

EyeBrow.-Thickness: minus. Concurrency: average. Lateral extent: average.

Nosf.-Profile: straight. Tip: depressed.

TEETH.-Wear: plus. Caries: minus. Lost: none.

EAr.-Lobe: free. Size: plus. Helix: average. Darwin's Point: average.

Musculature.-Fair.

HEAI،TH.-Excellent.

No. 103. Plate XI, Figs. 7, 8

Residence: camp of Sheikh Miniehil. Date: June 4, 1928.

Age: 20; unmarried.

Brothers: 2 living. Sisters: 2 living.

Tattoo marks: on back of right hand.

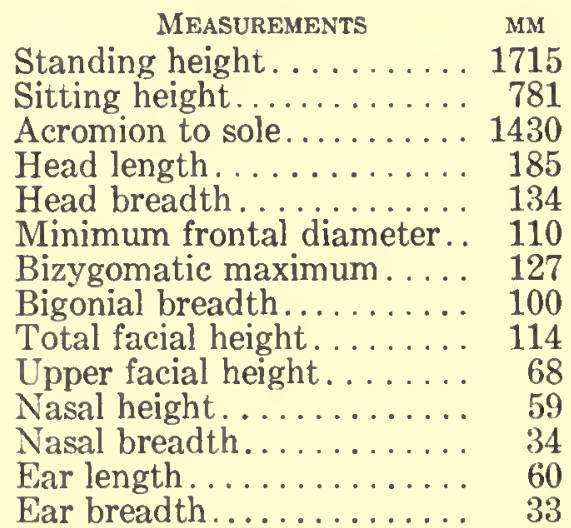

HAIR.-Texture: medium. Color: dark brown. Quantity: head, double plus; beard, minus; body, minus.

EYE.-Color: dark brown. Sclera: yellow. Iris: zoned.

Eymbrow.-Thickness: minus. Concurrency: average. Lateral extent: average. Browridges: median.

Nose.-Profile: straight. Tip: depressed. Wings: medium.

TEETH.-Wear: slight. Caries: average. Lost: none.

EAr.-Lobe: attached. Size: average. Helix: plus. Darwin's Point: plus.

Musculature.-Good.

HEALTH.-Excellent.

No. 104. Plate XII, Figs. 1, 2

Residence: camp of Sheikh Miniehil. Date: June 4, 1928.

Age: 25; unmarried.

Brothers: 2 living. Sisters: 2 living.

Tattoo marks: on back of right hand. 


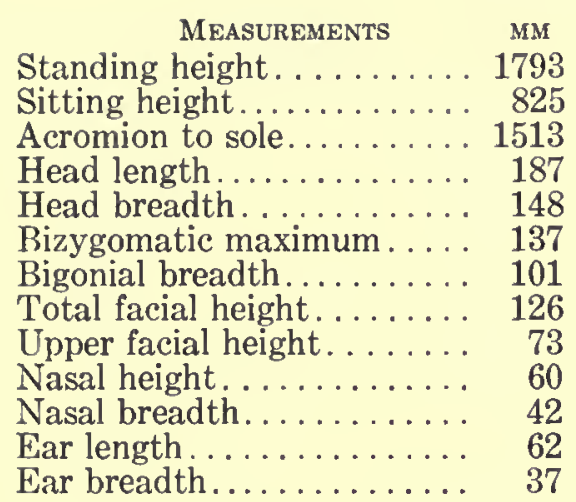

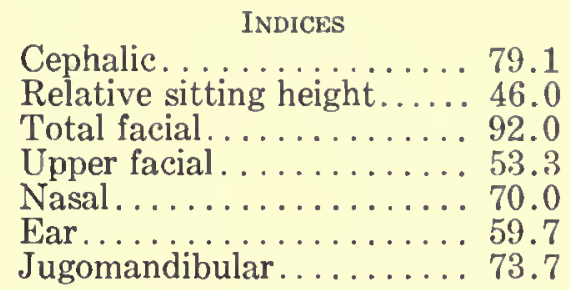

HAlR.-Form: low waves. Texture: medium. Quantity: head, plus; beard, plus; body, plus. EYE.-Color: dark brown. Sclera: speckled. Iris: zoned.

TEeTH.-Wear: none. Caries: none. Lost: none.

EAR.-Lobe: attached. Size: average. Helix: average. Darwin's Point: plus.

Chest.-Development: plus.

Musculature.-Good.

HEAItTH.-Excellent.

No. 105. Plate XII, Figs. 3, 4

Residence: camp of Sheikh Miniehil. Date: June 4, 1928.

Age: 22 ; unmarried.

Brothers: 2 living. Sisters: 1 living.

Tattoo marks: glove on right hand.

\begin{tabular}{|c|c|}
\hline \\
\hline \\
\hline \multicolumn{2}{|c|}{$\begin{array}{l}\text { Standing height } \ldots \ldots \ldots \ldots \ldots \\
\text { Sitting height. } 1590 \\
\end{array}$} \\
\hline $\begin{array}{l}\text { mion to sole. } \\
\text { I length. }\end{array}$ & \\
\hline & \\
\hline пе & \\
\hline & \\
\hline & \\
\hline & \\
\hline ht & \\
\hline & \\
\hline & \\
\hline & \\
\hline
\end{tabular}

HaIR.-Form: straight, low waves at ends. Texture: medium. Color: dark brown. EYE.-Sclera: clear. Iris: zoned.

EYeBrow.-Thickness: plus. Concurrency: plus. Lateral extent: plus.

Nose.-Wings: medium.

TEетH.-Wear: slight. Caries: plus. Lost: 2 right premolars.

EAR.-Lobe: attached. Helix: average. Darwin's Point: average.

Musculature.-Good.

HEALTH.-Good, except for yearly fever attacks.

No. 106. Plate XII, Figs. 5, 6

Residence: camp of Sheikh Miniehil. Date: June 4, 1928.

Age: 18; unmarried.

Brothers: 3 living. Sisters: 2 living.

Tattoo marks: none. 


\begin{tabular}{|c|c|}
\hline MEASUREMENTS & \\
\hline Standing height. & 172 \\
\hline height... & \\
\hline in to sole & \\
\hline a d & \\
\hline & \\
\hline neter & \\
\hline & \\
\hline Tot & \\
\hline height. & \\
\hline & \\
\hline & \\
\hline
\end{tabular}

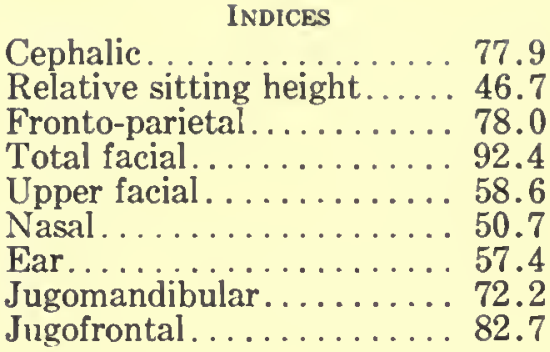

Cephalic............... 77.9

Total facial . . . . . .

Upper facial........... 58.6

HAIR.-Form: straight. Texture: fine. Color: dark brown. Quantity: head, average. EYE.-Color: dark brown. Sclera: clear. Iris: zoned.

NosE.-Profile: straight. Wings: medium.

TEETH.-Caries: none. Lost: none.

EAR.-Lobe: free. Size: average. Helix: average. Darwin's Point: double plus.

CHEST.-Development: average.

Musculature.-Fair.

HEALTH.-Good, fevered one year.

Cousin of Nos. 1 and 2. Bears family resemblance.

Very prominent malars.

\section{No. 107. Plate XII, Figs. 7, 8}

Residence: camp of Sheikh Miniehil. Date: June 4, 1928.

Age: 50; married twice, 30 years ago and 1 year ago. Sons: 3 living. Daughters: 2 dead. Brothers: none. Sisters: none.

Tattoo marks: on right wrist and gazelle on forearm.

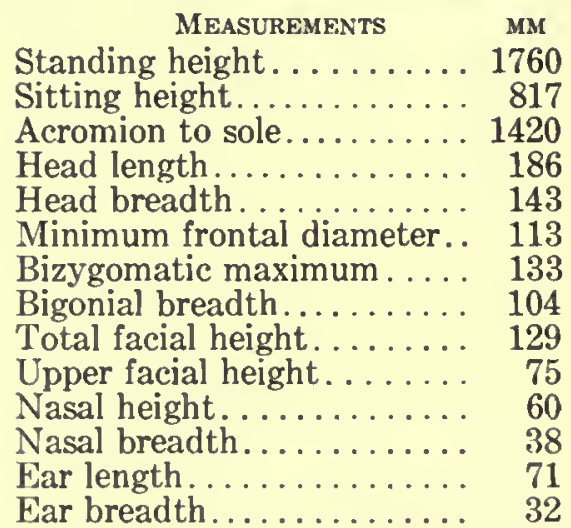

Cephalic. ............. 76.9

Relative sitting height..... 46.4

Fronto-parietal........ 79.0

Total facial..............9. 97.0

Upper facial. . . . . . . . . . 56.4

Nasal. . . . . . . . . . 63.4

Ear................ 45.1

Jugomandibular. . . . . . . 78.2

Jugofrontal . . . . . . . . 85.0

HAIR.-Texture: fine. Quantity: head, average; beard, double plus; body, plus.

EYE.-Color: dark brown. Sclera: clear. Iris: zoned.

EYEBrow.-Thickness: minus.

NosE.-Profile: straight. Tip: thickness, average. Wings: compressed.

TEeтн.-Wear: double plus. Caries: minus. Lost: 2 right lower molars, 2 left lower molars.

EAR.-Lobe: free. Helix: plus. Darwin's Point: double plus.

Musculature.--Excellent.

HEALTH.-Good.

No. 108. Plate XIII, Figs. 1, 2

Residence: camp of Sheikh Miniehil. Date: June 4, 1928.

Age: 30 ; married for 15 years. Sons: 2 living. Daughters: none. 
Brothers: 2 living. Sisters: none.

Tattoo marks: gazelle on right inside forearm.

\begin{tabular}{|c|c|c|}
\hline MFASUREMENTS & MM & INDICES \\
\hline Standing height. & 1766 & Cephalic. \\
\hline Sitting height. & 810 & Relative sitting height. \\
\hline Acromion to sole. & 1472 & Fronto-parietal.... \\
\hline Head length.... & 185 & Total facial... \\
\hline Head breadth. & 146 & Upper facial \\
\hline Minimum frontal diameter. & 105 & Nasal. \\
\hline Bizygomatic maximum.... & 128 & Ear. \\
\hline Bigonial breadth. & 100 & Jugomandibular. \\
\hline Total facial height & 126 & Jugofrontal \\
\hline
\end{tabular}

Upper facial height. . . . . . 78

Nasal height........... 58

Nasal breadth. . . . . . . . . . 34

Ear length............. 51

Ear breadth............... 33

HAIR.-Texture: fine. Quantity: head, plus.

EyE.-Color: dark brown. Sclera: clear. Iris: zoned.

Nose.-Profile: straight. Wings: compressed. Tip: horizontal.

Teетн.-Wear: plus. Caries: none. Lost: none.

EAR.-Lobe: free. Helix: double plus. Darwin's Point: average.

Chest.-Development: plus.

Musculature.-Good.

HEALTH.-Good, sometimes fevered.

\section{No. 109. Plate XIII, Figs. 3, 4}

Residence: camp of Sheikh Miniehil. Date: June 4, 1928.

Age: 20 ; unmarried.

Brothers: none. Sisters: 4 living.

Tattoo marks: on right wrist and spot on glabella.

\begin{tabular}{|c|c|c|}
\hline MEASUREMENTS & MM & INDICES \\
\hline Standing height....... & 1690 & Cephalic. \\
\hline Sitting height. & 784 & Relative sitting height. \\
\hline Acromion to sole. & 1404 & Fronto-parietal........ \\
\hline Head length.... & 187 & Total facial. . \\
\hline Head breadth. & 146 & Upper facial. \\
\hline Minimum frontal diameter. & 119 & Nasal. \\
\hline Bizygomatic maximum ..... & 138 & Ear. \\
\hline Bigonial breadth. & 102 & Jugomandibular \\
\hline Total facial height & 118 & Jugofrontal \\
\hline
\end{tabular}

Upper facial height....... 76

Nasal height. . . . . . . . . 63

Nasal breadth........... 35

Ear length............. 61

Ear breadth .............. 36

HAIR.-Texture: medium. Color: dark brown. Quantity: head, double plus; beard, minus; body, minus.

EYE.-Color: dark brown. Sclera: clear. Iris: zoned.

EYEBRow.-Thickness: double plus.

NosE.-Profile: straight. Tip: horizontal. Wings: medium.

TEETH.-Wear: none. Caries: none. Lost: none.

EAR.-Lobe: free. Size: average. Helix: average. Darwin's Point: plus.

CHEST.-Development: plus.

Musculature.-Good.

HEALTH.-Excellent, except for occasional fever attacks. 
No. 110. Plate XIII, FIgs. 5, 6

Residence: camp of Sheikh Miniehil. Date: June 4, 1928.

Age: 40 ; married for 20 years. Sons: 2 living, 2 dead. Daughters: 2 living, 2 dead. Brothers: 2 living. Sisters: 2 living.

Tattoo marks: none.

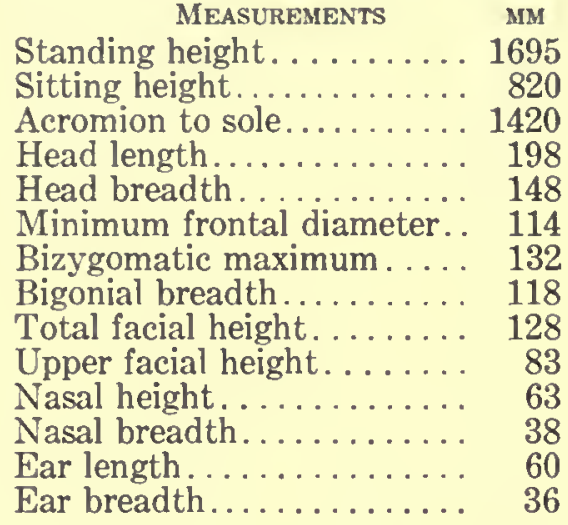

INDICES

Cephalic...............74.7

Relative sitting height..... 48.4

Fronto-parietal......... 77.0

Total facial...........9.9. 97.0

Upper facial . . . . . . . . . 6. 62.8

Nasal...............60.3

Ear................660. 60

Jugomandibular. . . . . . . . . . 89.4

Jugofrontal. . . . . . . . . . 86.4

HAIR.-Texture: fine. Color: dark brown.

EYE.-Color: dark brown. Sclera: clear. Iris: zoned.

EYEBRow.-Thickness: double plus.

NoSE.-Profile: straight. Tip: depressed. Wings: compressed.

TEETH.-Wear: double plus. Caries: none. Lost: none.

EAR.-Lobe: attached. Helix: average. Darwin's Point: double plus.

CHEST.-Development: double plus.

Musculature.-Good.

HEALTH.-Good, except for occasional attacks of fever.

\section{No. 111. Plate XIII, Figs. 7, 8}

Residence: camp of Sheikh Miniehil. Date: June 4, 1928.

Age: 60; married for 15 years, wife now dead. Sons: 2 living. Daughters: 1 dead.

Brothers: 1 living. Sisters: 5 living.

Tattoo marks: none.

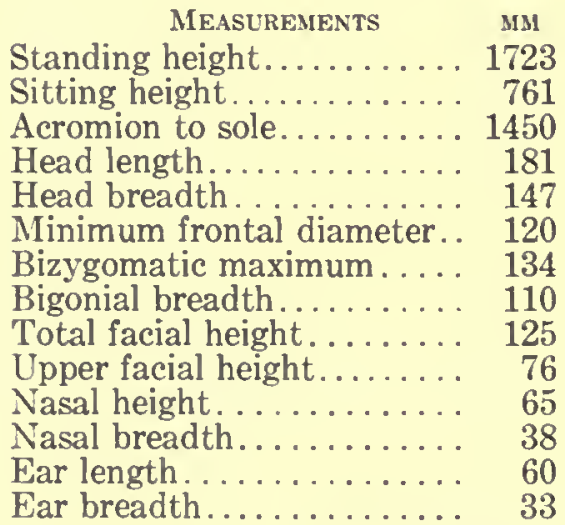

INDICES

Cephalic.............. 81.3

Relative sitting height..... 44.1

Fronto-parietal.........8 81.6

Total facial.............. 93.4

Upper facial. .......... 56.7

Nasal............... 58.5

Ear................ 55.0

Jugomandibular......... 82.1

Jugofrontal ............. 89.6

HAIR.-Texture: medium. Quantity: head, minus; beard, double plus.

EYE.-Color: dark brown. Sclera: clear. Iris: zoned.

NosE.-Profile: convex.

TEETH.-Wear: double plus. Caries: minus. Lost: third left lower molar and right lower molar. 
EAR.-Lobe: free. Helix: average. Darwin's Point: double plus.

Musculature.-Good.

HEALTH.-Excellent.

No. 112. Plate XIV, Figs. 1, 2

Residence: camp of Sheikh Miniehil. Date: June 4, 1928.

Age: 35; married for 15 years. Sons: none. Daughters: 3 living.

Brothers: 1 living. Sisters: 2 living.

Tattoo marks: none.

\begin{tabular}{|c|c|}
\hline \multicolumn{2}{|r|}{ nan } \\
\hline Standing heigh & 167 \\
\hline eight. & 81 \\
\hline to alo & 14 \\
\hline & \\
\hline$d+h$ & \\
\hline iameter. & \\
\hline 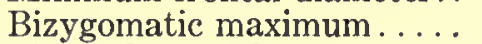 & \\
\hline & \\
\hline & \\
\hline IgII & \\
\hline & \\
\hline & \\
\hline & \\
\hline
\end{tabular}

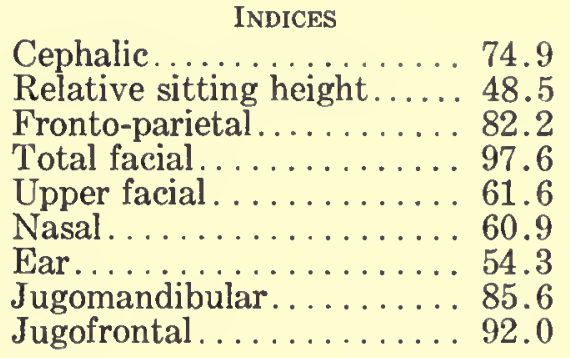

HAIR.-Texture: medium. Color: dark brown. Quantity: head, double plus; beard, double plus; body, double plus.

EYE.-Color: dark brown. Sclera: clear. Iris: zoned.

Nose.-Profile: straight. Tip: elevated. Wings: medium-flaring.

TEETH.-Wear: triple plus. Caries: triple plus. Lost: 3 right and 3 left lower molars.

EAR.-Lobe: free. Development: average. Helix: double plus. Darwin's Point: triple plus. Musculature.--Excellent.

HEaLTH.-Good, except for poor teeth.

No. 113. Plate XIV, Figs. 3, 4

Residence: camp of Sheikh Miniehil. Date: June 4, 1928.

Age: 60 ; married twice, 40 years ago and 5 months ago. Sons: 4 living. Daughters: 3 living, 2 dead.

Brothers: 1 living, 1 dead. Sisters: 4 living, 3 dead.

Tattoo marks: gazelle on right inside forearm.

\begin{tabular}{|c|c|}
\hline \multicolumn{2}{|l|}{$\begin{array}{l}\text { MEASUREMENTS } \\
\text { MEARt }\end{array}$} \\
\hline Standing height........ & 1712 \\
\hline Sitting height. & 820 \\
\hline Acromion to sole & 1438 \\
\hline Head length. & 187 \\
\hline Head breadth & 144 \\
\hline Minimum frontal diameter & 113 \\
\hline Bizygomatic maximum... & 134 \\
\hline Bigonial breadth...... & 94 \\
\hline Total facial height. & 130 \\
\hline Upper facial height & 82 \\
\hline Nasal height. . & 68 \\
\hline Nasal breadth & \\
\hline Ear length & \\
\hline$\pi$ & \\
\hline
\end{tabular}

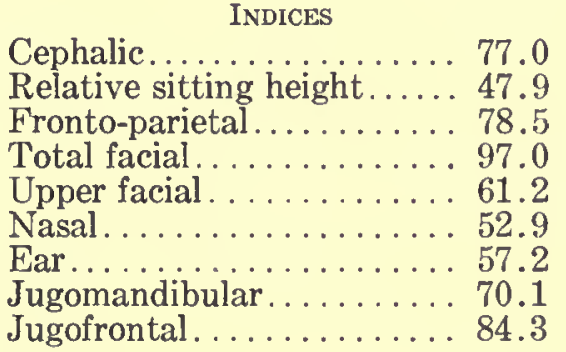

HAIR.-Color: dark brown and gray. Quantity: head, minus; beard, triple plus; body, double plus.

EYE.-Color: green brown. Sclera: clear. Iris: zoned. 
EYEBRow.-Thickness: double plus.

NosE.-Profile: convex. Tip: depressed. Wings: compressed.

TеEтн.—Wear: double plus. Caries: minus. Lost: 2 right lower molars, 1 left lower molar.

CHEST.-Development: minus.

Musculature.-Fair.

HEALTH.-Good now, formerly frequently fevered.

Father of No. 74. Very prominent malars. Cheeks very thin.

No. 114. Plate XIV, Figs. 5, 6

Residence: camp of Sheikh Miniehil. Date: June 4, 1928.

Age: 60 ; married for 30 years. Sons: 3 living. Daughters: 1 living, 1 dead.

Brothers: 1 living, 1 dead. Sisters: 1 living.

Tattoo marks: gazelle on inside of right forearm.

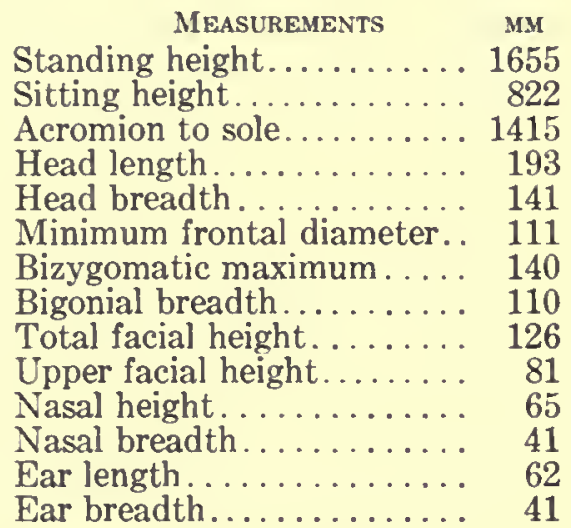

INDICES

Cephalic............ 73.1

Relative sitting height..... 49.7

Fronto-parietal......... 78.7

Total facial. . . . . . . . . 90.0

Upper facial. . . . . . . . 57.9

Nasal. ...............6 63.1

Ear................666.2

Jugomandibular......... 78.6

Jugofrontal. . . . . . . . 79.3

HAIR. - Form: low waves. Texture: fine. Color: dark brown. Quantity: head, average; beard, double plus; body, average.

EYF.-Color: green brown. Sclera: clear. Iris: zoned.

Nose.-Profile: convex. Tip: elevated. Wings: compressed.

TeEth.-Wear: triple plus. Caries: triple plus. Lost: nearly all.

EAR.-Lobe: attached. Helix: double plus. Darwin's Point: plus.

CHEST.-Development: plus.

Musculature.-Good.

HEALTH.-Good.

Father of Nos. 1 and 2.

No. 115

Residence: camp of Sheikh Miniehil. Date: June 4, 1928.

Age: 38; married for 15 years. Sons: 2 living, 2 dead. Daughters: 1 dead.

Brothers: 1 living. Sisters: 5 living.

Tattoo marks: none.

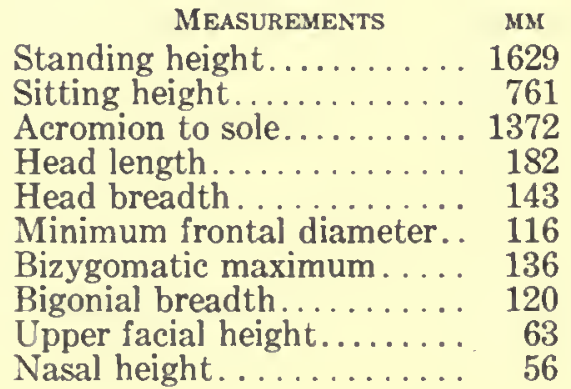

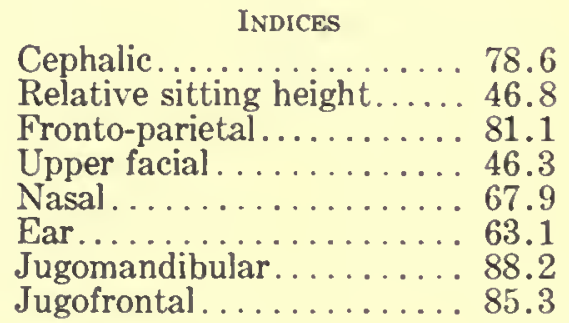




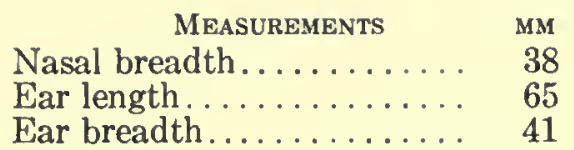

HaIR.-Texture: coarse. Color: dark brown. Quantity: head, plus; beard, double plus; body, plus.

EYE.-Color: dark brown. Sclera: bloodshot. Iris: zoned.

EYEBROW.-Thickness: double plus.

Nose.-Profile: convex. Tip: depressed. Wings: medium.

TEETH.-Wear: double plus. Caries: average. Lost: 1 right upper molar.

EAR.-Lobe: free. Size: plus. Helix: plus.

CHEST.-Development: average.

Musculature.-Good.

Health.-Good. Fevered last year.

No. 116. Plate XIV, Figs. 7, 8

Residence: camp of Sheikh Miniehil. Date: June 4, 1928.

Age: 35; married for 20 years. Sons: 1 living. Daughters: 2 living.

Brothers: 2 living. Sisters: 2 living.

Tattoo marks: slight line on back of right wrist.

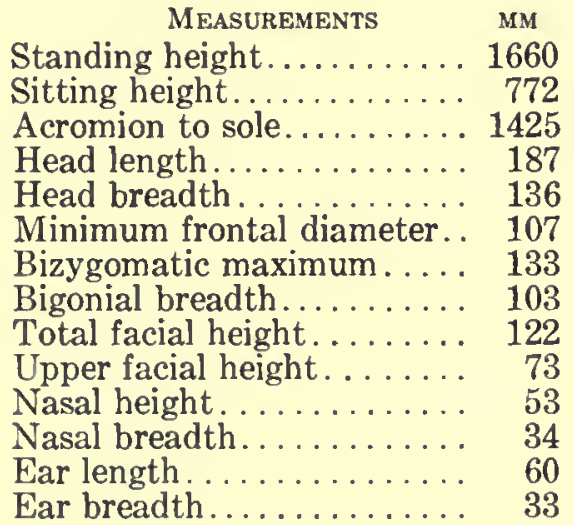

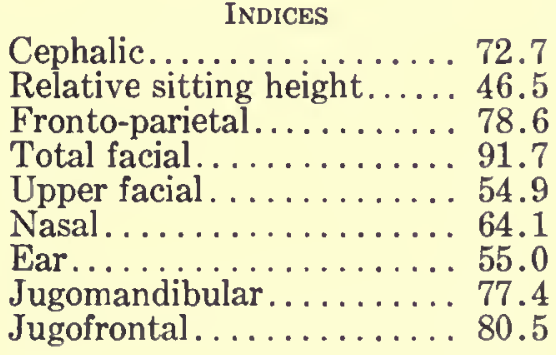

HAIR.-Form: straight. Texture: coarse.

EYE.-Color: dark brown. Sclera: clear. Iris: zoned.

NosE.-Profile: concave. Wings: compressed.

TEетн.-Wear: triple plus. Caries: triple plus. Lost: front lower teeth broken.

EAR.-Lobe: attached. Size: plus. Helix: double plus. Darwin's Point: double plus.

CHEST.-Development: minus.

Musculature.-Fair.

HEALTH.-Excellent.

No. 117

Residence: camp of Sheikh Miniehil. Date: June 4, 1928.

Age: 30 ; married for 6 years (wife now dead). Sons: none. Daughters: 1 living.

Brothers: 1 living. Sisters: 4 living.

Tattoo marks: on back of right wrist.

\begin{tabular}{|c|c|}
\hline MEasuRFments & MM \\
\hline Standing height... & 1676 \\
\hline Sitting height. . & 755 \\
\hline Acromion to sole. & 1328 \\
\hline Head length.. & 190 \\
\hline 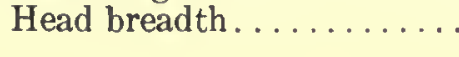 & 17 \\
\hline
\end{tabular}




\begin{tabular}{|c|c|c|}
\hline & & INDICES \\
\hline Minimum frontal diameter. . & 110 & 62.6 \\
\hline Bizygomatic maximum..... & 127 & Jugomandibular \\
\hline Bigonial breadth. & 103 & Jugofrontal. . . . \\
\hline Upper facial height. & 73 & \\
\hline Nasal height. ..... & 56 & \\
\hline Nasal breadth. & 33 & \\
\hline Ear length. & 64 & \\
\hline Ear breadth & 18 & \\
\hline
\end{tabular}

HAIR.-Texture: medium. Color: dark brown. Quantity: head, double plus; beard, minus; body, plus.

EYE.-Color: dark brown. Sclera: bloodshot. Iris: zoned.

EYEBROW.-Thickness: minus. Concurrency: minus.

Nose.-Profile: concavo-convex. Tip: thickness plus. Wings: medium.

TEETH.-Wear: none. Caries: none. Lost: none.

EAR.-Lobe: free. Helix: plus. Darwin's Point: plus.

Musculature.-Fair.

HEALTH.-Fair.

No. 118

Residence: camp of Sheikh Miniehil. Date: June 4, 1928.

Age: 65; married for 20 years. Sons: 1 living. Daughters: 1 living.

Brothers: none. Sisters: 3 living.

Tattoo marks: gazelle on right wrist.

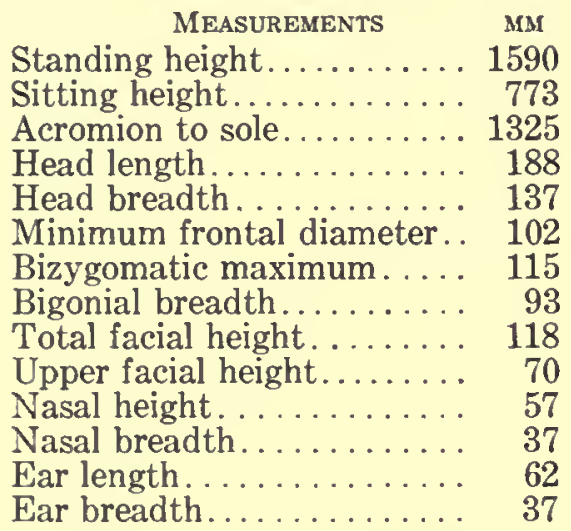

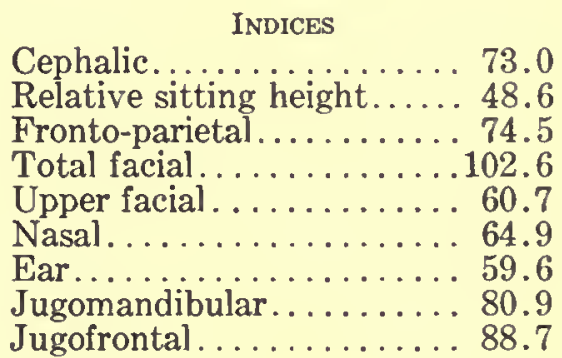

HAIR.-Form: low waves. Texture: medium. Color: dark brown.

EYE.-Color: dark brown. Sclera: clear. Iris: zoned.

EYEBROW.-Thickness: double plus. Concurrency: average. Lateral extent: average.

NoSE.-Profile: convex. Tip: depressed.

TEETH.-Wear: triple plus. Caries: triple plus. Lost: 10 or more.

EAR.-Size: plus.

Musculature.-Fair.

HEALTH.-Good. Formerly suffered from fever attacks.

No. 119

Residence: camp of Sheikh Miniehil. Date: June 4, 1928.

Age: 56; married for 20 years. Sons: 2 living. Daughters: 2 living.

Brothers: 1 dead. Sisters: 2 living, 2 dead.

Tattoo marks: none.

MEASUREMENTS MM

Standing height......... 1743

Sitting height........... 876 


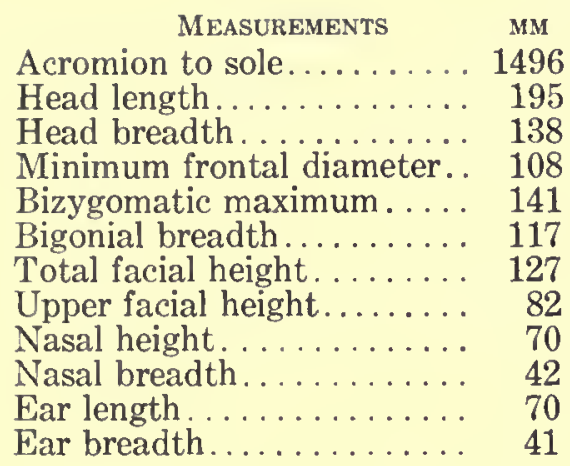

Fronto-parietal ....... 78.3

Total facial............. 90.1

Upper facial . ........... 58.2

Nasal. . . . . . . ...... 60.0

Ear................... 58.6

Jugomandibular.........88 83.0

Jugofrontal. ......... 76.6

HAIR. - Form: very low waves. Texture: medium.

EYE.-Color: dark brown. Sclera: bloodshot. Iris: zoned.

Nose.-Profile: convex. Tip: horizontal. Wings: flaring.

TEETH.-Wear: triple plus. Caries: triple plus. Lost: 1 right lower incisor.

EAR.-Lobe: free. Helix: plus. Darwin's Point: plus.

CHest.-Development: plus.

Musculature.-Good.

HEALTH.-Fair except for bad teeth.

No. 120

Residence: camp of Sheikh Miniehil. Date: June 4, 1928.

Age: 20 ; unmarried.

Brothers: 2 living. Sisters: 3 living.

Tattoo marks: on right wrist and gazelle on right inside forearm.

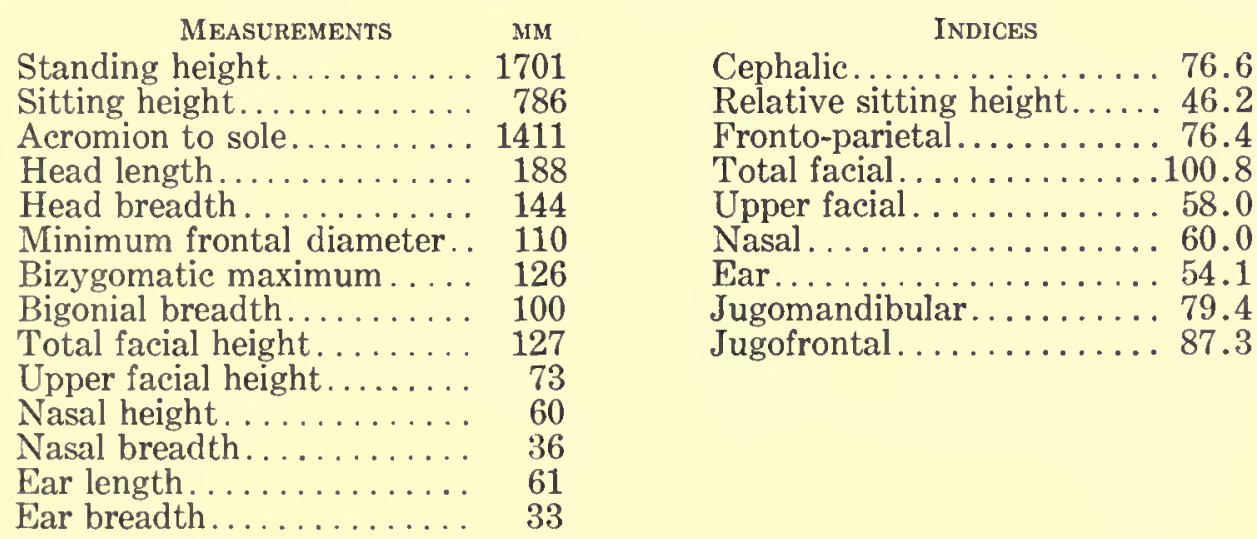

HAIR.-Texture: medium. Color: dark brown.

EYE.-Color: green brown. Sclera: clear. Iris: zoned.

Nose.-Profile: straight. Tip: horizontal. Wings: medium.

TeErH.-Wear: none. Caries: none. Lost: none.

EAR.-Lobe: free.

Chest.-Development: double plus.

Musculature.-Good.

HEALTH.-Good.

No. 121

Residence: camp of Sheikh Miniehil. Date: June 4, 1928.

Age: 30 ; unmarried.

Brothers: 2 living. Sisters: 1 living.

Tattoo marks: gazelle on inside of right wrist. 


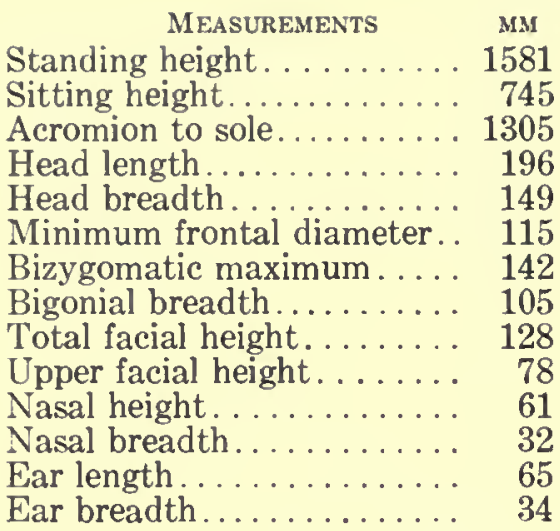

\begin{tabular}{|c|c|}
\hline Cephalic. & 76.0 \\
\hline Relative sitting height. & 47.1 \\
\hline Fronto-parietal....... & 77.2 \\
\hline Total facial. . & 90.1 \\
\hline Upper facial & 54.9 \\
\hline Nasal. . . . . & 52.5 \\
\hline Ear.. & 52.3 \\
\hline $\begin{array}{l}\text { Jugomandibular . . . . . . . . . . . . . } \\
\text { Jugofrontal . . }\end{array}$ & $\begin{array}{l}73.9 \\
80.9\end{array}$ \\
\hline
\end{tabular}

HAIR.-Form: low waves. Texture: fine. Color: dark brown. Quantity: head, double plus; beard, plus; body, plus.

EYE.-Sclera: bloodshot. Iris: zoned.

EYEBROw.-Thickness: plus.

Nose.-Profile: straight. Tip: horizontal. Wings: compressed.

EAR.-Lobe: free. Size: average. Darwin's Point: double plus.

CHEst.-Development: plus.

Musculature.--Excellent.

HEALTH.-Excellent.

No. 122

Residence: camp of Sheikh Miniehil. Date: June 4, 1928.

Age: 20 ; married for 3 years. No children.

Brothers: 3 living, 3 dead. Sisters: 3 living, 2 dead.

Tattoo marks: gazelle on inside of right forearm.

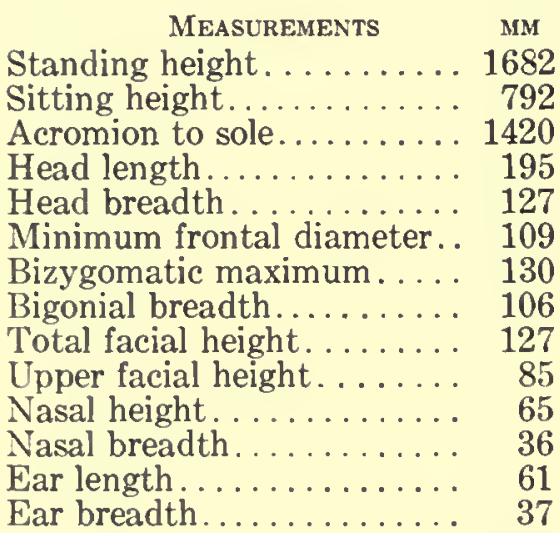

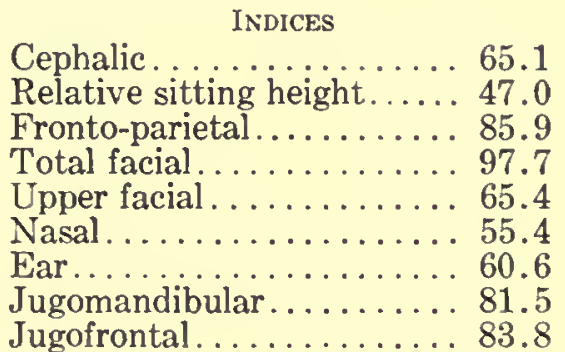

HaIR. - Form: low waves. Color: dark brown. Quantity: head, double plus; beard, minus; body, minus.

EYE.-Sclera: clear. Iris: zoned.

Nose.-Profile: straight. Tip: depressed. Wings: medium.

EAR.-Lobe: free. Size: plus. Helix: plus. Darwin's Point: plus.

CHEST.-Development: plus.

Musculature.-Good.

HEALTH.-Poor, always fevered.

No. 123

Residence: camp of Sheikh Miniehil. Date: June 4, 1928.

Age: 80 ; married for 60 years. Sons: 2 living, 1 dead. Daughters: none.

Brothers: 3 dead. Sisters: 1 living.

Tattoo marks: none. 


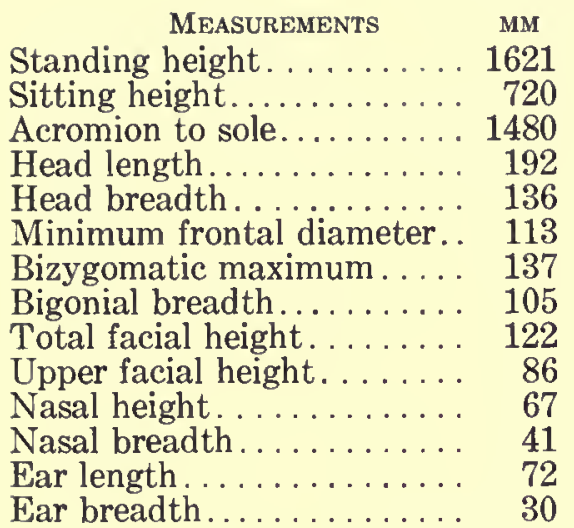

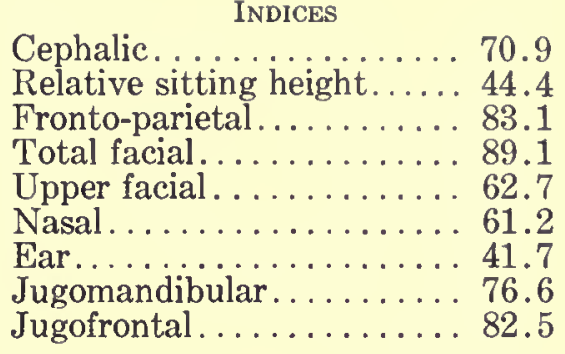

HAIR.-Form: straight. Color: white. Quantity: head, double minus; beard, double plus; body, plus.

EYE.-Color: dark brown. Sclera: bloodshot. Iris: zoned.

Nose.-Profile: convex. Tip: elevated. Wings: flaring.

TEETH.-Wear: triple plus. Caries: triple plus.

EAR.-Lobe: free. Helix: plus. Darwin's Point: double plus.

Chest.-Development: plus.

Musculature.--Poor.

HEALTH.-Poor.

No. 124

Residence: camp of Sheikh Miniehil. Date: June 4, 1928.

Age: 30 ; married for 10 years. Sons: 1 living. Daughters: 1 living.

Brothers: 2 living. Sisters: 1 living.

Tattoo marks: glove on right hand and gazelle on inside of right forearm.

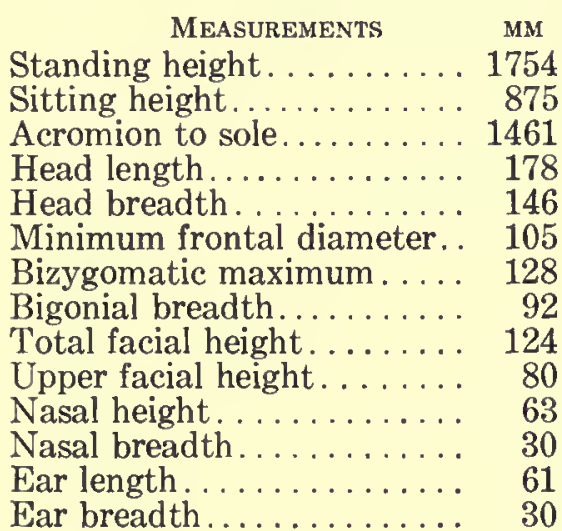

Cephalic............. 82.0

Relative sitting height..... 49.8

Fronto-parietal.......... 72.0

Total facial.............. 97.0

Upper facial..........6. 62.5

Nasal .................. 47.6

Ear............... 49.2

Jugomandibular........... 71.8

Jugofrontal. ........... 82.1

HAIR.—Color: dark brown. Quantity: head, shaved; beard, average; body, minus.

EYE.-Color: dark brown. Sclera: clear. Iris: zoned.

Nose.-Profile: straight. Wings: medium.

Chest.-Development: plus.

Musculature.-Poor.

Health.-Poor, due to stomach trouble.

No. 125

Residence: camp of Sheikh Miniehil. Date: June 4, 1928.

Age: 50 ; married for 17 years. Sons: 1 living. Daughters: 3 living.

Brothers: 1 living. Sisters: 1 living.

Tattoo marks: none. 


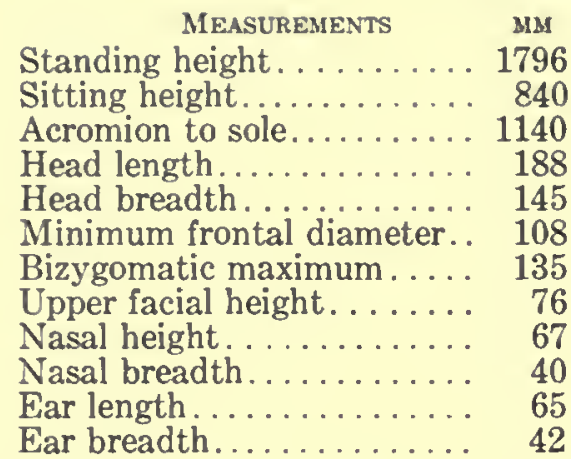

INDICES

Cephalic............. 77.0

Relative sitting height..... 46.8

Fronto-parietal......... 74.5

Nasal............... 59.7

Ear.............6.6 64.6

HAIR.-Form: very low waves. Color: dark brown.

EYE.-Sclera: clear. Iris: zoned. Blind: right.

Nose.-Profile: convex.

EAR.-Lobe: free. Size: average. Helix: triple plus.

CHEST.-Development: plus.

Musculature.-Good.

HEALTH.-Good.

No. 126. Plate XV, Figs. 1, 2

Residence: camp of Sheikh Miniehil. Date: June 4, 1928.

Age: 26 ; unmarried.

Brothers: 2 living, 3 dead. Sisters: 1 living, 2 dead.

Tattoo marks: none.

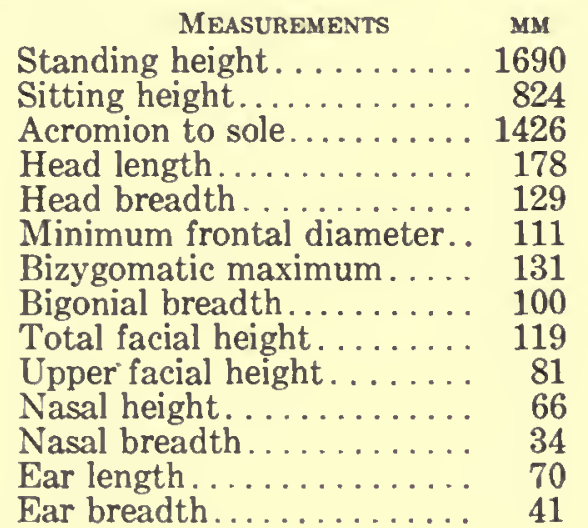

INDICES

Cephalic............. 72.5

Relative sitting height..... 48.7

Fronto-parietal........ 86.1

Total facial.............. 90.9

Upper facial. ..........6 61.8

Nasal. ............... 51.5

Ear................ 58.6

Jugomandibular.......... 76.3

Jugofrontal........... 84.7

HAIR.-Form: low waves. Texture: fine. Color: dark brown.

EyE.-Color: dark brown. Sclera: clear. Iris: zoned.

Nose.--Profile: convex. Tip: depressed.

EAR.-Lobe: free. Helix: double plus. Darwin's Point: plus.

CHEST.-Development: double plus.

Musculature.-Good.

HEALTH.-Excellent.

No. 127. Plate XV, Figs. 3, 4

Residence: camp of Sheikh Miniehil. Date: June 4, 1928.

Age: 27; married twice, 6 years ago and 4 years ago. Sons: 1 living. Daughters: 1 dead. Brothers: 2 living. Sisters: 5 living, 1 dead.

Tattoo marks: gazelle on back of right hand and inside of right forearm.

MaAsurements MM

Standing height. . . . . . . 1631

Sitting height........... 775
INDICES

Cephalic.............77.6

Relative sitting height...... 47.5 


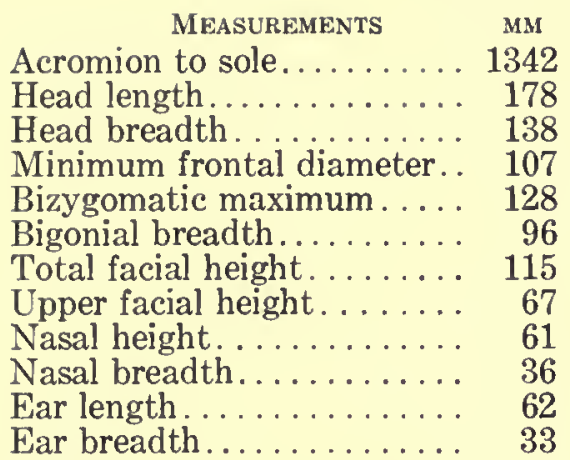

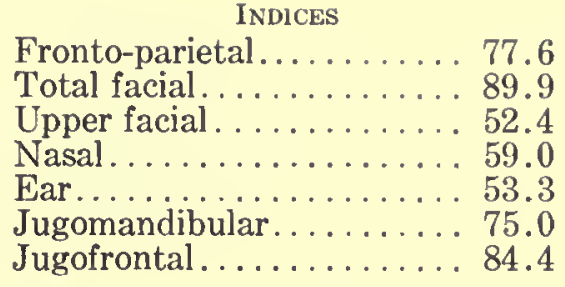

HaIR.-Form: low waves. Texture: fine. Color: dark brown. Quantity: head, plus; beard, minus; body, minus.

EYE.-Color: dark brown. Sclera: bloodshot. Iris: zoned.

Nose.-Profile: concavo-convex.

TeEth.-Wear: double plus. Caries: double plus. Lost: 2 left upper premolars.

EAR.-Lobe: free. Helix: plus. Darwin's Point: plus.

CHEst.-Development: plus.

Musculature.-Good.

HEALTH.-Excellent.

Son of Sheikh Hadji Miniehil.

No. 128. Plate XV, Figs. 5, 6

Locality: Jemdet Nasr. Date: June 4, 1928.

Birthplace: Kut al Hai. Age: 16; married for 4 years. Sons: 1 living. Daughters: 1 dead. Brothers: 1 living, 1 dead. Sisters: 1 living.

Tattoo marks: on right wrist.

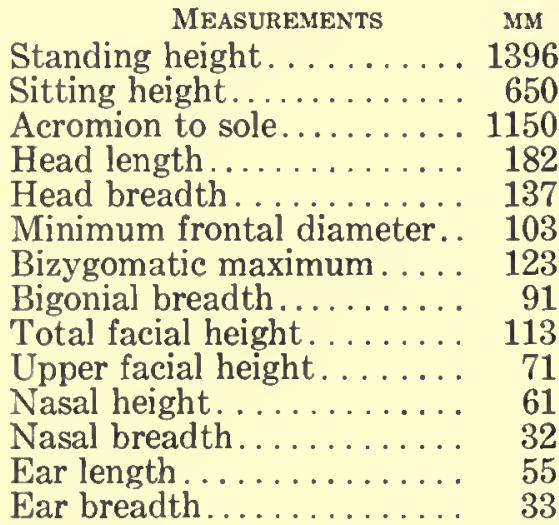

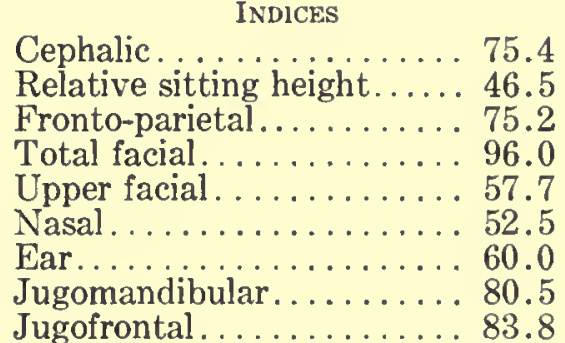

Jugofrontal

HAIR.-Form: low waves. Texture: medium. Color: dark brown. Quantity: head, minus; beard, plus; body, plus.

EYE.-Color: dark brown. Sclera: speckled. Iris: zoned.

EyeBrow.-Thickness: average. Concurrency: plus. Lateral extent: plus. Brow-ridges: median. Glabella: average.

NosE.-Profile: straight. Tip: thickness, average; depressed. Wings: medium.

TEETH.-Wear: plus. Caries: average. Lost: none.

EAR.-Lobe: free. Size: average. Helix: plus. Darwin's Point: minus.

CHEST.-Development: plus.

Musculature.-Fair.

HEALTH.- Yearly attacks of fever.

Nervous and rather stupid. Marked wrinkling on face. 


\section{No. 129. Plate XV, Fig. 7}

Residence: camp of Sheikh Miniehil. Date: June 4, 1928.

Age: 30 ; married for 12 years. Sons: 1 living. Daughters: 1 living, 2 dead.

Brothers: 5 living. Sisters: 1 living.

Tattoo marks: spot on right wrist and on end of nose.

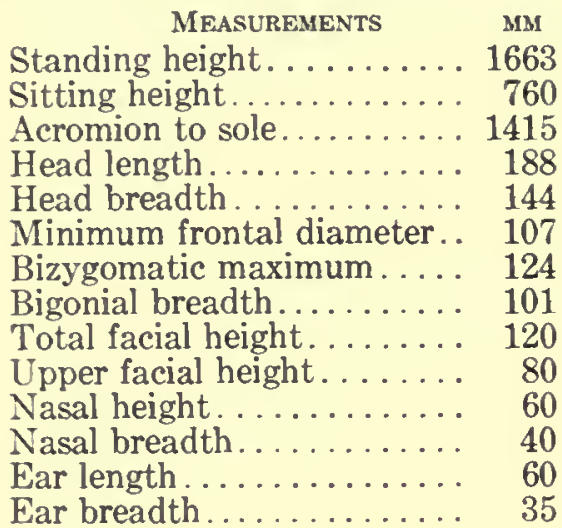

INDICES

Cephalic.............. 76.6

Relative sitting height..... 45.7

Fronto-parietal......... 74.4

Total facial............ 96.9

Upper facial. .........6. 64.5

Nasal.............. 66.6

Ear................ 58.4

Jugomandibular......... 81.4

Jugofrontal........... 86.3

HaIR.-Texture: coarse. Color: dark brown. Quantity: head, minus; beard, plus (white).

EYE.-Color: dark brown. Sclera: bloodshot. Iris: zoned.

Nose.-Profile: concave. Tip: thickness, double plus. Wings: flaring.

EAR.-Lobe: free. Helix: double plus. Darwin's Point: average.

CHEST.-Development: plus.

MUSCUlature.-Good.

HEALTH.-Excellent.

No. 130. Plate XVI, Figs. 1, 2

Residence: camp of Sheikh Miniehil. Date: June 4, 1928.

Age: 70; married for 40 years. Sons: none. Daughters: 1 living.

Brothers: 1 living. Sisters: 1 living, 1 dead.

Tattoo marks: none.

MEASUREMENTS

Standing height......... 1677

Sitting height......... 828

Acromion to sole... . . . . . 1442

Head length........... 180

Head breadth........... 145

Minimum frontal diameter. . 106

Bizygomatic maximum.... 134

Bigonial breadth......... 100

Total facial height........ 120

Upper facial height. . . . . . 76

Nasal height. ........... 60

Nasal breadth........... 36

Ear length.............. 71

Ear breadth............ 36
INDICES

Cephalic.............80.6

Relative sitting height..... 49.4

Fronto-parietal......... 73.2

Total facial........... 89.6

Upper facial. . . . . ...... 56.6

Nasal.............. 60.0

Ear................ 50.7

Jugomandibular. . . . . . 74.6

Jugofrontal . . . . . . . . . . 79.1

HAIR.-Color: gray and white. Quantity: head, double minus; beard, double plus; body, double plus.

EYE.-Color: green brown. Sclera: bloodshot. Iris: zoned.

NosE.-Profile: convex. Tip: depressed. Wings: medium.

TEETH.-Wear: extreme. Caries: triple plus. Lost: all but 2 incisors in both lower jaws.

Decay of teeth and absorption of gums.

EAR.-Lobe: free. Helix: plus. Darwin's Point: plus.

ChesT.-Derelopment: minus. 
Musculature.-Very poor.

HEalth.-Poor.

No. 131. Plate XVI, Figs. 3, 4

Residence: camp of Sheikh Miniehil. Date: June 4, 1928.

Age: 45 ; married once, wife died 13 years ago. No children.

Brothers: 1 living, 1 dead. Sisters: none.

Tattoo marks: three dots on right wrist.

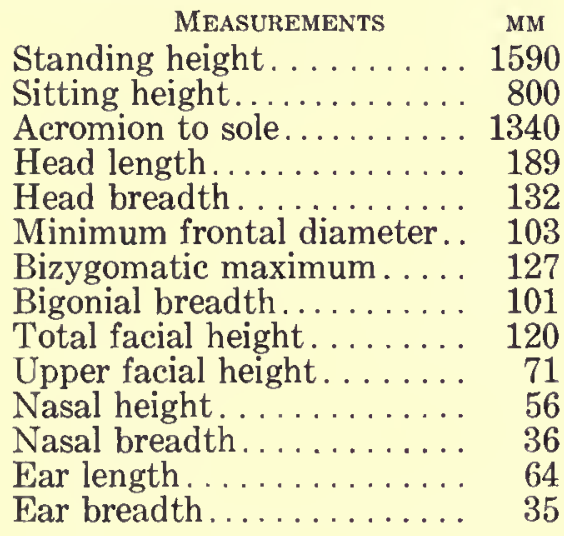

INDICES

Cephalic . . . . . . . 69.9

Relative sitting height..... 50.3

Fronto-parietal......... 78.0

Total facial ............. 94.5

Upper facial.......... 55.9

Nasal...............6. 64.4

Ear.................. 54.6

Jugomandibular......... 79.5

Jugofrontal............. 81.1

HAIR.-Color: dark brown; beard, gray. Quantity: head, double minus; beard, double plus; body, plus.

EYE.-Color: dark brown. Sclera: clear. Iris: zoned.

Nose.-Profile: concavo-convex. Tip: depressed.

TeETH.-Wear: double plus. Caries: double plus. Lost: 1 lower molar right and 1 left.

Ear.-Lobe: attached. Size: minus. Helix: double plus. Darwin's Point: plus.

Chest.-Development: plus.

Musculature.-Good.

Health.-Excellent.

In prison at Omsk, captured from Turkish army in 1914.

No. 132. Plate XVI, Figs. 5, 6

Residence: camp of Sheikh Miniehil. Date: June 4, 1928.

Age: 30 ; married for 4 years. No children.

Brothers: 1 living. Sisters: none.

Tattoo marks: on right wrist.

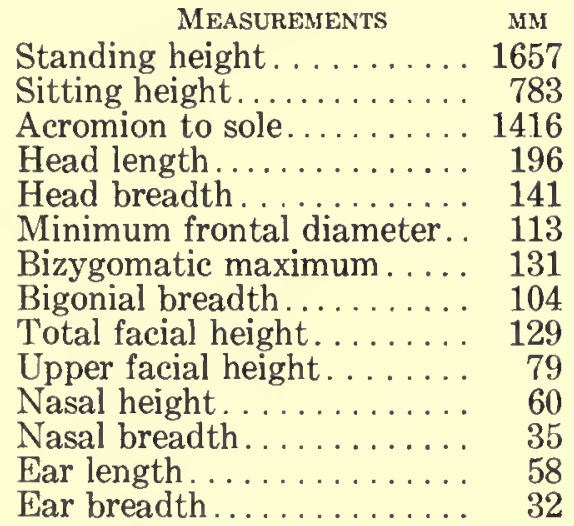

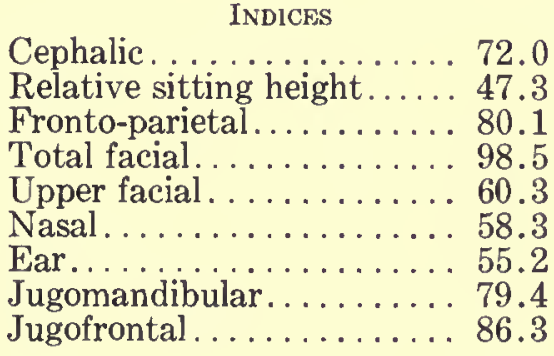

HaIR.-Form: curly frizzly. Texture: coarse. Color: dark brown. Quantity: head, double plus; beard, minus; body, average. 
EYE.-Sclera: clear. Iris: zoned.

Nose.-Profile: convex. Tip: thickness, double plus; depressed. Wings: medium. EAR.-Lobe: attached. Size: minus. Helix: average. Darwin's Point: average.

CHEST.-Development: plus.

Musculature.-Excellent.

HEalth.-Excellent.

\section{No. 133. Plate XVI, Figs. 7, 8}

Residence: camp of Sheikh Miniehil. Date: June 4, 1928.

Age: 31; married 6 years ago (wife now dead). Sons: none. Daughters: 1 living.

Brothers: 5 living. Sisters: 1 living.

Tattoo marks: gazelle on right forearm.

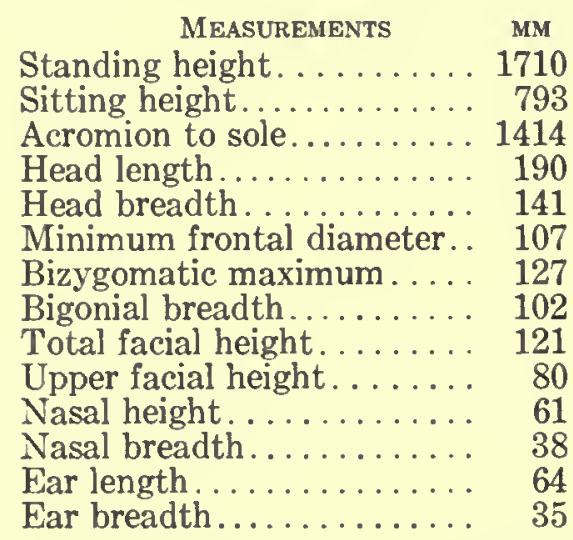

INDICES

Cephalic............ 74.3

Relative sitting height..... 46.3

Fronto-parietal......... 75.9

Total facial............. 95.3

Upper facial . . . . . . . . . 63.0

Nasal. ..............6. 62.3

Ear................ 54.6

Jugomandibular. . . . . . . . 80.3

Jugofrontal. . . . . . . . . 84.2

HAIR.-Texture: medium. Color: dark brown. Quantity: head, plus; beard, plus; body, plus. EYE.-Color: dark brown. Sclera: clear. Iris: zoned.

Nose.-Profile: concave. Tip: thickness, plus; horizontal. Wings: medium.

EAR.-Lobe: free. Helix: average. Darwin's Point: average.

CHEST.-Development: plus.

Musculature.-Good.

HEALTH.-Excellent.

Brother of No. 129.

No. 134. Plate XVII, Figs. 1, 2

Residence: camp of Sheikh Miniehil. Date: June 4, 1928.

Age: 30 ; married for 3 years. Sons: 1 living. Daughters: 2 living.

Brothers: 2 living. Sisters: none.

Tattoo marks: glove on back of right hand.

\begin{tabular}{|c|c|c|}
\hline MEASUREMENTS & MM & INDICES \\
\hline Standing height. . . . . & 1672 & Cephalic. \\
\hline Sitting height. & 782 & Relative sitting height. \\
\hline Acromion to sole. & 1380 & Fronto-parietal.........76. \\
\hline Head length. & 185 & Total facial............ 88 \\
\hline Head breadth. & 143 & Upper facial \\
\hline Minimum frontal diameter. & 109 & Nasal...... \\
\hline Bizygomatic maximum . . . . & 125 & Ear. \\
\hline ial breadth. & 97 & Jugomandibular. \\
\hline Total facial height. & 110 & Jugofrontal \\
\hline
\end{tabular}

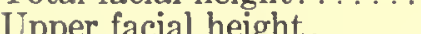

Nasal height

Nasal breadth............ 36

Ear length.............. 53

Ear breadth........... 38 
HaIR.-Form: low waves. Texture: medium. Color: dark brown. Quantity: head, plus; beard, plus; body, average.

EYE.-Color: dark brown. Sclera: bloodshot. Iris: zoned.

Nose.-Profile: concavo-convex. Wings: medium-compressed.

EAR.-Lobe: attached. Helix: double plus. Darwin's Point: plus.

CHEST.-Development: plus.

Musculature.-Good.

HEALTH.-Excellent.

No. 135. Plate XVII, Fig. 4

Residence: camp of Sheikh Miniehil. Date: June 4, 1928.

Age: 35; married for 13 years. Sons: 2 living. Daughters: 3 living.

Brothers: 1 dead. Sisters: 1 living.

Tattoo marks: none.

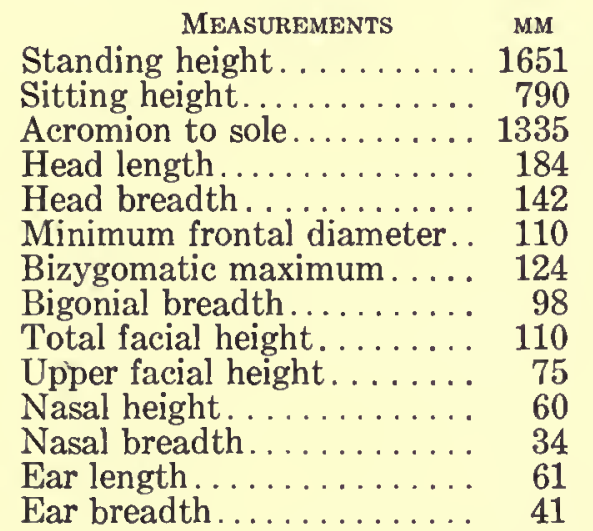

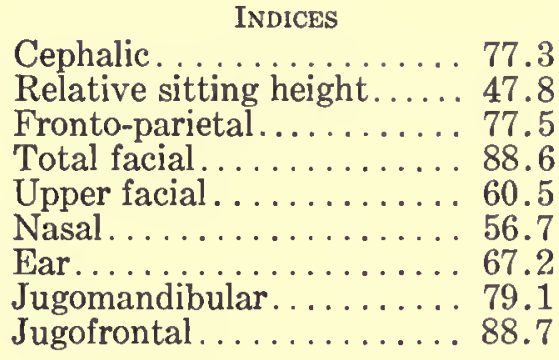

HAIR.-Color: black. Quantity: head, double minus; beard, double plus.

EYE.-Color: dark brown. Sclera: bloodshot. Iris: zoned. Blind: right.

Nose.-Profile: concavo-convex. Tip: thickness, plus; elevated. Wings: compressed.

TEETH.-Wear: double plus. Caries: double plus. Lost: one. Condition: poor.

EAR.-Lobe: free. Size: double plus. Helix: double plus. Darvin's Point: double plus.

CHEST.-Development: double minus.

MusculatuRE.-Poor.

HEALTH.-Poor, always sick.

No. 136. Plate XVII, Figs. 5, 6

Residence: camp of Sheikh Miniehil. Date: June 4, 1928.

Age: 38 ; married for 2 years. Sons: none. Daughters: 1 living.

Brothers: 3 living. Sisters: 2 living.

Tattoo marks: glove on back of right hand.

\begin{tabular}{|c|c|c|}
\hline Measurements & мм & INDICES \\
\hline Standing height. & 1601 & Cephalic. \\
\hline Sitting height. & 830 & Relative sitting height. \\
\hline Acromion to sole & 1330 & Fronto-parietal. \\
\hline Head length. & 185 & Total facial.. \\
\hline Head breadth. & 140 & Upper facial. \\
\hline Minimum frontal diameter. & 106 & Nasal... \\
\hline Bizygomatic maximum ..... & 130 & Ear. \\
\hline Bigonial breadth....... & 109 & Jugomandibular \\
\hline Total facial height. & 120 & Jugofrontal \\
\hline
\end{tabular}




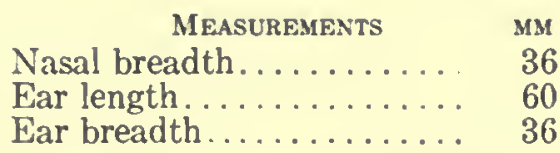

HaIR.-Form: low waves. Texture: medium.

EYE.-Color: dark brown. Sclera: bloodshot. Iris: zoned.

Nose.-Profile: straight. Tip: thickness, average; elevated. Wings: flaring.

TEFTH.-Wear: plus. Caries: double plus. Lost: 1 right lower molar.

EAR.-Lobe: free. Size: plus. Helix: double plus. Darwin's Point: double plus.

CHEst.-Development: double plus.

MUSCulature.-Excellent.

HEALTH.-Excellent.

Brother of No. 82 .

No. 137. Plate XVII, Figs. 7, 8

Residence: camp of Sheikh Miniehil. Date: June 4, 1928.

Age: 30 ; married for 2 years. No children.

Brothers: 1 living. Sisters: 2 living, 3 dead.

Tattoo marks: spot on right wrist.

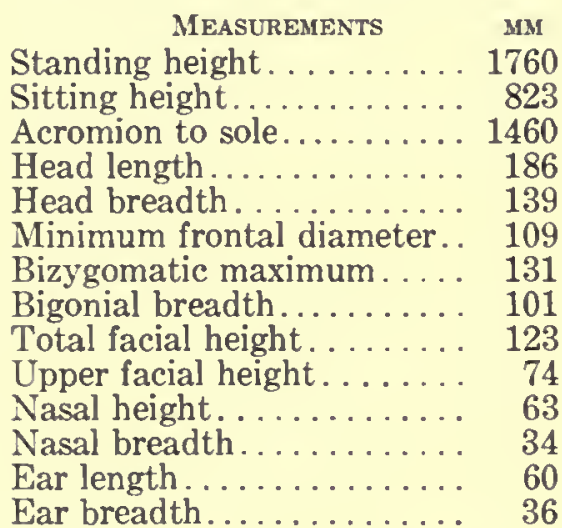

INDICES

Cephalic............74.7

Relative sitting height..... 46.7

Fronto-parietal........ 78.4

Total facial............ 93.9

Upper facial. . . . . . . . . 56.5

Nasal . . . . . . . . . . . 54.0

Ear..............6 60.0

Jugomandibular. . . . . . . 77.1

Jugofrontal. ......... 83.2

HAIR. - Color: dark brown. Quantity: head, minus; beard, minus; body, minus.

EYE.-Color: dark brown. Sclera: bloodshot. Iris: zoned.

EYEBRow.-Thickness: minus. Concurrency: minus. Lateral extent: average.

TEeTH.-Wear: double plus. Caries: double plus. Lost: 2 right lower molars.

EAR.-Lobe: free. Size: plus. Helix: average. Darwin's Point: double plus.

CHEST.-Development: double plus.

Musculature.-Excellent.

HEALTH.-Excellent.

No. 138. Plate XVIII, Figs. 1, 2

Residence: camp of Sheikh Miniehil. Date: June 4, 1928.

Age: 35; married for 5 years. Sons: 3 dead. Daughters: 1 dead.

Brothers: 4 living. Sisters: 1 living.

Tattoo marks: none.

$\begin{array}{cc}\text { MEasurements } & \text { MM } \\ \text { Standing height. . . . . . . } 1740 & 1740 .\end{array}$

Sitting height........... 821

Acromion to sole.......... 1443

Head length........... 182

Head breadth............ 144

Minimum frontal diameter.. 107

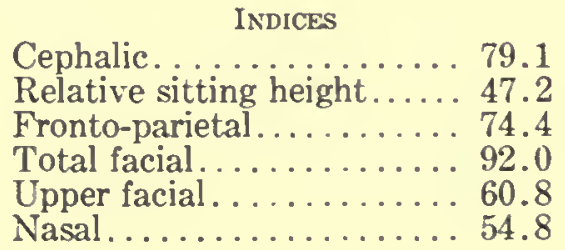




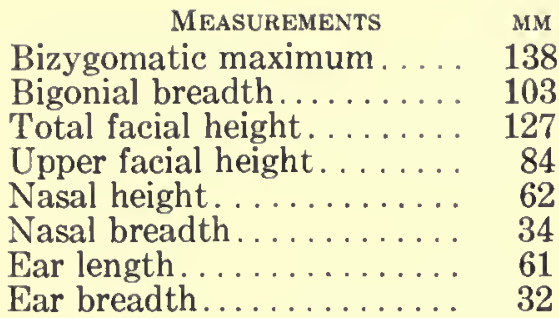

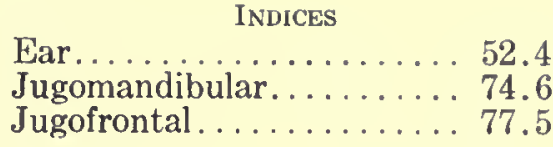

INDICES
Jugomandibular. . . . . . . . 74.6

Jugofrontal . . . . . . . . . . 77.5

HAIR. - Form: low waves. Texture: fine. Color: dark brown. Quantity: head, triple plus; beard, double plus; body, double plus.

EYe.-Color: green brown. Sclera: bloodshot. Iris: zoned.

Nose.-Profile: straight. Tip: thickness, plus; depressed.

TEETH. - Wear: plus. Caries: plus. Lost: 1 right lower molar.

EAR.-Lobe: free. Size: plus. Helix: plus. Darwin's Point: plus.

CHEST.-Development: double plus.

Musculature.-Excellent.

HEALTh.-Excellent.

No. 139. Plate XVIII, Figs. 3, 4

Residence: camp of Sheikh Miniehil. Date: June 4, 1928.

Age: 35; married for 16 years. Sons: 1 living, 2 dead. Daughters: 1 living, 1 dead. Brothers: 2 living, 1 dead. Sisters: 3 living, 1 dead.

Tattoo marks: 3 spots on back of right hand.

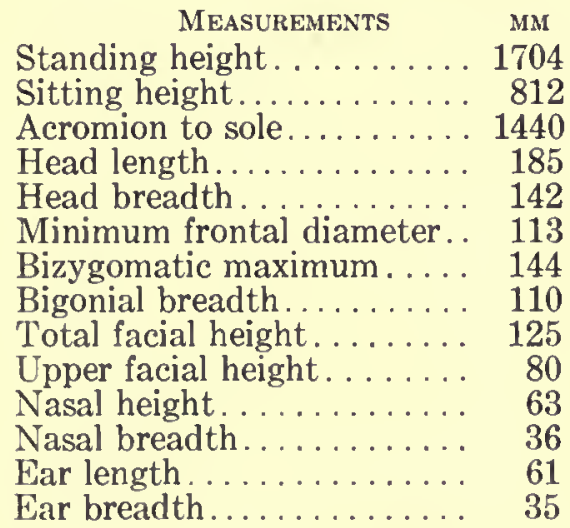

INDICES

Cephalic.............76.7

Relative sitting height..... 47.6

Fronto-parietal......... 72.5

Total facial............ 86.8

Upper facial. .......... 55.6

Nasal. .............. 57.2

Ear................ 57.4

Jugomandibular. . . . . . . 76.4

Jugofrontal . . . . . . . 78.5

Hair.-Form: low waves. Texture: medium. Quantity: head, minus; beard, double plus; body, plus.

EYE.-Color: dark brown. Sclera: bloodshot. Iris: zoned.

Nose.-Profile: concavo-convex. Tip: thickness, plus. Wings: medium.

TEETH.-Wear: double plus. Caries: triple plus. Lost: 2 left upper premolars, 1 right upper molar.

EAr.-Lobe: free. Size: plus. Helix: plus. Darwin's Point: plus.

CHest.-Development: double plus.

Musculature.-Excellent.

HEALTH.--Excellent.

Brother of No. 45.

No. 140. Plate XVIII, Figs. 5, 6

Residence: camp of Sheikh Miniehil. Date: June 4, 1928.

Age: 20 ; unmarried.

Brothers: 2 living. Sisters: 1 living.

Tattoo marks: dog on right inside forearm. 


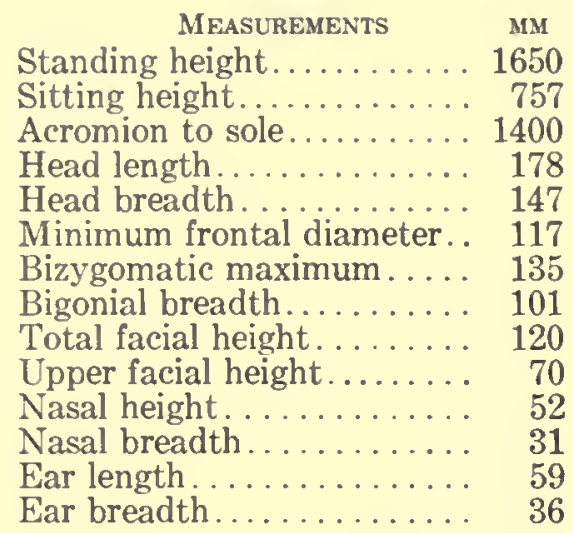

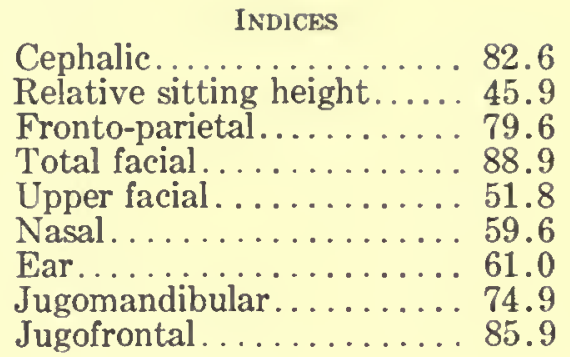

HAIR. - Form: low waves. Texture: fine. Color: dark brown.

EYE.-Color: dark brown. Sclera: clear. Iris: zoned.

Nose.-Profile: concave. Tip: thickness, average; horizontal.

EAR.-Lobe: free. Size: average. Helix: plus. Darwin's Point: plus.

CHEST.-Development: plus.

Musculature.-Excellent.

HEALTh.-Excellent.

No. 141. Plate XVIII, Figs. 7, 8

Residence: camp of Sheikh Miniehil. Date: June 4, 1928.

Age: 35 ; married for 15 years. Sons: 4 living, 2 dead. Daughters: 1 living, 1 dead. Brothers: 2 living. Sisters: 1 dead.

Tattoo marks: glove on back of right hand and gazelle on inside of right forearm.

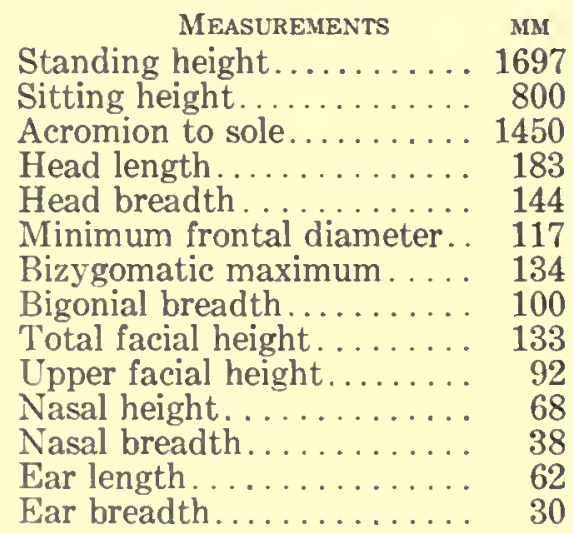

Cephalic................ 78.7

Relative sitting height..... 47.2

Fronto-parietal.......... 81.3

Total facial............ 99.3

Upper facial. . . . . . . . 68.6

Nasal. ............... 55.9

Ear................. 48.4

Jugomandibular......... 74.6

Jugofrontal. ........... 87.3

Halr.-Form: low waves. Texture: medium. Color: dark brown. Quantity: head, minus; beard, double plus; body, double plus.

EYE.-Color: dark brown. Sclera: clear. Iris: zoned. Slight cataract in left eye.

Nose.-Profile: straight. Tip: thickness, average; depressed.

TEETH.-Caries: plus. Lost: none.

EAR.-Lobe: attached. Size: minus. Helix: plus. Darwin's Point: triple plus.

Musculature.-Excellent.

HEALTh.-Excellent.

No. 142. Plate XIX, Figs. 1, 2

Residence: camp of Sheikh Miniehil. Date: June 4, 1928.

Age: 30 ; unmarried.

Brothers: 2 dead. Sisters: 1 living.

Tattoo marks: glove on back of right hand and gazelle on inside of right forearm. 


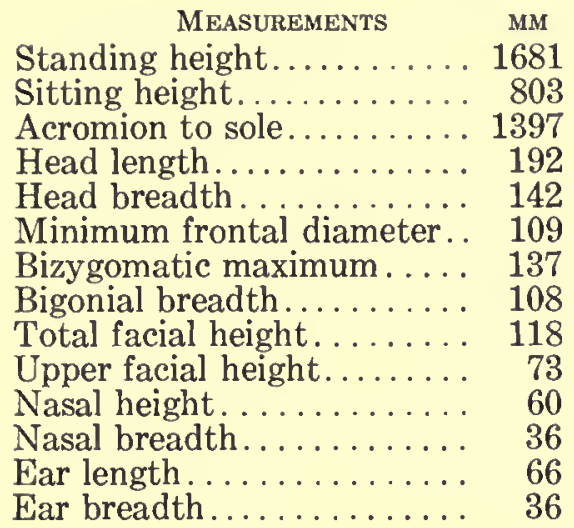

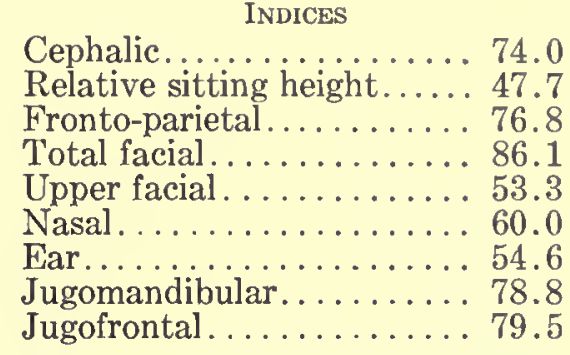

HAIR.-Form: curly frizzly. Texture: medium. Color: dark brown. Quantity: head, double plus; beard, minus; body, minus.

EYe.-Color: dark brown. Iris: zoned.

EYEBRow.-Thickness: double plus.

Nose.-Profile: straight. Tip: thickness, plus; depressed. Wings: medium.

TEETH.-Wear: average. Caries: average. Lost: none.

EAR.-Lobe: attached. Size: average. Helix: plus. Darwin's Point: double minus.

Chest.-Development: plus.

Musculature.-Good.

HEALTH.-Excellent.

No. 143. Plate XIX, Figs. 3, 4

Residence: camp of Sheikh Miniehil. Date: June 5, 1928.

Age: 55 ; married for 4 years. No children.

Brothers: 1 dead. Sisters: 2 living, 1 dead.

Tattoo marks: spot on left temporal.

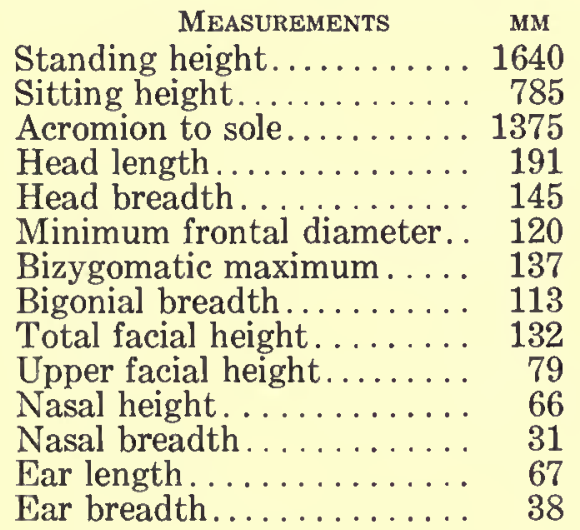

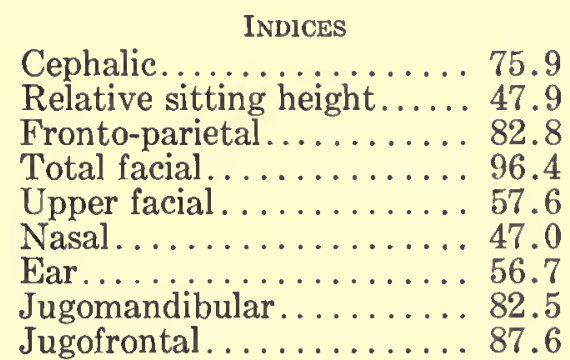

HAIR.-Color: dark brown and gray.

EYE.-Color: dark brown. Sclera: bloodshot. Iris: zoned.

Nose.-Profile: convex. Tip: thickness, plus; depressed. Wings: medium.

TeEтн.-Wear: plus. Caries: double plus. Lost: 1 left lower molar.

Chest.-Development: plus.

Musculature.-Good.

HEALTH.-Good.

\section{No. 144. Plate XIX, Figs. 5, 6}

Residence: camp of Sheikh Miniehil. Date: June 5, 1928.

Age: 50; married 4 times, the last time 4 years ago. Sons: 2 living, 1 dead. Daughters: 3 living, 1 dead. 
Brothers: 1 dead. Sisters: 1 living, 1 dead.

Tattoo marks: gazelle on inside of right forearm.

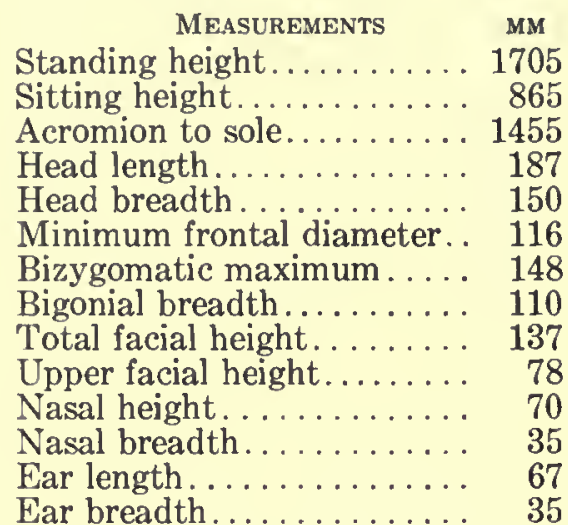

INDICES

Cephalic............. 80.2

Relative sitting height..... 50.7

Fronto-parietal......... 77.3

Total facial. . . . . . . . . . 92.6

Upper facial. . . . . . . . . 52.7

Nasal............... 50.0

Ear................. 52.3

Jugomandibular......... 74.3

Jugofrontal . . . . . . . . . . 78.4

HAIR.-Texture: medium. Color: dark brown. Quantity: head, minus; beard, double plus; body, double plus.

EYE.-Color: green brown. Sclera: bloodshot. Iris: zoned.

Nose.-Profile: convex. Tip: thickness, minus; depressed. Wings: compressed.

TEeth.-Wear: double plus. Caries: double plus. Lost: right lower canine.

EAR.-Lobe: free. Size: plus. Helix: plus. Darwin's Point: plus.

CHEst.-Development: double plus.

Musculature.--Excellent.

HEALTH.-Excellent.

No. 145. Plate XIX, Figs. 7, 8

Residence: camp of Sheikh Miniehil. Date: June 5, 1928.

Age: 50; married 3 times, all wives dead. Sons: 3 dead. Daughters: 2 living, 3 dead. Brothers: none. Sisters: 2 dead.

Tattoo marks: none.

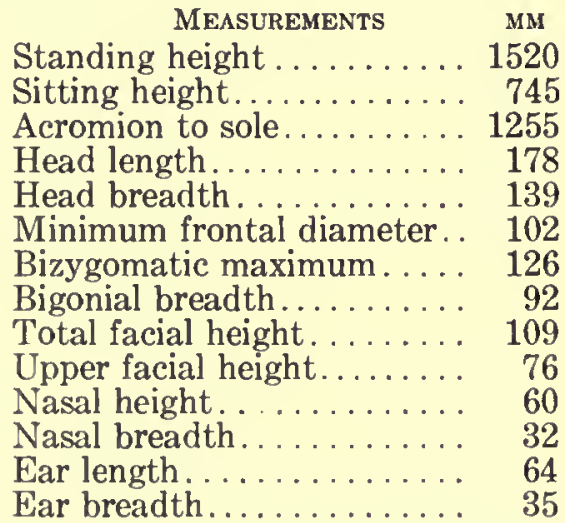

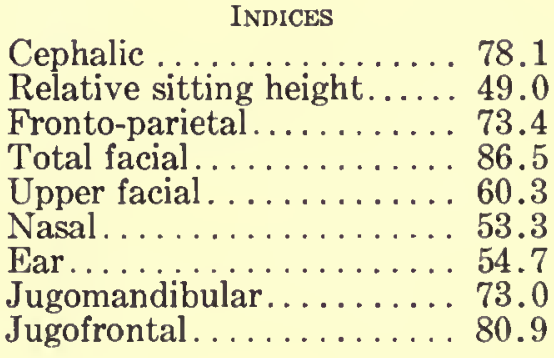

HaIr.-Form: low waves. Texture: medium. Color: dark brown. Quantity: head, minus; beard, double plus; body, minus.

EYE.-Color: green brown. Sclera: bloodshot. Iris: zoned.

NosE.-Profile: convex. Tip: thickness, double plus. Wings: medium-flaring.

TЕетн.-Wear: triple plus. Caries: triple plus. Lost: at least half.

EAR.-Lobe: free. Size: plus. Very protruding at top.

Chest.-Development: double minus.

Musculature.-Very poor.

HEALTH.-Good but looks weak. 
No. 146. Plate XX, Figs. 1, 2

Residence: camp of Sheikh Miniehil. Date: June 5, 1928.

Age: 25; married for 18 months. Sons: 1 living. Daughters: none.

Brothers: 2 living. Sisters: 2 living.

Tattoo marks: gazelle on inside of right wrist and 3 spots on each temporal.

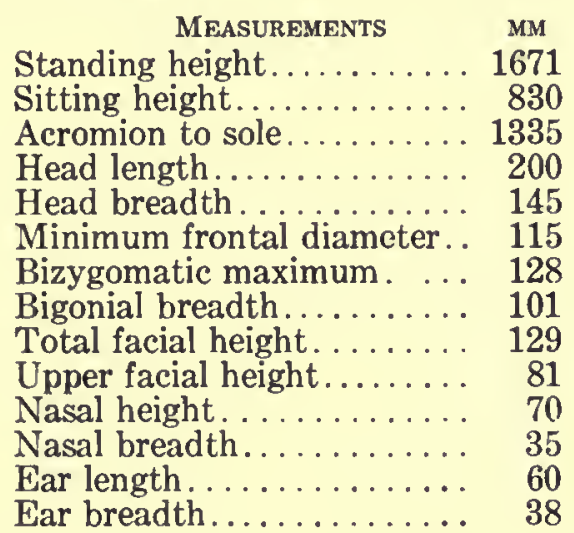

INDICES

Cephalic............. 72.5

Relative sitting height...... 49.7

Fronto-parietal.......... 79.4

Total facial .............. 100.8

Upper facial ..........6. 63.3

Nasal. . . . . . . . . . . 50.0

Ear................6. 63.4

Jugomandibular......... 78.9

Jugofrontal. . . . . . . . . . 89.8

HaIR.-Form: low waves. Texture: medium. Color: dark brown. Quantity: head, double plus; beard, minus; body, double plus.

EYE.-Color: dark brown. Sclera: bloodshot. Iris: homogeneous.

EYEBRow.-Thickness: double plus.

NosE.-Profile: straight. Wings: medium.

EAR.-Lobe: free. Size: plus. Helix: plus. Darwin's Point: double plus.

Chest.-Development: double plus.

Musculature.-Good.

HEALTH.-Excellent.

No. 147. Plate XX, Figs. 3,4

Residence: camp of Sheikh Miniehil. Date: June 5, 1928.

Age: 40; married for 20 years (wife now dead). Sons: 3 living, 2 dead. Daughters: 2 living. Brothers: 3 dead. Sisters: 2 living, 1 dead.

Tattoo marks: gazelle on right forearm.

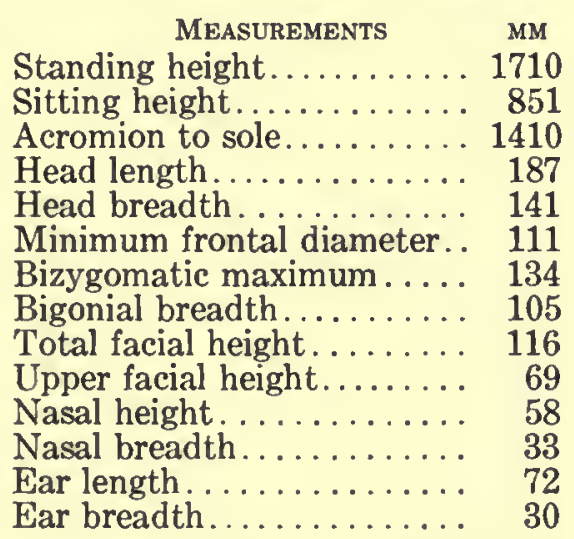

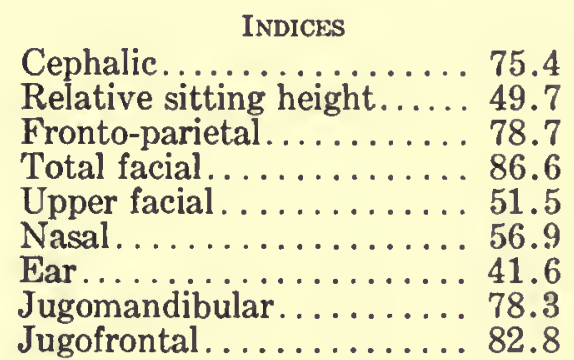

HaIR.-Texture: medium. Color: dark brown. Quantity: head, minus; beard, plus; body, plus. EYE.-Color: dark brown. Iris: zoned.

Nose.-Profile: straight. Tip: thickness, plus. Wings: medium.

TEEтH.-Wear: plus. Caries: double plus. Lost: 1 upper molar left and 1 right.

EAR.-Lobe: free. Helix: plus. Darwin's Point: double plus. 
CHest.-Development: plus.

Musculature.-Good.

HEALTH.-Good except for fever attacks.

No. 148

Residence: camp of Sheikh Miniehil. Date: June 5, 1928.

Age: 40 ; married for 20 years. Sons: 3 living, 1 dead. Daughters: 1 living.

Brothers: none. Sisters: none.

Tattoo marks: spots on back of left wrist.

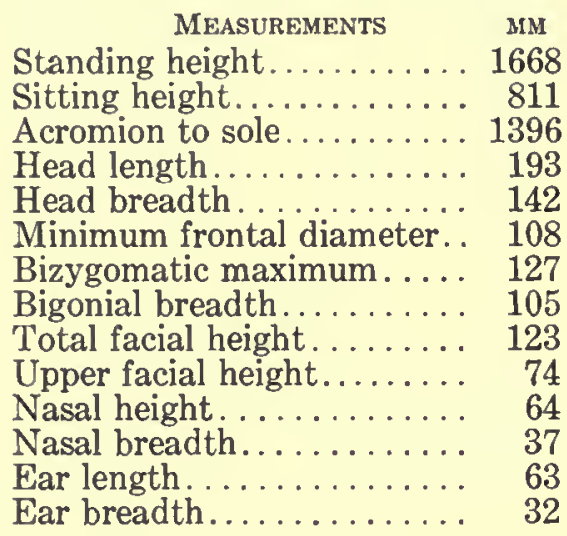

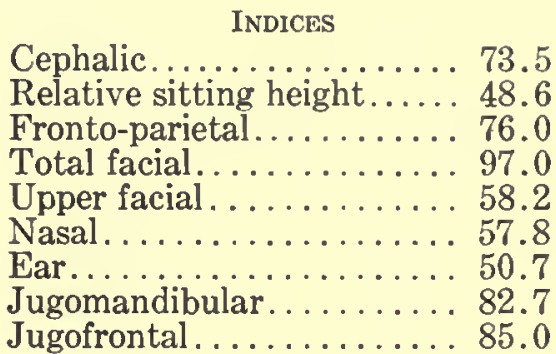

Relative sitting height....... 48.6

Fronto-parietal.........76.0

Total facial $\ldots . .97 .0$

Upper facial . . . . . . . . . . 58.2

.. 57.8

Jugomandibular......... 82.7

Jugofrontal. ............ 85.0

HAIR. - Form: low waves. Color: light brown. Quantity: head, double plus; beard, plus; body, plus.

EYE.-Color: green brown. Sclera: bloodshot. Iris: zoned.

NosE.-Profile: straight. Tip: depressed. Wings: medium.

TеЕтн.-Caries: double plus. Lost: upper left canine.

EAR.-Helix: plus. Darwin's Point: plus. Marked protrusion.

CHEST.-Development: double plus.

Musculature.-Excellent.

HeALTH.-Excellent.

\section{No. 149}

Residence: camp of Sheikh Miniehil. Date: June 5, 1928.

Age: 25; unmarried.

Brothers: 1 living. Sisters: 3 living.

Tattoo marks: gazelle on inside of right forearm, spots on back of left hand.

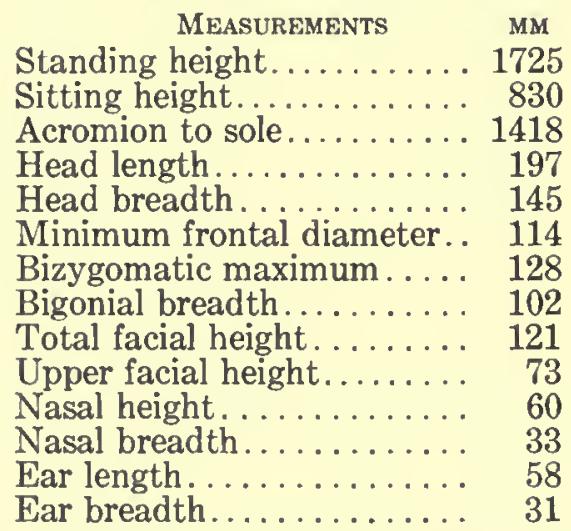

Cephalic.............. 73.6

Relative sitting height..... 48.1

Fronto-parietal.......... 78.7

Total facial............ 93.8

Upper facial. . . . . . . . . 57.1

Nasal............... 55.0

Ear................. 53.4

Jugomandibular......... 79.7

Jugofrontal............. 89.1

HAIR.-Form: low waves. Texture: medium. Color: dark brown. Quantity: head, double plus; beard, plus; body, plus. 
EYE.-Color: dark brown. Sclera: clear. Iris: zoned.

NosE.-Profile: convex. Tip: thickness, plus; elevated.

CHest.-Development: plus.

MUSCUlature.-Good.

HEALTH.-Good.

Lost part of right hallux in railway accident at Tell Inghara.

No. 152. Plate XX, Figs. 5, 6

Residence: camp of Sheikh Miniehil. Date: June 5, 1928.

Age: 45; married for 25 years. Sons: 3 living. Daughters: 2 dead.

Brothers: 1 living. Sisters: 3 living.

Tattoo marks: on back of left hand, spot on right upper lip, and spots on back of right hand.

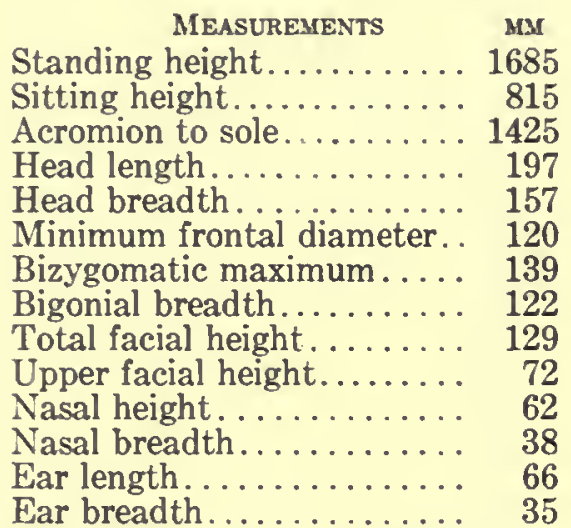

Cephalic...............79.7

Relative sitting height...... 48.4

Fronto-parietal.......... 76.0

Total facial................ 92.8

Upper facial. ........... 51.7

Nasal. ..............6. 61.3

Ear................ 53.0

Jugomandibular.........88 87.7

Jugofrontal. .......... 86.3

HaIR.-Form: low waves. Texture: medium. Color: dark brown. Quantity: head, minus; beard, double plus; body, plus.

EyE.-Color: gray brown. Sclera: bloodshot. Iris: zoned.

Nose.-Profile: straight. Tip: thickness, plus. Wings: medium.

CHEST.-Development: plus.

Musculature.-Excellent.

HEALTH.-Excellent.

No. 153. Plate XX, Figs. 7, 8

Residence: camp of Sheikh Abied. Date: June 5, 1928.

Age: 55; married 3 times, one wife now dead. Sons: 2 living, 2 dead. Daughters: 2 living, 2 dead.

Brothers: 1 living. Sisters: 1 living, 1 dead.

Tattoo marks: gazelle on inside of right wrist.

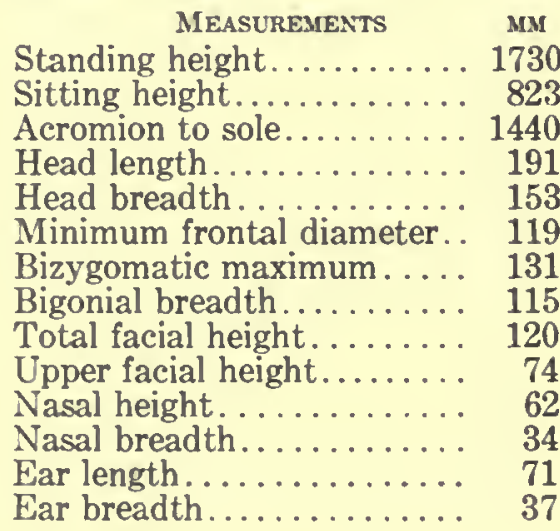

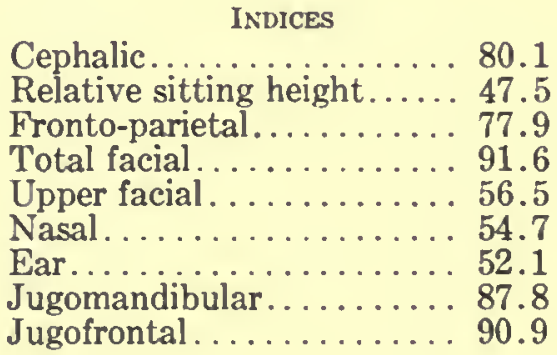


HAIR. - Color: dark brown and gray. Quantity: head, shaved; beard, double plus; body, plus. EYE.-Color: green brown. Sclera: speckled. Iris: zoned.

Nose.-Profile: convex. Tip: thickness, plus; depressed. Wings: medium.

TeETH.-Wear: triple plus. Caries: none. Lost: none.

CHEST.-Development: plus.

Musculature.-Excellent.

HeAlth.-Excellent except for fever attacks.

No. 154. Plate XXI, Figs. 1, 2

Residence: camp of Sheikh Abied. Date: June 5, 1928.

Age: 55; married for 2 years. Sons: 1 living. Daughters: none.

Brothers: 3 living. Sisters: none.

Tattoo marks: none.

\begin{tabular}{|c|c|}
\hline MEASUREMENTS & MM \\
\hline Standing height. & 1764 \\
\hline Sitting height. & 830 \\
\hline Acromion to sole. & 1505 \\
\hline Head length. & 193 \\
\hline Head breadth & 138 \\
\hline Minimum frontal diameter.. & 114 \\
\hline Bizygomatic maximum . . . . . & 133 \\
\hline Bigonial breadth. & 102 \\
\hline Total facial height. & 132 \\
\hline Upper facial height. & 77 \\
\hline Nasal height & 62 \\
\hline Nasal breadth & 35 \\
\hline Ear length & 70 \\
\hline$n$ hmodtl & 37 \\
\hline
\end{tabular}

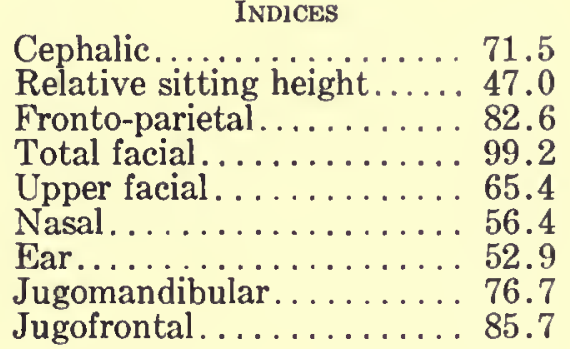

HAIR.-Texture: medium. Color: dark brown. Quantity: head, minus; beard, plus; body, minus.

Eye.-Color: dark brown. Sclera: clear. Iris: zoned.

Nose.-Profile: straight. Tip: thickness, plus; depressed. Wings: medium.

TEETH.-Lost: 1 lower molar left and 1 right.

CHest.-Development: plus.

Musculature.-Excellent.

HEALTH.-Excellent.

No. 155

Residence: camp of Sheikh Abied. Date: June 5, 1928.

Age: 20 ; unmarried.

Brothers: 2 dead. Sisters: 1 living.

Tattoo marks: none.

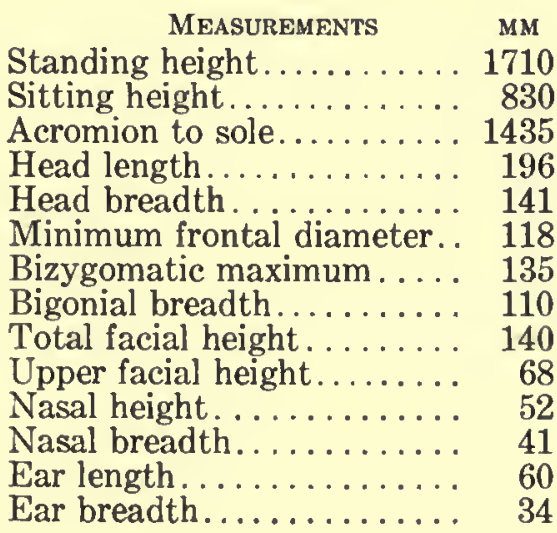

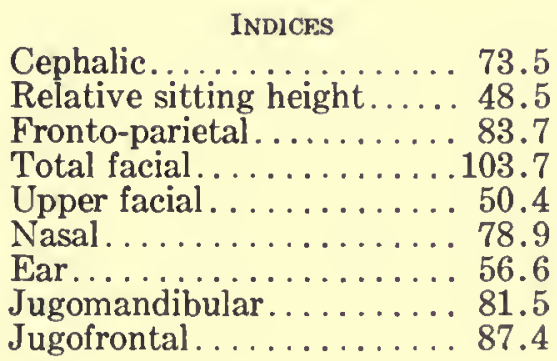


HAIR.-Form: low waves. Texture: fine. Quantity: head, plus; beard, minus; body, average. EYE.-Color: dark brown. Sclera: clear. Iris: zoned.

Nose.-Profile: convex. Tip: thickness, average; depressed.

Chest.-Development: plus.

Musculature.-Fair.

HEALTH.-Fair but frequent stomach trouble.

No. 156

Residence: camp of Sheikh Abied. Date: June 5, 1928.

Age: 30; unmarried.

Brothers: none. Sisters: 1 living.

Tattoo marks: none.

\begin{tabular}{|c|c|}
\hline MEASUREMENTS & MM \\
\hline Standing height. & 1702 \\
\hline Sitting height. & 740 \\
\hline Acromion to sole. & 1410 \\
\hline Head length..... & 191 \\
\hline Head breadth. & 144 \\
\hline Minimum frontal diameter. & 113 \\
\hline Bizygomatic maximum.... & 133 \\
\hline Bigonial breadth.......... & 103 \\
\hline Total facial height........ & 123 \\
\hline Upper facial height........ & 72 \\
\hline Nasal height. & 56 \\
\hline$\ldots \ldots \ldots$ & 35 \\
\hline & 59 \\
\hline & \\
\hline
\end{tabular}

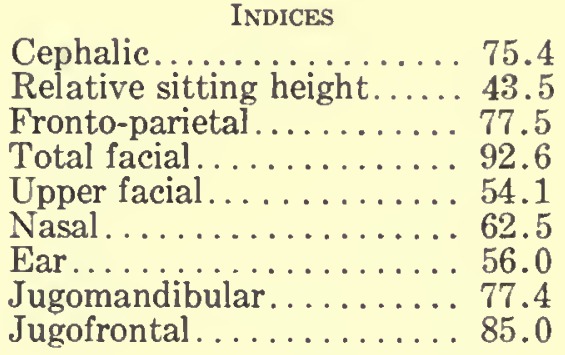

Cephalic................ 75.4

Relative sitting height..... 43.5

Relative sitting height..... 73.5

Upper facial.........

Nasal. ..............62.5

Jugomandibular............ 77.4

Jugofrontal. ........... 85.0

HalR. - Form: low waves. Texture: medium. Color: dark brown. Quantity: head, double plus; beard, minus; body, average.

EYE.-Color: dark brown. Sclera: bloodshot. Iris: zoned.

Nose.-Profile: concavo-convex. Wings: medium.

TeEth.-Wear: plus. Caries: none. Lost: none.

Chest.-Development: plus.

Musculature.-Excellent.

HEALTH._Excellent.

\section{No. 157. Plate XXI, Figs. 3, 4}

Residence: camp of Sheikh Abied. Date: June 5, 1928.

Age: 35; married for 15 years. Sons: 2 living. Daughters: 1 living.

Brothers: 1 dead. Sisters: 1 living.

Tattoo marks: gazelle on inside of right forearm.

\begin{tabular}{|c|c|}
\hline \multirow{3}{*}{\multicolumn{2}{|c|}{ MEasurements }} \\
\hline & \\
\hline & \\
\hline & \\
\hline & \\
\hline & \\
\hline & \\
\hline & \\
\hline & \\
\hline & \\
\hline & \\
\hline & \\
\hline & \\
\hline & \\
\hline & \\
\hline & \\
\hline & \\
\hline Standing height. & 1670 \\
\hline Sitting height. & 740 \\
\hline Acromion to sole & 1382 \\
\hline Head length & 196 \\
\hline Head breadth. & 151 \\
\hline Minimum frontal diameter. & 115 \\
\hline Bizygomatic maximum..... & 130 \\
\hline Bigonial breadth. . & 100 \\
\hline Total facial height. & 117 \\
\hline Upper facial height. & 64 \\
\hline Nasal height & 56 \\
\hline Nasal breadth. & 40 \\
\hline Ear length. & 65 \\
\hline $\mathrm{r}$ br & 36 \\
\hline
\end{tabular}

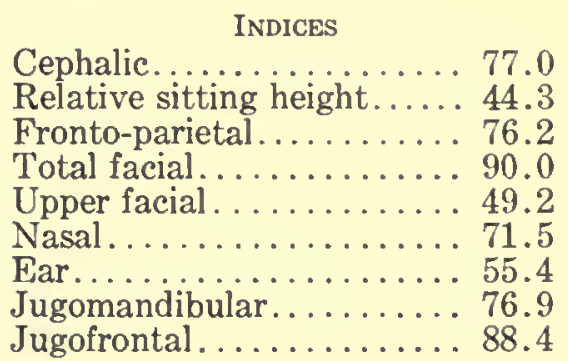


HaIr.-Form: low waves. Texture: medium. Color: dark brown. Quantity: head, plus; beard, plus; body, plus.

EYE.-Color: green brown. Sclera: bloodshot. Iris: zoned.

Nose.-Profile: convex. Tip: thickness, plus; depressed. Wings: medium.

TeEth.-Wear: double plus. Caries: plus. Lost: 1 left lower premolar.

CHEST.-Development: plus.

Musculature.-Excellent.

HEALTH.-Excellent.

No. 158. Plate XXI, Figs. 5, 6

Residence: camp of Sheikh Abied. Date: June 5, 1928.

Age: 40 ; married for 20 years (wife now dead). Sons: 1 living, 3 dead. Daughters: 2 living, 1 dead.

Brothers: 2 living, 3 dead. Sisters: 4 living.

Tattoo marks: none.

\begin{tabular}{|c|c|}
\hline MEASUREMENTS & \\
\hline Standing height & \\
\hline Sitting height. & \\
\hline Acromion to sole & \\
\hline Head length & \\
\hline Head breadth. & \\
\hline Minimum frontal diameter. & \\
\hline Bizygomatic maximum... & \\
\hline Bigonial breadth. & \\
\hline Total facial height & \\
\hline Upper facial height. & \\
\hline Nasal height & \\
\hline Nasal breadth & \\
\hline $\begin{array}{l}\text { Ear length } \\
\text { Ear breadtl }\end{array}$ & \\
\hline & \\
\hline
\end{tabular}

Cephalic............... 77.2

Relative sitting height..... 50.3

Fronto-parietal........... 79.7

Total facial............. 79.7

Upper facial . . . . . . . . . 53.6

Nasal................ 69.0

Ear.................. 59.7

Jugomandibular......... 79.7

Jugofrontal ............ 79.7

HAIR.-Texture: medium. Color: dark brown. Quantity: head, plus; beard, double plus.

EYE.-Color: gray brown. Sclera: bloodshot. Iris: zoned.

Nose.-Profile: concavo-convex. Wings: medium.

TEетн.-Wear: double plus. Caries: triple plus. Lost: 3 left lower molars, 2 right lower molars.

CHEST.-Development: plus.

Musculature.-Good.

HEALTH.-Excellent.

No. 159. Plate XXI, Figs. 7, 8

Residence: camp of Sheikh Abied. Date: June 5, 1928.

Age: 50; married for 14 years. Sons: 1 living. Daughters: 1 living, 2 dead.

Brothers: 2 living. Sisters: none.

Tattoo marks: glove on back of right hand, and gazelle on inside of right forearm.

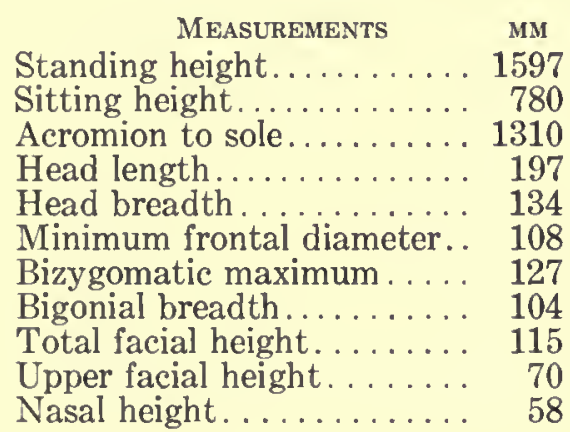

Cephalic..............6 68.0

Relative sitting height..... 48.9

Fronto-parietal......... 80.6

Total facial............ 90.6

Upper facial. . . . . . . . 55.2

Nasal $\ldots \ldots \ldots \ldots \ldots \ldots \ldots . .58 .6$

Ear................. 57.2

Jugomandibular........ 81.9

Jugofrontal. ............ 85.0 


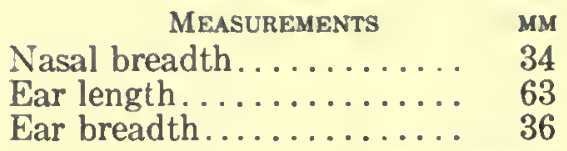

HAIR.-Texture: medium. Color: dark brown. Quantity: head, minus; beard, plus; body, plus.

EYE.-Color: dark brown. Sclera: bloodshot. Iris: zoned.

NosE.-Profile: straight. Tip: thickness, plus; elevated. Wings: medium.

Chest.-Development: plus.

Musculature.---Excellent.

Health.-Excellent.

No. 160. Plate XXII, Figs. 1, 2

Residence: camp of Sheikh Abied. Date: June 5, 1928.

Age: 20 ; unmarried.

Brothers: 4 living. Sisters: 3 living.

Tattoo marks: spot on inside of right wrist.

\begin{tabular}{|c|c|c|}
\hline MEASUREMENTS & MM & INDICES \\
\hline Standing height. . & 1635 & Cephalic. . \\
\hline Sitting height. & 800 & Relative sitting height..... 49.0 \\
\hline Acromion to sole. & 1360 & Fronto-parietal........ \\
\hline Head length..... & 191 & Total facial... \\
\hline Head breadth. . & 135 & Upper facial \\
\hline Minimum frontal diameter. . & 113 & Nasal... \\
\hline Bizygomatic maximum.... & 133 & 60.0 \\
\hline Bigonial breadth.......... & 97 & Jugomandibular..... \\
\hline Total facial height........ & 106 & Jugofrontal. . . . . . . . . \\
\hline Upper facial height........ & 63 & \\
\hline Nasal height............ & 49 & \\
\hline Nasal breadth. & 32 & \\
\hline Ear length. . & 60 & \\
\hline Ear breadth.... & 36 & \\
\hline
\end{tabular}

HAIR.-Form: low waves. Texture: medium. Color: dark brown. Quantity: head, double plus; beard, plus; body, plus.

Ere.-Color: dark brown. Sclera: bloodshot. Iris: zoned.

Nose.-Profile: straight. Tip: elevated.

TEETH.-Lost: 1 right upper molar.

CHEST.-Development: plus.

Musculature.-Good.

HEALTH.-Good.

No. 161. Plate XXII, Figs. 3, 4

Residence: camp of Sheikh Abied. Date: June 5, 1928.

Age: 30 ; married for 10 years. Sons: 2 living. Daughters: 2 living.

Brothers: none. Sisters: none.

Tattoo marks: glove on back of right hand and gazelle on inside of right forearm.

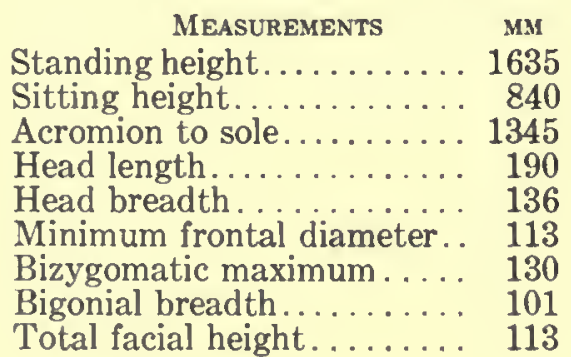

Cephalic............ 71.6

Relative sitting height..... 51.4

Fronto-parietal......... 83.1

Total facial............ 87.0

Upper facial. . . . . . . . 58.4

Nasal. ................. 64.4

Ear................. 55.2

Jugomandibular. . . . . . . 77.7

Jugofrontal. . . . . . . . . . 86.9 


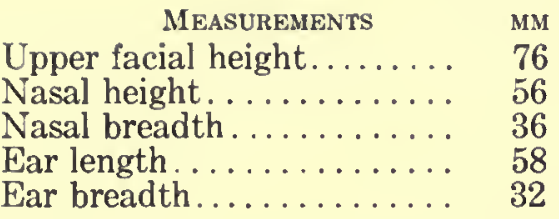

HaIR.-Form: low waves. Texture: medium. Color: dark brown. Quantity: head, double plus; beard, plus; body, plus.

EYe.-Color: dark brown. Sclera: bloodshot. Iris: zoned.

Nose.-Profile: convex. Tip: elevated.

TEeTH.-Lost: 1 right upper molar.

CHEST.-Development: plus.

Musculature.-Good.

HEALTH.-Good.

No. 162. Plate XXII, Figs. 5, 6

Residence: camp of Sheikh Abied. Date: June 5, 1928.

Age: 20; unmarried.

Brothers: 1 living. Sisters: 1 living.

Tattoo marks: glove and gazelle on right hand and forearm and four spots on each temporal.

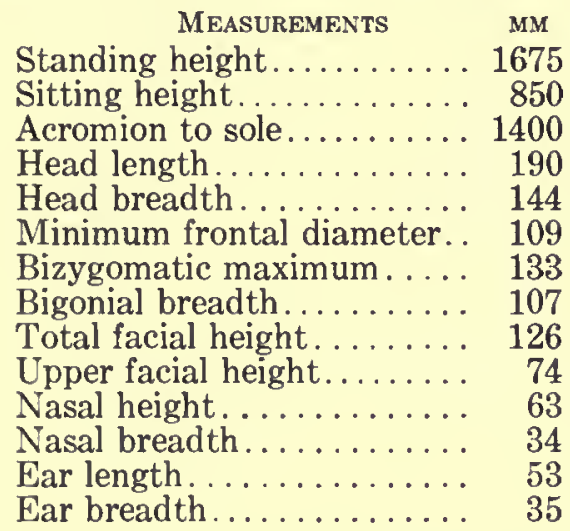

HaIR.-Form: low waves. Texture: fine. Color: dark brown.

EYE.-Color: dark brown. Sclera: clear. Iris: zoned.

Nose.-Profile: straight. Wings: medium-compressed.

Chest.-Development: plus.

Musculature.-Good.

HEalTH.-Good.

No. 163. Plate XXII, Figs. 7, 8

Residence: camp of Sheikh Abied. Date: June 5, 1928.

Age: 20; unmarried.

Brothers: 1 living. Sisters: 1 living.

Tattoo marks: spots in cluster on back of right hand.

\begin{tabular}{|c|c|c|}
\hline MEASUREMENTS & MM & INDICES \\
\hline Standing height... & 1655 & Cephalic. \\
\hline Sitting height. . & 785 & Relative sitting height. \\
\hline Acromion to sole. & 1400 & Fronto-parietal. \\
\hline Head length. & 194 & Total facial.... \\
\hline Head breadth & 141 & Upper facial. \\
\hline Minimum frontal diameter. & 115 & Nasal. \\
\hline Bizygomatic maximum.... & 125 & Ear. \\
\hline Bigonial breadth....... & 90 & Jugomand \\
\hline
\end{tabular}




\begin{tabular}{|c|c|c|}
\hline MEASUREMENTS & MM & INDICES \\
\hline Total facial height.... & 122 & Jugofrontal. ........... 92.0 \\
\hline $\begin{array}{l}\text { Upper facial height. } \\
\text { Nasal height....... }\end{array}$ & & \\
\hline Nasal breadth. & 36 & \\
\hline Ear length.... & 63 & \\
\hline Ear breadth. & 34 & \\
\hline
\end{tabular}

HAIR.-Texture: coarse. Color: dark brown. Quantity: head, double plus; beard, minus; body, plus.

EYE.-Color: dark brown. Sclera: clear. Iris: zoned.

NosE.-Profile: concavo-convex. Tip: thickness, plus; depressed. Wings: flaring.

TEETH.-Lost: none.

Chest.-Development: plus.

Musculature.-Excellent.

HEALTH.-Excellent.

No. 164. Plate XXIII, Figs. 1, 2

Residence: camp of Sheikh Abied. Date: June 5, 1928.

Age: 40 ; married twice, 20 years ago and 1 year ago. Sons: 2 living. Daughters: 3 living. Brothers: 1 living. Sisters: 2 living.

Tattoo marks: glove and gazelle on right hand and arm, spot on right side of upper lip.

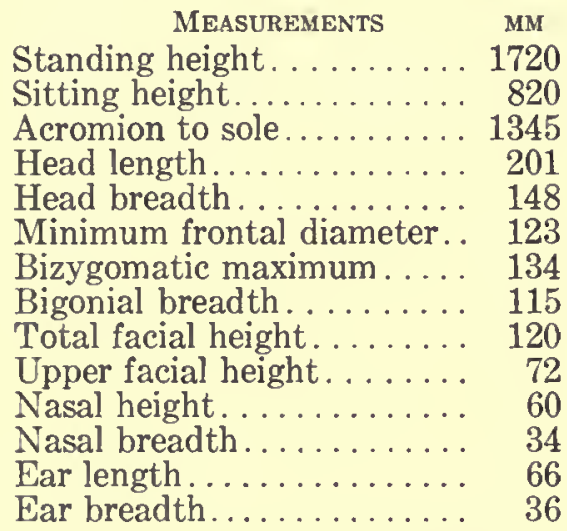

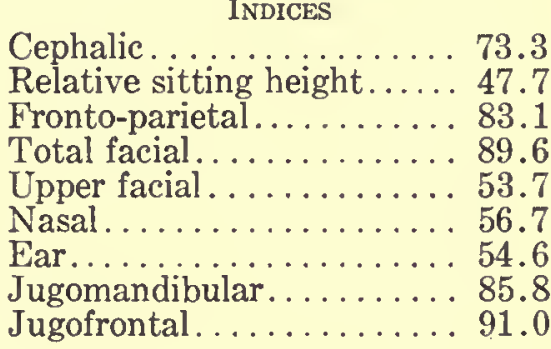

HaIr.-Form: low waves. Texture: medium. Color: gray. Quantity: head, plus; beard, plus; body, plus.

EYE.-Color: green brown. Sclera: clear. Iris: zoned.

NosE.-Profile: concavo-convex. Tip: thickness, double plus.

TEETH.-Wear: double plus. Caries: double plus. Lost: 1 left lower premolar.

CHEST.-Development: plus.

Musculature.--Excellent.

HEALTH.-Excellent.

No. 165

Residence: camp of Sheikh Abied. Date: June 5, 1928.

Age: 45; married for 25 years. Sons: 1 living. Daughters: 4 living.

Brothers: 1 living. Sisters: 2 living.

Tattoo marks: none.

MEASUREMENTS MM

Standing height. ........ 1744

Sitting height.......... 740

Acromion to sole.......... 1425

Head length........... 186

Head breadth........... 156
INDICES

Cephalic............. 83.9

Relative sitting height..... 42.4

Fronto-parietal ............ 77.6

Total facial........... 87.5

Upper facial. , . . . . . . 52.1 


\begin{tabular}{|c|c|}
\hline MEASUREMENTS & MM \\
\hline Minimum frontal diameter. & 12 \\
\hline Bizygomatic maximum.... & 14 \\
\hline Bigonial breadth. & 11 \\
\hline Total facial height. & 12 \\
\hline Upper facial height. . . & 7 \\
\hline Nasal height. . . . . . . . . . & \\
\hline Nasal breadth........... & \\
\hline Ear length & \\
\hline ar breadt & \\
\hline
\end{tabular}

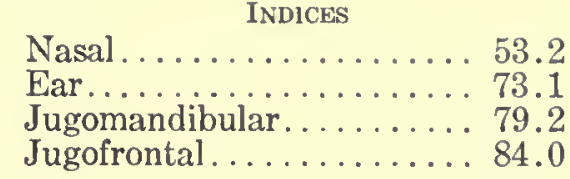

HAIR. - Form: low waves. Color: dark brown. Quantity: head, plus.

EYE.-Color: dark brown. Sclera: bloodshot. Iris: zoned.

NosE.-Profile: straight. Wings: medium.

TEETH.-Lost: 1 right upper premolar.

ChesT.-Development: plus.

Musculature.-Excellent.

HEALTH.—Excellent.

No. 166

Residence: camp of Sheikh Abied. Date: June 5, 1928.

Age: 30 ; married twice, 12 years ago and 8 years ago. Sons: 2,1 , living; 1,0 , dead. Daughters: 1,1 , living.

Brothers: 2 living. Sisters: 1 dead.

Tattoo marks: cross on right wrist following wrist injury.

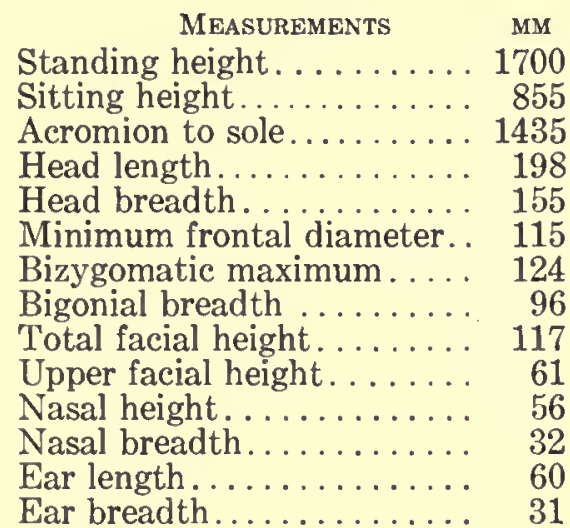

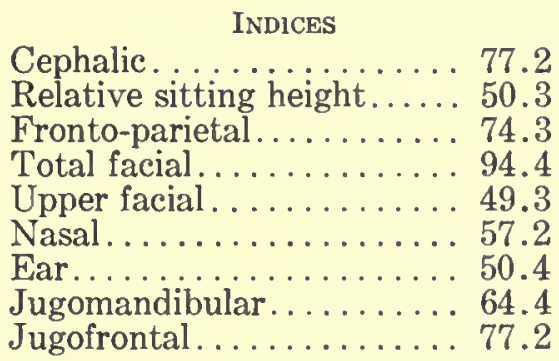

HaIr.-Form: low waves. Color: dark brown. Quantity: head, plus; beard, minus; body, average.

EYE.-Color: dark brown. Sclera: bloodshot. Iris: zoned.

NosE.-Profile: straight. Wings: medium.

TеEтн.-Lost: 2 upper and 2 lower molars.

CHEST.-Development: plus.

MUSCUlatURE.-Good.

HEALTH.-Excellent.

Son of Sheikh Abied.

No. 167

Residence: camp of Sheikh Abied. Date: June 5, 1928.

Age: 55; married for 20 years. Sons: 3 living, 2 dead. Daughters: 4 living.

Brothers: 1 dead. Sisters: 1 living.

Tattoo marks: none.

Measurements MM

Standing height. . . . . . . 1640

Sitting height............ 770
INDICES

Cephalic............... 71.7

Relative sitting height..... 46.9 


\begin{tabular}{|c|c|c|}
\hline Measurements & MM & INDICES \\
\hline Acromion to sole. & 1390 & Fronto-parietal. \\
\hline Head length..... & 198 & Total facial. . \\
\hline Head breadth. & 142 & Upper facial. \\
\hline Minimum frontal diameter. . & 115 & Nasal. \\
\hline Bizygomatic maximum..... & 140 & Ear. \\
\hline Bigonial breadth......... & 115 & Jugomandibular. \\
\hline Total facial height. & 139 & Jugofrontal. \\
\hline Upper facial height. . & 91 & \\
\hline Nasal height.. & 60 & \\
\hline Nasal breadth. & 36 & \\
\hline length & 58 & \\
\hline orea & 37 & \\
\hline
\end{tabular}

HAIR.-Form: low waves. Color: dark brown and gray. Quantity: head, plus; beard, double plus; body, plus.

Eye.-Color: dark brown. Sclera: clear. Iris: zoned.

Nose.-Profile: straight. Tip: thickness, doub!e plus; horizontal.

TEETH.-Wear: triple plus. Caries: triple plus. Lost: 6.

Chest.-Development: average.

Musculature.-Poor.

HEALTH.-Fair now, formerly often fevered and sick.

No. 168

Residence: camp of Sheikh Miniehil.

Age: 23 ; unmarried.

Brothers: 3 living. Sisters: 3 living.

Tattoo marks: glove on right hand and gazelle on outside of right wrist.

\begin{tabular}{|c|c|c|}
\hline MEASUREMENTS & мм & INDICES \\
\hline Standing height. ...... & 1620 & Cephalic. \\
\hline Sitting height.. & 730 & Relative sitting height \\
\hline Acromion to sole. & 1340 & Fronto-parietal....... \\
\hline Head length. & 192 & Total facial \\
\hline Head breadth. & 149 & Upper facial \\
\hline Minimum frontal diameter. & 117 & Nasal. \\
\hline Bizygomatic maximum. & 135 & Ear \\
\hline Bigonial breadth. & 105 & Jugomandibular \\
\hline Total facial height. & 120 & Jugofrontal. . . . . \\
\hline
\end{tabular}

.

Naser height height........ 67

Nasal breadth............... 38

Ear length................ 64

Ear breadth............... 37

HAIR. - Form: low waves. Texture: medium. Color: dark brown.

EYE.-Color: dark brown. Sclera: clear. Iris: zoned.

Nose.-Profile: concavo-convex. Tip: thickness, plus.

Chest.-Development: plus.

Musculature.-Excellent.

HEALTH.-Excellent.

No. 170. Plate XXIII, Figs. 5, 6

Residence: camp of Sheikh Eliowie. Date: June 5, 1928.

Age: 20; unmarried.

Brothers: 3 living. Sisters: none.

Tattoo marks: 3 spots on inside of right forearm.

\begin{tabular}{|c|c|}
\hline MEASUREMENTS & INDICES \\
\hline $\begin{array}{l}\text { Standing height. } \\
\text { Sitting height. }\end{array}$ & Cephalic....... \\
\hline
\end{tabular}




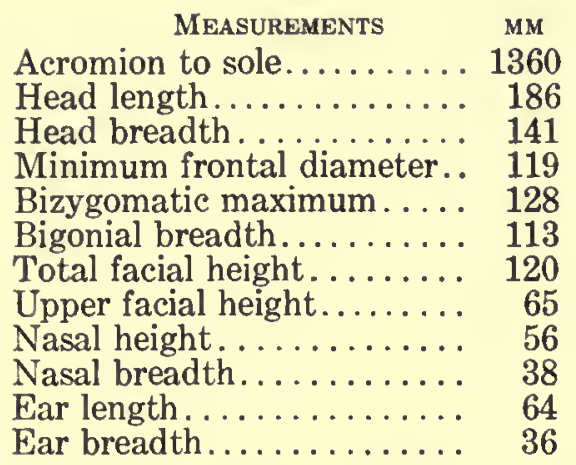

Fronto-parietal.......... 84.4

Total facial. ............ 93.8

Upper facial . . ........ 58.6

Nasal. ..............6. 67.9

Ear............... 56.3

Jugomandibular......... 88.3

Jugofrontal. .......... 93.0

HaIr.-Form: low waves. Texture: fine. Color: dark brown. Quantity: head, plus; beard, minus; body, plus.

EyE.-Color: dark brown. Sclera: clear. Iris: zoned.

Nose.-Profile: concavo-convex.

Chest.-Development: plus.

Musculature.-Excellent.

HEALTH.-Good.

No. 171. Plate XXIII, Figs. 7, 8

Residence: camp of Sheikh Eliowie. Date: June 5, 1928.

Age: 25; unmarried.

Brothers: 3 living. Sisters: 3 living.

Tattoo marks: design on right inside forearm.

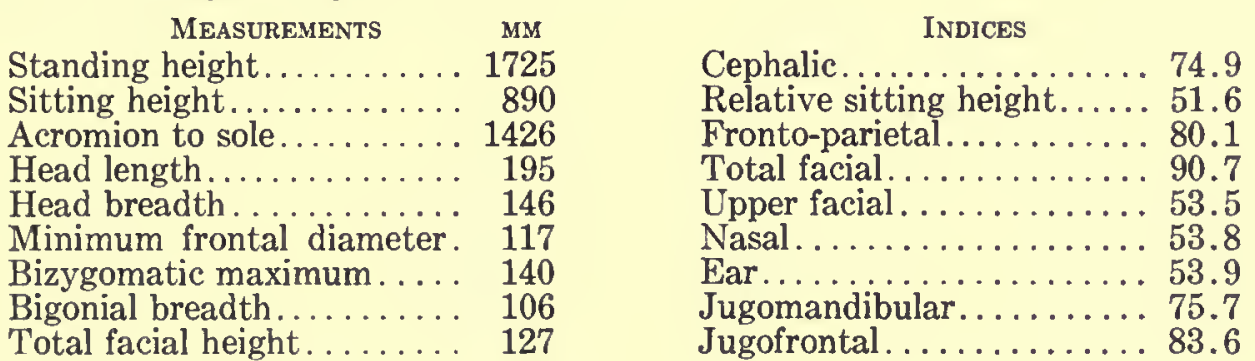

Upper facial height ......... 75

Nasal height. .......... 67

Nasal breadth........... 36

Ear length.............. 65

Ear breadth........... 35

HaIR.-Form: low waves. Texture: medium. Color: dark brown. Quantity: head, plus; beard, minus; body, minus.

EYE.-Color: dark brown. Sclera: bloodshot. Iris: zoned.

NosE.-Profile: convex. Wings: medium.

SCAPULAE.-Vertebral borders: straight.

CHEST.-Development: plus.

Musculature.-Excellent.

HEALTH.-Good.

No. 172. Plate XXIV, Figs. 1, 2

Residence: camp of Sheikh Eliowie. Date: June 5, 1928.

Age: 20 ; unmarried.

Brothers: 1 living. Sisters: 1 living.

Tattoo marks: on back of right hand.

MEASUREMENTS MM

Standing height......... 1700

Sitting height........... 845
INDICES

Cephalic..............74.9

Relative sitting height...... 49.7 


\begin{tabular}{|c|c|c|}
\hline MEASUREMENTS & мM & INDICES \\
\hline Acromion to sole...... & 1395 & Fronto-parietal..... \\
\hline Head length. & 179 & Total facial \\
\hline Head breadth. & 134 & Upper facial \\
\hline Minimum frontal diameter & 98 & Nasal. \\
\hline Bizygomatic maximum. & 124 & Ear. \\
\hline Bigonial breadth.. & 97 & Jugomandibular \\
\hline Total facial height & 106 & Jugofrontal..... \\
\hline
\end{tabular}

Upper facial height........ 67

Nasal height............. 48

Nasal breadth.......... 33

Ear length.............. 55

Ear breadth............. 33

HAIR.-Form: low waves. Texture: coarse. Color: reddish brown.

EYE.-Color: dark brown. Sclera: clear. Iris: zoned.

Nose.-Profile: straight. Tip: thickness, plus. Wings: medium.

TEETH.-Wear: average. Caries: none. Lost: none.

CHEST.-Development: fair.

MUSCULATURE.-Fair.

HEALTH.-Good.

No. 173. Plate XXIV, Figs. 3, 4

Residence: camp of Sheikh Eliowie. Date: June 5, 1928.

Age: 60 ; married twice, 20 years ago and 15 years ago. Sons: 2,0 , living. Daughters: 1 , 1 , living.

Brothers: 1 living. Sisters: 3 living.

Tattoo marks: on back of right hand.

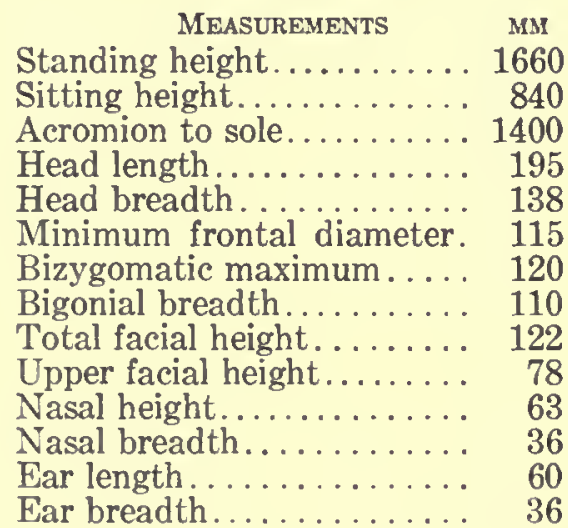

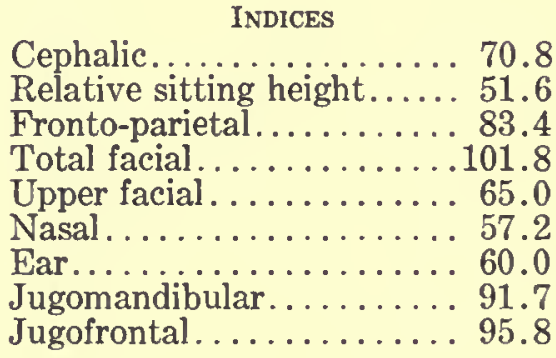

HAIR.-Texture: medium. Color: dark brown. Quantity: head, double minus; beard, double plus; body, plus.

EyE.-Color: green brown. Sclera: bloodshot. Iris: zoned.

NosE.-Profile: straight. Wings: medium.

TEETH.-Wear: triple plus. Caries: triple plus. Lost: 3 or more.

CHEST.-Development: poor.

Musculature.-Poor.

HEALTH.-Good.

Father of No. 43.

No. 174. Plate XXIV, Figs. 5, 6

Residence: camp of Sheikh Eliowie. Date: June 5, 1928.

Age: 30; unmarried. Brothers: 2 living. Sisters: none.

Tattoo marks: none. 


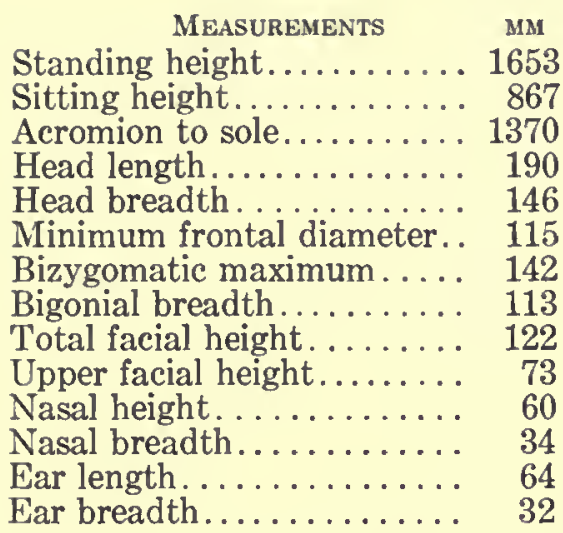

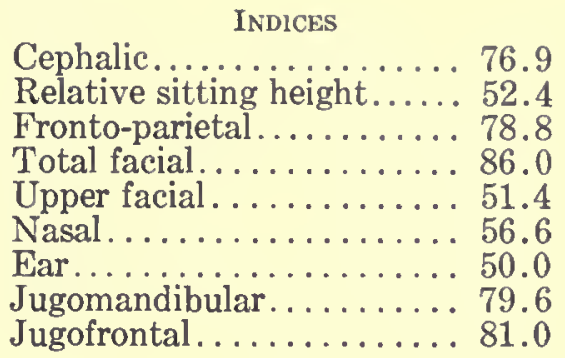

HAIR.-Form: low waves. Texture: medium. Color: dark brown.

EYE.-Color: dark brown. Sclera: clear. Iris: zoned.

Nose.-Profile: concavo-convex. Tip: thickness, plus. Wings: medium.

TEETH.-Lost: 1 left premolar.

CHEST.-Development: plus.

Musculature.-Good.

HEALTH.-Good.

No. 175. Plate XXIV, Figs. 7, 8

Residence: camp of Sheikh Eliowie. Date: June 5, 1928.

Age: 35 ; married for 5 years. No children.

Brothers: 5 living. Sisters: 1 living.

Tattoo marks: on back of right hand and inside of right forearm.

\begin{tabular}{|c|c|}
\hline MEASUREMENTS & MM \\
\hline Standing height. . & 1740 \\
\hline Sitting height. & 860 \\
\hline Acromion to sole. & 1440 \\
\hline Head length... & 19 \\
\hline Head breadth. & 142 \\
\hline Minimum frontal diameter. & 11 \\
\hline Bizygomatic maximum. . . . & 13 \\
\hline Bigonial breadth.......... & 10 \\
\hline Total facial height. ........ & 13 \\
\hline Upper facial height........ & 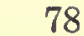 \\
\hline Nasal height & \\
\hline Nasal breadth. & \\
\hline Far le & \\
\hline & \\
\hline
\end{tabular}

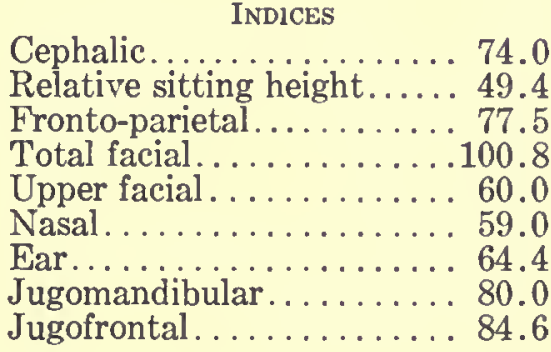

Cephalic.............. 74.0

Relative sitting height......49.4

Fronto-parietal....*......77.5

Upper facial. . . .

Nasal. . . . . ........ 59.0

Jugomandibular. . . . . . . . . 8. 80.0

Jugofrontal. . . . . . . . . 84.6

HAIR.-Form: low waves. Texture: medium. Color: dark brown. Quantity: head, double plus.

EYE. - Sclera: bloodshot. Iris: zoned.

Nose.-Profile: straight. Tip: thickness, plus; depressed. Wings: medium.

TEeth.-Wear: double plus. Caries: plus. Lost: 1 left lower premolar.

CHEST.-Development: plus.

Musculature.-Excellent.

HEALTH.-Excellent.

No. 176

Residence: camp of Sheikh Eliowie. Date: June 5, 1928.

Age: 70; married for 40 years. Sons: 2 living. Daughters: 2 living.

Brothers: none. Sisters: 2 dead.

Tattoo marks: none. 


\begin{tabular}{|c|c|c|}
\hline MEASUREMENTS & MM & INDICES \\
\hline Standing height. . & 1726 & Cephalic. \\
\hline Sitting height. & 890 & Relative sitting height. \\
\hline Acromion to sole. & 1440 & Fronto-parietal........ \\
\hline Head length. & 182 & Total facial.... \\
\hline Head breadth. & 131 & Upper facial \\
\hline Minimum frontal diameter. & 108 & Nasal. \\
\hline ygomatic maximum... & 130 & Ear. \\
\hline Bigonial breadth. & 101 & Jugomandibular. \\
\hline Total facial height. . . . . . . & 128 & Jugofronta \\
\hline
\end{tabular}

Upper facial height........ 75

Nasal height. .......... 62

Nasal breadth.......... 37

Ear length............... 70

Ear breadth............ 45

HAIR.-Color: gray and light brown. Quantity: head, double minus; beard, double plus; body, double plus.

EYE.-Color: dark brown. Sclera: bloodshot. Iris: zoned.

Nose.-Profile: straight. Tip: thickness, double plus.

TEETH.-Wear: triple plus. Caries: double plus. Lost: first lower molar on each side.

ChEST.-Development: minus.

Musculature.-Poor.

HEALTH.-Good.

\section{No. 177. Plate XXV, Figs. 1, 2}

Residence: camp of Sheikh Eliowie. Date: June 5, 1928.

Age: 35 ; unmarried.

Brothers: none. Sisters: none.

Tattoo marks: none.

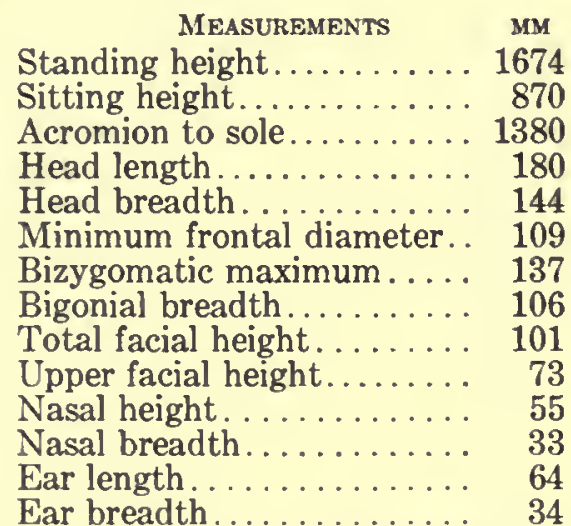

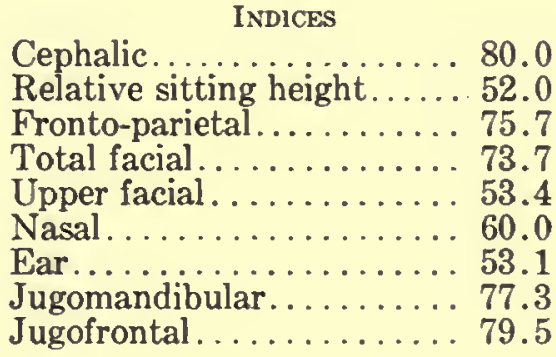

HAIR.-Form: low waves. Texture: fine. Color: reddish brown. Quantity: head, minus; beard, plus; body, average.

EYE.-Sclera: bloodshot. Iris: zoned.

Nose.-Profile: convex. Tip: thickness, minus; horizontal.

TEETH.-Wear: double plus.

CHEST.-Development: average.

Musculature.-Fair.

HEALTH.-Good.

No. 178. Plate XXV, Figs. 3,4

Residence: camp of Sheikh Eliowie. Date: June 5, 1928.

Age: 30 ; unmarried.

Brothers: 1 living. Sisters: none.

Tattoo marks: none. 


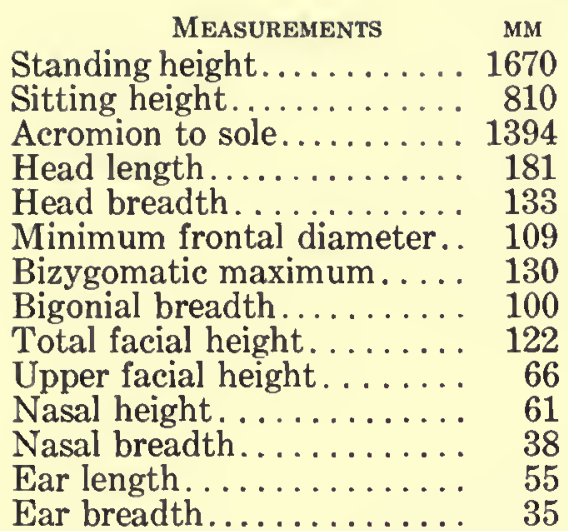

INDICES

Cephalic............ 73.5

Relative sitting height..... 48.5

Fronto-parietal......... 82.0

Total facial............... 93.9

Upper facial. . . . . ..... 50.8

Nasal. . . . . . . . . . 62.4

Ear.................6.6 63.6

Jugomandibular......... 77.0

Jugofrontal........... 83.9

HaIR. - Form: low waves. Texture: medium. Color: reddish brown. Quantity: head, double plus; beard, minus; body, average.

Eye.-Color: dark brown. Sclera: clear. Iris: zoned.

NosE.-Profile: straight.

CHEST.-Development: plus.

Musculature.-Fair.

HEALTH.-Good.

\section{No. 179}

Residence: camp of Sheikh Eliowie. Date: June 5, 1928.

Age: 18; unmarried.

Brothers: 2 living, 1 dead. Sisters: 3 living, 1 dead.

Tattoo marks: glove on back of right hand.

\begin{tabular}{|c|c|c|}
\hline MEASUREMENTS & MM & INDICES \\
\hline Standing height....... & 1625 & Cephalic. \\
\hline Sitting height. . & 813 & Relative sitting height. .... 50.0 \\
\hline Acromion to sole. & 1324 & Fronto-parietal........ \\
\hline Head length. . & 176 & Total facial. \\
\hline Head breadth. & 146 & Upper facial \\
\hline Minimum frontal diameter. . & 115 & Nasal \\
\hline omatic maximum.... & 134 & Ea \\
\hline $1 \mathrm{br}$ & 106 & Jugomandibular.... \\
\hline eight. & 113 & $\ldots \ldots \ldots \ldots$ \\
\hline
\end{tabular}

Upper facial height.......... 63

Nasal height........... 51

Nasal breadth.......... 36

Ear length............. 54

Ear breadth............ 33

HAIR.-Form: low waves. Color: reddish brown. Quantity: head, double plus; beard, minus.

EYE.-Color: dark brown. Sclera: clear. Iris: zoned.

NoSE.-Profile: straight. Tip: thickness, plus. Wings: medium-flaring.

CHEST.-Development: plus.

Musculature.-Good.

HEALTH.-Good.

No. 180. Plate XXV, Figs. 5, 6

Residence: camp of Sheikh Eliowie. Date: June 5, 1928.

Age: 55; married for 12 years. Sons: 2 living, 2 dead. - Daughters: 1 living.

Brothers: 4 living. Sisters: 3 dead.

Tattoo marks: glove on back of right hand and gazelle on inside of right forearm.

\begin{tabular}{|c|c|c|}
\hline MEASUREMENTS & MM & INDICES \\
\hline ng height.. & 1650 & Cephalic............ 72.0 \\
\hline
\end{tabular}




\begin{tabular}{|c|c|}
\hline \multicolumn{2}{|l|}{ MEASUREMENTS } \\
\hline Acromion to & \\
\hline gth.. & \\
\hline & \\
\hline al diamete & \\
\hline & \\
\hline & \\
\hline & \\
\hline & \\
\hline & \\
\hline $\mathrm{al} \mathrm{b}$ & \\
\hline & \\
\hline
\end{tabular}

Fronto-parietal........ 85.1

Total facial............ 79.6

Upper facial . . . . . . . . 47.2

Nasal............... 73.1

Ear................. 57.2

Jugomandibular......... 82.4

Jugofrontal. ........... 84.5

HAIr.-Color: gray. Quantity: head, double minus; beard, double plus; body, plus.

EYe.-Color: green brown. Sclera: bloodshot. Iris: zoned.

Nose,-Profile: convex. Tip: thickness, plus. Wings: flaring.

TеEтн.-Wear: double plus. Caries: minus. Lost: none.

CHEst.-Development: average.

Musculature.-Fair.

HEALTH.-Good.

\section{No. 181. Plate XXV, Figs. 7, 8}

Residence: camp of Sheikh Eliowie. Date: June 5, 1928.

Age: 40 ; unmarried.

Brothers: 1 living. Sisters: 2 living.

Tattoo marks: none.

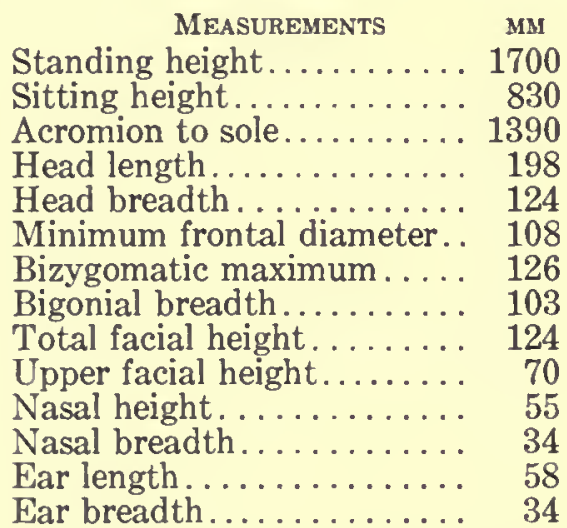

INDICES

Cephalic.............6 62.6

Relative sitting height....... 48.8

Fronto-parietal......... 87.1

Total facial............... 98.5

Upper facial. . . . . . . 55.6

Nasal. ............... 61.8

Ear............... 58.6

Jugomandibular.......... 81.7

Jugofrontal. ........... 85.7

HaIR.-Form: low waves. Texture: fine. Color: reddish brown.

EYE.-Color: dark brown. Sclera: clear. Iris: zoned.

NosE.-Profile: straight. Wings: medium.

TEETH.-Lost: 1 left lower.

CHEst.-Development: plus.

Musculature.-Good.

HEALTH.-Excellent.

No. 182. Plate XXVI, Figs. 1, 2

Residence: camp of Sheikh Eliowie. Date: June 5, 1928.

Age: 35; married for 10 years. Sons: 2 living. Daughters: 1 living.

Brothers: 3 living. Sisters: none.

Tattoo marks: back of right hand.

Measurements MM

Standing height........ 1760

Sitting height.......... 885
INDICES

Cephalic.............. 75.8

Relative sitting height..... 50.3 


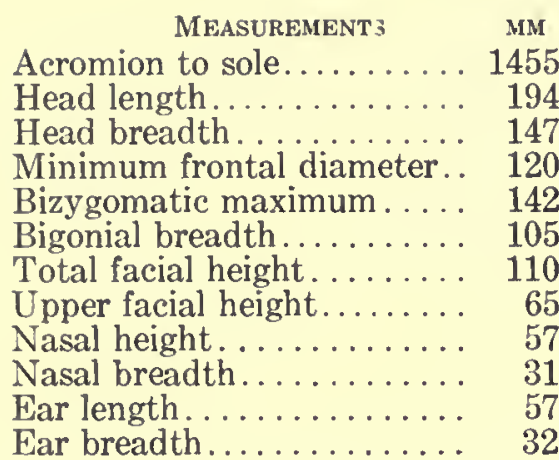

INDICES

Fronto-parietal........ 81.7

Total facial............. 77.5

Upper facial. ......... 45.7

Nasal. ............... 54.4

Ear............... 56.1

Jugomandibular. . . . . . . 73.9

Jugofrontal.......... 84.5

HAIR.-Texture: medium. Color: reddish brown.

EYE.-Color: dark brown. Sclera: bloodshot. Iris: zoned.

NosE.-Profile: convex.

CHEst.-Development: plus.

Musculature.-Good.

HEALTH.-Good.

\section{No. 183. Plate XXVI, Figs. 3, 4}

Residence: camp of Sheikh Eliowie. Date: June 5, 1928.

Age: 40 ; married for 5 years. No children.

Brothers: 2 living. Sisters: none.

Tattoo marks: none.

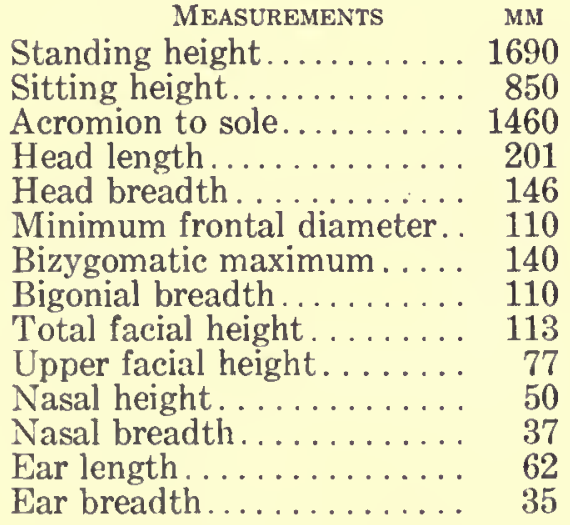

Cephalic.............. 72.4

Relative sitting height..... 50.3

Fronto-parietal........... 75.4

Total facial............ 80.7

Upper facial . . . . . . . . . . . 55.0

Nasal . . . . . . . . . . . . 74.0

Ear................. 56.5

Jugomandibular. . . . . . . 78.5

Jugofrontal.............. 78.5

HAIR.-Form: low waves. T'exture: medium.

EYE.-Color: dark brown. Sclera: clear. Iris: zoned.

Nose.-Profile: convex.

TEETH.-Lost: 3 right lower molars, 1 right upper molar.

Chest.-Development: plus.

Musculature.-Fair.

HEALTH.-Good.

No. 184. Plate XXVI, Figs. 5, 6

Residence: camp of Sheikh Eliowie. Date: June 5, 1928.

Age: 60 or more; married for 30 years. Sons: 2 living. Daughters: 1 dead.

Brothers: 1 living, 2 dead. Sisters: none.

Tattoo marks: none.

MEASUREMENTS MM

Standing height......... 1650

Sitting height........... 780

Acromion to sole......... 1365
INDICES

Cephalic............. 71.8

Relative sitting height..... 47.3

Fronto-parietal........... 77.0 


\begin{tabular}{|c|c|}
\hline Measurements & MM \\
\hline Head length. & \\
\hline Head bread th. . & \\
\hline $\begin{array}{l}\text { Minimum frontal diameter. } \\
\text { Bizygomatic maximum... }\end{array}$ & \\
\hline Bigonial breadth... & \\
\hline Total facial height & \\
\hline Upper facial height. & \\
\hline $\begin{array}{l}\text { Nasal height } \\
\text { Nasal breadth }\end{array}$ & \\
\hline length. & \\
\hline & \\
\hline
\end{tabular}

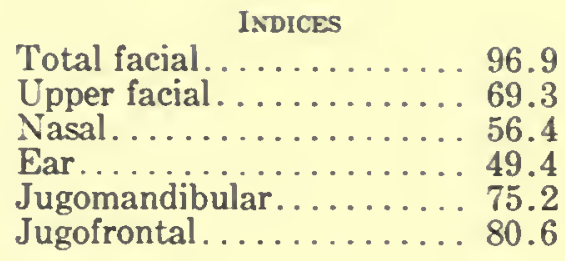

HAIR.-Form: low waves. Texture: medium. Color: gray. Quantity: head, plus; beard, double plus; body, triple plus.

EYE.-Color: green brown. Sclera: bloodshot. Iris: zoned.

Nose.-Profile: convex. Tip: thickness, minus; depressed.

TEeTH.-Wear: triple plus. Caries: triple plus. Lost: many.

CHEsT.-Development: minus.

Musculature.-Fair.

HEALTH.-Good.

\section{No. 185. Plate XXVI, Figs. 7, 8}

Residence: camp of Sheikh Eliowie. Date: June 5, 1928.

Age: 40 ; married for 20 years. Sons: 1 living, 1 dead. Daughters: 1 living.

Brothers: 1 living, 1 dead. Sisters: 2 living.

Tattoo marks: none.

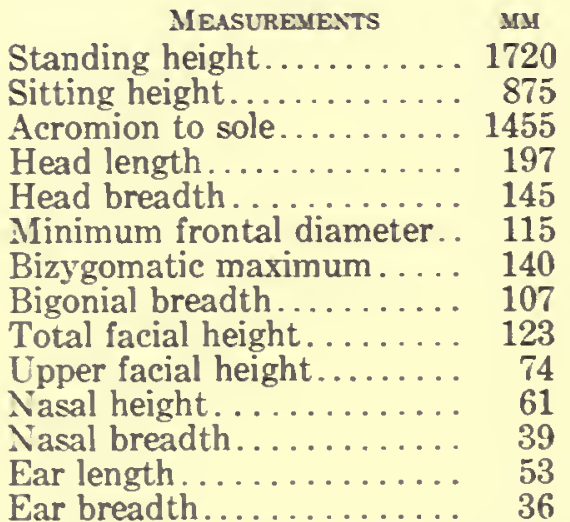

HAIR.-Form: low waves. Color: dark brown and gray. Quantity: head, plus; beard, double plus; body, plus.

EYE.-Color: green brown. Sclera: bloodshot. Iris: zoned.

NOSE.-Profile: straight. Tip: thickness, double plus. Wings: medium.

TEETH.-Lost: 1 left upper molar.

CHEST.-Development: plus.

Musculature.-Good.

HEALTH._Excellent.

No. 186. Plate XXVII, Figs. 1, 2

Residence: camp of Sheikh Eliowie. Date: June 5, 1928.

Age: 28; married for 5 years. Sons: 1 dead. Daughters: 1 living.

Brothers: 1 living. Sisters: 5 living.

Tattoo marks: a spot on each temple and each wrist. 


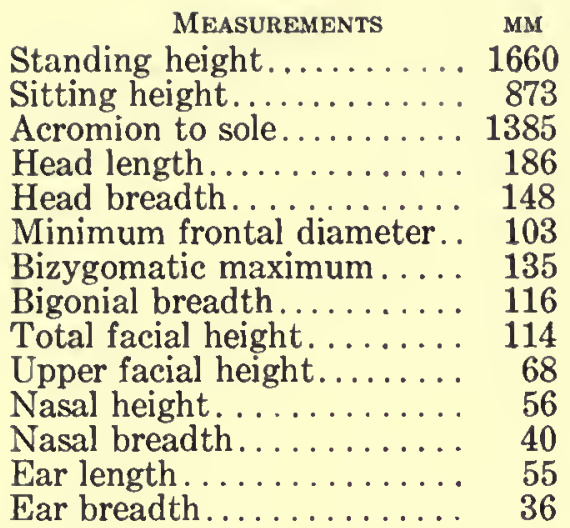

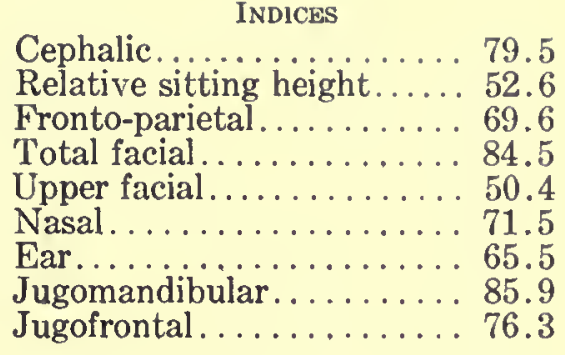

HaIR.-Form: low waves. Texture: medium. Color: dark brown. Quantity: head, double plus; beard, minus; body, plus.

EYE.-Color: dark brown. Sclera: clear. Iris: zoned.

Nose.-Profile: straight. Tip: thickness, minus; depressed.

TEETH.-Lost: none.

CHEST.-Development: average.

Musculature.-Fair.

HEALTH.-Good.

No. 187. Plate XXVII, Figs. 3, 4

Residence: camp of Sheikh Eliowie. Date: June 5, 1928.

Age: 25; married for 5 years. No children.

Brothers: 5 living. Sisters: 1 living.

Tattoo marks: glove on right wrist and gazelle on inside of right forearm.

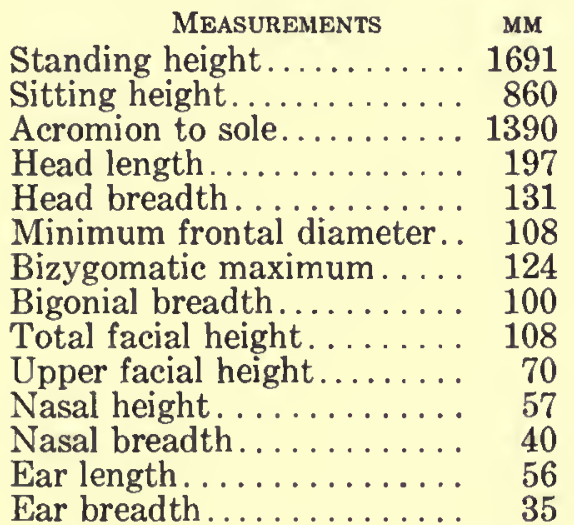

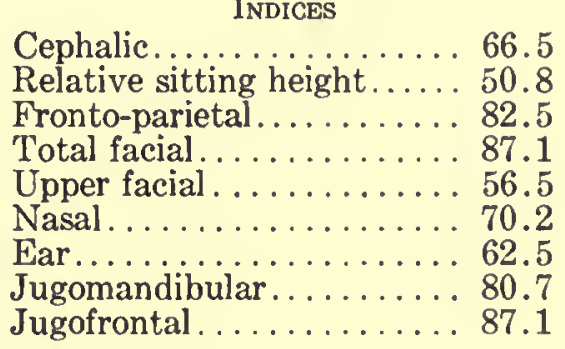

66.5

0.8

82.5

Jugomandibular. . . . . . . . . 87.1
Jugofrontal . . . . . . . . 87.9

HAIR.-Form: low waves. Texture: medium. Color: dark brown.

EYE.-Color: dark brown. Sclera: clear. Iris: zoned.

Nose.-Profile: concavo-convex. Wings: medium.

TEETH.-Lost: none.

Chest.-Development: double plus.

Musculature.-Good.

HEALTH.-Excellent.

No. 188. Plate XXVII, Figs. 5, 6

Residence: camp of Sheikh Eliowie. Date: June 5, 1928.

Age: 25; unmarried.

Brothers: 1 living, 1 dead. Sisters: none.

Tattoo marks: none. 


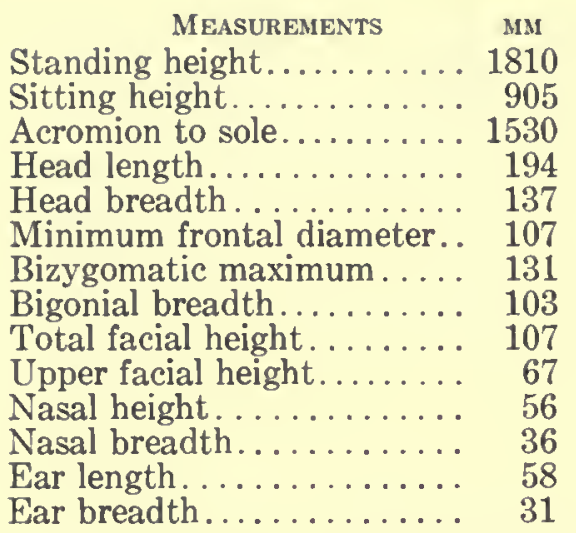

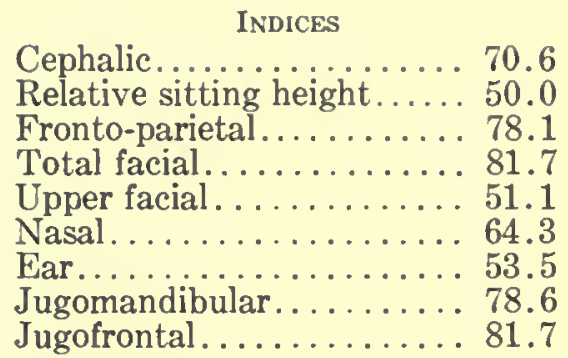

HaIR. - Form: low waves. Texture: medium-fine.

EYE.-Color: dark brown. Sclera: clear. Iris: zoned.

Nose.-Profile: straight.

TEETH.-Lost: none.

Chest.-Development: plus.

Musculature.-Good.

HEALTH.-Excellent.

No. 189

Residence: camp of Sheikh Swadi. Date: June 5, 1928.

Age: 25; unmarried.

Brothers: 1 living. Sisters: 2 living.

Tattoo marks: none.

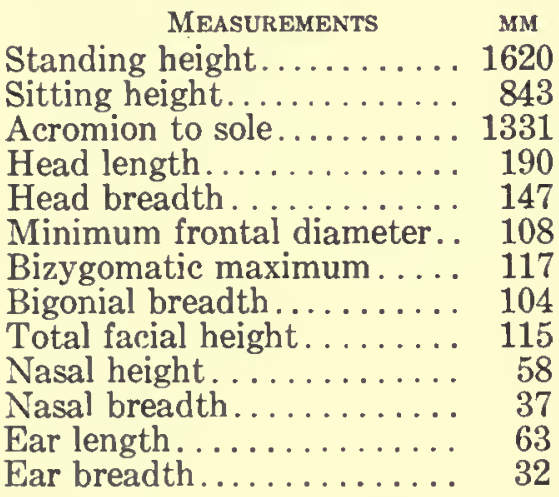

INDICES

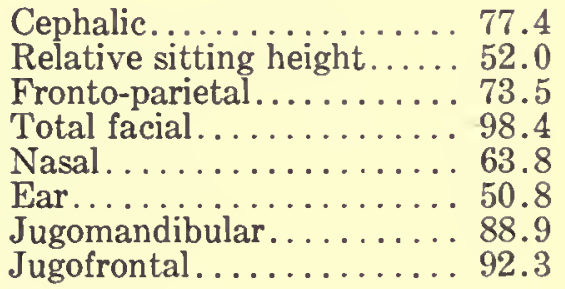

HaIR.-Form: low waves. Texture: medium. Color: dark brown. Quantity: head, double plus; beard, minus; body, plus.

Eye.-Color: dark brown. Sclera: clear. Iris: zoned.

Nose.-Profile: concavo-convex.

TEETH.-Lost: none.

CHEST.-Development: plus.

Musculature.-Good.

HEALTH.-Excellent.

No. 190

Residence: camp of Sheikh Swadi. Date: June 5, 1928.

Age: 48; married for 25 years. Sons: 2 living, 2 dead. Daughters: 2 living, 1 dead. Brothers: none. Sisters: 1 dead.

Tattoo marks: none.

Measurements MM

Standing height......... 1702

Sitting height........... 849

Acromion to sole......... 1456
INDICES

Cephalic............. 77.9

Relative sitting height..... 49.8

Fronto-parietal.......... 77.5 


\begin{tabular}{|c|c|}
\hline & \\
\hline Head length. & 194 \\
\hline Head breadth. & 15 \\
\hline Minimum frontal diameter. . & 11 \\
\hline Bizygomatic maximum..... & 14 \\
\hline Bigonial breadth.... . . . & 02 \\
\hline Total facial height. & 0 \\
\hline Upper facial height........ & \\
\hline Nasal height. ........... & \\
\hline asal breadth. & \\
\hline length.... & \\
\hline breadtl & \\
\hline
\end{tabular}

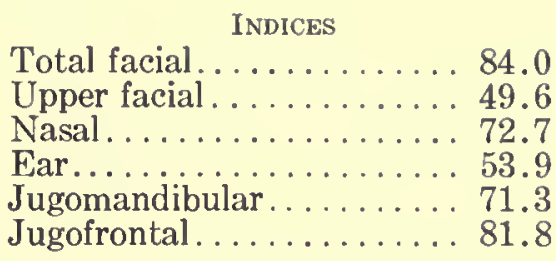

Harr.-Form: low waves. Texture: medium. Color: dark brown and gray. Quantity: head, double plus; beard, double plus; body, plus.

EYE.-Color: dark brown. Sclera: clear. Iris: zoned.

Nose.-Profile: concavo-convex.

TEETH.-Wear: double plus. Caries: plus. Lost: 1 upper molar on each side. Upper incisors very large.

CHest.-Development: average.

Musculature.-Poor.

HEALTH.-Good.

No. 191

Residence: camp of Sheikh Swadi. Date: June 5, 1928.

Age: 30 ; married for 10 years. Sons: 1 living, 1 dead. Daughters: none.

Brothers: 1 dead. Sisters: 1 living, 1 dead.

Tattoo marks: none.

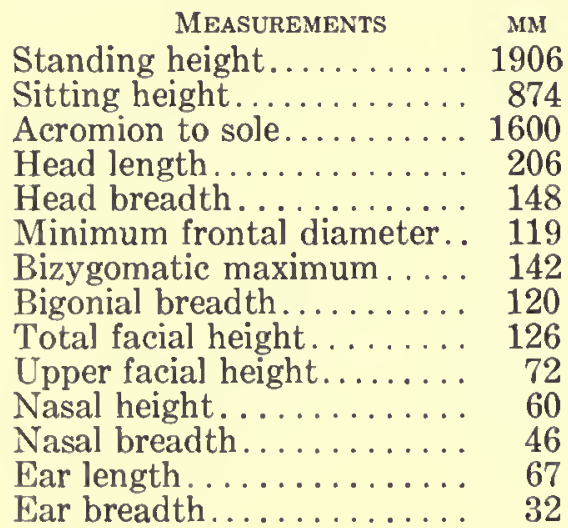

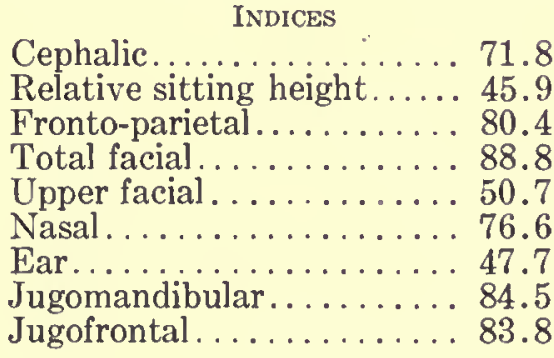

HAIR.-Form: low waves. Texture: medium. Color: dark brown. Quantity: head, double plus; beard, minus; body, average.

EYE.-Color: dark brown. Sclera: clear. Iris: zoned.

Nose.-Profile: concave. Tip: thickness, double plus. Wings: flaring.

CHest.-Development: triple plus.

Musculature.-Excellent.

HEALTH.-Excellent.

Overgrowth, probably due to unbalanced metabolism caused by irregular functioning of endocrine glands.

No. 192

Residence: camp of Sheikh Swadi. Date: June 5, 1928.

Age: 40 ; married for 4 years. Sons: 2 living. Daughters: none.

Brothers: none. Sisters: 1 living.

Tattoo marks: none. 


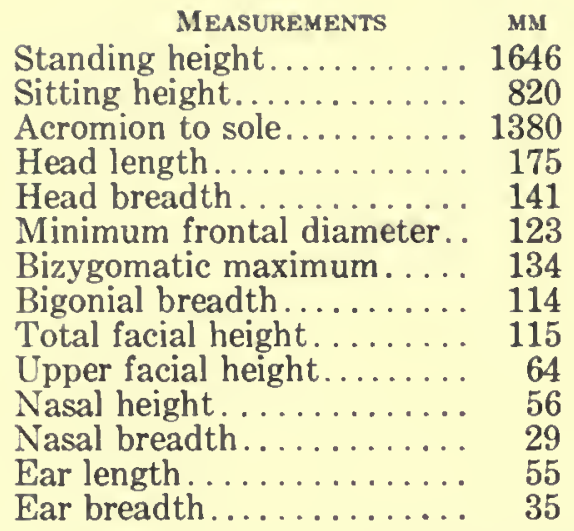

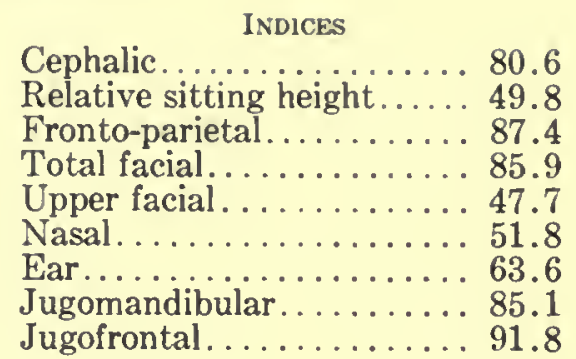

HaIr.-Form: low waves. Texture: medium. Quantity: head, double plus; beard, plus. EYE.-Color: green brown. Sclera: bloodshot. Iris: zoned.

NosE.-Profile: straight.

Teeth.-Wear: double plus. Caries: double plus. Lost: none.

CHEst.-Development: minus.

Musculature.-Poor.

HEALTH.-Good.

No. 193

Residence: camp of Sheikh Swadi. Date: June 5, 1928.

Age: 35; married for 10 years. Sons: 2 living. Daughters: 1 living.

Brothers: 2 living. Sisters: 4 living.

Tattoo marks: none.

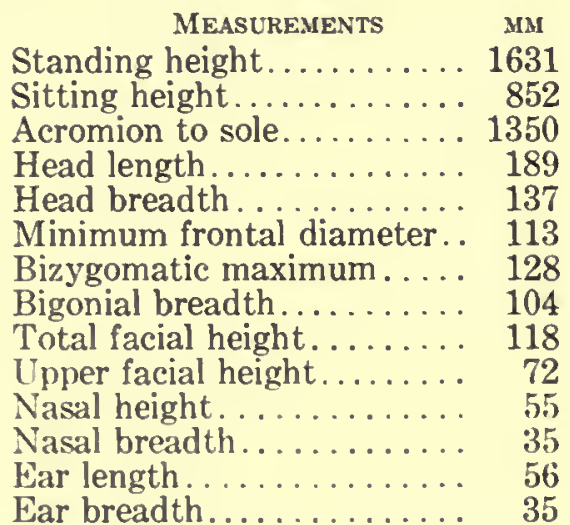

HaIr.-Form: low waves. Texture: fine. Color: dark brown. Quantity: head, double plus; beard, double plus; body, double plus.

EYE.-Color: dark brown. Sclera: clear. Iris: zoned.

Nose.-Profile: straight. Tip: thickness, minus. Wings: compressed.

TEETH.-Lost: none.

CHEST.-Development: plus.

Musculature.-Excellent.

HEALTH.--Excellent.

Residence: camp of Sheikh Swadi. Date: June 5, 1928.

Age: 60 or more; married for 40 years. Sons: 2 living, 2 dead. Daughters: 2 living, 1 dead. Brothers: 1 living, 3 dead. Sisters: 1 dead.

Tattoo marks: none. 


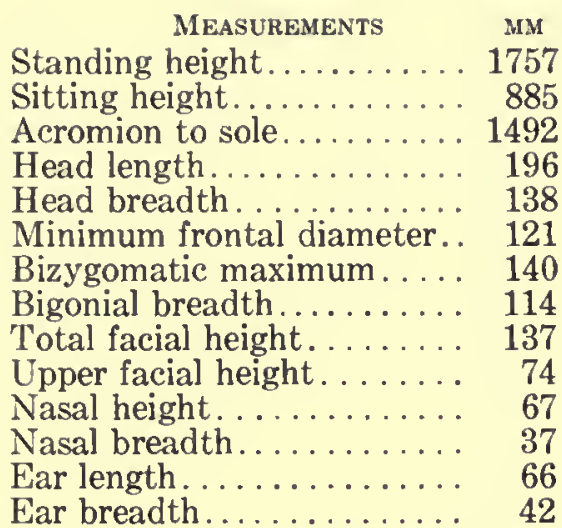

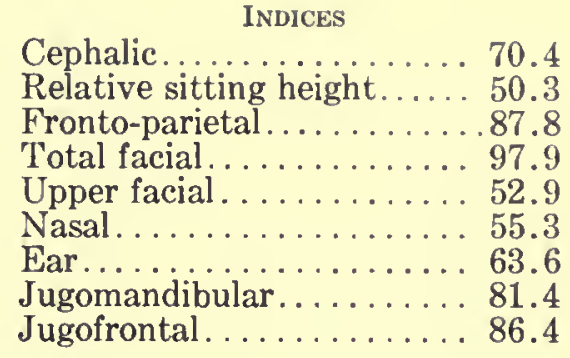

HaIr.-Form: low waves. Texture: medium. Color: gray. Quantity: head, plus. EYE.-Color: green brown. Sclera: clear. Iris: zoned.

Nose.-Profile: straight. Tip: thickness, double plus. Wings: medium.

TEETH.-Wear: plus. Lost: none.

CHEsT.-Development: plus.

Musculature.-Excellent.

Health.-Good.

No. 195

Residence: camp of Sheikh Swadi. Date: June 5, 1928.

Age: 40; married for 20 years. Sons: 2 living. Daughters: 1 living.

Brothers: none. Sisters: none.

Tattoo marks: glove on back of right hand, gazelle on inside of right forearm, and spots below nasion.

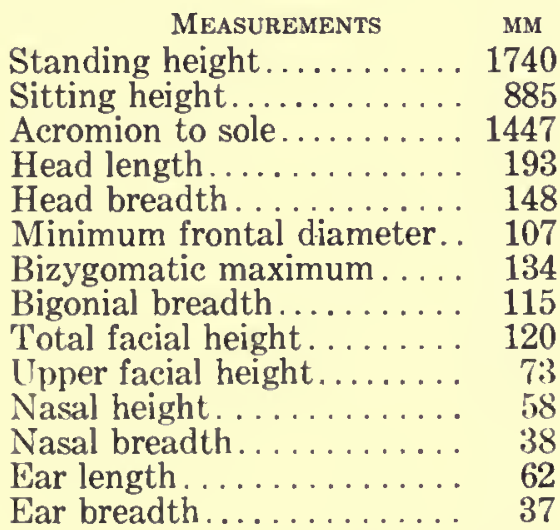

INDICES

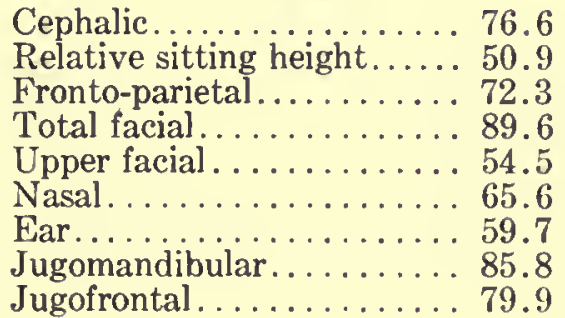

HaIR.-Form: low waves. Texture: medium. Color: dark brown and gray. Quantity: head, plus; beard, double plus; body, average.

EYE.-Color: green brown. Sclera: clear. Iris: zoned.

Nose.-Profile: convex. Wings: medium.

TEETH.-Wear: double plus.

CHEST.-Development: double plus.

Musculature.-Excellent.

HEALTH.-Excellent.

Residence: camp of Sheikh Swadi. Date: June 5, 1928.

Age: 50 ; married for 30 years. Sons: 1 living. Daughters: 2 living.

Brothers: 4 dead. Sisters: 1 dead.

Tattoo marks: none. 


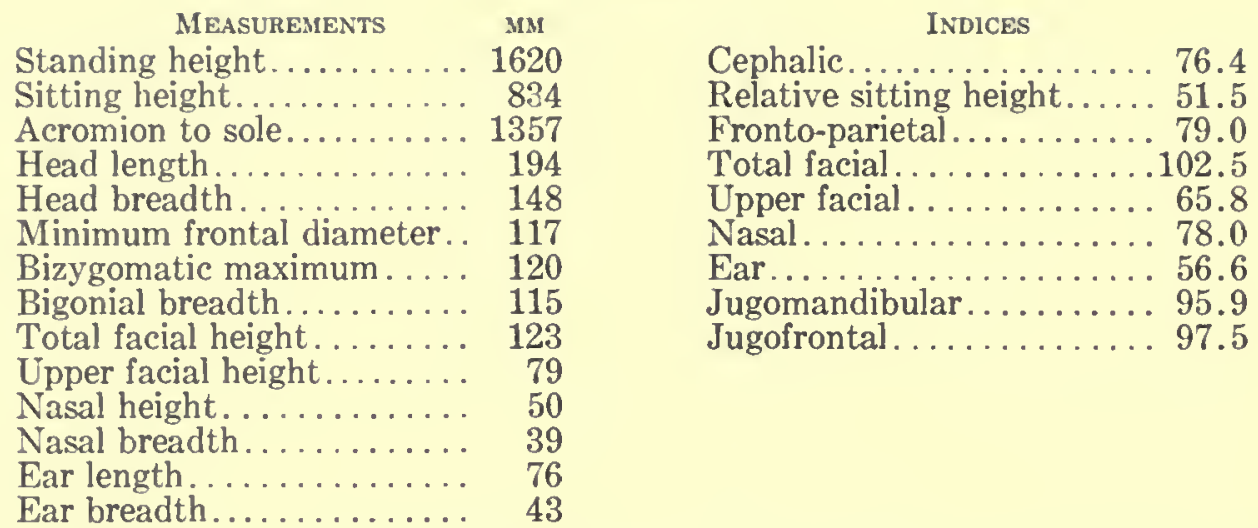

HAIR.-Form: low waves. Texture: medium. Color: dark brown and white. Quantity: head, plus; beard, plus; body, plus.

EYE.-Color: green brown. Sclera: clear. Iris: zoned.

NOSE.--Profile: straight. Wings: flaring.

TEETH._Lost: none. Condition: good.

CHEst.-Derelopment: plus.

Musculature.-Good.

HEALTH.-Excellent.

No. 197

Residence: camp of Sheikh Swadi. Date: June 6, 1928.

Age: 45; married for 20 years. Sons: 1 living. Daughters: 2 living.

Brothers: 3 living, 2 dead. Sisters: 1 living.

Tattoo marks: none.

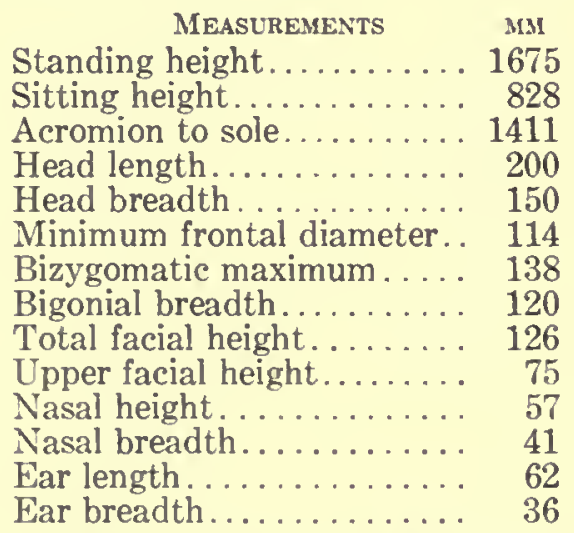

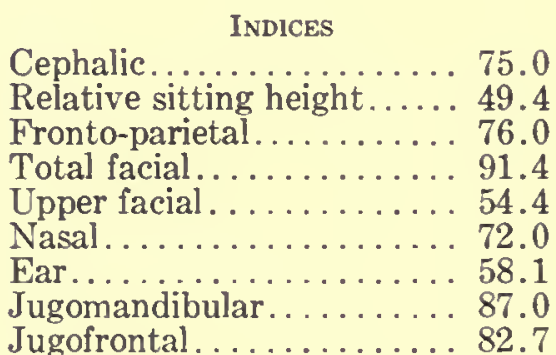

Jugofrontal . . . . . . . . . . 82.7

HAIR.-Form: low waves. Texture: medium. Color: dark brown. Quantity: head, plus. EYE.-Color: dark brown. Sclera: clear. Iris: zoned.

NosE.-Profile: convex. Tip: thickness, plus.

TEETH.-Lost: none.

CHEST.-Development: plus.

Musculature.-Excellent.

HEALTh.-Excellent.

\section{No. 198}

Residence: camp of Sheikh Swadi. Date: June 6, 1928.

Age: 65; married for 40 years. Sons: 2 living. Daughters: none.

Brothers: none. Sisters: none.

Tattoo marks: none. 


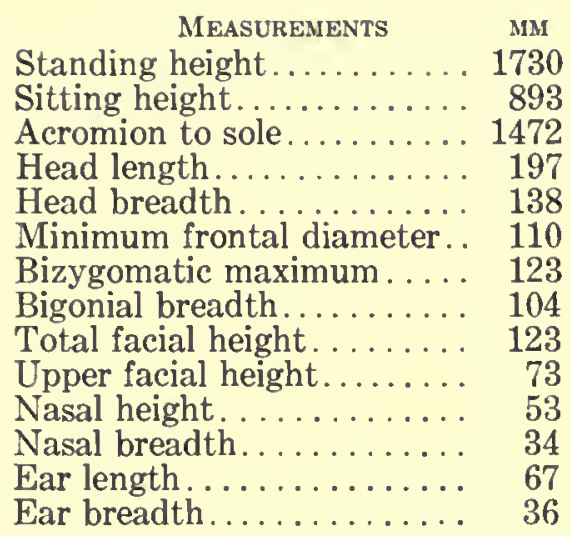

Cephalic........... 70.0

Relative sitting height..... 51.6

Fronto-parietal.......... 79.9

Total facial. . . . . . . . . . 100.0

Upper facial. ............ 59.4

Nasal. . . . . . . . . . . 64.2

Ear................... 53.7

Jugomandibular. . . . . . 84.6

Jugofrontal . . . . . . . . . . 89.4

HaIR.-Form: low waves. Texture: medium. Color: gray. Quantity: head, plus; beard, double plus; body, minus.

EYE.-Color: green brown. Sclera: bloodshot. Iris: zoned.

NosE.-Profile: concavo-convex. Tip: thickness, double plus; depressed. Wings: medium. TEETH.-Lost: none.

Chest.-Development: plus.

Musculature.-Good.

HEALTH.-Poor.

No. 199

Residence: camp of Sheikh Swadi. Date: June 6, 1928.

Age: 75; married for 50 years. Sons: 3 living. Daughters: 2 living.

Brothers: 4 dead. Sisters: none.

Tattoo marks: none.

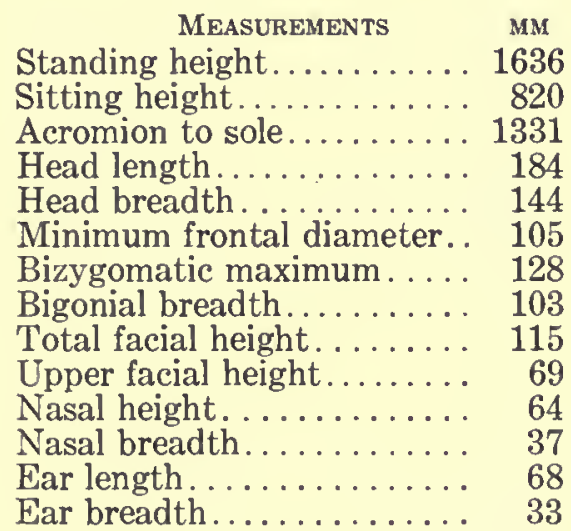

INDICES

Cephalic................ 78.3

Relative sitting height...... 50.1

Fronto-parietal.......... 73.0

Total facial............. 89.9

Upper facial. . . . . . . . 53.9

Nasal............... 57.9

Ear............... 48.5

Jugomandibular......... 80.5

Jugofrontal............ 82.1

HaIR.-F'orm: low waves. Texture: medium. Color: white. Quantity: head, double minus; beard, double plus; body, minus.

EYE.-Color: green brown. Sclera: clear. Iris: zoned.

Nose.-Profile: straight. Wings: medium.

TEETH.-Wear: triple plus. Caries: triple plus. Lost: half.

ChesT.-Development: minus.

MusCulature.-Very poor.

HEALTH.-Poor.

No. 200. Plate XXVII, Figs. 7, 8

Residence: camp of Sheikh Swadi. Date: June 6, 1928.

Age: 35; married for 8 years. Sons: 1 living. Daughters: 1 living.

Brothers: 4 living. Sisters: 3 living.

Tattoo marks: none. 


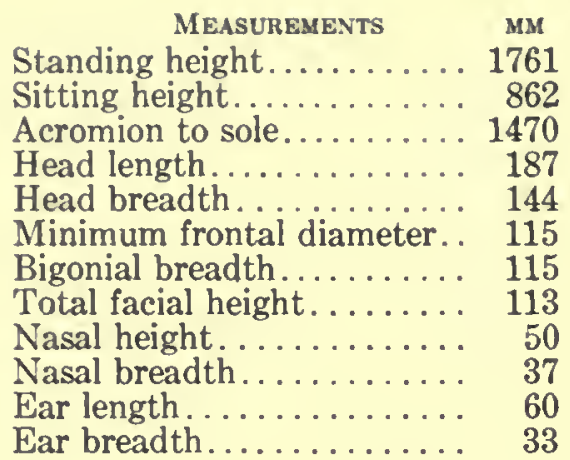

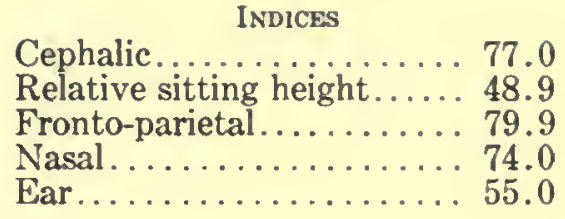

HAIR.-Form: curly frizzly. Texture: medium. Color: dark brown. Quanti!y: head, double plus; beard, plus; body, plus.

EYE.-Color: dark brown. Sclera: clear. Iris: zoned.

Nose.-Profile: convex. Wings: medium.

Chest.-Development: double plus.

Musculature.-Excellent.

HEALTH.-Excellent.

No. 201

Residence: camp of Sheikh Swadi. Date: June 6, 1928.

Age: 80; married for 60 years. Sons: 2 living. Daughters: 2 living.

Brothers: none. Sisters: 1 dead.

Tattoo marks: spots on back of left hand.

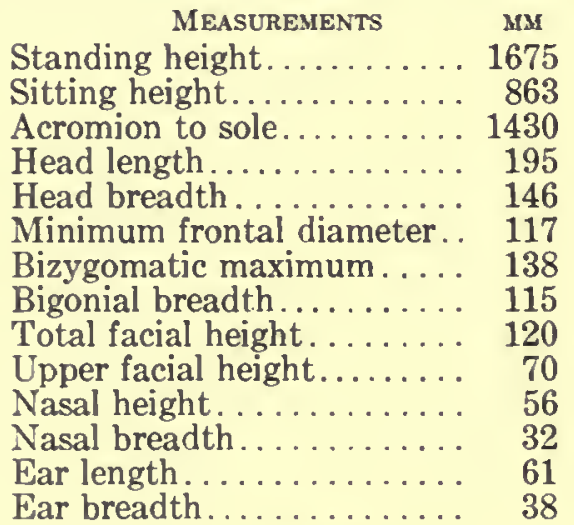

HAIR.-Color: white. Quantity: head, shaved; beard, plus; body, average.

EYE.-Color: green brown. Sclera: bloodshot. Iris: zoned.

Nose.-Profile: straight. Wings: medium.

TEетH.-Wear: triple plus. Caries: double plus. Lost: several.

Chest.-Development: plus.

Musculature.-Good.

Health.-Poor; eye trouble.

No. 202

Residence: camp of Sheikh Swadi. Date: June 6, 1928.

Age: 40 ; unmarried.

Brothers: 2 living. Sisters: 1 living.

Tattoo marks: 2 spots on back of right hand.

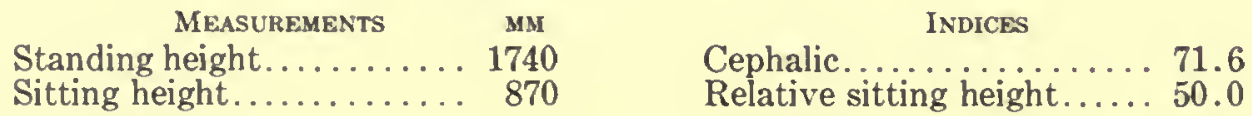




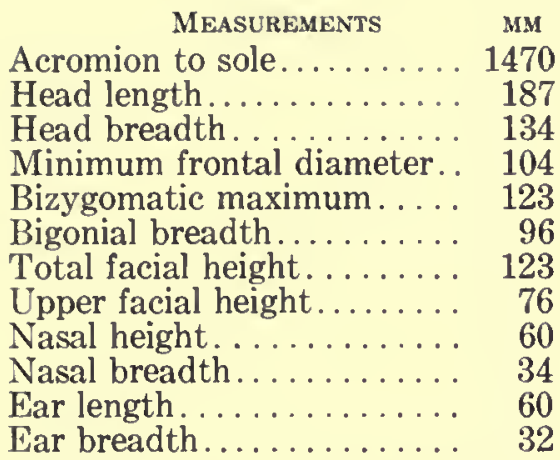

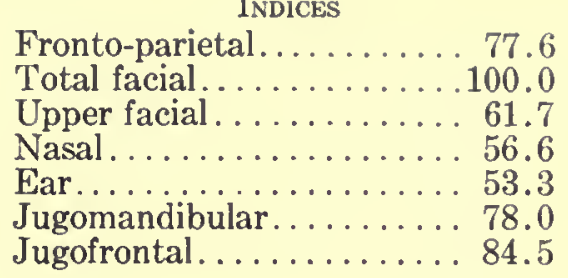

HAIR.-Texture: medium. Quantity: head, shaved.

EYE.-Color: dark brown. Sclera: speckled.

Nose.-Profile: concavo-convex. Tip: thickness, double plus.

TEETH.-Lost: none.

CHEST.-Development: minus.

MUSCUlatURE.-Poor.

HEALTH.-Poor; eye trouble.

No. 203

Residence: camp of Sheikh Swadi. Date: June 6, 1928.

Age: 75; married for 35 years. Sons: 2 living, 2 dead. Daughters: 2 dead.

Brothers: 1 living, 2 dead. Sisters: 1 dead.

Tattoo marks: spots on back of each hand and on each temple.

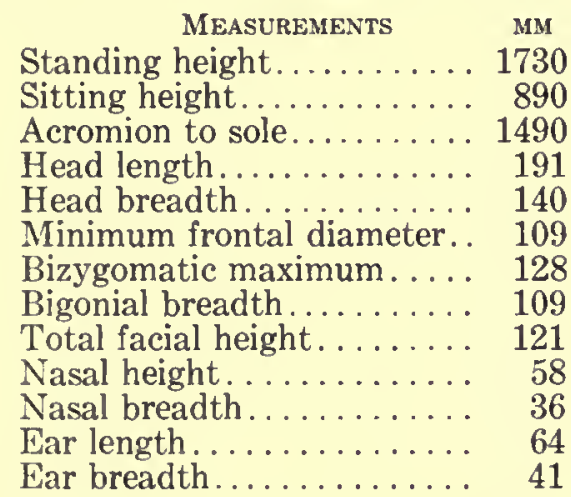

Cephalic............... 73.4

Relative sitting height...... 51.5

Fronto-parietal......... 77.9

Total facial. . ............. 94.6

Nasal. ..............62.0

Ear.................6 64.1

Jugomandibular........ 85.2

Jugofrontal. .......... 85.2

HAIR.-Texture: medium. Color: gray. Quantity: head, plus.

EYE.-Color: dark brown. Sclera: bloodshot. Iris: zoned.

Nose.-Profile: straight. Wings: medium.

TEETH.-Caries: double plus. Lost: 4.

Chest.-Development: average.

Musculature.-Poor.

HEALTH.-Good.

No. 204

Residence: camp of Sheikh Swadi. Date: June 6, 1928.

Age: 20 ; married for 8 years. Sons: 1 dead. Daughters: none.

Brothers: 1 dead. Sisters: none.

Tattoo marks: none.

MEASUREMENTS MM

Standing height. . . . . . . 1650

Sitting height............ 828

Acromion to sole........ 1390
INDICES

Cephalic.............. 78.5

Relative sitting height..... 50.2

Fronto-parietal.........76.0 


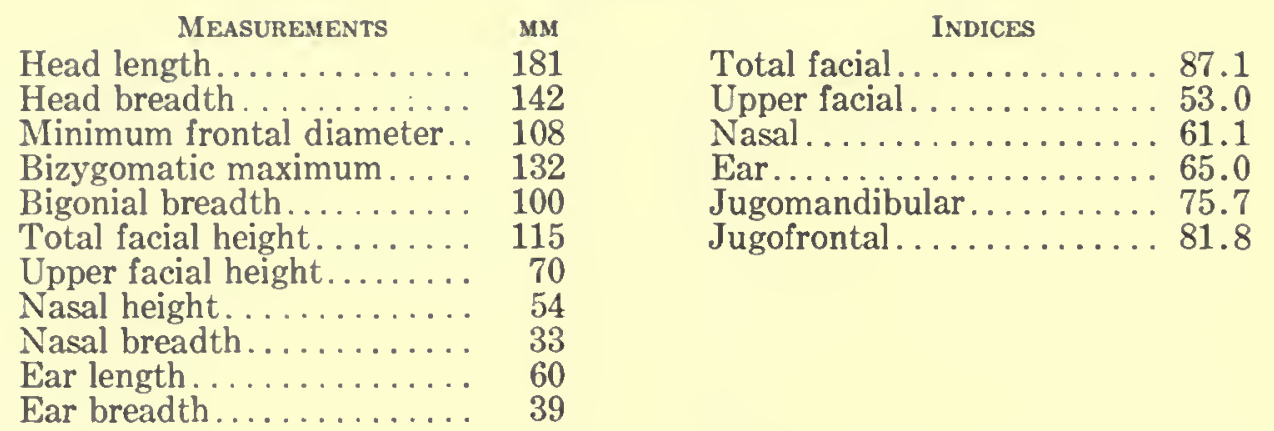

HAIR.-Texture: fine. Color: dark brown. Quantity: head, double plus; beard, minus; body, average.

EYE.-Color: dark brown. Sclera: clear. Iris: zoned.

Nose.-Profile: concavo-convex.

TEETH.-Lost: none.

Chest.-Development: plus.

Musculature.--Good.

HEALTH.-Excellent except for cataract in eye.

No. 205

Residence: camp of Sheikh Swadi. Date: June 6, 1928.

Age: 40 ; married twice, 25 years ago and 12 years ago. Sons: 1, 0, living. Daughters:

2 , 1 , living.

Brothers: none. Sisters: 1 living.

Tattoo marks: none.

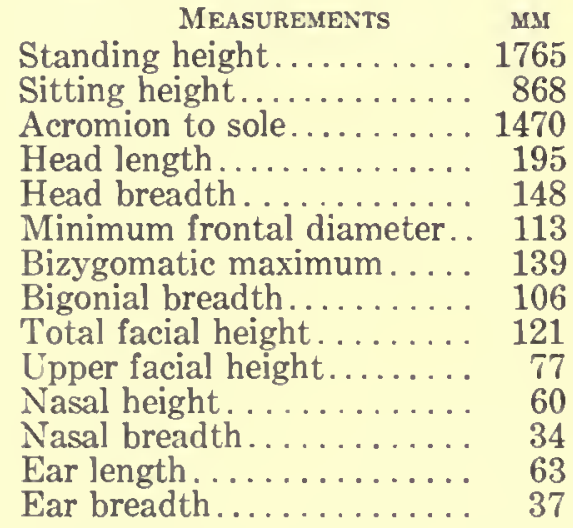

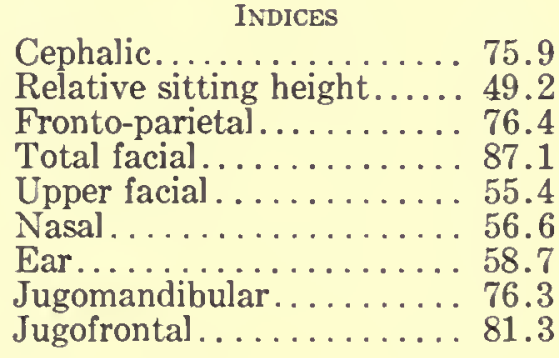

HAIR.-Form: low waves. Texture: medium.

EYE.-Color: dark brown. Sclera: clear. Iris: zoned.

Nose.--Profile: convex. Tip: thickness, plus.

Teetr.-Lost: none. Condition: excellent.

Chest.--Development: double plus.

Musculature.-Excellent.

HEALTH.---Excellent.

No. 206

Residence: camp of Sheikh Swadi. Date: June 6, 1928.

Age: 40; married for 30 years. Sons: 1 living. Daughters: 2 living.

Brothers: 3 living, 2 dead. Sisters: none.

Tattoo marks: none. 


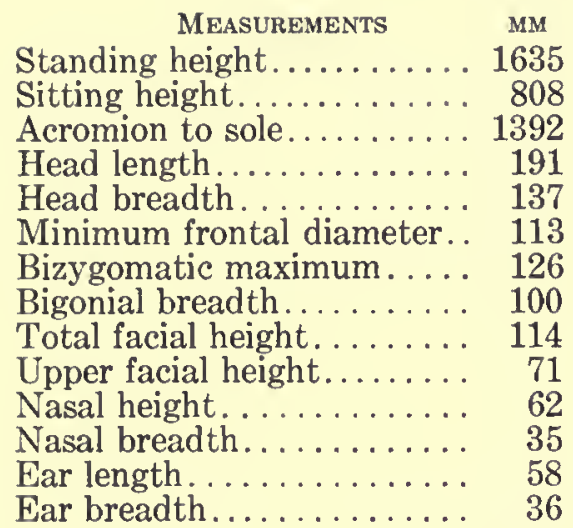

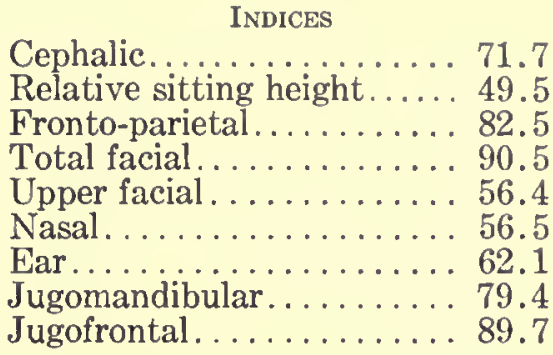

EYE.-Color: dark brown. Sclera: bloodshot. Iris: zoned.

Nose.-Profile: convex. Tip: thickness, double plus. Wings: medium.

TEETH,-Lost: none.

CHEsT.-Development: average.

Musculature.-Poor.

HEALTH.-Excellent.

No. 207. Plate XXVIII, Figs. 1, 2

Residence: camp of Sheikh Swadi. Date: June 6, 1928.

Age: 35 ; unmarried.

Brothers: 1 living, 1 dead. Sisters: 2 living.

Tattoo marks: none.

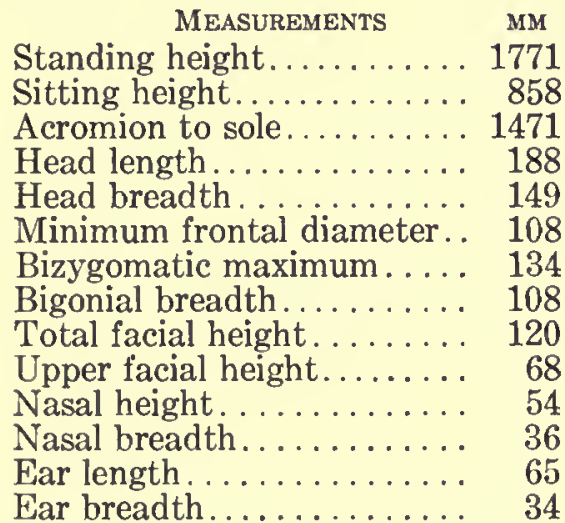

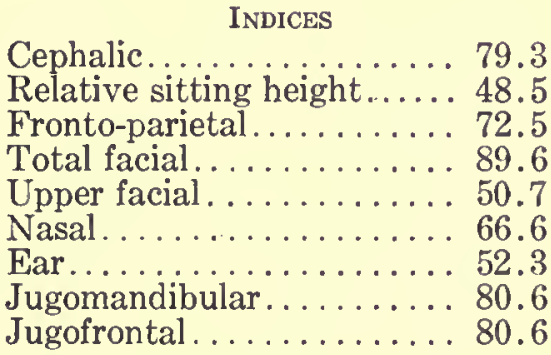

HAIR.-Form: low waves. Texture: medium. Color: dark brown.

EYE.-Color: dark brown. Sclera: clear. Iris: zoned.

Nose.-Profile: straight. Wings: medium.

TEETH.-Lost: none.

ChesT.-Development: plus.

Musculature.-Excellent.

HEALTH.-Excellent.

No. 208

Residence: camp of Sheikh Swadi. Date: June 6, 1928.

Age: 35 ; unmarried.

Brothers: 1 living. Sisters: 1 living.

Tattoo marks: none.

$\begin{array}{cc}\text { Measurements } & \text { мм } \\ \text { Standing height.......... } 1687\end{array}$

Sitting height........... 800

INDICES

Cephalic.............. 77.6

Relative sitting height...... 47.4 


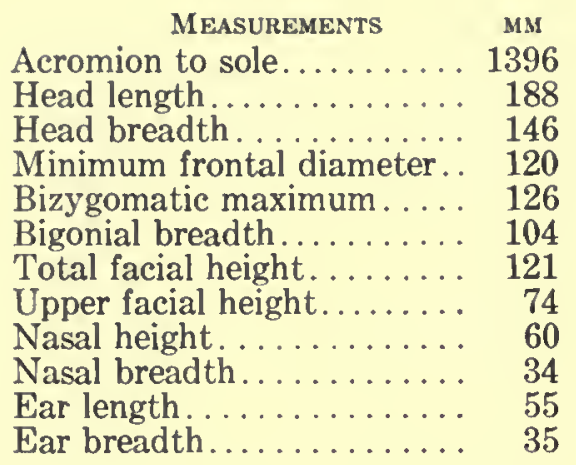

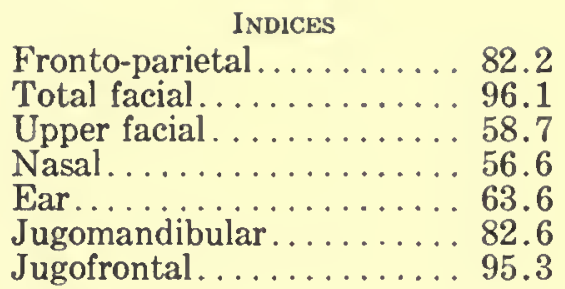

No. 209

Residence: camp of Sheikh Swadi. Date: June 6, 1928.

Age: 30 ; unmarried.

Brothers: 3 living. Sisters: 1 living, 1 dead.

Tattoo marks: none.

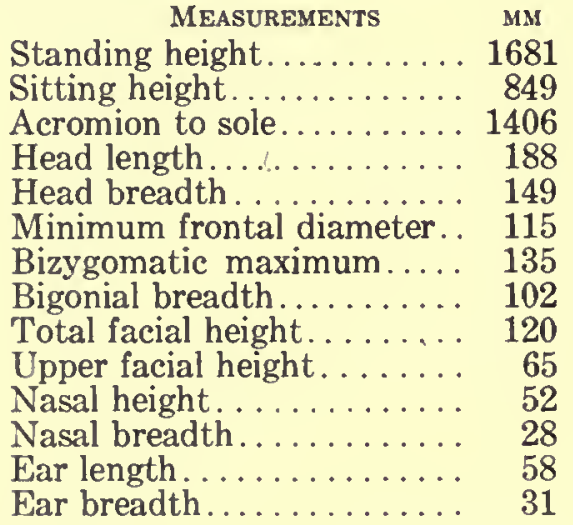

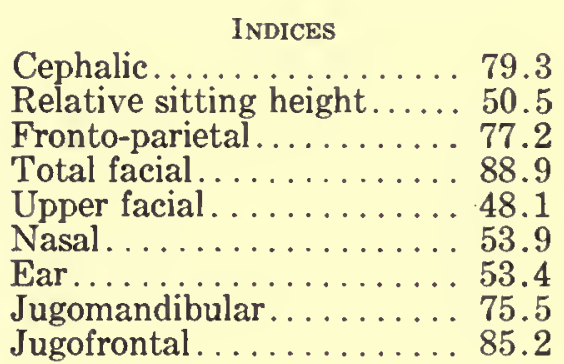

HaIR.-Form: low waves. Texture: fine. Color: dark brown. Quantity: head, double plus; beard, plus; body, plus.

EYE.-Color: dark brown. Sclera: clear. Iris: zoned.

Nose.-Profile: concavo-convex.

TeEth.-Wear: plus. Lost: none. First right upper incisor broken.

CHest.-Development: plus.

Musculature.-Good.

HEALTH.-Excellent.

No. 210

Residence: camp of Sheikh Swadi. Date: June 6, 1928.

Age: 20; unmarried.

Brothers: 1 living. Sisters: 3 living.

Tattoo marks: none.

MEASUREMENTS MM

Standing height......... 1620

Sitting height........... 820 


\begin{tabular}{|c|c|}
\hline MEASUREMENTS & $\operatorname{lng}$ \\
\hline nion to sole. & 1330 \\
\hline ngth & \\
\hline & \\
\hline ntal diameter & \\
\hline & \\
\hline & \\
\hline Tot & \\
\hline & \\
\hline sal height. & \\
\hline al $\mathrm{k}$ & \\
\hline ] & \\
\hline
\end{tabular}

Fronto-parietal ......... 81.5

Total facial ............ 86.0

Upper facial. . . . . . . . . . 51.6

Nasal. ............... 58.5

Ear................. 56.3

Jugomandibular........... 78.2

Jugofrontal. ............ 85.9

HAIR.-Form: low waves. Texture: medium. Quantity: head, double plus; beard, minus; body, minus.

EyE.-Color: dark brown. Sclera: clear. Iris: zoned.

NosE.-Profile: concavo-convex.

Chest.-Development: plus.

Musculature.-Good.

HEALTH._Excellent.

No. 211

Residence: camp of Sheikh Swadi. Date: June 6, 1928.

Age: 42 ; married for 20 years. Sons: 1 living, 1 dead. Daughters: 1 living.

Brothers: 1 living. Sisters: 1 dead.

Tattoo marks: none.

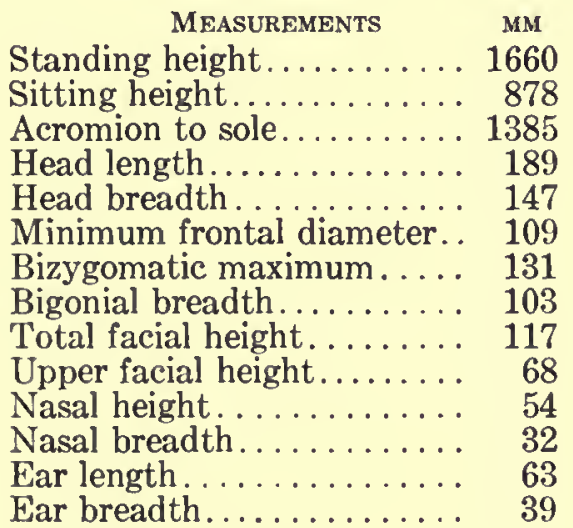

INDICES

Cephalic.............. 77.7

Relative sitting height..... 52.9

Fronto-parietal......... 74.2

Total facial............ 89.4

Upper facial. .......... 52.0

Nasal................. 59.3

Ear...............6. 61.9

Jugomandibular. . . . . . . 78.6

Jugofrontal. .......... 83.2

HAIR.-Texture: medium.

EYE.-Color: dark brown. Sclera: clear. Iris: zoned.

Nose.-Profile: concavo-convex.

TEETH.-Lost: none.

CHEST.-Development: plus.

Musculature.-Excellent.

HEALTH.--Excellent.

No. 212

Residence: camp of Sheikh Swadi. Date: June 6, 1928.

Age: 32 ; unmarried.

Brothers: none. Sisters: 5 living.

Tattoo marks: none.

Measurements MM

Standing height........ 1620

Sitting height............ 812

Acromion to sole........ 1345

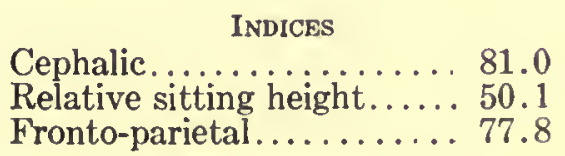




\begin{tabular}{|c|c|c|}
\hline MEASUREMENTS & MM & INDICES \\
\hline Head length. & 194 & Total facial. ......... \\
\hline Head breadth. . & 157 & Upper facial . . . . . . . . . 51.5 \\
\hline Minimum frontal diameter. & 122 & Nasal.............. \\
\hline Bizygomatic maximum ..... & 140 & 59.3 \\
\hline Bigonial breadth........... & 119 & Jugomandibular. . \\
\hline Total facial height... & 123 & Jugofrontal . . . . \\
\hline Upper facial height......... & 72 & \\
\hline Nasal height. ............ & 61 & \\
\hline Nasal breadth............ & 33 & \\
\hline Ear length..... & 54 & \\
\hline - & 32 & \\
\hline
\end{tabular}

HaIR.-Texture: coarse. Color: dark brown. Quantity: head, double plus; beard, minus; body, plus.

EYE.-Color: dark brown. Sclera: bloodshot. Iris: zoned.

Nose.-Profile: straight. Tip: thickness, minus.

TEETH.-Lost: 2 right upper premolars.

CHEST.-Development: plus.

Musculature.-Good.

HEALTH.-Excellent.

No. 213. Plate XXVIII, Figs. 3,4

Residence: camp of Sheikh Swadi. Date: June 6, 1928.

Age: 45; married for 5 years. No children.

Brothers: none. Sisters: none.

Tattoo marks: none.

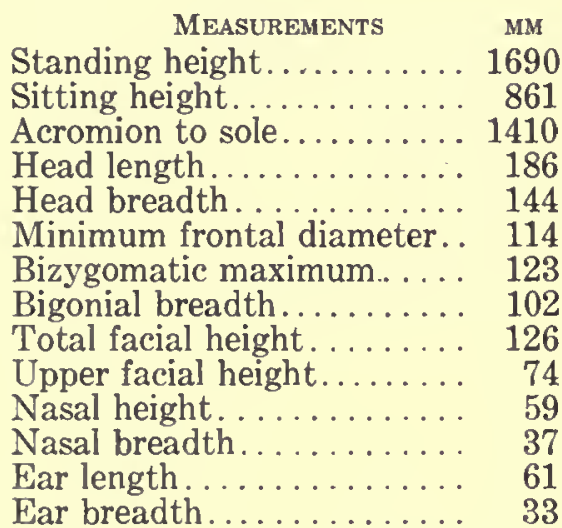

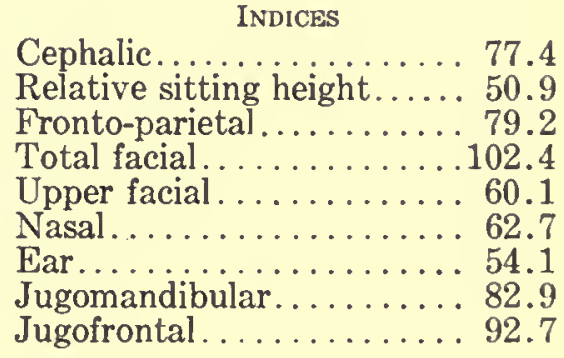

HaIR.-Texture: medium. Color: dark brown and gray. Quantity: head, plus. EYE.-Color: dark brown. Sclera: clear. Iris: zoned.

Nose.-Profile: straight. Wings: medium.

TeEth. - Caries: double plus. Lost: none.

CHEst.-Development: plus.

Musculature.-Good.

HEALTH.-Excellent.

No. 214

Residence: camp of Sheikh Swadi. Date: June 6, 1928.

Age: 30 ; unmarried.

Brothers: 1 dead. Sisters: none.

Tattoo marks: glove on back of right hand.

Measurements MM

Standing height........ 1860

1860
898

Cephalic............. 74.5 


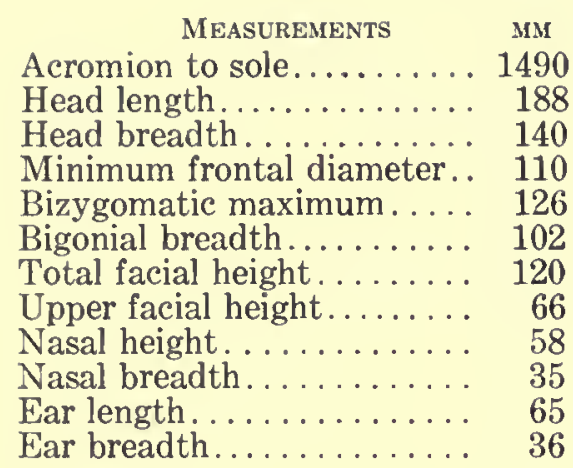

Fronto-parietal......... 78.6

Total facial............ 95.3

Upper facial. .......... 52.4

Nasal. ...............6 60.4

Ear................ 55.4

Jugomandibular......... 81.0

Jugofrontal . . . . . . . . . . 87.3

HAIR.-Form: low waves. Texture: medium. Color: dark brown. Quantity: head, double plus.

EYE.-Color: dark brown. Sclera: clear. Iris: zoned.

NosE.-Profile: straight.

TEETH.-Lost: none.

CHEST.-Development: plus.

Musculature.-Excellent.

HEALTH.-Excellent.

No. 215

Residence: camp of Sheikh Swadi. Date: June 6, 1928.

Age: 30 ; unmarried.

Brothers: 2 living. Sisters: none.

Tattoo marks: one spot on each temple.

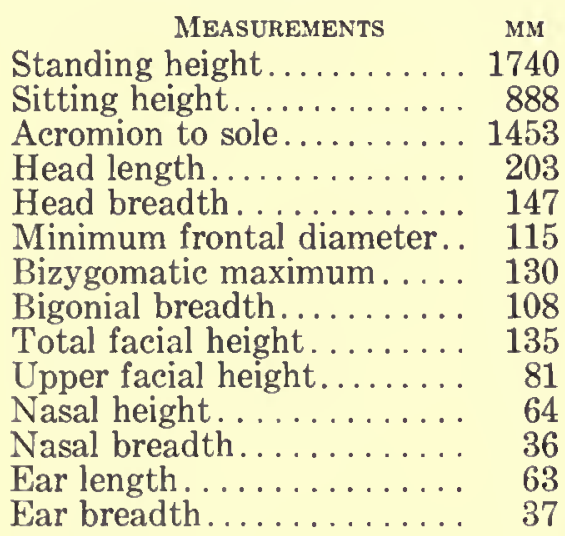

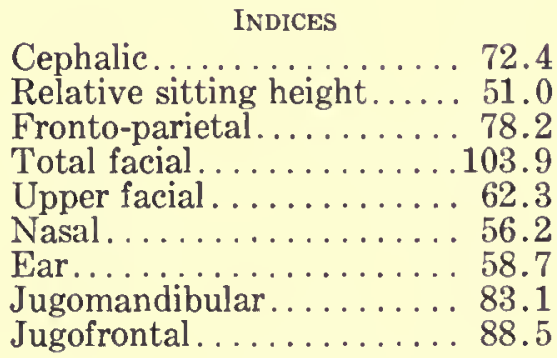

HaIR.-Form: low waves. Texture: fine. Quantity: head, double plus; beard, minus; body, plus.

EYE.-Color: black. Sclera: clear. Iris: zoned.

TEETH.-Lost: none.

CHEST.-Development: plus.

Musculature.-Excellent.

HEALTH.-Excellent.

No. 216

Residence: camp of Sheikh Swadi. Date: June 6, 1928.

Age: 58; married for 30 years. Sons: 1 living. Daughters: 1 living.

Brothers: 1 living. Sisters: none.

Tattoo marks: none.

MEASUREMENTS MM

Standing height......... 1711

Sitting height............ 902

INDICES
Cephalic................. 75.0
Relative sitting height..... 52.7 


\begin{tabular}{|c|c|}
\hline ENTS & \\
\hline ion to & 14 \\
\hline & \\
\hline & \\
\hline meter & \\
\hline & \\
\hline & \\
\hline & \\
\hline & \\
\hline & \\
\hline & \\
\hline & \\
\hline
\end{tabular}

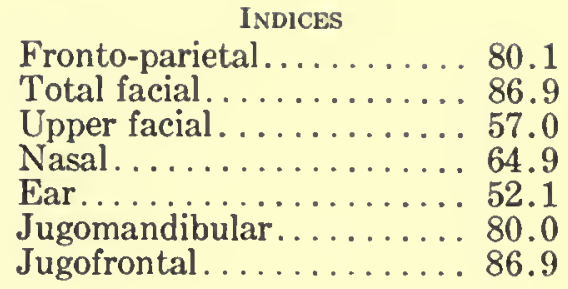

HAIR.-Form: low waves. Texture: medium.

EYE.-Color: dark brown. Sclera: clear. Iris: zoned.

NosE.-Profile: concavo-convex.

TEETH.-Lost: none.

Chest.-Development: plus.

Musculature.-Excellent.

Health.—Excellent.

No. 217

Residence: camp of Sheikh Swadi. Date: June 6, 1928.

Age: 62 ; married for 40 years. Sons: 1 living, 4 dead. Daughters: 3 living.

Brothers: 2 dead. Sisters: 2 dead.

Tattoo marks: glove on back of right hand and spots on inside of right forearm.

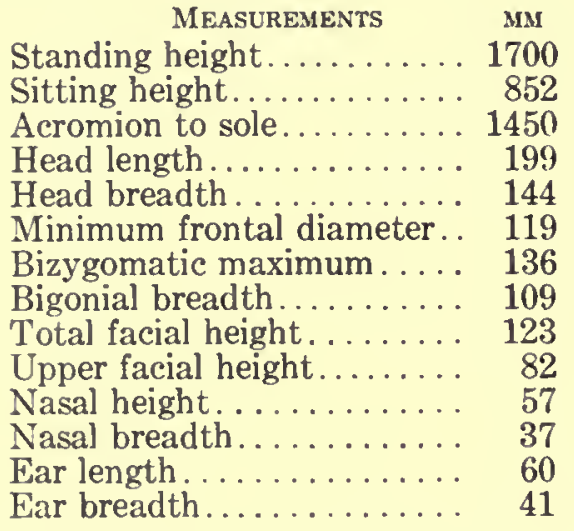

Cephalic............ 72.4

Relative sitting height..... 50.1

Fronto-parietal......... 82.6

Total facial........... 90.5

Upper facial. ........... 60.3

Nasal. ............. 64.9

Ear.................6. 68.4

Jugomandibular. . . . . 80.1

Jugofrontal . . . . . . . . 87.5

HAIR.-Texture: fine. Color: dark brown and gray. Quantity: head, minus; beard, double plus; body, minus.

EYE.-Color: dark brown. Sclera: clear. Iris: zoned.

Nose.-Profile: straight. Wings: medium.

TEETH.-Lost: none.

Chest.-Development: plus.

Musculature.--Excellent.

HEALTh.-Excellent.

\section{No. 218}

Residence: camp of Sheikh Swadi. Date: June 6, 1928.

Age: 30 ; married twice, 12 years ago and 1 year ago. Sons: 2 living, 2 dead. Daughters: 2 living.

Brothers: 1 living. Sisters: 1 living.

Tattoo marks: 3 spots on left side of glabella. 


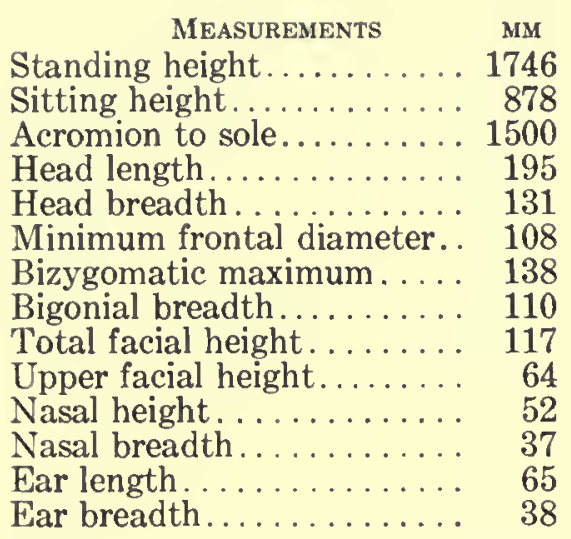

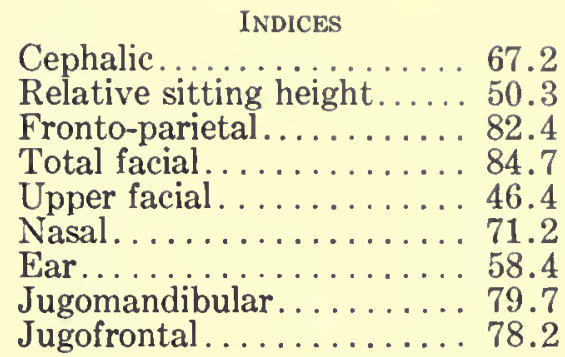

HAIR.-Form: low waves. Color: black. Quantity: head, double plus; beard, plus; body, plus.

EYE.-Color: dark brown. Sclera: bloodshot. Iris: zoned.

NosE.-Profile: straight.

TEETH.-Lost: none.

Chest.-Development: plus.

Musculature.-Excellent.

HEALTH.-Excellent.

\section{No. 219. Plate XXVIII, Figs. 5, 6}

Residence: camp of Sheikh Swadi. Date: June 6, 1928.

Age: 30 ; unmarried.

Brothers: 1 living. Sisters: 1 living.

Tattoo marks: none.

\begin{tabular}{|c|c|}
\hline MEASUREMENTS & MM \\
\hline Standing height....... & 1670 \\
\hline Sitting height. & 813 \\
\hline Acromion to sole. & 1375 \\
\hline Head length.... & 183 \\
\hline Head breadth & 143 \\
\hline Minimum frontal diameter. . & 109 \\
\hline Bizygomatic maximum . . . . . & 136 \\
\hline Bigonial breadth........... & 100 \\
\hline Total facial height. & 119 \\
\hline Upper facial height. & 64 \\
\hline Nasal height. ......... & 52 \\
\hline Nasal breadth & 30 \\
\hline Ear length.... & 58 \\
\hline For hroodth & 32 \\
\hline
\end{tabular}

INDICES

Cephalic..............78.2

Relative sitting height...... 48.6

Fronto-parietal......... 76.2

Total facial .............. 87.5

Upper facial . . . . . . . . 47.1

Nasal. .............. 57.7

Ear................. 55.2

Jugomandibular. . . . . . . . 73.5

Jugofrontal. . . . . . . . . 80.1

HaIR.-Form: low waves. Texture: coarse. Quantity: head, double plus; beard, minus. EYE.-Color: green brown. Sclera: bloodshot. Iris: zoned.

Nose.-Profile: straight.

TEETH._Lost: none.

CHEst.-Development: plus.

Musculature.-Excellent.

HeALTH.—Excellent.

No. 220. Plate XXVIII, Figs. 7, 8

Residence: camp of Sheikh Swadi. Date: June 6, 1928.

Age: 50; married for 15 years. Sons: 4 living, 2 dead. Daughters: 2 living.

Brothers: 3 dead. Sisters: 1 living.

Tattoo marks: none. 


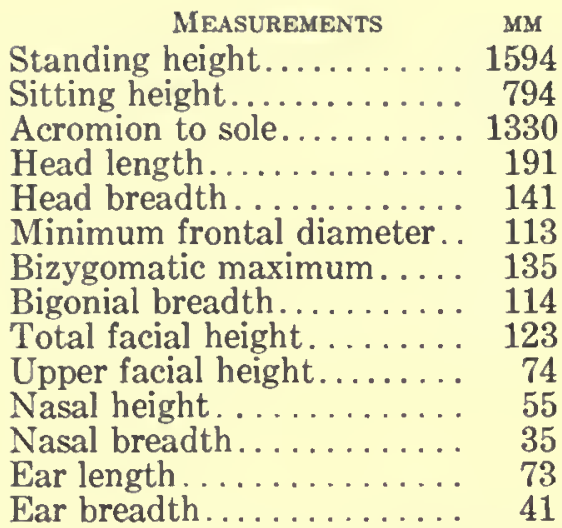

INDICES

Cephalic............. 73.8

Relative sitting height..... 49.7

Fronto-parietal ..........80.2

Total facial.............. 91.1

Upper facial. .......... 54.8

Nasal............... 63.6

Ear............... 56.2

Jugomandibular.......... 84.4

Jugofrontal. . . . . . . . 83.7

HAIR. - Color: dark brown. Quantity: head, plus; beard, double plus; body, plus. EYE.-Color: dark brown. Sclera: clear. Iris: zoned.

Nose.-Profile: straight.

TEeтн.-Wear: double plus. Caries: double plus. Lost: 4.

Chest.-Development: plus.

Musculature.-Good.

HEALTH.-Good.

No. 221. Plate XXIX, Figs. 1, 2

Residence: camp of Sheikh Swadi. Date: June 6, 1928.

Age: 20 ; unmarried.

Brothers: none. Sisters: none.

Tattoo marks: glove on back of right hand.

\begin{tabular}{|c|c|c|}
\hline MEASUREMENTS & MM & INDICES \\
\hline Standing height. & 1690 & Cephalic. \\
\hline Sitting height. & 813 & Relative sitting height..... 48.1 \\
\hline Acromion to sole. & 1386 & Fronto-parietal....... \\
\hline Head length..... & 187 & Total facial. . \\
\hline Head breadth . . . . . . . & 147 & Upper facial . . . . . . \\
\hline Minimum frontal diameter. . & 120 & Nasal. . . . . . . . . . . \\
\hline Bizygomatic maximum . . . . & 134 & Ear. \\
\hline Bigonial breadth......... & 104 & Jugomandibular......... 77. \\
\hline Total facial height. . . . . . . & 121 & Jugofrontal . . . . . . . \\
\hline Upper facial height. . . . . . . & 67 & \\
\hline Nasal height. . . . . . . . . . & 62 & \\
\hline Nasal breadth............ & 36 & \\
\hline$\ldots \ldots \ldots \ldots$ & 60 & \\
\hline ar breadth & 40 & \\
\hline
\end{tabular}

HAIR.-Form: low waves. Texture: coarse. Color: dark brown.

EyE.-Color: dark brown. Sclera: clear. Iris: zoned.

Nose.-Profile: convex.

TEETH.-Lost: none.

Chest.-Derelopment: plus.

Musculature.--Excellent.

HEALTH.-Excellent.

No. 222. Plate XXIX, Figs. 3,4

Residence: camp of Sheikh Swadi. Date: June 6, 1928.

Age: 17; unmarried.

Brothers: 2 living. Sisters: none.

Tattoo marks: none. 


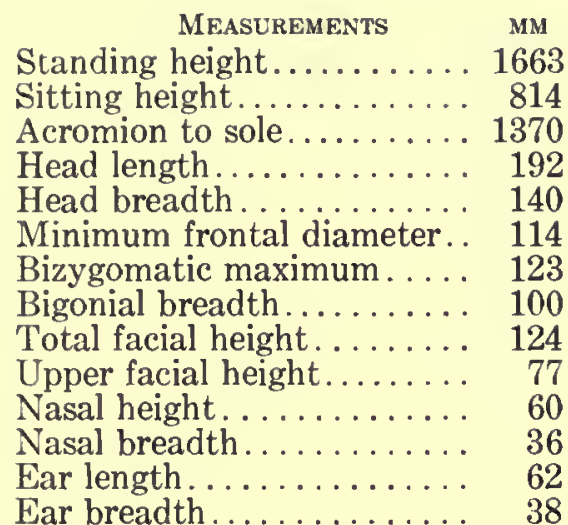

Cephalic............. 72.9

Relative sitting height. . . . 48.9

Fronto-parietal......... 81.4

Total facial. ............. 100.8

Upper facial. . . . . . . . . . 62.6

Nasal. . . . . . . . . . . . 60.0

Ear............... 61.3

Jugomandibular. . . . . . . 81.3

Jugofrontal. . . . . . . . . 92.7

HAIR.-Form: low waves. Texture: medium.

Eye.-Color: dark brown. Sclera: clear. Iris: zoned.

TEETH.-Caries: double plus. Lost: none.

CHEST.-Development: plus.

Musculature.-Excellent.

HEALTH.-Excellent.

No. 223

Residence: camp of Sheikh Swadi. Date: June 6, 1928.

Age: 32 ; married once. Sons: none. Daughters: 1 living.

Brothers: 2 living. Sisters: 1 living.

Tattoo marks: none.

\begin{tabular}{|c|c|}
\hline Measurements & MM \\
\hline Standing height. & 1727 \\
\hline Sitting height. & 898 \\
\hline Acromion to sole & 1445 \\
\hline Head length. & 19 \\
\hline Head breadth & 14 \\
\hline Minimum frontal diameter. & 11 \\
\hline Bizygomatic maximum ..... & 14 \\
\hline Bigonial breadth. & 13 \\
\hline Total facial height... & 107 \\
\hline Upper facial height......... & 73 \\
\hline Nasal height. & 07 \\
\hline Nasal breadth. & \\
\hline Ear length & \\
\hline & \\
\hline
\end{tabular}

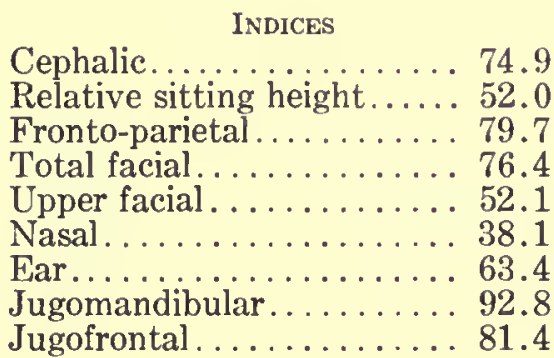

HAIR.-Form: low waves. Texture: fine. Color: black. Quantity: head, double plus; beard, minus; body, plus.

EYE.-Color: dark brown. Sclera: clear. Iris: zoned.

Nose.-Profile: straight.

TEETH.-Lost: none.

Chest.-Development: plus.

Musculature.-Excellent.

HEALTH.-Excellent.

No. 224. Plate XXIX, Figs. 5, 6

Residence: camp of Sheikh Swadi. Date: June 6, 1928.

Age: 40 ; married 3 times, 15 years ago, 6 years ago, and 2 years ago.

Sons: 1 living. Daughters: 5 living, 1 dead.

Brothers: 2 living. Sisters: 2 living, 2 dead.

Tattoo marks: none. 


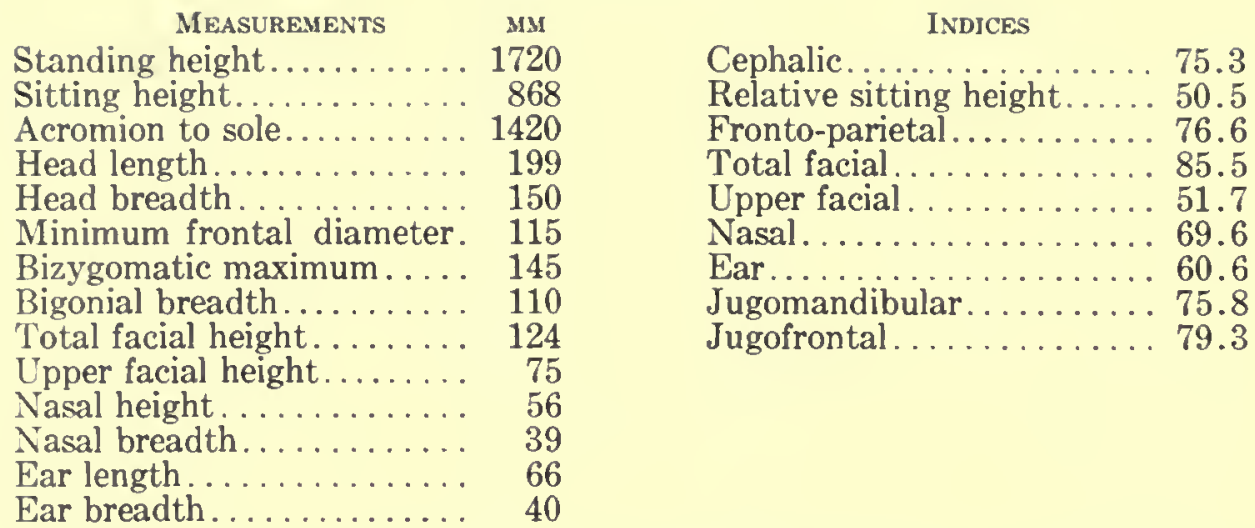

HaIR.-Form: low waves. Texture: fine. Quantity: head, minus; beard, plus; body, plus. EYE.-Color: green brown. Sclera: bloodshot. Iris: zoned.

Nose.-Profile: convex. Wings: medium.

TEETH.-Lost: 2.

Chest.-Development: plus.

Musculature.-Excellent.

HEALTH.--Excellent.

\section{No. 225. Plate XXIX, Figs. 7, 8}

Residence: camp of Sheikh Swadi. Date: June 6, 1928.

Age: 30 ; married for 13 years, wife now dead. No children.

Brothers: none. Sisters: none.

Tattoo marks: none.

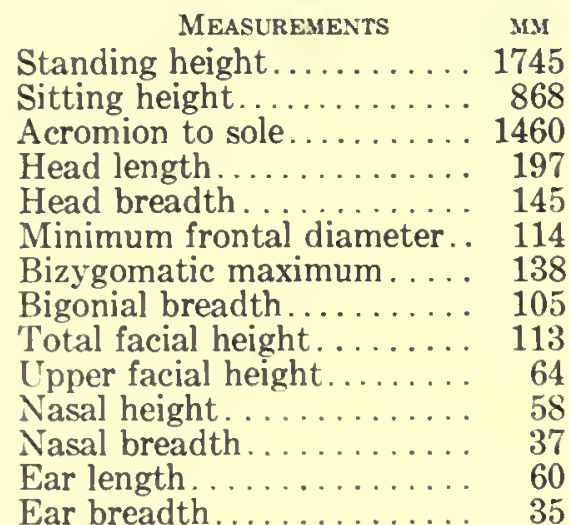

INDICES

Cephalic............. 73.6

Relative sitting height..... 49.7

Fronto-parietal......... 78.6

Total facial ............... 81.8

Upper facial. .......... 46.3

Nasal. ..............6 63.8

Ear................. 58.3

Jugomandibular. . . . . . . 76.1

Jugofrontal. .......... 82.6

HAIR.-Color: dark brown black.

EYE.-Color: dark brown. Sclera: clear. Iris: zoned.

NOSE.-Profile: straight. Wings: medium.

Chest.-Derelopment: plus.

Musculature.-Excellent.

HEALTH.-Excellent.

No. 226. Plate XXX, Figs. 1, 2

Residence: camp of Sheikh Swadi. Date: June 6, 1928.

Age: 20; unmarried.

Brothers: 1 living. Sisters: 2 living.

Tattoo marks: none. 


\begin{tabular}{|c|c|}
\hline MEasurements & MM \\
\hline Standing height. & 1600 \\
\hline Sitting height. & 818 \\
\hline Acromion to sole. & 1310 \\
\hline Head length. & 189 \\
\hline Head breadth. & 137 \\
\hline Minimum frontal diameter. & 117 \\
\hline Bizygomatic maximum. . . . & 131 \\
\hline Bigonial breadth. & 108 \\
\hline Total facial height. & 115 \\
\hline Upper facial height. & 69 \\
\hline Nasal height. & 60 \\
\hline Nasal breadth. & 35 \\
\hline Ear length. & 56 \\
\hline Ear breadth & \\
\hline
\end{tabular}

Cephalic............ 72.4

Relative sitting height.... 51.2

Fronto-parietal......... 85.4

Total facial. ........... 87.8

Upper facial . . . . . . . . . . 52.7

Nasal................ 58.3

Ear................6. 62.5

Jugomandibular. . . . . . 82.5

Jugofrontal . . . . . . . . 8. 89.3

HAIR.-Form: low waves. Texture: medium. Color: dark brown black. Quantity: head, plus; beard, minus.

EYE.-Color: dark brown. Sclera: clear. Iris: zoned.

Nose.-Profile: straight. Wings: medium.

TEETH.-Lost: none.

CHEST.-Development: plus.

Musculature.-Excellent.

HEALTH.-Excellent.

\section{No. 227. Plate XXX, Figs. 3, 4}

Residence: camp of Sheikh Swadi. Date: June 6, 1928.

Age: 30 ; married for 7 years. No children.

Brothers: 2 living. Sisters: none.

Tattoo marks: none.

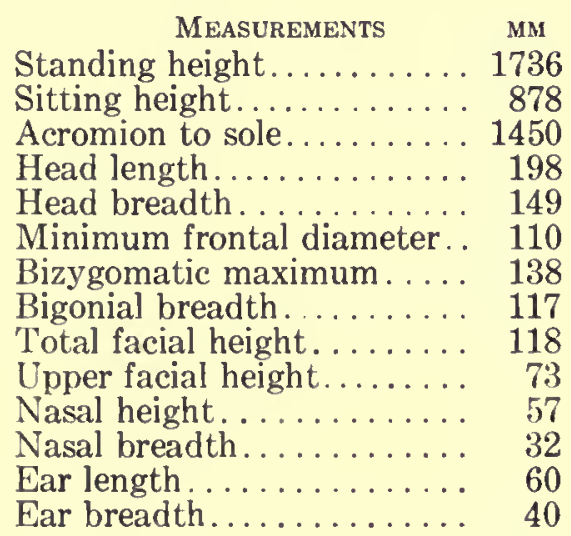

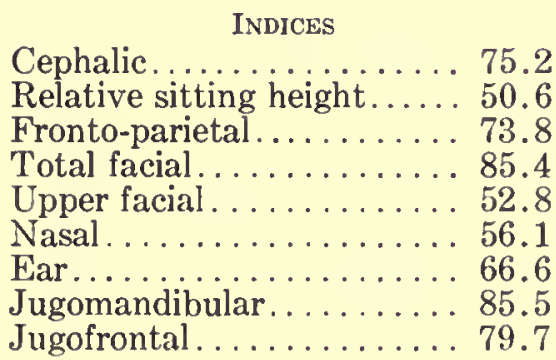

HaIR. -Form: low waves. Color: dark brown. Quantity: head, plus; beard, plus. EyE.-Color: dark brown. Sclera: clear. Iris: zoned.

Nose.-Profile: straight.

TEETH.-Lost: none.

CHEsT.-Development: double plus.

Musculature.-Excellent.

HEALTH.-Excellent.

No. 228. Plate XXX, Figs. 5, 6

Residence: camp of Sheikh Swadi. Date: June 6, 1928.

Age: 18; unmarried.

Brothers: 1 dead. Sisters: none.

Tattoo marks: glove on back of right hand, spot on glabella, and spots on both temples. 


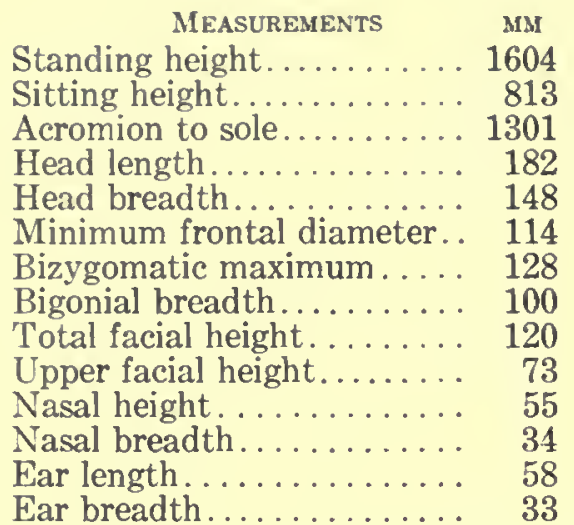

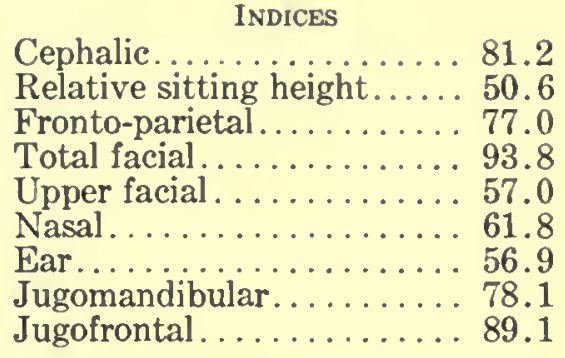

HAIR. - Form: low waves. Texture: fine. Quantity: head, double plus; beard, double minus. EYE.-Color: dark brown. Sclera: clear. Iris: zoned.

Nose.-Profile: convex. Wings: medium.

TEETH.-Wear: plus. Caries: double plus. Unerupted: third molar.

CHEst.-Development: plus.

Musculature.-Excellent.

HEALTH.-Good.

\section{No. 229. Plate XXX, Figs. 7, 8}

Residence: camp of Sheikh Swadi. Date: June 6, 1928.

Age: 35; married for 10 years. Sons: 2 dead. Daughters: 1 living.

Brothers: none. Sisters: 2 living.

Tattoo marks: none.

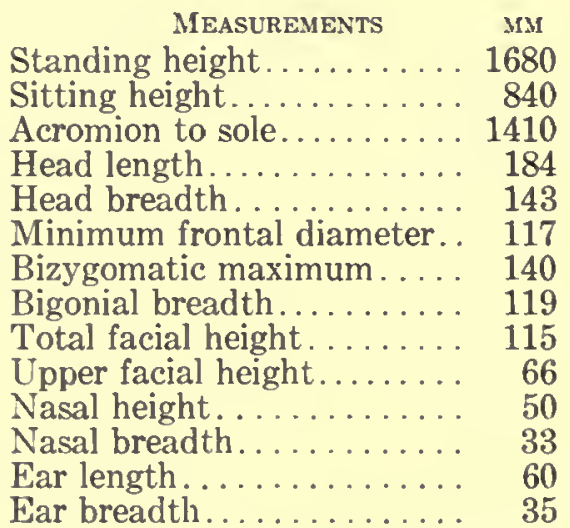

HAIR.—Form: low waves. Texture: fine. Color: black. Quantity: head, double plus; beard, double plus; body, double plus.

EYE.-Color: dark brown. Sclera: clear. Iris: zoned.

Nose.-Profile: straight. Wings: medium.

TEETH.-Lost: none.

ChesT.-Development: plus.

Musculature.-Excellent.

HEALTH.-Excellent.

\section{No. 230. Plate XXXI, Figs. 1, 2}

Residence: camp of Sheikh Swadi. Date: June 6, 1928.

Age: 50 ; married for 30 years. Sons: 1 living. Daughters: 2 living.

Brothers: 1 dead. Sisters: 2 living.

Tattoo marks: none. 


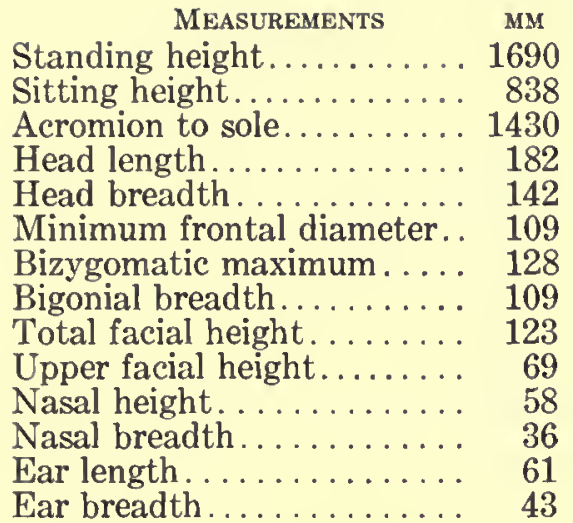

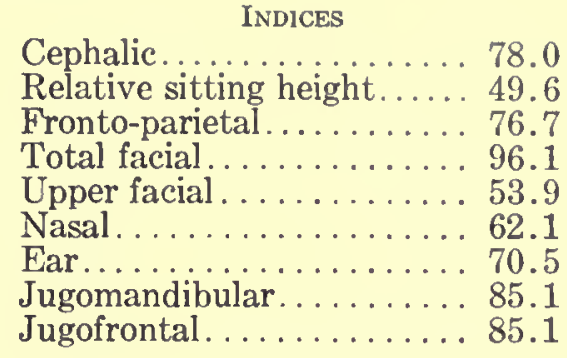

HaIR.-Form: low waves. Texture: medium. Color: dark brown and gray. Quantity: head, plus; beard, plus; body, average.

EYE.-Color: gray brown. Sclera: clear. Iris: zoned.

NosE.-Profile: straight. Wings: medium-compressed.

TEETH.-Lost: none.

Chest.-Development: plus.

Musculature.-Excellent.

HEALTH.-Good.

Deformed right hand, radius, and ulna.

No. 231. Plate XXXI, Figs. 3, 4

Residence: camp of Sheikh Swadi. Date: June 6, 1928.

Age: 55; married for 22 years. Sons: 1 living. Daughters: none.

Brothers: 3 living. Sisters: 1 dead.

Tattoo marks: two crosses on inside of left forearm.

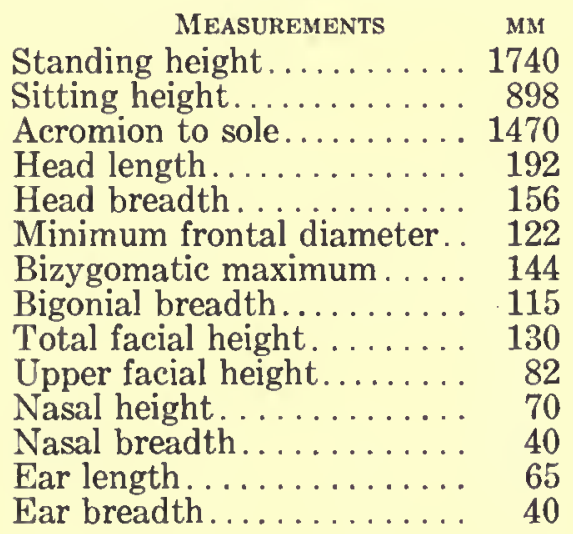

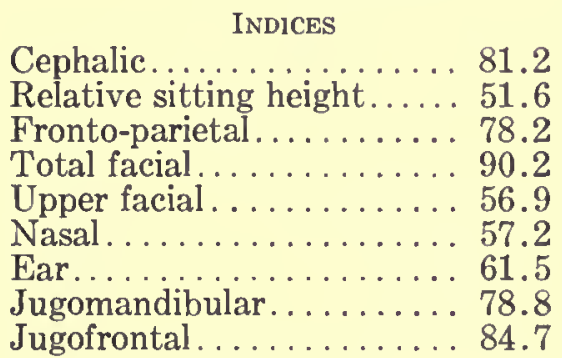

Jugomandibular......... 84.8

HAIR.-Texture: medium. Color: dark brown. Quantity: head, average; beard, plus; body, plus.

EYE.-Color: dark brown. Sclera: clear. Iris: zoned.

Nose.-Profile: straight. Tip: thickness, double plus. Wings: flaring.

TEETH.-Lost: none.

Chest.-Development: plus.

Musculature.-Excellent.

HEALTH.-Excellent.

No. 232. Plate XXXI, Figs. 5, 6

Residence: camp of Sheikh Swadi. Date: June 6, 1928.

Age: 25; unmarried. 
Brothers: 3 living. Sisters: 1 dead.

Tattoo marks: none.

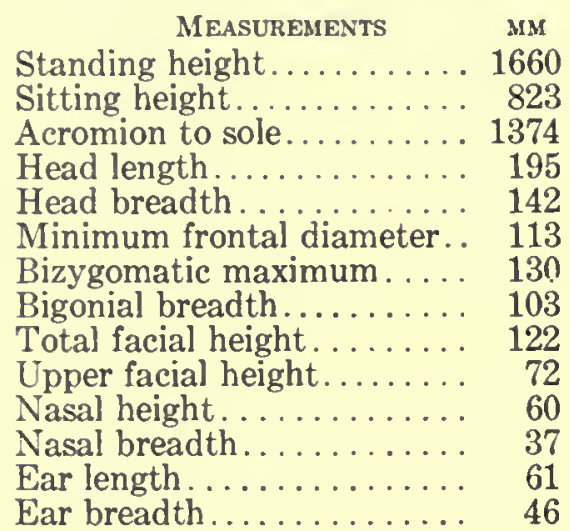

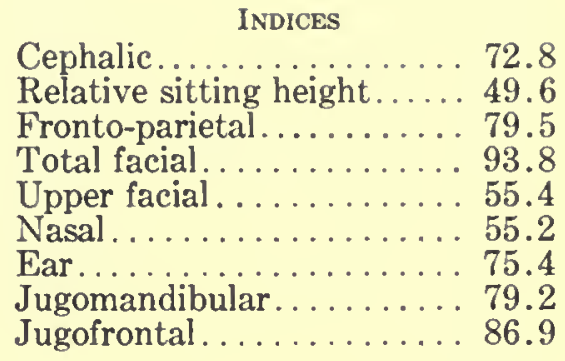

Halr.-Form: low waves. Texture: fine. Color: dark brown black. Quantity: head, triple plus; beard, plus; body, plus.

EYE.-Color: dark brown. Sclera: clear. Iris: zoned.

Nose.-Profile: straight. Wings: medium.

TEETH.-Lost: none.

CHEST.-Development: plus.

Musculature.-Excellent.

HEALTH.—Excellent.

No. 233

Residence: camp of Sheikh Swadi. Date: June 6, 1928.

Age: 100 ; married for 50 years.

Sons: 1 living. Daughters: none.

Brothers: none. Sisters: none.

Tattoo marks: none.

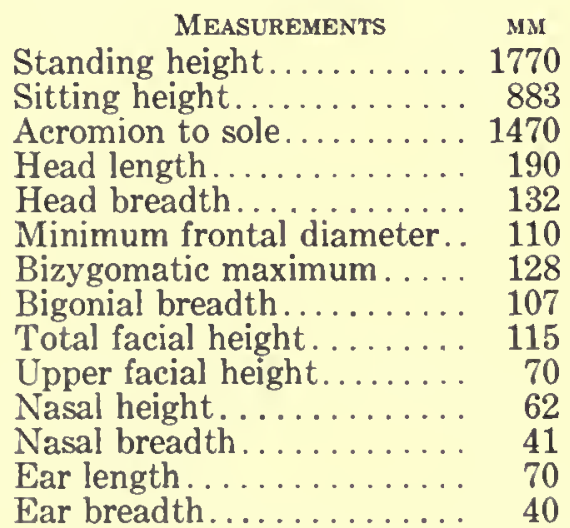

HaIR.-Form: low waves. Texture: medium. Color: gray. Quantity: head, minus; beard, triple plus; body, double plus.

EYE.-Color: dark brown. Sclera: bloodshot. Iris: zoned.

EYeBrow.-Thickness: double plus. Concurrency: double plus. Lateral extent: double plus. Brow-ridges: median.

Nose.-Profile: straight. Tip: depressed. Wings: flaring, plus.

TеEтн.-Wear: double plus. Caries: plus. Lost: none.

EAR.-Lobe: free. Size: average. Helix: double plus. Darwin's Point: double plus. 
Chest.-Development: plus.

Musculature.-Good.

HEALTh.-Good.

No. 234. Plate XXXI, Figs. 7, 8

Residence: camp of Sayyid Ruda. Date: June 7, 1928.

Age: 30 ; married for 10 years. Sons: 1 living. Daughters: none.

Brothers: none. Sisters: 1 living.

Tattoo marks: none.

\begin{tabular}{|c|c|}
\hline MEASUREMENTS & MM \\
\hline Standing height...... & 1764 \\
\hline Sitting height. & 940 \\
\hline Acromion to sole. & 1478 \\
\hline Head length. & 188 \\
\hline Head breadth. & 139 \\
\hline Minimum frontal diameter. & 113 \\
\hline Bizygomatic maximum. . . . & 121 \\
\hline Bigonial breadth.......... & 110 \\
\hline Total facial height. ........ & 119 \\
\hline Upper facial height........ & 74 \\
\hline Nasal height. . . . . . . . . . & 6 \\
\hline Nasal breadth. . . . . . . . . & \\
\hline Ear lengt & 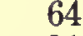 \\
\hline & \\
\hline
\end{tabular}

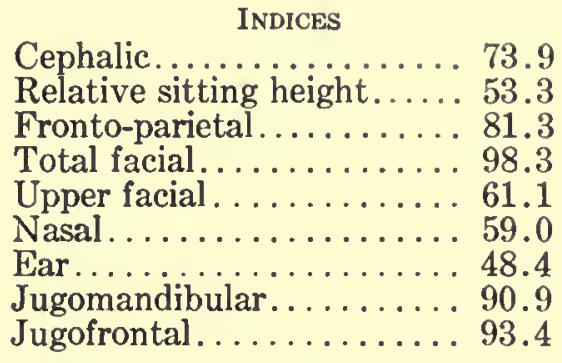

Halr.-Form: low waves. Texture: medium. Color: dark brown. Quantity: head, double plus; beard, plus; body, plus.

EYE.-Color: dark brown. Sclera: bloodshot. Iris: zoned.

NosE.-Profile: straight.

TEETH.-Lost: none.

Chest.-Development: plus.

Musculature.-Excellent.

HEALTH.--Excellent.

No. 235. Plate XXXII, Figs. 1, 2

Residence: camp of Sayyid Ruda. Date: June 7, 1928.

Age: 21; unmarried.

Brothers: 2 dead. Sisters: 2 dead.

Tattoo marks: none.

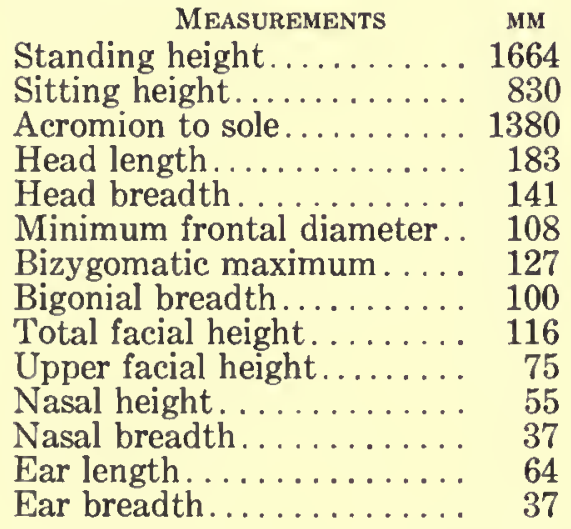

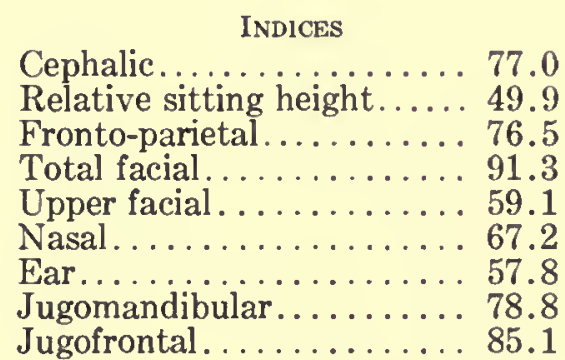

HAIR.-Form: low waves. Texture: medium.

Eye.-Color: dark brown. Sclera: bloodshot. Iris: zoned.

NosE.-Profile: straight. Wings: medium. 
TEeTH.-Lost: none.

CHEST.-Development: plus.

Musculature.-Excellent.

HEALTH.-Excellent.

No. 236. Plate XXXII, Figs. 3, 4

Residence: camp of Sayyid Ruda. Date: June 7, 1928.

Age: 20; unmarried.

Brothers: 1 living, 1 dead. Sisters: 2 living.

Tattoo marks: none.

\begin{tabular}{|c|c|}
\hline MEASUREMENTS & MM \\
\hline Standing height. & 1701 \\
\hline Sitting height. & 87 \\
\hline Acromion to sole. & 142 \\
\hline Head length & 19 \\
\hline Head breadth & 147 \\
\hline Minimum frontal diameter. . & 11 \\
\hline Bizygomatic maximum . . . . . & 13 \\
\hline Bigonial breadth. & 10 \\
\hline Total facial height & 1 \\
\hline Upper facial height..... & 66 \\
\hline Nasal height & \\
\hline Nasal bread & \\
\hline $\mathrm{Ea}$ & \\
\hline$\ldots$ & \\
\hline
\end{tabular}

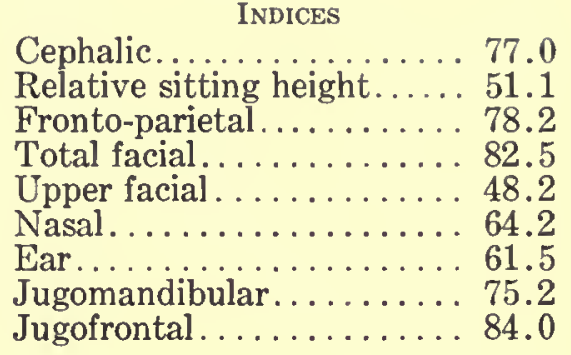

2.5

Jugomandibular......... 75.2

Jugofrontal. ........... 84.0

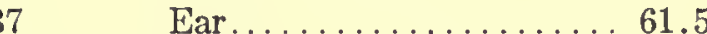

HAIR.-Form: low waves. Texture: medium. Color: black but dyed with henna.

EYE.-Color: dark brown. Sclera: bloodshot. Iris: zoned.

Nose.-Profile: concavo-convex.

TEETH.-Lost: none.

CHEST.-Development: plus.

Musculature.-Excellent.

HEALTH.-Excellent.

No. 237. Plate XXXII, Figs. 5, 6

Residence: camp of Sayyid Ruda. Date: June 7, 1928.

Age: 25; unmarried.

Brothers: 1 living. Sisters: 1 dead.

Tattoo marks: spot on right wrist.

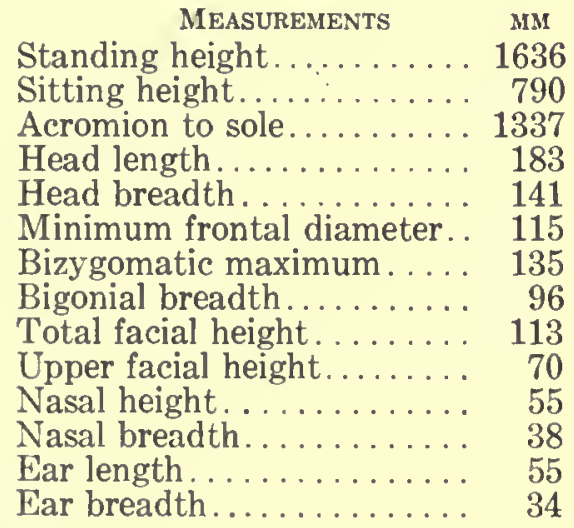

HAIR.-Form: curly frizzly. Texture: coarse. Color: black.

EYE.-Color: dark brown. Sclera: clear. Iris: zoned.

Nose.-Profile: convex. Wings: medium. 
TEeTH.-Lost: none.

Chest.-Development: plus.

Musculature.-Excellent.

HEALTH.-Excellent.

\section{No. 238. Plate XXXII, Figs. 7, 8}

Residence: camp of Sayyid Ruda. Date: June 7, 1928.

Age: 25; married for 8 years. No children.

Brothers: 1 living, 1 dead. Sisters: 1 living.

Tattoo marks: glove on right hand and gazelle on inside of right forearm.

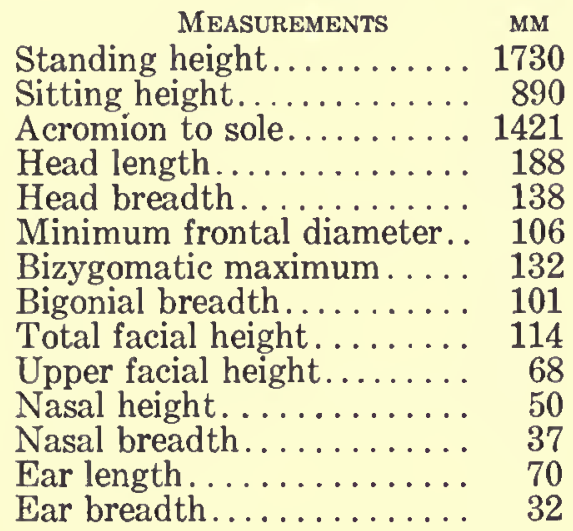

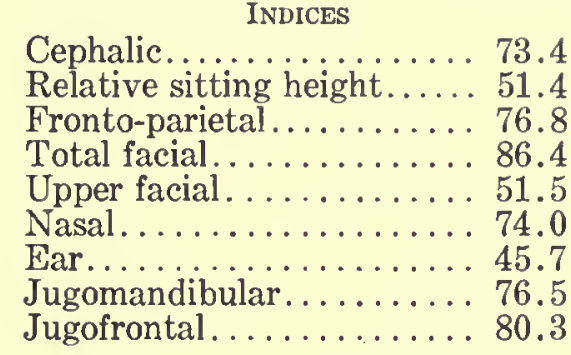

Cephalic............... 73.4

Relative sitting height..... 51.4

ronto-parietal ........76.8

Upper facial. ...........................

Nasal................ 74.0

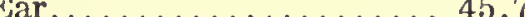

Jugofrontal. . . . . . . . . 80.3

Halk.-Form: low waves. Texture: medium. Color: dark brown, dyed with henna. Quantity: head, double plus; beard, average; body, average.

EYE.-Color: dark brown. Sclera: clear. Iris: zoned.

NosE.-Profile: straight.

TEETH.-Lost: none.

Chest.-Development: plus.

Musculature.-Excellent.

HEALTH.-Excellent.

No. 239. Plate XXXIII, Figs. 1, 2

Residence: camp of Sayyid Ruda. Date: June 7, 1928.

Age: 30; married for 10 years. Sons: 1 living. Daughters: 1 dead.

Brothers: 1 living, 1 dead. Sisters: none.

Tattoo marks: glove on back of right hand, spots on both temples.

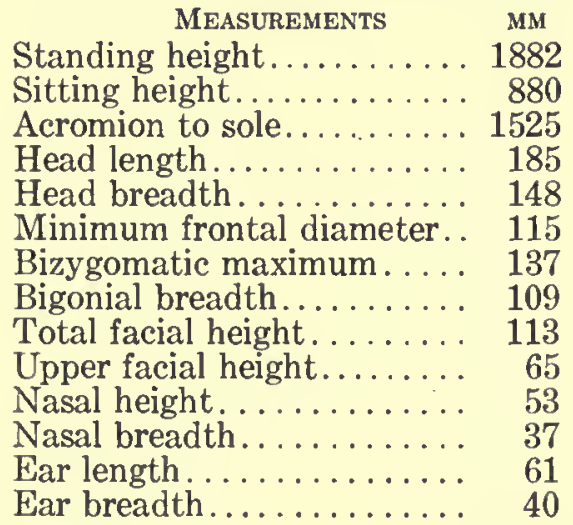

Cephalic............... 79.9

Relative sitting height..... 46.7

Fronto-parietal.......... 77.6

Total facial............ 82.4

Upper facial. . . . . . . . . 47.4

Nasal. . . . . . . . . 69.8

Ear.................65.6

Jugomandibular. . . . . 79.6

. Jugofrontal . . . . . . . . . . 83.9

HaIR.-Form: low waves. Texture: medium. Color: dark brown. Quantity: head, double plus; beard, average. 
EYE.-Color: dark brown. Sclera: bloodshot. Iris: zoned.

Nose.-Profile: straight. Tip: thickness, minus. Wings: medium.

TEETH.-Lost: none.

Chest.-Development: plus.

Musculature.--Excellent.

HEALTH.-Excellent.

No. 240

Residence: camp of Sayyid Ruda. Date: June 7, 1928.

Age: 30 ; married for 7 years. Sons: 2 living. Daughters: none.

Brothers: 1 living. Sisters: 4 living.

Tattoo marks: spots on back of right hand and one spot on each temple.

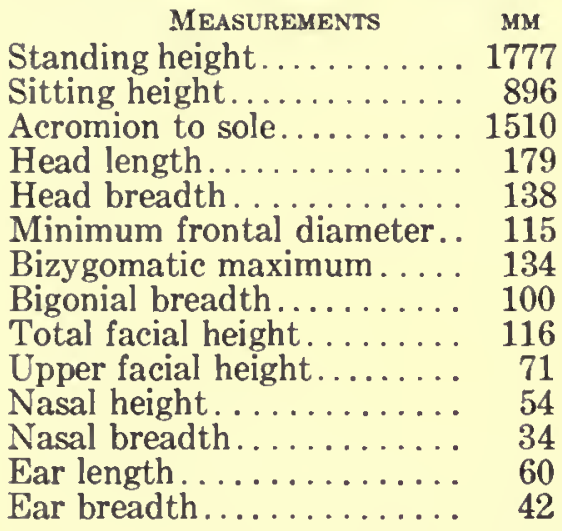

Cephalic............. 77.0

Relative sitting height ...... 50.4

Fronto-parietal.........88.3

Total facial........... 86.6

Upper facial. .......... 53.0

Nasal. . . . . . . . . . 62.9

Ear ............. 70

Jugomandibular.......... 74.7

Jugofrontal............. 85.8

HaIR.-Form: low waves. Texture: medium. Color: dark brown. Quantity: head, triple plus; beard, plus; body, plus.

EYE.-Color: dark brown. Sclera: bloodshot. Iris: zoned.

Nose.-Profile: concave.

TEETH.-Lost: first right upper molar.

Chest.-Development: plus.

Musculature.-Excellent.

HEALTH.-Excellent.

No. 241. Plate XXXIII, Figs. 3, 4

Residence: camp of Sayyid Ruda. Date: June 7, 1928.

Age: 26; married for 15 years. Sons: 1 living, 1 dead. Daughters: 1 dead.

Brothers: 2 dead. Sisters: 1 living.

Tattoo marks: glove on back of right hand.

\begin{tabular}{|c|c|c|}
\hline MEASUREMENTS & MM & INDICES \\
\hline Standing height. & 1666 & Cephalic. \\
\hline Sitting height.... & 870 & Relative sitting height \\
\hline omion to sole. & 1411 & Fronto-parietal..... \\
\hline d length. & 178 & Total facial. \\
\hline breadth. & 143 & Upper facial \\
\hline n frontal diameter. & 109 & Nasal...... \\
\hline natic maximum.... & 137 & Ear. \\
\hline lal br & 96 & Jugomandibular. . \\
\hline facia & 114 & Jugofrontal. \\
\hline
\end{tabular}

Upper facial height......... . 110

Nasal height............ 58

Nasal breadth........... 38

Ear length.................. 60

Ear breadth........... 36 
HaIr.-F orm: low waves. Texture: medium. Color: black. EYE.-Color: dark brown. Sclera: bloodshot. Iris: zoned.

Nose.-Profile: concave.

TеEтH.-Lost: none.

CHEST.-Development: plus.

Musculature.-Good.

HEALTH.-Poor.

No. 242. Plate XXXIII, Figs. 5, 6

Residence: camp of Sayyid Ruda. Date: June 7, 1928.

Age: 20; unmarried.

Brothers: 1 living. Sisters: 1 living.

Tattoo marks: none.

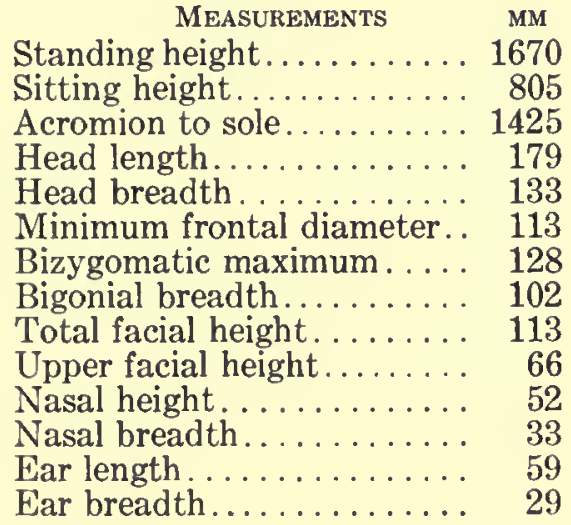

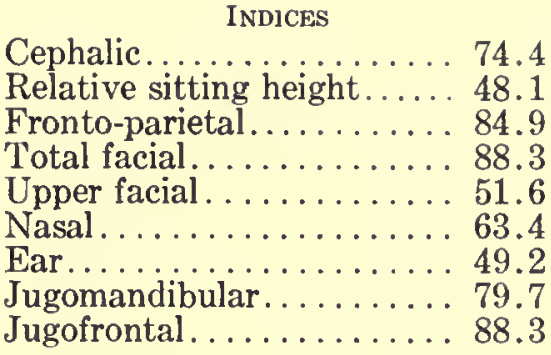

HAIR.-Form: low waves. Texture: fine. Color: black.

EYE.-Color: dark brown. Sclera: bloodshot. Iris: zoned.

NosE.-Profile: straight. Wings: medium.

TEETH.-Lost: none.

Chest.-Development: plus.

Musculature.-Good.

HEALTH.-Excellent.

No. 243. Plate XXXIII, Figs. 7, 8

Residence: camp of Sayyid Ruda. Date: June 7, 1928.

Age: 35 ; unmarried.

Brothers: 2 living, 2 dead. Sisters: 1 living.

Tattoo marks: none.

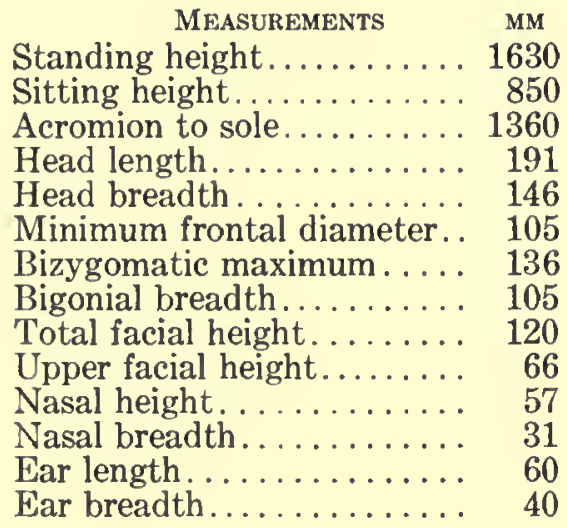

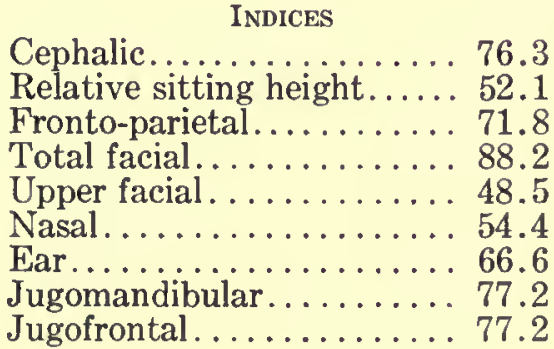


HaIr.-Form: low waves. Texture: coarse. Color: dark brown. Quantity: head, plus; beard, plus; body, double plus.

EYE.-Color: dark brown. Sclera: bloodshot. Iris: zoned.

NosE.-Profile: straight. Wings: medium.

TEETH.-Lost: none.

Chest.-Development: plus.

MUSCULATURE.-Good.

HEALTH.-Good.

No. 244. Plate XXXIV, Figs. 1, 2

Residence: camp of Sayyid Ruda. Date: June 7, 1928.

Age: 20; unmarried.

Brothers: none. Sisters: none.

Tattoo marks: glove on back of right hand.

\begin{tabular}{|c|c|}
\hline MEasurements & MM \\
\hline Standing height........ & 1727 \\
\hline Sitting height. & 860 \\
\hline Acromion to sole. & 1455 \\
\hline Head length.. & 190 \\
\hline Head breadth. & 138 \\
\hline Minimum frontal diameter. & 108 \\
\hline Bizygomatic maximum..... & 123 \\
\hline Bigonial breadth....... & 96 \\
\hline Total facial height.... & 124 \\
\hline Upper facial height..... & 70 \\
\hline Nasal height & 54 \\
\hline Nasal breadth & 35 \\
\hline Ear lengt & 61 \\
\hline & \\
\hline
\end{tabular}

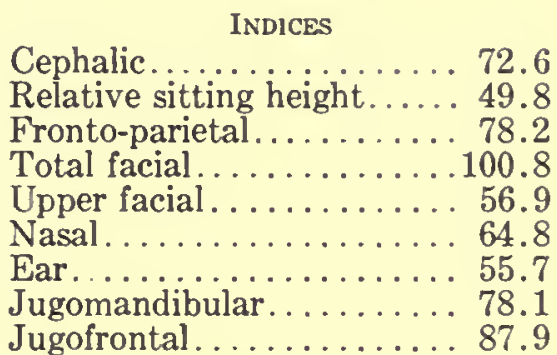

Cephalic..............72.6

Relative sitting height..... 49.8

Upper facial.$\ldots 56.9$

Nasal. ..............6. 64.8

Jugomandibular.......... 78.1

Jugofrontal............ 87.9

HaIR.-Form: low waves. Texture: fine. Color: dark brown.

EYE.-Color: dark brown. Sclera: clear. Iris: zoned.

Nose.-Profile: convex.

TEETH.-Lost: none.

CHEST.-Development: plus.

Musculature.-Good.

HEALTH.-Excellent.

No. 245. Plate XXXIV, Figs. 3,4

Residence: camp of Sayyid Ruda. Date: June 7, 1928.

Age: 35 ; married for 12 years. Sons: 1 living. Daughters: 1 living.

Brothers: 1 living. Sisters: 1 living.

Tattoo marks: none.

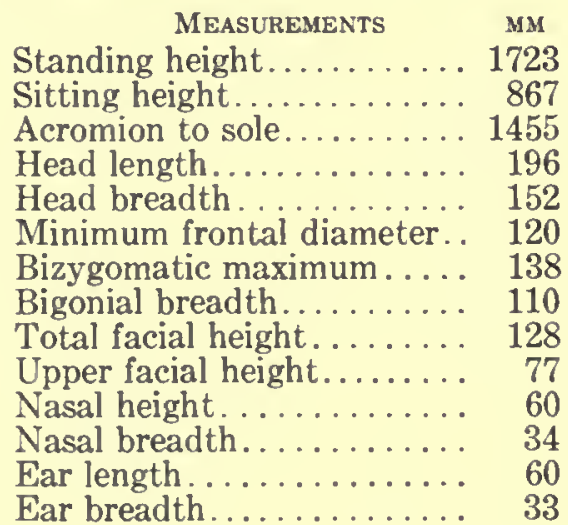

INDICES

Cephalic............... 77.5

Relative sitting height...... 50.3

Fronto-parietal......... 78.9

Total facial .............. 92.7

Upper facial........... 55.8

Nasal. ................ 56.7

Ear................ 54.9

Jugomandibular............ 79.7

Jugofrontal . . . . . . . . 87.0 
HaIr.-Form: low waves. Texture: fine. Color: dark brown and gray.

EYE.-Color: dark brown. Sclera: bloodshot. Iris: zoned.

Nose.-Profi'e: straight. Tip: thickness, minus; depressed. Wings: compressed.

CHEST.-Development: plus.

Musculature.-Excellent.

HEALTH.-Good.

No. 246. Plate XXXIV, Figs. 5, 6

Residence: camp of Sayyid Ruda. Date: June 7, 1928.

Age: 21 ; unmarried.

Brothers: none. Sisters: 1 living.

Tattoo marks: none.

\begin{tabular}{|c|c|}
\hline MEASUREMENTS & MM \\
\hline Standing height. & 1745 \\
\hline Sitting height. & 900 \\
\hline Acromion to sole. & 1465 \\
\hline Head length. & 190 \\
\hline Head breadth. & 143 \\
\hline Minimum frontal diameter.. & 110 \\
\hline Bizygomatic maximum..... & 130 \\
\hline Bigonial breadth....... & 110 \\
\hline Total facial height. . & 115 \\
\hline Upper facial height......... & 63 \\
\hline Nasal height & 53 \\
\hline Nasal breadth & 37 \\
\hline Ear lengt & 65 \\
\hline & \\
\hline
\end{tabular}

Indices
Cephalic............. .

HAIR.-Color: dark brown. Head shaved.

EYE.-Color: dark brown. Sclera: clear. Iris: zoned.

NosE.-Profile: concavo-convex.

CHEST.-Development: plus.

Musculature.-Excellent.

Health.-Poor, much fever.

\section{No. 247. Plate XXXIV, Figs. 7, 8}

Residence: camp of Sayyid Ruda. Date: June 7, 1928.

Age: 25 ; unmarried.

Brothers: 2 living, 2 dead. Sisters: none.

Tattoo marks: spots on back of left hand.

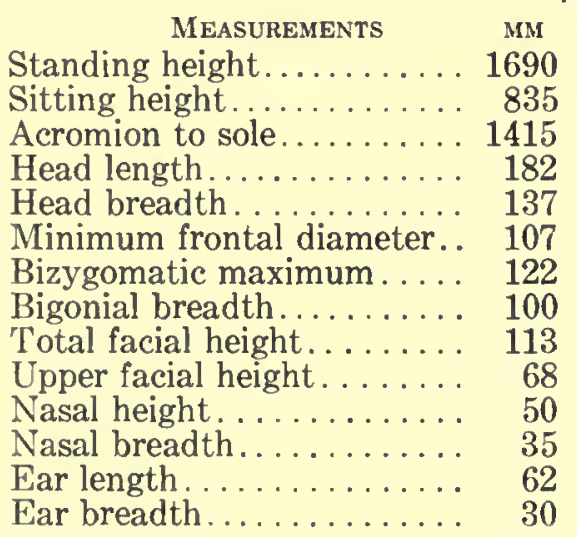

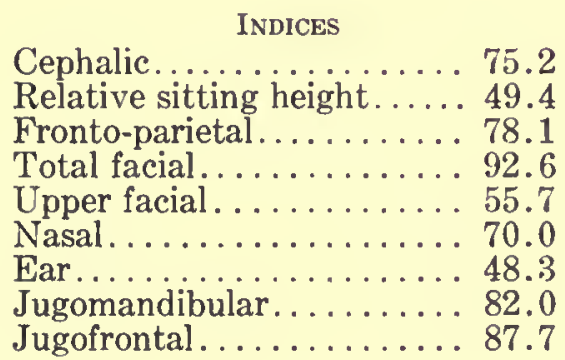

HalR.-Form: low waves. Texture: fine. Quantity: head, double plus; beard, plus; body, average. 
EYE.- Color: dark brown. Sclera: bloodshot. Iris: zoned.

Nose.-Profile: concavo-convex.

T'EETH.-Condition: good.

CHEST.-Development: plus.

Musculature.-Good.

HEALTH.--Excellent.

No. 248. Plate XXXV, Figs. 1, 2

Residence: camp of Sayyid Ruda. Date: June 7, 1928.

Age: 20 ; unmarried.

Brothers: 1 living. Sisters: 4 living.

Tattoo marks: spots on right wrist and one spot on nasion.

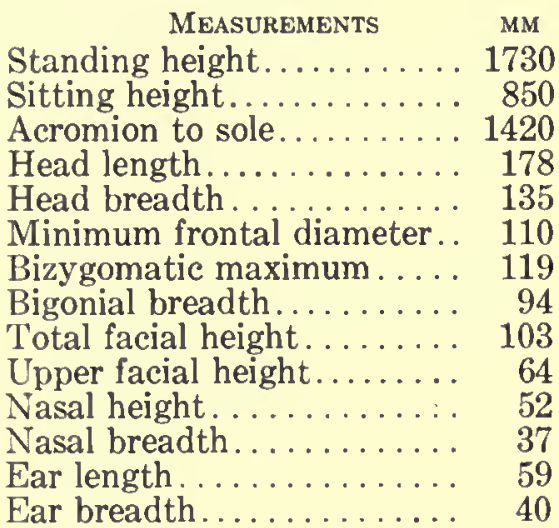

Cephalic.............. 75.8

Relative sitting height...... 49.2

Fronto-parietal..........81.4

Total facial............... 86.5

Upper facial. .......... 53.8

Nasal. . . . . . . . . . . 71.2

Ear..............6. 67.8

Jugomandibular. . . . . . 79.0

Jugofrontal. ........... 92.4

HalR. - Form: low waves. Color: dark brown. Quantity: head, triple plus; beard, minus. EYE.-Color: dark brown. Sclera: clear. Iris: zoned.

Nose.-Profile: concavo-convex.

TEETH.-Lost: none.

CHEST.-Development: plus.

Musculature.-Good.

HEALTH.-Excellent.

No. 249. Plate XXXV, Figs. 3, 4

Residence: camp of Sayyid Ruda. Date: June 7, 1928.

Age: 40 ; married twice, 20 years ago and 10 years ago. Sons: 2, 0, living. Daughters: 1, 1 , living.

Brothers: 1 living. Sisters: 2 dead.

Tattoo marks: none.

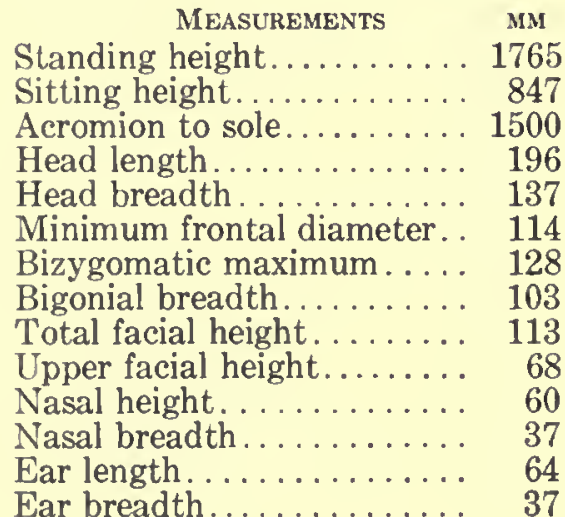

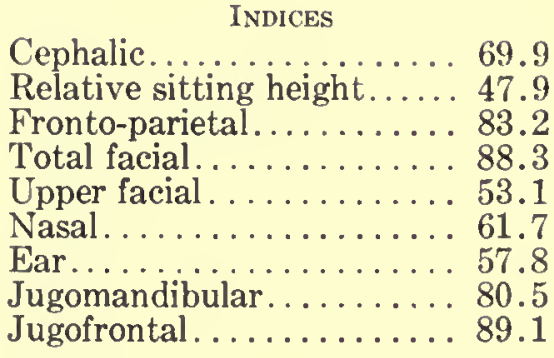


HAIR.-Texture: medium. Color: dark brown and gray. Quantity: head, average; beard, plus; body, plus.

EYE.-Color: dark brown. Sclera: clear. Iris: zoned.

Nose.-Profile: straight.

TEETH.-Lost: none.

CHEST.-Development: plus.

Musculature.-Good.

Health.-Good.

No. 250. Plate XXXV, Figs. 5, 6

Residence: camp of Sayyid Ruda. Date: June 7, 1928.

Age: 45; married twice, 20 years ago and 5 years ago. Sons: 2, 0 , living; 1 , 0 , dead. Daughters: 1,0 , living.

Brothers: none. Sisters: 1 dead.

Tattoo marks: spots on right wrist and spot on each temple.

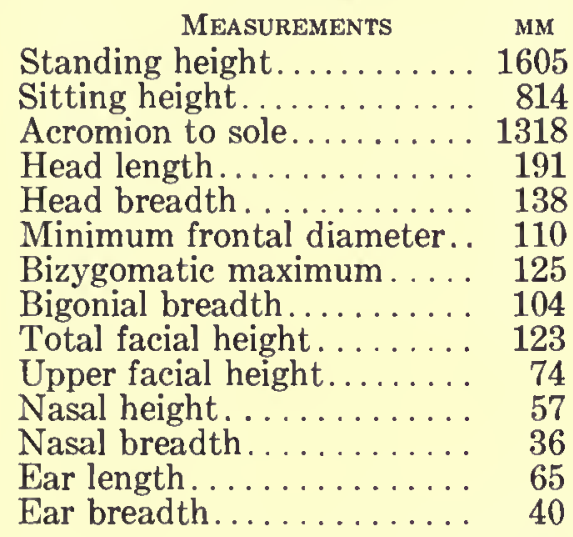

HaIR.-Form: low waves. Color: dark brown and gray.

EYE.-Color: dark brown. Sclera: bloodshot. Iris: zoned.

Nose.-Profile: concave. Tip: elevated. Wings: medium.

TEETH.-Lost: none.

CHEST.-Development: plus.

Musculature.-Good.

HEALTH.-Good.

Cephalic.............. 72.2

Relative sitting height ...... 50.6

Fronto-parietal......... 79.7

Total facial .............. 98.2

Upper facial . . . . . . . . 59.2

Nasal................ 63.1

Ear................6. 61.5

Jugomandibular......... 83.2

Jugofrontal. .......... 88.0

No. 251. Plate XXXV, Figs. 7, 8

Residence: camp of Sayyid Ruda. Date: June 7, 1928.

Age: 40; unmarried.

Brothers: none. Sisters: none.

Tattoo marks: spot on each temple.

MEASUREMENTS MM

Standing height......... 1672

Sitting height........... 810

Acromion to sole.......... 1410

Head length............ 188

Head breadth........... 145

Minimum frontal diameter.. 113

Bizygomatic maximum . . . . 141

Bigonial breadth.......... 107

Total facial height. ........ 118

Upper facial height......... 73

Nasal height............ 57

Nasal breadth............ 35

Ear length............. 58

Ear breadth............ 34

\section{INDICES}

Cephalic.............. 77.1

Relative sitting height...... 48.4

Fronto-parietal......... 77.9

Total facial............. 83.7

Upper facial. ........... 51.7

Nasal. ................. 61.4

Ear................ 58.6

Jugomandibular............ 75.9

Jugofrontal. .......... 80.1 
HalR.-Form: low waves. Texture: fine. Color: dark brown.

EYE.-Color: dark brown. Sclera: clear. Iris: zoned.

NosE.-Profile: convex.

TEETH.-Lost: none.

CHEST.-Development: plus.

Musculature.-Excellent.

HEALTH.-Good.

No. 252. Plate XXXVI, Figs. 1, 2

Residence: camp of Sheikh Miniehil. Date: June 7, 1928.

Age: 40 ; unmarried.

Brothers: 7 living. Sisters: 4 living.

Tattoo marks: glove on right hand and gazelle and spots on inside of right forearm.

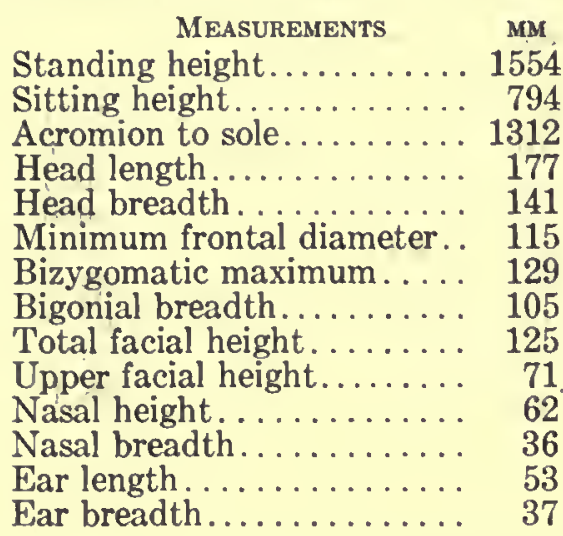

Cephalic............. 79.6

Relative sitting height..... 51.1

Fronto-parietal......... 81.5

Total facial ............ 96.8

Upper facial . . . . . . . . . 55.0

Nasal............... 58.1

Ear.................6. 69.8

Jugomandibular. . . . . . 81.4

Jugofrontal . . . . . . . . . . 89.2

HAIR.-Form: low waves. Texture: medium. Color: dark brown.

EYE.-Color: dark brown. Sclera: bloodshot: Iris: zoned.

NosE.-Profile: straight.

TEETH.-Lost: none.

Chest.-Development: plus.

Musculature.-Good.

HEALTH.-Good.

No. 253. Plate XXXVI, Figs. 3, 4

Residence: camp of Sayyid Ruda. Date: June 7, 1928.

Age: 35; married for 3 years. No children.

Brothers: 1 living, 1 dead. Sisters: none.

Tattoo marks: spots on both wrists.

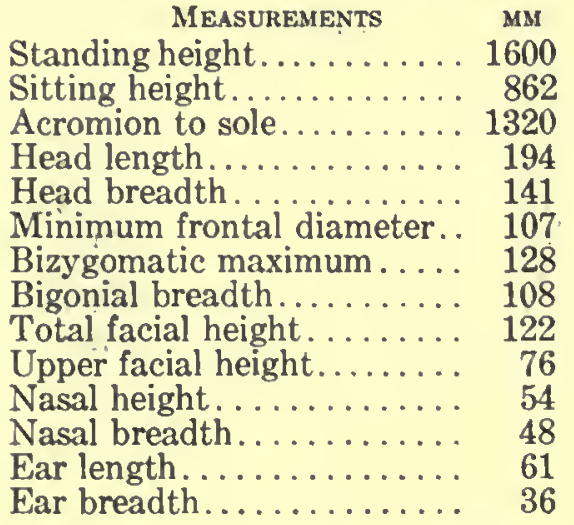

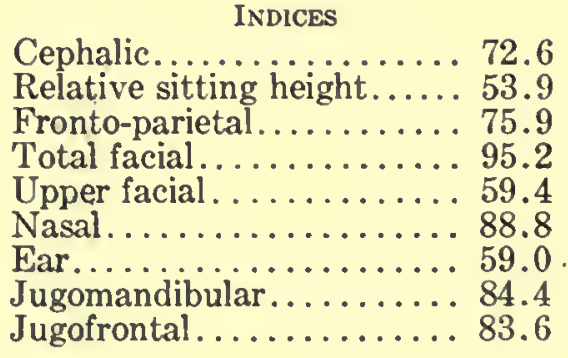


HAIR.-Form: low waves. Texture: fine. Color: dark brown. Quantity: head, double plús; beard, plus; body, plus.

EyE.-Sclera: bloodshot. Iris: zoned.

CHEsT.-Development: plus.

Musculature.-Excellent.

HEALTH.-Excellent.

No. 254. Plate XXXVI, Figs. 5, 6

Residence: camp of Sheikh Miniehil. Date: June 7, 1928.

Age: 55; married for 30 years. Sons: 2 living. Daughters: 1 living.

Brothers: none. Sisters: none.

Tattoo marks: glove and gazelle.

\begin{tabular}{|c|c|}
\hline \multicolumn{2}{|l|}{ Measurements } \\
\hline & 1744 \\
\hline Sitting height. & 850 \\
\hline Acromion to sole. & 1544 \\
\hline Head length.... & 187 \\
\hline Head breadth. & \\
\hline Minimum frontal diameter. & 115 \\
\hline Bizygomatic maximum. . & 138 \\
\hline Bigonial breadth..... & 106 \\
\hline Total facial height. & 130 \\
\hline Nasal height. & 00 \\
\hline Nasal breadth & \\
\hline Ear length & \\
\hline Ear breadt & 32 \\
\hline
\end{tabular}

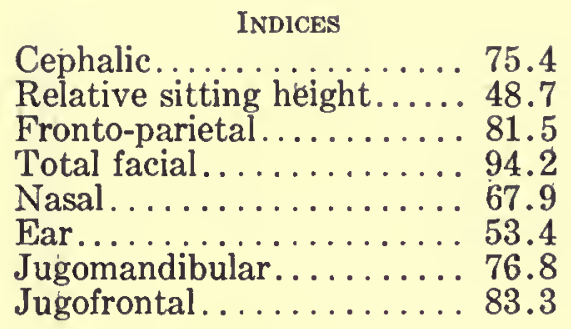

HaIR.-Form: low waves. Texture: medium. Color: dark brown and gray.

EYE.-Color: dark brown. Sclera: bloodshot. Iris: zoned.

NosE.-Profile: straight. Wings: medium.

TEETH.—Lost: 5.

Chest,-Development: plus.

MUSCUlature.-Good.

HEALTH.-Good.

No. 255

Residence: camp of Sheikh Khadem. Date: June 7, 1928.

Age: 40 ; married for 14 years. No children.

Brothers: 3 dead. Sisters: 2 dead.

Tattoo marks: none.

\begin{tabular}{|c|c|}
\hline MEASUREMENTS & MM \\
\hline Standing height. . & 1680 \\
\hline Sitting height. & 857 \\
\hline Acromion to sole. & 1425 \\
\hline Head length..... & 175 \\
\hline Head breadth & 131 \\
\hline Minimum frontal diameter. . & 105 \\
\hline Bizygomatic maximum ..... & 131 \\
\hline Bigonial breadth. & 105 \\
\hline Total facial height. & 128 \\
\hline Upper facial height. . & 83 \\
\hline height. & 66 \\
\hline Nas & 30 \\
\hline Ear len & 65 \\
\hline$\ldots \ldots \ldots$ & \\
\hline
\end{tabular}

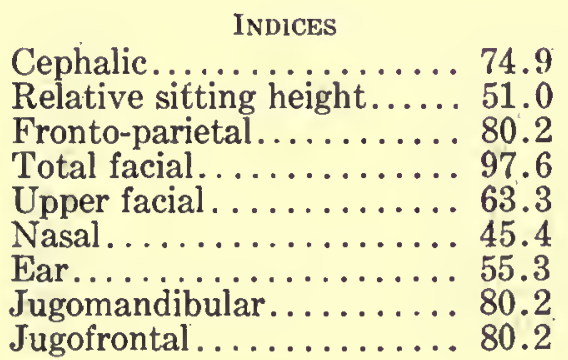

Jugofrontal .............. 80.2

HaIR.-Form: low waves. Texture: fine. Color: black. Quantity: head, double plus; beard, plus; body, plus. 
EYE.-Color: dark brown. Sclera: bloodshot. Iris: zoned.

Nose.-Profile: concavo-convex.

Teeth.-Wear: plus. Lost: 2. Condition: poor.

CHEsT.-Derelopment: plus.

Musculature.-Good.

HEALTH.-Good.

No. 256

Residence: camp of Sheikh Khadem. Date: June 7, 1928.

Age: 25; married for 10 years. Sons: none. Daughters: 1 living.

Brothers: 1 dead. Sisters: 1 living.

Tattoo marks: glove and gazelle on right hand and forearm.

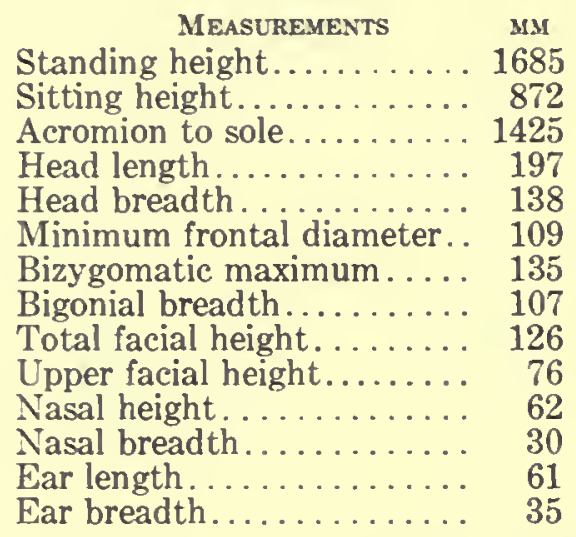

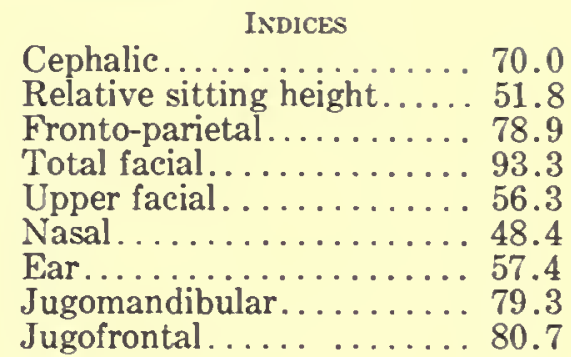

HaIR.-Form: low waves. Texture: medium. Color: black.

EYE.-Color: dark brown. Sclera: bloodshot. Iris: zoned.

Nose.-Profile: straight. Wings: medium-compressed.

TEETH. - Lost: none.

Chest.-Development: plus.

Musculature.-Excellent.

HEALTH.-Good.

No. 257

Residence: camp of Sheikh Khadem. Date: June 7, 1928.

Age: 42 ; unmarried.

Brothers: 3 living. Sisters: 1 living.

Tattoo marks: glove on right hand.

\begin{tabular}{|c|c|}
\hline MEASUREMENTS & MM \\
\hline Standing height. & 1710 \\
\hline Sitting height. & 892 \\
\hline Acromion to sole & 1455 \\
\hline Head length.. & 196 \\
\hline Head breadth. & 151 \\
\hline Minimum frontal diameter. & 116 \\
\hline Bizygomatic maximum.... & 138 \\
\hline Bigonial breadth. & 111 \\
\hline Total facial height. & 116 \\
\hline er facial height. . & 74 \\
\hline Nasal height. & 60 \\
\hline Tasal breadth. & 39 \\
\hline Car length. & 65 \\
\hline$\cdots$ & \\
\hline
\end{tabular}

HAIR.-Form: low waves. Color: dark brown black.

EYE.-Color: gray brown. Sclera: speckled and bloodshot. Iris: rayed. 
Nose.-Profile: concavo-convex.

TEETH.-Caries: double plus.

CHEsT.-Development: plus.

Musculature.-Good.

HEALTH.-Poor.

No. 258. Plate XXXVI, Figs. 7, 8

Residence: camp of Sheikh Khadem. Date: June 7, 1928.

Birthplace: camp of Sheikh Aziz. Age: 40; unmarried.

Brothers: 1 living, 1 dead. Sisters: 2 living.

Tattoo marks: spots on back of left wrist.

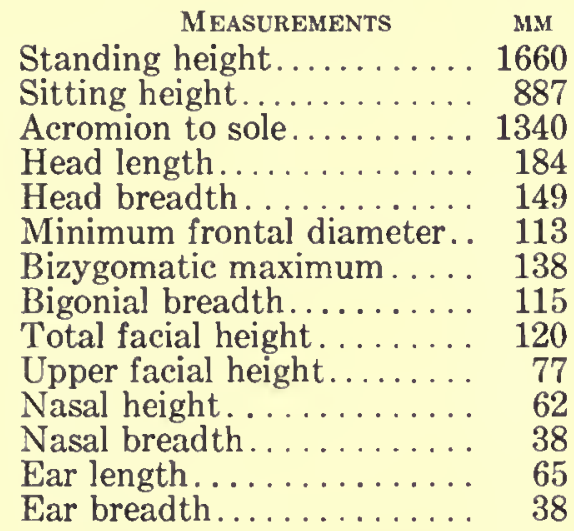

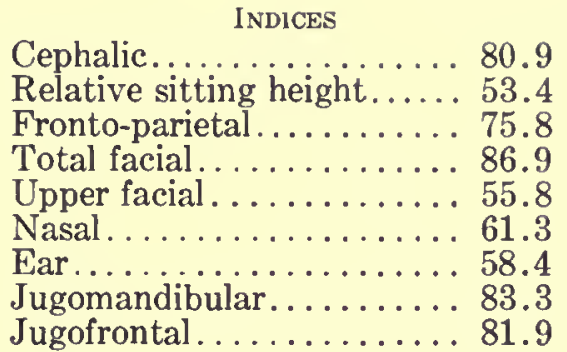

HAIR.-Quantity: head, average; beard, minus; body, average.

EYE.-Color: dark brown. Sclera: bloodshot. Iris: homogeneous.

Nose.-Profile: convex. Tip: thickness, double plus. Wings: flaring.

TEETH.-Lost: none.

Chest.-Development: plus.

Musculature.-Excellent.

HEALTH.-Good.

Skin very dark in color. Appears to have some Negro blood.

No. 259. Plate XXXVII, Figs. 1, 2

Residence: camp of Sheikh Khadem. Date: June 7, 1928.

Age: 30 ; married for 10 years. Sons: 1 dead. Daughters: 1 living.

Brothers: 3 dead. Sisters: none.

Tattoo marks: glove and gazelle on right hand and forearm.

\begin{tabular}{|c|c|c|}
\hline MEASUREMENTS & MM & INDICES \\
\hline Standing height.... & 1650 & Cephalic. \\
\hline Sitting height. & 867 & Relative sitting height..... 52 \\
\hline Acromion to sole. & 1370 & Fronto-parietal......... 76 \\
\hline Head length..... & 183 & Total facial..... \\
\hline Head breadth. & 138 & Upper facial. \\
\hline Minimum frontal diameter. & 106 & Nasal. . . . . . \\
\hline Bizygomatic maximum.... & 122 & Ear. \\
\hline Bigonial breadth......... & 95 & Jugomandibr \\
\hline Total facial height. . . . . . . & 113 & Jugofrontal \\
\hline
\end{tabular}

Upper facial height........ 78

Nasal height..................... 64

Nasal breadth........... 31

Ear length ........................... 61

Ear breadth............. 36

HAIR.-Texture: medium. Color: black. Quantity: head, plus; beard, plus; body, plus.

EYE.-Color: dark brown. Sclera: clear. Iris: zoned. 
NosE.-Profile: concave. Tip: elevated. Wings: medium.

TEETH.-Lost: none.

Chest.-Development: plus.

Musculature.--Excellent.

HEALTH.-Good.

\section{No. 260. Plate XXXVII, Figs. 3, 4}

Residence: camp of Sheikh Khadem. Date: June 7, 1928.

Birthplace: camp of Sheikh Ghatal. Age: 40; married for 20 years. Sons: 1 living, 2 dead. Daughters: 2 living.

Brothers: 2 living. Sisters: none.

Tattoo marks: glove and gazelle on right hand and forearm; spots on left hand; 6 spots over left eye, and 7 spots over right eye.

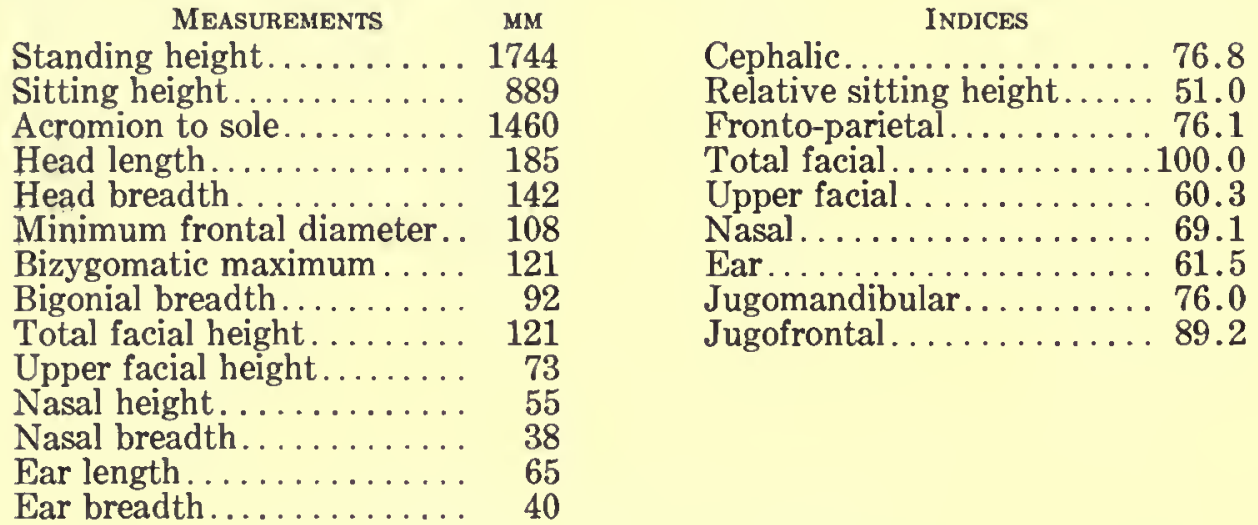

HAIR.-Form: low waves. Color: dark brown black. Quantity: head, plus; beard, plus; body, plus.

EyE.-Color: dark brown. Sclera: speckled and bloodshot. Iris: homogeneous.

Nose.-Profile: concave. Tip: elevated. Wings: medium.

TEETH.-Lost: none.

CHEST.-Development: double plus.

Musculature.-Excellent.

HEALTH.-Excellent.

No. 261. Plate XXXVII, Figs. 5, 6

Residence: camp of Sheikh Khadem. Date: June 7, 1928.

Birthplace: camp of Sheikh Abdullah on Tigris River.

Married for 6 years. Sons: 1 living. Daughters: none.

Brothers: 1 living. Sisters: none.

Tattoo marks: glove and gazelle on right hand and forearm.

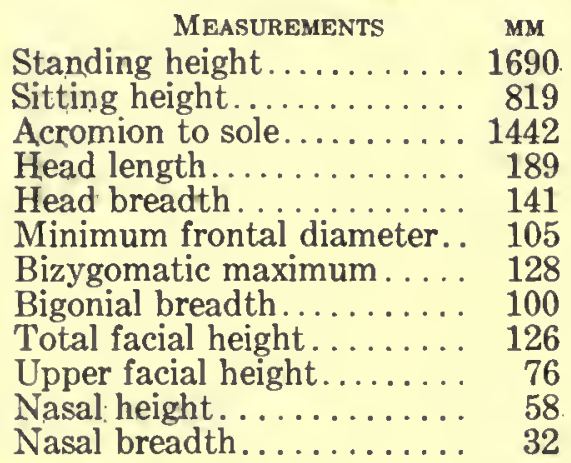

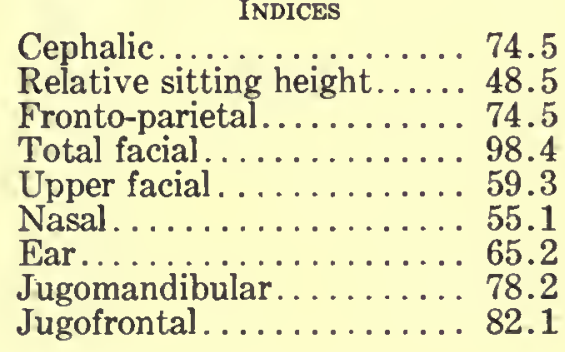

Cephalic............. 74.5

Relative sitting height..... 48.5

Fronto-parietal .......... 74.5

Nasal............... 55.1

Jugomandibular......... 78.2

Jugofrontal. .......... 82.1 


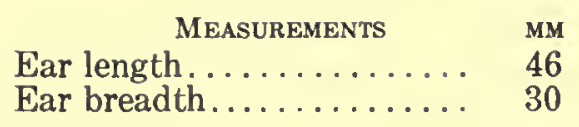

HAIR.-Texture: fine. Color: dark brown black. Quantity: head, average; beard, average; body, average.

EYE.-Color: dark brown. Iris: zoned.

NosE.-Profile: straight.

Chest.-Development: plus.

Musculature.-Good.

HEALTH.-Good.

No. 262

Residence: camp of Sheikh Khadem. Date: June 7, 1928.

Birthplace: camp of Sheikh Abdullah. Age: 26; unmarried:

Brothers: 1 living. Sisters: none.

Tattoo marks: spot on right wrist and one on each temple:

\begin{tabular}{|c|c|}
\hline MEASUREMENTS & MM \\
\hline Standing height. & 1690 \\
\hline Sitting height & 857 \\
\hline Acromion to sole & 1443 \\
\hline Head length & 187 \\
\hline Head breadth & 136 \\
\hline Minimum frontal diameter. . & 10 \\
\hline Bizygomatic maximum . . . . & 128 \\
\hline Bigonial breadth. & 100 \\
\hline Total facial height. & 122 \\
\hline Upper facial height. . . . . . . . & 81 \\
\hline Nasal height: & 70 \\
\hline 1 breadth & 38 \\
\hline 5 & 62 \\
\hline & \\
\hline
\end{tabular}

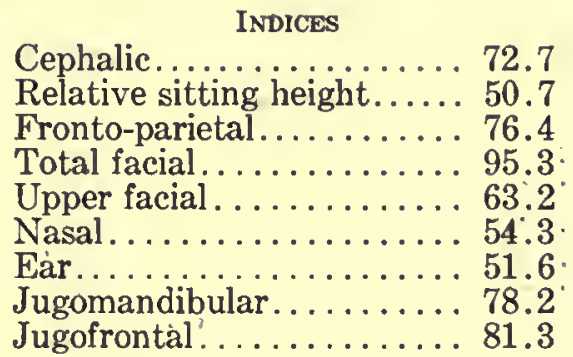

HAIR.-Form: low waves. Texture: medium. Color: dark brown.

EYE.-Sclera: bloodshot. Iris: zoned.

NosE.-Profile: straight. Wings: medium:

TEETH.-Lost: none.

CHEST.-Development: plus.

Musculature.-Good.

HEALTH.-Good.

No. 263. Plate XXXVII, Figs. 7, 8

Residence: camp of Sheikh Khadem. Date: June 7, 1928.

Birthplace: camp of Sheikh Abdullah. Age: 32 ; married twice, 13 years ago and 9 years ago. Sons: 1,1 , living. Daughters: 0,2 , living.

Brothers: 1 living. Sisters: none.

Tattoo marks: none.

\begin{tabular}{|c|c|}
\hline MEASUREMENTS & MM \\
\hline Standing height....... & 1720 \\
\hline Sitting height. & 847 \\
\hline Acromion to sole & 1450 \\
\hline Head length. & 192 \\
\hline eadth & 145 \\
\hline Minimum frontal diameter: & 114 \\
\hline atic maximum . . . . & 134 \\
\hline breadth. & 108 \\
\hline Tota & 128 \\
\hline height. & 80 \\
\hline
\end{tabular}

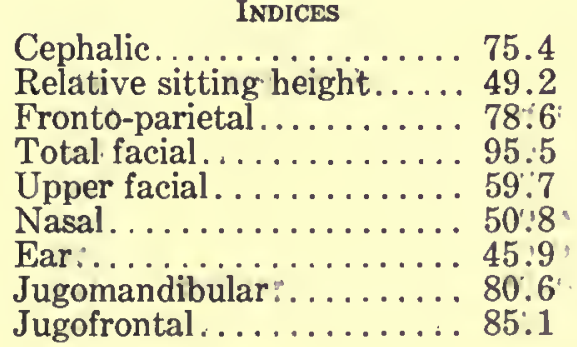




\begin{tabular}{|c|c|}
\hline MEASUREMENTS & MM \\
\hline sal height. & 63 \\
\hline l breadth. & \\
\hline ar length. . & \\
\hline ar breadth & \\
\hline
\end{tabular}

HAIR.-Texture: fine. Color: dark brown. Dyed with henna.

EYE.-Color: dark brown. Sclera: clear. Iris: zoned.

Nose.-Profile: straight.

TEETH.-Wear: plus. Caries: double plus. Lost: none.

Chest.-Development: average.

Musculature.-Good.

Health.-Good.

\section{No. 264. Plate XXXVIII, Figs. 1, 2}

Residence: camp of Sheikh Khadem. Date: June 7, 1928.

Age: 35; married twice, 15 years ago and 3 years ago. Sons: 0,1 , living. Daughters: none. Brothers: 2 living. Sisters: 1 living.

Tattoo marks: gazelle on inside of right forearm.

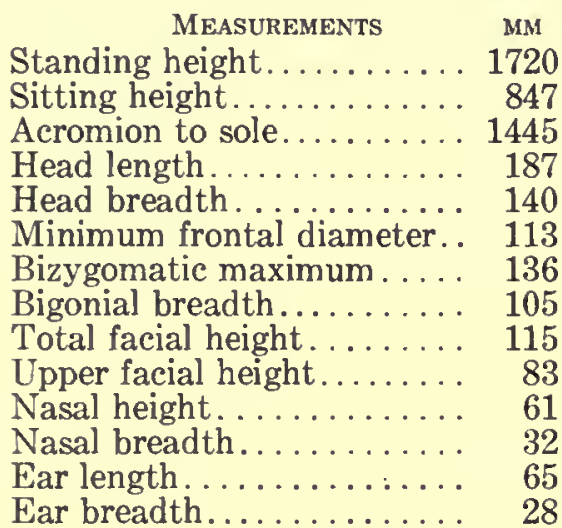

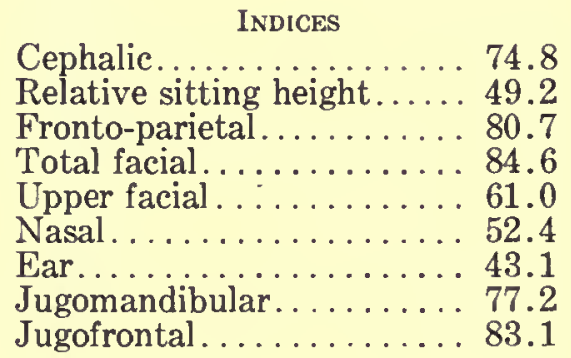

HAIR.-Form: low waves. Texture: medium. Color: black. Quantity: head, average; beard, plus; body, plus.

EYE.-Color: dark brown. Sclera: bloodshot. Iris: zoned.

Nose.-Profile: straight. Wings: compressed.

TEETH.-Lost: none.

Chest.-Development: plus.

Musculature.-Good.

HEALTH.-Good.

\section{No. 265. Plate XXXVIII, Figs. 3, 4}

Residence: camp of Sheikh Khadem. Date: June 7, 1928.

Birthplace: camp of Sheikh Abdullah. Age: 20; unmarried.

Brothers: 2 living. Sisters: 1 living.

Tattoo marks: spots on right wrist.

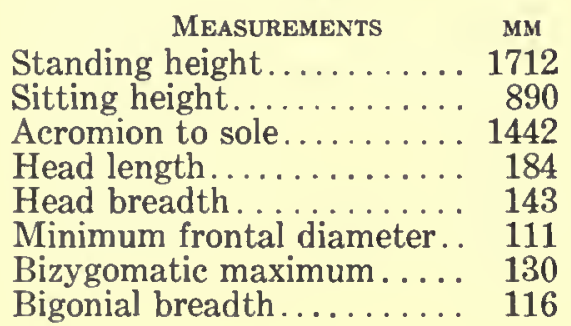




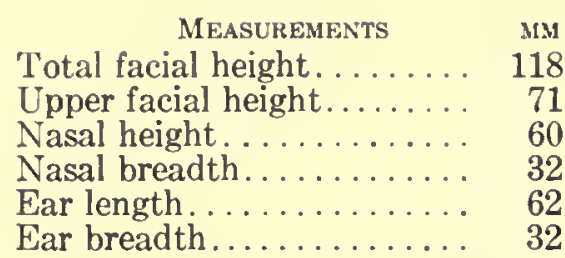

Jugofrontal. .......... 85.4

HaIR.-Form: low waves. Texture: medium. Color: dark brown. Quantity: head, double plus; beard, minus; body, minus.

EYE.-Color: blue brown. Sclera: speckled and bloodshot. Iris: zoned. Condition: fair.

Nose.-Profile: convex.

TEETH.-Lost: none.

Chest.-Development: plus.

Musculature.-Fair.

HEALTH.-Fair.

Right eye blind and left eye strained. Cataract forming in left eye.

No. 266

Locality: camp of Sheikh Khadem. Date: June 7, 1928.

Birthplace: camp of Sheikh Ghatal. Age: 50; married for 20 years. Sons: 2 living, 1 dead. Daughters: 3 living.

Brothers: 2 living. Sisters: none.

Tattoo marks: glove on right hand; line of spots across forehead (one-inch gap at glabella).

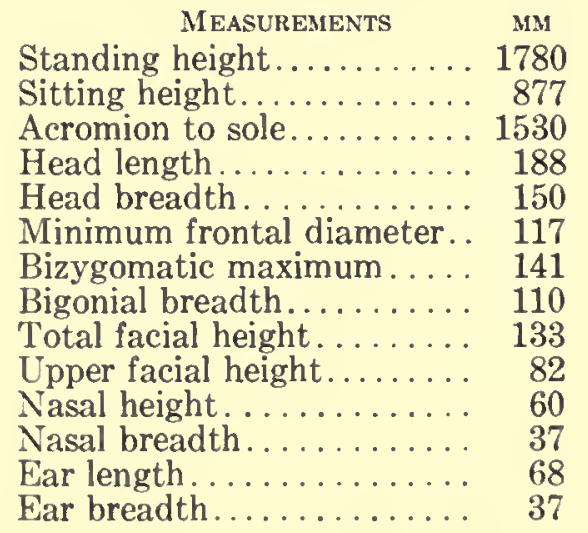

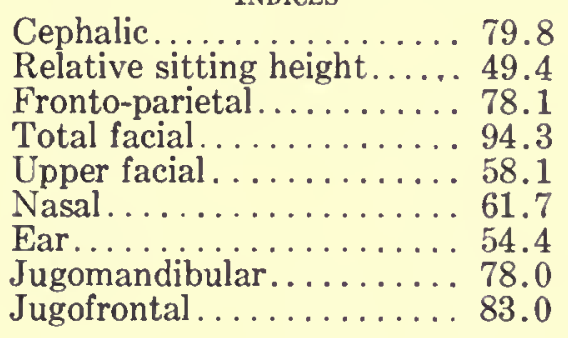

Cephalic............... 79.8

Relative sitting height..... 49.4

Fronto-parietal ............78.1

Upper facial. ..................

Nasal. ..............6. 61.7

Jugofrontal . . . . . . . . 83.0

HaIr.-Form: low waves. Texture: fine. Color: dark brown. Quantity: head, plus; beard, double plus; body, plus.

EYE.-Color: green brown. Sclera: bloodshot. Iris: homogeneous.

Nose.-Profile: straight. Tip: thickness, double plus. Wings: medium.

TEETH.-Lost: none.

Chest.-Development: double plus.

Musculature.-Excellent.

HEALTH.-Excellent.

No. 267

Residence: camp of Sheikh Khadem. Date: June 7, 1928.

Age: 80; married for 50 years. Sons: 1 living, 1 dead. Daughters: 2 dead.

Brothers: 4 dead. Sisters: none.

Tattoo marks: none.

MEASUREMENTS MM

Standing height......... 1730

Sitting height.......... 867

Acromion to sole.......... 1460

Head length........... 191

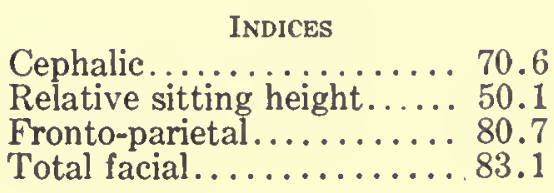




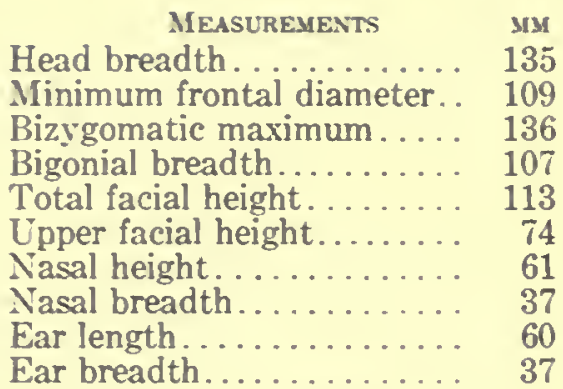

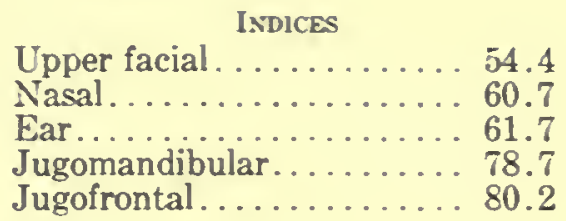

No. 269

Locality: camp of Sheikh Khadem. Date: June 7, 1928.

Birthplace: camp of Sheikh Abdullah. Age: 40; married for 30 years. Sons: none. Daughters: 2 living.

Brothers: 1 living. Sisters: 1 living.

Tattoo marks: glove on right hand.

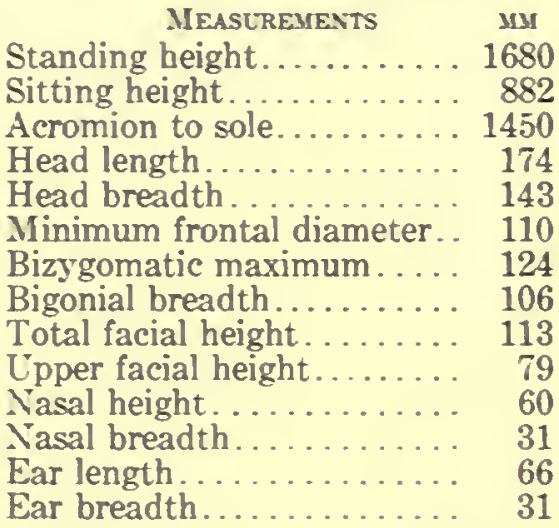

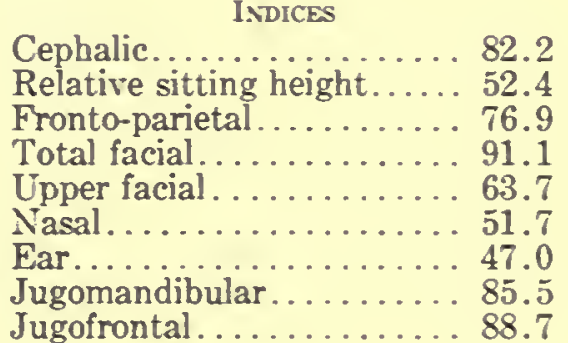

HAIR-Texture: medium. Color: dark brown. Quantity: head, average; beard, plus.

EYE.-Color: dark brown. Sclera: clear. Iris: zoned.

Nose.-Profile: straight. Wings: medium-compressed.

TEETH.-Lost: none.

CHEST.-Derelopment: plus.

Musculature.-Good.

HEALTH.-Good.

No. $270 \mathrm{~A}$

Locality: camp of Sheikh Khadem. Date: June 7, 1928.

Birthplace: camp of Sheikh Abdullah. Age: 34 ; married for 2 years. Sons: none. Daughters: 1 living.

Brothers: 1 living. Sisters: 1 living.

Tattoo marks: 2 spots on right wrist and on inside of right forearm.

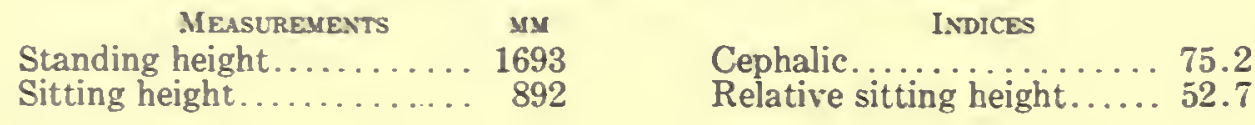




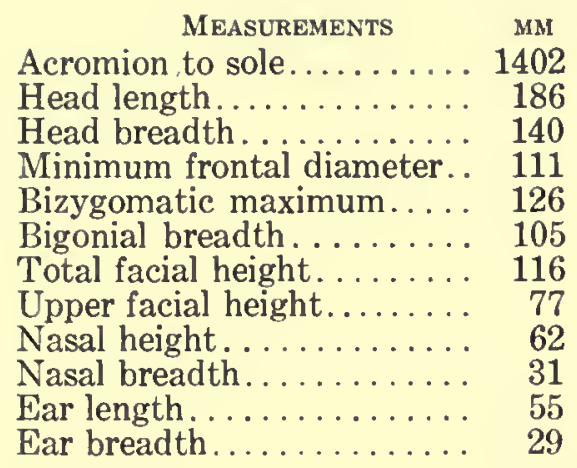

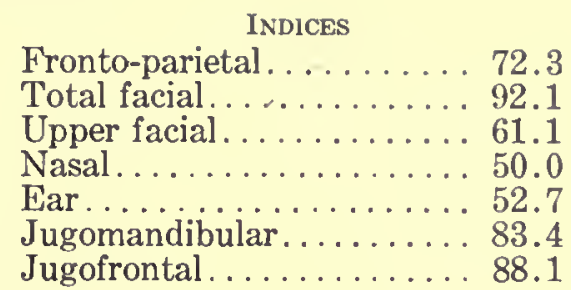

Fronto-parietal. . . . . . . 72.3

Total facial .................. 92.1

Upper facial. ..........6 61.1

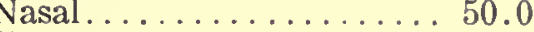

Jugomandibular. . . . . . . 8 83.4

Jugofrontal. . . . . . . . . . 88.1

HAIR.-Texture: medium. Color: dark brown. Quantity: head, double plus; beard, plus; body, plus.

EyE.-Color: dark brown. Sclera: clear. Iris: zoned.

Nose.-Profile: straight.

TEETH._Lost: none.

Chest.-Development: plus.

MUSCUlATURE.-Good.

Health.-Good.

No. $270 \mathrm{~B}$

Residence: camp of Sheikh Khadem. Date: June 7, 1928.

Age: 30; married for 15 years. Sons: 2 living, 1 dead. Daughters: none.

Brothers: 2 dead. Sisters: 1 living.

Tattoo marks: glove and gazelle on right hand and forearm.

\begin{tabular}{|c|c|}
\hline \multicolumn{2}{|l|}{ MEASUREMENTS } \\
\hline Head length. & \\
\hline $\begin{array}{l}\text { Head breadth......... } \\
\text { Minimum frontal diameter. }\end{array}$ & \\
\hline Bizygomatic maximum.... & \\
\hline Bigonial breadth.. & \\
\hline $\begin{array}{l}\text { Total facial height.. } \\
\text { Upper facial height. }\end{array}$ & \\
\hline $\begin{array}{l}\text { Upper facial height. } \\
\text { Nasal height....... }\end{array}$ & \\
\hline $\begin{array}{l}\text { Nasal height } \\
\text { Jasal breadth }\end{array}$ & \\
\hline len & \\
\hline 10 & \\
\hline
\end{tabular}

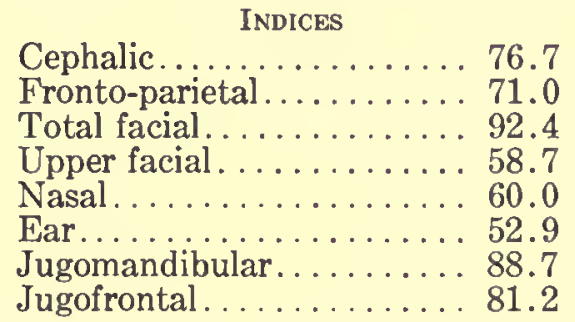

36

HaIr.-Form: low waves. Texture: medium. Color: dark brown. Quantity: head, triple plus; beard, plus; body, plus.

EYE.-Color: dark brown. Sclera: bloodshot. Iris: zoned.

Nose.-Profile: straight. Wings: medium.

TEETH.-Lost: 2.

CHEst.-Development: double plus.

Musculature.-Excellent.

HEALTH.-Good.

No. 271

Residence: camp of Sheikh Khadem. Date: June 7, 1928.

Age: 20 ; unmarried.

Brothers: 1 living. Sisters: none.

Tattoo marks: glove and gazelle on right hand and forearm.

\begin{tabular}{|c|c|c|}
\hline MEASUREMENTS & MM & INDICES \\
\hline Head length.......... & 178 & Cephalic........... \\
\hline 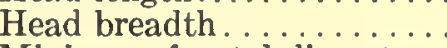 & 142 & Fronto-parietal...... \\
\hline Minimum frontal diameter. & 107 & Total facial. \\
\hline
\end{tabular}




\begin{tabular}{|c|c|c|}
\hline MEASUREMENTS & MMI & INDICES \\
\hline Bizygomatic maximum.... . & 130 & Upper facial.... \\
\hline Bigonial breadth. & 100 & Nasal. \\
\hline al facial height. & 119 & Ear. \\
\hline Upper facial height & 72 & Jugomandibular \\
\hline Nasal height..... & 58 & Jugofrontal \\
\hline
\end{tabular}

Nasal breadth........... 34

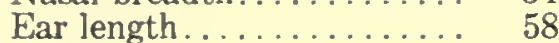

Ear breadth........... 38

HAIR.-Form: low waves. Texture: fine. Quantity: head, double plus; beard, average; body, average.

EYE.-Color: dark brown. Sclera: clear. Iris: zoned.

Nose.-Profile: straight. Wings: medium.

TEETH.-Lost: none. Condition: good.

Chest.-Development: plus.

Musculature.-Good.

HEALTH.-Good.

No. 272. Plate XXXVIII, Figs. 5, 6

Residence: camp of Sheikh Khadem. Date: June 7, 1928.

Age: 18; unmarried.

Brothers: none. Sisters: 1 dead.

Tattoo marks: none.

\begin{tabular}{|c|c|c|}
\hline MEASUREMENTS & MM & INDICES \\
\hline Head length. & 184 & Cephalic. \\
\hline Head breadth. & 139 & Fronto-parietal. \\
\hline Minimum frontal diameter. & 116 & Total facial. . \\
\hline Bizygomatic maximum.... & 128 & Upper facial \\
\hline onial breadth. & 97 & Nasal.... \\
\hline facial height. & 117 & Ear \\
\hline facial height. . & 72 & Jugomandibular. . . . \\
\hline & 58 & Jugofrontal \\
\hline
\end{tabular}

Nasal breadth........... 34

Ear length............... 64

Ear breadth............. 35

HAIR.-Form: low waves. Texture: medium.

EYE.-Color: dark brown. Sclera: clear. Iris: zoned.

NosE.-Profile: concavo-convex.

TEETH.-Lost: none. Third molars unerupted.

Chest.-Development: plus.

Musculature.-Good.

HEALTH.-Good.

No. 273. Plate XXXVIII, Fig. 7

Residence: camp of Sheikh Khadem. Date: June 7, 1928.

Age: 25; married for 10 years. No children.

Brothers: 2 living, 2 dead. Sisters: 1 living.

Tattoo marks: spot on nose.

\begin{tabular}{|c|c|}
\hline MEASUREMENTS & MM \\
\hline Head length. & 188 \\
\hline Head breadth. & 135 \\
\hline Minimum frontal diameter. . & 5 \\
\hline Bizygomatic maximum..... & \\
\hline Bigonial breadth.......... & \\
\hline Total facial height. & 120 \\
\hline Upper facial height. & \\
\hline
\end{tabular}

INDICES

Cephalic............. 71.8

Fronto-parietal.......... 77.7

Total facial...........99.0

Upper facial. . . . . . . . . . 60.8

Nasal. . . . . . . . . . . . . . 60.0

Ear....................... 54.8

Jugomandibular. . . . . . 80.9 


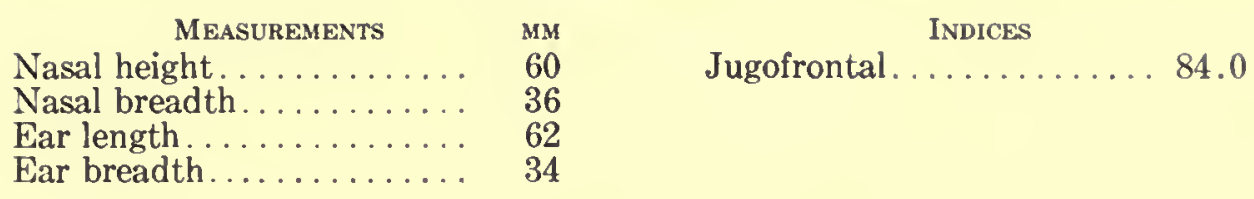

HaIR.-Form: low waves. Texture: medium. Color: dark brown.

EYE.-Color: dark brown. Sclera: clear. Iris: homogeneous.

Nose.--Profile: concavo-convex. Wings: medium.

TEETH.-Lost: none.

CHEST.-Development: double plus.

Musculature.-Excellent.

HEALTH.--Excellent.

No. 274

Residence: camp of Sheikh Khadem. Date: June 7, 1928.

Age: 30; married for 15 years. Sons: 1 living. Daughters: 2 living, 1 dead.

Brothers: 2 living. Sisters: none.

Tattoo marks: glove and gazelle; spots on right temple.

\begin{tabular}{|c|c|c|}
\hline MEASUREMENTS & MM & INDICES \\
\hline Head length. & 182 & Cephalic. \\
\hline Head breadth. & 142 & Fronto-parietal \\
\hline Minimum frontal diameter. & 101 & Total facial. \\
\hline Bizygomatic maximum . & 125 & Upper facial \\
\hline Bigonial breadth. & 90 & Nasal. \\
\hline Total facial height. & 118 & Ear. \\
\hline Upper facial height & 78 & Jugomandib \\
\hline Nasal height. & 66 & Jugofrontal \\
\hline
\end{tabular}

Nasal breadth........... 32

Ear length............ 58

Ear breadth............ 33

HaIR.-Form: low waves. Texture: fine. Color: dark brown. Quantity: head, plus; beard, plus; body, plus.

EYE.-Color: dark brown. Sclera: bloodshot. Iris: zoned.

NosE.-Profile: straight. Wings: compressed.

TEETH.-Lost: none.

CHEST.-Development: plus.

Musculature.-Good.

HEALTH.-Good.

Brother of No. 266.

No. 275. Plate XXXVIII, Fig. 8

Residence: camp of Sheikh Khadem. Date: June 7, 1928.

Age: 25; unmarried.

Brothers: 2 dead. Sisters: 1 dead.

Tattoo marks: cross on each temple.

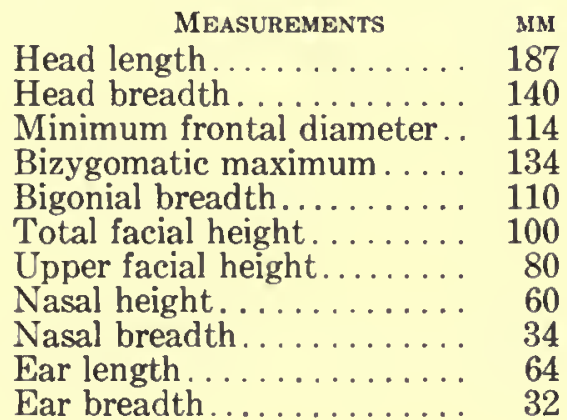

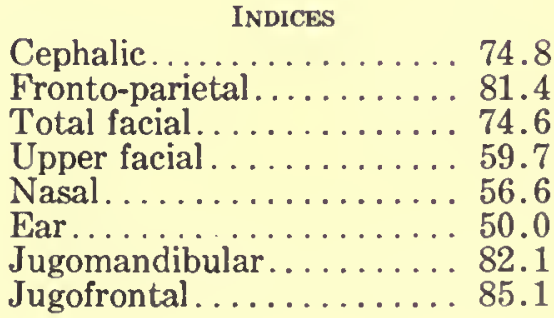

Cephalic............ 74.8

Fronto-parietal.........8 81.4

Total facial............ 74.6

Upper facial. . . . . . . 59.7

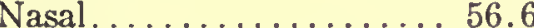

Jugomandibular. . . . . . . 8. 82.1

Jugofrontal. ........... 85.1 
HaIr.-Form: low waves. Texture: medium.

EYE.-Color: dark brown. Sclera: clear. Iris: zoned.

Nose.-Profile: straight. Wings: medium.

TEETH.-Lost: none.

Chest.-Development: plus.

Musculature.-Good.

HEALTH.-Good.

No. 276

Residence: camp of Sheikh Khadem. Date: June 7, 1928.

Age: 20; unmarried.

Brothers: none. Sisters: 1 living.

Tattoo marks: glove on right hand.

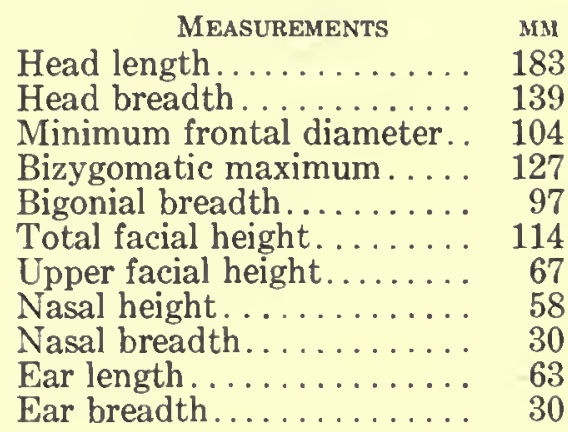

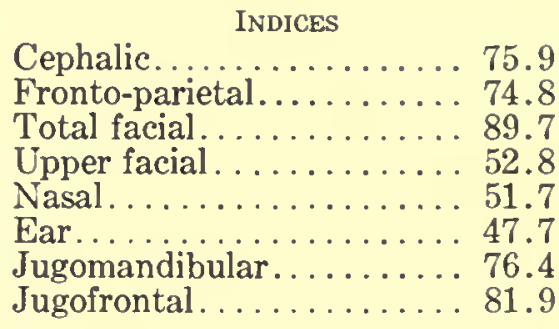

HaIr.-Form: low waves. Texture: medium.

EYE.-Color: dark brown. Sclera: bloodshot. Iris: zoned.

NosE.-Profile: concavo-convex. Tip: thickness, double plus.

TEETH.-Lost: none.

CHEST.-Development: plus.

Musculature.-Good.

HEALTH.-Good.

No. 277

Residence: camp of Sheikh Khadem. Date: June 7, 1928.

Age: 30 ; married for 2 years. Sons: 1 living. Daughters: none.

Brothers: 1 living. Sisters: 3 living.

Tattoo marks: line on left temple.

\begin{tabular}{|c|c|}
\hline MEASUREMENTS & MM \\
\hline Head length. & 198 \\
\hline Head breadth & 130 \\
\hline Minimum frontal diameter. . & 108 \\
\hline Bizygomatic maximum . . . . & 119 \\
\hline Bigonial breadth. & 100 \\
\hline Total facial height. & 117 \\
\hline Upper facial height. . . . . . . & 68 \\
\hline Nasal height. & 53 \\
\hline Nasal breadth. & 38 \\
\hline Ear length & 62 \\
\hline For hromdt & \\
\hline
\end{tabular}

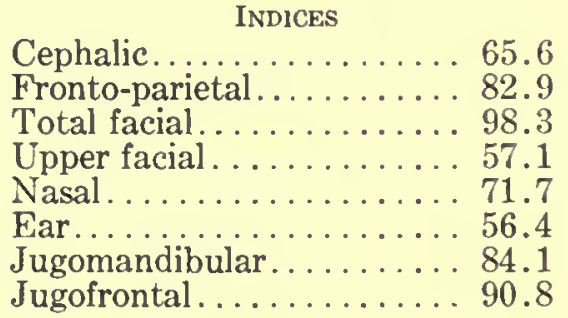

HaIR.-Form: low waves. Texture: fine. Color: dark brown. Quantity: head, double plus; beard, plus; body, plus.

Eye.-Sclera: clear. Iris: zoned.

Nose.-Profile: concavo-convex. Tip: thickness, plus. Wings: medium.

TEETH.-Lost: none.

CHEST.-Development: plus. 
Musculature.-Good.

HEALTH.-Good.

No. 278. Plate XXXIX, Figs. 1, 2

Residence: camp of Sheikh Khadem. Date: June 7, 1928.

Age: 70; married for 30 years. Sons: 1 living. Daughters: 1 living, 2 dead.

Brothers: none. Sisters: none.

Tattoo marks: none.

\begin{tabular}{|c|c|}
\hline \multicolumn{2}{|l|}{$\begin{array}{l}\text { MEASUREMENTS } \\
\text { MEASU }\end{array}$} \\
\hline Head length. & 1 \\
\hline Head breadth & \\
\hline Minimum frontal diameter. & \\
\hline zygomatic maximum . .... & \\
\hline breadth. & \\
\hline al facial height. & \\
\hline per facial height. & \\
\hline al height. ...... & \\
\hline al breadth & \\
\hline brea & \\
\hline
\end{tabular}

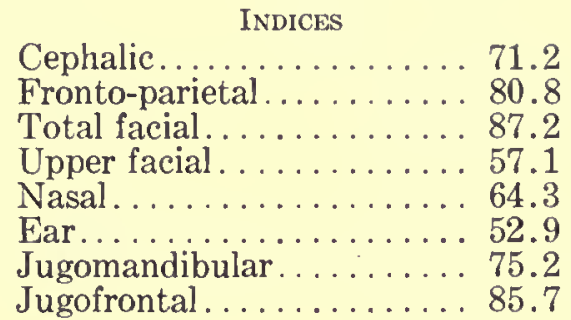

HAIR.-Color: gray. Quantity: head, average; beard, double plus.

EYE.-Color: green brown. Sclera: bloodshot. Iris: zoned.

Nose.-Profile: convex.

TEETH.-Wear: double plus. Caries: double plus. Lost: 2.

Chest.-Development: double plus.

Musculature.-Good.

HEALTH.-Good.

\section{No. 279. Plate XXXIX, Figs. 3, 4}

Residence: camp of Sheikh Khadem. Date: June 7, 1928.

Age: 50; married for 15 years. Sons: 2 living, 1 dead. Daughters: 1 dead.

Brothers: 1 dead. Sisters: none.

Tattoo marks: gazelle on inside of right forearm.

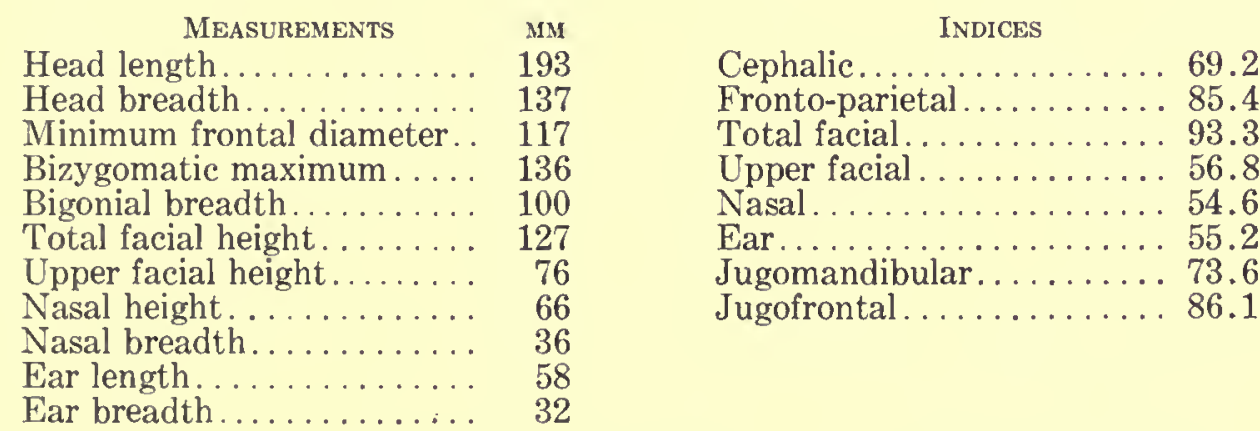

HAIR.-Color: dark brown. Quantity: head, average; beard, average; body, plus.

EYE.-Color: dark brown. Sclera: bloodshot. Iris: zoned.

NOSE.-Profile: straight. Wings: flaring.

TEETH.-Lost: 2.

Chest.-Development: double plus.

Musculature.-Excellent.

HEALTH.-Good.

No. 280. Plate XXXIX, Figs. 5, 6

Residence: camp of Sheikh Khadem. Date: June 7, 1928.

Age: 20; unmarried. 
Brothers: 1 living. Sisters: none.

Tattoo marks: none.

\begin{tabular}{|c|c|c|}
\hline MEASUREMENTS & MM & INDICES \\
\hline Head length. & 187 & Cephalic. \\
\hline Head breadth. & 134 & Fronto-parietal \\
\hline Minimum frontal diameter. & 117 & Total facial... \\
\hline Bizygomatic maximum . . . . & 134 & Upper facial \\
\hline Bigonial breadth. & 113 & Nasal. \\
\hline Total facial height. & 128 & Ear. \\
\hline er facial height..... & 76 & Jugomandibular. ... . \\
\hline al height. & 57 & Jugofrontal \\
\hline
\end{tabular}

Nasal breadth.......... 40

Ear length............ 61

Ear breadth.............. 32

HAIR.-Form: straight. Texture: medium. Color: black.

EYE.-Color: dark brown. Sclera: clear. Iris: homogeneous.

Nose.-Profile: convex. Wings: medium.

TEETH.-Lost: none.

CHEST.-Development: double plus.

Musculature.-Excellent.

HEALTH.-Excellent.

High cheek-bones suggest Mongoloid blood.

No. 281A. Plate XXXIX, Figs. 7, 8

Residence: camp of Sheikh Khadem. Date: June 7, 1928.

Age: 35 ; married for 15 years. Sons: 3 living. Daughters: 1 living, 2 dead.

Brothers: none. Sisters: none.

Tattoo marks: glove on right hand and gazelle on inside of right forearm.

\begin{tabular}{|c|c|}
\hline \multicolumn{2}{|l|}{ MEASUREMENTS } \\
\hline Head length & 193 \\
\hline Head breadth. & 136 \\
\hline Minimum frontal diameter. & 114 \\
\hline Bizygomatic maximum ..... & 131 \\
\hline Bigonial breadth.......... & 99 \\
\hline Total facial height. & 118 \\
\hline Upper facial height........ & 77 \\
\hline Nasal height. . ........... & 60 \\
\hline Nasal breadth & 36 \\
\hline Ear length. & 70 \\
\hline Ear breadtl & \\
\hline
\end{tabular}

HaIR.-Form: low waves. Texture: medium. Color: dark brown. Quantity: head, plus. EYE.-Color: dark brown. Sclera: clear. Iris: homogeneous.

NosE.-Profile: straight.

ТеЕтн.-Wear: double plus. Caries: double plus. Lost: 1.

CHEST.-Development: plus.

Musculature.-Good.

HEALTH.-Good.

Cephalic.............. 70.4

Fronto-parietal........... 83.8

Total facial. ......... 90.0

Upper facial. . . . . . . . . 58.8

Nasal. .............6 60.0

Ear................... 54.3

Jugomandibular........ 75.6

Jugofrontal. ............ 77.1

Residence: camp of Sheikh Khadem. Date: June 7, 1928.

Age: 55; married for 4 years. Sons: 1 living. Daughters: 2 living.

Brothers: none. Sisters: none.

Tattoo marks: glove on back of right hand.

\begin{tabular}{|c|c|c|}
\hline MEASUREMENTS & MM & INDICES \\
\hline Head length. & 193 & Cephalic...: \\
\hline Head breadth & 144 & Fronto-parietal. \\
\hline
\end{tabular}




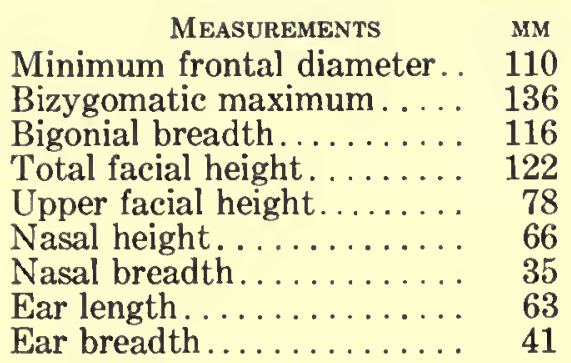

\begin{tabular}{|c|c|}
\hline Total facial......... & \\
\hline Upper facial. & 56.3 \\
\hline Nasal... & 53.0 \\
\hline Ear... & 65.1 \\
\hline Jugomandibular. & 85.3 \\
\hline Jugofrontal. . . . & 80.9 \\
\hline
\end{tabular}

HaIr.-Form: low waves. Texture: medium. Quantity: head, double plus; heard, double plus; body, plus.

EYE.-Color: green brown. Sclera: bloodshot. Iris: zoned.

NosE.-Profile: straight. Wings: medium.

TeEth.-Wear: double plus. Caries: double plus. Lost: 2.

EAR.-Darwin's Point: double plus.

Chest.-Development: plus.

Musculature.-Good.

HEALTH.-Good.

No. 282. Plate XL, Fig. 2

Residence: camp of Sheikh Khadem. Date: June 7, 1928.

Age: 80 ; married for 50 years. Sons: 1 living, 3 dead. Daughters: none.

Brothers: none. Sisters: none.

Tattoo marks: none.

\begin{tabular}{|c|c|}
\hline MEasurements & MM \\
\hline Head length. & 198 \\
\hline Head breadth & 153 \\
\hline Minimum frontal diameter. & 112 \\
\hline Bizygomatic maximum ..... & 146 \\
\hline Bigonial breadth ........... & 113 \\
\hline Total facial height. . & 127 \\
\hline Upper facial height......... & 66 \\
\hline Nasal height. . . . . . . . . . & 76 \\
\hline Nasal breadth. & 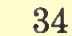 \\
\hline Ear length. & \\
\hline Ear breadth & \\
\hline
\end{tabular}

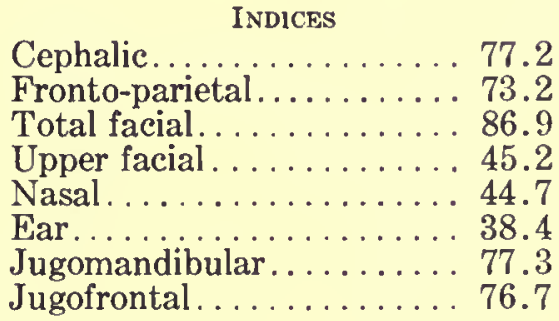

HAIR.-Color: gray. Quantity: head, minus; beard, double plus; body, average.

EYE.-Color: green brown. Sclera: bloodshot. Iris: zoned.

Nose.-Profile: straight. Wings: medium.

TEETH.-Lost: many.

CHEST.-Development: plus.

Musculature.-Fair.

HEALTH.-Good.

Very prominent malars. Cheeks full.

No. 283

Residence: camp of Sheikh Khadem. Date: June 7, 1928.

Age: 20; unmarried.

Brothers: 1 living. Sisters: none.

Tattoo marks: none.

Measurements MM

Head length........... 184

Head breadth............... 137

Minimum frontal diameter. . 114
INDICES

Cephalic............... 74.4

Fronto-parietal........... 83.2

Total facial. .......... 89.4 


\begin{tabular}{|c|c|}
\hline MEASUREMENTS & \\
\hline Bizygomatic maximum. & \\
\hline breadth. & \\
\hline 1 facial height. & \\
\hline Upper facial height. & \\
\hline & \\
\hline 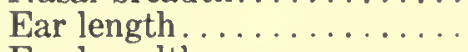 & \\
\hline & \\
\hline
\end{tabular}

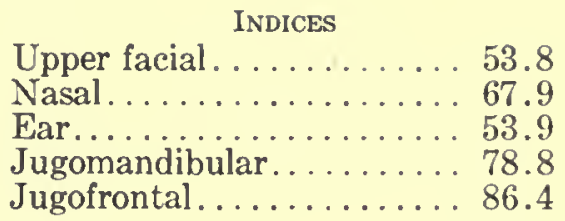

No. 285

Residence: camp of Sheikh Khadem. Date: June 7, 1928.

Age: 18; unmarried.

Brothers: none. Sisters: none.

Tattoo marks: spots on back of right hand.

\begin{tabular}{|c|c|c|}
\hline MEASUREMENTS & мм & INDICES \\
\hline Head length.......... & 191 & Cephalic. \\
\hline Head breadth. & 138 & Fronto-parietal........ \\
\hline Minimum frontal diameter. & 109 & Total facial............ \\
\hline Bizygomatic maximum.... & 127 & Upper facial. \\
\hline Bigonial breadth.......... & 101 & Nasal...... \\
\hline Total facial height. & 114 & 68.7 \\
\hline Upper facial height. ....... & 67 & Jugomandibular. . . . . \\
\hline Nasal height............ & 55 & Jugofrontal . . . . . . . . . . 85.9 \\
\hline Nasal breadth & 34 & \\
\hline Ear length. & 64 & \\
\hline Ear breadth & 44 & \\
\hline
\end{tabular}

HaIR.-Form: low waves. Texture: medium. Color: dark brown. Quantity: head, double plus; beard, minus; body, minus.

EyE.-Color: dark brown. Sclera: clear. Iris: zoned.

Nose.-Profile: straight. Wings: medium.

TEETH.-Lost: none. Unerupted: third molars.

CHEST.-Development: plus.

Musculature.-Good.

Healith.-Good.

No. 286. Plate XL, Figs. 3, 4

Residence: camp of Sheikh Khadem. Date: June 7, 1928.

Age: 90 ; married for 70 years. Sons: none. Daughters: 1 living.

Brothers: 1 living. Sisters: 2 dead.

Tattoo marks: none.

$\begin{array}{lr}\text { Measurements } & \text { MM } \\ \text { Head length............. } & 182 \\ \text { Head breadth........... } & 128 \\ \text { Minimum frontal diameter.. } & 107 \\ \text { Bizygomatic maximum..... } & 121 \\ \text { Bigonial breadth.......... } & 98 \\ \text { Total facial height. . . . . . } & 119\end{array}$

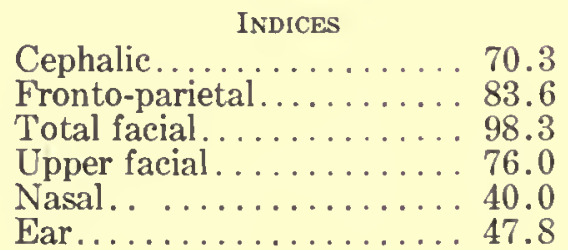




\begin{tabular}{|c|c|}
\hline MEASUREMENTS & $\mathrm{MM}$ \\
\hline Upper facial height.... & 92 \\
\hline Nasal height. . . . . & \\
\hline Nasal breadth... & \\
\hline ar length.... & \\
\hline ar breadth & \\
\hline
\end{tabular}

INDices
Jugomandibular . . . . . . . . 81.0

Jugofrontal . . . . . . . . . . 88.4

HAIR.-Quantity: head, minus; beard, double plus; body, plus.

EYE.-Color: green brown. Sclera: speckled and bloodshot. Iris: homogeneous.

NosE.-Profile: straight. Tip: thickness, double plus. Wings: medium.

TеEтн.-Wear: triple plus. Caries: triple plus. Lost: all but 1.

Chest.-Development: double minus.

Musculature.-Poor.

HEALTH.-Poor.

Deformed right hand. Condition of eyes poor. Extremely thin.

No. 287

Residence: camp of Sheikh Khadem. Date: June 7, 1928.

Age: 30; married for 4 years. Sons: none. Daughters: 2 living.

Brothers: none. Sisters: none.

Tattoo marks: none.

\begin{tabular}{|c|c|}
\hline MEASUREMENTS & MM \\
\hline Head length & 194 \\
\hline Head breadth. & 144 \\
\hline Minimum frontal diameter. & 106 \\
\hline Bizygomatic maximum.... & 114 \\
\hline Bigonial breadth . . . . . . . . & 105 \\
\hline Total facial height. & 114 \\
\hline Upper facial height.. & 70 \\
\hline Nasal height. . . . . . . . . . & 61 \\
\hline Nasal breadth............ & 34 \\
\hline Ear length. & 62 \\
\hline Won hec & \\
\hline
\end{tabular}

INDICES

Cephalic............... 74.2

Fronto-parietal ......... 73.6

Total facial ............. 100.0

Upper facial. . . . . . . . . 61.4

Nasal. .............. 55.7

Ear................. 59.6

Jugomandibular. . . . . . . 92.0

Jugofrontal. .......... 93.0

HAIR.-Color: dark brown. Quantity: head, average; beard, plus; body, plus.

EYE.-Color: dark brown. Sclera: clear. Iris: zoned.

Nose.-Profile: straight.

TEETH.-Lost: none.

Chest.-Derelopment: plus.

Musculature.-Excellent.

Health.-Good.

No. 288. Plate XL, Figs. 5, 6

Residence: camp of Sheikh Ghatal. Date: June 7, 1928.

Age: 20; unmarried.

Brothers: 1 living. Sisters: 1 living.

Tattoo marks: spots over glabella.

\begin{tabular}{|c|c|}
\hline MEASUREMENTS & MN \\
\hline Head length. & 18 \\
\hline Head breadth. & 13 \\
\hline Minimum frontal diameter. . & 1 \\
\hline Bizygomatic maximum $\therefore \ldots$ & \\
\hline Bigonial breadth . . . . . . . & \\
\hline Total facial height........ & \\
\hline Upper facial height........ & \\
\hline Nasal height............. & \\
\hline Nasal breadth............ & \\
\hline 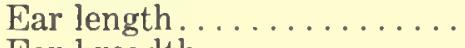 & \\
\hline & \\
\hline
\end{tabular}

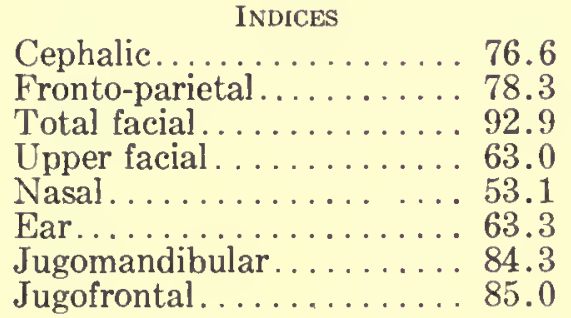


Halr.-Form: low waves. Texture: medium. Color: dark brown. EYE.-Color: dark brown. Sclera: clear. Iris: zoned.

NosE.-Profile: straight.

ChEst.-Derelopment: plus.

Musculature.-Good.

HEALTH.-Good.

\section{No. 350}

Residence: camp of Sheikh Aziz. Date: June 9, 1928.

Age: 40; married for 15 years. Sons: 1 living. Daughters: 4 living, 2 dead.

Brothers: 1 living. Sisters: 2 living.

Tattoo marks: glove on back of right hand.

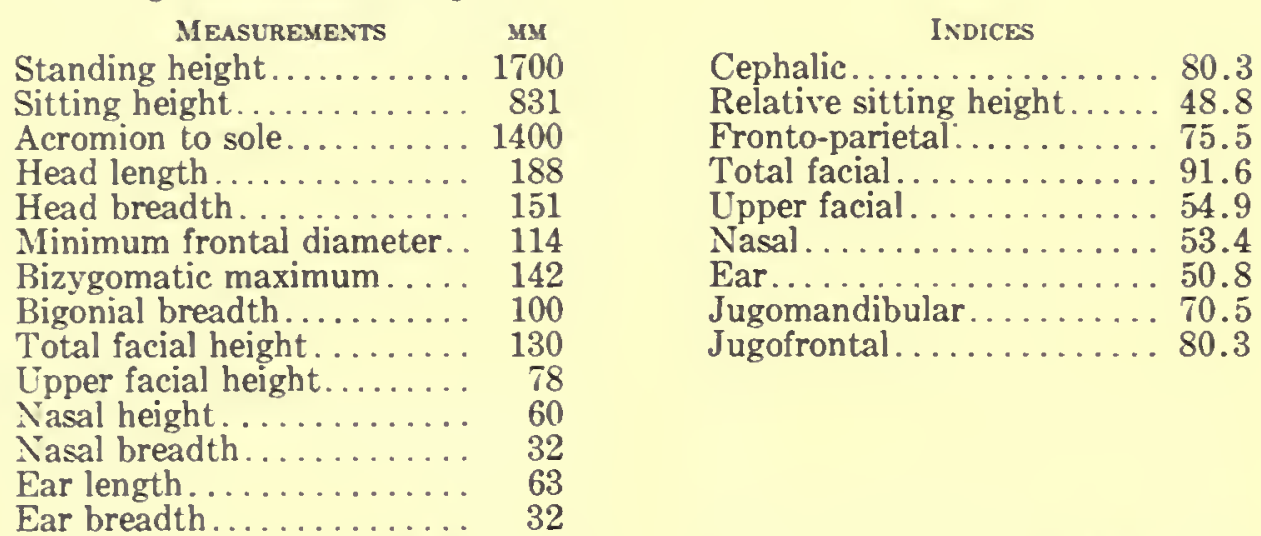

HAIR.-Form: low waves. Texture: medium. Color: dark brown. Quantity: head, plus; beard, plus; body, plus.

EYE.-Color: dark brown. Sclera: clear. Iris: homogeneous.

Nose.-Profile: straight. Wings: medium.

TEETH.-Lost: many.

CHEST.-Derelopment: plus.

Musculature.-Good.

HEALTH.-Good.

No. 351

Residence: camp of Sheikh Aziz. Date: June 9, 1928.

Age: 30 ; married for 3 years. Sons: 1 living. Daughters: none.

Brothers: 1 living. Sisters: 1 living.

Tattoo marks: none.

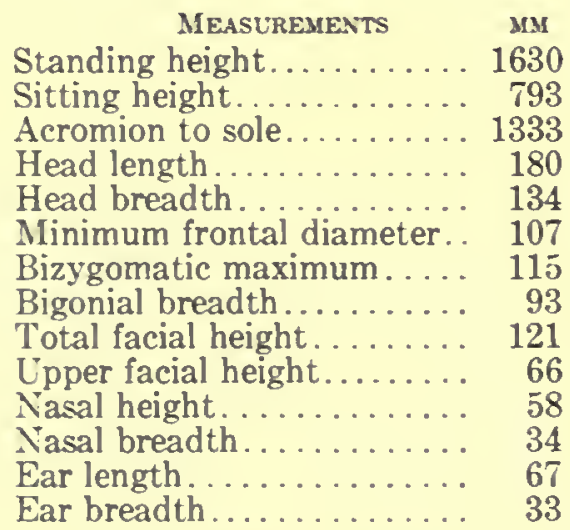

INDICES

Cephalic.............74.5

Relative sitting height..... 48.7

Fronto-parietal......... 79.9

Total facial............. 105.2

Upper facial.......... 57.4

Nasal .............. 58.6

Ear............... 49.3

Jugomandibular......... 80.9

Jugofrontal........... 93.1

Halr. -Form: low waves. Texture: medium. Color: black. Quantity: head, double plus; beard, plus; body, plus. 
EYE.-Color: green brown. Sclera: clear. Iris: zoned.

Nose.-Profile: convex. Wings: medium.

TeETH.-Lost: 1 .

CHEst.-Development: plus.

Musculature.-Good.

HEALTH.-Good.

No. 352

Residence: camp of Sheikh Aziz. Date: June 9, 1928.

Age: 20; married for 1 year. No children.

Brothers: 2 living. Sisters: 2 living.

Tattoo marks: cross and spot on back of right wrist.

\begin{tabular}{|c|c|c|}
\hline MEASUREMENTS & MM & INDICES \\
\hline Standing height. & 1695 & Cephalic. \\
\hline Sitting height. & 770 & Relative sitting height. \\
\hline Acromion to sole. & 1395 & Fronto-parietal........ \\
\hline Head length. & 195 & Total facial... \\
\hline Head breadth & 134 & Upper facial \\
\hline Minimum frontal diameter. . & 114 & Nasal.. \\
\hline Bizygomatic maximum. . . . . & 126 & Ear. \\
\hline Bigonial breadth....... & 102 & Jugomandibular. \\
\hline Total facial height......... & 115 & Jugofrontal. \\
\hline
\end{tabular}

Upper facial height....... 72

Nasal height........... 55

Nasal breadth............ 31

Ear length............ 62

Ear breadth............. 34

HAIR.-Form: low waves. Texture: fine. Color: dark brown. Quantity: head, double plus; beard, minus; body, average.

EyE.-Color: dark brown. Sclera: clear. Iris: zoned.

Nose.-Profile: straight. Wings: medium.

TEETH.-Lost: 1.

CHEST.-Development: plus.

Musculature.-Good.

HEALTH.-Good.

No. 353

Residence: camp of Sheikh Aziz. Date: June 9, 1928.

Age: 30 ; unmarried.

Brothers: 1 dead. Sisters: 1 living.

Tattoo marks: none.

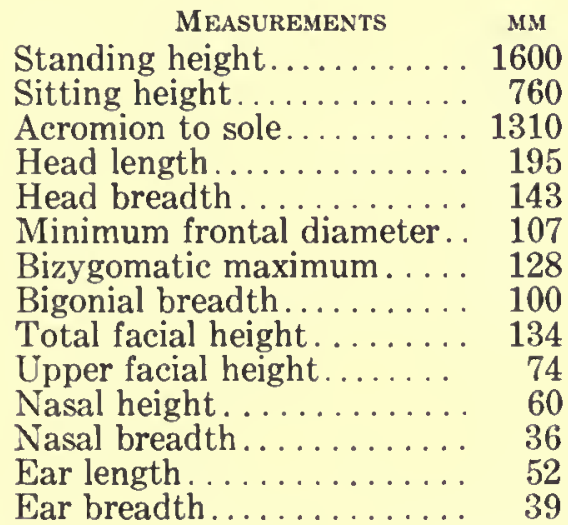

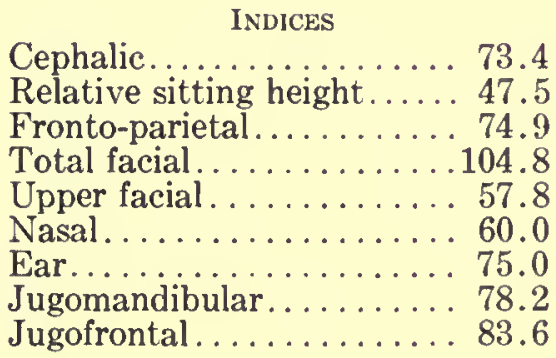

HAIR.-Form: deep waves. Texture: coarse. Quantity: head, plus; beard, minus; body, average. 
EYE--Color: green brown. Sclera: clear. Iris: zoned.

Nose.-Profile: concave.

TEETH.-Lost: none.

CHEst.-Development: plus.

Musculature.-Good.

HEALTH.-Good.

No. 354

Residence: camp of Sheikh Aziz. Date: June 9, 1928.

Age: 30; married for 2 years. No children.

Brothers: none. Sisters: 1 dead.

Tattoo marks: none.

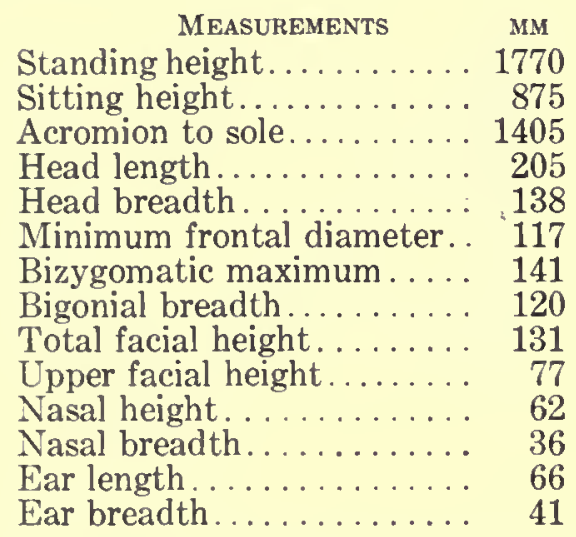

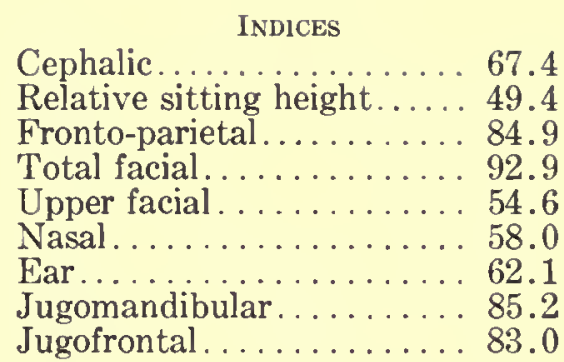

HAIR.-Texture: medium. Color: dark brown. Quantity: head, plus; beard, plus; body, plus. EYE.-Color: dark brown. Sclera: clear. Iris: homogeneous. Blind in right eye.

Nose.-Profile: straight. Wings: medium-compressed.

TEETH.-Lost: 1 .

CHEsT.-Development: plus.

Musculature.--Good.

HEALTH.-Good.

\section{No. 355. Plate XL, Figs. 7, 8}

Residence: camp of Sheikh Aziz. Date: June 9, 1928.

Age: 75; married for 8 years (wife now dead). Sons: none. Daughters: 2 living.

Brothers: none. Sisters: none.

Tattoo marks: on inside of right forearm.

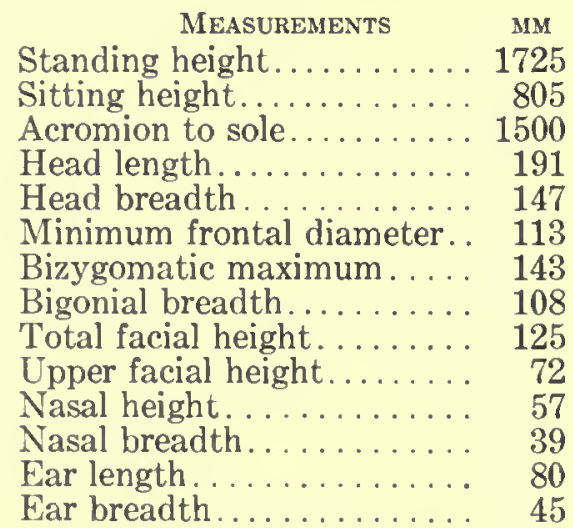

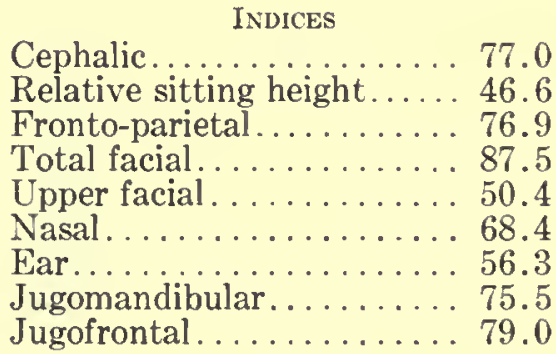

HAIR.-Quantity: head, minus; beard, double plus.

EYE.-Color: blue brown. Sclera: bloodshot. Iris: zoned. 
Nose.-Profile: concave. Wings: flaring.

TEETH.-Lost: many.

CHEST.-Development: plus.

Musculature.-Good.

HEALTH.-Good.

No. 356. Plate XLI, Figs. 1, 2

Residence: camp of Sheikh Aziz. Date: June 9, 1928.

Age: 20; unmarried.

Brothers: 2 living. Sisters: none.

Tattoo marks: none.

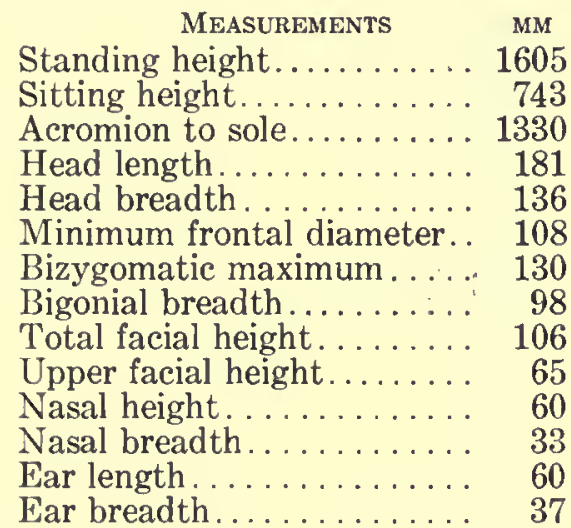

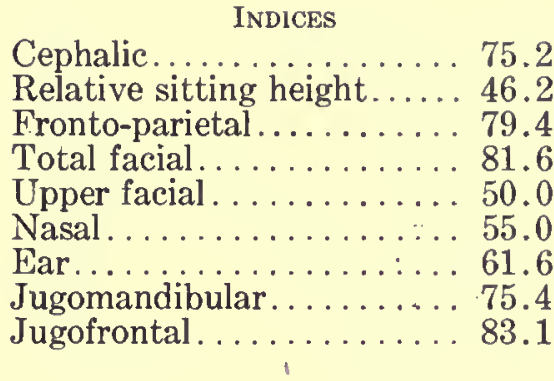

HaIR.-Form: low waves. Texture: medium. Color: dark brown.

EYE.-Color: dark brown. Sclera: clear. Iris: homogeneous.

NosE.-Profile: convex. Wings: medium-flaring.

CHEST.-Development: average.

Musculature.-Fair.

HEALTH.-Good.

\section{No. 357. Plate XLI, Figs. 3, 4}

Residence: camp of Sheikh Aziz. Date: June 9, 1928.

Age: 50 ; married for 20 years. No children.

Brothers: 2 living, 1 dead. Sisters: 1 living, 2 dead.

Tattoo marks: spots on back of left wrist.

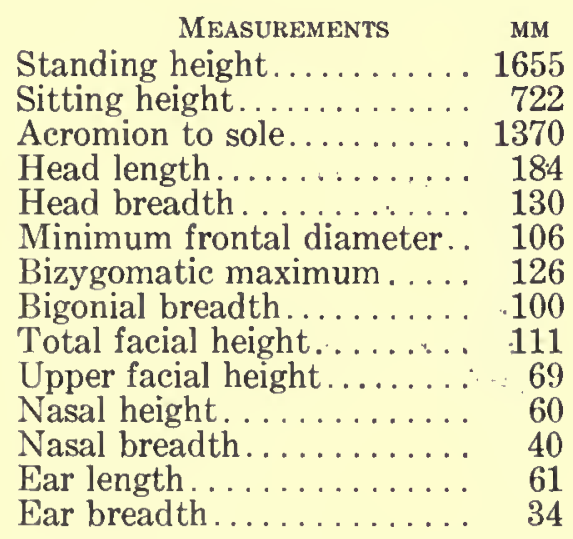

HAIR.-Color: dark brown. Quantity: head, average; beard, double plus; body, double plus. EyE.-Color: blue brown. Sclera: bloodshot. Nose.-Profile: concave. Wings: medium. 
TEETH.-Lost: 1.

CHEsT.-Development: average.

Musculature.-Fair.

Health.-Good.

No. 358. Plate Xli, Figs. 5, 6

Residence: camp of Sheikh Aziz. Date: June 9, 1928.

Age: 25; unmarried.

Brothers: 1 living, 2 dead. Sisters: 1 living, 4 dead.

Tattoo marks: none.

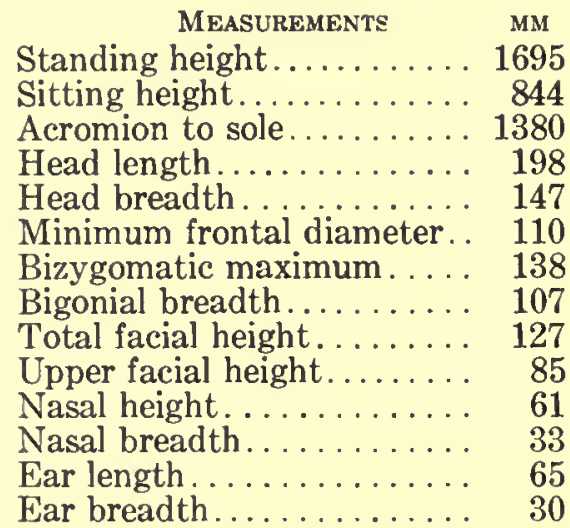

INDICES

Cephalic............. 74.2

Relative sitting height..... 49.8

Fronto-parietal........ 74.9

Total facial. . . . . . . . . . . 92.1

Upper facial . . . . ...... 61.6

Nasal. ................. 54.1

Ear................ 46.2

Jugomandibular. . . . . . . 77.6

Jugofrontal . . . . . . . 79.7

HAIR.-Form: low waves. Texture: medium. Color: dark brown. Quantity: head, double plus; beard, minus; body, minus.

EYE.-Color: dark brown. Sclera: clear. Iris: homogeneous.

NosE.-Profile: straight. Wings: medium.

TEETH.-Lost: none.

CHEST.-Development: plus.

MUSCULATURE.-Excellent.

HEALTH.-Good.

No. 359. Plate Xli, Figs. 7, 8

Residence: camp of Sheikh Aziz. Date: June 9, 1928.

Age: 20; unmarried.

Brothers: 1 living, 2 dead. Sisters: 2 living, 1 dead.

Tattoo marks: none.

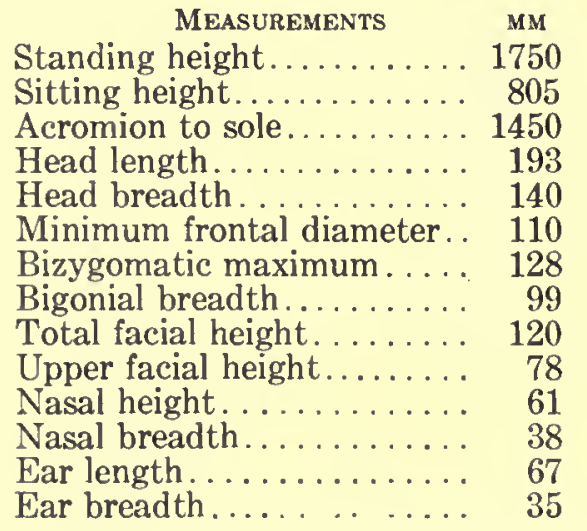

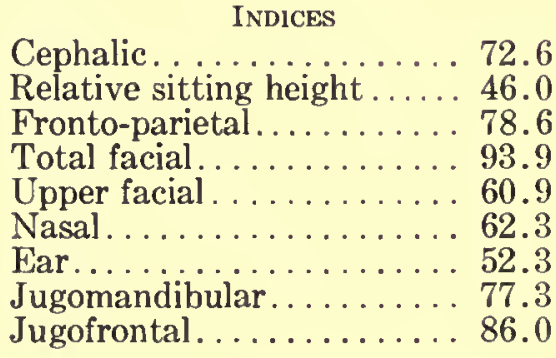

HAIR.-Form: frizzly woolly. Texture: coarse.

EYE.-Color: dark brown. Sclera: clear. Iris: homogeneous. 
Nose.-Profile: concave. Tip: thickness, double plus. Wings: flaring.

TEETH.-Lost: none.

Chest.-Development: plus.

Musculature.-Good.

HEALTH.-Good.

No. 360. Plate XlII, Figs. 1, 2

Residence: camp of Sheikh Aziz. Date: June 9, 1928.

Age: 40; married for 10 years. Sons: none. Daughters: 1 dead.

Brothers: none. Sisters: 1 living.

Tattoo marks: spot on chin.

\begin{tabular}{|c|c|c|}
\hline Measurements & MM & INDICES \\
\hline Standing height.. & 1710 & Cephalic. \\
\hline Sitting height. . & 830 & Relative sitting height. \\
\hline Acromion to sole. & 1405 & Fronto-parietal. \\
\hline Head length ..... & 197 & Total facial... \\
\hline Head breadth. & 145 & Upper facial \\
\hline Minimum frontal diameter. & 119 & Nasal. \\
\hline Bizygomatic maximum ..... & 136 & Ear. \\
\hline Bigonial breadth.. & 109 & Jugomandibular \\
\hline Total facial height. & 117 & Jugofrontal . . \\
\hline Upper facial height. & 66 & \\
\hline Nasal height. & 45 & \\
\hline Nasal breadth. & 31 & \\
\hline Ear length. & 60 & \\
\hline Ear breadth. & 32 & \\
\hline
\end{tabular}

HAIR.-Quantity: head, average.

EYE.-Color: green brown. Sclera: clear. Iris: homogeneous.

Nose.-Profile: straight. Wings: medium.

TEETH.-Lost: none.

Chest.-Development: plus.

Musculature.-Good.

HEALTH.-Good.

Very prominent malars. Cheeks full.

No. 361. Plate XlII, Figs. 3, 4

Residence: camp of Sheikh Aziz. Date: June 9, 1928.

Age: 30 ; married for 10 years. Sons: 1 living. Daughters: 1 living, 1 dead.

Brothers: 1 living. Sisters: 1 living, 2 dead.

Tattoo marks: spots on inside of right upper arm.

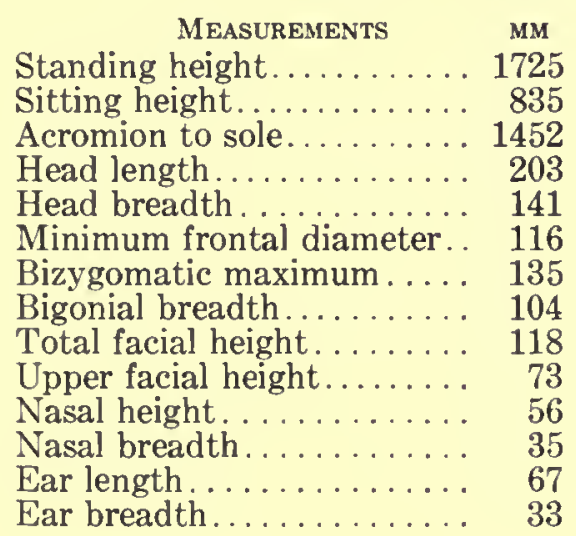

INDICES

Cephalic..............6. 69.4

Relative sitting height..... 48.4

Fronto-parietal......... 82.3

Total facial............. 87.4

Upper facial........... 54.1

Nasal. ................ 62.5

Ear................ 49.3

Jugomandibular........ 77.0

Jugofrontal . . . . . . . . . . 85.9 
HaIr.-Form: low waves. Texture: medium. Color: dark brown. Quantity: head, plus. EYE.-Color: dark brown. Sclera: clear. Iris: homogeneous.

NosE.-Profile: straight. Wings: medium.

TEETH.--Lost: none.

ChEsT.-Development: plus.

Musculature.-Good.

Health.-Good.

No. 364. Plate XLIII, Figs. 1, 2

Residence: camp of Sheikh Aziz. Date: June 9, 1928.

Age: 20 ; married for 3 years. Sons: 1 living. Daughters: none.

Brothers: 1 living. Sisters: 1 living, 1 dead.

Tattoo marks: none.

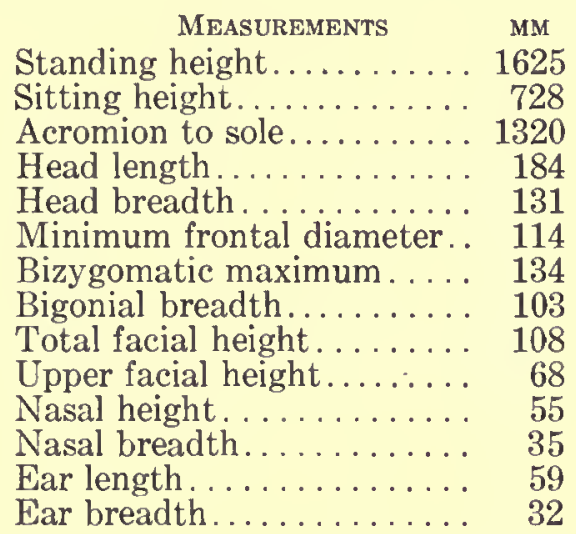

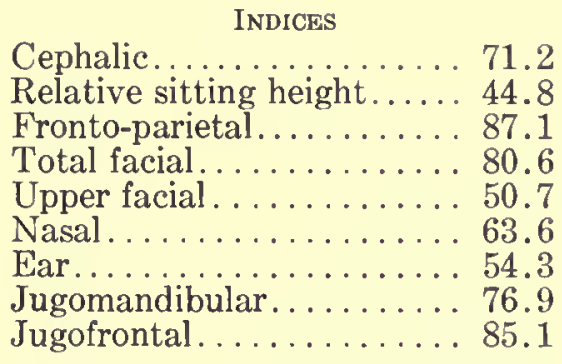

Cephalic.............. 71.2

Relative sitting height..... 44.8

Fronto-parietal.........,87.1

Nasal

Ear................ 54.3

Jugofrontal. .......... 85.1

HaIr.-Form: low waves. Texture: medium. Color: dark brown.

EYE. - Color: dark brown. Sclera: clear. Iris: homogeneous.

NosE.-Profile: convex. Wings: compressed.

TeEтH.-Lost: none. Condition: good.

Chest.-Development: plus.

Musculature.-Good.

Health.-Good.

\section{No. 365. Plate XlIII, Figs. 3, 4}

Residence: camp of Sheikh Aziz. Date: June 9, 1928.

Age: 19; unmarried.

Brothers: none. Sisters: none.

Tattoo marks: none.

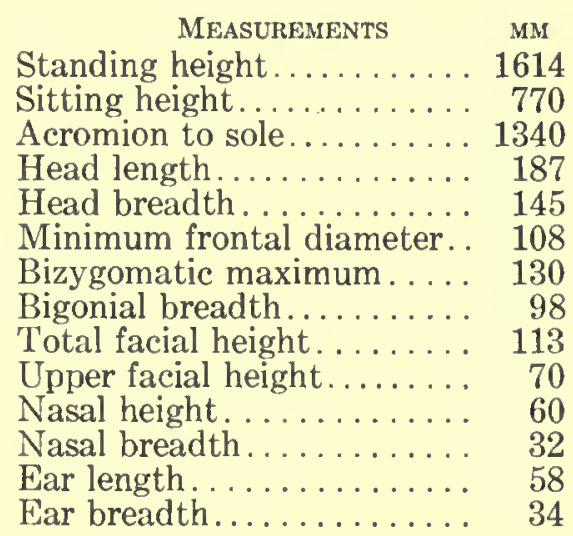

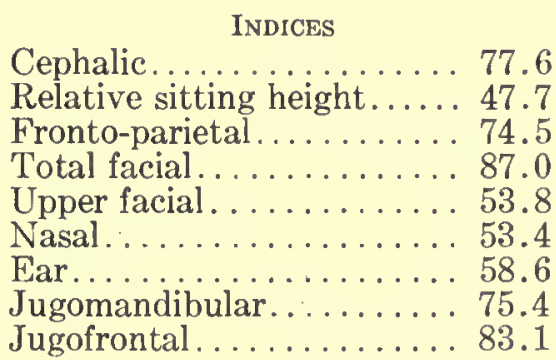

Cephalic............... 77.6

Relative sitting height..... 47.7

Fronto-parietal......... 74.5

Total facial............ 87.0

Upper facial. . . . . . . . 53.8

Nasal. ................ 53.4

Jugomandibular........ 75.4

Jugofrontal........... 83.1 
HAIR.-Texture: medium. Quantity: head, plus; beard, minus; body, average.

EYE.-Color: green brown. Sclera: clear. Iris: homogeneous.

Nose.-Profile: convex. Wings: medium.

TEETH.-Lost: none.

Chest.-Development: plus.

Musculature.-Good.

HEALTH.--Good.

Marked prominence of malars.

No. 366. Plate XliII, Figs. 5, 6

Residence: camp of Sheikh Aziz. Date: June 9, 1928.

Age: 17; unmarried.

Brothers: 2 living. Sisters: 2 living.

Tattoo marks: spot on nasion and gazelle on back of right wrist.

\begin{tabular}{|c|c|}
\hline MEASUREMENTS & MM \\
\hline Standing height. & 1610 \\
\hline Sitting height... & \\
\hline Acromion to sole & \\
\hline Head length. & \\
\hline Head breadth. & \\
\hline Minimum frontal diameter. & \\
\hline Bizygomatic maximum. .. & \\
\hline Bigonial breadth...... & \\
\hline Total facial height. & \\
\hline Upper facial height. & \\
\hline Nasal height. & \\
\hline Nasal breadth. & \\
\hline Ear length. & \\
\hline Ear breadth & \\
\hline
\end{tabular}

Cephalic............... 80.4

Relative sitting height..... 46.0

Fronto-parietal.......... 76.4

Total facial............ 84.4

Upper facial. . . . . . . . . . 49.2

Nasal. . . . . . . . . . 53.6

Ear................... 50.0

Jugomandibular........ 81.3

Jugofrontal ............. 88.3

HaIR.-Form: low waves. Texture: fine. Color: dark brown.

EYE.-Color: blue brown. Sclera: clear. Iris: zoned.

Nose.-Profile: convex. Wings: medium-compressed.

TEETH.-Lost: none. Third molars unerupted. Teeth in good condition.

Chest.-Development: plus.

Musculature.-Good.

HEALTH.-Good.

No. 367 . Plate XliII, Figs. 7,8

Residence: camp of Sheikh Aziz. Date: June 9, 1928.

Age: 90 ; married for 40 years (wife now dead). Sons: 1 living, 1 dead. Daughters: none.

Brothers: none. Sisters: none.

Tattoo marks: glove on right hand and gazelle on inside of right forearm.

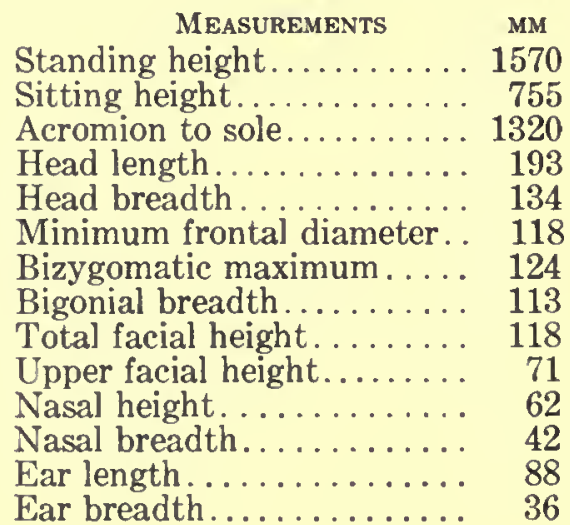

Cephalic..............6.69.5

Relative sitting height...... 48.1

Fronto-parietal......... 88.1

Total facial.............. 95.2

Upper facial. . . . . . . . . 57.3

Nasal. . . . . . . . . . . . . 67.7

Ear.............. 40.9

Jugomandibular. . ......... 91.2

Jugofrontal. . . . . . . . . 95.2 
HAIR.-Color: gray and white. Quantity: head, average; beard, double plus; body, plus. EYE.-Color: blue brown. Sclera: bloodshot. Iris: zoned.

Nose.-Profile: straight. Wings: medium.

TEETH.-Lost: all.

Chest.-Development: plus.

Musculature.-Good.

HeALTH.-Good.

No. 368. Plate XliV, Figs. 1, 2

Residence: camp of Sheikh Aziz. Date: June 9, 1928.

Age: 45 ; married twice, 15 years ago and 4 years ago.

Sons: 2, 1, living. Daughters: 3,1 , living.

Brothers: 1 dead. Sisters: 1 dead.

Tattoo marks: none.

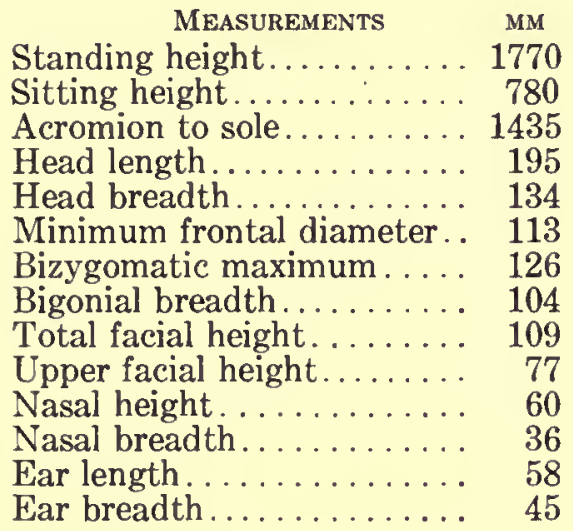

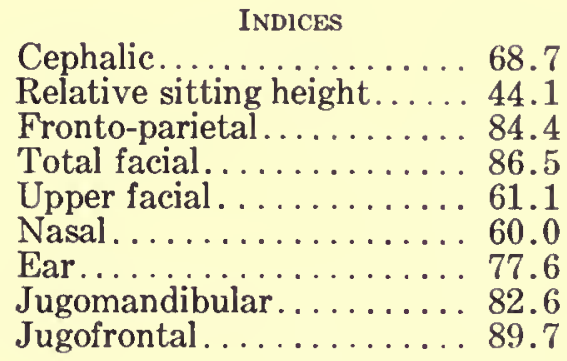

HAIR.-Form: low waves. Texture: fine. Color: dark brown.

Eye.-Color: blue brown. Sclera: clear. Iris: zoned.

Nose.-Profile: convex. Wings: medium-flaring.

TEETH.-Lost: none.

Chest.-Development: plus.

Musculature.--Good.

HEALTH.-Good.

No. 369

Residence: camp of Sheikh Aziz. Date: June 9, 1928.

Age: 50 ; married 4 times (all wives dead). Sons: 1, 0, 0, 1, living. Daughters: 1, 0, 0, 0 , living; $0,0,2,0$, dead.

Brothers: none. Sisters: 1 dead.

Tattoo marks: none.

\begin{tabular}{|c|c|c|}
\hline & MEASUREMENTS & MM \\
\hline Standi & g height. & 1680 \\
\hline Sitting & neight. & 760 \\
\hline Acrom & on to sole. & 1380 \\
\hline Head 1 & ngth..... & 206 \\
\hline Head & eadth. & 13 \\
\hline Mini & n frontal diameter. . & 116 \\
\hline Bizy & atic maximum . . . . & 137 \\
\hline Bigo & breadth.......... & 118 \\
\hline Total & cial height....... & 127 \\
\hline Uppe & cial height. . . . . . . & 78 \\
\hline Nasal & & 60 \\
\hline t & & \\
\hline
\end{tabular}

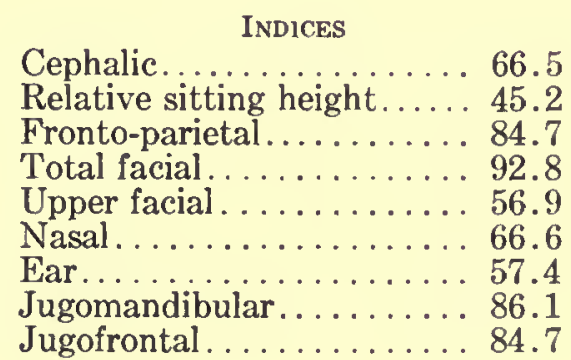

Cephalic.............66 66.5

Relative sitting height..... 45.2

Fronto-parietal.......... 84.7

Upper facial $\ldots \ldots \ldots \ldots . .6 \%$

Nasal. .............66.6

Jugomandibular......... 86.1

Jugofrontal. ........... 84.7 


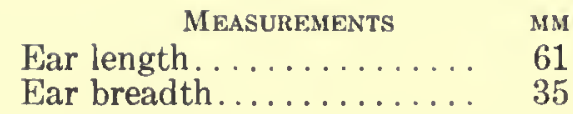

HAIR. - Form: low waves. Texture: medium. Color: dark brown.

EYE.-Color: blue brown. Sclera: clear. Iris: zoned.

NosE.-Profile: straight. Wings: flaring.

Chest.--Development: double plus.

Musculature.-Excellent.

HEALTH. - Good.

No. 370. Plate XliV, Figs. 3, 4

Residence: camp of Sheikh Aziz. Date: June 9, 1928.

Age: 50; married for 10 years. Sons: 1 living. Daughters: 1 living.

Brothers: 1 dead. Sisters: 1 dead.

Tattoo marks: none.

\begin{tabular}{|c|c|}
\hline MEASUREMENTS & MM \\
\hline Standing height. & 1728 \\
\hline Sitting height. & 780 \\
\hline Acromion to sole & 1480 \\
\hline Head length & 198 \\
\hline Head breadth & 136 \\
\hline Minimum frontal diameter. . & 114 \\
\hline Bizygomatic maximum . . . . . & 133 \\
\hline Bigonial breadth. & 110 \\
\hline Total facial height. & 115 \\
\hline Upper facial height. . . . . . & 71 \\
\hline Nasal height. & 63 \\
\hline Nasal breadth & 3 \\
\hline Ear len & 64 \\
\hline & $?$ \\
\hline
\end{tabular}

Cephalic...........6 68.7

Relative sitting height..... 45.2

Fronto-parietal.......... 83.9

Total facial........... 86.5

Upper facial . . . . . . ......... 53.4

Nasal. . . . . . . . . . 55.6

Ear................. 56.3

Jugomandibular. . . . . . . 8. 82.7

Jugofrontal. . . . . . . . . 85.7

HAIR.-Quantity: head, minus; beard, plus; body, plus.

EYE.-Color: blue brown. Sclera: clear.

Nose.-Profile: straight. Wings: medium.

TEETH.-Lost: 1.

Chest.-Development: plus.

Musculature.-Good.

HEALTH.-Good.

No. 371. Plate XliV, Figs. 5, 6

Residence: camp of Sheikh Aziz. Date: June 9, 1928.

Age: 50; married for 10 years. Sons: 2 dead. Daughters: 2 living, 1 dead.

Brothers: none. Sisters: 1 dead.

Tattoo marks: gazelle on right wrist.

\begin{tabular}{|c|c|}
\hline MEASUREMENTS & MM \\
\hline Standing height. ...... & 1720 \\
\hline Sitting height. & 800 \\
\hline Acromion to sole. & 1415 \\
\hline dength ..... & 191 \\
\hline eadth. & 144 \\
\hline frontal diameter. . & 117 \\
\hline tic maximum . ... & 138 \\
\hline dth.......... & 110 \\
\hline height. . . . . . . . & 115 \\
\hline height. . . . . . . & 77 \\
\hline & 66 \\
\hline & 40 \\
\hline
\end{tabular}

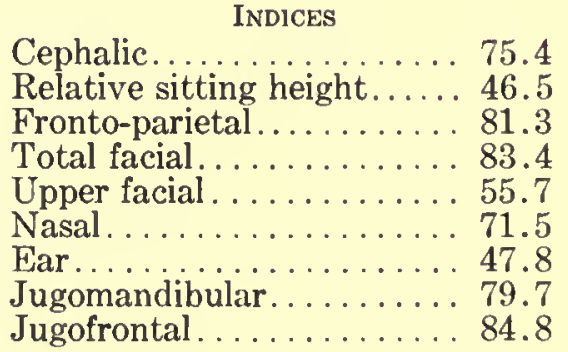


$\begin{array}{cr}\text { Measurements } & \text { MM } \\ \text { Ear length } \ldots \ldots \ldots \ldots \ldots . & 67\end{array}$

Ear breadth........... 32

HAIR.-Color: dark brown. Quantity: head, minus; beard, plus.

EyE.-Color: green brown. Sclera: clear. Iris: zoned.

Nose.-Profile: straight. Wings: medium-flaring.

TEETH.-Lost: none.

Chest.-Development: plus.

Musculature.-Good.

HEALTH.-Good.

No. 372

Residence: camp of Sheikh Aziz. Date: June 9, 1928.

Age: 40 ; unmarried.

Brothers: none. Sisters: 1 dead.

Tattoo marks: none.

\begin{tabular}{|c|c|c|}
\hline MEASUREMENTS & MM & INDICES \\
\hline Sitting height. & 750 & $\ldots \ldots \ldots 71.9$ \\
\hline Acromion to sole. & 1375 & Fronto-parietal..... \\
\hline Head length. & 192 & Total facial. \\
\hline Head breadth. & 138 & Upper facial \\
\hline Minimum frontal diameter. & 117 & . 57.4 \\
\hline Bizygomatic maximum... & 131 & 64.0 \\
\hline Bigonial breadth.......... & 100 & Jugomandibular. \\
\hline Total facial height. & 115 & Jugofrontal. \\
\hline Upper facial height........ & 69 & \\
\hline Nasal height. ........... & 61 & \\
\hline Nasal breadth & 35 & \\
\hline Ear length. & 64 & \\
\hline Ear breadth. & 41 & \\
\hline
\end{tabular}

HaIR.-Form: low waves. Texture: medium. Color: dark brown. Quantity: head, double plus; beard, double plus; body, plus.

EyE.-Color: blue brown. Sclera: clear. Iris: zoned.

NosE.-Profile: straight. Wings: medium-flaring.

TEETH.-Lost: none.

CHEST.-Development: plus.

Musculature.-Good.

HEALTH.-Good.

No. 373

Residence: camp of Sheikh Aziz. Date: June 9, 1928.

Age: 30 ; married for 4 years. No children.

Brothers: none. Sisters: 1 living.

Tattoo marks: spot on right wrist.

\begin{tabular}{|c|c|c|}
\hline Measurements & MM & INDICES \\
\hline Standing height. . & 1614 & Cephalic. \\
\hline Sitting height. & 753 & Relative sitting height. \\
\hline Acromion to sole. & 1350 & Fronto-parietal....... \\
\hline Head length. & 198 & Total facial. \\
\hline Head breadth. & 146 & Upper facial \\
\hline Minimum frontal diameter. . & 111 & Nasal. \\
\hline Bizygomatic maximum ..... & 136 & Ear. \\
\hline onial breadth. & 100 & Jugomandibular. \\
\hline al facia & 118 & Jugofrontal. . . . . . . \\
\hline
\end{tabular}

Uper facial height. . ..............

Nasal height.

Nasal breadth.......... 33 


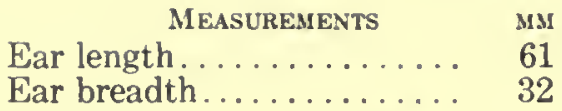

HAIr.-Form: low waves. Texture: medium. Color: black. Quantity: head, double plus; beard, plus; body, average.

EYE.-Color: green brown. Sclera: clear. Iris: zoned.

Nose.-Profile: straight. Wings: medium.

TEETH._Lost: none.

CHEST.-Development: plus.

Musculature.-Good.

HEALTH.-Good.

No. 374

Residence: camp of Sheikh Aziz. Date: June 9, 1928.

Age: 35; married for 6 years. Sons: none. Daughters: 2 living.

Brothers: none. Sisters: none.

Tattoo marks: 3 spots on each temple.

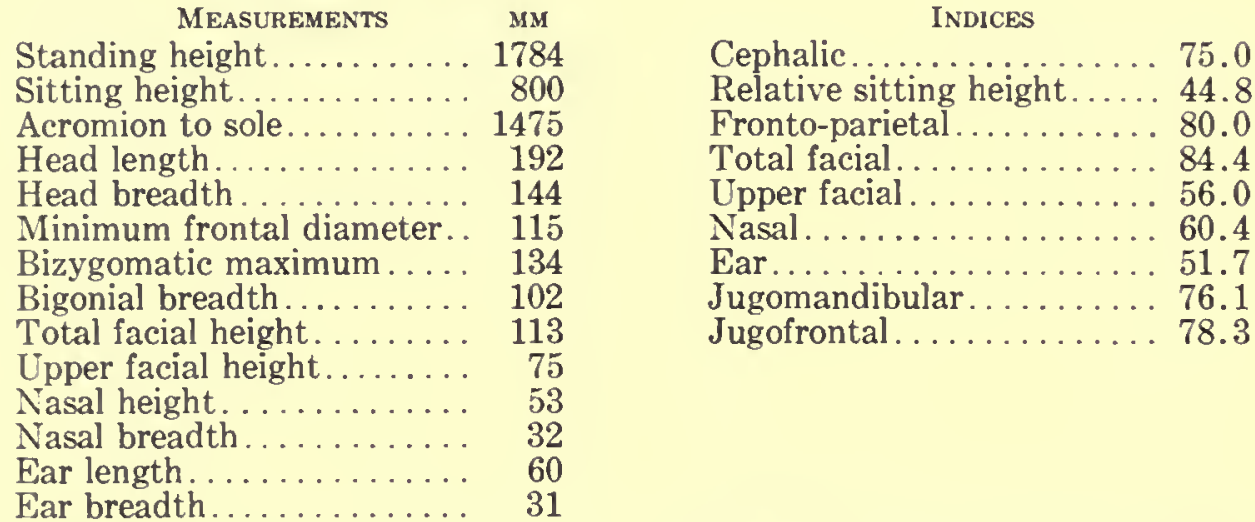

HAIR.-Texture: medium. Color: dark brown. Quantity: head, double plus; beard, plus; body, average.

EYE.-Color: dark brown. Sclera: clear. Iris: zoned.

Nose.-Profile: straight. lVings: medium.

TEETH.-Lost: none.

CHEST.-Development: double plus.

Musculature.-Excellent.

HEALTH.-Good.

No. 375

Residence: camp of Sheikh Aziz. Date: June 9, 1928.

Age: 40; married for 12 years. Sons: 1 dead. Daughters: 3 living.

Brothers: none. Sisters: 1 dead.

Tattoo marks: none.

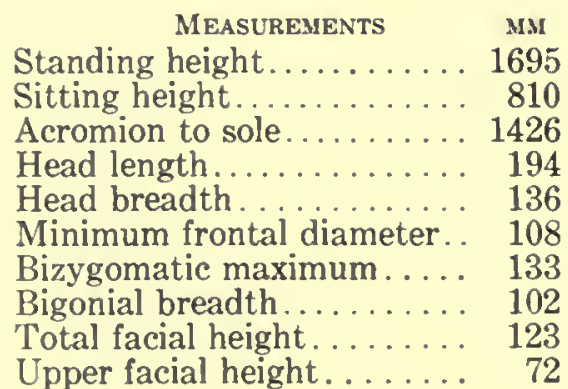

INDICES

Cephalic.............70.1

Relative sitting height..... 47.8

Fronto-parietal......... 79.4

Total facial. . . . . . . . . 92.5

Upper facial. . . . . . . . . . 54.1

Nasal. .............. 76.0

Ear................. 59.1

Jugomandibular........ 76.7

Jugofrontal. . . . . . . . 81.2 
MEASUREMENTS MM

Nasal height. .......... 54

Nasal breadth.......... 41

Ear length . . . . . . . . . . 66

Ear breadth............ 39

HAIR.-Form: low waves. Texture: fine, Color: black.

EYE.-Color: dark brown. Sclera: clear. Iris: zoned.

Nose.-Profile: straight.

TEeTH.-Lost: 1.

Chest.-Developmient: average.

Musculature.-Poor.

HeALTH.-Good.

No. 376. Plate XliV, Figs. 7, 8

Residence: camp of Sheikh Aziz. Date: June 9, 1928.

Age: 80 ; married 5 times, 40, 35, 31, 29, and 25 years ago. Sons: $0,1,0,0$, 0, living. Daughters: $0,1,0,0$, 0, living.

Brothers: 1 dead. Sisters: 1 dead.

Tattoo marks: none.

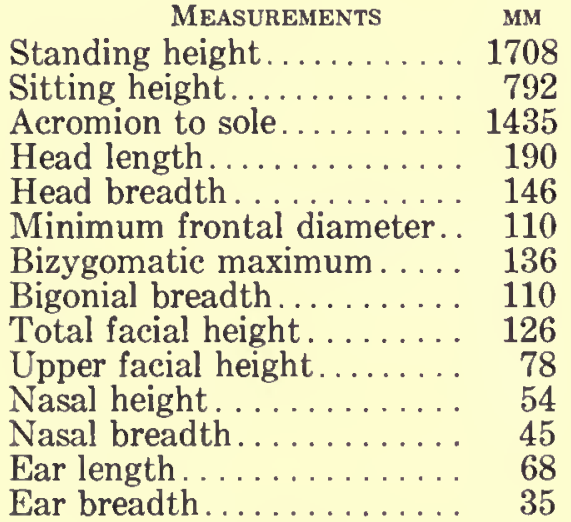

\begin{tabular}{|c|c|}
\hline \multicolumn{2}{|l|}{ INDICES } \\
\hline Cephalic............ & 76.9 \\
\hline Relative sitting height. & 46.3 \\
\hline Fronto-parietal....... & 75.4 \\
\hline Total facial. & 92.6 \\
\hline Upper facial & 57.3 \\
\hline Nasal... & 83.4 \\
\hline Ear. & 51.5 \\
\hline Jugomandibular & 80.9 \\
\hline Jugofrontal. . . . & 80.9 \\
\hline
\end{tabular}

HAIR.-Color: gray. Quantity: head, average; beard, plus.

EYE.-Color: blue brown. Sclera: bloodshot. Iris: zoned.

Nose.-Profile: straight. Wings: flaring.

TeEth.-Wear: double plus. Caries: double plus. Lost: 2.

Chest.-Development: plus.

Musculature.-Good.

HEALTH.-Good.

Very good teeth considering his age. Four wives are dead.

No. 377

Residence: camp of Sheikh Aziz. Date: June 9, 1928.

Age: 35; married once (wife now dead). Sons: none. Daughters: 1 living, 1 dead.

Brothers: none. Sisters: 1 living, 1 dead.

Tattoo marks: none.

\begin{tabular}{|c|c|}
\hline MEASUREMENTS & MM \\
\hline Standing height. & 173 \\
\hline Sitting height. & 83 \\
\hline Acromion to sole & 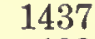 \\
\hline Head length & . \\
\hline Head breadth & \\
\hline ete & \\
\hline
\end{tabular}

Cephalic............. 71.9

Relative sitting height..... 47.9

Fronto-parietal......... 84.1

Total facial. ............ 93.3

Upper facial. . . . . . . . . . . 56.1

Nasal. . . . . . . . . . . 55.2 


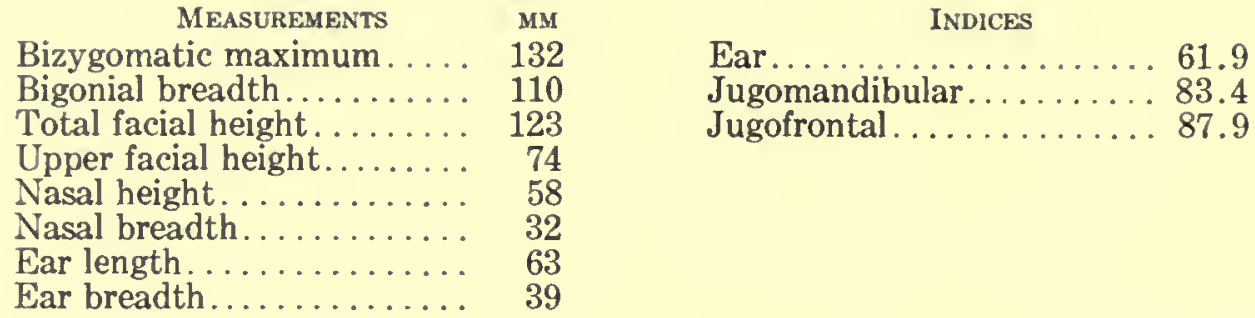

HaIR.-Form: deep waves. Texture: medium. Color: dark brown. Quantity: head, double plus; beard, plus; body, plus.

EYE.-Color: dark brown. Sclera: clear. Iris: zoned.

NosE.-Profile: concavo-convex. Wings: medium.

TEETH.-Lost: none.

CHEst.-Development: plus.

Musculature.-Good.

HEALTH.-Good.

No. 378. Plate XLV, Fig. 1

Residence: camp of Sheikh Aziz. Date: June 9, 1928.

Age: 25; unmarried.

Brothers: none. Sisters: none.

Tattoo marks: none.

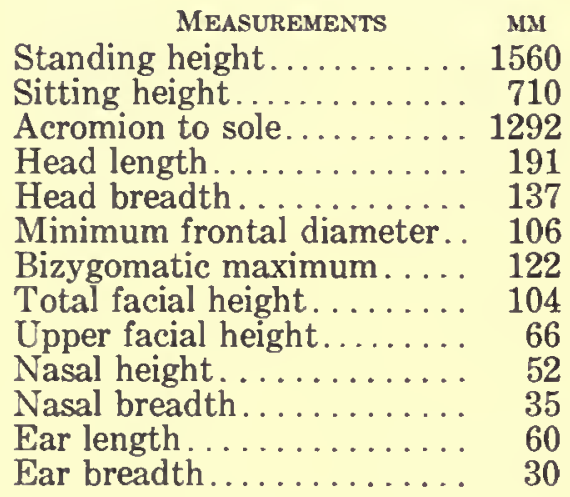

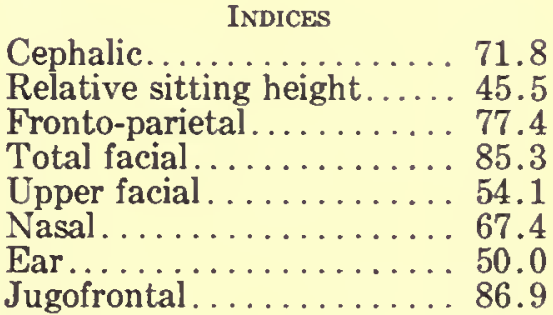

HalR.-Form: low waves. Texture: fine. Color: dark brown. Quantity: head, plus; beard, minus; body, average.

EYE.-Color: dark brown. Sclera: clear. Iris: zoned.

NosE.-Profile: straight. Tip: thickness, minus; depressed.

TEETH.-Lost: none.

Chest.-Development: plus.

MUSCUlATURE.-Good.

HEALTH.-Good.

\section{No. 379. Plate XLV, Fig. 2}

Residence: camp of Sheikh Aziz. Date: June 9, 1928.

Age: 50; married for 10 years. Sons: 1 living. Daughters: 2 living.

Brothers: 1 dead. Sisters: 1 dead.

Tattoo marks: glove on back of right hand, gazelle on inside of right forearm; spot on left wrist; 2 spots to the left of glabella.

\begin{tabular}{|c|c|}
\hline MEASUREMENTS & MM \\
\hline Standing height. . & 1685 \\
\hline $\begin{array}{l}\text { Sitting height... } \\
\text { Acromion to sole }\end{array}$ & $\begin{array}{r}775 \\
1415\end{array}$ \\
\hline
\end{tabular}

INDICES

Cephalic............... 70.4

Relative sitting height..... 45.9

Fronto-parietal......... 79.8 


\begin{tabular}{|c|c|}
\hline Measurements & MM \\
\hline Head length. & 196 \\
\hline Head breadth. & 138 \\
\hline Minimum frontal diameter. & 110 \\
\hline Bizygomatic maximum .... & 137 \\
\hline Bigonial breadth. . & 110 \\
\hline Total facial height. . & 121 \\
\hline Upper facial height. . & 75 \\
\hline Nasal height. . ..... & 58 \\
\hline Nasal breadth. . & 36 \\
\hline Ear length. & 70 \\
\hline Ear breadth & 35 \\
\hline
\end{tabular}

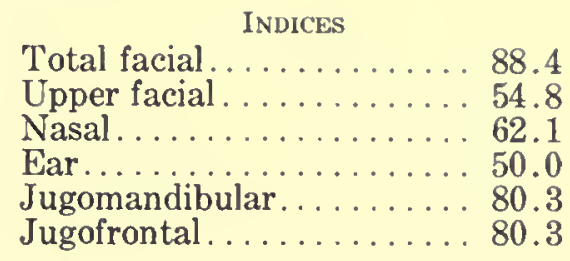

HAIR.-Form: low waves. Texture: medium. Color: dark brown.

EYE.-Color: blue brown. Sclera: bloodshot. Iris: zoned.

Nose.-Profile: concavo-convex. Tip: thickness, double plus; elevated. Wings: mediumflaring.

TEETH.-Lost: 1.

CHEST.-Development: plus.

Musculature.-Good.

HEALTH.-Good.

\section{No. 380. Plate XLV, Figs. 3,4}

Residence: camp of Sheikh Aziz. Date: June 9, 1928.

Age: 30 ; unmarried.

Brothers: 2 dead. Sisters: none.

Tattoo marks: 2 spots on left wrist and spot at nasion.

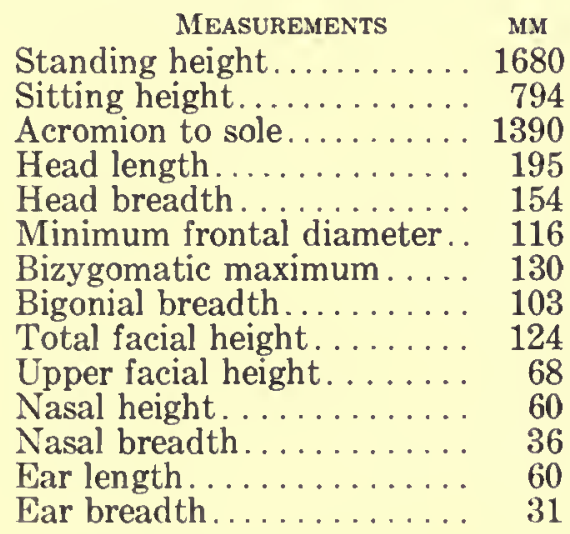

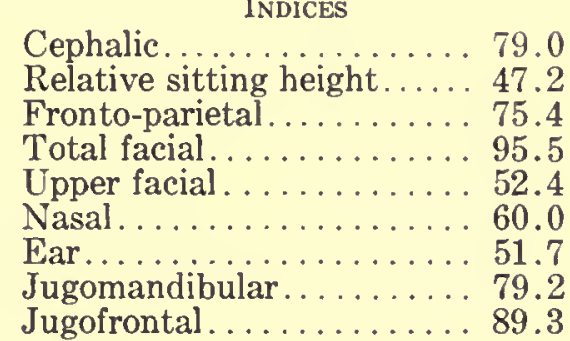

Cephalic............ 79.0

Relative sitting height.....47.2

Upper facial........ .52 .4$

Nasal. ..............6 60.0

Jugofrontal . . . . . . . . . . 89.3 


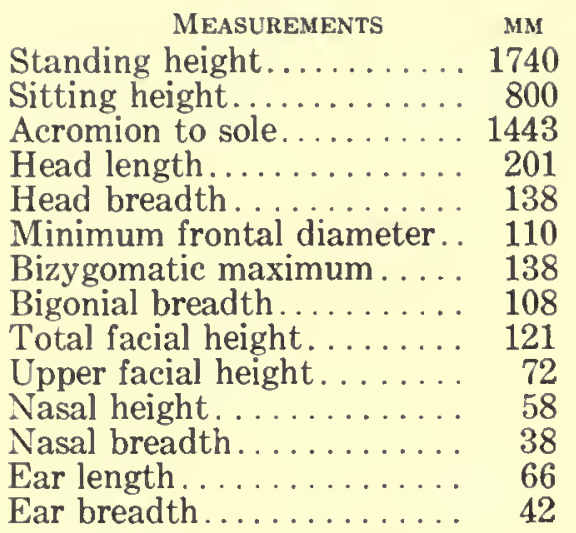

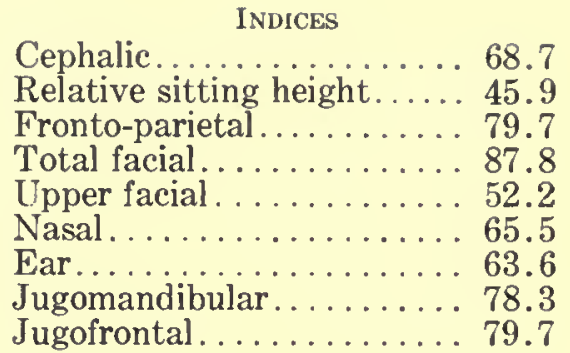

HAIR.-Form: low waves. Texture: medium. Color: dark brown. Quantity: head, double plus; beard, plus; body, plus.

EYE.-Color: dark brown. Sclera: clear. Iris: zoned.

Nose.-Profile: straight. Wings: medium-flaring.

TEETH.-Lost: 1.

CHEST.-Development: plus.

Musculature.-Good.

HEALTH.--Good.

No. 382. Plate XLV, Figs. 5, 6

Residence: camp of Sheikh Aziz. Date: June 9, 1928.

Age: 80 ; married for 50 years (wife now dead). Sons: 2 living. Daughters: 10 living, 7 dead. Brothers: 4 dead. Sisters: 1 living, 1 dead.

Tattoo marks: none.

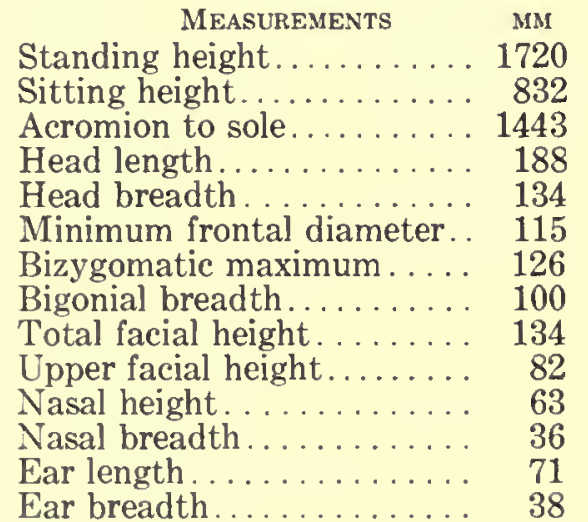

HAIR.-Color: gray. Quantity: head, minus; beard, double plus.

EYE.-Color: blue brown. Sclera: bloodshot. Iris: zoned.

Nose.-Profile: straight. Wings: compressed.

TeETH.-Wear: double plus. Caries: double plus. Lost: 4.

CHest.-Development: average.

Musculature.-Good.

HEALTH.-Good.

Lips very thick. Marked wrinkling on face.

No. 383

Residence: camp of Sheikh Aziz. Date: June 9, 1928.

Age: 45; married for 15 years. Sons: none. Daughters: 5 living, 2 dead. 
Brothers: 1 living. Sisters: 1 living, 1 dead.

Tattoo marks: bar with 3 lines on right wrist.

\begin{tabular}{|c|c|}
\hline & \\
\hline Standing height....... & 162 \\
\hline & 78 \\
\hline & 13 \\
\hline & \\
\hline & \\
\hline & \\
\hline & \\
\hline & \\
\hline & \\
\hline & \\
\hline & \\
\hline & \\
\hline & \\
\hline & \\
\hline MEASUREMENTS & \\
\hline Sitting height & \\
\hline Acromion to sole & \\
\hline Head length. & \\
\hline Head breadth. & \\
\hline Minimum frontal diameter. & \\
\hline Bizygomatic maximum & \\
\hline Bigonial breadth. & 10 \\
\hline Total facial height. & \\
\hline Upper facial height. & \\
\hline Nasal height. & \\
\hline Nasal breadth. & \\
\hline Ear breadth. & \\
\hline
\end{tabular}

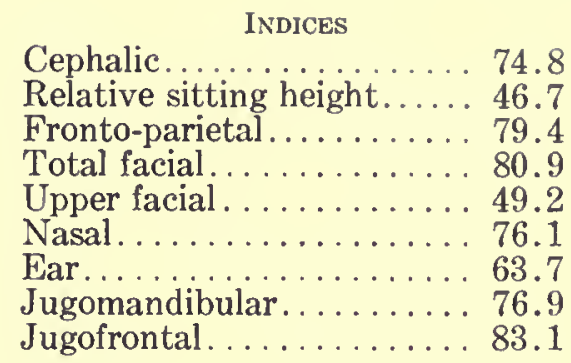

74.8

6.7

80.9

49.2

Jugofrontal. . . . . . . . . 83.1

HAIR.-Form: low waves. Texture: fine. Color: black. Quantity: head, plus; beard, plus; body, plus.

EYE.-Color: blue brown. Sclera: clear. Iris: zoned.

NosE.-Profile: straight. Wings: compressed.

TeETH.-Lost: 3.

CHEST.-Development: plus.

Musculature.-Good.

HEALTH.-Good.

No. 384

Residence: camp of Sheikh Aziz. Date: June 9, 1928.

Age: 43 ; married for 10 years. Sons: 1 living. Daughters: 2 living.

Brothers: none. Sisters: 3 living.

Tattoo marks: 4 spots on back of left hand.

\begin{tabular}{|c|c|c|}
\hline Measurements & мM & INDICES \\
\hline Standing height. & 1683 & Cephalic. \\
\hline Sitting height. & 796 & Relative sitting height. \\
\hline Acromion to sole. & 1384 & Fronto-parietal........ \\
\hline Head length.... & 195 & Total facial... \\
\hline Head breadth. & 137 & Upper facial. \\
\hline Minimum frontal diameter. & 119 & Nasal..... \\
\hline Bizygomatic maximum... & 136 & 64.1 \\
\hline Bigonial breadth...... & 106 & Jugomandibular. \\
\hline Total facial height. & 116 & Jugofrontal. . . \\
\hline Upper facial height. & 76 & \\
\hline Nasal height. & 56 & \\
\hline Nasal breadth. & 33 & \\
\hline Ear length. & 64 & \\
\hline Ear breadtl & 41 & \\
\hline
\end{tabular}

HAIR.-Texture: medium. Color: black.

EYE.-Color: dark brown. Sclera: clear. Iris: zoned.

NosE.-Profile: straight. Wings: medium.

TEETH.-Lost: none. Condition: good.

CHest.-Development: plus.

Musculature.-Good.

HEalth.-Good.

No. 385

Residence: camp of Sheikh Aziz. Date: June 9, 1928.

Age: 30 ; married for 4 years. Sons: none. Daughters: 1 living. 
Brothers: none. Sisters: none.

Tattoo marks: none.

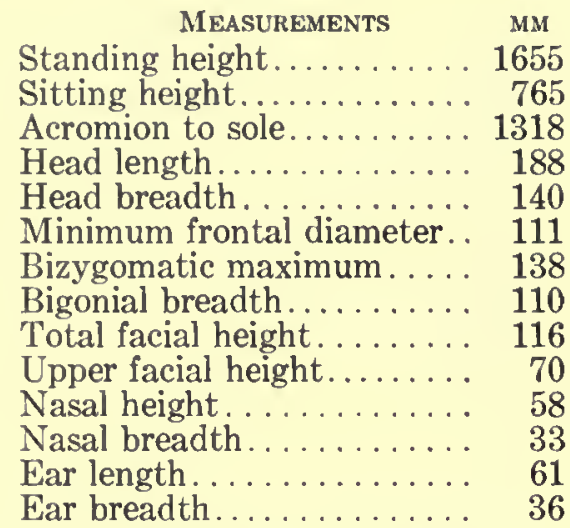

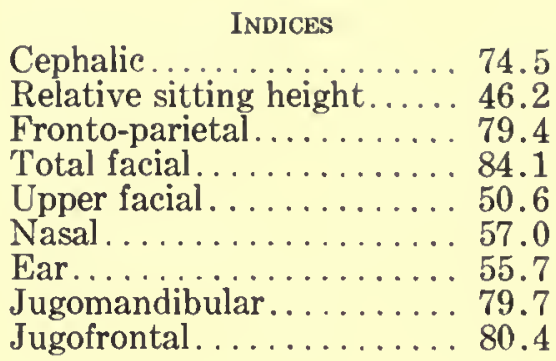

HAIR.-Form: deep waves. Texture: fine. Color: dark brown. Quantity: head, double plus; beard, plus; body, plus.

EYE.-Color: dark brown. Sclera: clear. Iris: zoned.

Nose.-Profile: straight. Wings: compressed.

TEETH.- Lost: none.

CHEst.-Development: double plus.

Musculature.-Excellent.

HEALTH.-Good.

No. 386

Residence: camp of Sheikh Aziz. Date: June 9, 1928.

Age: 40 ; married for 14 years. Sons: 2 living, 2 dead. Daughters: none.

Brothers: 1 living. Sisters: 2 living.

Tattoo marks: none.

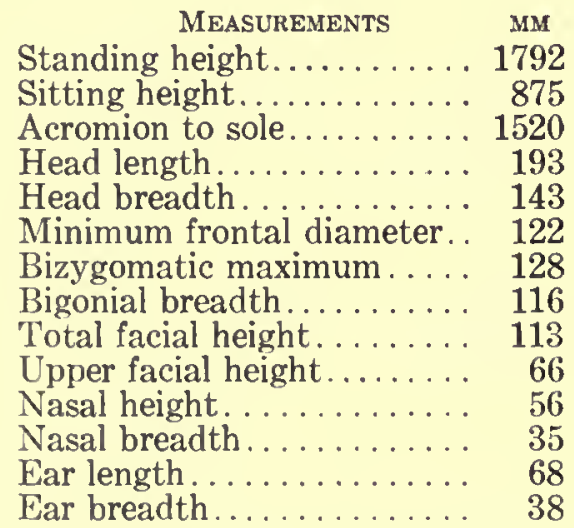

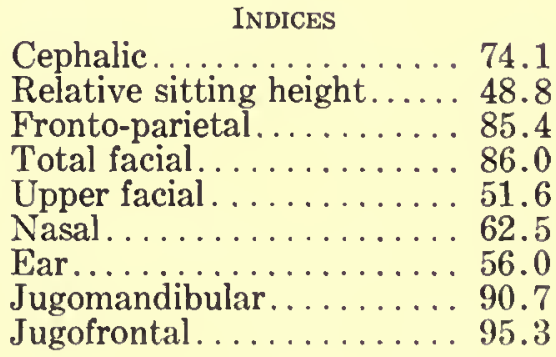

HAIR.-Form: low waves. Texture: medium. Color: dark brown.

EYE.-Color: dark brown. Sclera: clear.

Nose.-Profile: straight. Tip: depressed. Wings: compressed.

TEETH.-Lost: 1.

Chest.-Development: plus.

Musculature.-Good.

HEALTH.--Good.

No. 387

Residence: camp of Sheikh Aziz. Date: June 9, 1928.

Age: 30 ; unmarried. 
Brothers: 1 living, 1 dead. Sisters: 1 living, 1 dead.

Tattoo marks: none.

\begin{tabular}{|c|c|}
\hline MEASUREMENTS & MM \\
\hline Standing height... & 1570 \\
\hline Sitting height. & 740 \\
\hline Acromion to sole. & 1260 \\
\hline Head length..... & 186 \\
\hline Head breadth. & 144 \\
\hline Minimum frontal diameter. . & 102 \\
\hline Bizygomatic maximum . . . . & 124 \\
\hline Bigonial breadth. & 100 \\
\hline Total facial height. . . . . . . . & 111 \\
\hline Upper facial height......... & 73 \\
\hline Nasal height & 53 \\
\hline Nasal & \\
\hline Ear le & \\
\hline & \\
\hline
\end{tabular}

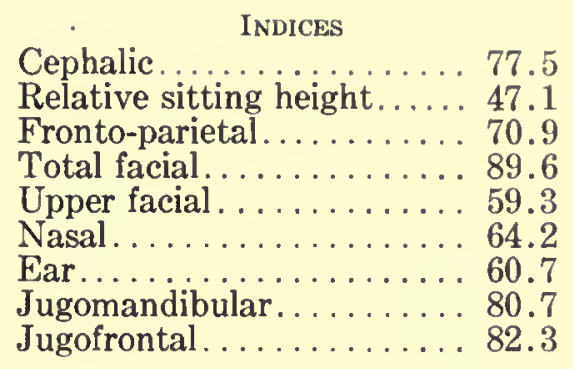

HAIR.-Form: curly frizzly. Texture: coarse. Quantity: head, double plus; beard, minus; body, average.

EYE.-Color: dark brown. Sclera: clear. Iris: zoned.

Nose.-Profile: straight. Wings: medium.

TEETH.-Lost: none.

Chest.-Development: average.

Musculature.-Good.

HEALTH.-Good.

Third molars unerupted. Marked prominence of malars.

No. 388

Residence: camp of Sheikh Aziz. Date: June 9, 1928.

Age: 30 ; married for 8 years. Sons: 1 living, 1 dead. Daughters: 1 dead.

Brothers: 2 living. Sisters: 2 dead.

Tattoo marks: glove and gazelle on hand and inside of right forearm.

\begin{tabular}{|c|c|}
\hline MEasurements & MM \\
\hline Standing height. & 1730 \\
\hline Sitting height. & 795 \\
\hline Acromion to sole. & 1435 \\
\hline Head length.... & 203 \\
\hline Head breadth. & 142 \\
\hline Minimum frontal diameter. & 120 \\
\hline Bizygomatic maximum ..... & 134 \\
\hline Bigonial breadth.......... & 110 \\
\hline Total facial height. & 124 \\
\hline Upper facial height. & 75 \\
\hline Nasal height. & 60 \\
\hline Nasal breadth & 36 \\
\hline Ear len & 64 \\
\hline Wom & \\
\hline
\end{tabular}

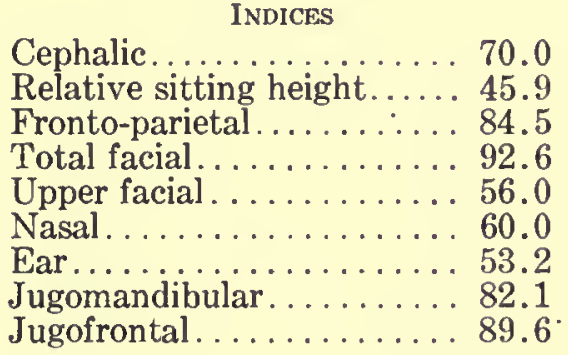

HaIR.-Form: low waves. Texture: medium. Color: dark brown. Quantity: head, plus; beard, double plus.

EYE.-Color: blue brown. Sclera: clear. Iris: zoned.

Nose.-Profile: straight. Wings: medium.

TEETH.-Lost: none.

Chest.-Development: plus.

Musculature.-Good.

Health.-Good. 
No. 389

Residence: camp of Sheikh Aziz. Date: June 9, 1928.

Age: 60 ; married twice, 30 years ago and 20 years ago. Sons: 1, 2, living. Daughters: 7, 1 , living.

Brothers: 2 living, 2 dead. Sisters: 1 living, 1 dead.

Tattoo marks: glove on back of right hand.

\begin{tabular}{|c|c|}
\hline MEASUREMENTS & MM \\
\hline Standing height. & 1570 \\
\hline Sitting height. & \\
\hline Acromion to sole. & 130 \\
\hline Head length. & \\
\hline Head breadth & \\
\hline Minimum frontal diameter. & \\
\hline Bizygomatic maximum.... & \\
\hline Bigonial breadth.. & \\
\hline Total facial height. & \\
\hline Upper facial height. & \\
\hline $\begin{array}{l}\text { Nasal height } \\
\text { Nasal breadth }\end{array}$ & \\
\hline $\begin{array}{l}\text { Nasal breadth } \\
\text { Ear length... }\end{array}$ & \\
\hline $\begin{array}{l}\text { Ear length.. } \\
\text { Ear breadth. }\end{array}$ & \\
\hline
\end{tabular}

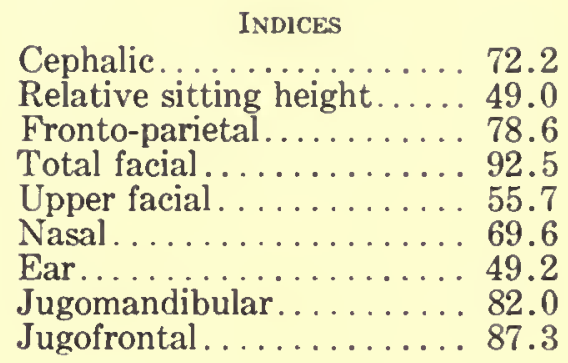

2.2

2.5

55.7

99.6

Jugofrontal. ...........8 87.3

HaIR.-Color: dark brown and gray. Quantity: head, minus; beard, plus; body, plus. EYE.-Color: blue brown. Sclera: bloodshot. Iris: zoned.

NosE.-Profile: concavo-convex. Wings: flaring.

TEETH.-Lost: 2.

Chest.-Development: average.

Musculature.-Fair.

HEALTH.-Good.

No. 390

Residence: camp of Sheikh Aziz. Date: June 9, 1928.

Age: 30; married for 10 years. Sons: 1 dead. Daughters: 2 living.

Brothers: 3 living. Sisters: 2 living.

Tattoo marks: glove on right hand.

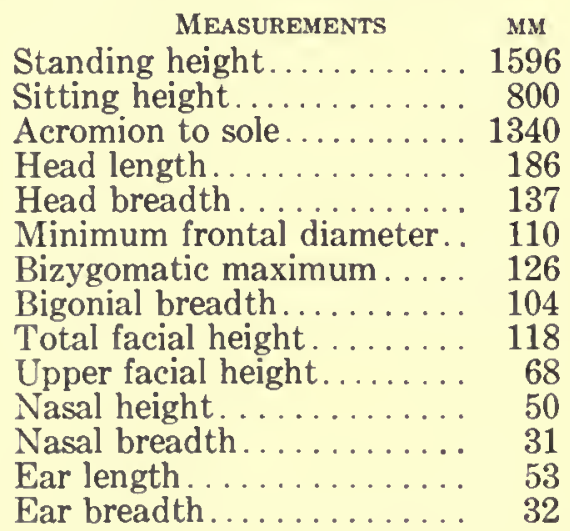

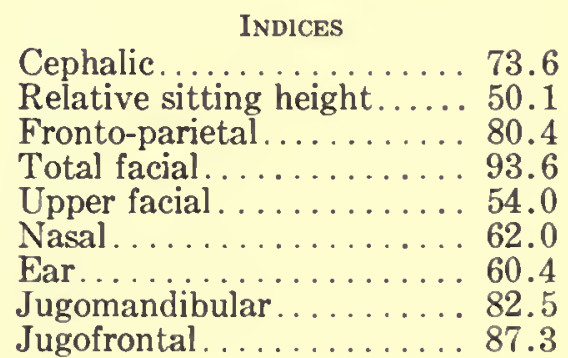

HaIR.-Form: low waves. Texture: medium. Color: dark brown. Quantity: head, double plus; beard, plus; body, plus.

Eye.-Color: blue brown. Sclera: clear. Iris: zoned.

Nose.-Profile: straight. Wings: medium.

TEETH.-Lost: none.

Chest.-Development: plus.

Musculature.-Good.

HEALTH.-Good. 
No. 391

Residence: camp of Sheikh Aziz. Date: June 9, 1928.

Age: 20 ; unmarried.

Brothers: none. Sisters: none.

Tattoo marks: none.

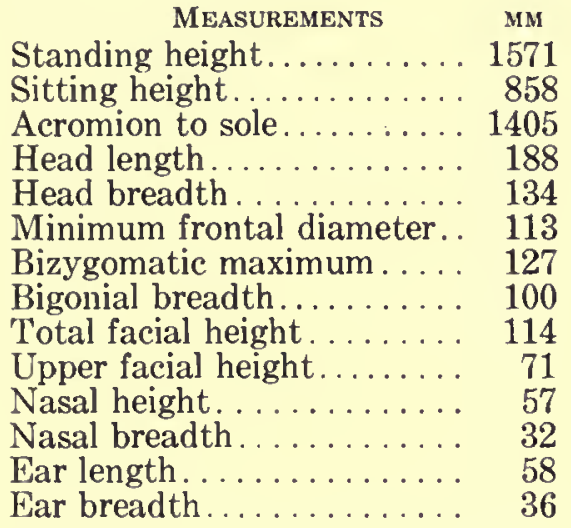

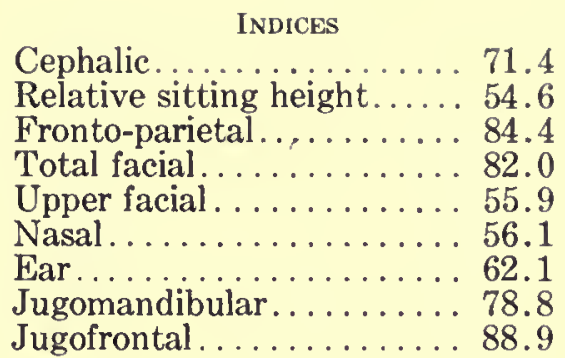

Hair.-Form: low waves. T'exture: fine. Color: black. Quantity: head, double plus; beard, double minus; body, minus.

Eye.-Color: dark brown. Sclera: clear. Iris: homogeneous.

Nose.-Profile: concave. Wings: medium.

TEETH.-Lost: none. Unerupted: third molars.

Chest.-Development: plus.

Musculature.-Good.

HEALTH.-Good.

No. 392. Plate XLV, Figs. 7, 8

Residence: camp of Sheikh Aziz. Date: June 9, 1928.

Age: 25; unmarried.

Brothers: none. Sisters: 2 living.

Tattoo marks: cross on inside of right forearm.

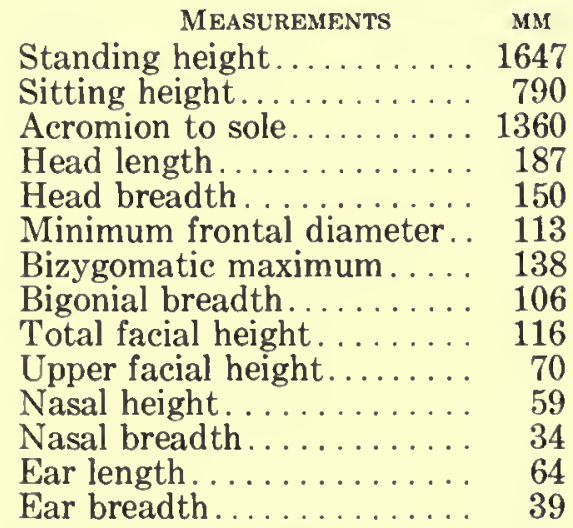

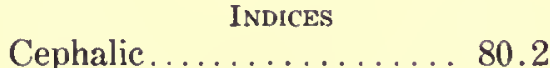

Relative sitting height..... 47.9

Fronto-parietal.......... 75.4

Total facial............. 84.1

Upper facial. .......... 50.7

Nasal. ..............6.61.0

Ear...............61.0

Jugomandibular. . . . . . . 76.8

Jugofrontal. . . . . . . . 81.9

HaIR.-Form: low waves. Texture: fine. Color: black. Quantity: head, triple plus; beard, minus; body, minus.

EYE.-Color: dark brown. Sclera: clear. Iris: zoned.

Nose.-Profile: concavo-convex.

TEETH.-Lost: none.

CHEST.-Development: average.

Musculature.-Fair.

HEALTH.-Good. 
Brothers: 2 living, 2 dead. Sisters: 7 living.

Tattoo marks: none.

\begin{tabular}{|c|c|c|}
\hline MEAsurements & мм & INDICES \\
\hline Standing height. & 1650 & Cephalic. \\
\hline Sitting height. & 800 & Relative sitting height \\
\hline Acromion to sole. & 1355 & Fronto-parietal. \\
\hline Head length.. & 191 & Total facial. \\
\hline Head breadth. & 147 & Upper facial \\
\hline Minimum frontal diameter. & 118 & Nasal. \\
\hline Bizygomatic maximum.... & 132 & Ear.. \\
\hline Bigonial breadth. & 99 & Jugomandibular. \\
\hline Total facial height. & 103 & Jugofrontal. . \\
\hline Upper facial height. & 68 & \\
\hline Nasal height. & 57 & \\
\hline Nasal breadth. & 36 & \\
\hline Ear length. & 60 & \\
\hline Ear breadth & 34 & \\
\hline
\end{tabular}

HAIR.-Form: low waves. Texture: fine. Color: black. Quantity: head, double plus; beard, double minus; body, double minus.

EYE.-Color: green brown. Sclera: clear. Iris: zoned.

Nose.-Profile: concave. Wings: flaring.

TEETH.-Lost: none.

Chest.-Development: average.

Musculature.-Good.

HEALTH.-Good.

No. 396

Residence: camp of Sheikh Aziz. Date: June 9, 1928.

Age: 35; married for 15 years. Sons: 2 living, 1 dead. Daughters: 1 living, 1 dead.

Brothers: 2 living. Sisters: 7 living.

Tattoo marks: glove on back of right hand and gazelle on inside of right forearm.

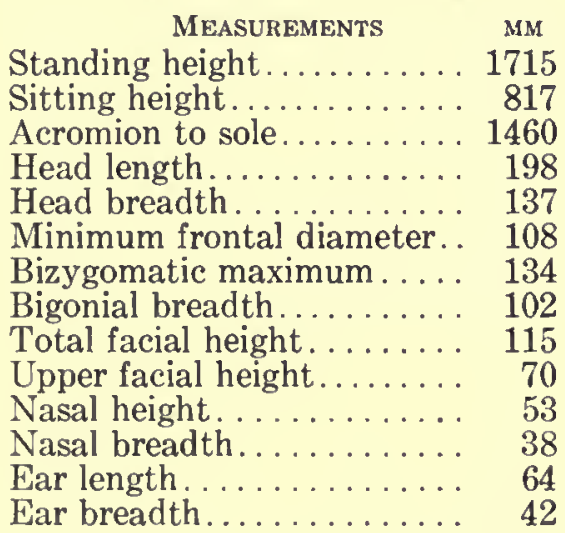

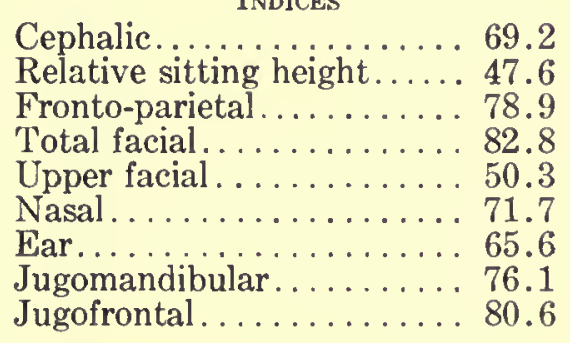

HAIR.-Form: low waves. Texture: medium. Color: black. Quantity: head, double plus; beard, plus; body, plus.

Eye.-Color: dark brown. Sclera: clear. Iris: zoned.

Nose.-Profile: straight. Wings: medium-flaring.

TEETH.-Lost: none.

Chest.-Development: double plus.

Musculature.-Excellent.

HEALTH.-Good.

No. 397

Residence: camp of Sheikh Aziz. Date: June 9, 1928.

Age: 30 ; married for 8 years. Sons: none. Daughters: 2 living, 1 dead. 
Brothers: none. Sisters: 2 living.

Tattoo marks: glove on back of right hand, and 2 dots and cross on inside of right forearm.

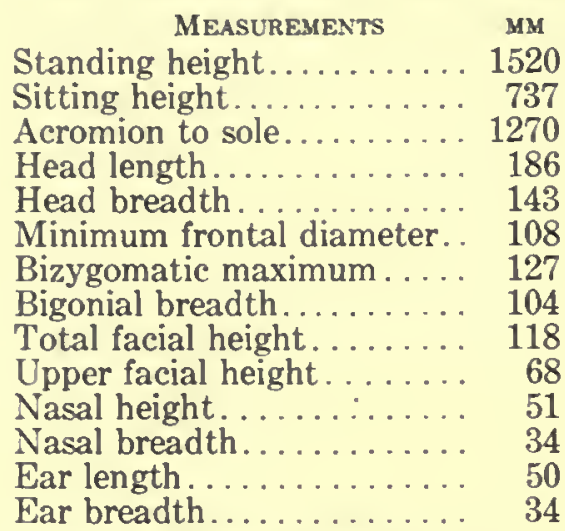

Cephalic.............. 76.9

Relative sitting height......48.4

Fronto-parietal.......... 75.5

Total facial........... 93.0

Upper facial . . . . . . 53.6

Nasal . . . . . . . . . . . 66.6

Ear...............6. 68.0

Jugomandibular........ 81.9

Jugofrontal . . . . . . . 83.1

HAIR.-Quantity: head, average; beard, plus.

EYE.-Color: dark brown. Sclera: clear. Iris: zoned.

Nose.-Profile: convex. Wings: medium.

TEETH. - Lost: none.

CHEsT.-Development: plus.

Musculature.-Good.

HEALTH.-Grood.

No. 398

Residence: camp of Sheikh Aziz. Date: June 9, 1928.

Age: 50; married for 20 years. Sons: 2 living. Daughters: 1 living, 1 dead.

Brothers: 2 dead. Sisters: none.

Tattoo marks: none.

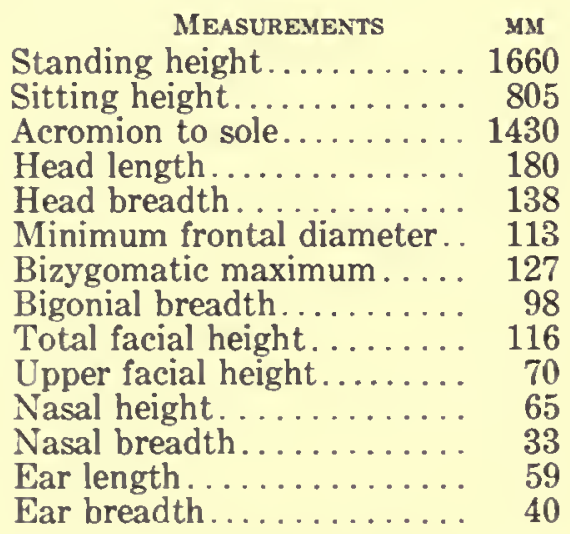

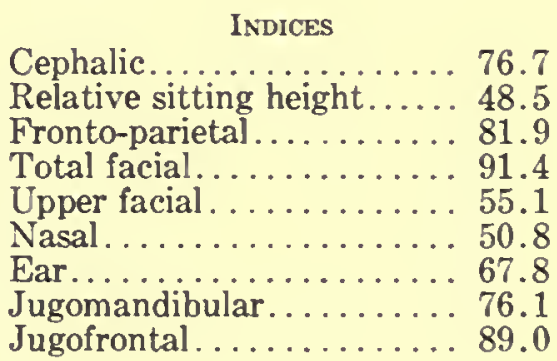

No. 399

HAIR.-Color: dark brown and gray.

EYE.-Color: blue brown. Sclera: bloodshot. Iris: zoned.

Nose.-Profile: straight. Wings: compressed.

TeETH.-Caries: plus. Lost: none.

CHEST.-Development: minus.

Musculature.-Poor.

HEALTH.-Good.

Residence: camp of Sheikh Aziz. Date: June 9, 1928.

Age: 18; unmarried.

Brothers: 1 living, 1 dead. Sisters: 1 dead.

Tattoo marks: glove and gazelle on right hand and inside of right forearm. 


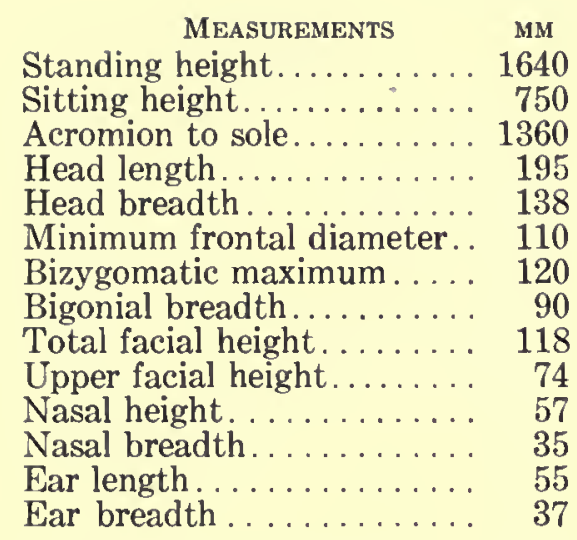

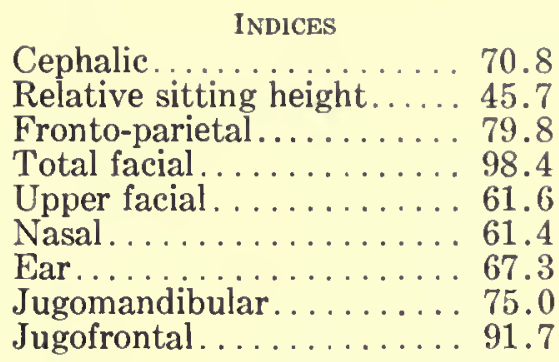

HAIR.-Texture: medium. Color: dark brown-black. Quantity: head, plus; beard, double minus; body, minus.

EYE.-Color: dark brown. Sclera: speckled. Blind in right eye, cataract in left eye.

Nose.-Profile: concavo-convex. Wings: medium.

TEETH.-Lost: none.

CHest.-Development: minus.

Musculature.-Poor.

HEALTH.-Fair.

\section{No. 400. Plate XlVI, Figs. 7, 8}

Residence: camp of Sheikh Hadji Hunta. Date: June 11, 1928.

Age: 45; married twice, 10 years ago and 3 years ago. Sons: 1, 0, living. Daughters: 2 , 1 , living; 4,1 , dead.

Brothers: 4 living. Sisters: 15 living, 12 dead.

Tattoo marks: none.

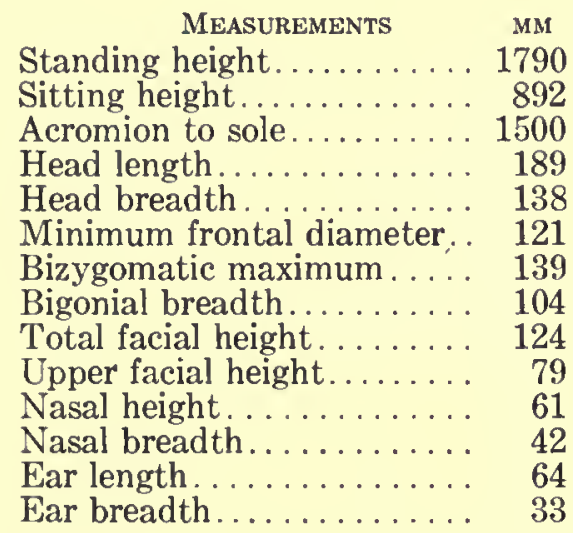

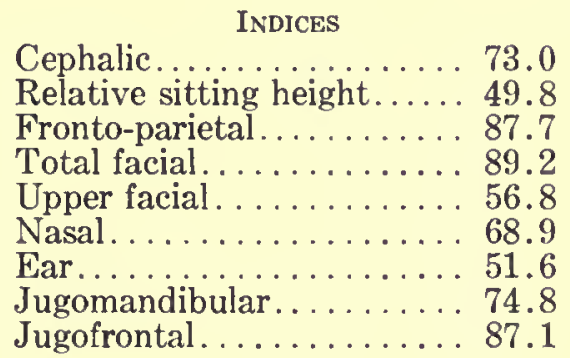

Halr.-Form: deep waves. Texture: medium. Color: black. Quantity: head, double plus; beard, double plus; body, double plus.

Eyw.-Color: dark brown. Sclera: clear. Iris: homogeneous.

NosE.-Profile: straight. Wings: flaring.

TEETH.-Lost: none.

Chest.-Development: double plus.

Musculature.-Excellent.

HEALTH.-Excellent.

No. 401. Plate XLVII, Figs. 1, 2

Residence: camp of Sheikh Hadji Hunta. Date: June 11, 1928.

Age: 23; unmarried. 
Brothers: 1 living. Sisters: 1 living.

Tattoo marks: none.

\begin{tabular}{|c|c|}
\hline MEASUREMENTS & MM \\
\hline Standing height....... & 1723 \\
\hline Sitting height. & 838 \\
\hline Acromion to sole. & 1435 \\
\hline Head length. & 185 \\
\hline Head breadth. & 147 \\
\hline Iinimum frontal diameter.. & 118 \\
\hline Bizygomatic maximum. . . . & 133 \\
\hline Bigonial breadth........... & 97 \\
\hline Total facial height. ........ & 118 \\
\hline Upper facial height. . . . . . . & 76 \\
\hline Nasal height & 60 \\
\hline 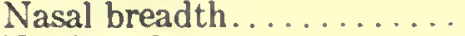 & 3 \\
\hline nor & 67 \\
\hline & \\
\hline
\end{tabular}

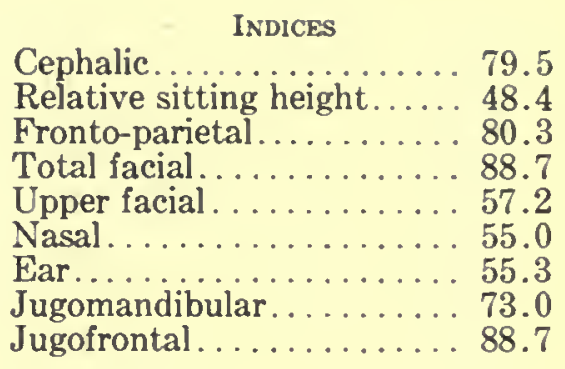
7

HAIR.-Form: deep waves. Texture: medium. Color: black. Quantity: head, double plus; beard, minus; body, minus.

EYE.-Color: dark brown. Sclera: clear. Iris: homogeneous.

NosE.-Profile: straight. Wings: medium.

TEETH.-Lost: none.

CHEst.-Development: plus.

Musculature.-Good.

HEALTH.-Good.

No. 402. Plate XLVII, Figs. 3, 4

Residence: camp of Sheikh Hadji Hunta. Date: June 11, 1928.

Age: 25; married for 10 years. Sons: 1 living. Daughters: 2 living. Brothers: 1 living. Sisters: 2 living.

Tattoo marks: none.

\begin{tabular}{|c|c|}
\hline MEASUREMENTS & MM \\
\hline Standing height. & 1723 \\
\hline Sitting height. & 843 \\
\hline Acromion to sole & 1430 \\
\hline Head length. & 194 \\
\hline Head breadth. & 141 \\
\hline Minimum frontal diameter. . & 107 \\
\hline Bizygomatic maximum . . . . & 137 \\
\hline Bigonial breadth.......... & 104 \\
\hline Total facial height...... & 126 \\
\hline Upper facial height....... & 70 \\
\hline Nasal height. & 5 \\
\hline asal hreadth & 35 \\
\hline r le & 5 \\
\hline & \\
\hline
\end{tabular}

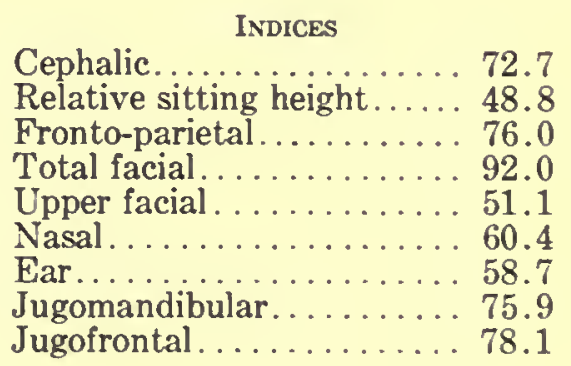

HAIR.-Texture: medium. Color: dark brown.

EYE.-Color: blue brown. Sclera: clear. Iris: zoned.

NosE.-Profile: straight. Wings: medium.

TEETH.-Lost: none.

Chest.-Development: plus.

Musculature.-Good.

HEALTH.-Good.

No. 403. Plate XlVII, Figs. 5, 6

Residence: camp of Sheikh Hadji Hunta. Date: June 11, 1928.

Age: 39 ; married for 8 years. Sons: 1 living. Daughters: 2 living. 
Brothers: 1 living. Sisters: 2 living.

Tattoo marks: none.

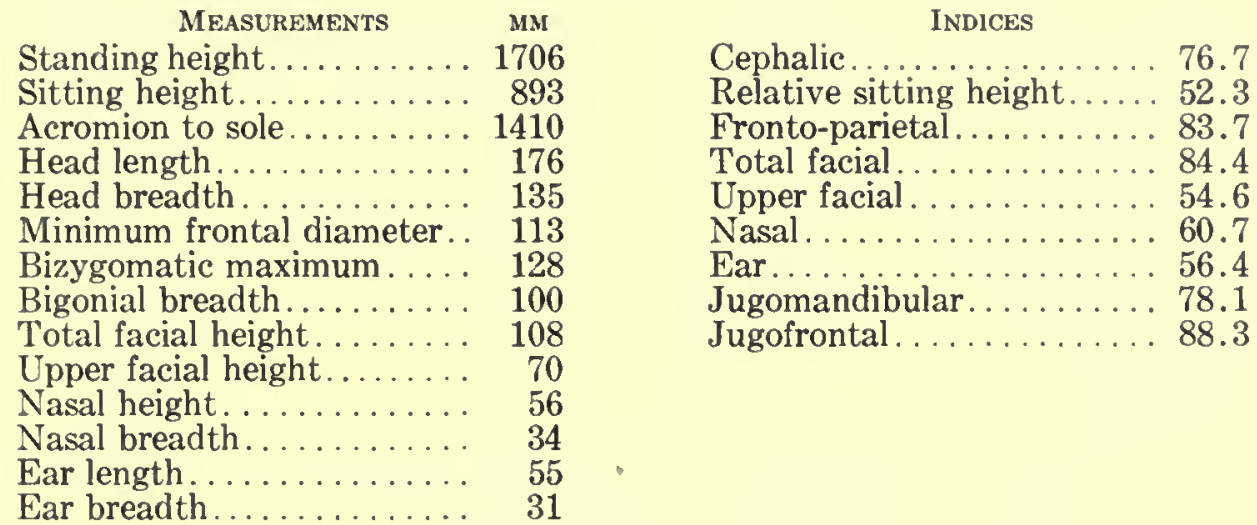

HAIR.-Color: black and gray. Quantity: head, minus.

EYE.-Color: dark brown. Sclera: clear. Iris: homogeneous.

Nose.-Profile: straight. Wings: medium.

TEETH.-Lost: none.

CHeST.-Development: plus.

Musculature.-Good.

HEALTH.-Good.

No. 404

Residence: camp of Sheikh Hadji Hunta. Date: June 11, 1928.

Age: 25; married for 10 years. Sons: 1 living, 1 dead. Daughters: 3 dead.

Brothers: 2 living. Sisters: 4 living.

Tattoo marks: 3 spots on back of right hand and 1 spot on inside of left wrist.

\begin{tabular}{|c|c|c|}
\hline MEASUREMENTS & MM & INDICES \\
\hline Standing height. & 1650 & Cephalic... \\
\hline Sitting height. & 833 & Relative sitting height..... 50.4 \\
\hline Acromion to sole. . & 1350 & Fronto-parietal......... 79.9 \\
\hline Head length..... & 196 & Total facial.... \\
\hline Head breadth. & 138 & Upper facial. \\
\hline Minimum frontal diameter. & 110 & Nasal. . . . . . \\
\hline Bizygomatic maximum. . . . & 126 & . 64.9 \\
\hline Bigonial breadth....... & 99 & Jugomandibular. . . . \\
\hline Total facial height. . . & 114 & Jugofrontal . . . . . . . . . . 87.3 \\
\hline Upper facial height. & 69 & \\
\hline Nasal height. & 60 & \\
\hline Nasal breadth & 35 & \\
\hline Ear length. . & 57 & \\
\hline Ear breadth & $?$ & \\
\hline
\end{tabular}

HAIR.-Texture: fine. Quantity: head, double plus; beard, average.

EYE.-Color: blue brown. Sclera: clear. Iris: homogeneous.

Nose.-Profile: straight. Wings: medium.

TEETH.-Lost: none.

CHEsT.-Development: plus.

Musculature.-Good.

HEALTH.-Good.

No. 405

Residence: camp of Sheikh Hadji Hunta. Date: June 11, 1928.

Age: 43 ; married twice, 20 years ago and 5 years ago. Sons: 1 , 0 , living; 2,0 , dead. Daughters: 1,0 , living; 0,1 , dead. 
Brothers: 1 dead. Sisters: 1 living.

Tattoo marks: none.

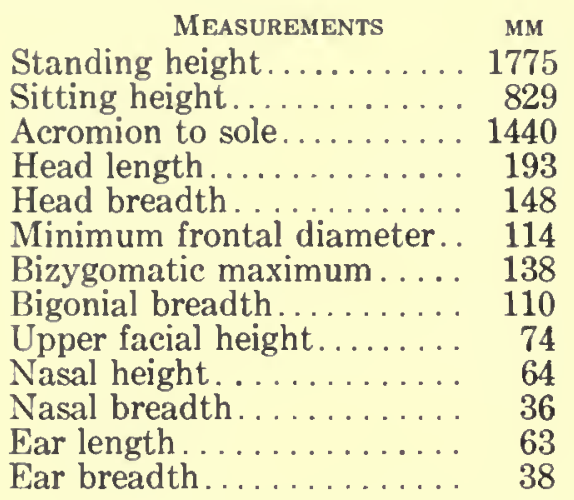

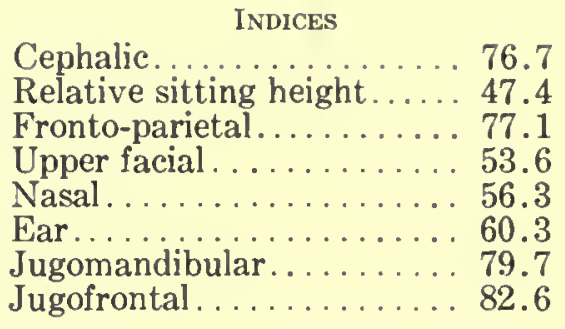

Cephalic.............. 76.7

Relative sitting height..... 47.4

Fronto-parietal.......... 77.1

. 53.6

Ear

Jugomandibular......... 79.7

Jugofrontal. . . . . . . . 82.6

HAIR.-Color: dark brown. Quantity: head, minus; beard, double plus; body, minus.

EYE.-Color: blue brown. Sclera: clear. Iris: zoned.

Nose.-Profile: straight. Tip: thickness, plus. Wings: flaring.

TEETH.-Lost: 4.

Chest.-Development: plus.

Musculature.-Good.

HEALTH.-Good.

\section{No. 406. Plate XLVII, Figs. 7, 8}

Residence: camp of Sheikh Hadji Hunta. Date: June 11, 1928.

Age: 23; married for several months. No children.

Brothers: none. Sisters: 1 living.

Tattoo marks: gazelle on inside of right forearm; glove on back of left hand.

\begin{tabular}{|c|c|c|}
\hline MEASUREMENTS & мм & INDICES \\
\hline Standing height. & 1670 & Cephalic. \\
\hline Sitting height. & 878 & Relative sitting height. \\
\hline Acromion to sole. & 1430 & Fronto-parietal. \\
\hline Head length. & 182 & Total facial. \\
\hline Head breadth & 147 & Upper facial \\
\hline Minimum frontal diameter. & 116 & Nasal. \\
\hline Bizygomatic maximum.... & 135 & Ear \\
\hline Bigonial breadth. & 101 & Jugomandibular \\
\hline Total facial height & 130 & Jugofrontal. \\
\hline
\end{tabular}

Ipper facial height........ 72

Nasal height. . . . . . . 61

Nasal breadth. . . . . . . . 33

Ear length ............ 63

Ear breadth............ 31

HaIR.-Form: low waves. Texture: medium. Quantity: head, plus; beard, minus; body, minus.

EYE.-Color: dark brown. Sclera: clear. Iris: homogeneous.

Nose.-Profile: straight. Wings: medium-flaring.

TеEтн.-Lost: none. Condition: good.

CHEST.-Development: double plus.

Musculature.-Excellent.

Health.-Good.

No. 407

Residence: camp of Sheikh Hadji Hunta. Date: June 11, 1928.

Age: 25; unmarried.

Brothers: 3 living. Sisters: 1 living.

Tattoo marks: circle with 8 rays and glove on right hand; designs on back of left wrist. 


\begin{tabular}{|c|c|}
\hline MEasurements & MM \\
\hline Standing height. . & 1670 \\
\hline Sitting height. & 809 \\
\hline Acromion to sole. & 1410 \\
\hline Head length.... & 197 \\
\hline Head breadth. & 144 \\
\hline Minimum frontal diameter. & 108 \\
\hline Bizygomatic maximum ..... & 129 \\
\hline Bigonial breadth. . & 97 \\
\hline Total facial height. & 118 \\
\hline Upper facial height. & 67 \\
\hline Nasal height. & 53 \\
\hline Nasal breadth. & 34 \\
\hline Ear length & 68 \\
\hline Ear bre & 36 \\
\hline
\end{tabular}

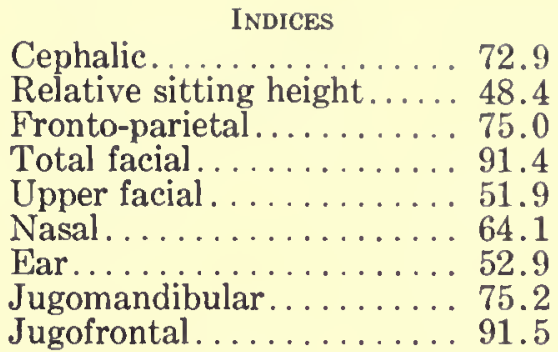

HAIR.-Form: deep waves. Texture: medium. Color: black.

EYE.-Color: dark brown. Sclera: clear. Iris: homogeneous.

Nose.-Profile: straight. Wings: medium-flaring.

TEETH.-Lost: none.

CHEST.-Development: average.

Musculature.-Good.

HEALTH.-Good.

No. 408. Plate XlVIII, Figs. 1, 2

Residence: camp of Sheikh Hadji Hunta. Date: June 11, 1928.

Age: 20; unmarried.

Brothers: none. Sisters: 1 living.

Tattoo marks: glove on back of right hand and design on back of right wrist.

\begin{tabular}{|c|c|c|}
\hline MEASUREMENTS & MM & INDICES \\
\hline Standing height. & 1690 & Cephalic. \\
\hline Sitting height. & 835 & Relative sitting height..... 49.4 \\
\hline Acromion to sole. & 1410 & Fronto-parietal.......... 85.8 \\
\hline Head length.... & 176 & Total facial. . \\
\hline Head breadth. & 141 & Upper facial \\
\hline Minimum frontal diameter. & 121 & Nasal. \\
\hline Bizygomatic maximum . . . . . & 135 & Ear. \\
\hline Bigonial breadth...... . & 98 & Jugomandibular. \\
\hline Total facial height. . . . . . . . & 115 & Jugofrontal \\
\hline
\end{tabular}

Upper facial height........ 72

Nasal height. .......... 62

Nasal breadth.......... 43

Ear length............. 54

Ear breadth............ 30

HaIR.-Form: low waves. Texture: medium. Color: black. Quantity: head, plus; beard, minus; body, minus.

EYE.-Color: dark brown. Sclera: clear. Iris: homogeneous.

NosE.-Profile: straight. Wings: flaring.

TEETH.-Lost: none. Condition: good.

CHEST.-Development: double plus.

Musculature.-Good.

HEALTH.-Good.

Skin dark in color; some Negro blood.

No. 409. Plate XlViII, Figs. 3, 4

Residence: camp of Sheikh Hadji Hunta. Date: June 11, 1928.

Age: 18; unmarried. 
Brothers: 3 living. Sisters: 1 living.

Tattoo marks: glove and cross on back of right hand.

\begin{tabular}{|c|c|c|}
\hline MEASUREMENTS & MM & INDICES \\
\hline Standing height. & 1665 & Cephalic. \\
\hline Sitting height. & 825 & Relative sitting height..... 49.5 \\
\hline Acromion to sole. & 1394 & Fronto-parietal.... \\
\hline Head length. & 184 & Total facial \\
\hline Head breadth. & 130 & Upper facial \\
\hline Minimum frontal diameter. & 104 & Nasal. \\
\hline Bizygomatic maximum . . . . . & 113 & 50.7 \\
\hline Bigonial breadth.......... & 90 & Jugomandibular. \\
\hline Total facial height. . . . . . . . & 115 & Jugofrontal. . . . . . . . . . . 92.0 \\
\hline
\end{tabular}

Upper facial height........ 64

Nasal height........... 56

Nasal breadth............ 37

Ear length............ 67

Ear breadth............ 34

HaIR. - Form: low waves. Texture: medium. Color: dark brown. Quantity: head, double plus; beard, minus; body, minus.

EYE.-Color: dark brown. Sclera: clear. Iris: homogeneous.

Nose.-Profile: straight. Wings: flaring.

TEETH.-Lost: none.

CHEST.-Development: plus.

Musculature.-Good.

HEALTH.-Good.

No. 410

Residence: camp of Sheikh Hadji Hunta. Date: June 11, 1928.

Age: 30 ; married for 9 years. Sons: 4 living. Daughters: none.

Brothers: 1 living. Sisters: 2 living, 1 dead.

Tattoo marks: none.

\begin{tabular}{|c|c|}
\hline MEASUREMENTS & MM \\
\hline $\begin{array}{l}\text { Standing height. } \\
\text { Sitting height }\end{array}$ & $\begin{aligned} 168 \\
8\end{aligned}$ \\
\hline Acromion to sole & 140 \\
\hline Head length. & \\
\hline Head breadth & \\
\hline Minimum frontal diameter. & \\
\hline $\begin{array}{l}\text { Bizygomatic maximum . . . . } \\
\text { Bigonial breadth }\end{array}$ & \\
\hline Total facial height & \\
\hline Upper facial height. & \\
\hline Nasal height. . & \\
\hline $\begin{array}{l}\text { Nasal breadth. } \\
\text { Ear length. . . }\end{array}$ & \\
\hline breadt & \\
\hline
\end{tabular}

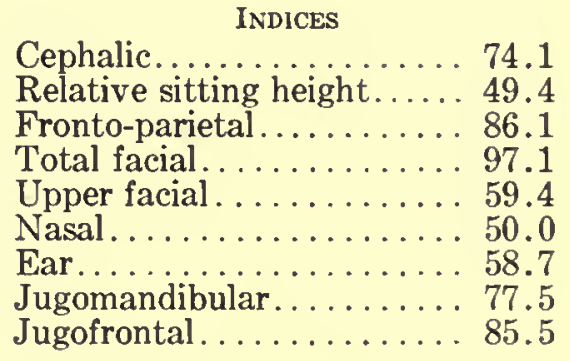

HAIR. - Form: low waves. Texture: medium. Color: black. Quantity: head, plus.

EYE. - Color: blue brown. Sclera: clear. Iris: zoned.

Nose.-Profile: straight. Tip: thickness, minus. Wings: medium-flaring.

ТЕETH.-Lost: none.

CHEsT.-Development: plus.

Musculature.-Good.

HEALTH.-Good.

No. 411

Residence: camp of Sheikh Hadji Hunta. Date: June 11, 1928.

Age: 75 ; married twice, 30 years ago and 25 years ago. Sons: 0 , 1, living; 1,0 , dead. Daughters: 1,1 , living. 
Brothers: 1 living, 2 dead. Sisters: 1 dead.

Tattoo marks: glove on right hand; design on inside of right forearm.

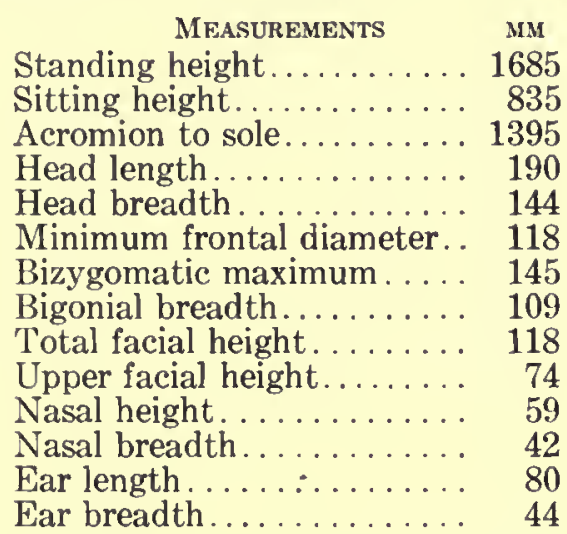

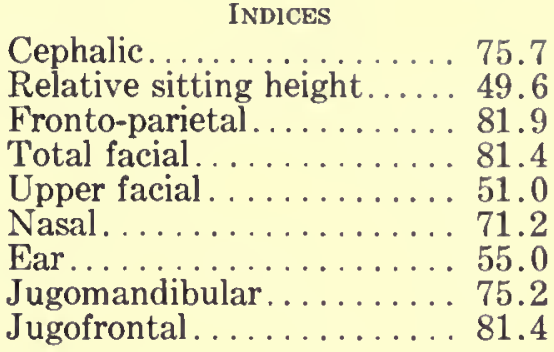

HAIR.-Color: gray.

EYE.-Color: blue brown. Sclera: bloodshot. Iris: zoned.

NosE.-Profile: straight. Wings: medium-flaring.

TEETH.-Lost: half.

Chest.-Development: plus.

Musculature.-Good.

HEALTH.-Good.

No. 412

Residence: camp of Sheikh Hadji Hunta. Date: June 11, 1928.

Age: 20; unmarried.

Brothers: 1 living. Sisters: 4 living.

Tattoo marks: glove on back of right hand.

\begin{tabular}{|c|c|c|}
\hline MEASUREMENTS & MM & INDICES \\
\hline Standing height. & 1534 & Cephalic. \\
\hline Sitting height... & 735 & Relative sitting height..... 47.9 \\
\hline Acromion to sole. & 1240 & Fronto-parietal..........88 \\
\hline Head length. & 180 & Total facial..... \\
\hline Head breadth. & 141 & Upper facial. \\
\hline Minimum frontal diameter. & 113 & Nasal \\
\hline zygomatic maximum. ... & 122 & $\mathrm{Ea}$ \\
\hline nial breadth. & 90 & nandibular... \\
\hline ght. & 110 & I \\
\hline
\end{tabular}

Upper facial height......... 63

Nasal height. ........... 54

Nasal breadth.......... 40

Ear length

Ear breadth........... 31

HAIR.-Form: deep waves. Texture: medium. Color: black.

EYE.-Color: dark brown. Sclera: clear. Iris: homogeneous.

NosE.-Profile: straight. Wings: medium.

TEETH.-Lost: none.

Chest.-Development: plus.

Musculature.-Good.

HEALTH.-Good.

No. 413

Residence: camp of Sheikh Hadji Hunta. Date: June 11, 1928.

Age: 40; married for 10 years. Sons: 1 living. Daughters: 1 living. 
Brothers: none. Sisters: none.

Tattoo marks: none.

\begin{tabular}{|c|c|}
\hline MEASUREMENTS & MM \\
\hline Standing height........ & 1730 \\
\hline Sitting height. & 860 \\
\hline Acromion to sole. & 1440 \\
\hline Head length.... & 191 \\
\hline Head breadth & 134 \\
\hline Minimum frontal diameter. & 115 \\
\hline Bizygomatic maximum . ... . & 125 \\
\hline Bigonial breadth.......... & 107 \\
\hline Total facial height. ........ & 116 \\
\hline Upper facial height........ & 66 \\
\hline Nasal height & 53 \\
\hline Nasal breadth & 37 \\
\hline Ear length & \\
\hline & \\
\hline
\end{tabular}

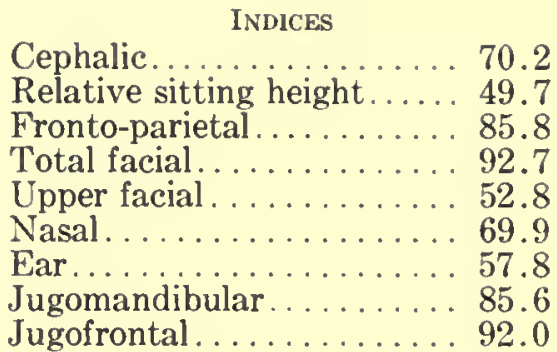

HAIR. - Form: low waves. Texture: medium. Color: black. Quantity: head, plus.

EYE.-Color: dark brown. Sclera: bloodshot. Iris: homogeneous.

Nose.-Profile: straight. Wings: medium.

TEETH.-Lost: 2.

CHEST.-Development: double plus.

Musculature.-Excellent.

HEALTH.-Good.

No. 414

Residence: camp of Sheikh Hadji Hunta. Date: June 11, 1928.

Age: 30 ; married twice, 12 years ago and 6 years ago. Sons: 0, 1, living. Daughters: 2,3 , living.

Brothers: 4 dead. Sisters: 4 living.

Tattoo marks: glove on back of right hand and gazelle on inside of right forearm.

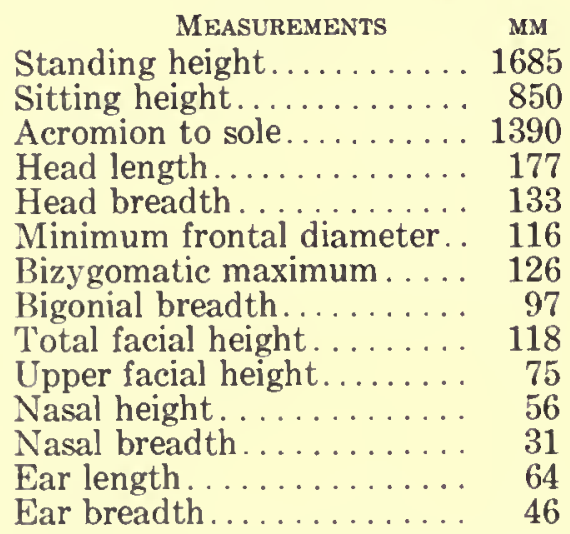

Cephalic.............. 75.1

Relative sitting height..... 50.4

Fronto-parietal.......... 87.2

Total facial............ 93.6

Upper facial. .......... 59.5

Nasal. ................ 55.4

Ear............... 71.9

Jugomandibular. . . . . . . 77.0

Jugofrontal. . . . . . . . . 9 92.1

HaIR.-Form: low waves. Texture: medium. Quantity: head, double plus.

EYE.-Color: dark brown. Sclera: clear. Iris: homogeneous.

Nose.-Profile: straight. Wings: compressed.

TEETH.-Lost: 3.

CHest.-Development: plus.

Musculature.-Good.

HEALTH.-Good.

No. 415

Residence: camp of Sheikh Hadji Hunta. Date: June 11, 1928.

Age: 22; unmarried. 
Brothers: none. Sisters: none.

Tattoo marks: none.

\begin{tabular}{|c|c|}
\hline MEASUREMENTS & 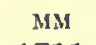 \\
\hline ding height. . & 17 \\
\hline Sitting height.... & \\
\hline n to sole & \\
\hline $\mathrm{H}$ & \\
\hline iameter. & \\
\hline & \\
\hline & \\
\hline $\begin{array}{l}\mathrm{To} \\
\mathrm{Ur}\end{array}$ & \\
\hline sal height. & \\
\hline & \\
\hline & \\
\hline
\end{tabular}

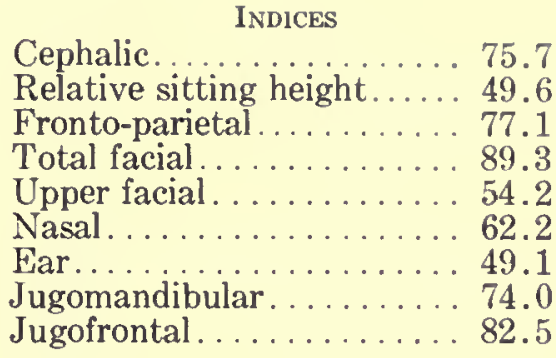

HAIr.-Form: deep waves. Texture: coarse. Color: black. Quantity: head, double plus; beard, minus; body, minus.

EyE.-Color: dark brown. Sclera: clear. Iris: homogeneous.

NosE.-Profile: straight. Wings: medium.

TEETH.-Lost: none.

CHEST.-Development: plus.

Musculature.-Good.

HEALTH.-Good.

No. 416

Residence: camp of Sheikh Hadji Hunta. Date: June 11, 1928.

Age: 75; married for 35 years. Sons: 1 living. Daughters: 1 living.

Brothers: none. Sisters: none.

Tattoo marks: glove and 2 spots on inside of right wrist; circle and spot on back of left hand.

\begin{tabular}{|c|c|}
\hline MEASUREMENTS & MM \\
\hline Standing height. & 1610 \\
\hline Sitting height. & 834 \\
\hline Acromion to sole. & 1380 \\
\hline Head length.... & 190 \\
\hline Head breadth. & 148 \\
\hline Minimum frontal diameter. . & 113 \\
\hline Bizygomatic maximum ..... & 141 \\
\hline Bigonial breadth. & 102 \\
\hline Total facial height. & 126 \\
\hline Upper facial height. . . & 76 \\
\hline Nasal height........ & 70 \\
\hline Nasal breadth. & 36 \\
\hline Ear length..... & 76 \\
\hline$\ldots \ldots$ & \\
\hline
\end{tabular}

HalR.-Form: low waves. Color: black and gray.

EYE.-Color: blue brown. Sclera: clear. Iris: zoned.

Nose.-Profile: convex.

TEETH.-Lost: 6.

CHEST.-Development: plus.

Musculature.-Good.

HEALTH._-Good.

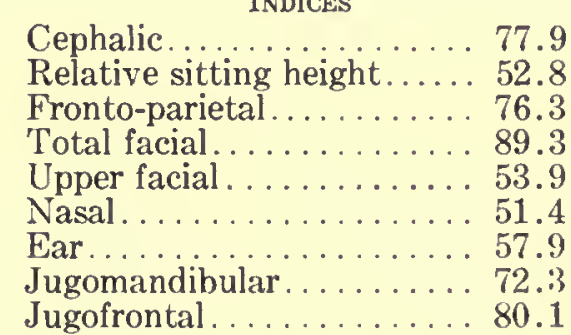

Cephalic ............... 77.9

No. 425

Residence: camp of Sheikh Aziz. Date: June 13, 1928.

Age: 50; married for 22 years. Sons: 2 living, 2 dead. Daughters: 1 living, 1 dead. 
Brothers: 1 dead. Sisters: 1 living.

Tattoo marks: spot on back of left wrist.

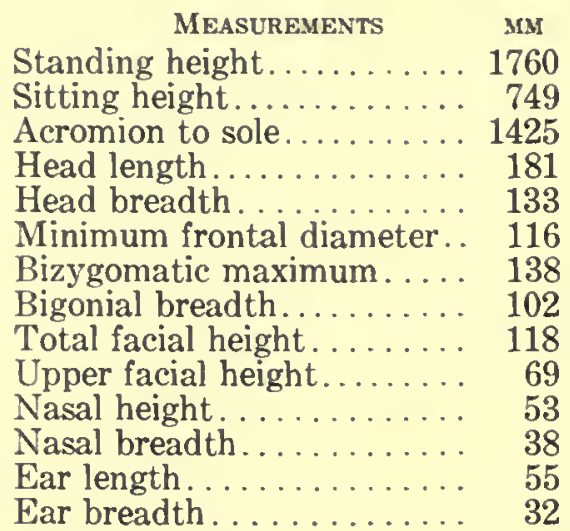

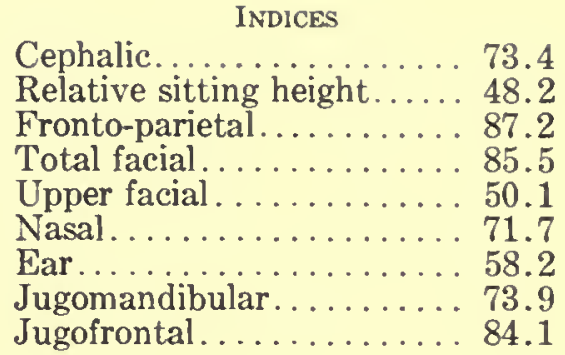

HAIR.-Form: low waves. Texture: medium. Color: black and gray. Quantity: head, plus; beard, plus; body, double plus.

EYE.-Color: dark brown. Sclera: clear. Iris: homogeneous.

NosE.-Profile: straight. Wings: flaring.

TEETH.-Lost: 2.

CHEST.-Derelopment: plus.

Musculature.-Good.

HEALTH.-Good.

\section{No. 426. Plate XLVIII, Figs. 5, 6}

Residence: camp of Sheikh Aziz. Date: June 13, 1928.

Age: 60 ; married three times, 30, 20, and 15 years ago. Sons: 1, 1, 0, living; 0, 1, 2, dead. Daughters: $3,1,2$, living; 0,0 , 2, dead.

Brothers: 1 living, 2 dead. Sisters: 2 dead.

Tattoo marks: none.

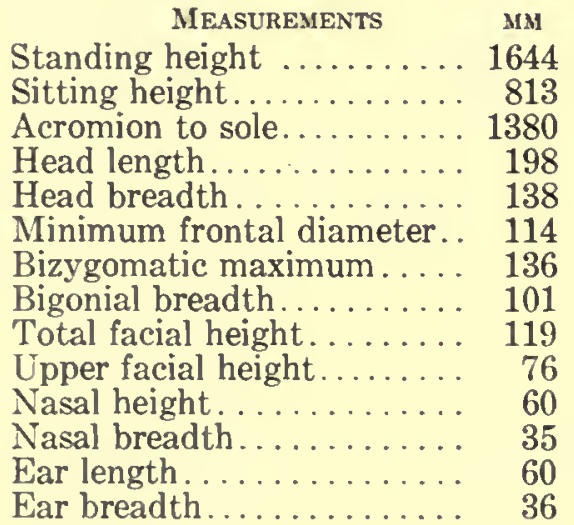

HaIR.-Form: low waves. Color: gray.

EYE.-Color: blue brown. Sclera: bloodshot. Iris: zoned.

Nose.-Profile: straight. Wings: medium-compressed.

TeETH.-Lost: 2.

Chest.-Development: average.

Musculature.-Fair.

Health.-Good. 
No. 429

Residence: camp of Sheikh Aziz. Date: June 13, 1928.

Age: 55; married twice, 20 years ago and 15 years ago. Sons: none. Daughters: 4, 4, living. Brothers: none. Sisters: 3 living.

Tattoo marks: none.

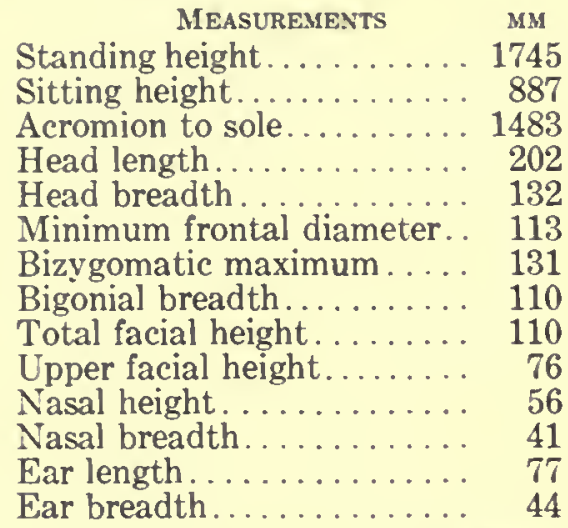

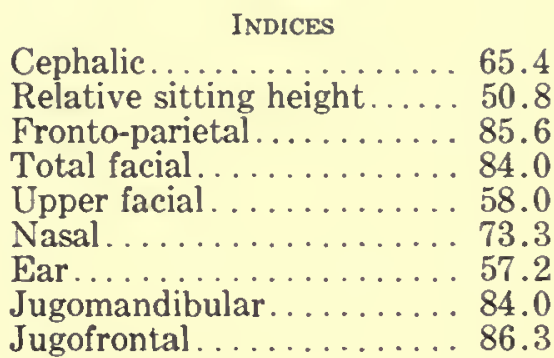

Cephalic..............6. 65.4

Relative sitting height..... 50.8

Upper facial . . . . . . . . . . 58.0

Nasal ................ 73.3

Jugomandibular........ 84.0

Jugofrontal............ 86.3

HAIR.-Color: black and gray. Quantity: head, average; beard, plus; body, plus.

EYE.-Color: dark brown. Sclera: clear. Iris: homogeneous. Slightly cross-eyed, left eye in bad condition.

Nose.-Profile: straight. Wings: medium-flaring.

TEETH.-Lost: none.

CHEST.-Development: average.

Musculature.-Fair.

HEALTH.-Good.

No. 430

Residence: camp of Sheikh Aziz. Date: June 13, 1928.

Age: 20; married for 3 years. No children.

Brothers: 2 living. Sisters: none.

Tattoo marks: none.

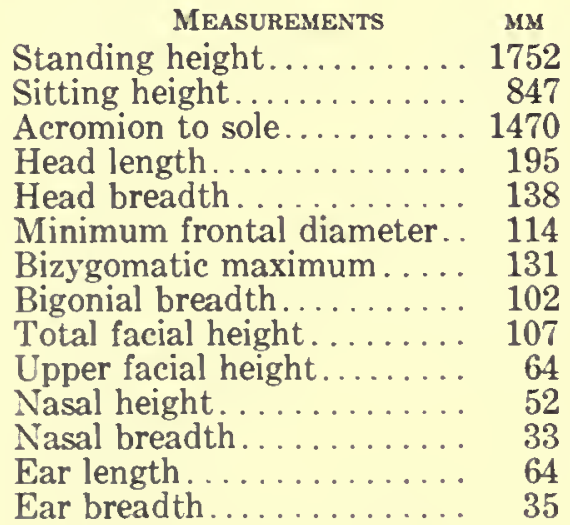

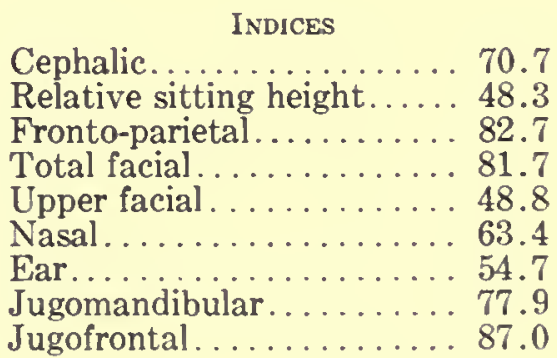

Cephalic............... 70.7

Relative sitting height..... 48.3

Fronto-parietal......... 82.7

Total facial $\ldots . . . .61 .7$

Ear...... 54.7

Jugofrontal............. 87.0

HAIR.-Form: low waves. Texture: medium. Color: dark brown. Quantity: head, double plus; beard, minus; body, minus.

EYE.-Color: blue brown. Sclera: clear. Iris: zoned.

Nose.-Profile: straight. Wings: medium.

TEETH._Lost: 4 . Unerupted: first lower molars.

CHEST.-Development: plus.

Musculature.-Good.

HEALTH.-Good. 
No. 431

Residence: camp of Sheikh Aziz. Date: June 13, 1928.

Age: 60; married for 15 years. Sons: 4 living, 1 dead. Daughters: 1 living.

Brothers: 1 dead. Sisters: none.

Tattoo marks: none.

\begin{tabular}{|c|c|}
\hline MEasurements & MM \\
\hline Standing height. & 1620 \\
\hline Sitting height. & 775 \\
\hline Acromion to sole & 1355 \\
\hline Head length & 18 \\
\hline Head breadth & 12 \\
\hline Minimum frontal diameter. . & 1 \\
\hline Bizygomatic maximum ..... & 143 \\
\hline Bigonial breadth. & 109 \\
\hline Total facial height. ........ & 113 \\
\hline Upper facial height........ & 74 \\
\hline Nasa] height. & 61 \\
\hline Nasal breadth & \\
\hline Ear lengtl & \\
\hline & \\
\hline
\end{tabular}

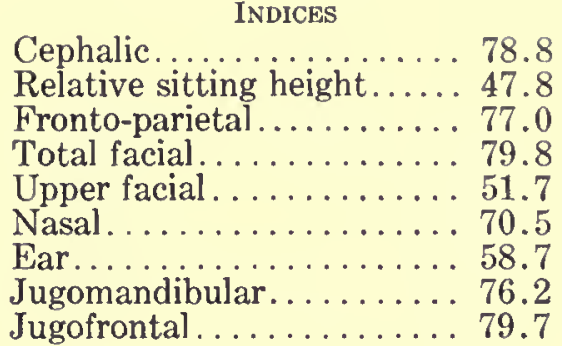

HAIR.-Texture: medium. Color: gray.

EYE.-Color: blue brown. Sclera: clear. Iris: zoned.

Nose.-Profile: straight. Wings: medium-flaring.

TEETH.-Lost: 5 .

Chest.-Development: average.

Musculature.-Fair.

Health. - Fair.

Marked prominence of malars.

No. 432

Residence: camp of Sheikh Aziz. Date: June 13, 1928.

Age: 23 ; married for 1 year. No children.

Brothers: 1 living. Sisters: none.

Tattoo marks: 5 spots on back of right wrist.

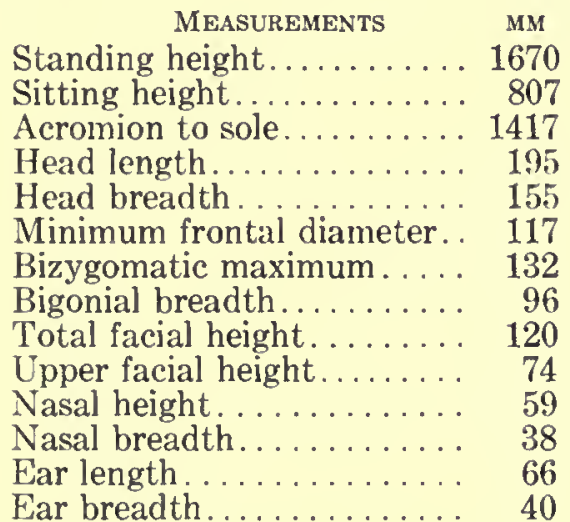

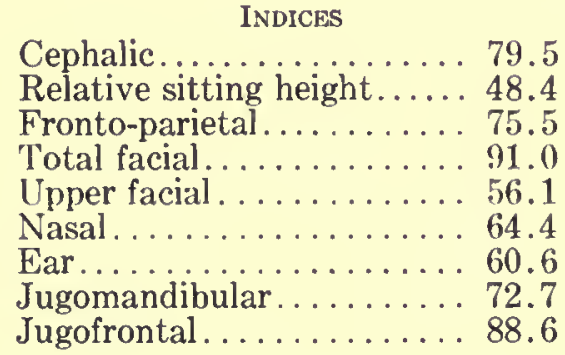

HaIR.-Form: low waves. Texture: medium. Color: dark brown. Quantity: head, double plus; beard, minus; body, minus.

EYE.-Color: dark brown. Sclera: clear. Iris: homogeneous.

Nose.-Profile: straight. Tip: thickness, double plus; elevated. Wings: flaring.

TEETH.-Lost: none.

Chest.-Development: plus.

Musculature.-Good.

Health.-Good. 
No. 433

Residence: camp of Sheikh Aziz. Date: June 13, 1928.

Age: 70; married for 50 years, wife now dead. Sons: 1 living. Daughters: 1 living, 1 dead. Brothers: 1 dead. Sisters: 1 living, 1 dead.

Tattoo marks: none.

\begin{tabular}{|c|c|}
\hline MEASUREMENTS & MM \\
\hline Standing height. . & 1628 \\
\hline Sitting height. & 795 \\
\hline Acromion to sole & 1410 \\
\hline Head length. & 196 \\
\hline Head breadth. & 148 \\
\hline Minimum frontal diameter. & 120 \\
\hline Bizygomatic maximum.... & 139 \\
\hline Bigonial breadth.......... & 120 \\
\hline Total facial height. . & 117 \\
\hline Upper facial height. . & 80 \\
\hline Nasal height. & 60 \\
\hline sal breadth. & 53 \\
\hline r length & 70 \\
\hline$n$ heodth & 11 \\
\hline
\end{tabular}

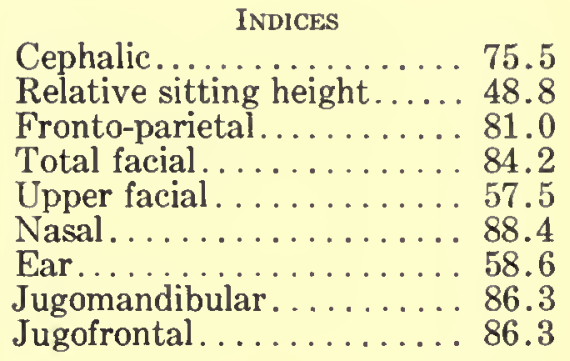

HAIR.—Color: gray. Quantity: head, minus; beard, double plus; body, double minus. EYE.-Blind.

NosE.-Profile: straight. Tip: thickness, double plus. Wings: flaring.

TEETH.-Lost: many.

EAR.-Lobe: free. Size: triple plus.

CHEST.-Development: minus.

Musculature.-Good.

HEALTH.-Fair.

\section{No. 434}

Residence: camp of Sheikh Aziz. Date: June 13, 1928.

Age: 30 ; married for 7 years. Sons: none. Daughters: 2 living.

Brothers: 1 dead. Sisters: 1 living.

Tattoo marks: none.

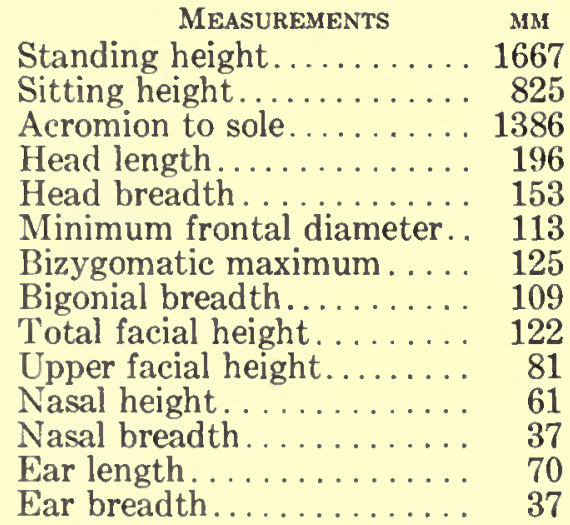

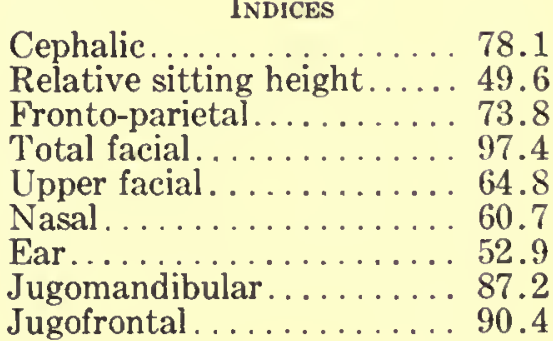

HAIR.-Form: low waves. Texture: medium. Color: dark brown. Quantity: head, plus; beard, plus; body, plus.

EyE.-Blind.

Nose.-Profile: straight. Wings: medium.

TEETH.-Lost: none.

Chest.-Development: plus. 
Musculature.-Good.

HEALTH.-Good.

No. 437

Residence: camp of Sheikh Aziz. Date: June 13, 1928.

Age: 30 ; married for 1 year. No children.

Brothers: none. Sisters: none.

Tattoo marks: none.

\begin{tabular}{|c|c|}
\hline MEASUREMENTS & MM \\
\hline Standing height... & 1730 \\
\hline Sitting height. & 846 \\
\hline Acromion to sole. . & 1430 \\
\hline Head length. & 195 \\
\hline Head breadth & 139 \\
\hline Minimum frontal diameter.. & 116 \\
\hline Bizygomatic maximum..... & 137 \\
\hline Bigonial breadth......... & 108 \\
\hline Total facial height. . & 124 \\
\hline Upper facial height........ & 72 \\
\hline Nasal height. & 53 \\
\hline Nasal breadth............ & 35 \\
\hline$\ldots \ldots \ldots$ & 66 \\
\hline & \\
\hline
\end{tabular}

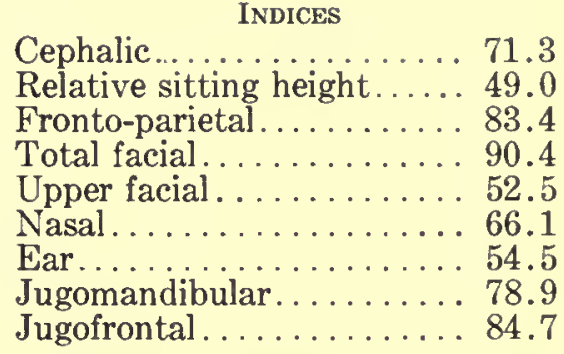

No. 438

Residence: camp of Sheikh Aziz. Date: June 13, 1928.

Age: 25; married for 1 year. No children.

Brothers: 1 living, 2 dead. Sisters: 1 living.

Tattoo marks: glove on back of left hand.

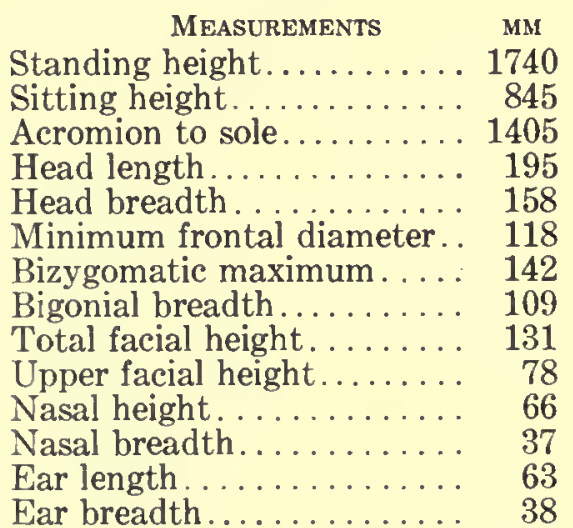

HAIR.-Form: low waves. Texture: coarse. Color: black. Quantity: head, plus; beard, plus; body, average.

EYE.-Color: dark brown. Sclera: clear. Iris: homogeneous.

Nosf.-Profile: straight. Wings: medium-compressed.

TEETH.-Lost: none.

Chest.-Development: plus. 
Musculature.-Good.

HEALTH.-Good.

No. 439

Residence: camp of Sheikh Aziz. Date: June 13, 1928.

Age: 65; married seven times. Sons: 2 living, 5 dead. Daughters: 3 living, 1 dead. Brothers: 2 dead. Sisters: none.

Tattoo marks: 3 spots on right wrist.

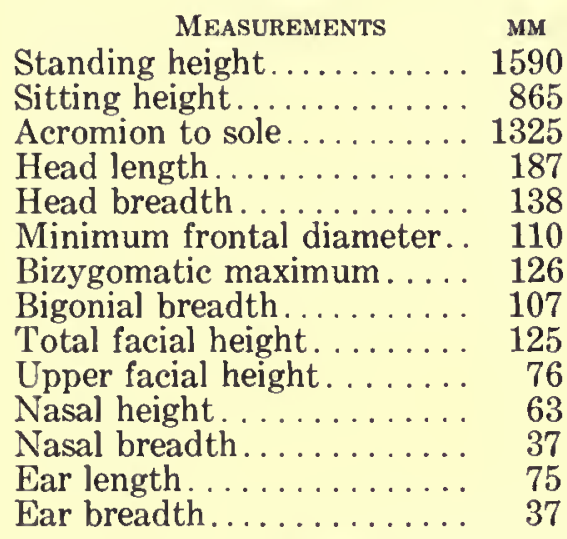

INDICES

Cephalic............... 73.8

Relative sitting height..... 54.4

Fronto-parietal.......... 79.8

Total facial. ............ 99.3

Upper facial . . . . . . . . . . 60.4

Nasal . . . . . . . . . . . . 58.8

Ear................. 49.4

Jugomandibular.........84.9

Jugofrontal . . . . . . . . . . . 87.3

HAIR.-Color: gray. Quantity: head, plus; beard, double plus; body, minus.

EYE.-Blind.

Nose.-Profile: straight. Wings: medium.

TEETH.-Lost: many.

Chest.-Development: average.

Musculature.-Poor.

HEALTh.-Poor.

No. 440

Residence: camp of Sheikh Aziz. Date: June 13, 1928.

Age: 40 ; married for 20 years. Sons: 1 living. Daughters: none.

Brothers: 2 dead. Sisters: 1 dead.

Tattoo marks: none.

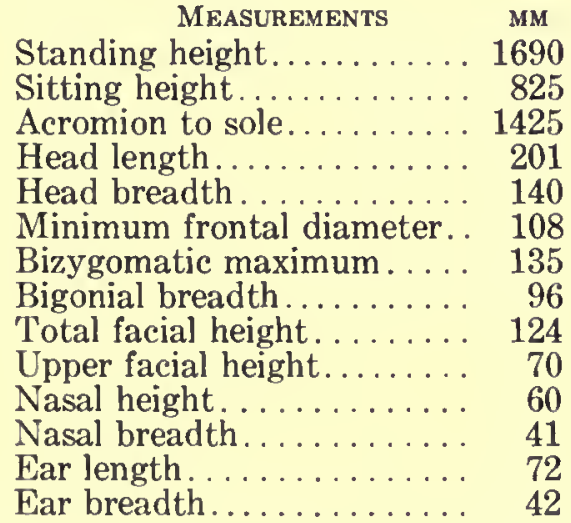

Cephalic............. 69.7

Relative sitting height.... 47.8

Fronto-parietal.......... 77.2

Total facial............ 92.0

Upper facia]. . . . . . . . . . 51.8

Nasal. . . . . . . . . . . 68.3

Ear........................... 58.3

Jugomandibular. . . . . . . 71.1

Jugofrontal . . . . . . . . . . 80.0

HaIr.-Form: low waves. Texture: medium. Color: dark brown.

EYE.-Color: green brown. Sclera: clear. Iris: homogeneous.

NOSE.-Profile: straight. Tip: thickness, double plus. Wings: flaring.

TEETH.-Lost: 2.

Chest.-Development: plus.

Musculature.-Good.

HEALTH.-Good. 
No. 441

Residence: camp of Sheikh Aziz. Date: June 13, 1928.

Age: 30 ; married for 8 years. Sons: 1 living, 5 dead. Daughters: none.

Brothers: 9 living, 6 dead. Sisters: 1 living.

Tattoo marks: glove on back of right hand and 12-rayed circle on right wrist.

\begin{tabular}{|c|c|}
\hline \multirow{2}{*}{\multicolumn{2}{|c|}{$\begin{array}{l}\text { MEasurements } \\
\text { Standing height }\end{array}$}} \\
\hline & \\
\hline $\begin{array}{l}\text { Sitting height... } \\
\text { Acromion to sole. }\end{array}$ & \\
\hline $\begin{array}{l}\text { Acromion to sole. } \\
\text { Head length..... }\end{array}$ & \\
\hline Head breadth. & \\
\hline Minimum frontal diameter & \\
\hline Bizygomatic maximum. & \\
\hline Bigonial breadth.. & \\
\hline $\begin{array}{l}\text { Total facial height. } \\
\text { Upper facial height. }\end{array}$ & \\
\hline $\begin{array}{l}\text { Upper facial height. } \\
\text { Nasal height..... }\end{array}$ & \\
\hline Nasal breadth. & \\
\hline & \\
\hline & \\
\hline
\end{tabular}

INDICES

Cephalic...............74.9

Relative sitting height..... 50.7

Fronto-parietal......... 80.7

Total facial..............100.0

Upper facial. ......... 59.4

Nasal. . . . . . . . . . 56.9

Ear................ 54.7

Jugomandibular. . . . . . 86.7

Jugofrontal. .......... 88.3

HaIR.—Form: low waves. Texture: medium-fine. Color: dark brown.

EYE.-Color: dark brown. Sclera: clear. Iris: homogeneous.

Nose.-Profile: straight. Wings: compressed.

Chest.-Development: plus.

Musculature.-Good.

HEALTH.-Good.

No. 442. Plate XLIX, Figs. 3, 4

Residence: camp of Sheikh Aziz. Date: June 13, 1928.

Age: 40 ; unmarried.

Brothers: none. Sisters: 3 living.

Tattoo marks: glove on right wrist.

\begin{tabular}{|c|c|}
\hline MEASUREMENTS & \\
\hline Standing height. & 166 \\
\hline Sitting height. & \\
\hline Acromion to sole & 139 \\
\hline Head length & \\
\hline Head breadth. & \\
\hline Minimum frontal diameter & \\
\hline Bizygomatic maximum .... & \\
\hline Bigonial breadth.. & \\
\hline Total facial height. & \\
\hline Upper facial height & \\
\hline Nasal height. & \\
\hline Nasal breadth. & \\
\hline $\begin{array}{l}\text { Head length.. } \\
\text { Head breadth. }\end{array}$ & \\
\hline
\end{tabular}

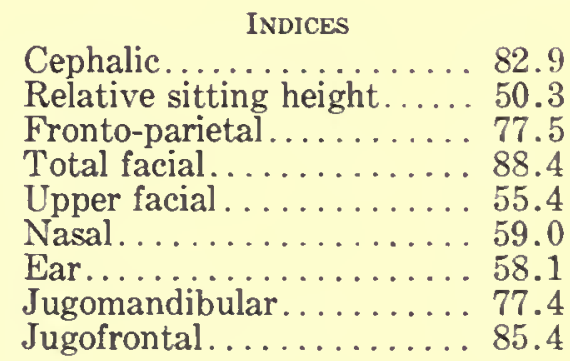

HAIR.-Form: low waves. Texture: medium. Color: black. Quantity: head, plus; beard, minus; body, minus.

EYE.-Color: dark brown. Sclera: clear. Iris: homogeneous.

Nose.-Profile: convex. Wings: medium-compressed.

TEEтH.-Lost: none.

Chest.-Development: plus.

Musculature.-Good.

Health.-Good. 
No. 443

Residence: camp of Sheikh Aziz. Date: June 13, 1928.

Age: 23; unmarried.

Brothers: 2 living. Sisters: none.

Tattoo marks: design.

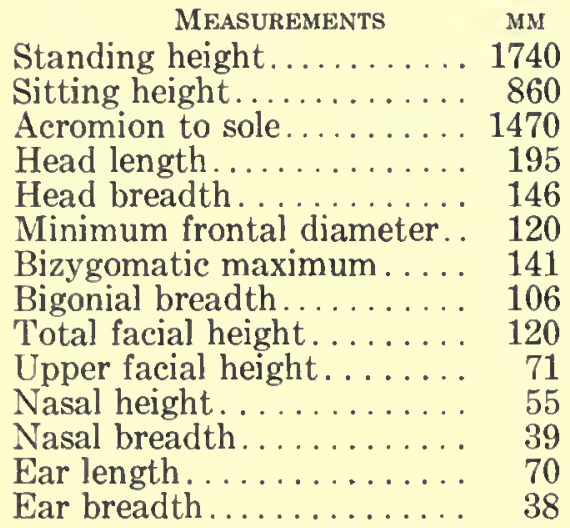

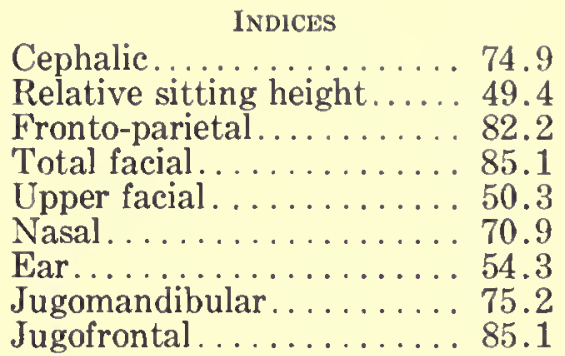

74.9

50.3

Jugofrontal ............. 85.1

HAIR.-Form: low waves. Texture: fine. Quantity: head, plus; beard, minus; body, minus.

EYE.-Color: dark brown. Sclera: clear. Iris: zoned.

NosE.-Profile: straight. Wings: medium-compressed.

TEETH.-Lost: none.

Chest.-Development: plus.

Musculature.-Good.

HEALTH.-Good.

No. 444

Residence: camp of Sheikh Aziz. Date: June 13, 1928.

Age: 18; unmarried.

Brothers: none. Sisters: none.

Tattoo marks: none.

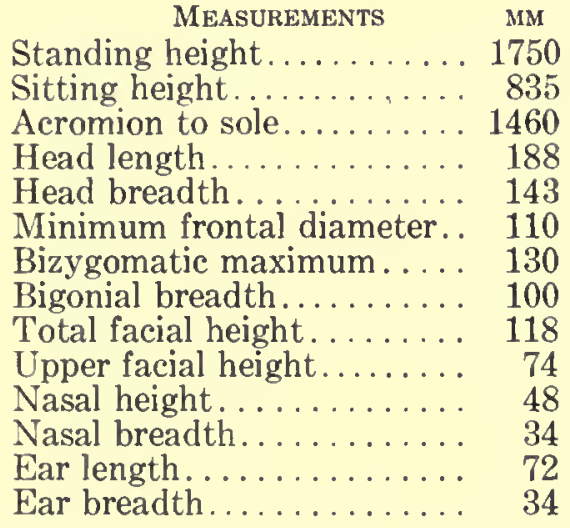

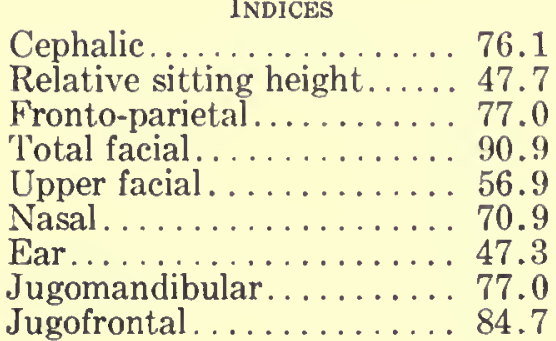

Cephalic.............. 76.1

Relative sitting height.....47.7

Upper facial . . . . . . . . . 56.9

Nasal. . . . ........... 70.9

Jugomandibular. . . . . . . . 77.0

Jugofrontal. . . . . . . . . . 84.7

HAIR.-Form: curly frizzly. Texture: medium. Color: dark brown.

EyE. Color: dark brown. Sclera: clear. Iris: zoned.

Nose.-Profile: straight. Wings: medium.

TEETH.-Lost: none.

CHest.-Development: plus.

Musculature.-Good.

HEALTH.-Good. 


\section{No. 445. Plate XliX, Figs. 5, 6}

Residence: camp of Sheikh Aziz. Date: June 13, 1928.

Age: 17 ; unmarried.

Brothers: none. Sisters: none.

Tattoo marks: none.

\begin{tabular}{|c|c|}
\hline MeAsuREMENTS & MM \\
\hline Standing height & 1702 \\
\hline Sitting height & 879 \\
\hline Acromion to sole & 1425 \\
\hline Head length. & 18 \\
\hline Head breadth & 140 \\
\hline Minimum frontal diameter. . & 110 \\
\hline Bizygomatic maximum . . . . & 128 \\
\hline Bigonial breadth & 101 \\
\hline Total facial height. ........ & 113 \\
\hline Upper facial height. . . . . . & 73 \\
\hline Nasal height. & 58 \\
\hline Nasal breadth & 32 \\
\hline Ear lengtl & 61 \\
\hline & \\
\hline
\end{tabular}

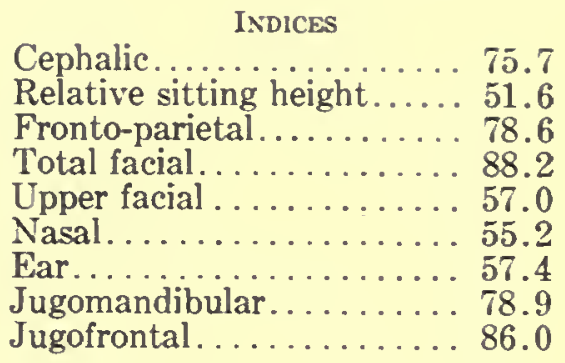

HAlR.-Form: low waves. Texture: coarse. Color: black. Quantity: head, double plus; beard, minus; body, minus.

EYE.-Color: dark brown. Sclera: clear. Iris: homogeneous.

Nose.-Profile: convex. Wings: medium.

TEETH.-Lost: none.

CHEsT.-Development: plus.

Musculature.-Good.

HEALTH.-Good.

\section{No. 446}

Residence: camp of Sheikh Aziz. Date: June 13, 1928.

Age: 30 ; unmarried.

Brothers: 1 living. Sisters: 1 living.

Tattoo marks: none.

\begin{tabular}{|c|c|}
\hline Measurements & MM \\
\hline Standing height....... . & 1705 \\
\hline Sitting height. & 860 \\
\hline Acromion to sole. & 1430 \\
\hline Head length. & 187 \\
\hline Head breadth. & 140 \\
\hline Minimum frontal diameter. & 113 \\
\hline Bizygomatic maximum.... & 127 \\
\hline Bigonial breadth......... & 102 \\
\hline Total facial height. ........ & 128 \\
\hline Upper facial height........ & 77 \\
\hline Nasal height. . . . . . . . . . & 66 \\
\hline Nasal breadth............. & \\
\hline leng & \\
\hline & \\
\hline
\end{tabular}

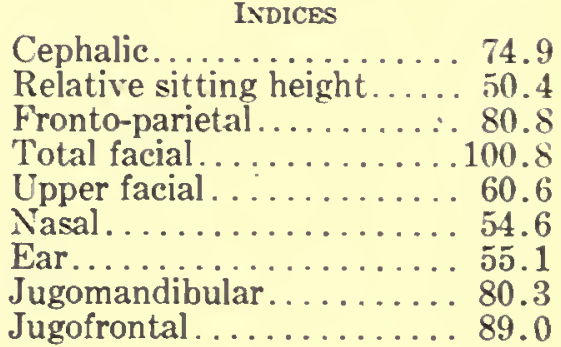

Cephalic.............. 74.9

Relative sitting height..... 50.4

.

Upper facial . 60.6

Nasal. ............. 54.6

Jugomandibular.......8 80.3

Jugofrontal . . . . . . . . 89.0

HAIR.-Form: low waves. Texture: coarse. Color: black.

EYE.-Color: black. Sclera: clear. Iris: homogeneous.

NosE.-Profle: straight. Wings: medium-flaring.

TEETH.-Lost: none.

CHEst.-Derelopment: plus.

Musculature.-Good.

HEALTH.-Good. 


\section{No. 447. Plate XliX, Figs. 7, 8}

Residence: camp of Sheikh Aziz. Date: June 13, 1928.

Age: 18; unmarried.

Brothers: 1 dead. Sisters: none.

Tattoo marks: glove on right hand and cross with 3 spots on inside of right forearm.

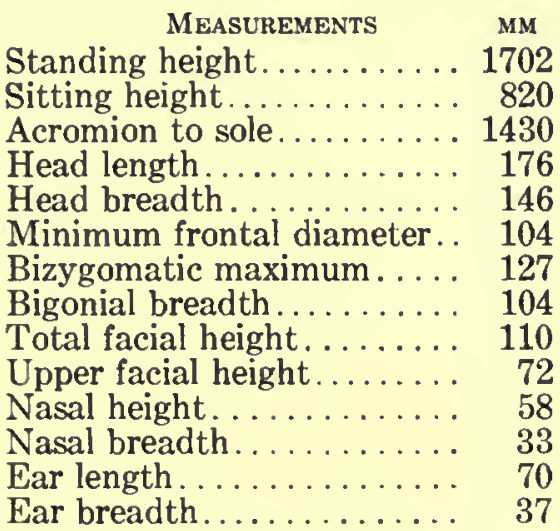

INDICES

Cephalic.............. 83.0

Relative sitting height..... 48.1

Fronto-parietal......... 71.3

Total facial............. 86.7

Upper facial ............ 56.7

Nasal . . . . . . . . . . . . . 56.9

Ear.................. 52.9

Jugomandibular......... 81.9

Jugofrontal. . . . . . . . . 81.9

HAIR.-Form: low waves. Texture: medium.

EYE.-Color: dark brown. Sclera: clear. Iris: homogeneous.

Nose.-Profile: concavo-convex. Wings: medium.

TEETH.-Lost: none. Unerupted: third molars.

CHEst.-Development: average.

MUSCULATURE.-Fair.

HEALTH.-Good.

No. 448

Residence: camp of Sheikh Aziz. Date: June 13, 1928.

Age: 20; unmarried.

Brothers: none. Sisters: none.

Tattoo marks: none.

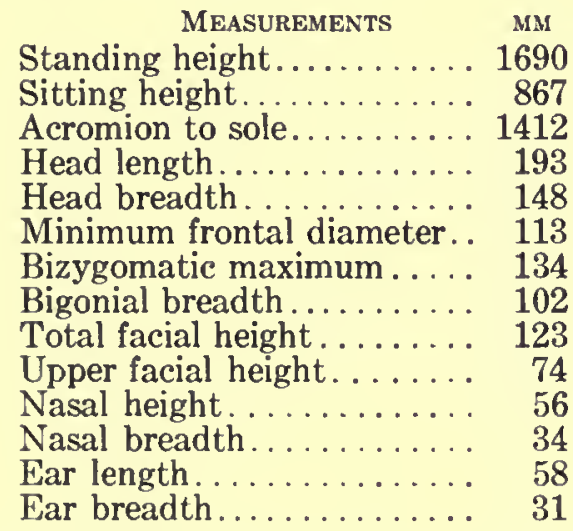

Cephalic................. 76.7

Relative sitting height..... 51.3

Fronto-parietal.......... 76.4

Total facial............. 91.9

Upper facial . . . . . . . . . . . 55.3

Nasal. .................6. 60.7

Ear.................... 53.5

Jugomandibular......... 73.4

Jugofrontal. ............ 81.3

HAIR.-Form: deep waves. Texture: medium. Quantity: head, double plus; beard, minus; body, average.

EYE.-Color: dark brown. Sclera: clear. Iris: homogeneous.

Nose.-Profile: straight. Wings: medium.

TEETH.-Lost: none.

CHEst.-Development: plus.

Musculature.-Good.

HEALTH.-Good. 
No. 449

Residence: camp of Sheikh Aziz. Date: June 13, 1928.

Age: 60 ; married for 34 years, wife died 1 year ago. No children.

Brothers: none. Sisters: none.

Tattoo marks: 5 spots on each temple.

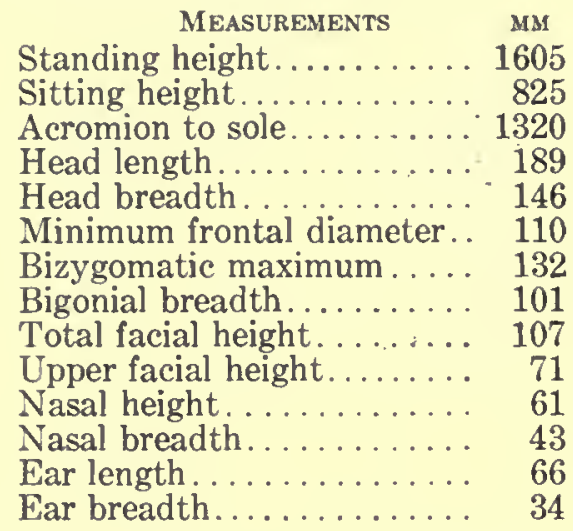

INDICES

Cephalic............... 77.2

Relative sitting height..... 51.4

Fronto-parietal......... 75.3

Total facial. . . . . . . . . . 81.0

Upper facial. .......... 53.8

Nasal............... 70.4

Ear................. 51.5

Jugomandibular......... 76.5

Jugofrontal........... 83.3

HaIR.-Form: low waves. Color: dark brown and gray. Quantity: head, plus; beard, plus; body, double plus.

EYE.-Color: blue brown. Sclera: clear. Iris: zoned.

Nose.-Profile: convex. Wings: medium-flaring.

TEETH.-Caries: double plus. Lost: 2.

CHEST.-Development: plus.

Musculature.-Good.

HEALTH.-Good.

Residence: camp of Sheikh Aziz. Date: June 13, 1928.

Age: 38 ; married three times, 24, 16, and 15 years ago, first wife now dead. Sons: 0, 2, 0 , living. Daughters: $0,1,0$, living.

Brothers: none. Sisters: none.

Tattoo marks: glove on back of right hand.

\begin{tabular}{|c|c|}
\hline Measurements & MM \\
\hline Standing height. . & 1752 \\
\hline Sitting height. & 845 \\
\hline Acromion to sole. & 1434 \\
\hline Head length. . & 197 \\
\hline Head breadth & 145 \\
\hline Minimum frontal diameter. & 120 \\
\hline Bizygomatic maximum .... . & 138 \\
\hline Bigonial breadth.......... & 110 \\
\hline Total facial height. & 118 \\
\hline Upper facial height......... & 70 \\
\hline Nasal height. & 60 \\
\hline Nasal breadtl & 36 \\
\hline Ear lengtl & 70 \\
\hline & \\
\hline
\end{tabular}

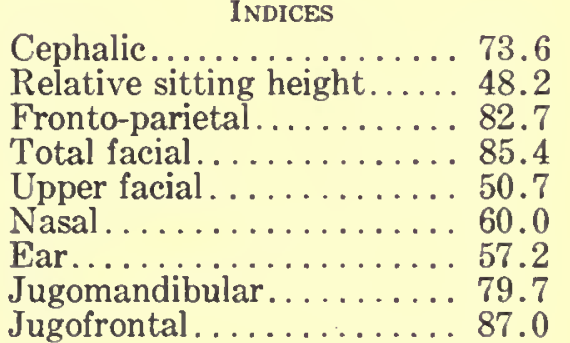

Cephalic............... 73.6

Relative sitting height..... 48.2

Total facial............... 85.4

Upper facial. ........... 50.7

Nasal...............6. 60.0

Jugomandibular. . . . . 79.7

Jugofrontal . .......... 87.0

HaIR.-Form: low waves. Texture: fine. Quantity: head, plus; beard, plus; body, plus. EYE.-Color: dark brown. Sclera: clear. Iris: homogeneous.

Nose.-Profile: straight. Wings: medium.

TEETH.-Lost: none.

CHEst.-Development: double plus.

Musculature.--Excellent.

HEALTH.-Good. 
No. 451

Residence: camp of Sheikh Aziz. Date: June 13, 1928.

Age: 20 ; unmarried.

Brothers: 1 dead. Sisters: none.

Tattoo marks: none.

\begin{tabular}{|c|c|c|}
\hline MEASUREMENTS & MM & INDICES \\
\hline Standing height. & 1670 & Cephalic. \\
\hline Sitting height. & 810 & Relative sitting height.. \\
\hline Acromion to sole & 1373 & Fronto-parietal........ \\
\hline Head length. & 186 & Total facial.. \\
\hline Head breadth & 137 & Upper facial \\
\hline Minimum frontal diameter. & 105 & Nasal. \\
\hline Bizygomatic maximum . . . & 124 & Ear. \\
\hline Bigonial breadth. & 96 & Jugomandibular....... \\
\hline Total facial height. & 116 & Jugofrontal. \\
\hline
\end{tabular}

Upper facial height........ 78

Nasal height. .................... 57

Nasal breadth.......... 37

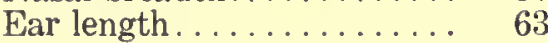

Ear breadth............ 37

HaIR.-Form: low waves. Texture: coarse. Color: dark brown. Quantity: head, plus; beard, minus; body, average.

EyE.-Color: dark brown. Sclera: clear. Iris: homogeneous.

Nose.-Profile: straight. Wings: medium-flaring.

ChEsT.-Development: average.

Musculature.-Fair.

HEALTH.-Good.

No. 452. Plate L, Figs. 1, 2

Residence: camp of Sheikh Aziz. Date: June 13, 1928.

Age: 20 ; unmarried.

Brothers: 3 dead. Sisters: none.

Tattoo marks: none.

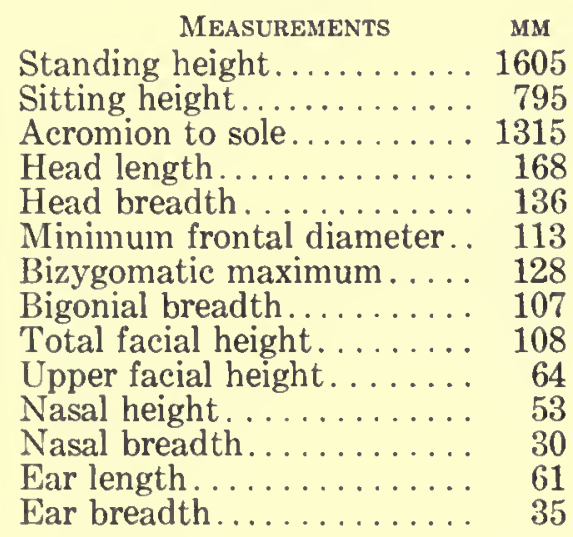

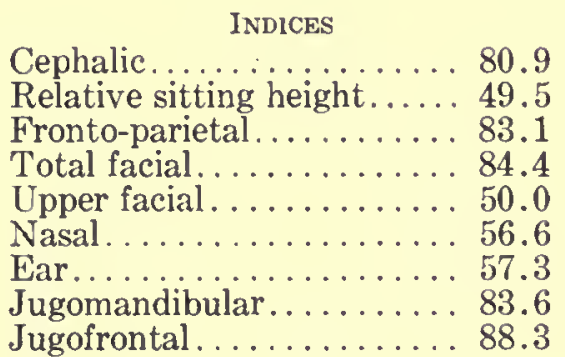

Jugofrontal . . . . . . . . . . 88.3

HAIR.-Form: low waves. Texture: medium. Color: dark brown. Quantity: head, plus; beard, double minus; body, double minus.

EYE.-Color: dark brown. Sclera: clear. Iris: homogeneous.

Nose.-Profile: straight. Wings: medium.

TEETH.-Lost: none.

CHEST.-Development: average.

Musculature.-Fair.

HEALTH.-Good. 
No. 453

Residence: camp of Sheikh Aziz. Date: June 13, 1928.

Age: 35; unmarried.

Brothers: none. Sisters: none.

Tattoo marks: none.

\begin{tabular}{|c|c|}
\hline MEASUREMENTS & MM \\
\hline Standing height. & 1730 \\
\hline Sitting height. & 815 \\
\hline Acromion to sole & 1480 \\
\hline Head length & 20 \\
\hline Head breadth & 13 \\
\hline Minimum frontal diameter. & 120 \\
\hline Bizygomatic maximum . ... . & 134 \\
\hline Bigonial breadth. & 10 \\
\hline Total facial height. . & 122 \\
\hline Upper facial height. & 78 \\
\hline Nasal height & 57 \\
\hline Nasal breadth & 37 \\
\hline Ear lengt & 63 \\
\hline & \\
\hline
\end{tabular}

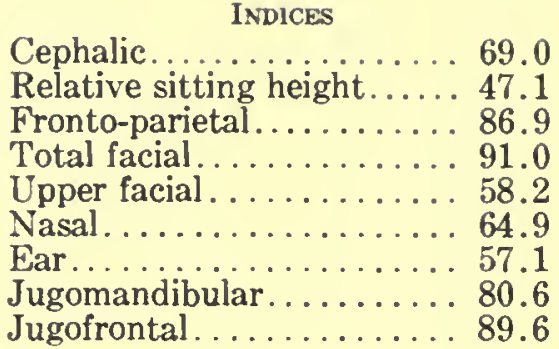

HAIR.-Form: low waves. Texture: coarse. Quantity: head, double plus; beard, plus; body, average.

EYE.-Color: dark brown. Sclera: clear. Iris: homogeneous.

NosE.-Profile: straight. Wings: medium.

TEETH.-Lost: 1.

CHEST.-Development: double plus.

Musculature.-Excellent.

HEALTH.--Excellent.

No. 454. Plate L, Figs. 3, 4

Residence: camp of Sheikh Aziz. Date: June 13, 1928.

Age: 30 ; unmarried.

Brothers: none. Sisters: none.

Tattoo marks: glove on back of right hand and 3 spots on each temple.

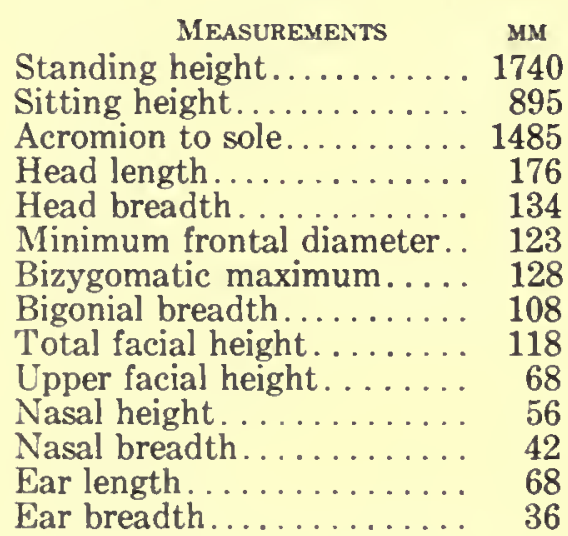

Cephalic............... 76.1

Relative sitting height..... 50.9

Fronto-parietal......... 91.7

Total facial............ 92.1

Upper facial. .............. 53.1

Nasal. . . . . . . . . . 75.0

Ear................... 52.9

Jugomandibular........ 84.4

Jugofrontal............96.0

Halr. - Form: low waves. Texture: medium. Color: black.

EYE.-Color: dark brown. Sclera: clear. Iris: homogeneous.

NosE.-Profile: convex. Wings: flaring.

TEETH.-Lost: 2.

CHEST.-Development: plus.

MusculaAture.-Good.

HEALTH.-Good. 
No. 455. Plate L, Figs. 5, 6

Residence: camp of Sheikh Aziz. Date: June 13, 1928.

Age: 23; unmarried.

Brothers: none. Sisters: none.

Tattoo marks: glove and 3 spots on back of right hand.

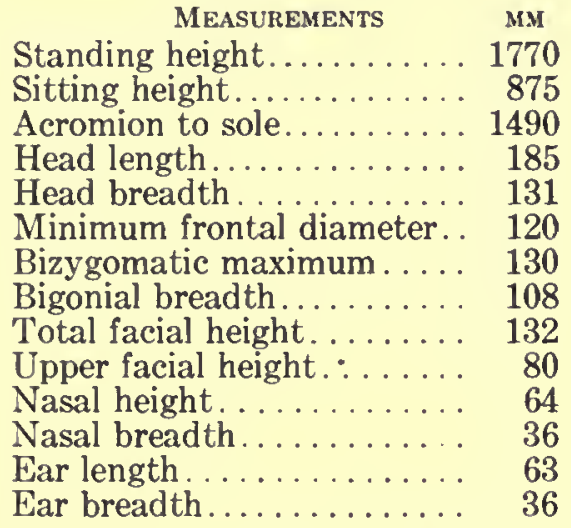

Cephalic............... 70.8

Relative sitting height..... 49.4

Fronto-parietal.........991.6

Total facial............101.7

Upper facial. ...........6. 61.5

Nasal. ............ 56.2

Ear.................. 57.2

Jugomandibular. . . . . . 8 83.1

Jugofrontal. . . . . . . . . . 92.3

HaIR.-Form: low waves. Texture: medium. Color: black. Quantity: head, plus; beard, minus; body, minus.

EyE.-Color: green brown. Sclera: clear. Iris: zoned.

NosE.-Profile: convex. Tip: depressed. Wings: medium.

CHEST.-Derelopment: double plus.

Musculature.-Excellent.

HEAlth.-Good.

\section{No. 456}

Residence: camp of Sheikh Aziz. Date: June 13, 1928.

Age: 55; married for 20 years. Sons: 1 living. Daughters: none.

Brothers: 1 dead. Sisters: none.

Tattoo marks: none.

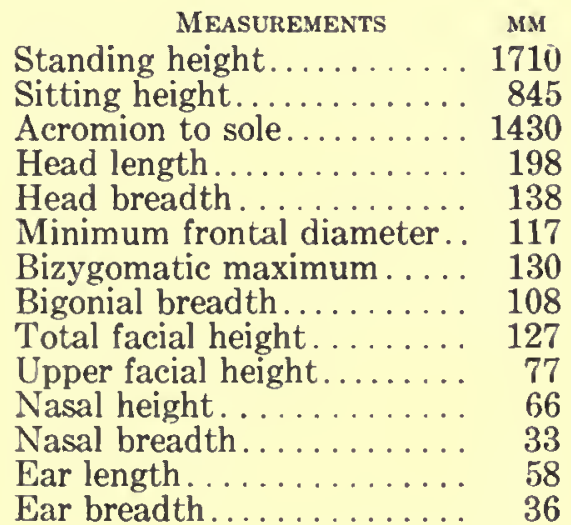

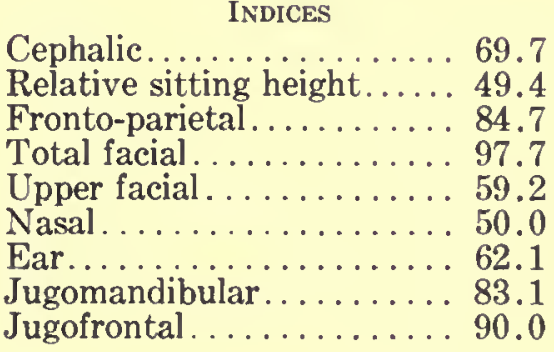

HaIR.-Color: gray. Head shaved.

EYE.-Color: dark brown. Sclera: clear. Iris: homogeneous.

NosE.-Profile: straight. Wings: compressed.

TеEтн.-Wear: triple plus. Caries: triple plus. Lost: many.

Chest.-Development: average.

Musculature.-Fair.

Health.-Fair. 
Residence: camp of Sheikh Aziz. Date: June 13, 1928.

Age: 65 ; married 30 years ago (wife now dead). No children.

Brothers: none. Sisters: none.

Tattoo marks: glove on back of right hand and gazelle on arm.

\begin{tabular}{|c|c|c|}
\hline MEASUREMENTS & MM & INDICES \\
\hline Standing height ......... & 1595 & Cephalic. \\
\hline 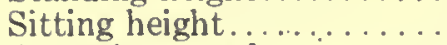 & 805 & Relative sitting height..... 50.4 \\
\hline Acromion to sole........ & 1340 & Fronto-parietal. . . . . . . 80.9 \\
\hline Head length............. & 192 & Total facial........... 86.4 \\
\hline Head breadth. & 142 & Upper facial. .......... 60 . \\
\hline Minimum frontal diameter. & 115 & Nasal. . . . . . . . . . . . 61.? \\
\hline Bizygomatic maximum. . . . & 133 & $\ldots \ldots \ldots \ldots 50$ \\
\hline Bigonial breadth........ & 114 & Jugomandibular......... 85.7 \\
\hline Total facial height....... & 115 & Jugofrontal. . . . . . . . . . 86. \\
\hline Upper facial height....... & 80 & - \\
\hline Nasal height. & 60 & \\
\hline Nasal breadth & 37 & \\
\hline & 77 & \\
\hline$\ldots \ldots$ & 39 & \\
\hline
\end{tabular}

HAIR.-Quantity: head, average; beard, double plus; body, double plus.

EYE.-Color: blue brown. Sclera: bloodshot. Iris: zoned.

Nose.-Profile: straight. Wings: medium-flaring.

TеEтн.-Wear: triple plus. Caries: triple plus. Lost: many.

CHEST.-Development: average.

Musculature.-Fair.

HEALTH.-Good.

No. 458. Plate L, Figs. 7, 8

Residence: camp of Sheikh Aziz. Date: June 13, 1928.

Age: 80 ; married for 25 years. Sons: 2 living, 2 dead. Daughters: 1 living, 1 dead. Brothers: 1 dead. Sisters: 1 living.

Tattoo marks: none.

\begin{tabular}{|c|c|}
\hline MEASUREMENTS & MM \\
\hline Standing height. & 1710 \\
\hline Sitting height. & 805 \\
\hline Acromion to sole. & 1455 \\
\hline Head length... & 191 \\
\hline Head breadth. & 137 \\
\hline Minimum frontal diameter. & 11 \\
\hline Bizygomatic maximum . . . . . & 133 \\
\hline Bigonial breadth. & 109 \\
\hline Total facial height. & 117 \\
\hline Upper facial height..... & 4 \\
\hline Nasal height. . . . . . . . & \\
\hline al breadth & \\
\hline$\ldots \ldots \ldots$ & \\
\hline & \\
\hline
\end{tabular}

Cephalic............. 71.7

Relative sitting height..... 47.1

Fronto-parietal......... 83.2

Total facial ............. 87.9

Upper facial . . . . . . . . 55.7

Nasal . . . . . . . . . . . . 70.7

Ear................. 89.2

Jugomandibular........ 82.0

Jugofrontal. . . . . . . . . . 85.7

Halr.-Color: white. Head shaved.

EYe.-Color: blue brown. Sclera: bloodshot. Iris: zoned.

Nose.-Profile: convex. Wings: medium-flaring.

TEeTH.-Lost: 3.

Chest.-Development: average.

Musculature.-Good.

HEALTH.-Fair.

Some Negro blood. 
No. 459

Residence: camp of Sheikh Aziz. Date: June 13, 1928.

Age: 30 ; married for 5 years. Sons: 1 living. Daughters: none.

Brothers: none. Sisters: none.

Tattoo marks: none.

\begin{tabular}{|c|c|}
\hline MEASUREMENTS & MM \\
\hline Standing height........ & 1675 \\
\hline Sitting height. & 830 \\
\hline Acromion to sole. & 1425 \\
\hline Head length.... & 196 \\
\hline Head breadth & 142 \\
\hline Minimum frontal diameter. . & 114 \\
\hline omatic maximum . . . . . & 126 \\
\hline ial breadth........... & 113 \\
\hline Total facial height......... & 120 \\
\hline Upper facial height........ & 72 \\
\hline$\ldots \ldots \ldots \ldots$ & 58 \\
\hline$\ldots \ldots \ldots$ & 40 \\
\hline & 64 \\
\hline & \\
\hline
\end{tabular}

\begin{tabular}{|c|c|}
\hline \multicolumn{2}{|l|}{ Cenhalic INDICES } \\
\hline Relative sitting height..... & 49.6 \\
\hline Fronto-parietal........ & 80.2 \\
\hline Total facial...... & 95.2 \\
\hline Upper facial & 57.1 \\
\hline Nasal..... & 69.0 \\
\hline Ear. & 64.1 \\
\hline Jugomandibular & 89.7 \\
\hline Jugofrontal .... & \\
\hline
\end{tabular}

HaIR.-Form: curly frizzly. Texture: coarse. Color: black. Quantity: head, plus; beard, double plus; body, plus.

EYE.-Color: dark brown. Sclera: clear. Iris: homogeneous.

NosE.-Profile: straight. Wings: flaring.

TEETH.-Wear: double plus. Caries: double plus.

Chest.-Development: plus.

Musculature.-Good.

HEALTH.-Good.

No. 460. Plate Li, Figs. 1, 2

Residence: camp of Sheikh Aziz. Date: June 13, 1928.

Age: 35 ; married twice, 6 years ago and this year (1928) (first wife now dead). Sons: 1 living. Daughters: none.

Brothers: 1 living. Sisters: 2 dead.

Tattoo marks: glove on back of right hand and gazelle on inside of right forearm.

\begin{tabular}{|c|c|}
\hline MEASUREMENTS & MM \\
\hline Standing height. & 1705 \\
\hline Sitting height. & 835 \\
\hline Acromjon to sole. & 1430 \\
\hline Head length.... & 198 \\
\hline Head breadth & 145 \\
\hline Minimum frontal diameter. & 108 \\
\hline Bizygomatic maximum .... & 133 \\
\hline Bigonial breadth.......... & 105 \\
\hline Total facial height. & 116 \\
\hline Upper facial height. . & 73 \\
\hline Nasal height. ........ & 64 \\
\hline asal breadt & 38 \\
\hline & 62 \\
\hline T & \\
\hline
\end{tabular}

INDICES

Cephalic............. 73.2

Relative sitting height..... 49.0

Fronto-parietal......... 74.5

Total facial. . . . . . . . . 87.2

Upper facial. . .......... 54.9

Nasal. .................... 59.3

Ear.............. 62.9

Jugomandibular. . . . . . 79.0

Jugofrontal. . . . . . . . 81.2

HAIR. -Quantity: head, average; beard, minus; body, average.

EYE.-Color: dark brown. Sclera: clear. Iris: homogeneous.

Nose.-Profile: convex. Wings: medium.

TEETH.-Lost: none.

Chest.-Development: plus.

Musculature.-Good.

HEALTH.-Good. 
No. 461. Plate LI, Figs. 3, 4

Residence: camp of Sheikh Aziz. Date: June 13, 1928.

Age: 55; married once (wife now dead). No children.

Brothers: 1 dead. Sisters: 1 living.

Tattoo marks: none.

\begin{tabular}{|c|c|}
\hline MEASUREMENTS & MM \\
\hline Standing height. & 1683 \\
\hline Sitting height. & 895 \\
\hline Acromion to sole. & 1420 \\
\hline Head length. & 190 \\
\hline Head breadth. & 14 \\
\hline Minimum frontal diameter. . & 11 \\
\hline Bizygomatic maximum . . . . . & 13 \\
\hline Bigonial breadth........... & 107 \\
\hline Total facial height. . . . . . . & 126 \\
\hline Upper facial height. ........ & 77 \\
\hline Nasal height & 63 \\
\hline Nasal breadtl & 34 \\
\hline Ear leng & 6 \\
\hline & \\
\hline
\end{tabular}

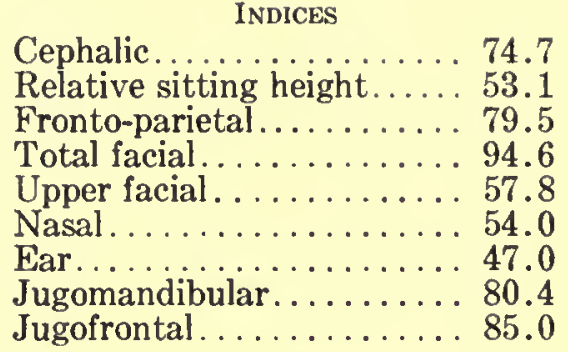

HAIR.-Color: gray. Head shaved.

EYE.-Color: dark brown. Sclera: clear. Iris: homogeneous.

Nose.-Profile: convex. Wings: medium.

TEETH.-Lost: none.

CHEsT.-Development: plus.

Musculature.-Good.

HEALTH.-Good.

\section{No. 462. Plate LI, Figs. 5, 6}

Residence: camp of Sheikh Aziz. Date: June 13, 1928.

Age: 35 ; married for 15 years. Sons: 1 living. Daughters: 2 living.

Brothers: 1 dead. Sisters: none.

Tattoo marks: none.

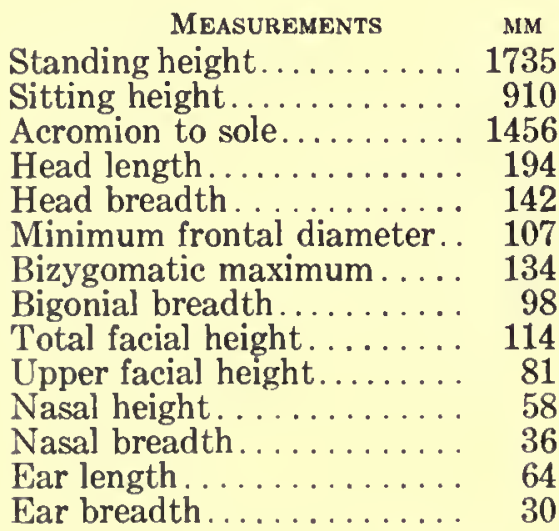

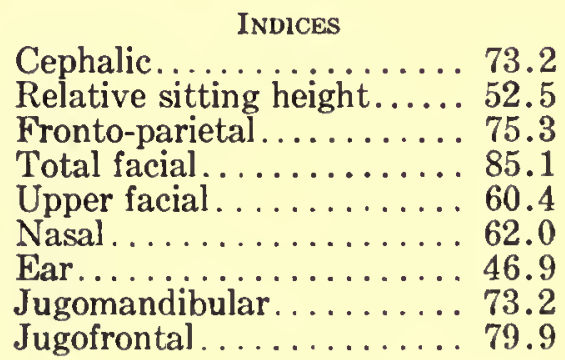

HAIR.-Form: low waves. Texture: medium. Color: black.

EYE.-Color: dark brown. Sclera: clear. Iris: homogeneous.

Nose.-Profile: straight. Wings: medium-compressed.

TEETH. - Lost: none.

Chest.-Development: plus.

Musculature.--Good.

HEALTH.-Good. 
No. 463

Residence: camp of Sheikh Aziz. Date: June 13, 1928.

Age: 27; unmarried.

Brothers: 1 living. Sisters: 3 living.

Tattoo marks: none.

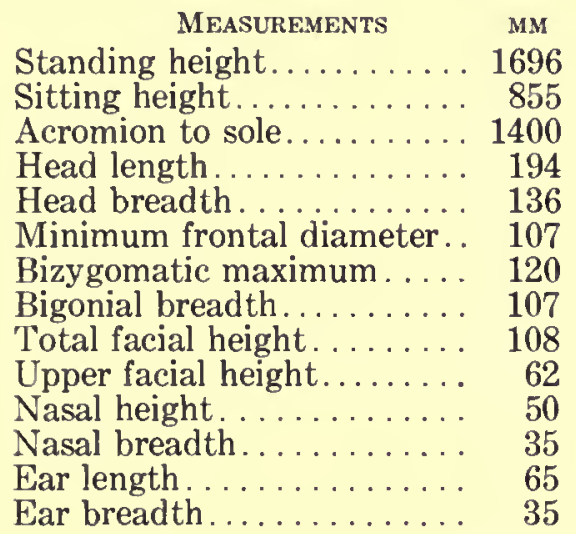

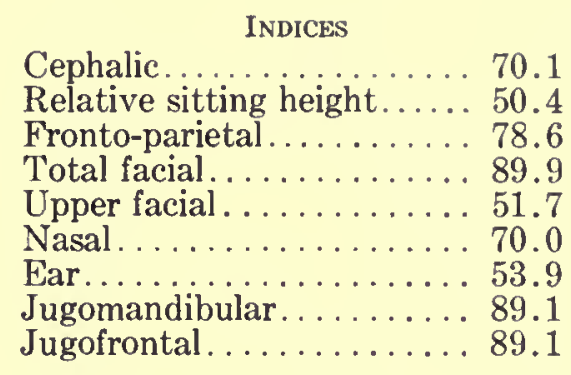

HaIR.-Form: low waves. Texture: medium. Quantity: head, plus; beard, minus; body, average.

EYE.-Color: dark brown. Sclera: clear. Iris: homogeneous.

Nose.-Profile: straight. Wings: medium-flaring.

TеEтн.-Wear: double plus. Caries: double plus. Lost: none.

Chest.-Development: plus.

Musculature.-Good.

HEALTH.--Good. Bad throat. Voice always hoarse.

No. 464

Residence: camp of Sheikh Aziz. Date: June 13, 1928.

Age: 20 ; unmarried.

Brothers: 2 living. Sisters: 1 living.

Tattoo marks: none.

\begin{tabular}{|c|c|}
\hline MEASUREMENTS & MM \\
\hline Standing height. & 170 \\
\hline Sitting height. & 850 \\
\hline Acromion to sole. & 1450 \\
\hline Head length & 18 \\
\hline ead breadth & 14 \\
\hline Iinimum frontal diameter. . & 11 \\
\hline latic maximum . . . . . & 12 \\
\hline al breadth. & 10 \\
\hline facial height. . . . . . . & 11 \\
\hline l height. . . . . . . & 66 \\
\hline $\mathrm{Na}$ & 53 \\
\hline & \\
\hline I & 64 \\
\hline & \\
\hline
\end{tabular}

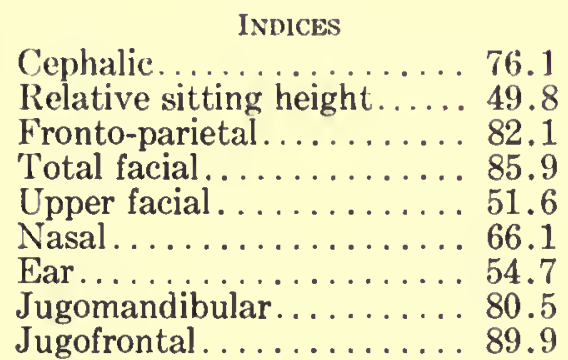

HAIR.-Form: curly frizzly. Texture: coarse. Color: dark brown.

EYE.-Color: dark brown. Sclera: clear. Iris: homogeneous.

NosE.-Profile: concave. Wings: medium-flaring.

TEETH.-Lost: none.

Chest.-Development: plus. 
Musculature.-Good.

HEALTH.-Good.

No. 465

Residence: cainp of Sheikh Aziz. Date: June 13, 1928.

Age: 20 ; unmarried.

Brothers: none. Sisters: 1 living, 1 dead.

Tattoo marks: none.

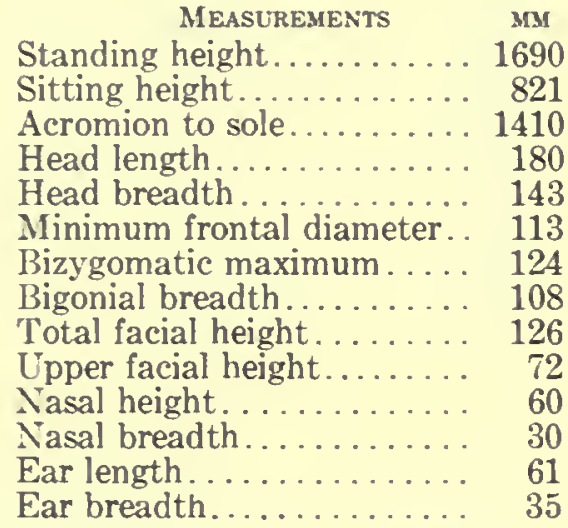

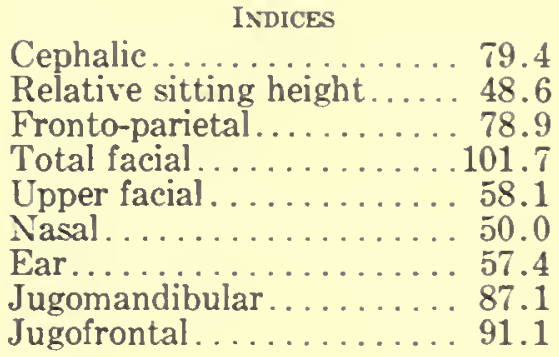

HAIR.-Form: deep waves. Texture: medium. Quantity: head, double plus; beard, ninus; body, average.

Ere.-Color: dark brown. Sclera: clear. Iris: homogeneous.

NOSE.-Profile: straight. Wings: medium.

TEETH.-Lost: none.

Chest.-Derelopinent: plus.

Musculature.-Good.

HEALTH.-Good.

No. 466

Residence: camp of Sheikh Aziz. Date: June 13, 1928.

Age: 30 ; married for 6 years. Sons: none. Daughters: 2 living.

Brothers: none. Sisters: 1 dead.

Tattoo marks: design on inside of right forearm.

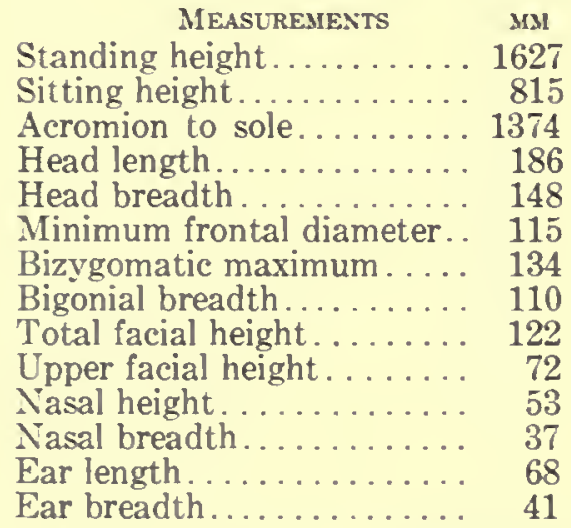

Cephalic........... 79.5

Relative sitting height..... 50.0

Fronto-parietal.......... 77.7

Total facial........... 91.0

Upper facial. ........... 53.7

Nasal..............6.6.8 69

Ear. . . 60.3

Jugomandibular. . . . . . 8. 82.1

Jugofrontal . . . . . . . . . 85.8

Eye.-Color: blue brown. Sclera: bloodshot. Iris: homogeneous.

Nose.-Profile: straight. Wings: medium.

TEETH. - Lost: none.

Chest.-Derelopment: arerage.

Musculature.-Fair.

HEALTH.-Good. 
No. 467

Residence: camp of Sheikh Aziz. Date: June 13, 1928.

Age: 20; married for 5 years. Sons: none. Daughters: 1 living, 1 dead.

Brothers: 1 living, 1 dead. Sisters: 1 dead.

Tattoo marks: none.

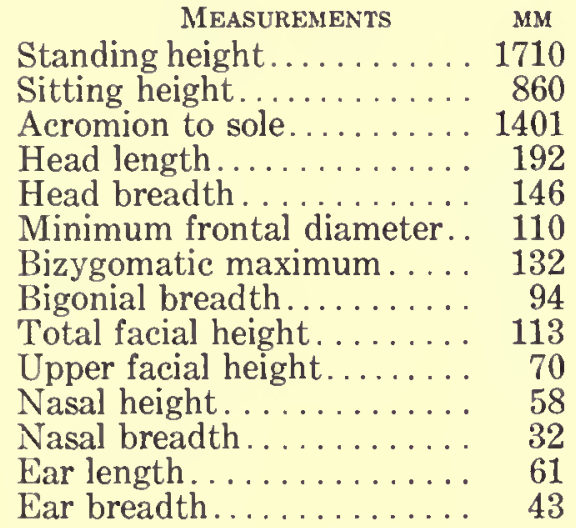

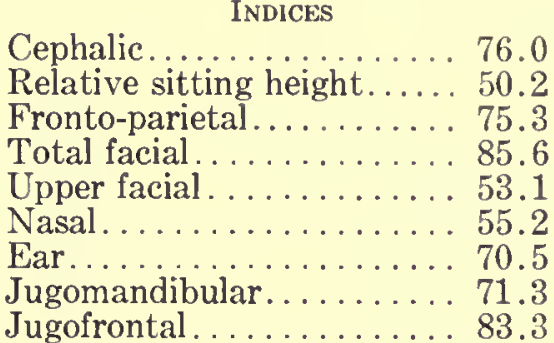

HAIR. - Form: curly frizzly. Texture: medium. Color: black. Quantity: head, double plus; beard, double minus; body, minus.

EYE. Color: dark brown. Sclera: clear. Iris: homogeneous.

Nose.-Profile: straight. Wings: medium.

TEETH.-Lost: 2.

Chest.-Development: plus.

Musculature.-Good.

HeAlth.-Good.

\section{No. 468}

Residence: camp of Sheikh Aziz. Date: June 13, 1928.

Age: 30 ; married for 10 years. Sons: 2 living, 1 dead. Daughters: 1 living.

Brothers: 1 dead. Sisters: 2 dead.

Tattoo marks: none.

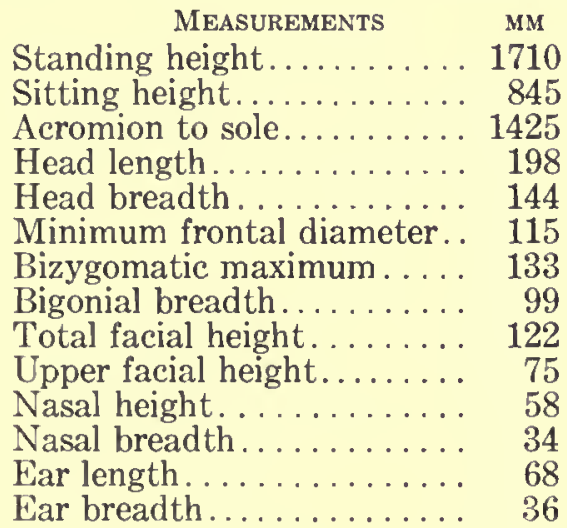

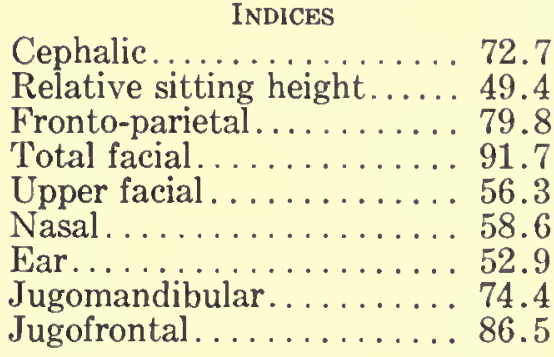

HaIR.-Form: low waves. Texture: medium. Quantity: head, plus; beard, plus; body, plus. EYE.-Color: dark brown. Sclera: clear. Iris: homogeneous.

Nose.-Profile: straight. Wings: medium.

TEETH.-Lost: none. Eruption: complete. Bite: marked over.

CHEST.-Development: plus.

Musculature.-Good. 
HEALTH.-Good.

Brother of No. 432.

\section{No. 469. Plate LI, Figs. 7, 8}

Residence: camp of Sheikh Aziz. Date: June 13, 1928.

Age: 20; unmarried.

Brothers: 1 living. Sisters: none.

Tattoo marks: none.

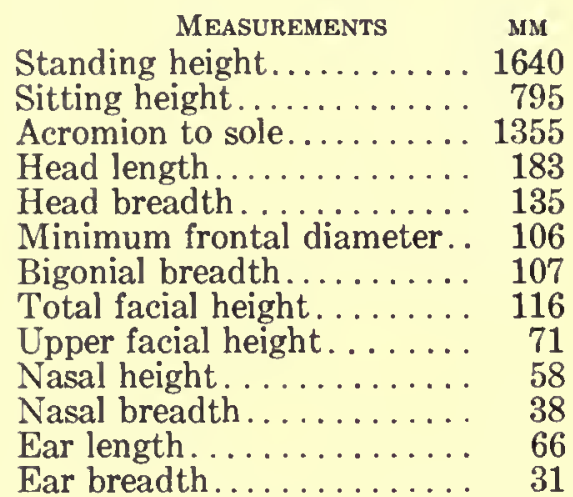

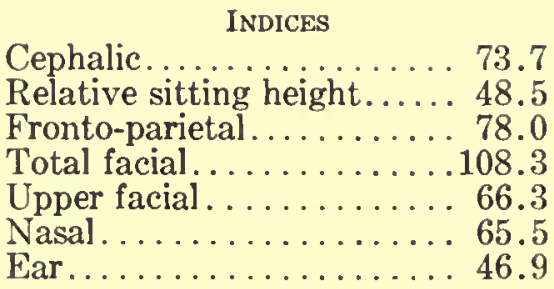

73.7

8. 3

HAIR.-Form: low waves. Texture: medium. Color: dark brown. Quantity: head, plus; beard, double minus; body, minus.

EYE.-Color: dark brown. Sclera: clear. Iris: homogeneous.

NoSE.-Profile: straight. Wings: medium.

TEETH.-Eruption: complete.

CHEst.-Development: plus.

Musculature.-Good.

HEALTH.-Good.

No. 470

Residence: camp of Sheikh Aziz. Date: June 13, 1928.

Age: 80 ; married for 35 years. Sons: 1 living, 1 dead. Daughters: 2 dead.

Brothers: 2 dead. Sisters: none.

Tattoo marks: none.

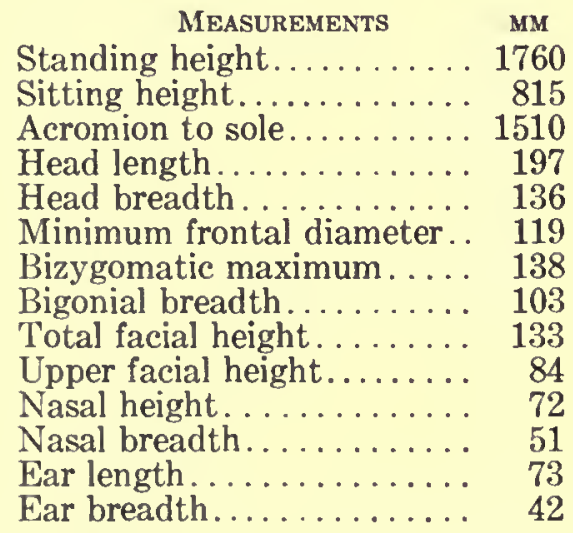

HAIR.-Quantity: head, double plus.

EYE.-Color: blue brown. Sclera: bloodshot. Iris: homogeneous. Condition: bad.

Nose.-Profile: straight. Wings: flaring.

TEETH.-Eruption: complete. Wear: double plus. Caries: triple plus. Lost: nearly all. Bite: under. 
CHEST.-Development: average.

Musculature.-Average.

HEALTH.-Fair.

Residence: camp of Sheikh Aziz. Date: June 13, 1928.

Age: 18; unmarried.

Brothers: none. Sisters: none.

Tattoo marks: none.

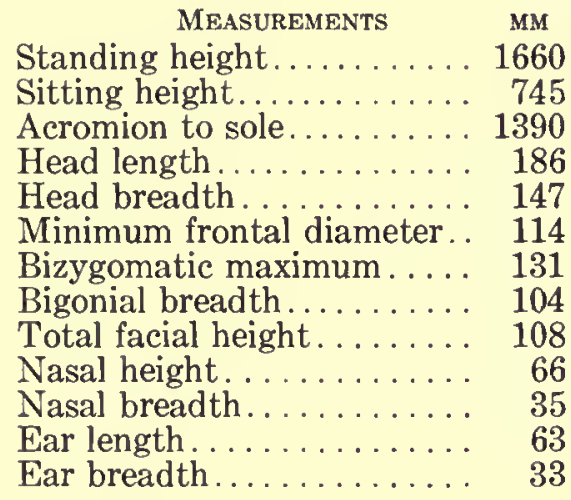

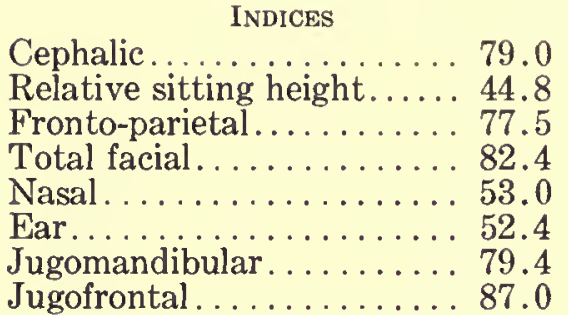

HAIR.-Form: low waves. Texture: medium. Quantity: head, double plus; beard, minus; body, average.

EyE.-Color: dark brown. Sclera: clear. Iris: homogeneous.

Nose.-Profile: straight. Wings: medium-flaring.

TEETH.-Eruption: complete. Lost: none. Bite: slight over.

CHEST.-Development: average.

Musculature.-Good.

HEALTH.--Good.

\section{SOLDIERS OF THE IRAQ ARMY}

\section{No. 500. Plate LII, Figs. 1, 2}

Place: Hilla Army Camp. Date: June 14, 1928.

Birthplace: Amara. Age: 20; unmarried.

Brothers: 1 living, 7 dead. Sisters: 2 living.

Tattoo marks: spot on left wrist.

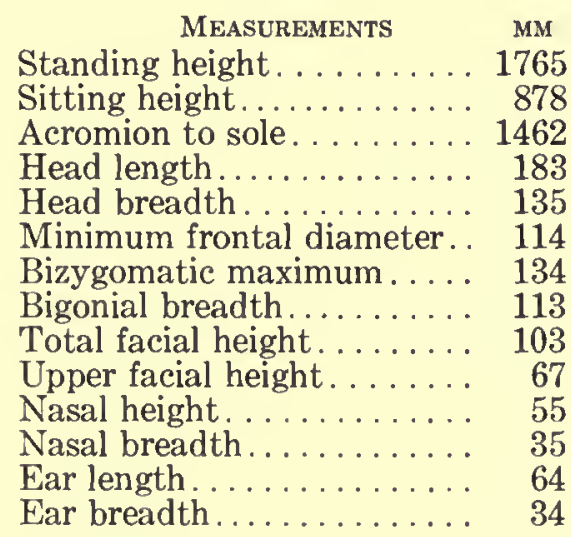

INDICES

Cephalic. .............73.8

Relative sitting height..... 49.7

Fronto-parietal. .......... 84.4

Total facial............ 76.8

Upper facial . . . . . . . . . . . . . 5 50.0

Nasal. . . . . ..........63.7

Ear...................... 53.2

Jugomandibular. . . . . . . 84.4

Jugofrontal . . . . . . . . . . . 85.1

HAIR.-Color: dark brown.

EYE.-Color: dark brown. Sclera: clear. Iris: homogeneous. 
Nose.-Profile: straight. Wings: medium.

TEETH.-Eruption: complete. Lost: none. Bite: slight over.

CHEST.-Development: plus.

Musculature.-Good.

HEALTH.-Good.

No. 501. Plate LiI, Figs. 3, 4

Place: Hilla Army Camp. Date: June 14, 1928.

Birthplace: Mahewil near Kish. Age: 20; unmarried.

Brothers: 2 living. Sisters: none.

Tattoo marks: none.

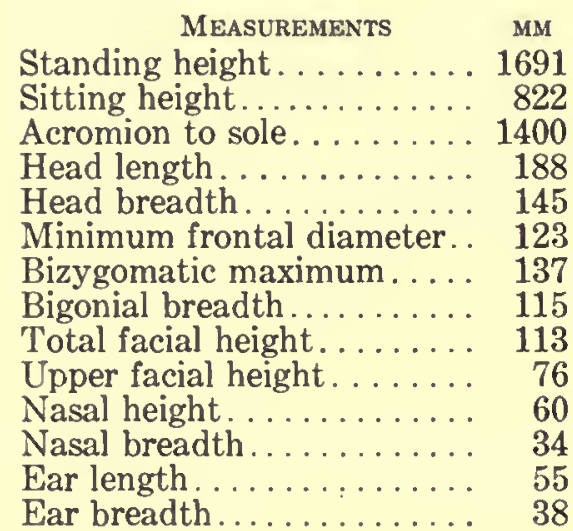

INDICES

Cephalic...............77.1

Relative sitting height...... 48.6

Fronto-parietal..........84.9

Total facial.............. 82.5

Upper facial. . . . . . . . 55.4

Nasal. . . . . . . . . . . . 56.7

Ear...............6. 69.1

Jugomandibular.......... 84.0

Jugofrontal . . . . . . . 89.8

HaIR. - Color: dark brown.

EYE.-Color: dark brown. Sclera: clear. Iris: homogeneous.

NosE.-Profile: straight. Wings: medium.

TEETH.-Eruption: complete. Lost: none. Bite: slight over.

Chest.-Development: plus.

Musculature.-Good.

HEALTH.-Good.

No. 502. Plate LiI, Figs. 5, 6

Place: Hilla Army Camp. Date: June 14, 1928.

Birthplace: Samawa. Age: 20; unmarried.

Brothers: 2 living. Sisters: 2 living.

Tattoo marks: none.

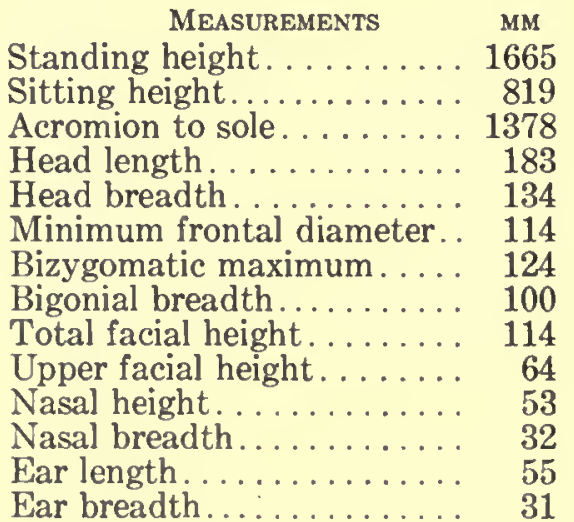

Cephalic.............. 73.2

Relative sitting height...... 49.1

Fronto-parietal......... 85.1

Total facial............. 91.8

Upper facial. . . . . . . . . . 51.7

Nasal. . . . . . . . . . . . . 60.3

Ear ...............56.3

Jugomandibular.......... 80.7

Jugofrontal. . . . . . . . . 91.9

HAIR.-Color: dark brown.

EYE.-Color: dark brown. Sclera: clear. Iris: zoned. 
NosE.-Profile: straight. Wings: medium.

TeEтн.-Eruption: complete. Lost: none. Bite: slight over.

CHEST.-Development: plus.

Musculature.-Good.

HEALTH.-Good.

\section{No. 503. Plate LII, Figs. 7, 8}

Place: Hilla Army Camp. Date: June 14, 1928.

Birthplace: Samawa. Age: 30; married for 15 years. Sons: 2 dead. Daughters: none. Brothers: none. Sisters: 2 living, 1 dead.

Tattoo marks: none.

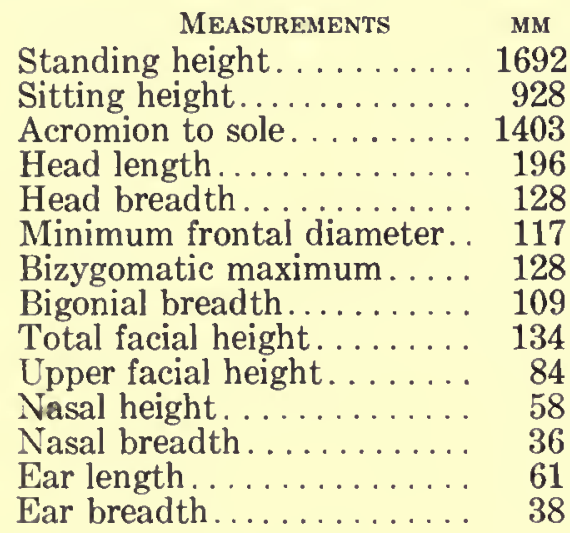

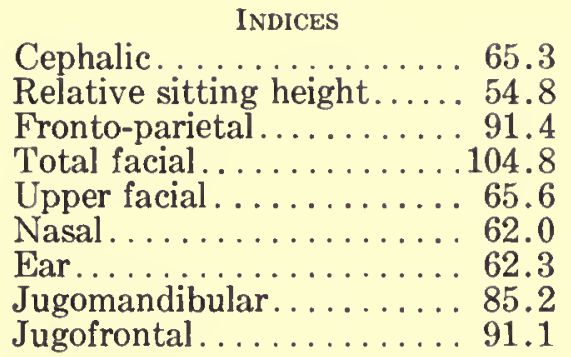

5.3 8 5.6 2.3 1.1

HAIR.-Color: dark brown.

EYE.-Color: dark brown. Sclera: clear. Iris: homogeneous.

NOSE.-Profile: straight. Wings: medium.

TEETH.-Eruption: complete. Bite: slight over.

Chest.-Development: plus.

Musculature.-Good.

HEALTH.-Good.

No. 504. Plate LiII, Figs. 1, 2

Place: Hilla Army Camp. Date: June 14, 1928.

Birthplace: near Hilla. Age: 25; married for 16 months. No children.

Brothers: 4 living. Sisters: 2 living.

Tattoo marks: 3 spots on right wrist.

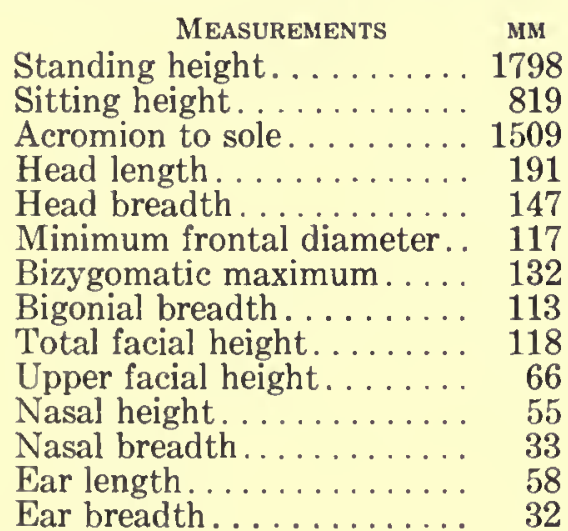

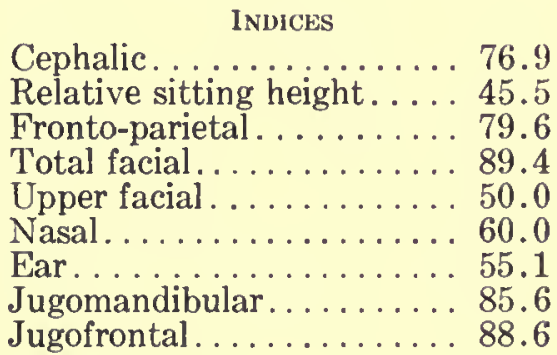

HAIR.-Color: black.

EyE.-Color: dark brown. Sclera: clear. Iris: homogeneous. 
Nose.-Profile: convex. Wings: medium.

TEETH.-Eruption: complete. Lost: 3.

ChEst.-Development: plus.

Musculature.-Good.

HEALTH.-Good.

No. 505. Plate liII, Figs. 3, 4

Place: Hilla Army Camp. Date: June 14, 1928.

Birthplace: Hindiya. Age: 25; unmarried.

Brothers: 2 living. Sisters: 2 living.

Tattoo marks: none.

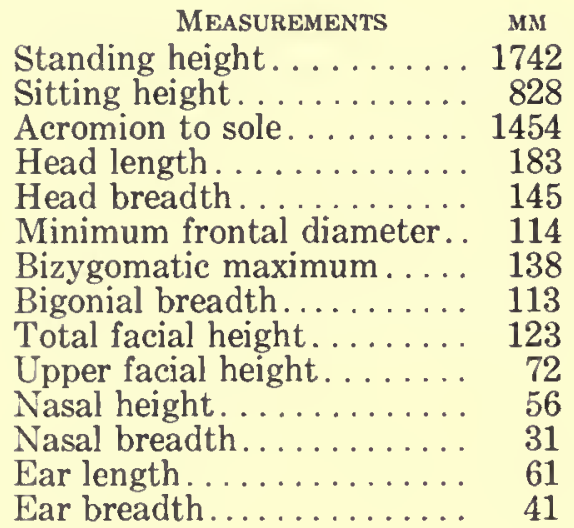

INDices

Cephalic.............79.2

Relative sitting height. . . . 47.5

Fronto-parietal..........78.6

Total facial. . . . . . . . . 89.1

Upper facial. . . . . . . . . . 52.2

Nasal. . . . . . . . . . . . . 55.3

Ear................ 67.2

Jugomandibular . . . . . . . . . 81.9

Jugofrontal. . . . . . . . . 82.6

HAIR.-Color: black.

EYE.-Color: dark brown. Sclera: clear. Iris: homogeneous.

NosE.-Profile: straight. Wings: medium.

TEETH.-Eruption: complete. Lost: 1 . Bite: slight over.

CHEST.-Development: average.

Musculature.-Fair.

HEALTH.-Good.

No. 506. Plate LiII, Figs. 5, 6

Place: Hilla Army Camp. Date: June 14, 1928.

Birthplace: Hilla. Age: 21; unmarried.

Brothers: 1 living, 2 dead. Sisters: 2 living, 3 dead.

Tattoo marks: glove on right hand, two spots and cross on inside of right forearm.

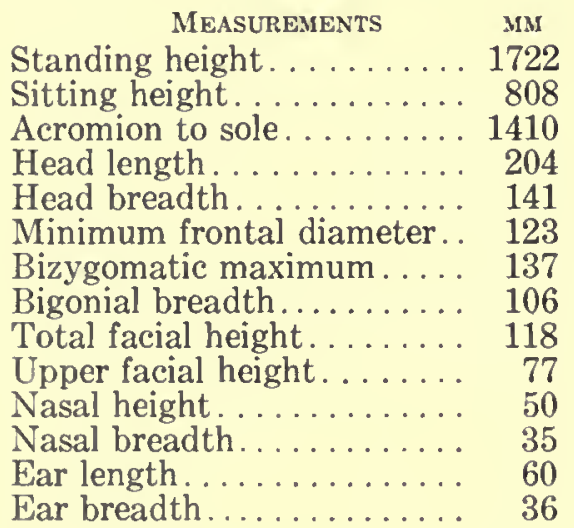

Cephalic. ............6.69.1

Relative sitting height. . . 46.9

Fronto-parietal......... 87.2

Total facial............ 86.1

Upper facial. ........... 56.2

Nasal.............. 70.0

Ear................6 60.0

Jugomandibular. . . . . . . . . 77.4

Jugofrontal . . . . . . . . 89.8

HAIR. - Color: dark brown.

EYE.-Color: dark brown. Sclera: clear. Iris: homogeneous. 
Nose.-Profile: straight. Wings: medium.

TEETH.--Eruption: complete. Lost: none. Bite: slight over.

CHEST.-Development: plus.

Musculature.-Good.

HEALTH.-Good.

\section{No. 507. Plate LiII, Figs. 7, 8}

Place: Hilla Army Camp. Date: June 14, 1928.

Birthplace: Hindiya. Age: 20; unmarried.

Brothers: 1 living, 1 dead. Sisters: 2 living.

Tattoo marks: spots on back of left hand.

\begin{tabular}{|c|c|c|}
\hline MEASUREMENTS & MM & INDICES \\
\hline Standing height. & 1683 & Cephalic. \\
\hline Sitting height. & 828 & Relative sitting height \\
\hline Acromion to sole. & 1400 & Fronto-parietal....... \\
\hline Head length. & 190 & Total facial. \\
\hline Head breadth. & 130 & Upper facial. \\
\hline Minimum frontal diameter. & 114 & Nasal. \\
\hline Bizygomatic maximum. & 128 & Ear. \\
\hline Bigonial breadth. & 106 & Jugomandibular \\
\hline otal facial height. & 113 & Jugofrontal . . . \\
\hline
\end{tabular}

Upper facial height....... 72

Nasal height.......... 58

Nasal breadth........... 35

Ear length............ 55

Ear breadth............ 35

HAIR.-Color: dark brown.

EYE.-Color: dark brown. Sclera: clear. Iris: homogeneous.

NosE.-Profile: straight. Wings: medium.

TEETH.-Eruption: complete. Lost: none. Bite: slight over.

Chest.-Development: plus.

Musculature.-Good.

HEALTH.-Good.

No. 508. Plate LiV, Figs. 1, 2

Place: Hilla Army Camp. Date: June 14, 1928.

Birthplace: Nasiriya. Age: 20; unmarried.

Brothers: 3 living. Sisters: none.

Tattoo marks: spots and line on right hand.

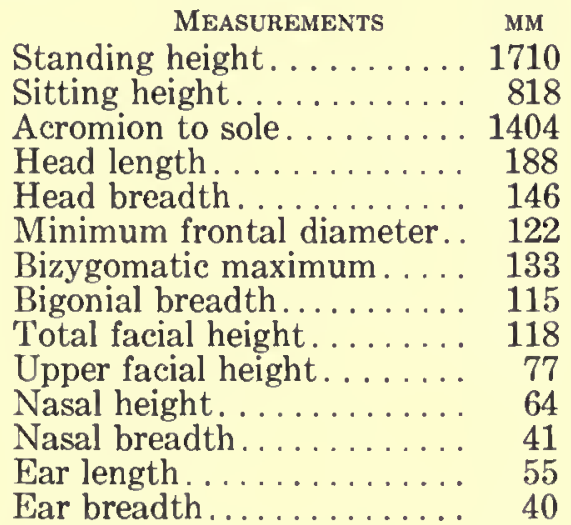

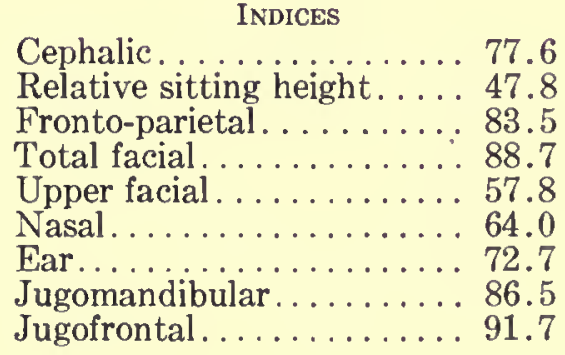

HAIR.-Color: dark brown.

EYE.-Color: dark brown. Sclera: clear. Iris: homogeneous. 
Nose.-Profile: straight. Wings: medium.

TEeth.-Eruption: complete. Lost: none. Bite: slight over.

Chest.-Development: plus.

Musculature.-Good.

HEALTH.--Good.

\section{No. 509. Plate LIV, Figs. 3, 4}

Place: Hilla Army Camp. Date: June 14, 1928.

Birthplace: Amara. Age: 25; married for 4 years. No children.

Brothers: 2 living. Sisters: 1 living.

Tattoo marks: spots and line on right wrist.

\begin{tabular}{|c|c|c|}
\hline MEASUREMENTS & MM & INDICES \\
\hline Standing height. ...... & 1770 & Cephalic. \\
\hline Sitting height... & 808 & Relative sitting height $\ldots . .45 .6$ \\
\hline Acromion to sole. & 1471 & Fronto-parietal.......... 76.7 \\
\hline Head length..... & 188 & Total facial............ \\
\hline Head breadth. & 150 & Upper facial \\
\hline Minimum frontal diameter. & 115 & Nasal. . . . . . \\
\hline Bizygomatic maximum ..... & 142 & $\therefore 72.9$ \\
\hline Bigonial breadth.......... & 115 & Jugomandibular. \\
\hline Total facial height... & 133 & Jugofrontal ....... \\
\hline Upper facial height. . . . . . . & 77 & \\
\hline Nasal height. . . . . . . . . . . & 58 & \\
\hline Nasal breadth............ & 38 & \\
\hline Ear length............. & 59 & \\
\hline Ear breadth. & 43 & \\
\hline
\end{tabular}

HAIR.-Color: dark brown. Quantity: head, plus.

EYE.-Color: dark brown. Sclera: clear. Iris: homogeneous.

NoSE.-Profile: straight. Wings: medium.

TEETH.-Eruption: complete. Lost: none. Bite: slight over.

Chest.-Development: plus.

Musculature.-Good.

HEALTH.-Good.

\section{No. 510. Plate LIV, Figs. 5, 6}

Place: Hilla Army Camp. Date: June 14, 1928.

Birthplace: Hilla. Age: 27; unmarried.

Brothers: 1 living. Sisters: 1 living.

Tattoo marks: spots on right wrist.

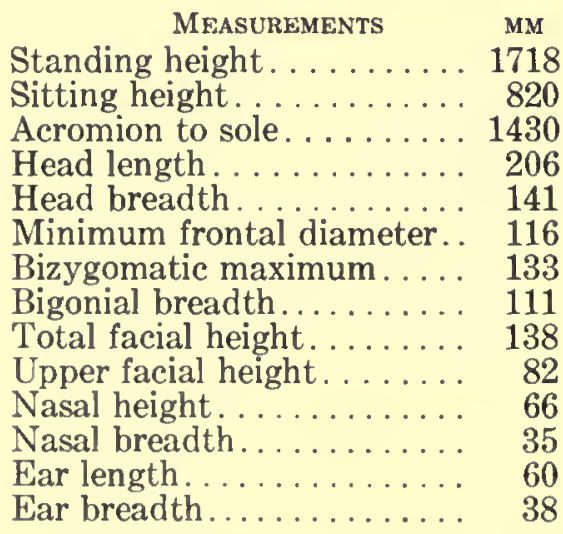

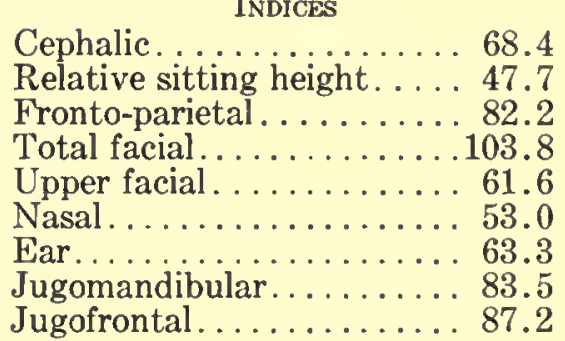

Cephalic............. 68.4 Relative sitting height. . . 47.7

Fronto-parietal........8 82.2

Total facial. ............. 103.8

Upper facial. . . . . . . . 61.6

Nasal. . . . 53.0

Jugomandibular........ 83.5

Jugofrontal ............. 87.2

HAIR.-Color: dark brown.

EYE.-Color: dark brown. Sclera: bloodshot. Iris: homogeneous. 
Nose.-Profile: straight. Wings: medium.

T'EетH.-Eruption: complete. Lost: none. Bile: slight over.

CHEST.-Development: plus.

Musculature.-Good.

HEALTH.-Good.

\section{No. 511. Plate LIV, Figs. 7, 8}

Place: Hilla Army Camp. Date: June 14, 1928.

Birthplace: Amara. Age: 19; unmarried.

Brothers: 6 living. Sisters: none.

Tattoo marks: glove on right hand.

\begin{tabular}{|c|c|c|}
\hline Measurements & мM & INDICES \\
\hline Standing height. & 1784 & Cephalic. \\
\hline Sitting height. & 868 & Relative sitting height. \\
\hline Acromion to sole.. & 1480 & Fronto-parietal..... \\
\hline Head length. & 184 & Total facial.. \\
\hline Head breadth. & 144 & Upper facial......... \\
\hline Minimum frontal diameter. & 118 & Nasal. \\
\hline omatic maximum .... & 132 & Ear \\
\hline dth. & 105 & Jugomandibular. . \\
\hline ight. & 118 & Jugofronta \\
\hline
\end{tabular}

Upper facial height. . . . . . 65

Nasal height. ......... 55

Nasal breadth........... 38

Ear length........... 63

Ear breadth........... 33

HAIR.-Color: dark brown.

EYE.-Color: dark brown. Sclera: clear. Iris: homogeneous.

Nose.-Profile: straight. Wings: medium.

TEETH.-Eruption: complete. Lost: none. Bite: slight over.

Chest.-Development: plus.

Musculature.-Good.

HEALTH.-Good.

No. 512. Plate LV, Figs. 1, 2

Place: Hilla Army Camp. Date: June 14, 1928.

Birthplace: Hilla. Age: 20; unmarried.

Brothers: 1 living. Sisters: none.

Tattoo marks: none.

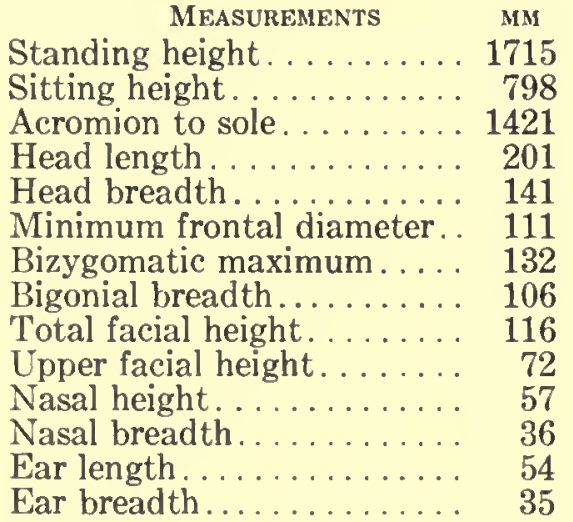

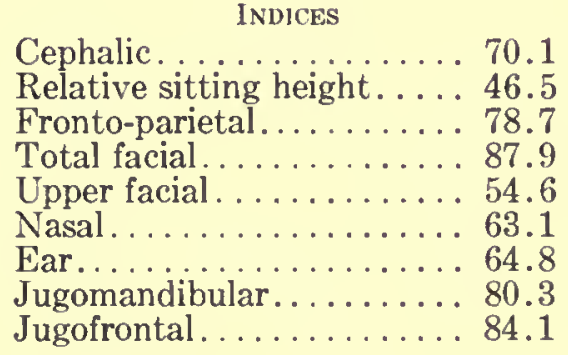

Cephalic.............70.1

Relative sitting height.... 46.5

Relonto-parietal. 78.7

Upper facial. ....... 54.6

Nasal. . . . . . ........6.63.1

Jugofrontal. ........... 84.1

HAIR. - Color: dark brown.

EYE.-Color: dark brown. Sclera: clear. Iris: homogeneous. 
Nose.--Profile: straight. Wings: medium-flaring.

TEETH.-Eruption: complete. Lost: none. Bite: slight over.

ChesT.-Development: plus.

Musculature.-Good.

HEALTH.-Good.

\section{No. 513. Plate LV, Figs. 3, 4}

Place: Hilla Army Camp. Date: June 14, 1928.

Birthplace: Hilla. Age: 25; unmarried.

Brothers: 1 living. Sisters: 1 living.

Tattoo marks: none.

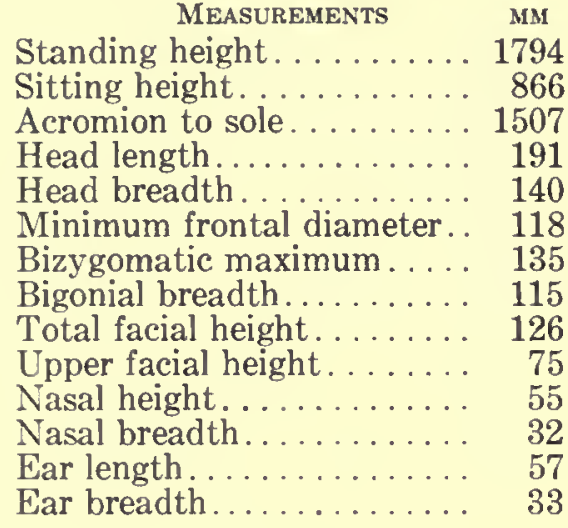

Cephalic............ 73.2

Relative sitting height. ... 48.2

Fronto-parietal.......... 84.2

Total facial........... 93.3

Upper facial............ 55.5

Nasal. . . . . . . . . 58.2

Ear.................... 57.9

Jugomandibular. . . . . . . 85.2

Jugofrontal . . . . . . . . 8. 87.4

HAIR.-Color: dark brown.

EYE.-Color: blue brown. Sclera: clear. Iris: zoned.

Nose.-Profile: convex. Wings: very compressed.

TEETH.-Eruption: complete. Lost: none. Bite: marked over.

CHEST.-Development: plus.

Musculature.-Good.

HEALTH.-Good.

\section{No. 514. Plate LV, Figs. 5, 6}

Place: Hilla Army Camp. Date: June 14, 1928.

Birthplace: Hindiya. Age: 20; unmarried.

Brothers: none. Sisters: none.

Tattoo marks: glove on back of right hand.

\begin{tabular}{|c|c|c|}
\hline MEASUREMENTS & MM & INDICES \\
\hline Standing height. . . . . . & 1782 & Cephalic. \\
\hline Sitting height. . & 888 & Relative sitting height. . . 49.8 \\
\hline Acromion to sole. & 1340 & Fronto-parietal......... 87.3 \\
\hline Head length.... & 188 & Total facial. .......... 90.7 \\
\hline Head breadth. & 135 & Upper facial \\
\hline Minimum frontal diameter. & 118 & Nasal...... \\
\hline Bizygomatic maximum.... & 128 & 63.5 \\
\hline Bigonial breadth......... & 100 & Jugomandibular. \\
\hline Total facial height..... & 116 & Jugofrontal . . . \\
\hline Upper facial height. . . . . . . & 68 & \\
\hline Nasal height. . . . . . . . . . . . & 55 & \\
\hline Nasal breadth. & 35 & \\
\hline Ear length. & 63 & \\
\hline Ear breadth & 40 & \\
\hline
\end{tabular}

EyE.-Color: dark brown. Sclera: clear. Iris: homogeneous.

NosE.-Profile: concave. Wings: flaring. 
Teeth.-Eruption: complete. Lost: none. Bite: slight over.

Chest.-Development: plus.

MUSCUlatURE.-Good.

HEalth.-Good.

No. 515. Plate LV, Figs. 7, 8

Place: Hilla Army Camp. Date: June 14, 1928.

Birthplace: Nasiriya. Age: 20; unmarried.

Brothers: 1 living. Sisters: 1 living.

Tattoo marks: none.

\begin{tabular}{|c|c|}
\hline MEASUREMENTS & MM \\
\hline Standing height. & 1772 \\
\hline Sitting height. & 898 \\
\hline Acromion to sole. & 1460 \\
\hline Head length.... & 194 \\
\hline Head breadth. & 148 \\
\hline Minimum frontal diameter. & 11 \\
\hline Bizygomatic maximum.... & 140 \\
\hline Bigonial breadth ......... & 11 \\
\hline Total facial height. & 131 \\
\hline Upper facial height. & $7^{\prime}$ \\
\hline Nasal height & \\
\hline Nasal breadth. & \\
\hline Ear length & \\
\hline & \\
\hline
\end{tabular}

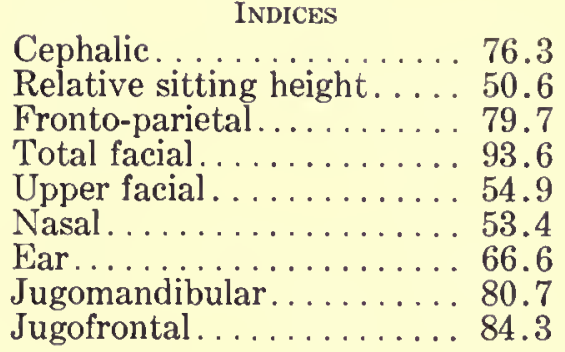

HAIR.-Color: dark brown.

EYE.-Color: dark brown. Sclera: clear. Iris: homogeneous.

Nose.-Profile: convex. Wings: medium-compressed.

TEETH.-Eruption: complete. Lost: 1. Bite: slight over.

CHEST.-Development: plus.

Musculature.-Good.

Health.-Good.

No. 516. Plate LVI, Figs. 1, 2

Place: Hilla Army Camp. Date: June 14, 1928.

Birthplace: Nasiriya. Age: 20; unmarried.

Brothers: 2 living. Sisters: none.

Tattoo marks: glove on back of right hand and gazelle on inside of right forearm.

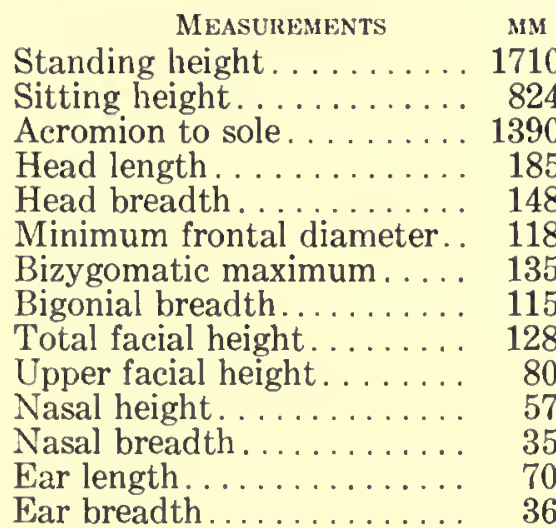

INDICES

Cephalic............. 79.9

Relative sitting height.... 48.2

Fronto-parietal. ........79.7

Total facial............ 94.7

Upper facial. .......... 59.3

Nasal. . . . . . . . . . . . . 61.4

Ear............... 51.4

Jugomandibular......... 85.2

Jugofrontal...........88 87.4

HAIR.-Color: dark brown.

EYE.-Color: dark brown. Sclera: clear. Iris: homogeneous.

Nose.-Profile: straight. Wings: compressed. 
TEETH.-Eruption: complete. Lost: none. Bite: slight over.

Chest.-Development: plus.

Musculature.-Good.

HEALTH.-Good.

No. 517. Plate LVI, Figs. 3, 4

Place: Hilla Army Camp. Date: June 14, 1928.

Birthplace: Hilla. Age: 25; unmarried.

Brothers: 2 living, 3 dead. Sisters: 1 living.

Tattoo marks: spots on right wrist.

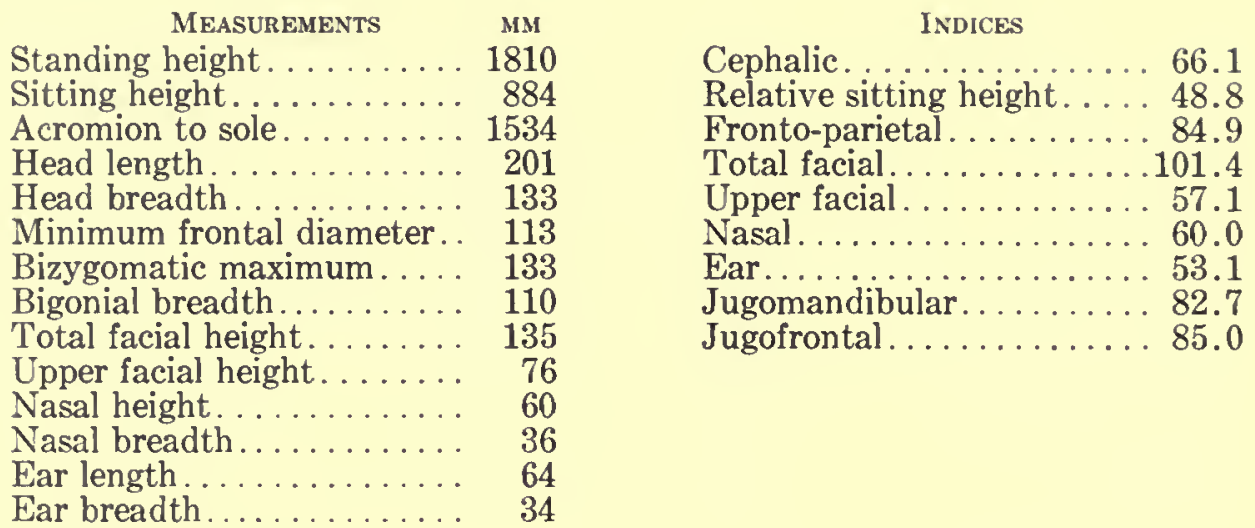

HAIR.-Color: dark brown.

EYE.-Color: green brown. Sclera: clear. Iris: zoned.

Nose.-Profile: convex. Tip: thickness, minus. Wings: compressed.

TEETH.-Eruption: complete. Lost: 1. Bite: slight over.

CHEST.-Derelopment: plus.

Musculature.-Good.

HEALTH.--Good.

No. 518. Plate LVI, Figs. 5, 6

Place: Hilla Army Camp. Date: June 14, 1928.

Birthplace: Hilla. Age: 20; unmarried.

Brothers: 2 living. Sisters: none.

Tattoo marks: gazelle and design on inside of right forearm.

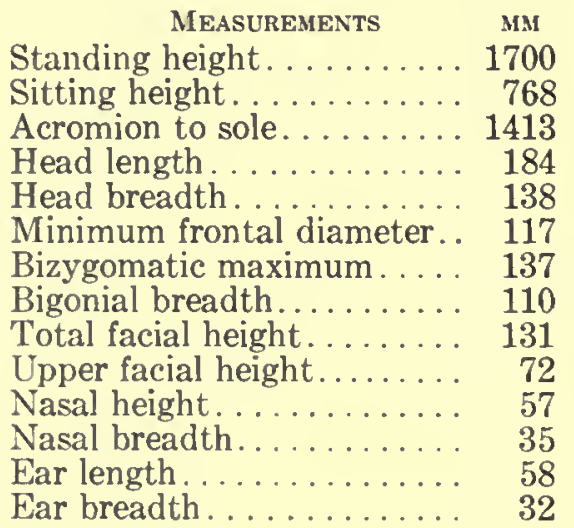

INDICES
Cephalic . . . . . . . .

EYE.-Color: dark brown. Sclera: clear. Iris: homogeneous.

Nose.-Profile: straight. Wings: medium-flaring.

TEETH.-Eruption: complete. Lost: none. Bite: slight over. 
CHEST.-Development: plus.

Musculature.-Good.

HEALTH.-Good.

No. 519. Plate LVI, Figs. 7, 8

Place: Hilla Army Camp. Date: June 14, 1928.

Birthplace: Amara. Age: 28; married for 4 years. Sons: 1 living. Daughters: none. Brothers: 2 living, 1 dead. Sisters: 1 living.

Tattoo marks: none.

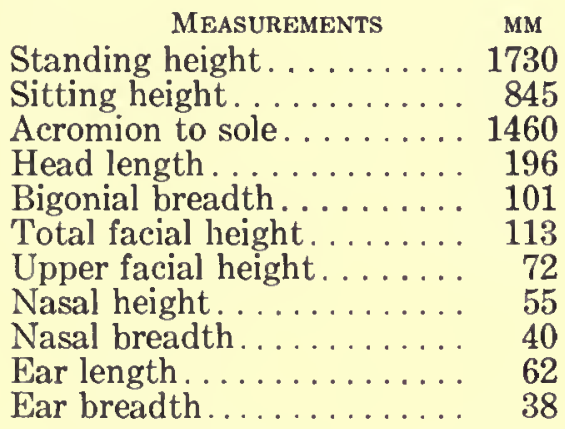

INDICES

Relative sitting height. . . . 48.8

Nasal. . . . . . . . . . . 72.8

Ear.............. 61.3

HAIR.-Color: dark brown.

EYE.-Color: green brown. Sclera: clear. Iris: zoned.

Nose.-Profile: convex. Wings: medium.

TEETH.-Eruption: complete. Lost: 1. Bite: slight over.

Chest.-Development: plus.

Musculature.-Good.

HEALTH.-Good.

No. 520. Plate LVII, Figs. 1, 2

Place: Hilla Army Camp. Date: June 14, 1928.

Birthplace: Nasiriya. Age: 22; unmarried.

Brothers: 1 living, 1 dead. Sisters: 1 living.

Tattoo marks: none.

\begin{tabular}{|c|c|c|}
\hline \multirow{9}{*}{\multicolumn{3}{|c|}{ 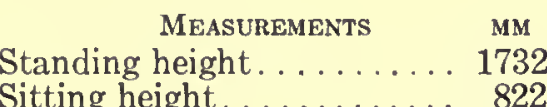 }} \\
\hline & & \\
\hline & \\
\hline \multirow{6}{*}{\multicolumn{2}{|c|}{ 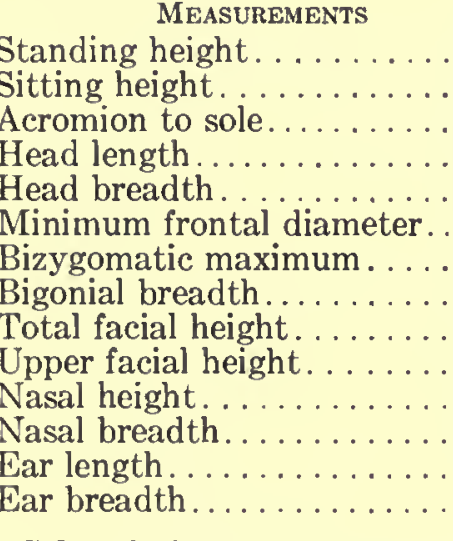 }} & \\
\hline \multirow{3}{*}{\multicolumn{3}{|c|}{ Head length $\ldots \ldots \ldots \ldots \ldots$}} \\
\hline \multirow{3}{*}{\multicolumn{3}{|c|}{$\ldots \ldots \ldots$}} \\
\hline \multirow{6}{*}{\multicolumn{3}{|c|}{ frontal diameter. }} \\
\hline \multirow{6}{*}{\multicolumn{3}{|c|}{$\begin{array}{l}\text { Bizygomatic maximum . . . . } \\
\text { Bigonial breadth . . . . }\end{array}$}} \\
\hline & & \\
\hline \multirow{3}{*}{\multicolumn{3}{|c|}{ Total facial height. ........ }} \\
\hline \multirow{6}{*}{\multicolumn{3}{|c|}{ Upper facial height. . . . . . }} \\
\hline & & \\
\hline \multirow{3}{*}{\multicolumn{3}{|c|}{ Nasal breadth........... }} \\
\hline \multirow{3}{*}{\multicolumn{3}{|c|}{ Ear length.............. }} \\
\hline & & \\
\hline & & \\
\hline & & \\
\hline & & \\
\hline & & \\
\hline & & \\
\hline & & \\
\hline & & \\
\hline & & \\
\hline & & \\
\hline & & \\
\hline & & \\
\hline & & \\
\hline & & \\
\hline & & \\
\hline & & \\
\hline & & \\
\hline & & \\
\hline & & \\
\hline & & 1 \\
\hline & & \\
\hline & & \\
\hline & & \\
\hline & & \\
\hline & & \\
\hline & & \\
\hline & & \\
\hline & & \\
\hline & & \\
\hline & & \\
\hline & & \\
\hline
\end{tabular}

INDICES

Cephalic ............ 73.7

Relative sitting height .... 47.4

Fronto-parietal......... 80.8

Total facial............. 82.6

Upper facial. . . . . . . . 50.7

Nasal. ................ 72.8

Ear.................... 61.1

Jugomandibular. . . . . . 74.6

Jugofrontal . . . . . . . . . . 84.0

HAIR. - Color: dark brown.

EYE.-Color: dark brown. Sclera: clear. Iris: homogeneous.

Nose.-Profile: convex. Wings: flaring.

TEeTH.-Eruption: complete. Lost: none. Bite: slight over.

Chest.-Development: plus.

Musculature.-Good.

HEALTH.-Good. 


\section{No. 521. Plate LVII, Figs. 3, 4}

Place: Hilla Army Camp. Date: June 14, 1928.

Birthplace: Hilla. Age: 22; married for 3 years. Sons: none. Daughters: 1 dead. Brothers: 1 living, 1 dead. Sisters: none.

Tattoo marks: 3 spots in line on right wrist.

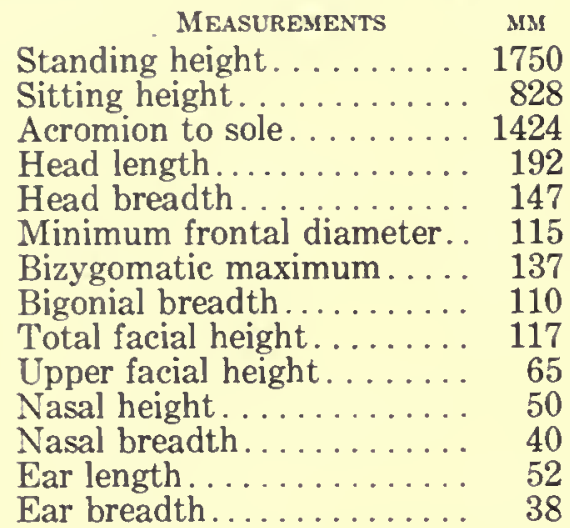

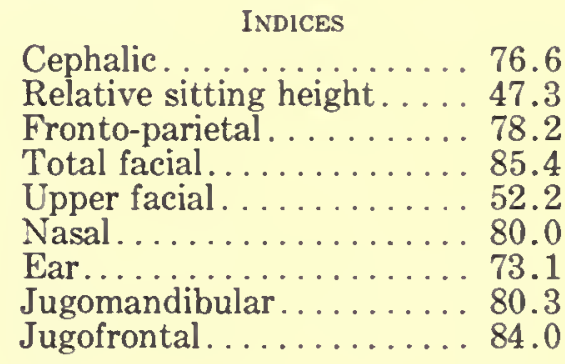

EYE.-Color: dark brown. Sclera: clear. Iris: homogeneous.

NosE.-Profile: concave. Wings: medium-flaring.

TEeTH.-Eruption: complete. Lost: 1. Bite: slight over.

ChEst.-Development: plus.

Musculature.-Good.

HEALTH.-Good.

\section{No. 522. Plate LVII, Fig. 5}

Place: Hilla Army Camp. Date: June 14, 1928.

Birthplace: Hilla. Age: 23; unmarried.

Brothers: 1 living. Sisters: none.

Tattoo marks: none.

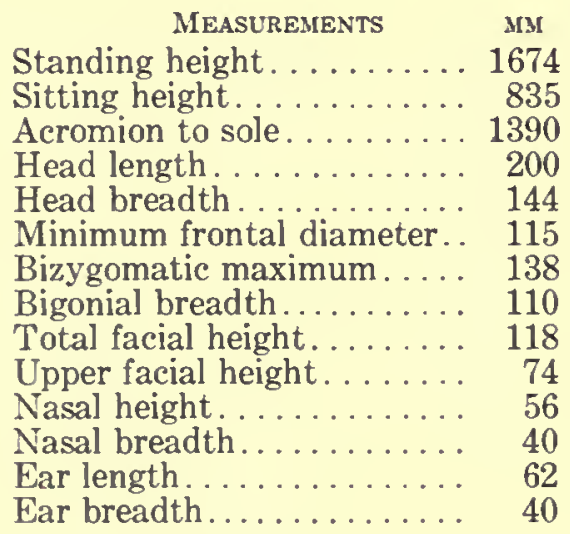

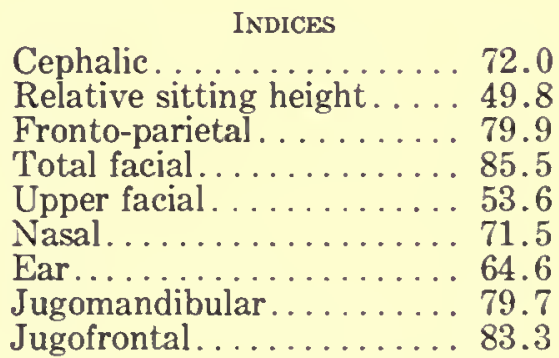

HAIR.-Color: dark brown.

EyE.-Color: dark brown. Sclera: clear. Iris: homogeneous.

Nose.-Profile: straight.

TEETH.-Eruption: complete. Lost: 1. Bite: slight over.

Chest.-Development: plus.

Musculature.-Good.

HEALTH.-Good. 
No. 523. Plate LVII, Figs. 6, 7

Place: Hilla Army Camp. Date: June 14, 1928.

Birthplace: Nasiriya. Age: 29; married for 4 years. Sons: 1 dead. Daughters: 2 dead. Brothers: 2 dead. Sisters: 2 living.

Tattoo marks: straight line on back of right hand.

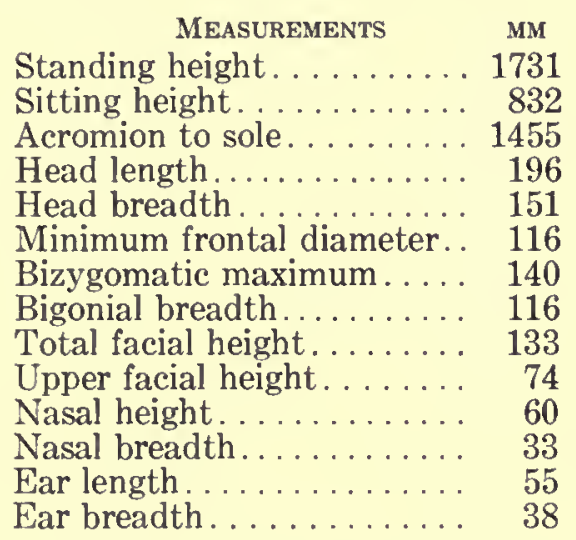

INDICES

Cephalic.............. 77.0

Relative sitting height.....48.1

Fronto-parietal . ....... 76.9

Total facial ............ 95.0

Upper facial . . . . . . . . 52.8

Nasal. . . . . . . . . 55.0

Ear.............6.6. 68.1

Jugomandibular. . . . . . . 82.9

Jugofrontal. ........... 82.9

Eye.-Color: dark brown. Sclera: clear. Iris: homogeneous.

Nose.-Profile: convex. Wings: medium.

TEeтH.-Eruption: complete. Lost: 1. Bite: slight over.

Chest.-Development: plus.

Musculature.-Good.

HEALTH.—Good.

No. 524. Plate LVIII, Figs. 1, 2

Place: Hilla Army Camp. Date: June 14, 1928.

Birthplace: Hilla. Age: 18; unmarried.

Brothers: 2 dead. Sisters: 3 living.

Tattoo marks: none.

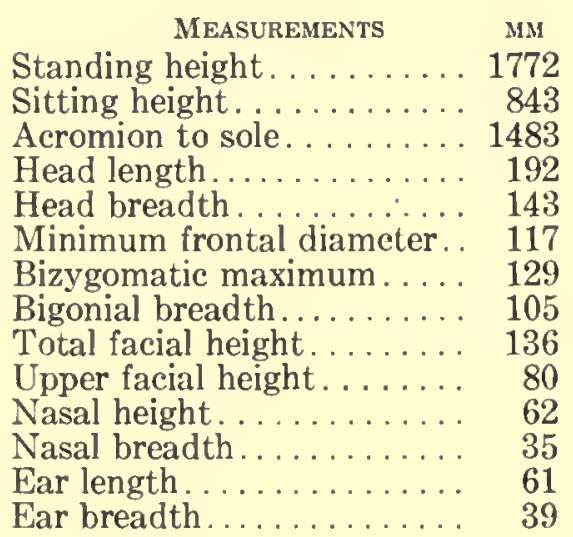

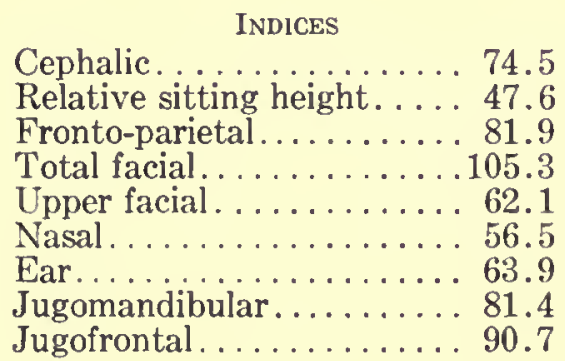

HAIR.-Color: dark brown.

EYE.-Color: dark brown. Sclera: clear. Iris: homogeneous.

NosE.-Profile: straight. Wings: medium.

TEeTH.-Eruption: complete. Lost: 2. Bite: slight over.

Chest.-Development: plus.

Musculature.-Good.

HEALTH.-Good. 
No. 525. Plate LVIII, Figs. 3, 4

Place: Hilla Army Camp. Date: June 14, 1928.

Birthplace: Amara. Age: 21; married for 2 years. Sons: 2 dead. Daughters: none. Brothers: 3 living, 1 dead. Sisters: 1 living, 1 dead.

Tattoo marks: none.

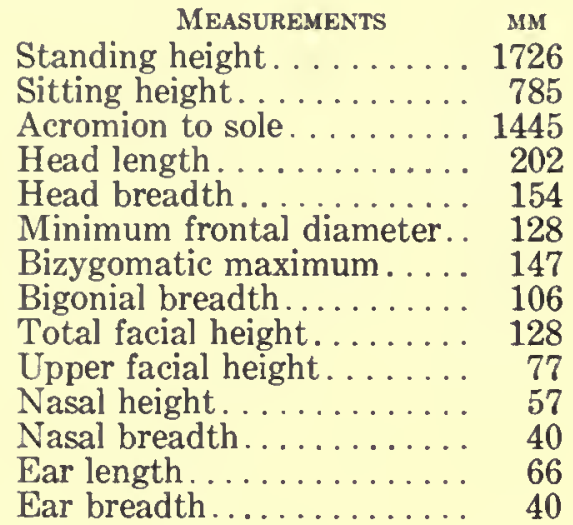

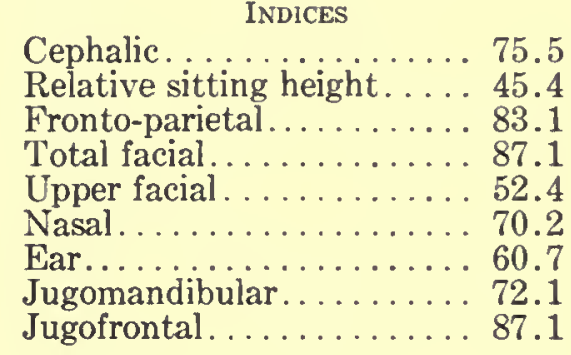

Cephalic............. 75.5

Relative sitting height.... 45.4

83.1

Upper facial. ......................

Nasal. . . . . . . . . . 70.2

60.7

Jugofrontal . . . . . . . . . 8. 87.1

HAIR.-Color: dark brown.

EYE.-Color: dark brown. Sclera: clear. Iris: homogeneous.

NosE.-Profile: concave. Wings: medium-flaring.

TEeth.-Eruption: complete. Lost: none. Bite: slight over.

CHEST.-Development: plus.

Musculature.-Good.

HEALTH.-Good.

\section{No. 526. Plate LVIII, Figs. 5, 6}

Place: Hilla Army Camp. Date: June 14, 1928.

Birthplace: Hilla. Age: 18; unmarried.

Brothers: 3 living. Sisters: 1 living.

Tattoo marks: glove on back of right hand.

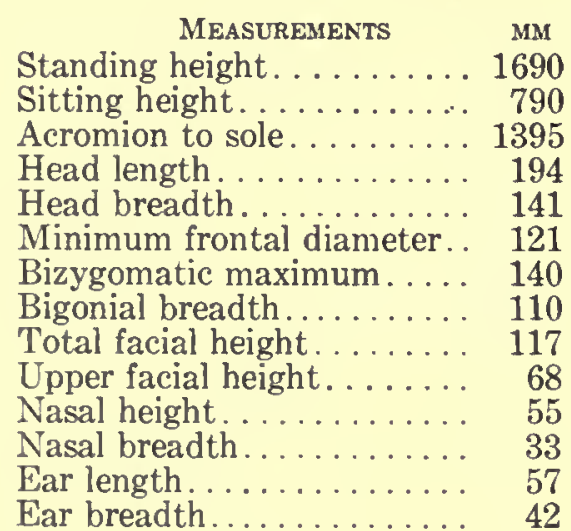

INDICES

Cephalic............ 72.7

Relative sitting height.... 46.7

Fronto-parietal......... 85.8

Total facial ........... 83.6

Upper facial . . . . . . . 48.6

Nasal ............... 60.0

Ear.................. 73.7

Jugomandibular . ........ 78.5

Jugofrontal. . . . . . . . . 86.5

HAIR.-Color: dark brown.

EYE.-Color: dark brown. Sclera: clear. Iris: homogeneous.

Nose.-Profile: straight. Wings: medium.

TEETH.-Lost: none.

CHEst.-Development: plus.

Musculature.-Good.

HEALTH.-Good. 
No. 527. Plate LVIII, Figs. 7, 8

Place: Hilla Army Camp. Date: June 14, 1928.

Birthplace: Hilla. Age: 18; unmarried.

Brothers: 2 living. Sisters: 1 living, 2 dead.

Tattoo marks: none.

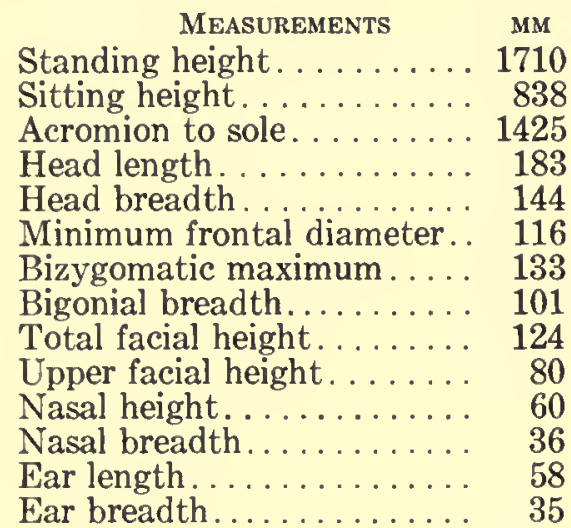

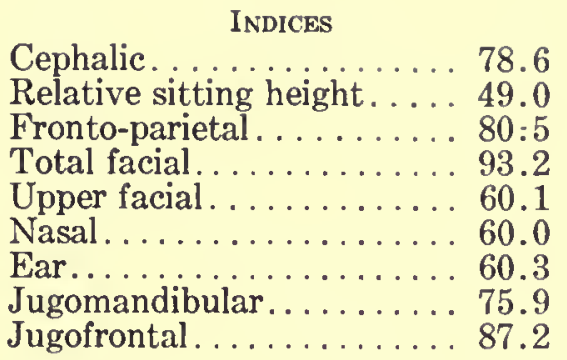

HAIR.-Color: dark brown.

EYE.-Color: blue brown. Sclera: clear. Iris: zoned.

Nose.-Profile: straight. Wings: medium.

TEETH._Lost: none.

Chest.-Development: plus.

Musculature.-Good.

HEALTH.-Good.

No. 528. Plate LIX, Figs. 1, 2

Place: Hilla Army Camp. Date: June 14, 1928.

Birthplace: Hilla. Married for 3 years. No children.

Brothers: 1 dead. Sisters: 1 living.

Tattoo marks: none.

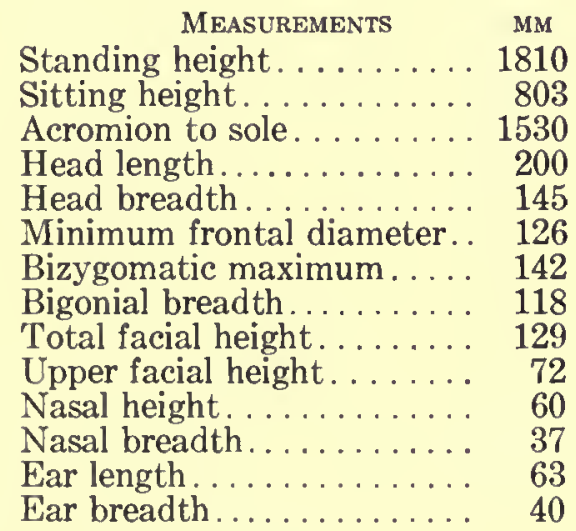

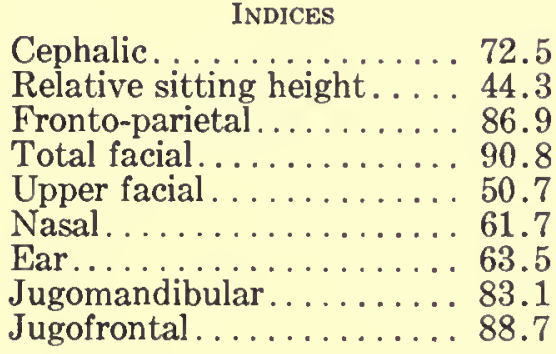

44.3

50.7

61.7

3.5

88.7

HAIR.-Color: dark brown.

Eye.-Color: dark brown. Sclera: clear. Iris: homogeneous.

NosE.-Profile: straight. Wings: medium-compressed.

TEETH.-Eruption: complete. Lost: none. Bite: slight over.

Chest.-Development: plus.

Musculature.-Good.

HEALTH.-Good. 
No. 529. Plate LiX, Figs. 3, 4

Place: Hilla Army Camp. Date: June 14, 1928.

Birthplace: Hilla. Age: 22; unmarried.

Brothers: 2 living. Sisters: 1 living, 1 dead.

Tattoo marks: none.

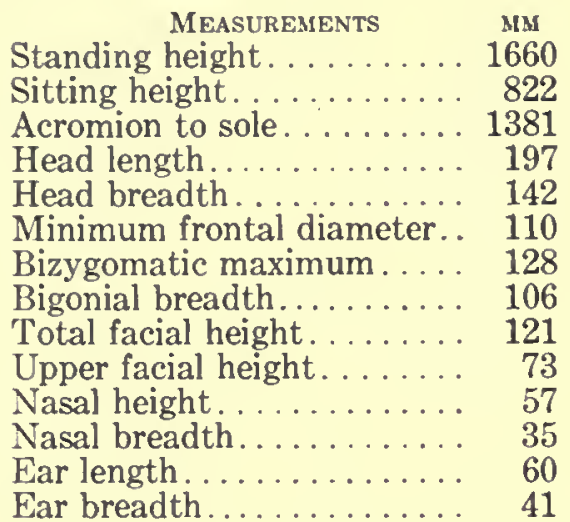

Cephalic............. 72.1

Relative sitting height.... 49.5

Fronto-parietal......... 77.4

Total facial............ 94.5

Upper facial. .......... 57.0

Nasal...............6.61.4

Ear..............6. 68.3

Jugomandibular. . . . . . . 82.8

Jugofrontal. . . . . . . . . 86.0

EyE.-Color: dark brown. Sclera: clear. Iris: homogeneous.

NosE.-Profile: straight. Wings: compressed.

TEETH.-Eruption: complete. Lost: 1. Bite: slight over.

Chest.-Development: average.

Musculature.-Fair.

HEALTH._Good.

No. 530. Plate LiX, Figs. 5, 6

Place: Hilla Army Camp. Date: June 14, 1928.

Birthplace: Hilla. Age: 18; unmarried.

Brothers: 1 living. Sisters: none.

Tattoo marks: spot on nasion.

Measurfments MM

Standing height. . . . . . . 1720

Sitting height........... 803

Acromion to sole......... 1451

Head length.............. 185

Head breadth .......... 136

Minimum frontal diameter. 113

Bizygomatic maximum.... 130

Bigonial breadth......... 113

Upper facial height. ...... 68

Nasal height. . . . . . . . . 57

Nasal breadth........... 38

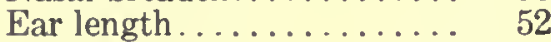

Ear breadth............ 37

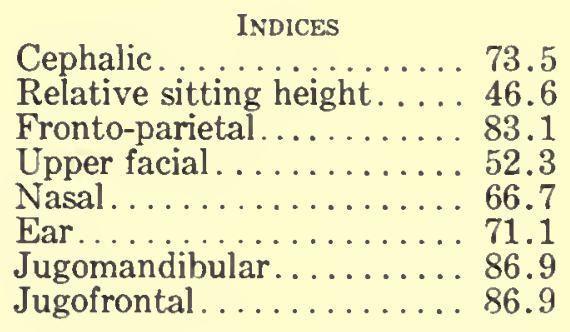

73.5

6

2.3

66.7

1.1

86.9

HAIR.-Color: dark brown.

EYE.-Color: dark brown. Sclera: clear. Iris: homogeneous.

Nose.-Profile: straight. Wings: medium.

TEETH.-Eruption: third molars unerupted. Lost: 1. Bite: slight over.

CHEST.-Development: average.

Musculature.-Fair.

HEALTH.-Good.

No. 531

Place: Hilla Army Camp. Date: June 14, 1928.

Birthplace: Hilla. Age: 25; married 14 years ago (wife now dead). Sons: 1 living. Daughters: none. 
Brothers: 2 living. Sisters: none.

Tattoo marks: glove on back of right hand.

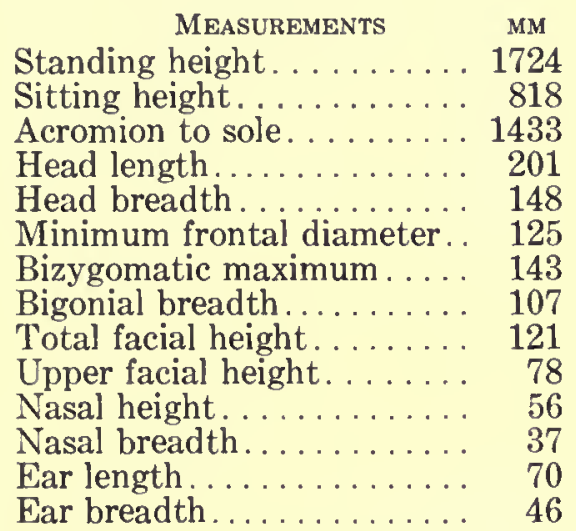

Cephalic............. 73.6

Relative sitting height. ... 47.4

Fronto-parietal.......... 83.4

Total facial............ 84.7

Upper facial. .......... 54.5

Nasal. ............66.1

Ear..................6. 65.7

Jugomandibular. . . . . . . 74.9

Jugofrontal . . . . . . . . . . 87.4

EyE.-Color: dark brown. Sclera: clear. Iris: homogeneous.

NosE.-Profile: straight. Wings: medium-flaring.

TEeTH.-Eruption: complete. Lost: none. Bite: slight over.

Chest.-Development: plus.

Musculature.-Good.

HEALTH.-Good.

\section{No. 532. Plate LiX, Figs. 7, 8}

Place: Hilla Army Camp. Date: June 14, 1928.

Birthplace: Hilla. Age: 19; unmarried.

Brothers: 2 living. Sisters: 3 living.

Tattoo marks: spot on nasion.

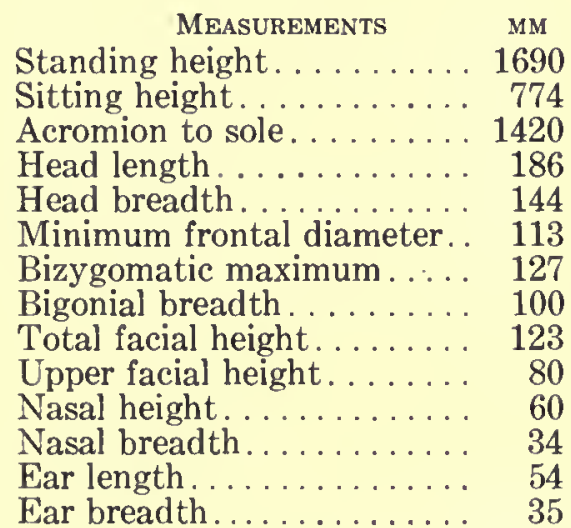

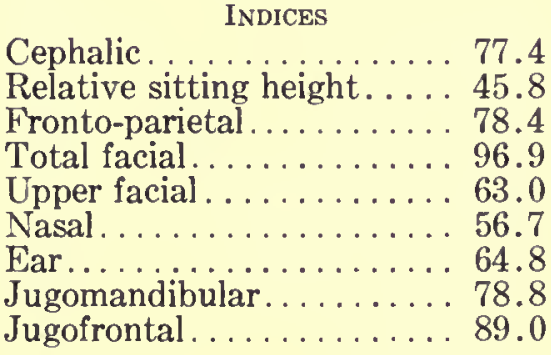

Cephalic............. 77.4

Relative sitting height. . . 45.8

Fronto-parietal ........... 78.4

Total facial. . . . . . . . . 96.9

Upper facial. .......... 63.0

Nasal. . . . . . . . . . . . 56.7

Jugomandibular ............. 78.8

Jugofrontal . . . . . . . . . . 89.0

HAIR.-Color: dark brown.

EYE.-Color: dark brown. Sclera: clear. Iris: homogeneous.

Nose.-Profile: convex. Wings: medium-compressed.

TEETH.-Eruption: third molars unerupted. Lost: none. Bite: slight over.

CHEsT.-Development: plus.

Musculature.-Good.

HEALTH.-Good.

No. 533. Plate LX, Figs. 1, 2

Place: Hilla Army Camp. Date: June 14, 1928.

Birthplace: Hilla. Age: 20; married in June, 1928.

Brothers: 5 living, 2 dead. Sisters: 1 dead.

Tattoo marks: spot on back of right wrist. 


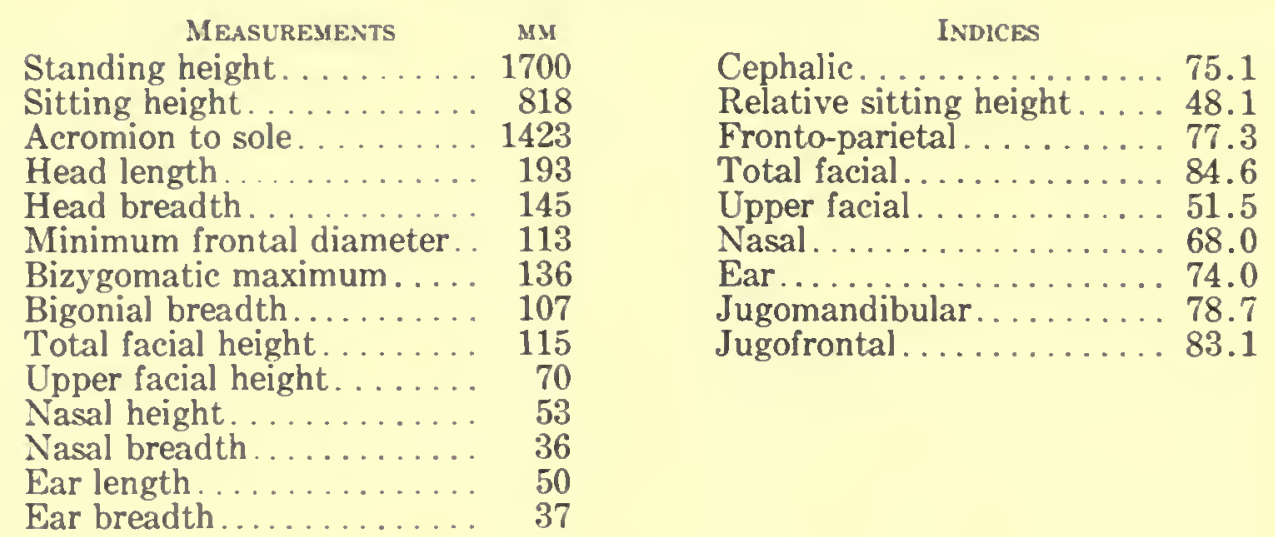

HAIR.-Color: dark brown.

EYE.-Color: dark brown. Sclera: clear. Iris: homogeneous.

NosE.-Profile: straight. Wings: medium.

TEETH.-Eruption: complete. Lost: none. Bite: slight over.

CHEST.-Development: plus.

Musculature.-Good.

HEALTH.-Good.

No. 534. Plate LX, Figs. 3, 4

Place: Hilla Army Camp. Date: June 14, 1928.

Birthplace: Hilla. Age: 22; unmarried.

Brothers: 2 living, 1 dead. Sisters: 1 living.

Tattoo marks: none.

\begin{tabular}{|c|c|c|}
\hline MEASUREMENTS & MM & INDICES \\
\hline Standing height. & 1666 & $\ldots \ldots \ldots 70.0$ \\
\hline Sitting height. & 832 & Relative sitting height.... 49.9 \\
\hline Acromion to sole. & 1410 & Fronto-parietal......... 78.6 \\
\hline Head length.... & 200 & Total facial. . . . . . . . 89.9 \\
\hline Head breadth. & 140 & Upper facial \\
\hline Minimum frontal diameter. & 110 & Nasal...... \\
\hline Bizygomatic maximum... & 128 & 64.5 \\
\hline Bigonial breadth......... & 121 & Jugomandibular. \\
\hline Total facial height. . . . . . . & 115 & Jugofrontal. . . . . . . . . 85.9 \\
\hline Upper facial height. . . . . . & 76 & \\
\hline Nasal height. & 56 & \\
\hline Nasal breadth & 37 & \\
\hline Ear length... & 62 & \\
\hline Ear breadth & 40 & \\
\hline
\end{tabular}

EYE.-Color: dark brown. Sclera: clear. Iris: homogeneous.

NosE.-Profile: straight. Wings: medium-compressed.

TeEth.-Eruption: complete. Lost: none. Bite: slight over.

Chest.-Development: plus.

Musculature.-Good.

HEALTH.-Good.

\section{No. 535. Plate LX, Figs. 5, 6}

Place: Hilla Army Camp. Date: June 14, 1928.

Birthplace: Hilla. Age: 18; married for 4 months.

Brothers: 3 living. Sisters: 1 living.

Tattoo marks: glove on right hand and gazelle on inside of right forearm.

MeasuremeNts MM

Standing height......... 1730

Sitting height............ 809
INDices

Cephalic............ 70.1

Relative sitting height. . . . 44.8 


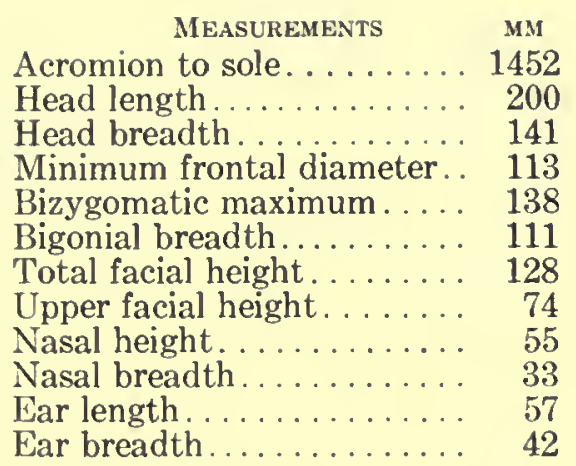

Fronto-parietal......... 80.1

Total facial. . . . . . . . . . 92.9

Upper facial. . ......... 53.6

Nasal. . . . . . . . . . . 60.0

Ear................ 73.7

Jugomandibular. . . . . . 8 80.5

Jugofrontal . . . . . . . . . . 81.9

HAIR.-Color: dark brown.

EYE.-Color: dark brown. Sclera: clear. Iris: homogeneous.

NosE.-Profile: straight. Wings: medium.

TEETH.-Eruption: third molars unerupted. Lost: none. Bite: slight over.

CHEsT,-Development: plus.

Musculature.-Good.

HEALTH.-Good.

No. 536. Plate LX, Figs. 7, 8

Place: Hilla Army Camp. Date: June 14, 1928.

Birthplace: Hilla. Age: 20; unmarried.

Brothers: 2 living, 1 dead. Sisters: 2 living.

Tattoo marks: none.

\begin{tabular}{|c|c|}
\hline MEASUREMENTS & MM \\
\hline Standing height. & 1734 \\
\hline Sitting height. & 818 \\
\hline Acromion to sole & 1440 \\
\hline Head length & 18 \\
\hline Head breadth. & 13 \\
\hline Minimum frontal diameter. . & 11 \\
\hline Bizygomatic maximum . .... & 13 \\
\hline Bigonial breadth . . . . . . . . . & $10^{\prime}$ \\
\hline Total facial height........ & 124 \\
\hline Upper facial height. . . . . . . & 72 \\
\hline Nasal height. & \\
\hline asal breadth & \\
\hline & \\
\hline & \\
\hline
\end{tabular}

INDICES

Cephalic.............74.1

Relative sitting height.....47.1

Fronto-parietal........ 82.5

Total facial . . . . . . . . . . 92.0

Upper facial . . . . . . . . . 57.0

Nasal............... 55.2

Ear................6. 64.3

Jugomandibular. . . . . . . . . 79.3

Jugofrontal . . . . . . . . . . 83.7

HAIR.-Color: dark brown.

EYE.-Color: blue brown. Sclera: clear. Iris: zoned.

Nose.-Profile: convex. Wings: medium.

TEETH.-Eruption: third molars unerupted. Lost: 1. Bite: slight over.

CHEST.-Development: average.

Musculature.-Fair.

HEALTH.-Good.

No. 537. Plate LXI, Figs. 1, 2

Place: Hilla Army Camp. Date: June 14, 1928.

Birthplace: Hilla. Age: 25; unmarried.

Brothers: 1 living, 1 dead. Sisters: 1 living.

Tattoo marks: none.

MEasurements MM

Standing height. . . . . . 1673

Sitting height .......... 791
INDICES

Cephalic.............67.4

Relative sitting height..... 47.2 


\begin{tabular}{|c|c|c|}
\hline MEASUREMENTS & MM & $\begin{array}{l}\text { INDICES } \\
\text { Fronto-narietal }\end{array}$ \\
\hline Acromion to sole.... . . & 1387 & Fronto-parietal..... \\
\hline Head length... & 193 & Total facial. \\
\hline Head breadth. & 130 & Upper facial \\
\hline Minimum frontal diameter. & 113 & Nasal. \\
\hline Bizygomatic maximum.... & 129 & Ear. \\
\hline Bigonial breadth. & 95 & Jugomandibular \\
\hline Total facial height. & 119 & Jugofrontal . . . \\
\hline Upper facial height. & 71 & \\
\hline $\begin{array}{l}\text { Nasal height. } \\
\text { Nasal breadth }\end{array}$ & 52 & \\
\hline $\begin{array}{l}\text { isal breadth } \\
\text { r length. }\end{array}$ & 35 & \\
\hline$r$ breadth ${ }_{r}^{r} \ldots \ldots \ldots$ & $\begin{array}{l}54 \\
36\end{array}$ & \\
\hline
\end{tabular}

EYE.-Color: very dark brown. Sclera: clear. Iris: homogeneous.

Nose.-Profile: straight. Wings: flaring.

TEETH.-Eruption: complete. Lost: none. Bite: slight over.

Chest.-Development: plus.

Musculature.-Good.

HEALTH.-Good.

Some Negro blood.

No. 538. Plate LXI, Figs. 3, 4

Place: Hilla Army Camp. Date: June 14, 1928.

Birthplace: Karbala. Age: 23; unmarried.

Brothers: 1 living, 1 dead. Sisters: 1 living.

Tattoo marks: glove on back of right hand.

\begin{tabular}{|c|c|c|}
\hline MEASUREMENTS & MM & INDICES \\
\hline Standing height. & 1686 & Cephalic. \\
\hline Sitting height. & 822 & Relative sitting height. \\
\hline Acromion to sole. & 1417 & Fronto-parietal....... \\
\hline Head length & 187 & Total facial. . \\
\hline Head breadth. & 141 & Upper facial \\
\hline Minimum frontal diameter. & 117 & Nasal. \\
\hline Bizygomatic maximum.... & 133 & Ear \\
\hline Bigonial breadth. & 108 & Jugomandibular \\
\hline tal facial height. & 122 & Jugofrontal. \\
\hline
\end{tabular}

Upper facial height....... 80

Nasal height. .......... 57

Nasal breadth............ 40

Ear length............. 62

Ear breadth ............... 35

Halk.-Color: dark brown.

EyE.-Color: green brown. Sclera: clear. Iris: zoned.

Nose.-Profile: straight.

TEETH.-Eruption: complete. Lost: none. Bite: slight over.

Chest.-Development: plus.

Musculature.-Good.

HEALTH.-Good.

No. 539. Plate LXI, Figs. 5, 6

Place: Hilla Army Camp. Date: June 14, 1928.

Birthplace: Hindiya. Age: 18; unmarried.

Brothers: 3 living. Sisters: 3 living.

Tattoo marks: glove on back of right hand.

\begin{tabular}{|c|c|c|}
\hline MEASUREMENTS & MM & INDICES \\
\hline $1 \mathrm{~g}$ height & $\begin{array}{r}1706 \\
781\end{array}$ & Cephalic. \\
\hline
\end{tabular}




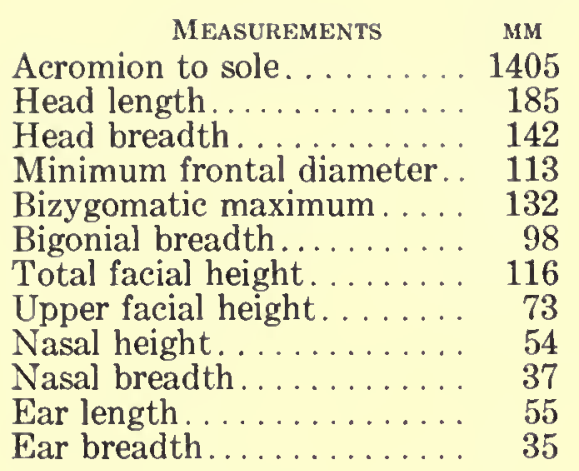

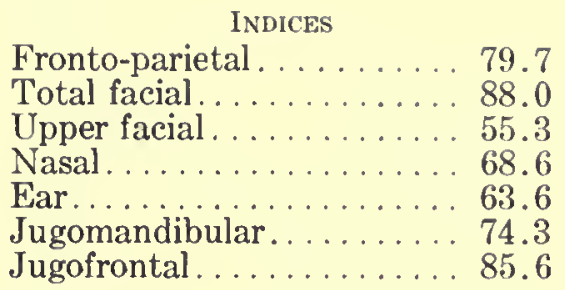

HAIR.-Color: dark brown.

EYE.-Color: dark brown. Sclera: clear. Iris: homogeneous.

Nose.-Profile: concave. Wings: flaring.

TEETH.-Eruption: third molars unerupted. Lost: none. Bite: slight over.

CHEst.-Development: average.

Musculature.-Fair.

HEALTH.-Good.

No. 540. Plate LXI, Figs. 7, 8

Place: Hilla Army Camp. Date: June 14, 1928.

Birthplace: Hindiya. Age: 25; unmarried.

Brothers: 3 living, 1 dead. Sisters: 2 living.

Tattoo marks: none.

\begin{tabular}{|c|c|}
\hline MEASUREMENTS & MM \\
\hline Standing height & 1711 \\
\hline Sitting height & 808 \\
\hline Acromion to sole & 1421 \\
\hline Head length & 191 \\
\hline Head breadth & 155 \\
\hline Minimum frontal diameter. & 120 \\
\hline Bizygomatic maximum . . . . & 136 \\
\hline Bigonial breadth. & 108 \\
\hline Total facial height. & 135 \\
\hline Upper facial height. & 78 \\
\hline Nasal height & 62 \\
\hline Nasal breadth & 36 \\
\hline Ear lengtl & 61 \\
\hline & 2 \\
\hline
\end{tabular}

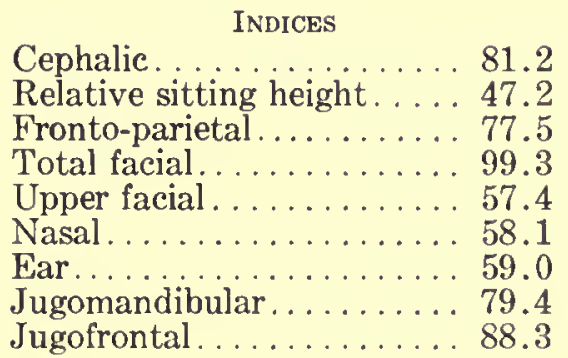

HAIR.-Color: dark brown.

EYE.-Color: dark brown. Sclera: clear. Iris: homogeneous.

Nose.-Profile: straight. Wings: medium.

TEeтH.-Eruption: complete. Lost: none. Bite: slight over.

Chest.-Development: plus.

Musculature.-Good.

HEALTH.-Good.

No. 541. Plate LXII, Figs. 1, 2

Place: Hilla Army Camp. Date: June 14, 1928.

Birthplace: Hindiya. Age: 22; unmarried.

Brothers: 2 living. Sisters: 3 living.

Tattoo marks: none.

Measurements MM

Standing height. . . . . 1690

Sitting height. .......... 853
INDICES

Cephalic............ 78.4

Relative sitting height.... 50.4 


\begin{tabular}{|c|c|c|}
\hline MeAsurements & MM & INDICES \\
\hline Acromion to sole. & 1445 & Fronto-parietal. \\
\hline Head length. & 189 & Total facial. \\
\hline Head breadth. & 148 & Upper facial \\
\hline Minimum frontal diameter. . & 113 & Nasal. \\
\hline Bizygomatic maximum.... & 132 & 63.4 \\
\hline Bigonial breadth. . & 103 & Jugomandibular. . . . \\
\hline Total facial height........ & 123 & Jugofrontal. . . . . . . . . 85.6 \\
\hline
\end{tabular}

HAIR.-Color: dark brown.

EYE.-Color: dark brown. Sclera: clear. Iris: homogeneous.

Nose.-Profile: straight. Wings: medium.

TEETH.-Eruption: complete. Lost: none. Bite: slight over.

CHEST.-Development: plus.

Musculature.-Good.

HEALTH.-Good.

No. 542. Plate LXII, Figs. 3,4

Place: Hilla Army Camp. Date: June 14, 1928.

Birthplace: Amara. Age: 23; unmarried.

Brothers: 1 living. Sisters: 2 living.

Tattoo marks: glove on right hand and gazelle on inside of right forearm.

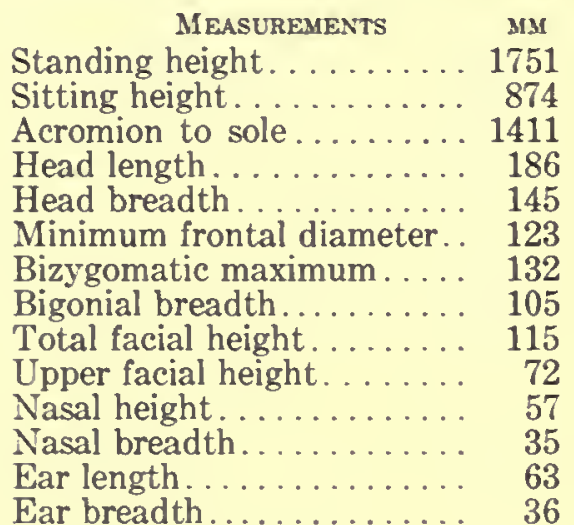

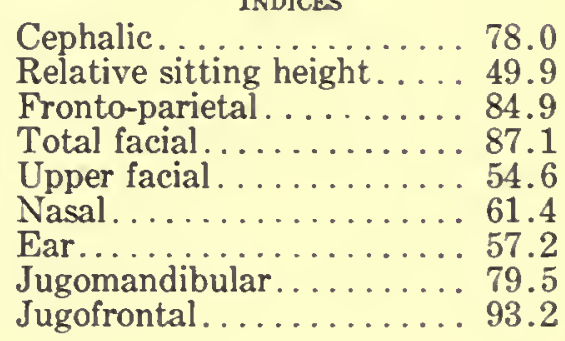

HAIR.-Color: dark brown.

EYE.-Color: dark brown. Sclera: clear. Iris: homogeneous.

NosE.-Profile: straight. Wings: medium.

TEETH.-Eruption: complete. Lost: none. Bite: slight over.

Chest.-Development: plus.

Musculature.-Good.

HEALTH.-Good.

No. 543. Plate LXII, Figs. 5, 6

Place: Hilla Army Camp. Date: June 14, 1928.

Birthplace: Amara. Age: 20; unmarried.

Brothers: 3 living. Sisters: 2 living.

Tattoo marks: none.

Measurements Mi

Standing height. ........ 1660

Sitting height.......... 824
INDICES

Cephalic.............. 74.4

Relative sitting height. . . . 49.6 


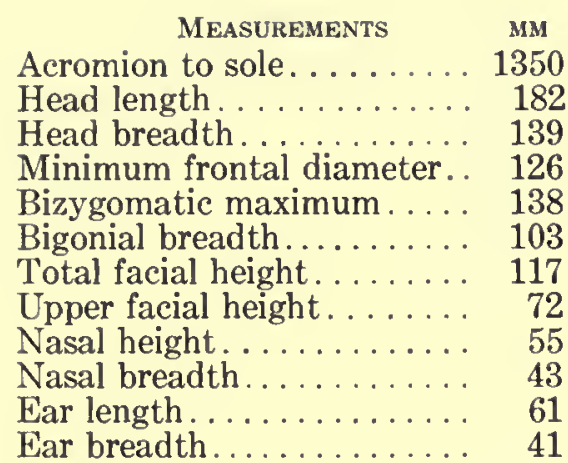

INDICES

Fronto-parietal. ....... 90.7

Total facial............ 84.9

Upper facial. . . . . . . 52.1

Nasal. .............. 78.2

Ear..............6. 67.2

Jugomandibular. . . . . . 74.6

Jugofrontal . . . . . . . 92.8

HaIR.-Color: dark brown.

EyE.-Color: dark brown. Sclera: clear. Iris: homogeneous.

Nose.-Profile: concave. Tip: thickness, double plus. Wings: flaring.

TEETH.-Eruption: third molars unerupted. Lost: none. Bite: slight over.

ChEST.-Development: plus.

Musculature.-Good.

HEALTH.-Good.

No. 544. Plate LXiI, Figs. 7, 8

Place: Hilla Army Camp. Date: June 14, 1928.

Birthplace: Amara. Age: 25; unmarried.

Brothers: 1 living. Sisters: 1 living.

Tattoo marks: glove on back of right hand and star on right temple.

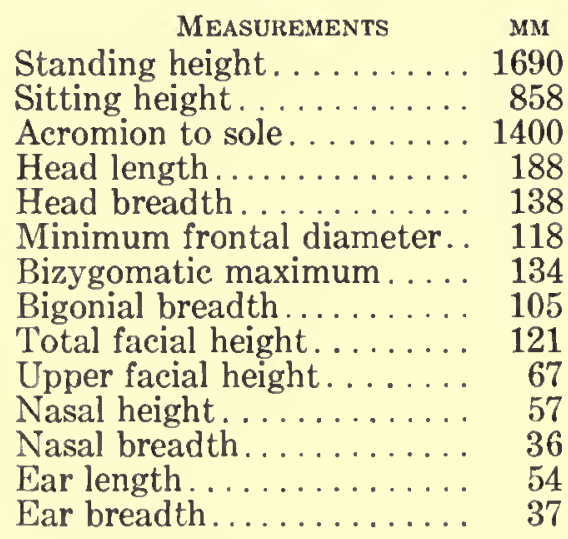

Cephalic.............. 73.4

Relative sitting height. . . 50.7

Fronto-parietal ........ 85.6

Total facial. ........... 90.3

Upper facial. .......... 50.0

Nasal. . . . . . . . . 63.2

Ear...............6. 68.5

Jugomandibular........ 78.3

Jugofrontal. .......... 86.6

HAIR.-Color: dark brown.

EYE.-Color: dark brown. Sclera: bloodshot. Iris: homogeneous.

Nose.-Profile: straight. Wings: medium-flaring.

TEETH.-Eruption: complete. Lost: none. Bite: slight over.

Chest.-Development: plus.

Musculature.-Good.

Health.-Good.

\section{No. 545. Piate LXIII, Figs. 1, 2}

Place: Hilla Army Camp. Date: June 14, 1928.

Birthplace: Amara. Age: 25; married for 6 years. No children.

Brothers: 1 living, 1 dead. Sisters: 2 living.

Tattoo marks: 2 spots on left temple.

MEASUREMENTS MM

Standing height. . . . . . 1764

Sitting height......... 909
INDICES

Cephalic............ 79.6

Relative sitting height.... 51.5 


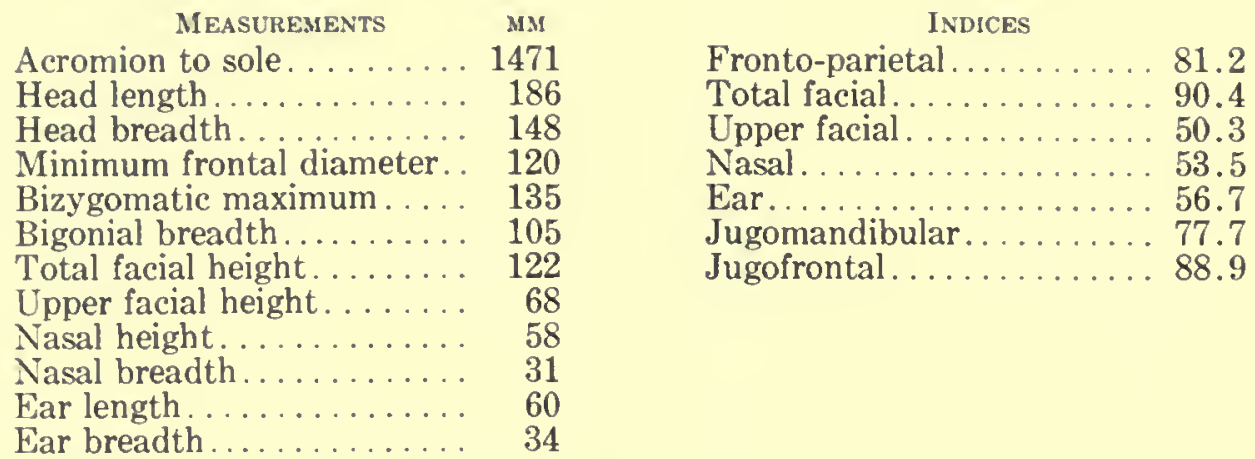

EYE.-Color: dark brown. Sclera: clear. Iris: homogeneous.

Nose.-Profile: convex. Wings: medium.

TEETH.-Eruption: complete. Lost: none. Bite: slight over.

CHEST.-Development: plus.

Musculature.-Good.

Health.-Good.

No. 546. Plate LXIII, Figs. 3, 4

Place: Hilla Army Camp. Date: June 14, 1928.

Birthplace: Nasiriya. Age: 24; unmarried.

Brothers: 2 living. Sisters: 8 living.

Tattoo marks: 4 spots on back of right hand; glove on back of left hand.

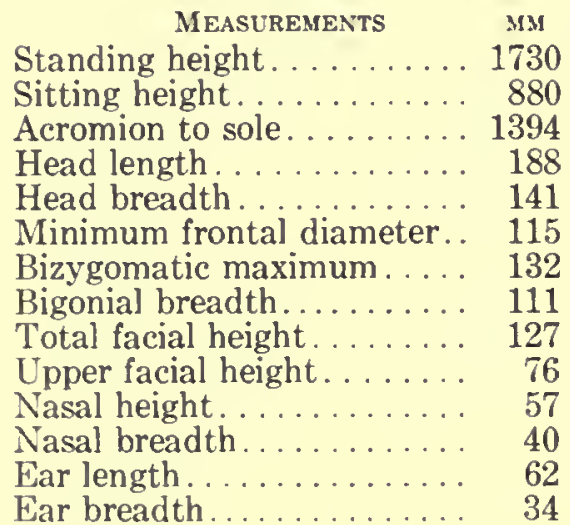

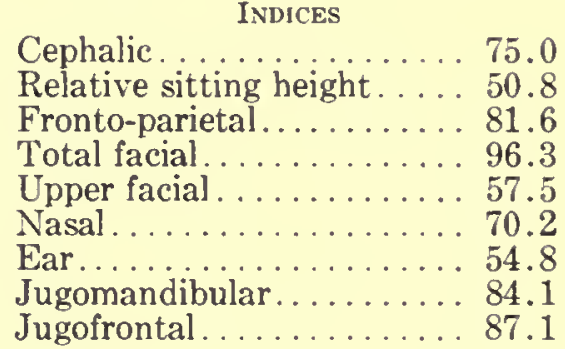

Hall. Color: dark brown.

EYE.-Color: dark brown. Sclera: clear. Iris: homogeneous.

Nose.-Profile: straight. Wings: flaring.

TeETH.-Eruption: complete. Lost: none. Bite: slight over.

CHEST.-Development: plus.

Musculature.-Good.

HEALTh.--Good.

No. 547. Plate LXIII, Figs. 5, 6

Place: Hilla Army Camp. Date: June 14, 1928.

Birthplace: Ad Diwaniya. Age: 20 ; unmarried.

Brothers: 2 living. Sisters: 1 living.

Tattoo marks: glove on back of right hand and spot on inside of right forearm.

\begin{tabular}{|c|c|c|}
\hline MEasurements & MM & INDICES \\
\hline Standing height. . . . . & 1643 & Cephalic... \\
\hline Sitting height. . & 835 & Relative sitting height. \\
\hline Acromion to sole. & 1355 & Fronto-parietal . \\
\hline
\end{tabular}




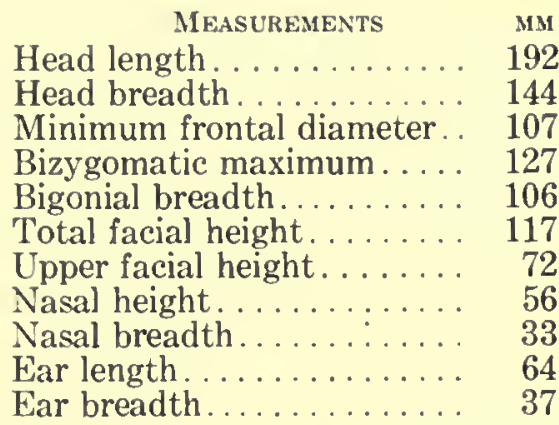

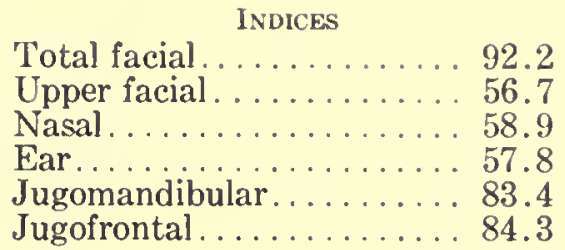

EYE.-Color: dark brown. Sclera: clear. Iris: homogeneous.

NosE.-Profile: convex. Wings: medium-compressed.

TEETH.-Eruption: third molars unerupted. Lost: none. Bite: slight over.

CHEST.-Development: plus.

Musculature.-Good.

HEALTH.-Good.

\section{No. 548. Plate LXIII, Figs. 7, 8}

Place: Hilla Army Camp. Date: June 14, 1928.

Birthplace: Ad Diwaniya. Age: 30; unmarried.

Brothers: 3 living. Sisters: 5 living.

Tattoo marks: spot and lines on right hand; spots on left hand.

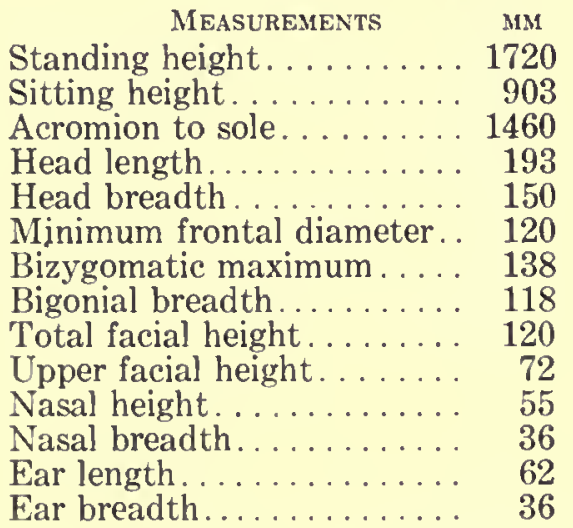

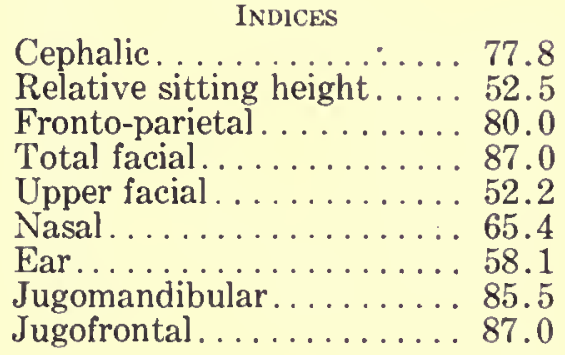

Halk.-Color: dark brown.

EYE. - Color: green brown. Sclera: clear. Iris: homogeneous.

Nose.-Profile: convex. Wings: medium.

TEETH.-Eruption: complete. Lost: 1. Bite: slight over.

CHEST.-Development: plus.

Musculature.-Good.

HEALTH.-Good.

No. 549. Plate LXIV, Figs. 1, 2

Place: Hilla Army Camp. Date: June 14, 1928.

Birthplace: Amara. Age: 25; unmarried.

Brothers: 1 living. Sisters: 1 living.

Tattoo marks: spot on left hand behind thumb.

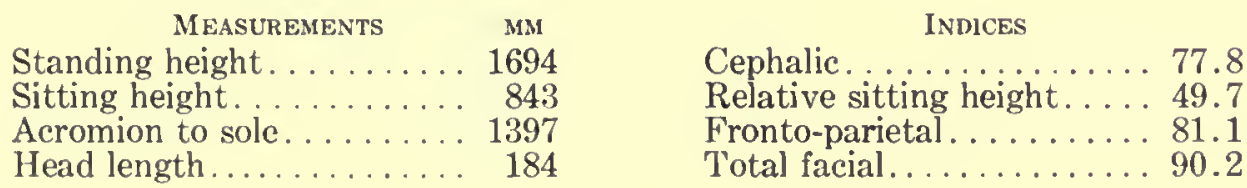




\begin{tabular}{|c|c|}
\hline \multicolumn{2}{|l|}{ MEASUREMENTS } \\
\hline breadth & \\
\hline xximum..... & \\
\hline & \\
\hline $\begin{array}{l}\text { Total facial height. } \\
\text { Upper facial height. }\end{array}$ & \\
\hline $\begin{array}{l}\text { er facial height. } \\
\text { al height...... }\end{array}$ & \\
\hline & \\
\hline 10 & \\
\hline & \\
\hline
\end{tabular}

INDICES

Upper facial.......... 57.1

Nasal. ............. 51.7

Ear............... 59.6

Jugomandibular. . . . . . 81.0

Jugofrontal. ......... 81.7

EyE.-Color: dark brown. Sclera: clear. Iris: homogeneous.

Nose.-Profile: straight. Wings: medium-flaring.

TEETH.-Eruption: complete. Lost: none. Bite: slight over.

CHEST.-Derelopment: plus.

Musculature.-Good.

HEALTH.-Good.

No. 550. Plate LXIV, Figs. 3,4

Place: Hilla Army Camp. Date: June 14, 1928.

Birthplace: Amara. Age: 20; unmarried.

Brothers: 1 living. Sisters: 3 living.

Tattoo marks: spot on back of right wrist; 1 spot on inside of right wrist.

\begin{tabular}{|c|c|c|}
\hline MEASUREMENTS & $3 \mathrm{M}$ & INDICES \\
\hline Standing height. . & 1758 & Cephalic. \\
\hline Sitting height. & 875 & Relative sitting height. \\
\hline Acromion to sole. & 1470 & Fronto-parietal....... \\
\hline Head length.... & 183 & Total facial....... \\
\hline Head breadth. . & 145 & Upper facial \\
\hline Minimum frontal diameter. . & 115 & Nasal. \\
\hline Bizygomatic maximum. . . . . & 133 & Ear. \\
\hline igonial breadth. & 106 & Jugomandibular \\
\hline otal facial height. & 113 & Jugofrontal. \\
\hline
\end{tabular}

(n)

Upper facial height....... 70

Nasal height............ 52

Nasal breadth............ 35

Ear length.............. 56

Ear breadth............. 32

EyE.-Color: dark brown. Sclera: clear. Iris: homogeneous.

Nose.-Profile: convex. Wings: medium.

TEETH.-Eruption: complete. Lost: 1. Bite: slight over.

CHEST.-Derelopment: plus.

Musculature.-Good.

HEALTH.-Good.

\section{No. 551. Plate LXIV, Figs. 5, 6}

Place: Hilla Army Camp. Date: June 14, 1928.

Birthplace: Amara. Age: 20; unmarried.

Brothers: 2 living. Sisters: 1 living.

Tattoo marks: none.

\begin{tabular}{|c|c|}
\hline Measurements & MM \\
\hline Standing height. & 1721 \\
\hline Sitting height. & 838 \\
\hline Acromion to sole & 1436 \\
\hline Head length. & 185 \\
\hline Head breadth. . & 14 \\
\hline Minimum fronta & \\
\hline
\end{tabular}

INDICES

Cephalic............ 76.3

Relative sitting height.... 48.6

Fronto-parietal......... 80.2

Total facial............. 89.7

Upper facial. ........... 53.3

Nasal. ................... 56.2 


\begin{tabular}{|c|c|}
\hline MEASUREMENTS & MM \\
\hline Bizygomatic maximum. & 135 \\
\hline Bigonial breadth. & 110 \\
\hline Total facial height.... & 121 \\
\hline Upper facial height. . . . . . . & 72 \\
\hline Nasal height. . . . . . . . . . & 57 \\
\hline Nasal breadth. & 32 \\
\hline Ear length & 55 \\
\hline$a r$ breadth & \\
\hline
\end{tabular}

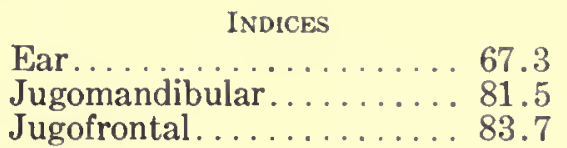

Jugomandibular........ 81.5

Jugofrontal. ........... 83.7

HAIR.-Color: dark brown.

EYE.-Color: dark brown. Sclera: bloodshot.

Nose.-Profile: straight. Wings: medium.

TeETH.-Eruption: complete. Lost: none. Bite: slight over.

Chest.-Development: plus.

Musculature.-Good.

HeAlth.-Good.

No. 552. Plate LXIV, Figs. 7, 8

Place: Hilla Army Camp. Date: June 14; 1928.

Birthplace: Basra. Race: Sudanese Negro. Age: 28; unmarried.

Brothers: 2 living. Sisters: 1 living, 1 dead.

Tattoo marks: snake and other designs on inside of right forearm.

\begin{tabular}{|c|c|c|}
\hline MEASUREMENTS & мM & INDICES \\
\hline Standing height. & 1812 & Cephalic \\
\hline Sitting height. & 850 & Relative sitting height. \\
\hline Acromion to sole & 1525 & Fronto-parietal... \\
\hline Head length.... & 186 & Total facial... \\
\hline Head breadth & 140 & Upper facial \\
\hline Minimum frontal diameter & 117 & Nasal. \\
\hline Bizygomatic maximum. . . & 144 & 57.4 \\
\hline Bigonial breadth...... & 116 & Jugomandibular. \\
\hline Total facial height. & 123 & Jugofrontal... \\
\hline Upper facial height. & 76 & \\
\hline Nasal height. & 61 & . \\
\hline Nasal breadth. & 41 & \\
\hline Ear length. & 61 & \\
\hline Ear breadth. & 35 & \\
\hline
\end{tabular}

HaIR.-Form: woolly. Texture: coarse. Color: black.

EYE.-Color: dark brown. Sclera: clear. Iris: homogeneous.

Nose.-Profile: concave. Wings: flaring plus.

TEETH.-Eruption: complete. Lost: one. Bite: marked over.

CHEST.-Development: double plus.

Musculature.-Excellent.

HEALTH.-Good.

Negro; therefore not included in statistics.

No. 553. Plate LXV, Figs. 1, 2

Place: Hilla Army Camp. Date: June 14, 1928.

Birthplace: Nasiriya. Age: 21 ; unmarried.

Brothers: 3 living. Sisters: 3 living.

Tattoo marks: cross on inside of right forearm.

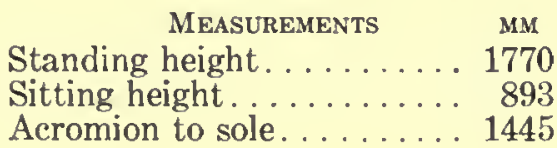

Cephalic............ 74.9

Relative sitting height.... 50.4

Fronto-parietal..........99.8 


\begin{tabular}{|c|c|}
\hline Measurements & ins \\
\hline Head length. & 18 \\
\hline Head breadth. & \\
\hline Minimum frontal diameter. & \\
\hline Bizygomatic maximum..... & \\
\hline Bigonial breadth.. & \\
\hline Total facial height. & \\
\hline Upper facial height. & \\
\hline Nasal $\mathrm{h}$ & \\
\hline Nasal breadth & \\
\hline Ear length & \\
\hline Ear breadth & \\
\hline
\end{tabular}

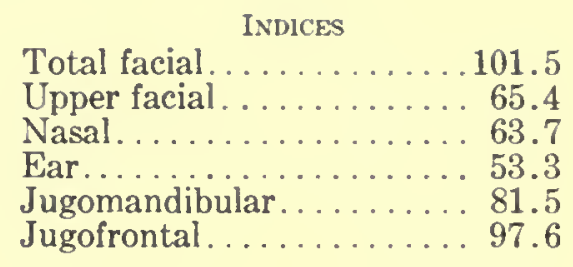

EYE.-Color: blue brown. Sclera: clear. Iris: zoned.

NosE.-Profile: concave. Wings: medium-flaring.

TEeTH.-Eruption: complete. Lost: none. Bite: slight over.

Chest.-Derelopment: plus.

Musculature.-Good.

HEALTh.-Good.

\section{No. 554. Plate LXV, Figs. 3, 4}

Place: Hilla Army Camp. Date: June 14, 1928.

Birthplace: Nasiriya. Age: 18; unmarried.

Brothers: 1 living. Sisters: 3 living.

Tattoo marks: glove on back of right hand and line on inside of right forearm.

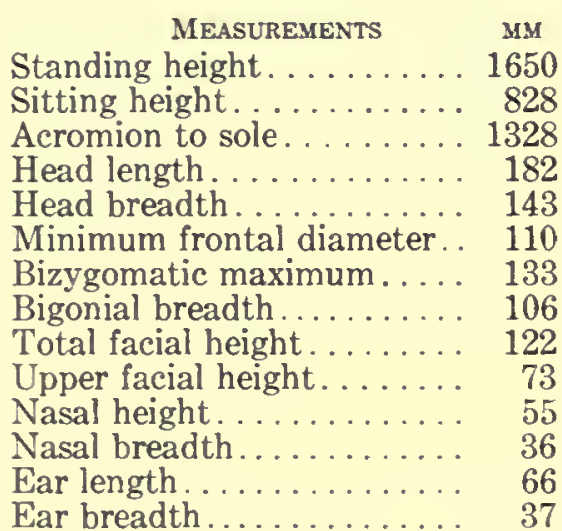

HAIR.-Color: dark brown.

EYE.-Color: dark brown. Sclera: clear. Iris: homogeneous.

Nose.-Profile: straight. Wings: medium.

TEETH.-Eruption: third molars unerupted. Lost: none. Bite: slight over.

CHEST.-Development: plus.

Musculature.-Good.

HEALTH.-Good.

No. 555. Plate LXV, Figs. 5, 6

Place: Hilla Army Camp. Date: June 14, 1928.

Birthplace: Karbala. Age: 20 ; married for 3 years. No children.

Brothers: 1 living. Sisters: 2 living.

Tattoo marks: none.

\begin{tabular}{|c|c|}
\hline MEASUREMENTS & мM \\
\hline Standing height. & 1670 \\
\hline Sitting height. & 853 \\
\hline mion to sc & 1335 \\
\hline
\end{tabular}

INDICES

Cephalic............ 80.1

Relative sitting height.... 51.0

Fronto-parietal.......... 80.6

Total facial........... 83.5 


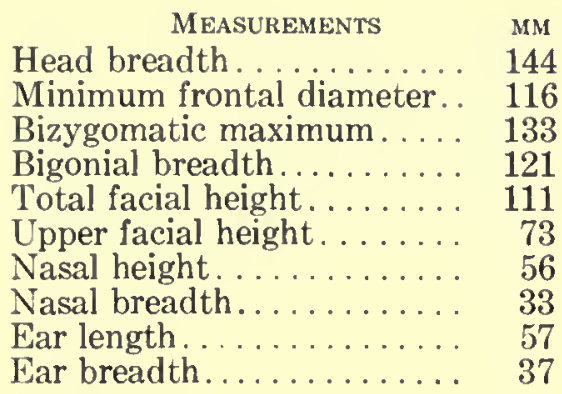

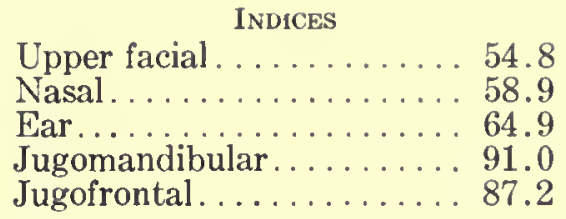

HAIR.-Quantity: head, plus.

EYE.-Color: green brown. Sclera: clear. Iris: zoned.

Nose.-Profile: convex. Tip: thickness, double plus. Wings: compressed.

TEETH.-Eruption: complete. Lost: none. Bite: slight over. Caries: double plus.

CHEST,-Development: plus.

Musculature.-Good.

HEALTH.-Good.

\section{No. 556. Plate LXV, Figs. 7, 8}

Place: Hilla Army Camp. Date: June 14, 1928.

Birthplace: Nasiriya. Age: 20; married in 1928.

Brothers: 3 living. Sisters: 1 living.

Tattoo marks: glove on back of right hand.

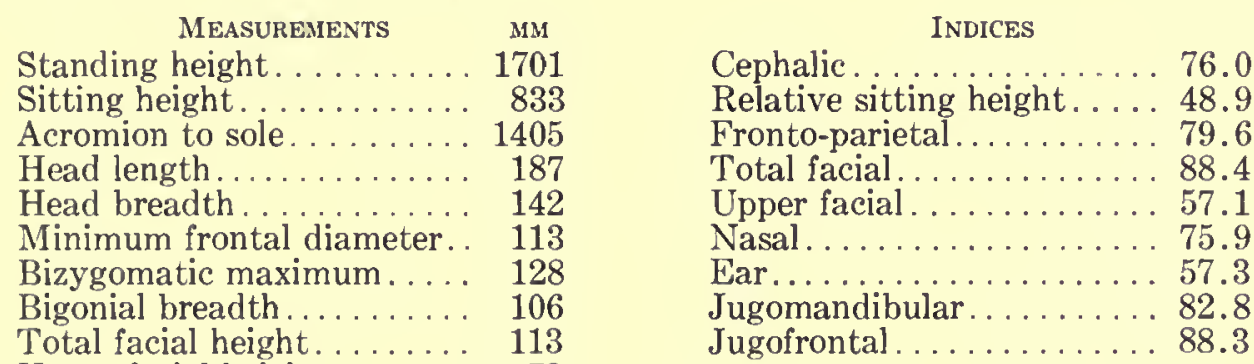

Lper facial height . ............

Nasal height. . . . . . . . . 54

Nasal breadth........... 41

Ear length............ 56

Ear breadth............ 32

EYk.--Color: dark brown. Sclera: clear. Iris: homogeneous.

Nose.-Profile: convex. Wings: flaring.

TEeTH.-Eruption: complete. Lost: none. Bite: slight over.

Chest.-Development: plus.

Musculature.-Good.

HEALTH.-Good.

No. 557. Plate LXVI, Figs. 1, 2

Place: Hilla Army Camp. Date: June 14, 1928.

Birthplace: Amara. Age: 25; married in 1928.

Brothers: 3 living. Sisters: 1 living.

Tattoo marks: glove on back of right hand, design in three colors; figures 7663 on inside of left forearm.

\begin{tabular}{lr} 
Measurements & \multicolumn{1}{c}{ MM } \\
Standing height. . . . . . . & 1736 \\
Sitting height. . . . . . . . & 898 \\
Acromion to sole . . . . . . & 1441
\end{tabular}

INDices

Relative sitting height. . . 51.7

Fronto-parietal.......... 81.9 


\begin{tabular}{|c|c|}
\hline Measurements & MM \\
\hline Head length. & 190 \\
\hline Head breadth. & \\
\hline Minimum frontal diameter. . & \\
\hline Bizygomatic maximum..... & 1. \\
\hline Bigonial breadth. & \\
\hline Total facial height. & 121 \\
\hline Upper facial height. . & \\
\hline Nasal height. ..... & \\
\hline Ear length.... & \\
\hline Ear bread & \\
\hline
\end{tabular}

EYE.-Color: dark brown. Sclera: clear. Iris: homogeneous.

NosE.-Profile: convex. Wings: medium.

TЕетн.-Eruption: complete. Lost: 1. Bite: slight over.

Chest.-Derelopment: plus.

Musculature.-Good.

HEALTH.-Good.

No. 558. Plate LXVI, Figs. 3, 4

Place: Hilla Army Camp. Date: June 14, 1928.

Birthplace: Nasiriya. Age: 22; married for 18 months. No children.

Brothers: none. Sisters: none.

Tattoo marks: none.

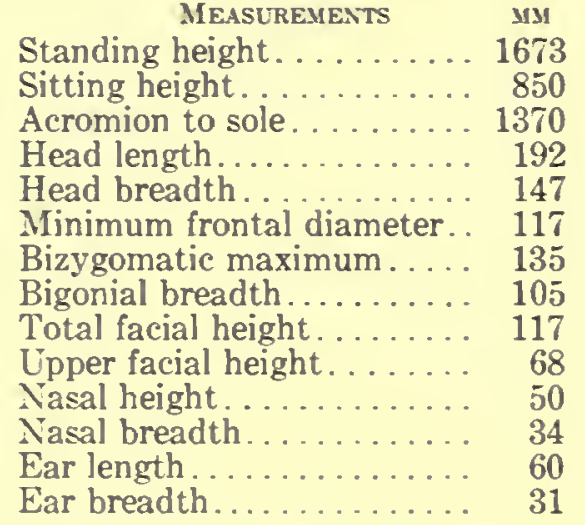

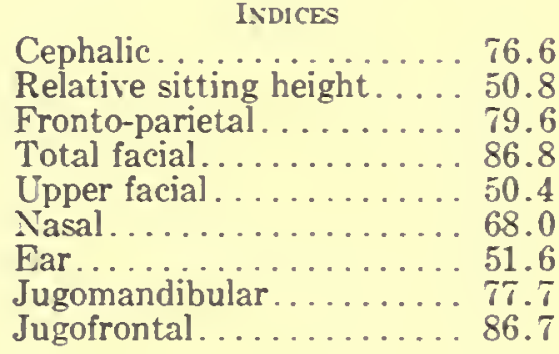

HAlR.-Color: dark brown.

EYE.-Color: dark brown. Sclera: clear. Iris: homogeneous.

Tose.-Profile: convex. Wings: medium-compressed.

TEETH.-Eruption: complete. Lost: none. Bite: slight over.

Chest.-Derelopment: plus.

Musculature.-Good.

HEALTH.-Good.

No. 559. Plate LXVI, Figs. 5, 6

Place: Hilla Army Camp. Date: June 14, 1928.

Birthplace: Nasiriya. Age: 20; unmarried.

Brothers: none. Sisters: 1 living.

Tattoo marks: line on back of left wrist.

MEasurements M

Standing height. . . . . . . 1673

Sitting height.......... 840

Acromion to sole........... 1410
INDICES

90.2

55.2

62.2

56.9

79.1

87.3 


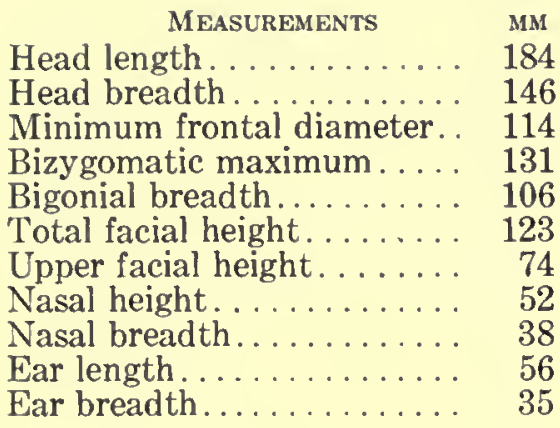

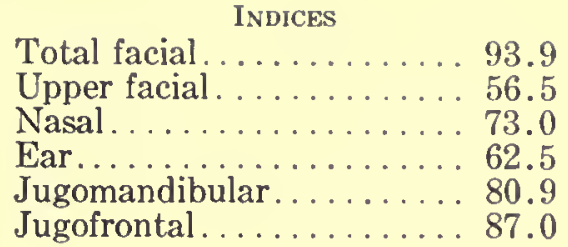

HAIR.-Color: dark brown.

EyE.-Color: dark brown. Sclera: clear. Iris: homogeneous.

NosE.-Profile: straight. Wings: medium.

TEETH.-Eruption: complete. Lost: 3. Bite: slight over.

Chest.-Development: plus.

Musculature.-Good.

HEALTH.-Good.

\section{No. 560. Plate LXVI, Figs. 7, 8}

Place: Hilla Army Camp. Date: June 14, 1928.

Birthplace: Amara. Age: 20; unmarried.

Brothers: 3 living. Sisters: 3 living.

Tattoo marks: slight line on back of left wrist.

\begin{tabular}{|c|c|c|}
\hline MEASUREMENTS & MM & INDICES \\
\hline Standing height. & 1662 & Cephalic . . . . . . . \\
\hline Sitting height. . & 801 & Relative sitting height. \\
\hline Acromion to sole. & 1393 & Fronto-parietal ....... \\
\hline Head length. & 188 & Total facial.... \\
\hline Head breadth. & 143 & Upper facial \\
\hline Minimum frontal diameter. & 114 & Nasal. ..... \\
\hline Bizygomatic maximum . . . . & 136 & Ear... \\
\hline Bigonial breadth.......... & 108 & Jugomandibular. \\
\hline Total facial height. & 117 & Jugofrontal. \\
\hline
\end{tabular}

Upper facial height ......... 78

Nasal height........... 54

Nasal breadth........... 33

Ear length.............. 61

Ear breadth........... 35

Cephalic.............. 76.1

Celative sitting height. 48.2

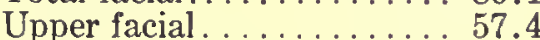

Nasal. ................6. 61.2

Jugomandibular......... 79.4

Jugofrontal. . . . . . . . . 83.8

HaIR.-Color: dark brown.

EYE.-Color: green brown. Sclera: clear. Iris: homogeneous.

Nose.-Profile: straight. Wings: medium-compressed.

TEетн.-Eruption: complete. Lost: none. Bite: slight over.

Chest.-Development: plus.

Musculature.-Good.

Health.-Good.

No. 561. Plate LXVII, Figs. 1, 2

Place: Hilla Army Camp. Date: June 14, 1928.

Birthplace: Hilla. Age: 25; unmarried.

Brothers: none. Sisters: none.

Tattoo marks: glove on back of right hand and gazelle on inside of right forearm.

MEASURements MM

Standing height. ........ 1671

INDICES

Sitting height. . . . . . . . 823

Cephalic.............. 75.0

Relative sitting height...... 49.3 


\begin{tabular}{|c|c|c|}
\hline Measurements & MM & INDICES \\
\hline Acromion to sole. & 1390 & Fronto-parietal..... \\
\hline Head length. & 188 & Total facial.... \\
\hline Head breadth. & 141 & Upper facial \\
\hline Minimum frontal diameter. & 108 & Nasal.... \\
\hline gomatic maximum . . . . & 127 & Ear... \\
\hline Bigonial breadth....... & 94 & Jugomandibular. . . . . . \\
\hline otal facial height. . . . . . & 116 & Jugofrontal. . . . . . . . 85.0 \\
\hline
\end{tabular}

HAIR. - Color: dark brown.

EYE.-Color: dark brown. Sclera: clear. Iris: homogeneous.

Nose.-Profile: straight. Wings: medium.

TEетн.-Eruption: complete. Lost: 2. Caries: plus. Bite: slight over.

CHEST.-Development: plus.

Musculature.-Good.

HEALTH.-Good.

No. 562. Plate LXVII, Figs. 3, 4

Place: Hilla Army Camp. Date: June 14, 1928.

Birthplace: Nasiriya. Age: 20; unmarried.

Brothers: 3 living. Sisters: 1 dead.

Tattoo marks: none.

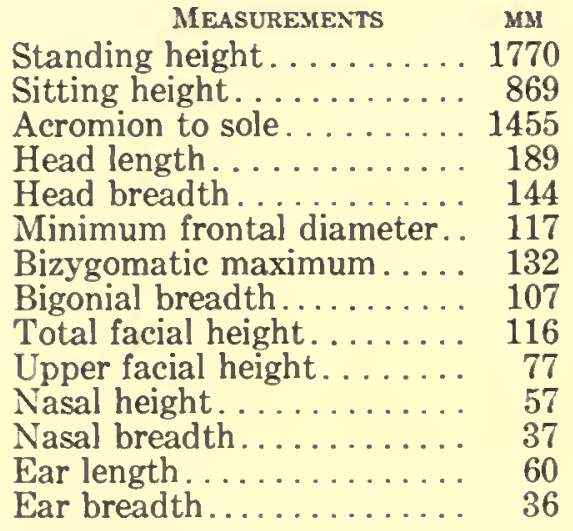

Cephalic............ 76.3

Relative sitting height.... 49.0

Fronto-parietal......... 81.3

Total facial........... 87.9

Upper facial. . . . . . . . . 58.3

Nasal..............64.9

Ear................ 60.0

Jugomandibular......... 81.1

Jugofrontal. . . . . . . 88.6

EYE.-Color: dark brown. Sclera: clear. Iris: homogeneous.

Nose.-Profile: straight. Wings: medium-flaring.

TEETH.-Eruption: complete. Lost: none. Bite: slight over.

Chest.-Development: plus.

Musculature.-Good.

HEALTH.-Good.

No. 563. Plate LXVII, Figs. 5, 6

Place: Hilla Army Camp. Date: June 14, 1928.

Birthplace: Nasiriya. Age: 20; unmarried.

Brothers: none. Sisters: none.

Tattoo marks: none.

Measurements MM

Standing height. . . . ... 1730

Sitting height. . . . . . . . 919
INDICES

Cephalic..............82.5

Relative sitting height. ... 53.1 


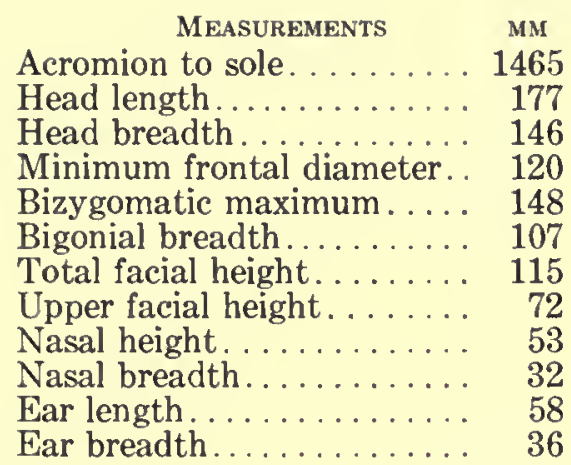

Fronto-parietal......... 82.2

Total facial. ................ 77.7

Upper facial. . . . . . . . . 48.6

Nasal. . . . . . . . . . . 60.4

Ear................ 62.1

Jugomandibular. . . . . . . . 72.3

Jugofrontal . . . . . . . . . 81.1

EYE.-Color: green brown. Sclera: clear. Iris: zoned.

Nose.-Profile: straight. Wings: medium.

TEETH.-Eruption: complete. Lost: none. Bite: slight over.

Chest.-Development: plus.

Musculature.-Good.

HEALTH.-Good.

No. 564. Plate LXVII, Figs. 7, 8

Place: Hilla Army Camp. Date: June 14, 1928.

Birthplace: Hindiya. Age: 22; unmarried.

Brothers: 2 dead. Sisters: 1 living.

Tattoo marks: girl in red and blue on back of right forearm.

\begin{tabular}{|c|c|c|}
\hline MEASUREMENTS & MM & INDICES \\
\hline Standing height. & 1764 & Cephalic. \\
\hline Sitting height. . & 908 & Relative sitting height. \\
\hline Acromion to sole. & 1452 & Fronto-parietal........ \\
\hline Head length..... & 189 & Total facial... \\
\hline Head breadth. . & 146 & Upper facial. \\
\hline Minimum frontal diameter. & 125 & Nasal..... \\
\hline Bizygomatic maximum . . . . & 143 & Ear. \\
\hline Bigonial breadth......... & 114 & Jugomandibular... \\
\hline Total facial height. ....... & 115 & Jugofrontal. . . . . . . . . . 87. \\
\hline Upper facial height. . . . & 70 & \\
\hline Nasal height. & 63 & \\
\hline Nasal breadth. & 40 & \\
\hline Ear length. & 56 & \\
\hline Ear breadth. & 35 & \\
\hline
\end{tabular}

EYE.-Color: dark brown. Sclera: clear. Iris: homogeneous.

NosE.-Profile: straight. Wings: flaring.

TEETH.-Eruption: complete. Lost: none. Caries: plus. Bite: slight over.

CHEST.-Development: plus.

Musculature.-Good.

HEALTH.-Good.

No. 565. Plate LXVIII, Figs. 1, 2

Place: Hilla Army Camp. Date: June 14, 1928.

Birthplace: Nasiriya. Age: 22; married 4 years ago (wife now dead). No children. Brothers: 2 living, 2 dead. Sisters: 1 living.

Tattoo marks: none.

$\begin{array}{lr}\text { Measurements } & \text { Mm } \\ \text { Standing height. . . . . . . } & 1685 \\ \text { Sitting height. . . . . . . . } & 838 \\ \text { Acromion to sole . . . . . . . . } & 1360 \\ \text { Head length . . . . . . } & 168\end{array}$

INDICES

Cephalic............ 86.9

Relative sitting height . . . 49.6

Fronto-parietal......... 71.2

Total facial............. 96.2 


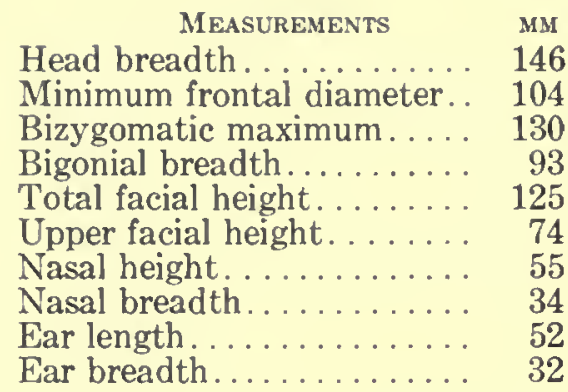

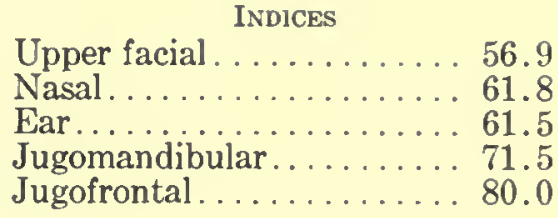

EYE.-Color: dark brown. Sclera: clear. Iris: homogeneous.

Nose.-Profile: convex.

TеЕтн.-Eruption: complete. Lost: none. Caries: plus. Bite: slight over.

Chest.-Development: plus.

Musculature.-Good.

HEALTH.-Good.

\section{No. 566. Plate LXVIII, Figs. 3, 4}

Place: Hilla Army Camp. Date: June 14, 1928.

Birthplace: Karbala. Age: 26 ; married for 5 years. Sons: 1 dead. Daughters: none. Brothers: 2 living. Sisters: 1 living.

Tattoo marks: glove on back of right hand and gazelle on inside of right forearm.

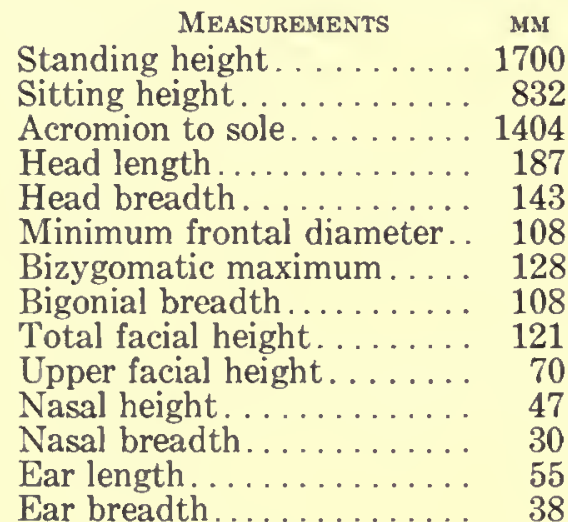

Cephalic..............76.5

Relative sitting height.... 48.9

Fronto-parietal......... 75.5

Total facial............. 94.5

Upper facial........... 54.7

Nasal. . . . . . . . . . . . . 6 63.8

Ear...............6. 69.1

Jugomandibular. . . . . . . . 84.4

Jugofrontal. .......... 84.4

HAIR.-Color: dark brown.

EYE.-Color: green brown. Sclera: clear. Iris: homogeneous.

Nose.-Profile: straight. Wings: medium.

TEETH.-Eruption: complete. Lost: 1. Bite: slight over.

Chest.-Development: plus.

Musculature.-Good.

HEALTH.-Good.

No. 567. Plate LXVIII, Figs. 5, 6

Place: Hilla Army Camp. Date: June 14, 1928.

Birthplace: Nasiriya. Age: 23; unmarried.

Brothers: 2 living. Sisters: 2 living.

Tattoo marks: none.

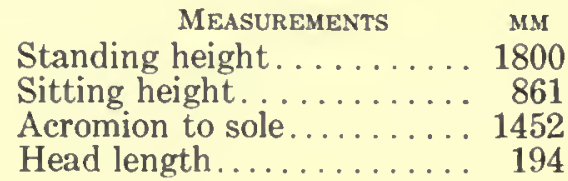

INDICES
Cephalic............. 72.6

Relative sitting height..... 47.8

Fronto-parietal ....... 83.0

Total facial.............. 103.6 


\begin{tabular}{|c|c|}
\hline MEASUREMENTS & MM \\
\hline Head breadth. & 141 \\
\hline Minimum frontal diameter. & 117 \\
\hline Bizygomatic maximum.... & 136 \\
\hline Bigonial breadth......... & 113 \\
\hline Total facial height. . & 141 \\
\hline Upper facial height... & 81 \\
\hline Nasal height......... & 62 \\
\hline Nasal breadth & 36 \\
\hline Ear length. & 63 \\
\hline Ear breadth & \\
\hline
\end{tabular}

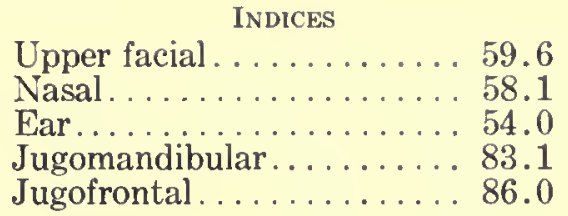

EYE.-Color: dark brown. Sclera: clear. Iris: homogeneous.

Nose.-Profile: straight. Wings: medium-flaring.

TEетн.-Eruption: complete. Lost: none. Bite: slight over.

Chest.-Development: plus.

Musculature.-Good.

Health.-Good.

\section{No. 568. Plate LXVIII, Figs. 7, 8}

Place: Hilla Army Camp. Date: June 14, 1928.

Birthplace: Nasiriya. Age: 17; unmarried.

Brothers: none. Sisters: 1 living.

Tattoo marks: glove on back of right hand, gazelle on inside of right forearm, and spot on nasal tip.

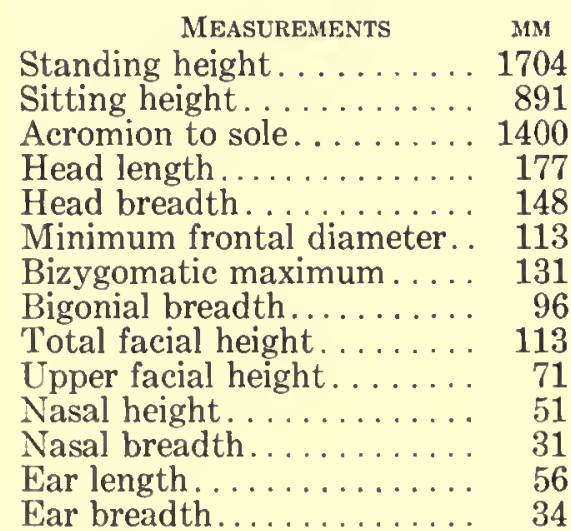

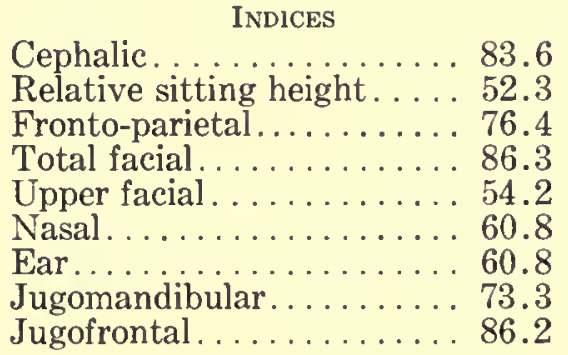

EYE.-Color: dark brown. Sclera: clear. Iris: homogeneous.

Nose.-Profile: convex. Wings: medium-compressed.

TEETH.-Eruption: third molars unerupted. Lost: none. Bite: slight over.

CHEST.-Development: plus.

Musculature.-Good.

HEALTH.-Good.

\section{No. 569. Plate LXIX, Figs. 1, 2}

Place: Hilla Army Camp. Date: June 14, 1928.

Birthplace: Nasiriya. Age: 17; unmarried.

Brothers: none. Sisters: none.

Tattoo marks: glove on back of right hand and snake on inside of right forearm.

\begin{tabular}{|c|c|c|}
\hline MEASUREMENTS & MM & INDICES \\
\hline ding height. . . . . . & 1740 & Cephalic. \\
\hline ng height. & 868 & Relative sitting height. \\
\hline Acromion to sole. & 1432 & Fronto-parietal....... \\
\hline Head length. & 187 & Total facial. \\
\hline
\end{tabular}




\begin{tabular}{|c|c|c|}
\hline MEASUREMENTS & MM & INDICES \\
\hline Head breadth. & 141 & Upper facial \\
\hline Linimum frontal diameter. & 111 & Nasal.... \\
\hline gomatic maximum. & 128 & Ear. \\
\hline nial breadth. & 103 & Jugomandibular. . \\
\hline froigl hoigh & 124 & Jugofrontal . \\
\hline
\end{tabular}

Upper facial height...... 81

Nasal height. . . . . . . . 62

Nasal breadth.......... 32

Ear length............ 60

Ear breadth........... 41

HAIR.-Color: dark brown.

EYE.-Color: dark brown. Sclera: clear. Iris: homogeneous.

NosE.-Profile: straight. Wings: medium.

TEETH.-Eruption: third molars unerupted. Lost: none. Bite: slight over.

Chest.-Development: plus.

Musculature.-Good.

HEALTH.--Good.

No. 570. Plate LXIX, Figs. 3, 4

Place: Hilla Army Camp. Date: June 14, 1928.

Birthplace: Nasiriya. Age: 20; unmarried.

Brothers: 2 living. Sisters: none.

Tattoo marks: glove on back of right hand and gazelle on inside of right forearm.

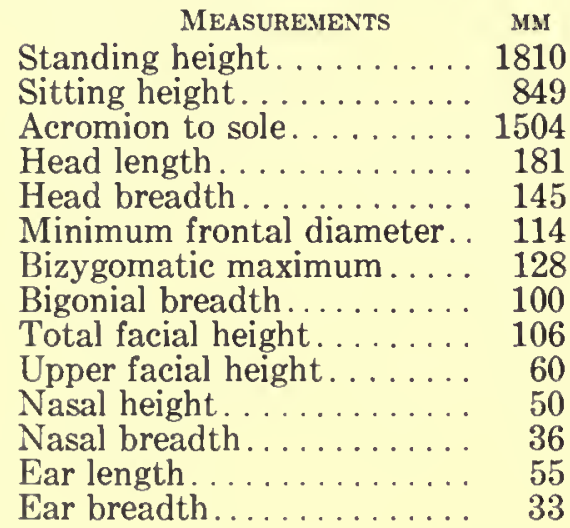

EYE.-Color: dark brown. Sclera: clear. Iris: homogeneous.

Nose.-Profile: straight. Wings: medium.

TeEтh.-Eruption: complete. Lost: none. Bite: slight over.

CHEsT.-Development: plus.

Musculature.-Good.

HEALTH. - Good.

Cephalic.............880.1

Relative sitting height. . . 46.9

Fronto-parietal . . . . . . 78.6

Total facial. . . . . . . 82.9

Upper facial. . . . . . . . 46.8

Nasal. . . . . . . . . . . . 72.0

Ear...............6 60.0

Jugomandibular. . . . . . . . 78.1

Jugofrontal . . . . . . . . 8 89.1

\section{No. 571. Plate LXIX, Figs. 5, 6}

Place: Hilla Army Camp. Date: June 14, 1928.

Birthplace: Ad Diwaniya. Age: 30 ; unmarried.

Brothers: 2 living. Sisters: none.

Tattoo marks: glove, small bird, spots and lines on back of left hand.

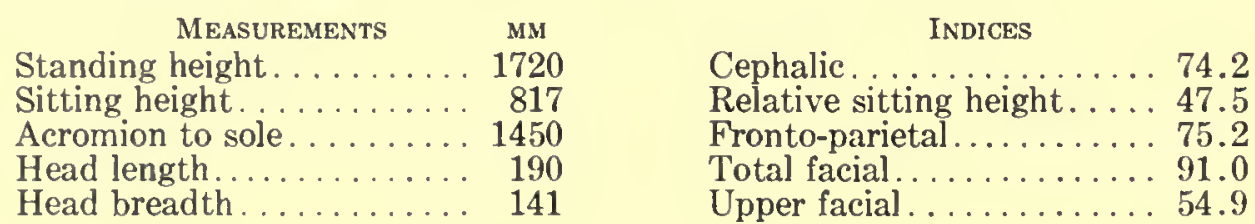




\begin{tabular}{|c|c|c|}
\hline MEASUREMENTS & MM & INDICES \\
\hline Minimum frontal diameter. & 106 & Nasal \\
\hline omatic maximum..... & 133 & Ear. \\
\hline Bigonial breadth. & 108 & Jugomandibular \\
\hline Total facial height & 121 & Jugofrontal \\
\hline
\end{tabular}

Total facial height........ 121

Upper facial height....... 73

Nasal height. .................... 53

Nasal breadth........... 30

Ear length............ 57

Ear breadth............. 34

Exe.-Color: dark brown. Sclera: clear. Iris: homogeneous.

Nose.-Profile: convex. Wings: medium.

Tenth.-Eruption: complete. Lost: none. Caries: plus. Bite: slight over.

Chest.-Development: plus.

Musculature.-Good.

HeaLTh.-Good.

No. 572. Plate LXIX, Fig. 7

Place: Hilla Army Camp. Date: June 14, 1928.

Birthplace: Nasiriya. Age: 21; unmarried.

Brothers: none. Sisters: none.

Tattoo marks: none.

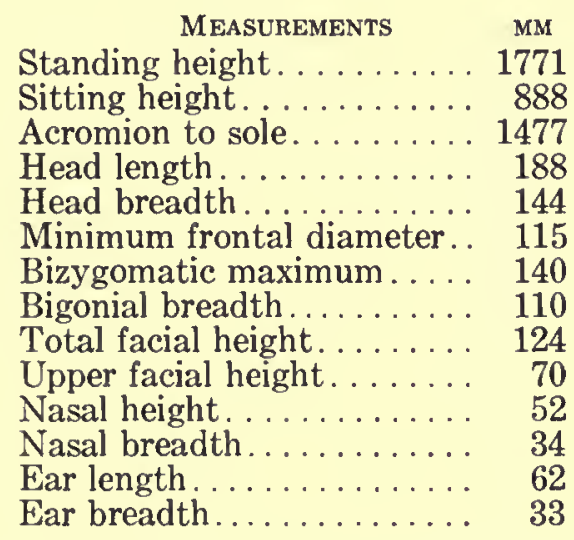

Cephalic. ............. 76.6

Relative sitting height. ... 50.1

Fronto-parietal........... 79.9

Total facial. . . . . . . . . 88.6

Upper facial ........... 50.0

Nasal . . . . . . . . . . . . 65.4

Ear................... 53.3

Jugomandibular. . . . . . 78.5

Jugofrontal . . . . . . . . . . . 82.1

Eye.-Color: dark brown. Sclera: clear. Iris: homogeneous.

NosE.-Profile: concave. Wings: flaring.

TEETH.-Eruption: complete. Lost: none. Bite: slight over.

CHEsT.-Development: plus.

Musculature.-Good.

HEALTH.-Good.

No. 573. Plate LXX, Figs. 1, 2

Place: Hilla Army Camp. Date: June 14, 1928.

Birthplace: Nasiriya. Age: 30; married twice, 2 and 5 years ago. Sons: 1, 0, living. Daughters: 1,1 , living.

Brothers: 1 living, 3 dead. Sisters: 2 dead.

Tattoo marks: 3 spots on back of left hand.

\begin{tabular}{|c|c|}
\hline MEASUREMENTS & MM \\
\hline Standing height. & 1790 \\
\hline Sitting height. & 882 \\
\hline Acromion to sole & 1511 \\
\hline Head length. & 197 \\
\hline Head breadth. & 140 \\
\hline Minimum frontal diamete & 110 \\
\hline
\end{tabular}

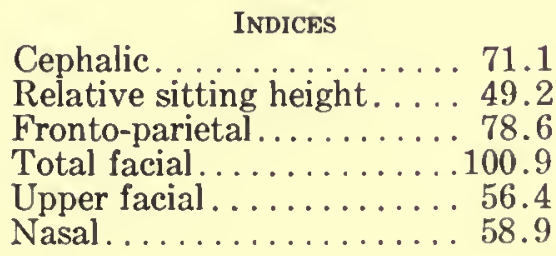




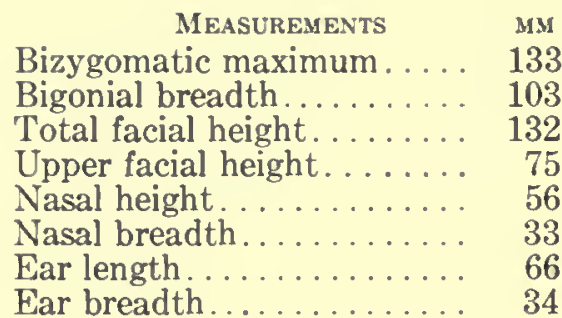

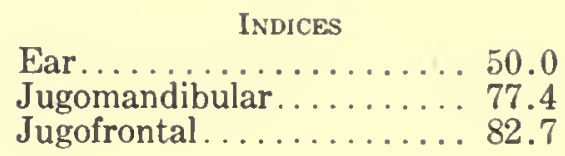

Jugomandibular......... 77.4

HAIR.-Color: dark brown.

EYE.-Color: dark brown. Sclera: clear. Iris: homogeneous.

NOSE.-Profile: straight. Wings: medium.

TEETH.-Eruption: complete. Lost: 1. Bite: slight over.

CHest.-Development: plus.

Musculature.-Good.

HEALTH.-Good.

No. 574. Plate LXX, Figs. 3, 4

Place: Hilla Army Camp. Date: June 14, 1928.

Birthplace: Nasiriya. Age: 25; unmarried.

Brothers: 3 living. Sisters: 3 living.

Tattoo marks: glove on back of right hand.

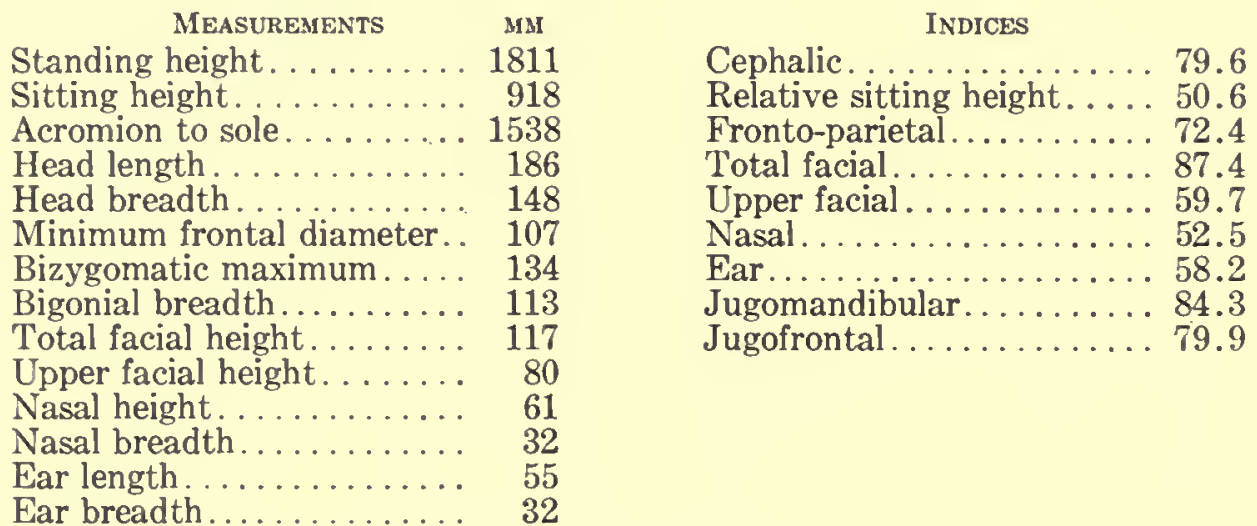

EYE.-Color: dark brown. Sclera: clear. Iris: homogeneous.

NosE.-Profile: straight. Wings: compressed.

TEETH.-Eruption: complete. Lost: none. Bite: slight over.

Chest.-Development: plus.

MUSCULATURE.-Good.

HeAlTH.-Good.

No. 575. Plate LXX, Figs. 5, 6

Place: Hilla Army Camp. Date: June 14, 1928.

Birthplace: Amara. Age: 25; married for 2 years. No children.

Brothers: 1 living. Sisters: none.

Tattoo marks: thick line on back of right wrist; large spot and line on inside of right forearm.

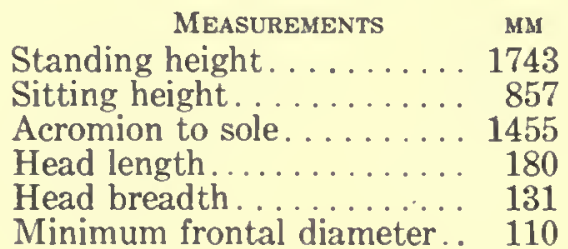

Cephalic.............. 72.8

Relative sitting height. . . 49.2

Fronto-parietal........ 84.0

Total facial . . . . . . . . . 93.0

Upper facial. . . . . . . . . 58.3

Minimum frontal diameter.. 110

Nasal. ............... 58.4 


\begin{tabular}{|c|c|c|}
\hline MEASUREMENTS & MM & INDICES \\
\hline Bizygomatic maximum.... & 127 & $\ldots \ldots$ \\
\hline Bigonial breadth.......... & 101 & Jugomandibular. . . . . \\
\hline Total facial height.... . & 118 & Jugofrontal..... \\
\hline Upper facial height. . . . . . . & 74 & \\
\hline Nasal height. . ........... & 60 & \\
\hline Nasal breadth. & 35 & \\
\hline Ear length.... & 66 & \\
\hline Ear breadth... & 41 & \\
\hline
\end{tabular}

HAIR.-Color: dark brown.

EYE.-Color: dark brown. Sclera: clear. Iris: homogeneous.

Nose.-Profile: straight. Tip: thickness, double plus. Wings: medium-flaring.

TEETH.--Eruption: complete. Lost: none. Bite: slight over.

CHEsT.-Development: plus.

Musculature.-Good.

HEALTH.-Good.

\section{No. 576. Plate LXX, Figs. 7, 8}

Place: Hilla Army Camp. Date: June 14, 1928.

Birthplace: Amara. Age: 25; unmarried.

Brothers: 1 living. Sisters: 2 living.

Tattoo marks: none.

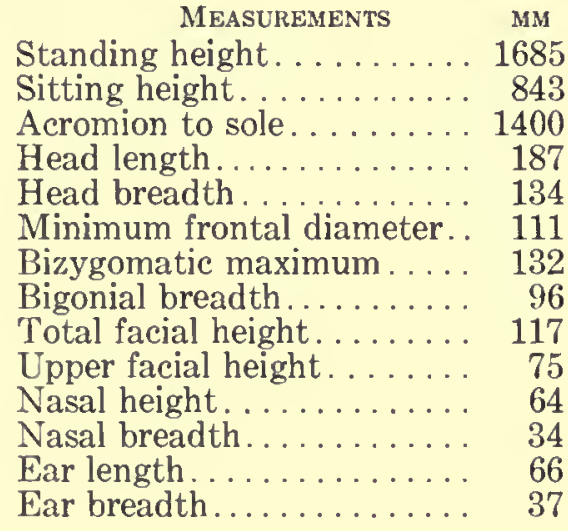

INDICES

Cephalic. ........... 71.6

Relative sitting height. . . . 50.0

Fronto-parietal. ......... 82.9

Total facial. . . . . . . . . . 88.6

Upper facial . . . . . . . . . . . . . 56.8

Nasal. . . . . . . . . . . . . 53.1

Ear...................... 56.1

Jugomandibular......... 72.7

Jugofrontal . . . . . . . . . . . 8 84.1

HAIR.-Color: dark brown.

EYE.-Color: dark brown. Sclera: clear. Iris: homogeneous.

Nose.-Profile: straight. Wings: medium-flaring. Tip: thickness, minus.

TEETH.-Eruption: complete. Lost: none. Bite: slight over.

Chest.-Development: plus.

Musculature.-Good.

HEALTH.-Good.

\section{No. 577. Plate LXXI, Figs. 1, 2}

Place: Hilla Army Camp. Date: June 14, 1928.

Birthplace: Amara. Age: 22; unmarried.

Brothers: none. Sisters: 2 living.

Tattoo marks: none.

\begin{tabular}{|c|c|}
\hline MEASUREMENTS & MM \\
\hline Standing height. & 1784 \\
\hline Sitting height. & 778 \\
\hline mion to sole & 149 \\
\hline length.. & \\
\hline 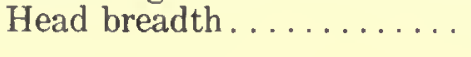 & \\
\hline
\end{tabular}

INDICES

Cephalic............7 71.8

Relative sitting height .... 43.6

Fronto-parietal........ 81.5

Total facial............. 94.7

Upper facial . . . . . . . . . . 60.6 


\begin{tabular}{|c|c|c|}
\hline MEASUREMENTS & MM & INDICES \\
\hline Minimum frontal diameter. . & 110 & Nasal. \\
\hline Bizygomatic maximum.... & 132 & Ear... \\
\hline Bigonial breadth......... & 100 & Jugomandibular. \\
\hline Total facial height......... & 125 & Jugofrontal . . . . . . . \\
\hline Upper facial height. . . . . . . & 80 & \\
\hline Nasal height. . . . . . . . . . . & 57 & \\
\hline Nasal breadth............ & 32 & \\
\hline ar length $\ldots \ldots \ldots \ldots \ldots$ & 60 & \\
\hline ar breadth............. & 41 & \\
\hline
\end{tabular}

HAIR.-Color: dark brown.

EYE.-Color: dark brown. Sclera: clear. Iris: homogeneous.

Nose.-Profile: straight. Wings: flaring.

TEETH.-Eruption: complete. Lost: none. Bite: slight over.

CHEsT.-Derelopment: plus.

Musculature.-Good.

HEALTH.-Good.

No. 578. Plate LXXI, Figs. 3, 4

Place: Hilla Army Camp. Date: June 14, 1928.

Birthplace: An Najaf. Age: 25; unmarried.

Brothers: 2 living. Sisters: none.

Tattoo marks: none.

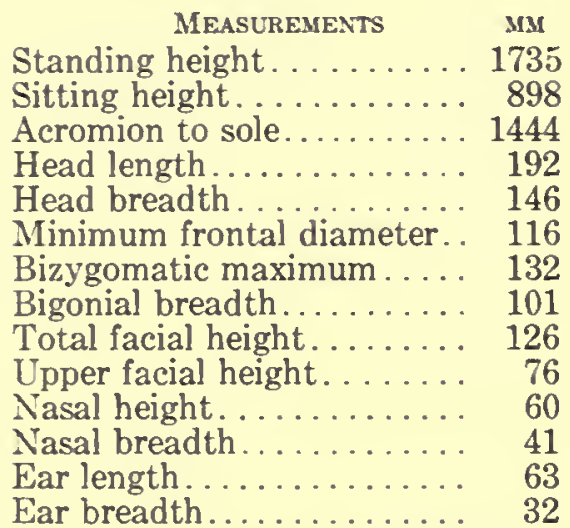

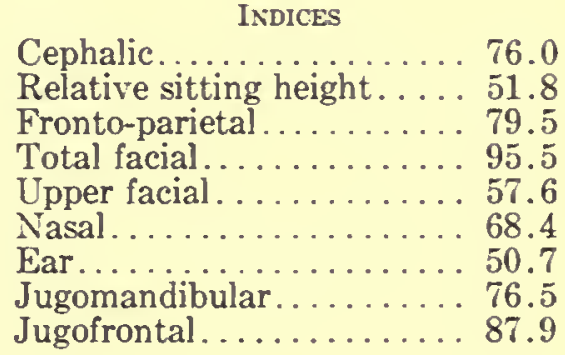

EYE.-Color: dark brown. Sclera: clear. Iris: homogeneous.

Nose.-Profile: concave. Tip: thickness, plus; elevated. Wings: flaring.

TeEтH.-Eruption: complete. Lost: none. Bite: slight over.

CHEST.-Derelopment: plus.

Musculature.-Good.

HEALTH.-Good.

No. 579. Plate LXXI, Figs. 5, 6

Place: Hilla Army Camp. Date: June 14, 1928.

Birthplace: Nasiriya. Age: 17; unmarried.

Brothers: 4 living. Sisters: 2 living.

Tattoo marks: none.

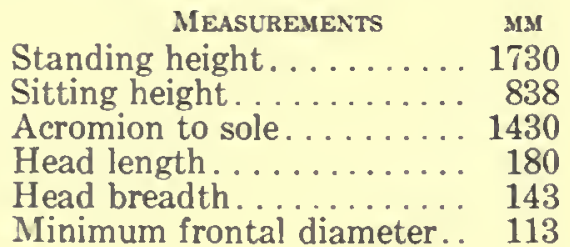

Cephalic............. 79.4 Relative sitting height.... 48.4 Fronto-parietal......... 79.0 Total facial............ 89.7 Upper facial. .........6.6.3 Nasal............... 57.1 


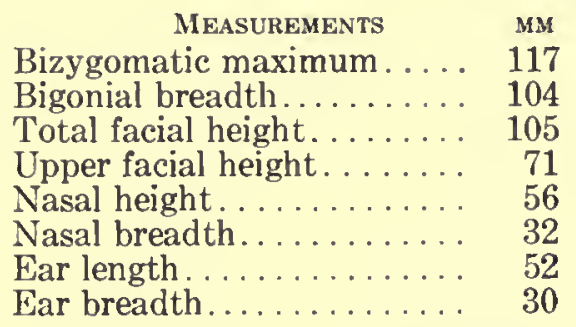

\author{
INDICES \\ Ear................ 57.7 \\ Jugomandibular. . . . . . 88.9 \\ Jugofrontal. .......... 96.5
}

EYE.-Color: dark brown. Sclera: clear. Iris: homogeneous.

Nose.-Profile: convex. Wings: medium-flaring.

TеEтн.-Eruption: third molars unerupted. Lost: none. Bite: slight over.

Chest.-Development: average.

Musculature.-Fair.

HEALTH.-Good.

\title{
No. 580. Plate LXXI, Figs. 7, 8
}

Place: Hilla Army Camp. Date: June 14, 1928.

Birthplace: Amara. Age: 20; unmarried.

Brothers: 2 living. Sisters: 1 living.

Tattoo marks: spot on back of right wrist.

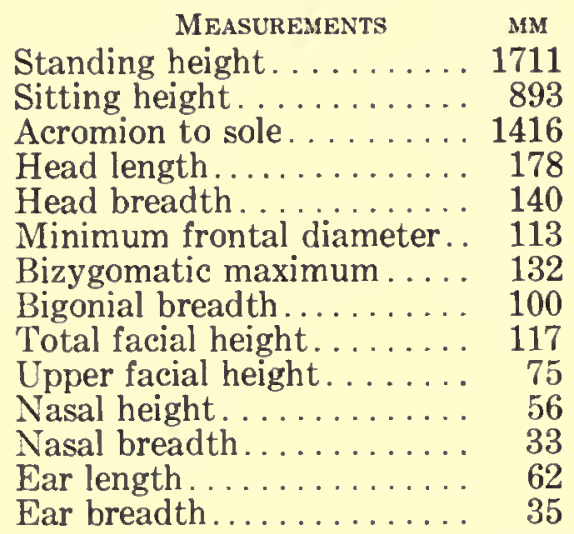

INDICES

Cephalic............. 78.7

Relative sitting height.... 52.1

Fronto-parietal......... 80.7

Total facial............ 88.6

Upper facial. . . . . . . . . 56.7

Nasal. . . . . . . . . . . 59.0

Ear.................. 56.5

Jugomandibular. . . . . . . . . 75.8

Jugofrontal. . . . . . . . . 85.6

EYE.-Color: dark brown. Sclera: clear. Iris: homogeneous.

Nose.-Profile: straight. Wings: medium-flaring.

TEETH.-Eruption: complete. Lost: none. Bite: slight over.

Chest.-Development: plus.

Musculature.-Good.

HEALTH.-Good.

No. 581. Plate LXXII, Figs. 1, 2

Place: Hilla Army Camp. Date: June 14, 1928.

Birthplace: Nasiriya. Age: 25; married for 10 years. Sons: 2 living. Daughters: none. Brothers: 2 living. Sisters: 1 dead.

Tattoo marks: none.

\begin{tabular}{|c|c|}
\hline MEASUREMENTS & MM \\
\hline Standing height . . . . . & 1760 \\
\hline Sitting height. & 910 \\
\hline Acromion to sole. & 1466 \\
\hline Head length. & 188 \\
\hline Head breadth. & 137 \\
\hline frontal diameter. & 114 \\
\hline latic maximum ... & 135 \\
\hline aniol hrondth & \\
\hline
\end{tabular}

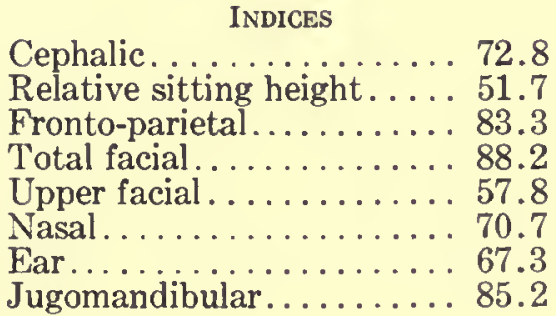




\begin{tabular}{|c|c|c|}
\hline MEASUREMENTS & MM & INDICES \\
\hline Total facial height. .... & 119 & Jugofrontal . \\
\hline Upper facial height. . & 78 & \\
\hline Nasal height. & 58 & \\
\hline Nasal breadth. & 41 & \\
\hline Ear length. & 61 & \\
\hline ar breadth. & 41 & \\
\hline
\end{tabular}

EYE.-Color: dark brown. Sclera: clear. Iris: homogeneous.

Nose.-Profile: straight. Wings: flaring.

TEETH.-Eruption: complete. Lost: 1. Bite: slight over.

CHest.-Development: plus.

Musculature.-Good.

HEALTH.--Good.

\section{No. 582. Plate LXXII, Figs. 3, 4}

Place: Hilla Army Camp. Date: June 14, 1928.

Birthplace: Shatra. Age: 22; unmarried.

Brothers: none. Sisters: 7 living.

Tattoo marks: 3 spots on back and 5 spots on inside of right wrist.

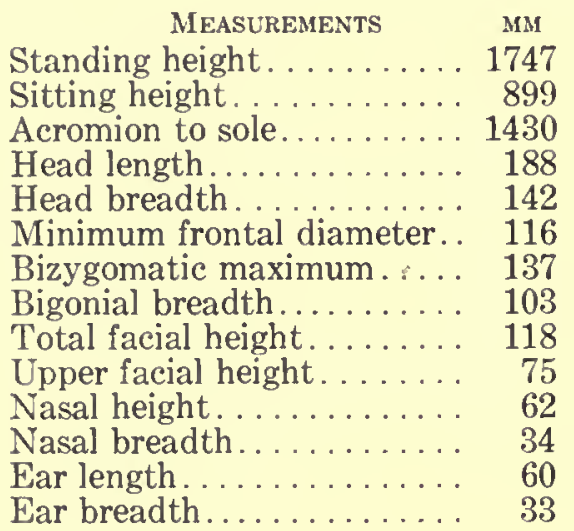

Cephalic .............. 75.6 Relative sitting height....,51.4 Fronto-parietal.........8 81.3 Total facial. .......... 86.1

Upper facial . . . . . . . . . 54.6 Nasal. . . . . . . . . . . 54.8

Ear................ 55.0

Jugomandibular. . . . . . . . 75.2

Jugofrontal . . . . . . . . . . 84.7

HAIR.-Color: dark brown.

EYE.-Color: dark brown. Sclera: clear. Iris: homogeneous.

NosE.-Profile: concave. Wings: flaring.

TEETH.--Eruption: complete. Lost: none. Bite: slight over.

Chest.-Development: plus.

Musculature.-Good.

HEALTH.-Good.

No. 583. Plate LXXII, Figs. 5, 6

Place: Hilla Army Camp. Date: June 14, 1928.

Birthplace: Nasiriya. Age: 22; unmarried.

Brothers: 2 living. Sisters: 2 living.

Tattoo marks: cross and two spots on right wrist.

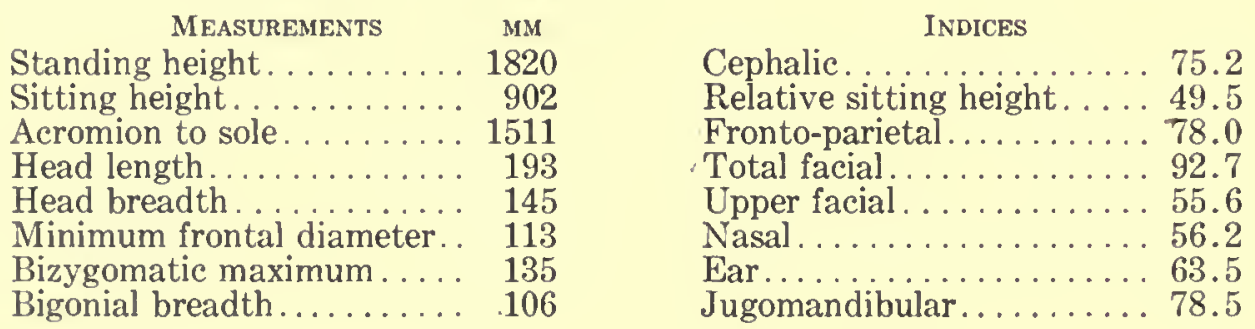




\begin{tabular}{|c|c|}
\hline MEASUREMENTS & MM \\
\hline Total facial height. & 125 \\
\hline Upper facial height. & 75 \\
\hline Nasal height. & 57 \\
\hline Nasal breadth. & 32 \\
\hline ar length. & 63 \\
\hline Ear breadth & 40 \\
\hline
\end{tabular}

EYE.-Color: dark brown. Sclera: clear. Iris: homogeneous.

NosE.-Profile: convex. Wings: medium.

TEETH.-Eruption: complete. Lost: none. Bite: slight over.

CHEST.-Development: plus.

Musculature.-Good.

Health.-Good.

No. 584. Plate LXXII, Figs. 7, 8

Place: Hilla Army Camp. Date: June 14, 1928.

Birthplace: Ad Diwaniya. Age: 25; married for 3 years. No children.

Brothers: 1 living. Sisters: none.

Tattoo marks: glove on back of left hand.

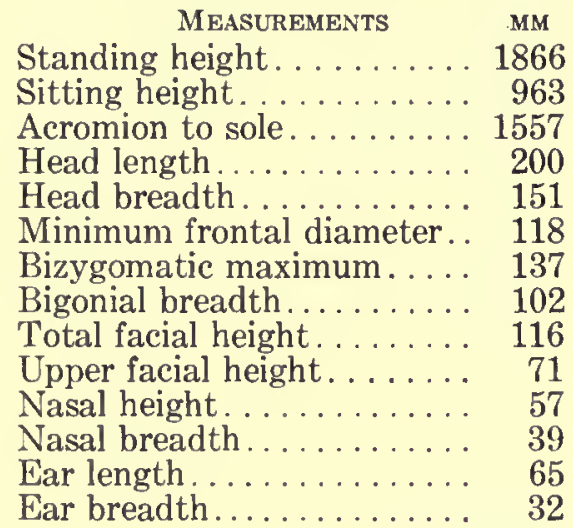

\section{INDICES}

Cephalic.............. 75.5

Relative sitting height. ... 51.6

Fronto-parietal......... 78.2

Total facial. . . . . . . . 84.6

Upper facial . . . . . . . . . . 51.8

Nasal. . . . . . . . . 68.4

Ear................. 49.3

Jugomandibular......... 74.4

Jugofrontal . . . . . . . . 85.1

EYE.-Color: green brown. Sclera: clear. Iris: zoned.

Nose.-Profile: convex. Wings: medium-flaring.

TEETH.-Eruption: complete. Lost: none. Bite: slight over.

CHEsT.-Development: double plus.

Musculature.-Excellent.

Health.-Good.

No. 585. Plate LXXIII, Figs. 1, 2

Place: Hilla Army Camp. Date: June 14, 1928.

Birthplace: near Baghdad. Age: 19; unmarried.

Brothers: 2 living. Sisters: 1 living.

Tattoo marks: none.

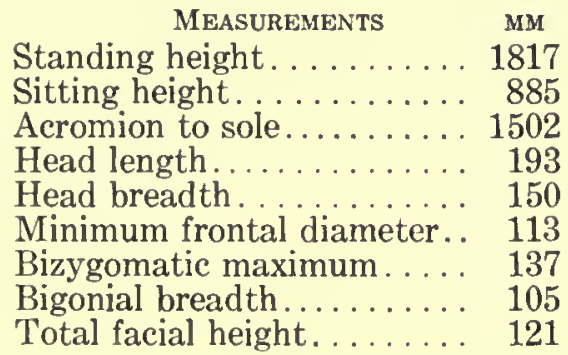

INDICES

Cephalic. ............777.8

Relative sitting height. .... 48.7

Fronto-parietal......... 75.4

Total facial............. 88.4

Upper facial. . . . . . . . 48.9

Nasal ................. 62.5

Ear.............6. 62.0

Jugomandibular. . . . . . . 76.6

Jugofrontal. .......... 82.5 


\begin{tabular}{|c|c|}
\hline MEASUREMENTS & MM \\
\hline Upper facial height. . & 67 \\
\hline Nasal height...... & 56 \\
\hline Nasal breadth. . & 35 \\
\hline Ear length. . & 58 \\
\hline Ear breadth. & 0 \\
\hline
\end{tabular}

EYE.-Color: dark brown. Sclera: clear. Iris: homogeneous.

Nose.-Profile: concavo-convex. Wings: medium.

TEETH.-Eruption: complete. Lost: none. Bite: slight over.

CHEST.-Development: plus.

Musculature.-Good.

HEALTH.-Good.

No. 586. Plate LXXIII, Figs. 3, 4

Place: Hilla Army Camp. Date: June 14, 1928.

Birthplace: Amara. Age: 22; unmarried.

Brothers: 3 living. Sisters: none.

Tattoo marks: glove on back of right hand; 2 lines with dot between on left hand.

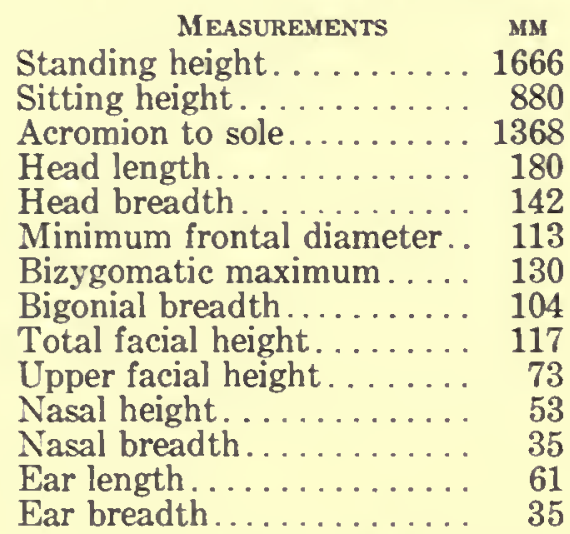

Cephalic............ 78.9

Relative sitting height..... 52.8

Fronto-parietal......... 79.6

Total facial ............ 90.0

Upper facial........... 56.1

Nasal. . . . . . . . . 66.1

Ear............... 57.4

Jugomandibular......... 80.0

Jugofrontal. .......... 86.9

EYE.-Color: dark brown. Sclera: clear. Iris: homogeneous.

NosE.-Profile: straight. Wings: medium.

TEетн.-Eruption: complete. Lost: none. Bite: slight over.

ChEST.-Development: plus.

Musculature.--Good.

HEALTH.-Good.

\section{No. 587. Plate LXXIII, Figs. 5, 6}

Place: Hilla Army Camp. Date: June 14, 1928.

Birthplace: Hilla. Age: 17; married for 2 years. Sons: none. Daughters: 1 living.

Brothers: 1 dead. Sisters: 1 living.

Tattoo marks: glove on back of right hand.

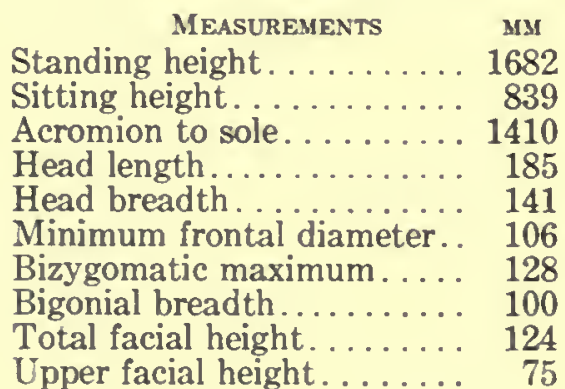

INDICES

Cephalic............. 76.3

Relative sitting height. . . 49.8

Fronto-parietal......... 75.3

Total facial. ........... 96.9

Upper facial. .......... 58.6

Nasal. ..............66.1 66.1

Ear................ 62.1

Jugomandibular. . . . . . . 78.2

Jugofrontal. . . . . . . . 82.8 


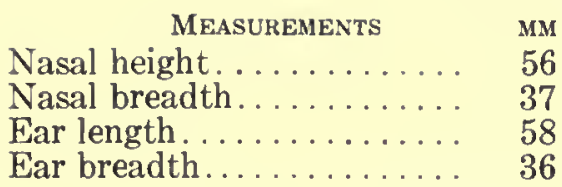

EyE.-Color: dark brown. Sclera: clear. Iris: homogeneous.

Nose.-Profile: straight. Tip: thickness, double plus. Wings: medium-flaring.

TEETH.-Eruption: third molars unerupted. Lost: none. Bite: slight over.

Chest.-Development: plus.

Musculature.-Good.

HEALTH.-Good.

No. 588. Plate LXXIII, Figs. 7, 8

Place: Hilla Army Camp. Date: June 14, 1928.

Birthplace: Amara. Age: 20 ; unmarried.

Brothers: 2 living. Sisters: 1 living.

Tattoo marks: spots forming snake, high upon inside of left arm.

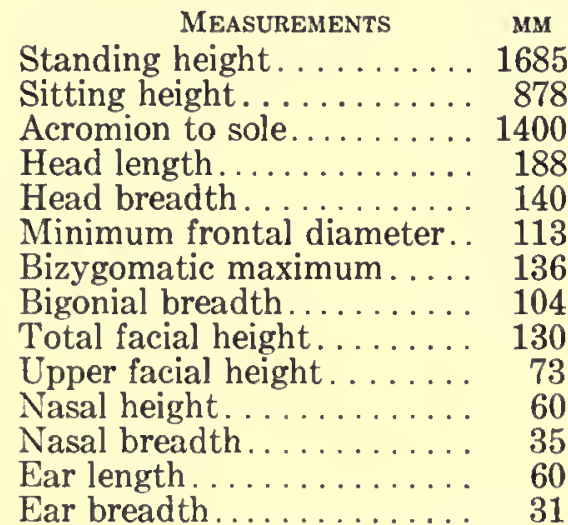

Cephalic............. 74.5

Relative sitting height.... 52.1

Fronto-parietal.......... 80.7

Total facial.............. 95.6

Upper facial. .......... 53.7

Nasal. .............. 58.4

Ear............... 51.6

Jugomandibular. . ...... 76.5

Jugofrontal. .......... 83.1

EyE.-Color: dark brown. Sclera: clear. Iris: homogeneous.

NOSE.-Profile: straight. Wings: medium.

TEETH.-Eruption: complete. Lost: none. Bite: slight over.

CHEST.-Development: plus.

Musculature.-Good.

Health.-Good.

No. 589. Plate LXXIV, Figs. 1, 2

Place: Hilla Army Camp. Date: June 14, 1928.

Birthplace: Nasiriya. Age: 19; unmarried.

Brothers: 2 living. Sisters: 1 living.

Tattoo marks: none.

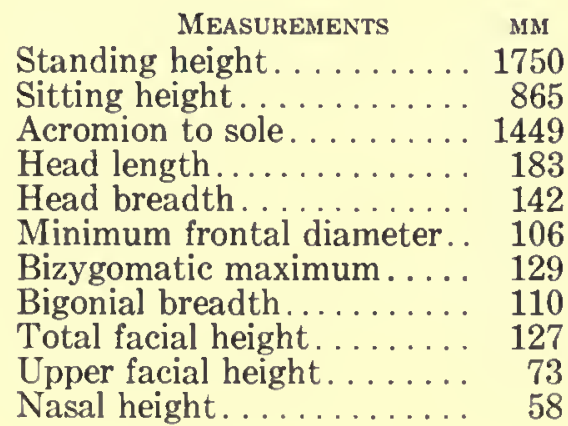

INDICES

Cephalic.............77.6

Relative sitting height. ... 49.4

Fronto-parietal......... 74.7

Total facial............. 98.5

Upper facial. . . . . . . . . . 56.6

Nasal ................ 59.7

Ear................. 59.6

Jugomandibular. . . . . . . 85.3

Jugofrontal . . . . . . . . . . 82.2 


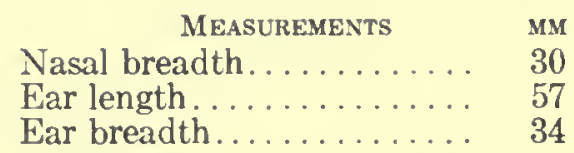

EYE.-Color: dark brown. Sclera: clear. Iris: homogeneous.

Nose.-Profile: convex. Wings: compressed.

TEETH.-Eruption: complete. Lost: none. Bite: slight over.

Chest.-Development: plus.

Musculature.-Good.

HEALTH.-Good.

No. 590. Plate LXXIV, Figs. 3, 4

Place: Hilla Army Camp. Date: June 15, 1928.

Birthplace: Hilla. Age: 18; unmarried.

Brothers: 2 living. Sisters: none.

Tattoo marks: none.

\begin{tabular}{|c|c|}
\hline MEASUREMENTS & \\
\hline $\begin{array}{l}\text { Standing height. } \\
\text { Sitting height... }\end{array}$ & $\begin{array}{r}170 \\
84\end{array}$ \\
\hline Acromion to sole & 14 \\
\hline Head length. & \\
\hline Head breadth. & \\
\hline Minimum frontal diameter. & \\
\hline Bizygomatic maximum . & \\
\hline Bigonial breadth & \\
\hline Total facial height. & \\
\hline Upper facial height. & \\
\hline Nasal height....... & \\
\hline $\begin{array}{l}\text { Nasal breadth. } \\
\text { Ear length... }\end{array}$ & \\
\hline ar brea & \\
\hline
\end{tabular}

INDICES

Cephalic............. 70.4

Relative sitting height..... 49.5

Fronto-parietal........ 80.7

Total facial............ 89.3

Upper facial. . . . . . . . 56.9

Nasal ............... 58.4

Ear................ 55.0

Jugomandibular........ 73.0

Jugofrontal.......... 81.5

EYE.-Color: dark brown. Sclera: clear. Iris: homogeneous.

Nose.-Profile: convex. Wings: medium.

TEETH.--Eruption: third molars unerupted. Lost: none. Bite: slight over.

Chest.-Development: plus.

Musculature.-Good.

HEALTh.-Good.

\section{No. 591. Plate LXXiV, Figs. 5, 6}

Place: Hilla Army Camp. Date: June 15, 1928.

Birthplace: Amara. Age: 22; unmarried.

Brothers: 3 living, 4 dead. Sisters: 2 dead.

Tattoo marks: none.

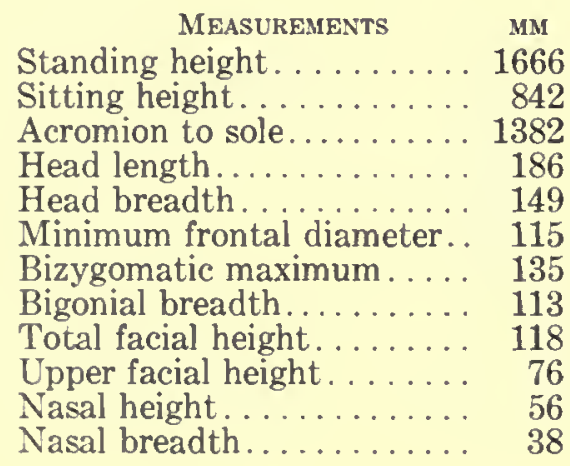

INDICES

Cephalic............88.1

Relative sitting height. . . . 50.5

Fronto-parietal......... 77.3

Total facial............. 87.4

Upper facial. . . . . . . . . . 56.3

Nasal................6.67.8

Ear............. 54.4

Jugomandibular . . . . . . 83.7

Jugofrontal. . . . . . . . . . . 85.1 


$\begin{array}{cr}\text { MEASUREMENTS } & \text { MM } \\ \text { Ear length............ } & \mathbf{5 7} \\ \text { Ear breadth ........... } & 31\end{array}$

EYE. - Color: dark brown. Sclera: clear. Iris: homogeneous.

Nose.-Profile: concave. Wings: medium-flaring.

TeEth.--Eruption: complete. Lost: none. Bite: slight over.

Chest.-Development: plus.

Musculature.-Good.

HEALTH.-Good.

No. 592. Plate LXXIV, Figs. 7, 8

Place: Hilla Army Camp. Date: June 15, 1928.

Birthplace: Nasiriya. Age: 17; unmarried.

Brothers: 3 living. Sisters: 1 living.

Tattoo marks: none.

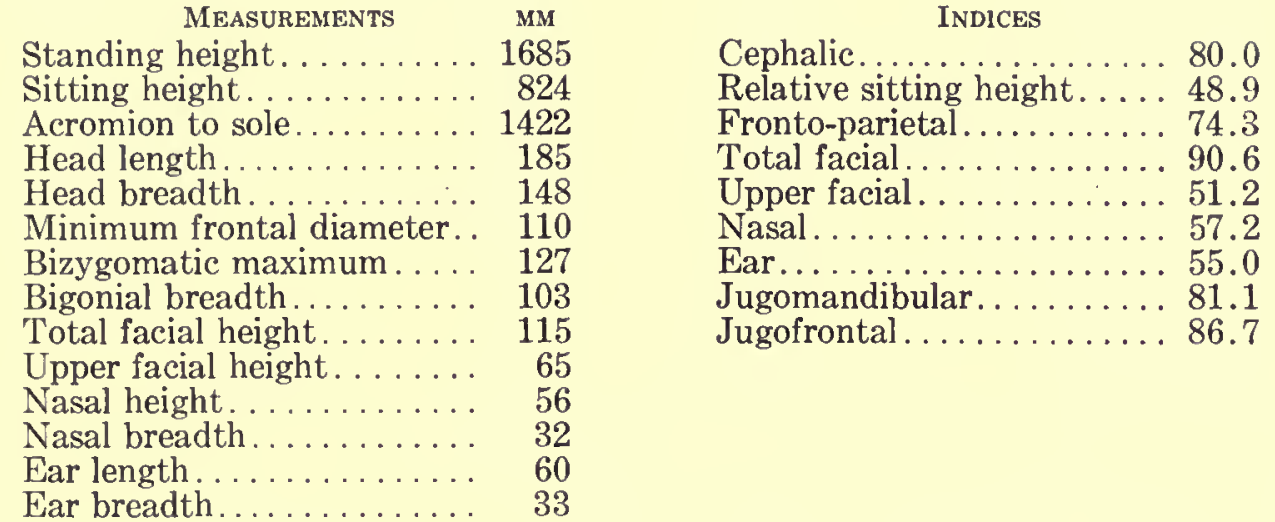

EYE.-Color: dark brown. Sclera: clear. Iris: homogeneous.

NOSE.-Profile: convex. Wings: medium.

TEETH.-Eruption: third molars unerupted. Lost: none. Bite: slight over.

Chest.-Development: plus.

Musculature.-Good.

HEALTH.-Good.

No. 593. Plate LXXV, Figs. 1, 2

Place: Hilla Army Camp. Date: June 15, 1928.

Birthplace: Nasiriya. Age: 20; unmarried.

Brothers: none. Sisters: none.

Tattoo marks: spots on each temple.

MEASUREMENTS MM

Standing height. . . . . . 1725

Sitting height . . . . . . . . . . 885

Acromion to sole. . . . . . . 1446

Head length.............. 194

Head breadth.......... 149

Minimum frontal diameter. . 117

Bizygomatic maximum ..... 137

Bigonial breadth.......... 114

Total facial height. . . . . . . 121

Upper facial height. ....... 76

Nasal height............ 58

Nasal breadth............ 34

Ear length............ 58

Ear breadth............. 36
INDICES

Cephalic............. 76.7

Relative sitting height. ... 51.3

Fronto-parietal.......... 78.6

Total facial............. 88.4

Upper facial. . . . . . . . . . . 55.4

Nasal................. 58.6

Ear...............6 62.0

Jugomandibular.......... 83.2

Jugofrontal. . . . . . . . 85.4 
EYE.-Color: dark brown. Sclera: clear. Iris: homogeneous.

Nose.-Profile: straight. Wings: medium.

TEетн.-Eruption: complete. Lost: none. Bite: slight over.

Chest.-Development: plus.

Musculature.-Good.

HEALTH.-Good.

\section{No. 594. Plate LXXV, Figs. 3, 4}

Place: Hilla Army Camp. Date: June 15, 1928.

Birthplace: Amara. Age: 18; unmarried.

Brothers: 1 living. Sisters: 1 living.

Tattoo marks: none.

\begin{tabular}{|c|c|}
\hline MEASUREMENTS & MM \\
\hline Standing height. & 1723 \\
\hline Sitting height. & 885 \\
\hline Acromion to sole & 1440 \\
\hline Head length... & 190 \\
\hline Head breadth. & 136 \\
\hline Minimum frontal diameter. & 113 \\
\hline Bizygomatic maximum . . . . . & 128 \\
\hline Bigonial breadth . . . . . . . & 98 \\
\hline Total facial height. & 106 \\
\hline Upper facial height. & 66 \\
\hline Nasal height. . . . . . . . & 57 \\
\hline Nasal breadth. & 1 \\
\hline Ear length.... & 57 \\
\hline For hreodth & \\
\hline
\end{tabular}

EYE.-Color: dark brown. Sclera: clear. Iris: homogeneous.

NosE.-Profile: straight. Wings: medium-flaring.

TEETH.-Eruption: third molars unerupted. Lost: none. Bite: marked over.

CHEST.-Development: average.

Musculature.-Fair.

HEALTH. - Good.

No. 595. Plate LXXV, Figs. 5, 6

Place: Hilla Army Camp. Date: June 15, 1928.

Birthplace: Amara. Age: 20; unmarried.

Brothers: 3 living. Sisters: 2 living.

Tattoo marks: none.

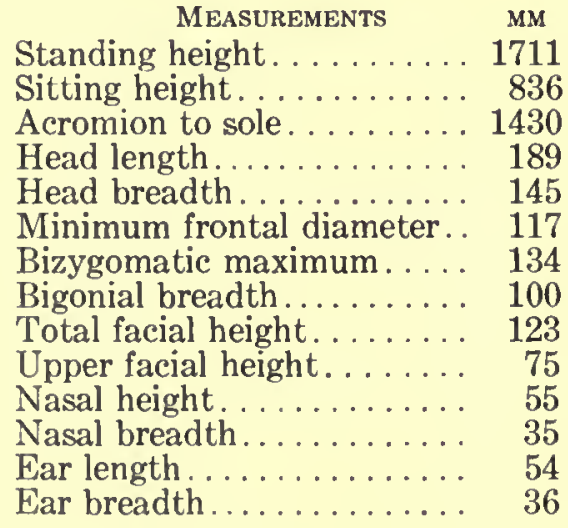

Cephalic............ 76.7

Relative sitting height. . . . 48.9

Fronto-parietal.......... 80.7

Total facial............ 91.8

Upper facial ............ 55.9

Nasal. . . . . . . . . . 63.7

Ear........................ 66.7

Jugomandibular. . . . . . . 74.7

Jugofrontal . . . . . . . . . . . . 87.3

EYE.-Color: dark brown. Sclera: clear. Iris: homogeneous.

Nose.-Profile: convex. Wings: medium-flaring. 
TEETH.-Eruption: complete. Lost: none. Bite: slight over.

Chest.-Development: plus.

Musculature.-Good.

HEALTH.-Good.

No. 596. Plate LXXV, Figs. 7, 8

Place: Hilla Army Camp. Date: June 15, 1928.

Birthplace: Amara. Age: 25; unmarried.

Brothers: 2 living. Sisters: 1 living.

Tattoo marks: none.

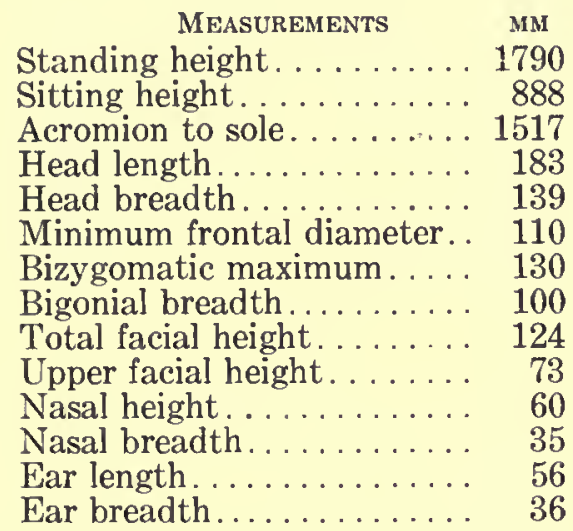

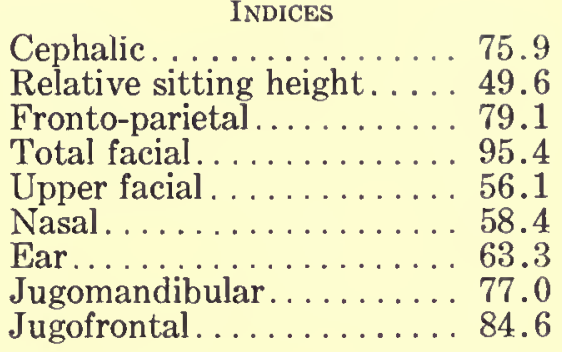

EyE.-Color: dark brown. Sclera: clear. Iris: homogeneous.

NosE.-Profile: straight. Wings: medium.

TEETH.-Eruption: complete. Lost: none. Bite: slight over.

Chest.-Development: plus.

Musculature.-Good.

Health.-Good.

\section{No. 597. Plate LXXVI, Figs. 1, 2}

Place: Hilla Army Camp. Date: June 15, 1928.

Birthplace: Hindiya. Age: 18; unmarried.

Brothers: 2 living. Sisters: 2 living.

Tattoo marks: 3 spots on back of right wrist.

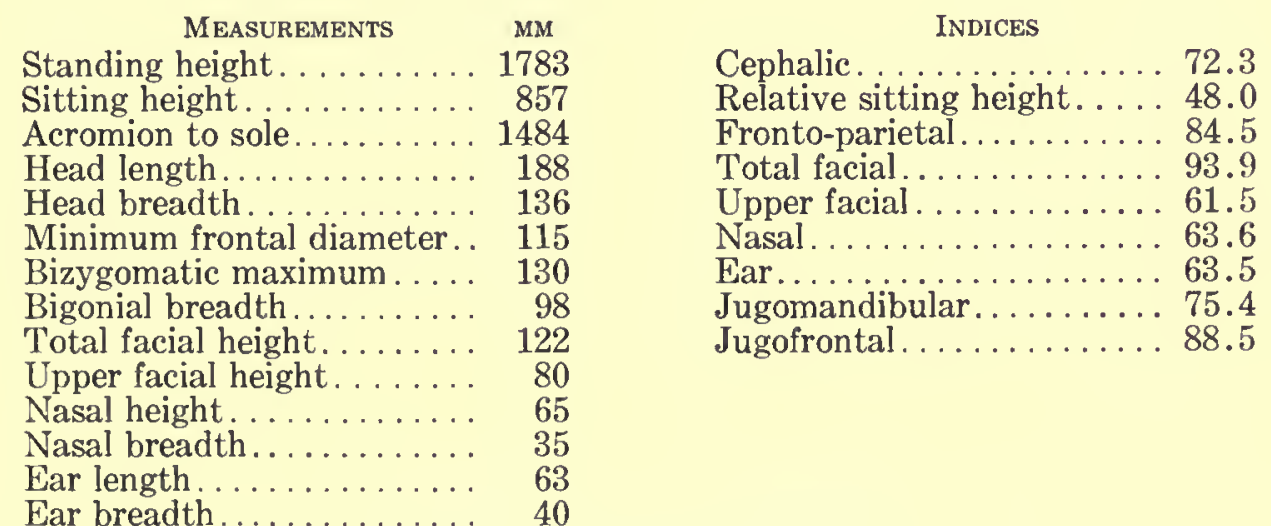

EYE.-Color: dark brown. Sclera: clear. Iris: homogeneous.

Nose.-Profile: convex. Tip: thickness, minus. Wings: medium-compressed.

TEETH.--Eruption: complete. Lost: none. Bite: slight over.

Chest.-Development: plus. 
Musculature.-Good.

HEALTH.-Good.

\section{No. 598. Plate LXXVI, Figs. 3, 4}

Place: Hilla Army Camp. Date: June 15, 1928.

Birthplace: Amara. Age: 20; unmarried.

Brothers: none. Sisters: 4 living.

Tattoo marks: two spots and animal on right arm.

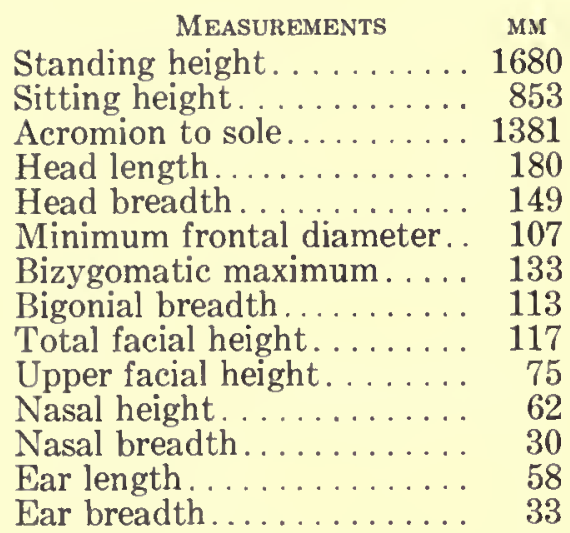

Eye.-Color: dark brown. Sclera: clear. Iris: homogeneous.

Nose.-Profile: concave. Wings: medium.

TeEтн.-Eruption: complete. Lost: none. Bite: slight over.

CHEST.-Development: plus.

Musculature.-Good.

HEALTH.--Good.

\section{No. 599. Plate LXXVI, Figs. 5, 6}

Place: Hilla Army Camp. Date: June 15, 1928.

Birthplace: Amara. Age: 19; unmarried.

Brothers: 1 living, 1 dead. Sisters: 1 dead.

Tattoo marks: 4 spots on right temple.

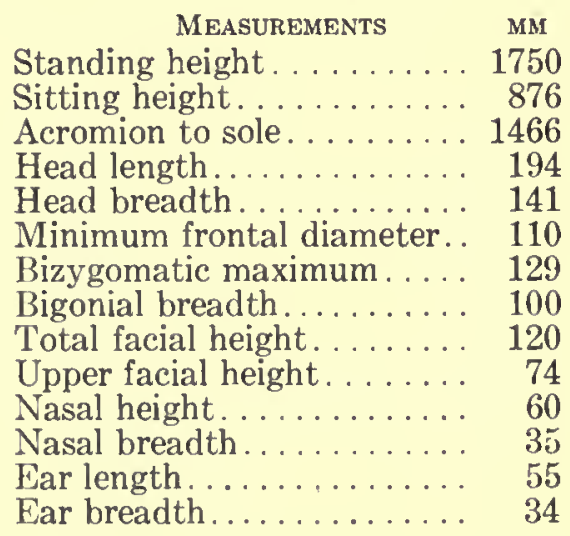

INDICES

Cephalic............. 72.6 Relative sitting height. ... 50.1 Fronto-parietal.......... 78.1 Total facial............ 93.1 Upper facial. . .......... 57.3 Nasal............... 58.4 Ear..............6. 61.7 Jugomandibular. . . . . . 77.5 Jugofrontal . . . . . . . . . . . 85.3

EYE.-Color: dark brown. Sclera: clear. Iris: homogeneous.

Nose.-Profile: straight. Wings: medium.

TEETH.-Eruption: complete. Lost: none. Bite: slight over.

CHEST.-Development: plus.

Musculature.-Good.

HEALTH.-Good. 
No. 600. Plate LXXVI, Figs. 7, 8

Place: Hilla Army Camp. Date: June 15, 1928.

Birthplace: Nasiriya. Age: 20; unmarried.

Brothers: 3 living. Sisters: none.

Tattoo marks: none.

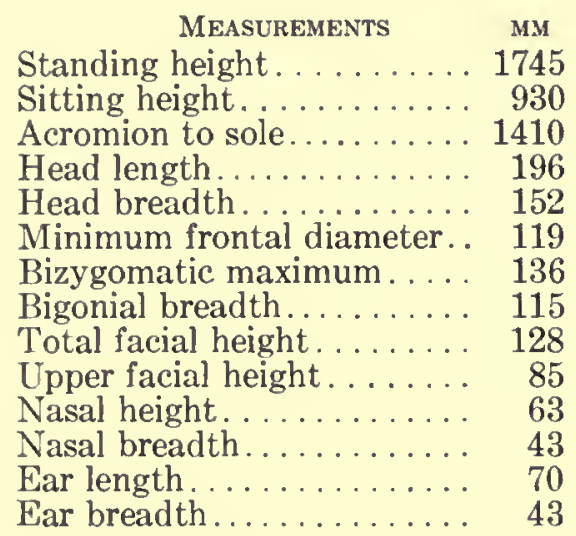

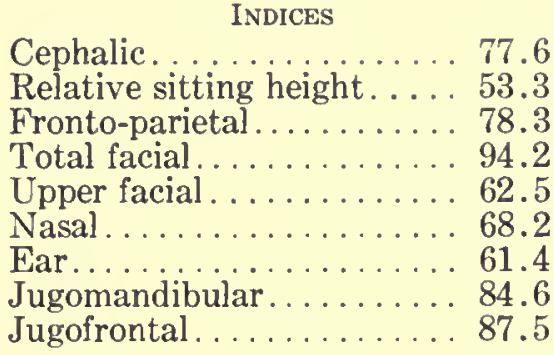

77.6

78.3

94.2

62.5

68.2

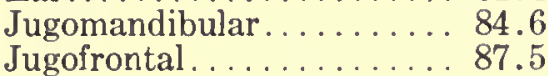

HAIR. - Color: dark brown.

EYE.-Color: dark brown. Sclera: clear. Iris: homogeneous.

Nose.-Profile: convex. Tip: depressed.

TEETH.-Eruption: complete. Caries: plus. Lost: 2.

Chest.-Development: plus.

Musculature.-Good.

HEALTH.-Good.

\section{No. 601. Plate LXXVII, Figs. 1, 2}

Place: Hilla Army Camp. Date: June 15, 1928.

Birthplace: near Kut al Imara. Age: 21; unmarried.

Brothers: 2 living. Sisters: none.

Tattoo marks: glove with circle and spot on back of right hand; gazelle on inside of right forearm; ring of spots on each temple.

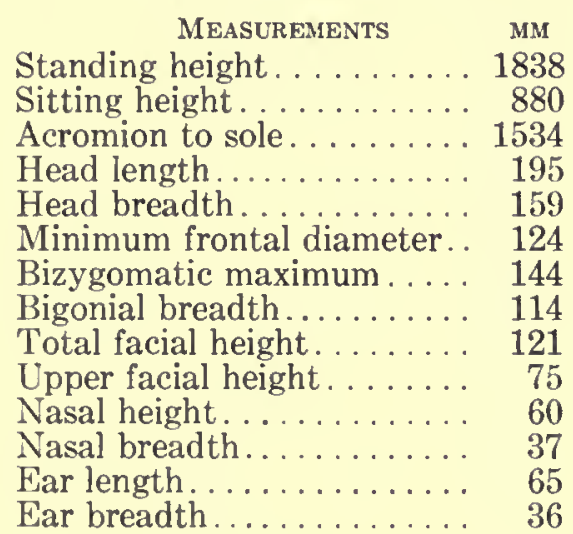

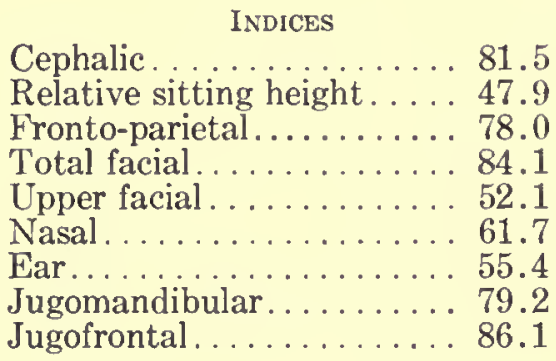

HAIR.-Color: dark brown.

EYE.-Color: dark brown. Sclera: clear. Iris: homogeneous.

Nose.-Profile: convex. Tip: thickness, minus. Wings: medium.

TеEтн.-Eruption: complete. Lost: 1. Bite: marked over.

Chest.-Development: plus.

Musculature.-Good.

Health.-Good. 


\section{No. 602. Plate LXXVII, Figs. 3, 4}

Place: Hilla Army Camp. Date: June 15, 1928.

Birthplace: Nasiriya. Age: 30; married for 3 years. Sons: none. Daughters: 1 living. Brothers: 1 living. Sisters: none.

Tattoo marks: spot on right side of nose.

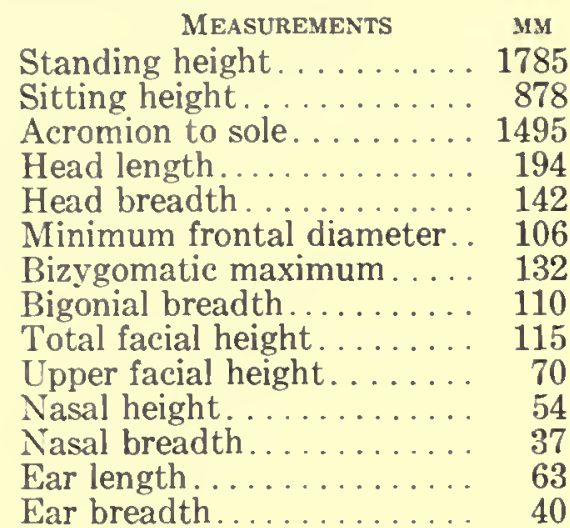

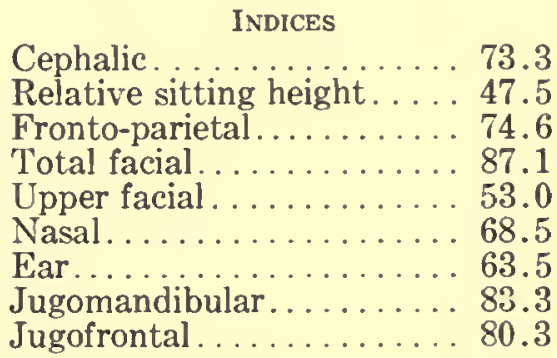

HAIR. - Color: dark brown.

EYE.-Color: dark brown. Sclera: clear. Iris: homogeneous.

Nose.-Profile: straight. Wings: medium-flaring.

TEетH.-Eruption: complete. Lost: none. Bite: slight over.

Chest.-Development: plus.

Musculature.-Good.

HEALTH.-Good.

No. 603. Plates LXXVII, Figs. 5, 6; LXXVIII, Fig. 6

Place: Hilla Army Camp. Date: June 15, 1928.

Birthplace: Nasiriya. Age: 18; unmarried.

Brothers: none. Sisters: none.

Tattoo marks: none.

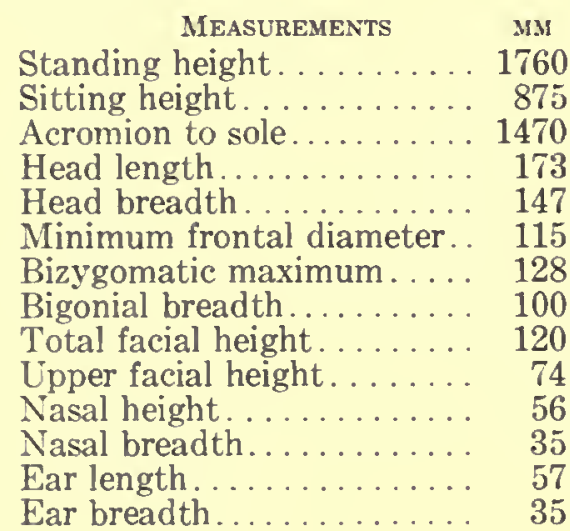

Cephalic. ............ 85.0

Relative sitting height. . . 49.6

Fronto-parietal......... 78.3

Total facial. . . . . . . . . . 93.9

Upper facial. ........... 57.7

Nasal. ............. 62.5

Ear. ..................61.5

Jugomandibular. . . . . . . 78.2

Jugofrontal . . . . . . . . 89.9

HAIR.-Color: dark brown.

EYE.-Color: dark brown. Sclera: clear. Iris: homogeneous.

NosE.-Profile: concave. Wings: flaring.

TEETH.--Eruption: complete. Lost: none.

CHEsT.-Development: plus.

Musculature.-Good.

HEALTH.-Good. 
No. 604. Plate LXXVII, Figs. 7, 8

Place: Hilla Army Camp. Date: June 15, 1928.

Birthplace: Amara. Age: 23; unmarried.

Brothers: 1 living, 2 dead. Sisters: 3 living.

Tattoo marks: none.

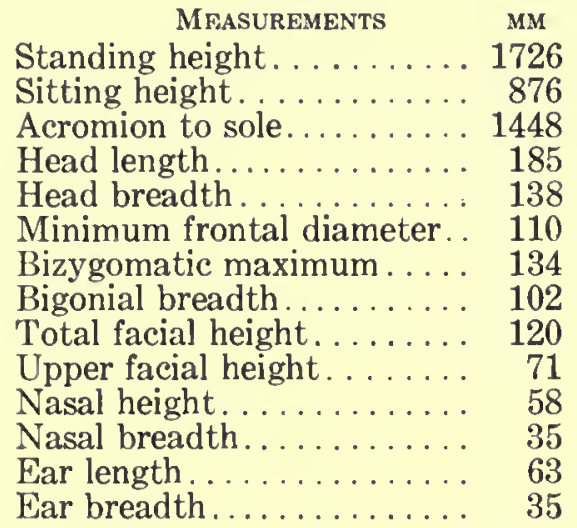

INDices
Cephalic............ 74.6

Relative sitting height.... 50.6

Fronto-parietal.......... 79.7

Total facial. . . . . . . . 89.6

Upper facial . . . . . . . . . . 53.0

Nasal. ............66 60.3

Ear.............. 55.6

Jugomandibular. . . . . . . 76.1

Jugofrontal . . . . . . . . 8 82.1

EYE.-Color: dark brown. Sclera: clear. Iris: homogeneous.

Nose.-Profile: straight. Wings: medium.

TEETH.-Eruption: complete. Lost: none. Bite: slight over.

Chest.-Development: plus.

Musculature.-Good.

Health.-Good.

No. 605. Plate LXXVIII, Figs. 1, 2

Place: Hilla Army Camp. Date: June 15, 1928.

Birthplace: near Kut al Imara. Age: 30; married for 6 years. Sons: 2 living. Daughters: 1 living.

Brothers: 9 living. Sisters: 2 living.

Tattoo marks: line on back of left wrist.

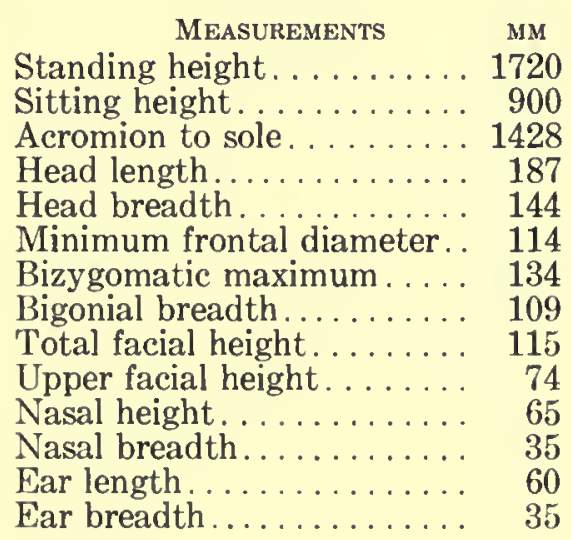

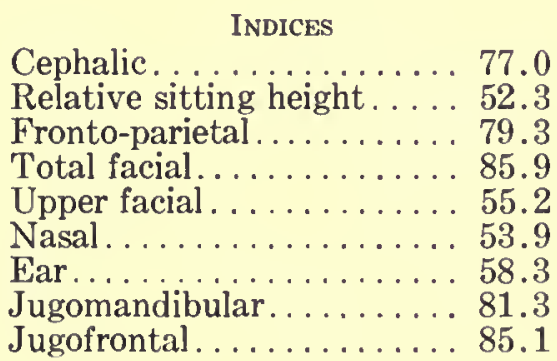

HAIR.-Color: dark brown.

EYE.-Color: dark brown. Sclera: clear. Iris: homogeneous.

Nose.-Profile: straight. Wings: medium.

TEETH.-Eruption: complete. Lost: none. Bite: slight over.

Chest.-Development: plus.

Musculature.-Good.

HEALTH.-Good. 


\section{No. 607. Plate LXXVIII, Fig. 5}

Place: Hilla Army Camp. Date: June 15, 1928.

Birthplace: Nasiriya. Age: 20; married for 3 years. Sons: 1 living. Daughters: none. Brothers: 2 living. Sisters: 6 living, 1 dead.

Tattoo marks: none.

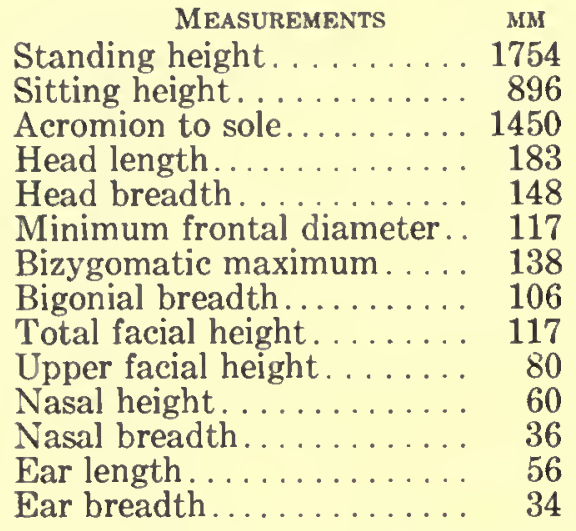

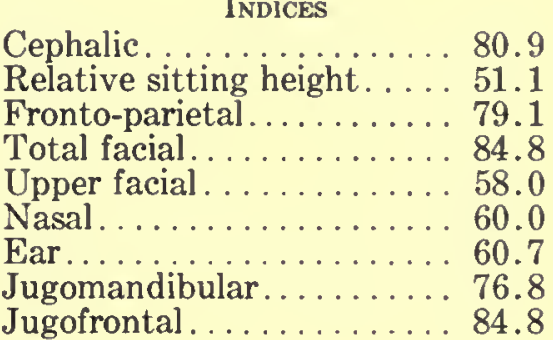

Cephalic............. 80.9

Relative sitting height.... 51.1

Upper facial ..... 58.0

Nasal. . . . . . . . . . . . . . 60.0

Jugomandibular. . . . . . 76.8

Jugofrontal . . . . . . . . . . . 84.8

EYe.-Color: dark brown. Sclera: clear. Iris: homogeneous.

Nose.-Profile: convex. Wings: medium.

TEETH.-Eruption: complete. Lost: none. Bite: slight over.

CHEST.-Development: plus.

Musculature.-Good.

HEALTH.-Good.

\section{No. 608. Plate LXXVIII, Figs. 7, 8}

Place: Hilla Army Camp. Date: June 15, 1928.

Birthplace: Nasiriya. Age: 20; unmarried.

Brothers: 2 living. Sisters: none.

Tattoo marks: none.

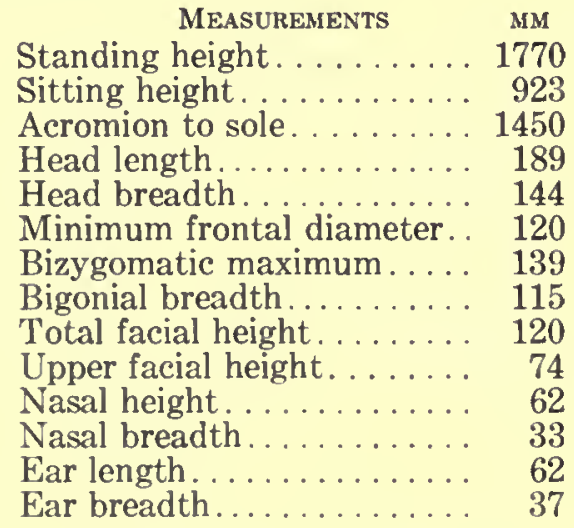

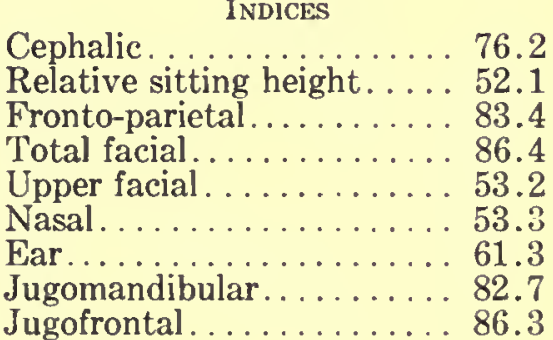

HAIR. - Color: dark brown.

EYE.-Color: dark brown. Sclera: clear. Iris: homogeneous.

Nose.-Profile: straight. Wings: medium.

TEETH.-Eruption: complete. Lost: none. Bite: slight over.

CHest.-Development: plus.

Musculature.-Good.

HEALTH.-Good. 
No. 609. Plate LXXIX, Figs. 1, 2

Place: Hilla Army Camp. Date: June 15, 1928.

Birthplace: Baghdad. Age: 21; unmarried.

Brothers: 2 living. Sisters: 2 living.

Tattoo marks: none.

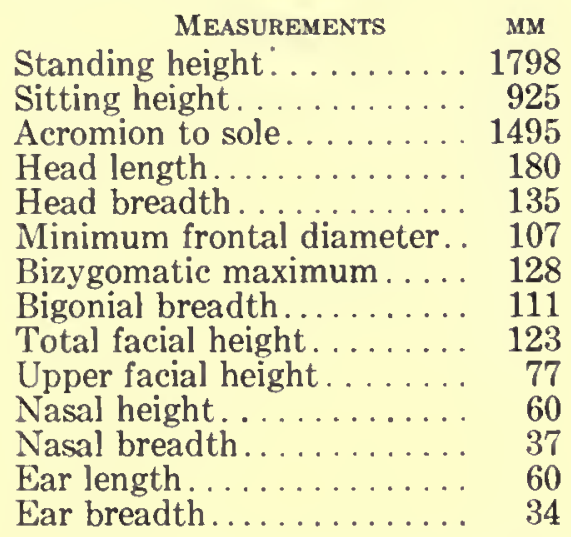

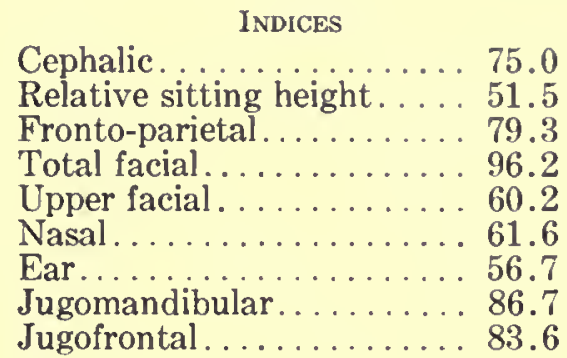

75.0

60.2

6.7
6.7

Jugomandrontal

EYE.-Color: dark brown. Sclera: clear. Iris: homogeneous.

NoSE.-Profile: straight. Wings: medium-compressed.

TEETH.-Eruption: complete. Lost: 1. Bite: slight over.

Chest.-Development: double plus.

Musculature.-Excellent.

HEALTH.-Good.

\section{No. 610. Plate LXXIX, Figs. 3, 4}

Place: Hilla Army Camp. Date: June 15, 1928.

Birthplace: Nasiriya. Age: 19 ; unmarried.

Brothers: 2 living. Sisters: 2 living.

Tattoo marks: none.

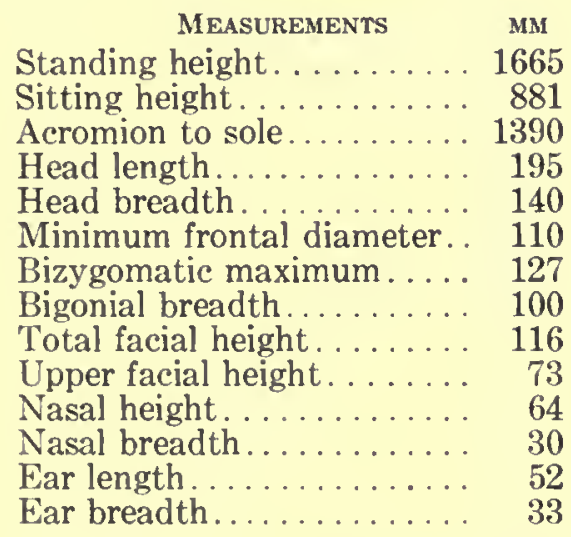

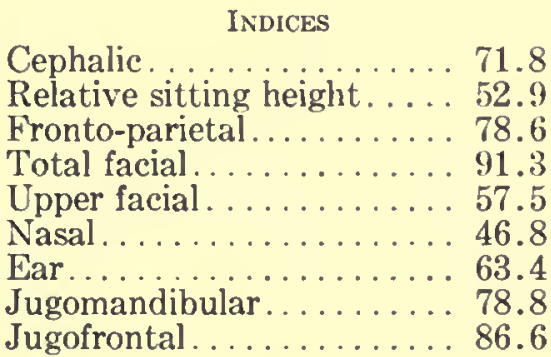

EYE.-Color: dark brown. Sclera: clear. Iris: homogeneous.

NosE.-Profile: convex. Wings: medium-compressed.

TEETH.-Eruption: complete. Lost: none. Bite: slight over.

ChEsT.-Development: plus.

Musculature.-Good.

HEALTH.-Good. 


\section{No. 611. Plate LXXIX, Figs. 5, 6}

Place: Hilla Army Camp. Date: June 15, 1928.

Birthplace: Shatra. Age: 19; unmarried.

Brothers: 1 living. Sisters: 1 living.

Tattoo marks: glove on back of right hand; animal design on inside of left forearm.

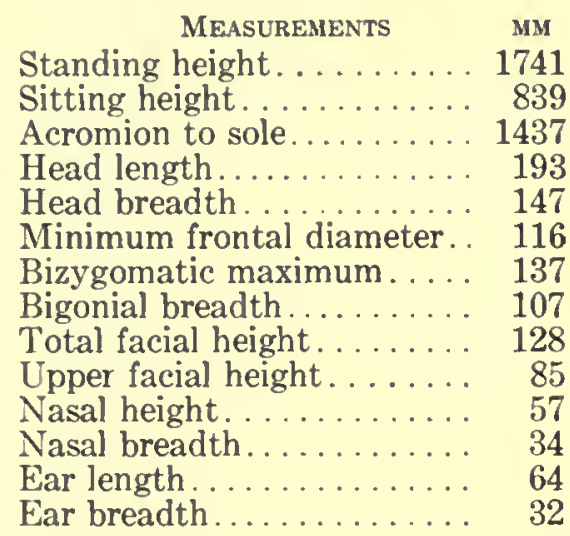

INDICES

Cephalic.............. 76.2

Relative sitting height.... 48.2

Fronto-parietal.......... 79.0

Total facial............ 93.5

Upper facial . . . . . . 62.0

Nasal. . . . . . . . . . 59.6

Ear............ 50.0

Jugomandibular . . . . . . . 78.1

Jugofrontal............ 84.7

EYE.-Color: dark brown. Sclera: clear. Iris: homogeneous.

Nose.-Profile: convex. Tip: thickness, double plus. Wings: medium-flaring.

Teeth.-Eruption: complete. Lost: none. Bite: slight over.

Chest.-Development: plus.

Musculature.-Good.

HEALTH.-Good.

\section{No. 612. Plate LXXiX, Figs. 7, 8}

Place: Hilla Army Camp. Date: June 15, 1928.

Birthplace: Khanaqin. Age: 30 ; married for 3 years. No children.

Brothers: 2 living. Sisters: 1 living.

Tattoo marks: triangles and lines on right wrist.

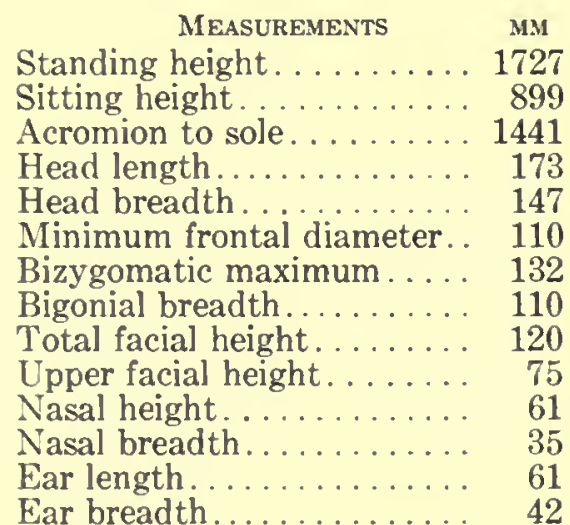

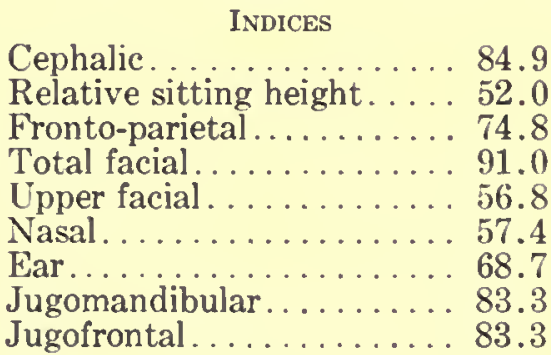

HAIR.-Color: light brown.

EYE.-Color: green brown. Sclera: clear. Iris: zoned.

Nose.-Profile: convex. Wings: medium-compressed.

TEETH.-Eruption: complete. Lost: none. Bite: slight over.

Chest.-Development: plus.

Musculature.-Good.

HEALTH.-Good.

Note: Omitted from averages due to artificial cranial deformation. 
No. 613. Plate LXXX, Figs. 1, 2

Place: Hilla Army Camp. Date: June 15, 1928.

Birthplace: Nasiriya. Age: 23; unmarried.

Brothers: 1 living. Sisters: 4 living.

Tattoo marks: 2 crosses on back of left wrist.

\begin{tabular}{|c|c|c|}
\hline MEASUREMENTS & MM & INDICES \\
\hline Standing height. & 1740 & Cephalic. \\
\hline Sitting height. & 885 & Relative sitting height. \\
\hline Acromion to sole. & 1457 & Fronto-parietal........ \\
\hline Head length..... & 189 & Total facial..... \\
\hline Head breadth & 149 & Upper facial \\
\hline Minimum frontal diameter. & 118 & Nasal. \\
\hline Bizygomatic maximum..... & 147 & Ear. \\
\hline Bigonial breadth.......... & 110 & Jugomandibular \\
\hline Total facial height. & 116 & Jugofrontal . \\
\hline
\end{tabular}

Upper facial height. . . . . . 75

Nasal height........... 57

Nasal breadth.

Ear length . . . . . . . . . . 57

Ear breadth............ 41

HAIR.-Color: black.

EyE.-Color: very dark brown. Sclera: clear. Iris: homogeneous.

Nose.-Profile: straight. Wings: medium-flaring.

TEETH.-Eruption: complete. Lost: none. Bite: marked over.

CHEST.-Development: plus.

Musculature.-Good.

HEALTH.-Good.

Some Negro blood.

\section{No. 614. Plate LXXX, Figs. 3, 4}

Place: Hilla Army Camp. Date: June 15, 1928.

Birthplace: Samarra. Age: 25; married for 11 years. Sons: 1 living, 3 dead. Daughters: 1 dead.

Brothers: 3 living. Sisters: 3 living.

Tattoo marks: glove on back of right hand.

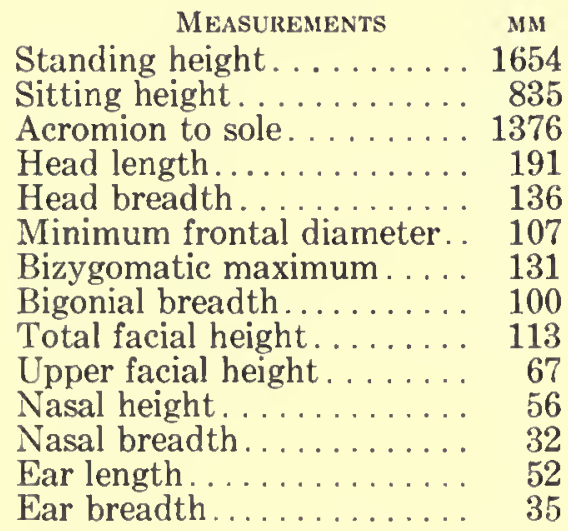

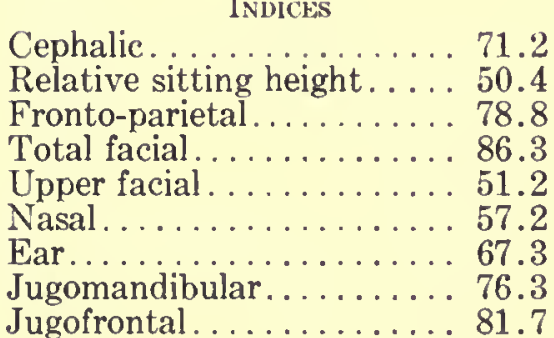


Musculature.-Good.

HEALTH.-Good.

\section{No. 615. Plate LXXX, Figs. 5, 6}

Place: Hilla Army Camp. Date: June 15, 1928.

Birthplace: Shatra. Age: 25; unmarried.

Brothers: 1 living. Sisters: 1 living.

Tattoo marks: 3 spots and line on back of right hand; 3 spots on back of left wrist.

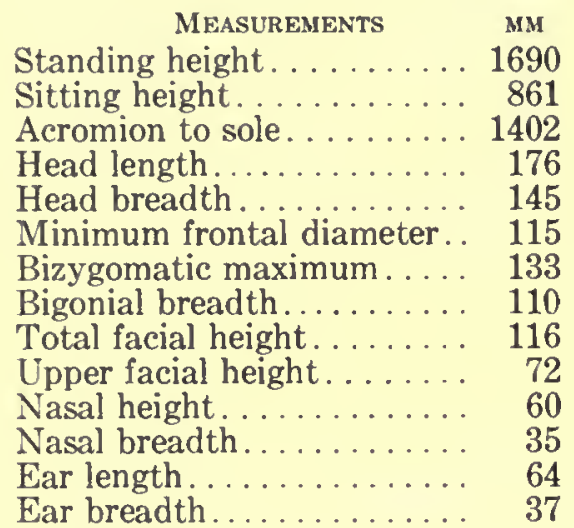

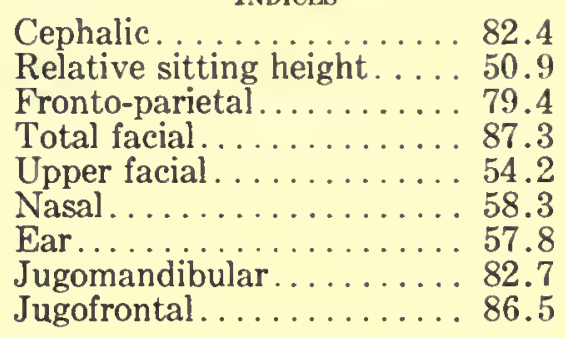

HAIR.-Color: dark brown.

EYE.-Color: dark brown. Sclera: clear. Iris: homogeneous.

NosE.-Profile: convex. Wings: medium.

TEETH.-Eruption: complete. Lost: none. Bite: slight over.

CHEST.-Development: plus.

Musculature.-Good.

HEALTH.-Good.

\section{No. 616. Plate LXXX, Figs. 7, 8}

Place: Hilla Army Camp. Date: June 15, 1928.

Birthplace: Nasiriya. Age: 25; unmarried.

Brothers: 2 living. Sisters: 1 living, 1 dead.

Tattoo marks: 6 spots on back of left hand.

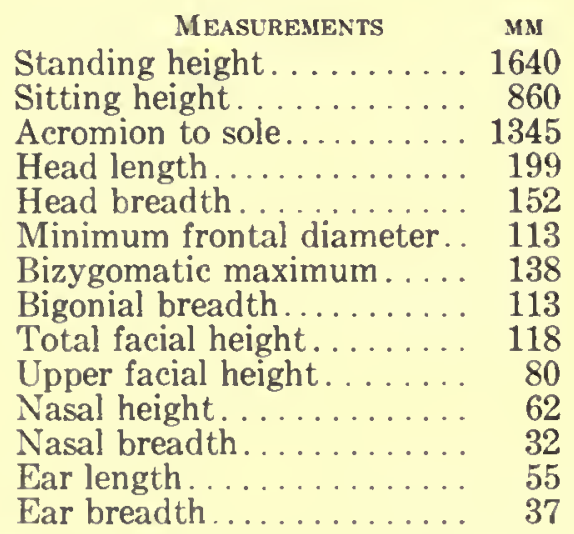

EYE.- Color: blue brown. Sclera: clear. Iris: zoned.

NoSE.-Profile: straight. Wings: medium.

TEeTH.-Eruption: complete. Lost: none. Bite: slight over.

Chest.-Development: plus. 
Musculature.-Good.

HEALTH.-Good.

No. 617. Plate LXXXI, Figs. 1, 2

Place: Hilla Army Camp. Date: June 15, 1928.

Birthplace: Nasiriya. Age: 23; unmarried.

Brothers: 4 living. Sisters: 1 living.

Tattoo marks: spot on each temple.

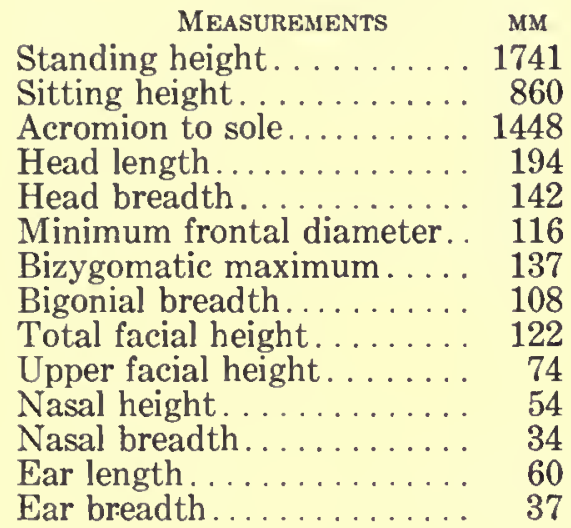

INDICES
Cephalic............ 73.2

Relative sitting height..... 49.4

Fronto-parietal. ......... 81.7

Total facial . . . . . . . . . 89.1

Upper facial . . . . . . . . . 53.6

Nasal. . . . . . . . . . . 63.0

Ear.............6.6 61.6

Jugomandibular . . . . . . . 78.9

Jugofrontal . . . . . . . 84.7

EYE.-Color: dark brown. Sclera: clear. Iris: homogeneous.

NOSE.-Profile: straight. Wings: medium-flaring.

TEETH.-Eruption: complete. Lost: none. Bite: slight over.

Chest.-Development: plus.

Musculature.-Good.

HEALTH.—Good.

No. 618. Plate LXXXI, Figs. 3, 4

Place: Hilla Army Camp. Date: June 15, 1928.

Birthplace: Mosul. Age: 24; unmarried.

Brothers: 2 living. Sisters: 2 living.

Tattoo marks: glove on back of right hand.

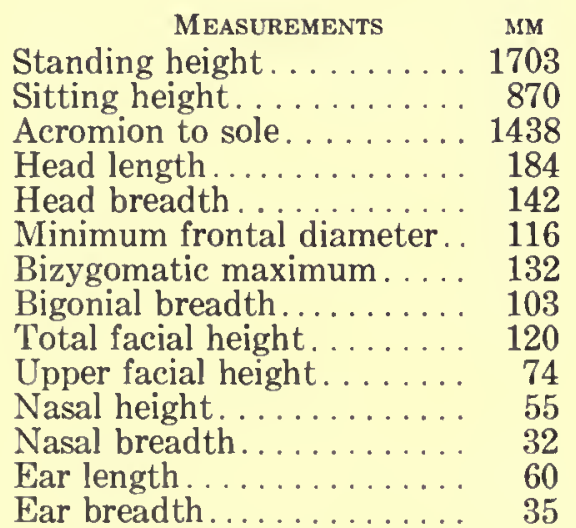

INDICES

Cephalic............77.3

Relative sitting height. . . 51.2

Fronto-parietal.........8 81.7

Total facial............ 91.0

Upper facial. .......... 56.1

Nasal. ............... 58.2

Ear................ 58.4

Jugomandibular. . . . . . . 78.0

Jugofrontal. . . . . . . . 87.9

EYE.-Color: dark brown. Sclera: clear. Iris: homogeneous.

Nose.-Profile: straight. Wings: medium.

TEETH.-Eruption: complete. Lost: none. Bite: slight over.

CHEST.-Development: plus.

Musculature.-Good.

HEALTH.-Good. 


\section{No. 619. Plate LXXXI, Figs. 5, 6}

Place: Hilla Army Camp. Date: June 15, 1928.

Birthplace: Mosul. Age: 25; married for 1 year. No children.

Brothers: 1 living. Sisters: 1 living.

Tattoo marks: none.

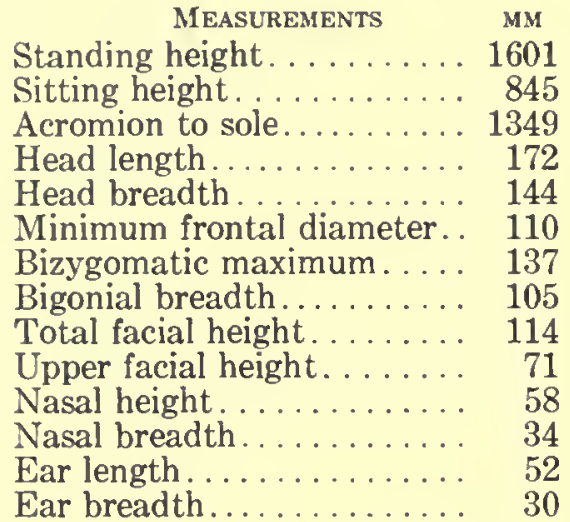

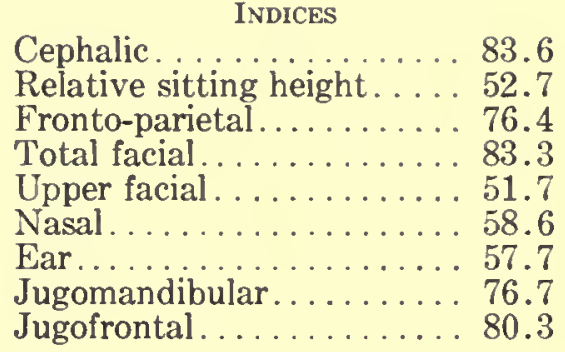

EYE.-Color: dark brown. Sclera: clear. Iris: homogeneous.

NosE.-Profile: convex. Wings: medium-flaring.

TEETH.-Eruption: complete. Lost: none. Bite: slight over.

CHest.-Development: plus.

Musculature.-Good.

HEALTH.-Good.

\section{No. 620. Plate LXXXI, Figs. 7, 8}

Place: Hilla Army Camp. Date: June 15, 1928.

Birthplace: Basra. Age: 24; unmarried.

Brothers: 2 living. Sisters: none.

Tattoo marks: none.

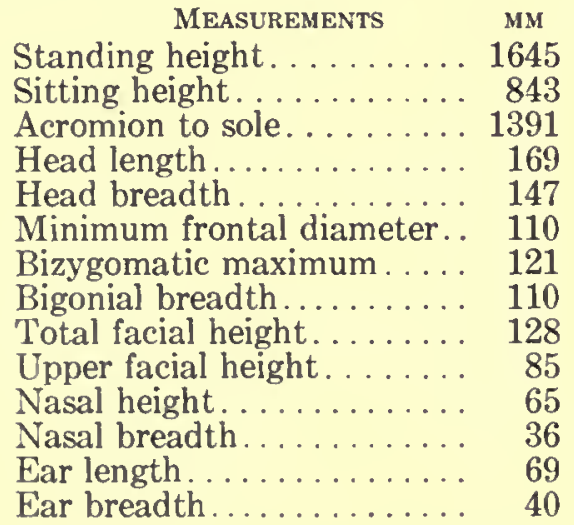

Cephalic............. 87.0

Relative sitting height. ... 51.2

Fronto-parietal......... 74.7

Total facial. . . . . . . . . . 105.8

Upper facial ............ 70.2

Nasal. . . . . . . . . . . . 55.4

Ear...................... 59.4

Jugomandibular . . . . . . . 90.9

Jugofrontal . . . . . . . . . . . . 90.9

EyE.-Color: gray brown. Sclera: clear. Iris: zoned.

Nose.-Profile: convex. Wings: medium.

TEETH.-Eruption: complete. Lost: 1. Bite: marked over.

CHEST.-Development: plus.

Musculature.-Good.

HEALTH.-Good.

Note: marked facial prognathism. 
No. 621. Plate LXXXII, Figs. 1, 2

Place: Hilla Army Camp. Date: June 15, 1928.

Birthplace: Amara. Age: 26; married for 3 years. No children.

Brothers: 1 living. Sisters: 2 living.

Tattoo marks: glove on back of right hand and gazelle and spots on inside of right forearm.

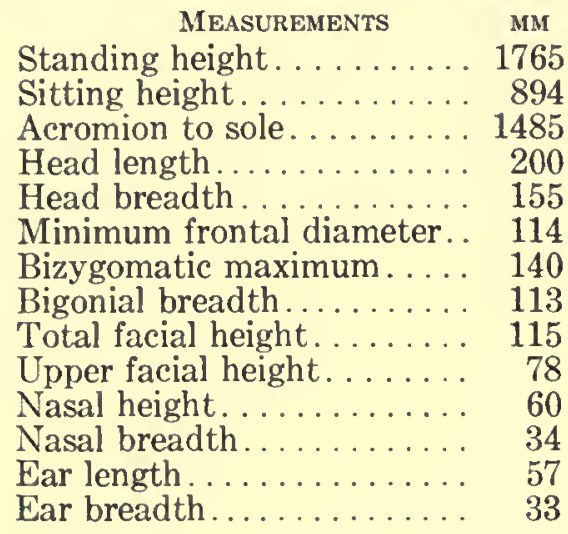

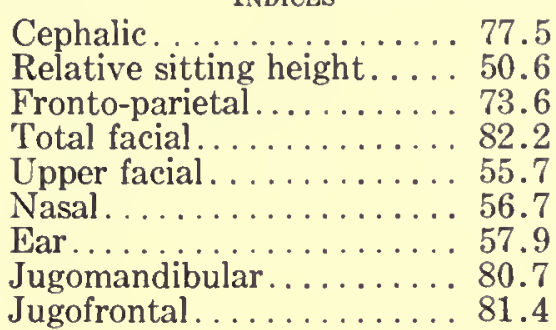

Cephalic............. 77.5

Relative sitting height. ... 50.6

Fronto-parietal.......... 73.6

Total facial ........... 82.2

Ear. .................... 57.9

Jugofrontal . . . . . . . . . . 81.4

EyE.-Color: dark brown. Sclera: clear. Iris: homogeneous.

Nose.-Profile: straight. Wings: medium.

TEETH.-Eruption: complete. Lost: none. Bite: slight over.

CHEST.-Development: plus.

Musculature.-Good.

Health.-Good.

No. 622. Plate LXXXII, Figs. 3, 4

Place: Hilla Army Camp. Date: June 15, 1928.

Birthplace: Amara. Age: 26; married for 3 years. No children.

Brothers: 1 living, 1 dead. Sisters: 3 living.

Tattoo marks: none.

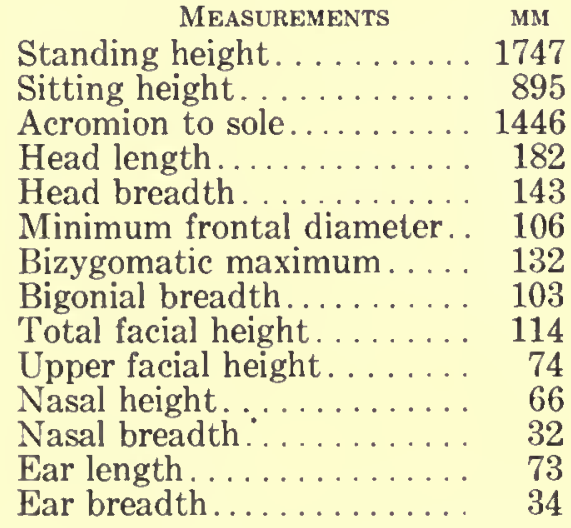

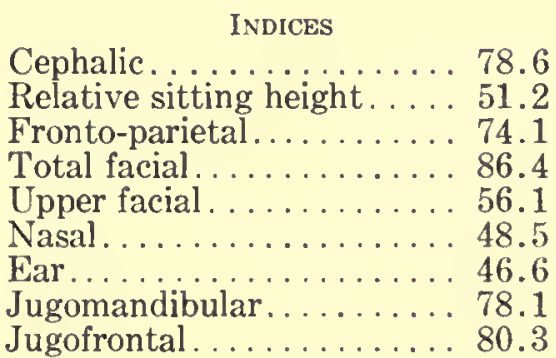

EYs.-Color: dark brown. Sclera: clear. Iris: homogeneous.

Nose.-Profile: convex. Wings: medium.

TEETH.-Eruption: complete. Lost: none. Bite: slight over.

CHest.-Development: plus.

Musculature.-Good.

HEALTH.-Good.

No. 623. Plate LXXXII, Figs. 5, 6

Place: Hilla Army Camp. Date: June 15, 1928.

Birthplace: Amara. Age: 26; unmarried. 
Brothers: 2 living. Sisters: 1 living.

Tattoo marks: line on right index finger and spot on each temple.

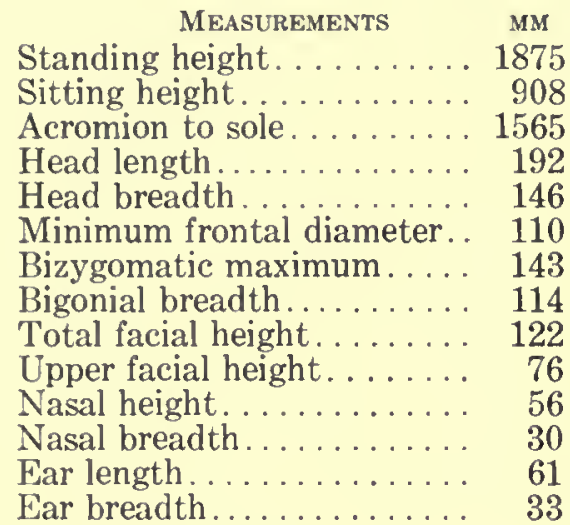

Cephalic............76 76.1

Relative sitting height..... 48.4

Fronto-parietal......... 75.4

Total facial............ 85.4

Upper facial. .......... 53.1

Nasal. .............. 53.6

Ear................ 54.2

Jugomandibular. . . . . . . 79.7

Jugofrontal. ......... 76.9

HAIR,-Color: dark brown.

EYE.-Color: dark brown. Sclera: clear. Iris: homogeneous.

Nose.-Profile: straight. Wings: medium-compressed.

TEETH.-Eruption: complete. Lost: none. Bite: slight over.

CHEst.-Development: plus.

Musculature.--Good.

HEALTH.-Good.

No. 624. Plate LXXXII, Figs. 7, 8

Place: Hilla Army Camp. Date: June 15, 1928.

Birthplace: Amara. Age: 20; unmarried.

Brothers: 1 living. Sisters: 1 dead.

Tattoo marks: glove on back of left hand.

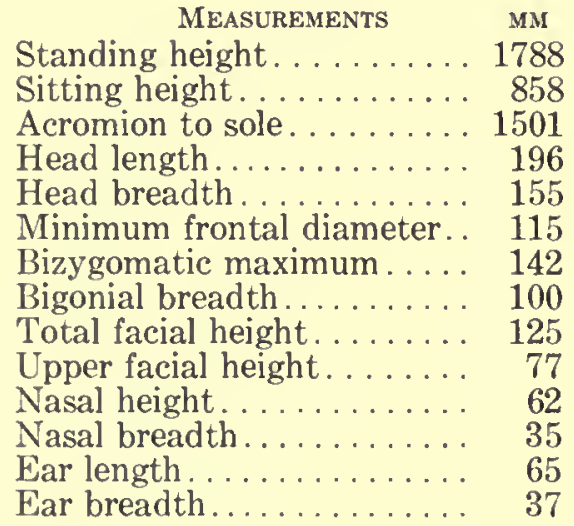

Cephalic.............79.1

Relative sitting height..... 48.0

Fronto-parietal..........74.3

Total facial............ 88.0

Upper facial. . . . . . . . . 54.2

Nasal. . . . . . . . . . . . 56.4

Ear................ 56.9

Jugomandibular . . . . . . . 70.4

Jugofrontal. .......... 81.0

EYE.-Color: dark brown. Sclera: clear. Iris: homogeneous.

Nose.-Profile: straight. Wings: medium.

TEeтH.-Eruption: complete. Lost: none. Bite: slight over.

Chest.-Development: plus.

Musculature.-Good.

Health.-Good.

No. 625. Plate LXXXIII, Figs. 1, 2

Place: Hilla Army Camp. Date: June 15, 1928.

Birthplace: Amara. Age: 23; married for 4 years. No children. 
Brothers: 1 living. Sisters: 3 living.

Tattoo marks: glove on back of right hand and gazelle on inside of right forearm.

\begin{tabular}{|c|c|c|}
\hline SUREMENTS & MM & INDICES \\
\hline Standing height. & 1747 & Cephalic \\
\hline Sitting height. & 908 & Relative sitting height. \\
\hline Acromion to sole. & 1456 & Fronto-parietal....... \\
\hline Head length. & 197 & Total facial. \\
\hline Head breadth. & 158 & Upper facial \\
\hline Minimum frontal diameter & 117 & Nasal. \\
\hline Bizygomatic maximum. & 148 & Ear. \\
\hline Bigonial breadth....... & 113 & Jugomandibular. \\
\hline Total facial height. & 123 & Jugofrontal. \\
\hline Upper facial height. & 74 & \\
\hline Nasal height. & 60 & \\
\hline Jasal breadth & 35 & \\
\hline Ear length & 7( & \\
\hline ea & & \\
\hline
\end{tabular}

EYE.-Color: dark brown. Sclera: clear. Iris: homogeneous.

Nose.-Profile: convex. Wings: flaring.

TEETH.-Eruption: complete. Lost: 1. Bite: slight over.

Chest.-Development: plus.

Musculature.-Good.

Health.-Good.

\section{No. 626. Plate LXXXIII, Figs. 3, 4}

Place: Hilla Army Camp. Date: June 15, 1928.

Birthplace: near Kirkuk. Age: 26; unmarried.

Brothers: none. Sisters: none.

Tattoo marks: long slanting line crossed by 8 short lines on left wrist.

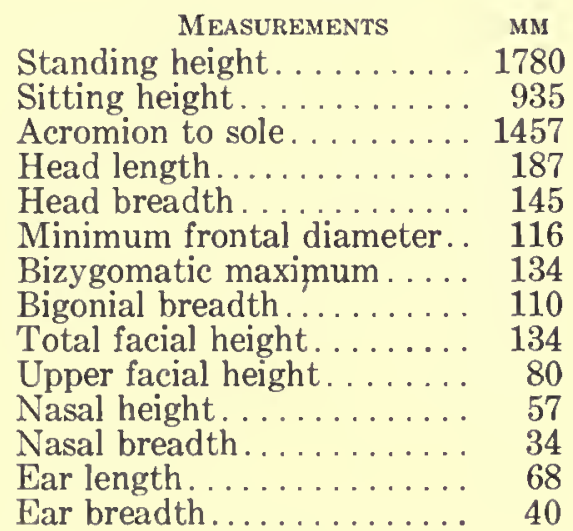

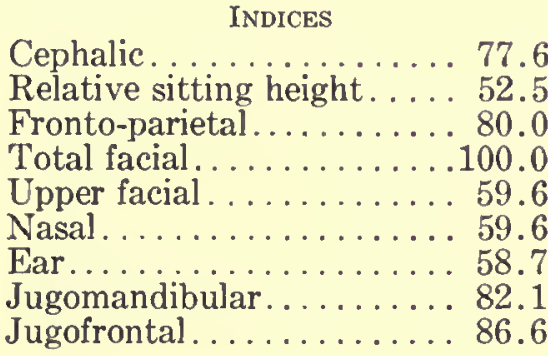

HAIR.-Color: dark brown.

EYE.-Color: dark brown. Sclera: clear. Iris: homogeneous.

Nose.-Profile: convex. Wings: medium.

TEETH.-Eruption: complete. Lost: none. Bite: slight over.

Chest,-Development: plus.

Musculature.-Good.

HEALTH.-Good.

No. 627

Place: Hilla Army Camp. Date: June 15, 1928.

Birthplace: Kirkuk. Age: 22 ; unmarried. 
Brothers: 2 living, 1 dead. Sisters: none.

Tattoo marks: none.

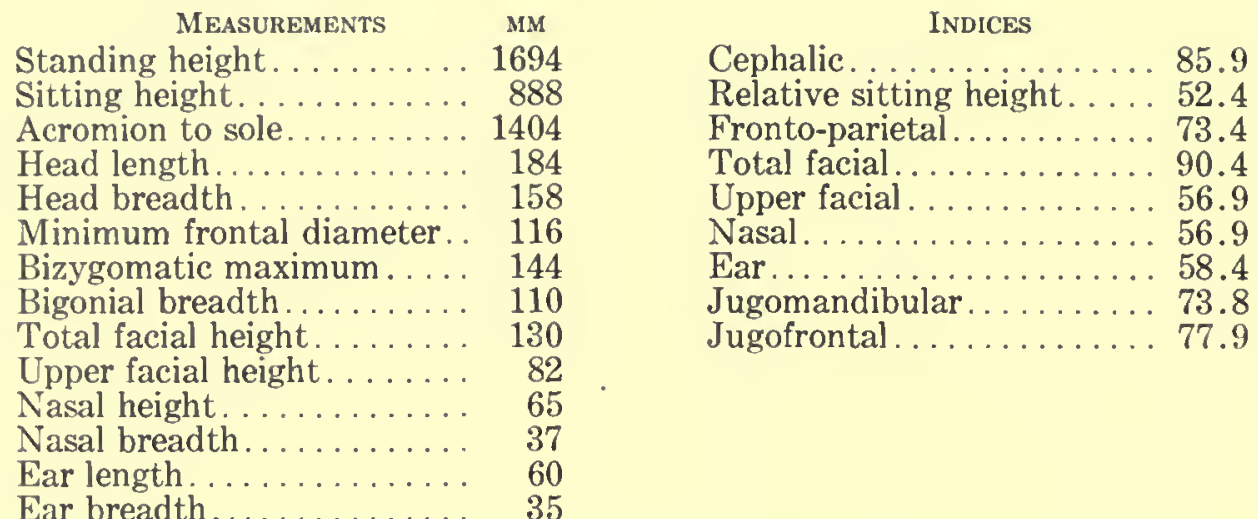

EYE.-Color: dark brown. Sclera: clear. Iris: homogeneous.

NosE.-Profile: concavo-convex. Wings: flaring.

TeETH.-Eruption: complete. Lost: none. Bite: slight over.

CHEST.-Development: double plus.

Musculature.-Excellent.

HEALTH.-Good.

No. 628. Plate LXXXIII, Figs. 5, 6

Place: Hilla Army Camp. Date: June 15, 1928.

Birthplace: Ad Diwaniya. Age: 28; married twice, 7 and 1 years ago. Sons: 1,0 , living. Daughters: none.

Brothers: 1 living, 3 dead. Sisters: 2 living.

Tattoo marks: glove on back of right hand.

\begin{tabular}{|c|c|c|}
\hline MEasurements & MM & INDICES \\
\hline Standing height. . . . . . & 1750 & Cephalic. \\
\hline Sitting height. & 918 & Relative sitting height. . \\
\hline Acromion to sole. . & 1465 & Fronto-parietal........ \\
\hline Head length.... & 188 & Total facial. \\
\hline Head breadth. & 147 & Upper facial \\
\hline Minimum frontal diameter. & 122 & Nasal. .... \\
\hline Bizygomatic maximum.... & 137 & . 54.8 \\
\hline Bigonial breadth.......... & 116 & Jugomandibular. . . . . . \\
\hline Total facial height. . . . . . . . & 116 & Jugofrontal. . . . . . . . . 89.1 \\
\hline Upper facial height. . . . . . . & 71 & \\
\hline Nasal height. . . . . . . . . . & 46 & \\
\hline Nasal breadth. . . . . . . . . . & 34 & \\
\hline 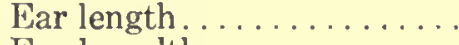 & 62 & \\
\hline breadth............. & 34 & \\
\hline
\end{tabular}

EYE.-Color: blue brown. Sclera: clear. Iris: zoned.

Nose.-Profile: concavo-convex. Wings: flaring.

TEETH.-Eruption: complete. Lost: none. Bite: slight over.

Chest.-Development: plus.

Musculature.-Good.

HEALTH.-Good.

No. 629. Plate LXXXIII, Figs. 7, 8

Place: Hilla Army Camp. Date: June 15, 1928.

Birthplace: Nasiriya. Age: 25; unmarried. 
Brothers: 2 living. Sisters: 1 living.

Tattoo marks: none.

\begin{tabular}{|c|c|}
\hline MEASUREMENTS & MM \\
\hline Standing height & 1773 \\
\hline Sitting height & $84 ?$ \\
\hline Acromion to sole & 1505 \\
\hline Head length & 191 \\
\hline Head breadth & 147 \\
\hline Minimum frontal diameter. & 123 \\
\hline Bizygomatic maximum ..... & 138 \\
\hline Bigonial breadth & 11 \\
\hline Total facial height. & 125 \\
\hline Upper facial height. & 74 \\
\hline Nasal height & 57 \\
\hline Nasal breadth & 34 \\
\hline Ear length & 68 \\
\hline & \\
\hline
\end{tabular}

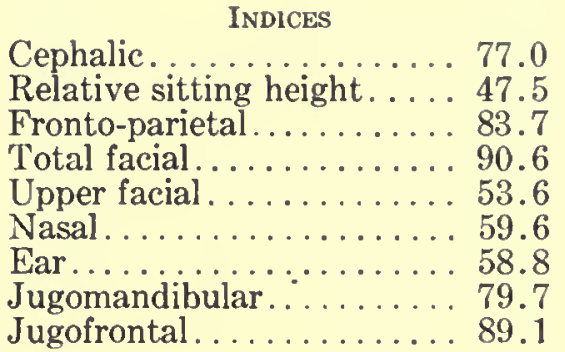

No. 630

Place: Hilla Army Camp. Date: June 15, 1928.

Birthplace: Nasiriya. Age: 30 ; unmarried.

Brothers: 2 living. Sisters: 2 living.

Tattoo marks: none.

\begin{tabular}{|c|c|}
\hline MEASUREMENTS & MM \\
\hline Standing height. & 1805 \\
\hline Sitting height. & 910 \\
\hline Acromion to sole. & 1531 \\
\hline Head length. & 191 \\
\hline Head breadth & 142 \\
\hline Minimum frontal diameter. . & 11 \\
\hline Bizygomatic maximum . . . . . & 134 \\
\hline Bigonial breadth........ & 10. \\
\hline Total facial height. & 12 \\
\hline Upper facial height. . . . & $7 ?$ \\
\hline Nasal height & 64 \\
\hline Nasal breadth & 33 \\
\hline Ea & $6^{\prime}$ \\
\hline & \\
\hline
\end{tabular}

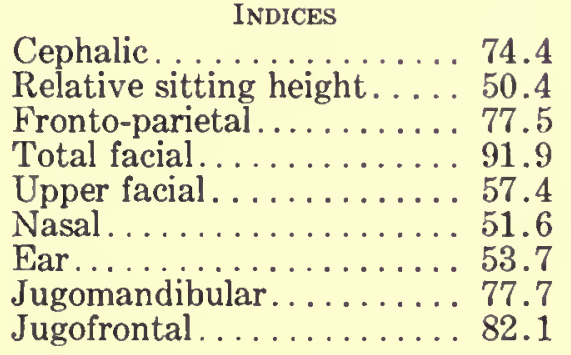

EYE.-Color: blue brown. Sclera: clear. Iris: zoned.

Nose.-Profile: convex. Tip: thickness, double plus. Wings: medium.

TEeтн.-Eruption: complete. Lost: none. Bite: slight over.

Chest.-Development: plus.

Musculature.-Good.

HEALTH.-Good.

No. 631

Place: Hilla Army Camp. Date: June 15, 1928.

Birthplace: Hilla. Age: 30; married for 5 years. Sons: none. Daughters: 2 living.

Brothers: 1 living. Sisters: 1 living.

Tattoo marks: spot on back of right wrist. 


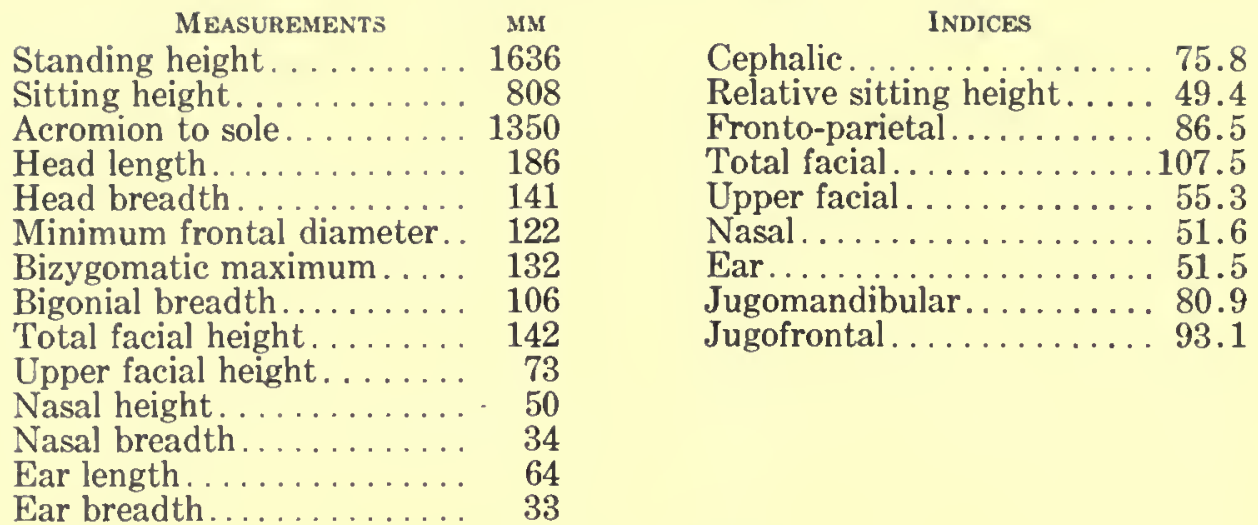

EYE.-Color: dark brown. Sclera: clear. Iris: homogeneous.

NosE.-Profile: concavo-convex. Wings: medium-flaring.

TEETH.-Eruption: complete. Lost: 1. Bite: slight over.

CHEST.-Development: plus.

Musculature.-Good.

HEALTH.-Good.

No. 632. Plate LXXXIV, Figs. 1, 2

Place: Hilla Army Camp. Date: June 15, 1928.

Birthplace: near Kirkuk. Age: 25; unmarried.

Brothers: 6 living. Sisters: none.

Tattoo marks: none.

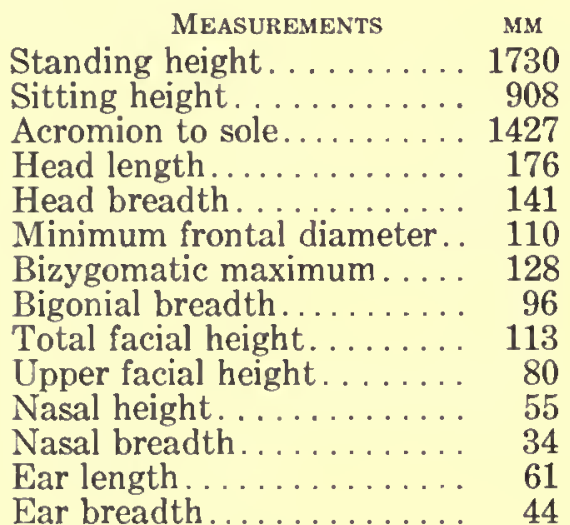

INDICES

Cephalic............. 80.1 Relative sitting height..... 52.4 Fronto-parietal........ 77.0 Total facial............ 88.4 Upper facial. . . ........ 61.7 Nasal. . . . . . . . . . . 61.8

Ear............... 72.2 Jugomandibular. . . . . . . 75.0

Jugofrontal. . . . . . . 85.9

HAIR.-Color: dark brown.

EYE.-Color: blue brown. Sclera: clear. Iris: zoned.

Nose.-Profile: straight. Wings: medium.

TEETH.-Eruption: complete. Lost: none. Bite: slight over.

Chest.-Development: plus.

Musculature.-Good.

HEALTH.-Good.

\section{No. 633. Plate LXXXIV, Figs. 3, 4}

Place: Hilla Army Camp. Date: June 15, 1928.

Birthplace: Nasiriya. Age: 25; unmarried.

Brothers: 4 living. Sisters: 2 living.

Tattoo marks: glove on back of right hand and circle of spots on each temple. 


\begin{tabular}{|c|c|}
\hline MEASUREMENTS & MM \\
\hline Standing height & 1650 \\
\hline Sitting height. & 858 \\
\hline Acromion to sole. & 1480 \\
\hline Head length.... & 186 \\
\hline Head breadth & 142 \\
\hline Minimum frontal diameter. & 113 \\
\hline Bizygomatic maximum . ... & 130 \\
\hline Bigonial breadth . . . . . . . . & 106 \\
\hline Total facial height & 122 \\
\hline Upper facial height. . . . . . & 73 \\
\hline Nasal height & 60 \\
\hline brea & 38 \\
\hline Ea & 63 \\
\hline & 42 \\
\hline
\end{tabular}

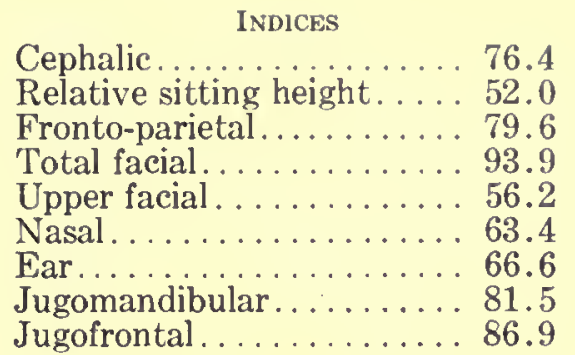

EYE.-Color: dark brown. Sclera: clear. Iris: homogeneous.

Nose.-Profile: convex. Wings: medium.

TeETH.-Eruption: complete. Lost: none. Bite: slight over.

Chest.-Development: plus.

Musculature.-Good.

HEALTH.-Good.

No. 634. Plate LXXXIV, Figs. 5, 6

Place: Hilla Army Camp. Date: June 15, 1928.

Birthplace: near Ramadi. Age: 20; unmarried.

Brothers: 2 living. Sisters: 1 living.

Tattoo marks: none.

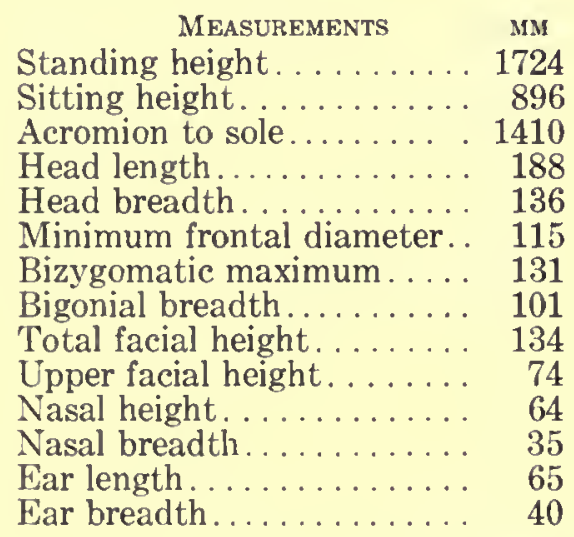

INDICES

Cephalic............ 72.3

Relative sitting height .... 52.0

Fronto-parietal..........84.6

Total facial..............102.1

Upper facial. . . . . . . . . . . 56.4

Nasal. . . . . . . . . . . . 55.7

Ear.................6. 61.5

Jugomandibular. . . . . . . . 77.1

Jugofrontal . . . . . . . . . . 87.8

EYE.-Color: dark brown. Sclera: clear. Iris: homogeneous.

Nose.-Profile: convex. Wings: medium-flaring.

TЕEтH.-Eruption: complete. Lost: none. Bite: slight over.

CHEST.-Development: plus.

Musculature.-Good.

HEALTH.-Good.

No. 635

Place: Hilla Army Camp. Date: June 15, 1928.

Birthplace: Amara. Age: 20; unmarried.

Brothers: 2 living. Sisters: 2 living.

Tattoo marks: line on back of right wrist.

MEASUREMENTS

Standing height........ 1733

Sitting height........... 873
INDICES

Cephalic............. 75.0

Relative sitting height.... 50.3 


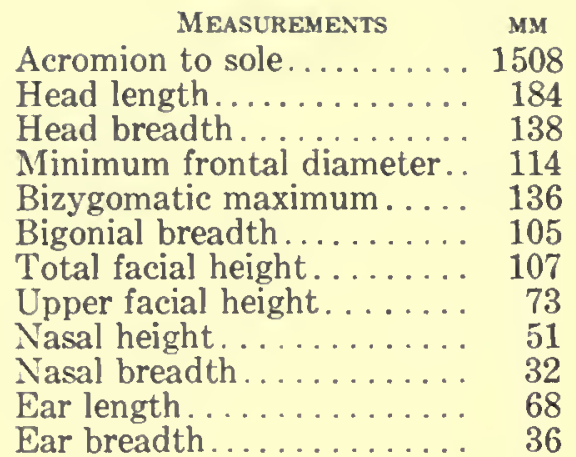

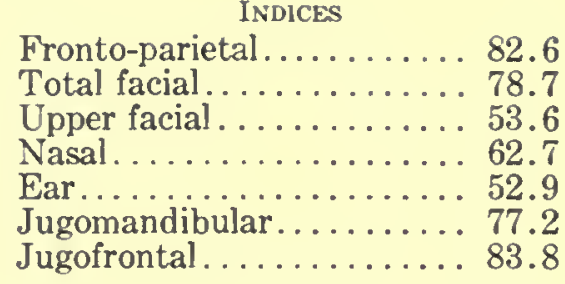

EYE.-Color: dark brown. Sclera: clear. Iris: homogeneous.

Nose.-Profile: straight. Tip: thickness, minus.

TEETH.-Eruption: complete. Lost: none. Bite: slight over.

ChEsT.-Development: plus.

Musculature.-Good.

HEALTH.-Good.

No. 636. Plate LXXXIV, Figs. 7, 8

Place: Hilla Army Camp. Date: June 15, 1928.

Birthplace: Basra. Age: 30 ; married for 3 years. No children.

Brothers: 1 living. Sisters: 1 living.

Tattoo marks: none.

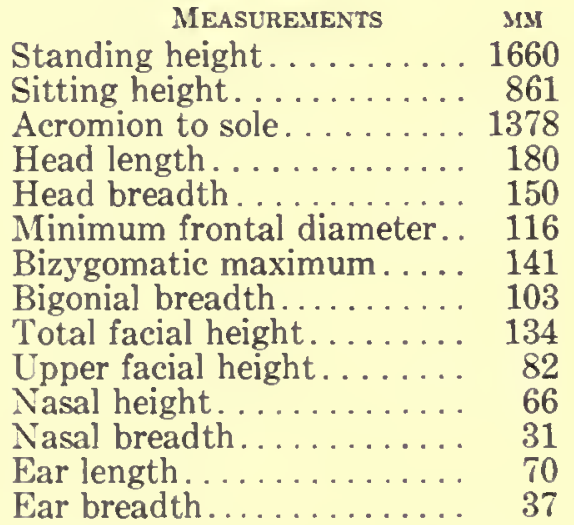

El'E.-Color: dark brown. Sclera: clear. Iris: homogeneous.

Nose.-Profile: convex. Wings: medium-compressed.

TEETH.-Eruption: complete. Lost: none. Bite: slight over.

CHEst.-Development: plus.

Musculature.-Good.

HEALTH.-Good.

No. 637. Plate LXXXV, Figs. 1, 2

Place: Hilla Army Camp. Date: June 15, 1928.

Birthplace: Amara. Age: 30; unmarried.

Brothers: 3 living, 1 dead. Sisters: 2 living.

Tattoo marks: glove on back of right hand.

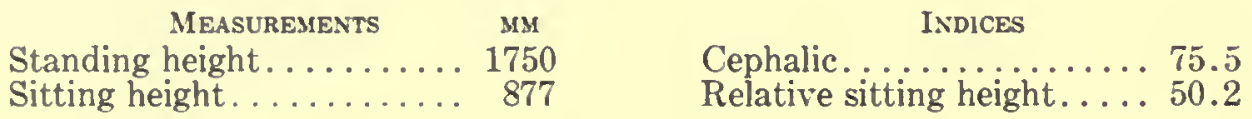




\begin{tabular}{|c|c|}
\hline MEASUREMEnTS & MM \\
\hline Acromion to sole. & 1490 \\
\hline Head length & 188 \\
\hline Head breadth & 142 \\
\hline Minimum frontal diameter. & 113 \\
\hline Bizygomatic maximum . ... . & 131 \\
\hline Bigonial breadth.......... & 110 \\
\hline Total facial height. . . . . . . & 120 \\
\hline Upper facial height. . . . . . & 80 \\
\hline Nasal height. & 50 \\
\hline Nasal breadth & 30 \\
\hline Ear length & 54 \\
\hline & 3 \\
\hline
\end{tabular}

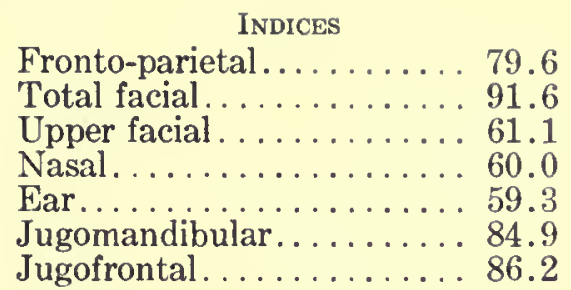

EYE.-Color: dark brown. Sclera: clear. Iris: homogeneous.

Nose.-Profile: straight. Wings: medium-compressed.

TEETH.-Eruption: complete. Lost: none. Bite: slight over.

Chest.-Development: plus.

Musculature.-Good.

HEALTH.-Good.

No. 638. Plate LXXXV, Figs. 3, 4

Place: Hilla Army Camp. Date: June 15, 1928.

Birthplace: Nasiriya. Age: 30 ; married twice, 5 and 1 years ago. No children.

Brothers: none. Sisters: 1 living.

Tattoo marks: glove on back of right hand.

\begin{tabular}{|c|c|}
\hline MEasurements & MM \\
\hline Standing height. & 1730 \\
\hline Sitting height. & 909 \\
\hline Acromion to sole. & 1441 \\
\hline Head length. & 180 \\
\hline Head breadth & 143 \\
\hline Minimum frontal diameter. & 113 \\
\hline Bizygomatic maximum. .... & 137 \\
\hline Bigonial breadth.......... & 107 \\
\hline Total facial height......... & 122 \\
\hline Upper facial height. . . . . . & 78 \\
\hline Nasal height. & 63 \\
\hline Nasal breadth & 40 \\
\hline Ear length & 71 \\
\hline & 34 \\
\hline
\end{tabular}

INDICES

Cephalic............. 79.5

Relative sitting height. . . . 52.5

Fronto-parietal......... 79.1

Total facial........... 89.1

Upper facial. ............. 55.4

Nasal. ............63.5

Ear................. 47.9

Jugomandibular......... 78.1

Jugofrontal . . . . . . . . 82.5

HAIR.-Color: dark brown and gray.

EyE.-Color: dark brown. Sclera: clear. Iris: homogeneous.

Nose.-Profile: concave. Tip: thickness, double plus. Wings: medium-flaring.

TEETH.-Eruption: complete. Lost: none. Bite: slight over.

ChEST.-Development: plus.

Musculature.-Good.

HEALTH.-Good.

No. 639. Plate LXXXV, Figs. 5, 6

Place: Hilla Army Camp. Date: June 15, 1928.

Birthplace: Shatra. Age: 24 ; married for 5 months.

Brothers: 1 living. Sisters: none.

Tattoo marks: line on back of right wrist.

Measurements MM

Standing height. . . . . . 1685

Sitting height. . . . . . . 859
INDICES

Cephalic.............78.3

Relative sitting height.... 51.0 


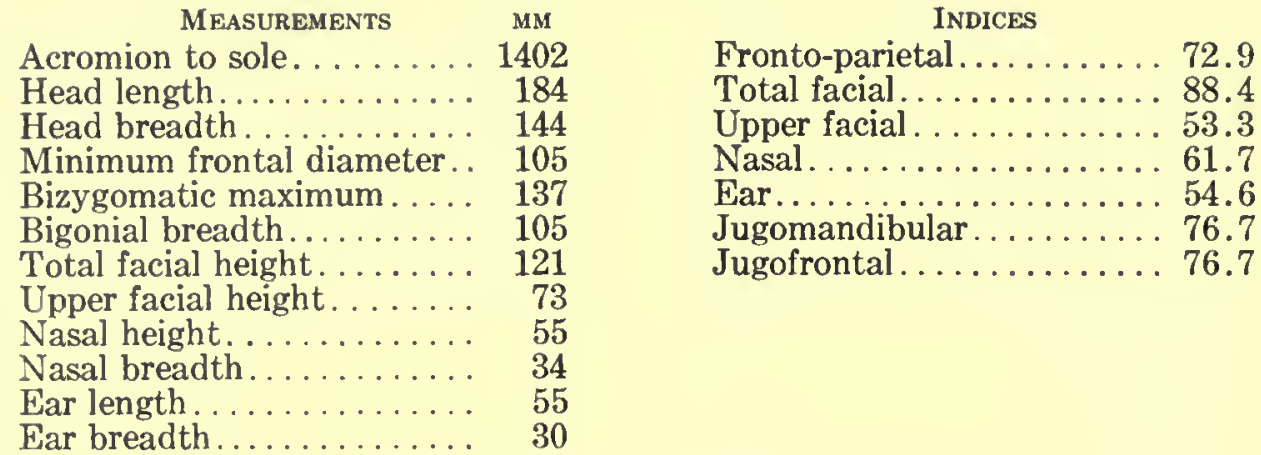

HAIR.-Color: dark brown.

EYE.-Color: dark brown. Sclera: clear. Iris: homogeneous.

Nose.-Profile: straight. Wings: medium-compressed.

TEETH.-Eruption: complete. Lost: none. Bite: slight over.

CHEsT.-Development: plus.

Musculature.-Good.

HEALTH.--Good.

No. 640

Place: Hilla Army Camp. Date: June 15, 1928.

Birthplace: Nasiriya. Age: 25; unmarried.

Brothers: 2 living. Sisters: 2 living.

Tattoo marks: none.

\begin{tabular}{|c|c|}
\hline MEASUREMENTS & MM \\
\hline Standing height. & 1698 \\
\hline Sitting height. & 884 \\
\hline Acromion to sole. & 1436 \\
\hline Head length. & $188^{\circ}$ \\
\hline Head breadth & 143 \\
\hline Minimum frontal diameter. . & 113 \\
\hline Bizygomatic maximum . . . . & 137 \\
\hline Bigonial breadth. & 104 \\
\hline Total facial height. & 131 \\
\hline Upper facial height. . . & 86 \\
\hline Nasal height & 61 \\
\hline breadt & 35 \\
\hline $\mathrm{Ea}$ & 64 \\
\hline & \\
\hline
\end{tabular}

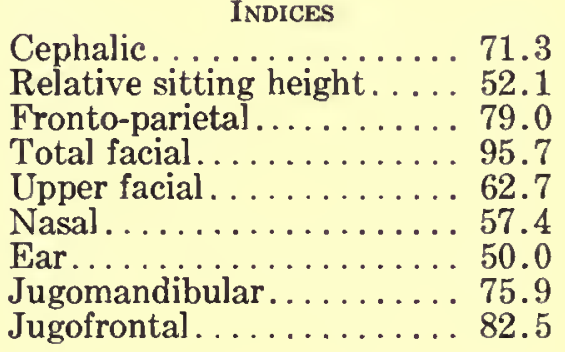

EYE.-Color: dark brown. Sclera: clear. Iris: homogeneous.

NosE.-Profile: straight. Wings: medium-flaring.

TEETH.-Eruption: complete. Lost: none. Bite: slight over.

CHEST.-Development: plus.

Musculature.-Good.

HEALTH.-Good.

No. 641

Place: Hilla Army Camp. Date: June 15, 1928.

Birthplace: Nasiriya. Age: 20; unmarried.

Brothers: 3 living. Sisters: 2 living.

Tattoo marks: none.

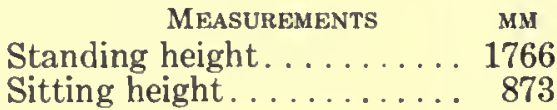

Cephalic............ 75.0

Relative sitting height.... 49.4 


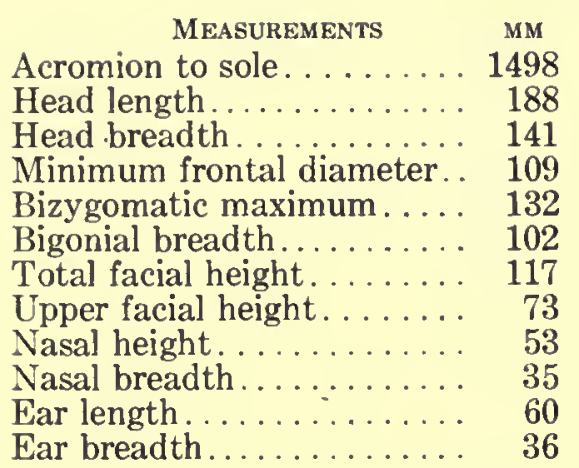

Fronto-parietal......... 77.4

Total facial ............ 88.7

Upper facial. . . . . . . . . 55.4

Nasal. .............66.1 66.1

Ear................6 60.0

Jugomandibular. . . . . . . . 77.3

Jugofrontal . . . . . . . . . . 82.6

EYE.-Color: dark brown. Sclera: clear. Iris: homogeneous.

Nose.-Profile: straight. Wings: medium.

TEETH.-Eruption: complete. Lost: none. Bite: slight over.

CHEST.-Development: plus.

Musculature.-Good.

HEALTH.-Good.

No. 642. Plate LXXXV, Figs. 7, 8

Place: Hilla Army Camp. Date: June 15, 1928.

Birthplace: Shatra. Age: 22; married for several months.

Brothers: 2 living. Sisters: 3 living.

Tattoo marks: 2 spots on back of right hand.

\begin{tabular}{|c|c|}
\hline MEASUREMENTS & $\mathrm{MM}$ \\
\hline Standing height. & 1727 \\
\hline Sitting height. & 858 \\
\hline Acromion to sole. & 1460 \\
\hline Head length. & 193 \\
\hline Head breadth & 157 \\
\hline Minimum frontal diameter. & 127 \\
\hline Bizygomatic maximum. .... & 148 \\
\hline Bigonial breadth.......... & 113 \\
\hline Total facial height. & 126 \\
\hline Upper facial height. . & 76 \\
\hline Nasal height & 52 \\
\hline Nasal breadth. . & 35 \\
\hline Ear length & 53 \\
\hline & 3 \\
\hline
\end{tabular}

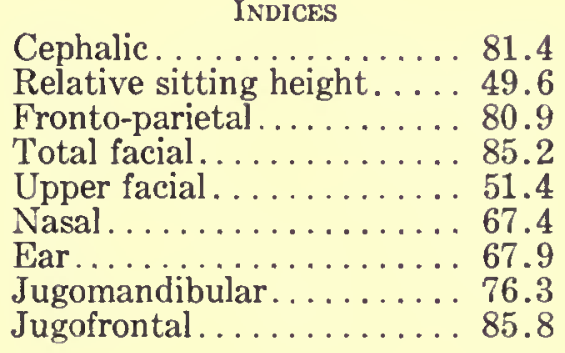

EYE.-Color: dark brown. Sclera: clear. Iris: homogeneous.

Nose.-Profile: straight. Wings: medium-compressed.

TEETH.-Eruption: complete. Lost: 1. Bite: slight over.

Chest.-Development: plus.

Musculature.-Good.

HEALTH.-Good.

No. 643. Plate LXXXVI, Figs. 1, 2

Place: Hilla Army Camp. Date: June 15, 1928.

Birthplace: Nasiriya. Age: 20; unmarried.

Brothers: 2 living. Sisters: 2 living.

Tattoo marks: none.

\begin{tabular}{|c|c|}
\hline MEASUREMENTS & мM \\
\hline Standing height. & 1702 \\
\hline Sitting height. & 859 \\
\hline
\end{tabular}

Cephalic........ 77.5

Relative sitting height. . ... 50.4

Fronto-parietal......... 84.9 


\begin{tabular}{|c|c|c|}
\hline MEASUREMENTS & MM & INDICES \\
\hline Head length. & 178 & Total facial... \\
\hline Head breadth. & 138 & Upper facial. \\
\hline Minimum frontal diameter. & 117 & Nasal. \\
\hline Bizygomatic maximum.... & 140 & Ear. \\
\hline Bigonial breadth...... & 108 & Jugomandibular. \\
\hline Total facial height. & 116 & Jugofrontal.... \\
\hline Upper facial height. & 71 & \\
\hline Nasal height. & 61 & \\
\hline Nasal breadth. & 30 & \\
\hline Ear length. & 60 & \\
\hline Ear breadth. & 36 & \\
\hline
\end{tabular}

HAIR.-Color: dark brown.

EYE.-Color: dark brown. Sclera: clear. Iris: homogeneous.

Nose.-Profile: straight.

TEETH.-Eruption: complete. Lost: none. Bite: slight over.

CHEsT.-Development: plus.

Musculature.-Good.

HEALTH.-Good.

No. 644. Plate LXXXVI, Figs. 3, 4

Place: Hilla Army Camp. Date: June 15, 1928.

Birthplace: Samawa. Age: 24; unmarried.

Brothers: 2 living. Sisters: 1 living.

Tattoo marks: none.

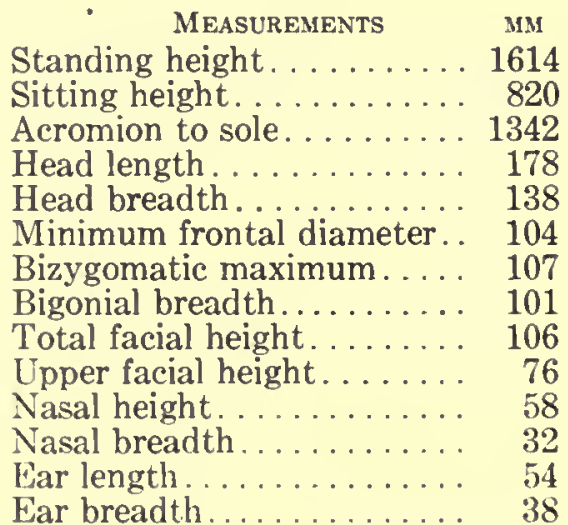

INDICES

Cephalic........... 77.5

Relative sitting height.... 50.7

Fronto-parietal......... 75.3

Total facial. . . . . . . . . . 99.1

Upper facial . . . . . . . . . 71.1

Nasal. .............. 55.2

Ear.................. 70.4

Jugomandibular. . . . . . . 9. 94.3

Jugofrontal. . . . . . . . . 97.1

HAIR.-Color: black.

EYE.-Color: dark brown. Sclera: clear. Iris: homogeneous.

NosE.-Profile: convex. Wings: medium-compressed.

TEETH.--Eruption: complete. Lost: none. Bite: slight over.

CHEst.-Development: plus.

Musculature.--Good.

HEALTH.-Good.

No. 645. Plate LXXXVI, Figs. 5, 6

Place: Hilla Army Camp. Date: June 15, 1928.

Birthplace: Karbala. Age: 25; married for 1 year. No children.

Brothers: 2 living. Sisters: 1 living.

Tattoo marks: none.

MEASUREMENTS MM

Standing height. . . . . . 1730

Sitting height. ......... 861
INDICES

Cephalic............. 77.8

Relative sitting height. . . 4 49.8 


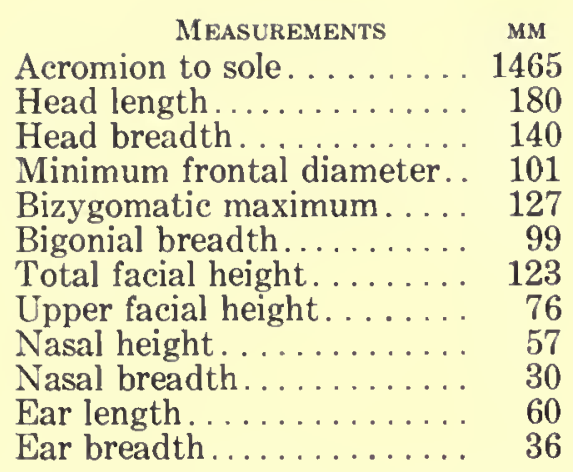

Fronto-parietal. ........ 72.1

Total facial............ 96.9

Upper facial ............ 59.7

Nasal ................. 52.6

Ear...............6. 60.0

Jugomandibular. . . . . . . 77.9

Jugofrontal. ............ 79.6

HAIR.-Color: dark brown.

EYk.-Color: dark brown. Sclera: clear. Iris: homogeneous.

Nose.-Profile: straight. Wings: medium.

TEETH.-Eruption: complete. Lost: none.

CHEST.-Development: plus.

Musculature.-Good.

HEALTH.-Good.

No. 646. Plate LXXXVI, Figs. 7, 8

Place: Hilla Army Camp. Date: June 15, 1928.

Birthplace: Mosul. Age: 22; unmarried.

Brothers: 1 living, 1 dead. Sisters: 1 dead.

Tattoo marks: figures 8090 in Arabic on inside of right forearm.

\begin{tabular}{|c|c|c|}
\hline MEASUREMENTS & MM & INDICES \\
\hline Standing height. . . . . . & 1668 & Cephalic. \\
\hline Sitting height. & 886 & Relative sitting height.... 53.1 \\
\hline Acromion to sole. & 1366 & Fronto-parietal........ \\
\hline Head length..... & 194 & Total facial. \\
\hline Head breadth. & 152 & Upper facial. \\
\hline Minimum frontal diameter. . & 113 & Nasal. \\
\hline Bizygomatic maximum.... & 140 & Ear. \\
\hline Bigonial breadth. ......... & 117 & Jugomandibular. . . \\
\hline Total facial height..... & 116 & Jugofrontal. . . . . \\
\hline Upper facial height. . . . . . . & 74 & \\
\hline Nasal height. & 57 & \\
\hline Nasal breadth. . & 35 & \\
\hline Ear length.... & 62 & \\
\hline Ear breadth... & 00 & \\
\hline
\end{tabular}

HAIR.-Color: dark brown.

EYE.-Color: dark brown. Sclera: clear. Iris: homogeneous.

Nose.-Profile: convex. Wings: medium-flaring.

TEETH.-Eruption: complete. Lost: none. Bite: slight over.

Chest.-Development: plus.

Musculature.-Good.

Health.-Good.

No. 647. Plate LXXXVII, Figs. 1, 2

Place: Hilla Army Camp. Date: June 15, 1928.

Birthplace: Shatra. Age: 22; unmarried.

Brothers: 2 living. Sisters: none.

Tattoo marks: none.

Measurements MM

Standing height. . . . . . 1707

Cephalic. ............... 74.3

Sitting height. . . . . . . . 843

Relative sitting height..... 49.4 


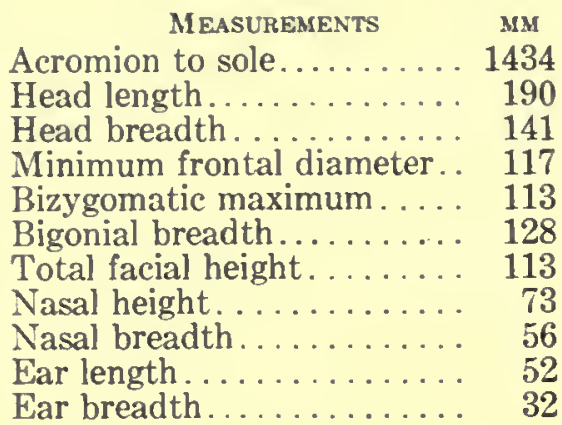

Fronto-parietal.......... 83.0

Total facial. . . . . . . 100.0

Nasal. . . . . . . . . . . . . . 76.7

Ear...............6.6 61.6

Jugomandibular. . . . . . 8. 88.3

Jugofrontal . . . . . . . . 88.3

EYE.-Color: dark brown. Sclera: clear. Iris: homogeneous.

Nose.-Profile: concave. Tip: thickness, triple plus. Wings: medium-flaring.

TEETH.-Eruption: complete. Lost: none. Bite: slight over.

CHEST.-Development: plus.

Musculature.-Good.

HEALTH.-Good.

\section{No. 648. Plate LXXXVII, Figs. 3, 4}

Place: Hilla Army Camp. Date: June 15, 1928.

Birthplace: Mandali. Age: 30 ; unmarried.

Brothers: none. Sisters: none.

Tattoo marks: gazelle on inside of right forearm.

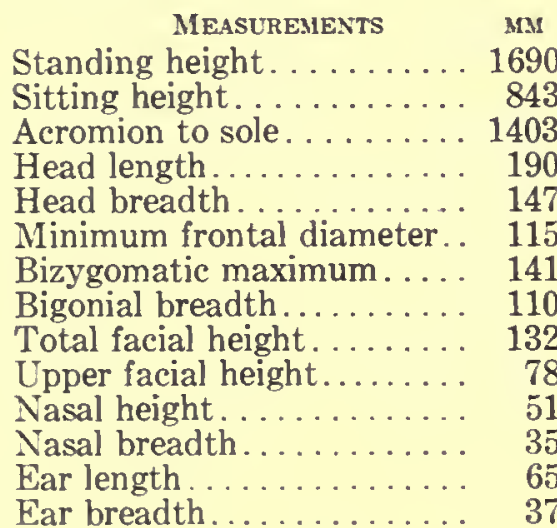

INDICES

Cephalic........... 77.4

Relative sitting height. . . . 49.8

Fronto-parietal........78.3

Total facial . . . . . . . . . . 93.6

Upper facial. ......... 55.4

Nasal................. 68.6

Ear.............. 56.9

Jugomandibular......... 78.0

Jugofrontal. . . . . . . . 81.5

HAIR.-Color: dark brown.

EYE.-Color: dark brown. Sclera: clear. Iris: homogeneous.

Nose.-Profile: convex. Wings: medium.

TEETH.-Eruption: complete. Lost: none. Bite: slight over.

CHEst.-Development: plus.

Musculature.-Good.

HEALTH.-Good.

Place: Hilla Army Camp. Date: June 16, 1928.

Birthplace: Hilla. Age: 20; unmarried.

Brothers: 1 living, 1 dead. Sisters: 2 living.

Tattoo marks: none.

\begin{tabular}{|c|c|}
\hline MEASUREMENTS & $M$ \\
\hline ding height. & 1661 \\
\hline Sitting height. & 811 \\
\hline Acromion to sc & 1345 \\
\hline Head length & 189 \\
\hline
\end{tabular}

Cephalic............. 74.1

Relative sitting height..... 48.8

Fronto-parietal......... 83.6

Total facial........... 92.4 


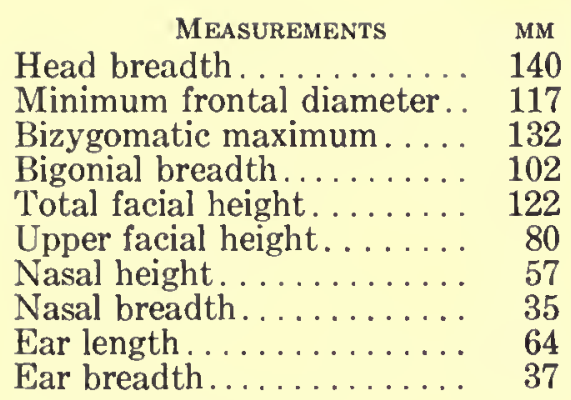

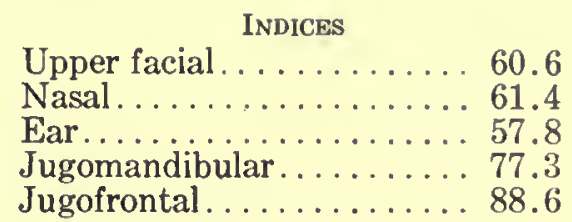

Upper facial. . . . . . . . 6 6 6.6.

Nasal................ 61.4

Jugomandibular. . . . . . 77.3

Jugofrontal . . . . . . . 88.6

HAIR.-Color: dark brown.

EYE.-Color: dark brown. Sclera: clear. Iris: homogeneous.

NosE.-Profile: straight. Wings: medium-compressed.

TEETH.-Eruption: complete. Lost: none. Bite: slight over.

\section{No. 651. Plate LXXXVII, Figs. 5, 6}

Place: Hilla Army Camp. Date: June 16, 1928.

Birthplace: Khanagin. Age: 22 ; married for 3 years. Sons: 1 living. Daughters: none. Brothers: 2 living. Sisters: 2 living.

Tattoo marks: none.

\begin{tabular}{|c|c|}
\hline \multirow{2}{*}{\multicolumn{2}{|c|}{ MEasurements }} \\
\hline \multirow{2}{*}{\multicolumn{2}{|c|}{ Standing height }} \\
\hline & \\
\hline & \\
\hline & \\
\hline & \\
\hline & \\
\hline & \\
\hline & \\
\hline & \\
\hline & \\
\hline & \\
\hline & \\
\hline & \\
\hline & \\
\hline & \\
\hline & \\
\hline Sitting height. ... & 832 \\
\hline Acromion to sole. & 1450 \\
\hline Head length. & 188 \\
\hline Head breadth. & 135 \\
\hline Minimum frontal diameter. & 105 \\
\hline Bizygomatic maximum. & 128 \\
\hline Bigonial breadth. & 108 \\
\hline Total facial height & 111 \\
\hline Upper facial height. & 66 \\
\hline Nasal height. & 55 \\
\hline Nasal breadth & 36 \\
\hline Ear length & 57 \\
\hline Far hroodtl & \\
\hline
\end{tabular}

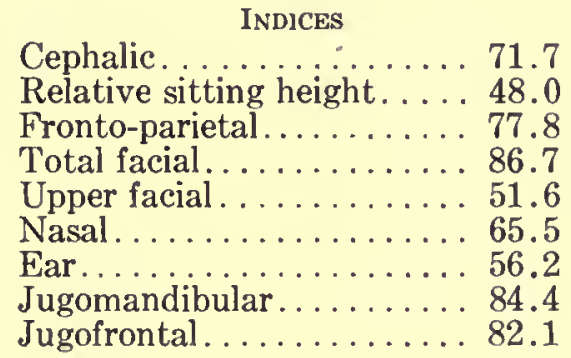

EYE.-Color: dark brown. Sclera: clear. Iris: homogeneous.

Nose.-Profile: straight. Wings: medium-flaring.

\section{No. 652. Plate LXXXVII, Figs. 7, 8}

Place: Hilla Army Camp. Date: June 16, 1928.

Birthplace: Amara. Age: 25; married for 1 year. No children.

Brothers: 4 living. Sisters: 1 living.

Tattoo marks: none.

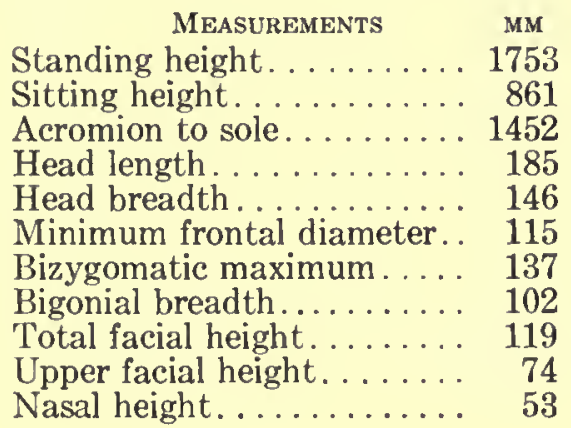

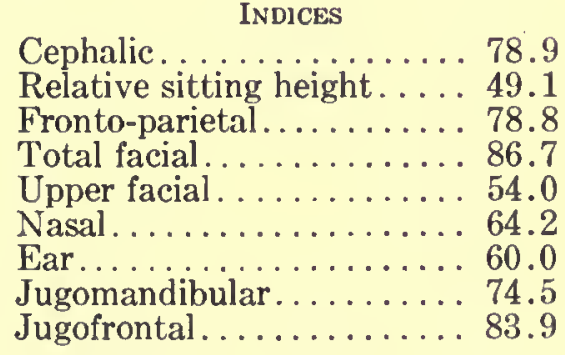

Cephalic............. 78.9

Rronto-parietal........... 78.8

Total facial. . . . . . . . . 86.7

Upper facial. . . . . . . . . 54.0

Nasal. . . . . . . . . . 64.2

Jugomandibular. . . . . . . . . 74.5

Jugofrontal. . . . . . . . 83.9 


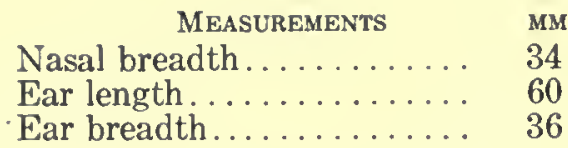

HaIR.-Form: low waves. Color: dark brown.

Eye.-Color: green brown. Sclera: clear. Iris: zoned.

Nose.-Profile: straight. Wings: medium.

\section{No. 653. Plate LXXXVIII, Figs. 1, 2}

Place: Hilla Army Camp. Date: June 16, 1928.

Birthplace: Amara. Age: 25; married for 2 years. Sons: none. Daughters: 1 living. Brothers: 3 living. Sisters: 1 living.

Tattoo marks: glove on back of right hand.

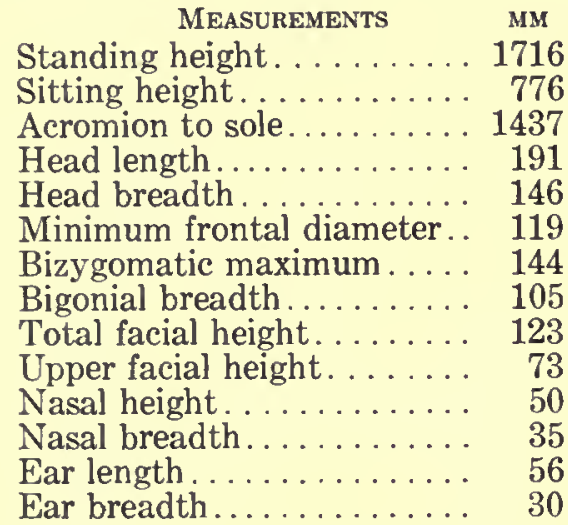

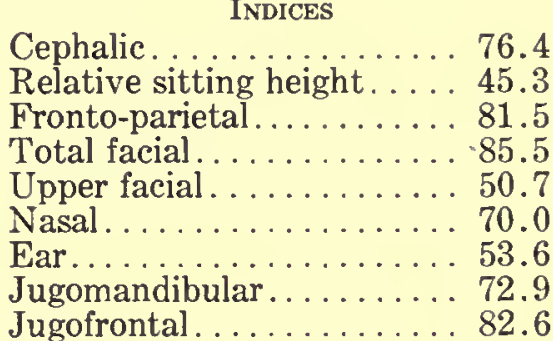

Jugomandibular.......... 72.9

HAIR.-Color: black.

EYE.-Color: dark brown. Sclera: clear. Iris: homogeneous.

Nose.-Profile: convex. Wings: medium.

\section{No. 654. Plate LXXXVIII, Figs. 3, 4}

Place: Hilla Army Camp. Date: June 16, 1928.

Birthplace: Basra. Age: 21; unmarried.

Brothers: 3 living. Sisters: 1 living.

Tattoo marks: line on back of right wrist.

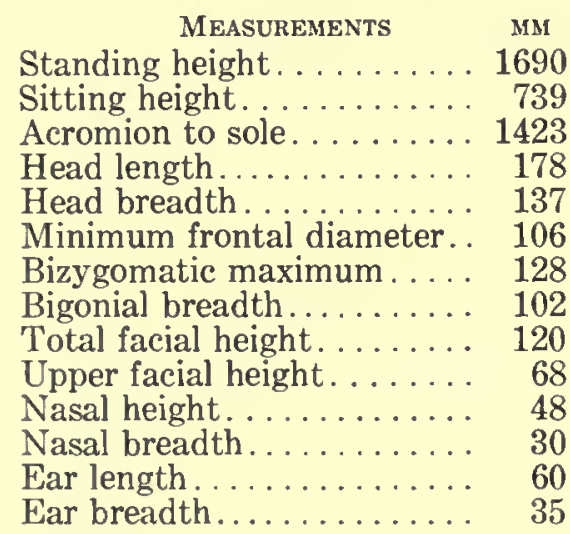

INDICES

Cephalic............. 77.0

Relative sitting height.... 43.6

Fronto-parietal......... 77.4

Total facial. . . . . . . . . . 93.9

Upper facial . . . . . . . . . . 53.2

Nasal

Ear................. 58.4

Jugomandibular. . . . . . . . 79.7

Jugofrontal. . . . . . . . . 82.8

HAIR.-Form: low waves. Texture: medium. Color: dark brown.

EYE.-Color: dark brown. Sclera: clear. Iris: homogeneous.

Nose.-Profile: straight. Wings: medium. 
No. 655. Plate LXXXVIII, Figs. 5, 6

Place: Hilla Army Camp. Date: June 16, 1928.

Birthplace: Sulaimaniya. Age: 20; married for 1 year. No children.

Brothers: 2 living. Sisters: 1 living.

Tattoo marks: none.

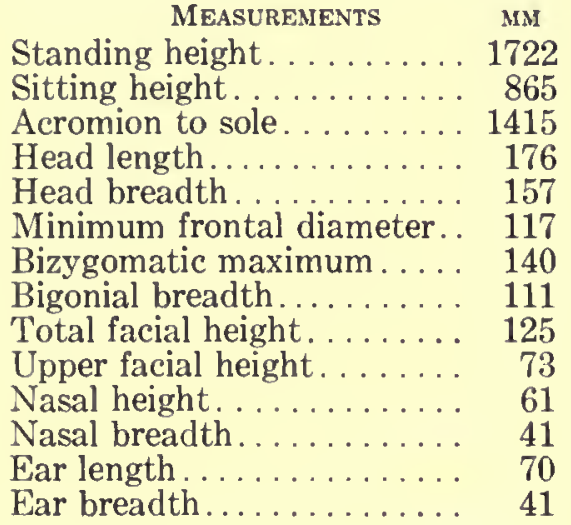

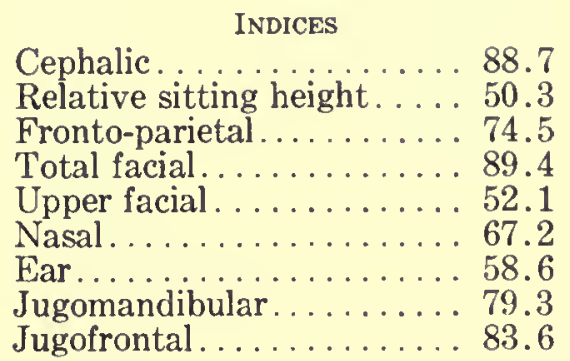

HAIR. - Form: low waves. Texture: medium.

EYE.-Color: dark brown. Sclera: clear. Iris: homogeneous.

Nose.-Profile: convex. Wings: flaring.

Chest.-Development: double plus.

Musculature.-Excellent.

\section{No. 656. Plate LXXXVIII, Figs. 7, 8}

Place: Hilla Army Camp. Date: June 16, 1928.

Birthplace: Mosul. Age: 20; married for 3 years. Sons: 1 living. Daughters: none. Brothers: 1 living. Sisters: 2 living.

Tattoo marks: glove on back of right hand and circle with 8 rays on back of right wrist.

\begin{tabular}{|c|c|c|}
\hline MEASUREMENTS & MM & INDICES \\
\hline Standing height. . & 1682 & Cephalic. \\
\hline Sitting height. & 761 & Relative sitting height. . . . 45.2 \\
\hline Acromion to sole. & 1405 & Fronto-parietal........ \\
\hline Head length. & 192 & Total facial. \\
\hline Head breadth. & 145 & Upper facial \\
\hline Minimum frontal diameter. & 113 & Nasal. \\
\hline Bizygomatic maximum . . . . & 134 & Ear. \\
\hline Bigonial breadth. & 107 & Jugomandibular. . \\
\hline Total facial height. & 120 & Jugofrontal. \\
\hline
\end{tabular}

Upper facial height....... 72

Nasal height........... 51

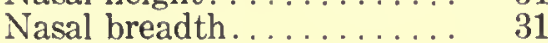

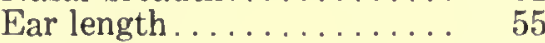

Ear breadth

No. 657. Plate LXXXIX, Figs. 1, 2

Place: Hilla Army Camp. Date: June 16, 1928.

Birthplace: Amara. Age: 20; married for 1 year. No children.

Brothers: 1 living, 1 dead. Sisters: 1 living.

Tattoo marks: glove on back of right hand and animal on inside of right forearm.

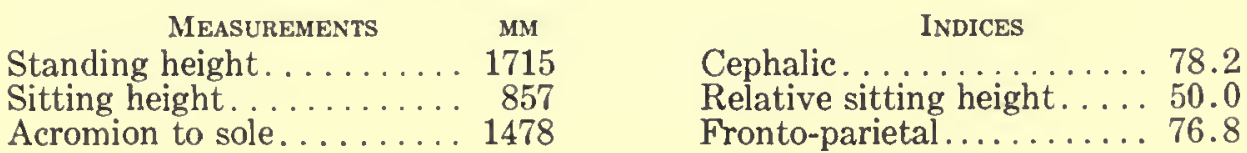




\begin{tabular}{|c|c|c|}
\hline MEASUREMENTS & MM & INDICES \\
\hline Head length. & 188 & Total facial \\
\hline Head breadth. & 147 & Upper facial. \\
\hline Minimum frontal diameter. & 113 & Nasal... \\
\hline Bizygomatic maximum . . . & 134 & . 64.3 \\
\hline onial breadth. & 106 & Jugomandibular...... \\
\hline al facial height & 120 & Jugofrontal. . . . . . \\
\hline
\end{tabular}

Upper facial height ........ 72

Nasal height............ 56

Nasal breadth.......... 36

Ear length ............. 56

Ear breadth........... 36

\section{No. 658. Plate LXXXIX, Figs. 3, 4}

Place: Hilla Army Camp. Date: June 16, 1928.

Birthplace: near Kut al Imara. Age: 20; unmarried.

Brothers: 2 living. Sisters: 3 living.

Tattoo marks: his name and figures 7020 on inside of right forearm.

\begin{tabular}{|c|c|}
\hline MEASUREMENTS & $\mathbf{M M}$ \\
\hline Standing height. . . . . & 1750 \\
\hline Sitting height. & 841 \\
\hline Acromion to sole & 1450 \\
\hline Head length.... & 182 \\
\hline Head breadth. & 137 \\
\hline Minimum frontal diameter. & 111 \\
\hline Bizygomatic maximum.... & 131 \\
\hline Bigonial breadth.......... & 97 \\
\hline Total facial height. . & 125 \\
\hline Upper facial height. . . . . . . & 77 \\
\hline Nasal height. . . . . . . . . . . & 56 \\
\hline sal breadth & \\
\hline length.... & \\
\hline bundth & \\
\hline
\end{tabular}

INDICES

Cephalic............. 75.2

Relative sitting height.... 48.1

Fronto-parietal........ 81.0

Total facial........... 95.5

Upper facial. . . . . . . . . 58.7

Nasal............... 55.4

Ear..................6. 60.7

Jugomandibular......... 74.0

Jugofrontal. ............ 84.7

\section{No. 659. Plate LXXXIX, Figs. 5, 6}

Place: Hilla Army Camp. Date: June 16, 1928.

Birthplace: Amara. Age: 20; married for 1 year. No children.

Brothers: 1 living. Sisters: 3 living.

Tattoo marks: his name and figures 6424 on inside of left forearm.

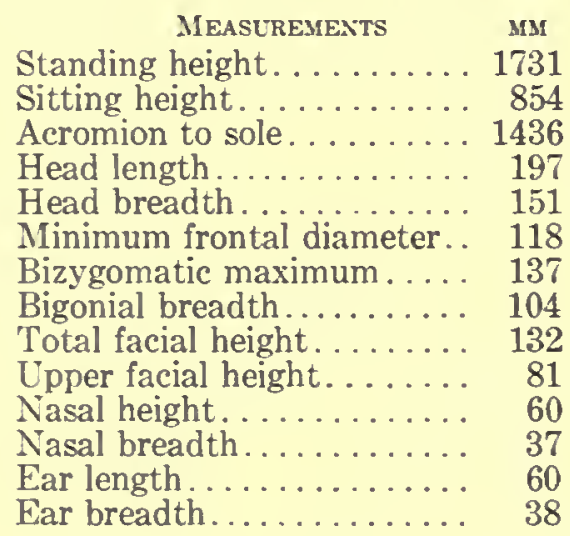

INDICES

Cephalic............. 76.6

Relative sitting height ....49.4

Fronto-parietal......... 78.2

Total facial . . . . . . . . 96.4

Upper facial. . . . . . . . . 58.4

Nasal. ............. 61.7

Ear................ 63.4

$\begin{array}{llll}\text { Jugomandibular. . . . . . } & 75.9\end{array}$

Jugofrontal.......... 86.1

\section{No. 660. Plate LXXXIX, Figs. 7, 8}

Place: Hilla Army Camp. Date: June 16, 1928.

Birthplace: Amara. Age: 21; unmarried. 
Brothers: 4 living. Sisters: 1 living.

Tattoo marks: none.

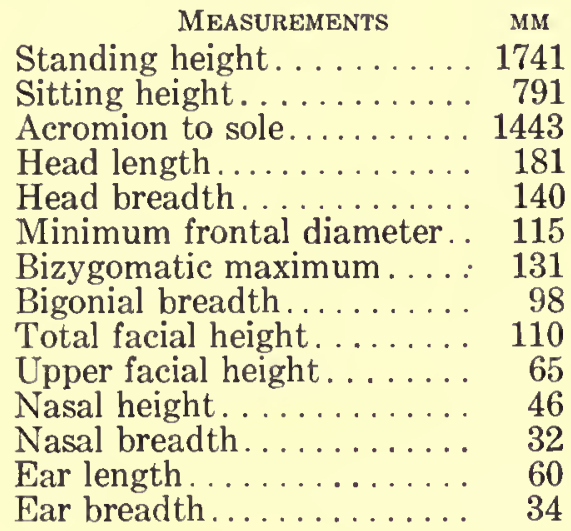

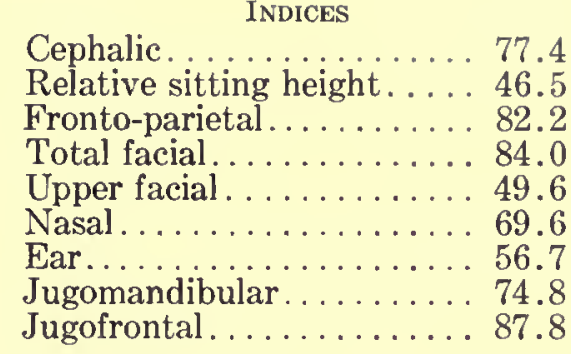

No. 661. Plate XC, Figs. 1, 2

Place: Hilla Army Camp. Date: June 16, 1928.

Birthplace: Nasiriya. Age: 22; unmarried.

Brothers: 3 living, 1 dead. Sisters: 3 living.

Tattoo marks: 3 spots on back of right hand; spot on each temple.

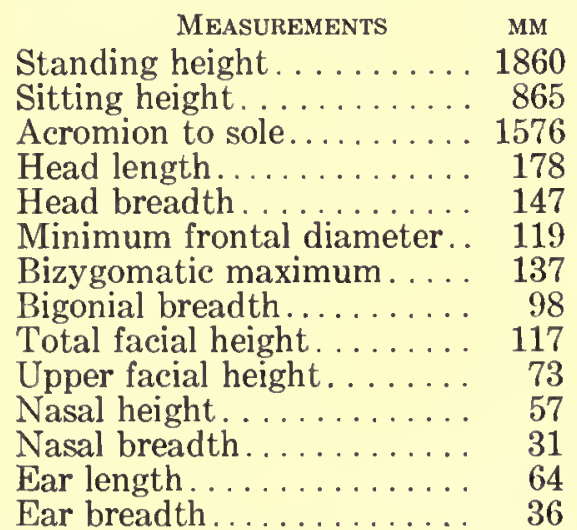

Cephalic. ......... 82.6

Relative sitting height..... 46.5

Fronto-parietal......... 81.0

Total facial .............. 85.4

Upper facial. . . . . . . . 53.2

Nasal. .............. 54.4

Ear................ 56.3

Jugomandibular . . . . . . . 71.5

Jugofrontal . . . . . . . 86.9

Extreme height, very large hands, and feet of abnormal size suggest unbalanced metabolism caused by irregular functioning of endocrine glands.

\section{No. 662. Plate XC, Figs. 3, 4}

Place: Hilla Army Camp. Date: June 16, 1928.

Birthplace: Amara. Age: 24; married for 3 years. No children.

Brothers: none. Sisters: 2 living.

Tattoo marks: large spot on each temple.

\begin{tabular}{|c|c|}
\hline \\
\hline Standing height $\ldots \ldots$ & \\
\hline & \\
\hline n lo sole & \\
\hline & \\
\hline & \\
\hline ter & \\
\hline 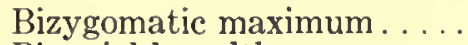 & \\
\hline & \\
\hline & \\
\hline & \\
\hline
\end{tabular}

Cephalic. ............ 78.6

Relative sitting height. ... 47.0

Fronto-parietal. ....... 77.0

Total facial............ 83.4

Upper facial........... 49.3

Nasal. . . . . . . . . . 80.0

Ear...............6. 61.5

Jugomandibular........ 76.8

Jugofrontal.............. 82.6 


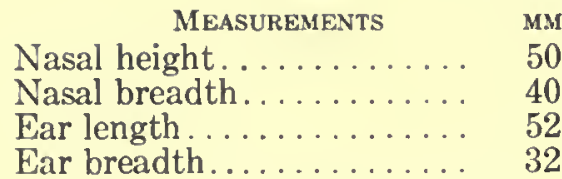

\section{No. 663. Plate XC, Figs. 5, 6}

Place: Hilla Army Camp. Date: June 16, 1928.

Birthplace: Amara. Age: 20; unmarried.

Brothers: 1 living, 2 dead. Sisters: 3 living.

Tattoo marks: glove on back of right hand and spots on temple.

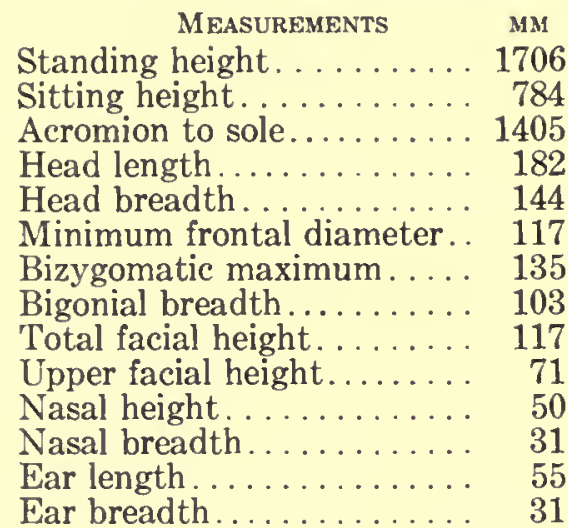

Cephalic............. 79.2

Relative sitting height. . . . 45.9

Fronto-parietal.........8 81.3

Total facial............ 86.7

Upper facial . . . . . . . . . 52.6

Nasal . . . . . . . . . . . 62.0

Ear................. 56.4

Jugomandibular............. 76.3

Jugofrontal . . . . . . . 86.6

\section{No. 664. Plate XC, Figs. 7, 8}

Place: Hilla Army Camp. Date: June 16, 1928.

Birthplace: Amara. Age: 22; unmarried.

Brothers: 4 living. Sisters: 1 living.

Tattoo marks: spot on back of right wrist.

\begin{tabular}{|c|c|c|}
\hline MEASUREMENTS & MM & INDICES \\
\hline Standing height. & 1675 & Cephalic. \\
\hline Sitting height. . & 769 & Relative sitting height. . . . 44 \\
\hline Acromion to sole. & 1390 & Fronto-parietal......... 74 \\
\hline d length. & 188 & Total facial. \\
\hline breadth. & 148 & Upper facial \\
\hline imum frontal diameter. & 110 & Nasal. \\
\hline gomatic maximum . . . . & 132 & Ear \\
\hline height. & 118 & $\mathrm{I}$ \\
\hline
\end{tabular}

Upper facial height. ....... 74

Nasal height.......... 52

Nasal breadth............. 41

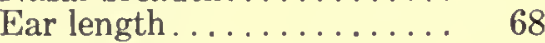

Ear breadth............ 41

No. 665. Plate XCI, Figs. 1,2

Place: Hilla Army Camp. Date: June 16, 1928.

Birthplace: Amara. Age: 22; unmarried.

Brothers: 4 living. Sisters: 2 living.

Tattoo marks: gazelle and selugi on inside of right forearm.

MEASUREMENTS MM

Standing height........ 1734

Sitting height. . . . . . . . 825

Acromion to sole.......... 1470
INDICES

Cephalic............76.2

Relative sitting height.... 47.6

Fronto-parietal.......... 76.7 
Head length............ 185

Head breadth.......... 141

Minimum frontal diameter.. 108

Bizygomatic maximum .... 130

Bigonial breadth......... 110

Total facial height........ 113

Upper facial height. . . . . . 71

Nasal height. . . . . . . . . 48

Nasal breadth.......... 32

Ear length............ 57

Ear breadth........... 31

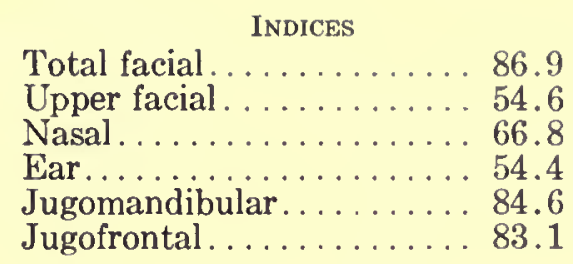

Right ear: length 46 , breadth 29 , due to part having been cut off.

\section{No. 666. Plate XCI, Figs. 3, 4}

Place: Hilla Army Camp. Date: June 16, 1928.

Birthplace: west of Mosul. Age: 20; married for 8 months.

Brothers: 1 living. Sisters: 1 dead.

Tattoo marks: "Ghasim Sinjar" and figures 8242 on inside of right forearm.

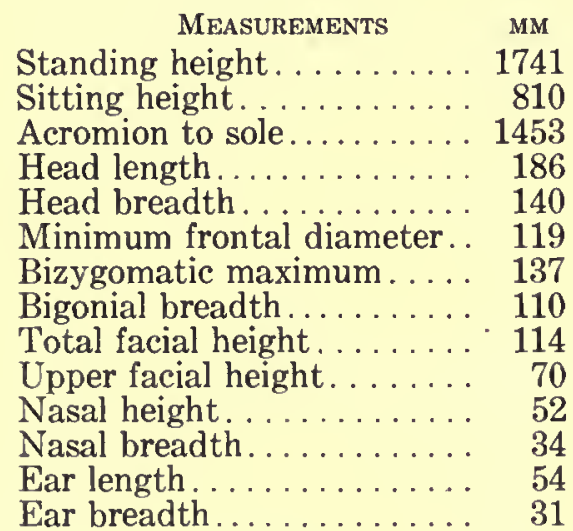

INDICES

Cephalic.............75.3

Relative sitting height. . . . 46.5

Fronto-parietal. ........ 85.0

Total facial. . . . . . . . 83.2

Upper facial . . . . . . . . . 51.1

Nasal. .............6. 65.3

Ear.................... 57.4

Jugomandibular. . . . . . . . 80.3

Jugofrontal . . . . . . . . . . 86.9

No. 667. Plate XCI, Figs. 5, 6

Place: Hilla Army Camp. Date: June 16, 1928.

Birthplace: Amara. Age: 25; married three times, 6, 4, and 1 years ago. Sons: 0, 1, 0, dead.

Daughters: 1, 0, 0, living.

Brothers: 2 living. Sisters: 3 living.

Tattoo marks: line on back of right wrist; also spots on each temple.

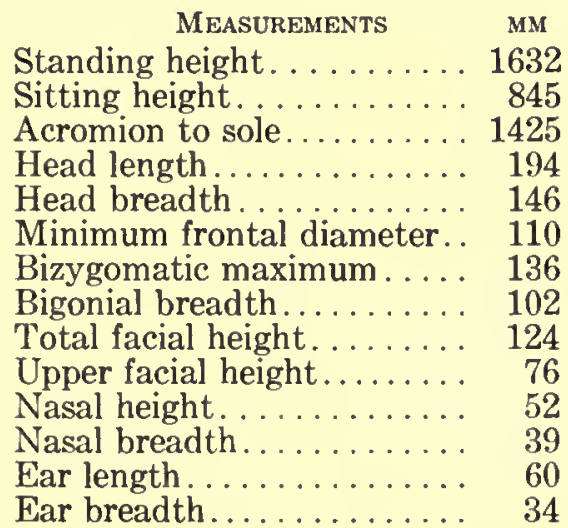

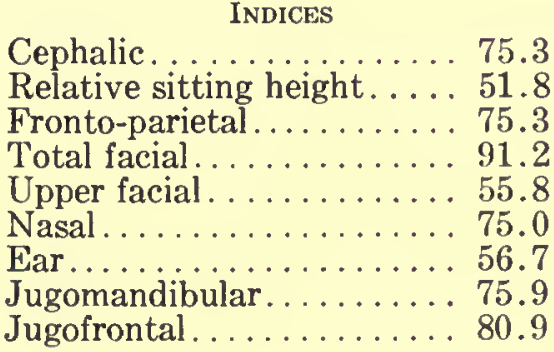


No. 668. Plate XCI, Figs. 7, 8

Place: Hilla Army Camp. Date: June 16, 1928.

Birthplace: Nasiriya. Age: 21 ; married for 5 years. Sons: 1 living. Daughters: none. Brothers: 3 living, 1 dead. Sisters: 2 living.

Tattoo marks: none.

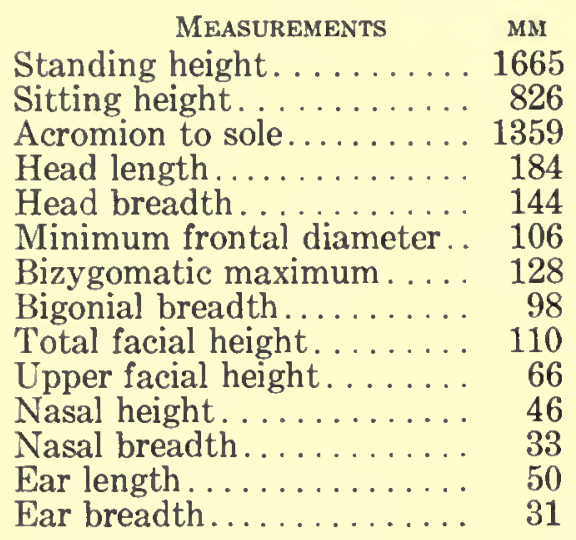

INDICES

Cephalic.............. 78.2

Relative sitting height. . . . 49.6

Fronto-parietal .......... 73.6

Total facial............ 86.0

Upper facial. . . . . . . . 51.6

Nasal ................. 71.7

Ear.............. 62.0

Jugomandibular. . . . . . . 76.5

Jugofrontal. ......... 84.4

No. 669. Plate XCII, Figs. 1, 2

Place: Hilla Army Camp. Date: June 16, 1928.

Birthplace: Amara. Age: 20; unmarried.

Brothers: 4 living. Sisters: 2 living.

Tattoo marks: line on back of right wrist.

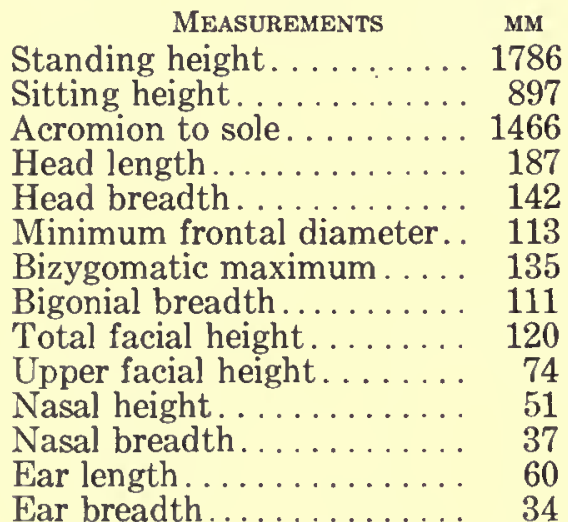

INDICES

Cephalic............ 76.0

Relative sitting height. . . 50.2

Fronto-parietal. ........ 79.6

Total facial............. 88.9

Upper facial . . . . . . . . . . 54.7

Nasal................... 72.6

Ear.............. 56.6

Jugomandibular......... 82.2

Jugofrontal. . . . . . . . 83.7

\section{No. 670. Plate XCII, Figs. 3, 4}

Place: Hilla Army Camp. Date: June 16, 1928.

Birthplace: An Najaf. Age: 19; unmarried.

Brothers: 2 living. Sisters: 3 living, 1 dead.

Tattoo marks: none.

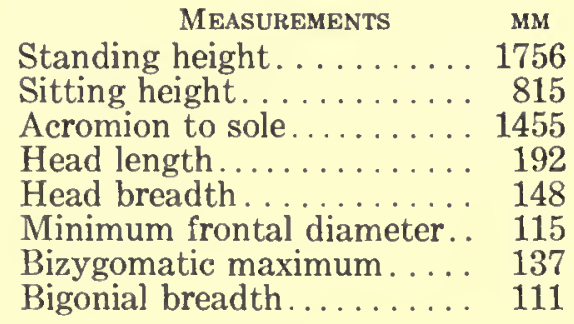

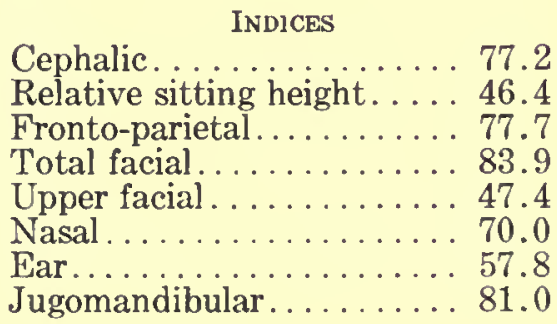




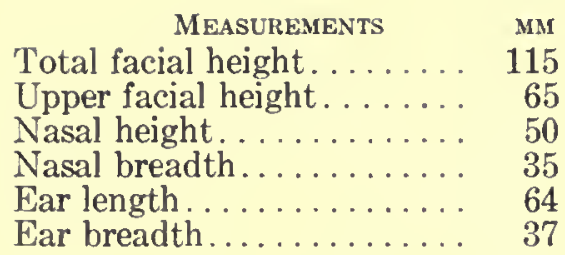

INDICES

Jugofrontal . . . . . . . . . 83.9

No. 671. Plate XCII, Figs. 5, 6

Place: Hilla Army Camp. Date: June 16, 1928.

Birthplace: Nasiriya. Age: 20; unmarried.

Brothers: 2 living, 1 dead. Sisters: none.

Tattoo marks: none.

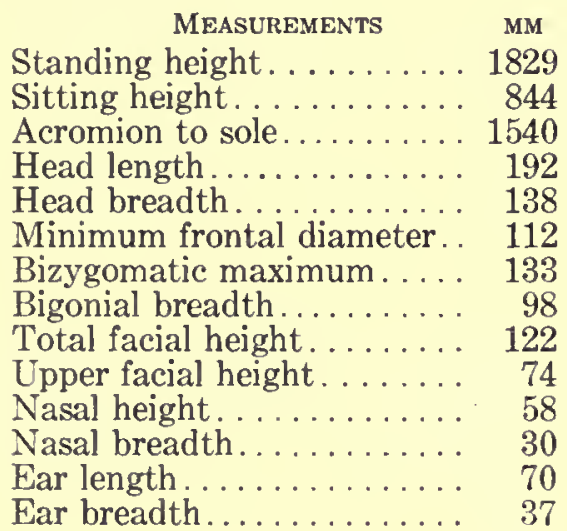

INDICES

Cephalic........... 71.9

Relative sitting height. . . . 46.2

Fronto-parietal......... 81.2

Total facial............ 91.8

Upper facial. . . . . . . . . 55.6

Nasal . . . . . . . . . . . . 51.7

Ear................ 52.8

Jugomandibular. . . . . . . 73.7

Jugofrontal . . . . . . . . . . . 84.2

\section{No. 672. Plate XCII, Figs. 7, 8}

Place: Hilla Army Camp. Date: June 16, 1928.

Birthplace: Mosul. Age: 18; unmarried.

Brothers: 1 living. Sisters: 1 living, 1 dead.

Tattoo marks: sun on back of right hand; bird carrying letter on inside of right forearm.

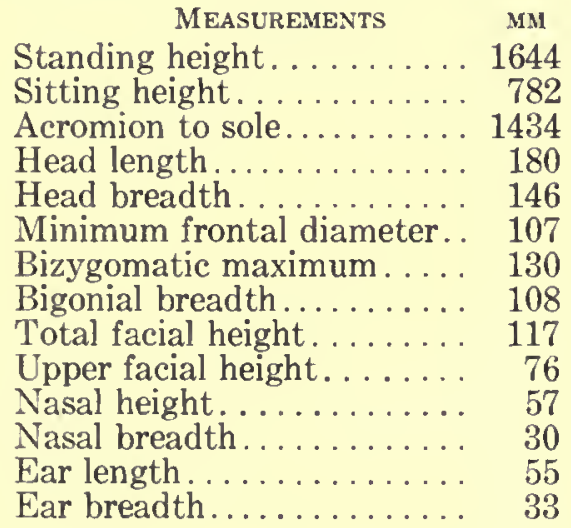

Cephalic. ............ 81.2

Relative sitting height. ... 47.5

Fronto-parietal......... 73.3

Total facial ............. 90.0

Upper facial . . . . . . . . . 58.5

Nasal. . . . . . . . . . . 52.7

Ear......................... 60

Jugomandibular. . . . . . . 83.1

Jugofrontal. . . . . . . . . 82.3

\section{No. 673. Plate XCIII, Figs. 1,2}

Place: Hilla Army Camp. Date: June 16, 1928.

Birthplace: Kut al Imara. Age: 20 ; unmarried.

Brothers: 1 living. Sisters: 2 living.

Tattoo marks: gazelle and two spots on inside of right forearm. 
No. 676. Plate XCIII, Figs. 7, 8

Place: Hilla Army Camp. Date: June 16, 1928.

Birthplace: Amara. Age: 24; married for 2 years. Sons: none. Daughters: 1 living. Brothers: 1 living. Sisters: 3 living.

Tattoo marks: spot on glabella and spot on each temple.

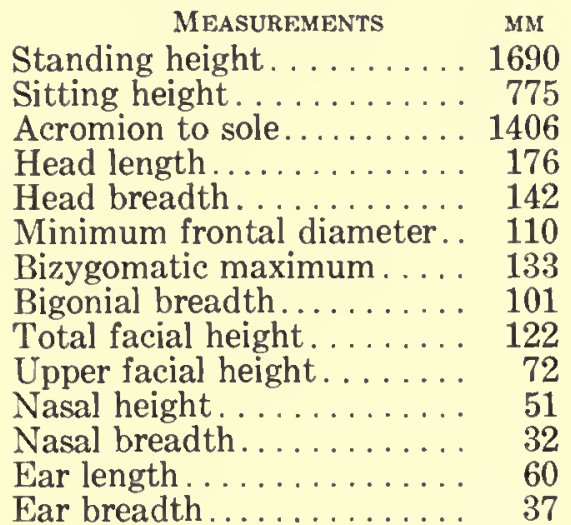

\begin{tabular}{|c|c|}
\hline \multirow{2}{*}{\multicolumn{2}{|c|}{ Cephalic INDICES }} \\
\hline & \\
\hline Relative sitting height & 46 \\
\hline Fronto-parietal...... & \\
\hline Total facial ... & 91 \\
\hline Upper facial & \\
\hline $\begin{array}{l}\text { Nasal. } \\
\text { Ear }\end{array}$ & \\
\hline $\begin{array}{l}\text { Ear........... } \\
\text { Jugomandibular. }\end{array}$ & \\
\hline $\begin{array}{l}\text { Jugomandibular. } \\
\text { Jugofrontal..... }\end{array}$ & 8 \\
\hline
\end{tabular}

No. 677. Plate XCIV, Figs. 1, 2

Place: Hilla Army Camp. Date: June 16, 1928.

Birthplace: Nasiriya. Age: 20 ; married for 6 years. Sons: 2 living. Daughters: none. Brothers: 10 living. Sisters: 3 living.

Tattoo marks: none.

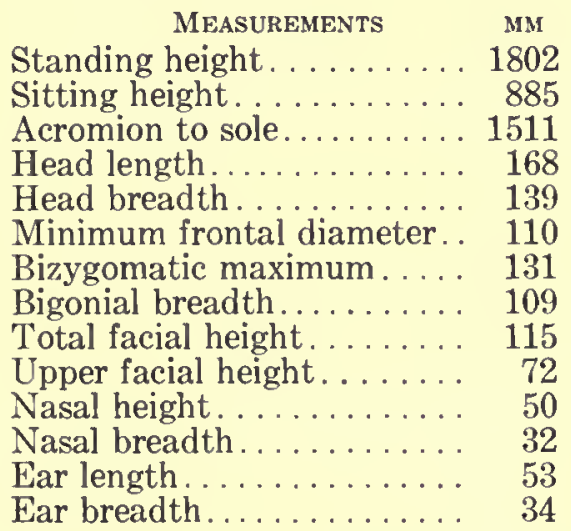

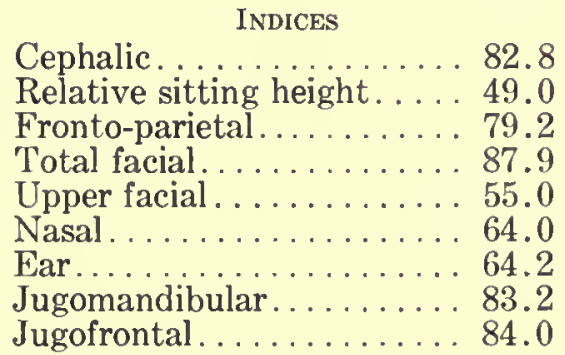

No. 678. Plate XCIV, Figs. 3, 4

Place: Hilla Army Camp. Date: June 16, 1928.

Birthplace: Amara. Age: 20; unmarried.

Brothers: 1 living. Sisters: none.

Tattoo marks: spot below thumb on back of right hand.

\begin{tabular}{|c|c|c|}
\hline MEASUREMENTS & MM & INDICES \\
\hline Standing height. & 1735 & Cephalic. \\
\hline Sitting height. & 822 & Relative sitting height. . \\
\hline Acromion to sole. & 1448 & Fronto-parietal........ \\
\hline Head length. & 182 & Total facial. \\
\hline Head breadth. & 147 & Upper facial \\
\hline imum frontal diameter. & 113 & Nasal. \\
\hline ygomatic maximum.... & 136 & 54.0 \\
\hline onial breadth... & 111 & Jugomandik \\
\hline
\end{tabular}




\begin{tabular}{|c|c|c|}
\hline MEASUREMENTS & MM & INDICES \\
\hline Total facial height. . . . . . . . & 121 & Jugofrontal . . . . . . . . . . 83.0 \\
\hline Upper facial height. . . . . . . & 76 & \\
\hline Nasal height. . . . . . . . . . & 60 & \\
\hline Nasal breadth. . . . . . . . . . & 32 & \\
\hline Ear length. & 63 & \\
\hline Ear breadth. & 34 & \\
\hline
\end{tabular}

\section{No. 679. Plate XCIV, Figs. 5, 6}

Place: Hilla Army Camp. Date: June 16, 1928.

Birthplace: Amara. Age: 21 ; unmarried.

Brothers: 3 living. Sisters: 2 living.

Tattoo marks: glove on back of right hand and gazelle on inside of right forearm.

\begin{tabular}{|c|c|c|}
\hline MEASUREMENTS & MM & INDICES \\
\hline Standing height. & 1801 & Cephalic. \\
\hline Sitting height. & 909 & Relative sitting height.... 50.4 \\
\hline Acromion to sole. & 1505 & Fronto-parietal........ \\
\hline Head length... & 180 & Total facial... \\
\hline Head breadth. & 150 & Upper facial \\
\hline Minimum frontal diameter. & 120 & Nasal. \\
\hline Bizygomatic maximum. ... & 140 & Ear. \\
\hline Bigonial breadth . . . . . . . & 114 & Jugomandibular . \\
\hline Total facial height. . . . . . . & 117 & Jugofrontal . . . . . . . . . 85.7 \\
\hline Upper facial height. . . . . . & 70 & \\
\hline Nasal height. . . . . . . . . . . & 60 & \\
\hline Nasal breadth. & 36 & \\
\hline Ear length. & 53 & \\
\hline Ear breadth & 36 & \\
\hline
\end{tabular}

\section{No. 680. Plate XCIV, Figs. 7, 8}

Place: Hilla Army Camp. Date: June 16, 1928.

Birthplace: Kut al Imara. Age: 21; unmarried.

Brothers: 2 dead. Sisters: 3 dead.

Tattoo marks: 2 spots and cross on right hand below base of third finger.

\begin{tabular}{|c|c|c|}
\hline Measurements & MM & INDICES \\
\hline Standing height. & 1747 & Cephalic. \\
\hline Sitting height. & 798 & Relative sitting height. \\
\hline Acromion to sole. & 1447 & Fronto-parietal........ \\
\hline Head length.. & 184 & Total facial.. \\
\hline Head breadth. & 144 & Upper facial \\
\hline Minimum frontal diameter. & 109 & Nasal. \\
\hline gomatic maximum. ... & 134 & Ear. \\
\hline onial breadth. & 106 & Jugomandibular. . . . . \\
\hline tal facial height. ....... & 129 & Jugofrontal. \\
\hline
\end{tabular}

Upper facial height. . . . . . 78

Nasal height........... 55

Nasal breadth.............. 36

Ear length............ 65

Ear breadth............ 42

\section{No. 681. Plate XCV, Figs. 1, 2}

Place: Hilla Army Camp. Date: June 16, 1928.

Birthplace: Amara. Age: 20; unmarried.

Brothers: 5 living. Sisters: 6 living.

Tattoo marks: elaborate glove on back of right hand. 


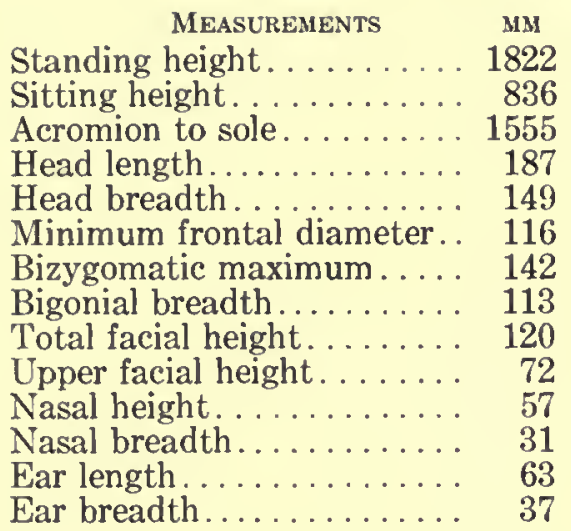

INDICES

Cephalic.............79.7

Relative sitting height.... 46.0

Fronto-parietal ......... 77.9

Total facial. . . . . . . . . . 8. 84.6

Upper facial. . . . . . . . . 50.7

Nasal. . . . . . . . . . . . . . . 54.4

Ear.................. 58.7

Jugomandibular . . . . . . . . 79.6

Jugofrontal . . . . . . . . 81.7

\section{No. 682. Plate XCV, Figs. 3, 4}

Place: Hilla Army Camp. Date: June 16, 1928.

Birthplace: near Ba'quba. Age: 20; unmarried.

Brothers: 1 living, 2 dead. Sisters: 1 dead.

Tattoo marks: cross on inside of left forearm and gazelle on back of right hand.

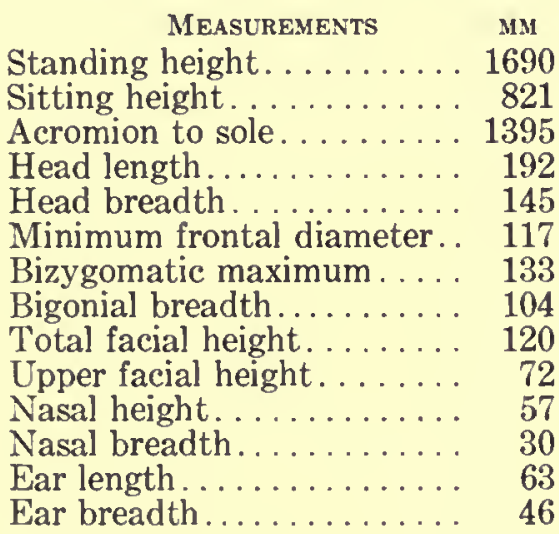

INDICES

Cephalic............ 75.5

Relative sitting height. .... 48.5

Fronto-parietal......... 80.7

Total facial. ........... 90.4

Upper facial. . . . . . . . . 54.1

Nasal................ 52.6

Ear................ 73.0

Jugomandibular. . . . . . . 78.2

Jugofrontal . . . . . . . . . . 88.0

No. 683. Plate XCV, Figs. 5, 6

Place: Hilla Army Camp. Date: June 16, 1928.

Birthplace: Basra. Age: 23; unmarried.

Brothers: 1 living. Sisters: 1 dead.

Tattoo marks: none.

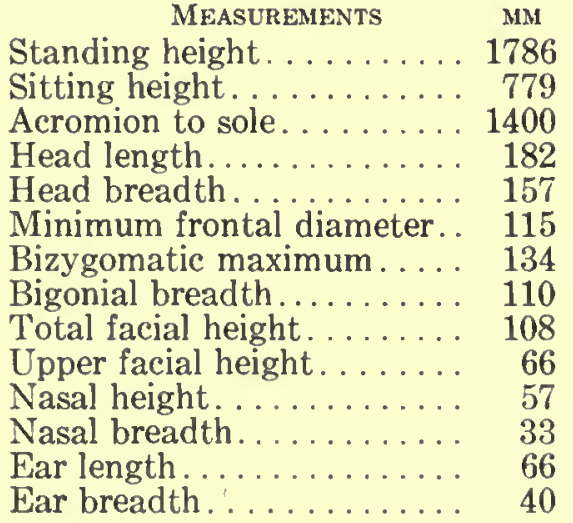

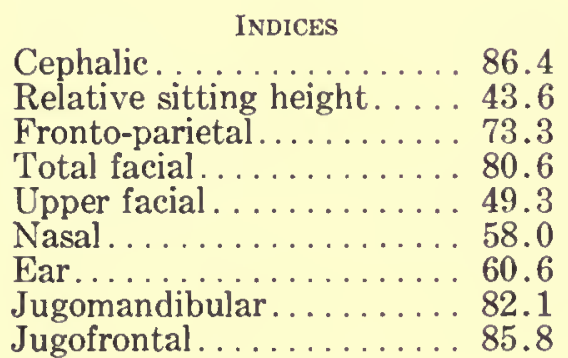

HAIR.-Form: curly frizzly. Texture: medium. Quantity: head, plus; beard, minus; body, plus. 


\section{No. 684. Plate XCV, Figs. 7, 8}

Place: Hilla Army Camp. Date: June 16, 1928.

Birthplace: Ba‘quba. Age: 30; married for 10 years. Sons: 2 living. Daughters: none. Brothers: 1 living, 5 dead. Sisters: 5 living.

Tattoo marks: none.

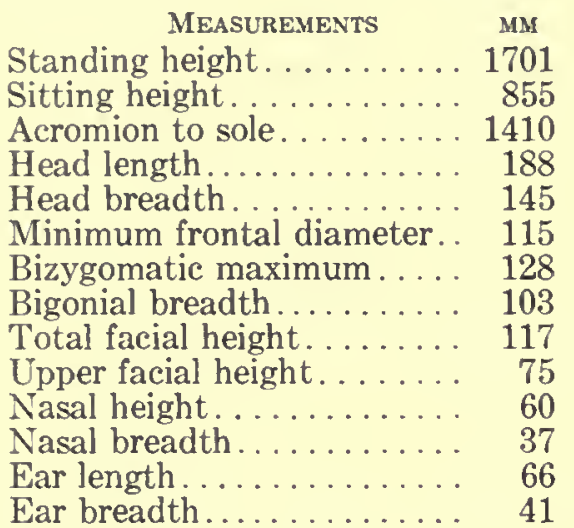

INDICES

Cephalic. ............. 77.1

Relative sitting height. ... 50.2

Fronto-parietal......... 79.4

Total facial. . . . . . . . . . 91.8

Upper facial. . .......... 58.6

Nasal. . . . . . . ...... 61.7

Ear................. 62.1

Jugomandibular. . . . . . . . 80.5

Jugofrontal. . . . . . . . . 89.8

HaIr.-Form: low waves. Texture: fine. Quantity: head, double plus; beard, average; body, average.

\section{No. 685. Plate XCVI, Figs. 1, 2}

Place: Hilla Army Camp. Date: June 16, 1928.

Birthplace: near Ba'quba. Age: 25; unmarried.

Brothers: 1 living, 1 dead. Sisters: 1 living.

Tattoo marks: none.

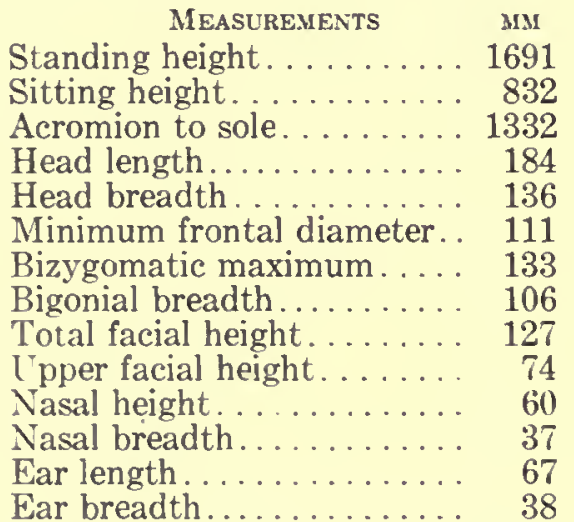

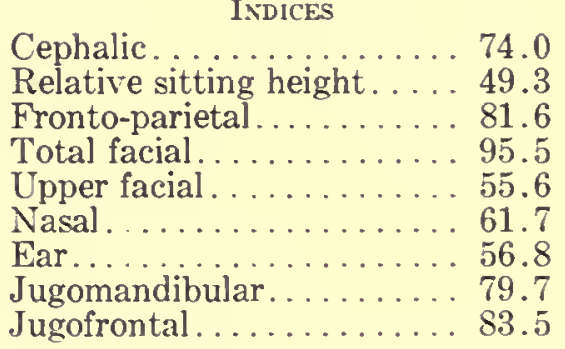

\section{No. 686. Plate XCVI, Figs. 3, 4}

Place: Hilla Army Camp. Date: June 17, 1928.

Birthplace: Baghdad. Age: 26 ; unmarried.

Brothers: 1 living. Sisters: 2 living.

Tattoo marks: none.

\begin{tabular}{|c|c|}
\hline MEASUREMENTS & MM \\
\hline Standing height. & 1720 \\
\hline Sitting height. & 809 \\
\hline Acromion to sole. & 1445 \\
\hline Head length. & 176 \\
\hline Head breadth . . . . . . . . & 147 \\
\hline Minimum frontal diamete & 115 \\
\hline
\end{tabular}

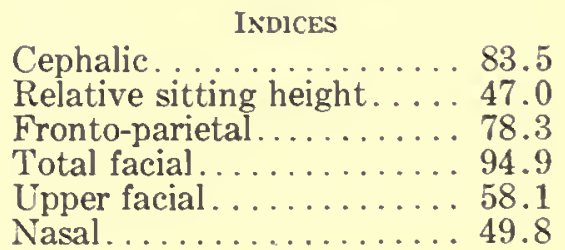




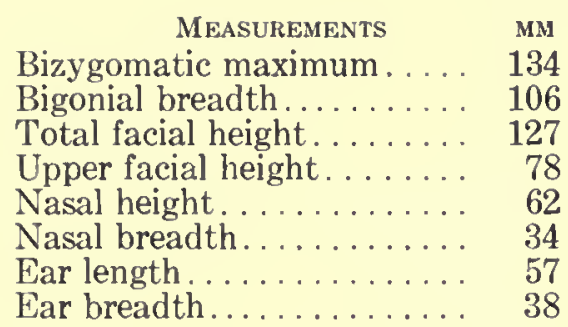

INDICES

Ear.................66.7

Jugomandibular. . . . . . . 79.1

Jugofrontal. . . . . . . 85.8

\section{No. 687. Plate XCVI, Figs. 5, 6}

Place: Hilla Army Camp. Date: June 17, 1928.

Birthplace: Ad Diwaniya. Age: 21; unmarried.

Brothers: 2 living. Sisters: 3 living.

Tattoo marks: glove on back of right hand, gazelle on inside of right forearm, and spot on nasal tip.

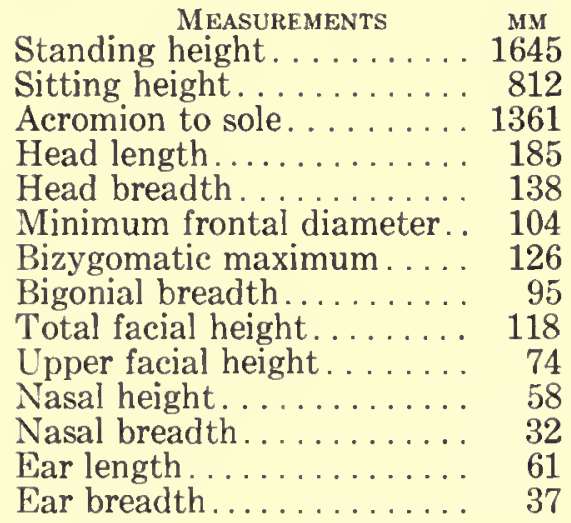

Cephalic............ 74.6

Relative sitting height. . . . 49.4

Fronto-parietal.......... 75.4

Total facial. ........... 93.7

Upper facial . . . . . . . . . . 58.7

Nasal. . . . . . . . . . . 55.2

Ear.................. 60.7

Jugomandibular. . . . . . . 75.4

Jugofrontal . . . . . . . . . . 82.5

EyE.-Color: green brown. Sclera: clear. Iris: zoned.

\section{No. 688. Plate XCVI, Figs. 7, 8}

Place: Hilla Army Camp. Date: June 17, 1928.

Birthplace: Mosul. Age: 25; unmarried.

Brothers: 4 dead. Sisters: none.

Tattoo marks: line and 4 spots on back of right wrist and spots on each temple.

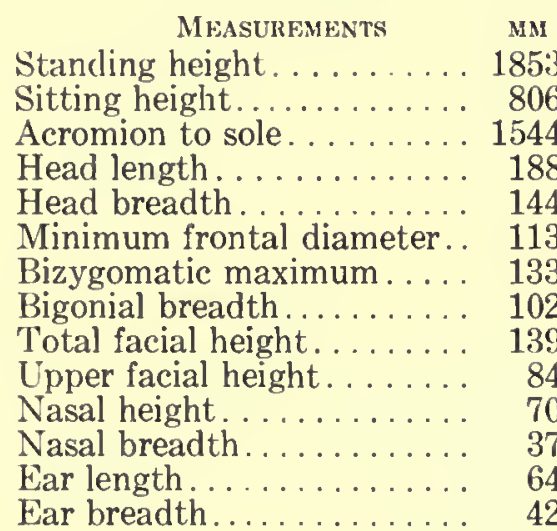

INDICES

Cephalic.............76.7

Relative sitting height..... 43.5

Fronto-parietal......... 78.5

Total facial............... 104.5

Upper facial . . . . . . . 63.2

Nasal ............... 52.8

Ear..............6. 65.6

Jugomandibular . . . . . . . . . 76.7

Jugofrontal. . . . . . . . 85.0

\section{No. 689. Plate XCVII, Figs. 1, 2}

Place: Hilla Army Camp. Date: June 17, 1928.

Birthplace: Samawa. Age: 20 ; married for 6 months. 
Brothers: 1 living. Sisters: 1 living, 2 dead.

Tattoo marks: glove on back of right hand.

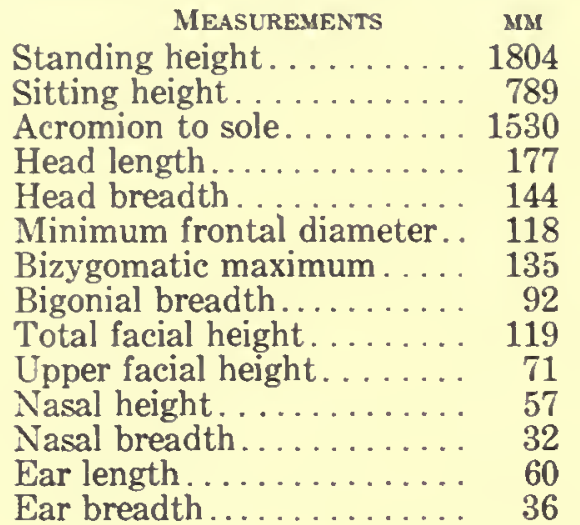

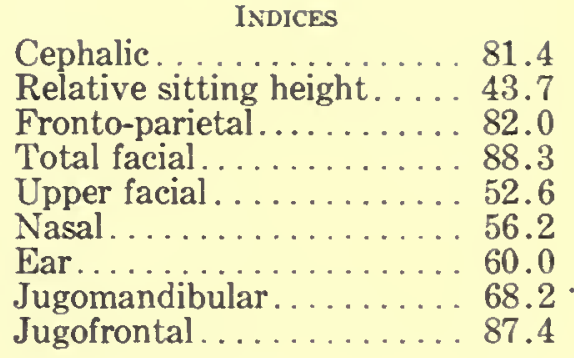

No. 690. Plate XCVII, Figs. 3,4

Place: Hilla Army Camp. Date: June 17, 1928.

Birthplace: Amara. Age: 23; married for 2 years.

Sons: 1 living. Daughters: none.

Brothers: 1 living, 1 dead. Sisters: 2 living.

Tattoo marks: none.

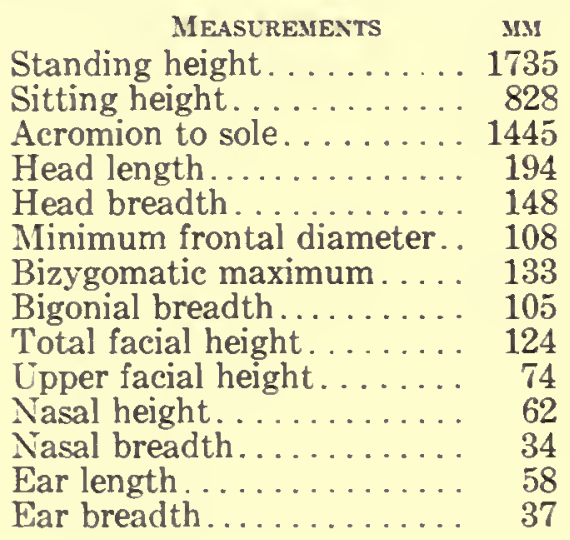

INDICES

Cephalic ........... 76.3

Relative sitting height....47.7

Fronto-parietal......... 73.0

Total facial . . . . . . . . . . 93.2

Upper facial . . . . . . . 55.6

Nasal. . . . . . . . . 54.7

Ear...............6. 63.8

Jugomandibular. . . . . 78.9

Jugofrontal............. 81.2

\section{No. 691. Plate XCVII, Figs. 5, 6}

Place: Hilla Army Camp. Date: June 17, 1928.

Birthplace: Baghdad. Age: 20; married for 1 year. Sons: 1 living. Daughters: none. Brothers: 4 living. Sisters: 2 living.

Tattoo marks: none.

Measurements MM

Standing height......... 1711

Sitting height........... 772

Acromion to sole......... 1430

Head length............ 188

Head breadth.......... 128

Minimum frontal diameter. . 106

Bizygomatic maximum . . . . 123

Bigonial breadth.......... 100

Total facial height........ 123

Upper facial height....... 72

Nasal height........... 58

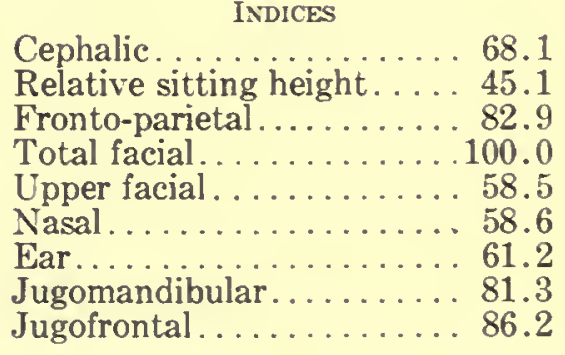

Cephalic..............68.1

Fronto-parietal....... 82.9

Total facial............. 100.0

Upper facial. . . . . . . . . . 58.5

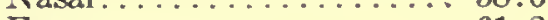

Jugomandibular. . . . . . . . 81.3

Jugofrontal. . . . . . . . 86.2 


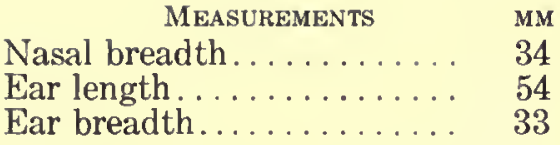

EYE.-Color: green brown. Sclera: clear. Iris: zoned.

\section{No. 692. Plate XCVII, Figs. 7, 8}

Place: Hilla Army Camp. Date: June 17, 1928.

Birthplace: Hilla. Age: 25; unmarried.

Brothers: 1 living. Sisters: 1 living.

Tattoo marks: none.

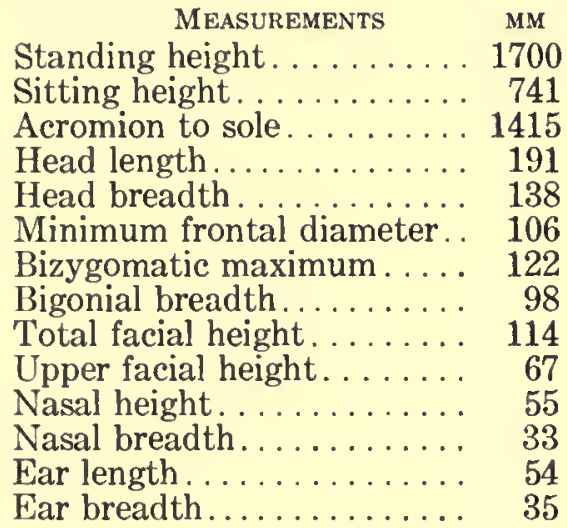

\section{INDICES}

Cephalic . ........... 72.2

Relative sitting height. . . . 43.6

Fronto-parietal.......... 76.8

Total facial. ........... 93.5

Upper facial . . . . . . . . . 54.9

Nasal. . . . . . . . . . . . . 60.0

Ear............... 64.8

Jugomandibular . . . . . . . . 8 80.3

Jugofrontal . . . . . . . . . . 86.9

\section{No. 693. Plate XCVIII, Figs. 1, 2}

Place: Hilla Army Camp. Date: June 17, 1928.

Birthplace: Nasiriya. Age: 22; unmarried.

Brothers: 4 dead. Sisters: 2 living.

Tattoo marks: none.

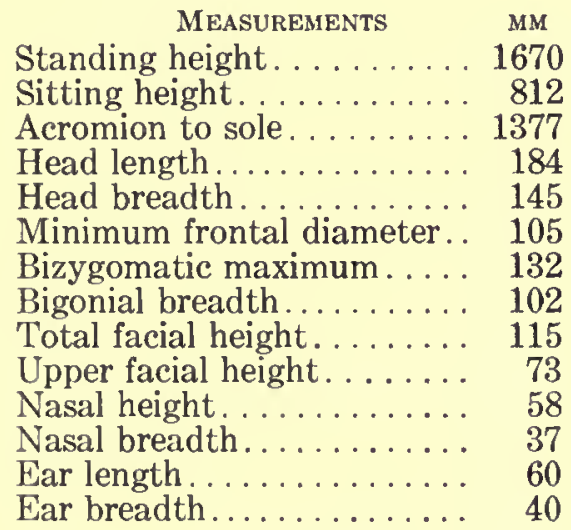

INDICES

Cephalic.............78.8

Relative sitting height. . . . 48.6

Fronto-parietal......... 72.5

Total facial. . . . . . . . . . . 87.2

Upper facial . . . . . . . . . . . 55.2

Nasal. . . . . . . . . . . 6 63.8

Ear...............666.7

Jugomandibular . . . . . . . 77.3

Jugofrontal. . . . . . . . . 79.5

\section{No. 694. Plate XCVIII, Figs. 3, 4}

Place: Hilla Army Camp. Date: June 17, 1928.

Birthplace: Nasiriya. Age: 20; unmarried.

Brothers: 1 living. Sisters: 2 living.

Tattoo marks: none.

MEASUREMENTS MM

Standing height. ....... 1692

Sitting height.......... 781

Acromion to sole........ 1394
INDICES

Cephalic............ 73.4

Relative sitting height. ... 45.1

Fronto-parietal.......... 75.7 


\begin{tabular}{|c|c|c|c|}
\hline MEASUREMENTS & MM & \multicolumn{2}{|c|}{ Drc } \\
\hline Head length. & 191 & Total facial & 86.5 \\
\hline breadth & 140 & Upper facial & 56. \\
\hline Minimum frontal diameter & 106 & Nasal..... & 50.0 \\
\hline Bizygomatic maximum.... & 125 & Ear & 69.3 \\
\hline Bigonial breadth & 99 & Jugomandibula & .2 \\
\hline Total facial heigh & 108 & Jugofrontal. . & \\
\hline
\end{tabular}

Upper facial height. ...... 70

Nasal height. ............. 60

Nasal breadth.......... 30

Ear length............ 52

Ear breadth............. 36

\section{No. 695. Plate XCVIII, Figs. 5, 6}

Place: Hilla Army Camp. Date: June 17, 1928.

Birthplace: Amara. Age: 20; married twice, 4 and 1 years ago. Sons: 1, 0, living.

Daughters: none.

Brothers: 2 living. Sisters: 3 living.

Tattoo marks: none.

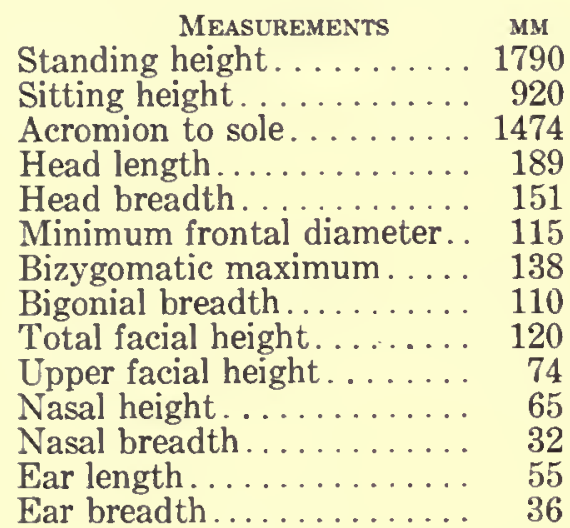

INDICES

Cephalic............. 79.9

Relative sitting height.... 51.4

Fronto-parietal......... 76.2

Total facial ............. 87.0

Upper facial . . . . . . . . . 53.6

Nasal. .............. 49.3

Ear...............6. 65.4

Jugomandibular . . . . . . . 79.7

Jugofrontal. . . . . . . 83.4

No. 696. Plate XCVIII, Figs. 7, 8

Place: Hilla Army Camp. Date: June 17, 1928.

Birthplace: Nasiriya. Age: 30; unmarried.

Brothers: 3 living, 2 dead. Sisters: 2 living, 1 dead.

Tattoo marks: spots on back of right hand and single spot on each temple.

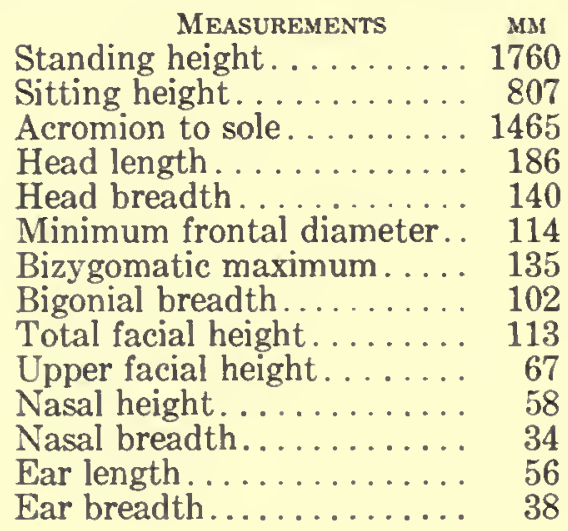

INDICES

Cephalic............. 75.3

Relative sitting height.... 45.8

Fronto-parietal......... 81.5

Total facial $\ldots 83.7$

Upper facial. .......... 49.6

Nasal .............. 58.6

Ear................6. 67.9

Jugomandibular........ 75.6

Jugofrontal............. 84.5

No. 697. Plate XCIX, Figs. 1, 2

Place: Hilla Army Camp. Date: June 17, 1928.

Birthplace: Baghdad. Age: 22; unmarried. 
Brothers: 4 dead. Sisters: 1 dead.

Tattoo marks: none.

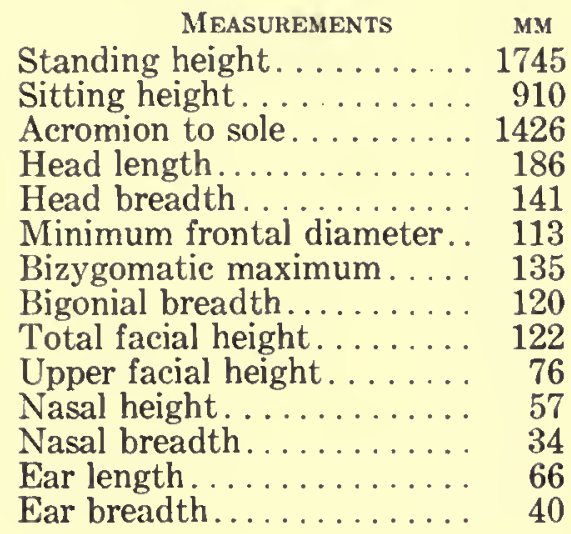

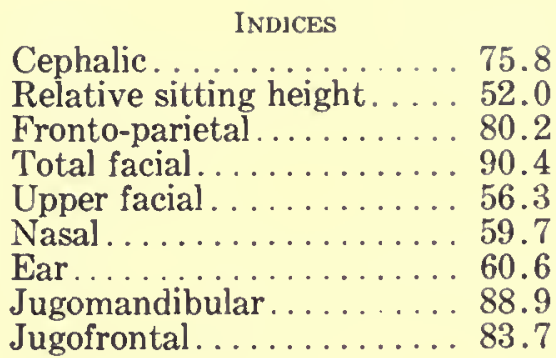

No. 698. Plate XCIX, Figs. 3, 4

Place: Hilla Army Camp. Date: June 17, 1928.

Birthplace: Nasiriya. Age: 21 ; married twice, 2 and 4 years ago. Sons: 1, 1 , living. Daughters: none.

Brothers: 2 living. Sisters: 1 living, 2 dead.

Tattoo marks: 2 spots on back of right wrist.

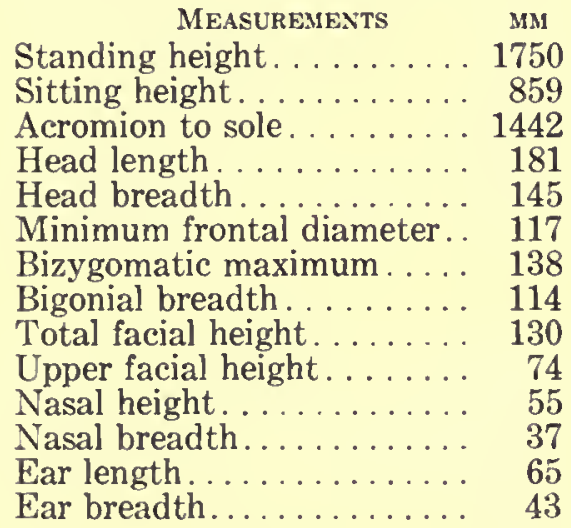

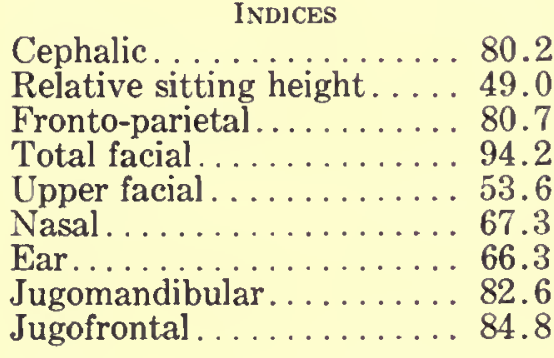

No. 699. Plate XCIX, Figs. 5, 6

Place: Hilla Army Camp. Date: June 17, 1928.

Birthplace: Amara. Race: Sudanese Negro. Age: 20; unmarried.

Brothers: 1 living. Sisters: 3 living.

Tattoo marks: none.

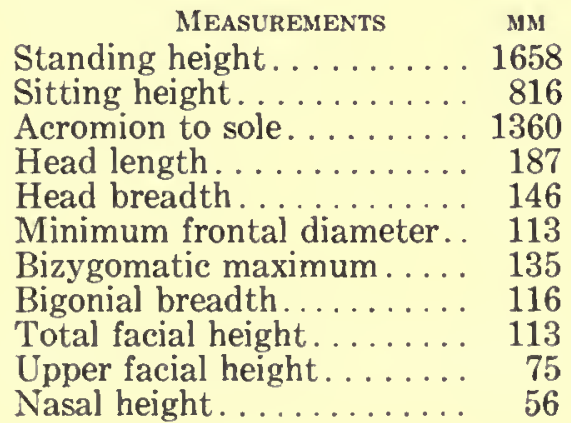

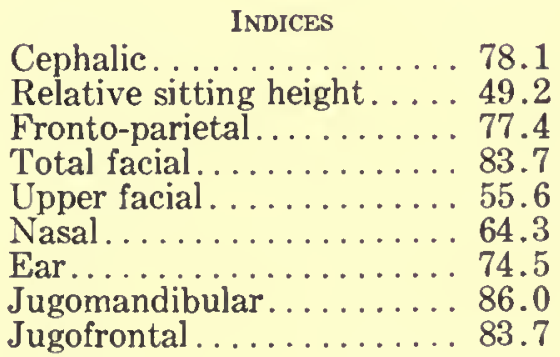




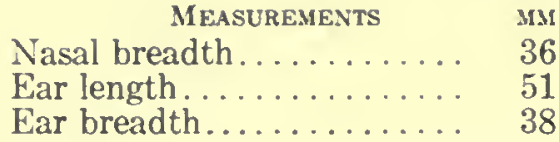

Negro; therefore, not included in statistics.

\section{No. 700. Plate XCIX, Figs. 7, 8}

Place: Hilla Army Camp. Date: June 17, 1928.

Birthplace: Amara. Age: 17; married for 2 years. No children.

Brothers: 2 living. Sisters: none.

'Tattoo marks: cross enclosed by circle on back of left hand.

\begin{tabular}{|c|c|c|}
\hline Measurements & MM & INDICES \\
\hline Standing height. . . . . . & 1745 & Cephalic. \\
\hline Sitting height... & 795 & Relative sitting height.... 45.5 \\
\hline Acromion to sole. & 1466 & Fronto-parietal......... 84.3 \\
\hline Head length... & 185 & Total facial. \\
\hline Head breadth. & 140 & Upper facial \\
\hline Minimum frontal diameter. & 118 & Nasal. . . . \\
\hline Bizygomatic maximum. . . . & 135 & . 60.0 \\
\hline Bigonial breadth.......... & 111 & Jugomandibular. . \\
\hline Total facial height........ & 123 & Jugofrontal.... \\
\hline Upper facial height. . . . & 62 & \\
\hline Nasal height. & 53 & \\
\hline Nasal breadth. & 33 & \\
\hline Ear length $\ldots \ldots \ldots \ldots \ldots$ & 65 & \\
\hline Ear breadth.. & 39 & \\
\hline
\end{tabular}

No. 701. Plate C, Figs. 1, 2

Place: Hilla Army Camp. Date: June 17, 1928.

Birthplace: Ba'quba. Age: 30 ; married for 7 years. Sons: 1 dead. Daughters: none. Brothers: 2 living. Sisters: 3 living.

Tattoo marks: none.

MEASUREMENTS MM

Standing height. . . . . . . 1758

Sitting height. . . . . . . 852

Acromion to sole......... 1470

Head length............. 188

Head breadth............ 147

Minimum frontal diameter.. 113

Bizygomatic maximum.... 138

Bigonial breadth......... 108

Total facial height........ 120

Upper facial height....... . 78

Nasal height........... 63

Nasal breadth........... 30

Ear length............. 55

Ear breadth.......... 37
INDICES

Cephalic............. 78.4

Relative sitting height.... 48.5

Fronto-parietal......... 76.9

Total facial ............ 87.0

Upper facial . . . . . . . . . . 56.5

Nasal. . . . . . . . . . . . . 47.6

Ear................. 67.3

Jugomandibular. . . . . . . 78.2

Jugofrontal . . . . . . . . . 81.9

No. 702

Place: Hilla Army Camp. Date: June 17, 1928.

Birthplace: Baghdad. Age: 18; married for 3 years. Sons: 1 living. Daughters: 1 dead.

Brothers: 1 living. Sisters: none.

Tattoo marks: none.

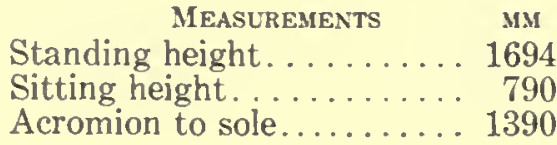

INDices
Cephalic............. 77.7
Relative sitting height.... 46.6
Fronto-parietal......... 74.0




\begin{tabular}{|c|c|}
\hline Measurements & MM \\
\hline Head length. & 188 \\
\hline Head breadth & 146 \\
\hline Minimum frontal diameter. & 18 \\
\hline Bizygomatic maximum .... . & 138 \\
\hline Bigonial breadth.......... & 1 \\
\hline Total facial height. . & 12 \\
\hline Upper facial height. . . . . . . & \\
\hline Nasal height. & \\
\hline Nasal breadth........... & \\
\hline Ear length & \\
\hline$d t$ & \\
\hline
\end{tabular}

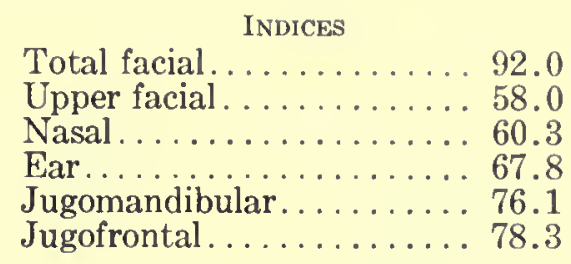

\section{No. 703. Plate C, Figs. 3,4}

Place: Hilla Army Camp. Date: June 17, 1928.

Birthplace: Amara. Age: 26; married for 5 years. Sons: none. Daughters: 1 living. Brothers: 2 living. Sisters: 1 living.

Tattoo marks: none.

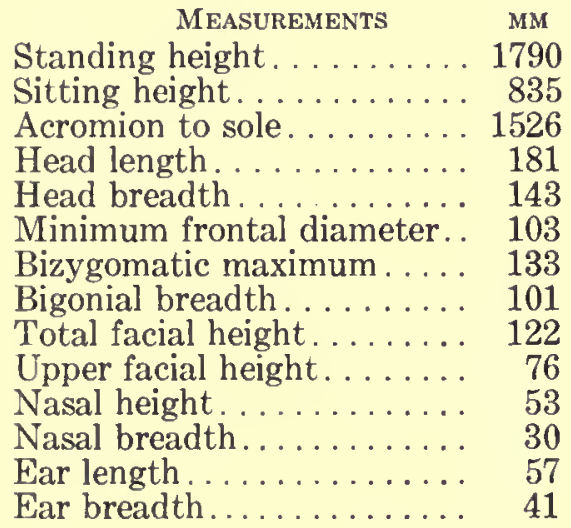

INDICES

Cephalic............. 79.1

Relative sitting height. ... 46.6

Fronto-parietal.......... 72.1

Total facial............ 91.7

Upper facial. . . . . . . . . 5 57.1

Nasal. ............. 56.6

Ear. . . . . . . . 72.0

Jugomandibular......... 75.9

Jugofrontal. . . . . . . . 77.5

Place: Hilla Army Camp. Date: June 17, 1928.

Birthplace: Shatra. Age: 20; unmarried.

Brothers: 7 living. Sisters: 3 living.

Tattoo marks: glove on back of right hand and spot at nasion.

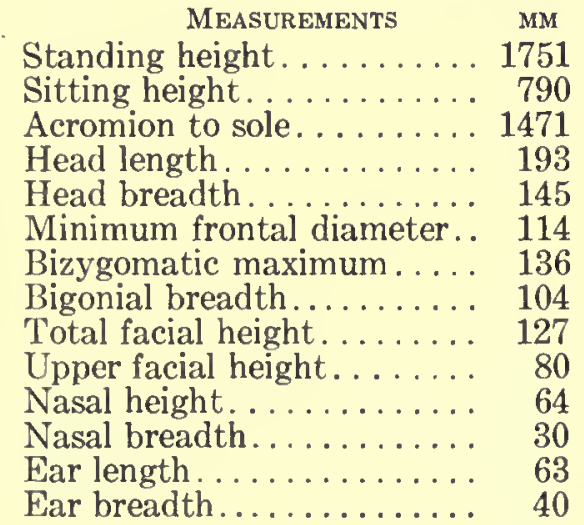

Cephalic............ 75.1

Relative sitting height. ... 45.0

Fronto-parietal.......... 78.6

Total facial............ 93.5

Upper facial. . . . . . . . . . . . 58.7

Nasal. . . ........... 46.9

Ear.................6. 63.5

Jugomandibular........ 76.5

Jugofrontal . . . . . . . . 83.8

\section{No. 705. Plate C, Figs. 7, 8}

Place: Hilla Army Camp. Date: June 17, 1928.

Birthplace: Sulaimaniya. Age: 25; unmarried. 


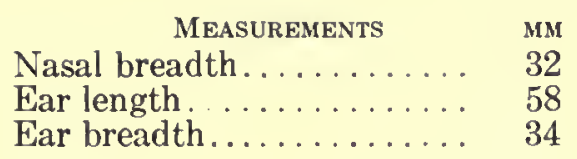

No. 708. Plate CI, Figs. 5, 6

Place: Hilla Army Camp. Date: June 17, 1928.

Birthplace: Baghdad. Age: 25; unmarried.

Brothers: 1 living. Sisters: 1 living.

Tattoo marks: none.

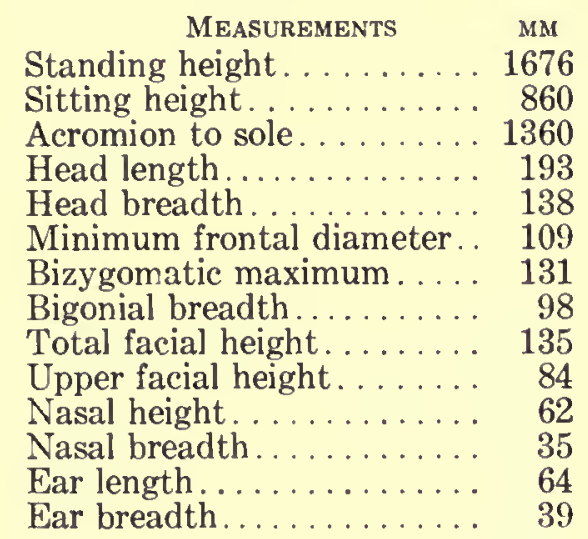

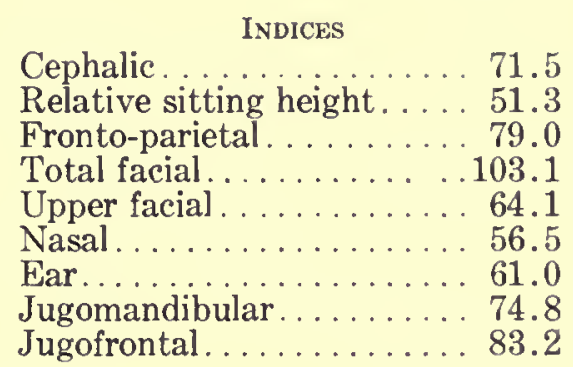

No. 709. Plate CI, Figs. 7, 8

Place: Hilla Army Camp. Date: June 17, 1928.

Birthplace: Baghdad. Age: 29; married for 4 years. Sons: 1 living. Daughters: none. Brothers: 1 living, 5 dead. Sisters: 1 living.

Tattoo marks: none.

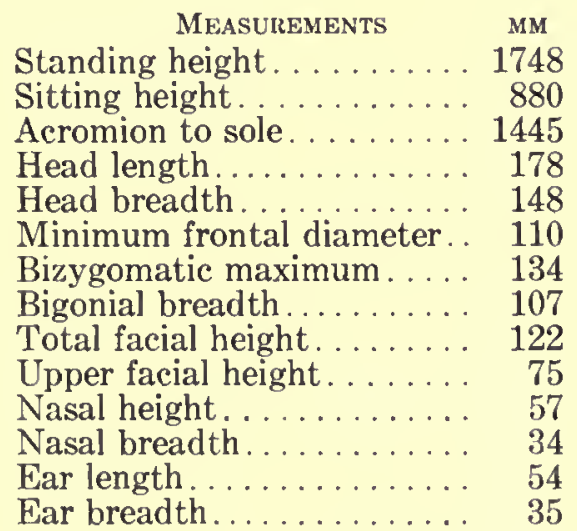

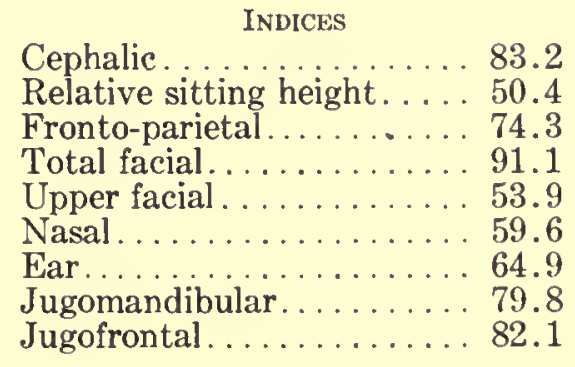

No. 710. Plate CII, Figs. 1, 2

Place: Hilla Army Camp. Date: June 17, 1928.

Birthplace: Baghdad. Age: 25; unmarried.

Brothers: 2 living, 1 dead. Sisters: 2 living.

Tattoo marks: girl in red and blue tattooed on inside of right forearm.

\begin{tabular}{|c|c|c|}
\hline MEASUREMENTS & MM & INDICES \\
\hline Standing height. . & 1672 & Cephalic. \\
\hline Sitting height. . & 846 & Relative sitting height. \\
\hline Acromion to sole... & 1377 & Fronto-parietal......... \\
\hline
\end{tabular}




\begin{tabular}{|c|c|c|}
\hline MEASUREMENTS & MM & INDICES \\
\hline Head length. & 186 & Total facial \\
\hline Head breadth. & 142 & Upper facial \\
\hline Minimum frontal diameter. & 116 & Nasal. . . . . \\
\hline Bizygomatic maximum.... & 136 & Ear. \\
\hline Bigonial breadth. & 108 & Jugomandibular... . \\
\hline Total facial height & 120 & Jugofrontal. . . . . . . \\
\hline
\end{tabular}

Upper facial height. . . . . . 70

Nasal height............ 53

Nasal breadth........... 36

Ear length ............. 50

Ear breadth........... 30

\section{No. 711. Plate CII, Figs. 3, 4}

Place: Hilla Army Camp. Date: June 17, 1928.

Birthplace: Baghdad. Age: 45; married for 25 years. Sons: 1 living. Daughters: 1 living. Brothers: 2 living. Sisters: 1 living.

Tattoo marks: glove on back of right hand.

\begin{tabular}{|c|c|c|}
\hline MEASUREMENTS & MM & INDICES \\
\hline Standing height. . . . . . & 1687 & Cephalic. \\
\hline Sitting height. & 895 & Relative sitting height. . . . 53.0 \\
\hline Acromion to sole. & 1403 & Fronto-parietal....... \\
\hline Head length.... & 190 & Total facial. . \\
\hline Head breadth. & 146 & Upper facial \\
\hline Minimum frontal diameter. . & 113 & Nasal..... \\
\hline Bizygomatic maximum . . . . . & 128 & Ear. \\
\hline Bigonial breadth .......... & 107 & Jugomandibular. . . . . \\
\hline Total facial height. ....... & 124 & Jugofrontal . . . . . . . \\
\hline Upper facial height. . . . & 76 & \\
\hline Nasal height. & 50 & \\
\hline Nasal breadth. & 38 & \\
\hline Ear length.... & 71 & \\
\hline Ear breadth & 37 & \\
\hline
\end{tabular}

\section{No. 712. Plate CII, Figs. 5, 6}

Place: Hilla Army Camp. Date: June 17, 1928.

Birthplace: Baghdad. Age: 25; unmarried.

Brothers: 2 living, 2 dead. Sisters: 1 living.

Tattoo marks: on right arm, two hands joined, girl's head, Turkish armored car; on left arm, armored car and nude woman.

\begin{tabular}{|c|c|c|}
\hline MEASUREMENTS & MM & INDICES \\
\hline Standing height. . . . . . & 1736 & Cephalic. \\
\hline Sitting height... & 875 & Relative sitting height. \\
\hline Acromion to sole. & 1460 & Fronto-parietal ........ 84.6 \\
\hline Head length..... & 192 & Total facial....... \\
\hline Head breadth. & 143 & Upper facial \\
\hline Minimum frontal diameter. . & 121 & Nasal. \\
\hline Bizygomatic maximum . . . . . & 140 & Ear. \\
\hline Bigonial breadth.......... & 114 & Jugomandibular. . \\
\hline Total facial height. . . . . . . & 124 & Jugofrontal . . . . . \\
\hline Upper facial height. . . . . . . & 65 & \\
\hline Nasal height............ & 55 & \\
\hline Nasal breadth & 37 & \\
\hline Ear length & 65 & \\
\hline Ear breadth & 40 & \\
\hline
\end{tabular}

\section{No. 713. Plate CII, Figs. 7, 8}

Place: Hilla Army Camp. Date: June 17, 1928.

Birthplace: Baghdad. Age: 27; unmarried. 
Brothers: 3 living, 7 dead. Sisters: 3 living, 2 dead.

Tattoo marks: spot on back of right hand.

\begin{tabular}{|c|c|c|}
\hline MEASUREMENTS & мм & INDICES \\
\hline Standing height. . . . . & 1701 & Cephalic. ........... \\
\hline Sitting height. & 907 & Relative sitting height. \\
\hline Acromion to sole & 1440 & Fronto-parietal...... \\
\hline Head length. & 178 & Total facial. \\
\hline Head breadth & 145 & Upper facial \\
\hline Minimum frontal diameter. & 111 & Nasal \\
\hline Bizygomatic maximum.... & 128 & Ear. \\
\hline Bigonial breadth. & 108 & Jugomandibular \\
\hline Total facial height & 121 & Jugofrontal. . \\
\hline
\end{tabular}

Upper facial height. ...... 70

Nasal height ............ 50

Nasal breadth........... 31

Ear length.............. 52

Ear breadth............ 31

\section{No. 714. Plate CIII, Figs. 1, 2}

Place: Hilla Army Camp. Date: June 17, 1928.

Birthplace: Mosul. Age: 22; unmarried.

Brothers: 2 living, 1 dead. Sisters: 2 living, 1 dead.

Tattoo marks: large spot on outside of right forearm.

\begin{tabular}{|c|c|c|}
\hline MEASUREMENTS & MM & INDICES \\
\hline Standing height. & 1673 & Cephalic. \\
\hline Sitting height. & 857 & Relative sitting height. \\
\hline Acromion to sole. & 1380 & Fronto-parietal. \\
\hline Head length..... & 183 & Total facial... \\
\hline Head breadth. & 149 & Upper facial \\
\hline Minimum frontal diameter. & 111 & Nasal. \\
\hline Bizygomatic maximum ..... & 138 & Ear. \\
\hline Bigonial breadth. & 107 & Jugomandibula \\
\hline Total facial height & 115 & Jugofrontal \\
\hline
\end{tabular}

Upper facial height. . . . . . 76

Nasal height. ........... 56

Nasal breadth............. 36

Ear length ............. 57

Ear breadth............. 32

No. 715. Plate CIII, Figs. 3, 4

Place: Hilla Army Camp. Date: June 17, 1928.

Birthplace: Nasiriya. Age: 20; unmarried.

Brothers: 2 living, 2 dead. Sisters: 2 living, 2 dead.

Tattoo marks: glove on back of right hand.

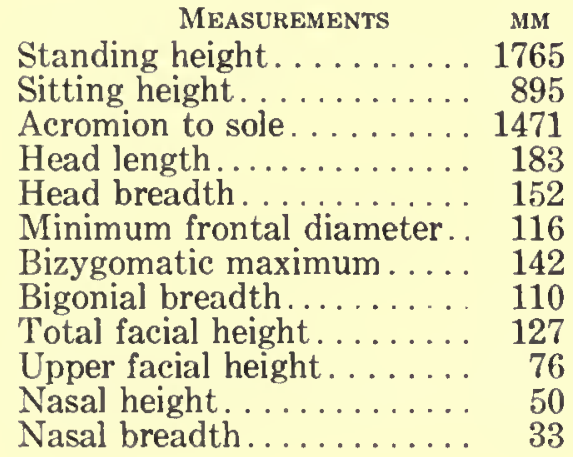

Indices
Cephalic............. 83.1

Relative sitting height..... 50.7

Fronto-parietal.......... 76.4

Total facial. ............ 89.4

Upper facial . . . . . . . . . . . . 53.1

Nasal. . . . . . . . . . . . . . 66.0

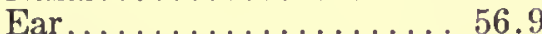

Jugomandibular. . . . . . . . 77.5

Jugofrontal. . . . . . . . 8 81.7 


$\begin{array}{cr}\text { MEasurements } & \text { MM } \\ \text { Ear length } \ldots \ldots \ldots \ldots \ldots \ldots & 58 \\ \text { Ear breadth } \ldots \ldots \ldots \ldots \ldots & 33\end{array}$

\section{No. 716. Plate CIII, Figs. 5, 6}

Place: Hilla Army Camp. Date: June 17, 1928.

Birthplace: Baghdad. Age: 22; married for 3 years. Sons: 1 living. Daughters: none. Brothers: 3 living, 4 dead. Sisters: 3 living.

Tat too marks: none.

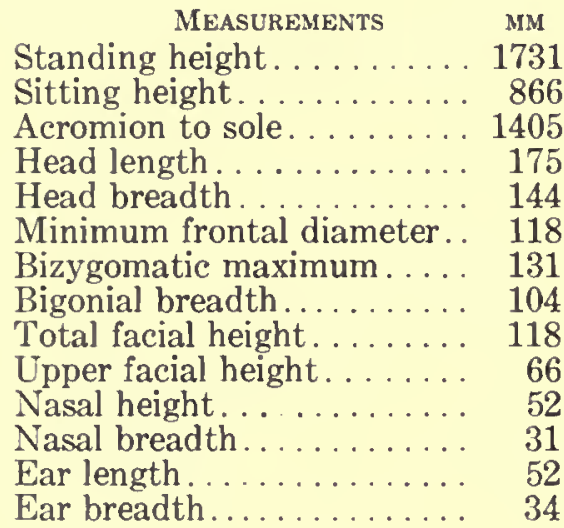

INDiCES

Cephalic............. 82.3

Relative sitting height.... 50.1

Fronto-parietal. ........ 82.0

Total facial ............. 90.1

Upper facial. .......... 50.4

Nasal ................ 59.6

Ear...............6. 65.4

Jugomandibular......... 79.4

Jugofrontal. ......... 90.1

\section{No. 717. Plate CIII, Figs. 7, 8}

Place: Hilla Army Camp. Date: June 17, 1928.

Birthplace: Baghdad. Age: 33 ; married for 2 years. No children.

Brothers: 3 living, 2 dead. Sisters: 3 living, 1 dead.

Tattoo marks: none.

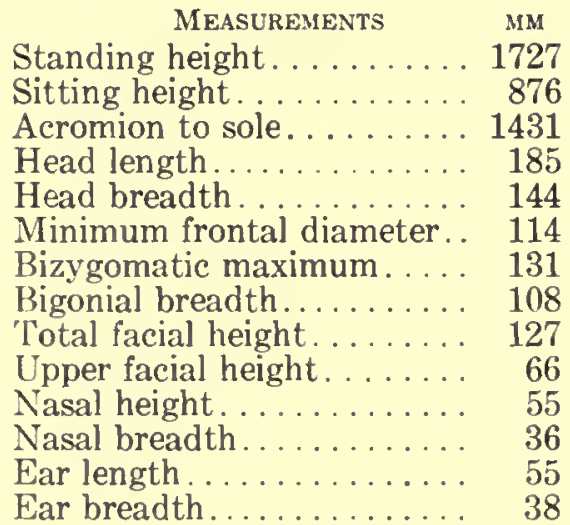

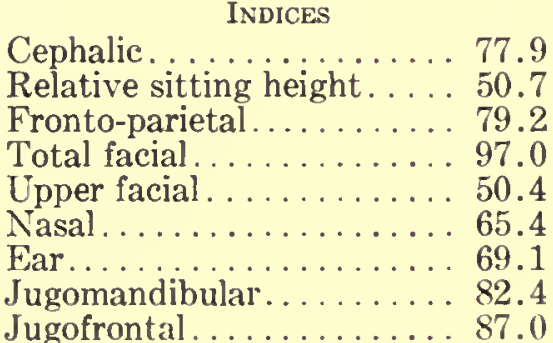

INDICES

Cephalic.............. 75.1

Relative sitting height..... 50.4

Fronto-parietal.........8 81.2

Total facial............. 88.5 


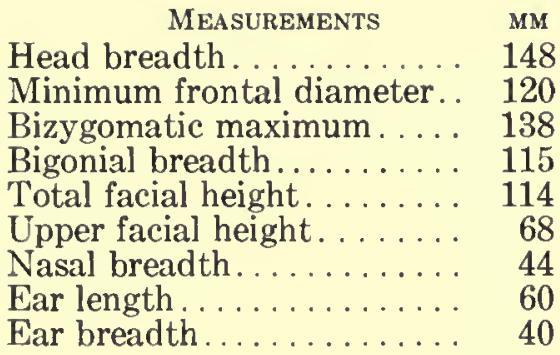

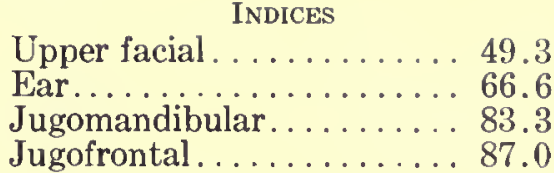

No. 719. Plate CIV, Figs. 3, 4

Place: Hilla Army Camp. Date: June 17, 1928.

Birthplace: Nasiriya. Age: 20; unmarried. Brothers: 1 living, 5 dead. Sisters: 3 dead. Tattoo marks: none.

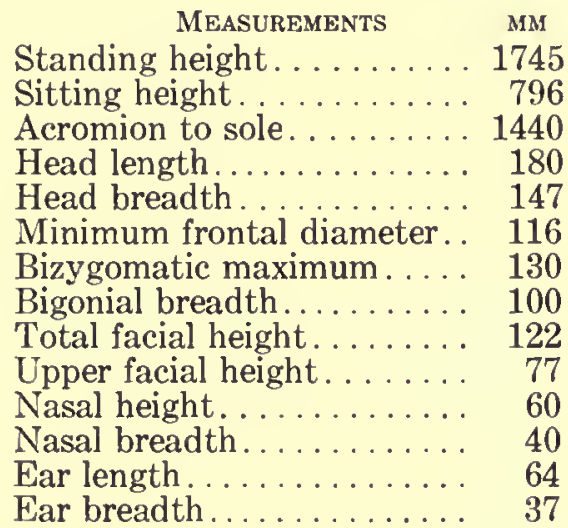

INDICES

Cephalic ............. 81.7

Relative sitting height. . . 45.6

Fronto-parietal......... 79.0

Total facial.............. 93.9

Upper facial. . . . . . . . 59.2

Nasal ................6. 66.7

Ear............... 57.8

Jugomandibular. ........... 77.0

Jugofrontal . . . . . . . . 89.2

\section{No. 720. Plate CIV, Figs. 5, 6}

Place: Hilla Army Camp. Date: June 17, 1928.

Birthplace: Ba'quba. Age: 20; unmarried.

Brothers: 3 living, 1 dead. Sisters: 1 living, 2 dead.

Tattoo marks: none.

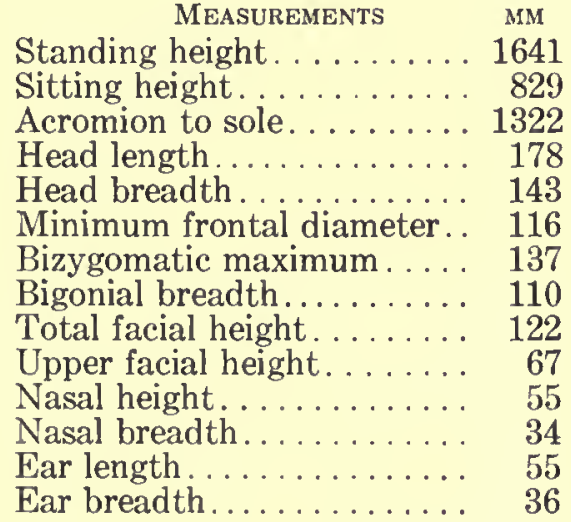

Cephalic.............80.2

Relative sitting height... 50.5

Fronto-parietal..........8 81.2

Total facial........... 89.1

Upper facial. . . . . . . . . . . 48.9

Nasal. .............61.8

Ear............... 63.6

Jugomandibular......... 80.3

Jugofrontal . . . . . . . . . . . . 8 84.7

\section{No. 721. Plate CIV, Figs. 7, 8}

Place: Hilla Army Camp. Date: June 17, 1928.

Birthplace: Ba‘quba. Age: 25 ; unmarried.

Brothers: 2 living. Sisters: 1 living.

Tattoo marks: none. 


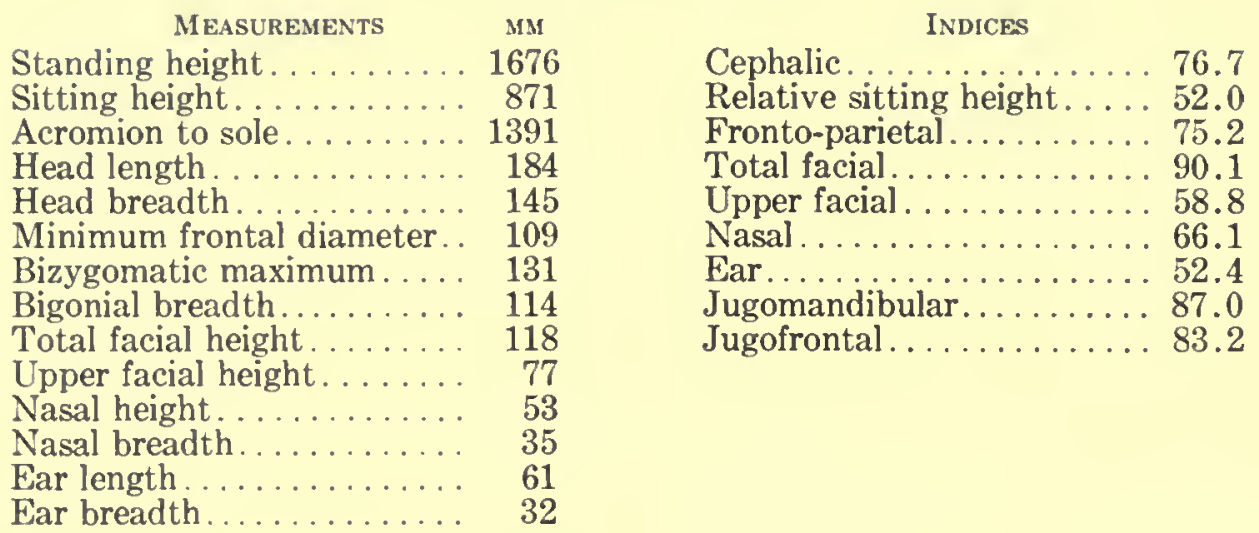

\section{No. 722. Plate CV, Figs. 1,2}

Place: Hilla Army Camp. Date: June 17, 1928.

Birthplace: Kirkuk. Age: 20; unmarried.

Brothers: 2 dead. Sisters: 1 living, 1 dead.

Tattoo marks: none.

\begin{tabular}{|c|c|}
\hline MEASUREMENTS & MM \\
\hline Standing height. & 1816 \\
\hline Sitting height. & 921 \\
\hline Acromion to sole & 1511 \\
\hline Head length... & 178 \\
\hline Head breadth. & 146 \\
\hline Minimum frontal diameter. . & 113 \\
\hline Bizygomatic maximum..... & 138 \\
\hline Bigonial breadth.......... & 105 \\
\hline Total facial height. & 124 \\
\hline Upper facial height. . & 75 \\
\hline Nasal height........ & 53 \\
\hline asal breadth & 37 \\
\hline Ear length & 54 \\
\hline For hr & 42 \\
\hline
\end{tabular}

\section{INDICES}

Cephalic............. 79.2

Relative sitting height. . . . 50.8

Fronto-parietal . . . . . . . 77.4

Total facial............. 89.9

Upper facial. . . . . . . . . 54.3

Nasal. ................ 69.9

Ear.................. 77.8

Jugomandibular............. 76.1

Jugofrontal. .......... 81.9

\section{No. 723. Plate CV, Figs. 3, 4}

Place: Hilla Army Camp. Date: June 17, 1928.

Birthplace: Ba'quba. Age: 20; unmarried.

Brothers: 4 living. Sisters: 1 living.

Tattoo marks: none.

\begin{tabular}{|c|c|}
\hline MEASUREMENTS & MM \\
\hline Standing height. & 1651 \\
\hline Sitting height. & 839 \\
\hline Acromion to sole & 1386 \\
\hline Head length..... & 184 \\
\hline Head breadth. & 143 \\
\hline Minimum frontal diameter. & 11 \\
\hline Bizygomatic maximum. . . . & \\
\hline Bigonial breadth.......... & 10 \\
\hline Total facial height. & 124 \\
\hline Upper facial height. . . . . . & \\
\hline Nasal height. . . . . . . . . . & \\
\hline Nasal breadth & \\
\hline Ear length & \\
\hline - hod & \\
\hline
\end{tabular}

INDICES

Cephalic............. 77.7

Relative sitting height.... 50.8

Fronto-parietal......... 79.0

Total facial. .............. 90.6

Upper facial. . . . . . . . . 49.6

Nasal. .................61.1

Ear.................. 54.0

Jugomandibular. . . . . . . 72.5

Jugofrontal. ........... 81.9 
No. 724. Plate CV, Figs. 5, 6

Place: Hilla Army Camp. Date: June 17, 1928.

Birthplace: Ba'quba. Age: 28; married for 10 years. Sons: 1 living. Daughters: 1 living. Brothers: 3 living, 1 dead. Sisters: 1 living, 1 dead.

Tattoo marks: designs on inside of upper part of right forearm.

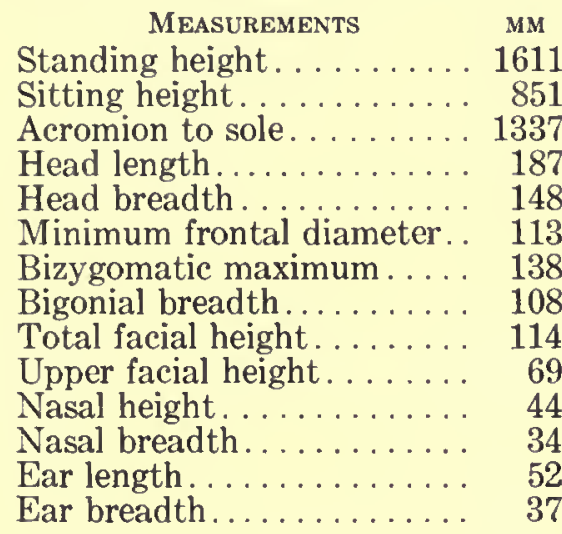

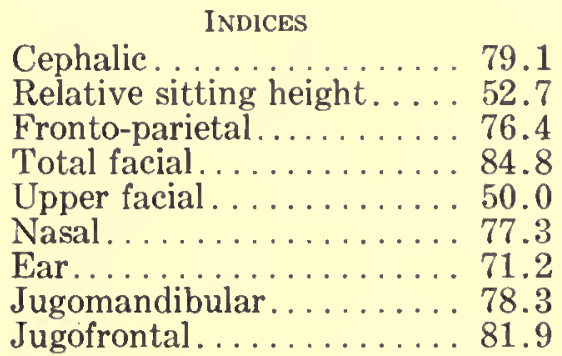

No. 725. Plate CV, Figs. 7, 8

Place: Hilla Army Camp. Date: June 17, 1928.

Birthplace: Ba'quba. Age: 25; unmarried.

Brothers: 3 living, 1 dead. Sisters: 2 dead.

Tattoo marks: 3 spots on back of right wrist.

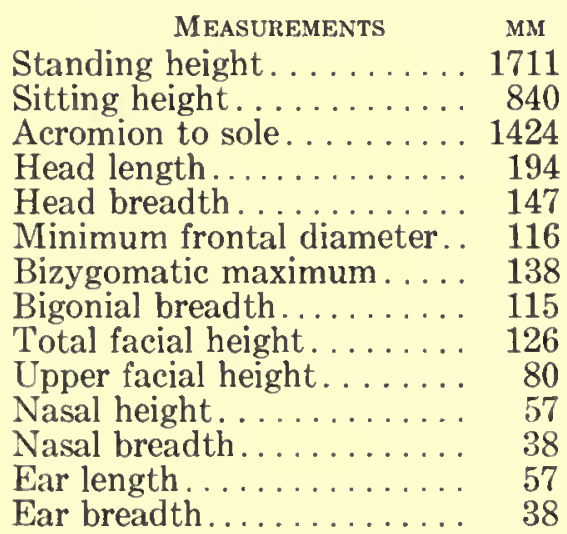

INDICES

Cephalic............ 75.7

Relative sitting height.... 49.1

Fronto-parietal........ 78.9

Total facial ............ 91.4

Upper facial. ......... 58.0

Nasal. ...............66.7 66.6

Ear..............6.6.6 66.6

Jugomandibular . . . . . . . . 83.3

Jugofrontal . . . . . . . 84.1

\section{No. 726. Plate CVI, Figs. 1, 2}

Place: Hilla Army Camp. Date: June 17, 1928.

Birthplace: Ba'quba. Age: 25; unmarried.

Brothers: 2 living. Sisters: none.

Tattoo marks: none.

\begin{tabular}{|c|c|}
\hline MEASUREMENTS & MM \\
\hline Standing height. & 1714 \\
\hline Sitting height. & 873 \\
\hline Acromion to sole. & 1410 \\
\hline Head length. & 196 \\
\hline Head breadth. & 1 \\
\hline frontal diameter. & 116 \\
\hline atic maximum ... & 137 \\
\hline gonial breadth. & \\
\hline
\end{tabular}

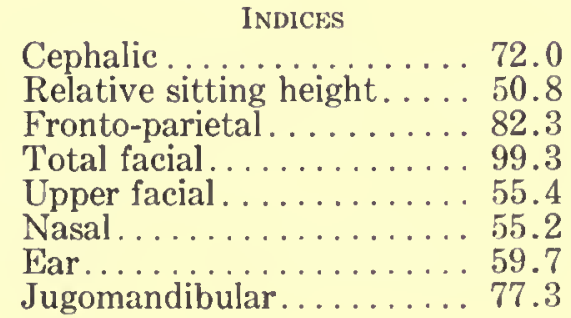




\begin{tabular}{|c|c|c|}
\hline MEASUREMENTS & MM & INDICES \\
\hline Total facial height...... & 136 & Jugofrontal . . . . . . . . . . 84.6 \\
\hline Upper facial height. . . . . & 76 & \\
\hline Nasal height. ............. & 58 & \\
\hline Nasal breadth............. & 32 & \\
\hline Ear length. . & 62 & \\
\hline Ear breadth & 37 & \\
\hline
\end{tabular}

\section{No. 727. Plate CVI, Figs. 3, 4}

Place: Hilla Army Camp. Date: June 17, 1928.

Birthplace: Ba'quba. Age: 35; married for 10 years. Sons: 1 dead. Daughters: 1 dead. Brothers: 4 dead. Sisters: 1 dead.

Tattoo marks: none.

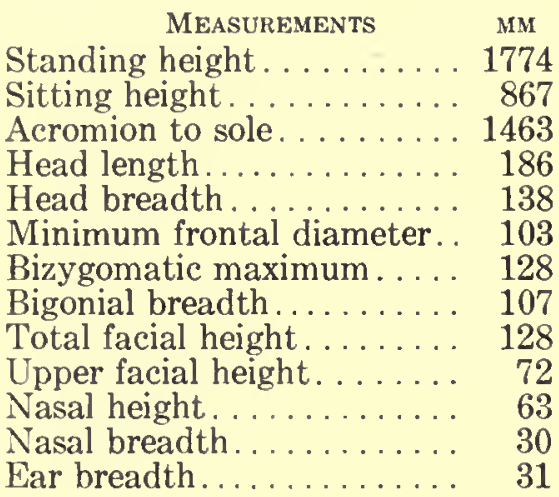

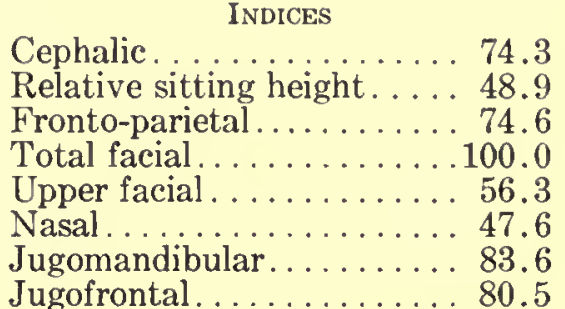

\section{No. 728. Plate CVI, Figs. 5, 6}

Place: Hilla Army Camp. Date: June 17, 1928.

Birthplace: Hilla. Age: 20 ; married for 1 year. No children.

Brothers: 7 living. Sisters: 1 living.

Tattoo marks: glove on back of right hand; name and figures 15815 on left arm.

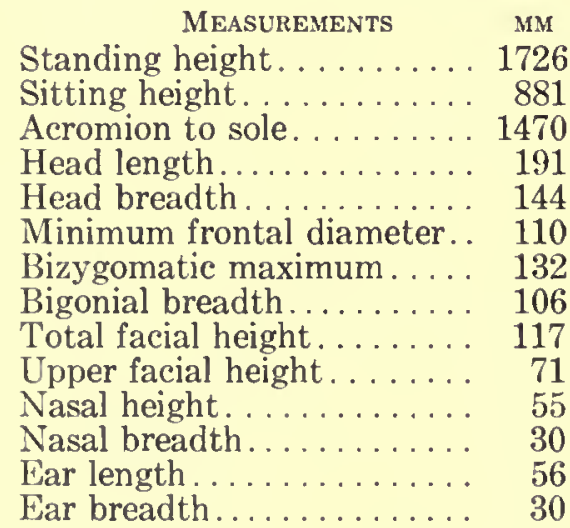

Cephalic............. 75.3

Relative sitting height.... 51.1

Fronto-parietal......... 76.4

Total facial. ............ 88.7

Upper facial. . . . . . . . 53.7

Nasal .................. 54.6

Ear................. 53.6

Jugomandibular. . . . . . . 80.3

Jugofrontal. ........... 83.4

\section{No. 729. Plate CVI, Figs. 7, 8}

Place: Hilla Army Camp. Date: June 17, 1928.

Birthplace: Hilla. Age: 22; unmarried.

Brothers: 2 living, 2 dead. Sisters: 1 dead.

Tattoo marks: 3 spots on back of right hand.

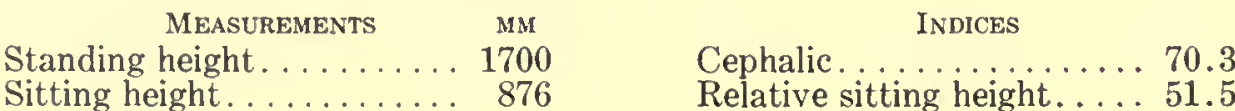




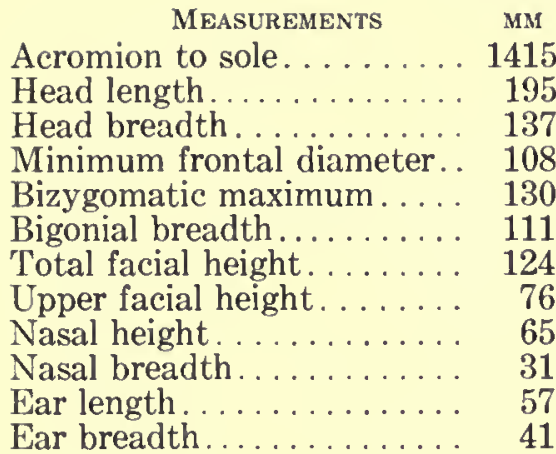

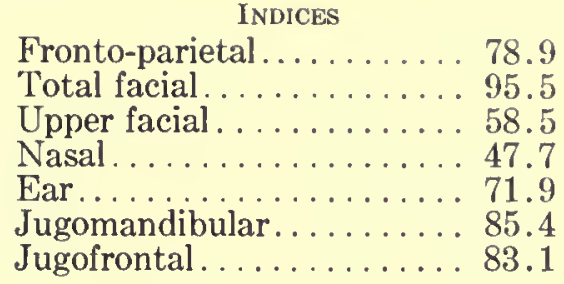

\section{No. 730. Plate CVII, Figs. 1, 2}

Place: Hilla Army Camp. Date: June 17, 1928.

Birthplace: An Najaf. Age: 21; unmarried.

Brothers: 1 living, 3 dead. Sisters: 1 living, 2 dead.

Tattoo marks: none.

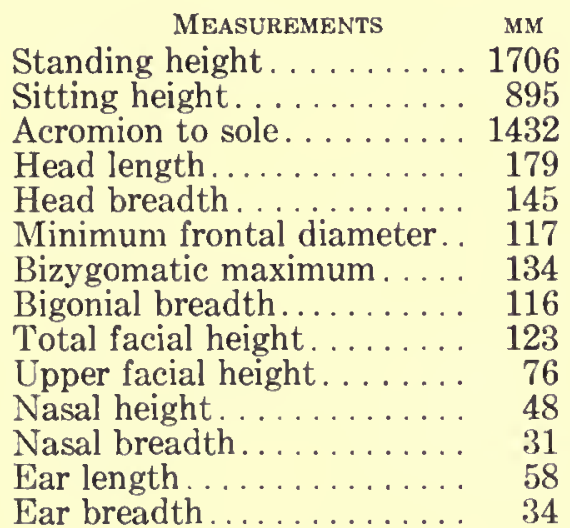

INDICES

Cephalic. ...........8 81.0

Relative sitting height.... 52.4

Fronto-parietal......... 80.7

Total facial ............... 91.8

Upper facial. . . . . . . . 56.7

Nasal . . . . . . . . . . . . . . 64.6

Ear............... 58.6

Jugomandibular. . . . . . 86.6

Jugofrontal ........... 87.3

\section{No. 731. Plate CVII, Figs. 3, 4}

Place: Hilla Army Camp. Date: June 17, 1928.

Birthplace: Mandali. Age: 28; unmarried.

Brothers: 1 dead. Sisters: 1 living.

Tattoo marks: none.

MEasurements MM

Standing height. . . . . . . 1751

Sitting height. .......... 892

Acromion to sole. . . . . . . . 1460

Head length............ 202

Head breadth............ 153

Minimum frontal diameter. . 114

Bizygomatic maximum ..... 136

Bigonial breadth......... 103

Total facial height. . . . . . . . 124

Upper facial height. . . . . . 76

Nasal height. . . . . . . . . 58

Nasal breadth.......... 34

Ear length . . . . . . . . . . 56

Ear breadth........... 35
INDICES

Cephalic............. 75.0

Relative sitting height. . . 5 51.0

Fronto-parietal.......... 74.5

Total facial............ 91.3

Upper facial. . . . . . . . . . . . 55.9

Nasal. ............ 58.6

Ear................ 62.5

Jugomandibular . . . . . . . 75.8

Jugofrontal. . . . . . . . . . 83.8

No. 732

Place: Hilla Army Camp. Date: June 17, 1928.

Birthplace: Kirkuk. Age: 25; unmarried. 
Brothers: 5 living. Sisters: none.

Tattoo marks: none.

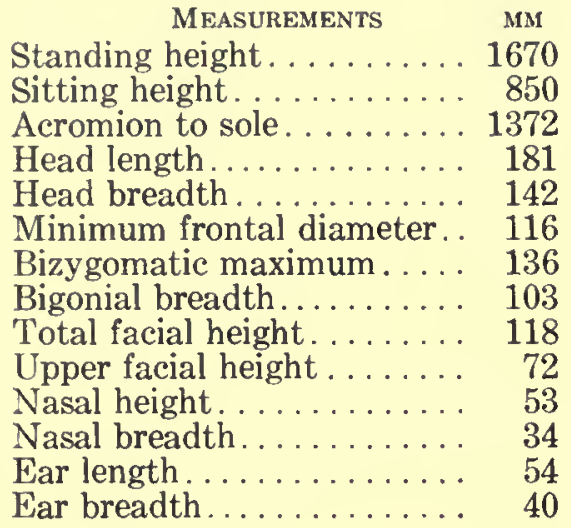

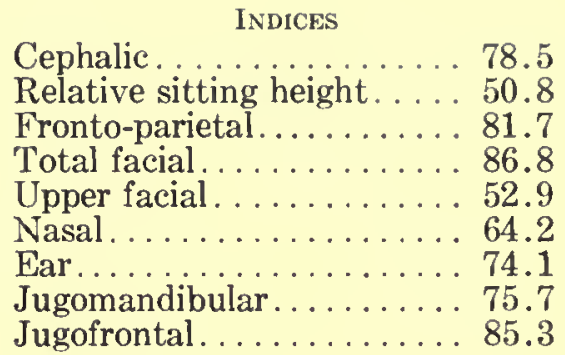

\section{BA'IJ BEDUINS}

\section{No. 300. Plate CVIII, Figs. 1, 2}

Locality: near Jemdet Nasr. Date: July 10, 1928.

Age: 35; married 20 years. Sons: 2 living. Daughters: 1 living.

Brothers: none. Sisters: none.

Tattoo marks: none.

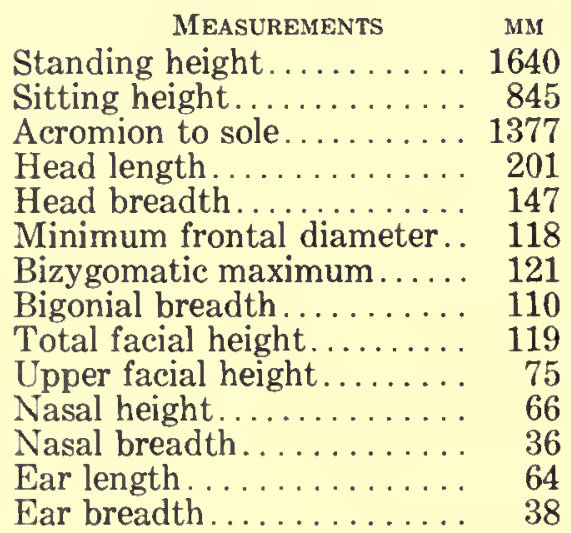

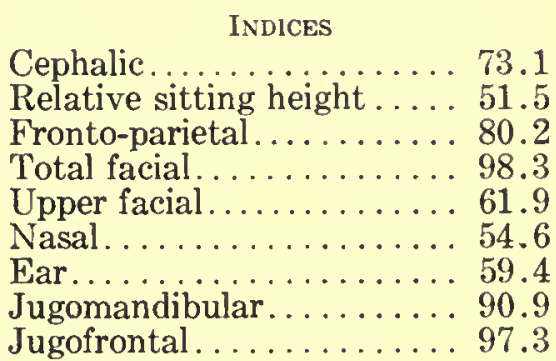

HAIR.-Form: low waves. Texture: medium. Color: dark brown. EYE.-Color: dark brown. Sclera: bloodshot. Iris: zoned.

Nose.-Profile: concavo-convex.

TEeтH.-Eruption: complete. Lost: none. Bite: slight over.

Chest.-Development: plus.

Musculature.-Excellent.

HEALTH.-Excellent.

\section{No. 301. Plate CVIII, Figs. 3, 4}

Locality: near Jemdet Nasr. Date: July 10, 1928.

Age: 60; married five times. Sons: 2 living, 5 dead. Daughters: 2 living, 3 dead. Brothers: 4 dead. Sisters: 2 dead.

Tattoo marks: none.

MEASUREMENTS MM

Standing height.... . . . . . 1775

Sitting height........... 861
INDICES

Cephalic...............6. 68.4

Relative sitting height.... 48.5 


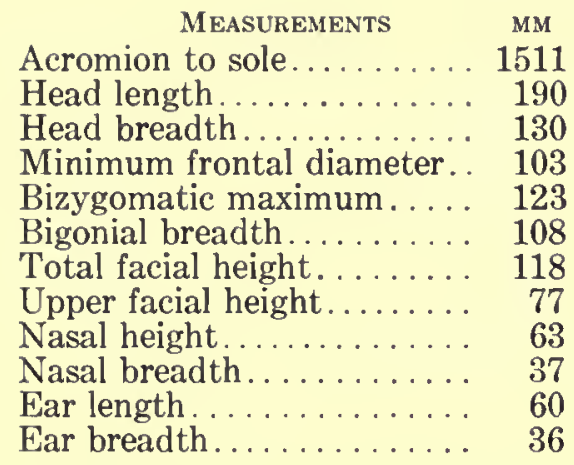

Fronto-parietal.......... 79.2

Total facial. . . . . . . . . 95.8

Upper facial............. 62.6

Nasal. ............. 58.7

Ear................. 60.0

Jugomandibular........ 87.8

Jugofrontal . . . . . . . . 82.9

HAIR.-Quantity: head, average; beard, double plus.

EYE.-Color: green brown. Sclera: bloodshot. Iris: zoned.

Nose.-Profile: straight.

TEETH.-Eruption: complete. Wear: plus. Caries: double plus. Bite: slight over. Lost: 2. Chest.-Development: average.

Musculature.-Poor.

HEALTH.-Poor; extremely thin.

No. 302. Plate CVIII, Figs. 5, 6

Locality: near Jemdet Nasr. Date: July 10, 1928.

Age: 20 ; married 2 years. Sons: none. Daughters: none.

Brothers: 1 living, 3 dead. Sisters: 2 living, 1 dead.

Tattoo marks: none.

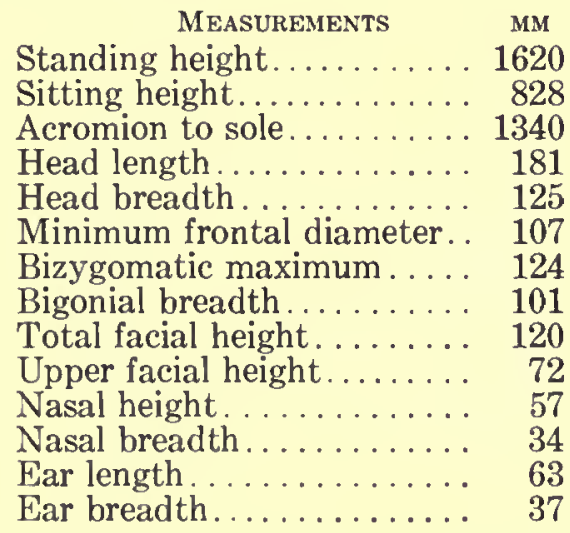

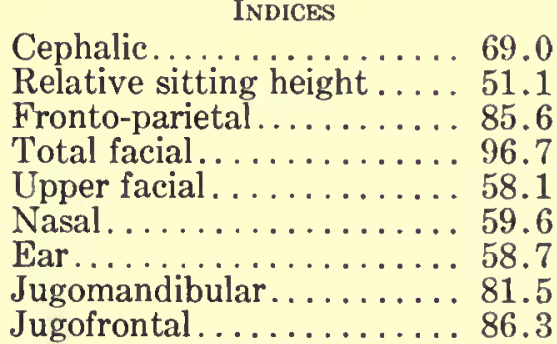

Relative sitting height .... 51.1

Fronto-parietal........ 85.6

Total facial............ 96.7

.

Ear.........................

Jugofrontal ............ 86.3

HAIR.-Form: curly frizzly. Texture: coarse. Color: reddish brown. Quantity: head, double plus; beard, plus; body, plus.

EYE.-Color: dark brown. Sclera: clear. Iris: homogeneous.

Nose.-Profile: straight. Wings: medium.

TEETH.-Eruption: complete. Lost: none.

CHEsT.-Development: double plus.

Musculature.-Excellent.

HEALTH.-Excellent.

\section{No. 303. Plate CVIII, Figs. 7,8}

Locality: near Jemdet Nasr. Date: July 10, 1928.

Age: 20; unmarried.

Brothers: none. Sisters: none.

Tattoo marks: spot on right side of upper lip. 


\begin{tabular}{|c|c|}
\hline MEASUREMENTS & MM \\
\hline Standing height.... & 1745 \\
\hline Sitting height..... & \\
\hline $\begin{array}{l}\text { Acromion to sole. } \\
\text { Head length }\end{array}$ & \\
\hline $\begin{array}{l}\text { Head length. } \\
\text { Head breadth }\end{array}$ & \\
\hline Minimum frontal diameter. & \\
\hline Bizygomatic maximum..... & \\
\hline Bigonial breadth. & \\
\hline Total facial height & \\
\hline Upper facial height & \\
\hline Nasal height. & \\
\hline $\begin{array}{l}\text { Nasal breadth } \\
\text { Ear length... }\end{array}$ & \\
\hline Car breadth & \\
\hline
\end{tabular}

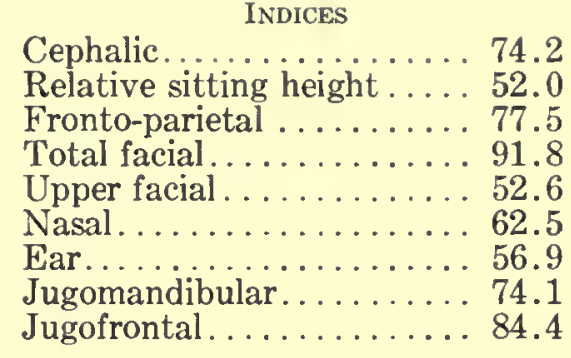

HAIR.—Form: deep waves. Texture: medium. Quantity: head, double plus; beard, plus. EYE.-Color: dark brown. Sclera: clear. Iris: zoned.

Nose.-Profile: straight. Wings: medium.

TEETH.-Eruption: complete. Lost: none. Bite: slight over.

Chest.-Development: plus.

Musculature.-Excellent.

HEALTH.-Excellent.

\section{No. 304. Plate CIX, Figs. 1, 2}

Locality: near Jemdet Nasr. Date: July 10, 1928.

Age: 25 ; unmarried.

Brothers: 2 living. Sisters: 1 living.

Tattoo marks: none.

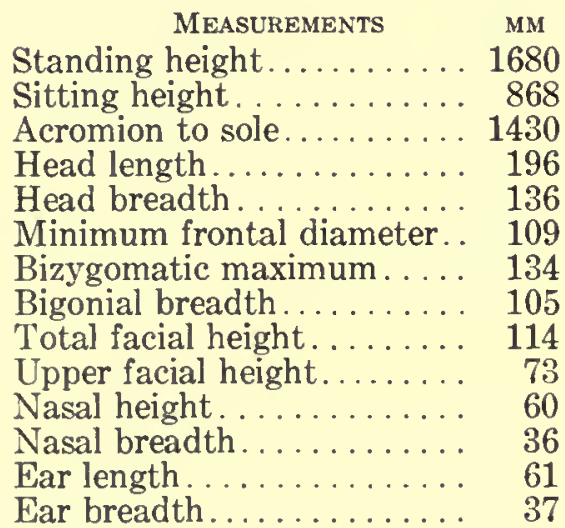

HAIR.-Form: deep waves. Texture: coarse. Color: dark brown. Quantity: head, double plus; beard, double plus; body, double plus.

EYE.-Color: dark brown. Sclera: clear. Iris: homogeneous.

NosE.-Profile: straight. Wings: medium.

TEETH.-Eruption: complete. Lost: none.

CHEsT.-Development: double plus.

Musculature.-Excellent.

HEALTH.-Excellent.

No. 305. Plate CIX, Figs. 3, 4

Locality: near Jemdet Nasr. Date: July 10, 1928.

Age: 40; married (wife now dead). Sons: none. Daughters: none. 
Brothers: 1 living. Sisters: none.

Tattoo marks: none.

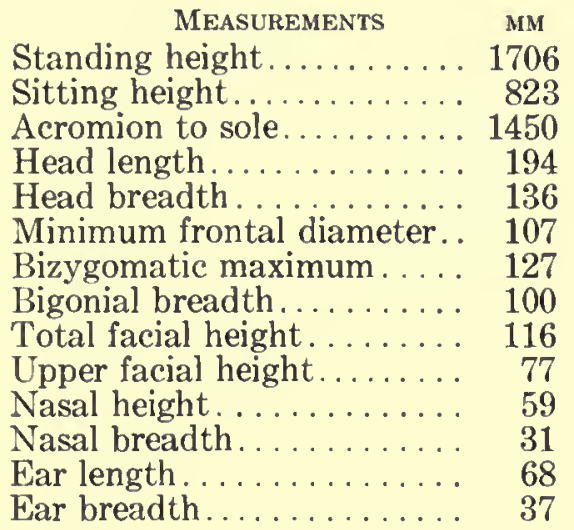

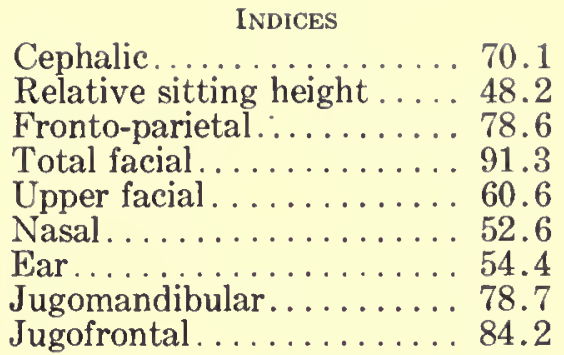

HAIR.-Texture: medium.

EYE.-Sclera: clear. Iris: homogeneous.

Nose.-Profile: concave. Tip: elevated. Wings: compressed.

TeEтн.-Eruption: complete. Wear: double plus. Lost: none. Bite: slight over.

CHEST.-Development: plus.

Musculature.-Good.

HEALTH.-Good.

No. 306. Plate CIX, Figs. 5, 6

Locality: near Jemdet Nasr. Date: July 10, 1928.

Age: 30 ; unmarried.

Brothers: none. Sisters: none.

Tattoo marks: none.

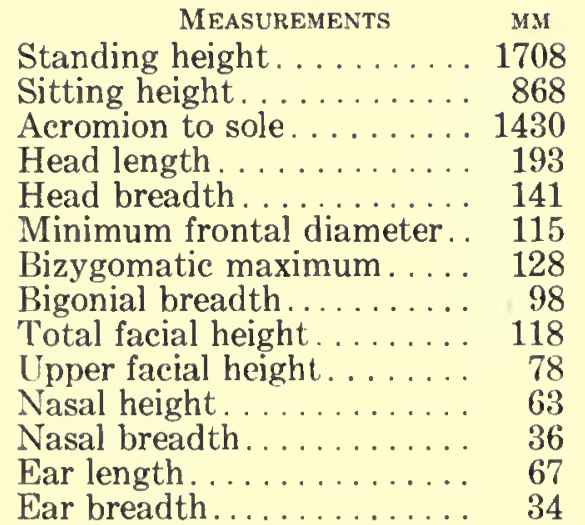

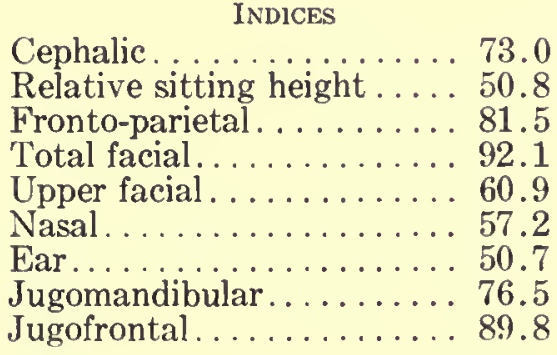

Cephalic............... 73.0

Relative sitting height.... 50.8

Fronto-parietal ........ 81.5

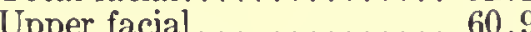

Nasal. . . ........... 57.2

Jugofrontal . . . . . . . . 89.8

HAIR.-Form: curly frizzly. Texture: medium. Quantity: head, double plus; beard, double plus; body, double plus.

EYE.-Color: green brown. Sclera: bloodshot. Iris: zoned.

Nose.-Profile: straight. Tip: depressed. Wings: compressed.

TEeTH.-Eruption: complete. Lost: 1. Bite: slight over.

CHEST.-Development: plus.

Musculature.-Good.

HEALTH.-Good.

No. 307. Plate CIX, Figs. 7, 8

Locality: near Jemdet Nasr. Date: July 10, 1928.

Age: 74; married 40 years. Sons: 2 living. Daughters: 1 living. 
Brothers: none. Sisters: none.

Tattoo marks: none.

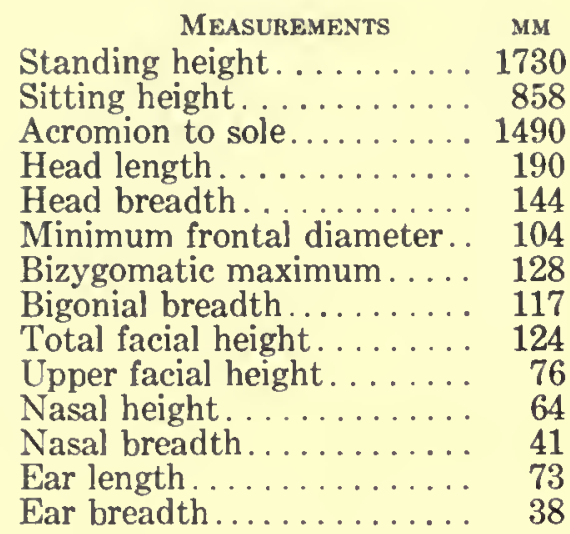

INDICES

Cephalic............ 75.7

Relative sitting height ..... 49.5

Fronto-parietal........ 72.2

Total facial. . . . . . . . . . . 96.9

Upper facial . . . . . . . . 59.3

Nasal . . . . . . . . . . . . . 64.0

Ear............... 52.1

Jugomandibular. . . . . . . . . 91.4

Jugofrontal. . . . . . . . 81.2

HAIR.-Color: gray and white. Quantity: head, minus; beard, plus; body, plus.

EYE.-Color: green brown. Sclera: bloodshot. Iris: zoned.

Nose.-Profile: concavo-convex. Tip: thickness, double plus. Wings: flaring.

TеEтн.-Eruption: complete. Lost: none. Bite: slight over.

Chest.-Development: average.

Musculature.-Average.

HEALTH.-Good.

No. 308. Plate CX, Figs. 1, 2

Locality: near Jemdet Nasr. Date: July 10, 1928.

Age: 20 ; unmarried.

Brothers: 2 living. Sisters: 1 living.

Tattoo marks: none.

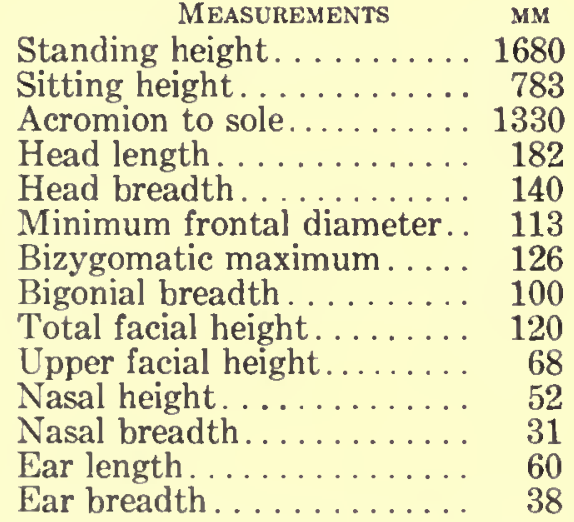

Cephalic............ 76.9

Relative sitting height.... 46.0

Fronto-parietal......... 80.7

Total facial............ 95.2

Upper facial. . . . . . . . . 54.0

Nasal. . . . . . . . . . . 54.7

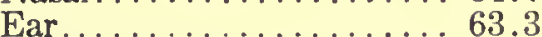

Jugomandibular . . . . . . . . 79.3

Jugofrontal . . . . . . . . . 89.6

HAIR.-Form: low waves. Texture: coarse. Color: reddish brown. Quantity: head, double

plus; beard, double minus.

EYE.-Color: dark brown. Sclera: clear. Iris: zoned.

Nose.-Profile: straight. Wings: medium.

TEETH.-Eruption: complete. Lost: none. Bite: marked over.

CHest.-Development: plus.

Musculature.-Good.

Health.-Good.

\section{No. 309. Plate CX, Figs. 3, 4}

Locality: near Jemdet Nasr. Date: July 10, 1928.

Age: 18; unmarried. 
Brothers: none. Sisters: none.

Tattoo marks: none.

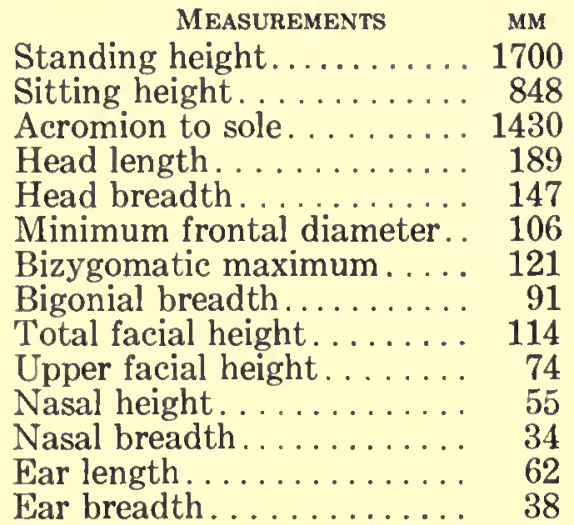

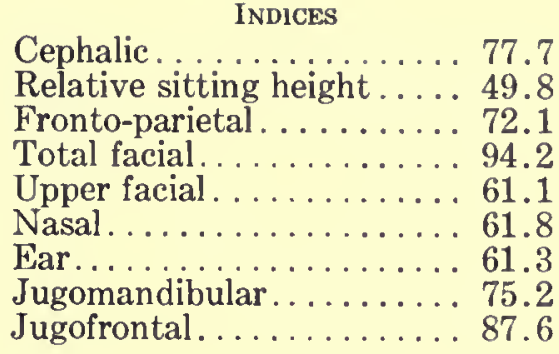

Cephalic. ............. 77.7

Relative sitting height.....49.8

ronto-parietal

Total facial. . . . . . . . . 94.2

Upper facial . . . . . . 61.1

Nasal.............. 61.8

Jugomandibular . . . . . . . . . 75.2

Jugofrontal. ...........88 87.6

HAIR.-Form: low waves. Texture: coarse. Color: dark brown. Quantity: head, double plus; beard, minus; body, minus.

EYE.-Color: green brown. Sclera: clear. Iris: homogeneous.

Nose.-Profile: concavo-convex.

TEETH.-Eruption: complete. Lost: none. Bite: marked over.

Chest.-Development: plus.

Musculature.-Good.

HEALTH.-Good.

No. 310. Plate CX, Figs. 5, 6

Locality: near Jemdet Nasr. Date: July 10, 1928.

Age: 25; married 5 years. Sons: none. Daughters: 2 dead.

Brothers: none. Sisters: none.

Tattoo marks: glove on back of right hand.

\begin{tabular}{|c|c|c|}
\hline MEASUREMENTS & MM & INDICES \\
\hline Standing height. & 1690 & Cephalic. \\
\hline Sitting height. & 844 & Relative sitting height \\
\hline Acromion to sole. & 1375 & Fronto-parietal. . \\
\hline Head length. & 194 & Total facial. . \\
\hline Head breadth. & 143 & Upper facial \\
\hline Minimum frontal diameter. & 109 & Nasal. . \\
\hline Bizygomatic maximum.... & 125 & Ear. \\
\hline Bigonial breadth....... & 96 & Jugomandibular. \\
\hline Total facial height. & 114 & Jugofrontal..... \\
\hline Upper facial height. & 68 & \\
\hline Nasal height. & 54 & \\
\hline Nasal breadth & 38 & \\
\hline Ear length. & 70 & \\
\hline Ear breadth & 41 & \\
\hline
\end{tabular}

HAIR.-Form: low waves. Texture: coarse. Color: black.

EYE.-Color: dark brown. Sclera: clear. Iris: homogeneous.

Nose.-Profile: concavo-convex. Tip: thickness, minus. Wings: flaring.

TEETH.-Eruption: complete. Lost: none. Bite: slight over.

Chest.-Development: plus.

Musculature.-Good.

HEALTH.-Good.

No. 311. Plate CX, Figs. 7, 8

Locality: near Jemdet Nasr. Date: July 10, 1928.

Age: 30 ; unmarried. 
Brothers: none. Sisters: none.

Tattoo marks: none.

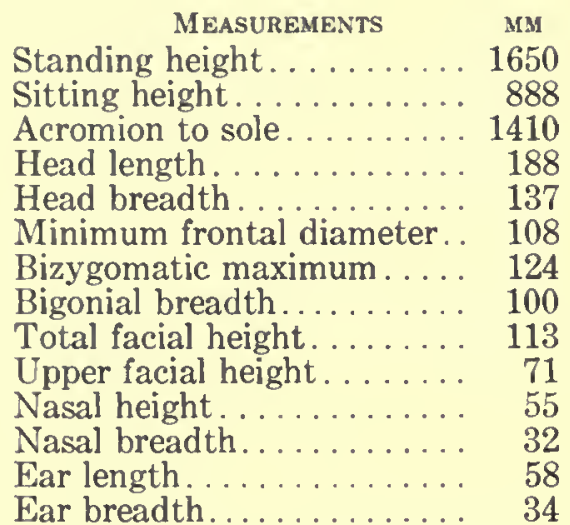

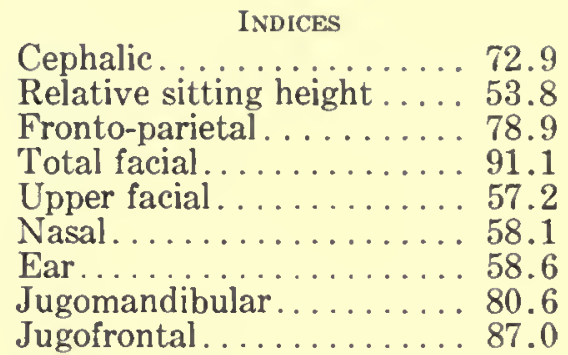

HaIr.-Form: low waves. Texture: medium. Color: black. Quantity: head, double plus; beard, double plus; body, plus.

EYE.-Color: dark brown. Sclera: clear. Iris: zoned.

NosE.-Profile: straight. Wings: medium-compressed.

TEETH.-Eruption: complete. Lost: none. Bite: marked over.

CHEst.-Development: plus.

Musculature.-Good.

HEALTH.-Good.

No. 312. Plate CXI, Figs. 1, 2

Locality: near Jemdet Nasr. Date: July 10, 1928.

Age: 25; married 1 year. Sons: none. Daughters: none.

Brothers: 1 living. Sisters: none.

Tattoo marks: none.

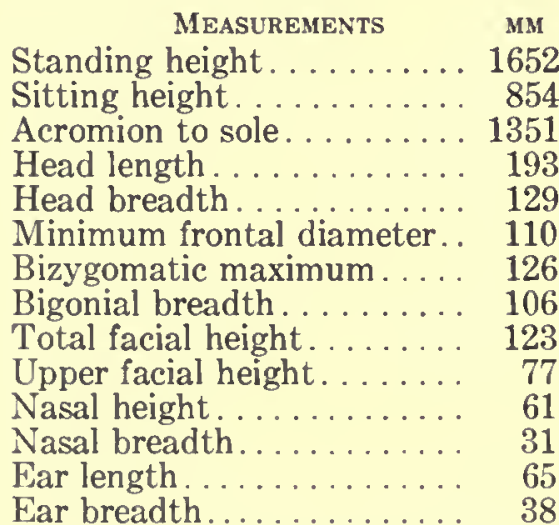

HAIR.-Form: low waves. Texture: coarse. Color: dark brown.

EYE.-Color: green brown. Sclera: clear. Iris: homogeneous.

NosE.-Profile: straight. Tip: thickness, plus. Wings: medium.

TEETH.-Eruption: complete. Lost: none. Bite: slight over.

Chest.-Development: plus.

Musculature.-Good.

HEALTH.-Good.

\section{No. 313. Plate CXI, Figs. 3, 4}

Locality: near Jemdet Nasr. Date: July 10, 1928.

Age: 35; married 20 years. Sons: none. Daughters: 2 living. 
Brothers: none. Sisters: none.

Tattoo marks: gazelle on back of right wrist.

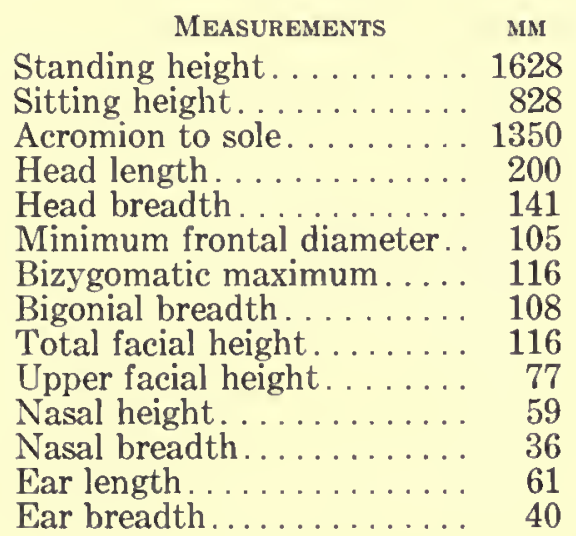

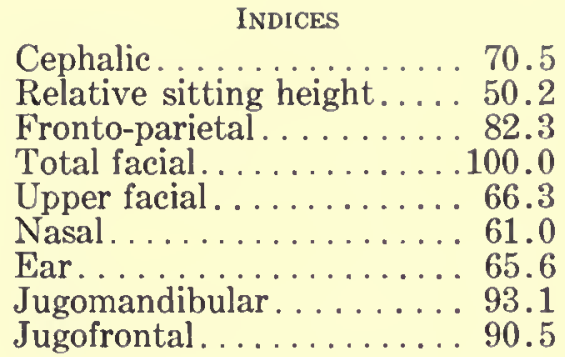

HaIR.-Form: deep waves. Texture: medium. Color: black. Quantity: head, double plus; beard, double plus.

EYE.-Color: green brown. Sclera: clear. Iris: zoned.

Nose.-Profile: straight. Wings: compressed.

TEETH.-Eruption: complete. Lost: none. Bite: slight over.

CHEst.-Development: double plus.

Musculature.-Excellent.

HEALTH.-Good.

\section{No. 314. Plate CXI, Figs. 5, 6}

Locality: near Jemdet Nasr. Date: July 10, 1928.

Age: 30 ; married 4 years. Sons: none. Daughters: 1 living.

Brothers: none. Sisters: none.

Tattoo marks: none.

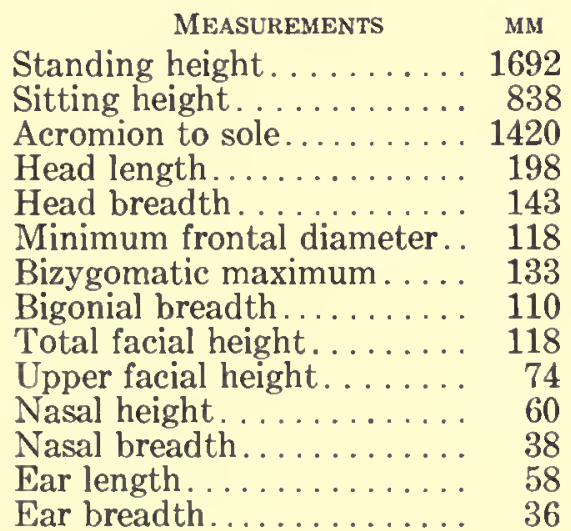

HaIR.-Form: deep waves. Texture: coarse. Color: black. Quantity: head, double plus; beard, double plus; body, plus.

EYE.-Color: green brown. Sclera: bloodshot. Iris: zoned.

NosE.-Profile: concave. Wings: medium-flaring.

TEETH.-Eruption: complete. Lost: none. Bite: slight over.

CHEST.-Development: plus.

Musculature.-Good.

HEALTh.-Good. 


\section{No. 315. Plate CXI, Figs. 7, 8}

Locality: near Jemdet Nasr. Date: July 10, 1928.

Age: 25 ; unmarried.

Brothers: none. Sisters: none.

Tattoo marks: none.

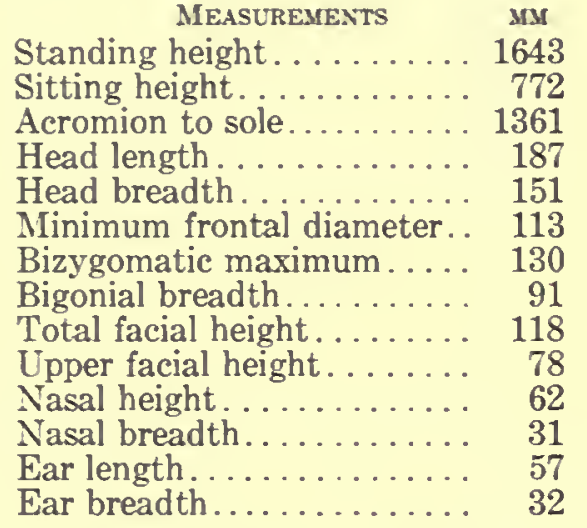

INDICES

Cephalic............. 80.7

Relative sitting height .... 46.9

Fronto-parietal.......... 74.8

Total facial. ........... 90.7

Uoper facial. . . . . . . . . . 60.0

Nasal. ............. 50.0

Ear................ 56.2

Jugomandibular........ 70.0

Jugofrontal. . . . . . . . . . 86.9

HAIR.-Form: low waves. Texture: fine. Color: black. Quantity: head, double plus; beard, plus; body, plus.

EYE.-Color: blue brown. Sclera: speckled. Iris: homogeneous. Blind: right.

Nose.-Profile: straight. Wings: medium.

TEETH.-Eruption: complete. Lost: none. Bite: slight over.

Chest.-Derelopment: plus.

Musculature.-Good.

HeALTH.-Good.

\section{No. 316. Plate CXII, Figs. 1, 2}

Locality: near Jemdet Nasr. Date: July 10, 1928.

Age: 45; married twice, 19 years and 2 years. Sons: 3 living. Daughters: 1 living. Brothers: 2 dead. Sisters: 1 dead.

Tattoo marks: none.

\begin{tabular}{|c|c|}
\hline \multirow{3}{*}{\multicolumn{2}{|c|}{ MEASUREMENTS }} \\
\hline & \\
\hline & \\
\hline & \\
\hline & \\
\hline & \\
\hline & \\
\hline & \\
\hline & \\
\hline & \\
\hline & \\
\hline & \\
\hline & \\
\hline & \\
\hline & \\
\hline & \\
\hline & \\
\hline Standing height. & 1635 \\
\hline Sitting height. & 816 \\
\hline Acromion to sole & 1347 \\
\hline Head length & 196 \\
\hline Head breadth & 128 \\
\hline Minimum frontal diameter. & 108 \\
\hline Bizygomatic maximum.... & 123 \\
\hline Bigonial breadth......... & 100 \\
\hline Total facial height. & 114 \\
\hline Upper facial height. . & 78 \\
\hline Nasal height. & 63 \\
\hline sal breadth & 0 \\
\hline length & 56 \\
\hline n hmod th & \\
\hline
\end{tabular}

INDICES

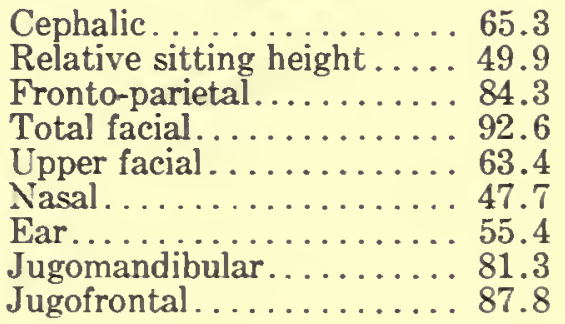

HAIR.-Form: curly frizzly. Texture: medium. Color: dark brown. Quantity: head, double plus; beard, triple plus.

EYE.-Color: blue brown. Sclera: bloodshot. Iris: zoned.

Nose.-Profile: straight. Wings: medium.

TEETH.-Eruption: complete.

CHEST.-Derelopment: plus. 
Musculature.-Good.

HEALTH.-Good.

\section{No. 317. Plate CXII, Figs. 3, 4}

Locality: near Jemdet Nasr. Date: July 10, 1928.

Age: 90 ; married 60 years. Sons: none. Daughters: 1 living.

Brothers: none. Sisters: none.

Tattoo marks: glove on back of right hand.

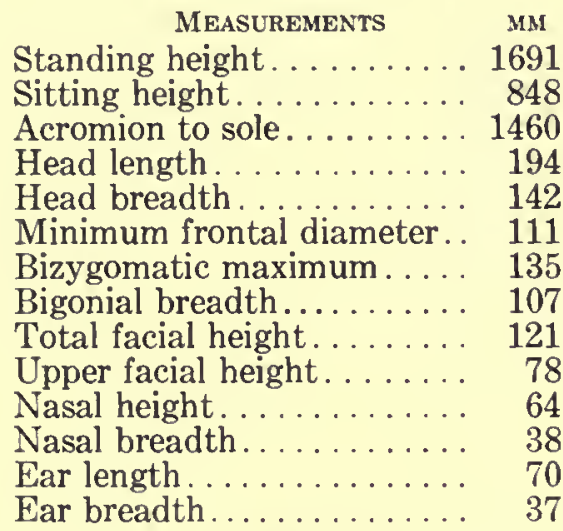

\section{INDICES}

Cephalic............. 73.2

Relative sitting height . . . . 50.1

Fronto-parietal. ........ 78.2

Total facial. . . . . . . . . 89.6

Upper facial . . . . . . . . . 57.7

Nasal. . . . . . . . . . . . 59.4

Ear................... 52.9

Jugomandibular. . . . . . 79.2

Jugofrontal. . . . . . . 82.2

HAIR.-Color: gray. Quantity: head, minus; beard, double plus.

EYE.-Color: blue brown. Sclera: bloodshot. Iris: zoned. Blind: left.

NosE.-Profile: straight. Tip: thickness, double plus. Wings: medium.

TEETH.-Eruption: complete. Lost: all but 2. Bite: slight over.

CHEst.-Development: average.

Musculature.-Poor.

HEALTH.-Poor.

No. 318. Plate CXII, Figs. 5, 6

Locality: near Jemdet Nasr. Date: July 10, 1928.

Age: 45; married twice. Sons: none. Daughters: none.

Brothers: none. Sisters: none.

Tattoo marks: none.

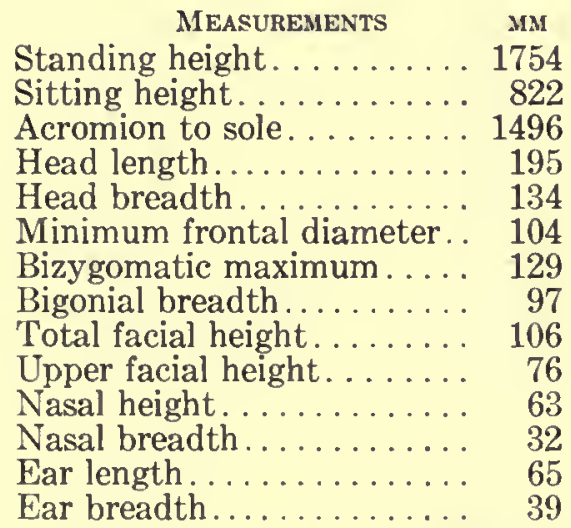

INDICES

Cephalic.............68.7

Relative sitting height .... 46.8

Fronto-parietal. ........ 77.6

Total facial............ 82.1

Upper facial. . . . . . . . . 58.9

Nasal.............. 50.8

Ear...............6. 60.0

Jugomandibular. . . . . . . 75.2

Jugofrontal. . . . . . . 8 80.7

HAIR.-Color: black and gray. Quantity: head, plus; beard, double plus; body, plus. EYE.-Color: blue brown. Sclera: clear. Iris: zoned.

Nose.-Profile: straight. Wings: compressed.

TEETH.-Eruption: complete. Bite: marked over. 
CHest.-Development: plus.

Musculature.-Good.

HEALTH.-Good.

No. 319. Plate CXII, Figs. 7, 8

Locality: near Jemdet Nasr. Date: July 10, 1928.

Age: 40; married 16 years. Sons: 1 living. Daughters: 1 living, 1 dead.

Brothers: 2 living, 1 dead. Sisters: 1 living.

Tattoo marks: none.

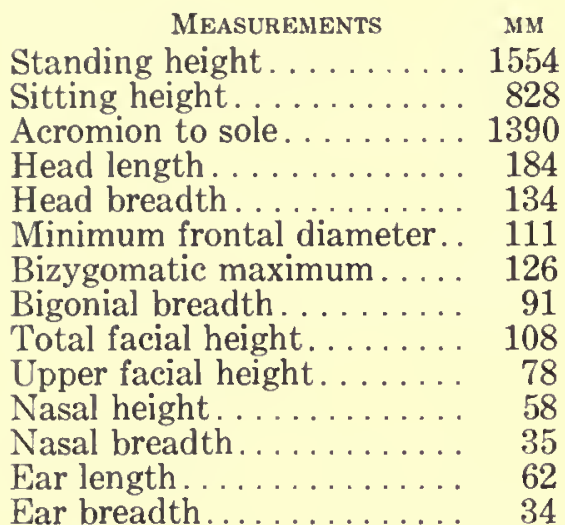

INDICES

Cephalic............ 72.9

Relative sitting height .... 53.2

Fronto-parietal........ 82.9

Total facial............. 85.7

Upper facial. . . . . . . 61.9

Nasal. . . . . . . . . . . . 60.4

Ear.................. 54.8

Jugomandibular. . . . . . 72.2

Jugofrontal. . . . . . . . 80.1

HAIR.-Color: dark brown and gray. Quantity: head, average; beard, double plus; body, plus.

EyE.-Color: blue brown. Sclera: clear. Iris: zoned.

Nose.-Profile: concave. Tip: thickness, double plus.

TEETH.-Eruption: complete. Lost: 3. Bite: marked over.

CHEST.-Development: plus.

Musculature.-Good.

HEALTH.-Good.

No. 320. Plate CXIII, Figs. 1, 2

Locality: near Jemdet Nasr. Date: July 10, 1928.

Age: 45 ; married twice, 25 years and 15 years. Sons: none. Daughters: 2, 0 , living: 0 , 2, dead.

Brothers: none. Sisters: none.

Tattoo marks: glove on back of right hand.

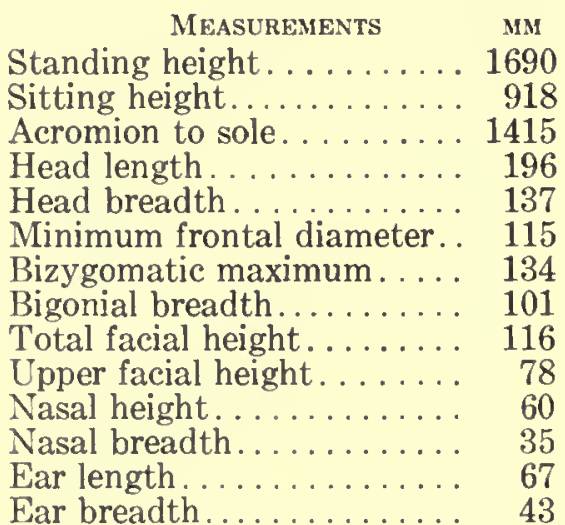

INDICES

Cephalic. . . . . . .....69.9

Relative sitting height..... 54.3

Fronto-parietal. ........ 84.0

Total facial. ........... 86.6

Upper facial. .......... 58.2

Nasal. ............... 58.4

Ear..................6. 64.2

Jugomandibular. . . . . . . 75.3

Jugofrontal. ......... 85.8

HAIR.-Color: black and gray.

EYE.-Color: blue brown. Sclera: bloodshot. Iris: zoned.

NosE.-Profile: straight. Wings: compressed.

TEETH.-Eruption: complete. Caries: double plus. Lost: none. Bite: slight over. 
Chest.-Development: plus.

Musculature.-Good.

HEALTH.-Good.

No. 321

Locality: near Jemdet Nasr. Date: July 10, 1928.

Age: 40; unmarried.

Brothers: none. Sisters: none.

Tattoo marks: two spots on inside of right hand.

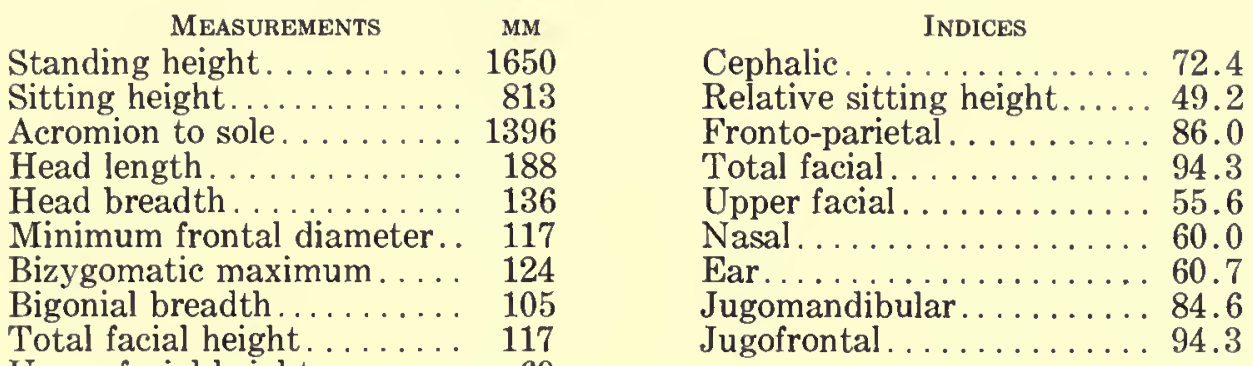

HAIR.-Quantity: head, minus; beard, double plus.

EyE.-Color: blue brown. Sclera: clear. Iris: zoned.

NosE.-Profile: concave. Wings: medium.

TEETH.-Eruption: complete. Lost: none. Bite: slight over.

Chest.-Development: plus.

Musculature.-Good.

HEALTH.-Good.

\section{No. 322. Plate CXIII, Figs. 3, 4}

Locality: near Jemdet Nasr. Date: July 10, 1928.

Age: 35; married 3 years. Sons: 1 living. Daughters: 1 living.

Brothers: 1 dead. Sisters: 1 living.

Tattoo marks: 3 spots and cross on back of right hand.

\begin{tabular}{|c|c|c|}
\hline MEASUREMENTS & MM & INDICES \\
\hline Standing height...... & 1690 & Cephalic. \\
\hline Sitting height. & 818 & Relative sitting height..... 48 \\
\hline Acromion to sole. & 1430 & Fronto-parietal.........78 \\
\hline Head length. & 188 & Total facial............ \\
\hline Head breadth. & 146 & Upper facial \\
\hline Minimum frontal diameter. . & 116 & Nasal. .... \\
\hline Bizygomatic maximum ..... & 131 & Ear.. \\
\hline Bigonial breadth. & 102 & Jugomandib \\
\hline Total facial height. & 123 & Jugofrontal \\
\hline
\end{tabular}

Upper facial height. . . . . .....

Nasal height.

Nasal breadth.................. 37

Ear length.............. 58

Ear breadth........... 34

HAIR.-Form: curly frizzly. Texture: coarse. Color: black. Quantity: head, double plus; beard, plus; body, plus.

Eye.-Color: blue brown. Sclera: clear. Iris: zoned.

NosE.-Profile: straight. Wings: medium. 
TeETH.-Eruption: complete. Lost: none.

Chest.-Development: plus.

Musculature.-Good.

HEALTH.-Good.

No. 323. Plate CXIII, Figs. 5, 6

Locality: near Jemdet Nasr. Date: July 10, 1928.

Age: 18; unmarried.

Brothers: 1 living. Sisters: none.

Tattoo marks: spot at nasion, spot on each temple and behind each ear.

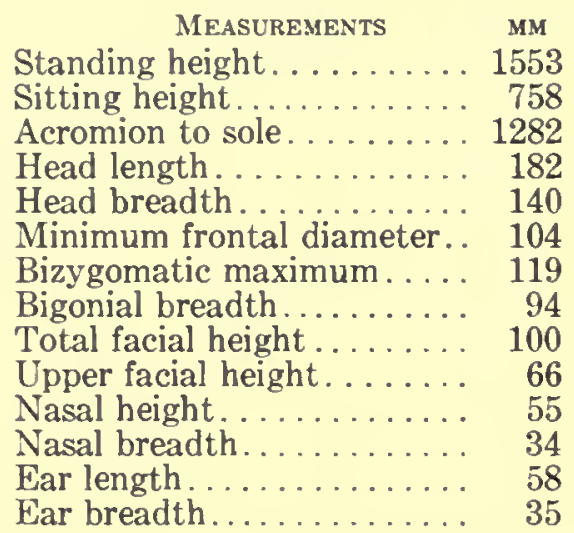

INDICES

Cephalic............. 77.0

Relative sitting height..... 48.8

Fronto-parietal......... 74.4

Total facial. . . . . . . . . 84.0

Upper facial. . . . . . . . 55.5

Nasal. . . . .......... 61.9

Ear.................. 60.4

Jugomandibular. . . . . . . . 79.0

Jugofrontal . . . . . . . . 8. 87.3

HaIr. - Form: low waves. Texture: fine. Color: black. Quantity: head, plus; beard, double minus; body, minus.

Eye.-Color: dark brown. Sclera: clear. Iris: homogeneous.

Nose.-Profile: concave. Tip: thickness, double plus. Wings: flaring.

TЕетн.-Lost: none. Bite: slight over.

Chest.-Development: plus.

Musculature.-Good.

HEALTH.-Good.

Some Negro blood.

No. 324. Plate CXIII, Figs. 7, 8

Locality: near Jemdet Nasr. Date: July 10, 1928.

Age: 50; married twice, 14 years (wife dead) and one year. Sons: 2, 0, dead. Daughters: none. Brothers: none. Sisters: none.

Tattoo marks: none.

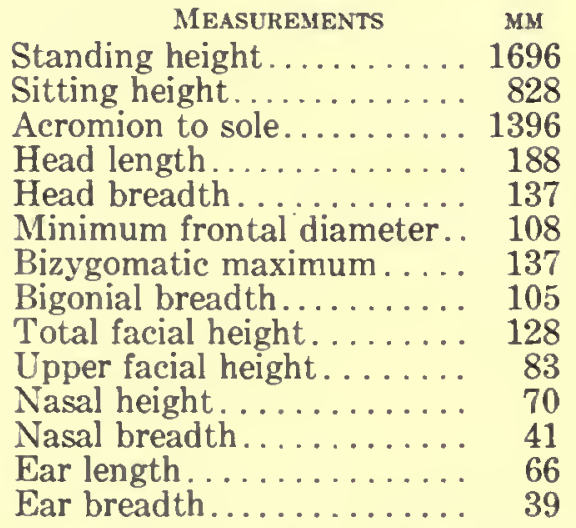

INDICES

Cephalic.............72.9

Relative sitting height..... 48.8

Fronto-parietal.......... 78.9

Total facial. .......... 94.5

Upper facial. . . . ........ 60.6

Nasal.............. 58.6

Ear..................... 59.1

Jugomandibular. . . . . . 76.6

Jugofrontal. . . . . . . . 78.8

HAIR. - Form: deep waves. Texture: fine. Color: dark brown.

EYE.-Color: blue brown. 
Nose.-Profile: convex. Tip: thickness, double plus. Wings: flaring.

TEETH.-Eruption: complete. Lost: none. Bite: slight over.

CHEsT.-Development: plus.

Musculature.-Good.

HEALTH.-Good.

No. 325. Plate CXIV, Figs. 1, 2

Locality: near Jemdet Nasr. Date: July 10, 1928.

Age: 65 ; married 25 years. Sons: 1 dead. Daughters: 5 dead.

Brothers: none. Sisters: none.

Tattoo marks: none.

\begin{tabular}{|c|c|}
\hline MEASUREMENTS & MM \\
\hline Standing height. & 1682 \\
\hline Sitting height. & 782 \\
\hline Acromion to sole. & 1432 \\
\hline Head length. . & 198 \\
\hline Head breadth. & 148 \\
\hline Minimum frontal diameter. & 114 \\
\hline Bizygomatic maximum . . . . & 132 \\
\hline Bigonial breadth....... & 100 \\
\hline Total facial height.... & 121 \\
\hline Upper facial height. . . . . . & 70 \\
\hline Nasal height. . . . . . . . & 65 \\
\hline Nasal breadth. & 39 \\
\hline Ear length.... & 67 \\
\hline Ear breadth & \\
\hline
\end{tabular}

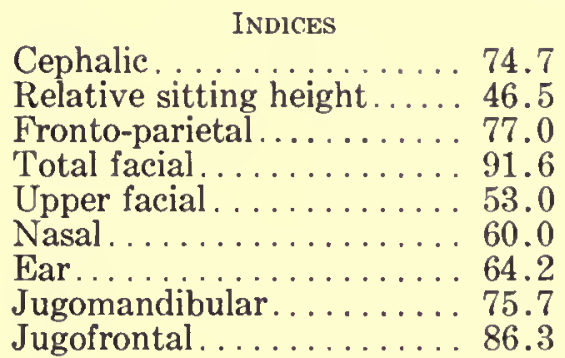

4.7

. 5

91.6

3.0

4.2

Jugomandibul

HAIR. - Color: black and gray. Quantity: head, average; beard, plus; body, plus.

EYE.-Color: blue brown. Sclera: bloodshot. Iris: zoned.

Nose.-Profile: straight. Wings: medium-flaring.

TEETH.-Eruption: complete. Lost: many.

CHEST.-Development: plus.

Musculature.-Good.

HEALTH.-Good.

No. 326. Plate CXIV, Figs. 3, 4

Locality: near Jemdet Nasr. Date: July 10, 1928.

Age: 30 ; unmarried.

Brothers: none. Sisters: none.

'lattoo marks: none.

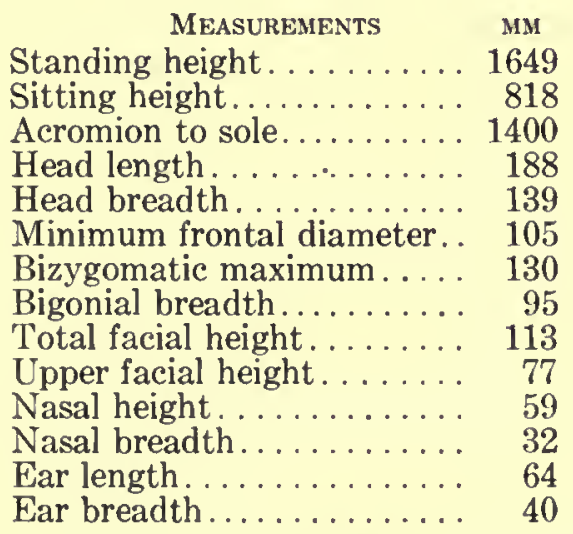

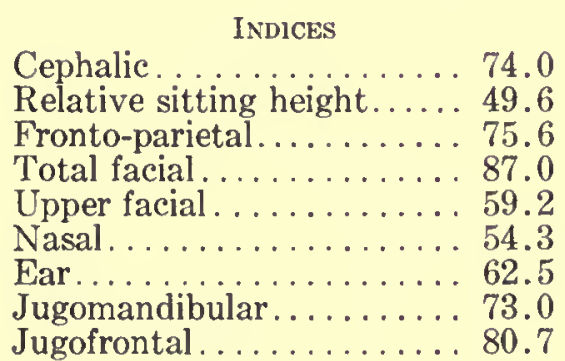

HAIR. - Color: black. Quantity: head, plus; beard, double plus; body, plus.

EYE. - Color: blue brown. Sclera: clear. Iris: zoned. 
NOSE.-Profile: straight. Wings: compressed.

Teетн.-Eruption: complete. Lost: 2 . Bite: slight over.

Chest.-Development: plus.

Musculature.-Good.

HEALTH.-Good.

No. 327. Plate CXIV, Figs. 5, 6

Locality: near Jemdet Nasr. Date: July 10, 1928.

Age: 35 ; married 5 years. Sons: none. Daughters: none.

Brothers: none. Sisters: none.

Tattoo marks: none.

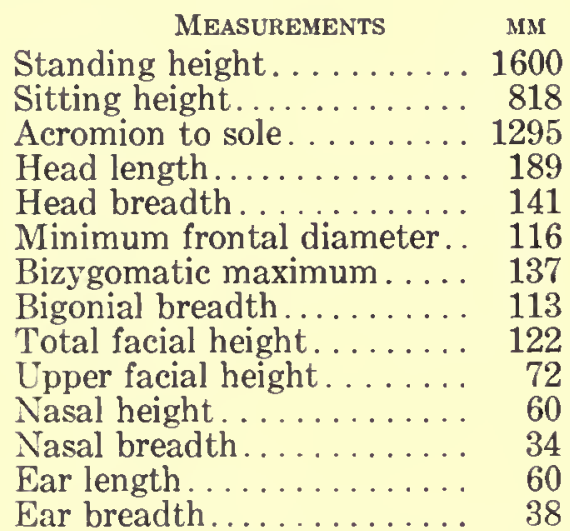

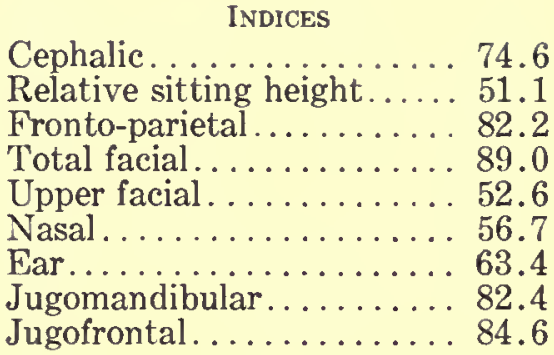

HaIR.-Form: low waves. Texture: medium. Color: dark brown. Quantity: head, plus; beard, double plus; body, plus.

EYE.-Color: blue brown. Sclera: clear. Iris: zoned.

NosE.-Profile: straight. Wings: medium.

TEETH.-Eruption: complete. Bite: slight over.

Chest.-Development: plus.

Musculature.-Good.

HEALTH.-Good.

\section{No. 328. Plate CXIV, Figs. 7, 8}

Locality: near Jemdet Nasr. Date: July 10, 1928.

Age: 50; married 15 years, wife now dead. Sons: 3 dead. Daughters: 1 dead.

Brothers: none. Sisters: none.

Tattoo marks: glove on back of right hand.

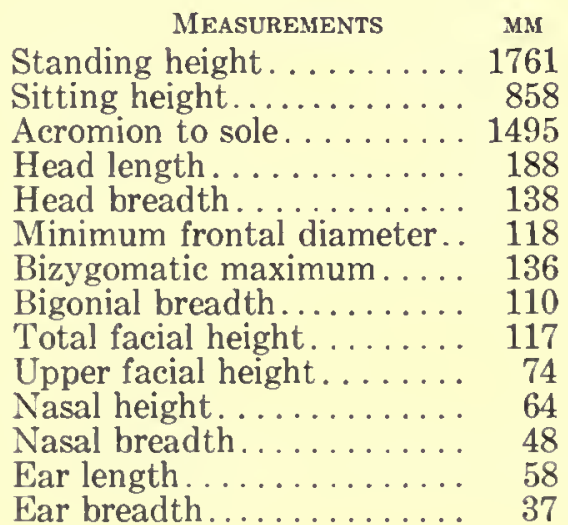


HAIR.-Color: gray.

EYE.-Color: blue brown. Sclera: clear. Iris: zoned.

Nose.-Profile: straight. Tip: thickness, double plus. Wings: flaring.

TEETH.-Eruption: complete. Lost: none. Bite: slight over.

CHEST.-Development: plus.

Musculature.-Average.

HEALTH.-Good.

No. 329. Plate CXV, Figs. 1, 2

Locality: near Jemdet Nasr. Date: July 10, 1928.

Age: 35 ; unmarried.

Brothers: none. Sisters: none.

Tattoo marks: none.

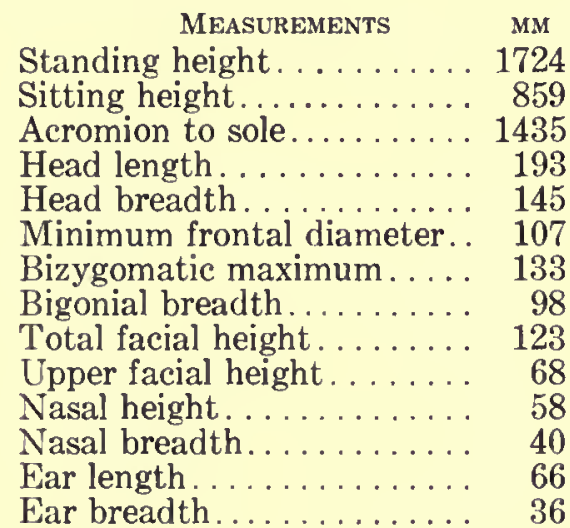

INDICES

Cephalic.............. 75.1

Relative sitting heigh

Fronto-parietal.......... 73.8

Total facial ............... 92.4

Upper facial. ........... 51.1

Nasal. . . . . . . . . . . . 68.9

Ear................ 54.5

Jugomandibular........... 73.6

Jugofrontal. .......... 80.4

HAIR.-Texture: coarse. Color: reddish brown. Quantity: head, double plus; beard, plus; body, plus.

EYE.-Color: blue brown. Sclera: clear. Iris: zoned.

NosE.-Profile: straight. Wings: medium.

TeEтн.-Eruption: complete. Lost: none. Bite: slight over.

CHEST.-Development: plus.

Musculature.-Good.

HEALTh.-Good.

No. 330. Plate CXV, Figs. 3,4

Locality: near Jemdet Nasr. Date: July 10, 1928.

Age: 30 ; unmarried.

Brothers: none. Sisters: none.

Tattoo marks: spots on each temple.

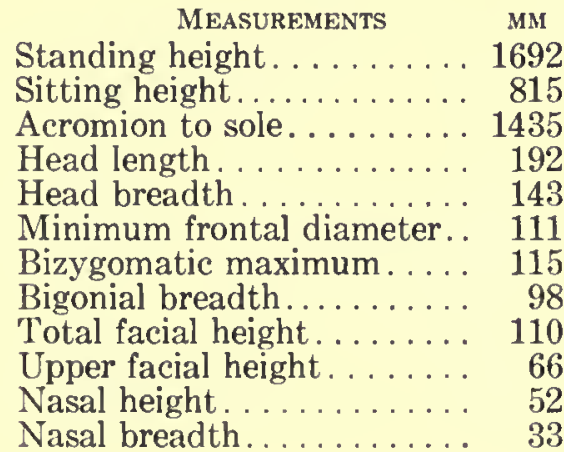

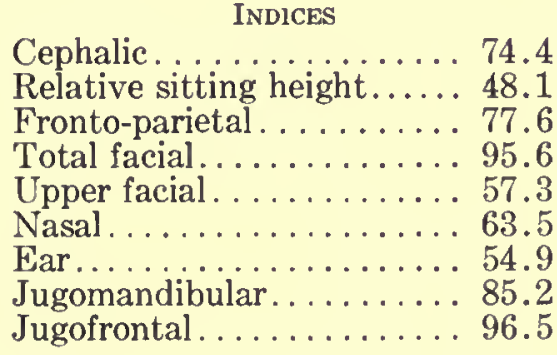

Cephalic.............. 74.4

Relative sitting height..... 48.1

Fronto-parietal ...........77.6

Upper facial ............... 57.3

Nasal. ..............6.6.5

Ear.................. 54.9

Jugofrontal. . . . . . . 96.5 


\begin{tabular}{|c|c|}
\hline MEASUREMENTS & MM \\
\hline Total facial height..... & 118 \\
\hline Upper facial height. & 70 \\
\hline Nasal height....... & 60 \\
\hline Nasal breadth & \\
\hline Ear length. & \\
\hline Ear breadth & \\
\hline
\end{tabular}

Jugofrontal . . . . . . . 79.4

18

HAIR.-Form: low waves. Texture: medium. Color: reddish brown. Quantity: head, double plus; beard, plus; body, plus.

EYE.-Color: blue brown. Sclera: clear. Iris: zoned.

Nose.-Profile: straight. Wings: medium.

TEETH.-Eruption: complete. Lost: none. Bite: slight over.

Chest.-Development: average.

Musculature.-Average.

HEALTH.-Good.

\section{No. 333. Plate CXVI, Figs. 1, 2}

Locality: near Jemdet Nasr. Date: July 10, 1928.

Age: 35 ; married four times: $12,5,4$, and 2 years. Sons: none. Daughters: none.

Brothers: none. Sisters: 1 living.

Tattoo marks: none.

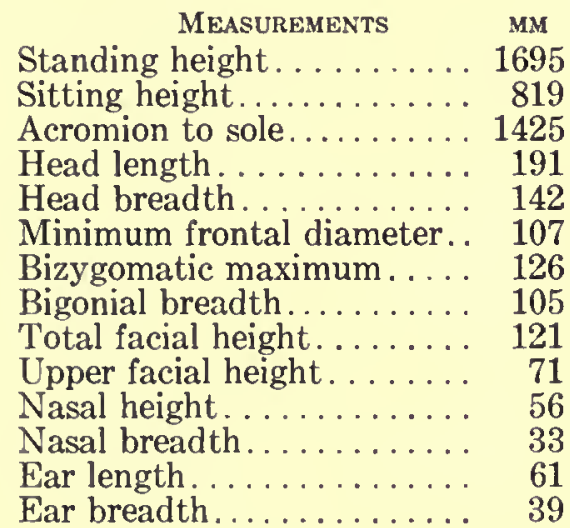

INDICES

Cephalic............74.3

Relative sitting height..... 48.3

Fronto-parietal......... 75.3

Total facial............... 96.1

Upper facial . . . . . . . . . 56.3

Nasal .................. 58.9

Ear................ 63.9

Jugomandibular. . . . . . . 8. 83.3

Jugofrontal. . . . . . . . 84.9

HAIR.-Form: low waves. Texture: medium. Color: black.

EYE.-Color: blue brown. Sclera: bloodshot. Iris: zoned.

Nose.-Profile: straight. Wings: medium.

TEETH.-Eruption: complete. Lost: none. Bite: marked over.

Chest.-Development: plus.

Musculature.-Good.

HeAlth.-Good.

No. 334. Plate CXVI, Figs. 3, 4

Locality: near Jemdet Nasr. Date: July 10, 1928.

Age: 45 ; married twice, both wives dead. Sons: 1, 0 , living; 0 , 1, dead. Daughters: 2, 0 , living.

Brothers: 2 dead. Sisters: 3 dead.

Tattoo marks: glove on back of right hand.

MEASUREMENTS MM

Standing height. ....... 1760

Sitting height............. 779

Acromion to sole. . . . . . . . 1410

Head length............ 194
INDICES

Cephalic............. 75.8

Relative sitting height...... 44.2

Fronto-parietal......... 73.4

Total facial ............... 85.7 


\begin{tabular}{|c|c|c|}
\hline Measurements & мM & INDICES \\
\hline readth. & 147 & Upper facial \\
\hline m frontal diameter.. & 10 & Nasal \\
\hline natic maximum. & 140 & Ear \\
\hline ial breadth. & 105 & Jugomandibular. \\
\hline al facial hei & 120 & Jugofrontal \\
\hline
\end{tabular}

Upper facial height. . . . . . 72

Nasal height........... 67

Nasal breadth........... 32

Ear length............. 64

Ear breadth........... 37

HaIR. - Form: low waves. Texture: medium. Color: black and gray.

EYE.-Color: blue brown. Sclera: clear. Iris: zoned.

NosE.-Profile: straight. Wings: medium.

TеEтн.-Eruption: complete. Wear: double plus. Caries: average plus. Lost: 4. Bite: slight over.

Chest.-Development: plus.

Musculature.-Good.

HEALTH.-Good.

\section{No. 335. Plate CXVI, Figs. 5, 6}

Locality: near Jemdet Nasr. Date: July 10, 1928.

Age: 38; married 16 years. Sons: none. Daughters: none.

Brothers: none. Sisters: none.

Tattoo marks: spot on back of right wrist.

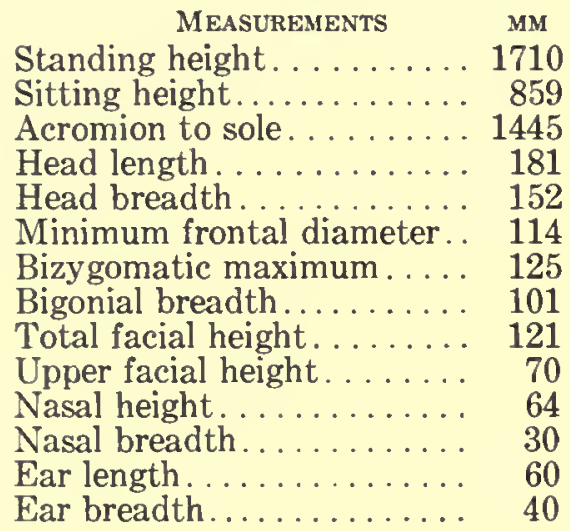

INDICES

Cephalic............ 83.9

Relative sitting height..... 50.2

Fronto-parietal......... 74.9

Total facial. ............. 96.7

Upper facial. . . . . . . . . . 56.0

Nasal ..................... 46.9

Ear............... 66.6

Jugomandibular. . . . . . . 80.8

Jugofrontal . . . . . . . . 91.2

HAIR.-Form: low waves. Texture: medium. Color: black.

EYE.-Color: blue brown. Sclera: bloodshot. Iris: zoned.

Nose.-Profile: straight. Wings: medium.

TEETH.-Eruption: complete. Lost: none.

Chest.-Development: plus.

Musculature.-Good.

Health.-Good.

No. 336. Plate CXVI, Figs. 7, 8

Locality: near Jemdet Nasr. Date: July 10, 1928.

Age: 80 ; married 50 years. Sons: 1 living, 2 dead. Daughters: 2 living, 1 dead.

Brothers: none. Sisters: 2 dead.

Tattoo marks: none.

Measurements MM

Standing height........ 1650

Sitting height.......... 779
INDICES

Cephalic.............. 71.7

Relative sitting height..... 47.2 


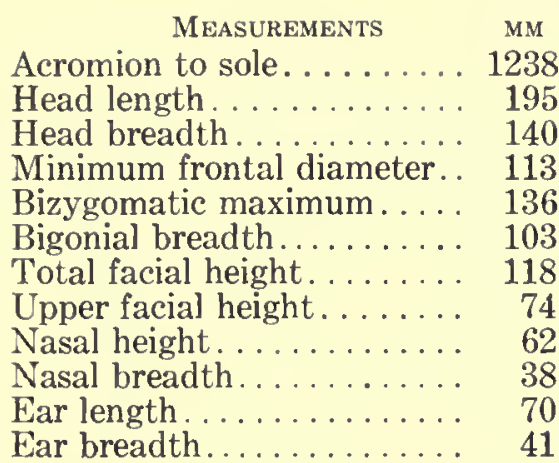

INDICES

Fronto-parietal........ 80.6

Total facial. . . . . . . . . . . 86.7

Upper facial. ........... 54.4

Nasal . . . . . . . . . . . . . 61.3

Ear

Jugomandibular............ 75.7

Jugofrontal. ........... 83.0

HAIR. - Color: gray and white.

EYE.-Color: blue brown. Sclera: bloodshot. Iris: zoned.

Nose.-Profile: straight. Wings: medium.

TеEтH.-Eruption: complete. Lost: half. Bite: marked over.

CHEsT.-Development: average.

Musculature.-Average.

HEALTH.-Fair.

\section{No. 337. Plate CXVII, Figs. 1, 2}

Locality: near Jemdet Nasr. Date: July 10, 1928.

Age: 40 ; married 2 years. Sons: none. Daughters: none.

Brothers: none. Sisters: none.

Tattoo marks: 2 dots and cross on back of right hand.

\begin{tabular}{|c|c|c|}
\hline MEASUREMENTS & M & INDICES \\
\hline Standing height. . . . . & 1687 & Cephalic........... \\
\hline Sitting height.. & 819 & Relative sitting height. \\
\hline Acromion to sole. & 1410 & Fronto-parietal. . . . . . . . 87.9 \\
\hline Head length.... & 188 & Total facial........... \\
\hline Head breadth. & 140 & Upper facial \\
\hline Minimum frontal diameter. & 123 & Nasal. ..... \\
\hline Bizygomatic maximum.... & 132 & Ear... \\
\hline Bigonial breadth........... & 110 & Jugomandibular \\
\hline Total facial height & 111 & Jugofrontal..... \\
\hline
\end{tabular}

Upper facial height....... 80

Nasal height. ........... 67

Nasal breadth. ........... 35

Ear length............. 64

Ear breadth........... 36

HAIR.-Form: low waves. Texture: medium. Color: black. Quantity: head, double plus; beard, double plus; body, plus.

EYE.-Color: blue brown. Sclera: bloodshot. Iris: zoned.

Nose.-Profile: straight. Wings: medium.

Teетн.-Eruption: complete. Lost: none. Bite: slight over.

CHEST.-Development: plus.

Musculature.-Good.

HEALTH.-Good. 


\section{STATISTICAL SUMMARIES}

This chapter contains the tabulated summaries of vital statistics and anthropometric data as well as some notes on the Chaldean village of Qaraqosh. The graphs in this section are frequency graphs, with the exception of Figure 41 , which records the cumulative percentages of the cephalic indices of 396 Kish Arabs, 227 Iraq soldiers, and thirty-eight Ba'ij Beduins. In plotting the index numbers, an index, for example, falling between 74.5 and 75.5 is indicated as one unit in the 75-frequency group. Those indices falling on 74.5 are split, a count of half an individual being credited to the 74 group and half to the 75 group. There is a brief summary of anthropometric data obtained in southwestern Asia during the past decade. A general description of the Field Museum Anthropological Expedition to the Near East during 1934, together with a list of the 3,056 individuals measured, is to be found in the Appendix.

The bibliography does not include references to the early explorations and excavations at Tell-el-Uhaimir (Kish). For this information the reader is referred to "Excavations at Kish" by S. Langdon, Paris, 1924, pp. 43-55, where excerpts from the travels and scientific reports of Benjamin of Tudela, Buckingham, Bellino, Mignon, Oppert, Baillie, Layard, de Genouillac, Jensen, and others will be found. A list of bibliographical references will be published in the Report of the Field Museum Anthropological Expedition to the Near East, 1934.

\section{VITAL STATISTICS}

With the assi_tance of $\mathrm{C}$. Champion de Crespigny the vital statisties have been summarized as follows:

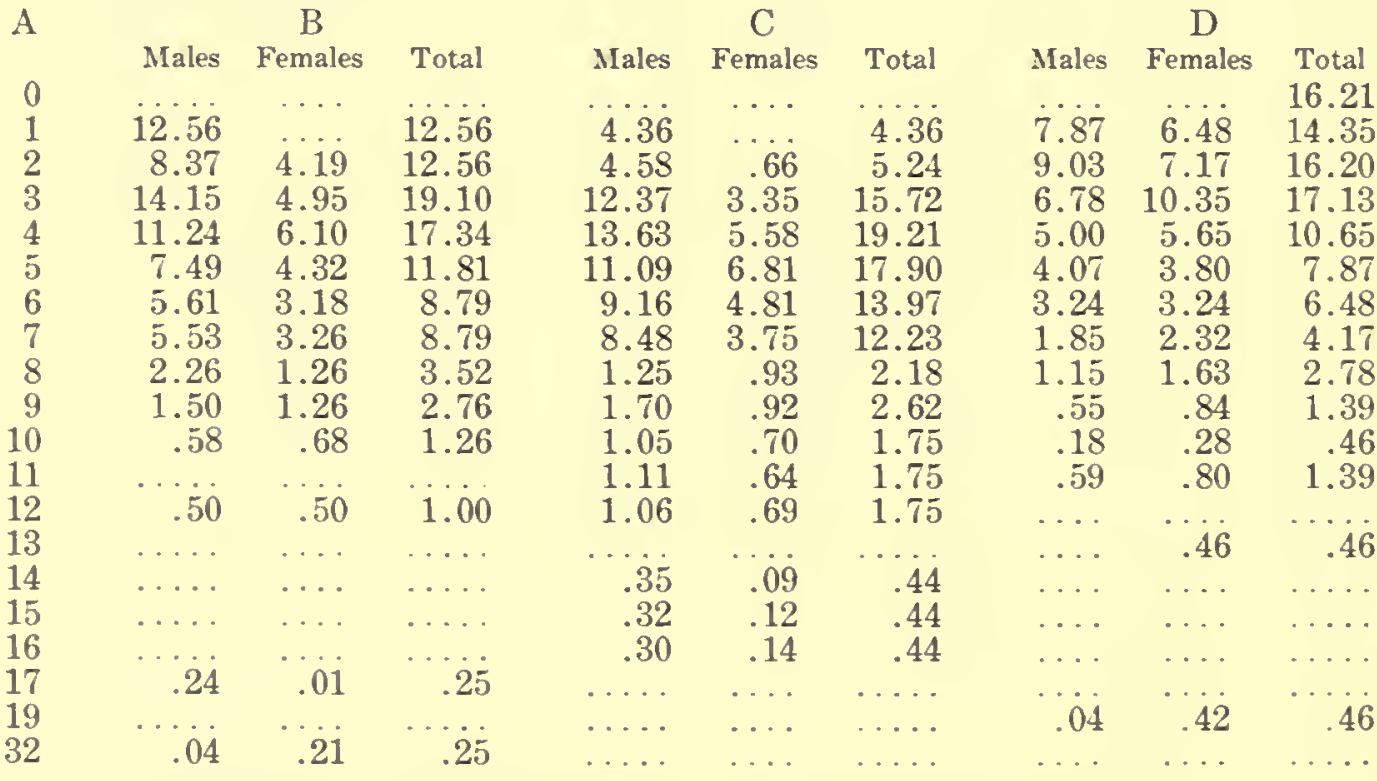

$A=$ number of children in each family.

$\mathrm{B}=$ percentage frequency of brothers and sisters in families of $398 \mathrm{Kish}$ Arabs.

$\mathrm{C}=$ percentage frequency of brothers and sisters in families of 229 soldiers in Iraq army.

$\mathrm{D}=$ percentage frequency of children in families of $216 \mathrm{Kish}$ Arabs. 
A random selection of individuals throughout a comparatively small area, made necessary by the condition of the country at that time, prohibits definite conclusions. But from this material may be drawn fairly accurate generalizations of interest and value, which can only be confirmed when a census of the territory is undertaken by the government of Iraq.

One group of statistics is based on 398 male Kish Arabs. Each individual in reply to routine questions gave the number of his brothers and sisters, alive or dead, so that the size of the families of his father's generation could be determined, and distinction was made between his male and female offspring.

It is surprising that the families are so small. In an agricultural community it might well be expected that the higher frequency percentages would fall in the five, six, and seven children groups. On the contrary, there is a noticeable drop from a high of 19.10 per cent in families of three, to only 11.81 per cent in families of five. Further, the astounding frequency of 12.56 per cent of families containing only one child suggests that preventive measures, generally more common in urban sections, are also used widely in the agricultural settlements.

Upon examination the figures reveal that male children were usually recorded in families where only one child was present. This is due to the fact that an Arab father wishes above all else to have a son, who shall perpetuate his name and tradition. The important factor is for the first-born to be a male. Thus the disappointment of a female first-born child is often concealed from the anthropologist by denying the very existence of the girl. For example, a man who is disappointed by the arrival of a girl will be so delighted with his new-born son that he may not even mention the former when questioned. Furthermore, to admit of only one child and that a daughter would test the veracity of an Arab of the Kish area almost beyond the breaking point.

If a first-born female child dies in infancy its death is considered little cause for sympathy; but the death of a young son is a major tragedy. As a direct result of this a female baby receives less care and attention and is therefore more subject to death. In the Kish area the infant mortality rate is high due to the lack of medical knowledge and sanitation.

The frequency distribution of children, rather than brothers and sisters, of 216 of these $398 \mathrm{Kish}$ Arabs was tabulated. The trend toward small families is intensified by a percentage of 16.21 of childless families. The fact that some of the men reported upon had hardly been married long enough to have children is to some degree responsible for this distortion. A comparison of figures in the tables shows a distinct gain in females in the progeny of the 216 married Arabs, over the relatively small percentage of females in the families into which they were born.

The majority of the males were married after the age of thirty and after the forty-first year all had taken wives. These late marriages are due to economic conditions outlined in the section dealing with the social life and customs (p. 88).

In the group of 229 soldiers, the much larger percentages of families containing four, five, six, and seven children in the fathers' families and the rare 
occurrence of families of only one and two children predispose the conclusion that the army is a good resting place for surplus sons in families overburdened beyond the point of having enough to eat. This conclusion, however, is liable to error, since, if conscription be in force, other factors would contribute to this distribution.

The number of children by each wife was recorded in the cases where a man had more than one wife. For example, "Sons: 2, 0" and "Daughters: 0, 1" mean that the first wife had borne two sons and no daughters while the second wife had no sons and one daughter.

\section{QARAQOSH}

During the summer of 1928 accompanied by S. Y. Showket I visited northern Iraq. The road from Baghdad to Mosul passes through Erbil, the ancient Arbela (Babylonian, Arba-ilu), and continues across the plain to the village of Qaraqosh. The inhabitants were friendly as soon as they learned that we were Christians (Nasrani). Mr. Showket addressed them in Chaldean, their native tongue, and they were overjoyed to find a visitor who could talk to them in their own language. The physical features of these people, different from the Arabs, Beduins, and Kurds, indicate that they are a homogeneous group. Except for the young girls, who were shy in posing before the camera, both men and women wished to be photographed. This seemed a valuable opportunity to take a large series of photographs; so Showket and I, each with a camera, exposed as many negatives as possible in the hour and a half at our disposal.

Time did not permit us to stay overnight in this village, and consequently we were unable to take anthropometric observations or measurements. Kappers (Kon. Akad. van Wet., XXXIII, No. 8, p. 806) refers to measurements on nineteen male and female Chaldeans from Qaraqosh. The cephalic index for males was 86.01 and for females 86.17 . The people appeared to me to be taller in stature and stronger in general physique than the Arabs of the Kish area. The men possess fine features which suggest an Arab-Kurdish mixture without any trace of the long, thin features of the Beduins. They wear head-dresses, kaffiyah (Kurd, aghabanu), wrapped about the head in Turkish fashion and the 'agal was little seen. The faces are hard and stern in appearance, and wrinkled, particularly in the region of the glabella. Masseter muscles, which are well developed in many cases, broaden the lower portion of their faces. The nose is straight and leptorrhine with a suggestion of a downward curve at the tip. Some of the men have coarser features, more flaring nostrils, and thicker integumental layers of the lips. The ear is medium in size and the helix lies close to the head. The eyes are dark brown in color and set relatively close together. The teeth are clean and white; caries is less conspicuous than among the Arabs of the Kish area. The color of the skin shows a tendency toward medium brown, and there appears to be an entire absence of the negroid element in the population.

The older men wear beards and the younger ones wear mustaches, dark brown in color. This is the usual shade of the hair also, neither light- nor blackhaired individuals being seen. 
Dressed in Arab clothes of various colors, many of the men wear leather shoes resembling Dutch sabots (yamani). These shoes are made by hand in the suk at Mosul and brought to the village by traveling salesmen. The men smoke stone pipes (sabil) which are ornamented, or cigarettes which they roll for themselves. Although European cigarettes are considered too strong and cause irritation in their throats, the men smoke tobacco (titin), a light green mixture which is harsh to the palate. The tobacco is carried in a cloth pouch (chis). Older men often carry a rosary (sibha).

Though shorter in stature than the men, the women are strong and willing workers and the men do little manual labor. The women have pleasant faces, round in shape, wide in the zygomatic arches and the bigonial breadth. The hair is dark brown in color and relatively coarse in quality. The nose is broader and shorter than that of the men, and the majority of the girls wear a golden nose ring (khazzamah) through the right nostril (arnabah). In a few cases we observed the nose ring worn in the left nostril. Dressed in black heavy robes (izar) which hang down to the ground, the older women cover their heads with a black cloth (kaffiyah) so that only the face, hands, and poorly shod feet are visible. The young girls are decorated with golden ornaments which include nose, ear (idhin; Kurd, guwarhe), and finger rings (mahbas; Kurd, hangustir), bracelets (kahwas; Kurd, kol-bend), and anklets (hijul; Kurd, pa-bend), and a circlet or diadem of golden coins (Kurd, karmak) around the forehead, fastening beneath the chin (Kurd, cheneghé). Their long robes are covered with many designs while the headcloth is often tucked in at the back of the neck. The teeth appeared to be clean and strong, but among the older women there was considerable evidence of dental decay.

Due perhaps to carrying pottery jars (kuwaza) filled with water on their heads or left shoulders, the girls have straight and well-proportioned figures. They draw water from the wells by means of a rope (habil) and pulley wheel (bakara) attached to a leathern bucket (dalu).

The people of this village appeared homogeneous; at some future date I should like to obtain anthropometric data and additional historical information on this interesting Christian group.

We asked one of the old men if there were any books (makatib) in the village. He replied that one of the religious elders possessed a very old and valuable manuscript. Although we made numerous attempts to examine this manuscript, thinking that it might be of important historical interest, the owner refused us permission, explaining courteously but firmly that we might be of the same faith, yet our foreign eyes would defile the holy book.

The site of the battle of Gaugamela in which Alexander overthrew Darius Codomanus on October 1, 331 B.C., was named after Arbela, the modern Erbil. The exact location of the battlefield has remained in doubt. According to Ptolemy and Aristobulus the fighting took place at Gaugamela and not at Arbela. Furthermore, the word Gaugamela means "the black bird" or "the black eagle," which is exactly the same meaning implied by the modern name of Qaraqosh 
in Turkish (cf. Kara-qush as transliteration). Thus, on the basis of philology and a detailed study of the historical sources, it seems probable that the battle of Gaugamela took place near the modern village of Qaraqosh. Dr. Alexander Sushko of the University of Chicago brought this information to my attention.

\section{ARABS OF THE KISH AREA STATISTICAL SUMMARIES}

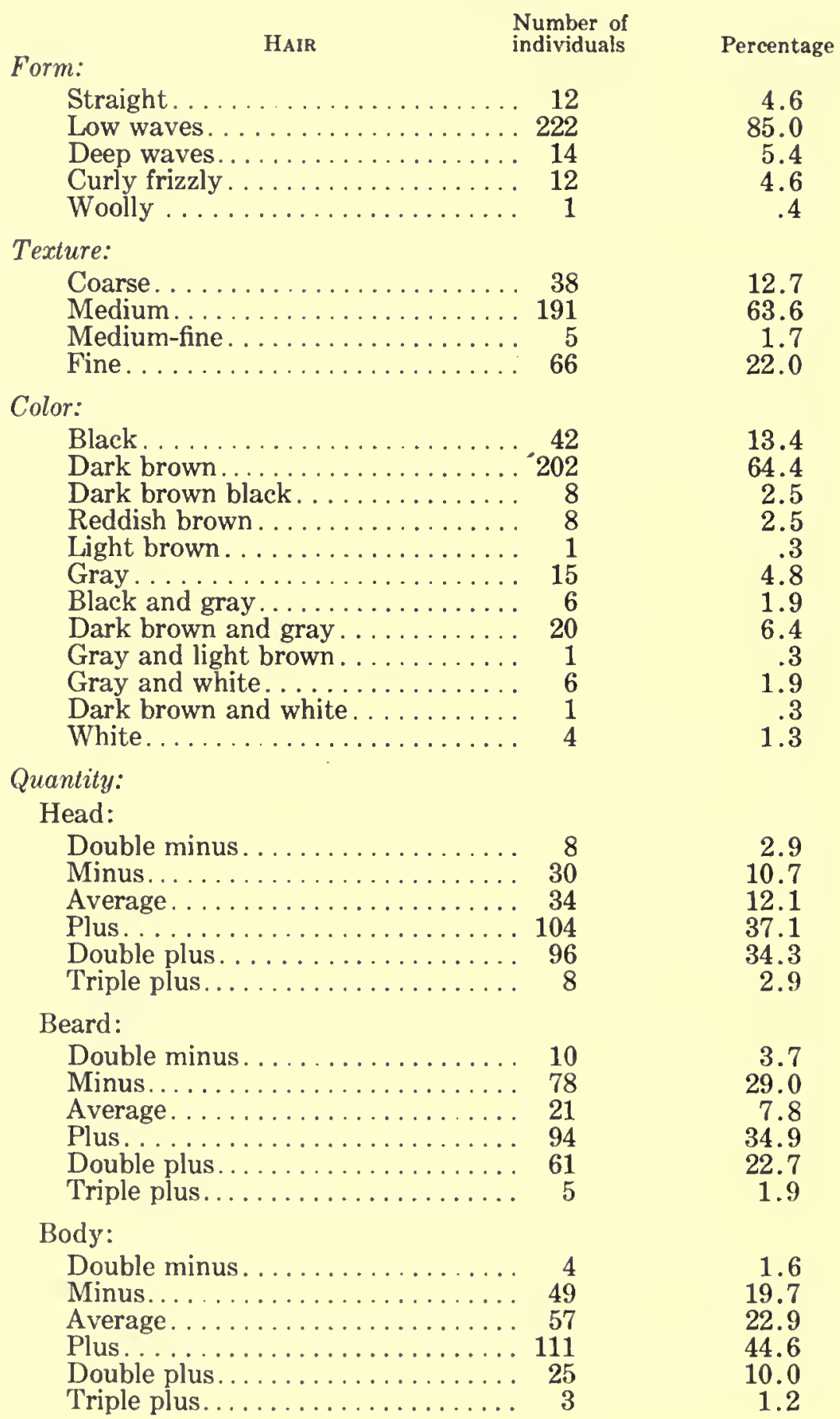


Color:

EYE

Number of individuals

Percentage

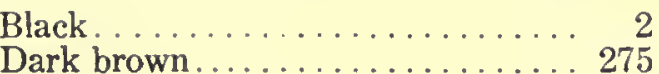

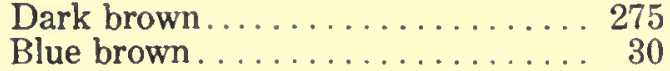

Gray brown .................. 9

Green brown................ 43

Sclera:

Clear.....................2 230

Speckled................... . 26

Yellow.....

Bloodshot................. 95

Speckled and yellow............ 1

Speckled and bloodshot.......... 10

Iris:

Homogeneous.............. 100

Rayed....................... 2

Zoned.................. 247

.6

76.6

8.3

2.5

12.0

63.2

7.1

0.5

26.1

.3

2.8

28.6

70.6

EYEBROW

Thickness:

Minus................... 14

Average. . . . . . . . . . . . . . 7

Plus....................... 26

Double plus................. 16

Concurrency:

Minus.................. 6

Average.................. 11

Plus .................... 27

Double plus................ 4

Lateral extent:

Minus..................... 3

Average ................. 15

Plus. ................................... 29

Double plus................ 1

22.2

11.1

41.3

25.4

12.5

22.9

56.3

8.3

6.2

31.3

60.4

2.1

BROW-RIDGES

Median................... 59

96.7

Continuous................. 2

Glabella:

Average.................. 12

60.0

Plus..................... 8

40.0

Profile:

Nose

Straight................. 209

Concave.................. 40

Convex.................... 73

Concavo-convex.............. 41

Tip:

Thickness:

Minus................... 10

Average.................

Double plus................. 24

Depression:

Depressed................ 80

Horizontal. . . . . . . . . . . . . . . 14

Elevated. 
Medium-compressed ............. 13

Medium................. 166

Medium-flaring. . . . . . . . . . . 31

Flaring. ................ 39

Percentage

12.3

4.6

58.5

10.9

13.7

Wear:

TEETH

None. . . . . ............... 16

Slight. . . . . . . . . . . . . . . . . 14

Average... . . . . . . . . . . . . . . 12

Plus .................. 33

Double plus............... 32

Triple plus. . . . . . . . . . . . . 19

Caries:

None... . . . . . . . . . . . . . . . 37

Minus ...................... 7

Average......................... 10

Plus..................... 25

Double plus ......................... 29

Triple plus............... 20

Lost:

None... . . . . . . . . . . . . . . . 197

One.................... 40

Two..................... 29

Three................... 10

Four................... 11

Five ..................... 2

Six .......................

Seven .................. 1

Ten ..................... 1

Half . . . . . . . . . . . . . . . 2

Several ..................... 1

Many ...................... 8

Nearly all.................. 3

All................... 1

Lobe:

EAR

Attached .................. 51

Free...................... 64 Size:

Minus..................... 7

Average. ..................... 29

Plus.

Double plus. . . . . . . . . . . . . 3

Triple plus................. 1

Roll of helix:

Minus....................... 2

Average.................. 27

Plus. 44

Double plus............... 12

Triple plus.................. 1

Darwin's Point:

Double minus. . . . . . . . . . . . . . 1

Minus ..................... 4

Average ........................ 25

Plus. . . . . . . . . . . . . . . . . . 39

Double plus ........................ 18

Triple plus.................. 2
12.7

11.1

9.5

26.2

25.4

15.1

28.9

5.5

7.8

19.5

22.7

15.6

64.0

13.0

9.4

3.2

3.5

.7

.7

.3

.3

.7

2.6

1.0

.3

44.4

55.6

8.5

35.4

51.2

3.7

1.2

2.3

31.4

51.2

13.9

1.2

1.1

4.5

28.1

43.8

20.2

2.3 


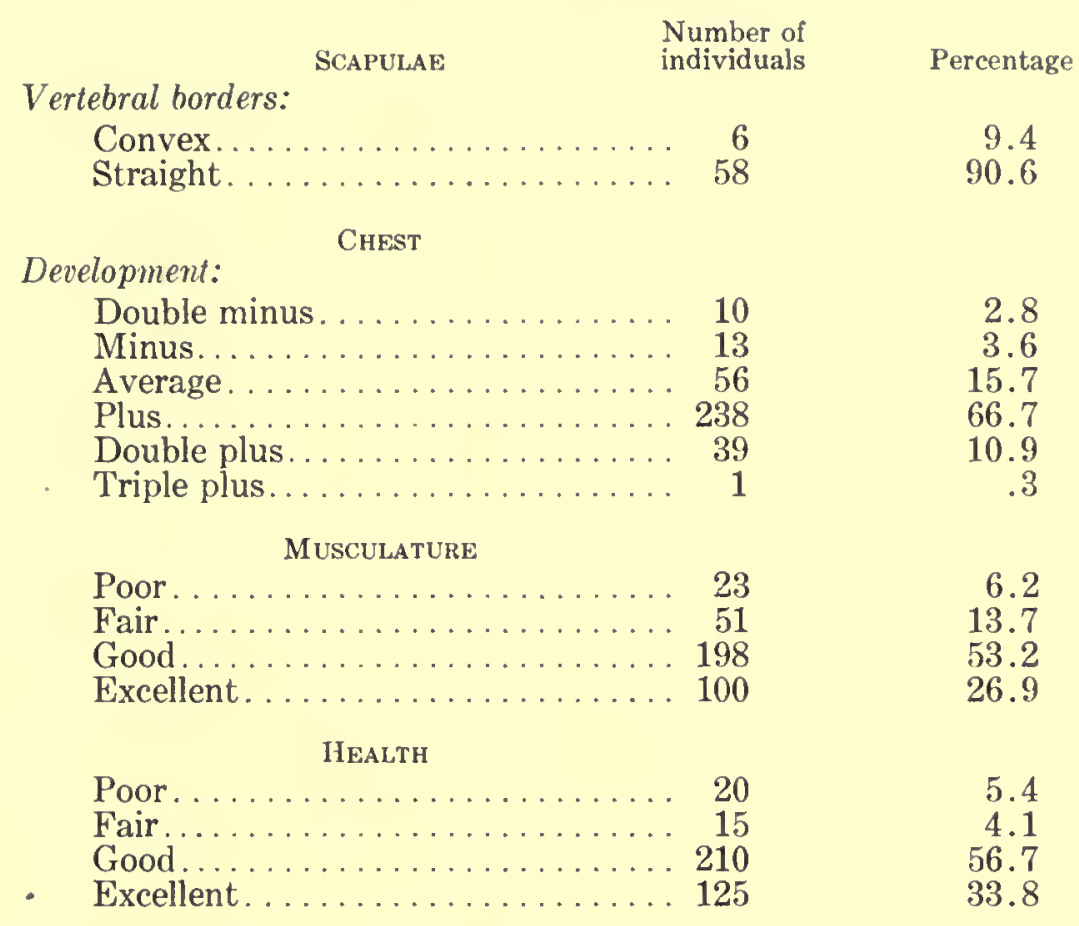

In recording the data of the Kish Arabs it was necessary to estimate the ages of many individuals since after thirty years they did not have any exact knowledge of their ages. For this reason, among the older men the greater numbers of individuals are grouped at ages $35,40,45,50$, and 55 . The statistical summaries follow:

\begin{tabular}{|c|c|c|c|c|c|c|c|}
\hline Age & $\begin{array}{l}\text { Number of } \\
\text { individuals }\end{array}$ & Age & $\begin{array}{l}\text { Number of } \\
\text { individuals }\end{array}$ & Age & $\begin{array}{l}\text { Number of } \\
\text { individuals }\end{array}$ & Age & $\begin{array}{l}\text { Number of } \\
\text { individuals }\end{array}$ \\
\hline 15 & 3 & 25 & 37 & 38 & 4 & 58 & 1 \\
\hline 16 & 4 & 26 & 3 & 39 & 1 & 60 & 11 \\
\hline 17 & 13 & 27 & 4 & 40 & 33 & 62 & 1 \\
\hline 18 & 25 & 28 & 4 & 42 & 2 & 65 & 4 \\
\hline 19 & 6 & 30 & 60 & 43 & 2 & 70 & 4 \\
\hline 20 & 61 & 31 & 1 & 45 & 11 & 75 & 5 \\
\hline 21 & 2 & 32 & 3 & 48 & 2 & 80 & 8 \\
\hline 22 & 3 & 33 & 2 & 50 & 20 & 90 & 3 \\
\hline 23 & 9 & 34 & 1 & 55 & 12 & 100 & 1 (No. 23 \\
\hline 24 & 4 & 35 & $2 \overline{6}$ & 56 & 1 & & \\
\hline
\end{tabular}

The average age for 397 individuals is 33.70 years. No age was recorded on No. 261.

Hair samples were obtained from all individuals except those listed below. Many of the men were shaved so that it was impossible to cut a specimen long enough to be of value. Permission was refused by No. 60 because he was in mourning. In the case of No. 71 a sample was not taken because the hair was diseased. No hair samples were obtained from the following individuals: Nos. $25,49,58,60,62,63,71,89,97,111,113,119,123,124,125,128,130,131,135$, $137,143,152,153,154,158,159,162,166,167,173,176,180,192,198,199$, $201,202,206,217,225,231,246,258,267,274,286,355,357,367,370,371,376$, $382,389,393,405,411,429,433,456,457,458,459,460,461,466$, and 470. 


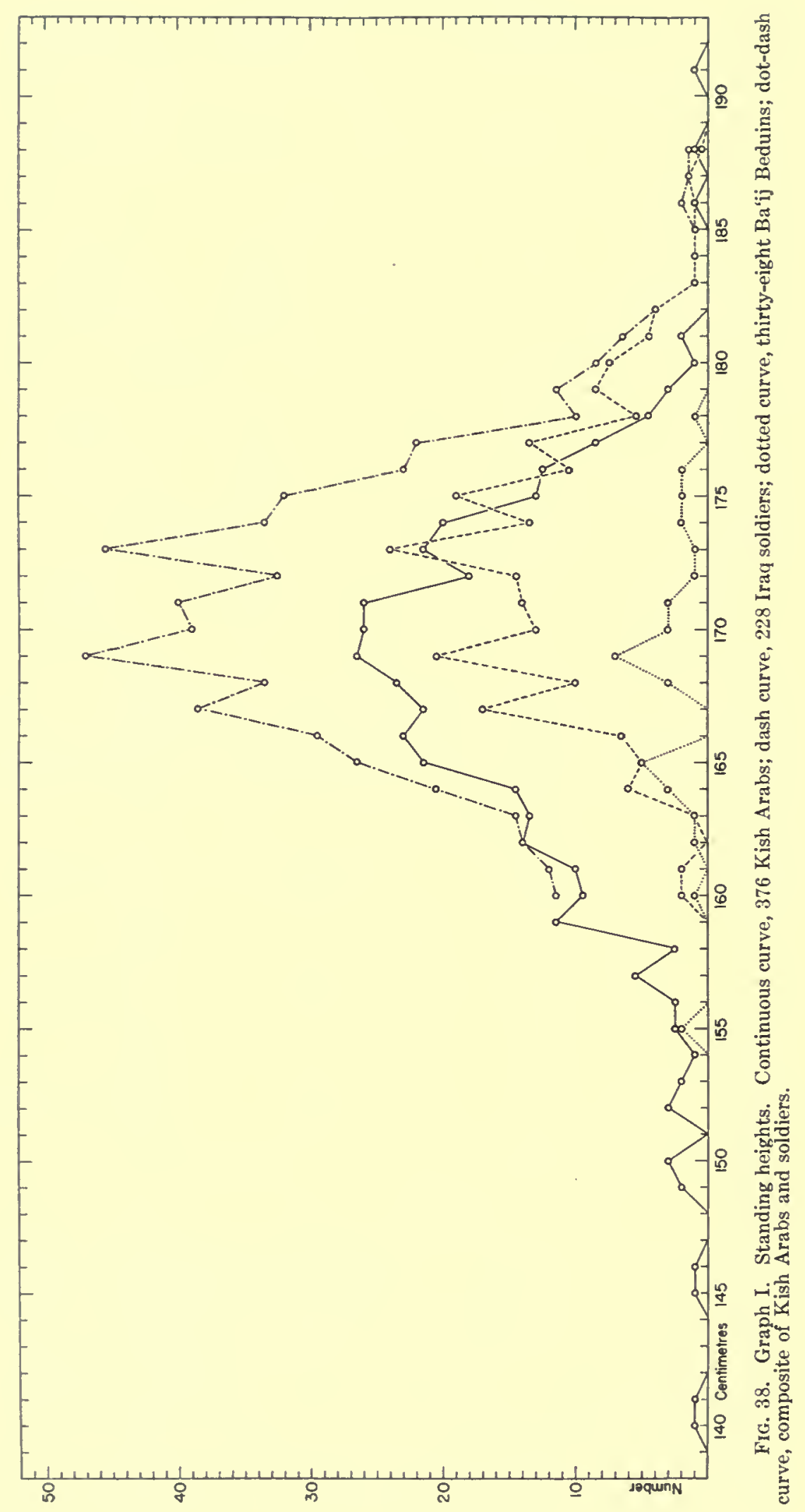




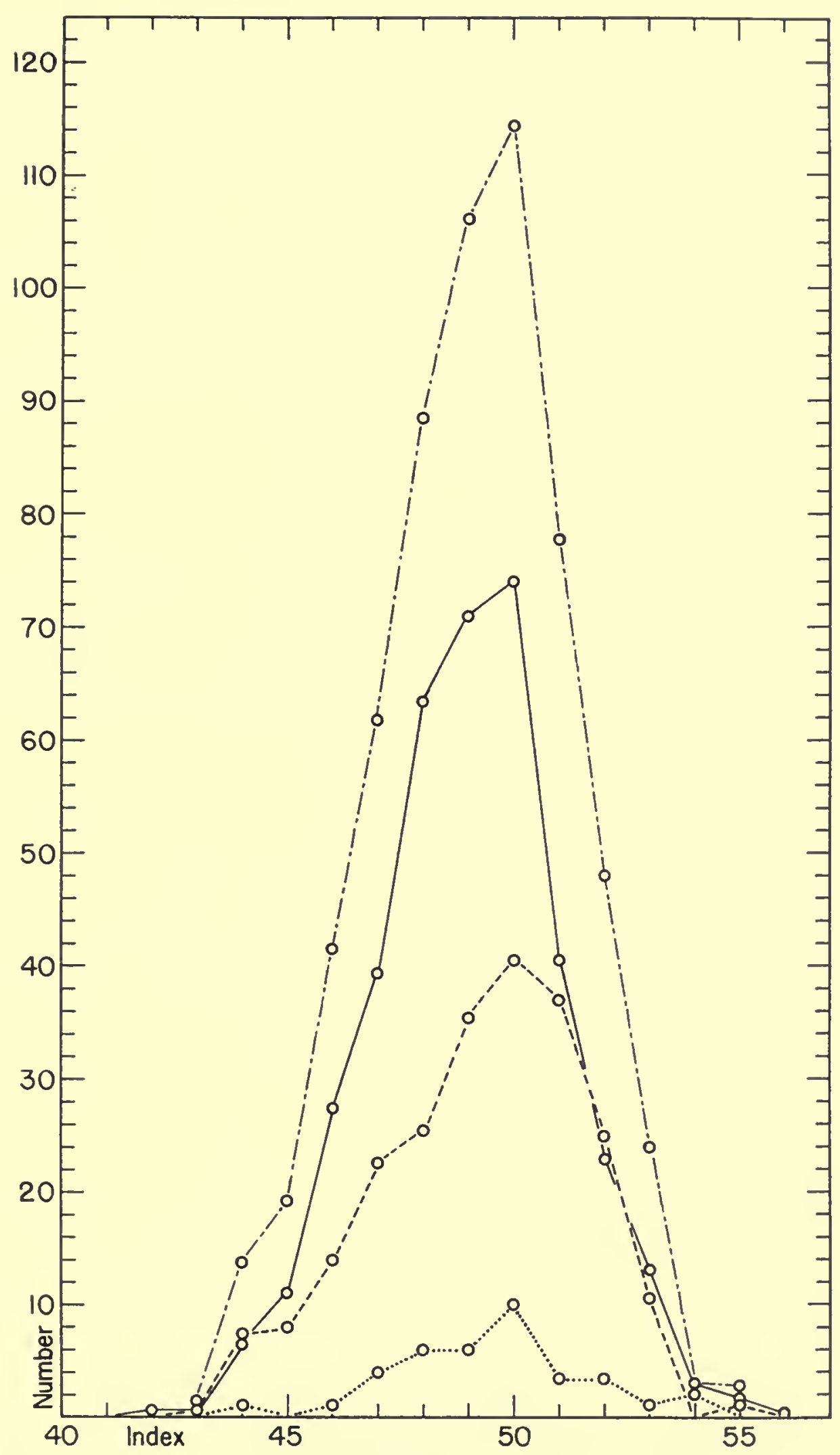

FIG. 39. Graph II. Index of relative sitting heights. Continuous curve, 376 Kish Arabs; dash curve, 228 Iraq soldiers; dotted curve, thirty-eight $\mathrm{Ba}$ ' $\mathrm{ij}$ Beduins; dot-dash curve, composite of $\mathrm{K}$ ish Arabs and soldiers. 
No. 14 had a remarkable abundance of hair all over his forehead but his face was not hirsute. In contrast to the long plaits of hair often worn under the head-dress (Nos. 43 and 54) No. 15 was slightly bald. No. 171 had a marked absence of pilosity all over his body.

Nos. $1,7,9,24,69,236,238,263,267$, and 407 had applied henna to their hair. No. 1 said he used it to encourage growth.

The following individuals had mustaches: Nos. 3, 4, 8, 9, 10, 11, 16, 22, $25,26,27,31,32,38,39,40,43,44,45,48,49,50,57,61,65,66,67,68,69,70$, $74,75,95,97,105,107,126,127,138,146,171,269,350$, and 388 .

Nos. 7, 17, 20, 28, 30, 34, 46, 52, and 112 wore a mustache and a slight beard. Nos. 12, 15, 21, 33, 42, 55, 63, 77, 92, 96, 108, 118, 129, 130, 131, 147, 167,173 , and 383 had mustaches and full beards.

No. 4 had a small shaved area in the region behind the bregma. No. 100 had a circular shaved area on the top of his head.

Nos. 68,286 , and 470 had bad eyes. In the case of No. 361 the right eye was defective and Nos. 23, 189, and 429 had poor vision in the left eye. A white spot impaired the sight of the left eye in the case of No. 31 and the right eye of No. 150 was missing. The eyes of Nos. 189, 361, and 429 were slightly crossed.

The eyes of Nos. 28 and 47 were in poor condition, practically closed.

Cataract occurs frequently among these people. No. 257 had cataracts in both eyes while the left eyes of Nos. 104, 141, 195, 265, and 399, and the right eyes of Nos. 202 and 204 were similarly afflicted.

Nos. 433,434 , and 439 were blind in both eyes. Nos. 195, 202, and 428 were blind in the left eye, No. 202 since childhood. Nos. 135, 201, 265, 354, and 399 had lost the sight of their right eyes, No. 201 since childhood.

The teeth of Nos. 10, 109, and 376, who was 80 years old, were in excellent condition. Those of No. 255 were bad and Nos. 65, 123, and 135 had very bad teeth. Nos. 12, 112, and 119 complained of trouble with their teeth and dental decay and absorption of the gums was very apparent in the case of No. 130. The teeth of No. 1 were stained with nicotine.

No. 190 had markedly large front incisors. No. 135 had irregular teeth. The first right incisor of No. 209 was broken while No. 116 had a front lower tooth broken.

The following numbers of individuals had lost molars: 1 , one left lower; 2 , one left upper; 1 , first right upper; 3 , one upper on each side; 1 , two upper on each side; 1 , three right lower and one right upper; 1 , two right lower and two left lower; 1 , two right lower and one left lower; 1 , two right upper and one right lower; 1, three right lower and three left lower; 1 , third left lower and third right lower; 1 , two upper and two lower; 8 , two right lower; 3 , one right upper; 1 , first right lower; 3 , one lower on each side; 1 , first lower on each side.

The following numbers of individuals had lost premolars: 5 , one left lower; 1, two left upper; 1 , one left; 1 , first lower; 1 , one lower on each side; 1 , one right upper; 1 , two right upper; 1 , two right. 
The following numbers of individuals had lost incisors: 1, all but two in both lower jaws; 1 , one right lower.

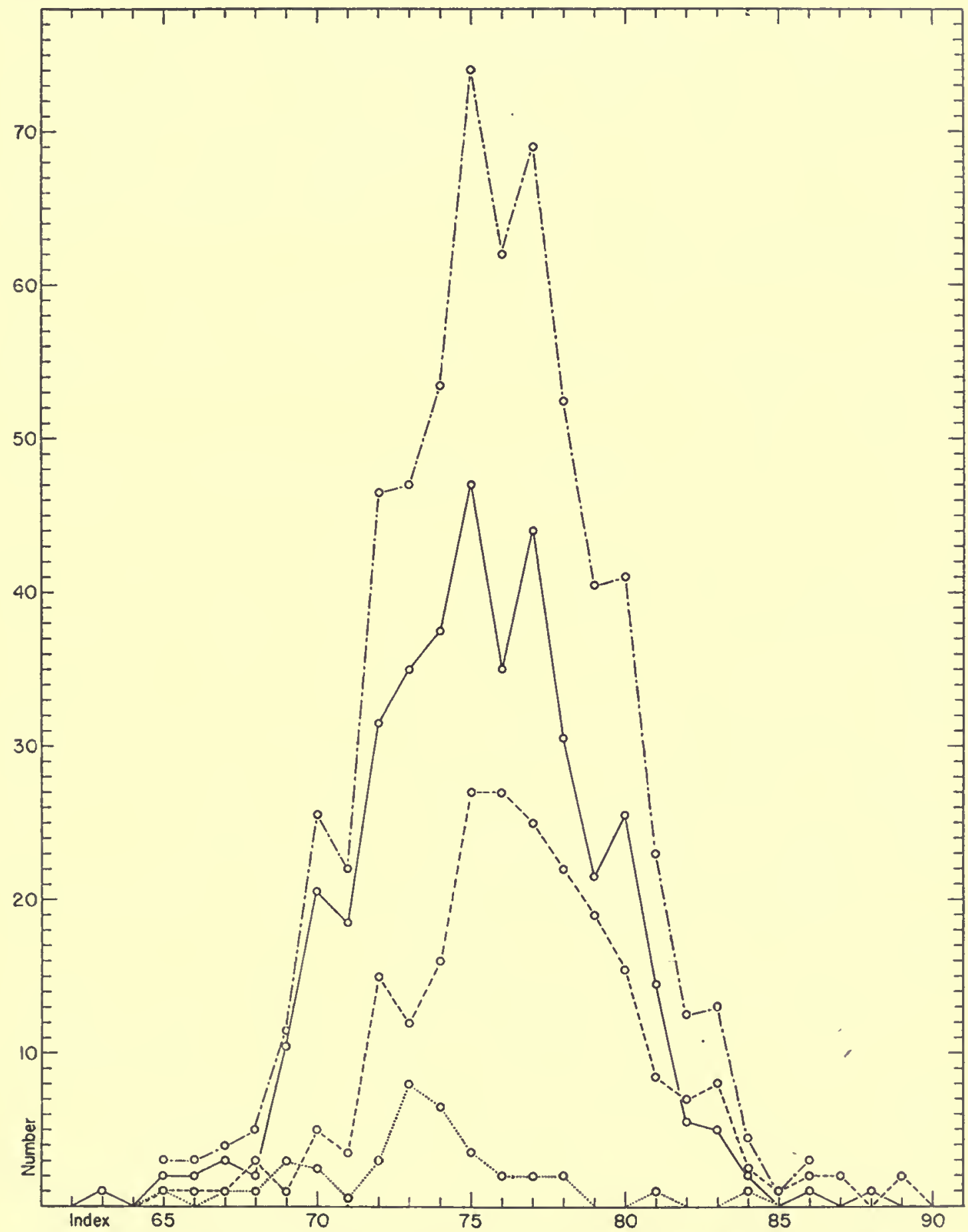

FIG. 40. Graph III. Cephalic indices. Continuous curve, 396 Kish Arabs; dash curve, 227 Iraq soldiers; dotted curve, thirty-eight Baij Beduins; dot-dash curve, composite of Kish Arabs and soldiers.

The following numbers of individuals had lost canines: 1, one left upper; 1 , one right lower. 
The following special cases of teeth lost were noted for individuals: one lower molar on each side, one lower left premolar; one upper and lower premolar on right side and two lower molars; two right upper incisors, one canine, and two premolars; one molar and one right lower canine; two right lower premolars and two molars; two left upper premolars and one right upper molar.

No. 50 had a diseased left ear. The right ear of No. 125 was peculiarly flexed in the apical region. No. 192 had his left ear slightly punctured near the lobe.

The following individuals suffered from recurrent fever attacks which lasted usually from three to eight days: Nos. $6,11,12,13,15,16,23,25,27,28,29$, $34,36,40,42,43,44,45,46,50,52,54,55,60,61,62,66,68,69,70,105,108$, $109,110,122,128,147,153$, and 246.

Nos. 29, 47, 48, 64, 67, and 115 had fever attacks during 1927.

Nos. 113, 118, and 167 had completely recovered from such fever attacks. Nos. 15, 68, and 201 were subject to headaches. Nos. 124 and 155 complained of stomach trouble.

For four successive months three years ago No. 2 had fever, headaches, and stomach ache. No. 4 had bad headaches for a continuous period of one year. No. 16 suffered from bi-yearly fever; he said that coffee made him ill and that the east wind affected him. No. 17 was sick for two years with fever. No. 25 said that his recurrent fever was due to the heat twelve years ago. No. 26 had fever and vomiting attacks during the cholera outbreak in 1927-28; he turned yellow then, perhaps from jaundice. Before his marriage No. 33 claimed that he had been often fevered but was now wholly recovered. No. 106 had fever for one year. No. 122 said he was always fevered. He had "Baghdad boils" on his left upper lip near the nasal orifice. There were lined scars on the right temple of No. 32 where blood was let to cure his headaches. No. 463 had a bad throat and said his voice was always harsh. Nos. 28 and 145 were sick-looking and Nos. 96 and 135 complained that they were often sick. No. 123 said his health had improved since Ramadan.

An admixture of Negro blood was apparent in the case of individuals Nos. $30,54,59,258,359,408$, and 458 . In No. 30 this was suggested by thickness of lips and in No. 59 by darkness of pigmentation.

Wide bizygomatic areas suggested a Mongoloid strain in Nos. 1, 2, and 393. No. 26 bears a close resemblance to the type portrayed by Sumerian artists. Smallpox scars were noted in the case of Nos. $19,42,44,51,58,97,102$, $105,108,117,132,143,174,196,208,222,232,381,411,453$, and 469.

No. 13 (age 20) was physically undeveloped. No. 191 was obviously a case of overgrowth which was due to irregular functioning of the endocrine glands.

The right hand, radius and ulna of No. 230 was deformed. No. 286 also had a deformed right hand. A cut on the upper lip of No. 398 caused a slight disfiguration.

The following individuals had scars: No. 22, across nose; Nos. 24 and 29, on left cheek from boils; No. 32, on left part of chin; No. 50, under 
left eye; No. 60, on glabella; No. 170, above glabella on right side; No. 233, on right side of nose, due to kick from mule; No. 284, behind right ear, not due to mastoid. No. 35 had a mark on his right wrist from burning, and No. 155 had a scar over the right squamosal due to burning.

No. 14 had warts in profusion on his hands and feet. No. 358 had warts on his right wrist.

A mole over the left eye was observed on No. 11, and No. 43 had a mole scar on the left side of his nose.

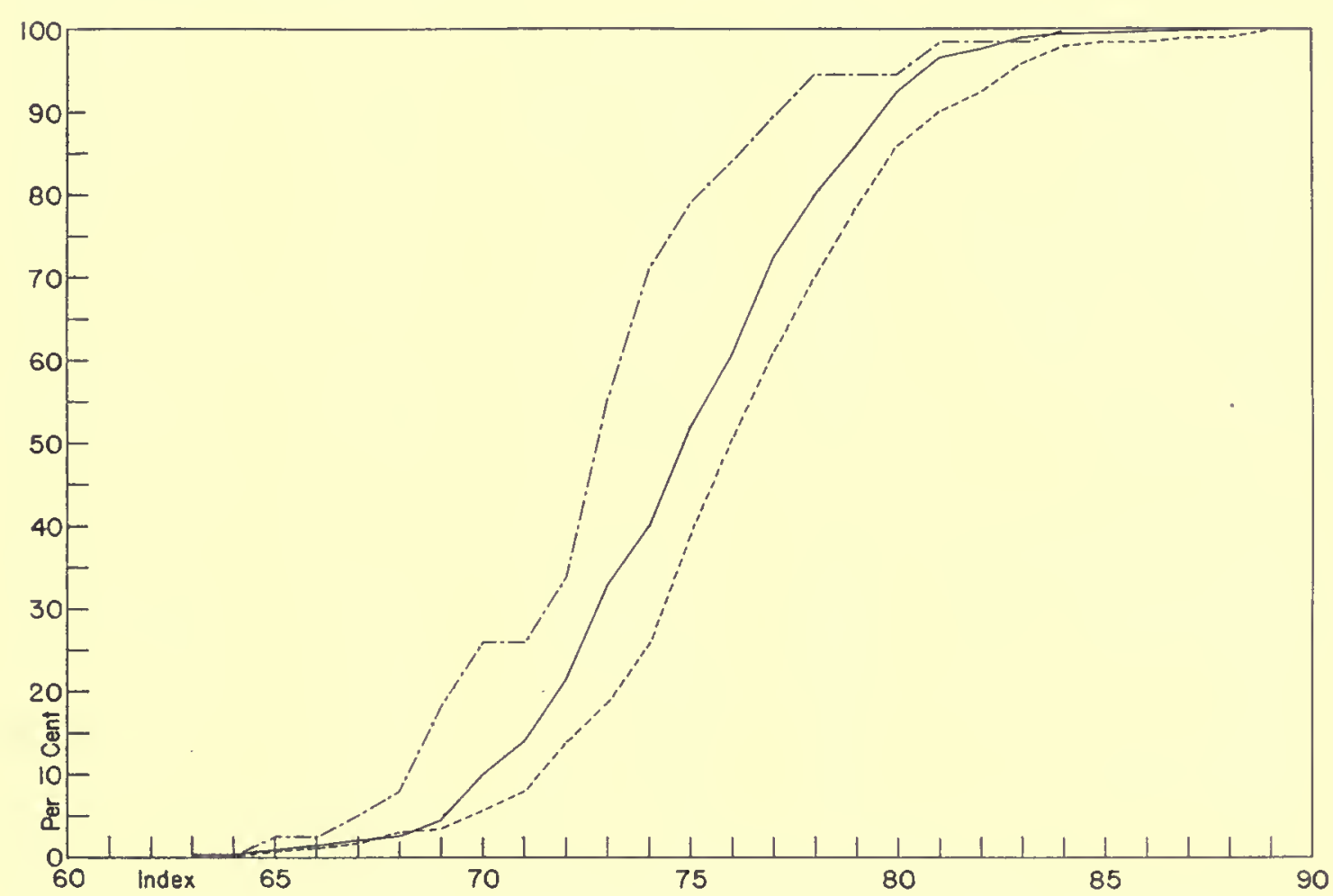

FIG. 41. Graph IV. Cumulative percentages of cephalic indices. Continuous curve, 396 Kish Arabs; dash curve, 227 Iraq soldiers; dot-dash curve, thirty-eight Ba ${ }^{\circ i j}$ Beduins.

No. 151 lost part of the right hallux in a railway accident. No. 154 had recovered from a fractured leg. No. 198 wore a kind of truss.

There were two individuals who were reputed to be insane, Nos. 59 and 62 . No. 62 was indeed slow-minded and rather stupid but he was a careful worker. Nos. 48, 63, 64, and 67 were good workmen. No. 37 was unusually intelligent. No. 34 (Achmed Hamadi) had a fair working knowledge of mechanics and was useful in light railway work on excavations. No. 178, on the other hand, was a very poor workman who had to be dismissed for attempting to hit the foreman (Rais) with a shovel (misha).

No. 76 was very talkative. No. 128 was nervous and rather stupid. Among the workers No. 49 was known as a good dancer and No. 41 could imitate animals, especially the barks of a dog, with exceptional skill. 
No. 131 had been in prison at Omsk after he was captured from the Turkish army in 1914. Formerly No. 264 had been a policeman in Ad Diwaniya for six years.

No. 13 wore a string on his right wrist to show that he had grown thinner.

In addition to those who had applied henna to their hair No. 45 had tinted his nails to make them beautiful and painted the palms of his hands to harden them. No. 158 had applied kohl to his under eyelids.

\begin{tabular}{|c|c|c|c|c|}
\hline & ANTH & OMETRIC & & \\
\hline Measurements & $\begin{array}{l}\text { Number of } \\
\text { individuals }\end{array}$ & Mean & $\begin{array}{l}\text { Standard } \\
\text { deviation }\end{array}$ & $\begin{array}{l}\text { Coefficient } \\
\text { of variation }\end{array}$ \\
\hline Standing height. & 376 & $1676.80 \pm .23$ & $6.68 \pm .16$ & $3.98 \pm .10$ \\
\hline Sitting height. & 378 & $821.30 \pm .16$ & $4.64 \pm .11$ & $5.65 \pm$ \\
\hline Acromion to sole. & 377 & $1402.80 \pm .23$ & $6.55 \pm .16$ & $4.66 \pm$. \\
\hline Head length. & 396 & $188.44 \pm .24$ & $7.01 \pm .17$ & $3.71 \pm .09$ \\
\hline Head breadth & 397 & $141.73 \pm .19$ & $5.69 \pm .14$ & $4.01 \pm$. \\
\hline Minimum frontal diamete & 395 & $111.13 \pm .18$ & $5.28 \pm .13$ & $4.75 \pm$. \\
\hline Bizygomatic breadth. & 396 & $129.46 \pm .26$ & $7.79 \pm .19$ & $6.01 \pm .14$ \\
\hline Bigonial breadth.... & 394 & $102.54 \pm .27$ & $7.84 \pm .19$ & $7.64 \pm$. \\
\hline Total facial height. & 393 & $119.81 \pm .25$ & $7.30 \pm .18$ & $6.09 \pm$. \\
\hline Upper facial height. & 392 & $72.97 \pm .26$ & $5.51 \pm .18$ & $7.55 \pm$. \\
\hline Nasal height. . .... & 393 & $58.33 \pm .17$ & $5.09 \pm .12$ & $8.72 \pm$ \\
\hline Nasal breadth. & 396 & $35.32 \pm .12$ & $3.48 \pm .08$ & $9.85 \pm .2$ \\
\hline Ear length. & 396 & $62.32 \pm .18$ & $5.43 \pm .13$ & $8.71 \pm$. \\
\hline Ear breadth & 395 & $35.31 \pm .12$ & $3.60 \pm .09$ & $10.19 \pm .24$ \\
\hline Indices & & & & \\
\hline Relative sitting height. & 376 & $48.98 \pm .07$ & $2.11 \pm .05$ & $4.31 \pm .11$ \\
\hline Cephalic. & 396 & $75.26 \pm .13$ & $3.71 \pm .09$ & $4.92+$ \\
\hline Fronto-parietal & 396 & $78.56 \pm .14$ & $4.32 \pm .10$ & $5.49 \pm$. \\
\hline Total facial. & 392 & $92.73 \pm .25$ & $7.35 \pm .18$ & $7.92 \pm$. \\
\hline Upper facial & 391 & $56.62 \pm .17$ & $5.02 \pm .12$ & $8.86 \pm$ \\
\hline & 397 & $61.15 \pm .26$ & $7.69 \pm .18$ & $12.57 \pm .30$ \\
\hline Ear. & 396 & $56.91 \pm .21$ & $6.16 \pm .15$ & $10.82 \pm$. \\
\hline Jugomandibular & 394 & $79.21 \pm .17$ & $4.92 \pm .12$ & $6.21 \pm .15$ \\
\hline Jugofrontal. & 394 & $85.89 \pm .15$ & $4.45 \pm .11$ & $5.24 \pm .12$ \\
\hline
\end{tabular}

\section{SOLDIERS OF THE IRAQ ARMY}

In dealing with this group of soldiers a number of statistics were recorded which show that these men were selected for their good physique and health. The average age of this series is low; all the individuals with three exceptions fall between the ages of 17 and 30 . In general I have avoided obtaining anthropometric data on immature individuals but to avoid this was impossible in the Iraq Army group, a factor which must be given due consideration.

AGE

The age of each soldier was carefully noted and checked against the official army records so that the results can be tabulated as follows:

$\begin{array}{cccccc}\text { Age } & \begin{array}{c}\text { Number of } \\ \text { individuals }\end{array} & \text { Age } & \begin{array}{c}\text { Number of } \\ \text { individuals }\end{array} & \text { Age } & \begin{array}{c}\text { Number of } \\ \text { individuals }\end{array} \\ 17 & 6 & 23 & 10 & 29 & 2 \\ 18 & 13 & 24 & 7 & 30 & 15 \\ 19 & 8 & 25 & 44 & 33 & 1 \\ 20 & 61 & 26 & 7 & 35 & 1 \\ 21 & 15 & 27 & 2 & 45 & 1 \\ 22 & 26 & 28 & 4 & & \end{array}$


No age was recorded for individual No. 528. The average age for 223 individuals is 22.16 years.

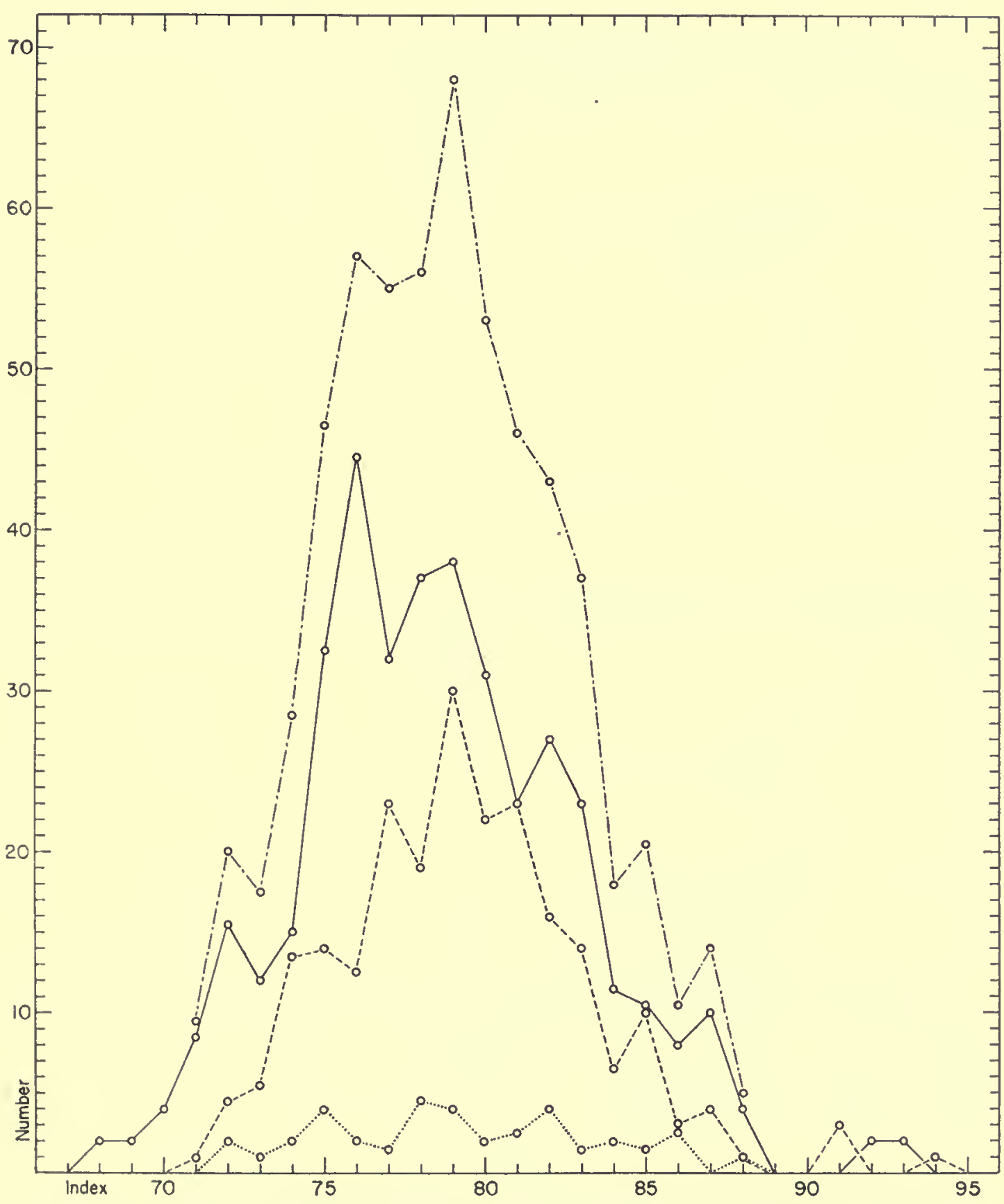

Fig. 42. Graph V. Fronto-parietal indices, Continuous curve, 395 Kish Arabs; dash curve, 227 Iraq soldiers; dotted curve, thirty-eight Ba'ij Beduins; dot-dash curve, composite of Kish Arabs and soldiers.

HAIR

The majority of the soldiers had dark brown hair of medium texture with the form low waves. Sixty-four individuals had dark brown hair. Nos. 504, 505, 613,644 , and 653 had black hair. No. 612 , who had a very peculiarly shaped 
head, had light brown hair. Nos. 509 and 555 had an unusual quantity of hair on the head. There were no observations recorded on No. 514.

\section{EYE}

The majority of the soldiers had dark brown eyes with clear sclera and homogeneous irises. The following information was recorded:

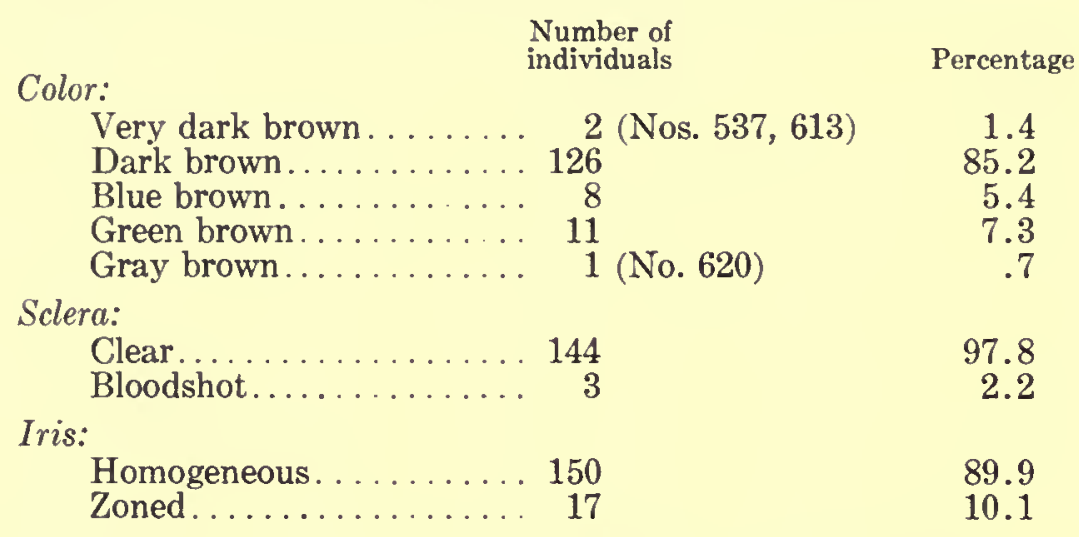

No. 525 was cross-eyed and had the right eye partly closed.

\section{NOSE}

The majority of individuals had straight noses with medium wings. The following information was recorded:

\begin{tabular}{|c|c|c|}
\hline & $\begin{array}{l}\text { Number of } \\
\text { individuals }\end{array}$ & Percentag \\
\hline \multicolumn{3}{|l|}{ Profile: } \\
\hline Straight. . & 81 & 57.0 \\
\hline Concave. & 14 & 9.9 \\
\hline Concavo-c & 3 & 2.1 \\
\hline Convex... & 44 & 31.0 \\
\hline \multicolumn{3}{|l|}{ Wings: } \\
\hline Medium & 66 & 53.3 \\
\hline Medium-c & 6 & 4.8 \\
\hline Compresse & 6 & 4.8 \\
\hline Medium-fl & 29 & 23.4 \\
\hline Flaring... & 17 & 13.7 \\
\hline
\end{tabular}

In regard to thickness of the nasal tip No. 647 was triple plus; Nos. 543, $555,575,587,611$, and 638 were double plus; No. 578 was average. Nos. 517, $576,597,601$, and 635 were thin. No. 578 had an elevated nasal tip and Nos. 600 and 629 had depressed tips.

\section{TEETH}

The average condition of the teeth was good. The occlusion was normal and the majority of the soldiers had lost no teeth. The following statistics were recorded:

$$
\begin{aligned}
& \text { individuals } \\
& \text { Bite: } \\
& \text { Under ............. } 1 \\
& \text { Slight over............. } 125 \\
& \text { Marked over ........... } 11 \\
& \text { Edge-to-edge .......... } 2
\end{aligned}
$$


Lost:

$$
\begin{aligned}
& \text { Number of } \\
& \text { individuals }
\end{aligned}
$$

None................ 106

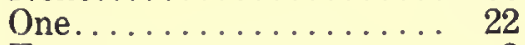

Two............. 3 (Nos. 524, 561, 600)

Three.............. 2 (Nos. 504, 559)

Eruption:

Complete.............. 131

Incomplete.......... 13

Caries:

Plus............. 5 (Nos. 561, 564, 565, 571, 600)

Double plus............ 1 (No. 555)

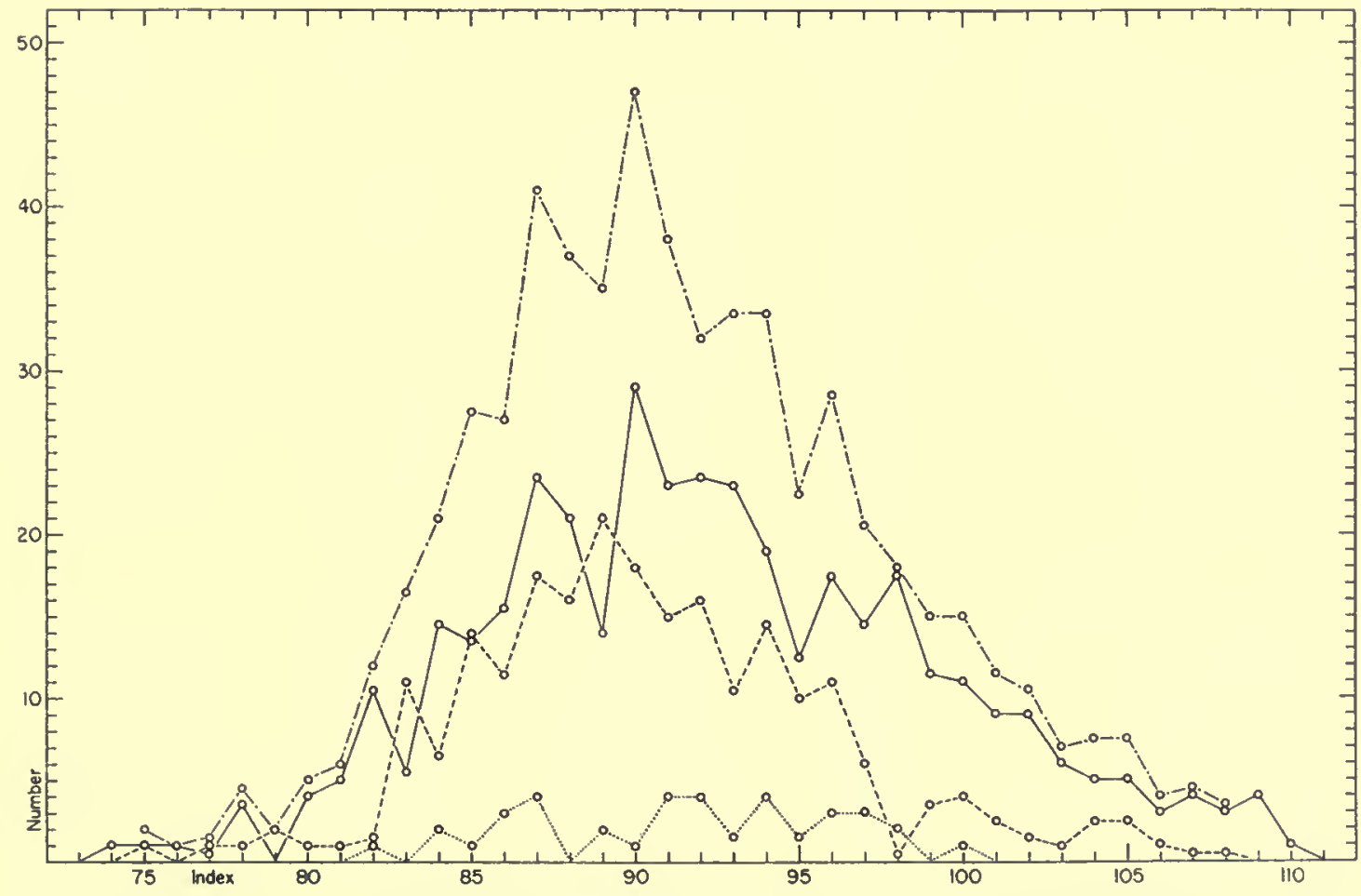

FIG. 43. Graph VI. Total facial indices. Continuous curve, 385 Kish Arabs; dash curve, 227 Iraq soldiers; dotted curve, thirty-eight Ba ${ }^{\mathrm{ij}}$ Beduins; dot-dash curve, composite of Kish Arabs and soldiers.

\section{CHEST}

The average development as recorded on 132 soldiers was good. Nos. 609,627 , and 655 were excellent and Nos. 505, 529, 530, 536, 539, 579, and 594 were average.

\section{MUSCULATURE}

The average muscular development as recorded on 133 individuals was good. Nos. 584, 609, 627, and 655 were excellent and Nos. 505, 529, 530, 539, 579, and 594 were average.

\section{HEALTH}

The statistics recorded on 142 soldiers showed them to be in good health. 
SMALLPOX

Nos. 531, 537, and 603 had smallpox scars.

\section{PATHOLOGICAL CASES}

No. 661 was extremely tall and his general development suggested a pathological condition due to unbalanced metabolism as the result of irregular functioning of the endocrines.

\section{NEGRO BLOOD}

Nos. 537, 552 (Sudanese), and 613.

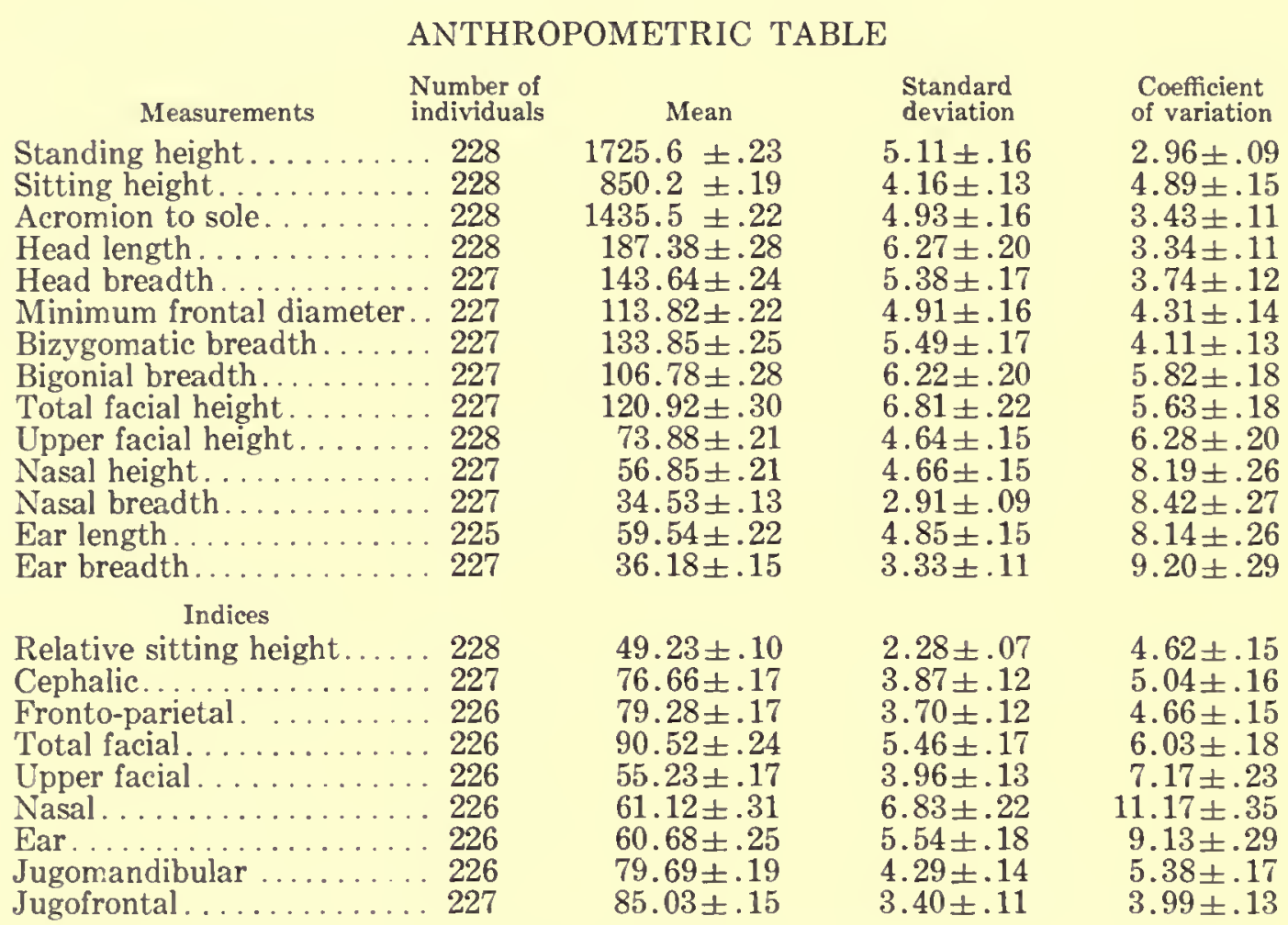

\section{BA'IJ BEDUINS}

During June, 1928, a sub-tribe of the Ba'ij Beduins was encamped between Kish and Jemdet Nasr. Twelve miles from Kish, on the desert track toward Tell Barguthiat, about fifty black tents were grouped. Sheikh Abbas ibn Diab, leader of this Beduin sub-tribe, which wanders between the Euphrates and Tigris rivers, possesses large herds of camels and sheep and is much feared by the fallahin or pastoral Arabs. The Beduins feel contempt and scorn for the settled Arabs. When desert pasturage is poor, they do not hesitate to drive their camels to feed in fields sown with corn. The Arabs, however, can only rain down curses on the heads of the marauding Beduins, for they dare not attempt to drive them away.

Sheikh Abbas gave us a cordial reception although the trappings of his tent indicated great poverty for the sheikh of a large tribe. After tea and coffee 
had been served, I invited Sheikh Abbas to examine the head calipers and the cameras. He then allowed me to make anthropometric observations and measurements on him as well as frontal and profile photographs. A hair sample was obtained only after much persuasion.

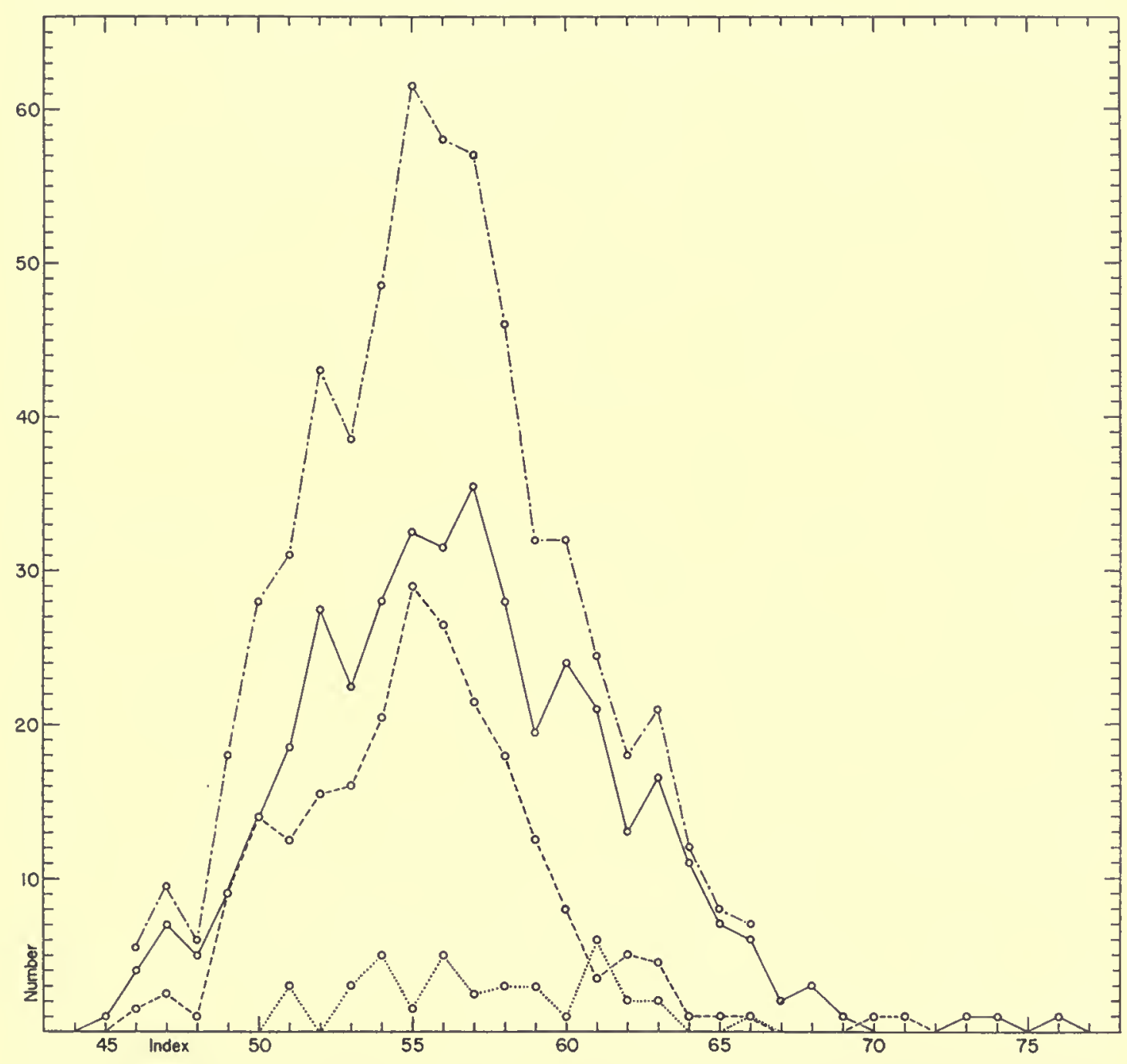

FIG. 44. Graph VII. Upper facial indices. Continuous curve, 391 Kish Arabs; dash curve, 226 Iraq soldiers; dotted curve, thirty-eight $\mathbf{B a}^{6} \mathrm{ij}$ Beduins; dot-dash curve, composite of Kish Arabs and soldiers.

The anthropometric data on his tribesmen are of particular interest since they were obtained from a group of Beduins from the jazirah lying between the Euphrates and Tigris rivers. While the number of individuals upon whom measurements and observations were taken is small, and therefore no definite conclusions can be drawn from them, the racial indications suggested are of importance.

It seems probable that the Beduins, including the hundreds of individuals with whom I came into contact in the various camps of the Huwaitat, Ruwalla, Bani Sukhr, Anaiza, and Sba'a visited by the Field Museum North Arabian 
Desert Expeditions, 1927-28, are directly connected racially with the earliest inhabitants of the Kish area.

Racial admixture among the Beduins is at a minimum owing to their independent nomadic life and to the resultant tribal inbreeding. The latter thus tends to preserve the ancient dominant strain and would leave little room for racial variation. 'The outline of the face, the stature, and the general musculature of the body at once separate the Beduins from the dwellers in cities who have become racially mixed. The only exception to the purity of the Beduin peoples is the infiltration of Negro blood through the importation of slaves. Negroid characteristics are apparently more dominant than are those of the Beduins, and thus usually appear in the progeny. One of the Beduin children appeared to have a relatively high percentage of Negro blood in his veins. Inquiries revealed that he was the son of an unattractive young Beduin girl and one of the big Negroes forming the Sheikh's bodyguard. As soon as the other children saw that we were particularly interested in this child, they began to make fun of his flaring nostrils and thick lips, and to shout that he was no true Beduin boy.

The eyes of the majority of the Ba'ij Beduins were blue brown in color with clear sclera and zoned irises. This eye color was observed occasionally among the Kish Arabs, but was not predominant among them. Several individuals complained of eye soreness due to wind and dust storms.

The teeth were in fairly good condition considering that they never blush nor take any care of them. 'The soft diet, however, consisting of starches and sugar, with the rare addition of proteins, tends to soften the gums and to cause dental caries and general decay at an early age. The smoking of the narghile is a general pastime and the nicotine and other injurious tobacco ingredients stain the teeth and cover them with a yellow film. The Negroes and those members of the community with Negro blood possess excellent, white teeth.

As will be seen from the anthropometric data recorded on this group of $\mathrm{Ba}$ 'ij Beduins, they are dolichocephalic, but the cephalic index of the Kish Arabs is only slightly higher (1.89), probably due to the arrival of a brachycephalic element, which forced itself racially as well as culturally upon the original population. A preliminary study of the skeletal material excavated at Kish from the earlier graves belonging to the period about 3250 B.C., suggests that the physical features of the peoples of the Kish area have become little changed during the past five thousand years.

The ages of the Ba'ij Beduins can only be estimated, since no individual could give an exact figure.

$\begin{array}{cccccc}\text { Age } & \begin{array}{c}\text { Number of } \\ \text { individuals }\end{array} & \text { Age } & \begin{array}{c}\text { Number of } \\ \text { individuals }\end{array} & \text { Age } & \begin{array}{c}\text { Number of } \\ \text { individuals }\end{array} \\ 18 & 2 & 38 & 1 & 65 & 1 \\ 20 & 3 & 40 & 4 & 74 & 1 \\ 25 & 5 & 45 & 4 & 80 & 1 \\ 30 & 6 & 50 & 2 & 90 & 1 \\ 35 & 6 & 60 & 1 & & \end{array}$

The average age of the thirty-eight $\mathrm{Ba}$ 'ij Beduins is 38.37 years. 
HAIR

CoLOR.-Dark brown: Nos. 300, 302, 309, 312, 316, 324, 327. Dark brown and gray: No. 319. Reddish brown: Nos. 302, 308, 329, 332. Black: Nos. 310 , $311,313,315,322,323,326,330,331,333,335,337$. Black and gray: Nos. 318 , 319, 325, 334. Gray: Nos. 307, 317, 328, 336.

Form.-Low waves: Nos. 300, 305, 307-312, 315, 323, 327, 331-335, 337. Deep waves: Nos. 303, 304, 313, 314, 324, 330. Curly frizzly: Nos. 302, 306, 316,322 .

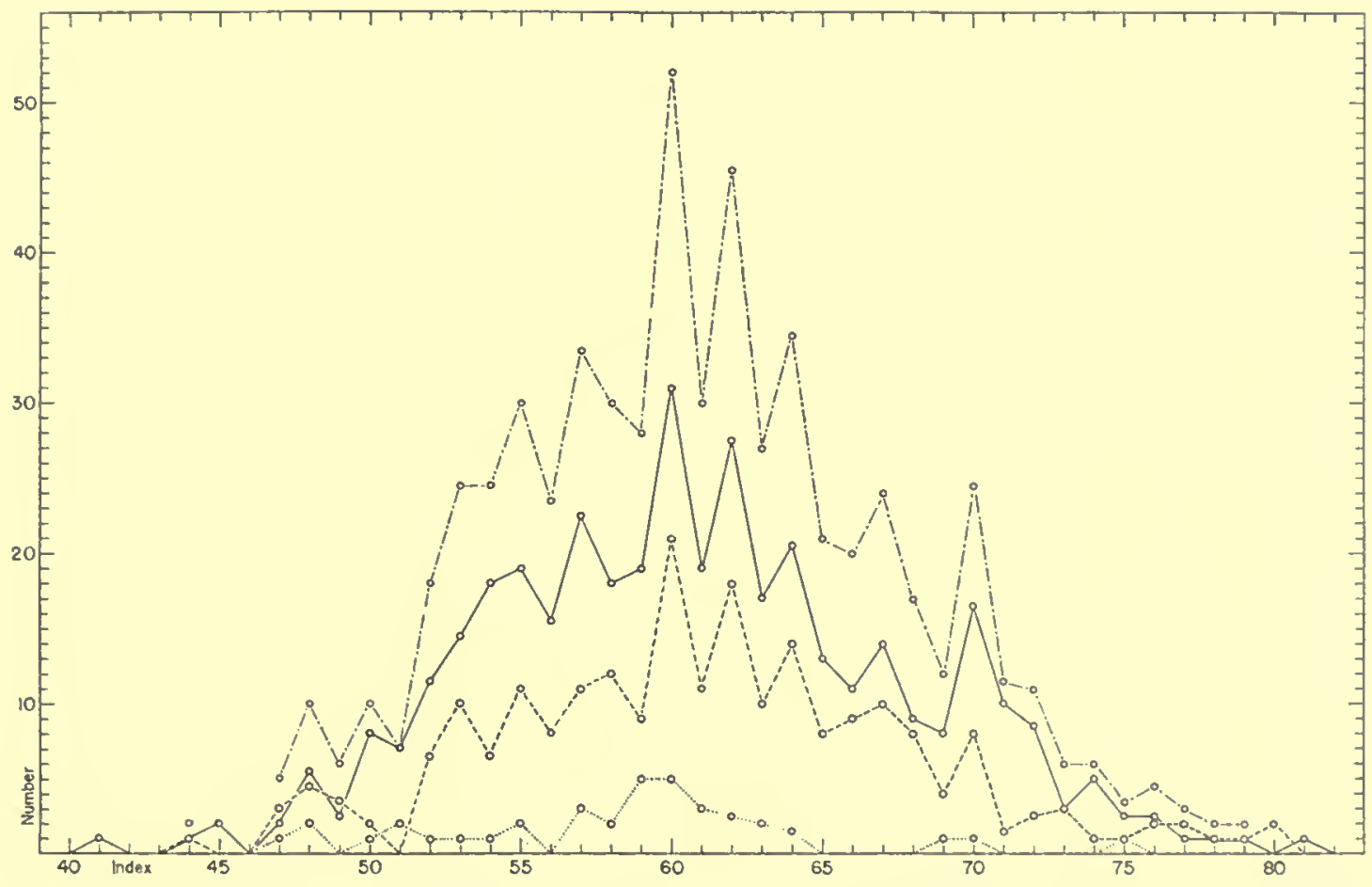

FIG. 45. Graph VIII. Nasal indices. Continuous curve, 389 Kish Arabs; dash curve, 226 Iraq soldiers; dotted curve, thirty-eight Ba'ij Beduins; dot-dash curve, composite of Kish Arabs and soldiers.

Texture.-Fine: Nos. 315, 323, 324, 330. Medium: Nos. 300, 303, 305, 306, 311, 313, 316, 327, 331-335, 337. Coarse: Nos. 302, 304, 308-310, 312, $314,322,329$.

Quantity.-Head: Minus: Nos. 307, 317, 321. Average: Nos. 301, 319, 325. Plus: Nos. 318, 323, 326, 327. Double plus: Nos. 302-304, 306, 308, 309, 311, 313-316, 322, 329, 330, 331, 332, 337. Beard: Double minus: Nos. 308, 323. Minus: Nos. 309, 331. Plus: Nos. 302, 303, 307, 315, 322, 325, 329, 330, 332. Double plus: Nos. 301, 304, 306, 311, 313, 314, 317-319, 321, 326, 327, 337. Triple plus: No. 316. Body: Minus: Nos. 309, 323. Plus: Nos. 302, 307, 311 , 314, 315, 318, 319, 322, 325-327, 329, 330, 337. Double plus: Nos. 304, 306.

Hair samples from twenty-three individuals were obtained. It was impossible to obtain specimens from Nos. 301, 305, 307, 313, 317, 319-323, 325, 329, 336, and 337. 
EYE

CoLOR.-Dark brown: Nos. 300, 303-305, 308, 310-313. Blue brown: Nos. 315-322, 324-337. Green brown: Nos. 301, 306, 307, 309, 312-314.

SClerA.-Clear: Nos. 302-305, 308-313, 318, 319, 321-323, 326-332, 334. Bloodshot: Nos. 300, 301, 306, 307, 314, 316, 317, 320, 325, 333, 335-337. Speckled: No. 315.

IRIS.-Homogeneous: Nos. 302, 304, 305, 309, 310, 312, 315, 323. Zoned: Nos. 300, 301, 303, 306-308, 311, 313, 314, 316-322, 325-330, 332-337.

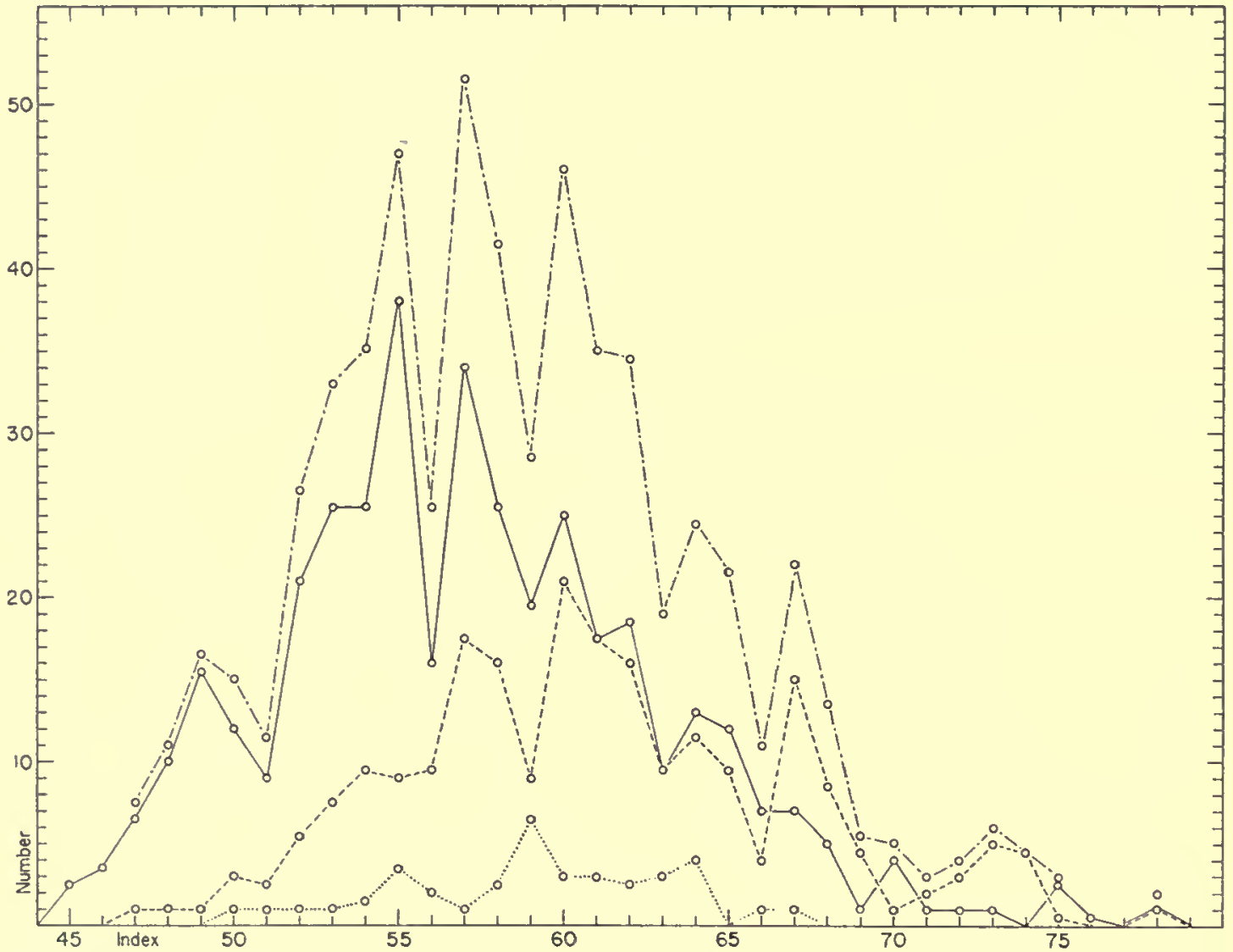

FIG. 46. Graph IX. Ear indices. Continuous curve, 390 Kish Arabs; dash curve, 226 Iraq soldiers; dotted curve, thirty-eight Ba'ij Beduins; dot-dash curve, composite of Kish Arabs and soldiers.

No. 315 was blind in the right eye and No. 317 in the left eye. No. 329 had a spot under the right eye.

\section{NOSE}

Profile.-Concave: Nos. 300, 305, 314, 319, 321, 323. Straight: Nos. 301-304, 306, 308, 311-313, 315-318, 320, 322, 325-337. Convex: No. 324. Concavo-convex: Nos. 307, 309, 310.

TiP.-Thickness: Minus: No. 310. Plus: No. 312. Double plus: Nos. 307, 317, 319, 323, 324, 328. Depression: Elevated: No. 305. Depressed: No. 306.

Wings.-Compressed: Nos. 305, 306, 313, 318, 320, 326, 331. Compressedmedium: No. 311. Medium: Nos. 302-304, 308, 312, 315-317, 321, 322, 327 , 
329, 330, 332-337. Medium-flaring: Nos. 314, 325. Flaring: Nos. 307, 310, $323,324,328$.

\section{TEE'TH}

ERUPTION.-Complete: Nos. 300-314, 316-322, 324-334, 336, 337.

Wear.-Average: No. 332. Plus: No. 301. Double plus: Nos. 305, 334.

CARIES.-Nos. 301, 320, 334.

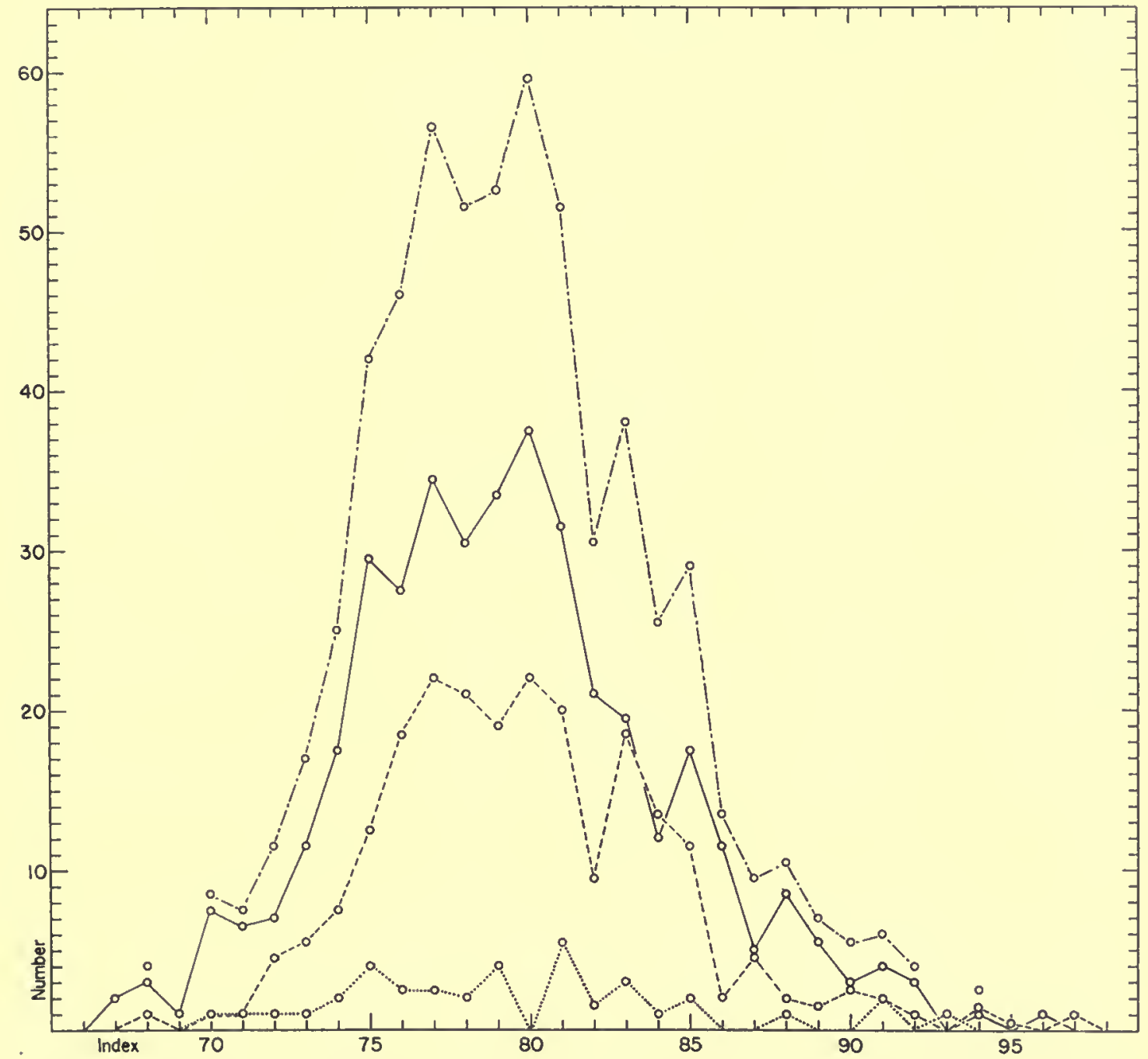

FIG. 47. Graph X. Jugomandibular indices. Continuous curve, 393 Kish Arabs; dash curve, 227 Iraq soldiers; dotted curve, thirty-eight Ba ${ }^{i j}$ Beduins; dot-dash curve, composite of Kish Ârabs and soldiers.

Lost.-None: Nos. 300, 302-305, 307-314, 320-322, 324, 328-330, 332, 333, 337. One: No. 306. Two: Nos. 301, 317, 326. Three: No. 319. Four: No. 334. Many: Nos. 325, 336.

BITE.-Slight over: Nos. 300, 301, 303, 305-307, 310, 312-314, 317, 320 , 321, 324, 326-332, 334, 337. Marked over: Nos. 308, 309, 311, 318, 333, 336.

\section{CHEST}

Development.-Average: Nos. 301, 307, 317, 336. Plus: Nos. 300, 303, 305, 306, 308-312, 314-316, 318-335, 337. Double plus: Nos. 302, 304, 313. 


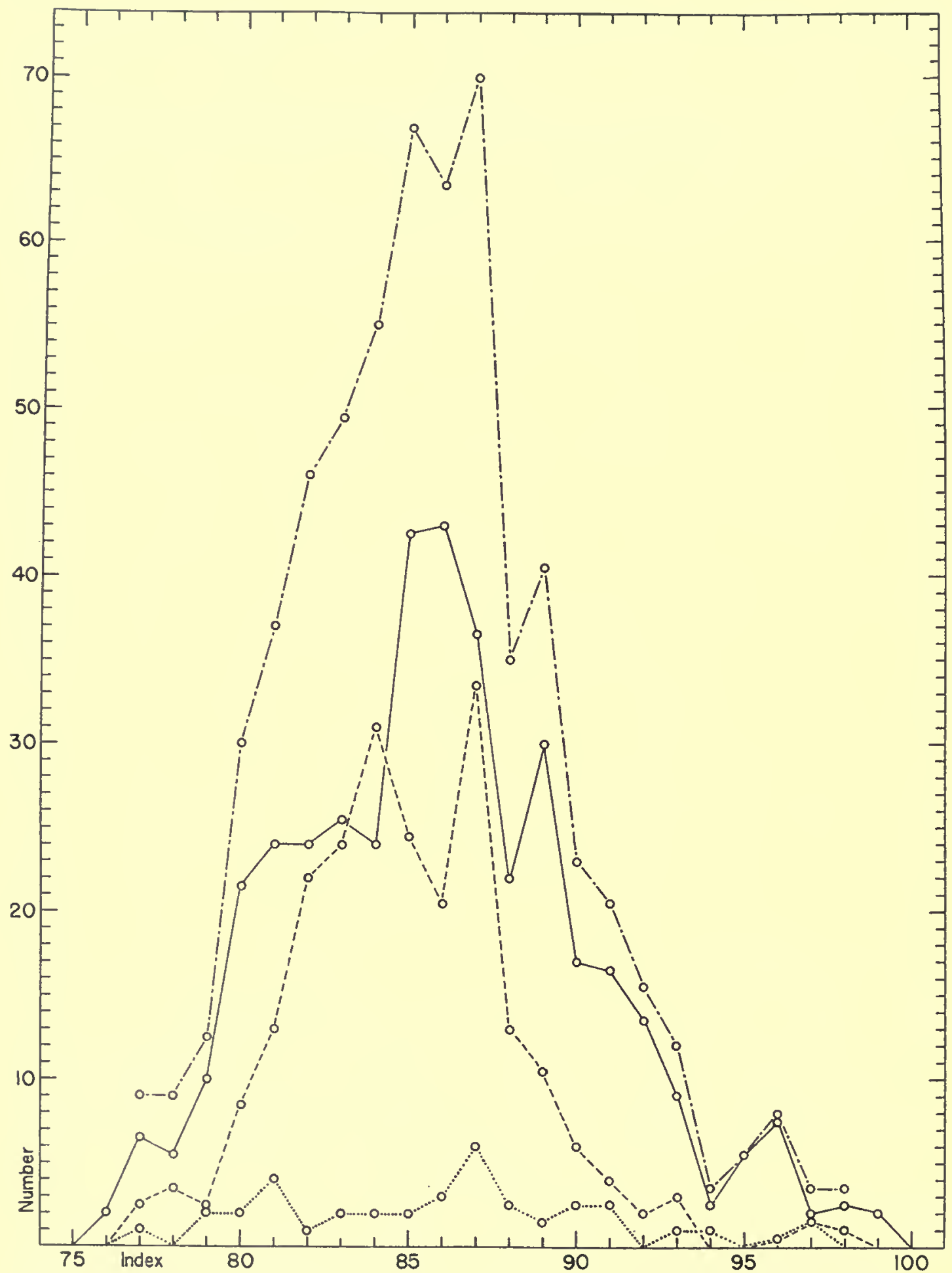

FIG. 48. Graph XI. Jugofrontal indices. Continuous curve, 395 Kish Arabs; dash curve, 227 Iraq soldiers; dotted curve, thirty-eight Ba'ij Beduins; dot-dash curve, composite of Kish Arabs and soldiers.

\section{MUSCULATURE}

Minus: Nos. 301, 317. Average: Nos. 307, 328, 336. Plus: Nos. 305, 306, 308-312, 314-316, 318-327, 329-335, 337. Double plus: Nos. 300, 302-304, 313. 
HEALTH

Poor: Nos. 301, 317. Fair: No. 336. Good: Nos. 305-316, 318-335, 337. Excellent: Nos. 300, 302-304.

No. 327.

\title{
SMALLPOX SCARS
}

No. 323.

\author{
NEGRO BLOOD
}

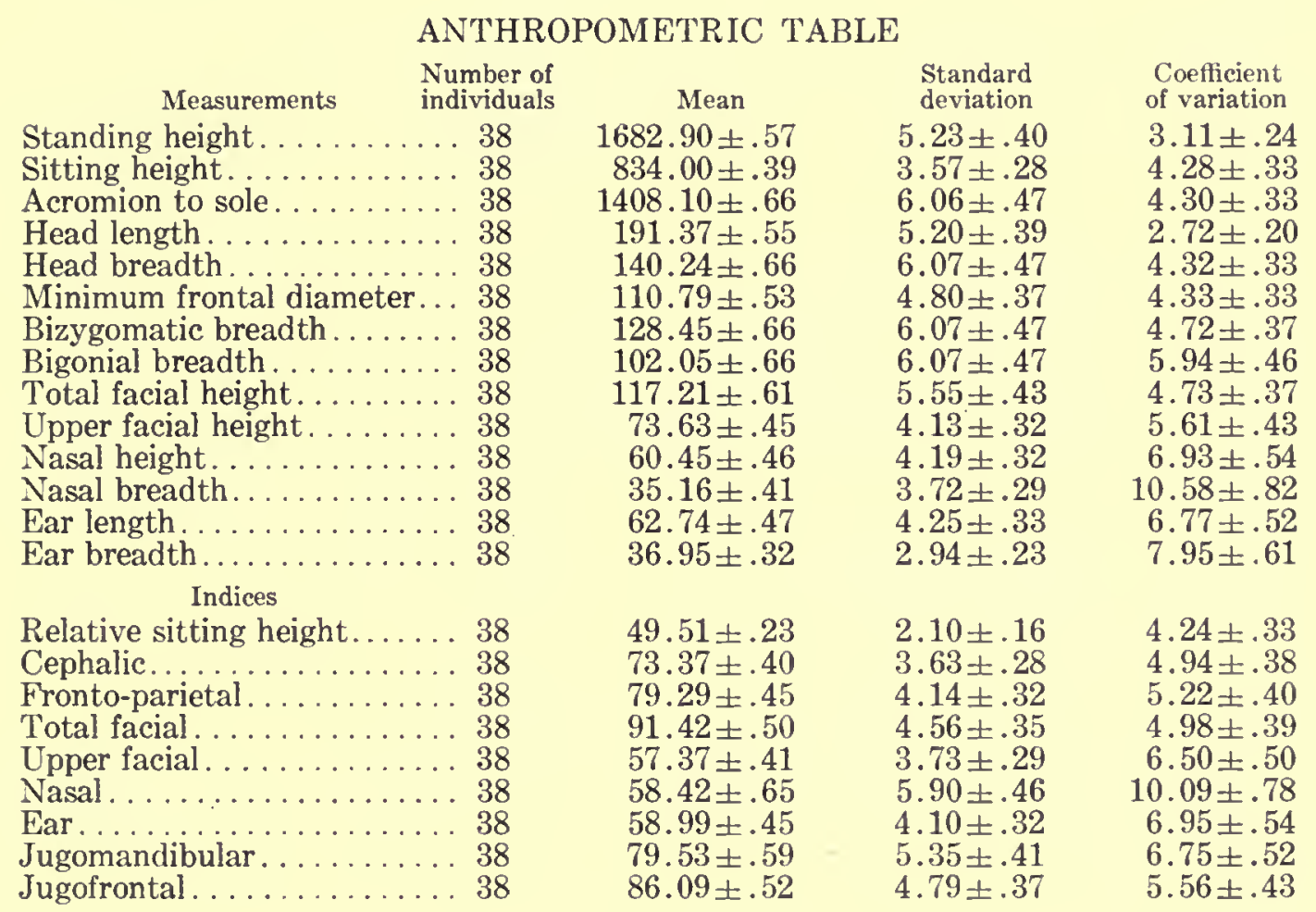

\section{TATTOOING}

A detailed report on the tattooed designs will be published later. The word "glove" refers to an elaborate design, consisting of a line across the wrist and a line of spots on the back of the hand leading to the base of the thumb and to each finger, forming a kind of mitten, a pattern which the Arabs call a glove (chaff).

\section{TABULAR SUMMARY OF TATTOOING}

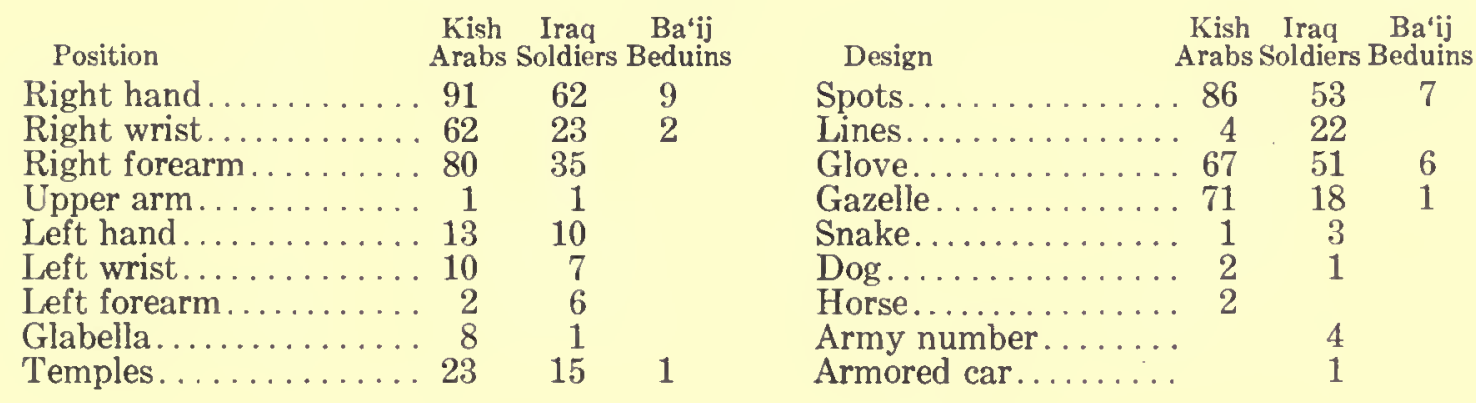




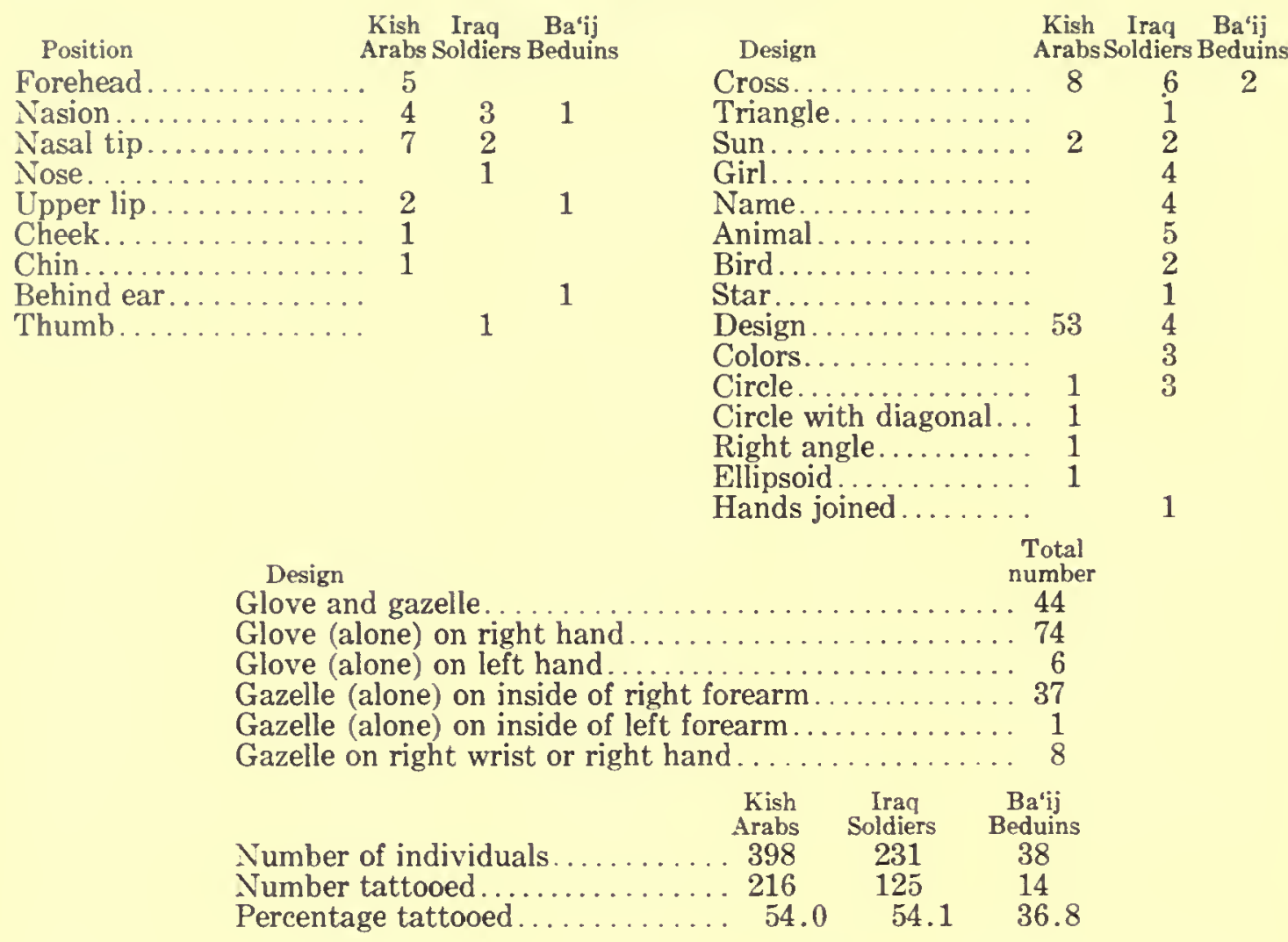

\section{SUMMARY OF ANTHROPOMETRIC DATA FROM SOUTHWESTERN ASIA}

In surveying the anthropometric data from southwestern Asia we find that during the past decade a wealth of statistical data has been obtained. C. U. Ariens Kappers has published reports on the head length, head breadth, and cephalic indices of numerous groups, including Kurds, Circassians, Persians, Turks, Greeks, Assyrians, Armenians, etc. (see Bibliography). Harald and M. Krischner of the American University, Beirut, published reports on the physical anthropology of Iraq and Iran, including measurements of head length and breadth, and the cephalic indices of 363 Armenians, 197 Chaldeans, 83 Assyrians, 91 Yezidis, 60 Kurds, 52 Jews, 32 Beduins and 690 Arabs of Iraq, and 1,140 "Persians" and 343 Armenians of Iran. In Iraq studies have been made on 5,696 individuals, which form definite contributions to the racial problems of this region.

\begin{tabular}{|c|c|c|}
\hline & Years & $\begin{array}{l}\text { Number of } \\
\text { individuals } \\
\text { measured }\end{array}$ \\
\hline Buxton and Field. & 1926 & 165 \\
\hline Krischner........ & $1927-31$ & 1568 \\
\hline Field...... & 1928 & 667 \\
\hline Field. . & 1934 & 1817 \\
\hline Smeaton... & 1934 & 731 \\
\hline Rassam... & $1933-34$ & 500 \\
\hline Smeaton.. & 1935 & 248 \\
\hline Total. & & 5696 \\
\hline
\end{tabular}


Krischner studied a number of Persians (see Bibliography) and I recorded observations on 246 individuals in Iran and 53 Lurs from Pusht-i-Kuh, who were measured in Baghdad. From South Arabia come the small but important series measured by Captain Bertram Thomas (see Arabia Felix, 1932) and the unpublished measurements made by Dr. Carleton Coon of Harvard.

In Syria Dr. Nejla Izzeddin of the Oriental Institute of the University of Chicago measured about one thousand Druze men and women.

In Trans-Jordan Dr. William M. Shanklin of the American University in Beirut measured 791 tribesmen and villagers (see Bibliography). During the spring of 1935 he made blood group studies on more than four hundred members of the Lazadat and the Mowali tribes. He also took measurements on three hundred of these tribesmen.

In Turkey groups have been measured by Dr. Shevket Aziz of the University of Istanbul and by Professor Eugène Pittard of the University of Geneva.

The greater portion of this material still remains unpublished but that is a matter of time. Furthermore, additional anthropometric research is being conducted in southwestern Asia and, provided that the political situation remains unaltered and the good will of the local governments toward anthropometric work remains favorable, there is every reason to hope that the racial composition of the peoples of the Near East will be determined. Similarly, the physical relationship of the peoples of southwestern Asia to the inhabitants of Asia, Africa, and Europe will become known. In conclusion, when the measurements of ancient skeletal material from the eastern borders of Iran to the Mediterranean and from Anatolia to the Arabian Sea are compared with anthropometric data on the modern inhabitants of southwestern Asia light will be thrown on many historical problems. 


\section{APPENDIX: FIELD MUSEUM ANTHROPOLOGICAL EXPEDITION TO THE NEAR EAST, 1934}

This expedition, sponsored by Mr. Marshall Field, began work in Baghdad, Iraq, on April 2, 1934. I was accompanied by Mr. Richard A. Martin, of Chicago, who was in charge of photography and the collection of zoological specimens. The expedition continued the anthropometric studies of the peoples of the Near East which had been begun at Kish, in December, 1925, by Dr. L. H. Dudley Buxton, who measured 164 Arabs of the Kish area.

In 1928, I had measured 398 Arabs of the Kish area, 231 members of the Iraq Army stationed at Hilla, and thirty-eight Ba'ij Beduins near Jemdet Nasr. These data are included in this publication. During a period of ten months in 1934 we traveled approximately 17,000 miles and made anthropometric measurements and studies on 3,056 individuals in Syria, Iraq, Iran, and the Caucasus region of the Union of Soviet Socialist Republics. I submitted each subject to the following measurements and observations, following the technique of Rudolf Martin: standing height, sitting height, head length, head breadth, bizygomatic and bigonial breadths, total facial height, upper facial height, nasal height, nasal breadth, left ear length and breadth. From these data the following indices have been calculated: relative sitting height, cephalic, fronto-parietal, total facial, upper facial, nasal, ear, jugomandibular, and jugofrontal. Observations were made on the color, form, and texture of the hair; eye color, sclera, and iris; nasal profile, tip, and wings; prominence of malars; teeth eruption, wear, caries, number lost and type of bite; chest development; general musculature; state of health; anomalies. There are also pathological notes and special remarks. The sex, age, birthplace, marriage, number of children, brothers, and sisters, living and dead, tattooed marks, and use of henna were noted. Apart from these measurements and observations every individual was photographed front and profile, a hair sample taken, a blood sample wherever possible, teeth smears, pulse, weight, and temperature of certain groups. The sixteen groups studied in Iraq, with the number of individuals in each, are listed in the following table:

INDIVIDUALS STUDIED IN IRAQ DURING 1934

\begin{tabular}{|c|c|c|}
\hline Marsh Arabs & Males & Females \\
\hline $\begin{array}{l}\text { Albu Muhammed. } \\
\text { Al Suwa'id....... }\end{array}$ & $\begin{array}{r}221 \\
50\end{array}$ & $\begin{array}{l}3 \\
0\end{array}$ \\
\hline \multicolumn{3}{|l|}{ Beduins } \\
\hline Shammar.. & 298 & 129 \\
\hline Anaiza..... & 23 & 0 \\
\hline Dulaim.. & 137 & 0 \\
\hline \multicolumn{3}{|l|}{ Non-Beduin nomads } \\
\hline Sulubba (Sleyb). & 39 & 10 \\
\hline Gypsies (Nawar). & 6 & 4 \\
\hline Groups near Beiru & 37 & 0 \\
\hline
\end{tabular}




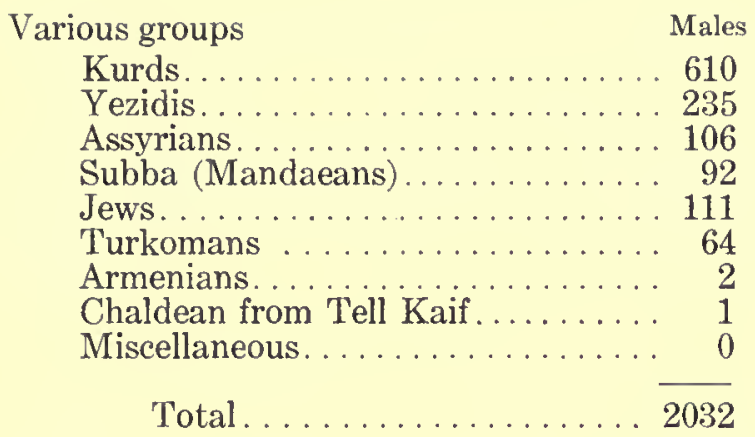

$$
\begin{gathered}
\text { Females } \\
32 \\
77 \\
137 \\
33 \\
52 \\
31 \\
4 \\
0 \\
3 \\
\hline 515
\end{gathered}
$$

The males were measured by the writer, the females and some of the males by Miss Winifred Smeaton, of Ann Arbor, Michigan.

It will be interesting to compare the various elements of the modern Iraqi population and particularly to see if the Kurds from each of the mountain valleys of Zakho, Aqra, Rowandiz, and Sulaimaniya show variations or whether they appear to be a homogeneous people. There is a marked difference in the average cephalic index of the Yezidis of Balad Sinjar and that of the Sheikh 'Adi district. The measurements on the group of 427 Shammar Beduins under Sheikh 'Ajil are valuable since they are the largest racial group in the northwestern part of Iraq.

Dr. Carl Rassam of the Royal College of Medicine in Baghdad gave me his measurements (head length and head breadth) on 500 males, females, and children, the majority of which were obtained in the Royal Hospital.

The results of this and former anthropometric studies in Iraq should enable the anthropologist to study the relationship of the Arab to the peoples of Africa, Europe, and Asia. A comparison between the measurements of ancient crania and those of the modern inhabitants of Iraq reveals the fact that there has been little change in the physical characteristics of these people during the past five thousand years. There is, however, still further work to be done on many other groups in this region.

In Iraq several specialists collected data for the expedition. Miss Smeaton accompanied the expedition for three months in order to make anthropometric measurements and studies on 515 women in various parts of Iraq. She is also preparing a detailed report of tattooing in Iraq. Mrs. E. S. Drower, of Baghdad, has completed an ethnological and linguistic report on the Marsh Arabs (Albu Muhammed) of the Chahala district, east of Amara. Mrs. M. Rue Clawson, of Beirut, Syria, made a special study of the teeth of 300 Kurds and Shammar Beduins. The report on these teeth smears has been prepared for publication by Dr. M. D. Clawson of the Iraq Petroleum Company. At the suggestion of Sir Arthur Keith, blood samples were obtained wherever possible. About 500 samples were taken from Kurds, Shammar, Anaiza, Dulaim, Yezidis, and Jews in Iraq and Iran. These were sent to Dr. Walter P. Kennedy, of the Royal College of Medicine in Baghdad, where they are being studied. Publication of the polynuclear count in 121 individuals (Kurds, Dulaim, and 
Jews) has been made in the Journal of the Royal Society of Tropical Medicine and Hygiene (XXVIII, No. 5, pp. 475-480, London, March, 1935).

According to Dr. Kennedy "the results differed considerably from the two sets of normal counts made in Britain. The average weighted mean was $1.980 \pm$ 0.171 and the standard deviation was 0.279 . This corresponds to counts which actually occurred:

$\begin{array}{ccccc}\text { I } & \text { II } & \text { III } & \text { IV } & \text { V } \\ 31 & 46 & 19 & 2 & 2 \\ 31 & 42 & 25 & 2 & 0\end{array}$

"The results gave an average mean of $2.628 \pm 0.135$, with a standard deviation of 0.19 . The difference between the means of Iraq is thus 0.648 . This is more than three times the probable errors of either, and therefore the difference is statistically significant."

Dr. Kennedy summarizes his results, based on a simple modification of Arneth's count and its application to the study of 121 male Iraqis, in the following words: "The average of the counts shows a marked shift to the left when compared with the health standard of the polynuclear count as laid down in Britain." Dr. H. F. Brewer, of St. Bartholomew's Hospital in London, very kindly arranged for the purchase of the haemagglutinating sera sent to Dr. Kennedy.

Dr. James Macfarlane, Senior Medical Officer of the Iraq Petroleum Company at Kirkuk, and Dr. Kennedy have recently concluded a joint paper entitled, "Investigations into the Human Blood Groups in Iraq," based on blood samples collected by the Field Museum Anthropological Expedition to the Near East, 1934, and on other samples obtained in Baghdad and in the Anthropological Research Laboratory in Kirkuk. The following information and tables are quoted from this forthcoming paper.

"The bloods were typed by the three spot method on slides, and all agglutinations were checked by microscopic observation.

"Table I gives the percentage of each type in the five racial groups we have tested, and also the frequencies of the three genetic factors, $\mathrm{O}, \mathrm{A}, \mathrm{B}$, using the equations: $r=0: p=0-A-0$ : and $q=0-B-0$. As a check $p-q-r=10$, and the deviation of the sum of the frequencies from this figure gives a measure of the equilibrium of the population, assuming that the agglutinations have been performed without error.

\section{TABLE I}

\begin{tabular}{|c|c|c|c|c|c|c|c|c|c|}
\hline \multirow[b]{2}{*}{ Group } & \multirow{2}{*}{$\begin{array}{c}\text { Number of } \\
\text { persons } \\
\text { typed }\end{array}$} & \multicolumn{4}{|c|}{ Percentage in each group } & \multicolumn{4}{|c|}{ Frequencies } \\
\hline & & 0 & A & $\mathrm{B}$ & $A B$ & $\mathrm{P}$ & $\mathbf{Q}$ & $r$ & $p-q-r$ \\
\hline Kurds. & . 737 & 42.3 & 30.9 & 16.7 & 10.1 & 2.04 & 1.18 & 6.51 & 9.73 \\
\hline Arabs & 367 & 33.8 & 35.7 & 24.3 & 6.2 & 2.53 & 1.81 & 5.81 & 10.15 \\
\hline Jew & 97 & 41.2 & 25.8 & 26.8 & 66.2 & 1.77 & 1.83 & 6.42 & 10.02 \\
\hline Turkomans & 67 & 41.8 & 41.8 & 14.9 & 1.5 & 3.21 & 1.06 & 6.47 & 10.74 \\
\hline Christians. & 150 & 33.3 & 39.3 & 17.4 & 11.0 & 2.88 & 1.47 & 5.78 & 10.13 \\
\hline
\end{tabular}




\section{SUMMARY OF RESULTS}

\begin{tabular}{|c|c|c|c|c|c|}
\hline Group & A & B & $\mathrm{AB}$ & o & Total \\
\hline Arabs. & 44 & 29 & 8 & 40 & 121 \\
\hline Kurds. & 125 & 66 & 41 & 164 & 396 \\
\hline Turkomans & 25 & 9 & 1 & 26 & 61 \\
\hline Christians. & 6 & & & 4 & 10 \\
\hline Assyrians. & 13 & 1 & & 10 & 24 \\
\hline Armenians. & 4 & 1 & 2 & 3 & 10 \\
\hline Jews.. & 2 & & & 1 & 3 \\
\hline & 219 & 106 & 52 & 248 & 25 \\
\hline
\end{tabular}

"In Table II the actual numbers and constitution of the first three groups are given, but as some of the sub-groups are rather small the frequencies are not calculated as their significance would be doubtful.

TABLE II

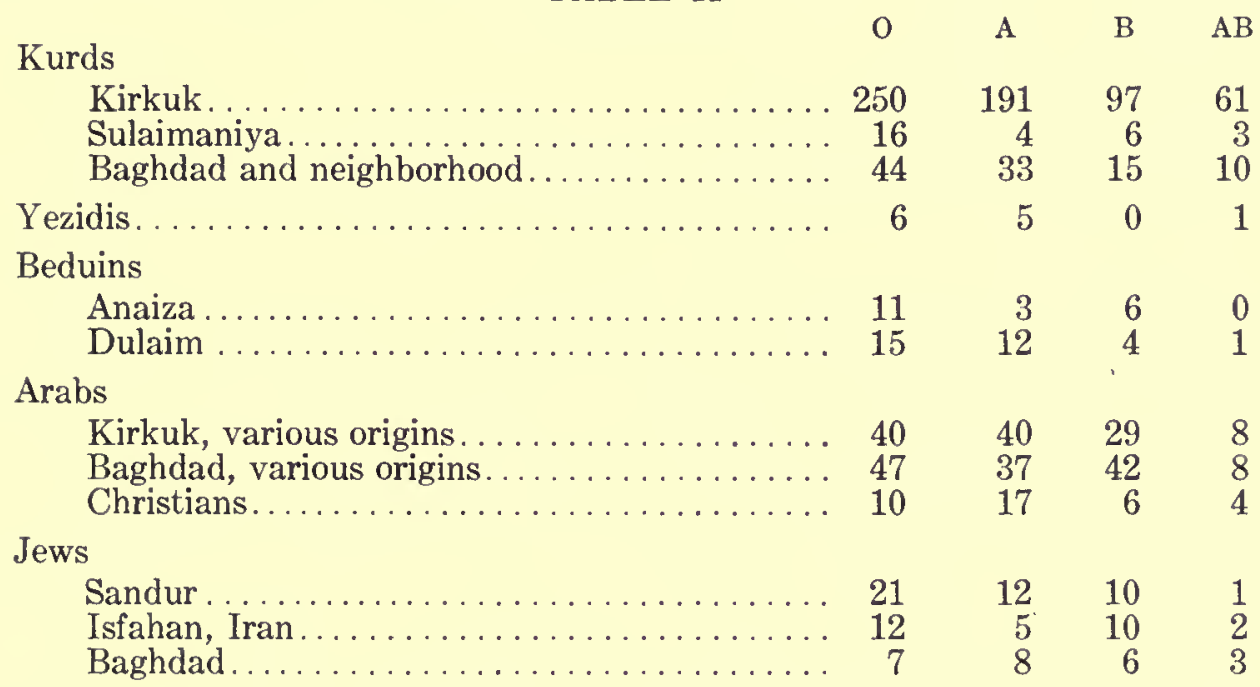

"The significant differences between the various groups in the above data indicate that fruitful results may be expected from an extension of blood typing investigations in Iraq."

Mr. Khedoory Muallim, whose services were lent to the expedition by Dr. Sinderson of the Royal College of Medicine in Baghdad, collected birds in the Amara marshes; Mr. Albert Meymourian, entomologist of the Rustam Agricultural Experimental Farm, collected insects in the Amara marshes; Mr. S. Y. Showket, of Basra, acted as interpreter and general assistant from April until the end of July; Mr. Yusuf Lazar made a valuable collection of plants and insects in Iraq, Trans-Jordan, Syria, and Iran.

In addition, collections of zoological and geological specimens were made in every district visited.

The expedition received unusual cooperation from Iraq officials, as well as from many private individuals. Outstanding among the many persons who rendered valuable assistance are the Prime Minister; Ali Jaudat Beg; the Minister of the Interior; Sir Kinahan Cornwallis; Mr. C. Grice, of the Ministry of the 
Interior; Major W. C. F. Wilson, adviser to the Iraq Government at Mosul; Sir John Burnett, the Air Vice-Marshal; the American Minister, Mr. Paul Knabenshue; Squadron Leader A. R. M. Rickards, of the Royal Air Force; Dr. Walter P. Kennedy, of the Royal College of Medicine, Baghdad; Dr. T. H. McLeod, of the Royal Hospital, Mosul; and the Mutasarrifs of the Mosul, Kirkuk, Erbil, and Amara Liwas.

Through the courtesy of Professor James H. Breasted, Dr. H. Frankfort, director in Iraq of the Oriental Institute Expeditions of the University of Chicago, very kindly lent the expedition a station-wagon for general use in Iraq. Mr. Gabriel Malak also gave generous assistance.

A search was made for archaeological sites in the North Arabian desert, lying in Iraq, Trans-Jordan, and Syria. Flint implements collected on the surface prove the former existence of paleolithic and neolithic man in that region. The Iraq Petroleum Company invited the members of the expedition to use their pipe-line stations and cooperated in every possible way.

In Kurdistan flint implements of upper paleolithic types were found in the gorges of Zakho, Aqra, Rowandiz, and Sulaimaniya, proving that ancient man once roamed the territory between Kurdistan and the Mediterranean.

At the end of July, we proceeded to Iran, accompanied by Dr. Walter P. Kennedy and Mr. Yusuf Lazar. The members of the expedition were guests of Dr. and Mrs. Erich Schmidt for four weeks at Rayy, near Tehran, during work in that vicinity. At Isfahan we visited Mr. and Mrs. Myron B. Smith and accompanied them to Persepolis, where Professor Ernst Herzfeld, field director of the Oriental Institute Expedition to Iran, cordially received the party. Anthropometric data were obtained on 74 Persians in the village of Kinnareh, near Persepolis; 99 Jews in Isfahan; 48 Persians in Yezd-i-Khast; 18 workmen at the Rayy excavations; and 3 Assyrians, 3 Kurds and 1 Moslem. In Baghdad 53 Lurs from Pusht-i-Kuh were measured. Zoological, botanical, and geological specimens were also collected. Cordial cooperation was received from the American Minister, Mr. William H. Hornibrook; the Prime Minister; the Minister of the Interior; the Minister of Education; the Chief of Police; and the Governor of Isfahan.

On September 14, we entered the Union of Soviet Socialist Republics at Baku on the Caspian Sea. At the request of the United States Department of State and Ambassador William C. Bullitt, the Soviet officials allowed free entry into the Soviet Union for all the expedition equipment. Traveling was greatly facilitated by VOKS (the Society for Cultural Relations with Foreign Countries) and Intourist (the Soviet travel organization), whose representatives cooperated in every possible way. With the assistance of VOKS fifty-one male Yezidis were measured in the Kurd Club, Tiflis. These observations will form valuable material for comparison with the data obtained on the two groups of Iraqi Yezidis.

Ordzhonikidze was reached by automobile over the Georgian Military Highway. The peoples of Northern Ossetia in the Caucasus have been little studied from the standpoint of physical anthropology. Through the assistance 
of T. Demurov, local chairman of Northern Ossetian Education, anthropometric observations, measurements, and photographs of 107 men and fifty women were made. In addition, a staff of medical assistants was provided by the Soviet government to obtain specimens of blood, hair samples, weight, pulse, temperature, and hand pressure of these individuals. The 156 blood samples were sent to Dr. Walter P. Kennedy, Royal Hospital, Baghdad, for study. At Ordzhonikidze nineteen deformed skulls from Nal'chik were measured and photographed in the Ossetian Museum. In the Dargavskaia Valley near Koban twenty skulls were measured in the tombs of the "Valley of the Dead."

Classifying the data and photographs obtained by the expedition is now in progress and the zoological, botanical, and geological specimens have been distributed to the various departments of the Museum. The Reports are now being prepared for publication. 


\section{BIBLIOGRAPHY}

Altounyan, E. H. R.

1927. Blood Transfusion in Syria. The Lancet, II, 1342.

Buxton, H. L. Dudley, and Rice, David Talbot

1931. Report on the Human Remains Found at Kish. Jour. Roy. Anthr. Inst., LXI, pp. 57-119.

Chantre, E.

1895. Recherches anthropologiques dans l'Asie occidentale. Arch. du Mus. d'Hist. Nat., VI, Lyons.

FIELD, HENRY

1926. New Discoveries at Kish; a Great Temple, 5,000 Year-Old Pottery. Illus. London News, Sept. 4, p. 395.

1929a. The Field Museum-Oxford University Joint Expedition to Kish, Mesopotamia, 1923-29. Field Mus. Nat. Hist., Anthr. Leaflet No. 28.

1929b. Early Man in North Arabia. Amer. Mus. Nat. Hist., Natural History, XXIX, pp. 33-44.

1931a. The Field Museum-Oxford University Joint Expedition at Kish. Art and Arch., XXXI, Nos. 5 and 6, pp. 243-252 and pp. 323-324, Washington.

1931b. Among the Beduins of North Arabia. Open Court, XLV, pp. 577-595.

1932a. Human Remains from Jemdet Nasr, Mesopotamia. Jour. Roy. Asiat. Soc., pp. 967-970.

1932b. The Cradle of Homo Sapiens. Amer. Jour. Arch., XXXVI, pp. 426-430.

1932c. Ancient Wheat and Barley from Kish. Amer. Anthr., new ser., XXXIV, pp. 303-309.

1932d. The Ancient and Modern Inhabitants of Arabia. Open Court, XLVI, pp. $847-871$.

1932e. Threshing Sledges in Kurdistan. Man, XXXII, No. 75.

1933. The Antiquity of Man in Southwestern Asia. Amer. Anthr., new ser., XXXV, pp. 51-62.

1934. Sulle caratteristiche geografiche dell' Arabia settentrionale. Boll. della Reale Soc. Geogr. Ital., ser. VI, XII, Jan., pp. 3-13.

1935a. The Field Museum Anthropological Expedition to the Near East, 1934. Science, Feb. 8, vol. 81, No. 2093, p. 146.

1935b. Ibid. The Oriental Institute Archaeological Report on the Near East, Fourth Quarter, Amer. Jour. Semitic Languages, LI, No. 3, April, pp. 207-209.

Field, Henry, and Martin, Richard A.

1935. Painted Pottery from Jemdet Nasr, Iraq. Amer. Jour. Arch., XXXIX, pp. $312-320$.

Fleeure, H. F.

1932. An Early Chapter of the Story of Homo Sapiens. Bull. John Ryland's Library, XVI, No. 2, July, Manchester, England.

FRANKFORT, H.

1932. Archaeology and the Sumerian Problem. Oriental Inst. Publ., IV, Chicago.

Genouillad, Henri DE

1924-25. Premières recherches archéologiques à Kich. 2 vols. Paris.

Jensen, Peter

1900. Kis. Zeitschrift für Assyriologie, XV, pp. 210-256.

KAPPERS, C. U. ARIENS

1930. Contributions to the Anthropology of the Near East. Kon. Akad. van Weten., I, II, vol. XXXIII, Amsterdam.

1931. Ibid. III, IV, V, VI, vol. XXXIV. 
Kappers, C. U. Ariens, and Parr, Leland W.

1934. An Introduction to the Anthropology of the Near East in Ancient and Recent Times. Amsterdam.

KEITH, Sir ARThUR

1926. Report on the Human Remains, Ur Excavations. I, Excavations at Al 'Ubaid, by Hall and Woolley, London.

1932. See Thomas, Bertram (1932b).

Kennedy, Walter P.

1935. The Polynuclear Count in an Iraq Population. Roy. Soc. Trop. Med. and Hygiene, XXVIII, No. 5, pp. 475-480.

KRISCHNER, H. and M.

1932. The Anthropology of Mesopotamia and Persia. Kon. Akad. van Weten., XXXV, Nos. 2 and 3, Amsterdam.

KrogMan, W. M.

1932. See Thomas, Bertram (1932b).

1933. See Schmidt, Erich F.

LANGDON, STEPHEN

1924. Excavations at Kish. I, Paris.

1928a. Pictographic Inscriptions from Jemdet Nasr, Excavated by the Oxford and Field Museum Expedition. Cuneiform Texts, VII, Oxford.

1928b. Ausgrabungen in Kisch und Charsagkalama. Der Alte Orient, XXVI, pp. $55-75$.

1930. Excavations at Kish. III, Paris.

1934. Ibid., IV, Paris.

LUSCHAN, F. VON

1911. The Early Inhabitants of Western Asia. Jour. Roy. Anthr. Inst., XLI, pp. 221-244.

MACKAY, DOROTHY

1927. Painted Potsherds. The English Review, July, pp. 81-89.

MACKAY, ERNEST

1925. Report on the Excavation of the "A" Cemetery at Kish, Mesopotamia. 'Field Mus. Nat. Hist., Anthr. Mem., I, No. 1.

1929. A Sumerian Palace and the "A" Cemetery at Kish, Mesopotamia. Field Mus. Nat. Hist., Anthr. Mem., I, No. 2.

1931. Report on Excavations at Jemdet Nasr, Iraq. Field Mus. Nat. Hist., Anthr. Mem., I, No. 3.

MARTIN, RichaRd A.

1935. See Field, Henry, and Martin, Richard A.

Mocchi, A.

1907. Sulla antropologia degli Arabi. Archiv. per l'Antropo. e l'Etnolog., XXXVII.

Olmstead, A. T.

1923. History of Assyria. New York.

1931. History of Palestine and Syria. New York.

PARR, LELAND W.

1929. Studies in Isohaemagglutination. Jour. of Immunology, XVI.

1930. Die Blutgruppenverteilung in der Bevölkerung des nähen ostens und Nordafrikas. Ukrainisches Zentralblatt für Blutgruppenforschung, IV, part 2.

1931. Blood Studies on Peoples of Western Asia and North Africa. Amer. Jour. of Phys. Anthr., XVI, p. 15.

1934. See Kappers, C. U. Ariens, and Parr, Leland W. 
Penniman, T. K.

1934. Excavations at Kish. IV, pp. 64-72, Paris.

Prentice, William K.

1931. A Greek Inscription from Kasr Burka. Amer. Jour. Arch., XXXV, pp. 48-50.

SCHMIDT, ERICH F.

1933. Alishar Hüyük, Season of 1928-1929. Part II, Oriental Inst. Publ., XX, Chicago. See pp. 122-137, by W. M. Krogman.

Seligmann, C. G.

1917. The Physical Characters of the Arabs. Jour. Roy. Anthr. Inst., XLVII, pp. 214-237.

SHANKLin, William M.

1934. The Anthropology of Transjordan Arabs. Psych. en Neurol. Bladen, Nos. 3-4, Amsterdam.

1935. Blood Grouping of the Rwala Arabs. Proc. Soc. Exper. Biol. and Med., 32, pp. $754-755$.

SPEISER, E.

1930. Mesopotamian Origins. Philadelphia.

THOMAS, BERTRAM

1929. Among Some Unknown Tribes of South Arabia. Jour. Roy. Anthr. Inst., LIX, pp. 97-111.

1930. Alarms and Excursions in Arabia. London.

1932a. Anthropological Observations in South Arabia. Jour. Roy. Anthr. Inst., LXII, pp. 83-104.

1932b. Arabia Felix. New York. See Appendix by Sir Arthur Keith and W. M. Krogman.

UNGNAD, A.

1933. Die ältesten Völkerwanderungen Vorderasiens. Breslau.

WATELin, L. C.

1929a. Rapport sur les fouilles de Kich. Jour. Asiat., CCXV, pp. 103-116, Paris.

1929b. Notes sur l'industrie lithique de Kich (Iraq). L'Anth., XXXIX, pp. 65-76.

1930. Excavations at Kish. III, Paris.

1931. Essai de coordination des periodes archaiques de la Mesopotamie et de l'Elam. L'Anthr., XLI, pp. 265-273.

1934. Excavations at Kish. IV, Paris. 


\section{GLOSSARY}

The colloquial words as used by the Arabs of the Kish area have been listed with the classical forms in parentheses, followed by page references in text. In some cases alternative spellings have been added. As in other parts of Iraq, the letter $k$ is usually pronounced $c h$ and the letter $q$ as $g$. In the glossary the diacritical marks have been added by Dr. A. Frayha of the Oriental Institute of the University of Chicago. All words and phrases are Arabic unless designated as follows: $\mathrm{B}=$ Babylonian; $\mathrm{G}=$ Greek; $\mathrm{H}=$ Hindustani; $\mathrm{K}=\mathrm{Kurdish}$; L=Latin; $\mathrm{S}=$ Spanish.

'Abā, 87, 95. Iong robe which forms outer garment.

'Agāl (' $I q \bar{a} l), 87,432$. Head-dress of camel's hair, sometimes of gold or silver thread, used to keep kaffiyah in place.

Aghabanu (K), 432. Head-dress (see 'Agāl). Al-Forja (S), 87. Saddlebag.

'A raq, 89. Alcoholic beverage distilled from dates or rice.

Arba-ilu (B), 432. Town of Erbil (G, Arbela), $75 \mathrm{~km}$ east of Mosul.

Arnabah, 433. Tip of nose through which nose-ring is inserted.

Bakara, 433. Pulley wheel used for drawing water.

Bugwa, 91. Brass water jar with handle. Water for coffee-making is kept in this container, which stands beside hearth.

Chaff (Kaff), 455. Glove or mitten. This refers to an elaborate design in tattooing, consisting of line across wrist and lines of spots on back of hand leading to base of thumb and each finger, forming a kind of mitten, a pattern which the Kish Arabs call a chaff (for kaff).

Chāi (Shāi), 92. Tea.

Chakow (K). Steel blades of threshing sledge (kotan).

Cheneghé (K), 433. Chin.

Chināgh, 93. Metal finger guard used to protect finger and back of hand from sickle blade during reaping.

Chiss (Kìs), 93, 433. Small leather bag used for mirror or money. Worn over right shoulder and hangs by left side. This is also the general word for any kind of bag.

Chmāgh, 93. Club with wooden head.

Chupatties (H), 89. Unleavened cakes.

Dallah (pl. Dilāl), 91. Spouted vessel used for making coffee.

Dalu, 433. Leather bucket.
Dishdāshi, 87, 88, 92. Long cotton garment generally white in color (same as thob).

Duzahle (K), 90. End-flute (see Mutlug).

Fallāhīn, 448. Peasants.

Fass, 123. Pickman employed at excavations.

$F_{\bar{\imath}}$ al-'ajalah al-nadāmah, 92. Means literally "in haste there is repentance." which is our proverb, "haste makes waste."

Finjān, 91, 92. China coffee cup or decorated metal holder for cup.

Furn, 89. Oven.

Gahwah (Qahwah), 89, 92. Coffee.

Gamīs (Qamiss), 87. Saddlecloth.

Gubbah (Qubbah), 95. Sacred building containing bones of holy man.

Gumgum (Qumqum), 91. Water for coffee is kept in this large metal vessel with curved spout, which stands beside hearth.

Guwarhe (K) (Idhin), 433. Ear.

Habil, 433. Rope.

Hadji (Hajji). A man who has made the pilgrimage to Mecca.

Hangustir (K) (Mahbas), 433. Finger rings.

I $a t a b, 85$. General word for firewood. Kish Arabs use this term for camel's thorn.

Hāwan, 91. Brass mortar in which coffee beans are ground with rhythmic beats of pestle.

Hijl (pl. Hijūl) (K, Pa-bend), 433. Anklet. Hunțah (IIințah), 89. Wheat.

$\bar{I} d$ al-hāwan, 91. Iron pestle used for pounding coffee beans (see Häwan).

Idhin (K, Guwarhe), 433. Ear.

Imām, 95. A holy man.

Imām $S a^{4} \bar{\imath} d, 95$. Tomb of holy man called $\mathrm{Sa}^{6} \mathrm{i} d$ near Kish.

Istikān, 91, 92. Thick tea glass. 
Izār, 433. Garment worn by women.

Jazīrah, 82, 89, 449. Desert. In Kish area this refers to wilderness beyond irrigation canals.

Jisr, 86. Bridge.

Jogah (pl. Jogat) (Jūqah or Jawqah), 97. Gang of workmen employed at excavations (for description see Field Mus. Nat. Hist., Anthro. Leaflet 28).

Kaffiyah (K, Aghabanu), 87, 88, 432, 433. Cloth worn over head.

Kahwas (K, Kol-bend). Bracelets.

Kalhe (K), 87. Leather-soled shoes with string uppers worn by Kurdish shepherds and Beduins.

Karmak (K), 433. Circlet or diadem of golden coins.

Khanjar (pl. Khanājir), 90, 92, 93. Dagger (see p. 93).

Khazzāmah, 433. Nose ring.

Khubz, 89. Bread.

Khurj, 87. Saddlebags.

Kol-bend (K) (Kahwas), 433. Bracelets.

Kotan (K). Threshing sledge with steel blades (chakow).

$K u w \bar{a} z a(K \bar{u} z), 433$. Pottery jar for water.

Laban, 89. Sour milk.

Liwa, 462. Administrative district.

Maghzal, 93. Wooden spindle ornamented with bands of red and yellow.

Magwār, 92, 93. General name for club; only correctly employed when head is made of bitumen (see Mazrak, K).

Mahbas ( $K$, Hangustir), 433. Finger rings.

Mahmüs, 91. Long-handled flat metal dish used for roasting coffee.

Makātīb (sing. Māktūb), 433. Books.

Mamzah (K), 90. Reed in end-flute (see Mutlug).

Mazrak (K), 92. Striking club (see Magwār).

Menū hädha, 90. "Who goes there?" Regular challenge of sentry or watchman.

Minjal, 93. Sickle with wooden handle. Shape resembles pottery sickles to which saw-toothed flints were attached by bitumen to form blade. Many pottery sickle handles and thousands of flint blades were excavated at Kish and Jemdet Nasr. The example in Figure 37 was purchased near Erbil.

Mishā, 443. Long-handled wooden shovel used at excavations and for digging irrigation ditches.
Mĩzān, 97. Ursa Major, the constellation of the Great Bear.

Musīgā (Musīqā), 90. Music.

Mutaşarrif, 462. Governor of a district, liwa. Mutlug, 90. End-flute.

$N \bar{a} r, 90$. Fire.

Nașrānī, 432. Christian.

Pa-bend (K) (Hijl), 433. Anklet.

Pecten (L), 88. Small shells used for kohl. Found in Sumerian graves.

$R a b \bar{a} b a h, 90 . \quad$ Single-stringed instrument played with horsehair bow. Obtained from Beduins.

Rais (Rayyis or Ra'̄'s), 97, 443. Foreman at excavations.

Rashmah, 93. Donkey halter usually decorated with cowrie shells and with small blue beads sewn on leather for protection against evil eye.

Sabīl, 433. Pipe.

Sage (K), 89. Long-handled iron dish for roasting coffee (see Mahmüş).

Şakhariya, 93. Club with stone head. Ancient maceheads are sometimes used. They are attached to handle by means of large nail.

Sayyid. Descendant of Prophet. A Sayyid wears a green band around his head or around his waist, as a mark of distinction.

Selügi (Salūqū), 384. Sometimes written slughi. Hound used by Beduins and Arabs for hunting gazelle.

Sha'ì $r, 89$. Barley.

Shenhé (K). Wooden fork used in harvest. Sibha, 433. Rosary, usually of amber beads. $S \bar{u} k(S \bar{u} q), 89,433$. Native market.

Tamr, 89. Dates.

Telgama (pl. Telgamat), 91. Coffee pots.

Thob (Thawb), 87. Long cotton garment (same as dishdäshi).

Timmin, 89. Rice.

Titin, 433. Tobacco.

$W \bar{a} d \bar{\imath}, 78$. Dry river or stream.

Yamani, 433. Leather shoes worn by men.

Zibūn, 87. Tight-fitting colored garment worn over dishdāshi and under ' $a b \bar{a}$. 


\section{INDEX}

Abu Sudaira (Kish), 83

Agade, 80

Albu Muhammed (Marsh Arabs), 458, 459

Alexander the Great, 433

Al Suwa'id (Marsh Arabs), 458

Amara, see Kut al Imara

American Indian traits among Kish Arabs, 15

American University, Beirut, 457

Anaiza Beduins, 449, 458; blood samples of, 459 ; blood typing of, 461

Anthropological Research Laboratory, Kirkuk, 460

Anthropometric method, 11, 12, 98-101; graphic analysis in, $20,21,37,38,54$, 67 ; photographic, $12,13,16,17$; technique of Rudolph Martin, 458

Anthropometric tables, see Ba'ij Beduins, Iraq soldiers, Kish Arabs

Aqra, Kurds from, 459; paleolithic site near, 462

Arabia, home of Semites, 19; invasion of by brachycephals, 38; Negroid population in, 17; original inhabitants of, 75,76 ; Pleistocene period in, 75,76

Arabs, age changes among, 17; ancestry of, 76; Armenoid traits among, 15, 16; Aryo-Dravidian traits among, 29, 43, 51 ; beard of, 16; blood typing of, 460 , 461 ; brachycephaly among, $27,38,74$; Caucasian traits among, 14, 16, 18, 19, 52,75 ; Dravidian traits among, 14,17 , $18,27,51,75,76$; eccentric forms among, 27 ; European traits among, 17, 25, 75; facial characteristics of, $16,60,61,75$, "ram-faced" type, 52, 53, 60; Hamitic traits among, 17, 75; head form and size of, 25, 27, 74, compared to Pathans, 29, 30 ; Jewish traits among, 16; leg length of, 70, 73; Mongoloid traits among, 14, 15; Negroid traits among, 14, 17, 18, 52,75 ; nose of, $15,16,45$, compared to Chatris, Egyptians, 51, Jews, 16, Pathans, 51 ; origin of, 19 ; pigmentation of, 18,75 ; racial affinities of, $14,15,19$, $25,29-31,56,75,76$; racial evolution of, 19; southern Chinese type among, 15; South European traits among, 17, 25,75 ; stature of, 73 ; variability of racial characters in, $27,64,65,68-70$, 73; see Anaiza Beduins, Ba'ij Beduins, Beduins, Egyptians, Iraq soldiers, Kish Arabs, North Arabs, South Arabs

Arbela, see Erbil

Armenians, blood typing of, 461; of Iraq, 456, 459; of Iran, 456

Armenoid characters and type, 15, 16

Arneth's count, 460
Aryo-Dravidians, 19, 27, 28; home of, 19; traits of among Arabs, 29, 43, 51; see Pathans

Assyrians, 456, 459; blood typing of, 461

Astragals, game of, 94

Astronomy, 97

Aziz, Shevket, 457

Babylon, 77, 81, 84

Baghdad, see Iraq soldiers from

"Baghdad boil," 442

Ba'ij Beduins, 448-455; acromion to sole measurements of, 455; age changes among, 13, 16, 17; ages of, 450; anthropometric table of, 455 ; beard of, 16 ; brachycephaly among, 23,25 ; cephalic indices of, $20-23,37,441,450$, compared to Iraq soldiers, Kish Arabs, 37, to Somalis, South Arabs, 23, cumulative percentages of, 443; chest of, 453; ear indices and measurements of, 452,455 ; European features among, 16-19; eyes, 450,452 , color of, $18,19,450,452$; facial indices of, relative, 53,54 , total, $60-62$, 447,455 , upper, 449,455 ; facial measurements of, 53, 58-60, 455; facial types of, long-faced type, 17," "ram-faced," 54,56 , short-faced, 17 ; fronto-parietal indices of, $39,445,455$; hair of, 451 , color of, 18 , 451; head form and size of, $20-23,27$, 28, 36, compared to Chatris, 27, to Egyptians, 26, 27, to Iraq soldiers, 25, 27 , to Kish Arabs, 36,37 , to Kish crania, 450 , to South Arabs, 22, 23, correlation of with stature, 28 ; health of, 455 ; jugofrontal indices of, 454,455 ; jugomandibular indices of, 453,455 ; minimum frontal diameter of, 38-41, 455; musculature of, 454; Negroid traits among, 450,455 ; nose of, $16,452,453$, indices and measurements of, 45-47, 451, 455; numerical series of, 11 ; relative sitting height indices of, $69-70,439,455$; sitting height of, 68-70, 455; smallpox among, 455; statistical tables on, 448-455; stature of, $68-70,438,455$, compared to Egyptians, Iraq soldiers, Kish Arabs, Pathans, 28; tattooing of, 455, 456; teeth of, 67, 450, 453; see North Arabs

Balad Sinjar Yezidis, 459

Balfour, Henry, 9

Bani Sukhr Beduins, 449

Ba‘quba, European type from, 18

Basra, European type from, 18

Bayir Wells, Trans-Jordan, Chellean implements at, 78

Beduins, descendants of Proto-Mediterraneans, 78,79 ; racial relationships of, 
449, 450; see Anaiza, Ba'ij, Bani Sukhr, Dulaim, Huwaitat, Ruwalla, Sba'a, Shammar

Beduin type, evolution of, 19

Beirut, American University at, 457 ; nomads near, 458

Blood, Arneth's count, 460; polynuclear count, 459 ; samples of, 459-461, 463; typing of, 460,461

Brachycephaly, see Arabs, Ba'ij Beduins, Chatris, Egyptians, Iraq soldiers, Kish Arabs, North Arabs, Pathans, South Arabs

Bread, 89

Breasted, James H., 462

Brewer, H. F., 460

Brick-making, 85

Building techniques, ancient brick-making, 85 ; bridge, 86 ; mud hut, 85,86

Bullitt, IVilliam C., 462

Burnett, Sir John, 462

Buxton, L. H. Dudley, 9, 11, 456, 458

Camels, see Domestic animals

Caucasian race, evolutionary center of, 75 , 76 ; migrations of, 76 ; traits of, see Armenoid characters, Arabs, European, South European

Caucasus, anthropological data obtained in, $458,462,463$

Cephalic indices, grouping of, 20; see $\mathrm{Ba}^{\circ} \mathrm{ij}$ Beduins, Chatris, Egyptians, Iraq soldiers, Kish Arabs, North Arabs, Pathans, Yezidis

Chaldeans, ancient, compared to Iraq soldiers, 24, 25; modern, 456, 459; see Qaraqosh

Champion de Crespigny, C., 430

Chatris, brachycephaly among, 27; cephalic indices of, 27, 28; Dravidians, 27; fronto-parietal indices of compared to Pathans, 42; head form and size of, 27 28, compared to Ba'ij Beduins, 27, to North Arabs, 30, to Pathans, 30; minimum frontal diameter of, 41-43; nose of, 50, 51, compared to Arabs, Egyptians, Pathans, 51; stature of compared to Pathans, 28

Chellean implements, Bayir Wells, TransJordan, 78

Chinese features among Kish Arabs, 15

Christians, Iraq, blood typing of, 460,461

Clawson, M. D., 459

Clawson, M. Rue, 459

Climate, influence of on culture of Egypt, Mesopotamia, North Arabia, 78

Coon, Carleton, 457

Cornwallis, Sir Kinahan, 461

Craniometry, see Anthropometric method

Cutha (Tell Ibrahim), 85

Cyrus, 81

Dargavskaia Valley, Koban, U.S.S.R., skulls measured in, 463
Darius, 433

Demurov, T., 463

Dogs in Kish area, 85

Domestic animals, 85-87, 89, 92

Donkeys, see Domestic animals

Dravidians, home of, 19; traits of among Arabs, 14, 17, 18, 27, 51, 75, 76, among Kish Arabs, 46; see Chatris

Dress, see Kish Arabs, Qaraqosh Chaldeans

Drower, E. S., 459

Druze, 457

Dulaim Beduins, 458; blood samples of, 459; blood typing of, 461

Ear indices and measurements, see $\mathrm{Ba}$ 'ij Beduins, Iraq soldiers, Kish Arabs

Education, 96, 97

Egypt, climatic influence on, 78

Egyptians, Khargha Oasis, brachycephaly among, 27; cephalic indices of, 26,27 ; facial indices and measurements of, 6264 ; head form and size of, 26,27 , compared to Ba'ij Beduins, Iraq soldiers, 26,27 ; nose of, $16,43-45$, compared to Arabs, Chatris, 51, Hamitic character of, 44; relative sitting height indices of, 71 ; sitting height of, 70 ; stature of, 70 , 71 , compared to Ba'ij Beduins, Iraq soldiers, Kish Arabs, Pathans, 28

Endocrine glands, 442, 448

Erbil (Arbela), 432

European features, see Arabs, Ba'ij Beduins, Caucasian, Iraq soldiers, South Europeans

Evil eye, 89

Eye and eye color, see Ba'ij Beduins, Iraq soldiers, Kish Arabs

Facial characteristics, indices, measurements, types, see Arabs, Ba'ij Beduins, Egyptians, Iraq soldiers, Kish Arabs

Field, Marshall, 458

Food, 89, 90

Frankfort, H., 462

Frayha, A., 467

Fronto-parietal indices, see Ba'ij Beduins, Chatris, Iraq soldiers, Kish Arabs, Pathans

Games and pastimes, 92, 94

"Garden of Eden," 77

Gaugamela, battle of, 433,434

Graphic analyses of types, description of, see Anthropometric method

Grice, C., 461

Gutium, barbarians of, 80

Gypsies (Nawar), 458

Hair and hair color, see Ba'ij Beduins, Iraq soldiers, Kish Arabs

Hamites, Egyptian nose characteristic of, 44; head form and size of, 23; home of, 19; Somalis typical of, 23 
Hamitic traits among Arabs, 17, 75; among South Arabs, 74

Head form and size, see cephalic indices and head measurements under Arabs, Ba'ij Beduins, Chatris, Hamites, Iraq soldiers, Kish Arabs, Pathans; see Kish crania Health, see Ba'ij Beduins, Iraq soldiers, Kish Arabs

Henna, 88, 440, 444

Herzfeld, Ernst, 462

Hilla, see Iraq soldiers from

Hooton, E. A., 9

Hornibrook, William H., 462

Horse, 86, 87, 92; harness of, 87

Household utensils, 91

Houses, Kish area, 85, 86

Hrdlicka, A., 26, 43, 44, 62, 64, 70, 71

Huwaitat Beduins, Trans-Jordan, 449

India, natives of, see Aryo-Dravidians, Chatris, Dravidians, Pathans; racial relationship of with Arabs, 75

Indo-Afghans, racial relationship of with Arabs, 75

Indo-Aryans, racial relationship of with Arabs, 75

Irani ("Persians"), 456, 457, 462

Iranian group, ethnological definition of, 19

Iraq, anthropometric data obtained in (1934), 458-461; blood samples from, 459 ; blood typing in, 460, 461; ethnological location of, 19; geographical position of, 77; migrations into, 77; modern inhabitants of compared with Mesopotamians, 459; neolithic period in, 77; paleolithic period in, 77

Iraqis, 456-461; see Baij Beduins, Iraq soldiers, Kish Arabs

Iraq Petroleum Company, 459, 460, 462

Iraq soldiers, 444-448, acromion to sole measurements of, 445; ages of, 444, 445; anthropometric table of, 448; Armenoid traits among, 15, 16; brachycephaly among, 25, 31; cephalic indices of, 24, $25,27,31,32,37,38,448$, compared to Ba'ij Beduins, Kish Arabs, 37, graph of, 38,441 , cumulative percentages of, 443 , chest of, 447; ear indices and measurements of, 448, 452; endocrine abnormalities in, 448; European features among, 18,31 ; eyes, 446 , color of, 19,446 ; facial indices of, relative, $53,59,60$, total, 53 , 447,448 , upper, 448,449 ; facial measurements of, $53,54,59,60,448$; facial types of, long-faced type, 17, "ram-faced," $53,54,59,60$, short-faced, 17 ; frontoparietal indices of, $39,445,448$; hair, 445,446 , color of, 445 ; head form and size of, $24,25,27,31,32$, compared to ancient Chaldeans, 24, 25, to Ba'ij Beduins, 25, 27, to Egyptians, 26, 27, to Kish Arabs, 36, 37, to Pathans, 28, 31, to South Arabs, 32, correlation of with stature, 28; health of, 447; jugofrontal indices of, 448, 454; jugomandibular indices of, 448, 453; Mediterranean type among, 13; minimum frontal diameter of, 38, 39, 41; Mongoloid traits among, 14, 15; musculature of, 447; Negroid traits among, 14, 15, 17, 448; nose of, 16,446 , indices and measurements of, $45,48-50,448,451$; numerical series of, 11; pathological conditions of, 448; relative sitting height indices of, 439,448 ; sitting height of, 448; smallpox among, 448; statistical tables on, 444-448; stature of, $68,438,448$, compared to Ba'ij Beduins, Egyptians, Kish Arabs, Pathans, 28; tattooing of, 455, 456; teeth of, $67,446,447$; vital statistics of, $430-432$

Iraq soldiers, from Baghdad, 50; from Baghdad, Kirkuk, Mosul, Sulaimaniya, cephalic indices of, 31, 32, 36, European features in, 18, 31, head form and size of, 32; from Hilla, Nasiriya, cephalic indices of, 30,31 , head form and size of, 30,31 ; from Kut al Imara, cephalic indices of, 31,32 , European features of, 18, head form and size of, 31,32

Irrigation, Kish area, 84

Isfahan, Iran, Jews in, 462

Izzeddin, Nejla, 457

Jaudat, Ali, 461

Jemdet Nasr, 9, 84

Jemdet Obeid, 84

Jews, blood typing of, 460,461 ; nose of compared to Arabs, 16

Jews from Iran, 459; anthropometric data on, 462

Jews from Iraq, 456, 459

Jugofrontal indices, see Ba'ij Beduins, Iraq soldiers, Kish Arabs

Jugomandibular indices, see Ba‘ij Beduins, Iraq soldiers, Kish Arabs

Kappers, C. U. Ariens, 432, 456

Keith, Sir Arthur, 10, 24, 459

Kennedy, Walter P., 459, 462, 463

Khargha Oasis, see Egyptians

Kinnareh, Iran, anthropological data from, 462

Kirkuk, see Iraq soldiers from

Kish, ancient human remains at, 11; crania from, 11, compared to Ba ij Beduins, 450 ; earliest inhabitants at, $79,81,82$; founding of, 77, 79; history of, 79-82; location of, 77; neolithic phase at, 78; pottery, neolithic at, 78, 79; ProtoMediterraneans' arrival at, 79; Semites' arrival at, 79, 80; Sumerians' arrival at, 79

Kish Arabs, 82-97, 434-444; acromion to sole measurements of, 444; ages of, 437 ; 
American Indian traits among, 15; anthropometric table of, 444; Armenoid traits among, 15, 16; "Baghdad boil" among, 442 ; brachycephaly among, 30 , $33-35,37,450$; brow-ridges of, 435 ; cephalic indices of, 32-38, 444, compared to $\mathrm{Ba}{ }^{\prime} \mathrm{ij}$ Beduins, Iraq soldiers, 37 , graph of, 38,441 , cumulative percentages of, 443 ; chest of, 437 ; children of, 96,430 , 431 ; Chinese features among, 15; Dravidian traits among, 46 ; dress of, 87,88 ; ear indices and measurements of, 436 , $442,444,452$; education of, 96,97 ; endocrine abnormalities of, 442; eyebrow of, 435 ; eyes, $88,435,440$, color of, 19, 435; facial indices of, relative, 53-58, 61 , total, 53, 62-67, 444, 447, upper, 444, 449; facial measurements of, 53, 60 67; facial types of, long-faced type, 17 , "ram-faced," 56-58, short-faced, 17 ; food of, 89, 90; fronto-parietal indices of, 444 , 445; games and pastimes of, 92,94 ; hair, $434,437,440$, color of, 18,434 ; head form and size of, 28, 32-37, compared to $\mathrm{Ba}^{\prime} \mathrm{ij}$ Beduins, 36, 37, to Iraq soldiers, 36, 37, to Pathans, 30 , correlation of with stature, 28; health of, 437,442 ; henna used by, 88, 440, 444; household utensils of, 91 ; jugofrontal indices of, 444,454 ; jugomandibular indices of, 444, 453; kohl used by, 88, 444; marriage customs of, 88 ; minimum frontal diameter of, 38-40, 42, 43, 444; Mongoloid traits among, 15, 442; musculature of, 437 ; music of, 90; Negroid traits among, 17, 442 ; nose of, $16,435,436$, indices and measurements of, $45-48,444,451$; numerical series of, 11; pathological conditions of, 442,443 ; racial characteristics of, 82 ; relative sitting height indices of, $70-74,439,444$; religion of, 95, 96; scapulae of, 437 ; scars of by burning, 443 ; sitting height of, 70-74, 444 ; smallpox among, 442; social life and customs of, 82-97; statistical tables on, 434-444; stature of, $68,70-74,438,444$, compared to $\mathrm{Ba}$ ij Beduins, Egyptians, Iraq soldiers, Pathans, 28, correlation of with head size, 28; Sumerian type among, 442; superstitions of, 89 ; tattooing of, $88,455,456$; taxation of, 94,95 ; teeth of, $436,440-442$; tobacco used by, 92 ; variability of racial characters in, 71 ; vital statistics of, $430-432$; weapons of, 90-93

Kish area, 77-97; domestic animals of, $85-$ $87,89,92$; geographical character of, 84; irrigation of, 84 ; map of, 83 ; mud huts used as houses in, 85,86 ; villages of, 85 ; women of, 88

Knabenshue, Paul, 462

Koban, U.S.S.R., see Dargavskaia Valley
Kohl, 88, 444

Krischner, Harald, 456, 457

Krischner, M., 456

Krogman, W. M., 10, 23

Kug-Bau, 80

Kurdistan, paleolithic sites in, 462

Kurds, 456, 459; blood samples of, 459 ; blood typing of, 460,461

Kut al Imara, see Iraq soldiers from

Lagash, 80

Langdon, Stephen, 79, 430

Laufer, Berthold, 9

Lazadat tribe, 'Trans-Jordan, 457

Lazar, Yusuf, 461, 462

Leg length, see sitting height and stature under Ba'ij Beduins, Egyptians, Iraq soldiers, Kish Arabs

Lurs from Pusht-i-Kuh, Iran, 457, 462

Macfarlane, James, 460

Mackay, Ernest, 80

McLeod, T. H., 462

Maer, battle of, 80

Mahewil, 84, 95

Malak, Gabriel, 462

Mancala, 94

Mandaeans (Subba), 459

Marett, R. R., 9

Marriage customs, 88

Marsh Arabs, 458, 459

Martin, Richard A., 10, 458

Martin, Rudolph, technique of, 458

Mediterranean type among Iraq soldiers, 13

Mesopotamia, 77; climatic influence on, 78 ; see Iraq

Meymourian, Albert, 461

Minimum frontal diameter, see Ba'ij Beduins, Chatris, Iraq soldiers, Kish Arabs, Pathans

Mongoloid traits among Arabs, 14, 15; among Iraq soldiers, 14, 15; among Kish Arabs, 15, 442; type, evolutionary center of, 75

Mosul, see Iraq soldiers from

Mowali tribe, Trans-Jordan, 457

Muallim, Khedoory, 461

Mud hut, 85,86

Music, 90

Nabunidus, 81

Nal'chik, deformed skulls from, 463

Nasiriya, see Iraq soldiers from

Navar (Gypsies), 458

Nebuchadnezzar, 81

Negroid, population, see Arabia; traits, see Arabs, Ba'ij Beduins, Iraq soldiers, Kish Arabs

Neolithic phase, in Iraq, 77; at Kish and in North Arabian Desert, 78, 79

North Arabian Desert, archaeological sites in, 462; climatic influence on, 78; 
earliest inhabitants of, 78; paleolithic man in, 78

North Arabs, brachycephaly among, 30, 74; cephalic indices of, 23 ; head form and size of, 22, 23, compared to Chatris, Pathans, 30, to South Arabs, 22, 23, 30, 74,75 ; physical characters of compared to South Arabs, 75; see Ba'ij Beduins

North Ossetians, anthropometric studies of, 462,$463 ;$ blood samples of, 463

Nose, Armenoid, 15, development of, indices, measurements of, etc., see Arabs, Ba'ij Beduins, Chatris, Egyptians, Iraq soldiers, Kish Arabs, Pathans; growth changes of, 16

Ordzhonikidze, U.S.S.R., 462, 463

Oriental Institute, University of Chicago, 457,462

Ossetian Museum, Ordzhonikidze, U.S.S.R., 463

Ossetians, see North Ossetians

Paleolithic period, see Aqra, Bayir Wells, Iraq, Kurdistan, North Arabian Desert, Rowandiz, Rutba, Sulaimaniya, Zakho

Pathans, Aryo-Dravidians, 27, 28; brachycephaly among, 30; cephalic indices of, 29,30 ; fronto-parietal indices of compared to Chatris, 42; head form and size of, 27-30, compared to Arabs, 29, 30, to Chatris, 30, to Iraq soldiers, 28,31 , to Kish Arabs, North Arabs, South Arabs, 30 ; minimum frontal diameter of, 42,43 ; nose of, compared to Arabs, Chatris, 51, indices and measurements of, 51, 52; stature of compared to $\mathrm{Ba}^{\prime} \mathrm{ij}$ Beduins, Chatris, Egyptians, Iraq soldiers, Kish Arabs, 28

Pathological conditions, see Iraq soldiers, Kish Arabs

"Persians" (Irani), 456, 457, 462

Photographic records, anthropometric analyses by, 12, 13, 16, 17

Pigmentation, $18,75,442$; see eye, hair, under Ba'ij Beduins, Iraq soldiers, Kish Arabs

Pittard, Eugène, $457^{\circ}$

Pleistocene period, 75; in Arabian peninsula, 75,76

Polynuclear count, 459

Pottery, neolithic, from Kish, 78, 79

Proto-Mediterraneans, ancestors of Beduins, 78,79 ; conquered by Sumerians, 79 migrations of, 79

Punjab natives, see Pathans

Pusht-i-Kuh, Iran, Lurs from, 457, 462

Qaraqosh, Chaldean village of, 430, 432-434; modern Chaldeans at, dress of, 432, 433, physical characteristics of, 432,433 , women of, 433; see Gaugamela

Qasr-el-Burqu', Roman occupation of, 78
Race study by means of photographs, 12 , 13,17

Ramadan, 95-97

"Ram-faced" types, see facial types under Arabs, Ba'ij Beduins, Iraq soldiers, Kish Arabs

Rassam, Carl, 456, 459

Ratzel, F., 19

Rayy excavations, Iran, anthropometric studies on workmen at, 462

Relative facial indices, see facial indices under Ba'ij Beduins, Iraq soldiers, Kish Arabs

Relative sitting height indices, see $\mathrm{Ba}{ }^{6} \mathrm{ij}$ Beduins, Egyptians, Iraq soldiers, Kish Arabs

Religion, 95, 96

Rickards, A. R. M., 462

Risley, H. H., 27, 28, 29, 50, 52

Rowandiz, 77; Kurds from, 459; paleolithic site near, 462

Royal College of Medicine, Baghdad, 459, 461

Rustam Agricultural Experimental Farm, Hinaidi near Baghdad, 461

Rutba, paleolithic site near, 78

Ruwalla Beduins, 449

Sargon, sons and descendants of, 80

Sayyid Ruda, camp of, 34, 35

Sba'a Beduins, 449

Scars by burning, 443

Schmidt, Erich F., 462

Schroeder, Eric, 9

Semites, Arabia home of, 19; Armenoid traits in, 16; arrival at Kish of, 79, 80; racial relationship of with Arabs, 76

Shammar Beduins, 458, 459

Shanklin, William M., 457

Sheikh Abbas ibn Diab of the Ba'ij Beduins, 448,449

Sheikh 'Adi Yezidis, 459

Sheikh 'Ajil ibn al-Yawir, Paramount Sheikh of the Shammar, 459

Sheikh Aziz, camp of, 35, 36

Sheikh Hadji Hunta, 9; camp of, 35, 36

Sheikh Hadji Miniehil, 9; camp of, 32-34, 85

Sheikhs of Kish area, 82

Sheikh Swadi, camp of, 34, 35

Showket, S. Y., 9, 432, 461

Sitting height, see Ba'ij Beduins, Egyptians, Iraq soldiers, Kish Arabs

Sleyb (Sulubba), 458

Smallpox, 442, 448, 455

Smeaton, Winifred, 456, 459

Smith, Myron B., 462

Somalis, cephalic indices of compared to Ba'ij Beduins, South Arabs, 23; representative of Hamites, 23

South Arabia, anthropometric data from, 457

South Arabs, brachycephaly among, 22-24, $30,74,75$; cephalic indices of, 23,24 , 
compared to Ba'ij Beduins, Somalis, 23; Hamitic traits among, 74; head form and size of, 22, 23, compared to Ba'ij Beduins, 22, 23, to Iraq soldiers, 32, to North Arabs, 22, 23, 30, 74, 75; physical characters of compared to North Arabs, 75

Southern Chinese type among Arabs, 15

South European features, among Arabs, 17, 25, 75; among Iraq soldiers, 18, 31; see European features

Southwestern Asia, anthropometric data from, 456, 457

Stature, see Arabs, Ba'ij Beduins, Chatris, Egyptians, Iraq soldiers, Kish Arabs

Subba (Mandaeans), 459

Sulaimaniya, 77; Kurds from, 459; paleolithic site near, 462; see Iraq soldiers from

Sulubba (Sleyb), 458

Sumerians, conquest of Kish by, 79, of Maer by, 80; type of among Kish Arabs, 442 Superstitions, 89

Sushko, Alexander, 434

Suwa'id, see Al Suwa'id

Tattooing, 88, 455, 456

Taxes, 94, 95

Teeth, occlusion of in primitive and civilized races, 67 ; see $\mathrm{Ba}{ }^{6} \mathrm{ij}$ Beduins, Iraq soldiers, Kish Arabs

Tell Barguthiat, 84, 448

Tell-el-Uhaimir (Kish), 80, 82-84

Tell Ibrahim (Cutha), 85

Tell Inghara (Kish), 83, 84

Tell Kaif, Chaldean from, 459

Thomas, Bertram, 73, 457; Somalis measured by, 23; South Arabs measured by, 22

Tobacco, 92

Total facial indices, see Ba'ij Beduins, Egyptians, Iraq soldiers, Kish Arabs
Trans-Jordan, anthropometric data from, 457

Trunk length, see Relative sitting height

Turkomans, 459; blood typing of, 460, 461

United Provinces, India, see Chatris

University of Chicago, 434, 457, 462

Upper face length, see "ram-faced" under Arabs, Ba'ij Beduins, Iraq soldiers, Kish Arabs

Ur, ancient inhabitants, cephalic indices of, 24,25 , cranial capacity of, 25 , skeletal remains of, 11; dynasty of, at Kish, 80 ; kingdom of, 81

U.S.S.R., anthropological data obtained in, $458,462,463$

Variability of racial characters, see Arabs, Kish Arabs

Villages of Kish area, description of, 85

Vital statistics, $430-432$

VOKS (The Society for Cultural Relations with Foreign Countries), U.S.S.R., 462

Watelin, L. C., 9

Weapons, 90-93

Wilson, W. C. F., 462

Women, status of in Kish area, 88; Chaldean, see Qaraqosh

Woolley, Sir Leonard, 11, 24

Yezd-i-Khast, Iran, anthropological data from, 462

Yezidis, 450; blood typing of, 461 ; cephalic indices of, 459 ; studied in 'Tiflis, U.S.S.R., 462

Zakho, Kurds from, 459; paleolithic site near, 462 


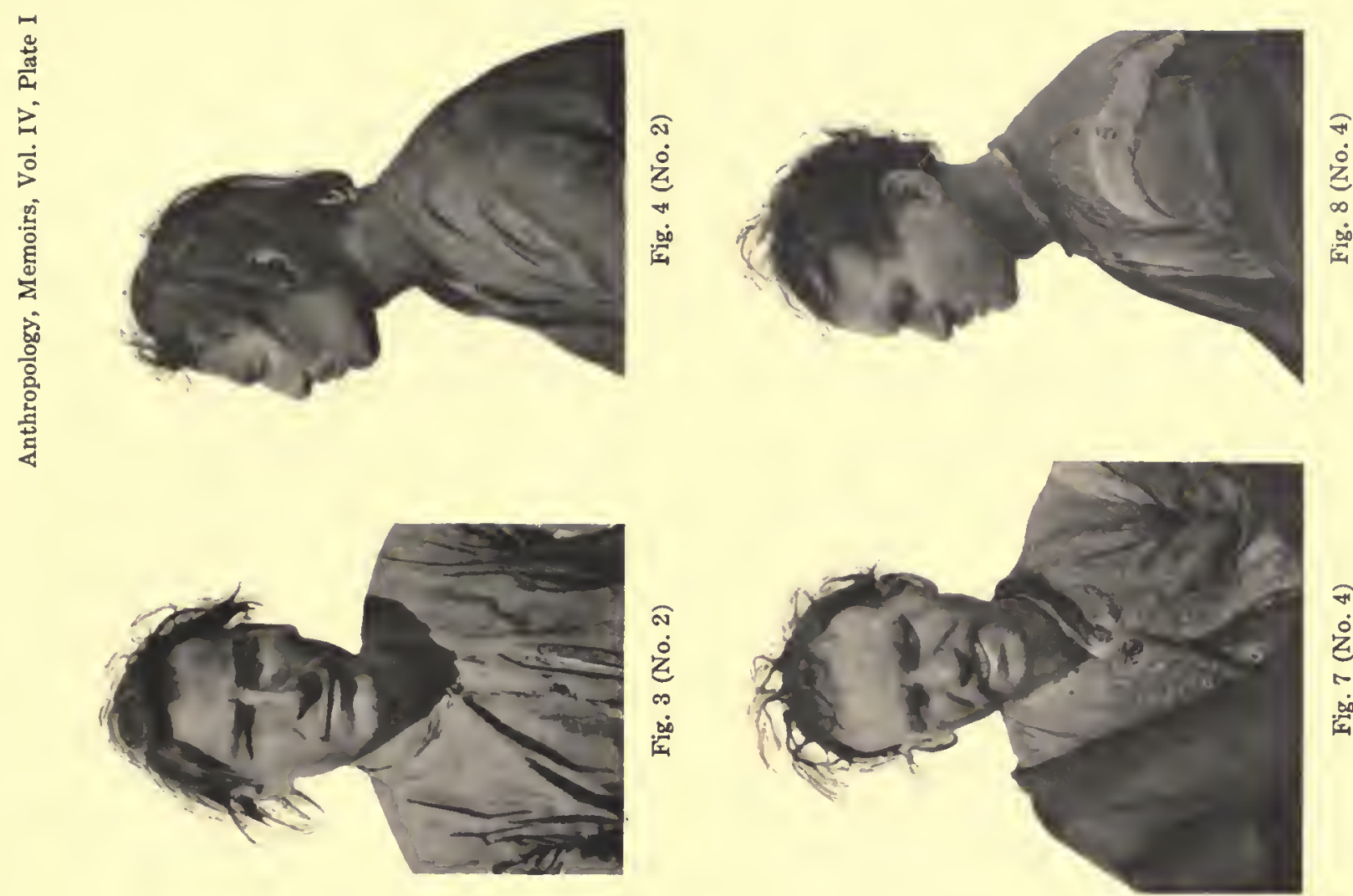

के
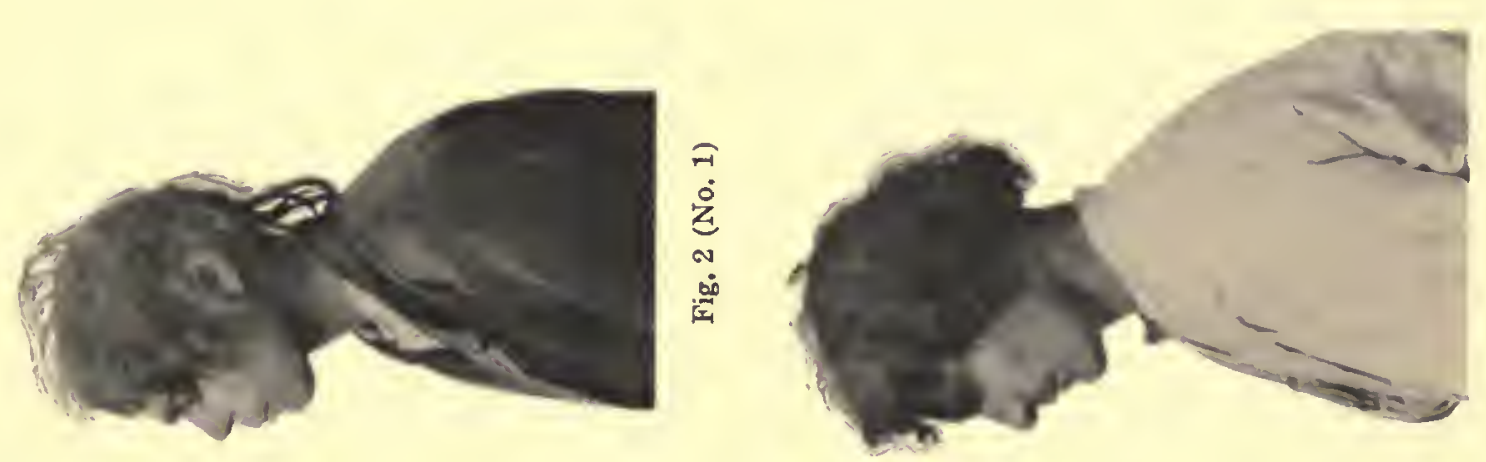

委

कิ
$\dot{0}$
$\dot{z}$
0
0
0
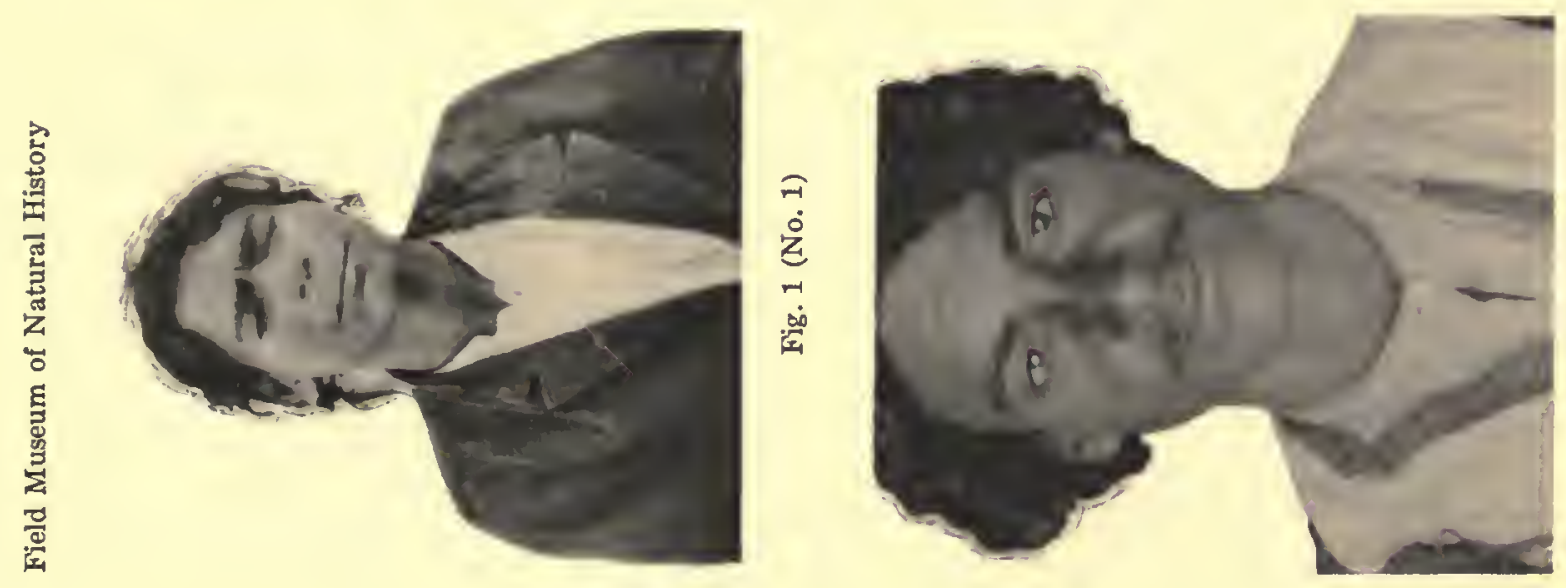

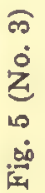

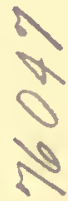



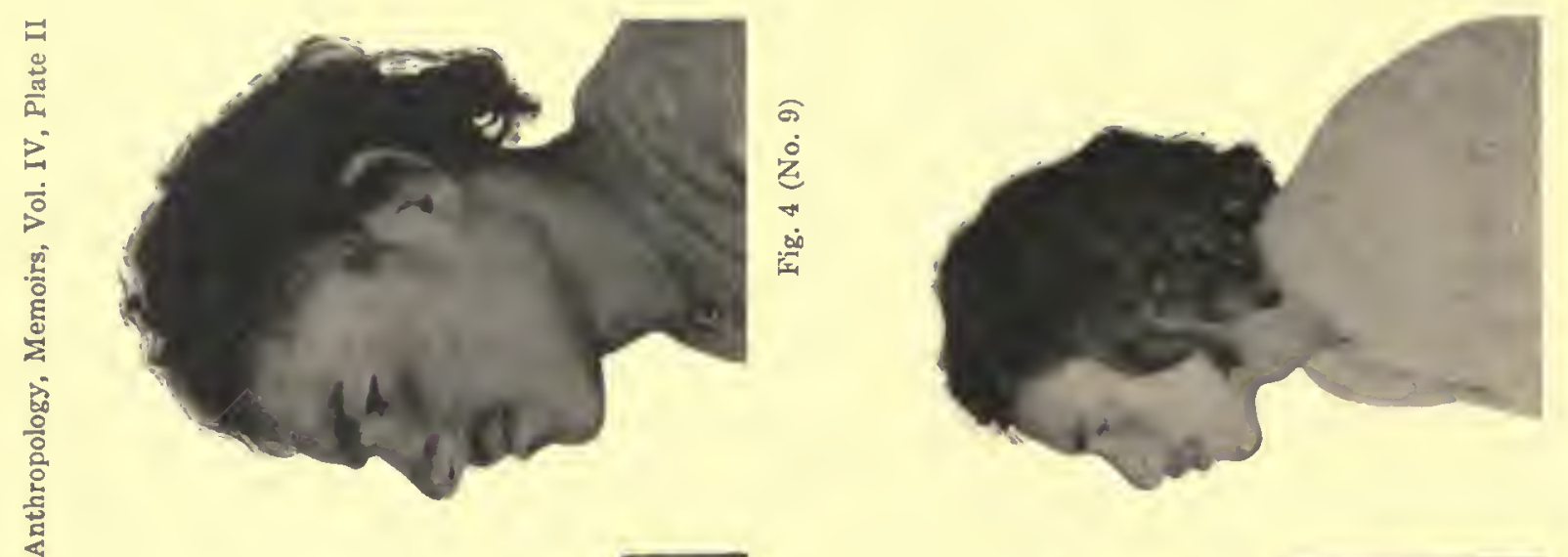

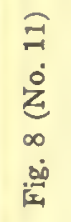
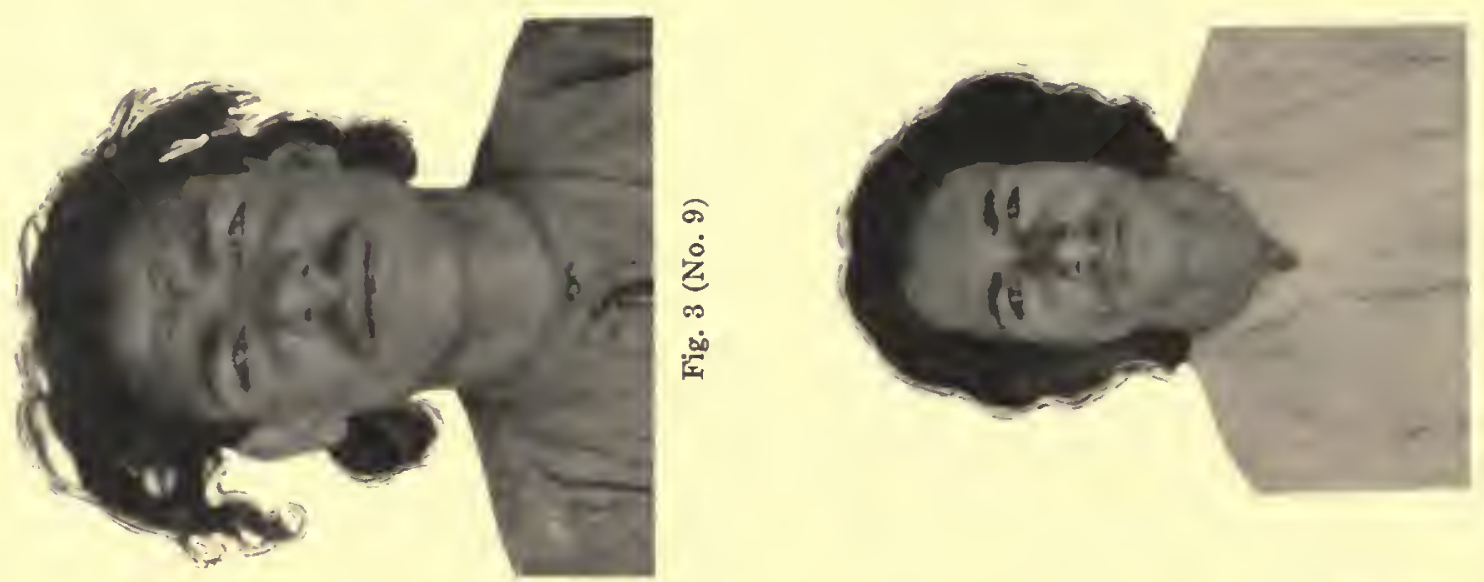

בิ
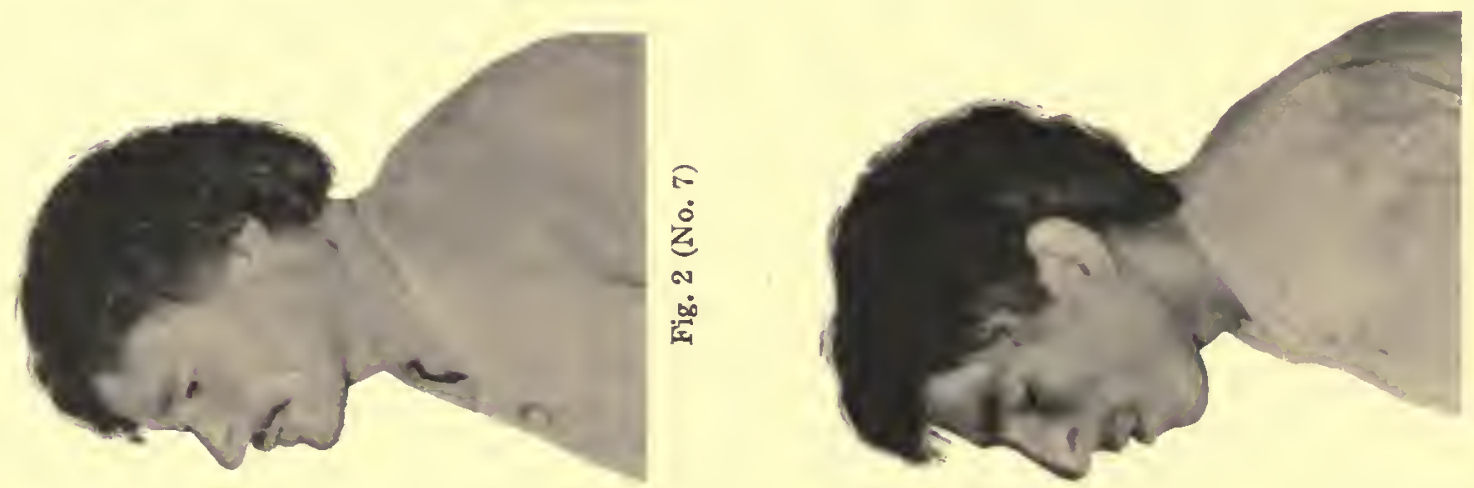

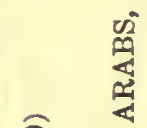
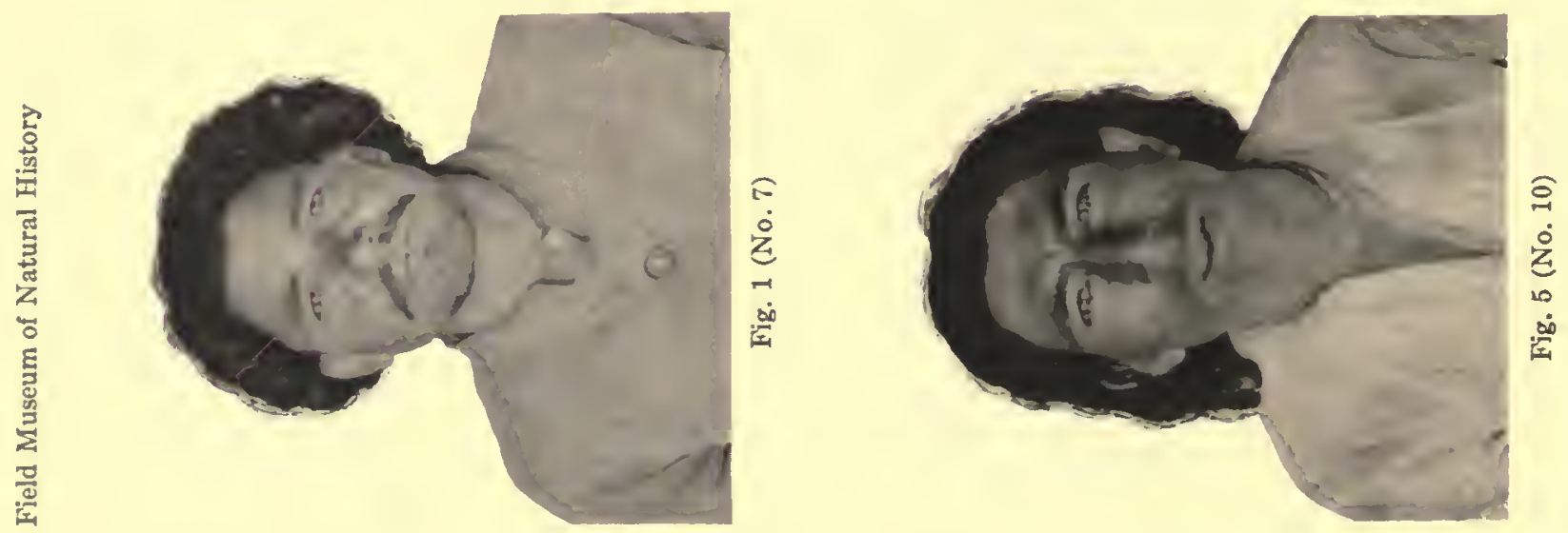

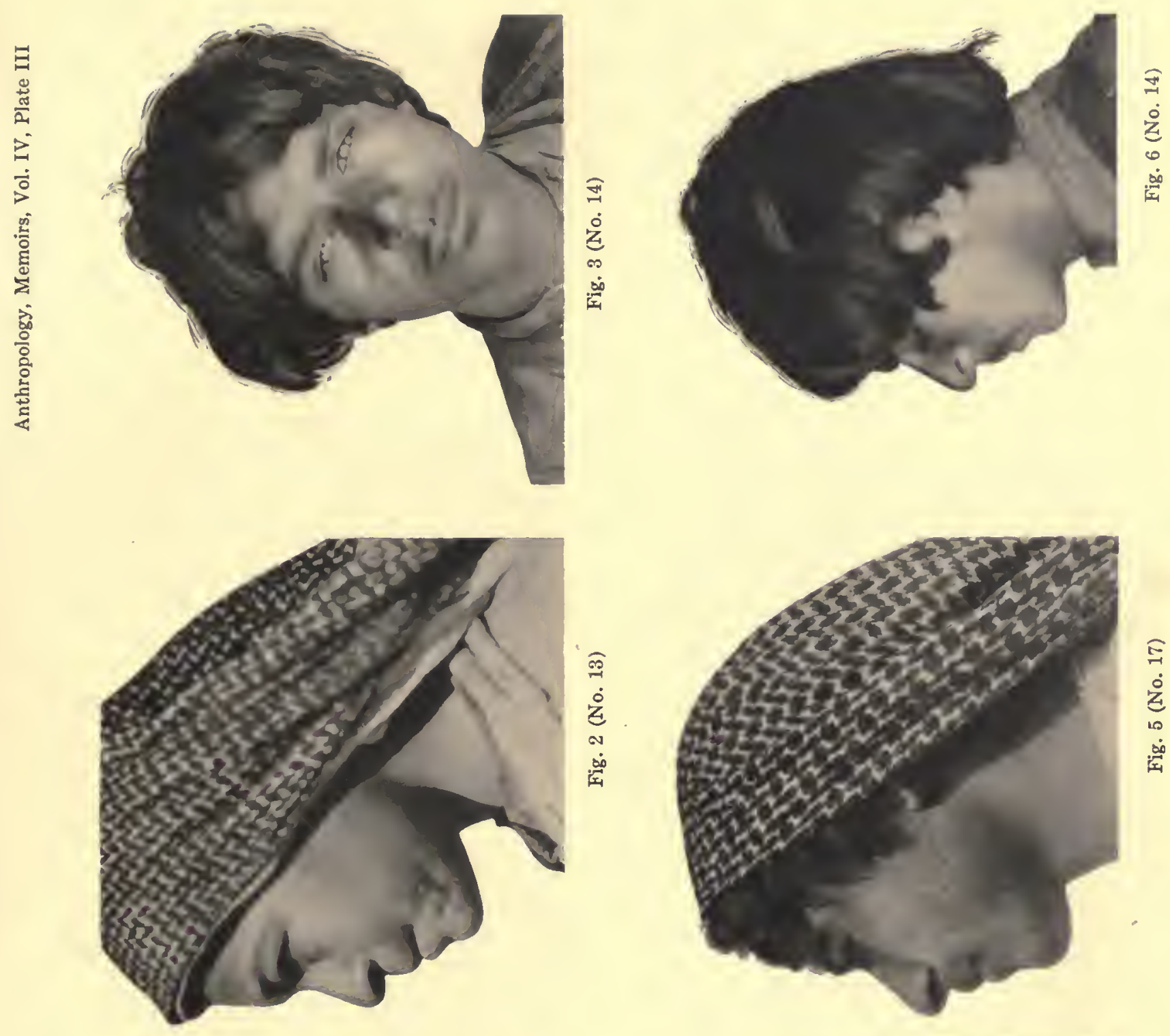

E
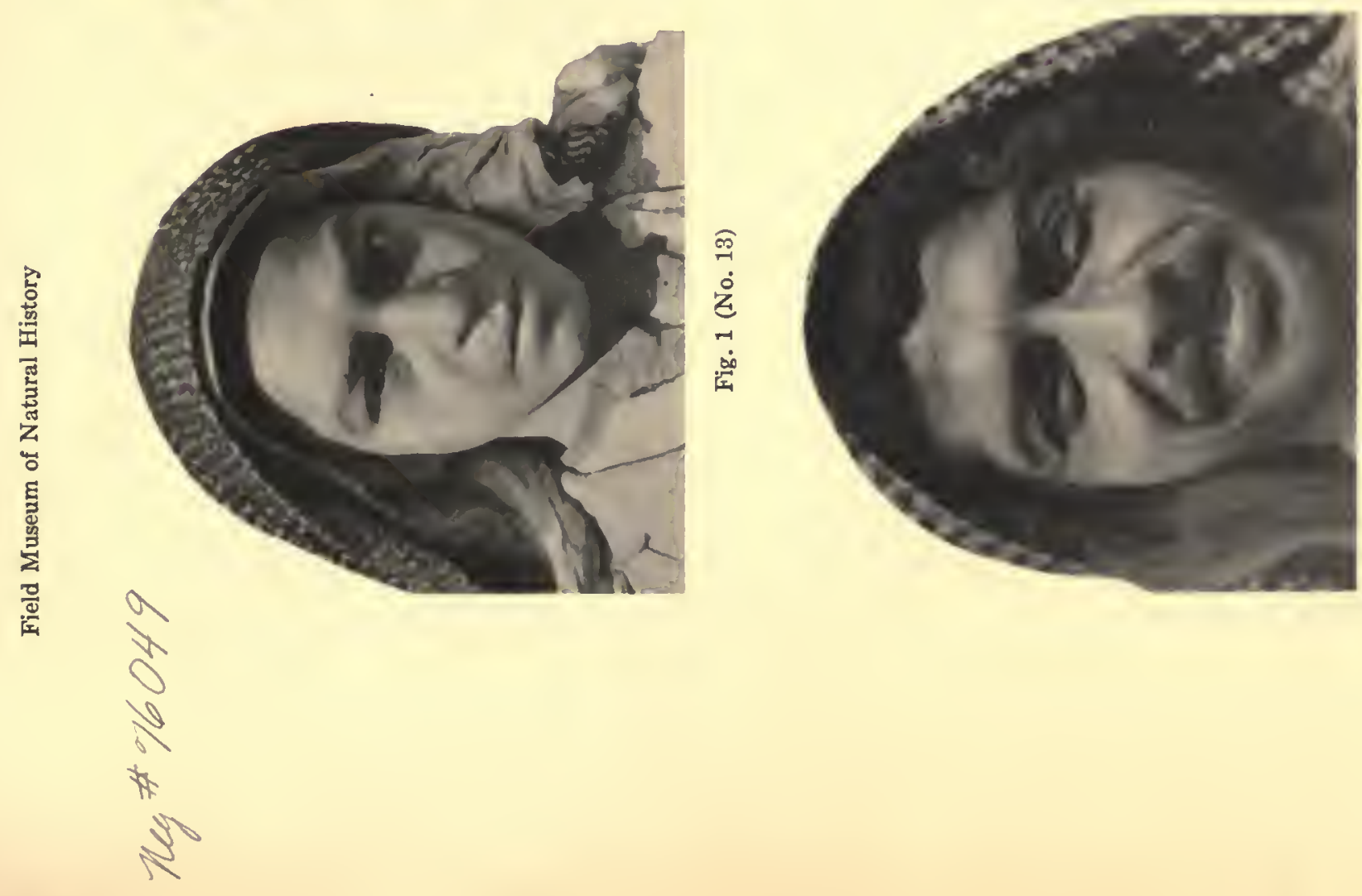

ت્ટ 

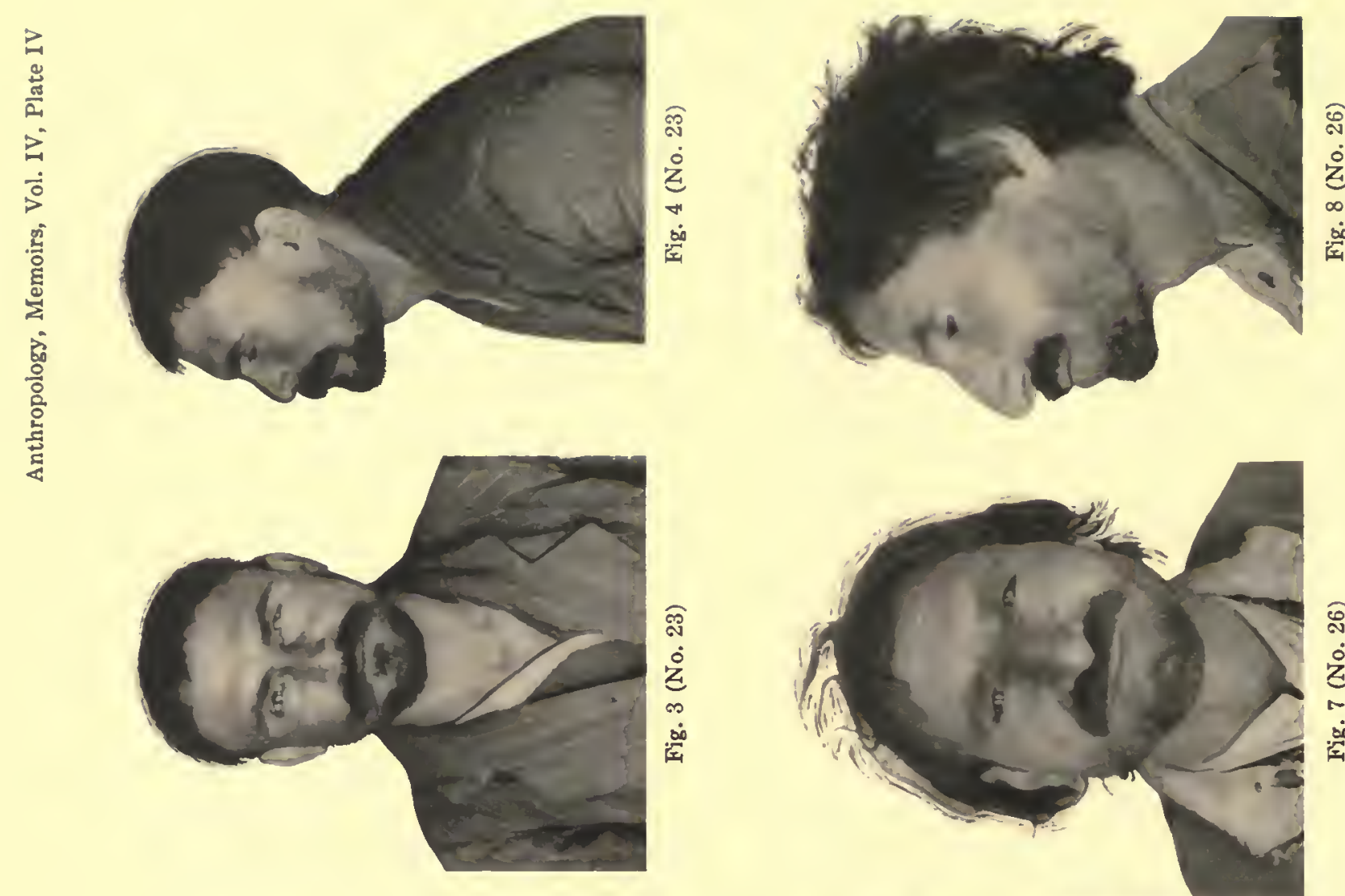

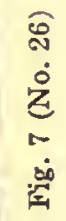
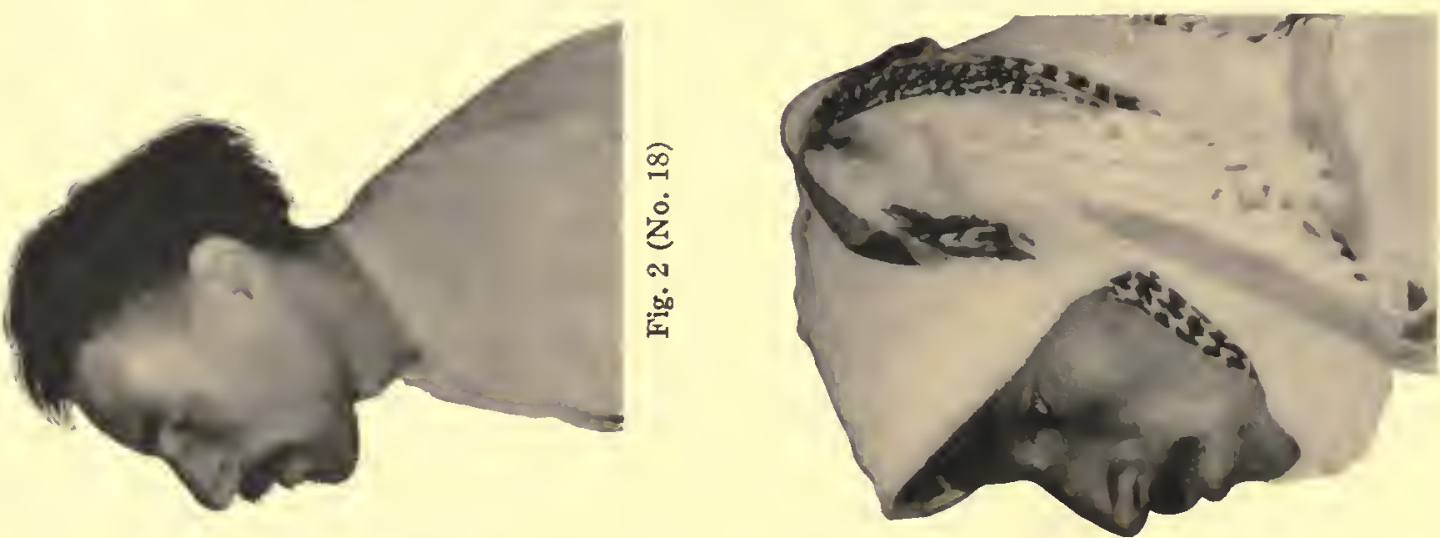

秃

5
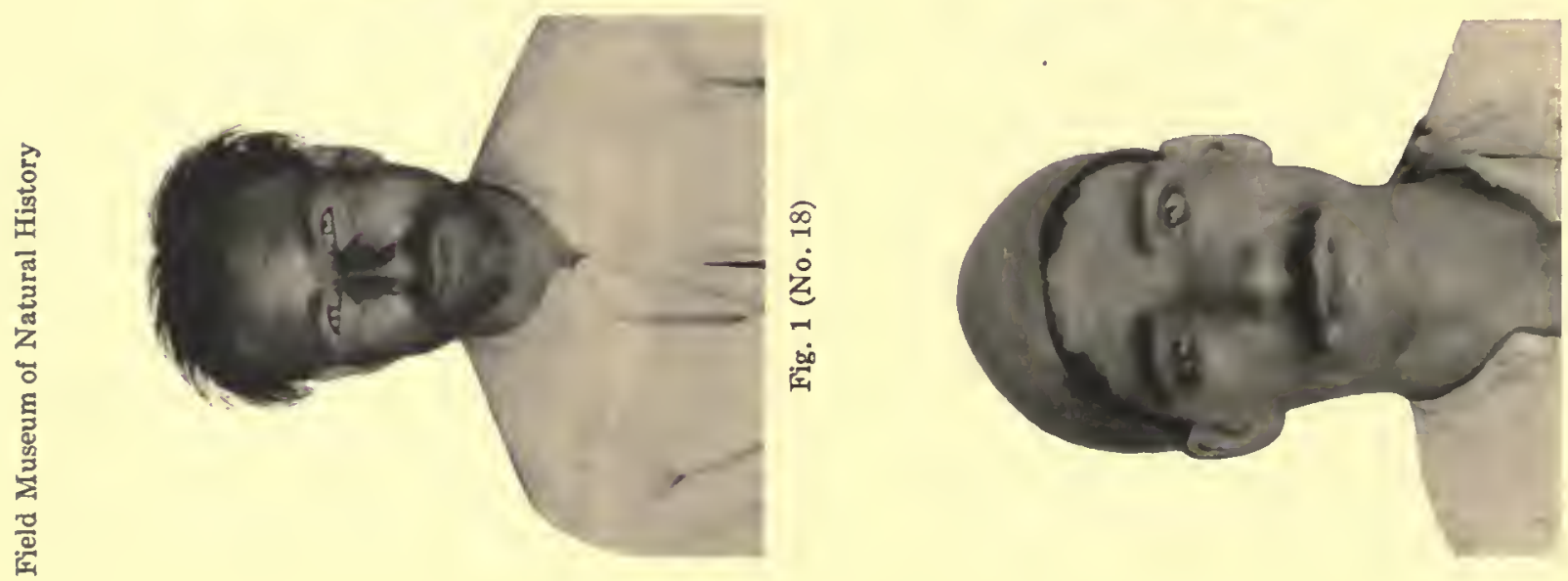

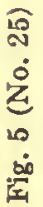



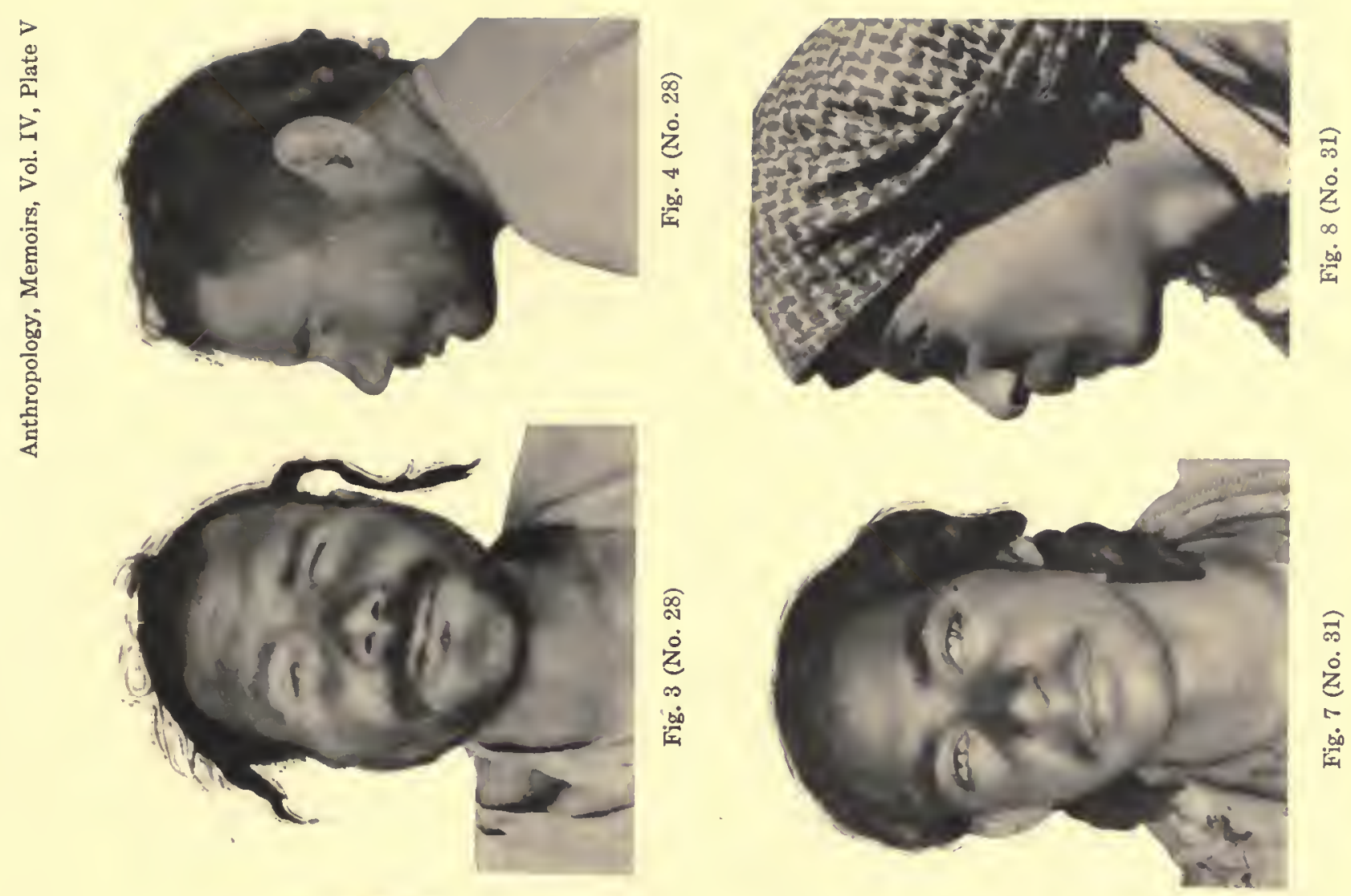

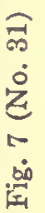
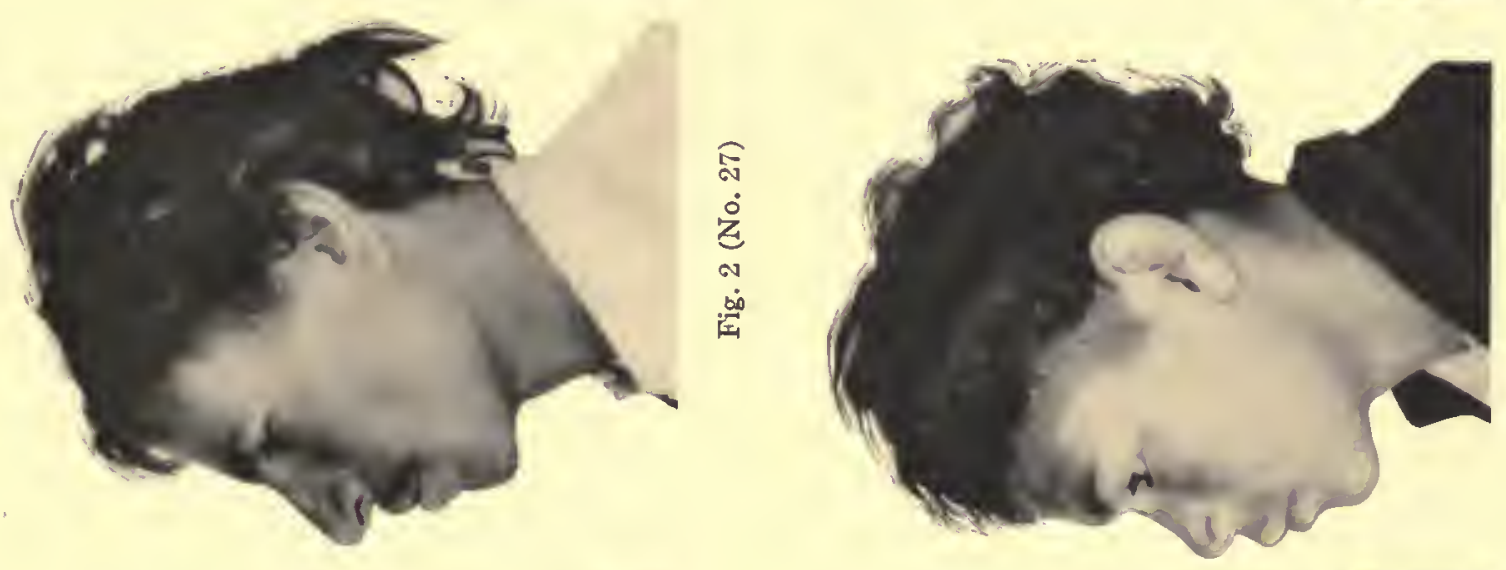

舀
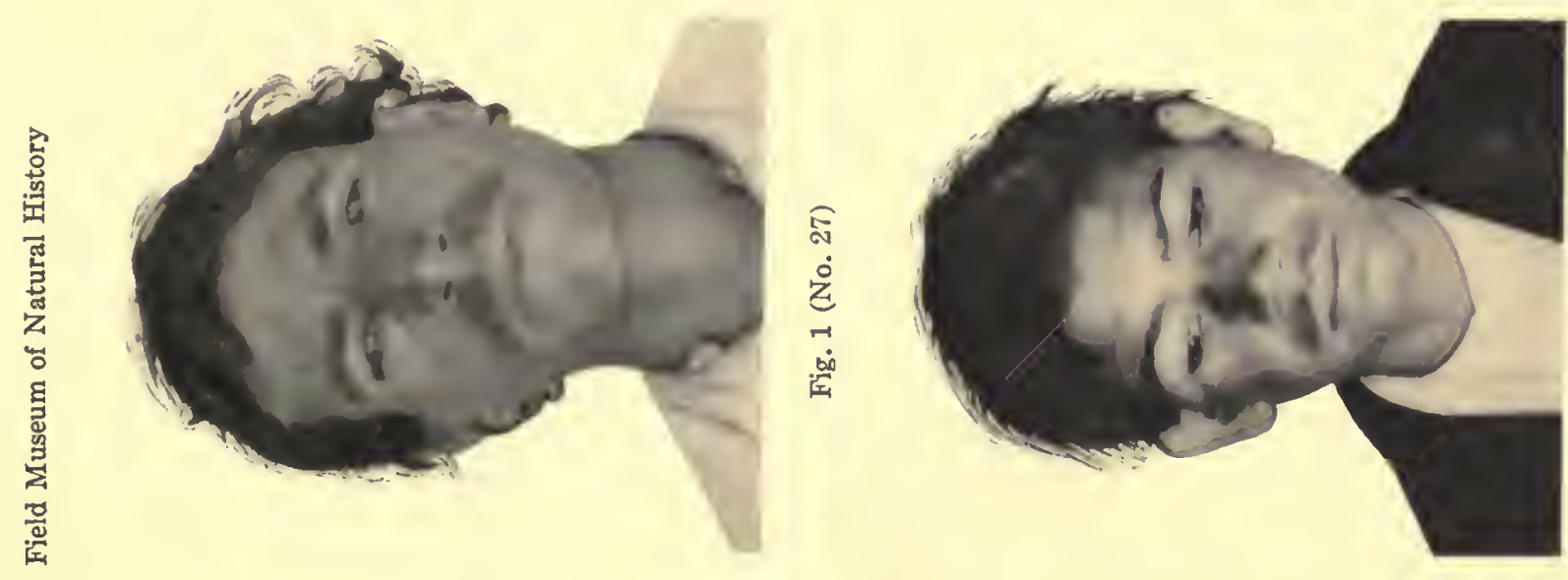

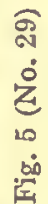

品

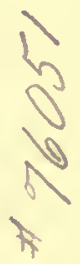




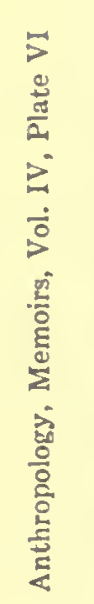
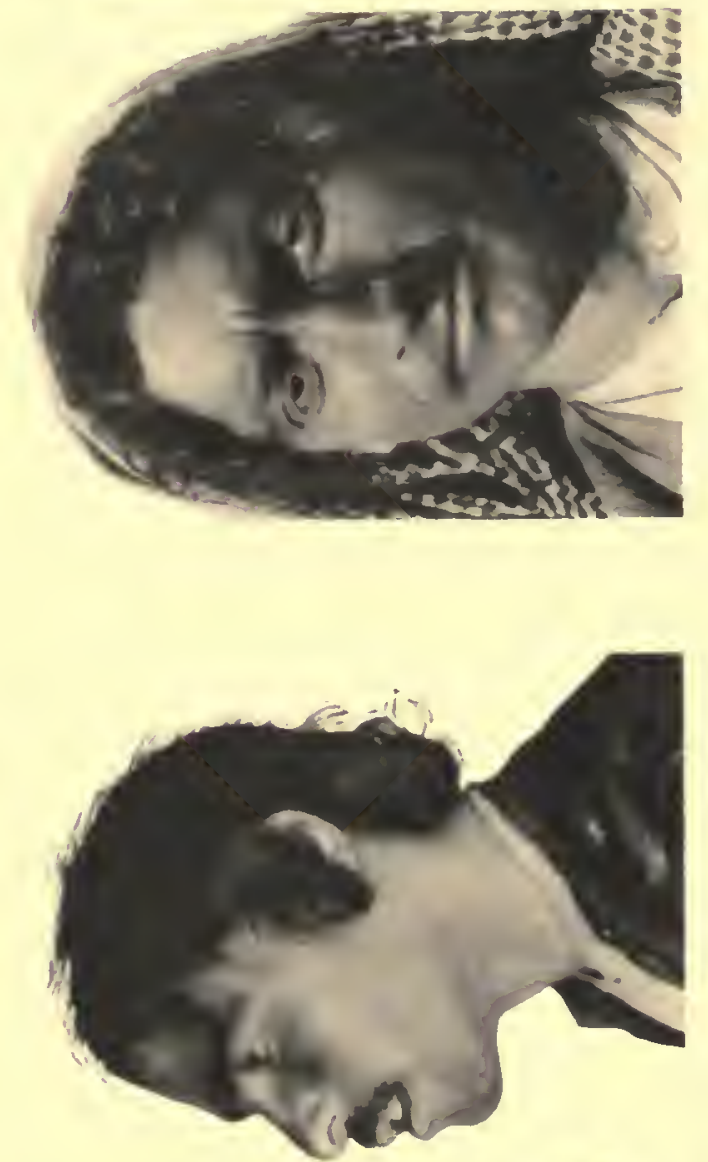

b N

3

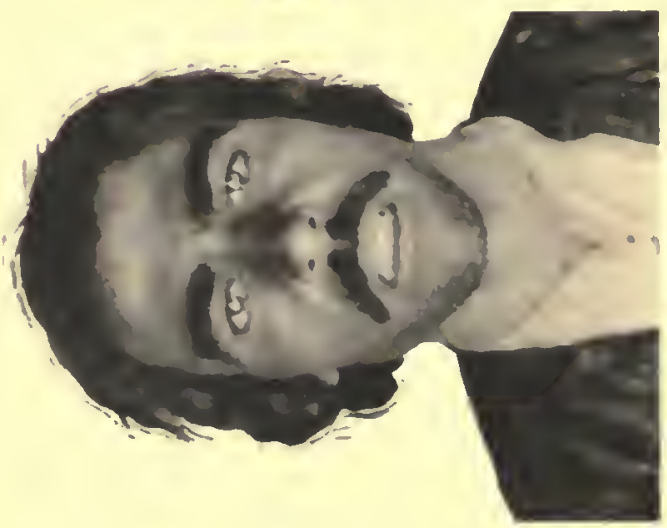

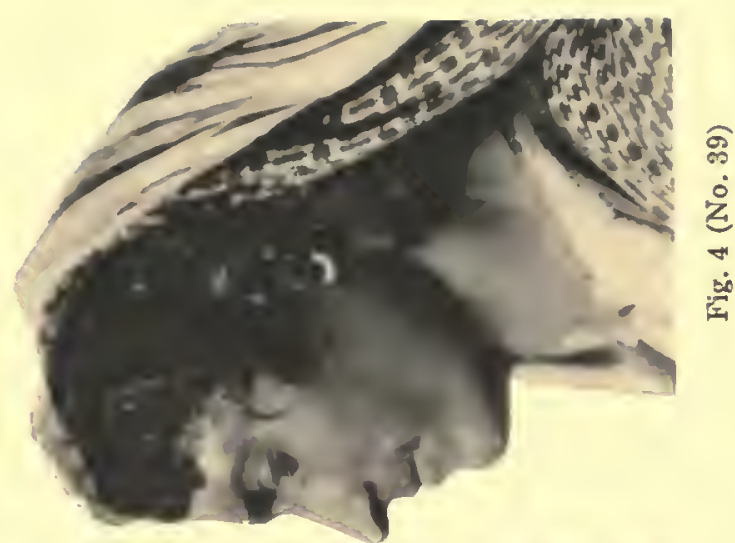
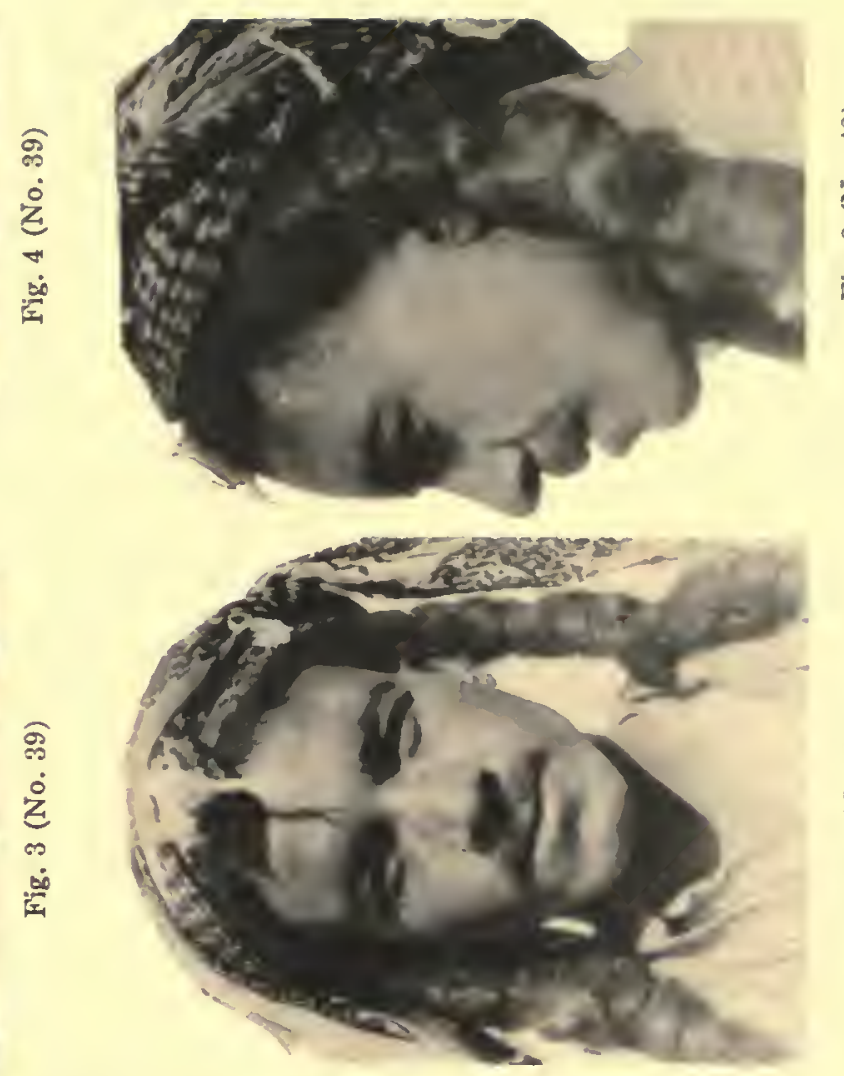

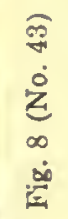

产

䁬

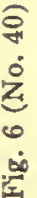

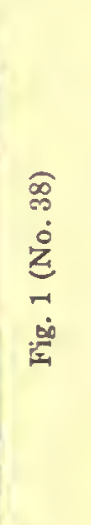

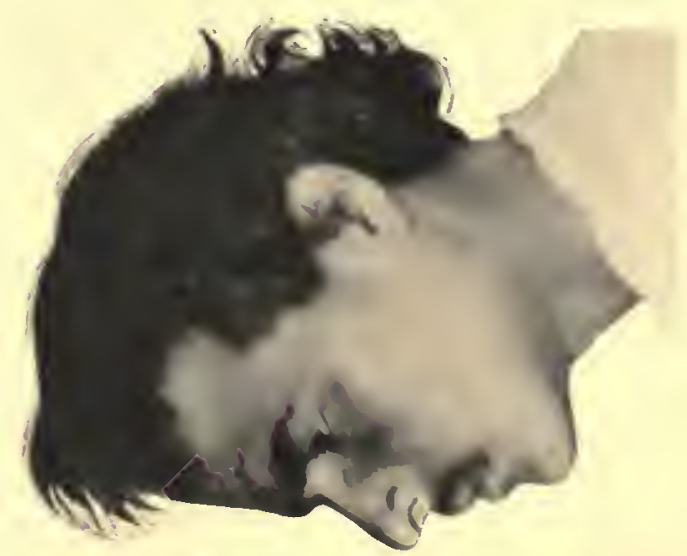

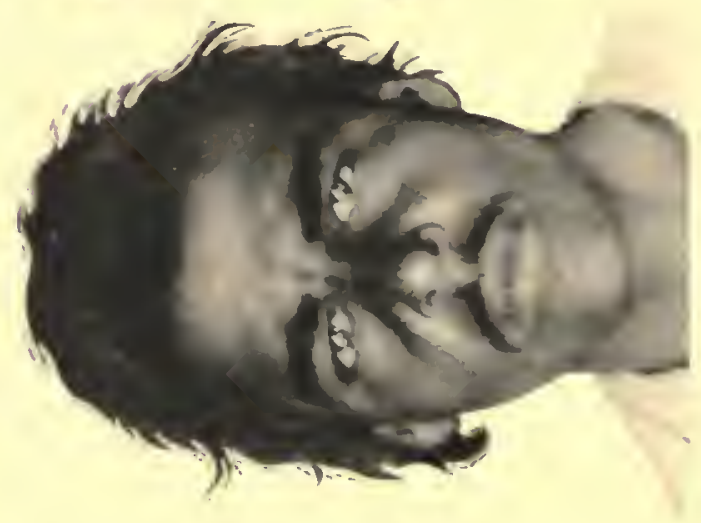



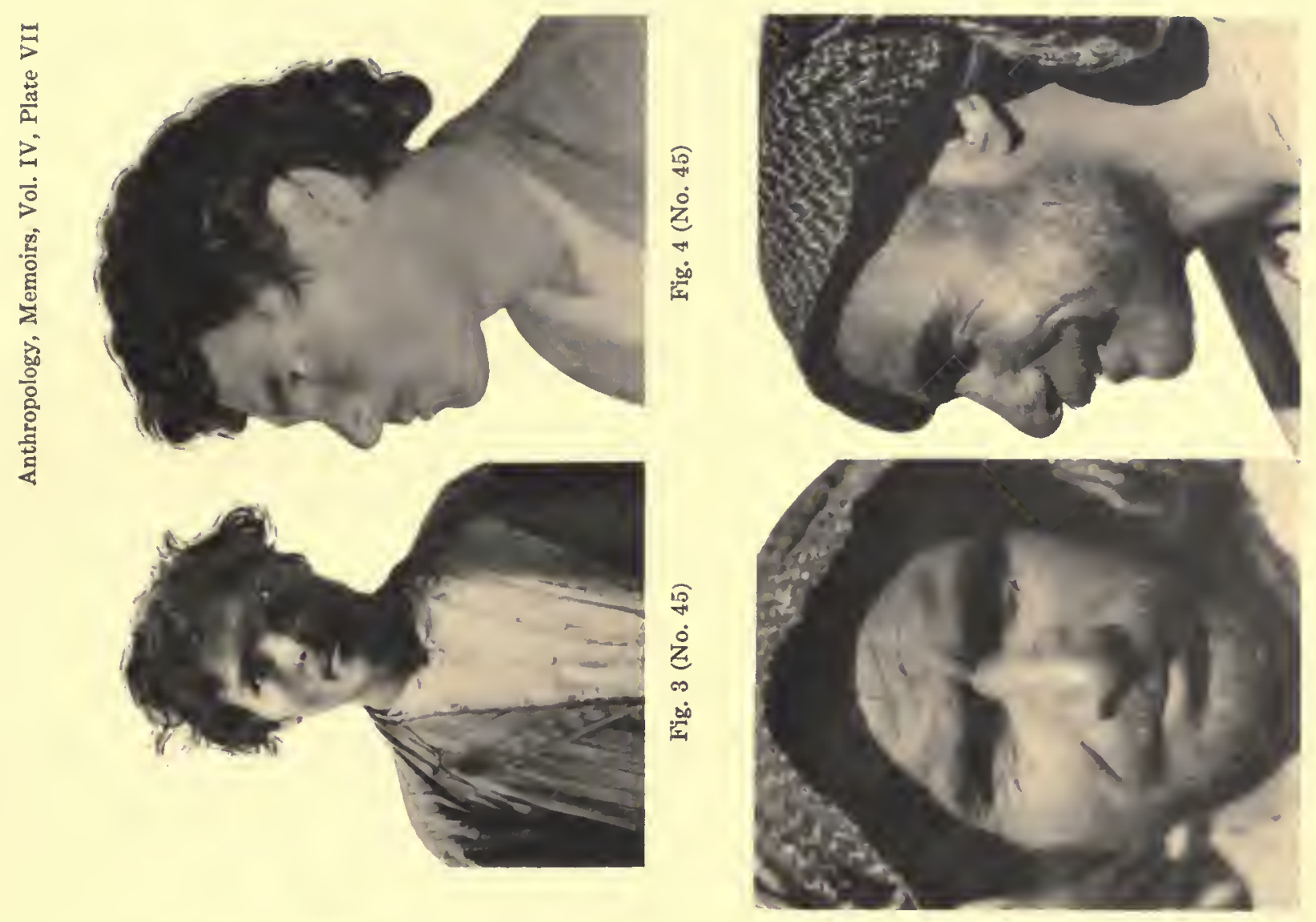

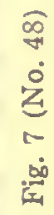

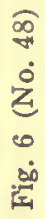

委
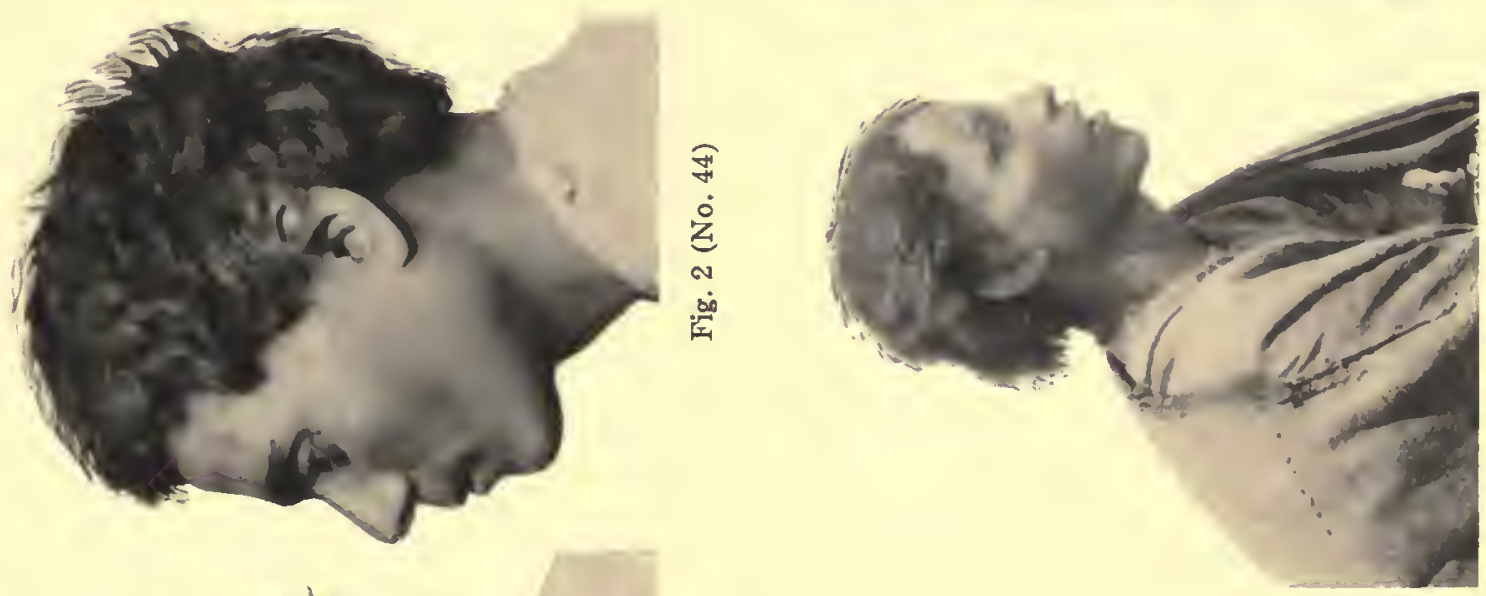

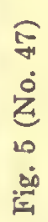
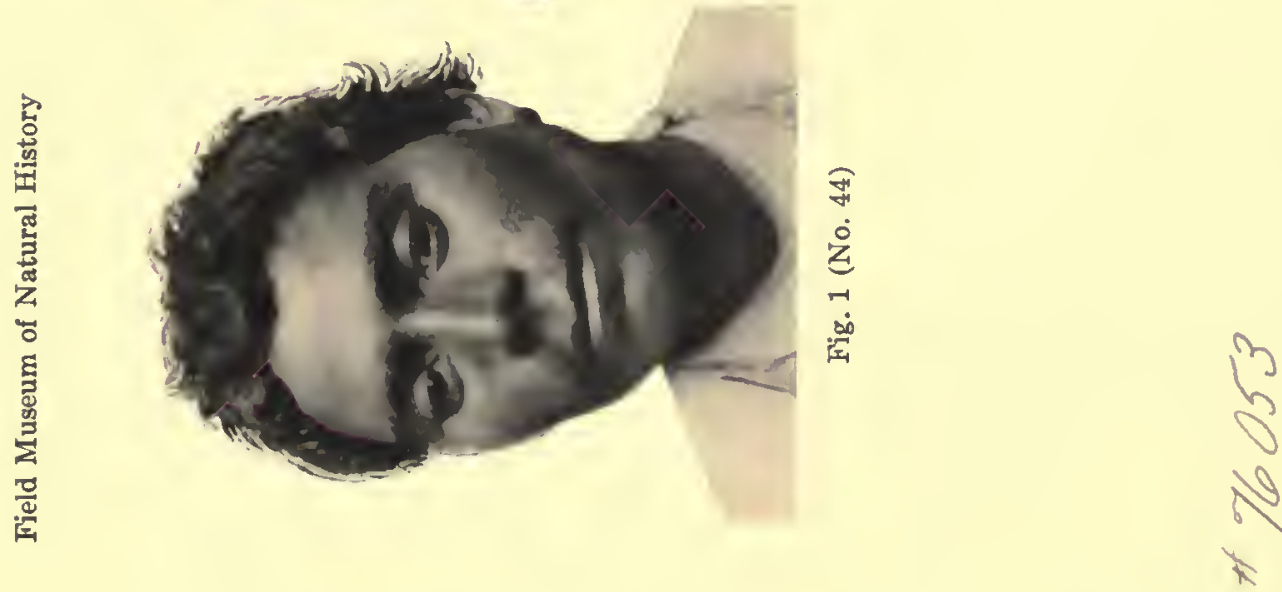

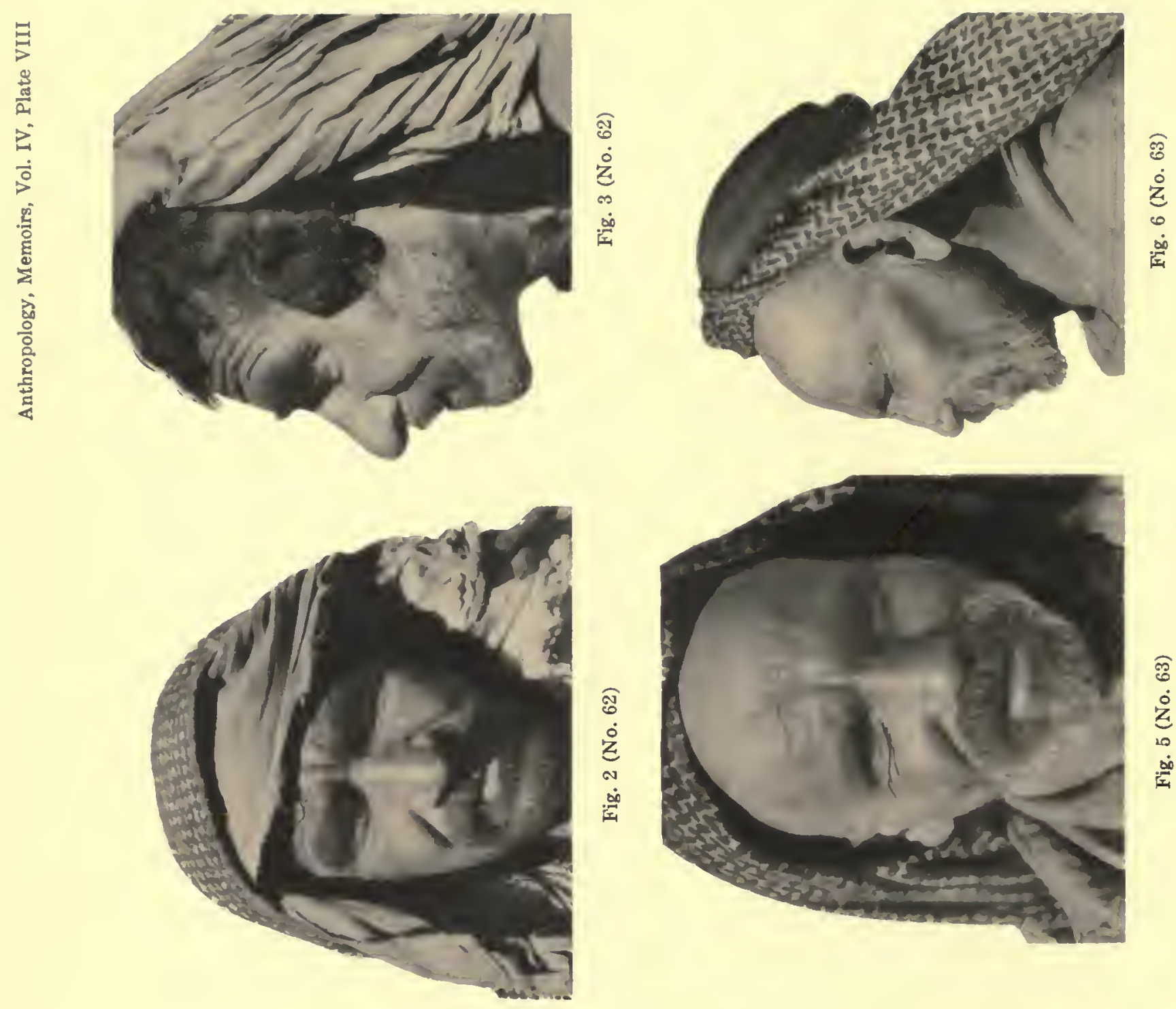

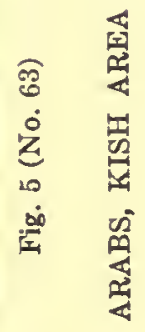
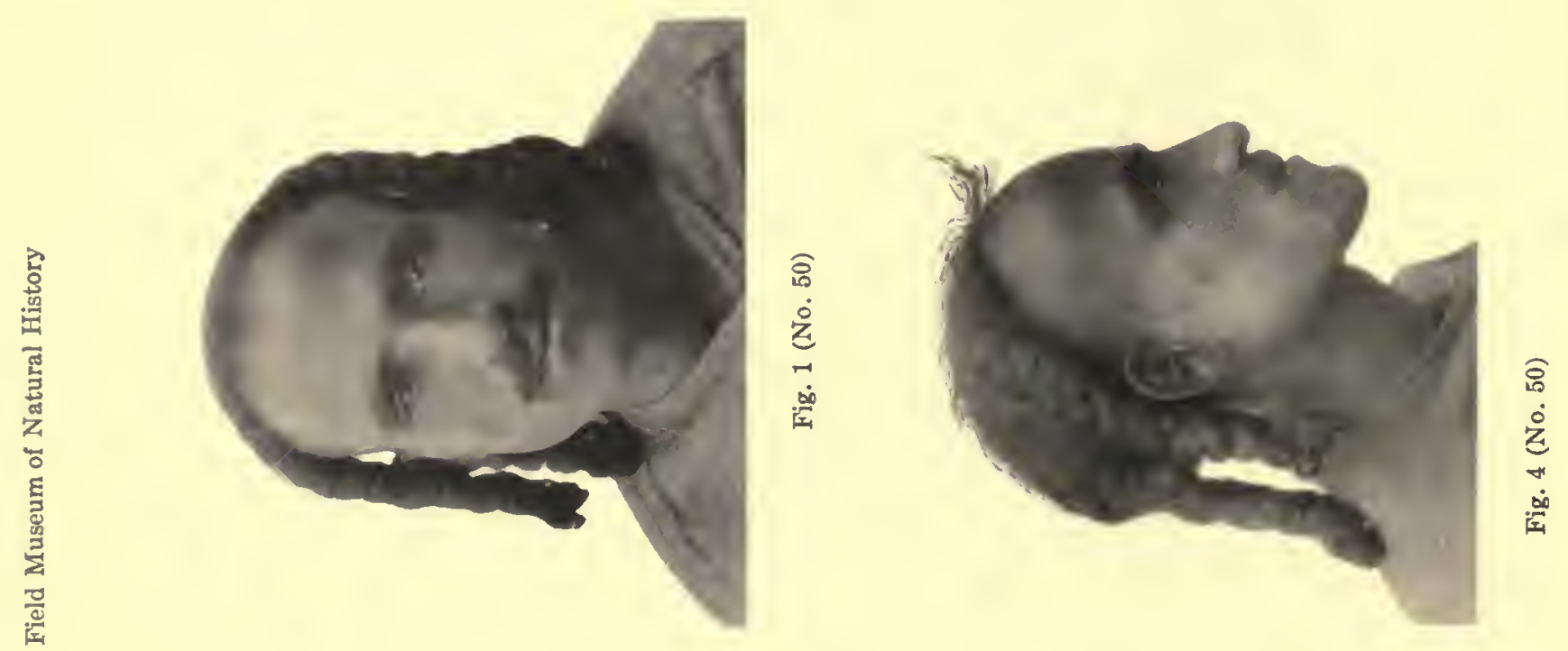

N 

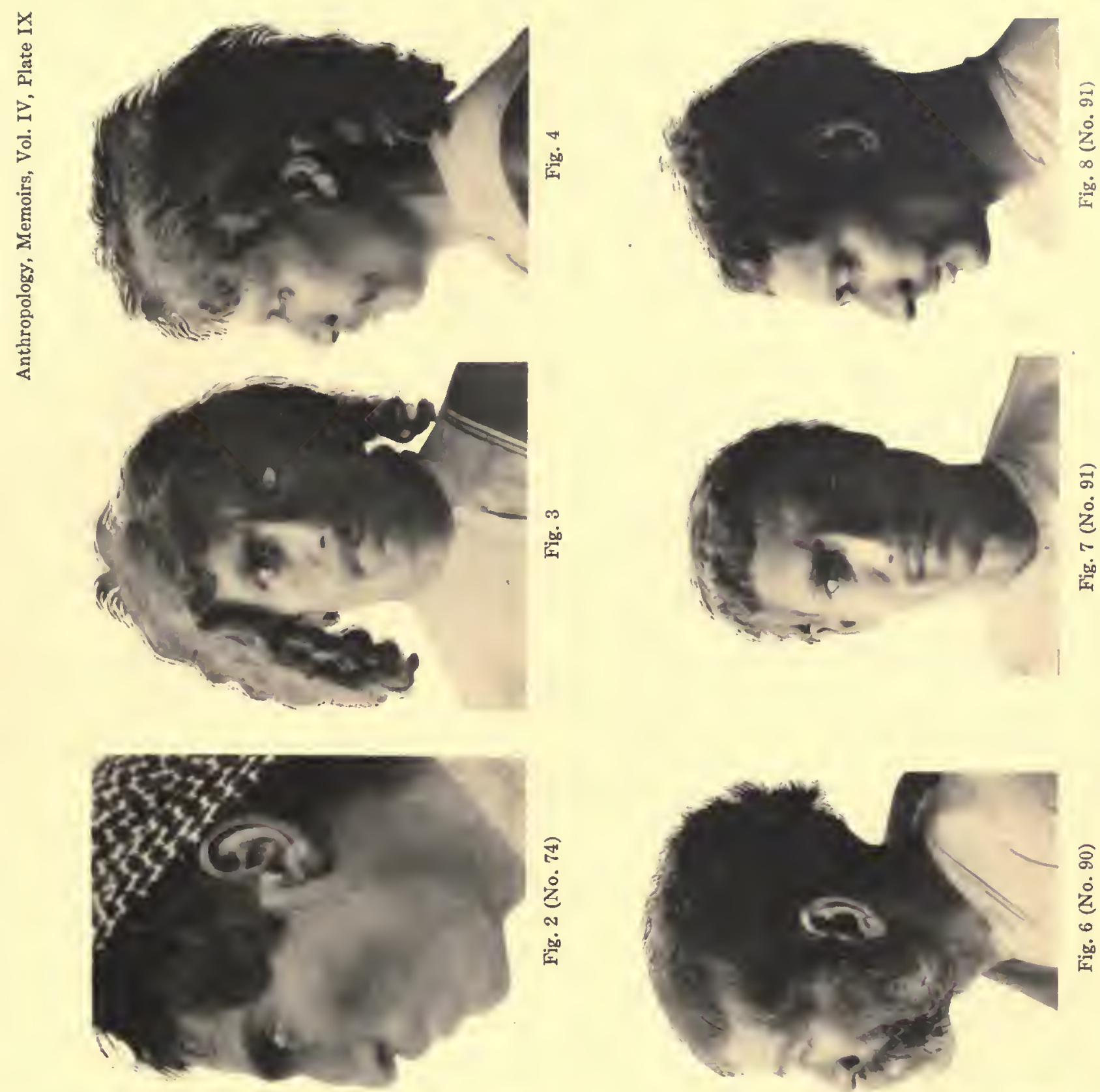

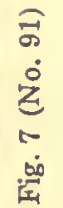

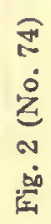

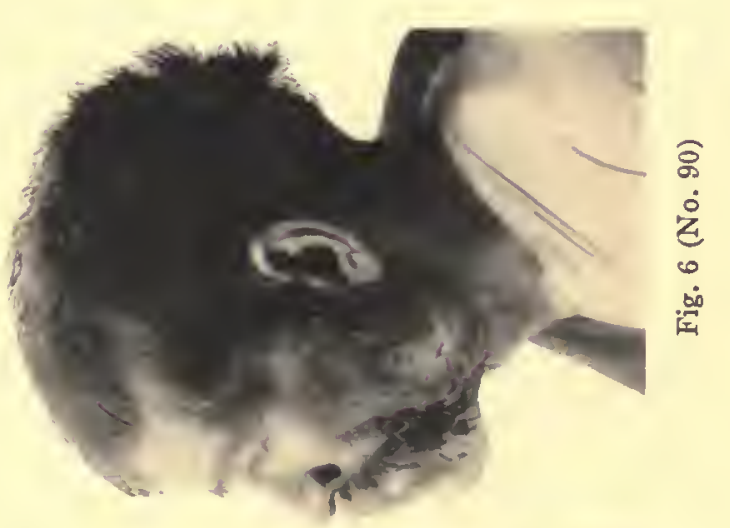

孚
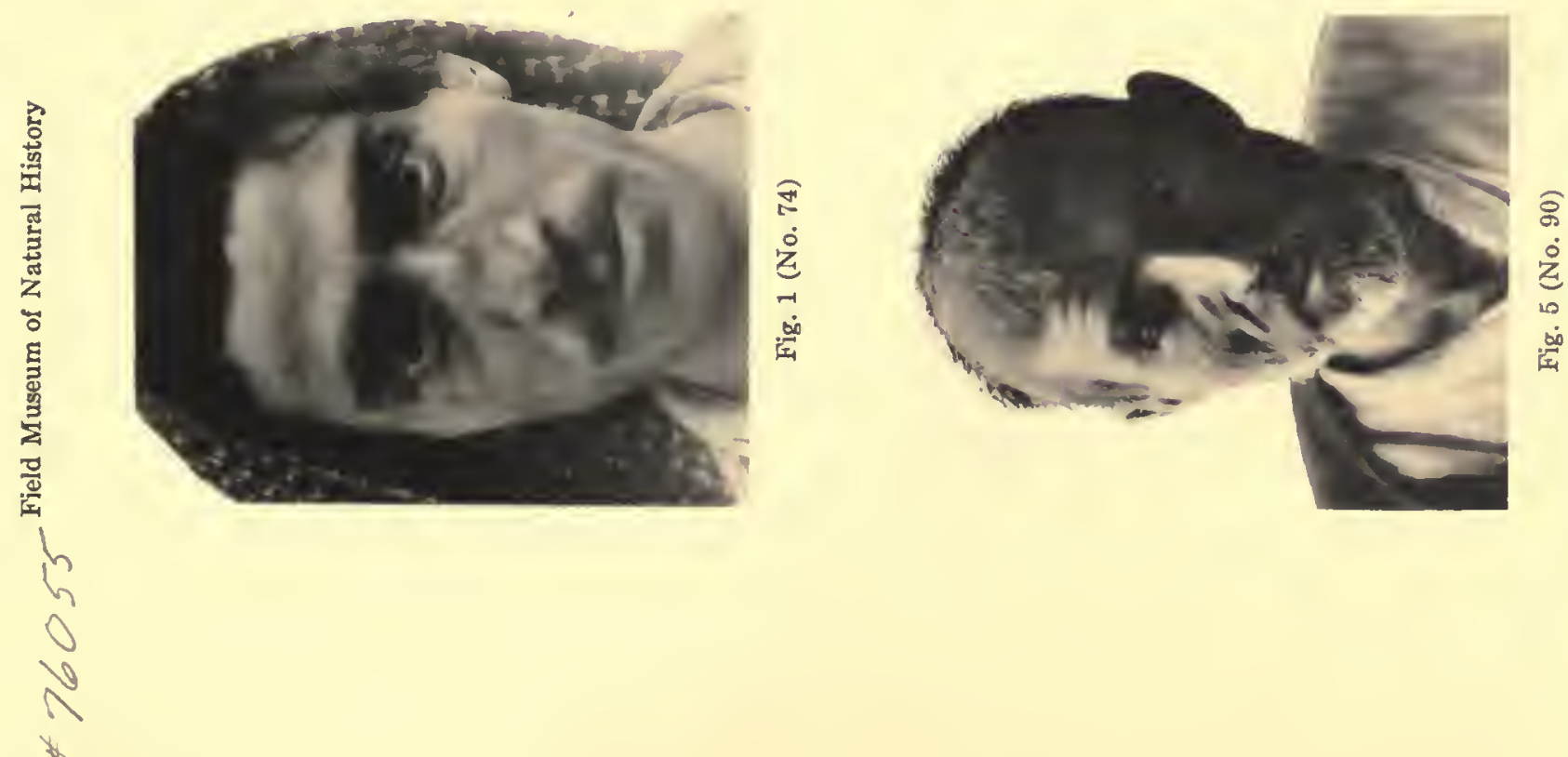

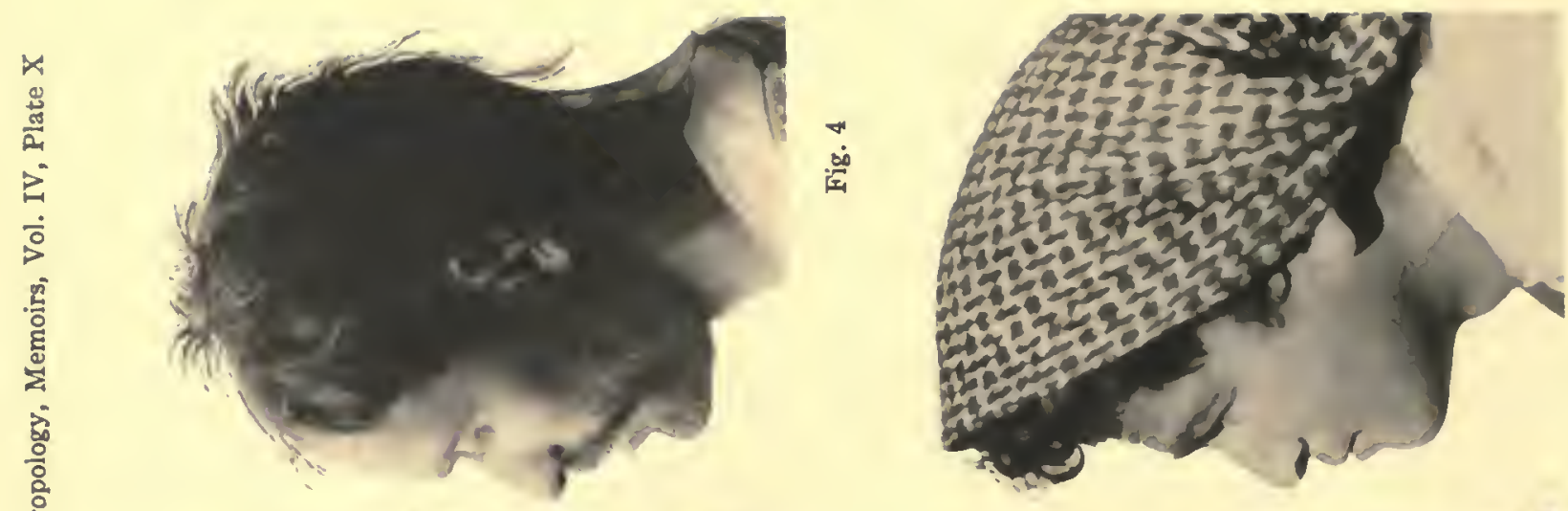

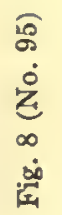
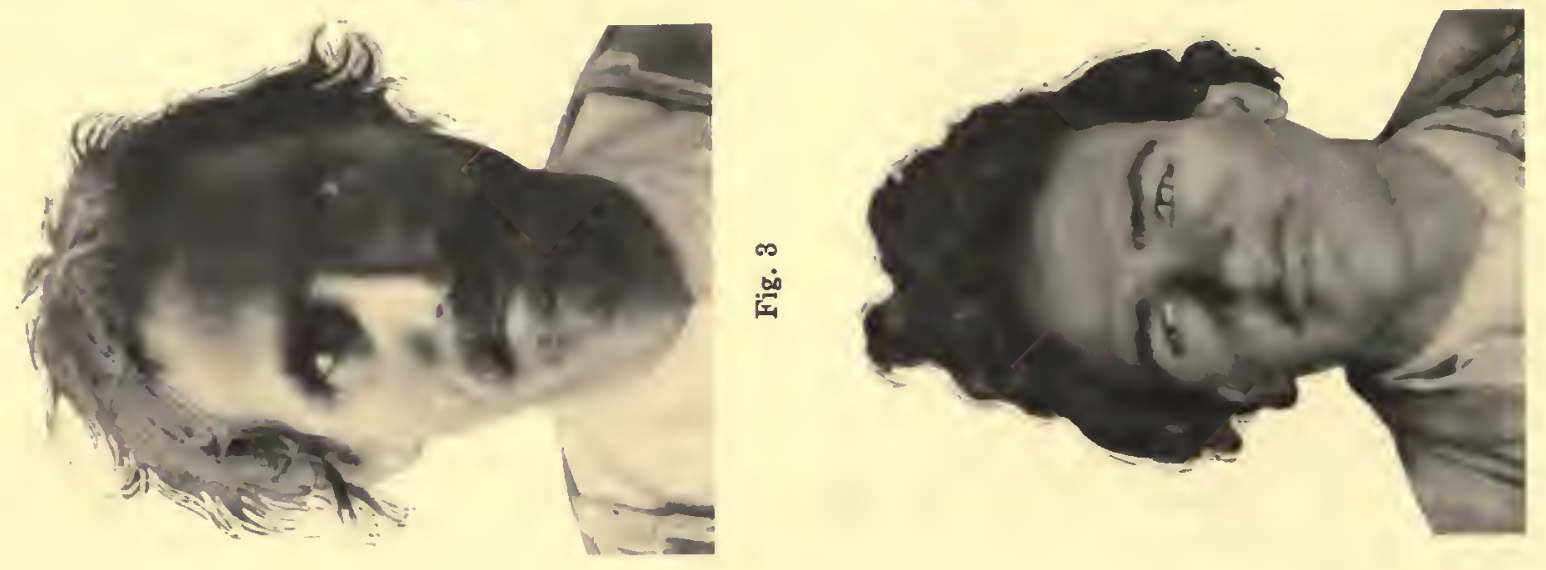

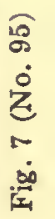
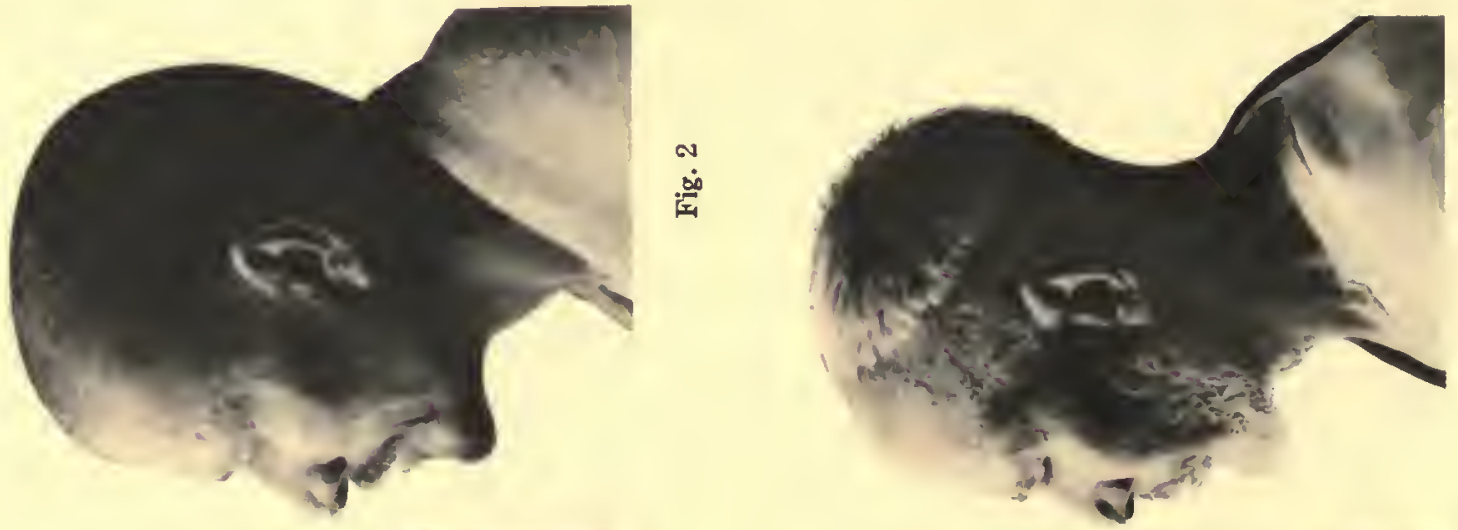

ส
$\dot{0}$
$\vdots$
0
$\dot{0}$

空
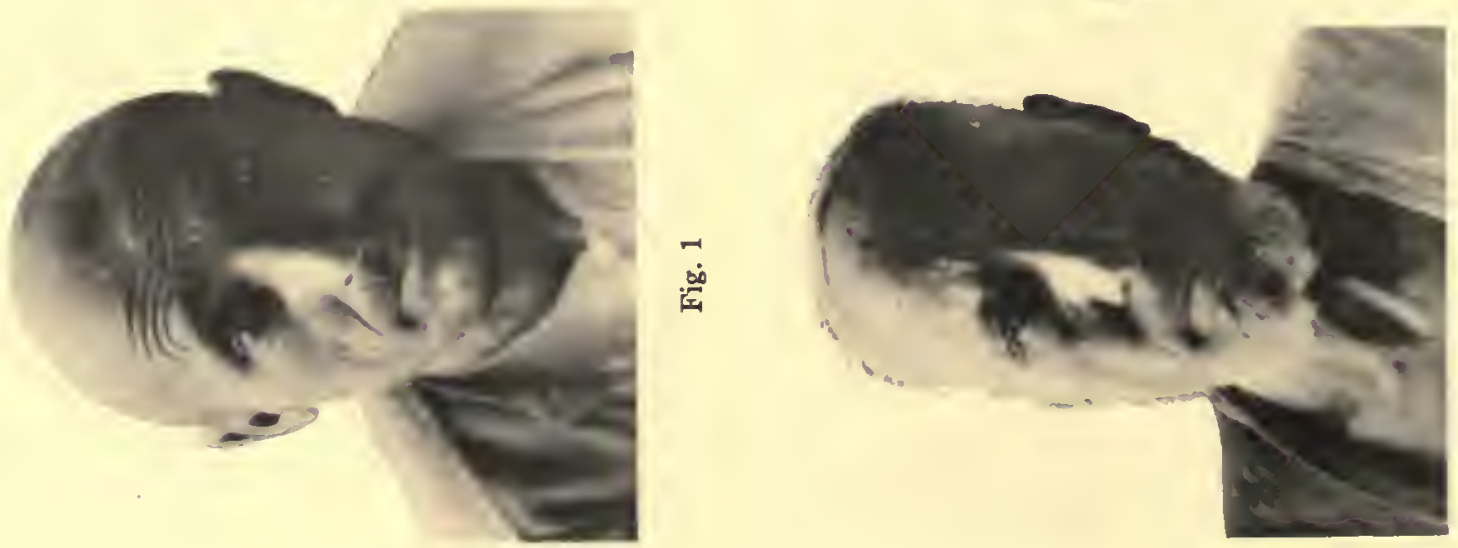

สิ
$\dot{0}$
$\dot{2}$
10
10
0

趁 N 

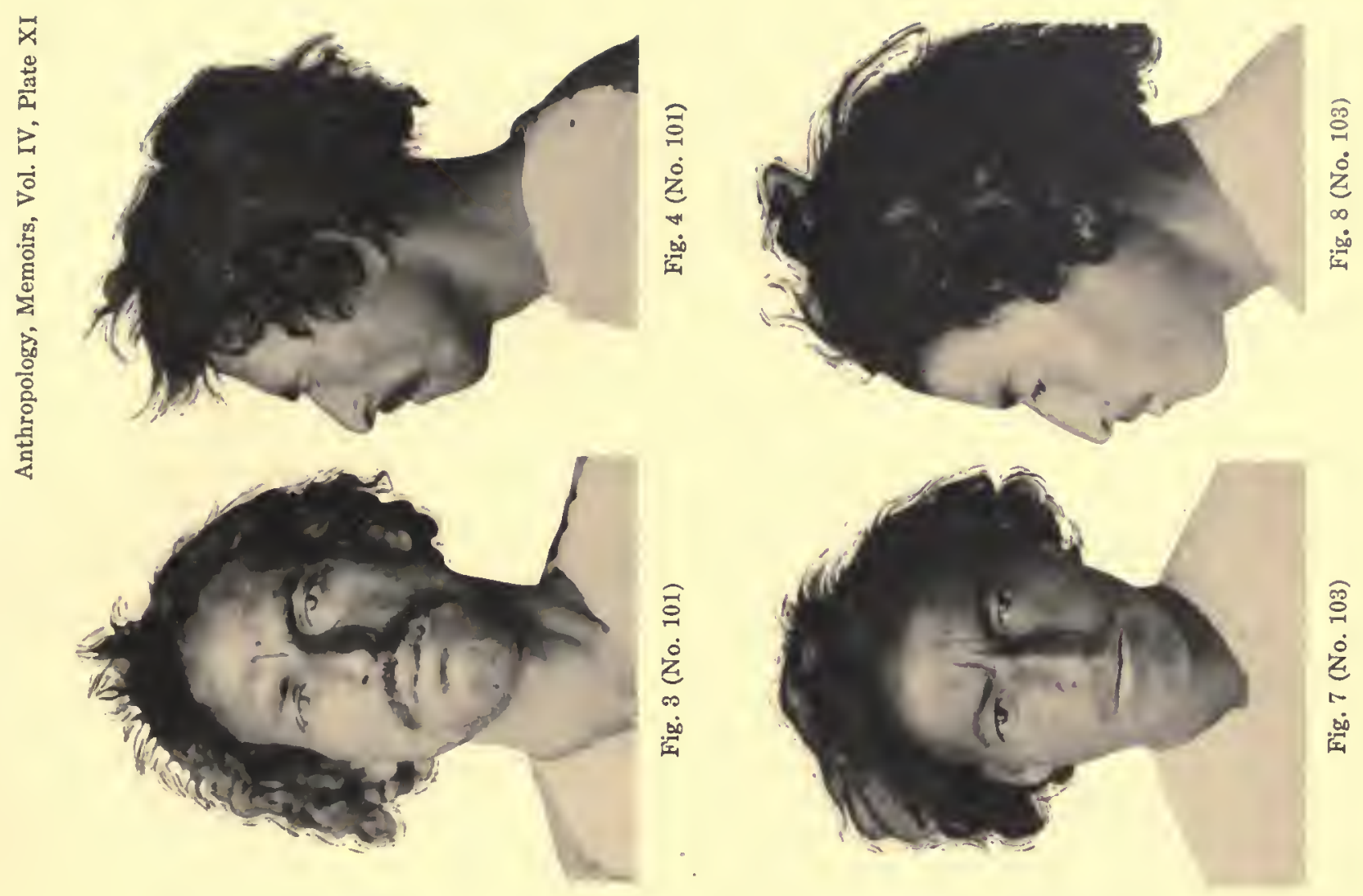

空
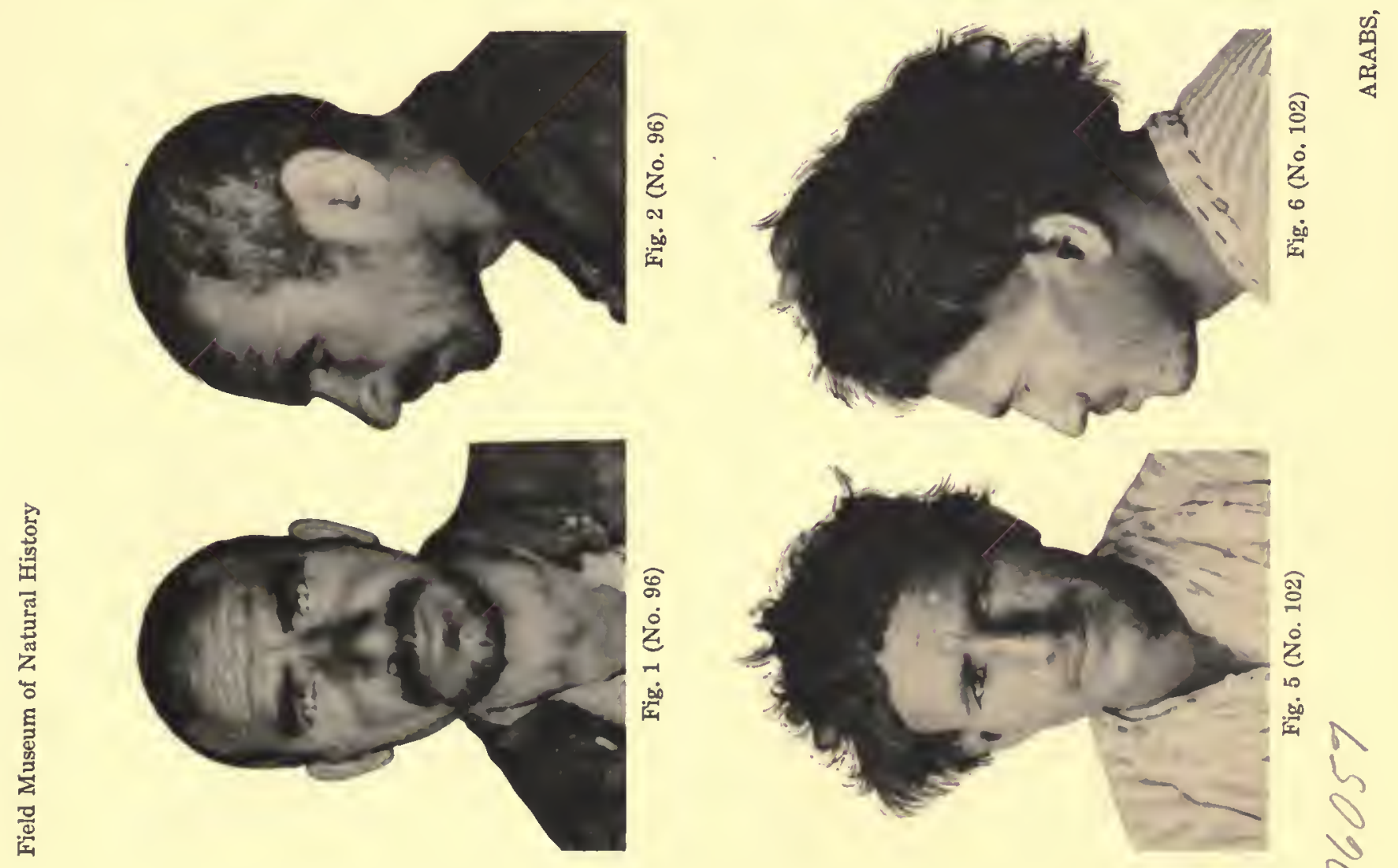

v $x$ 

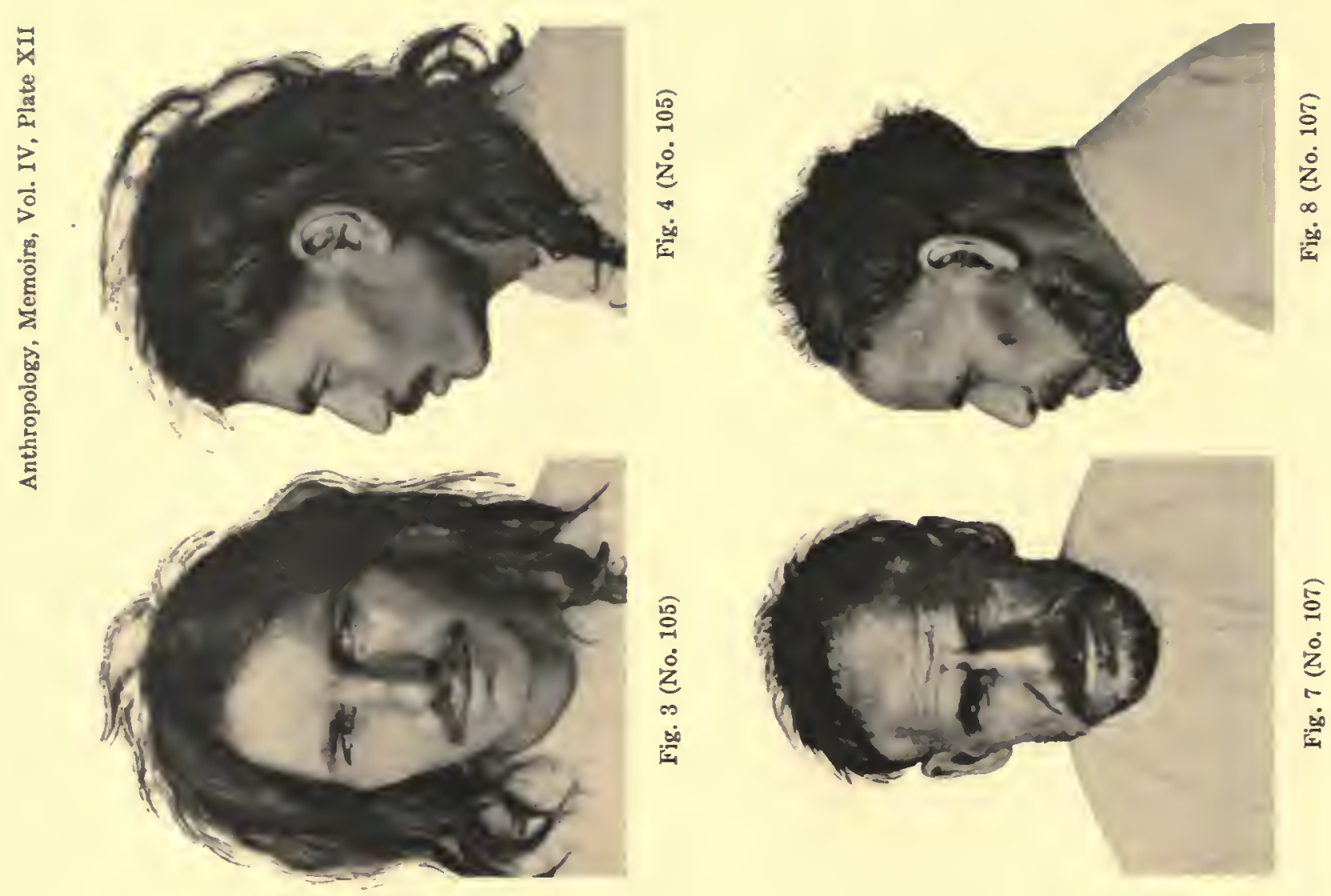

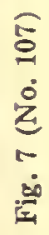
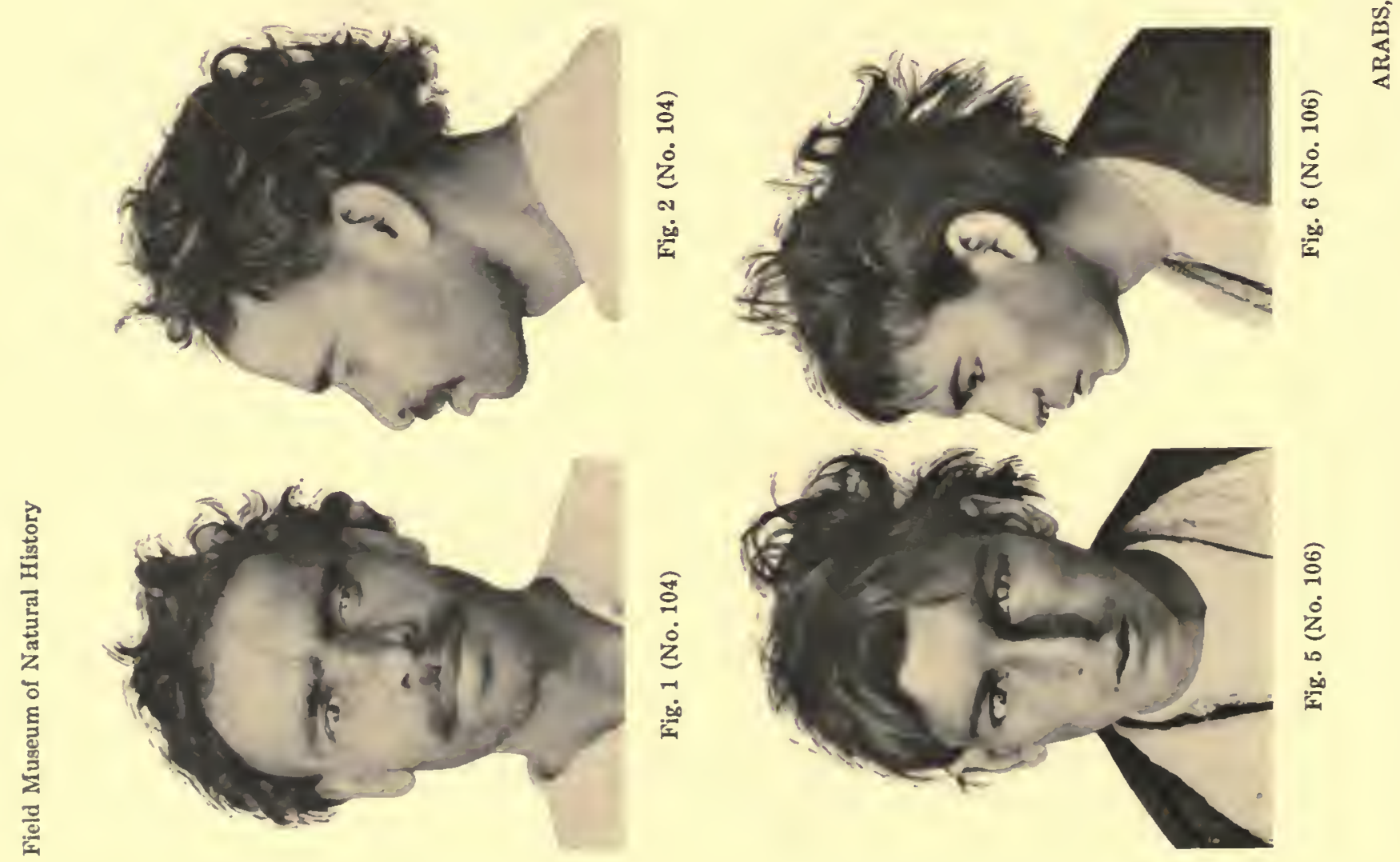
볼
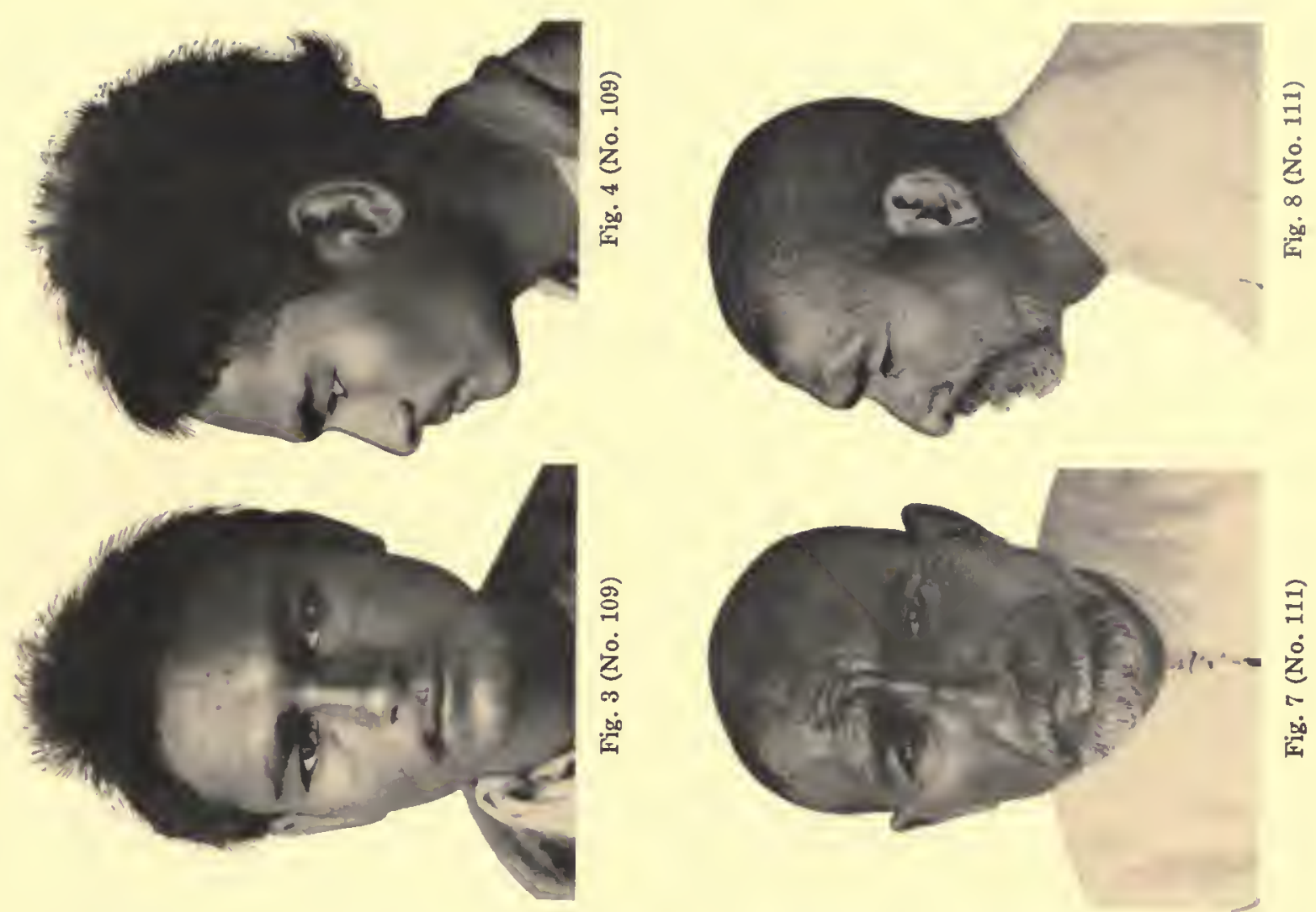

臿
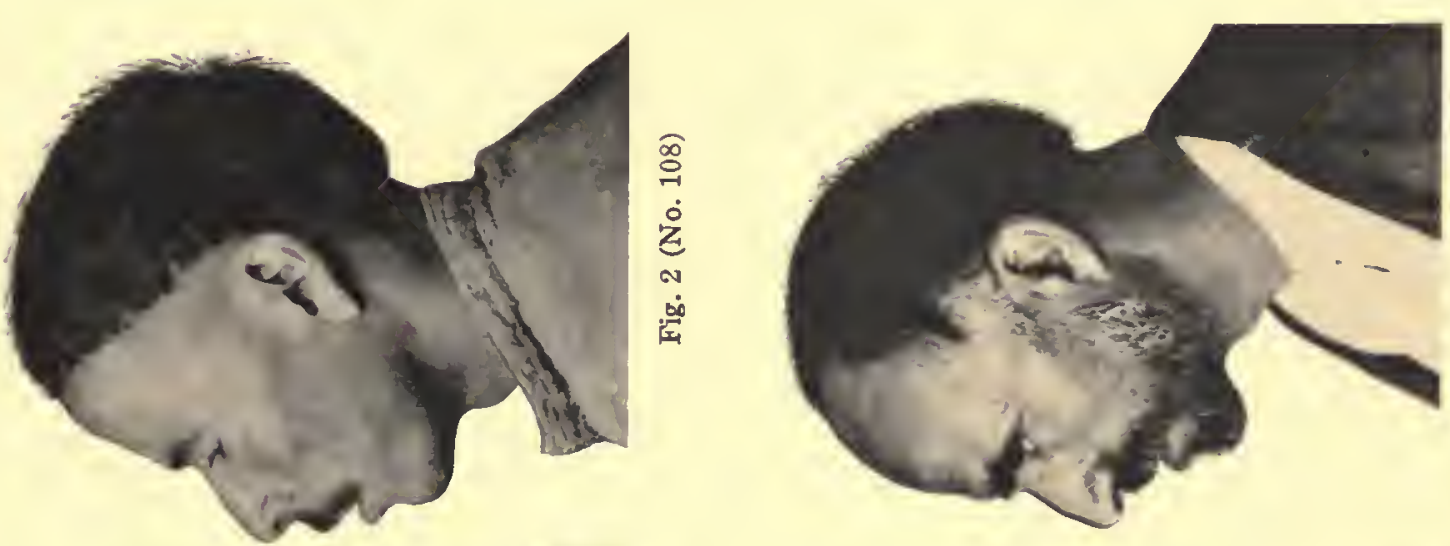

造

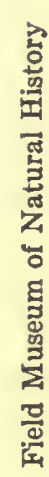
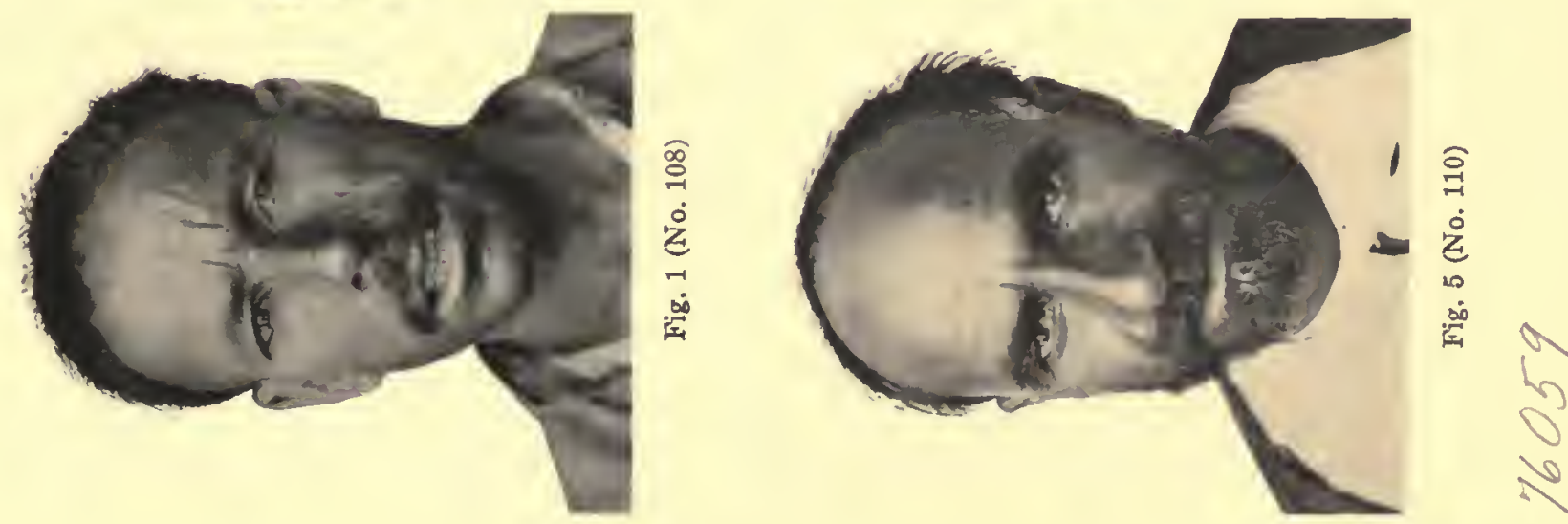

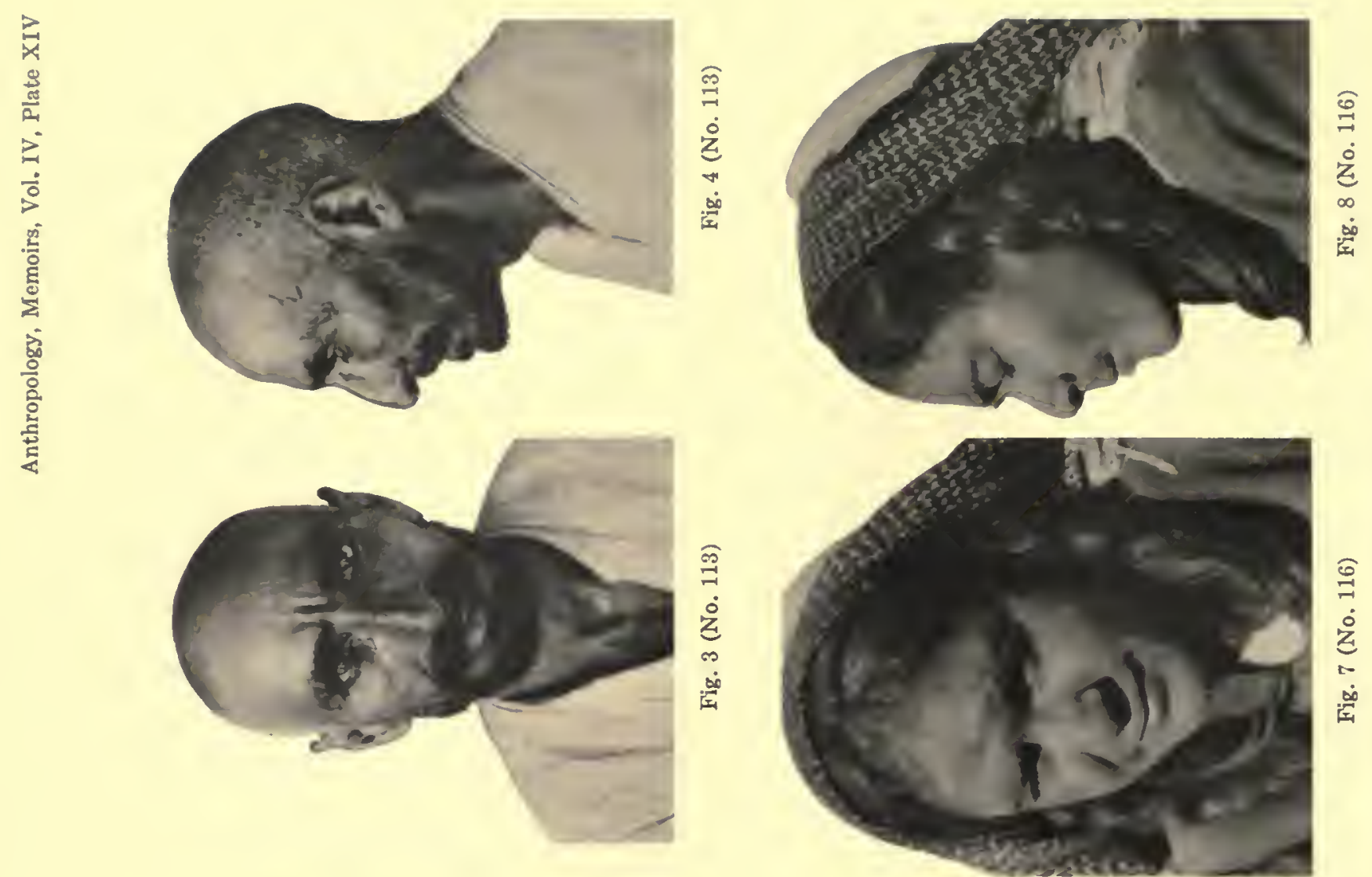

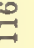

눈

車
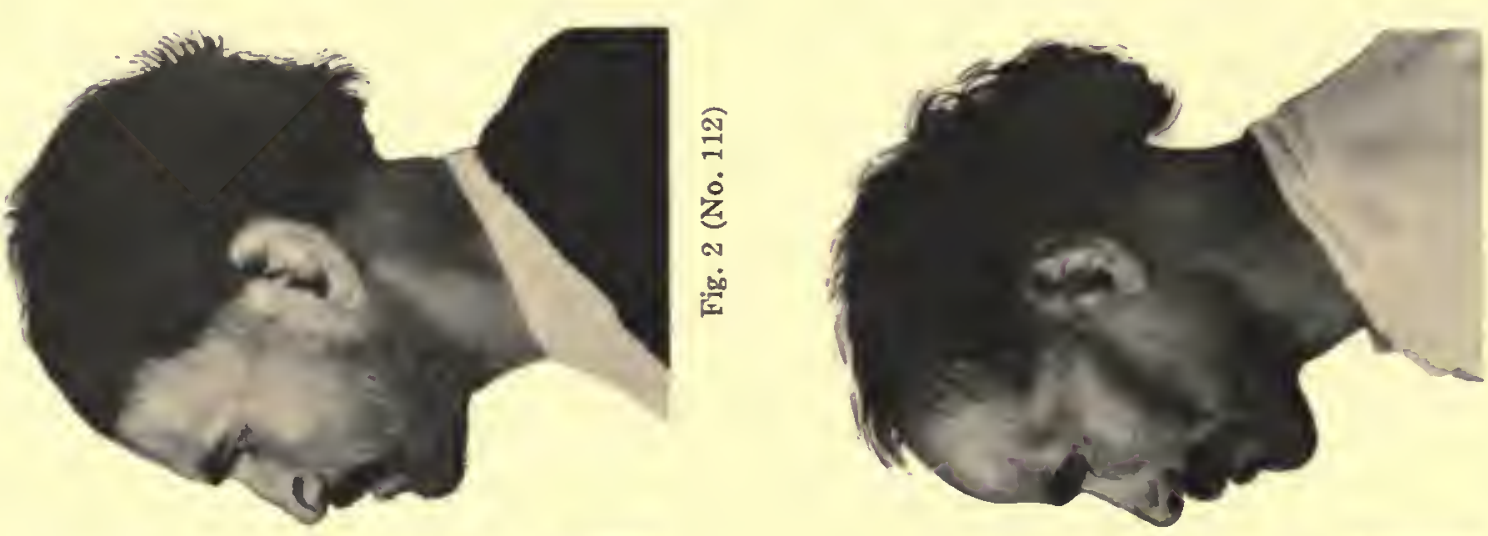

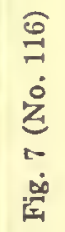

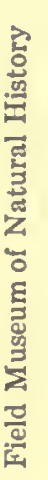
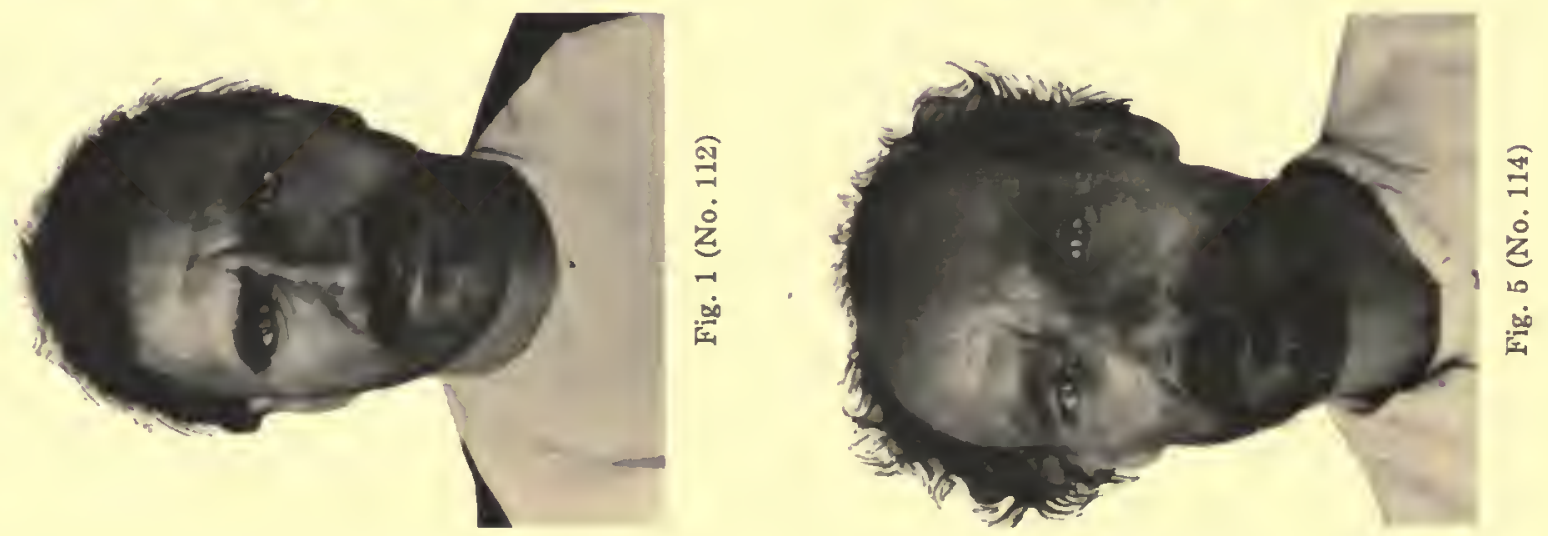

1 

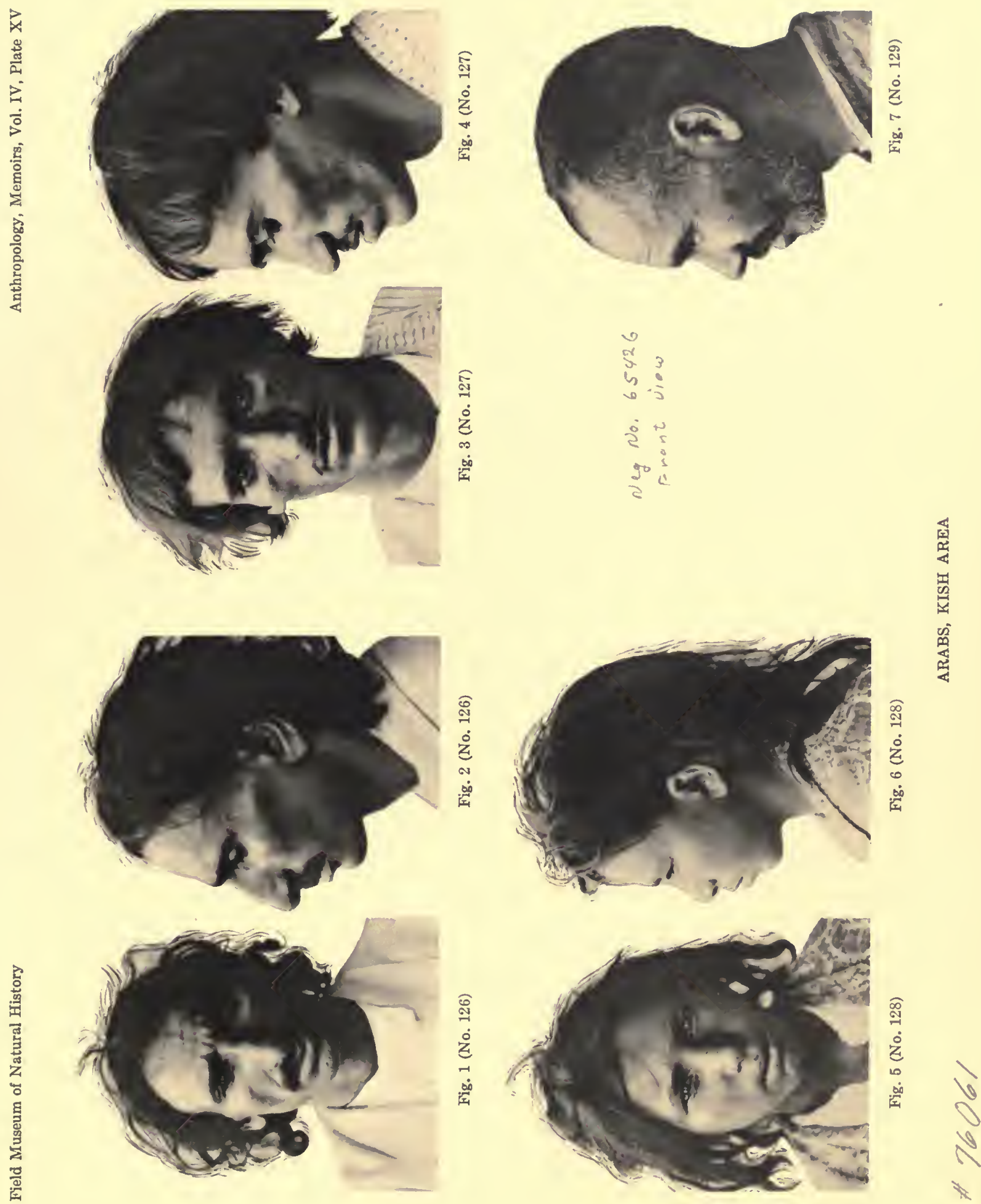

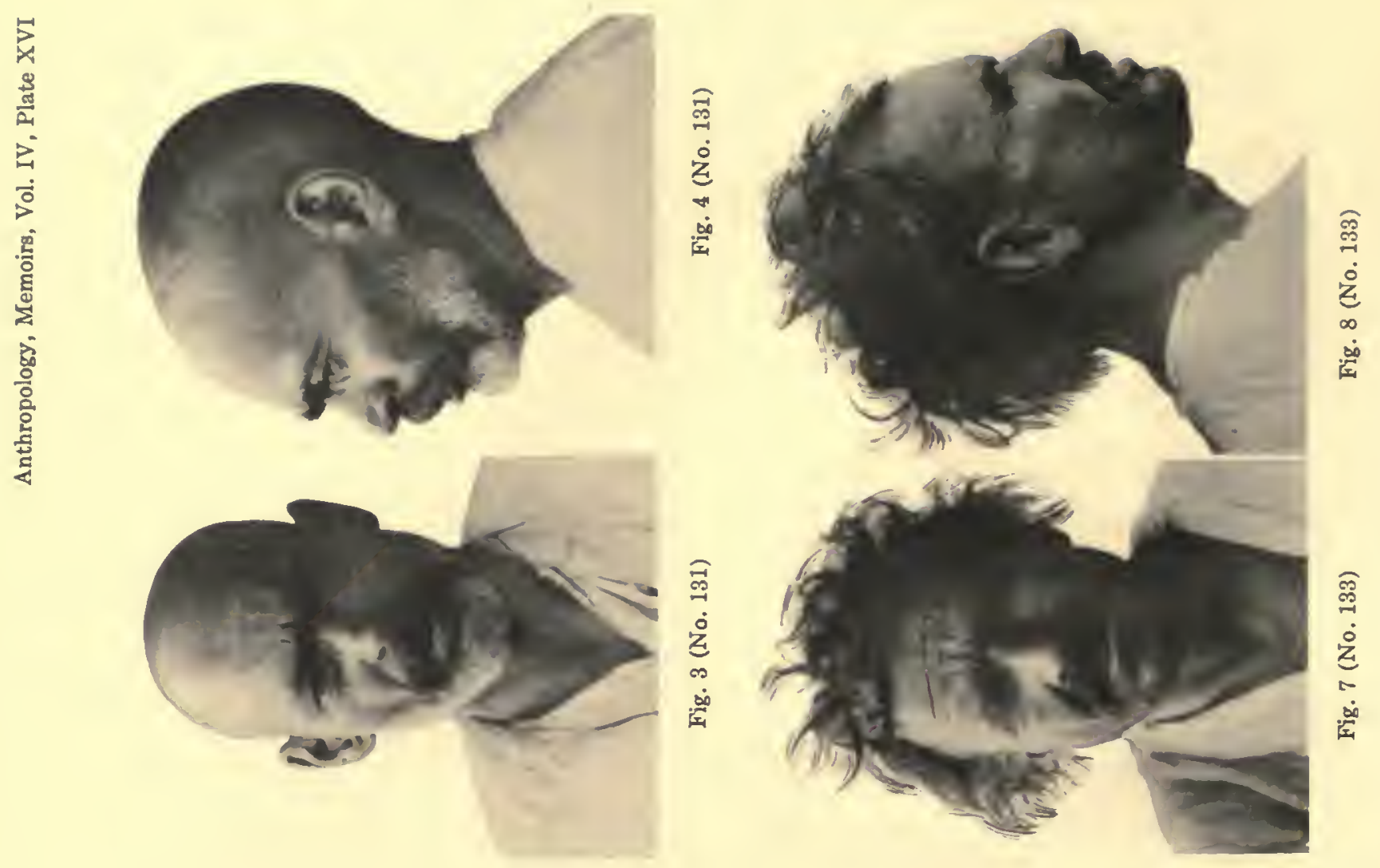

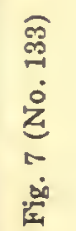
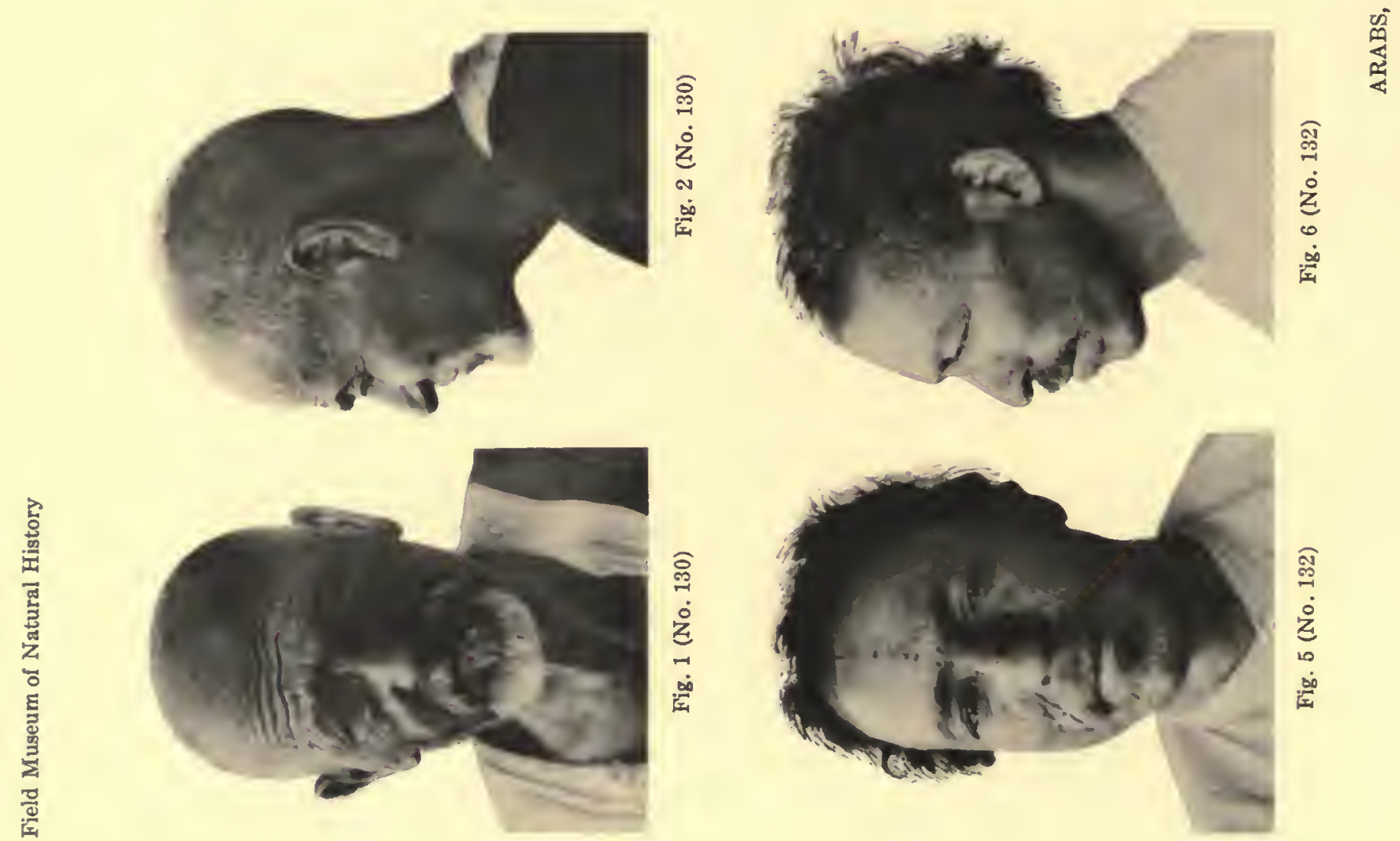

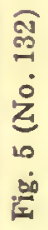




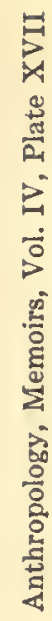
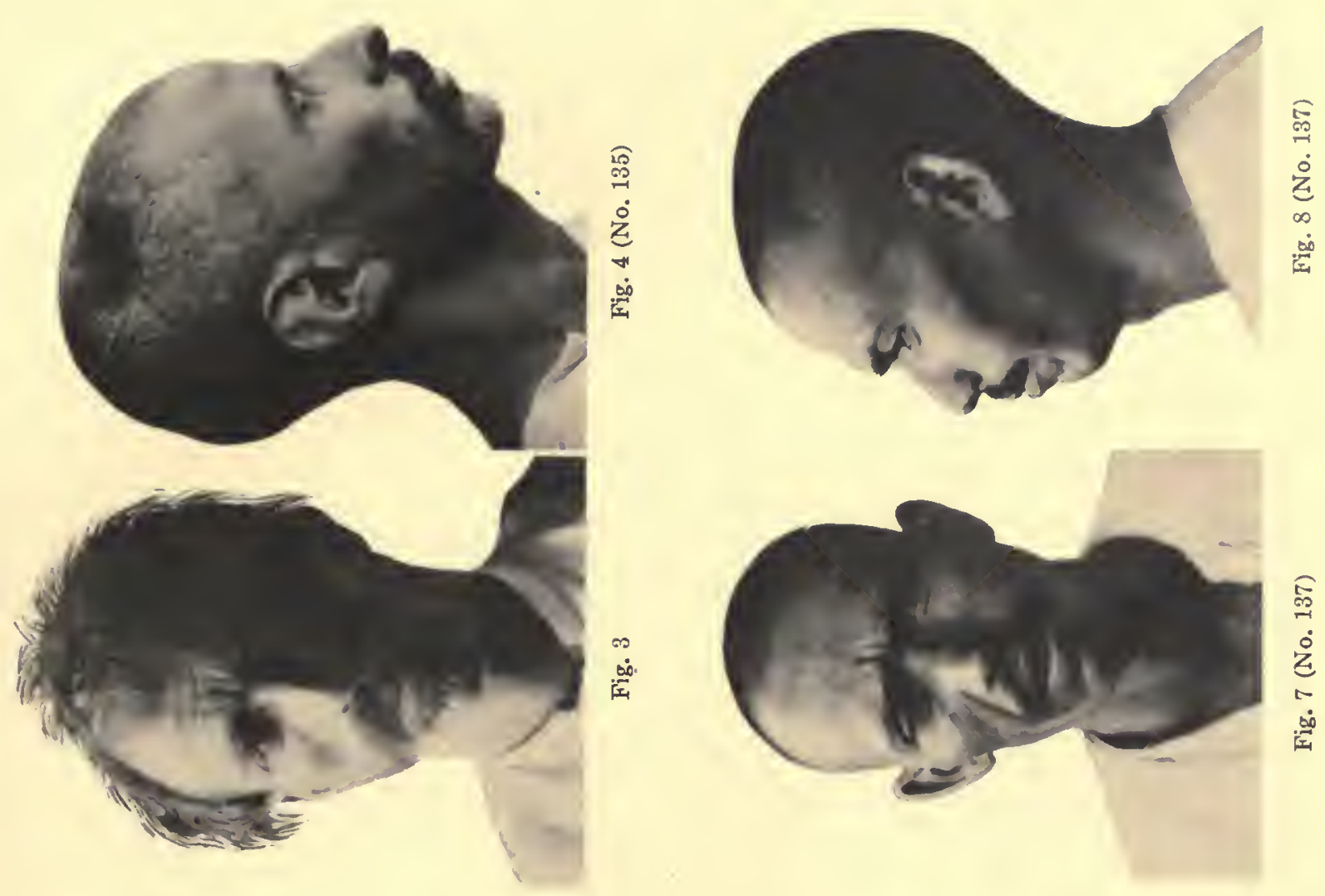

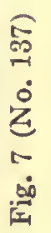
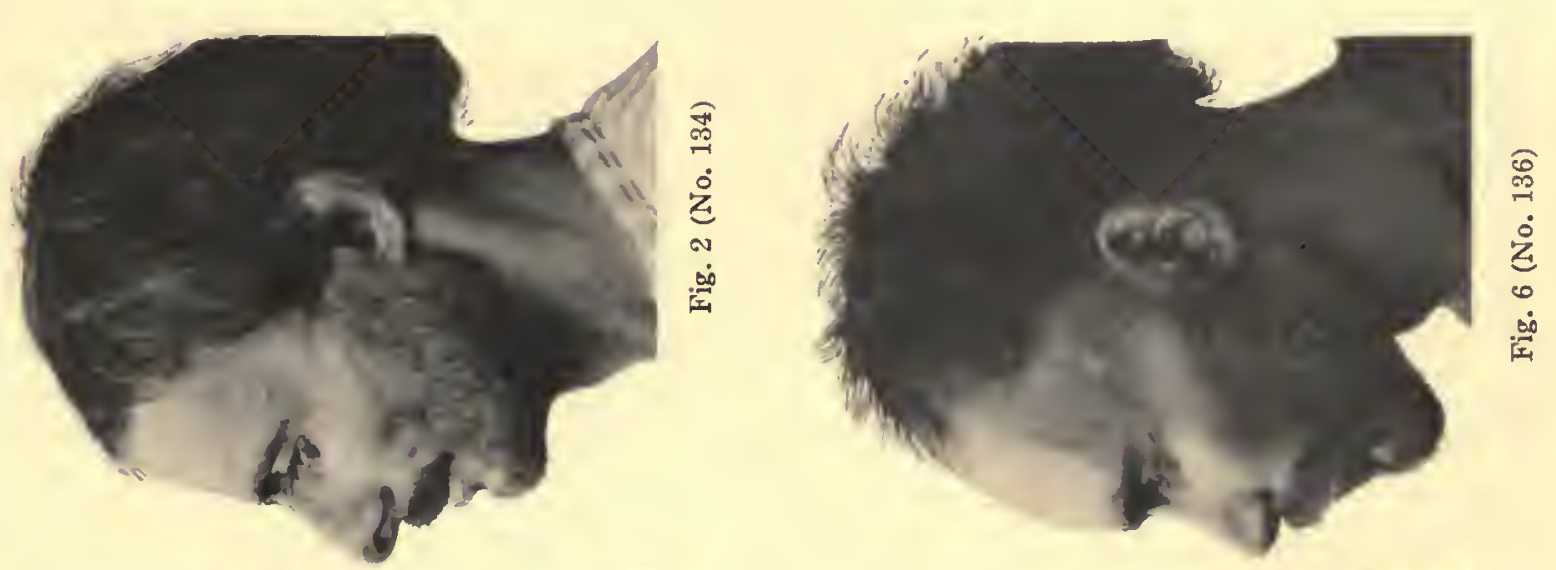

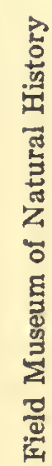
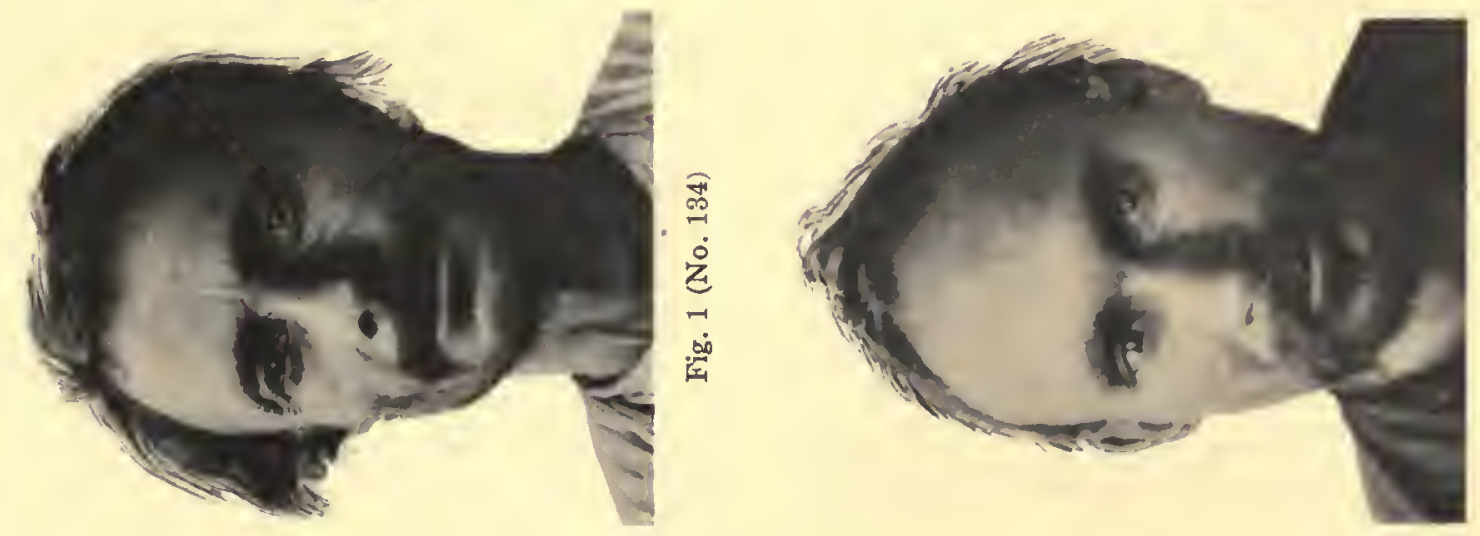

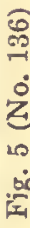

造

追 


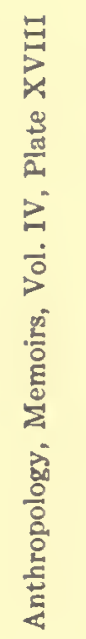
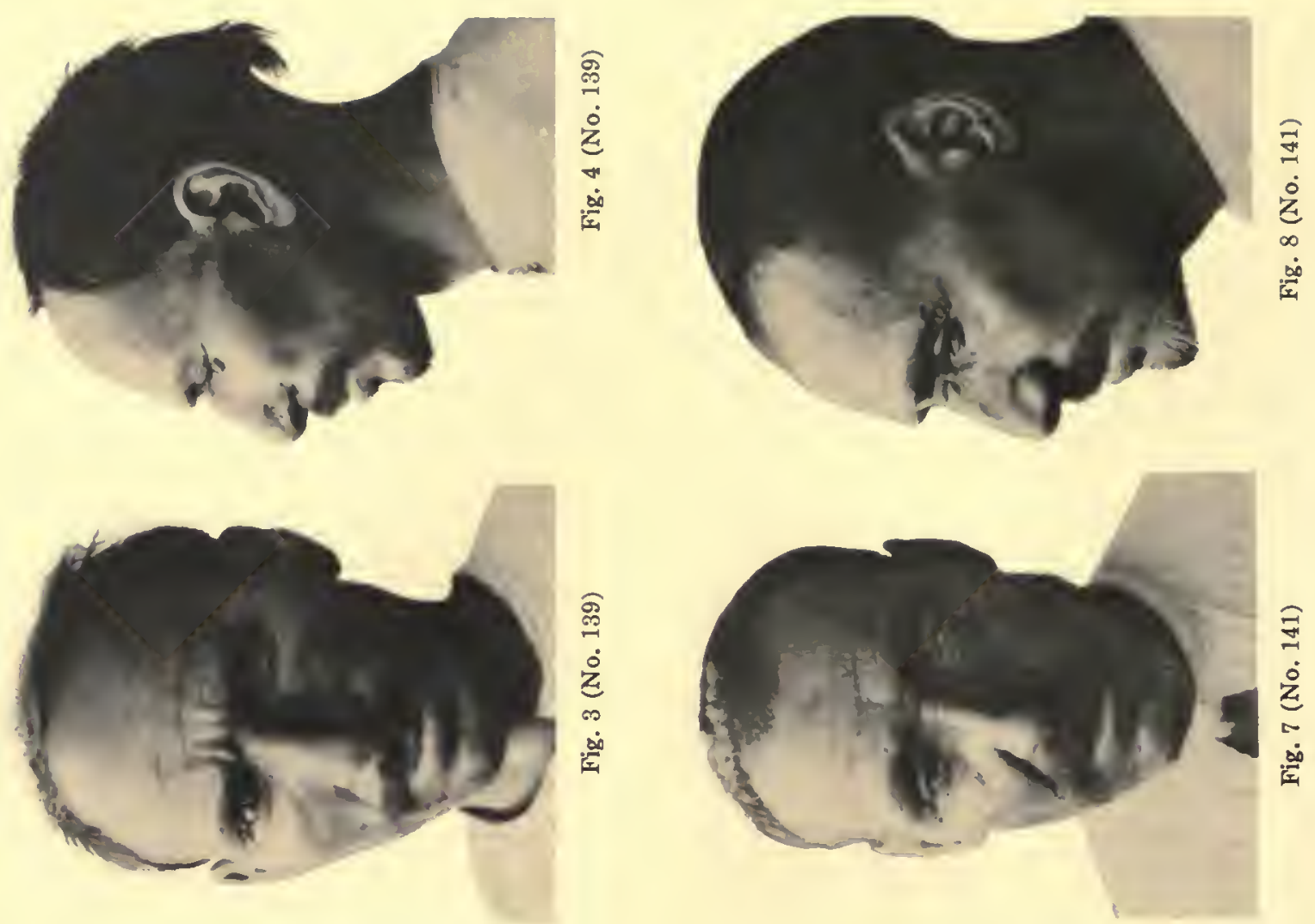

亲
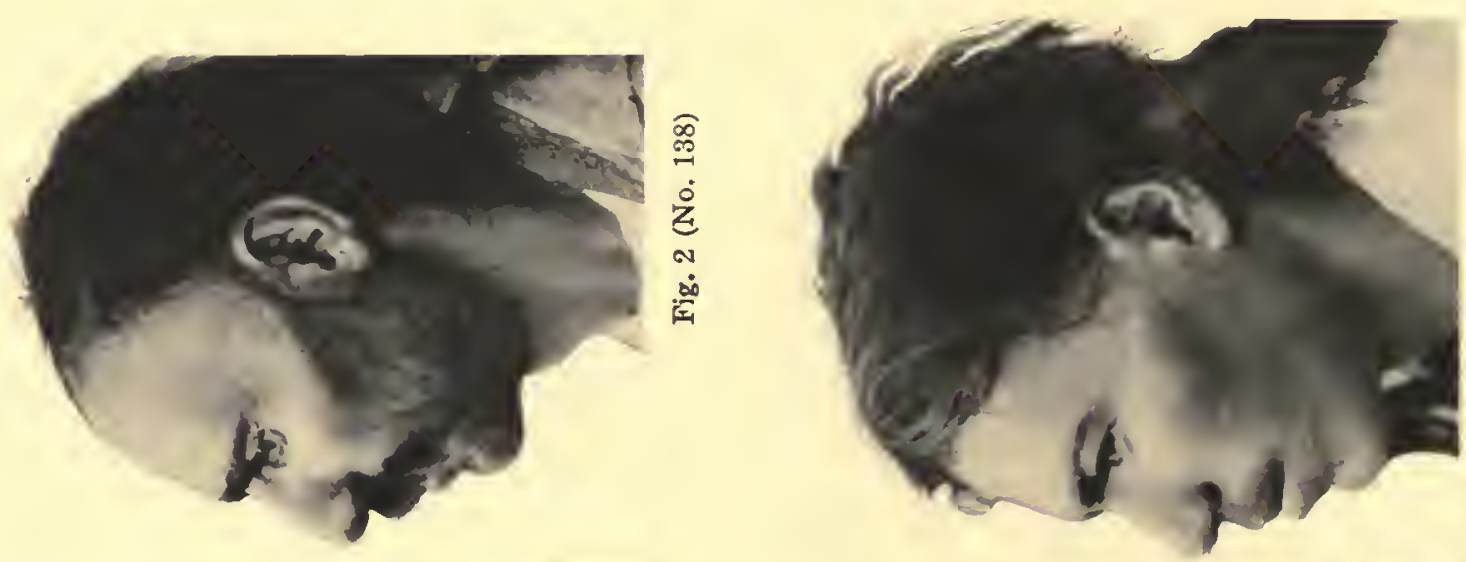

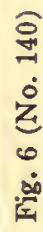

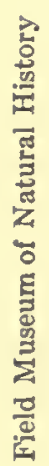
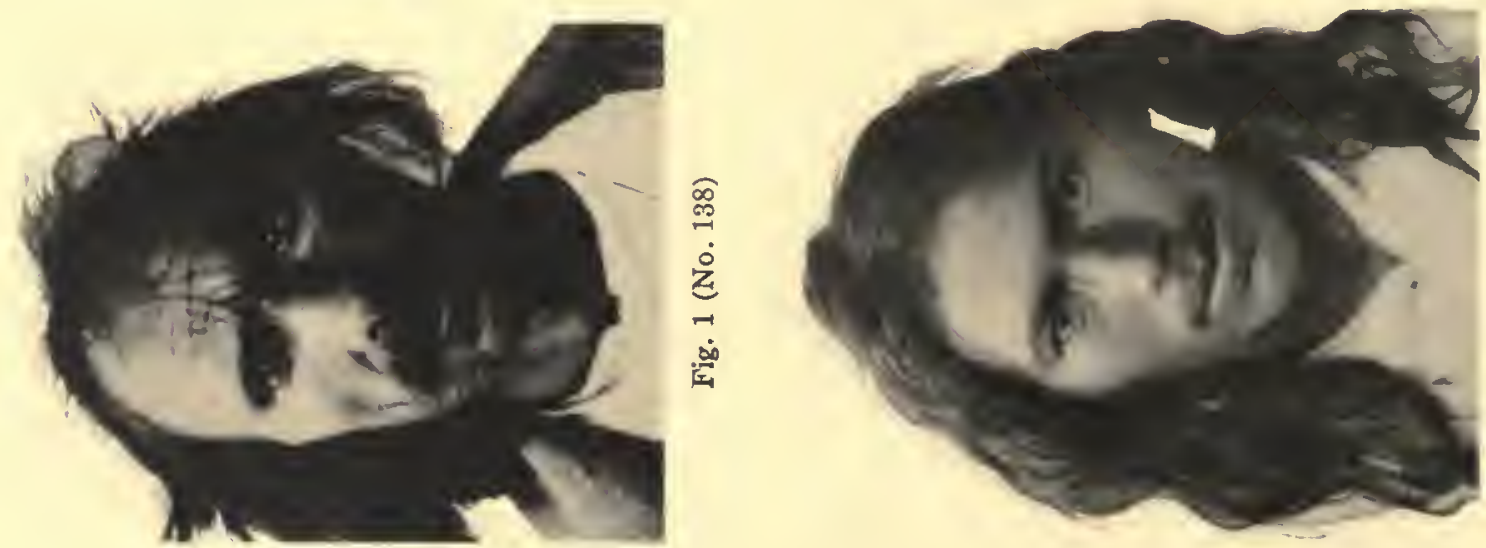

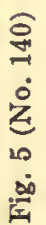




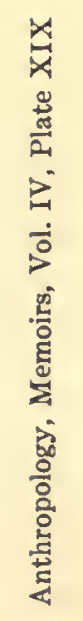
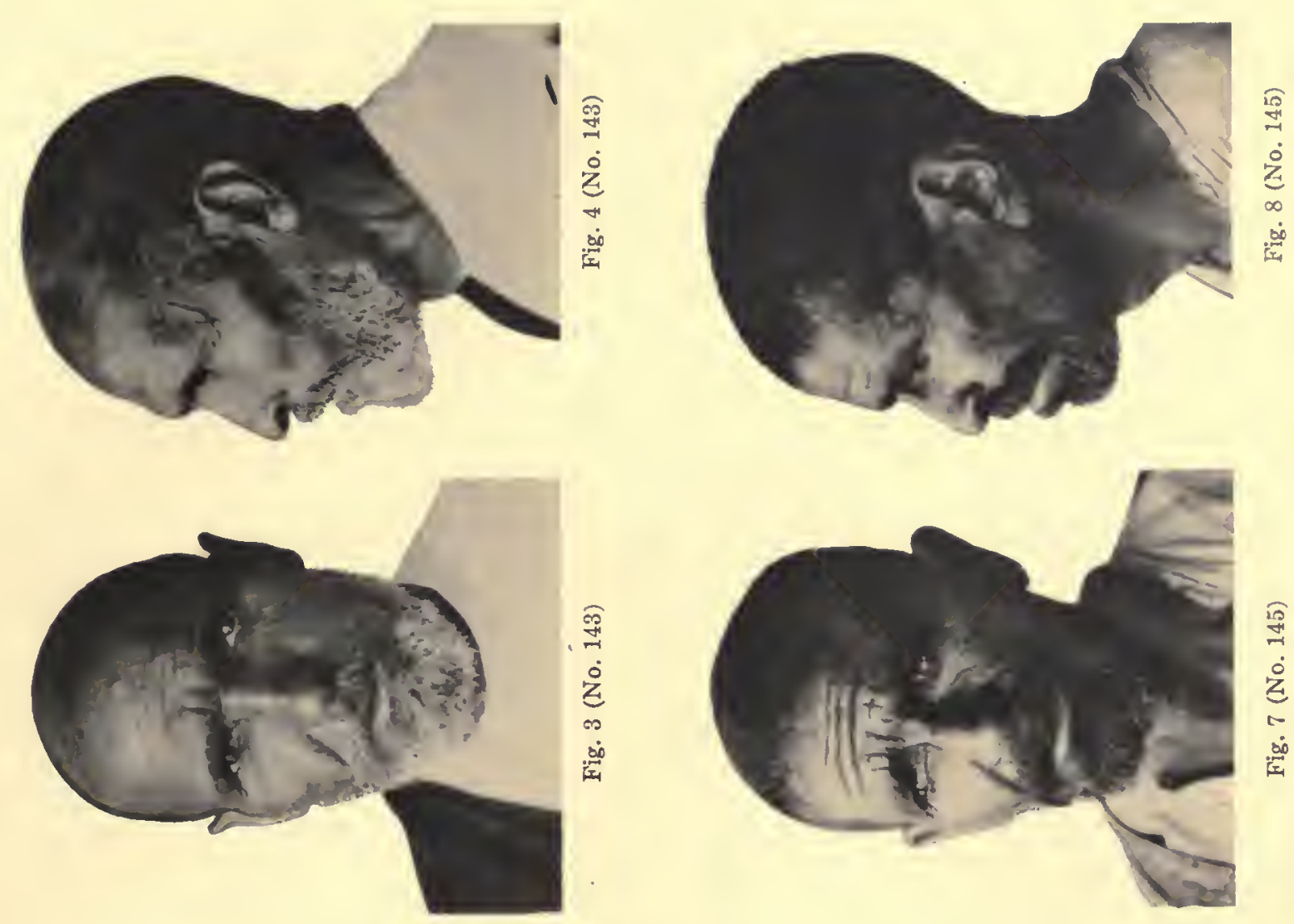

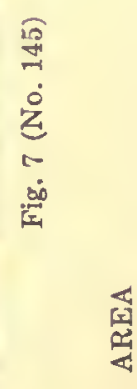
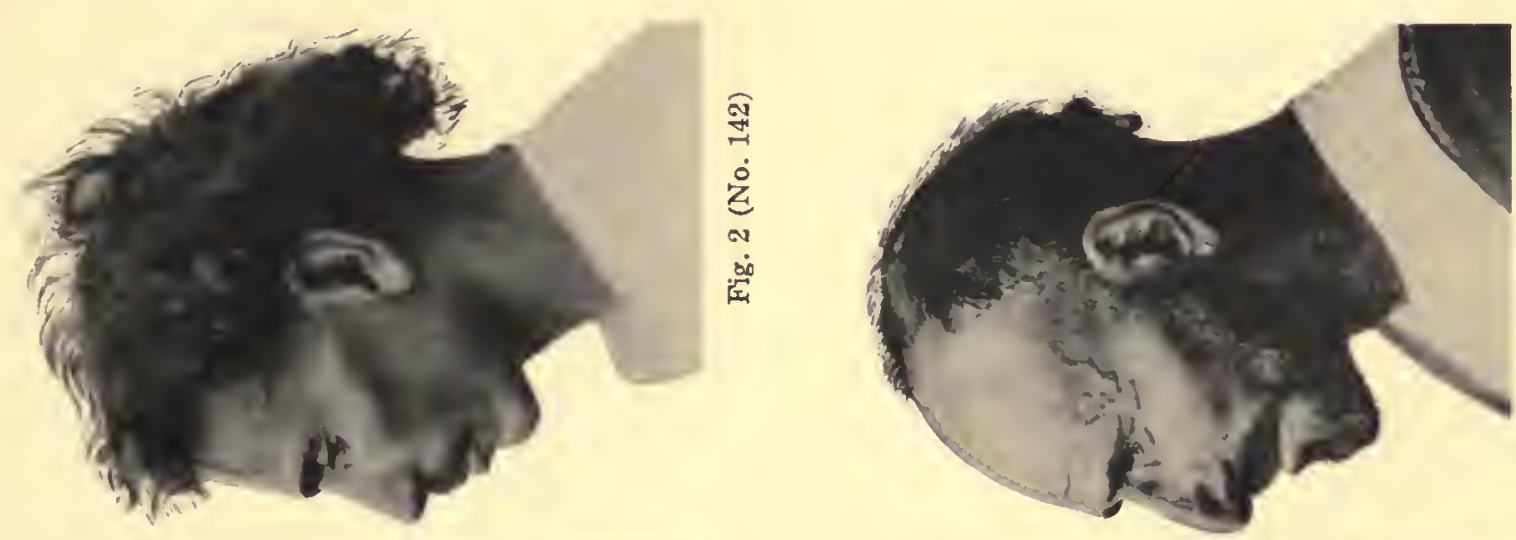

盟
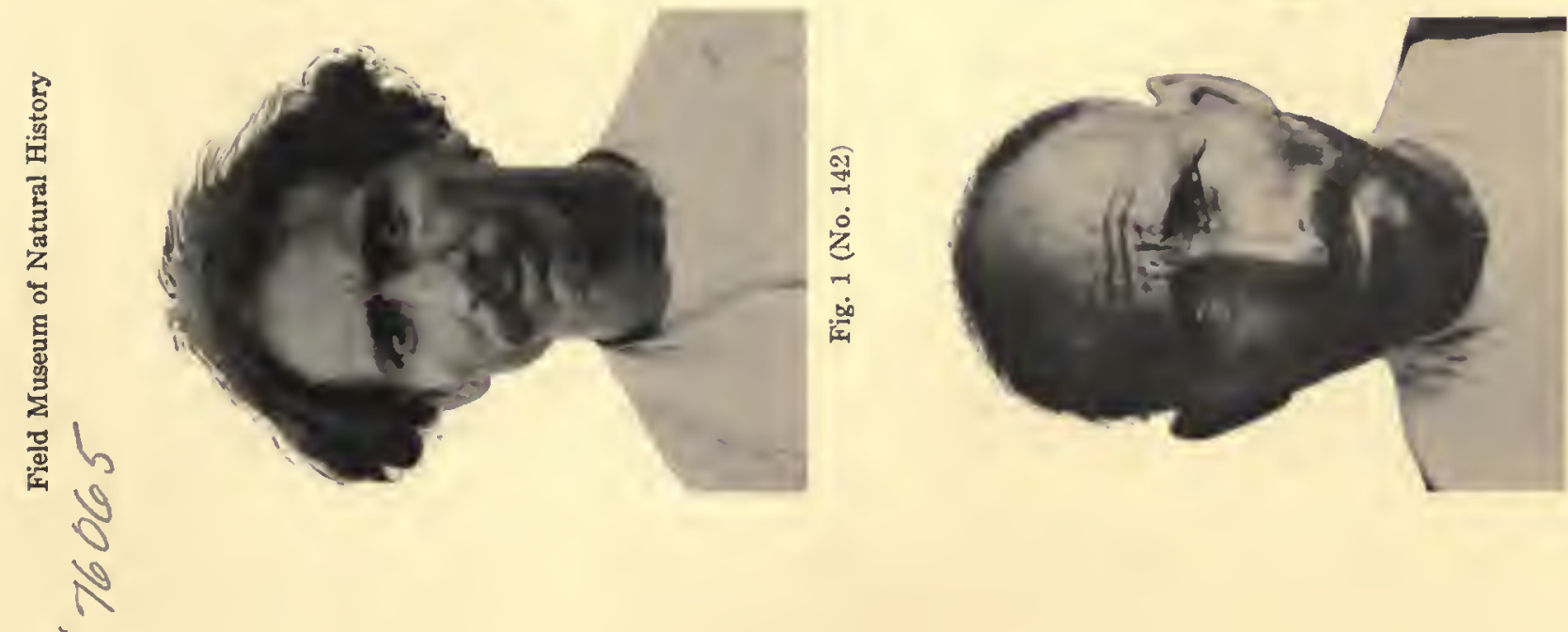

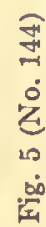
신 

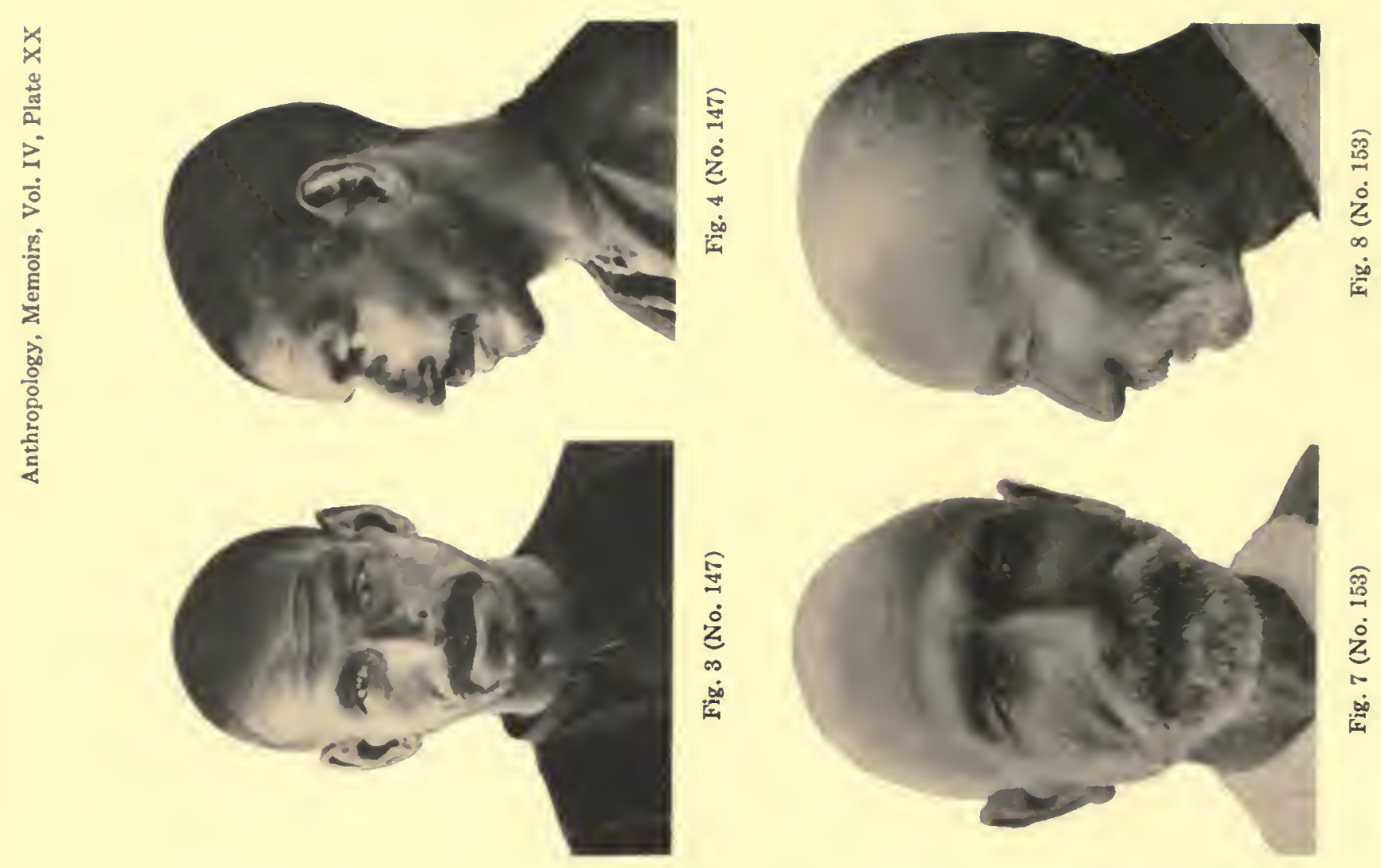

总
$\dot{0}$
$\dot{z}$
$\vdots$
$\dot{0}$
$\dot{a}$
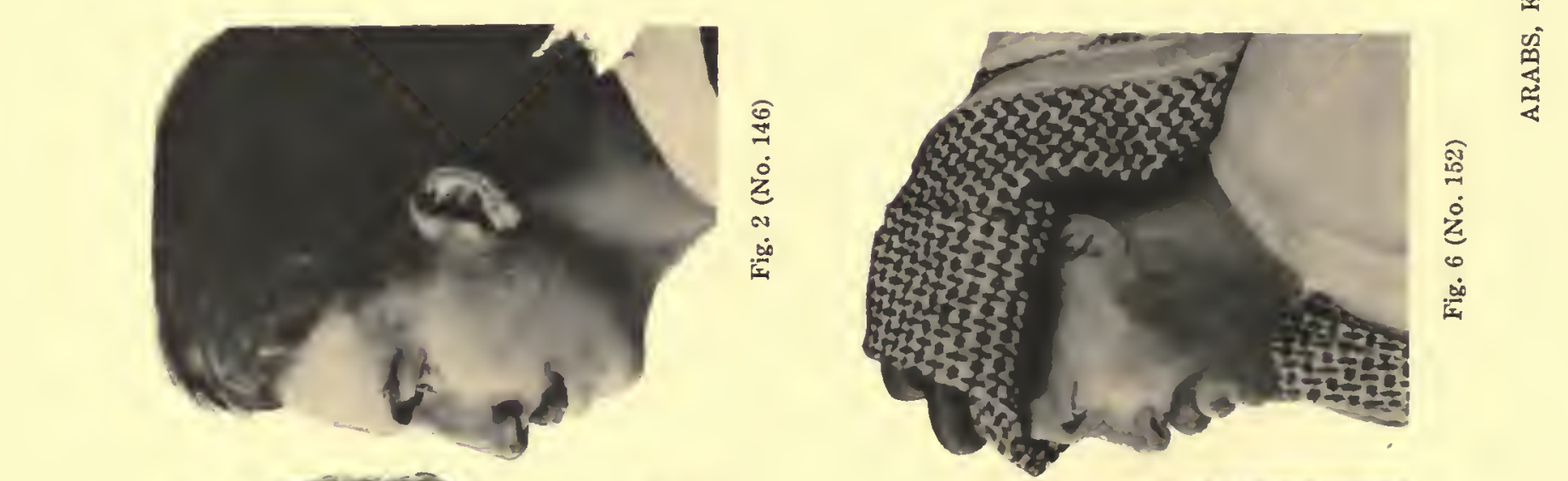

㽰
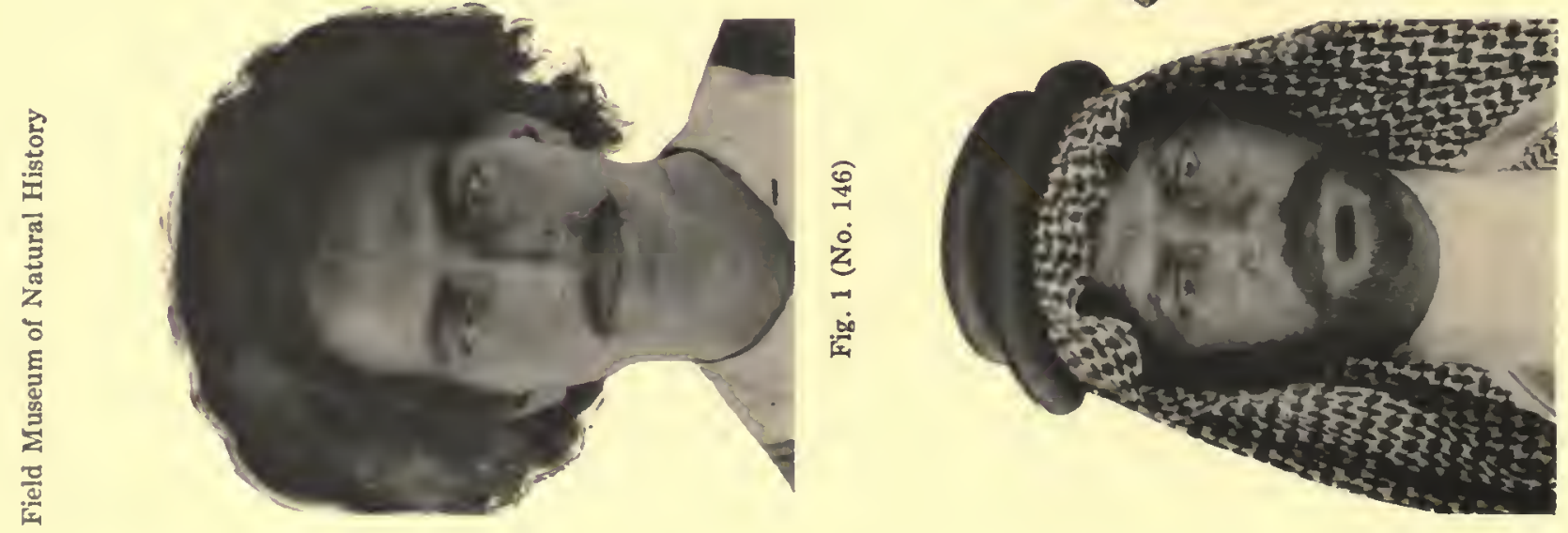

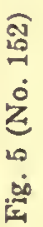




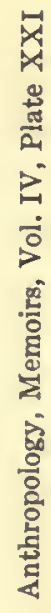
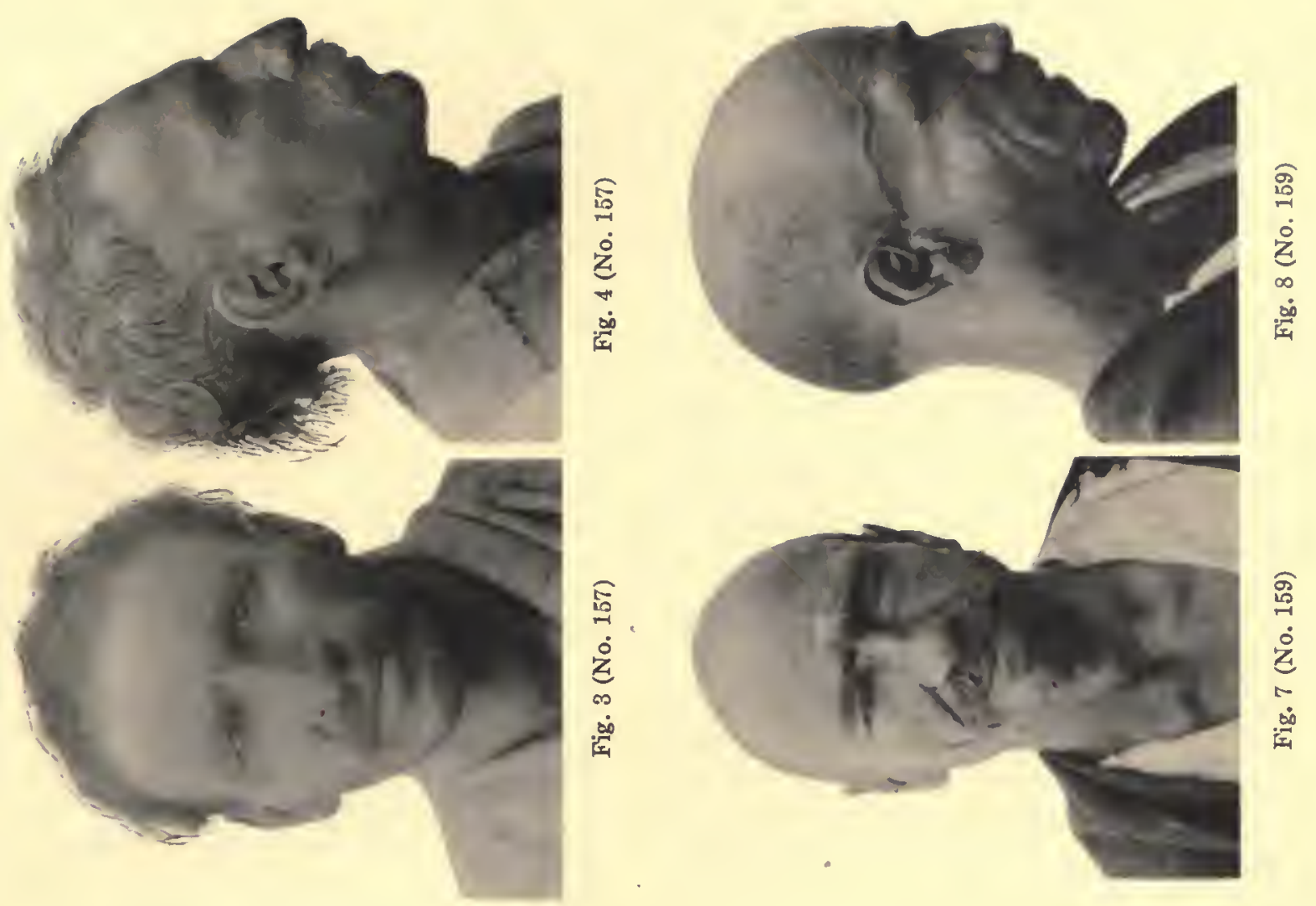

嵒
$\dot{0}$
$\dot{8}$
0
离
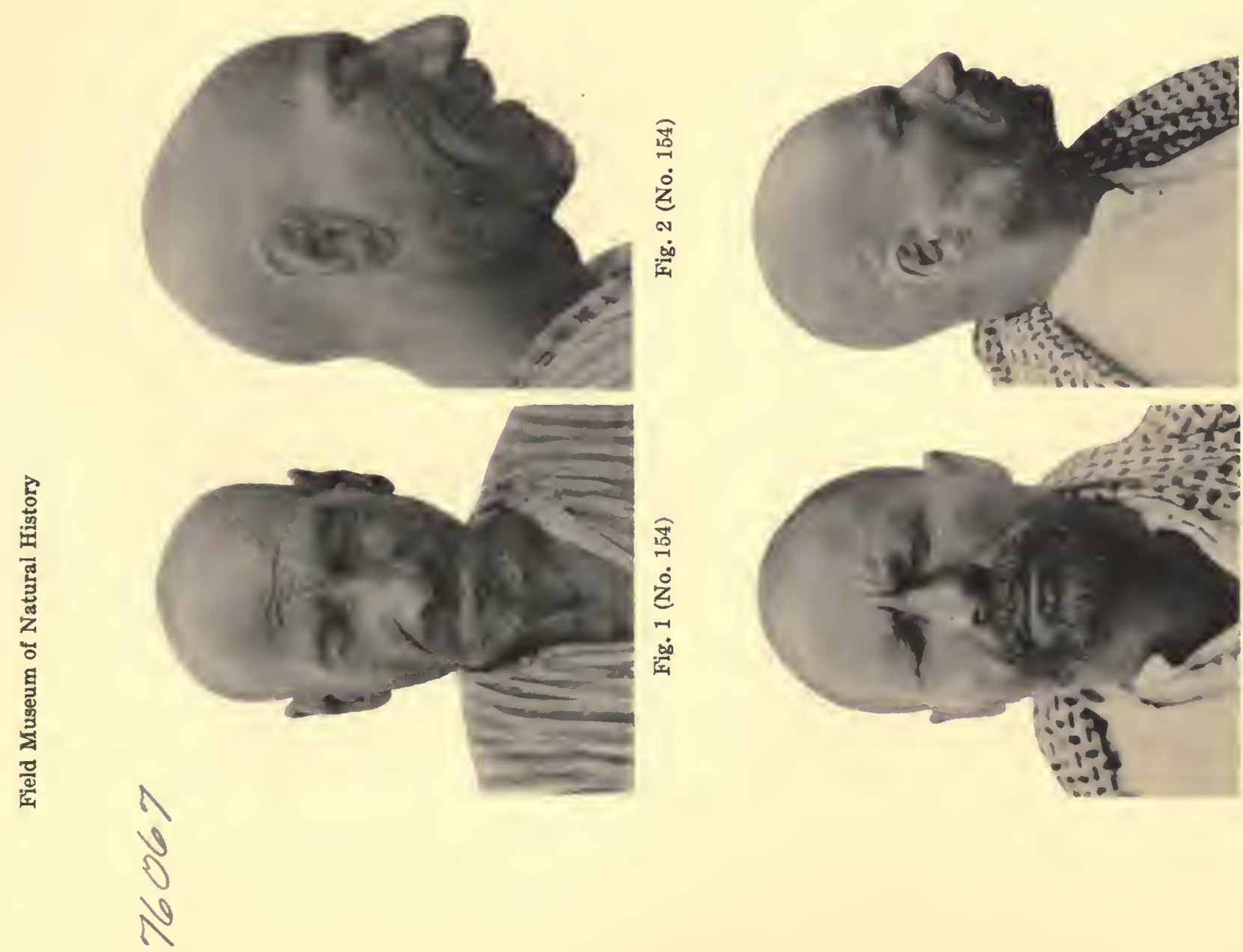

急 营 

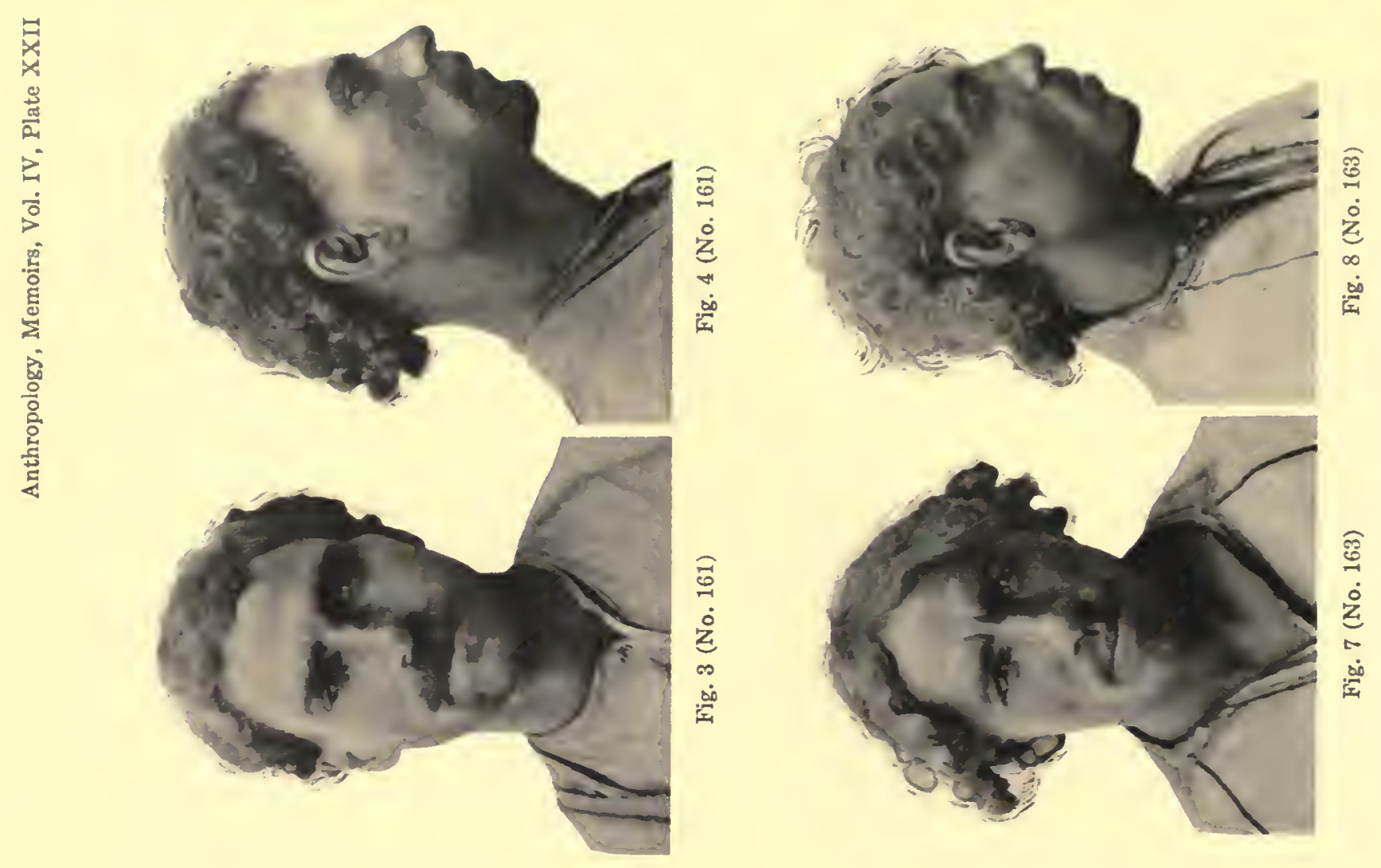

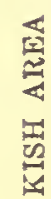

8

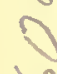

in

th

N
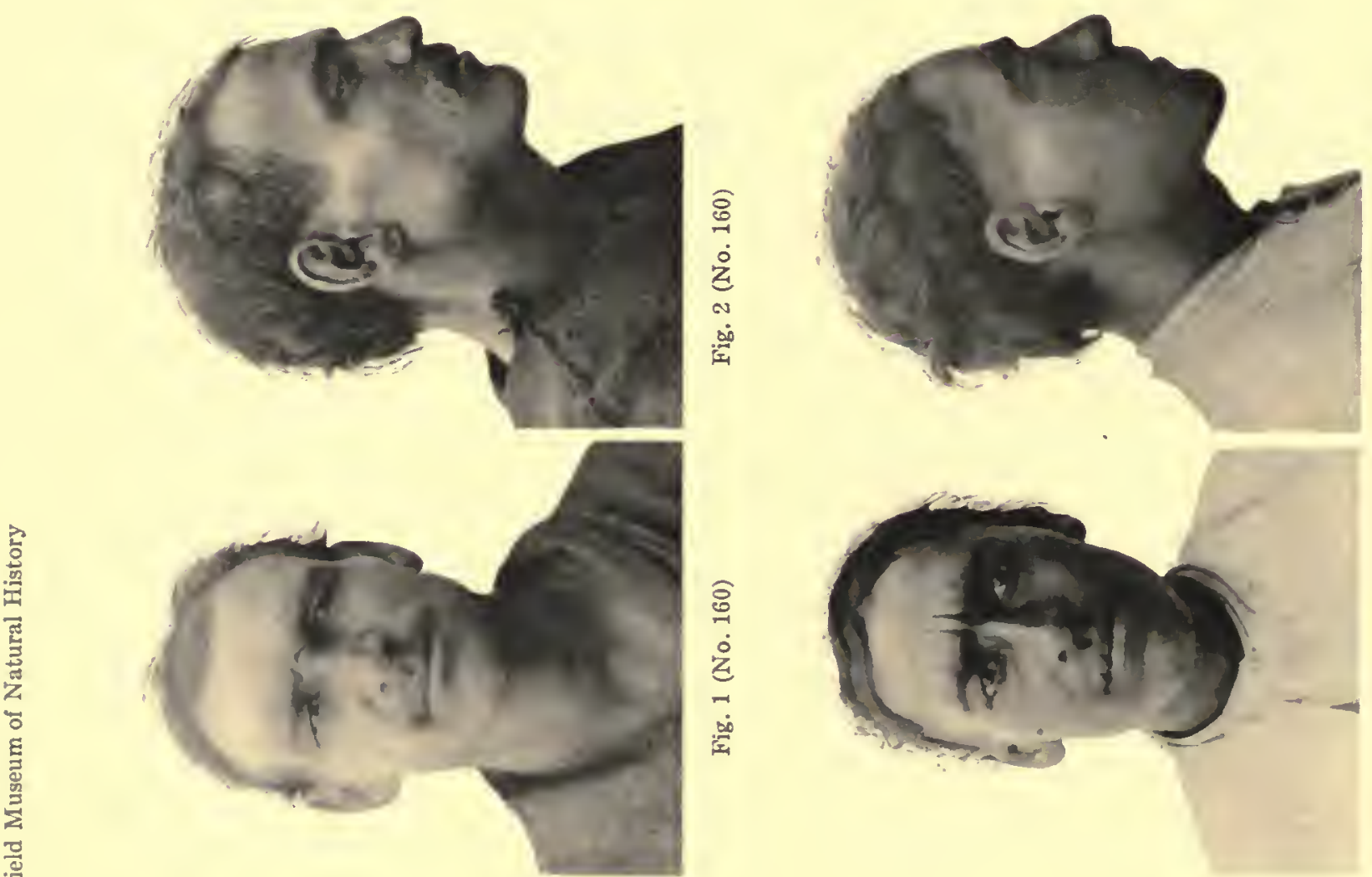

จิ
$\vdots$
$\dot{0}$
$\dot{z}$
0
0
0

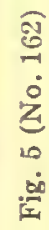


责
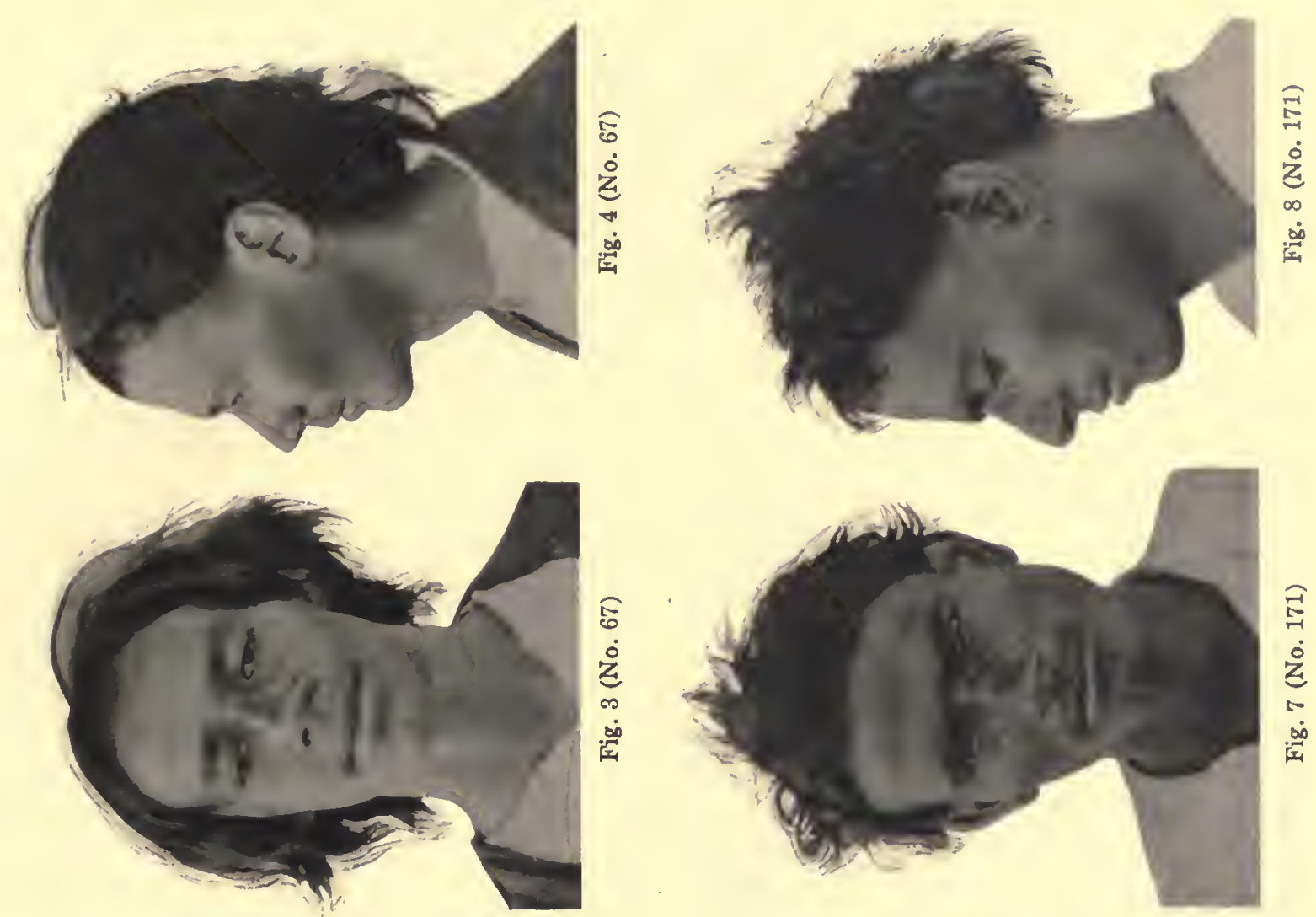

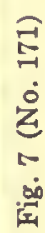

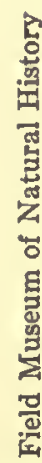
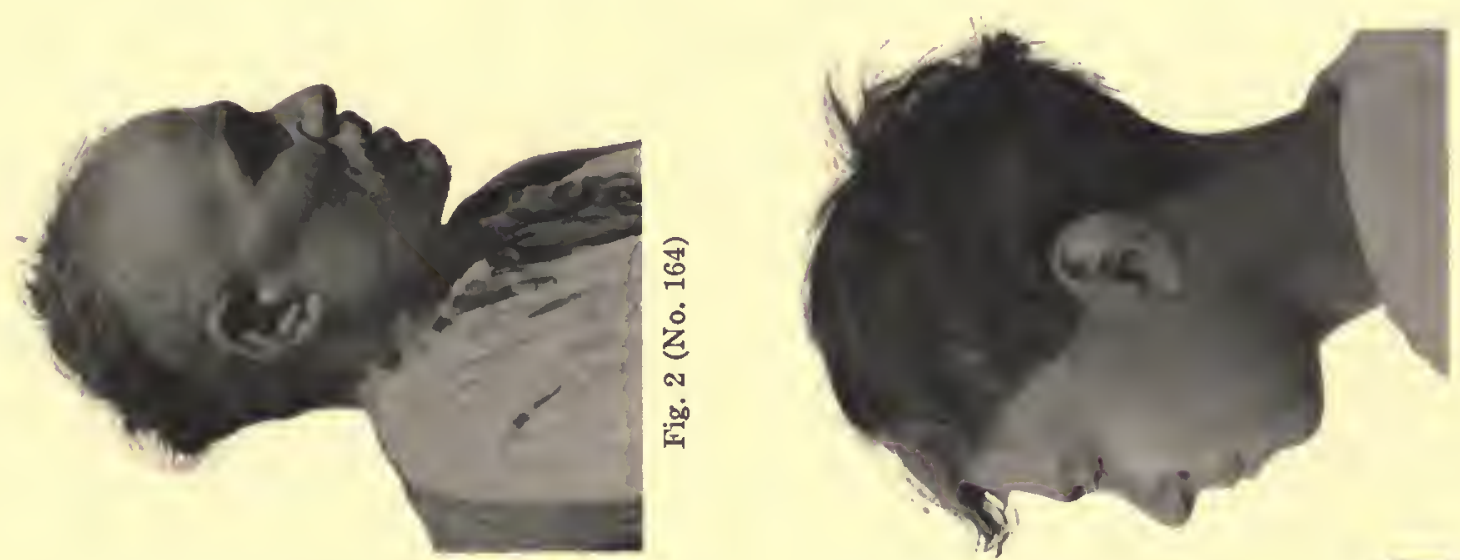

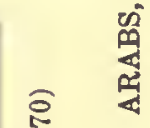
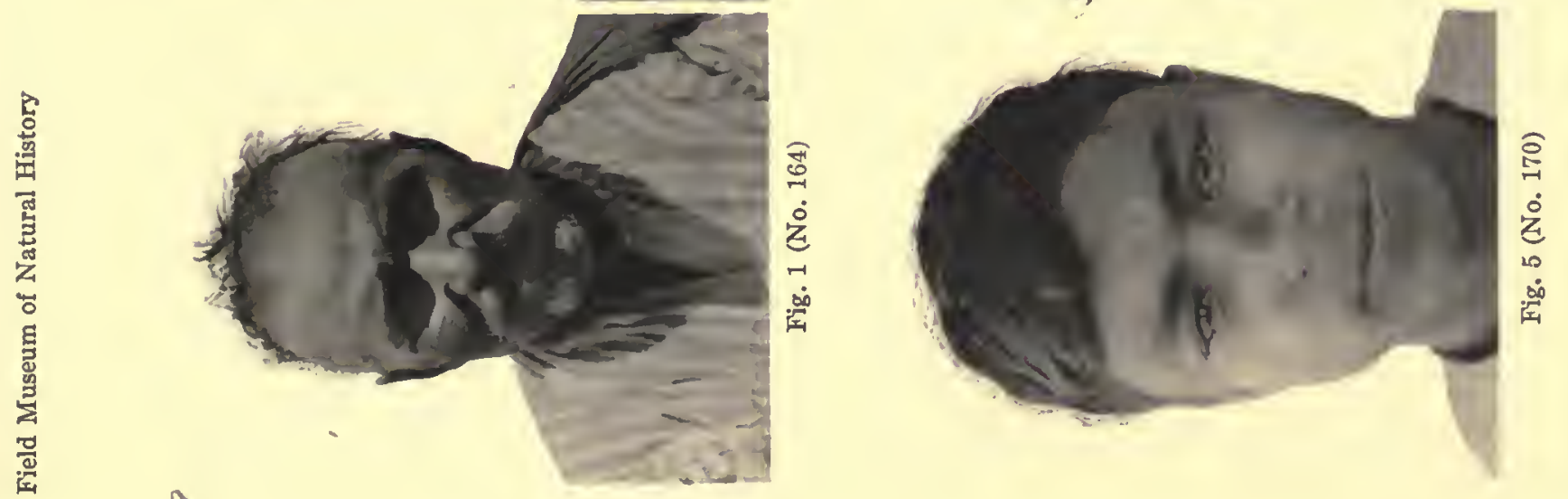

10

$\dot{8}$
0
0
0

送

N1 

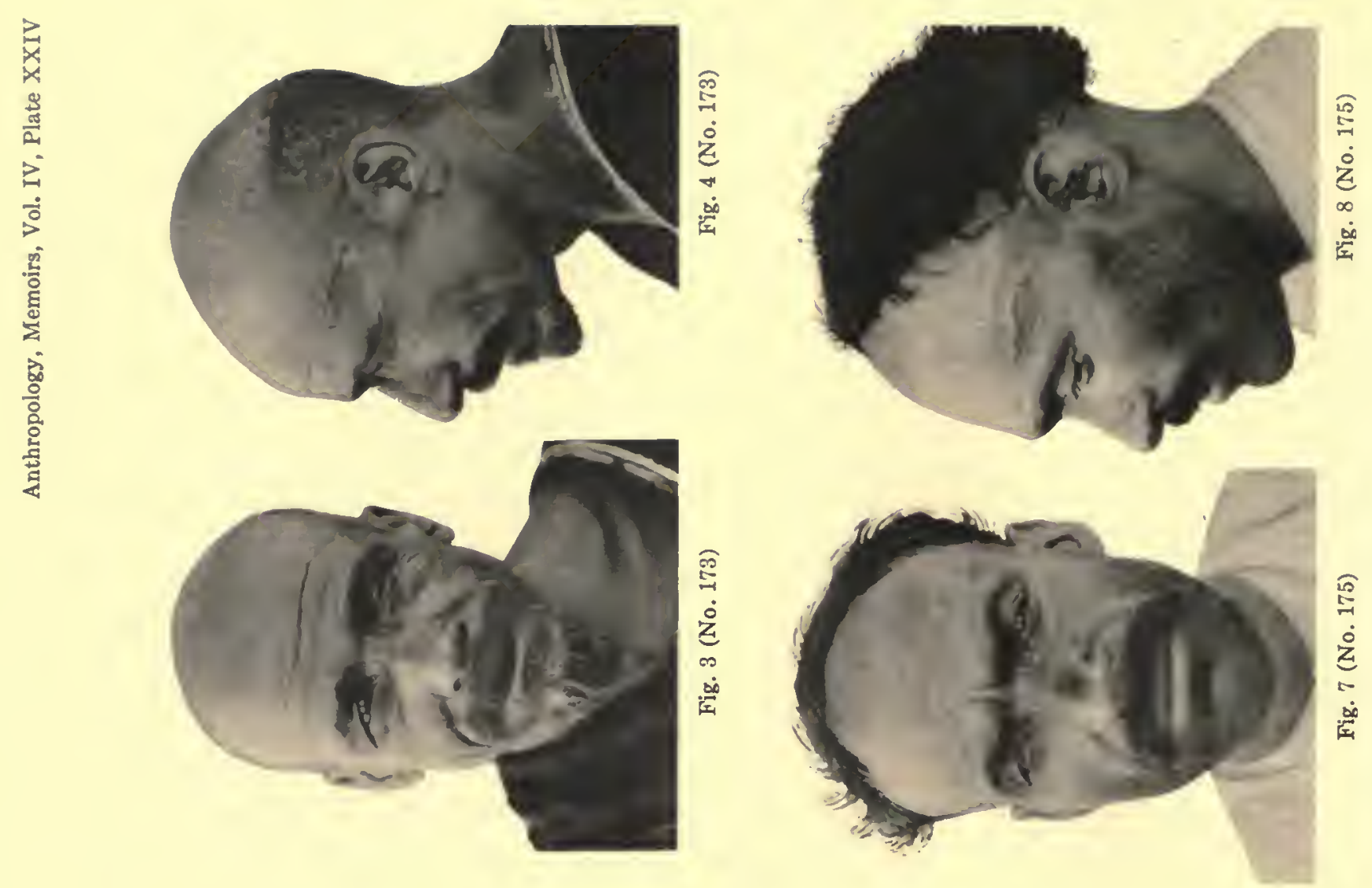

息
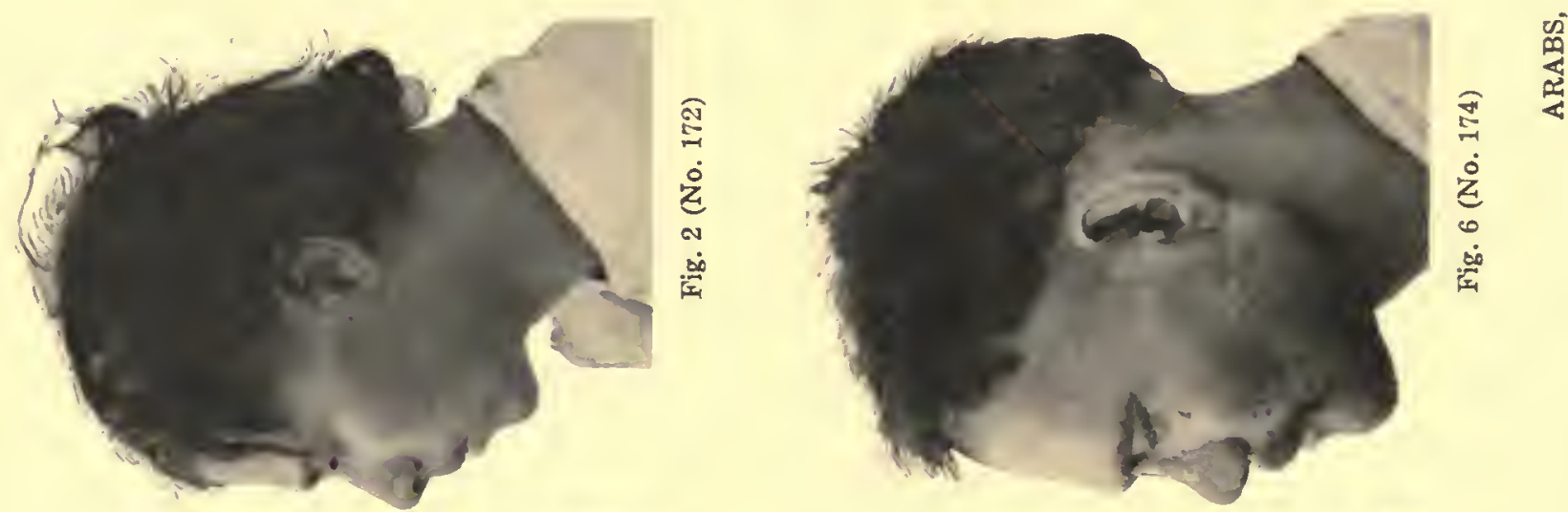

8
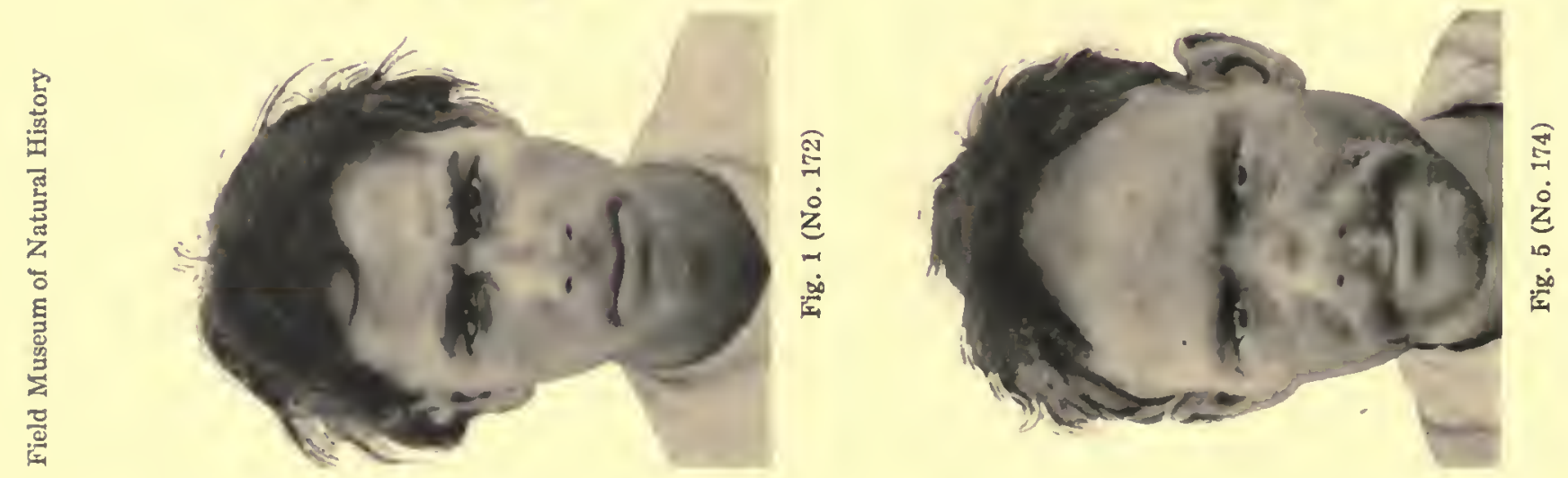


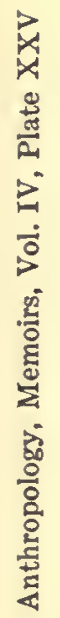
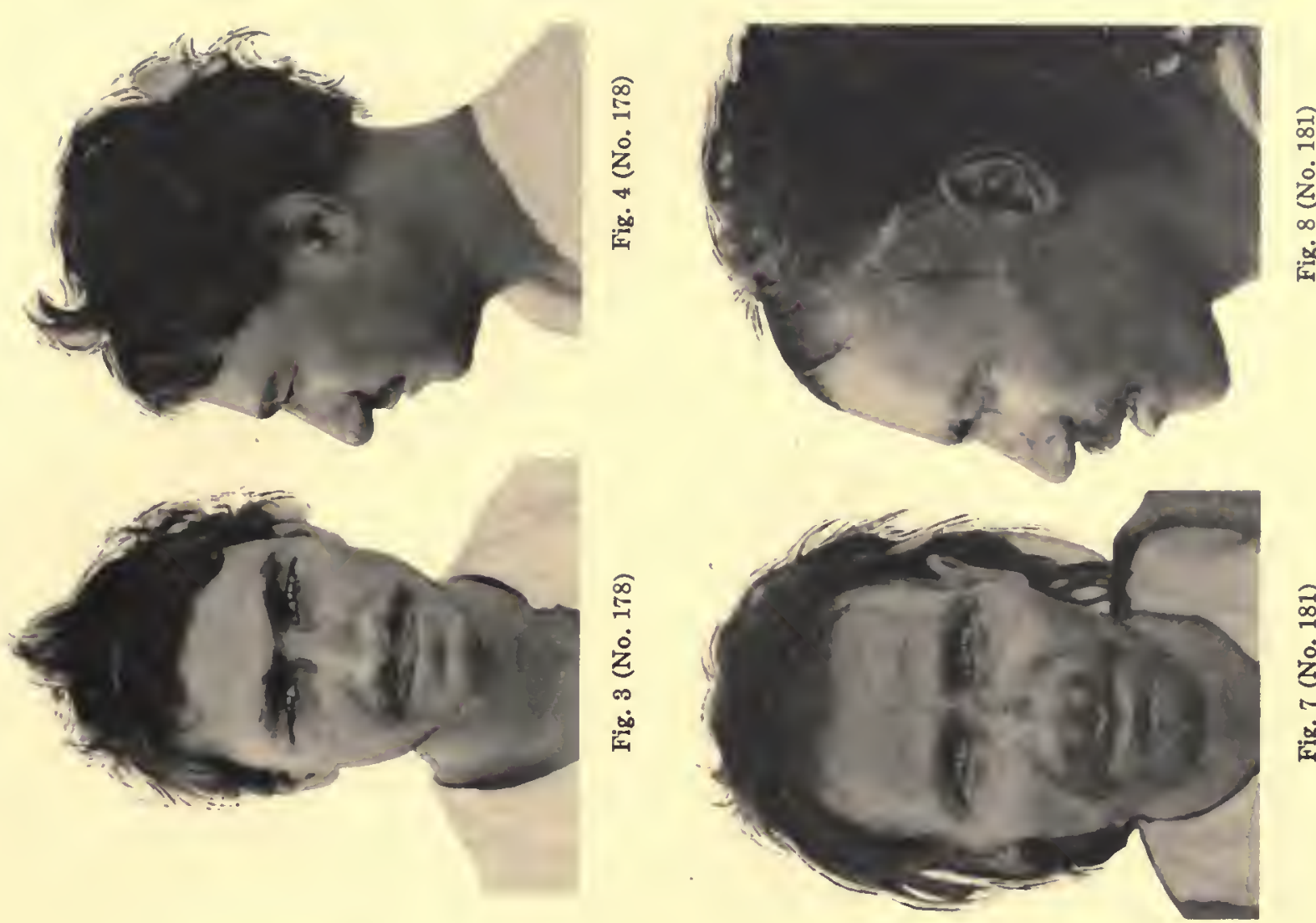

兽
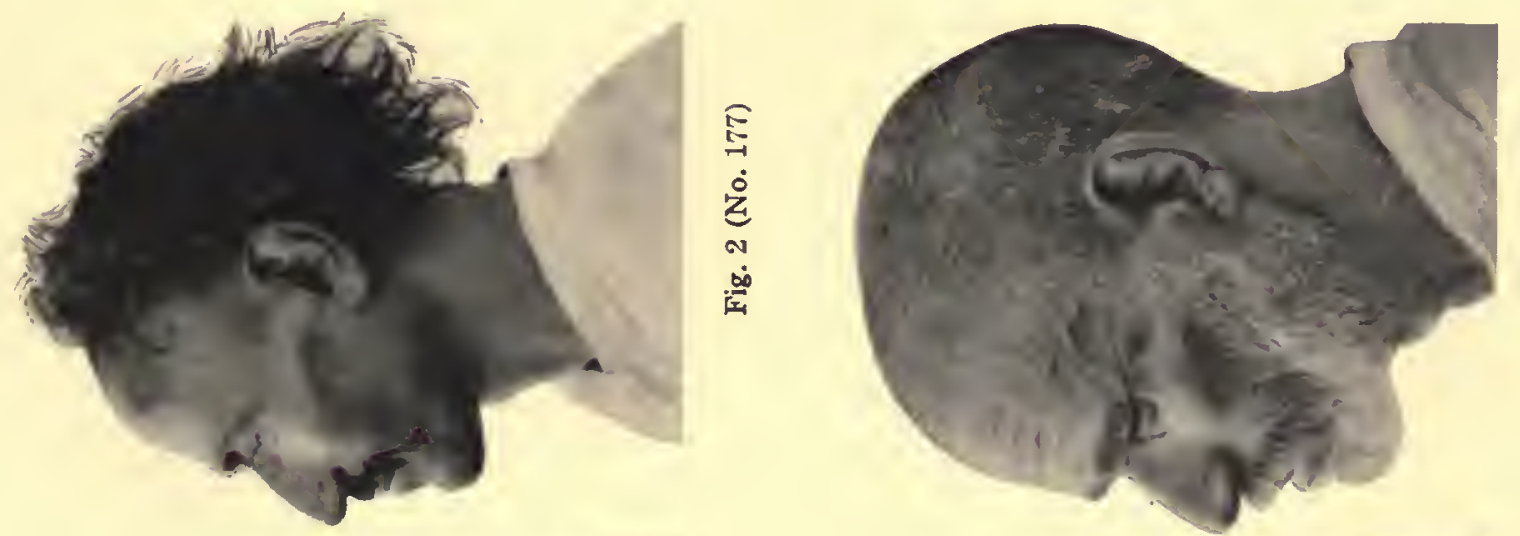

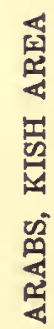

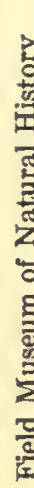
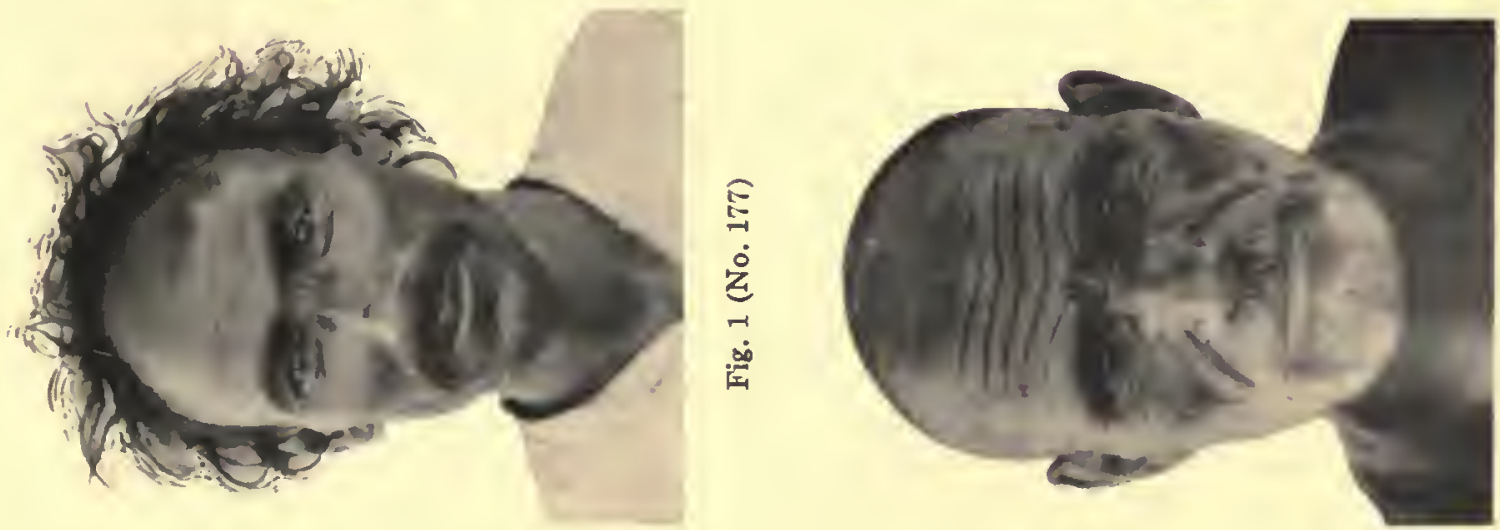

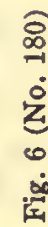

$N$

N 

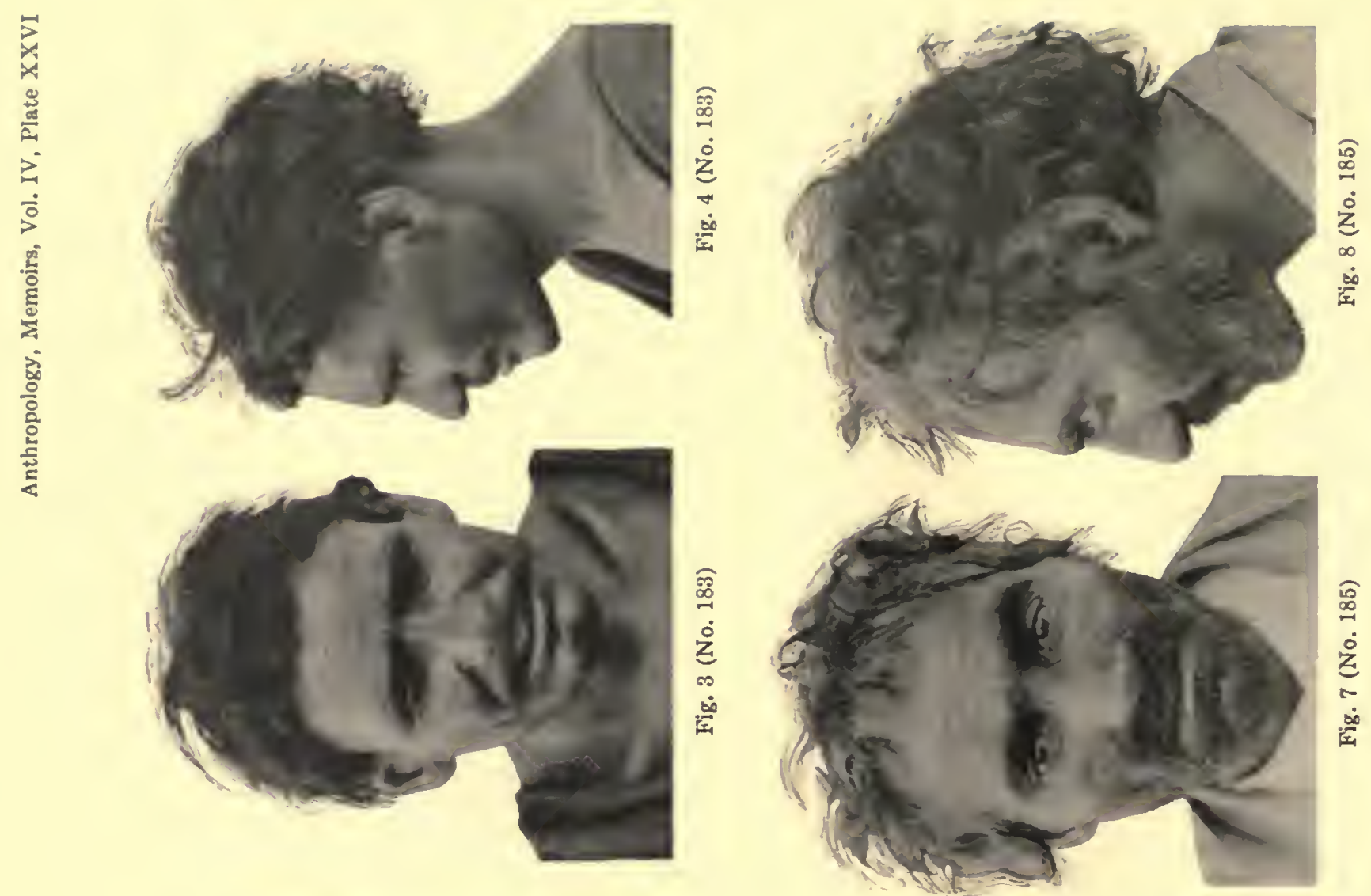

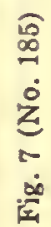
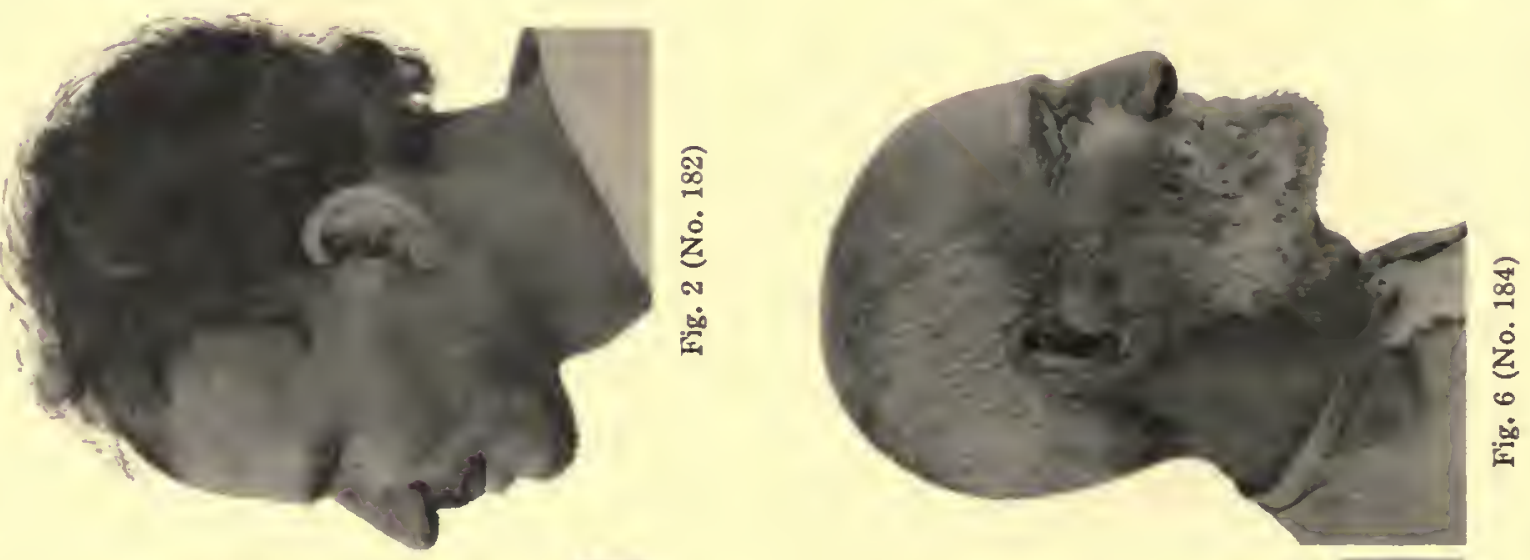

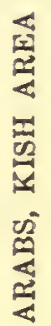

$y$
$N$
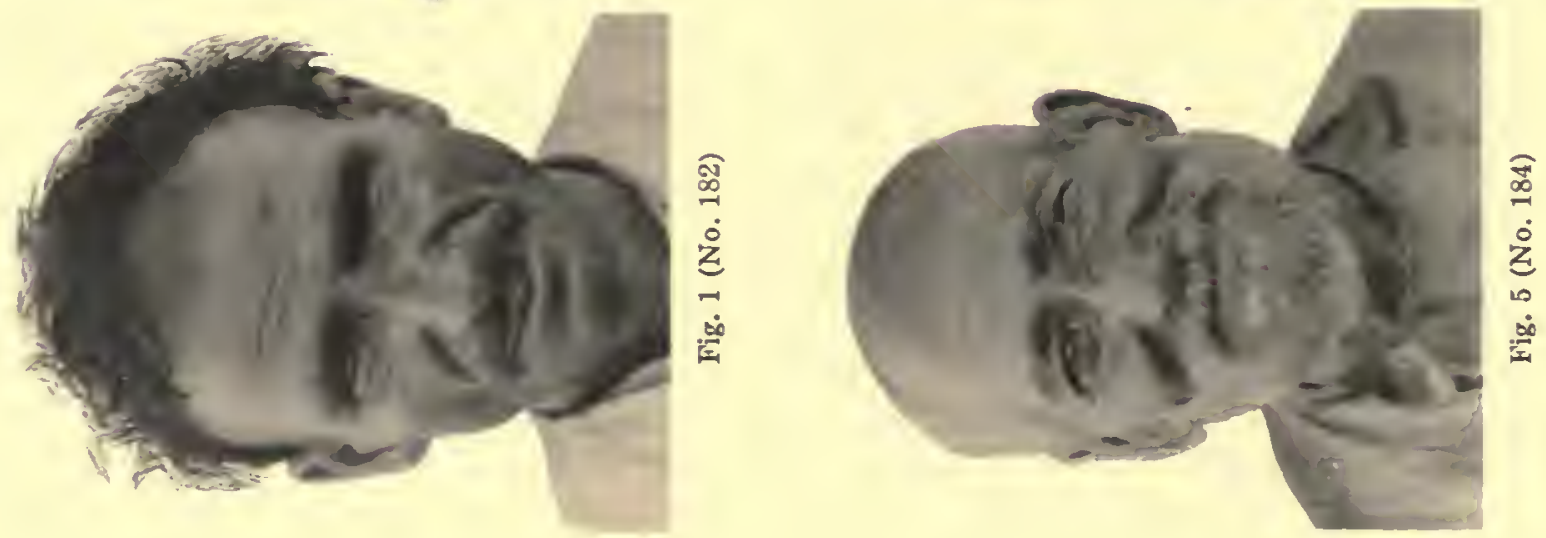

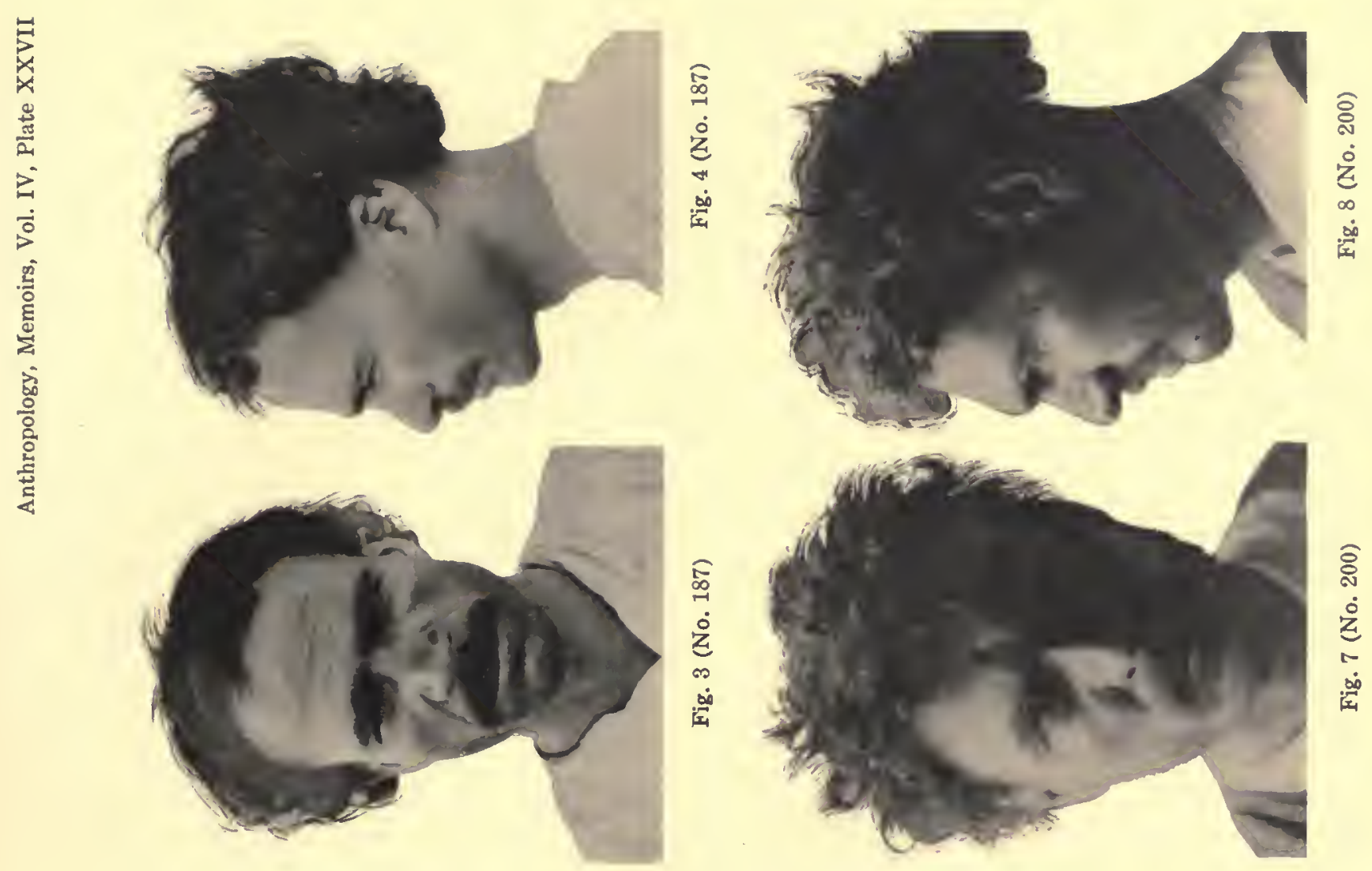

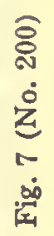
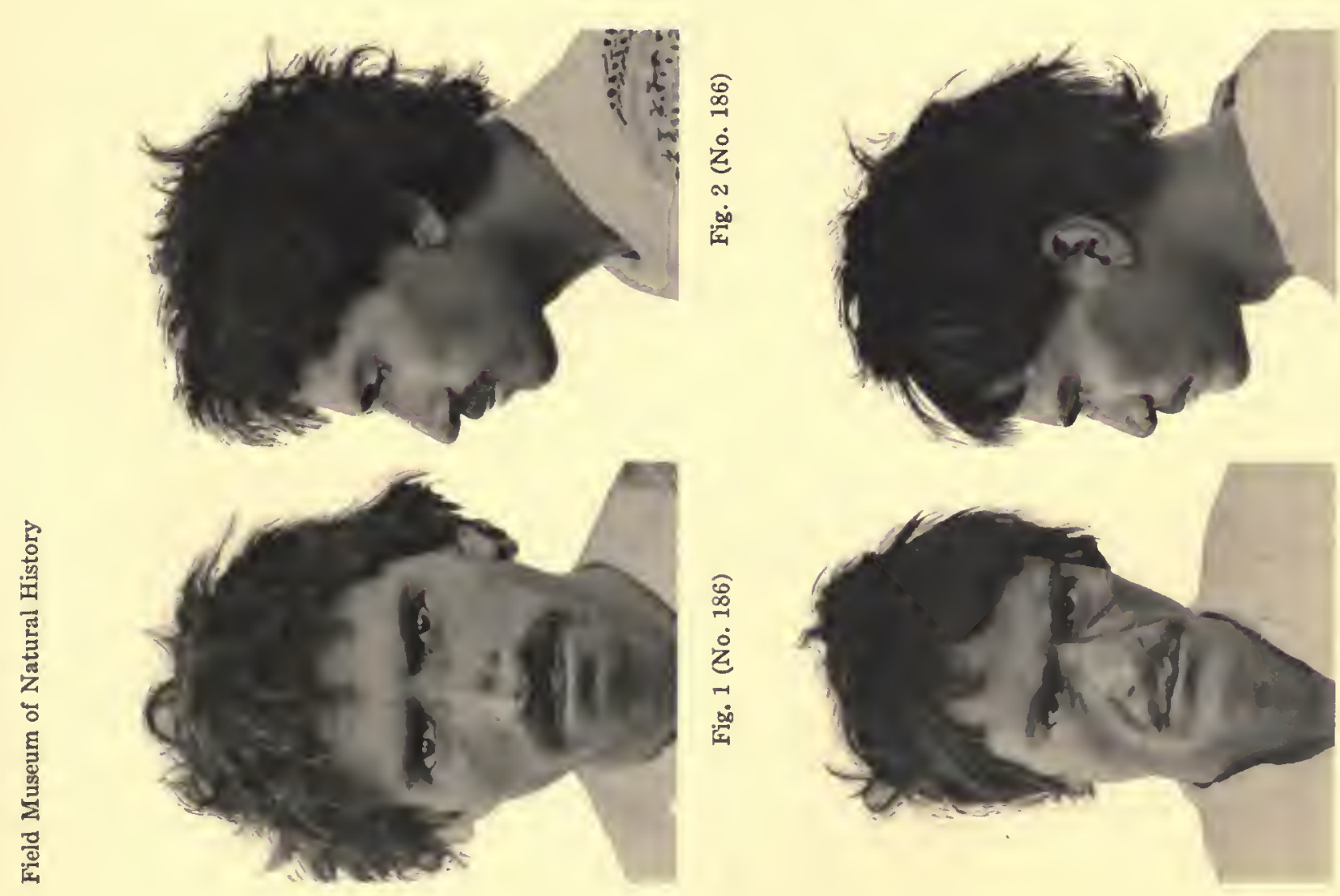

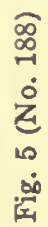

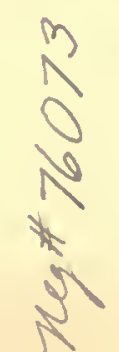



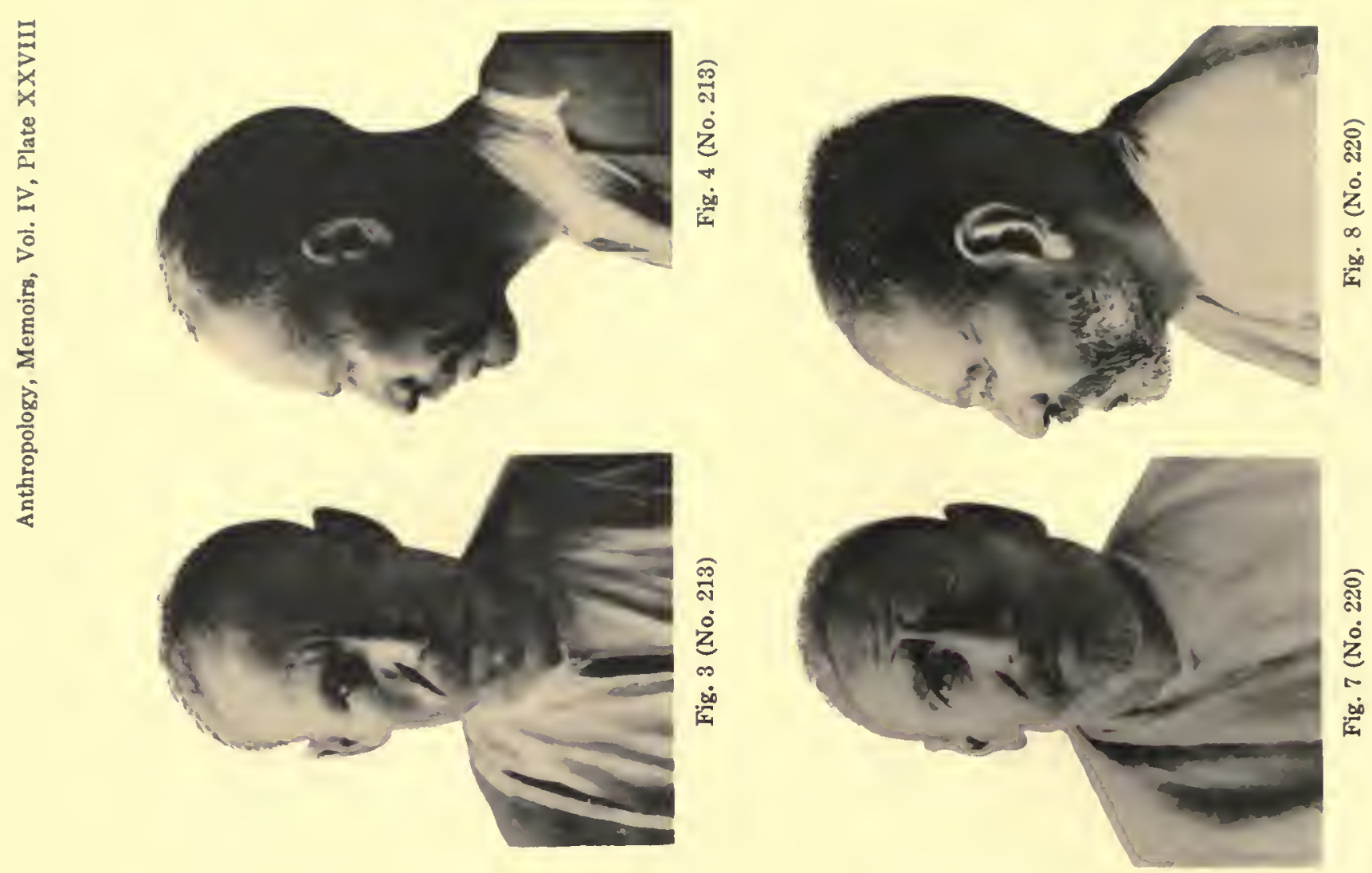

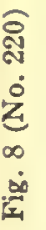

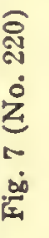
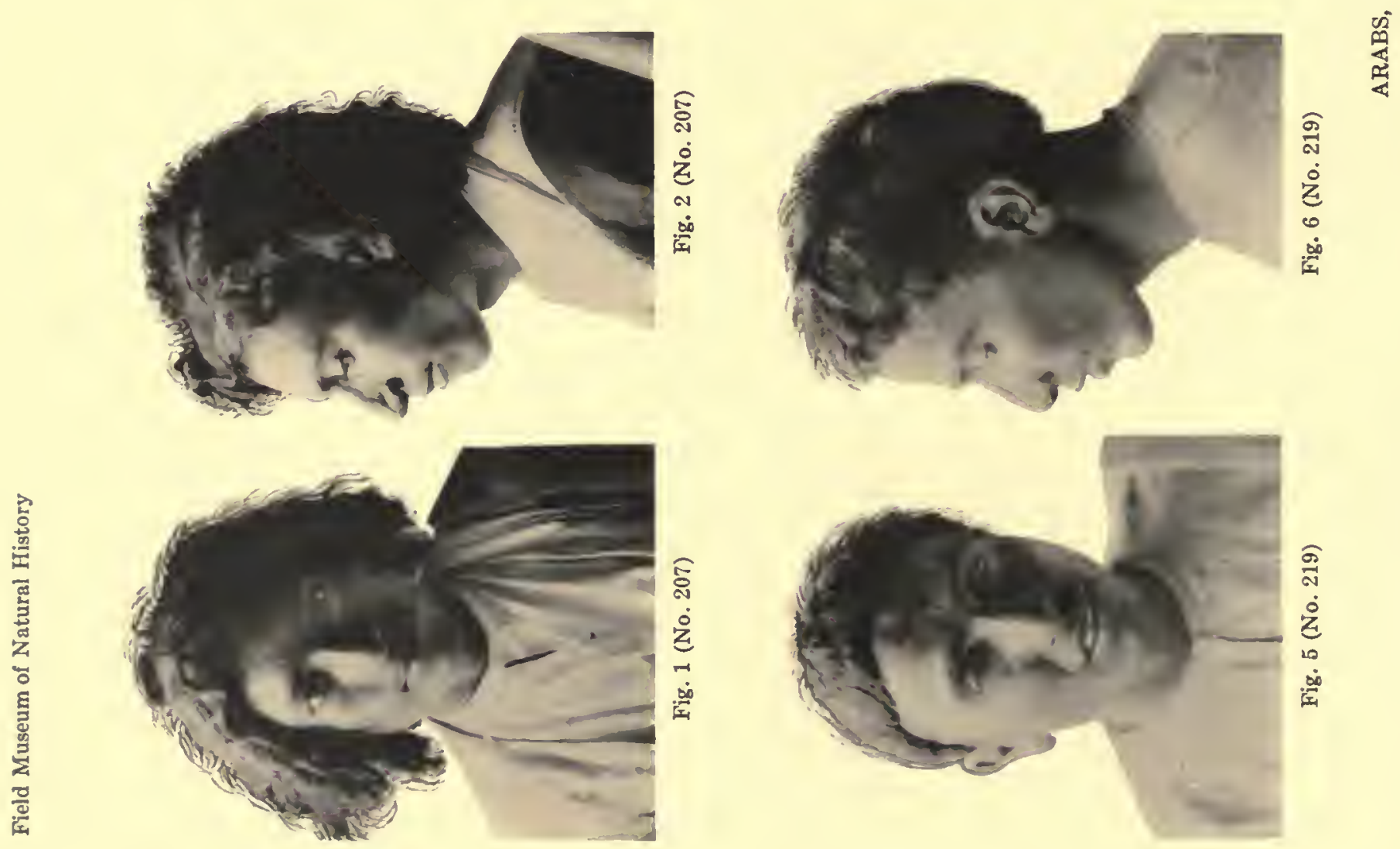

난 

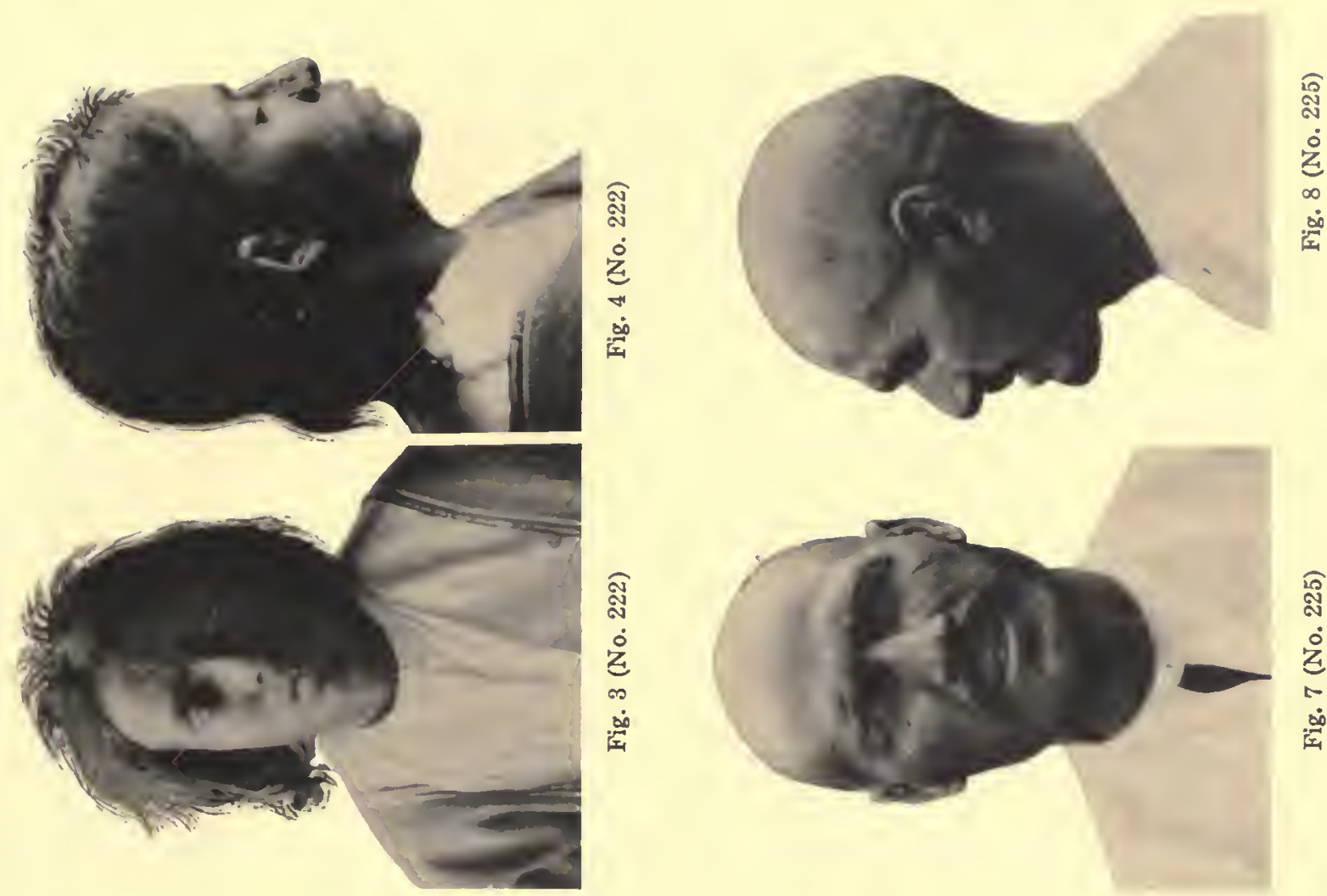

N
$\vdots$
$\vdots$
$\infty$
$\infty$
$\dot{0}$

ลิ

它 空
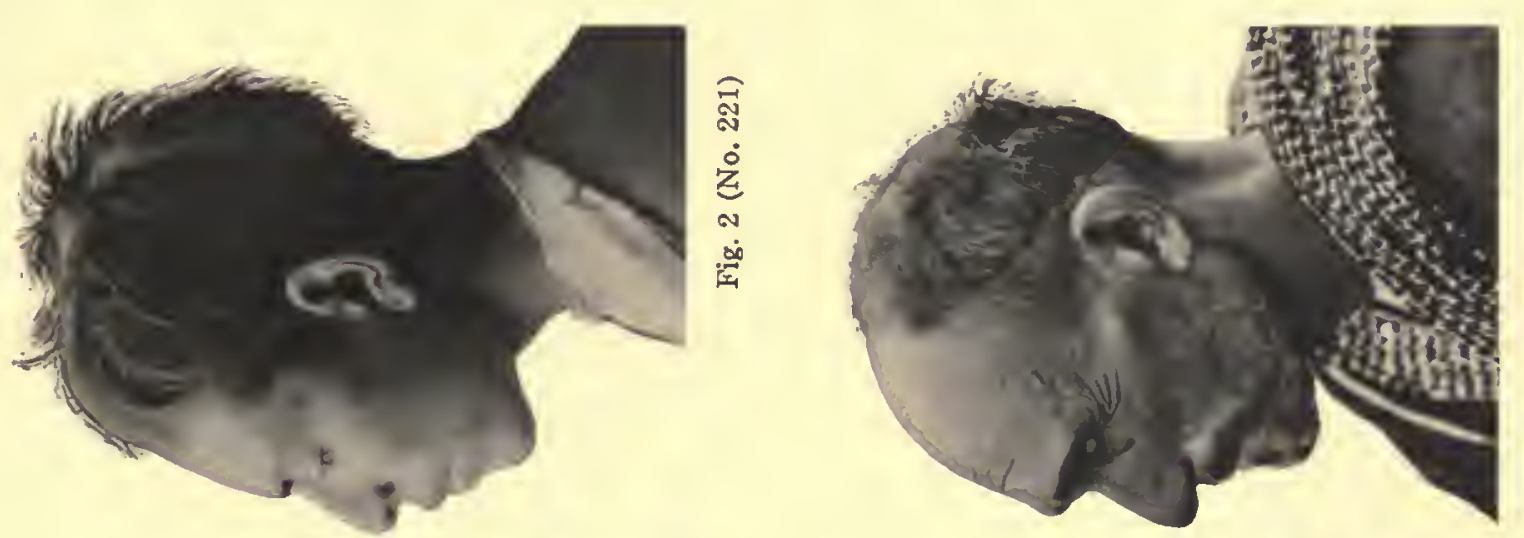

㟧

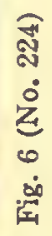

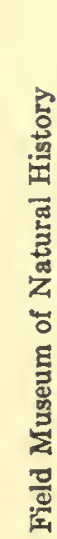
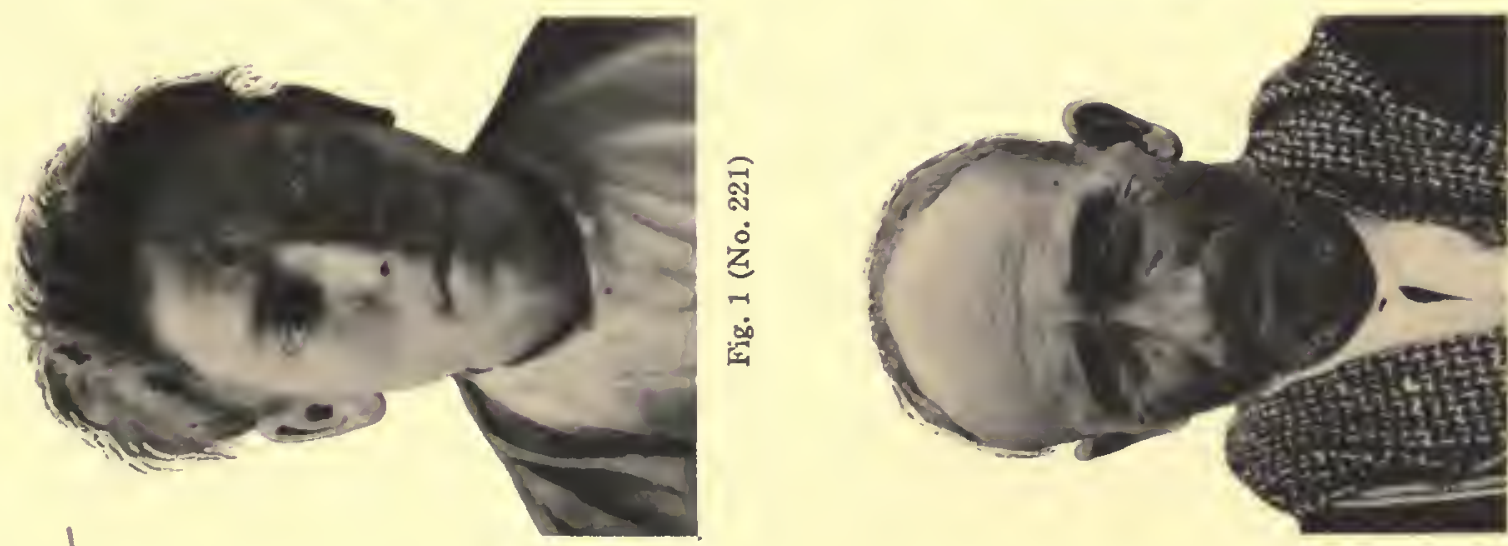

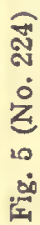

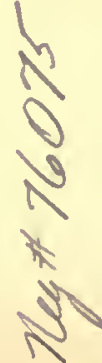



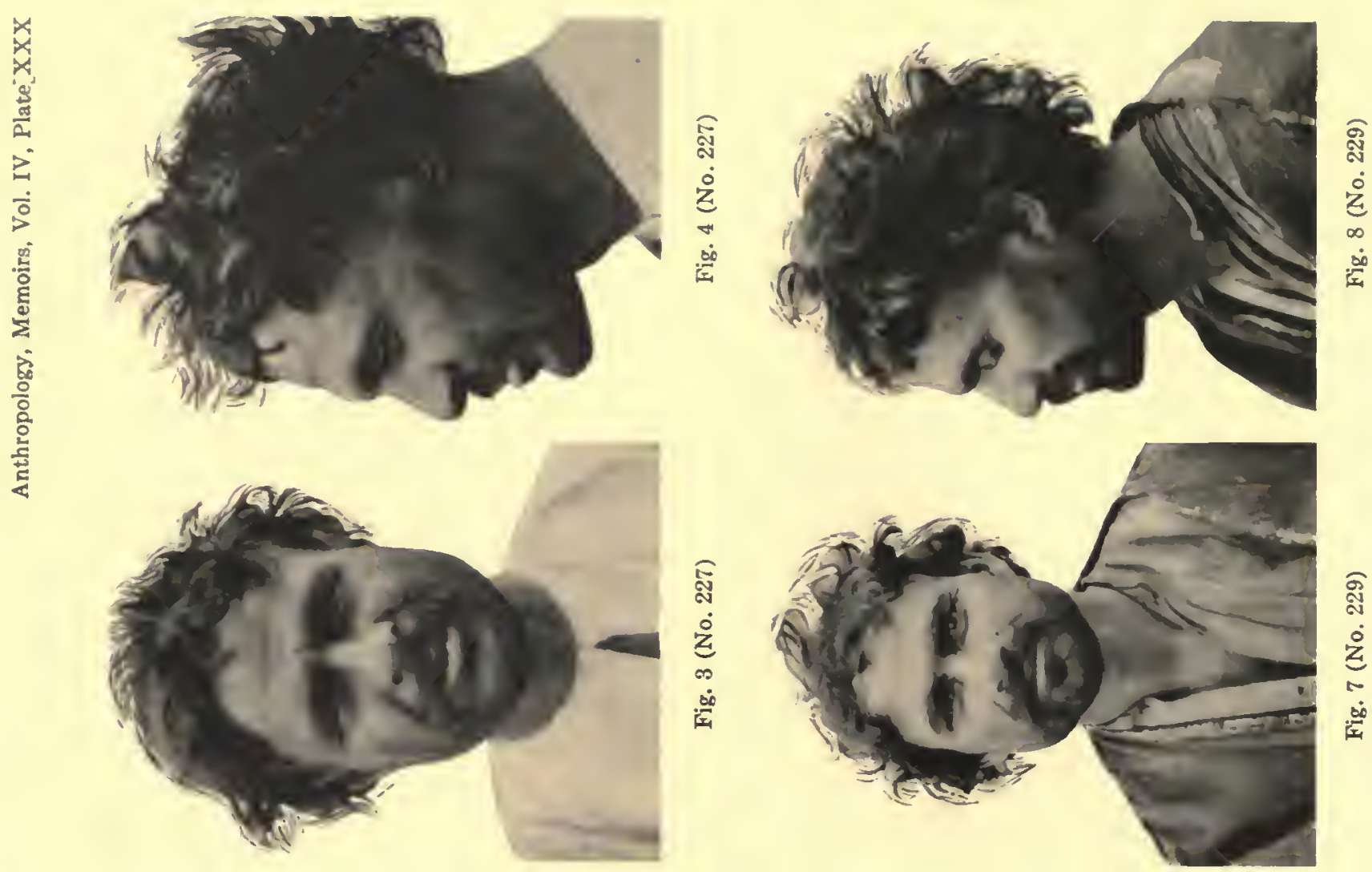

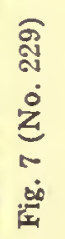

1
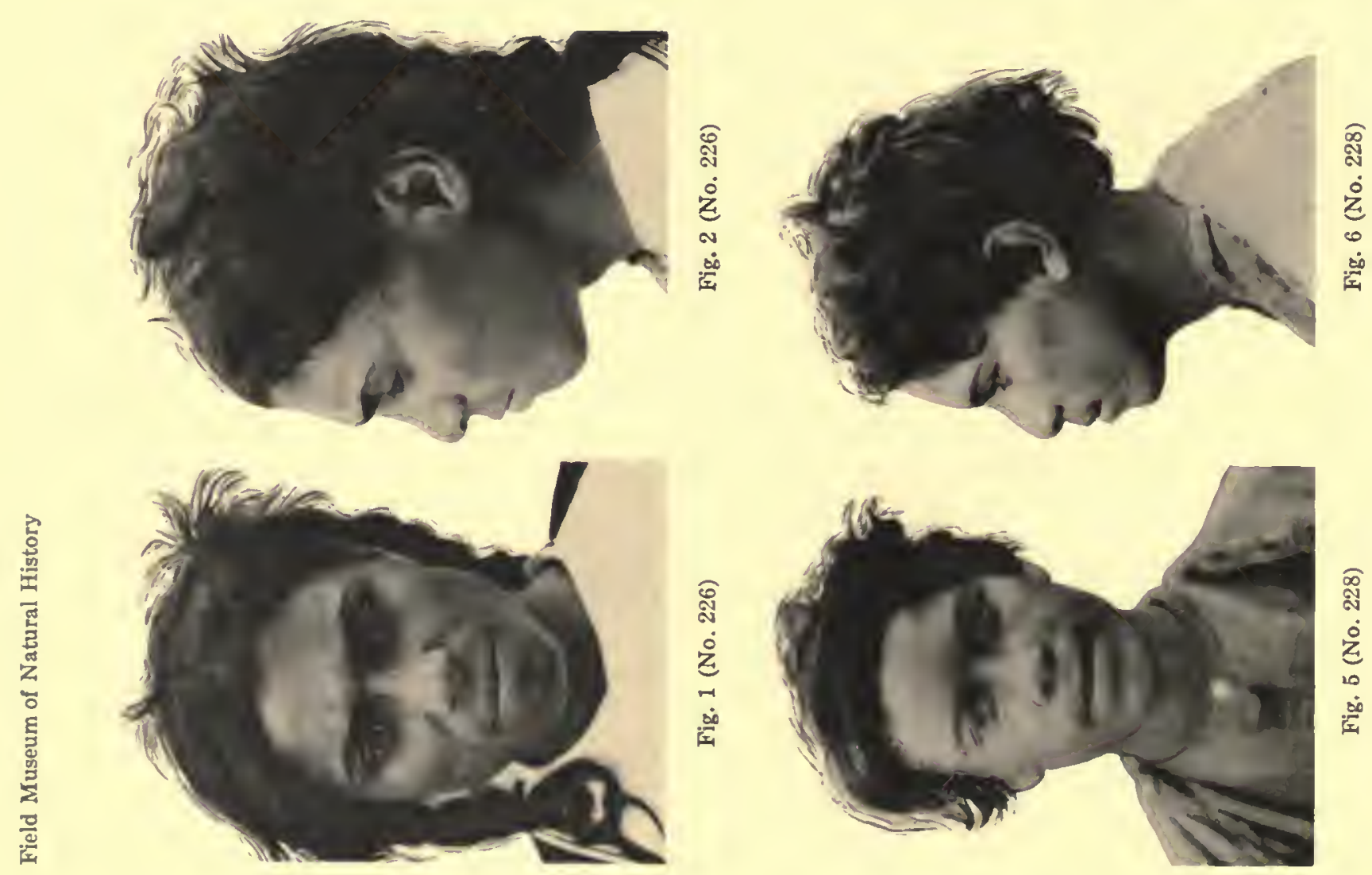


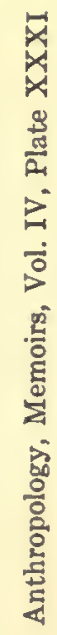
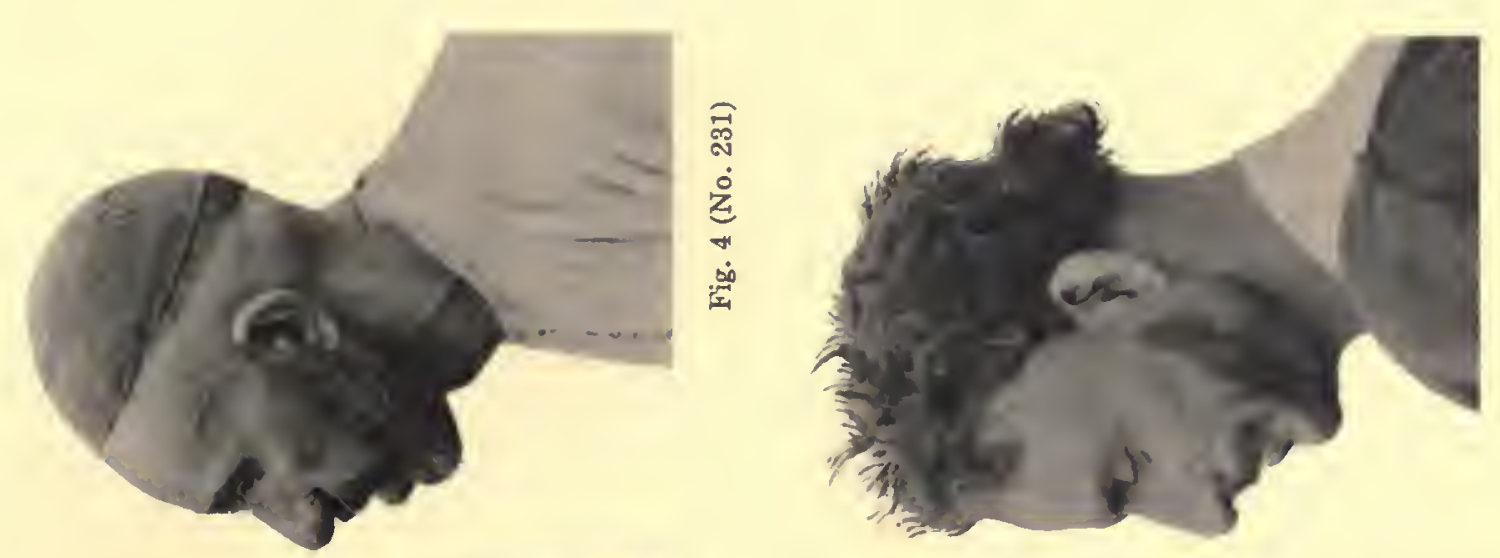

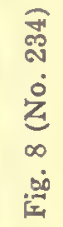
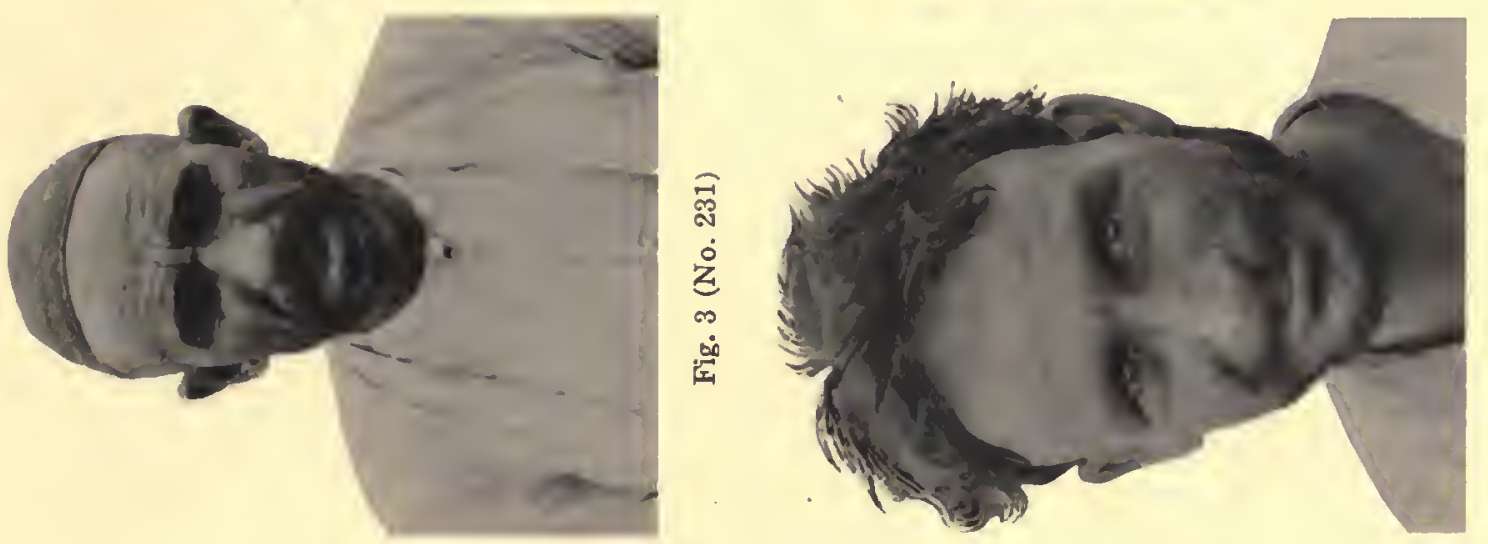

羿
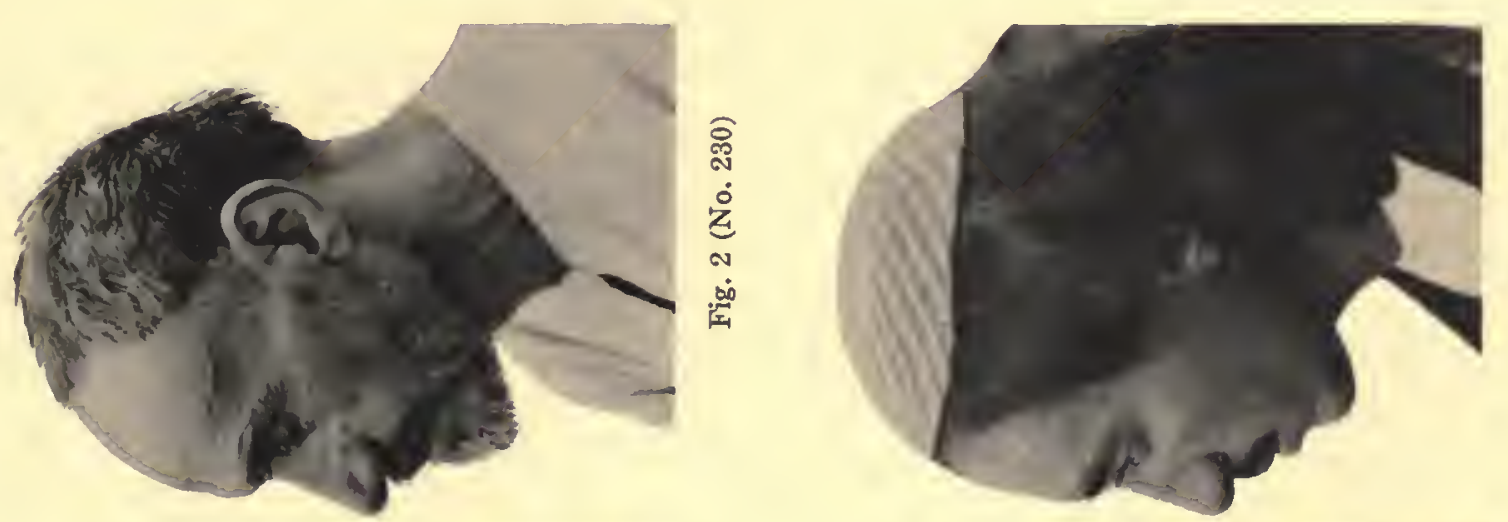

梀

总
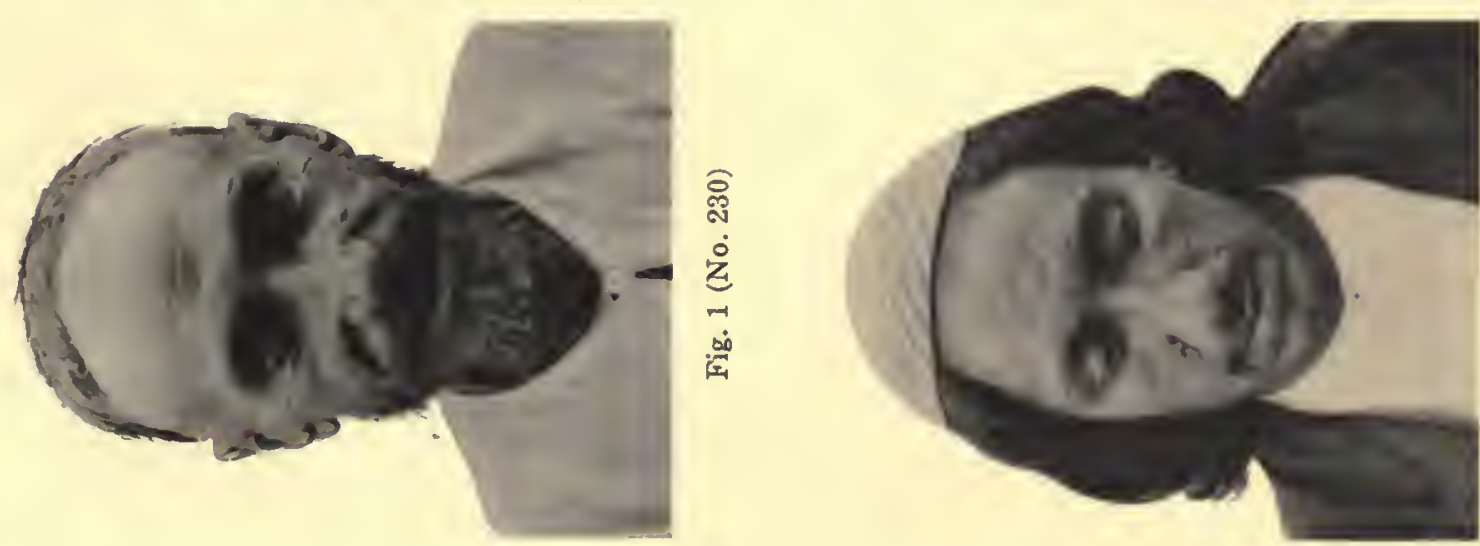

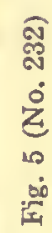

0 

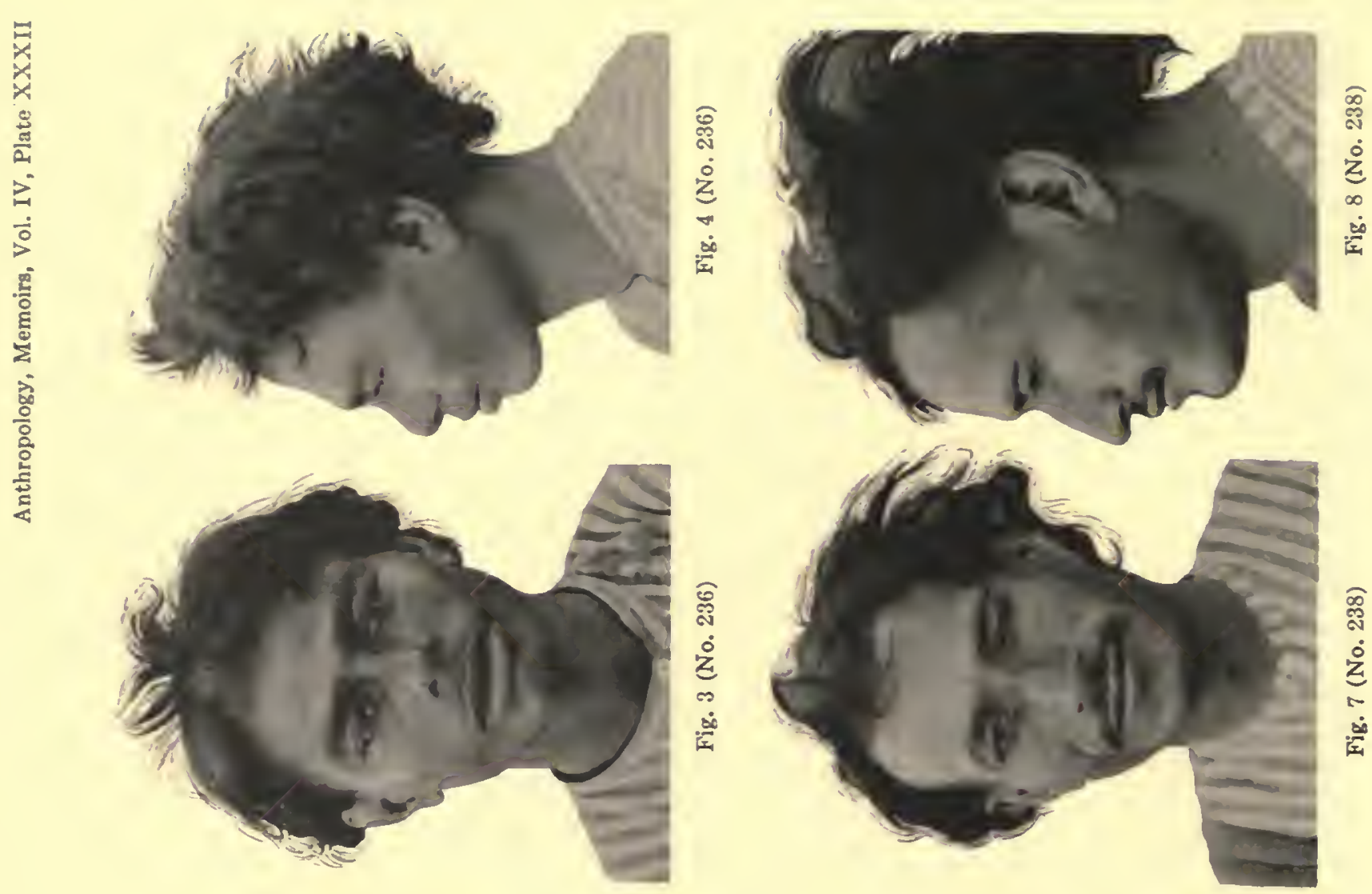

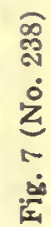
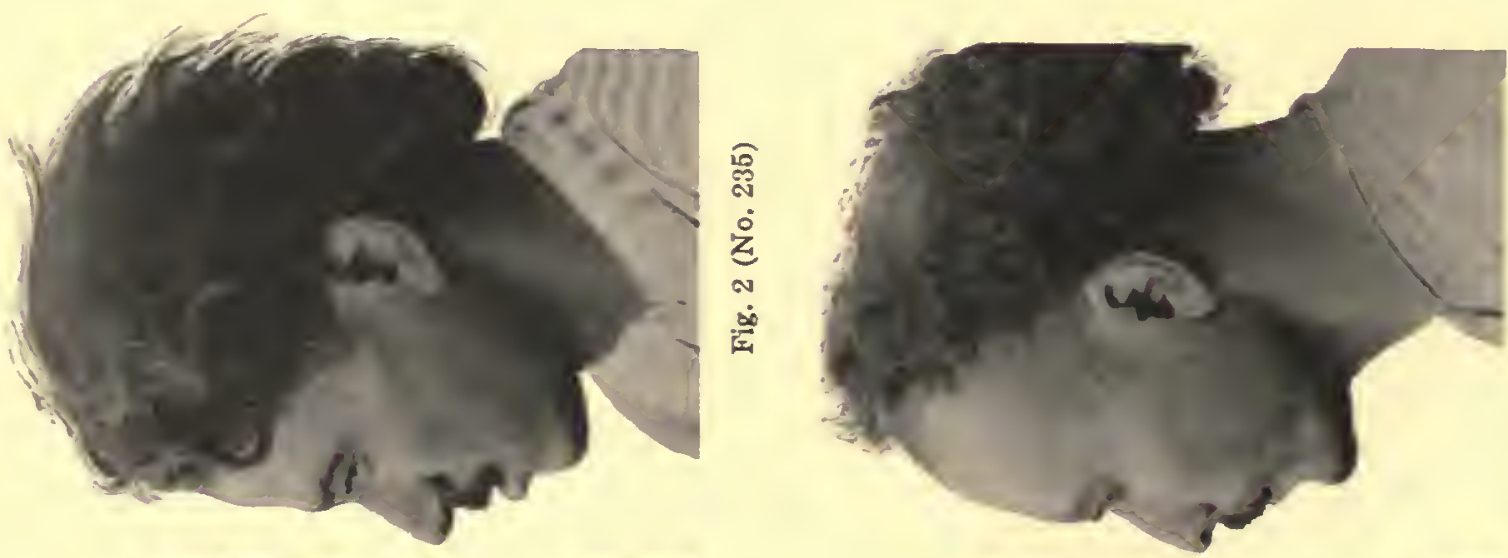

范 器

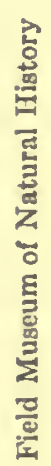
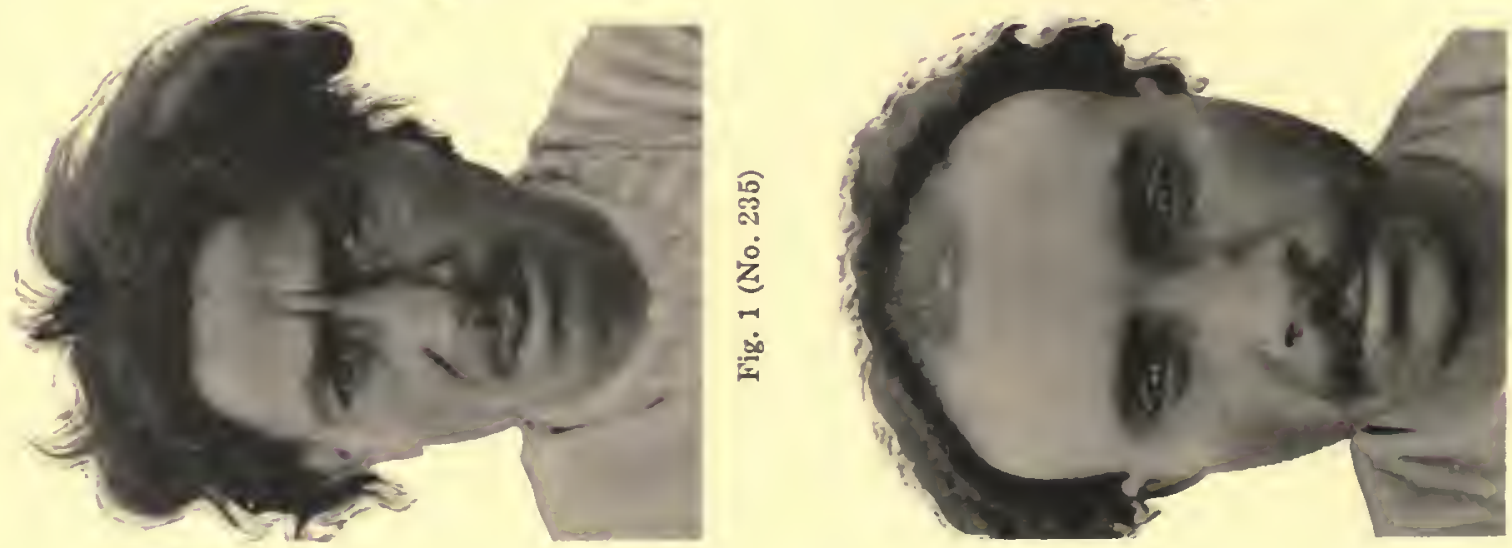

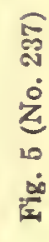



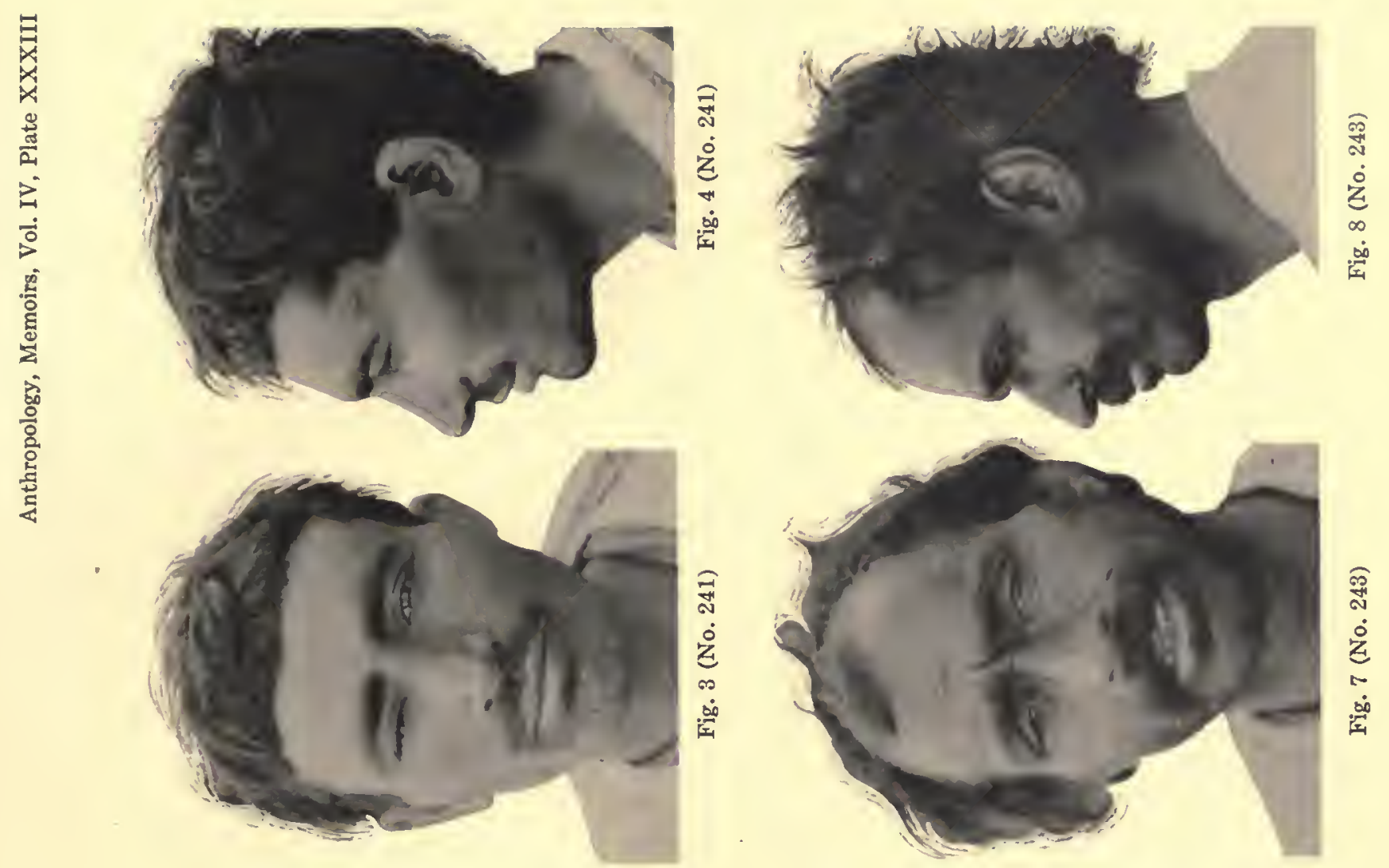

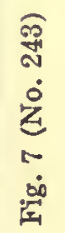
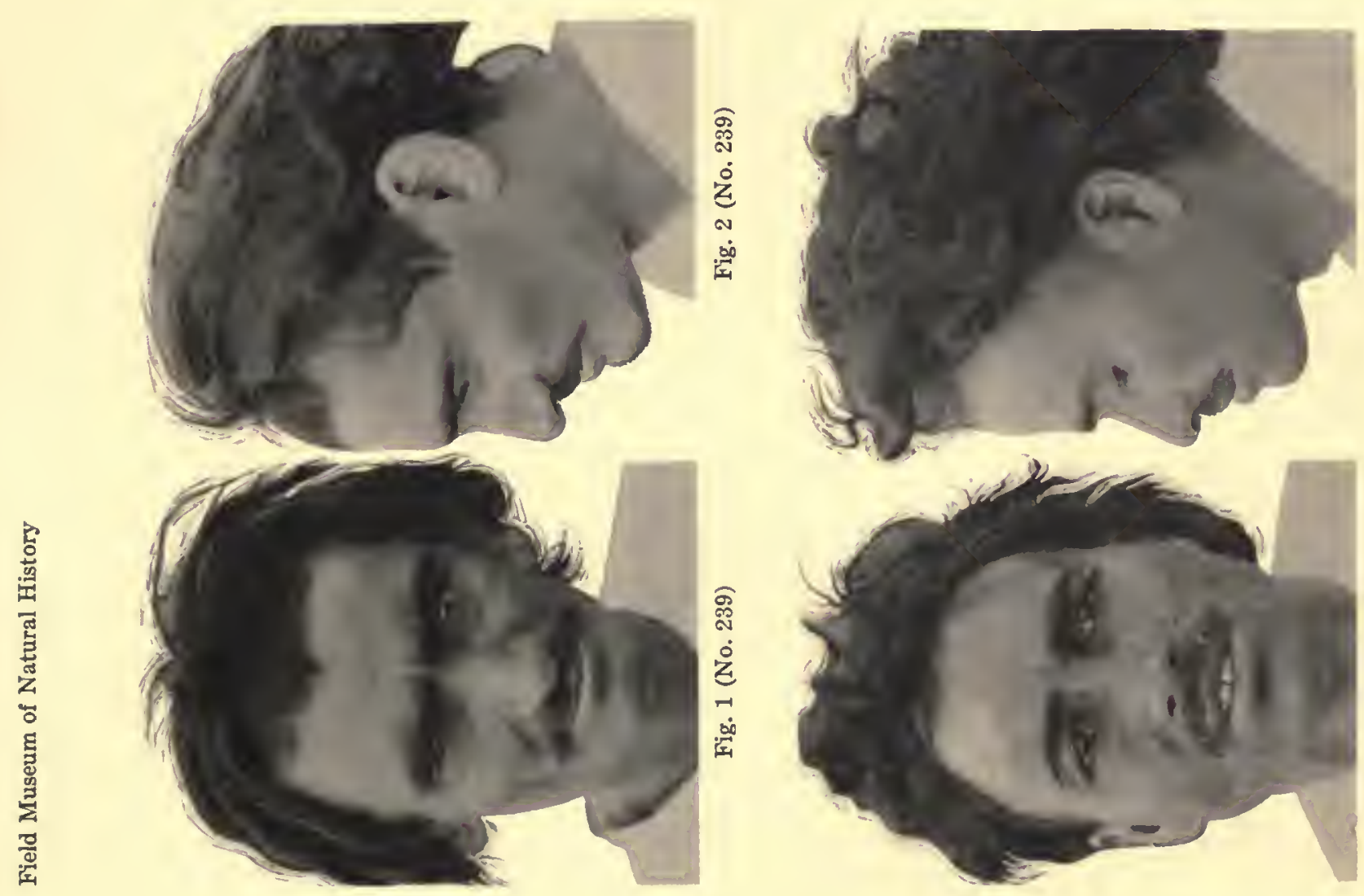

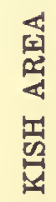

照

3
$a^{3}$
$n^{2}$
$n^{3}$

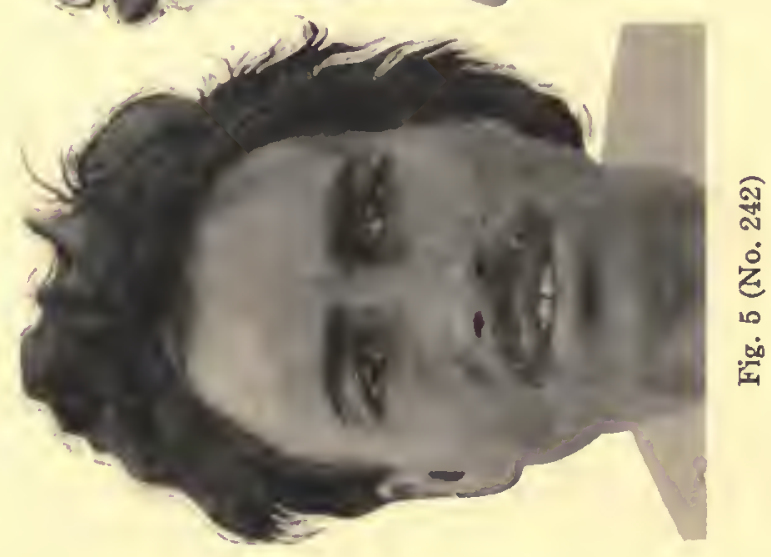



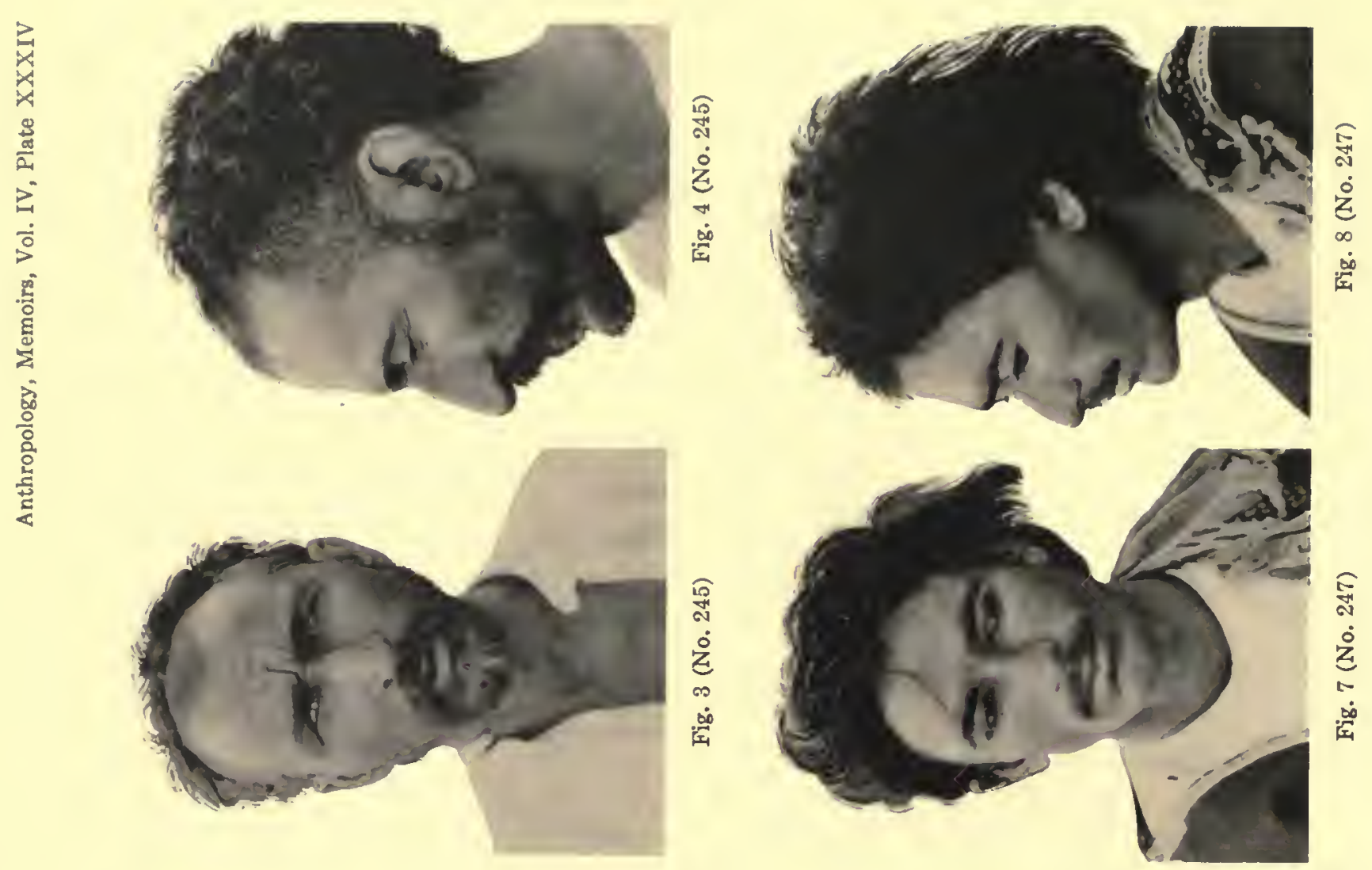

है
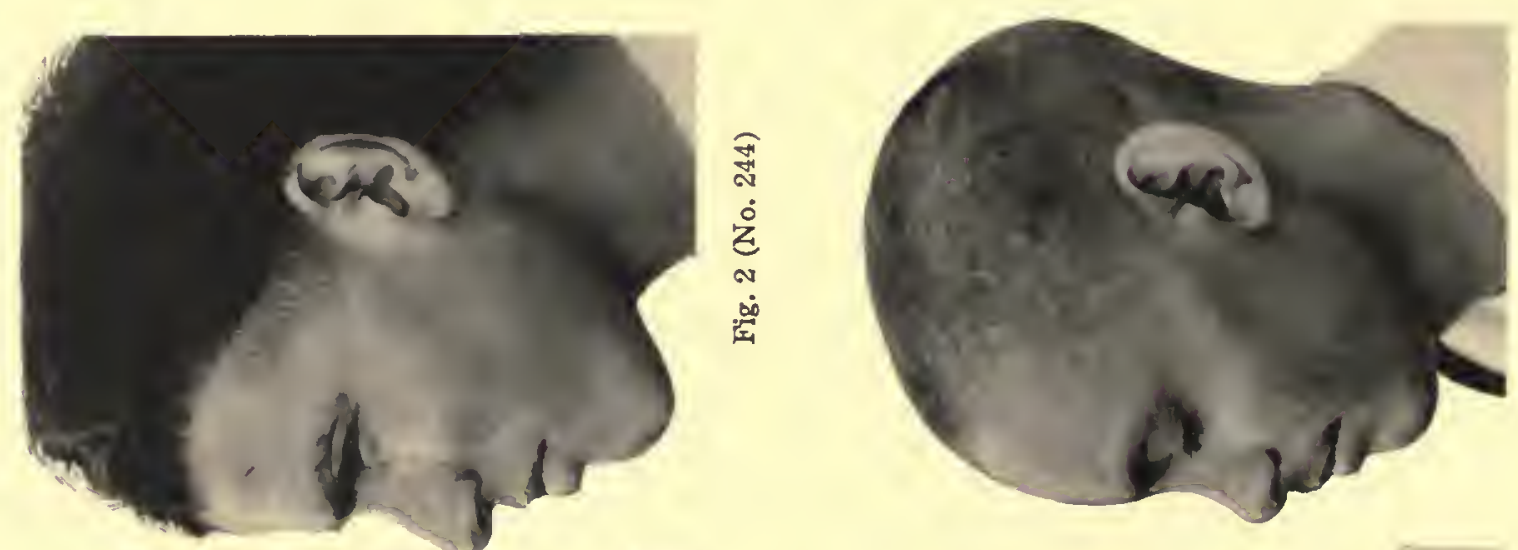

焉

종
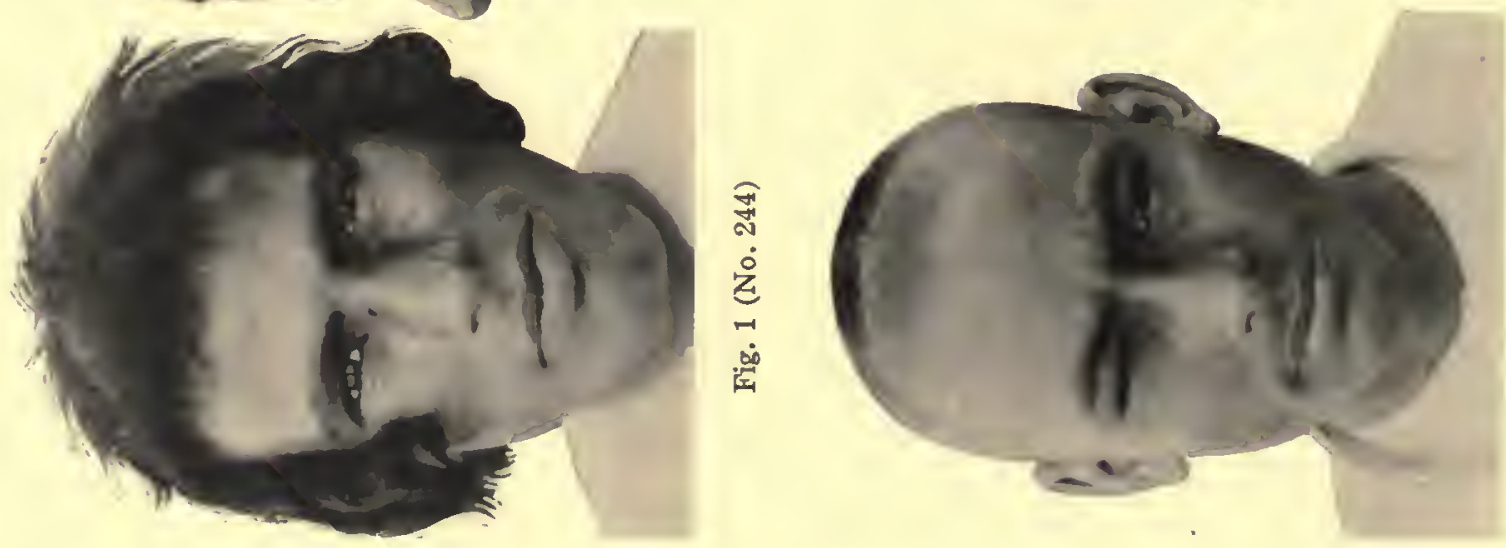

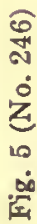



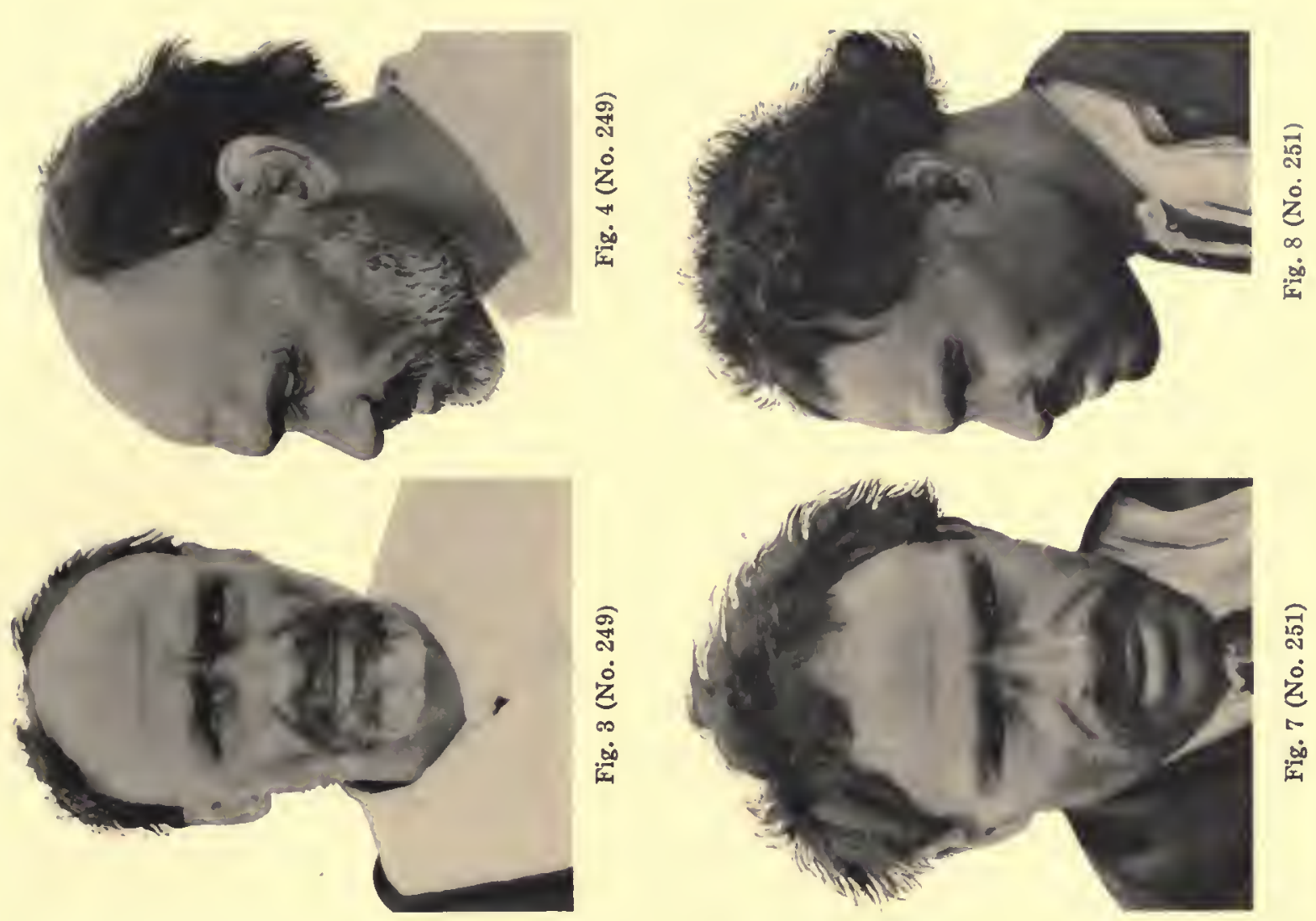

ลิำ

¿ุ

5
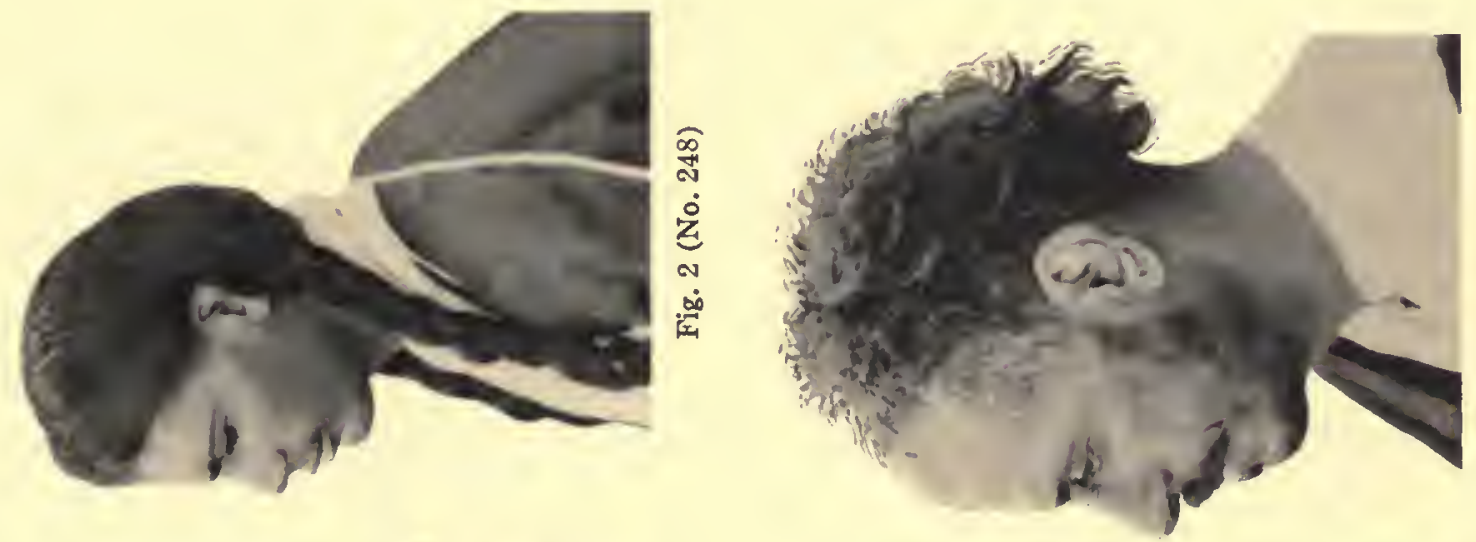

學


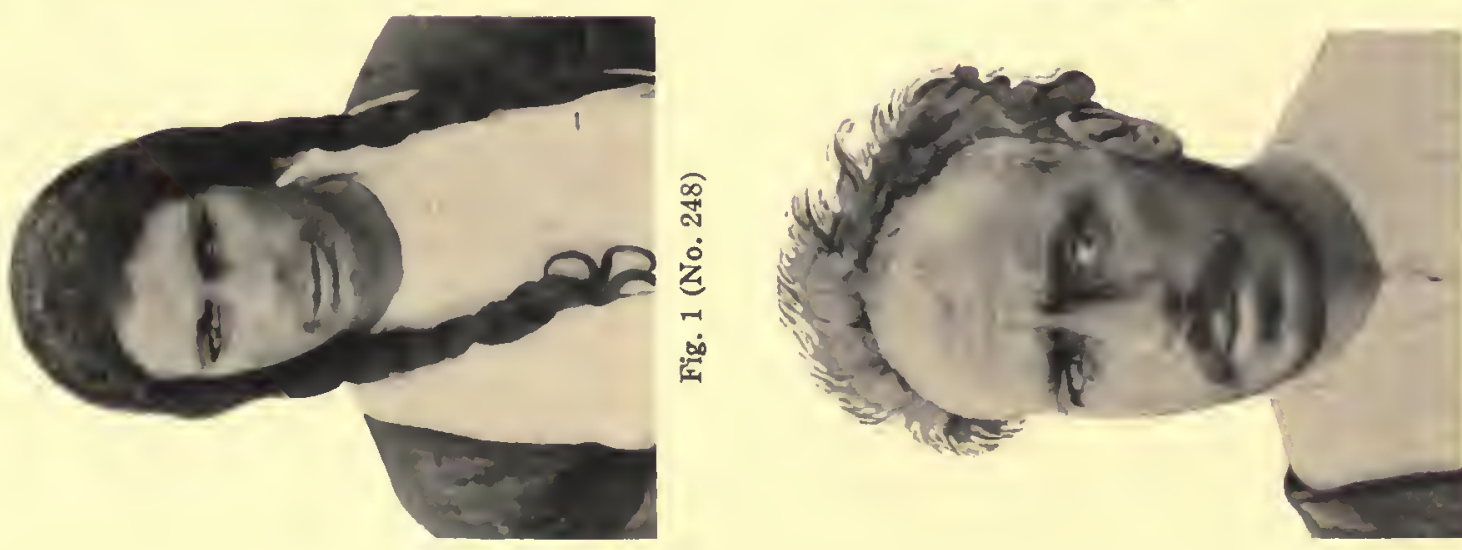

옹

a

m

1

1

N. 

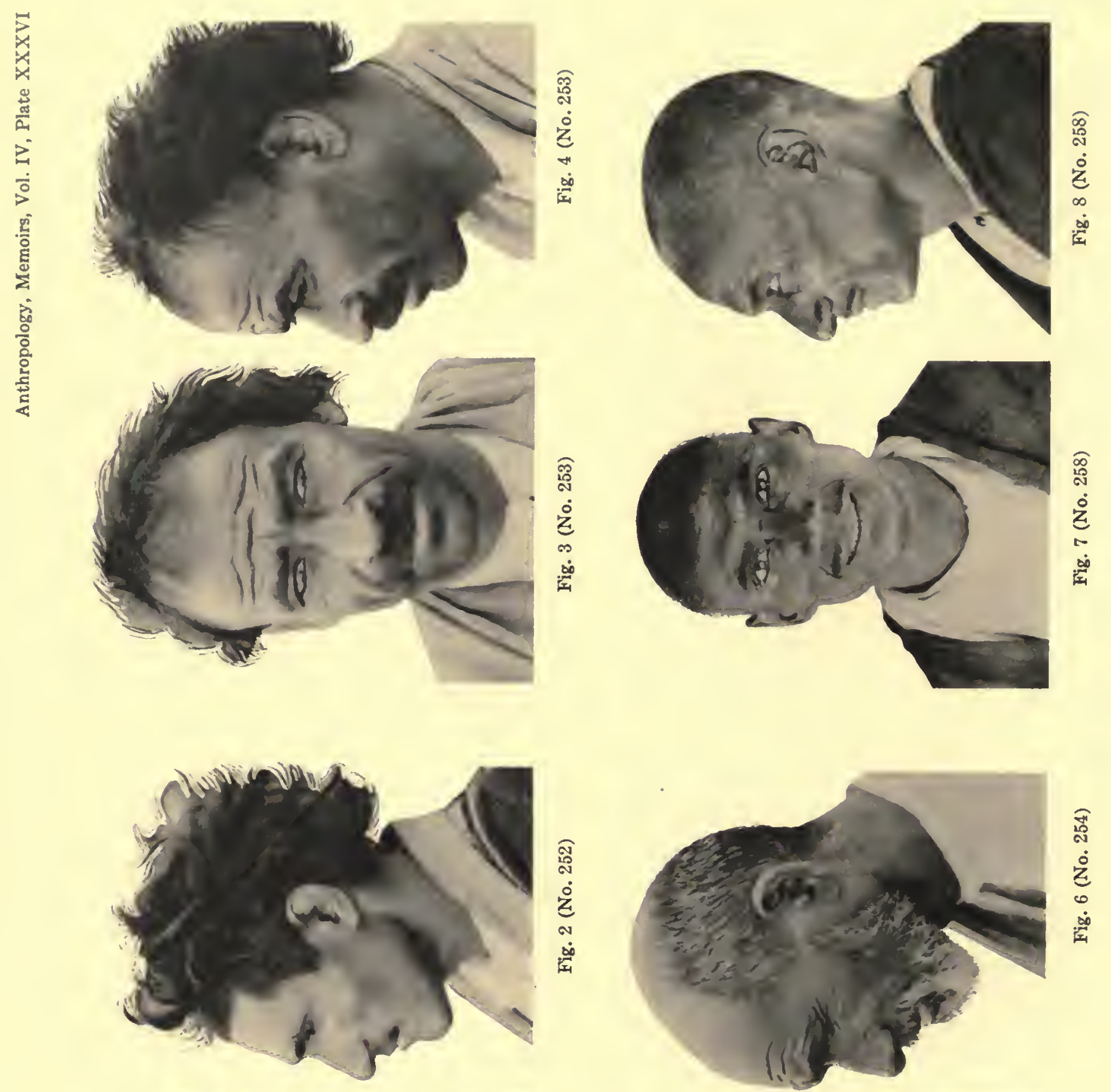

बा

8
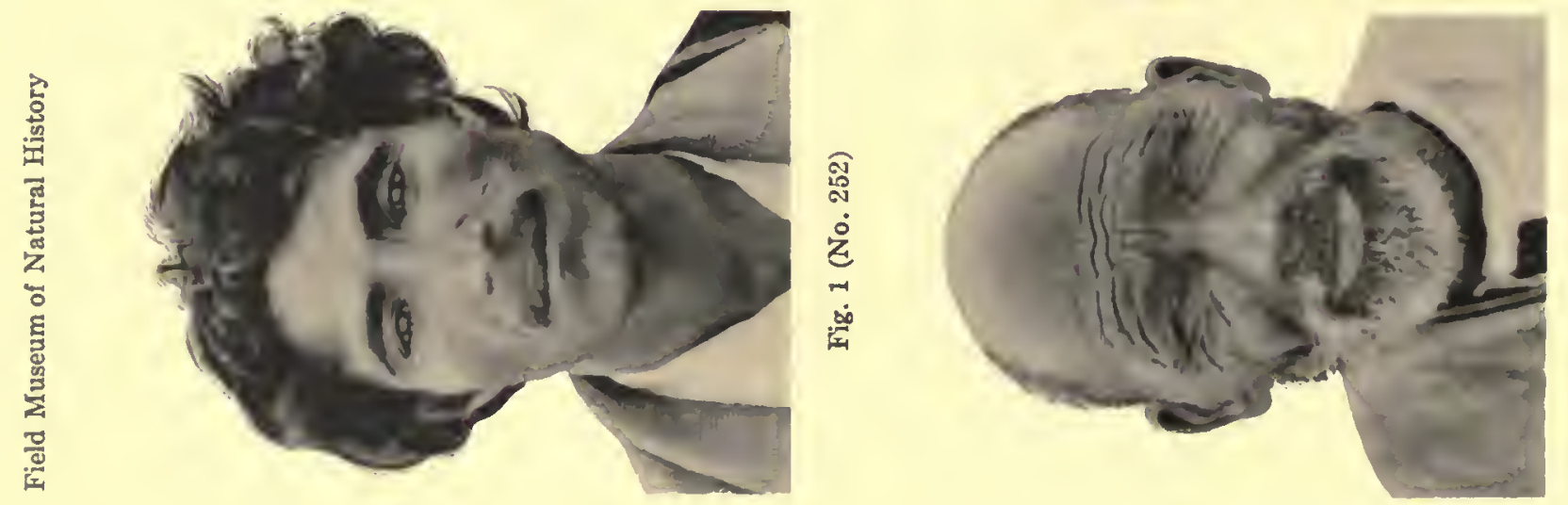

忿 
离
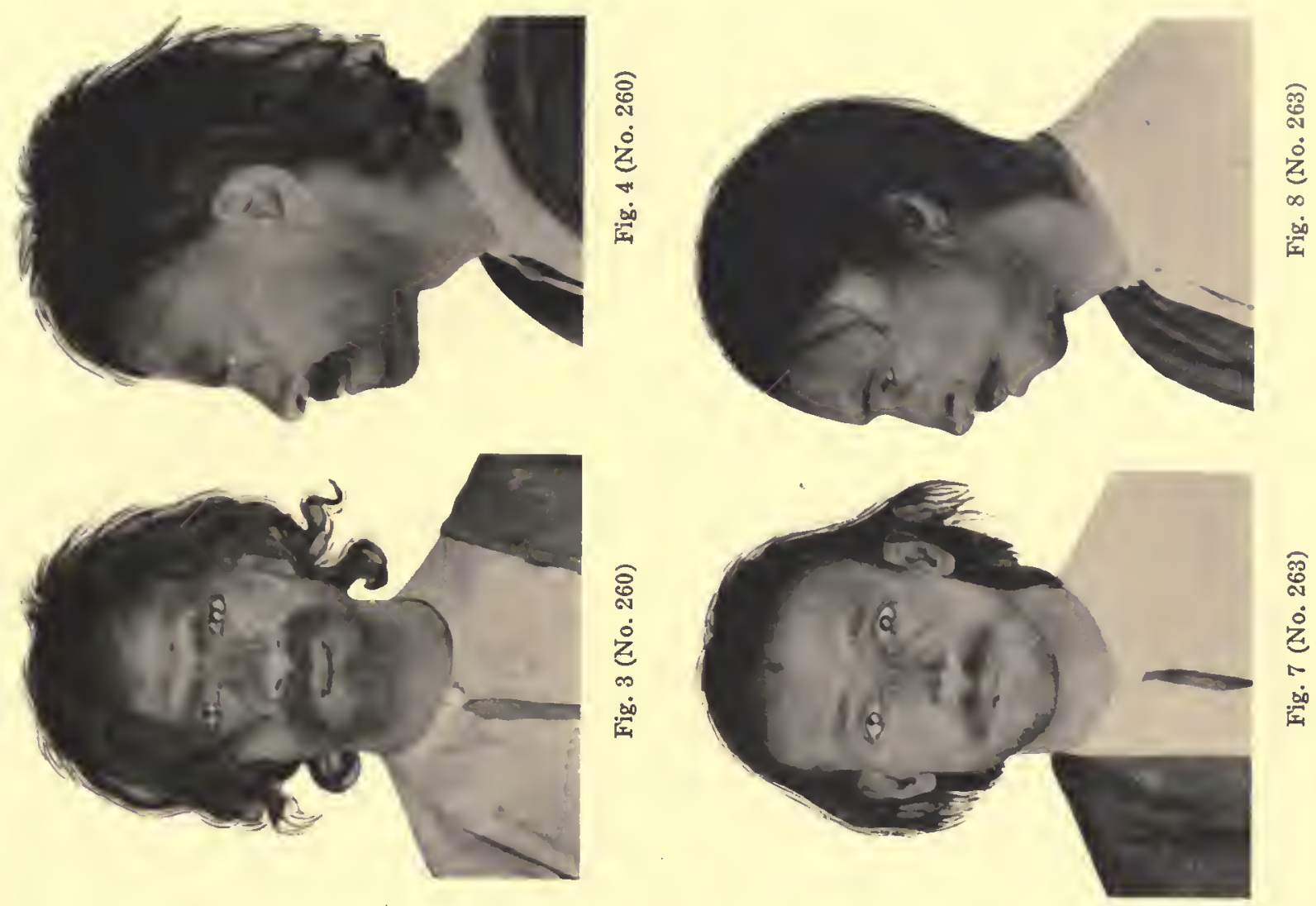

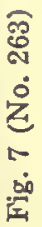
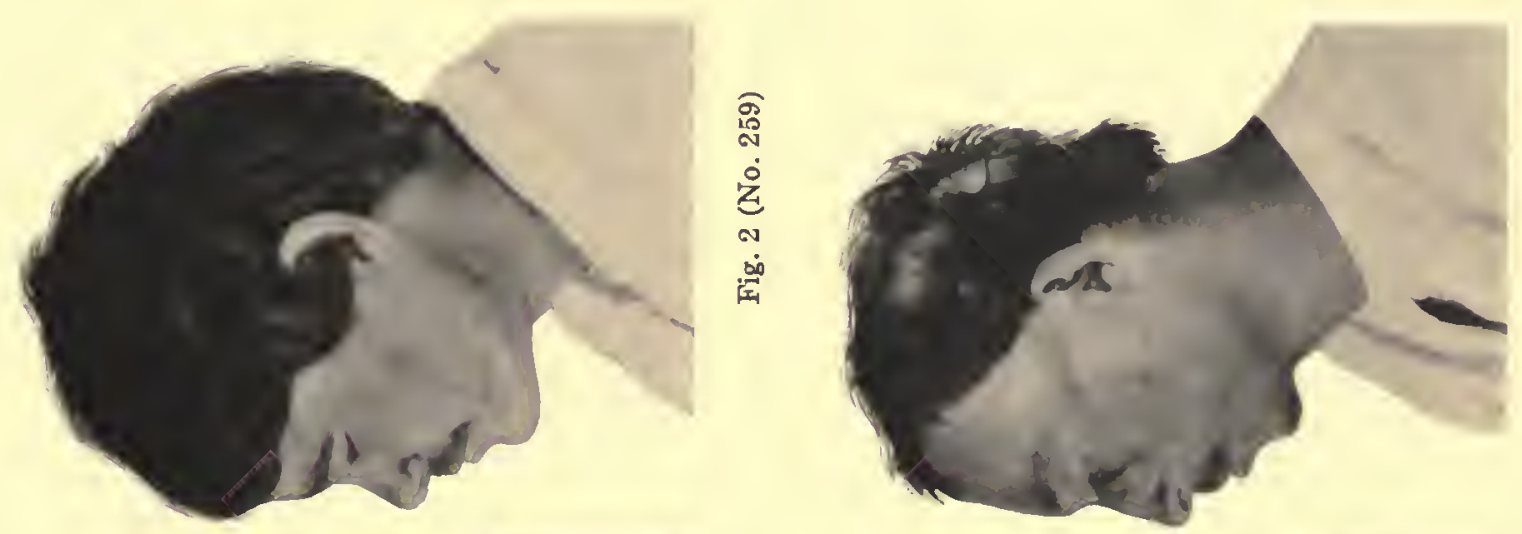

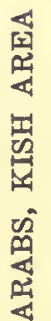
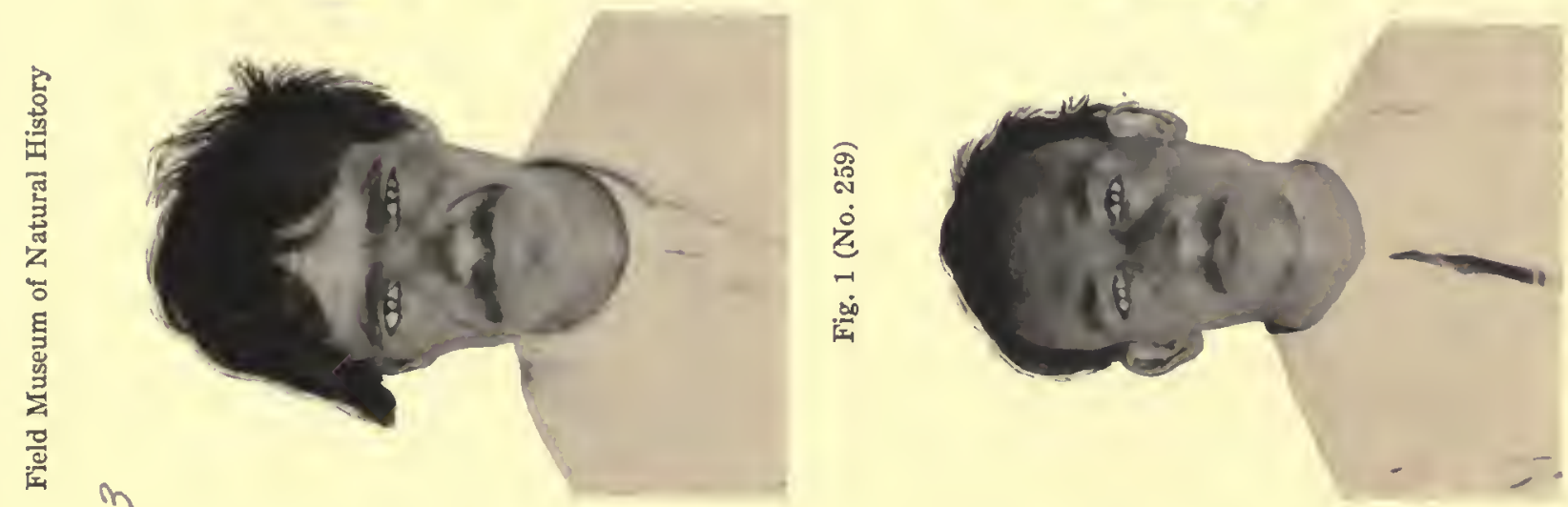

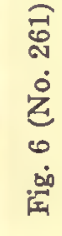

3
2
1
1

No 
是
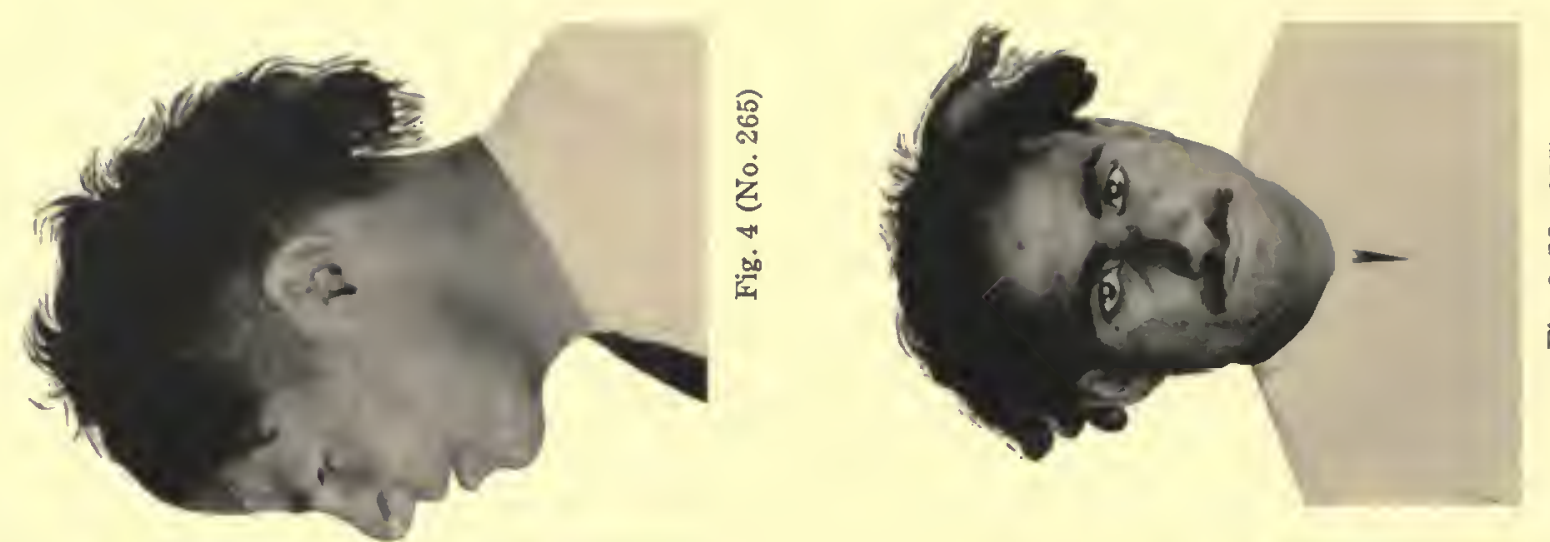

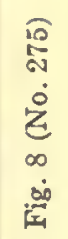
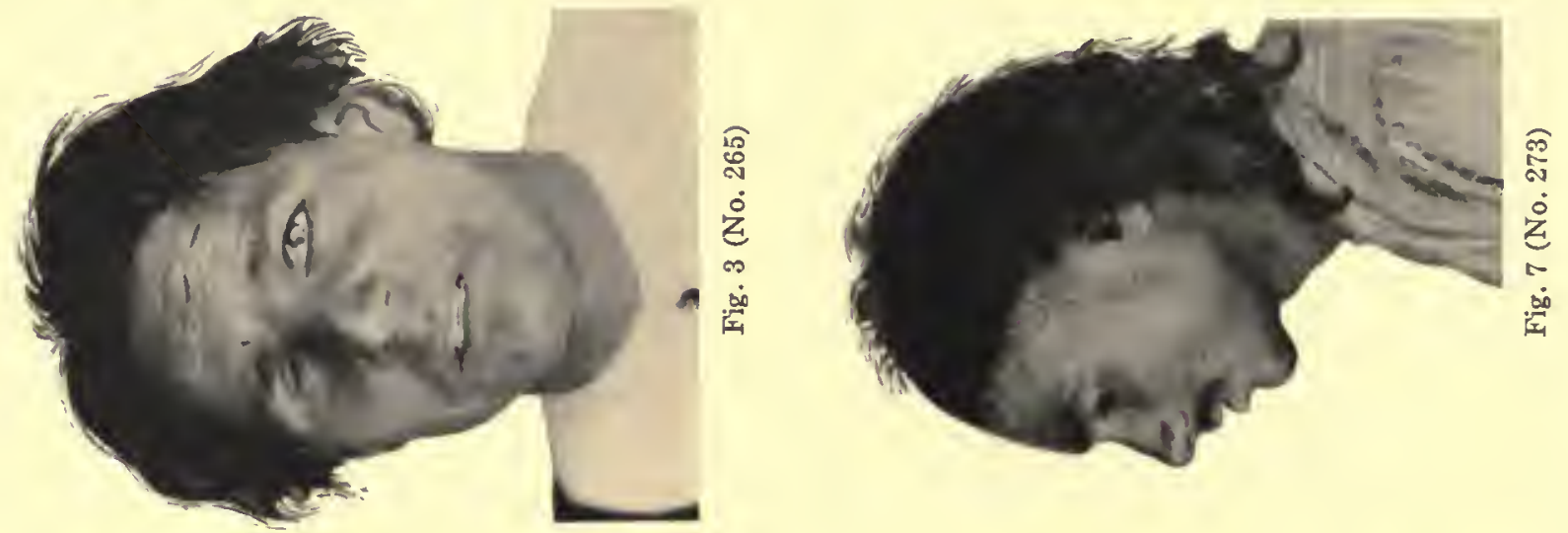

空

을
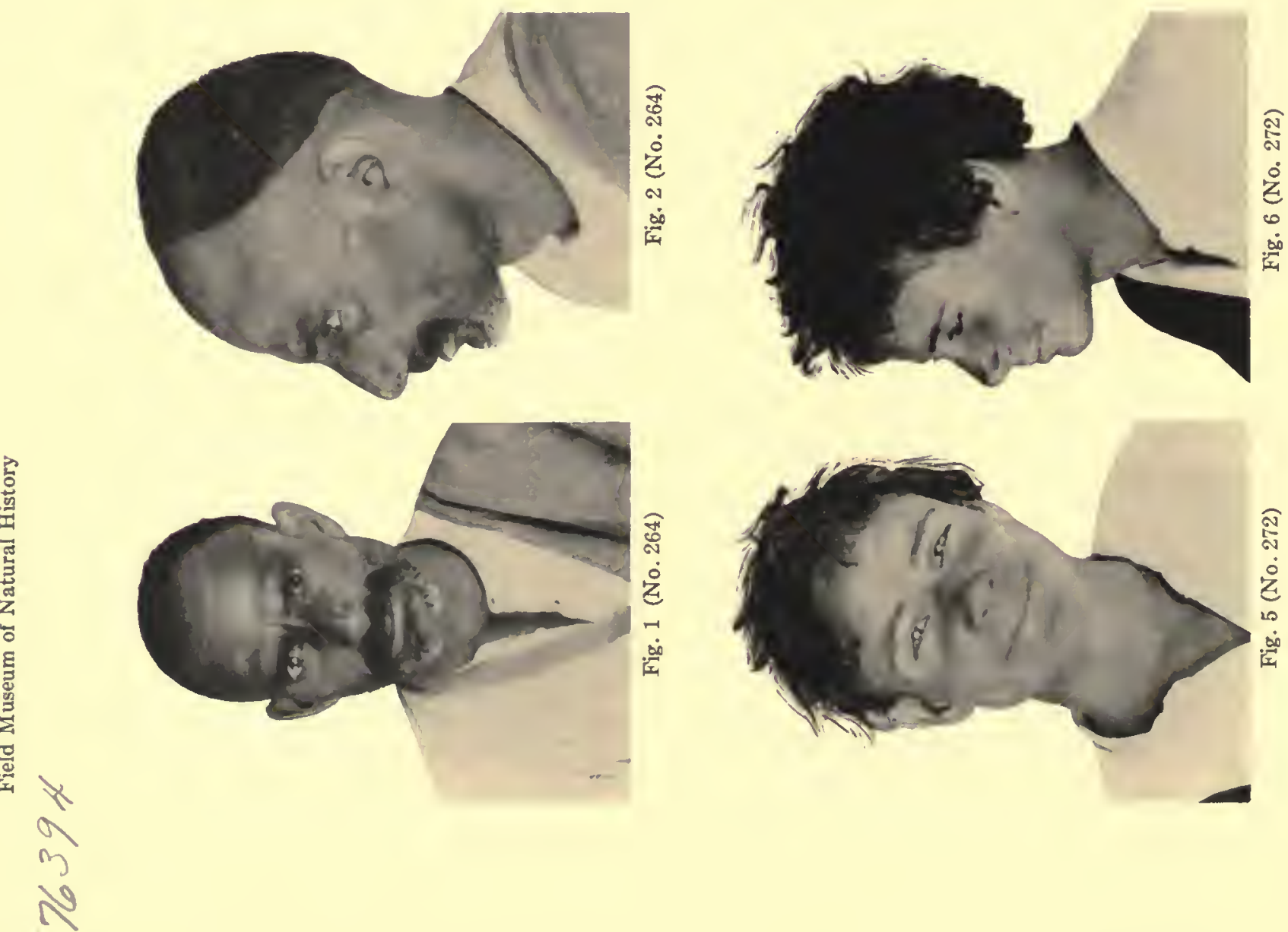

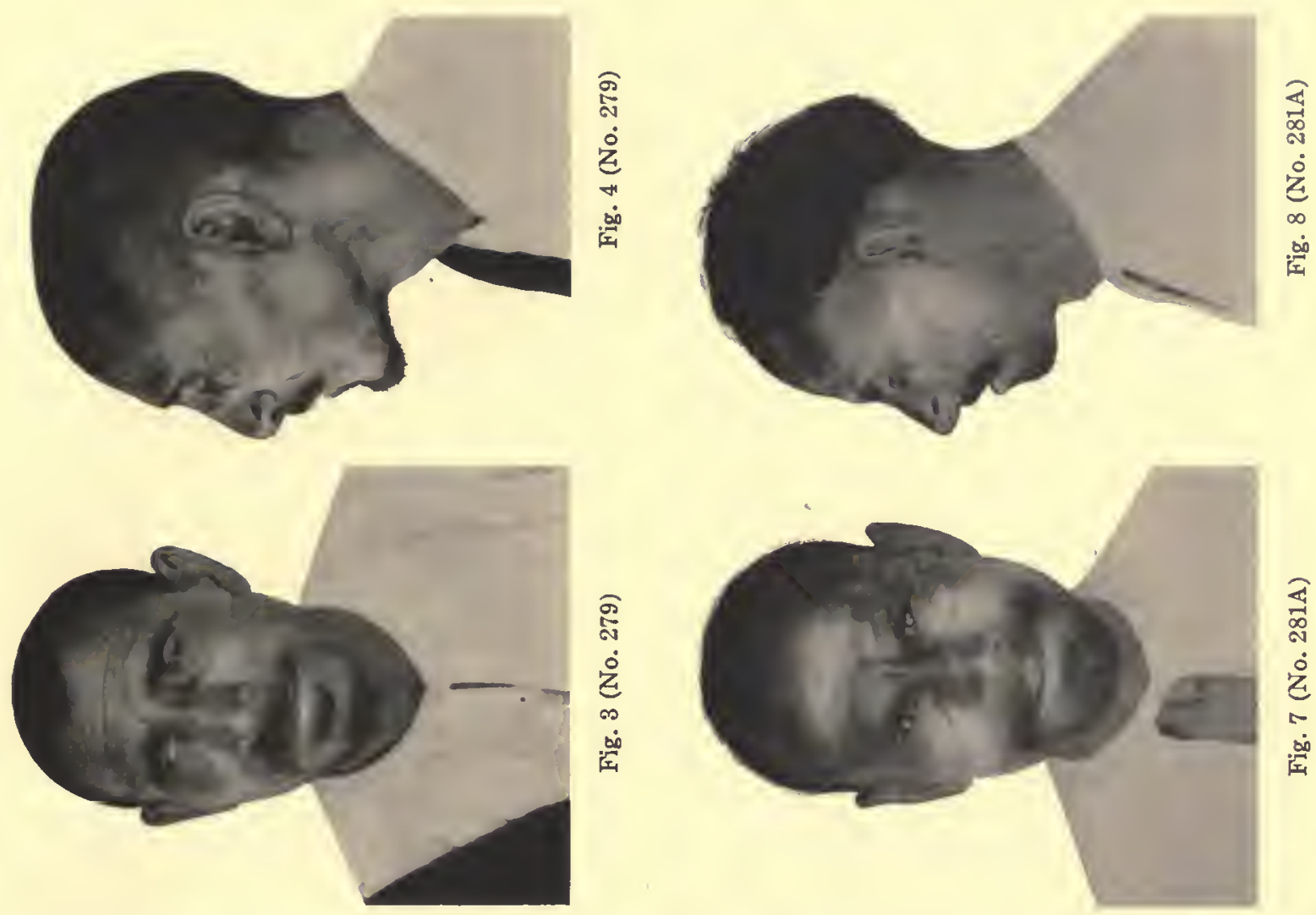

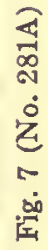
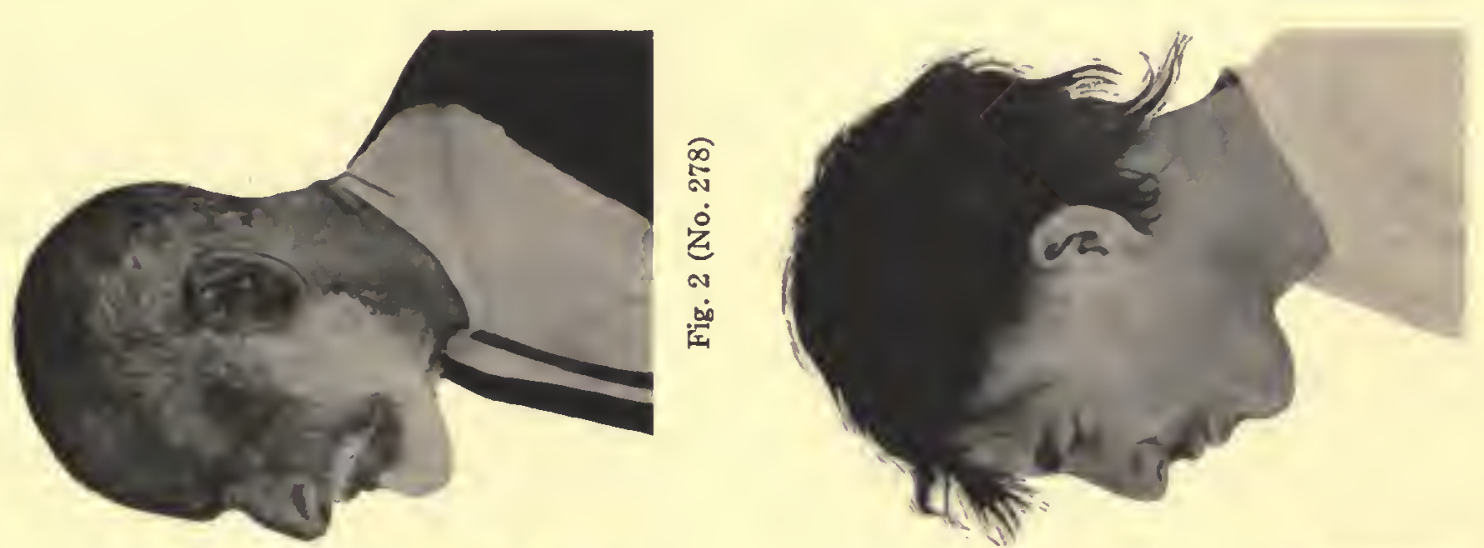

ฮิ

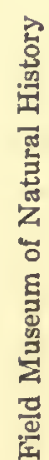
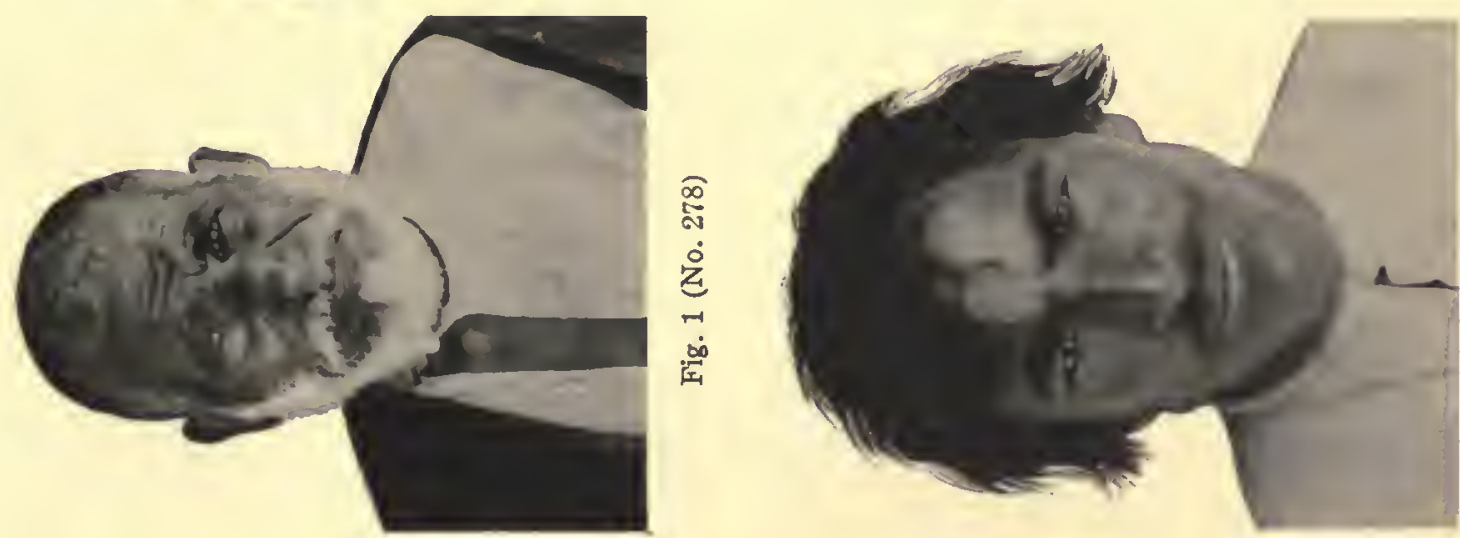

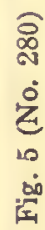

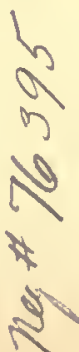



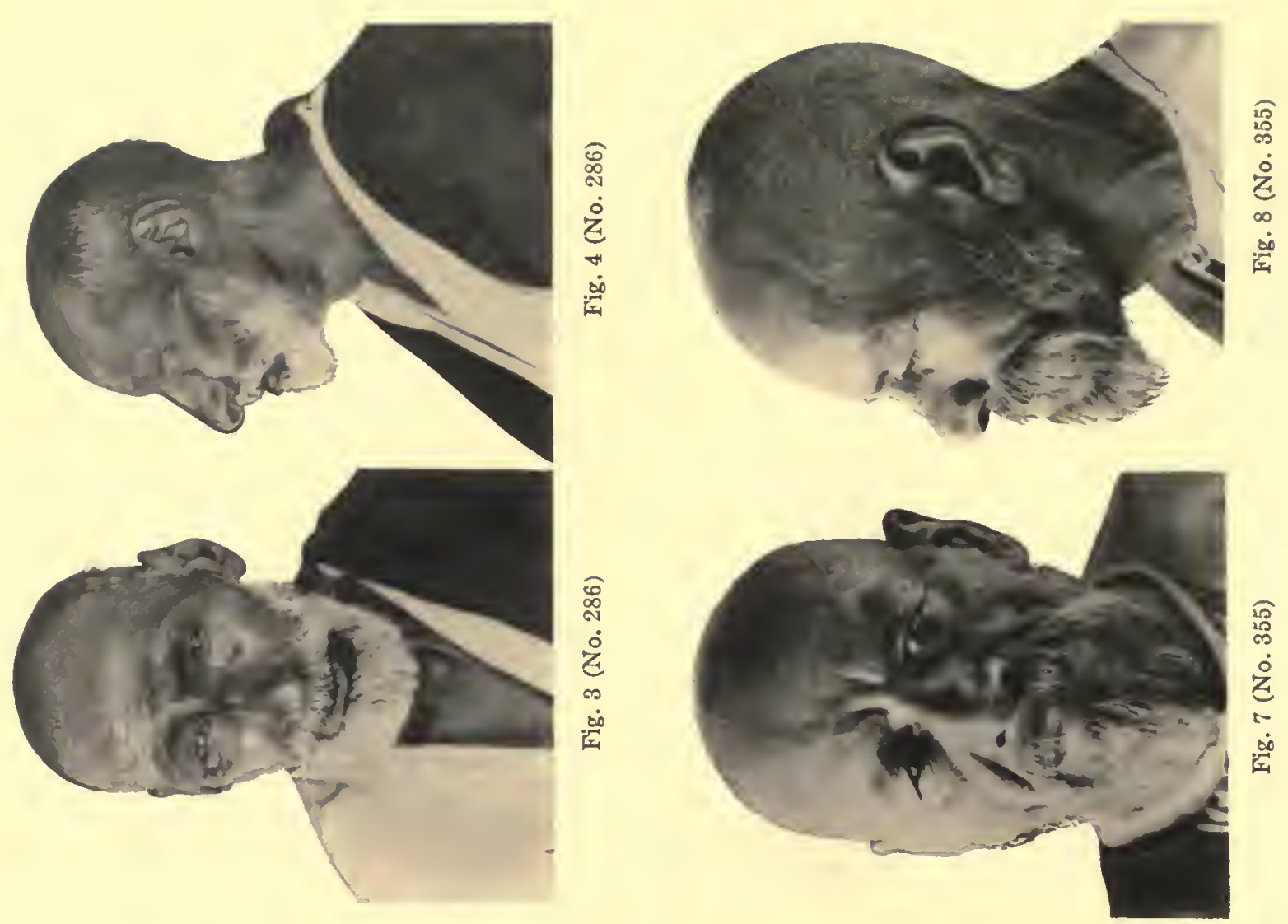

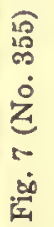
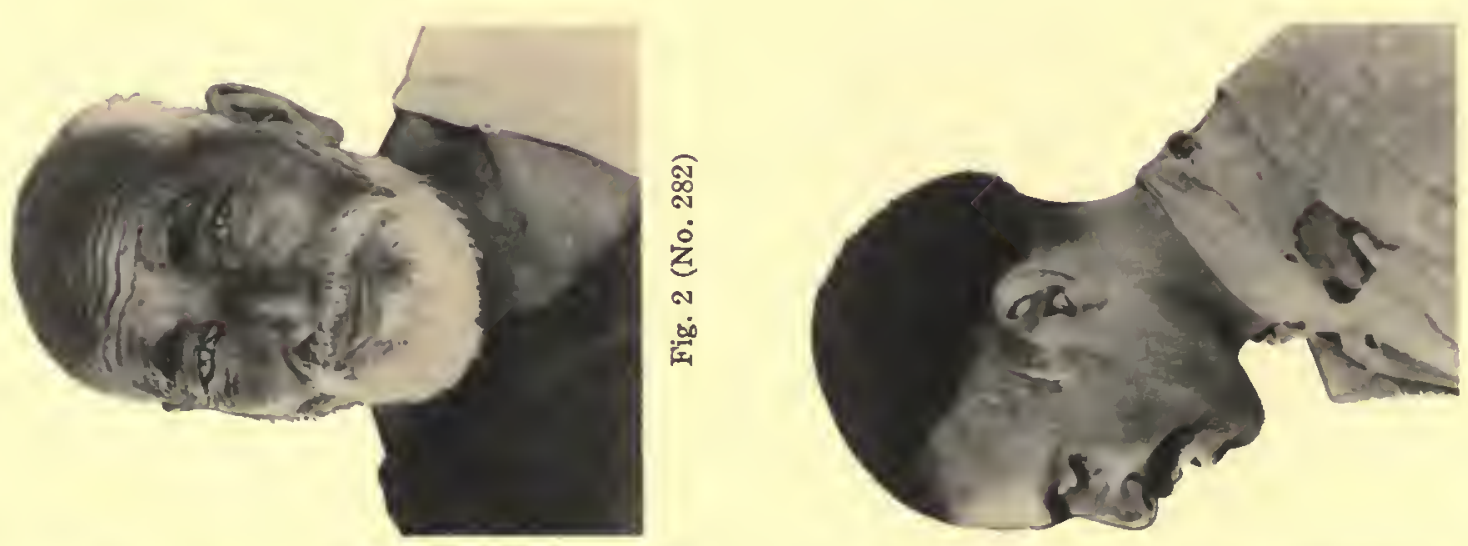

䞡
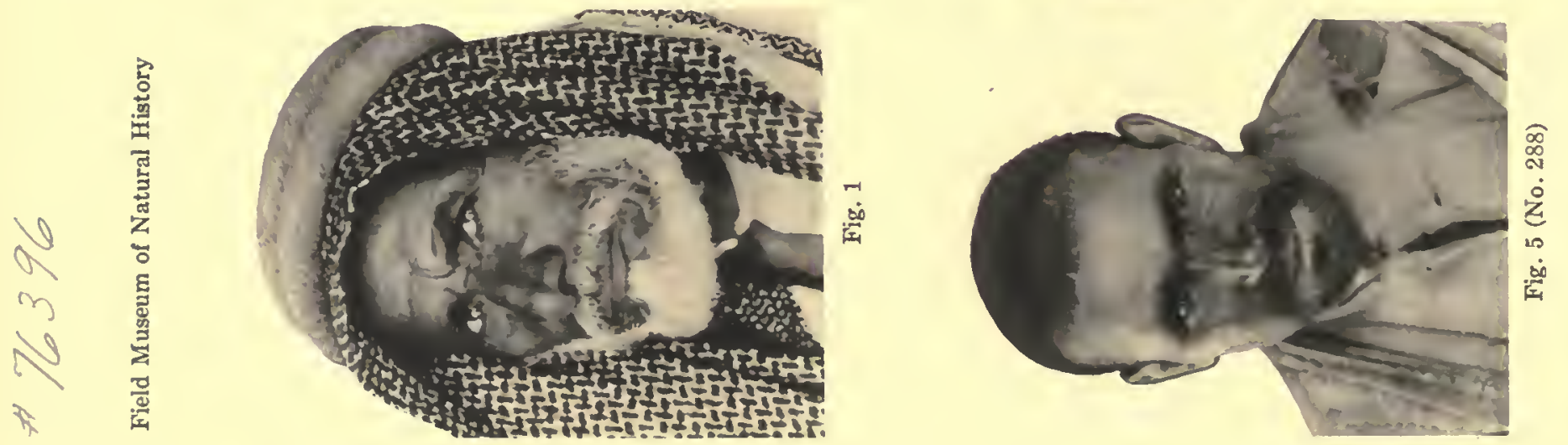

त) 

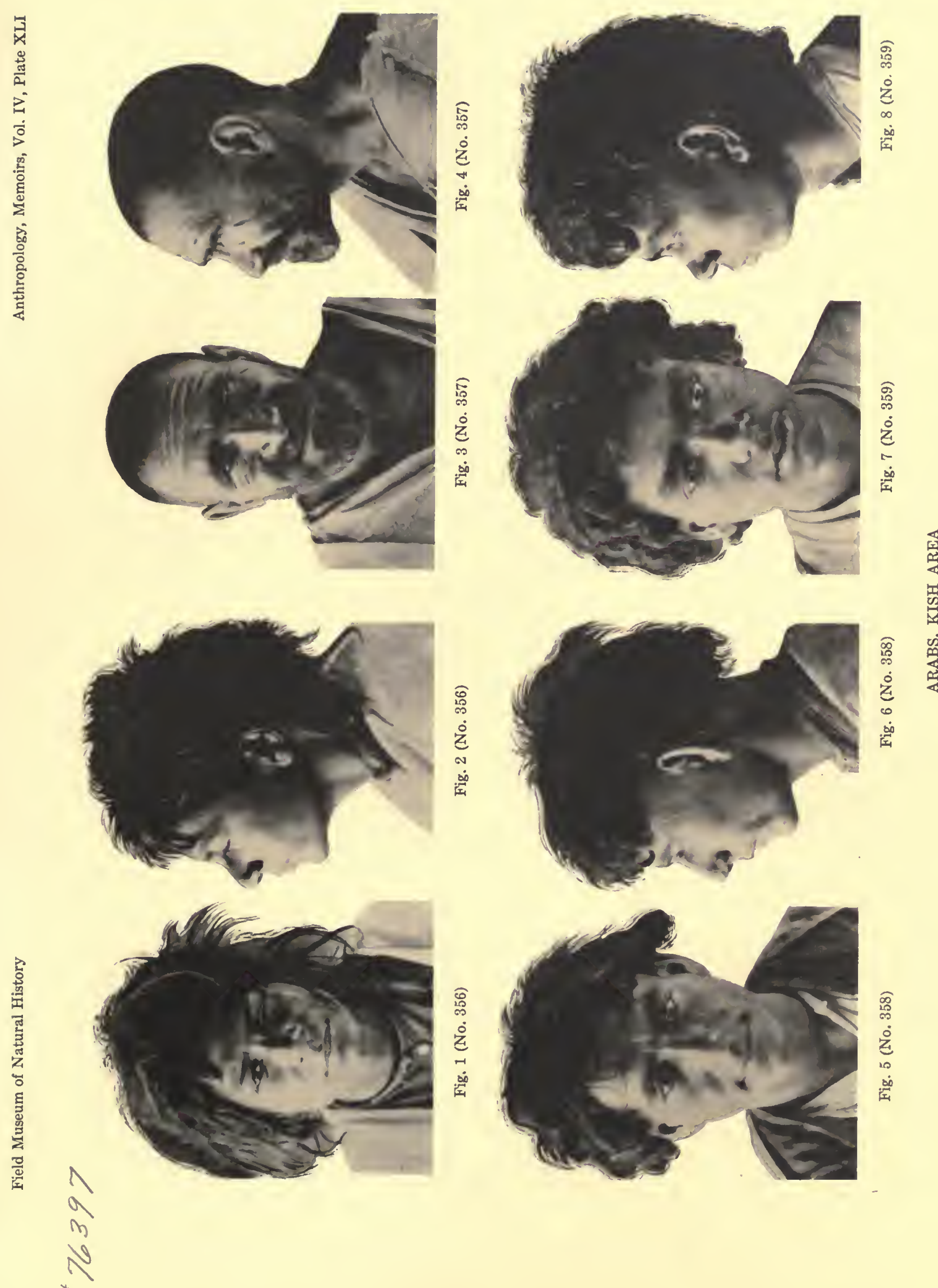

窝

合 留

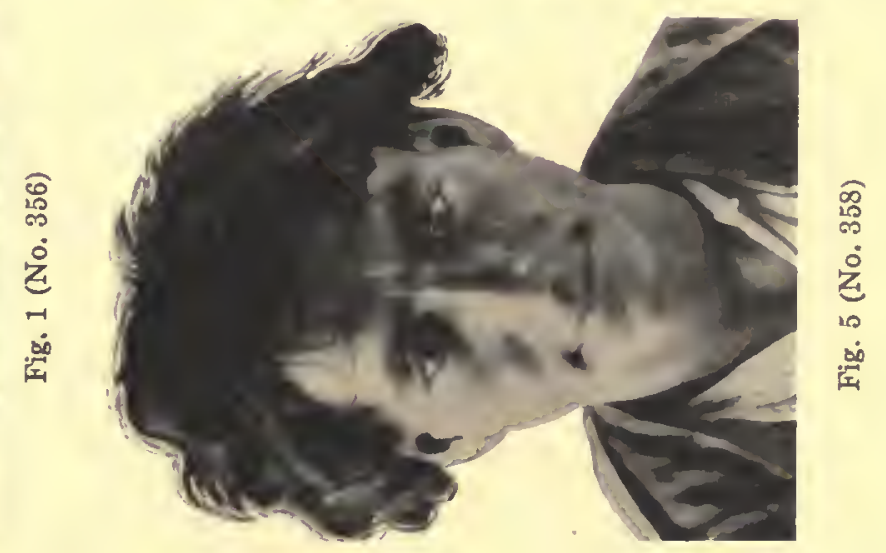



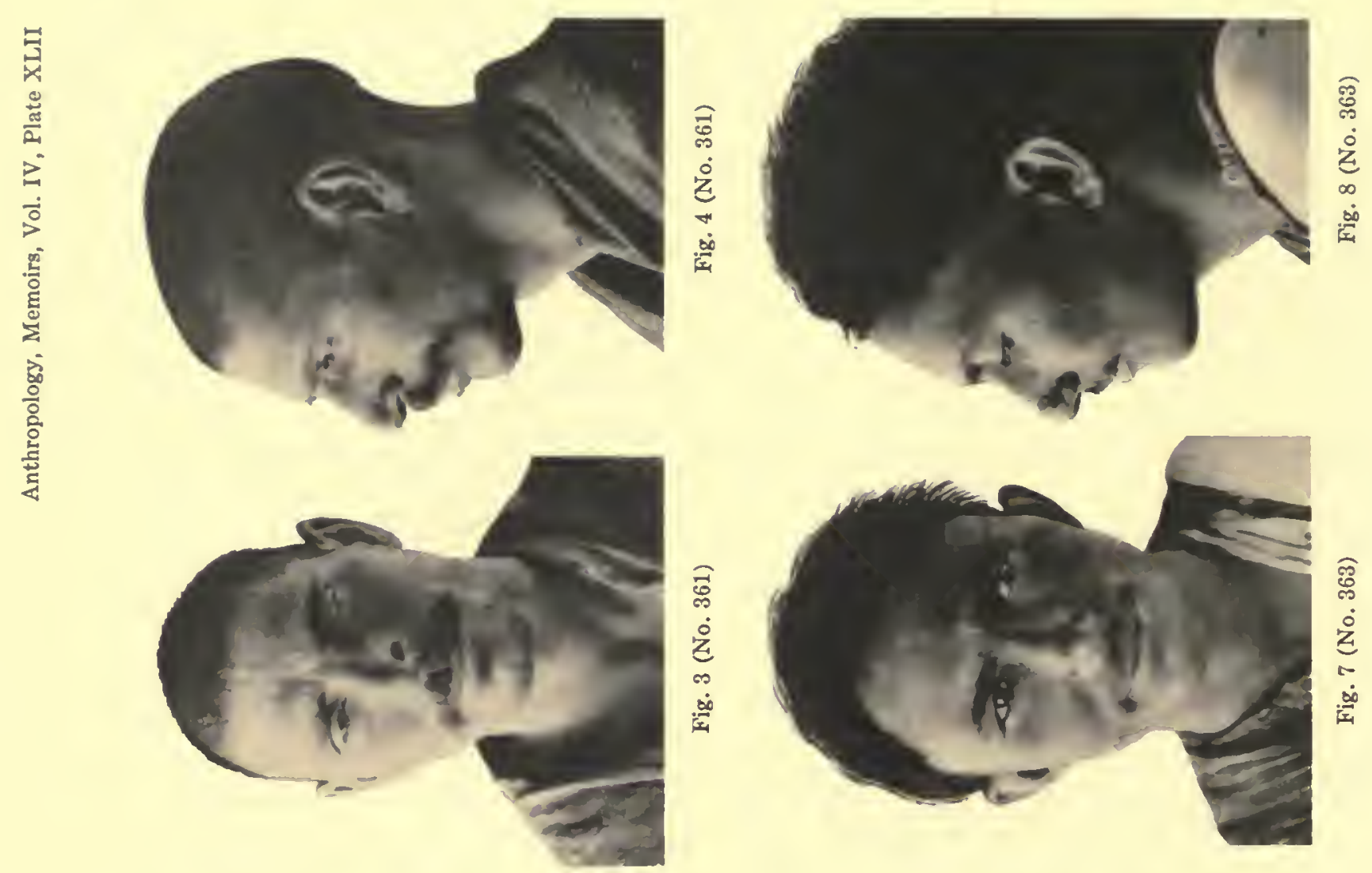

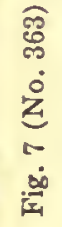
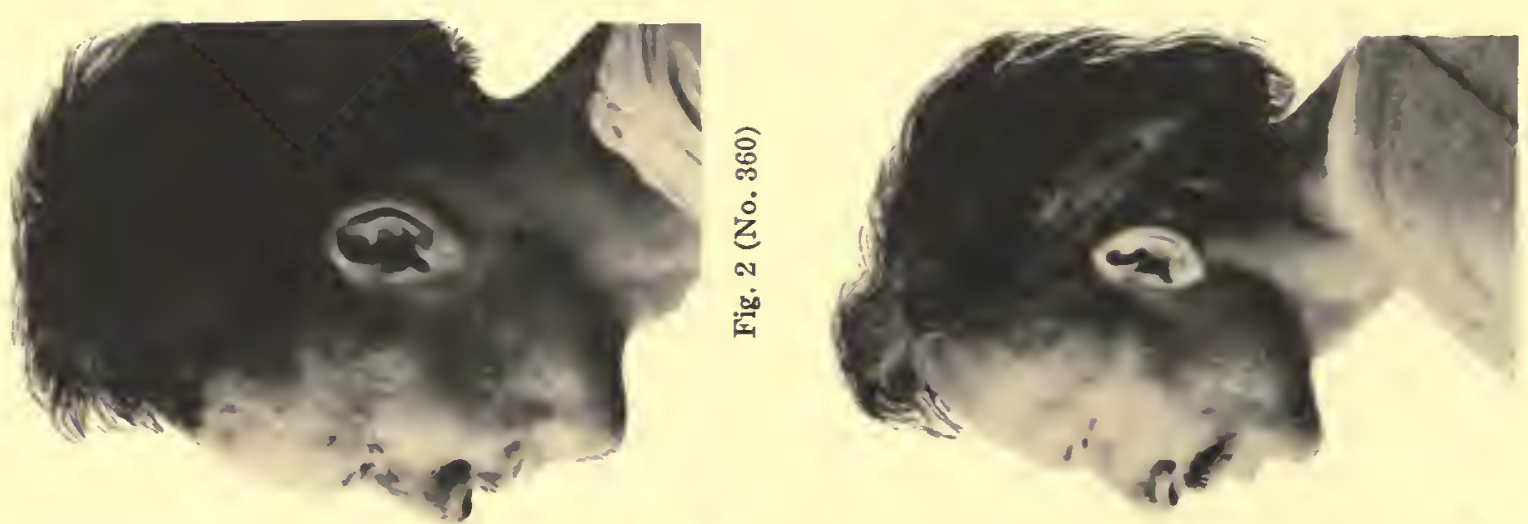

造

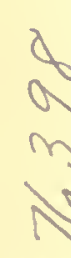
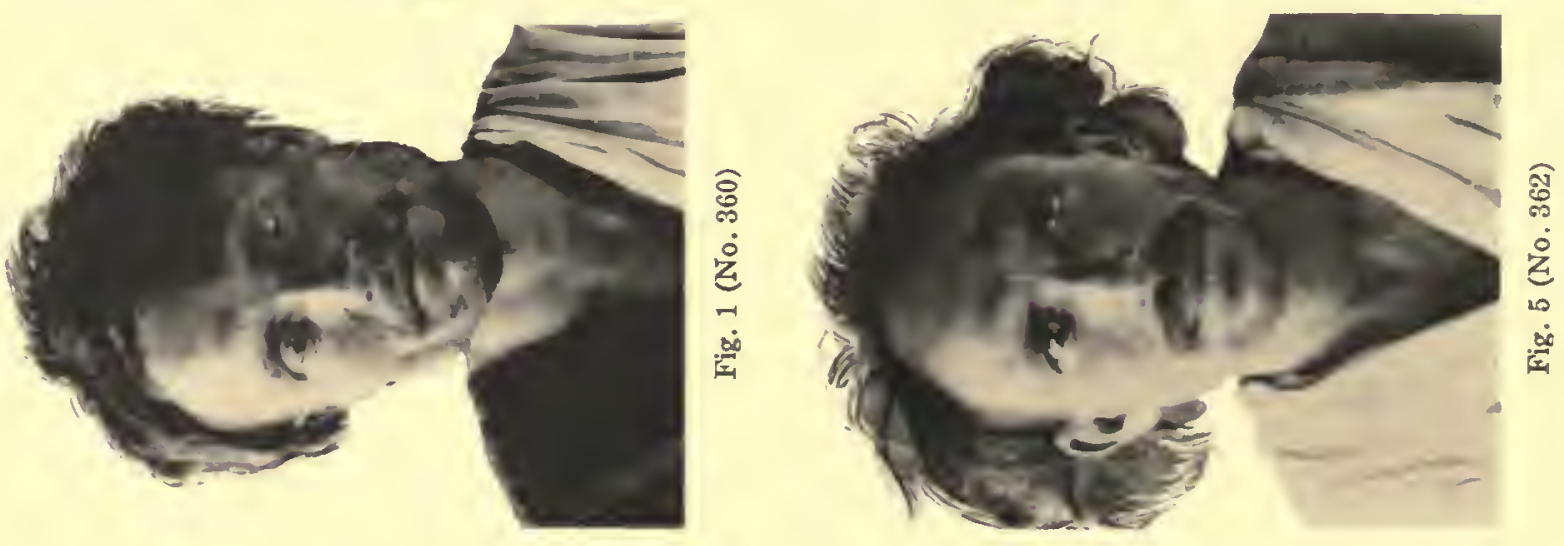

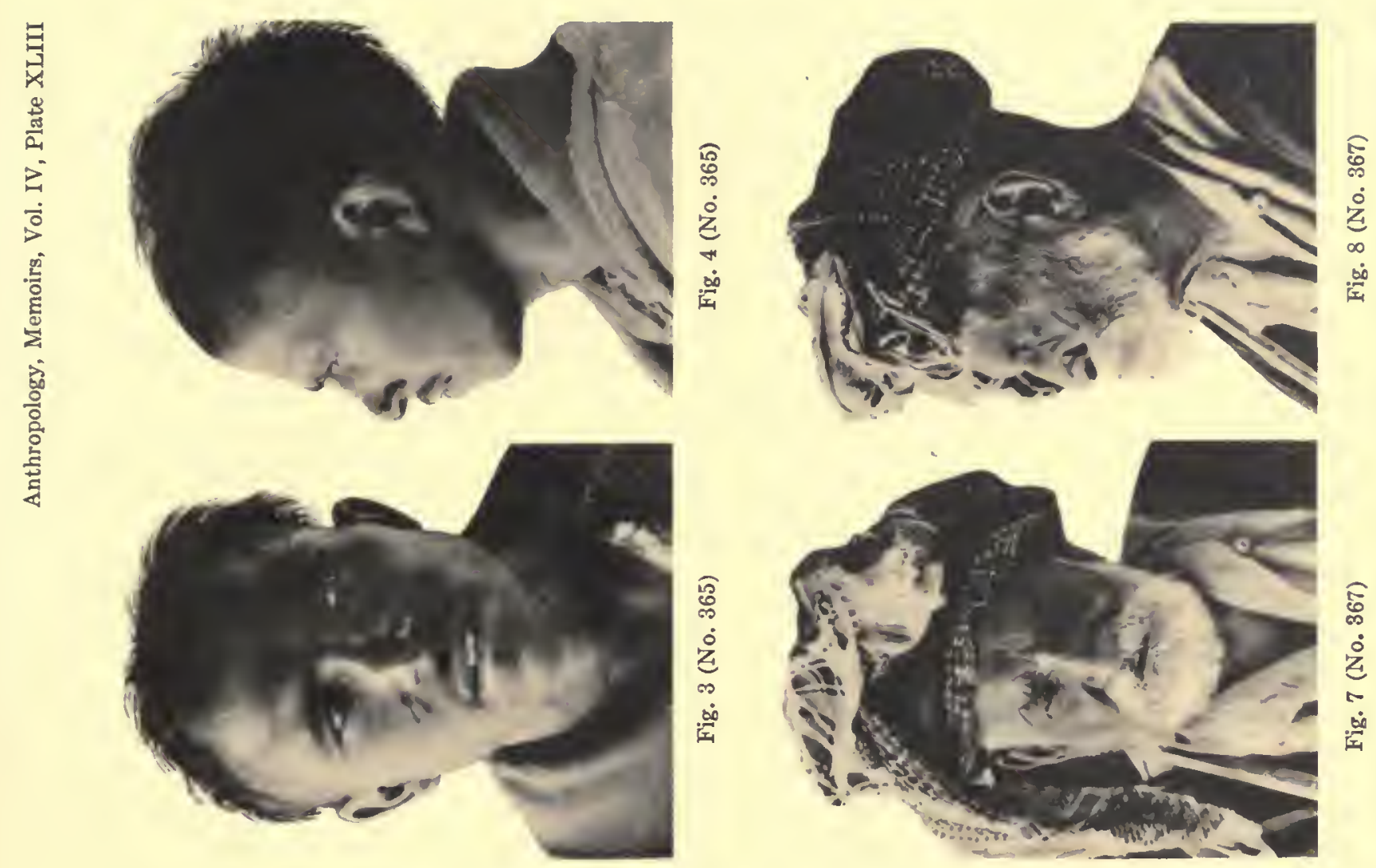

空
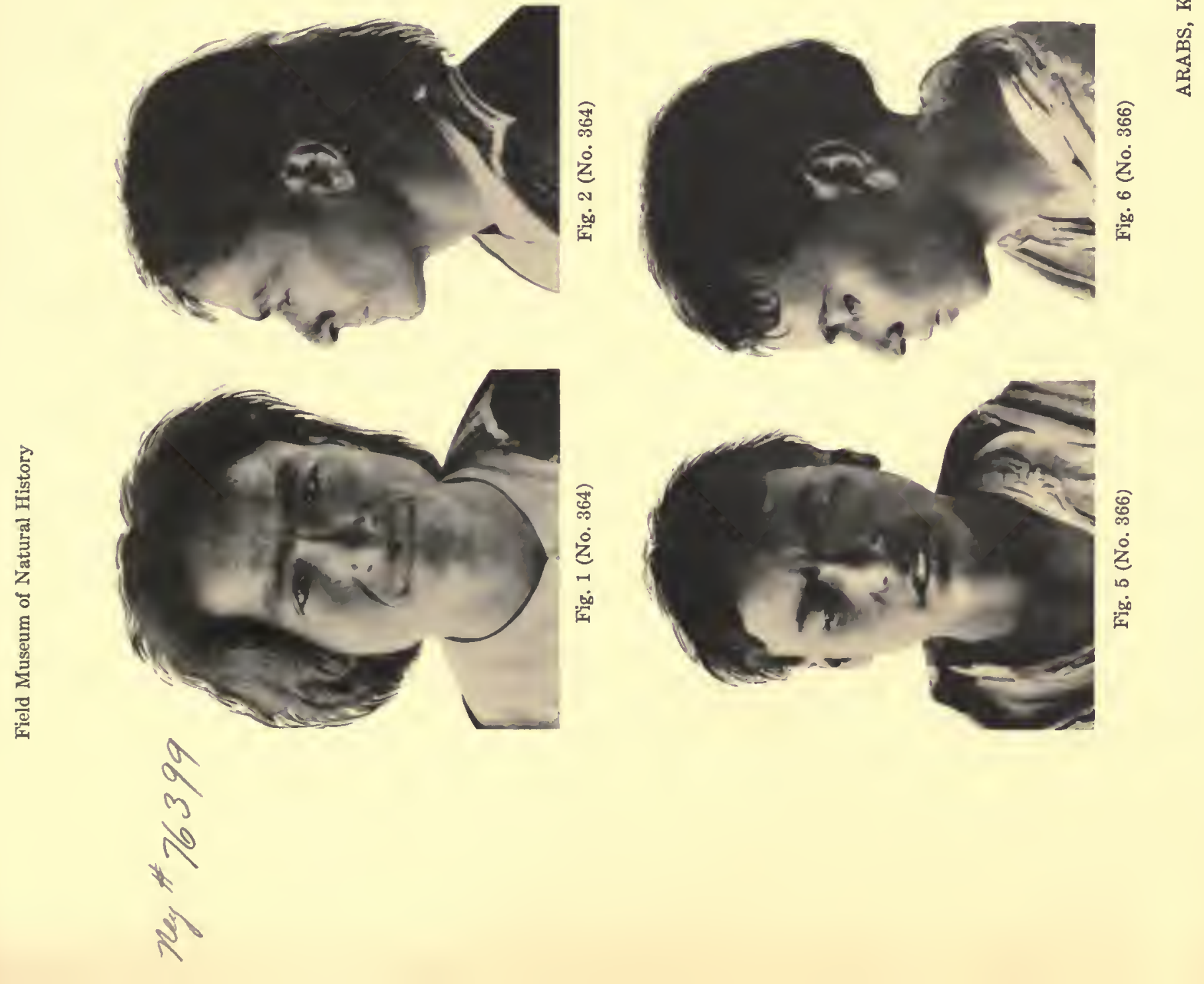

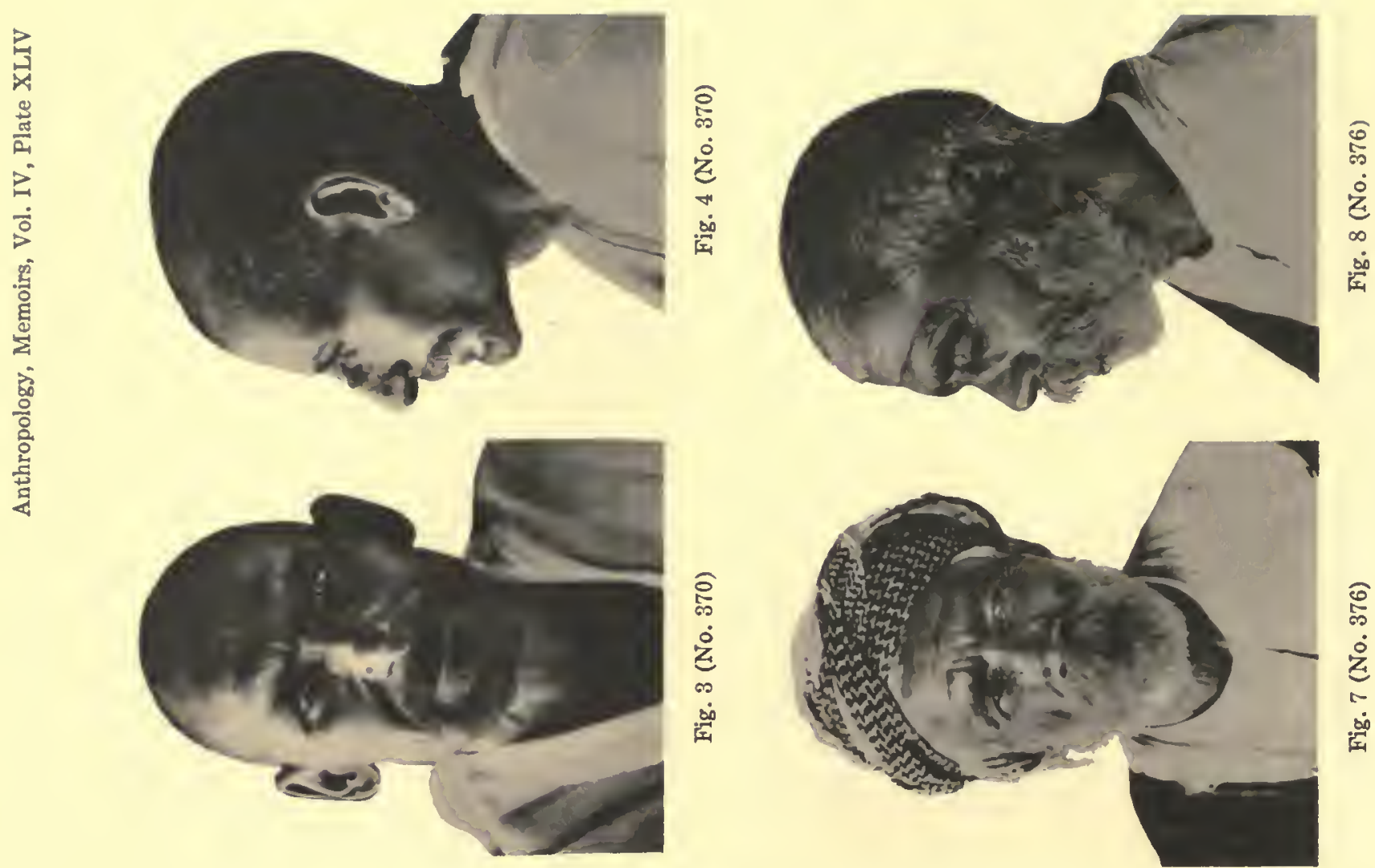

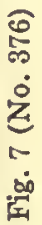
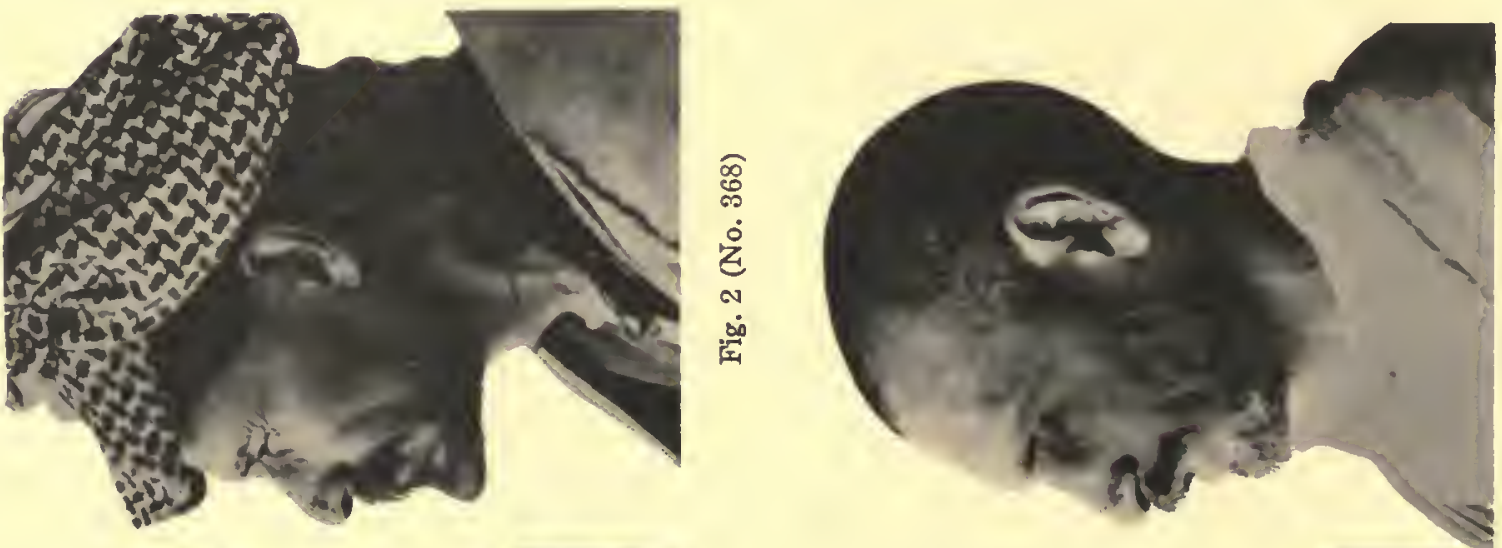

焉
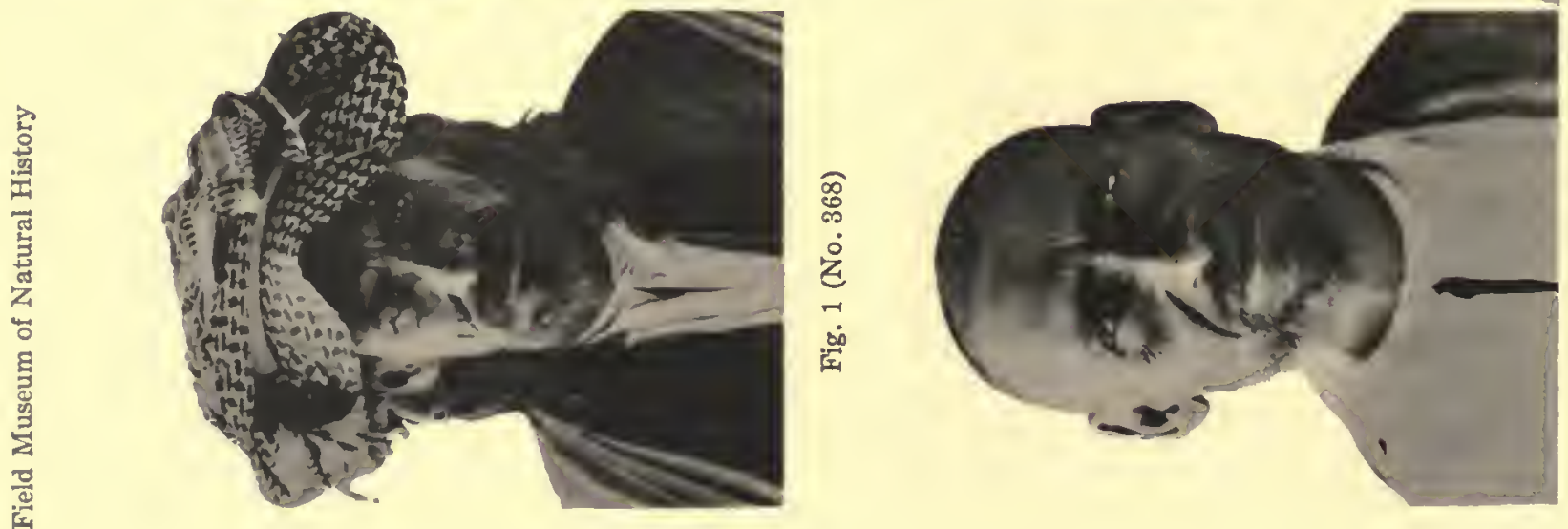

$\hat{0}$
0
$\dot{0}$
0
0
0

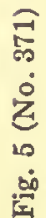
st 

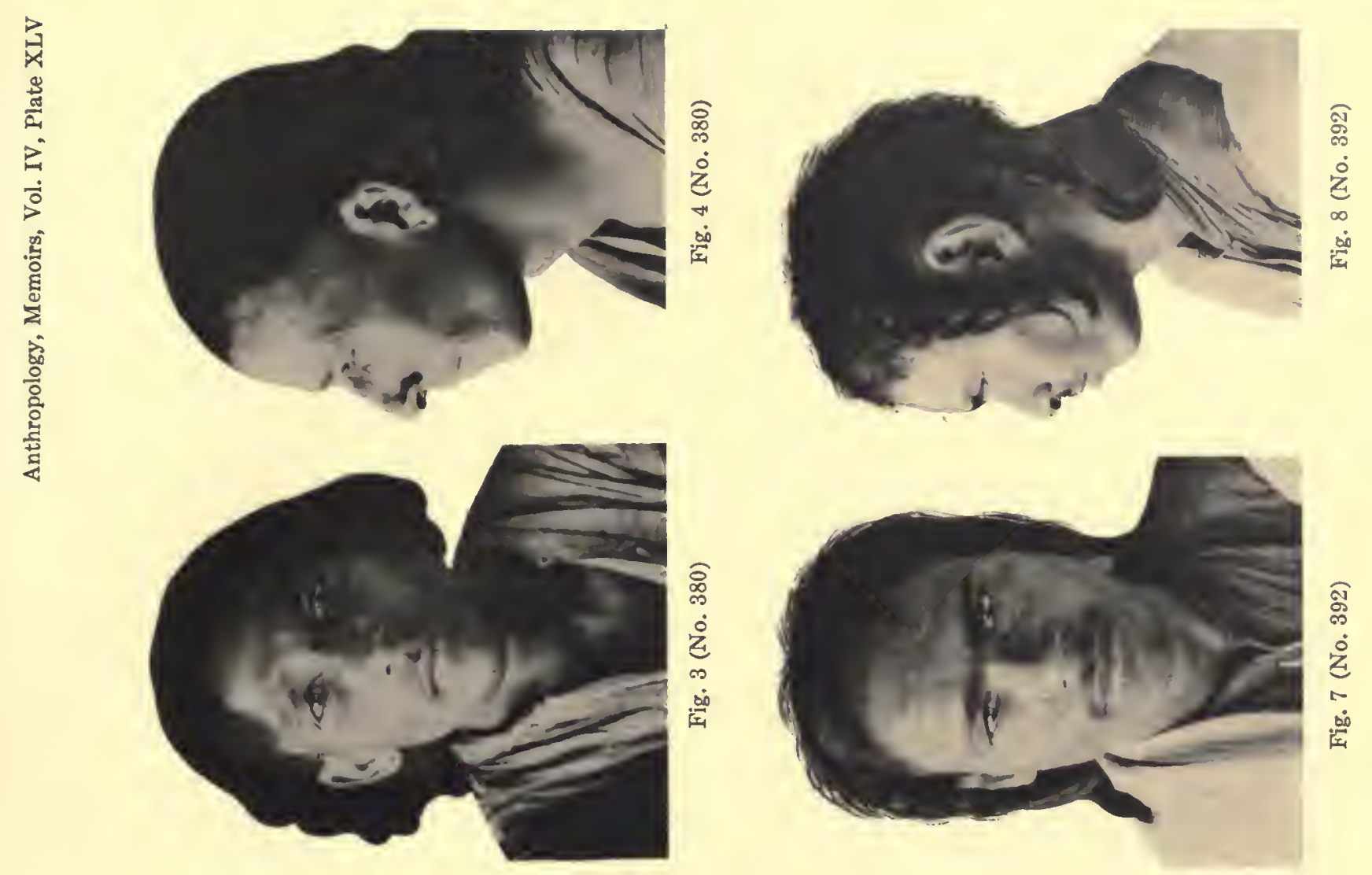

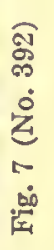
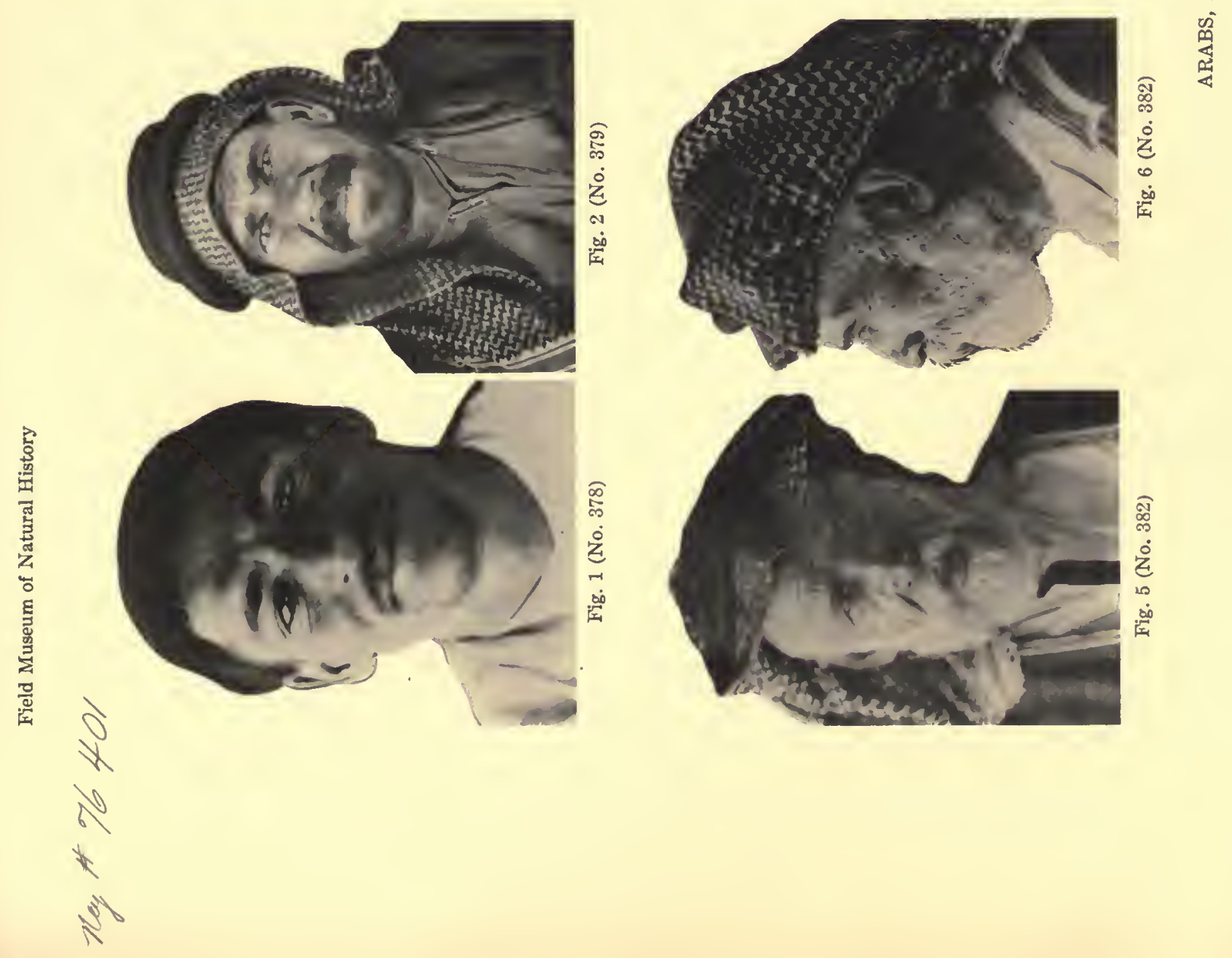

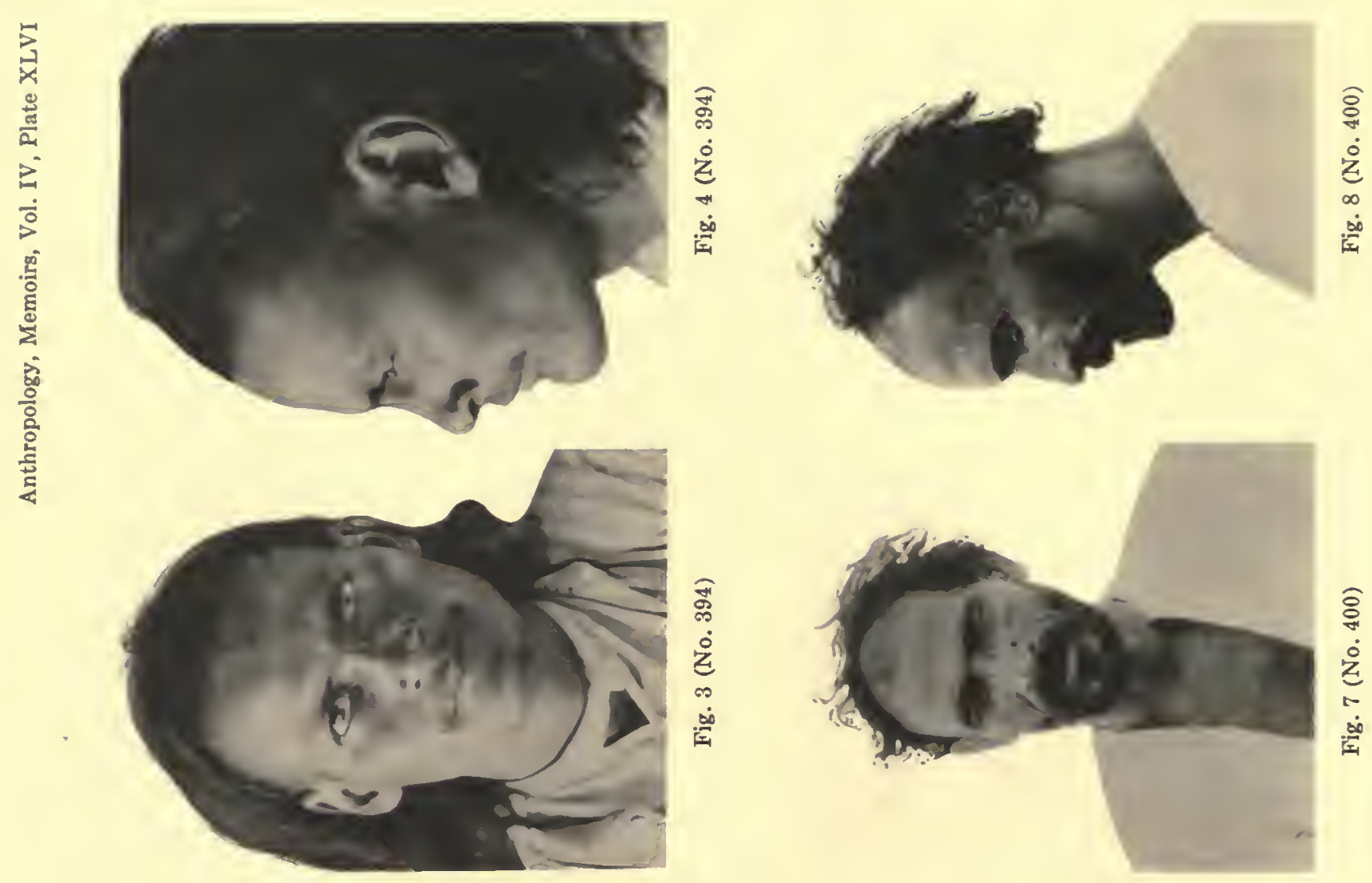

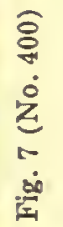
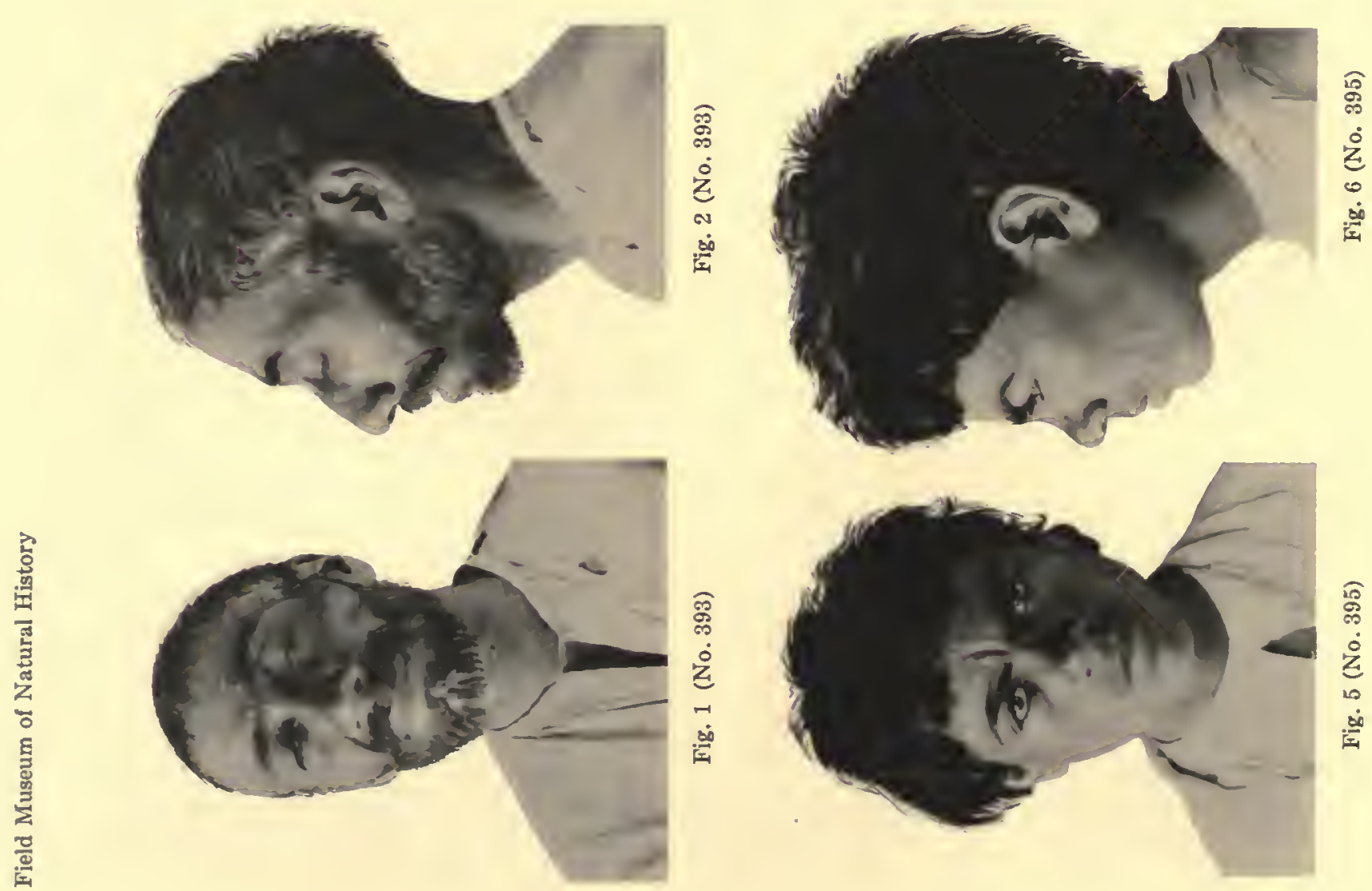

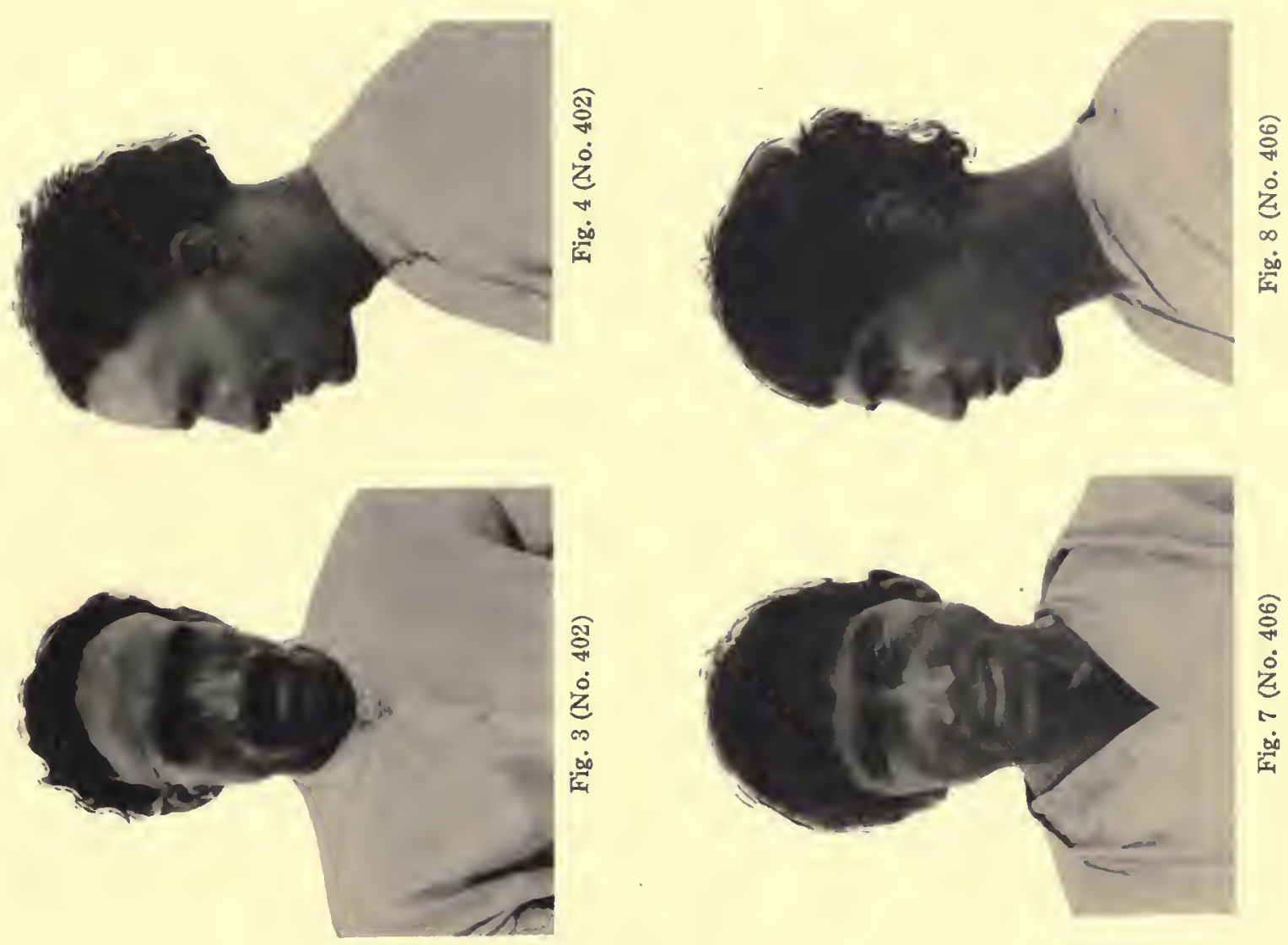

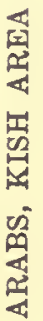
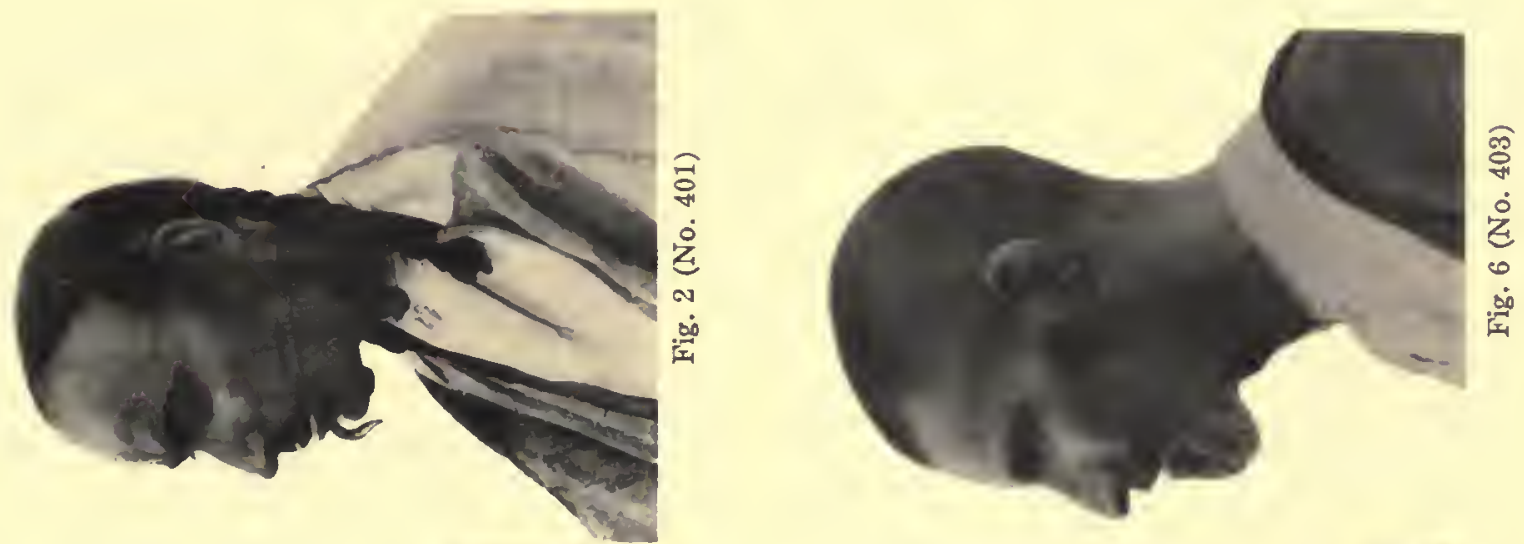

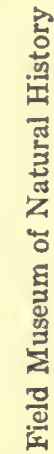

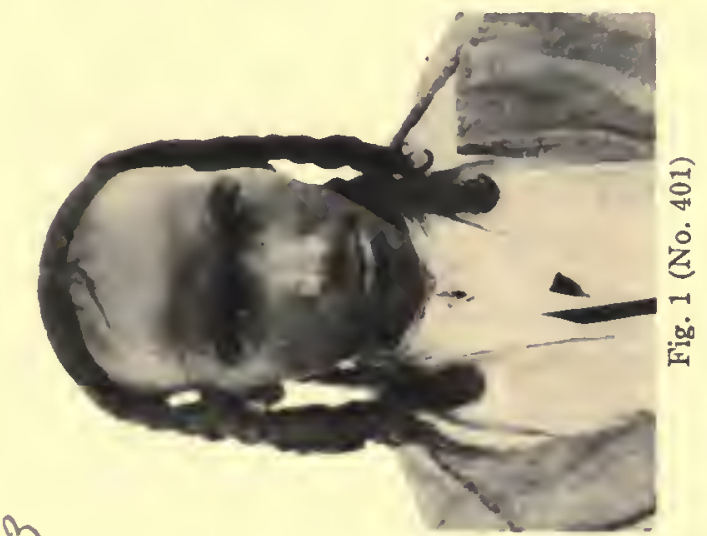

3
0
1

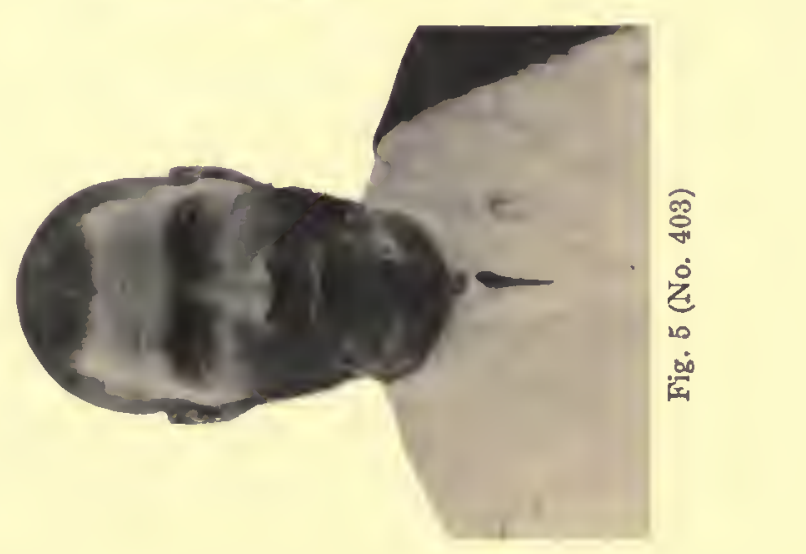

진 

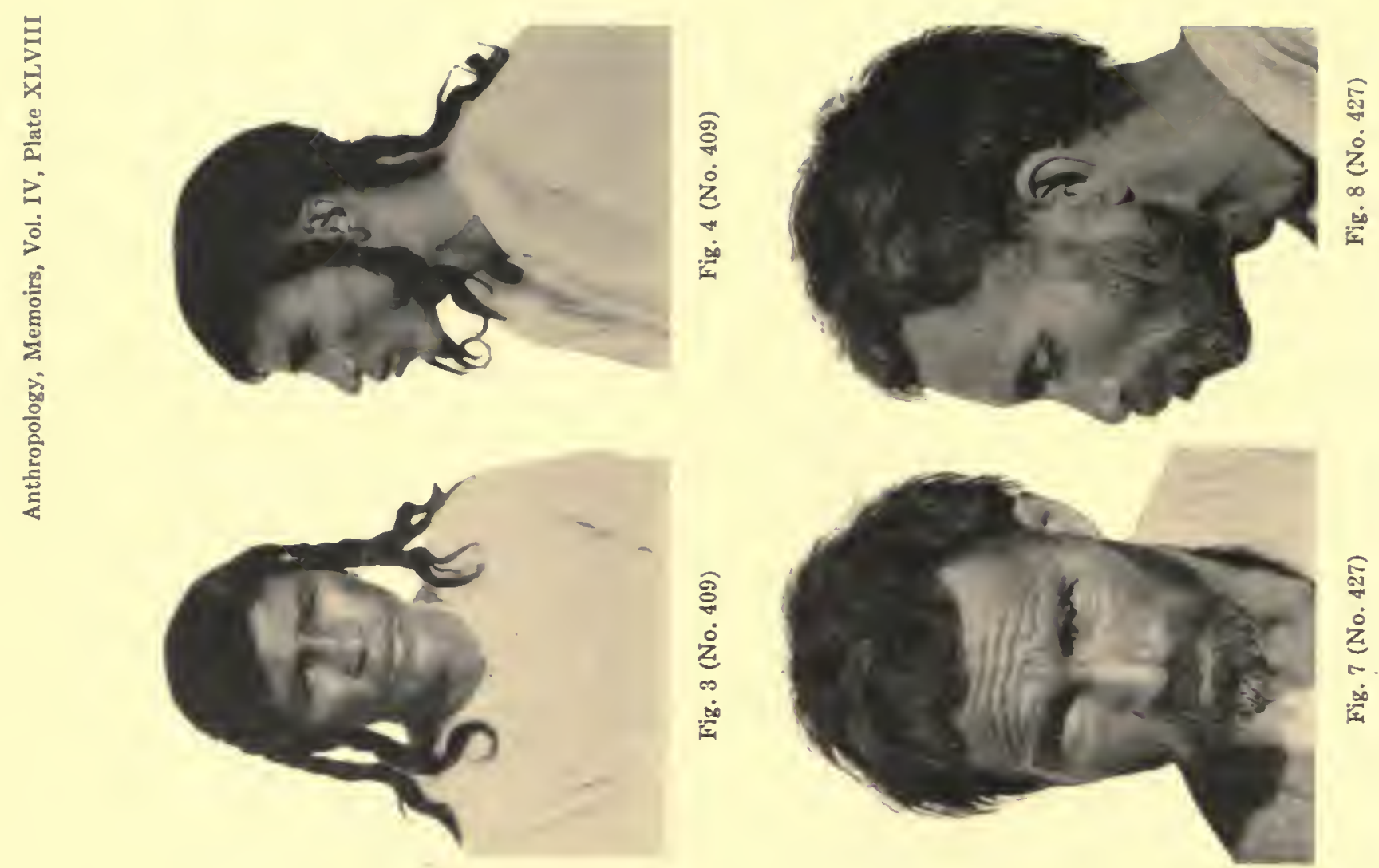

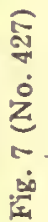
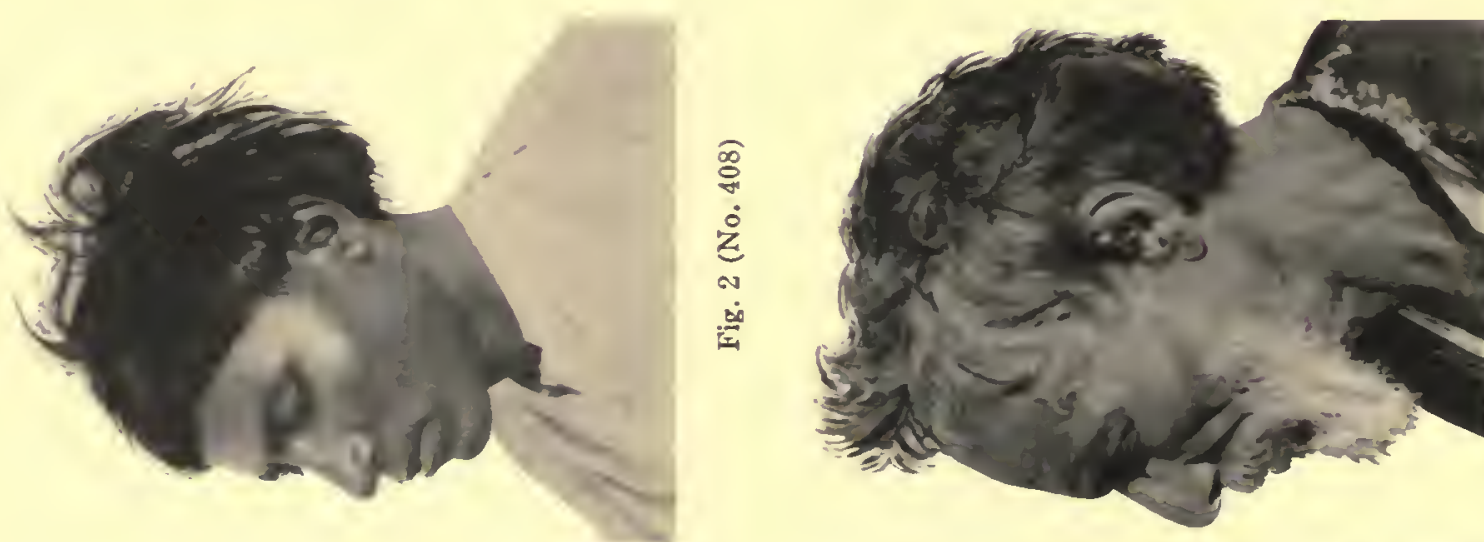

壳

W
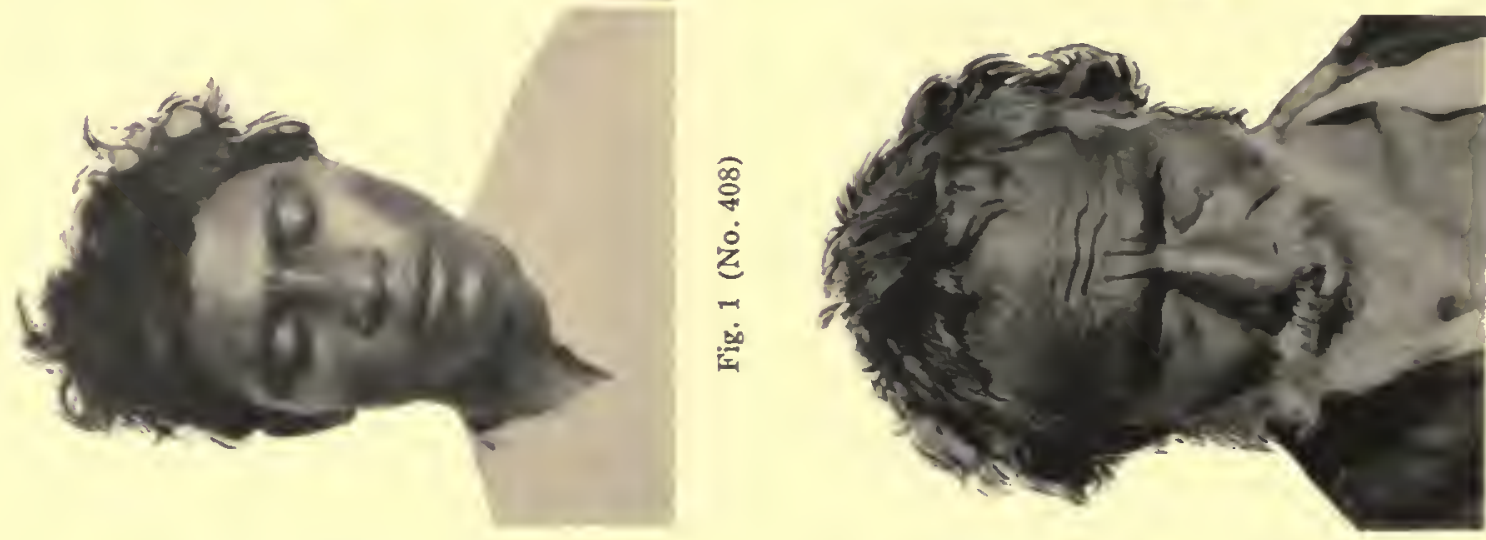

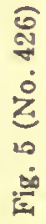




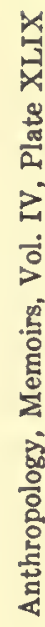
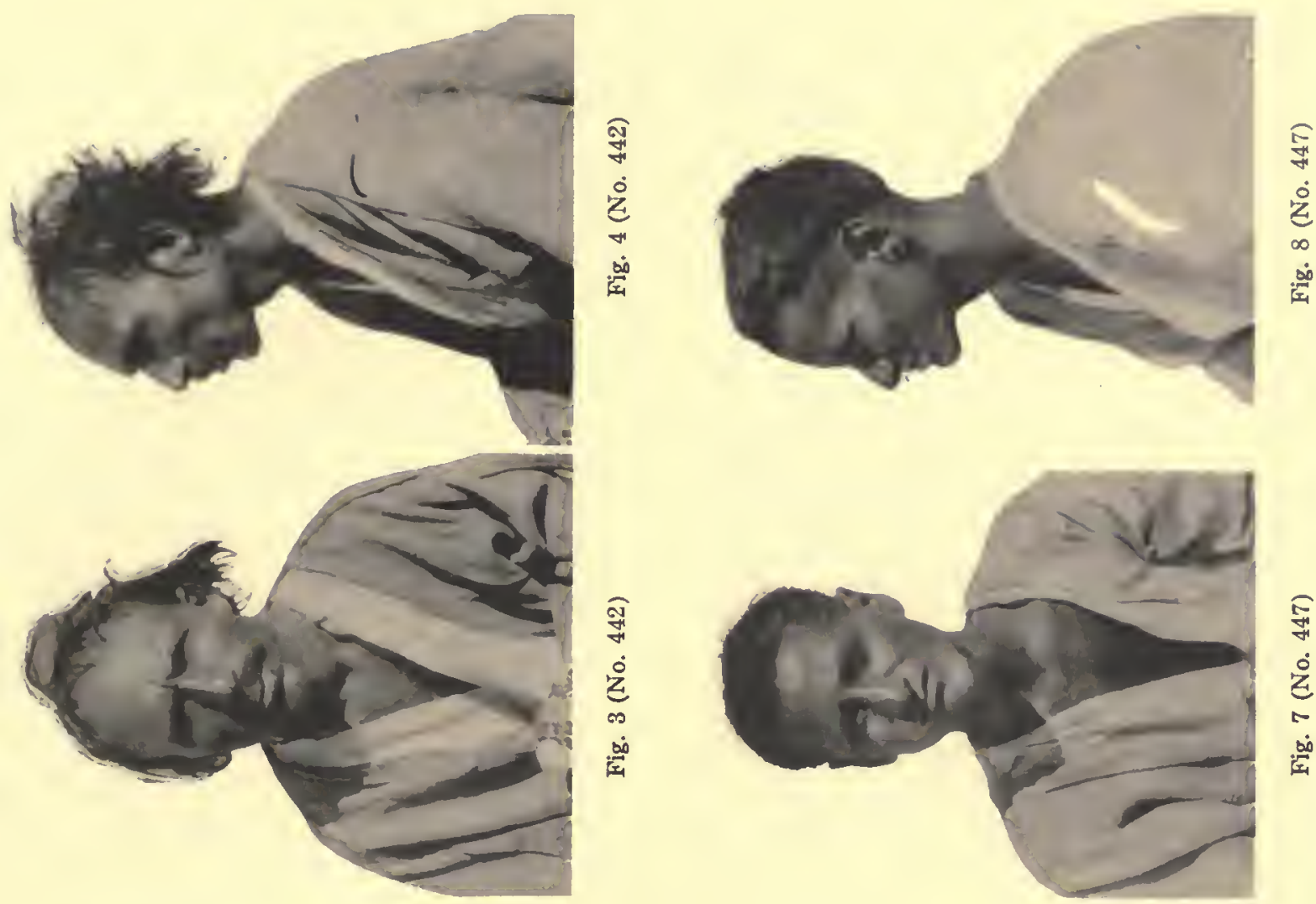

E⿱
$\dot{3}$
$\dot{2}$
$\infty$
$\infty$

客

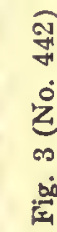

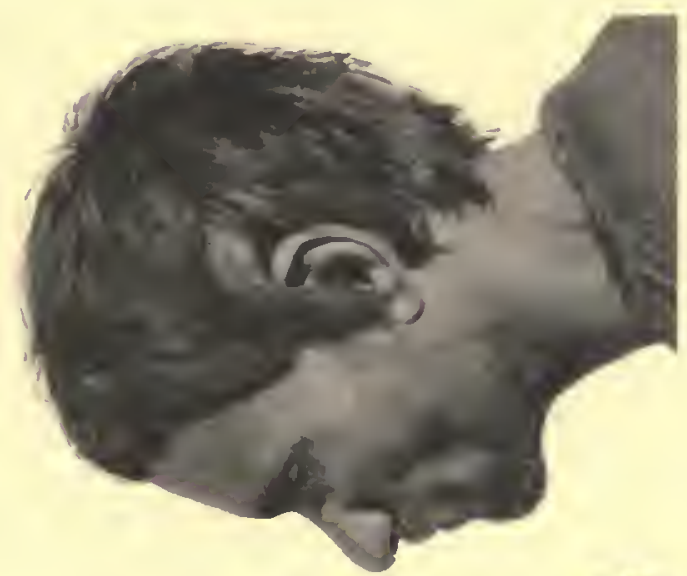

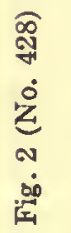

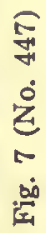
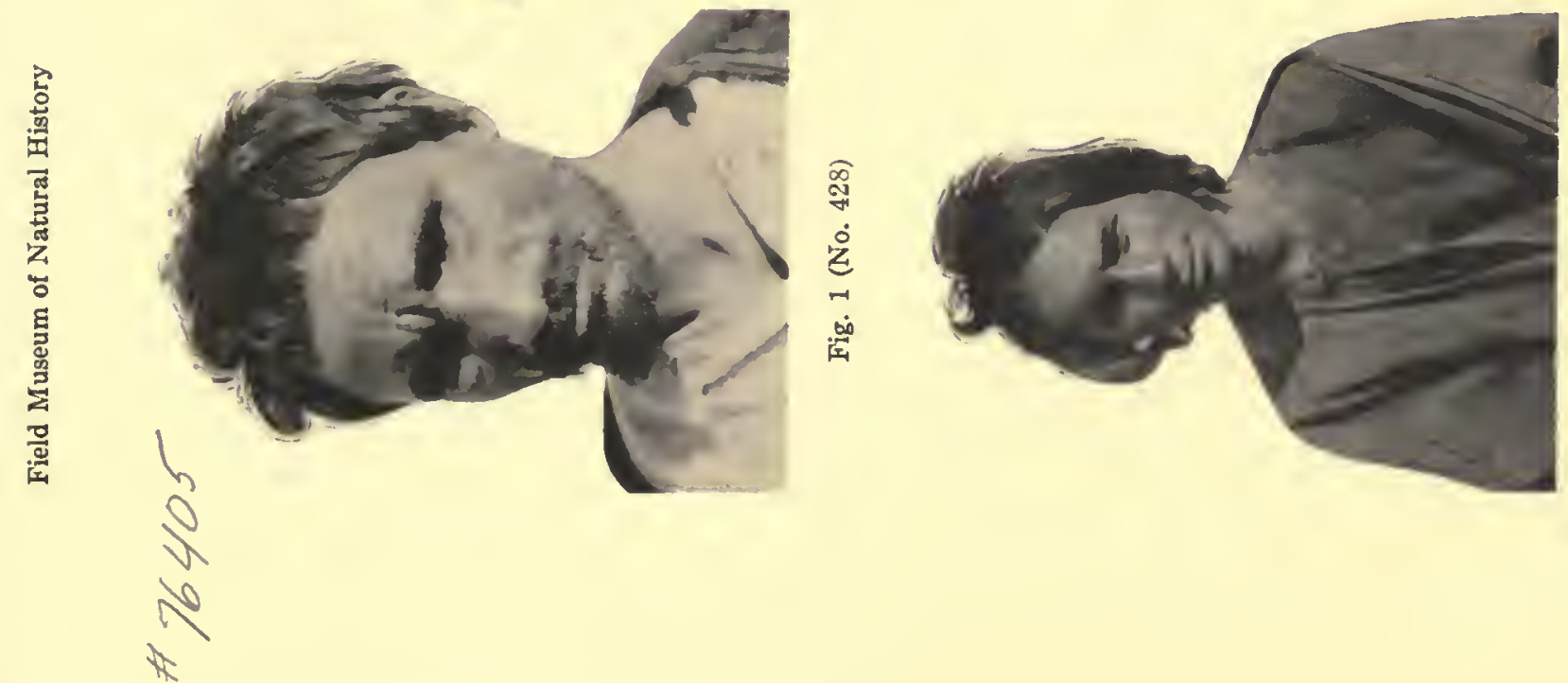

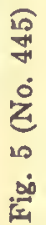

No 
굴
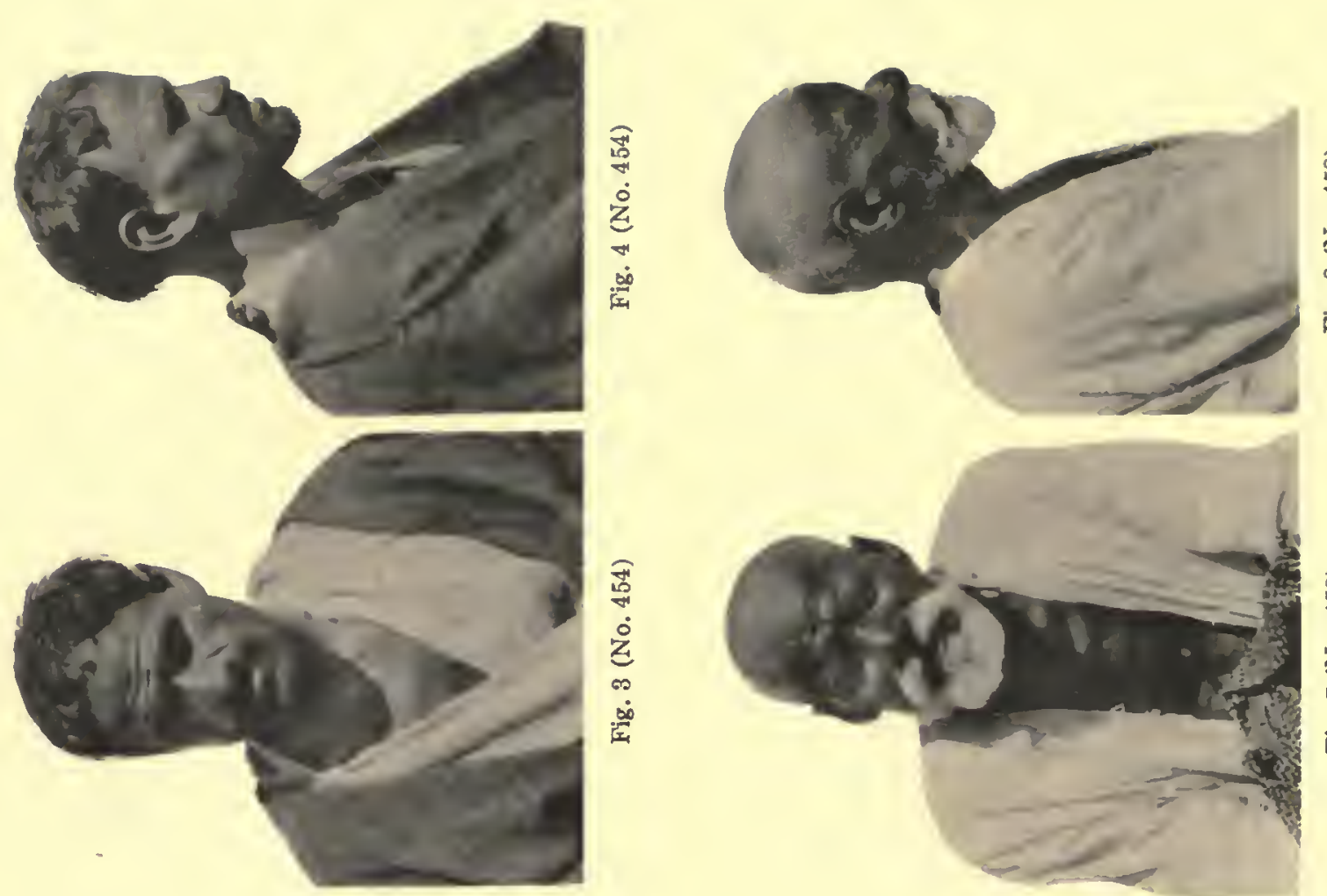

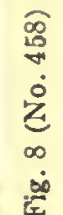

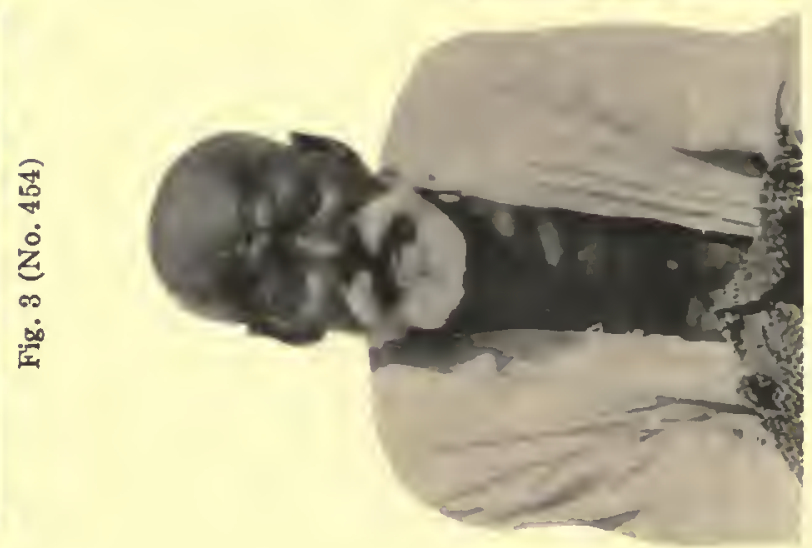

总

贈

6

is

औt

กิ
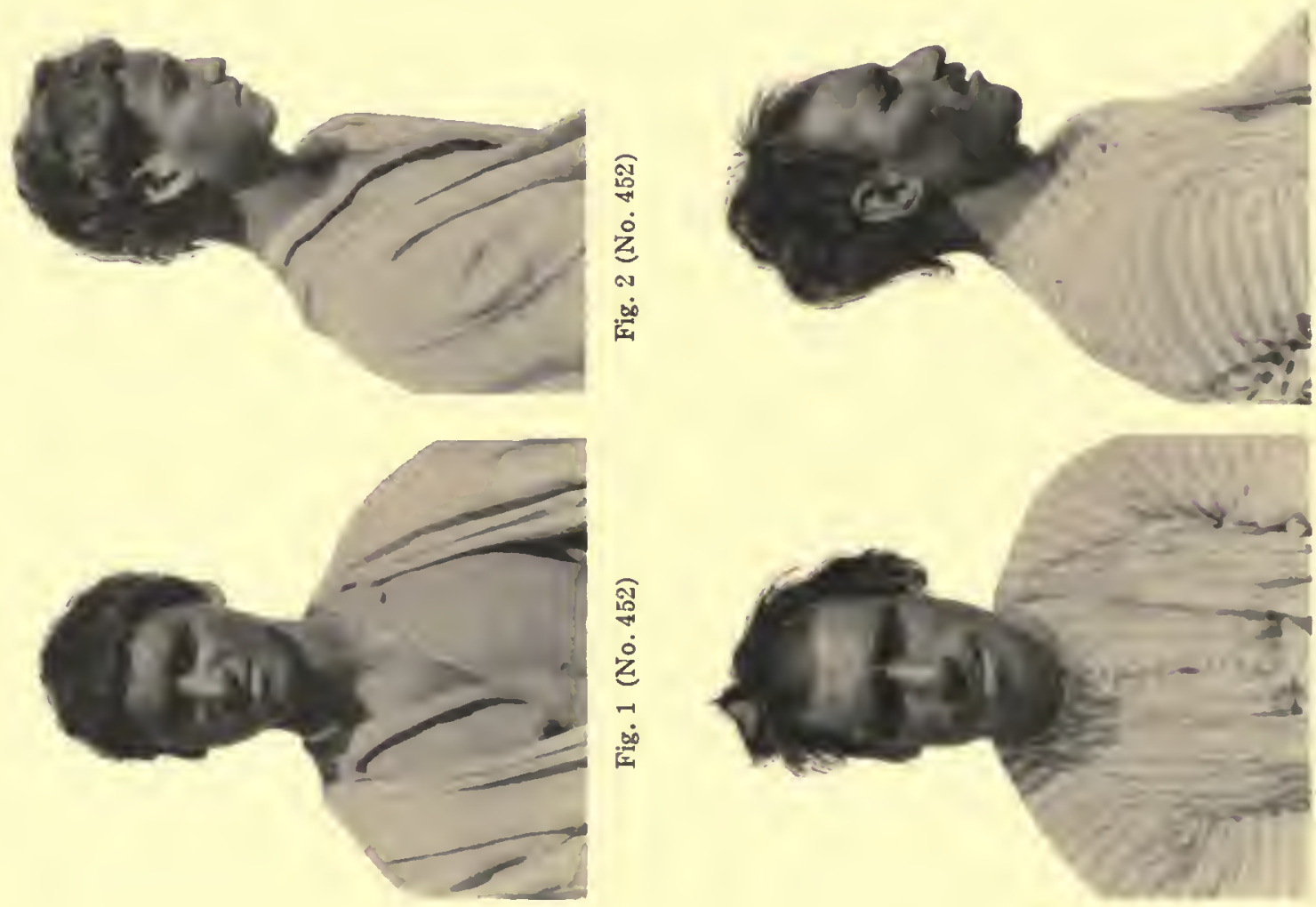
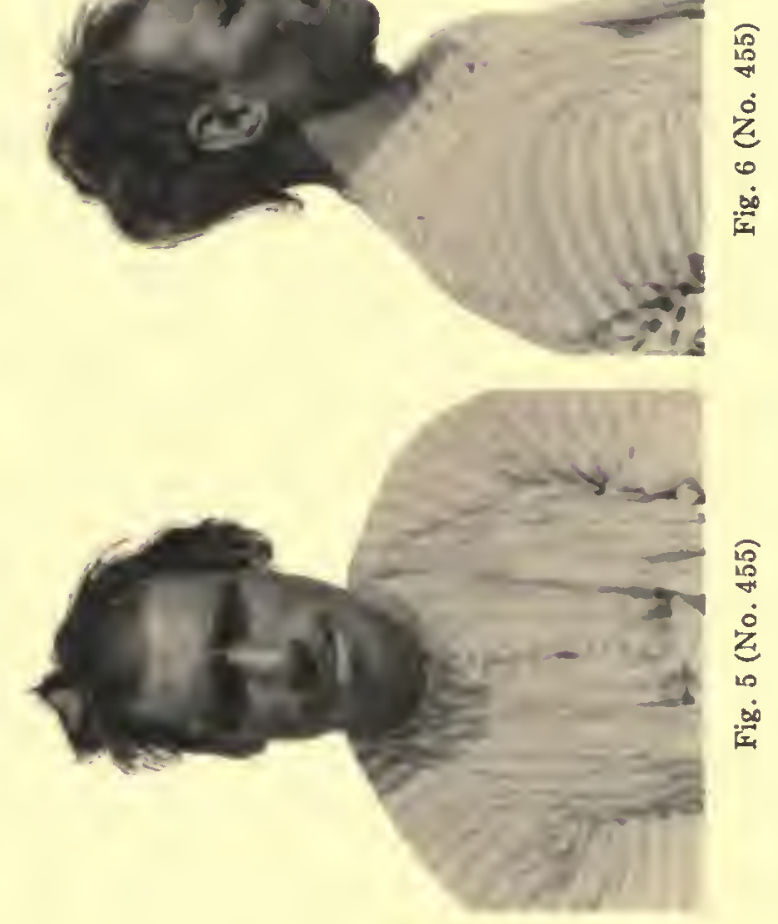
年
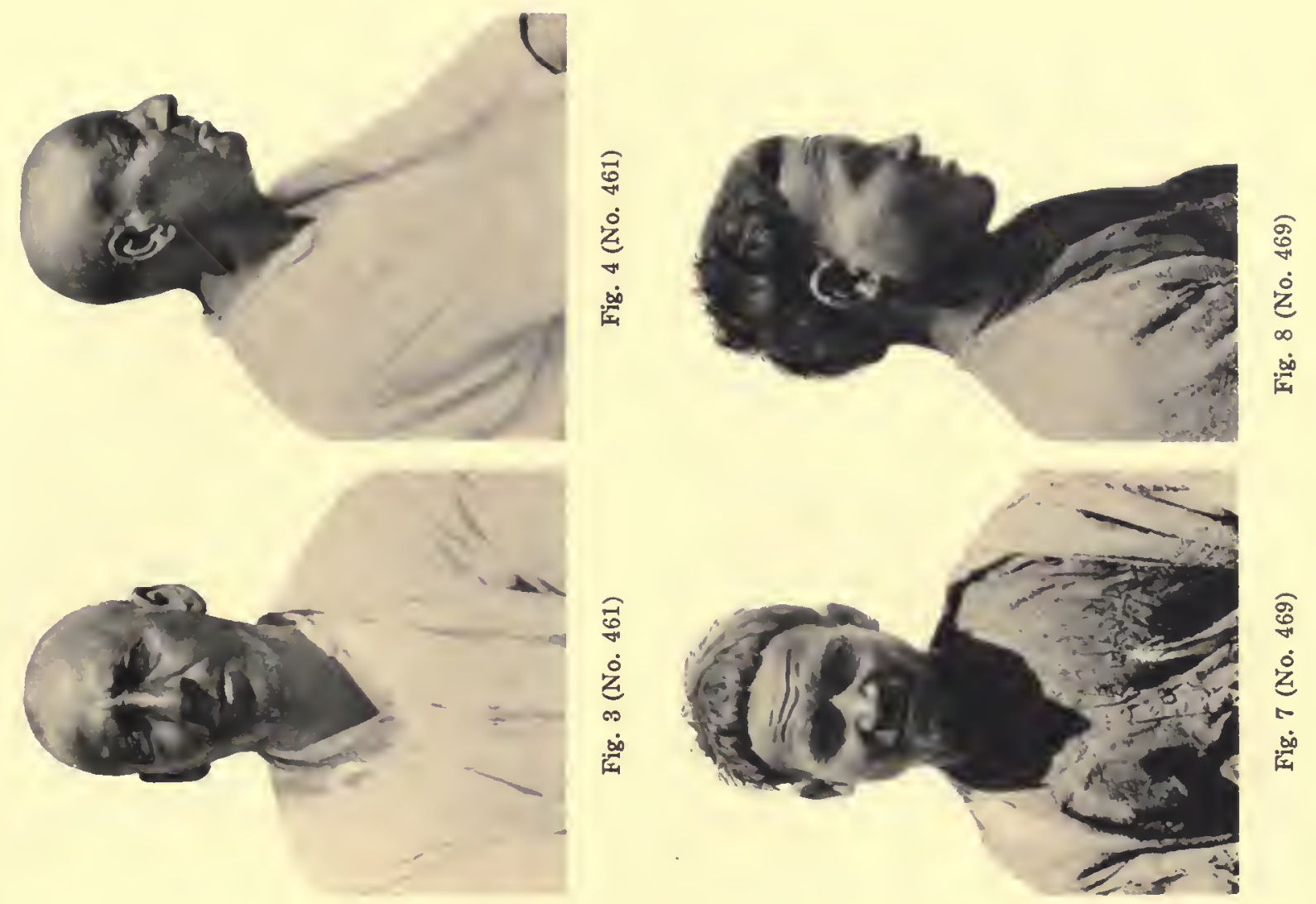

䍃
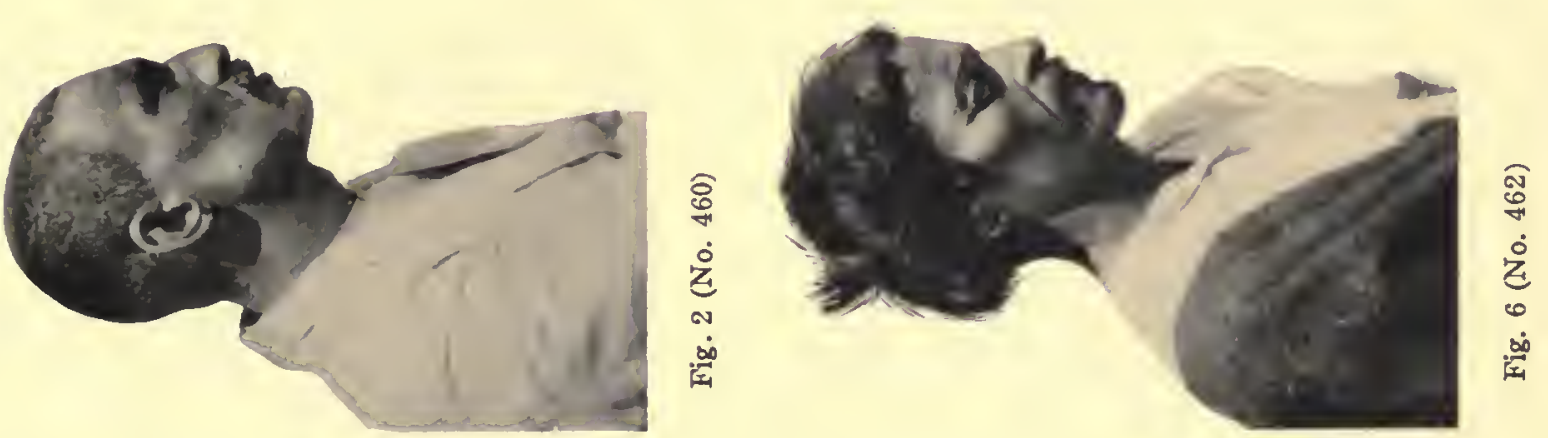

옹
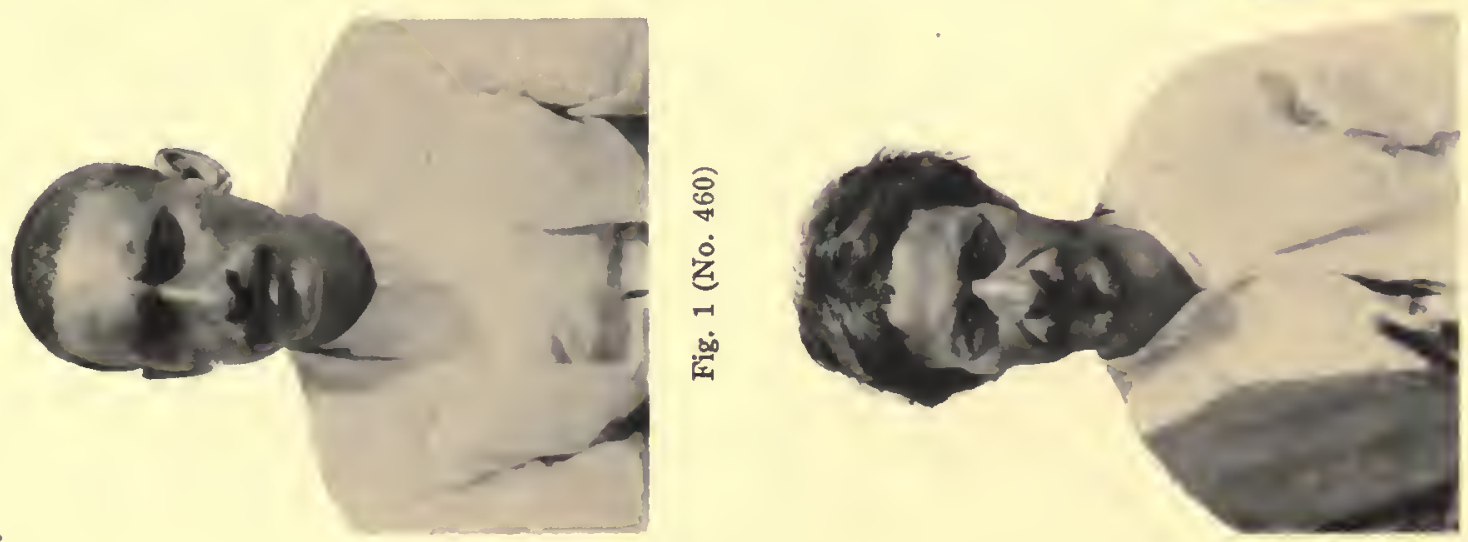

สิ

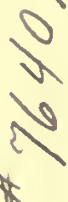

x

N) 


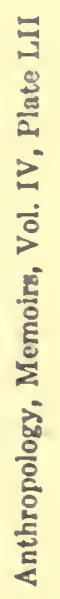
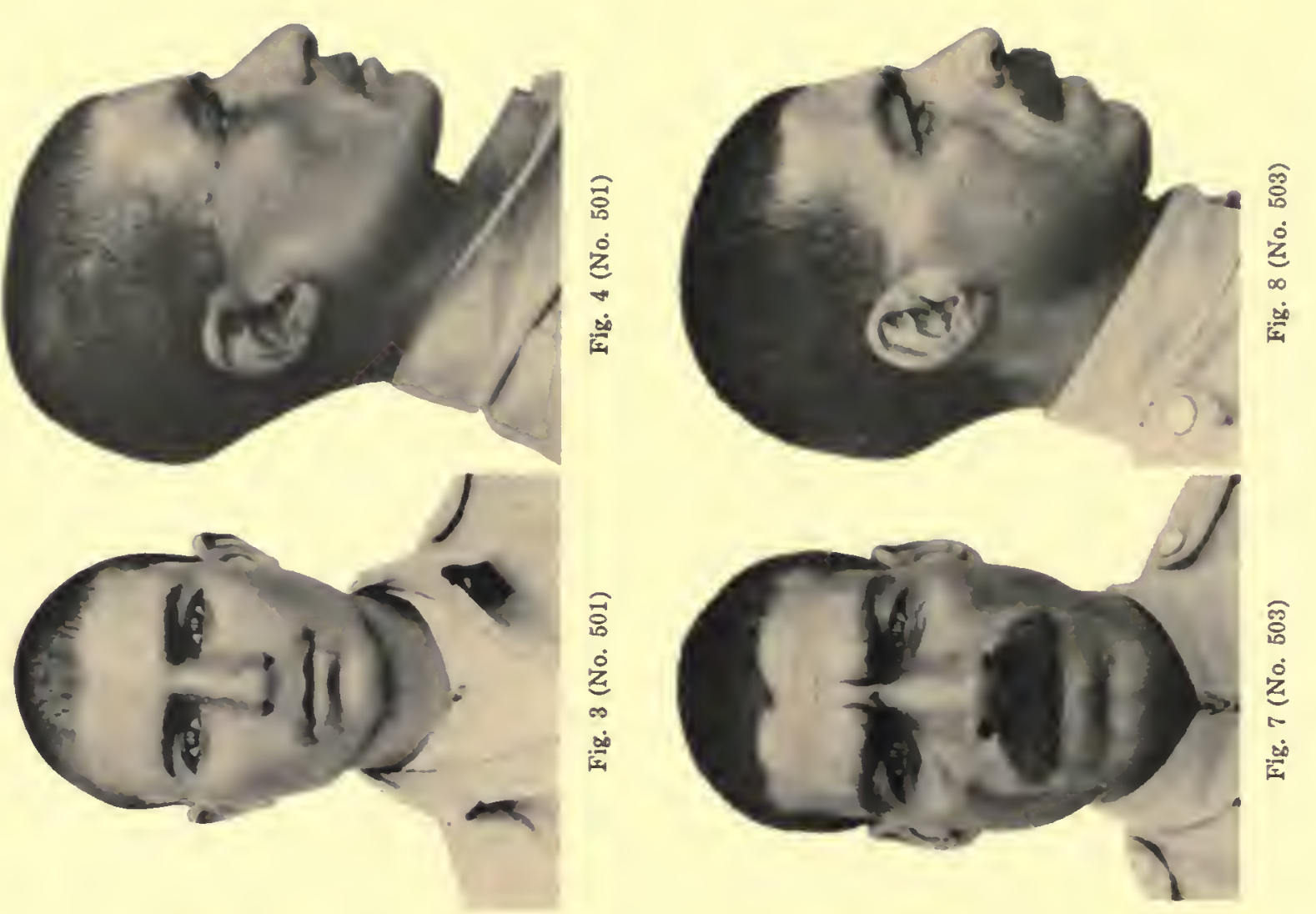

范
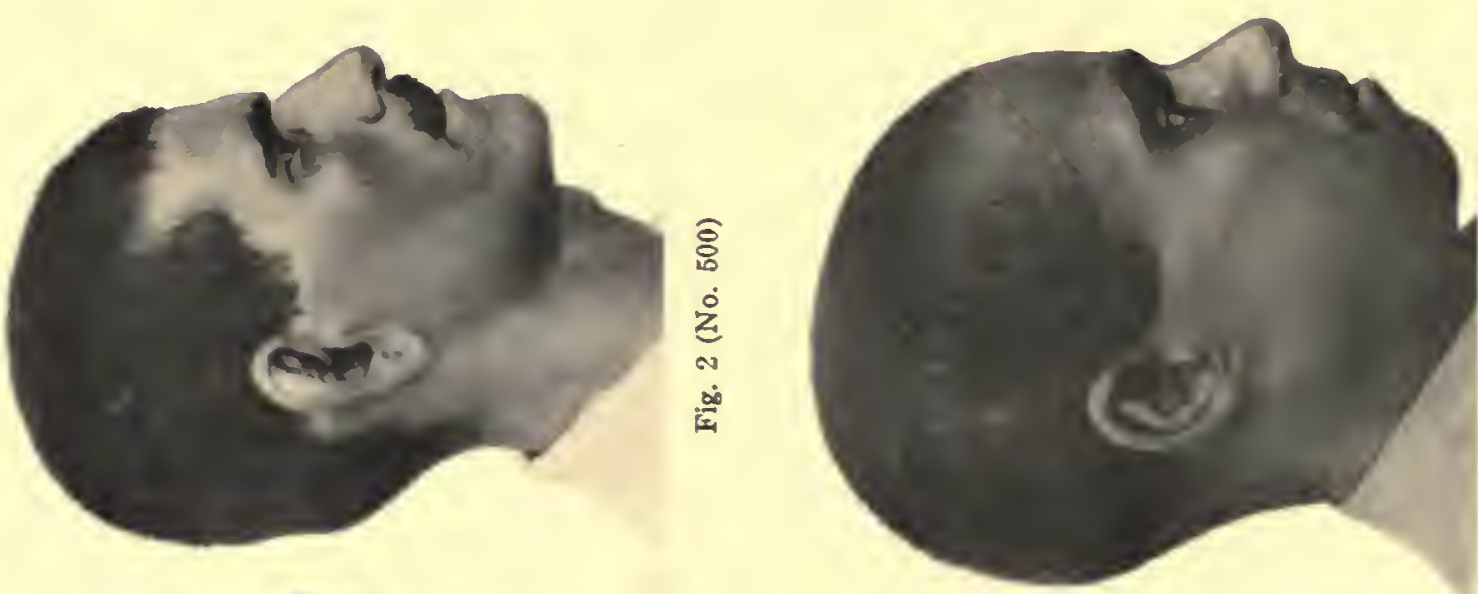

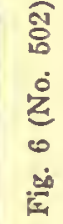

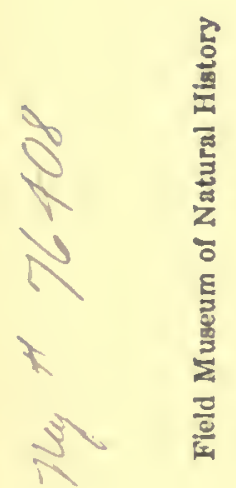
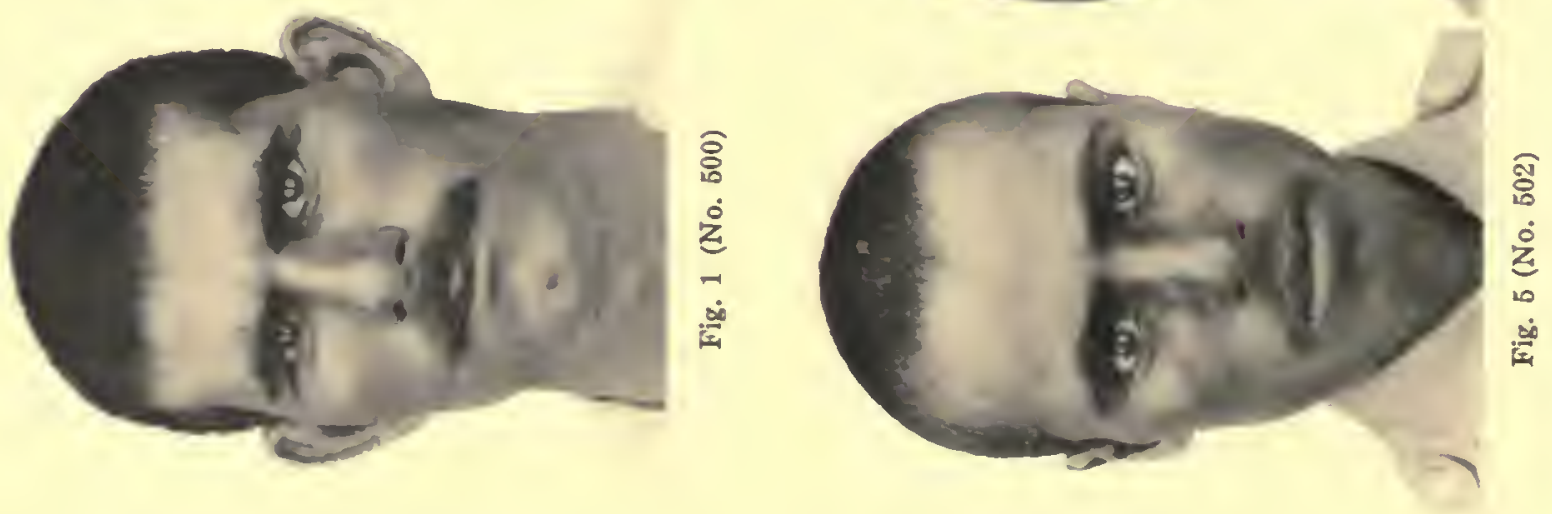
䚾
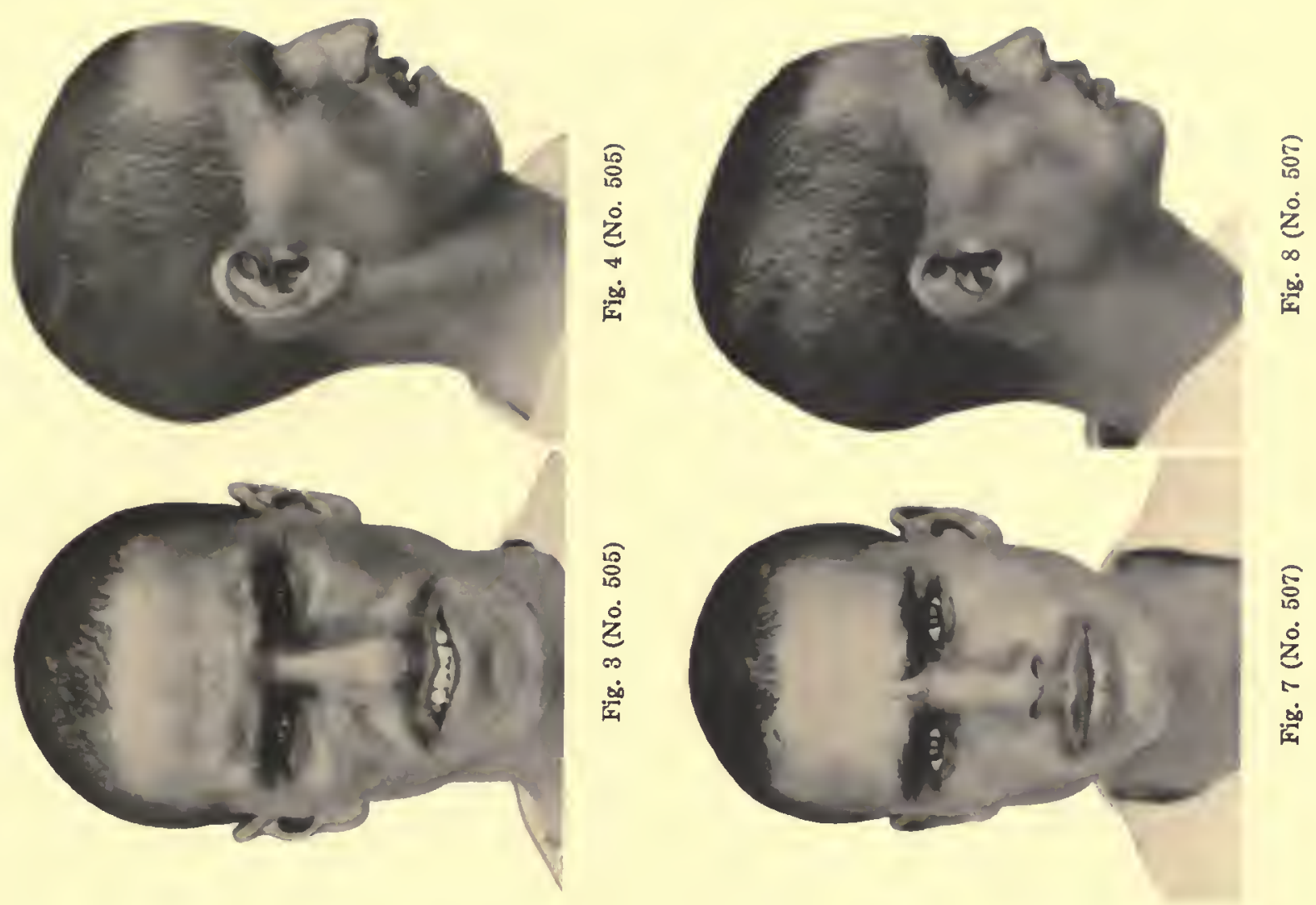

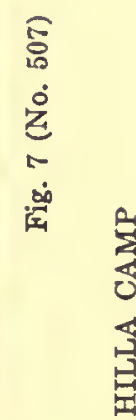
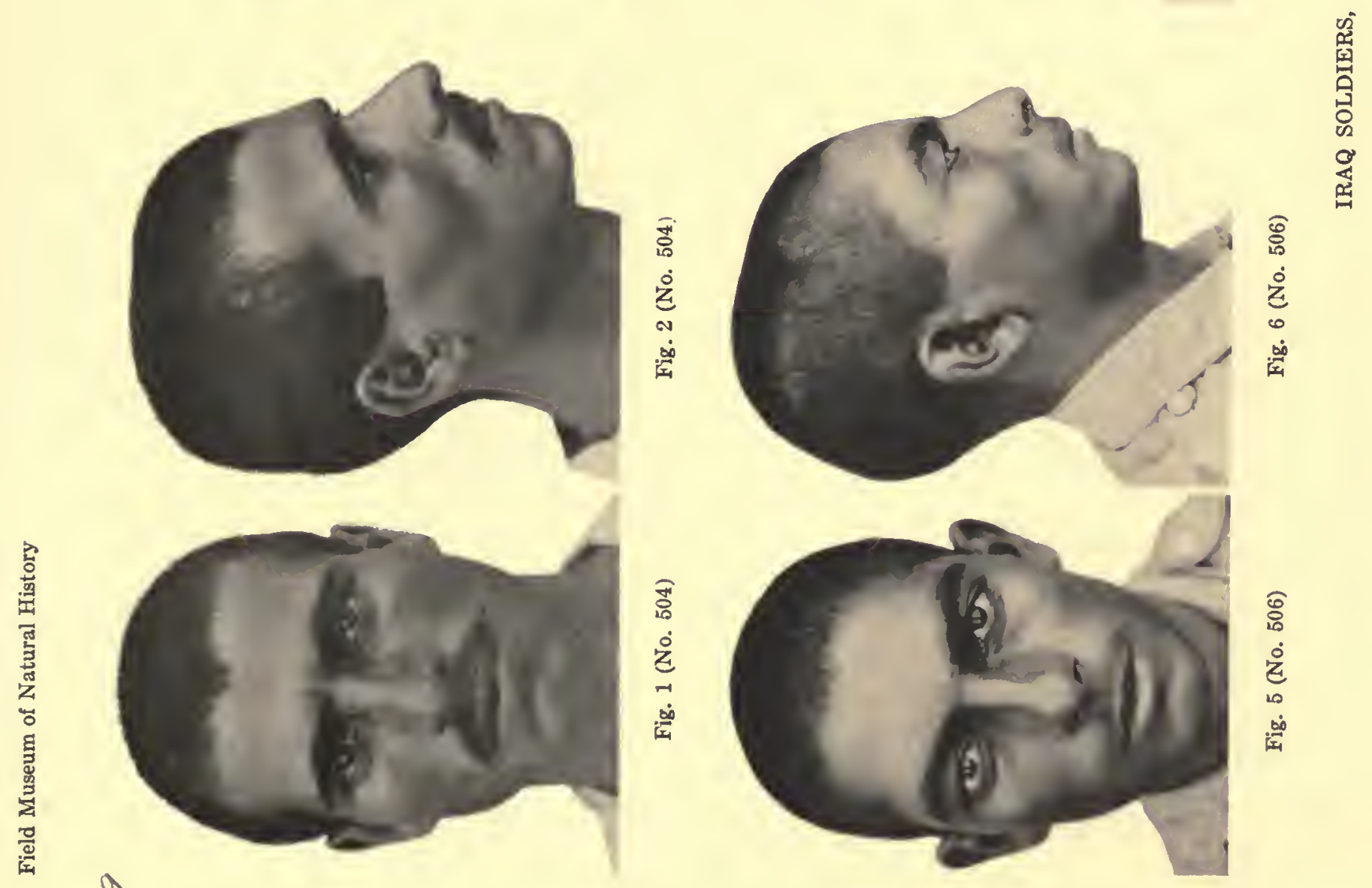

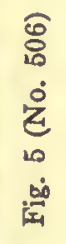

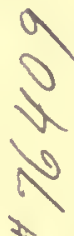

옹

दे

覀 

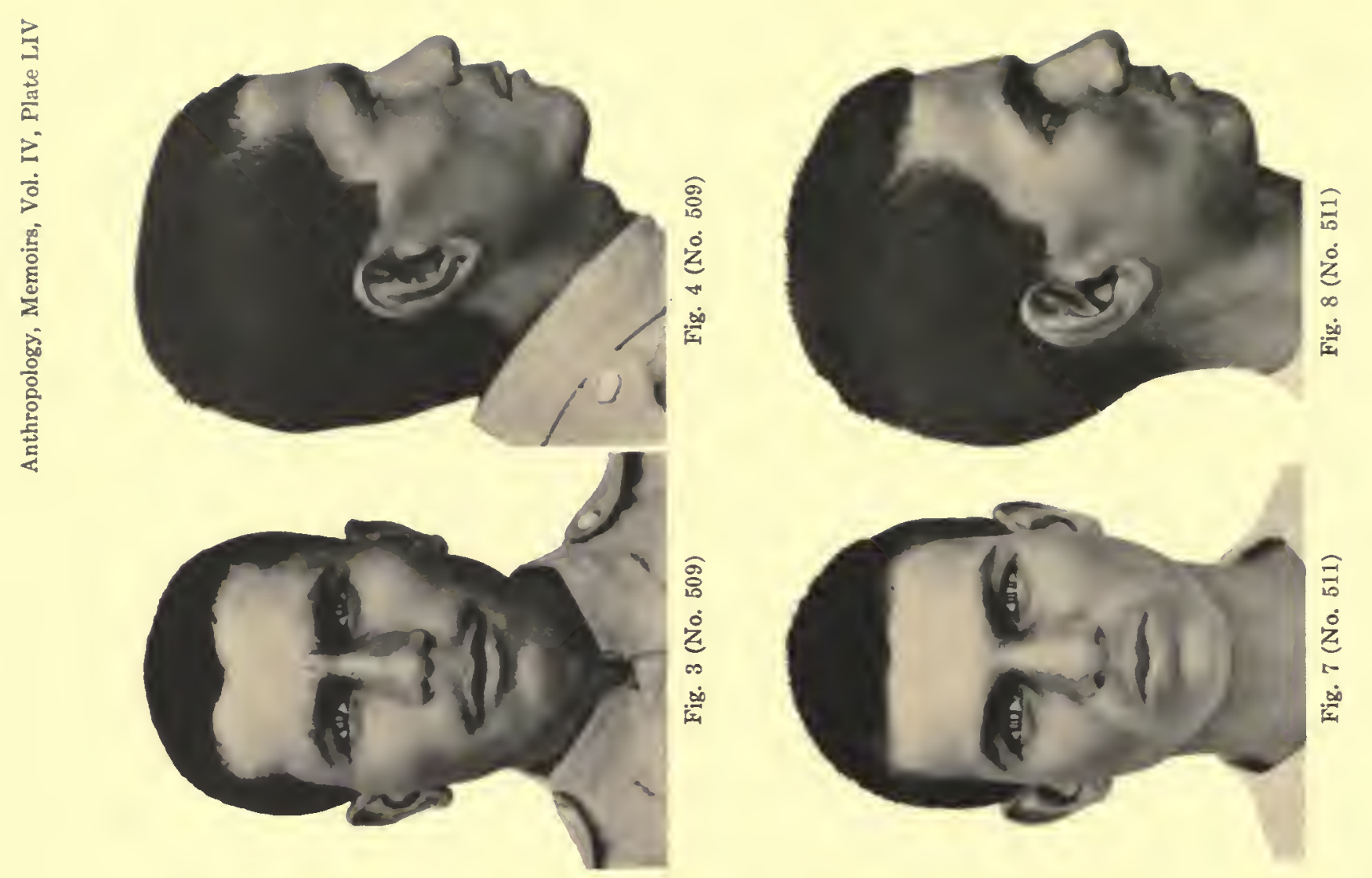

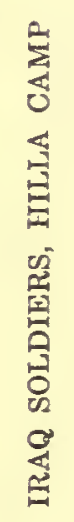

$\frac{1}{x}$

No
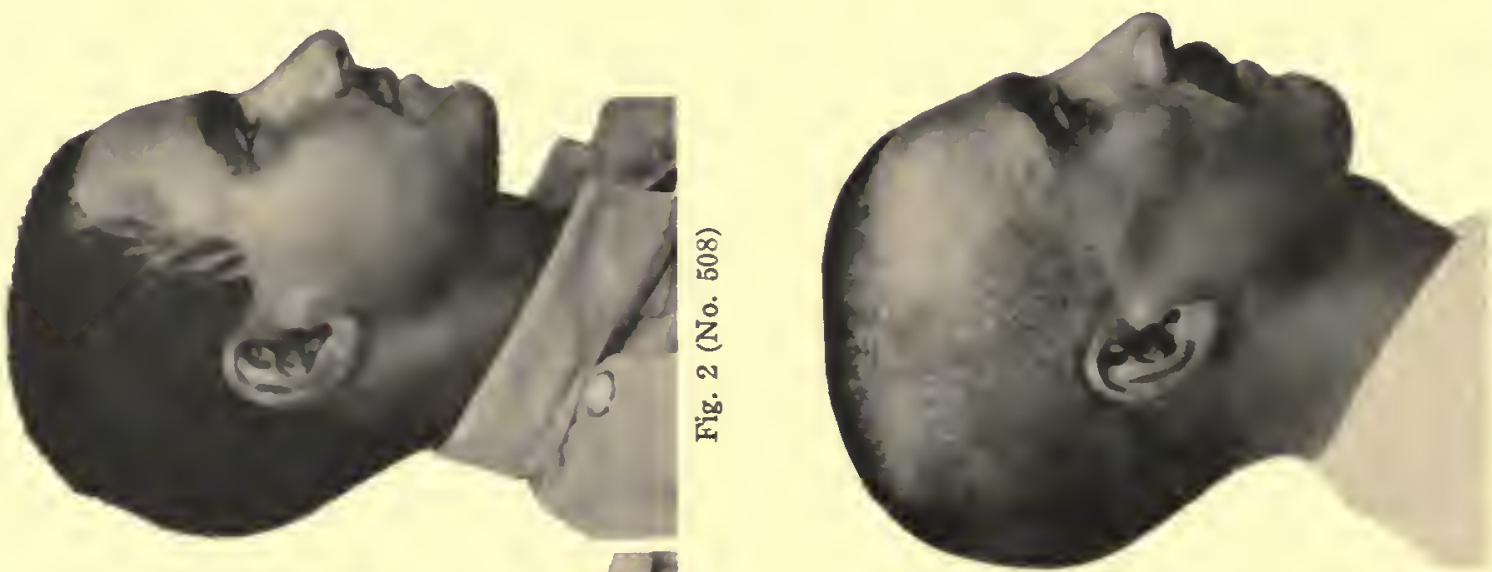

Oิ
is
$\dot{0}$
2
0
$\dot{0}$

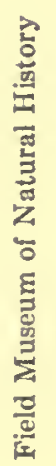
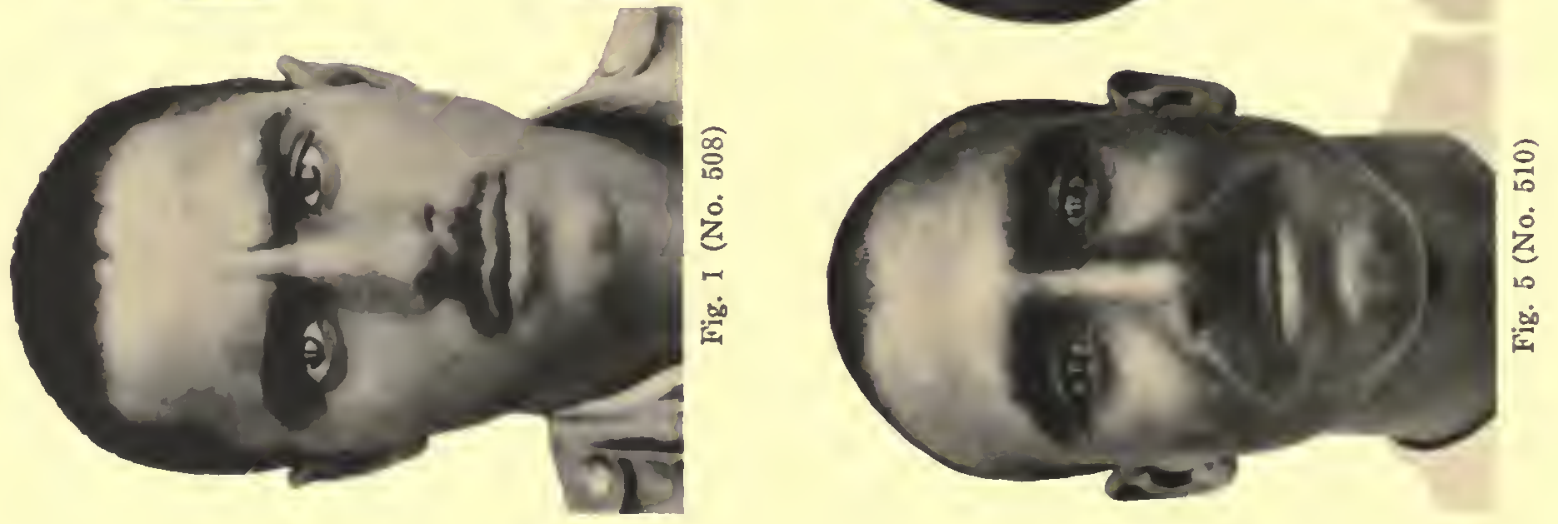

N 
要
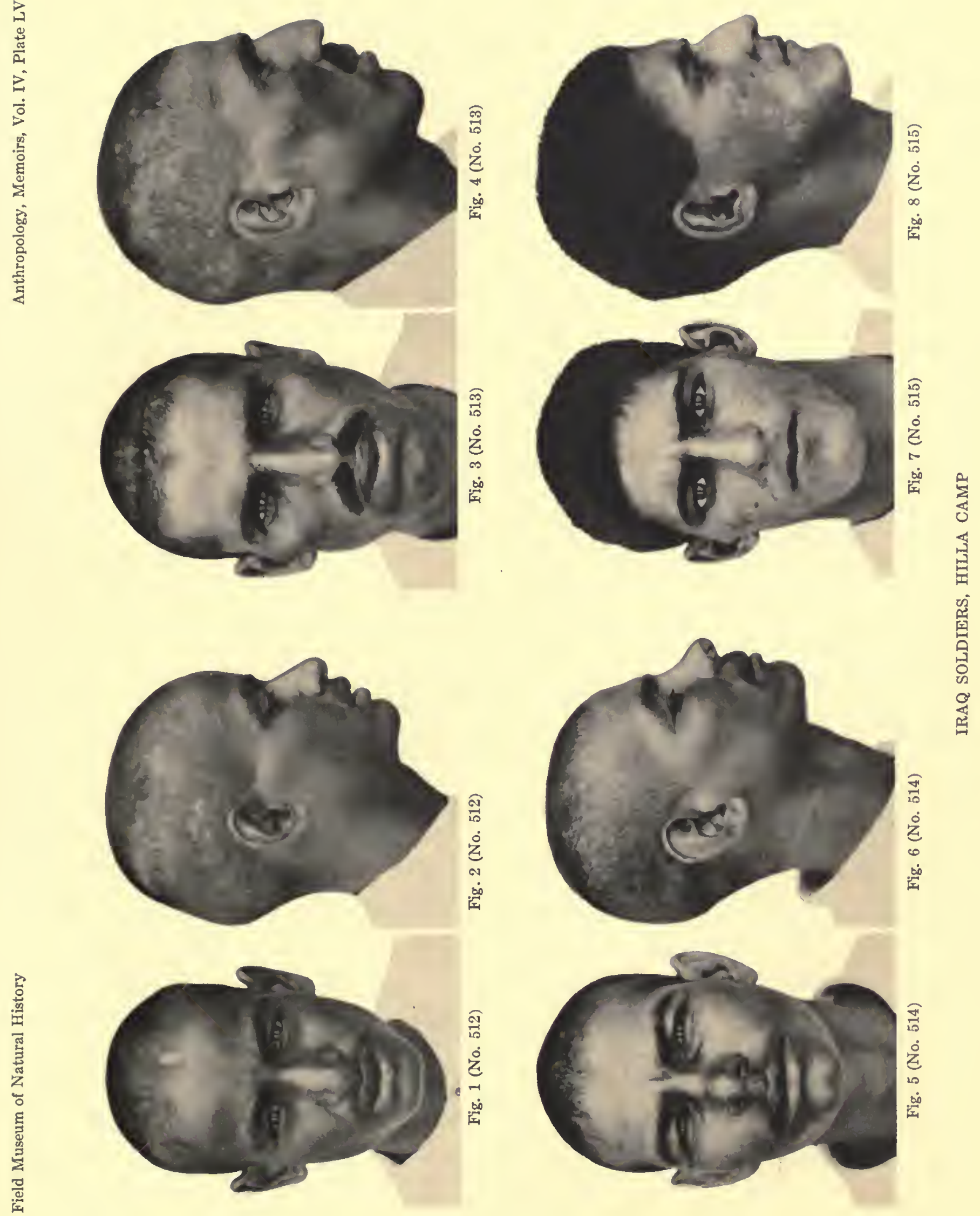

是

ฟ

1

$x$

$N$ 

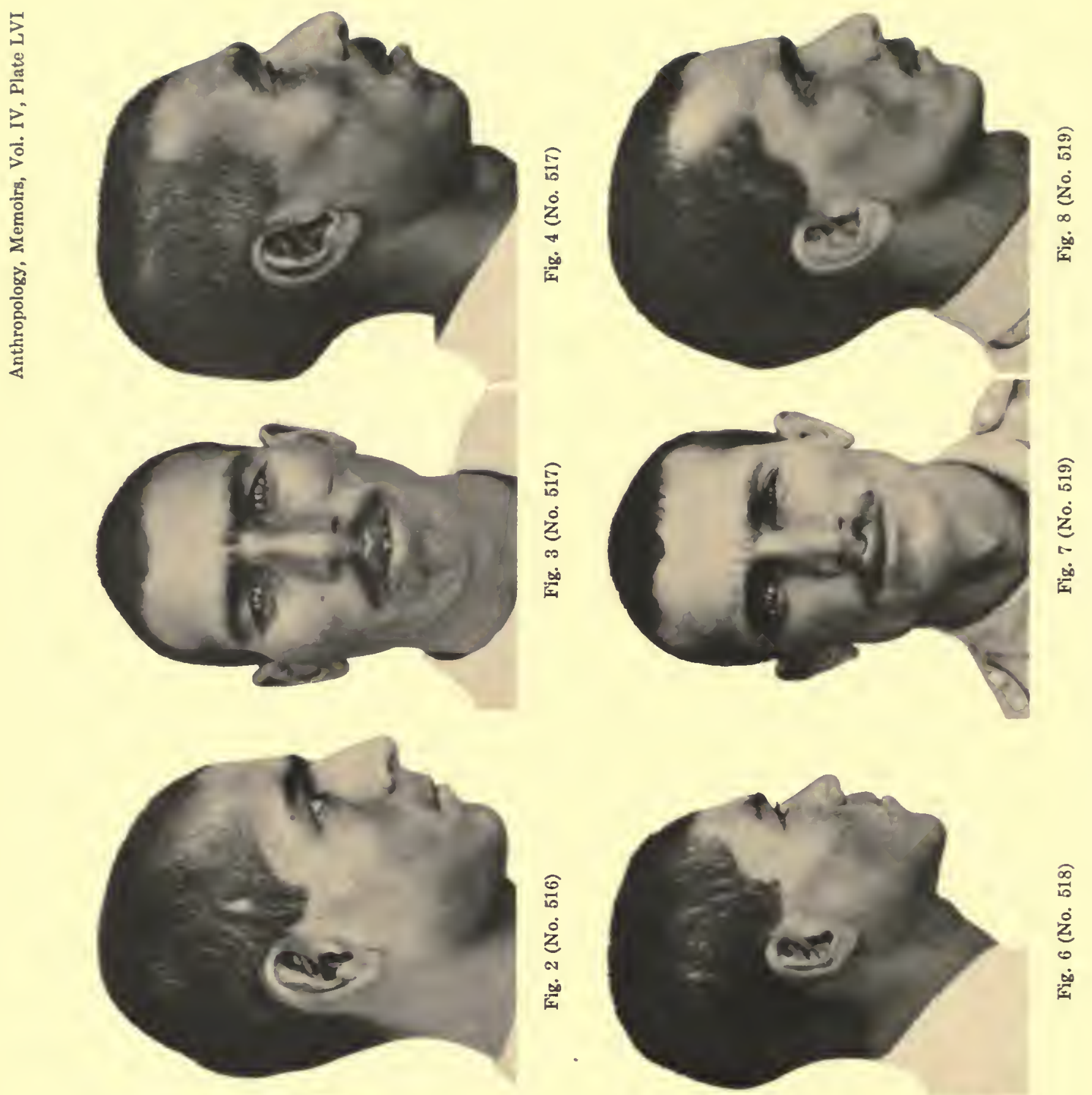

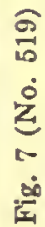

$\gamma$
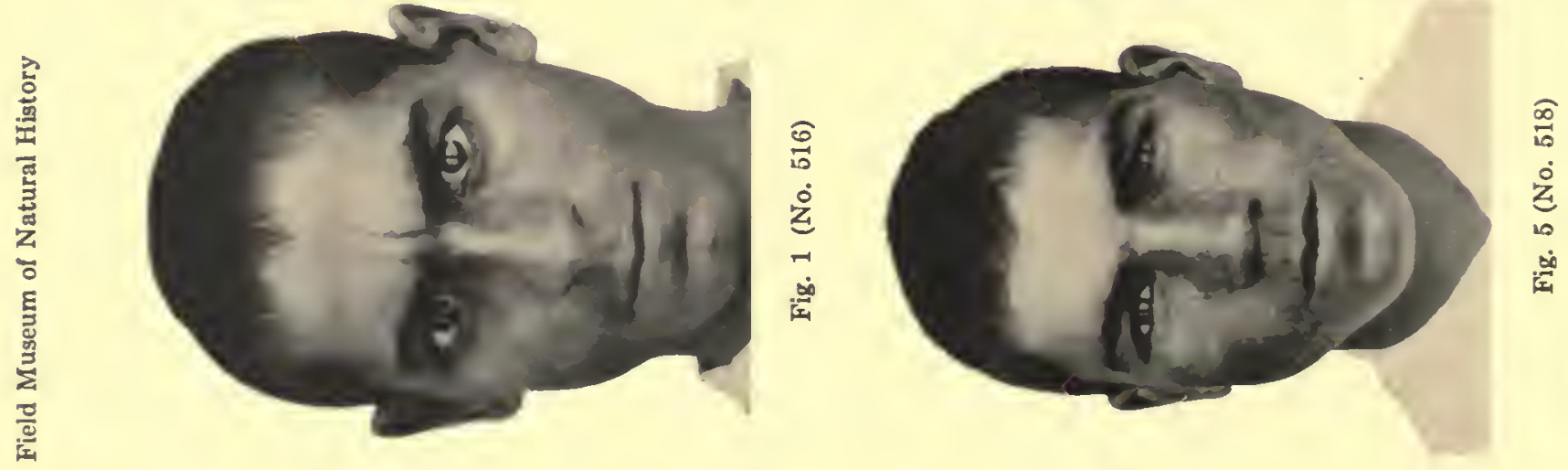

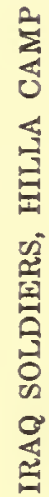




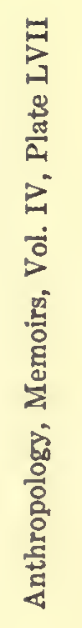
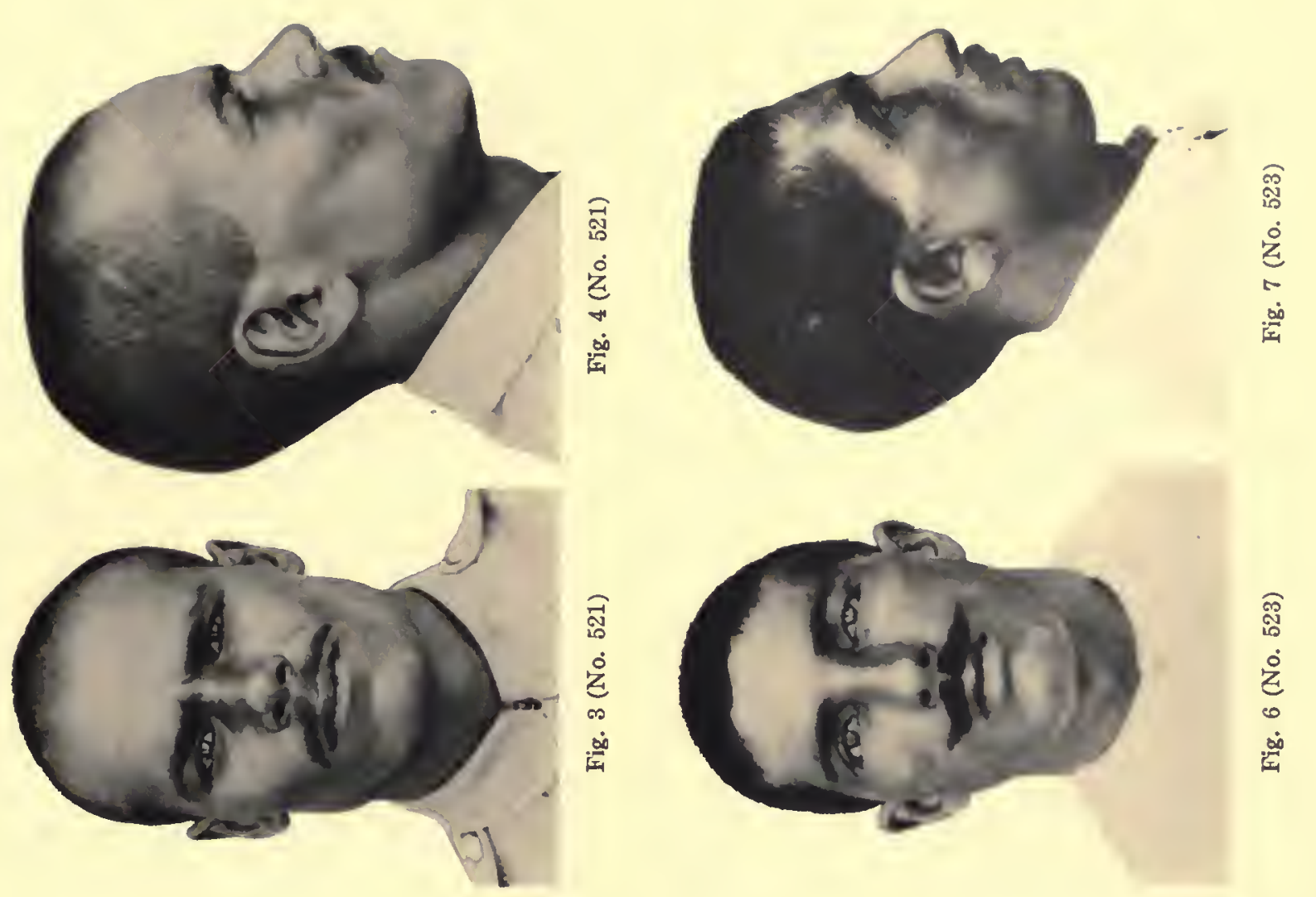

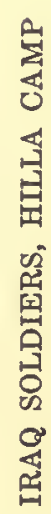

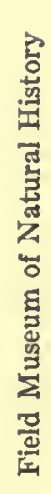

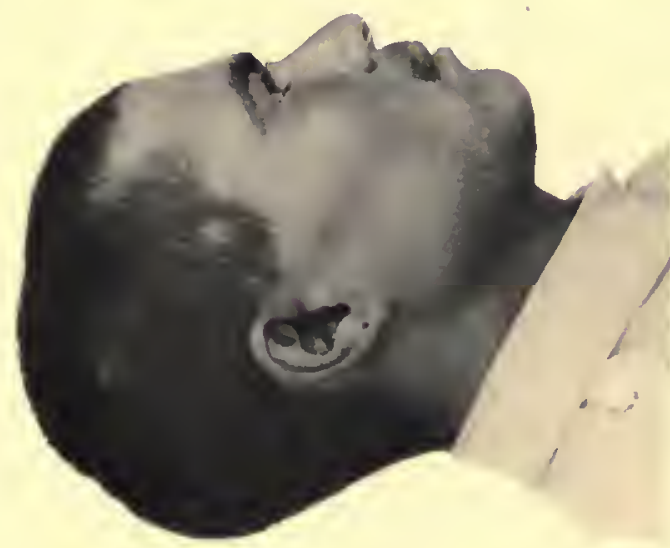

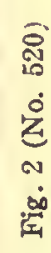
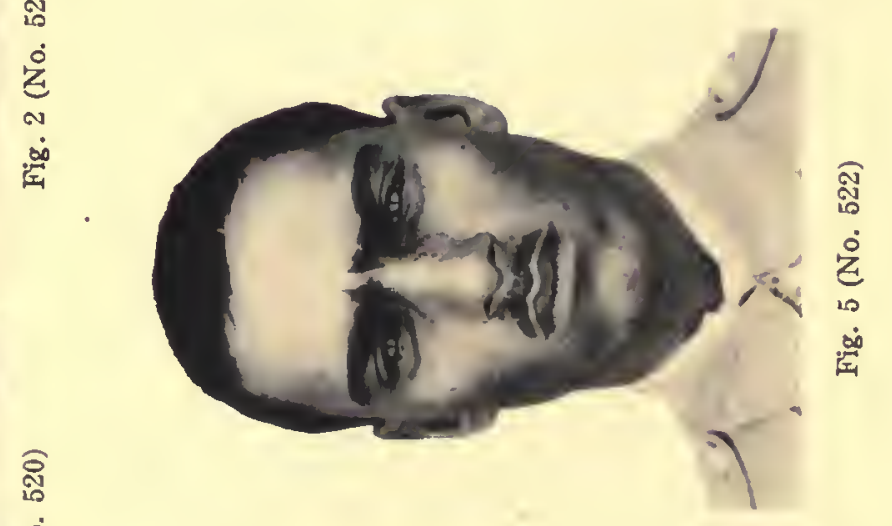

令

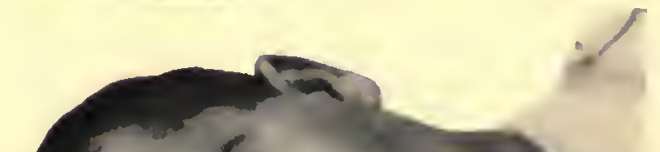

(8)

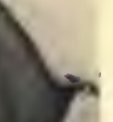

(a)

落 


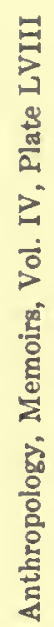
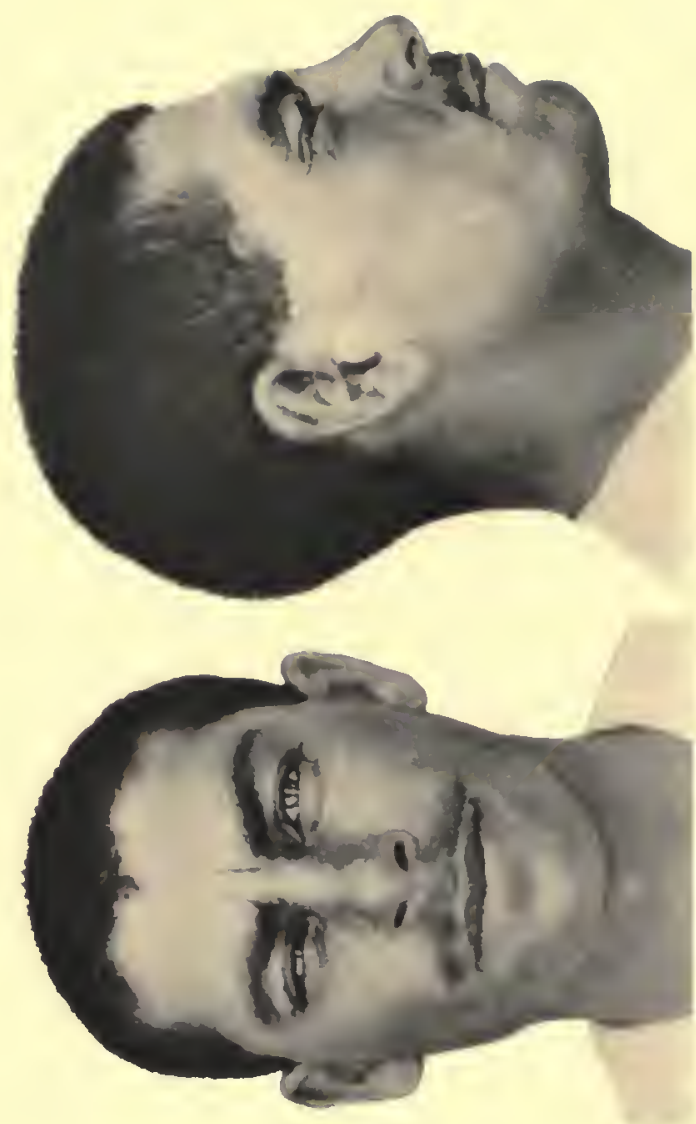

से

N

t)

닌

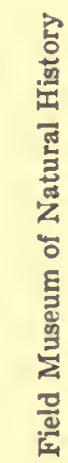

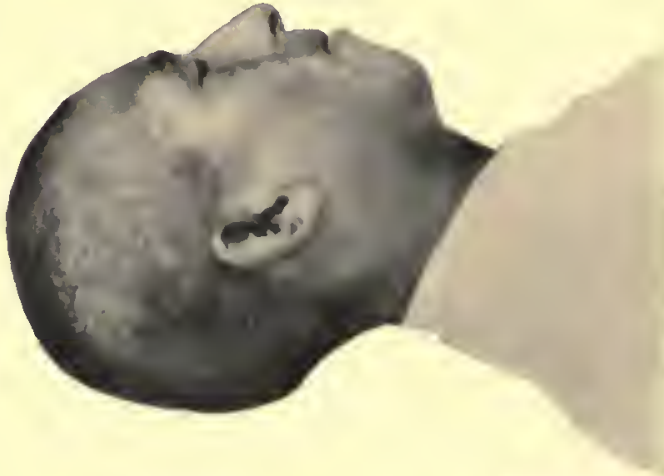

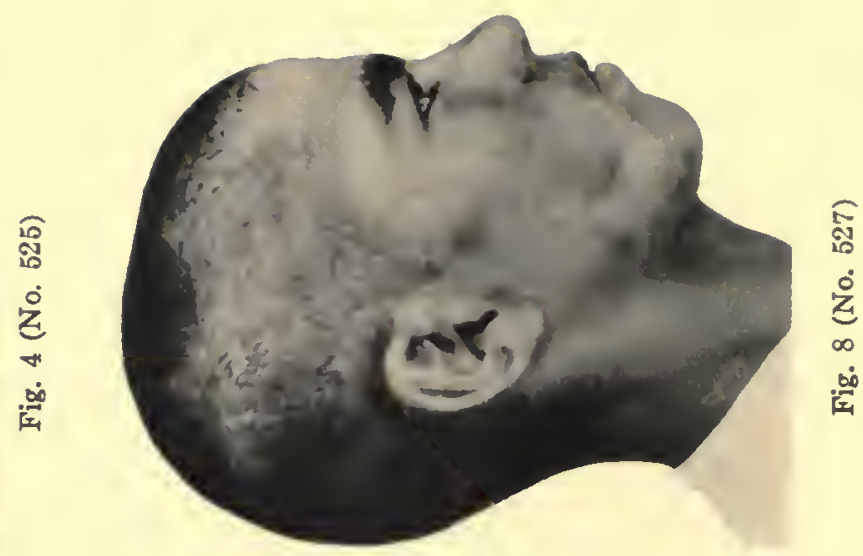

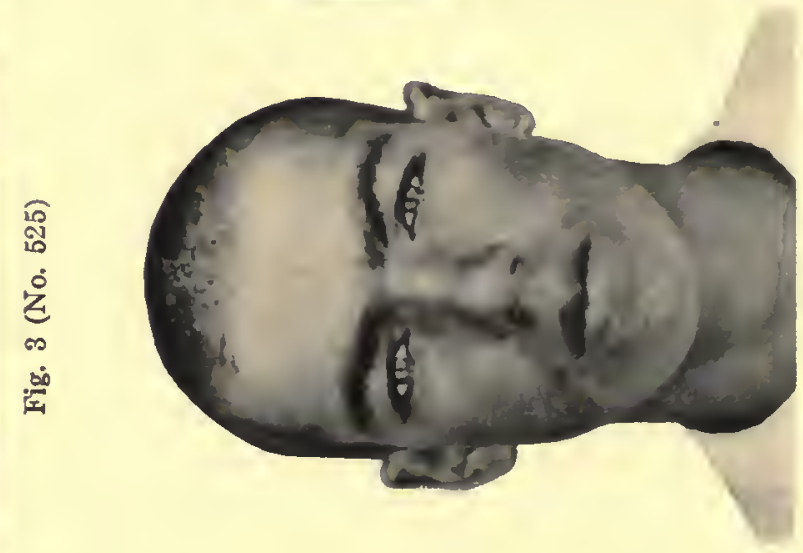

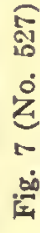
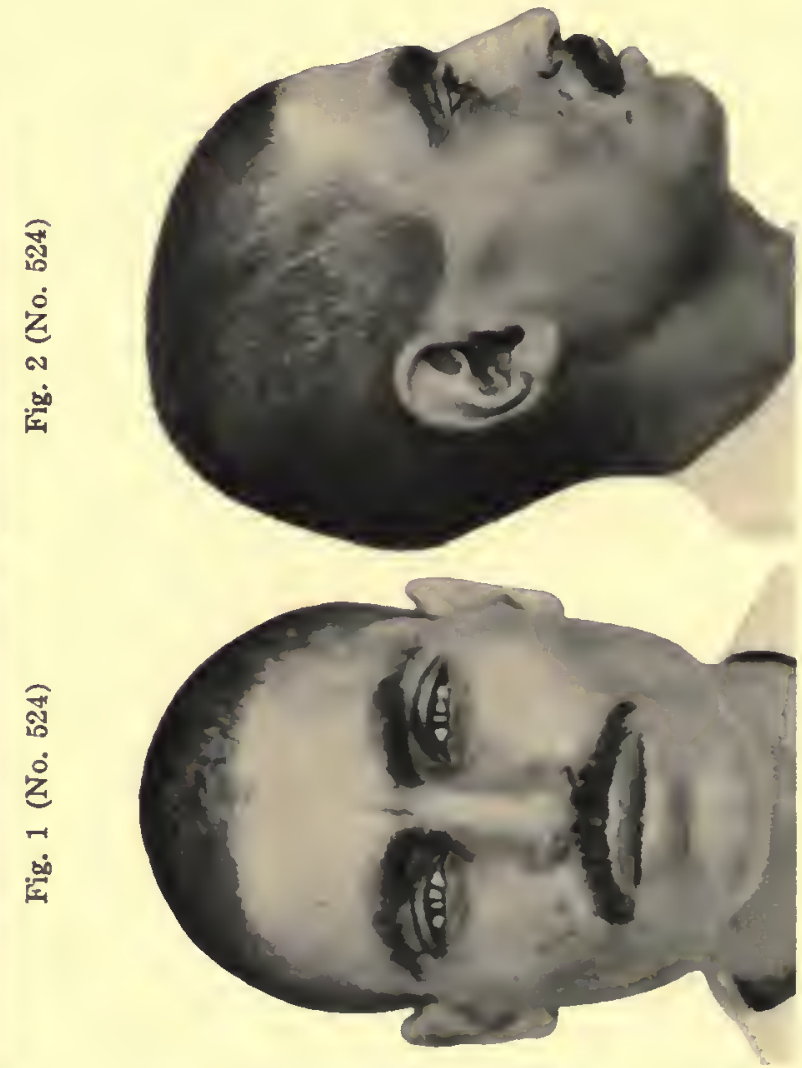

完

哭

¿े 它

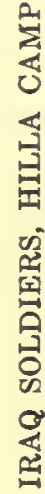


2
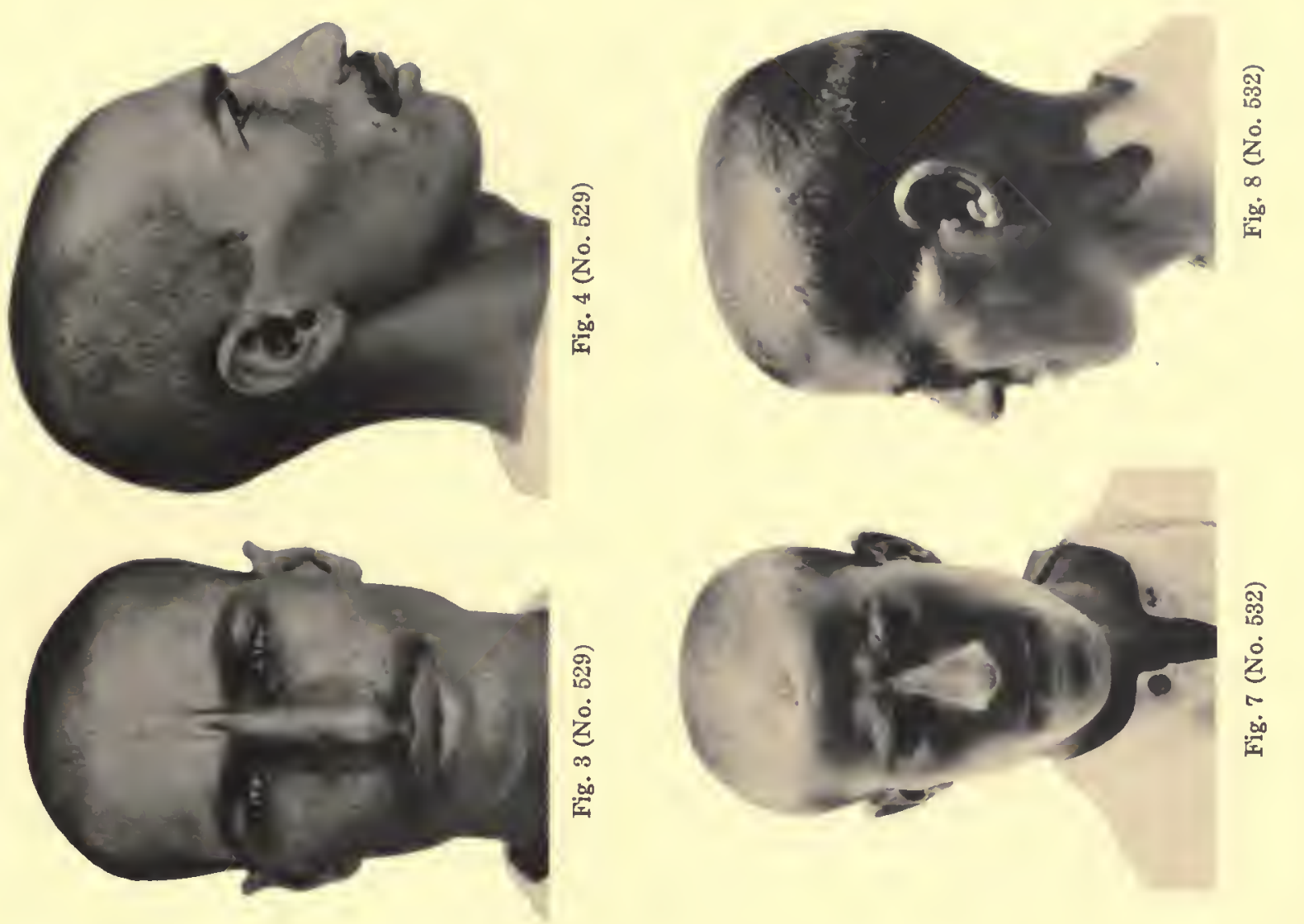

运

录
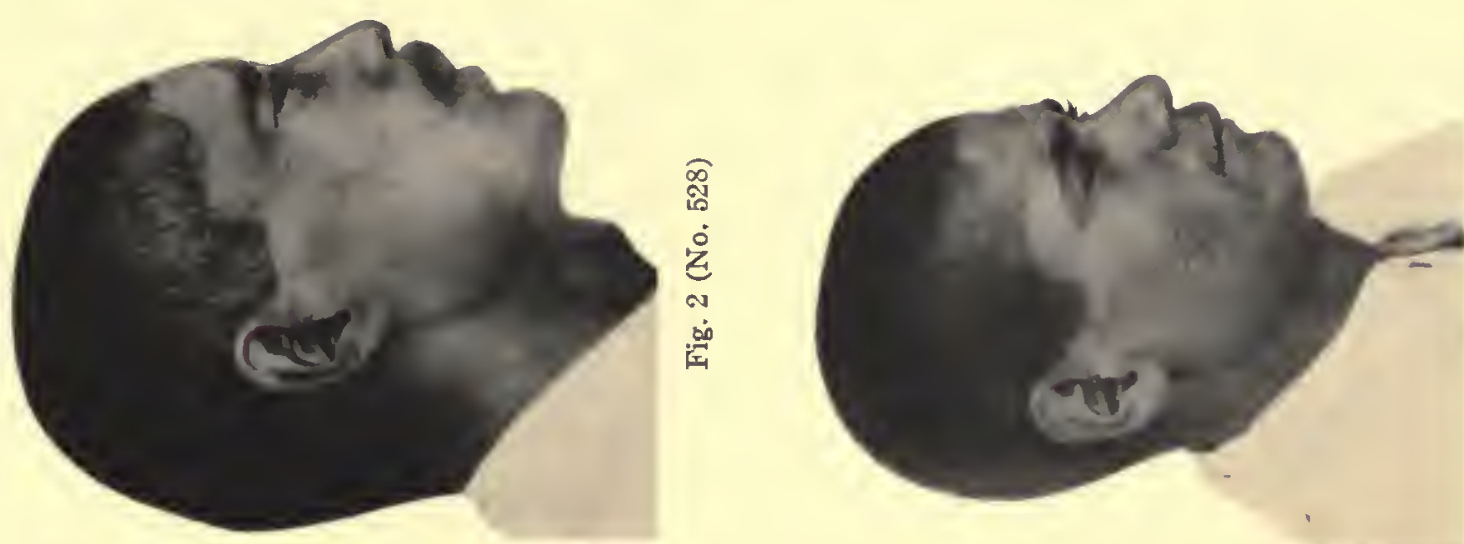

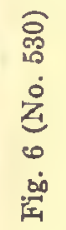

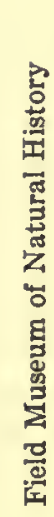

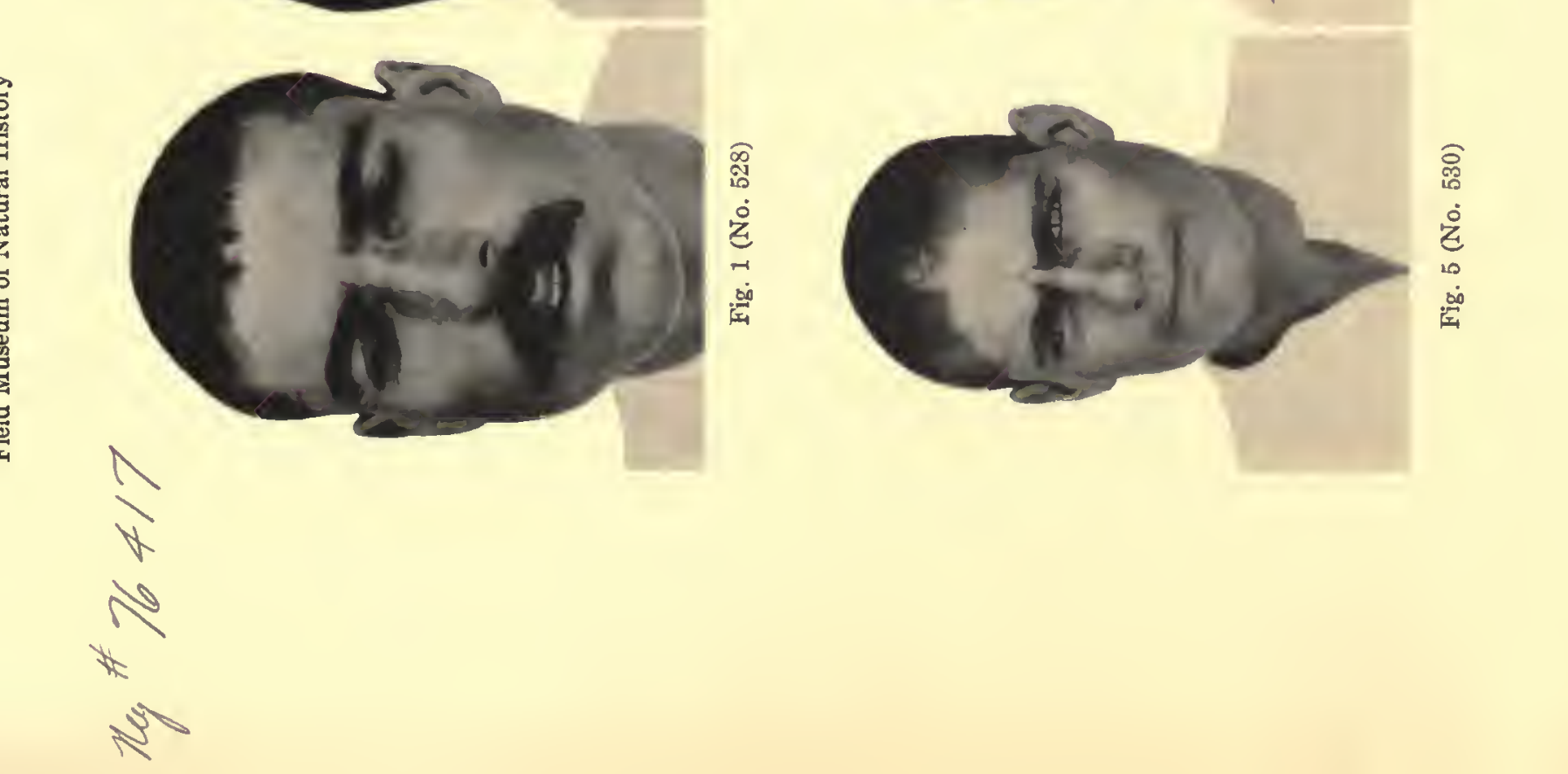



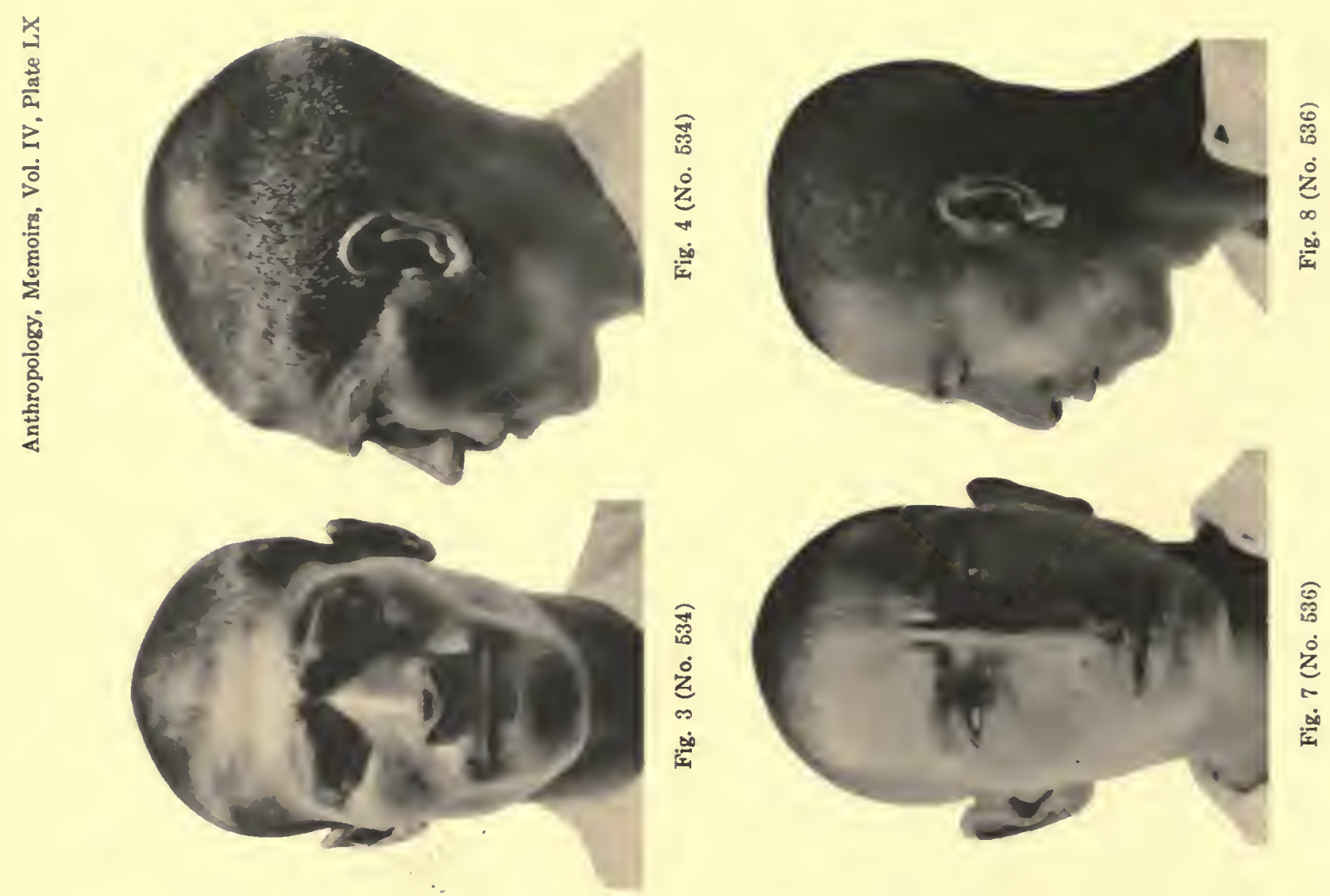

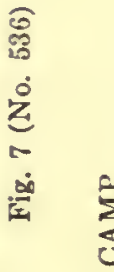
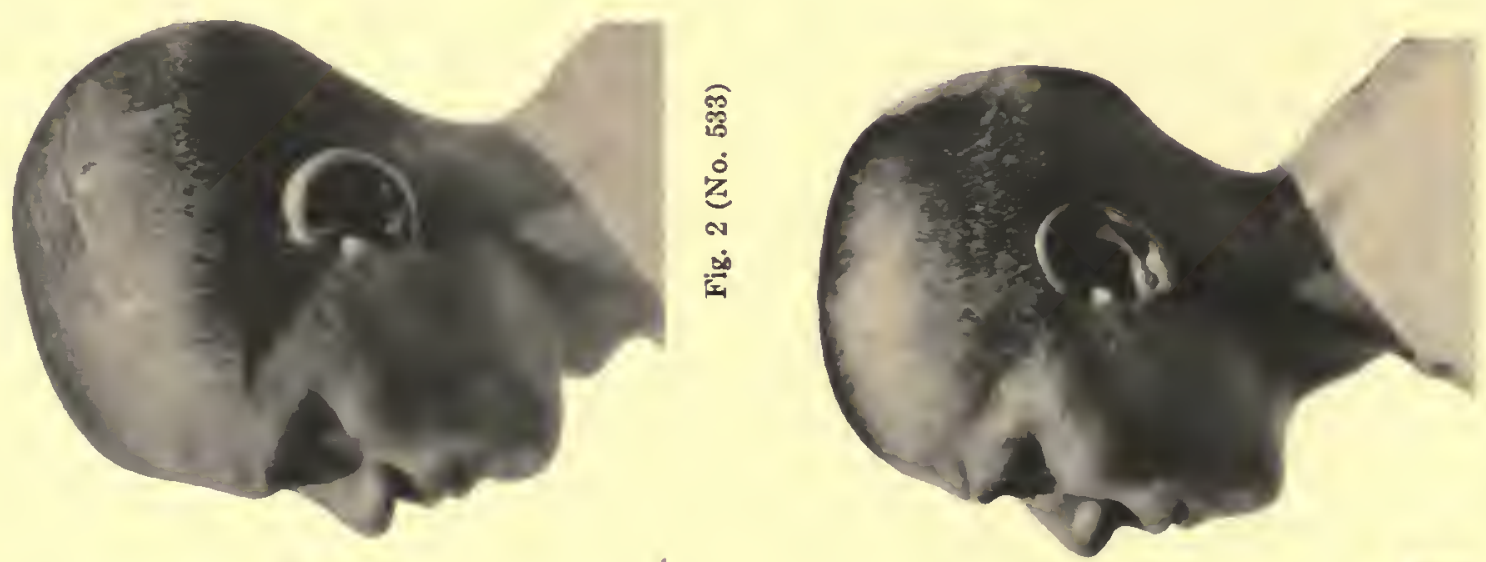

秴㤩
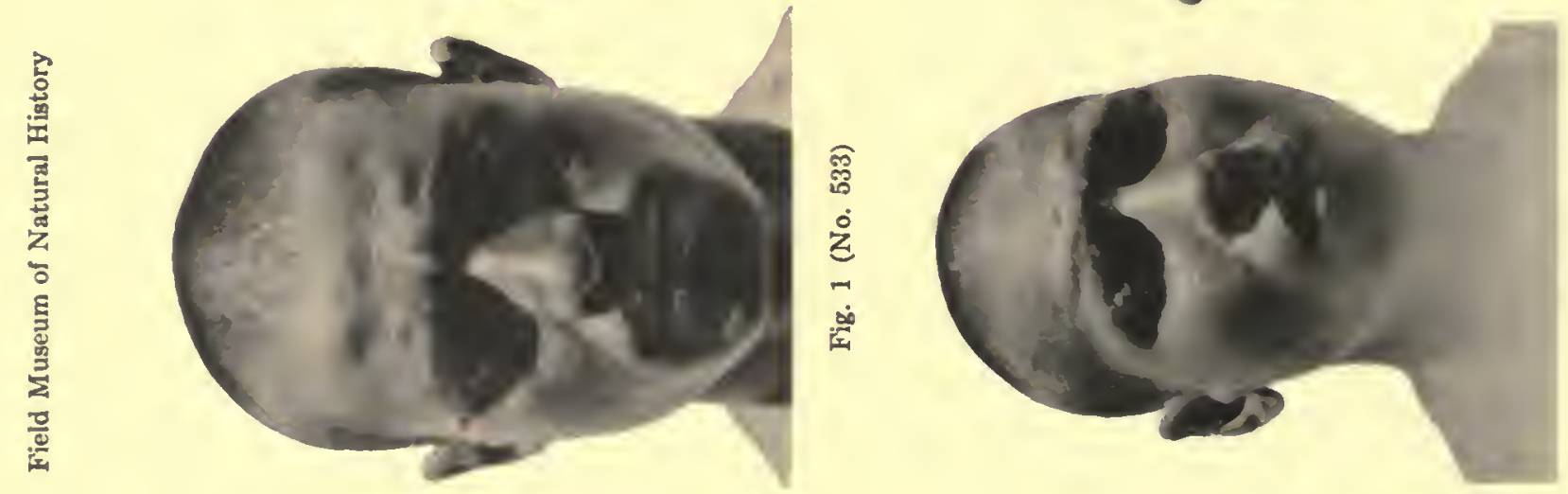

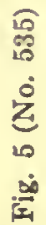




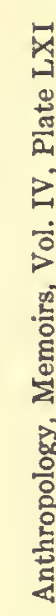
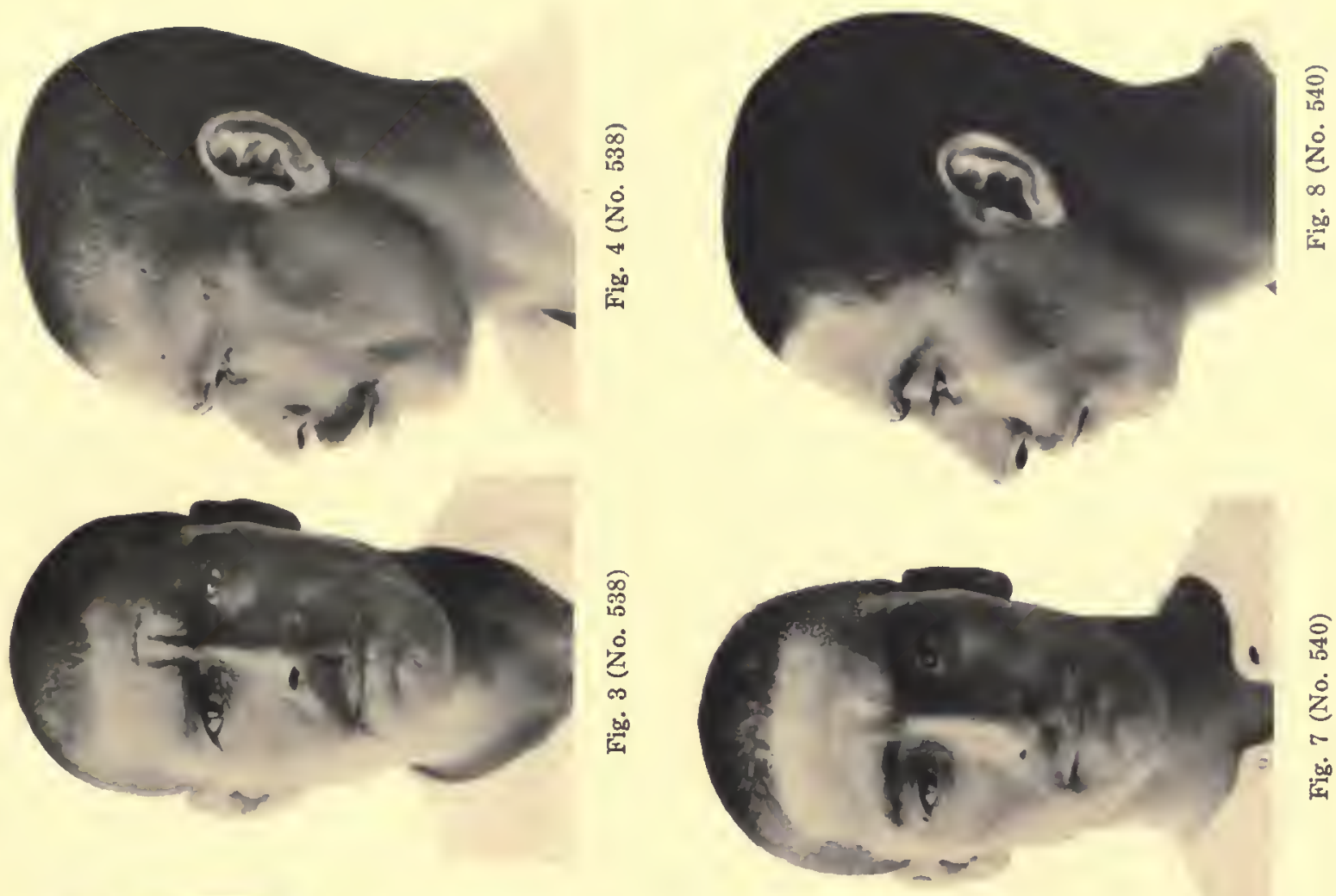

ㅇํㅇ

宅 谣

넌
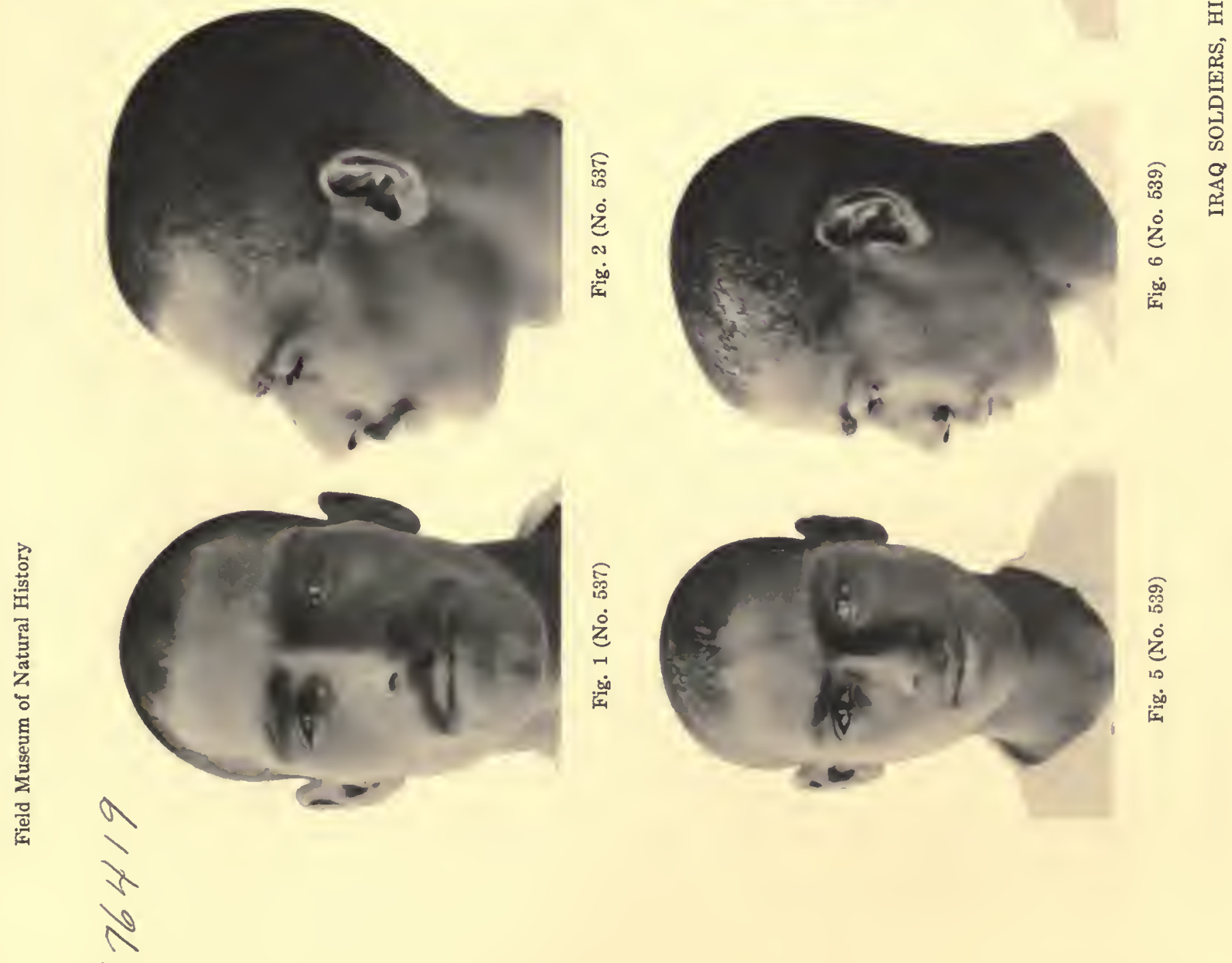


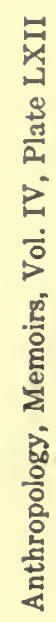
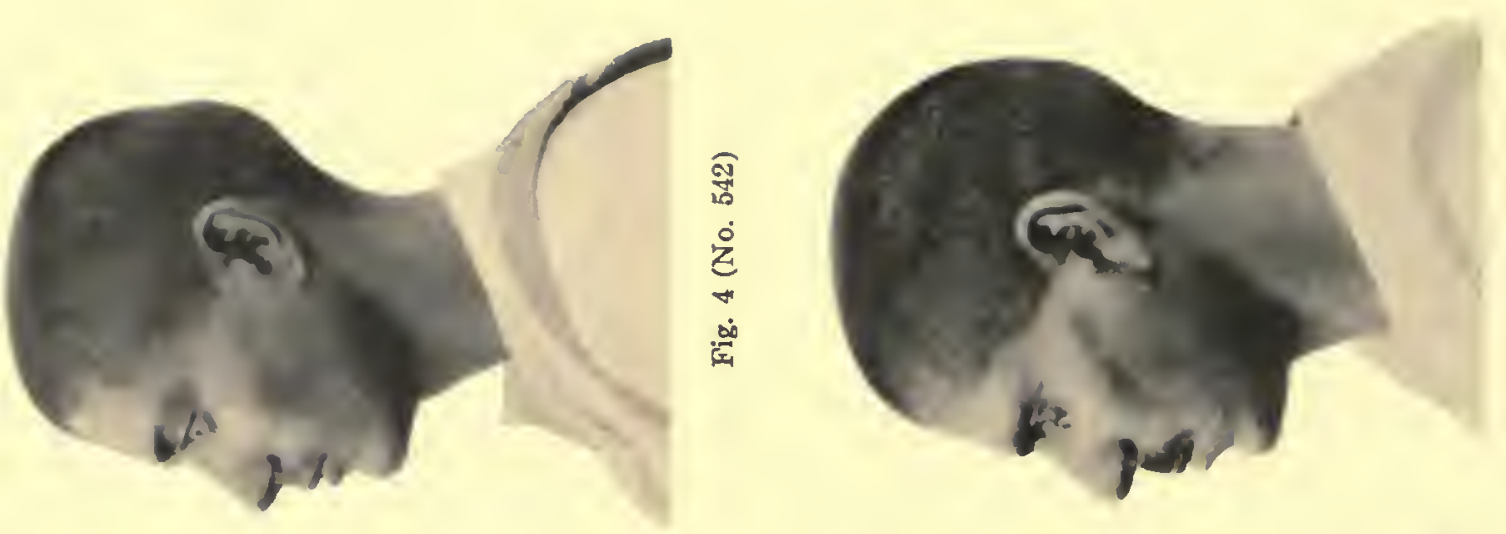

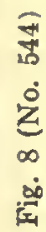
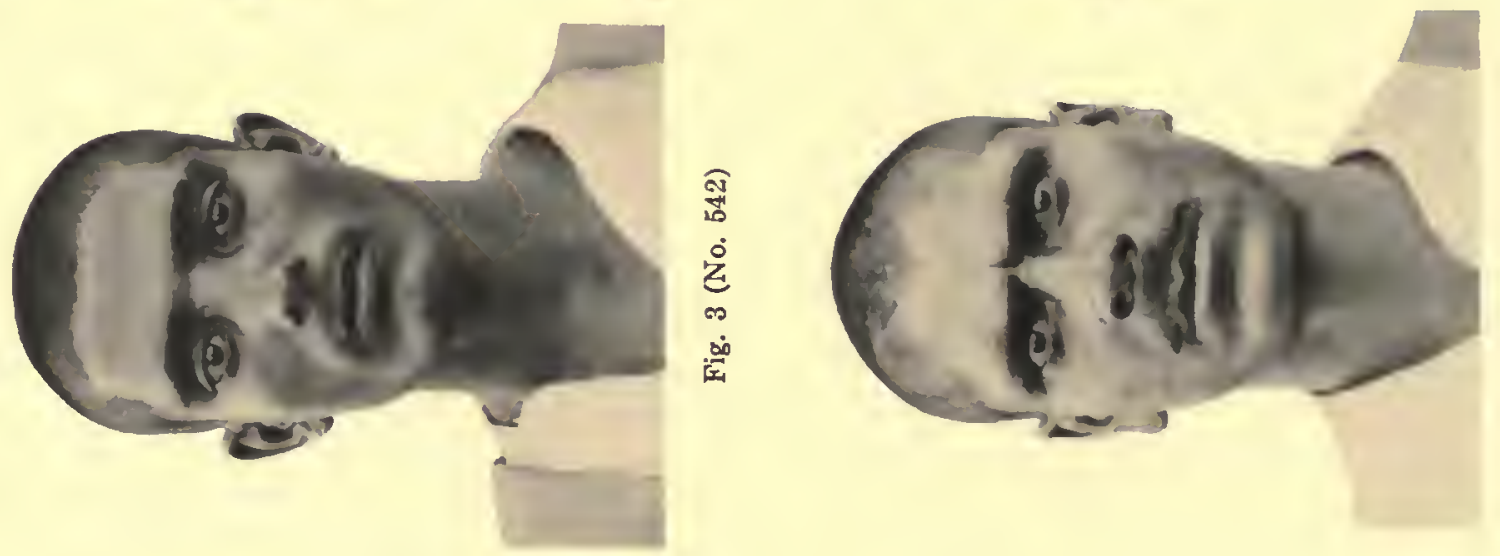

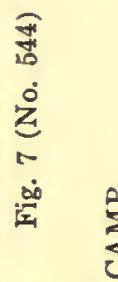
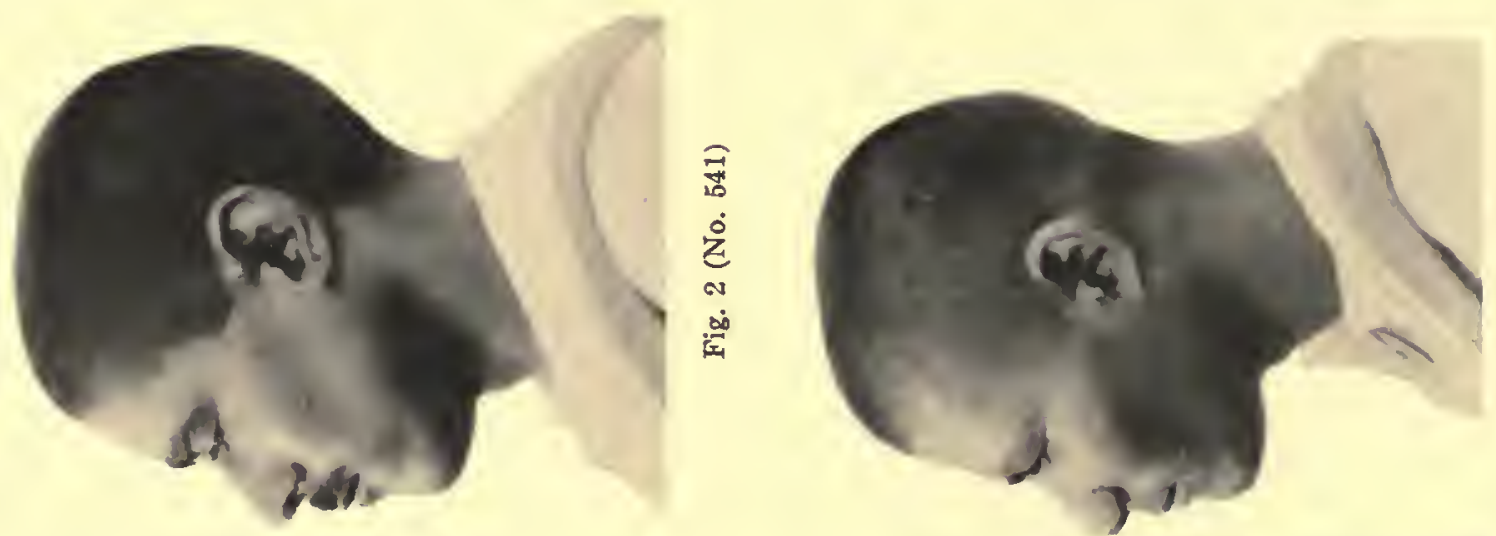

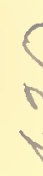
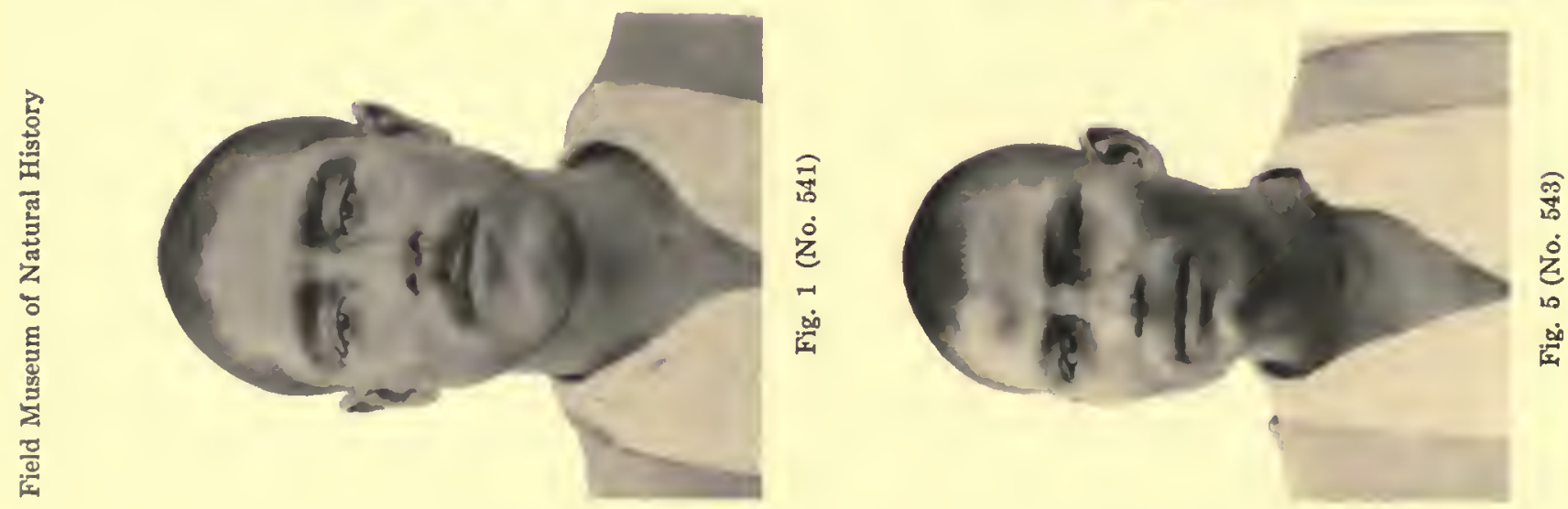

铔 N 

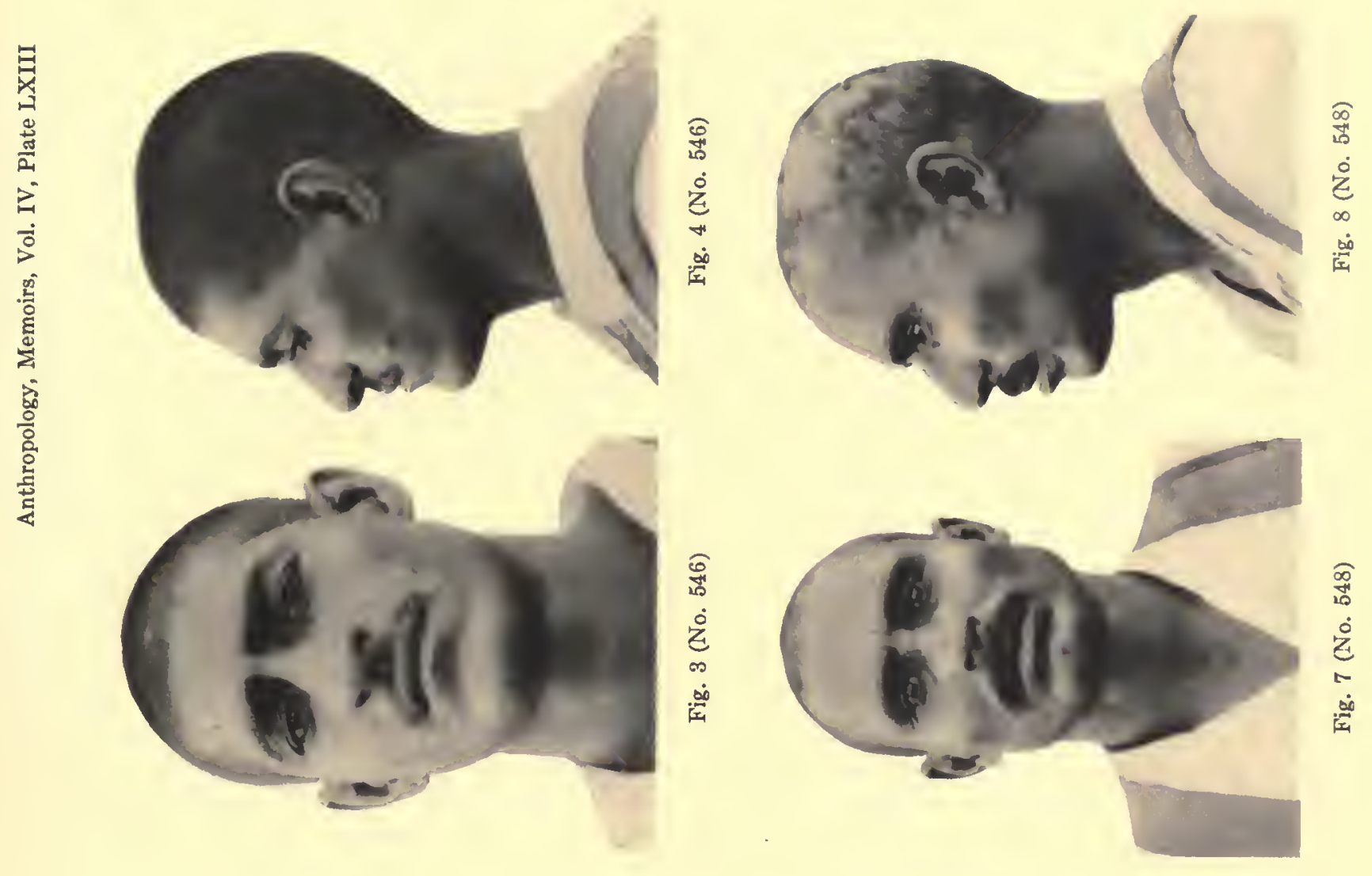

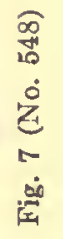
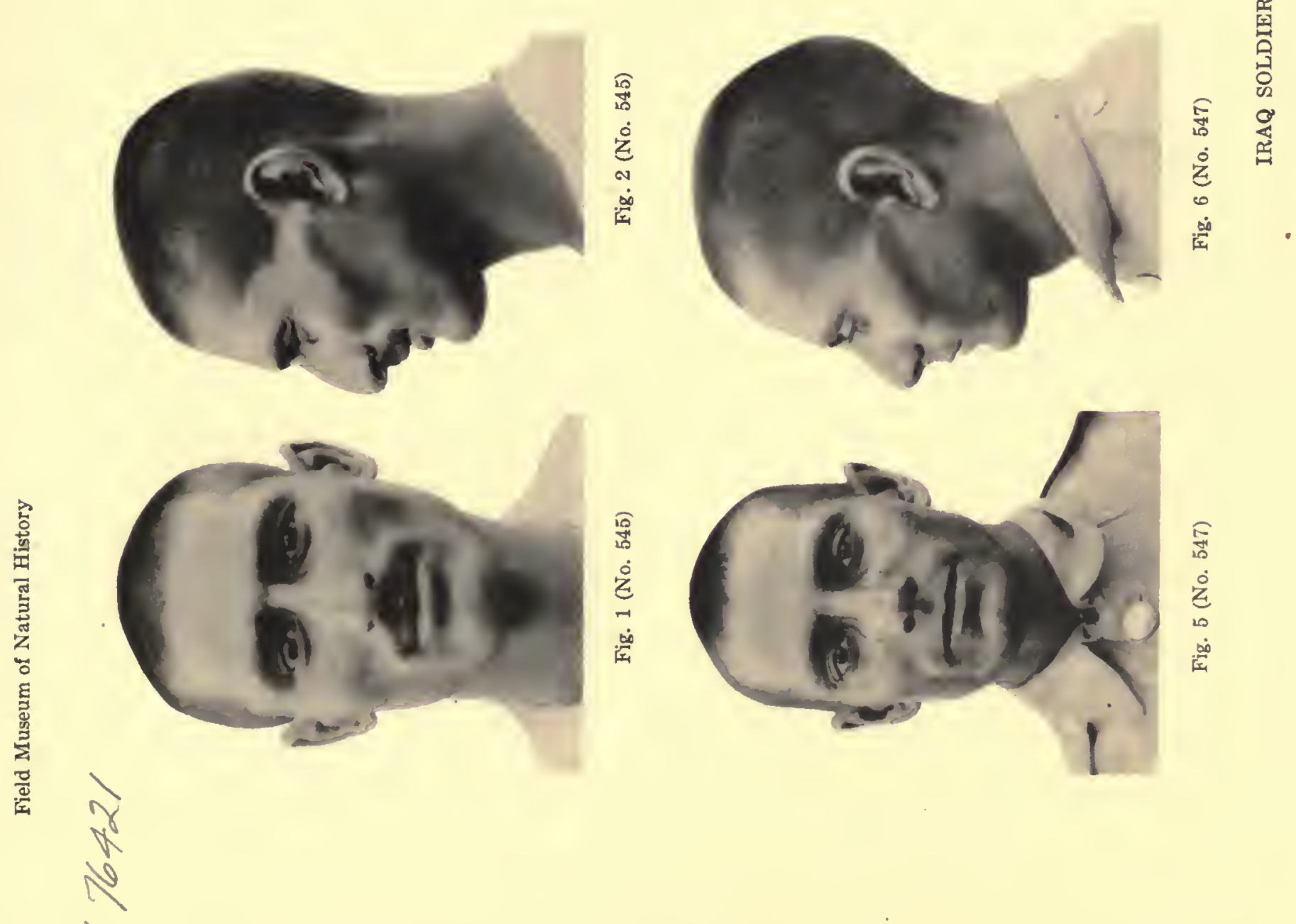

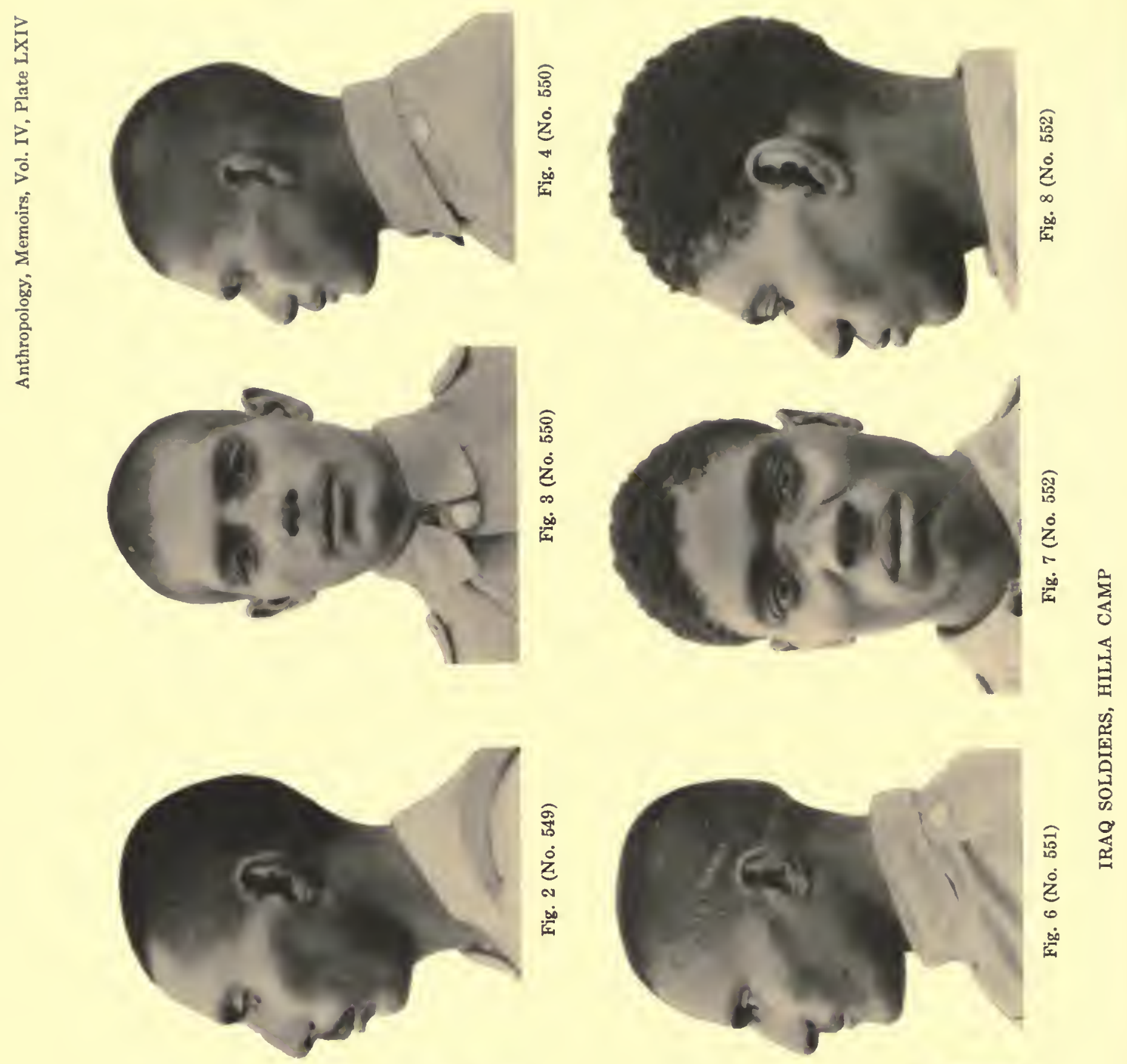

$y$
$y$
$y$
$y$
$y$
$x$
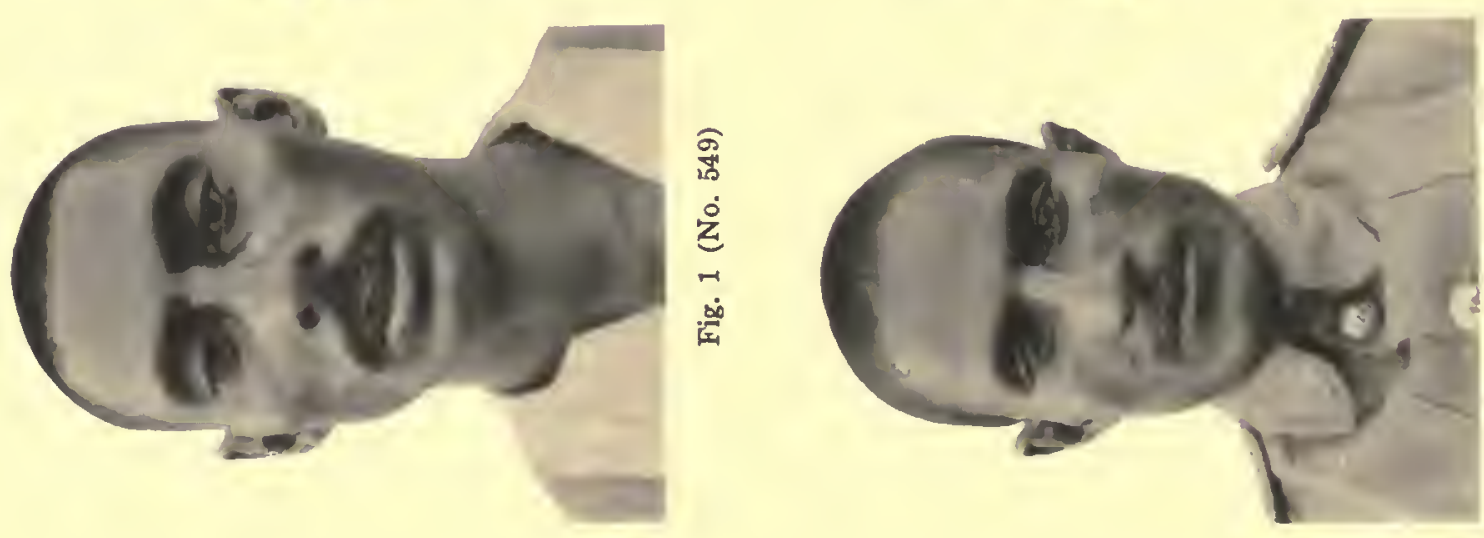

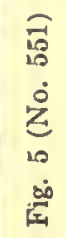



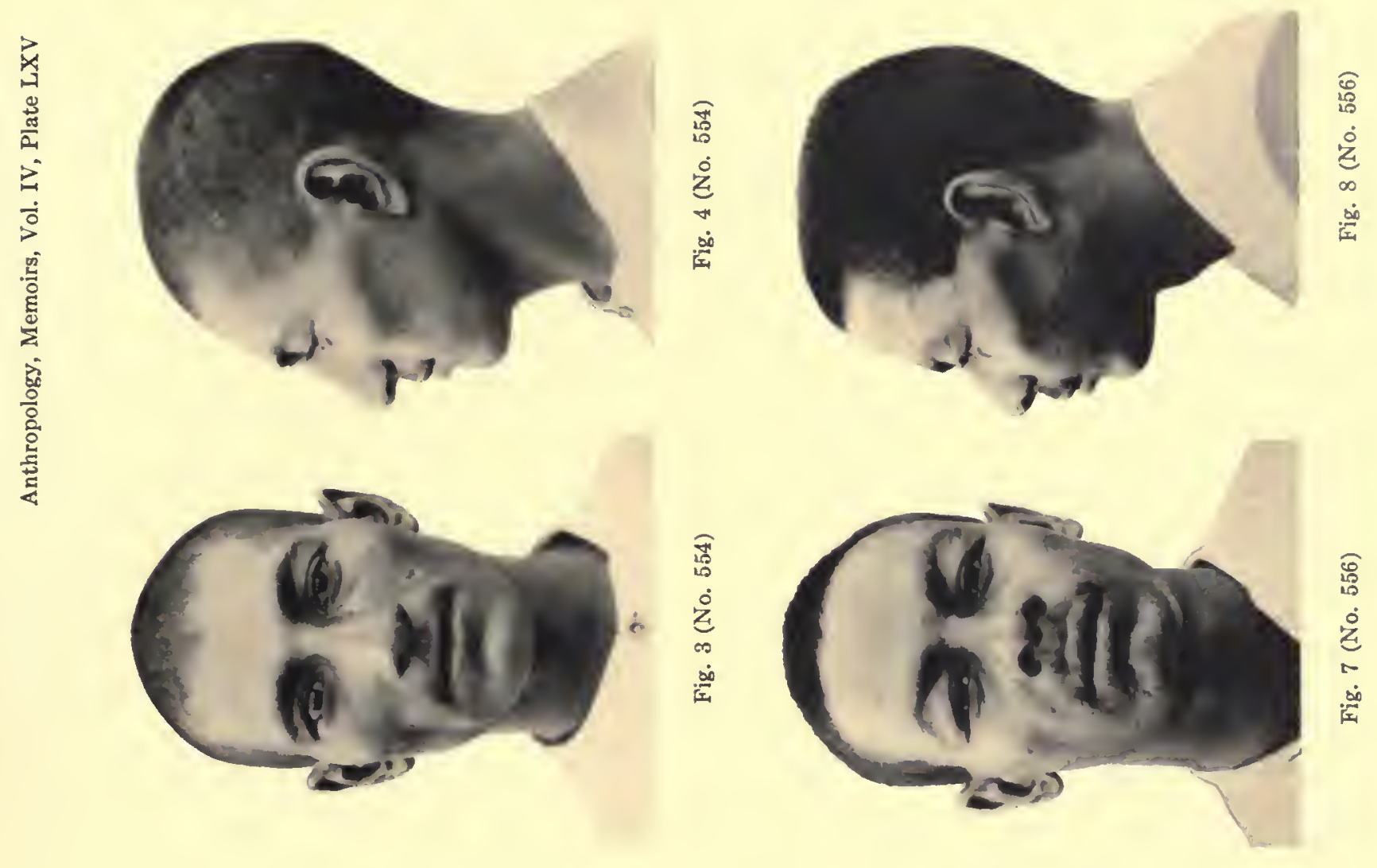

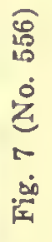

嵌
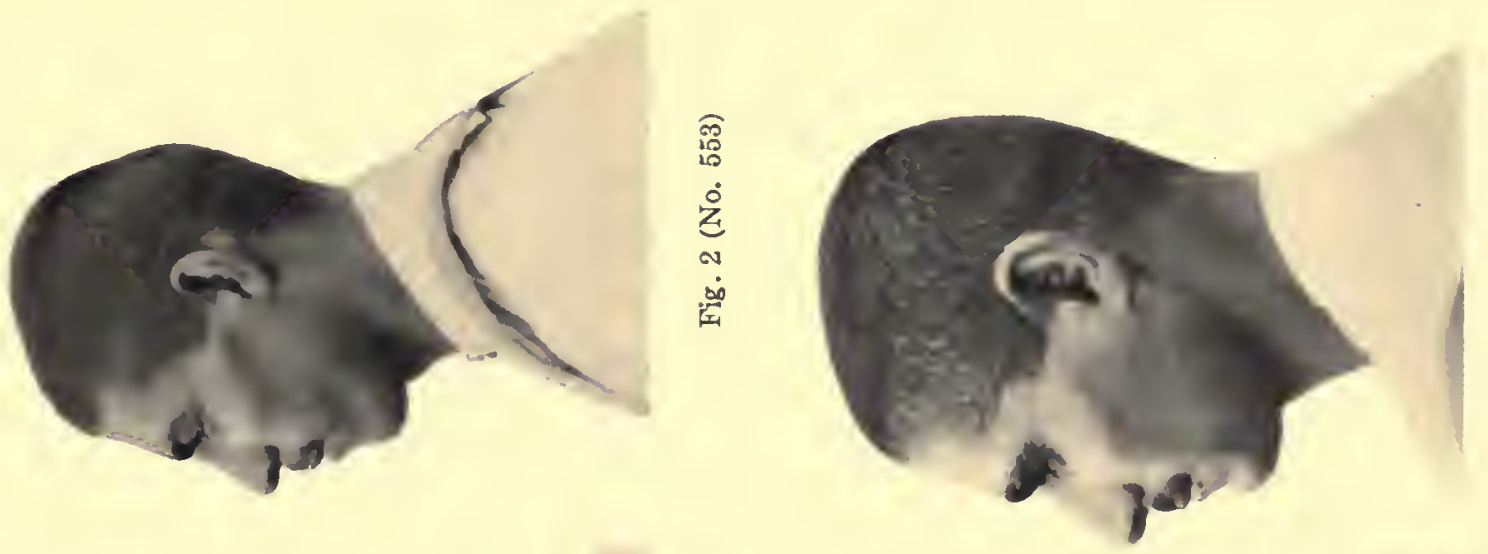

焉


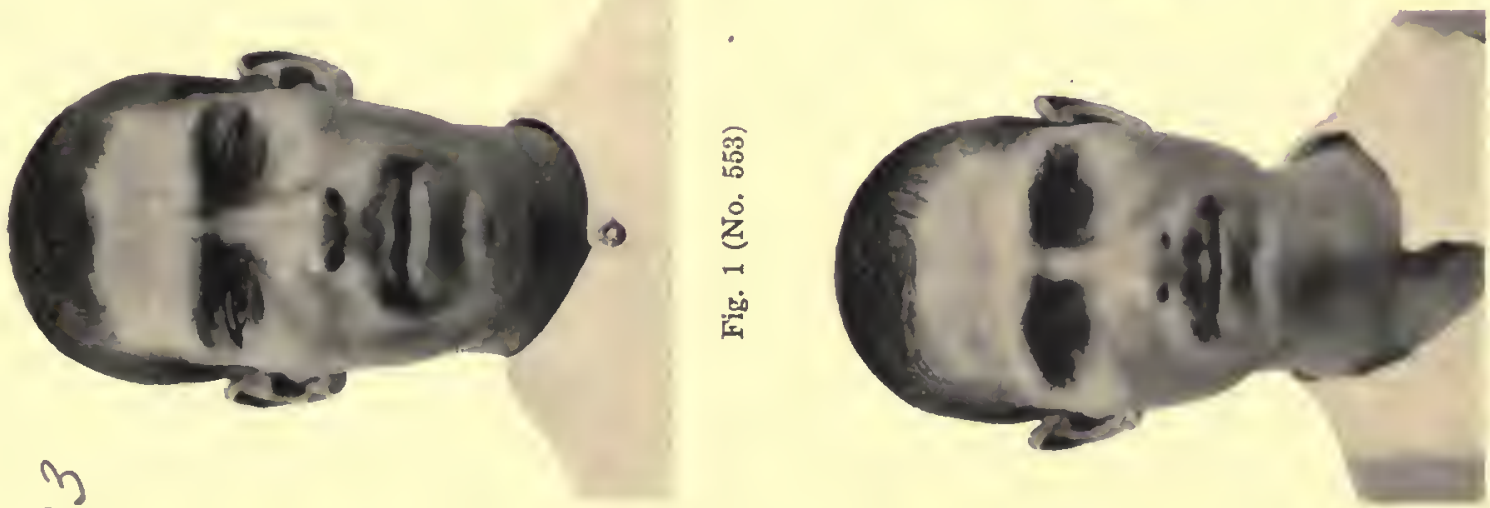

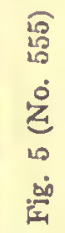

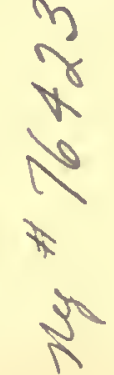



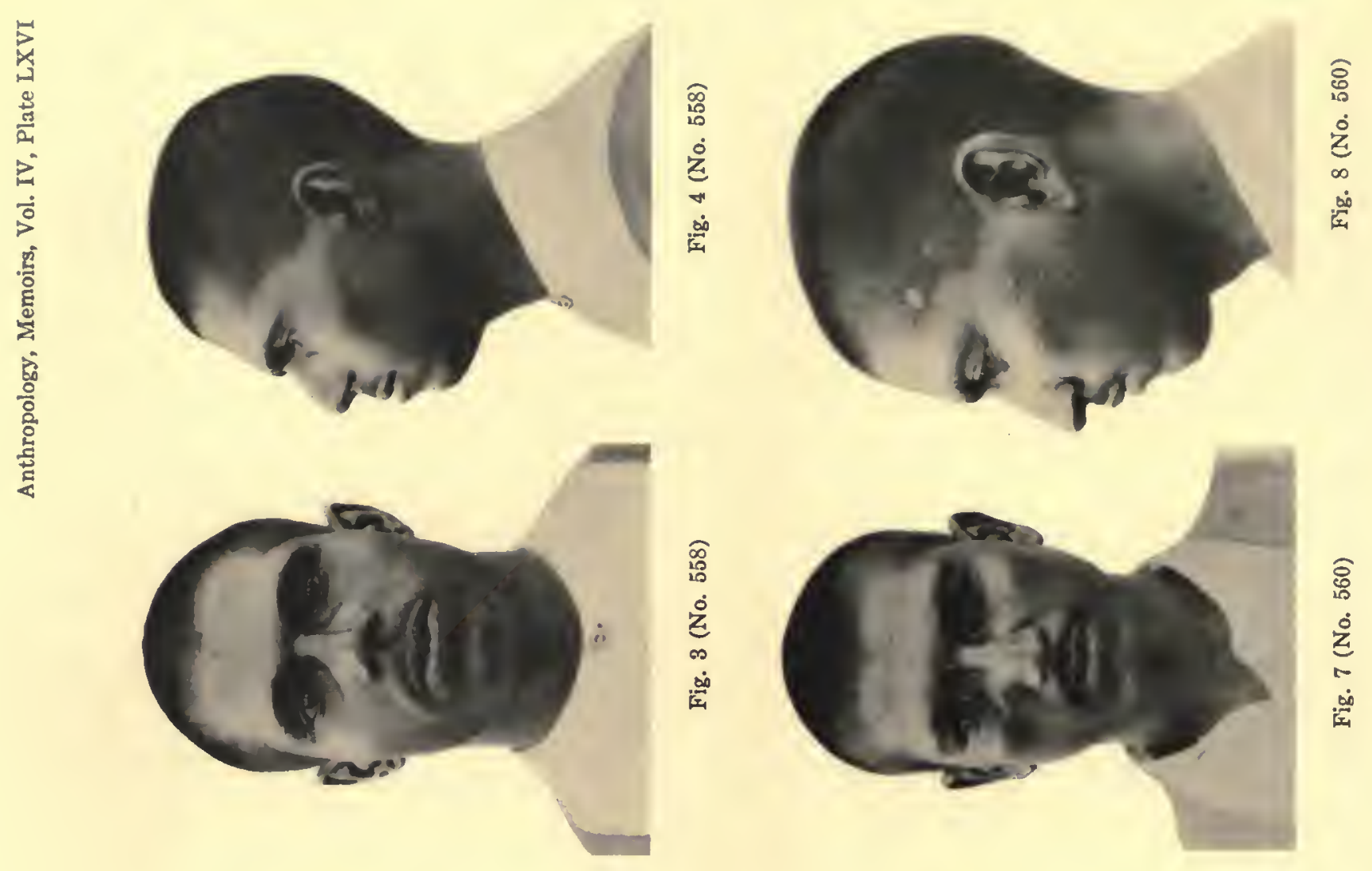

高
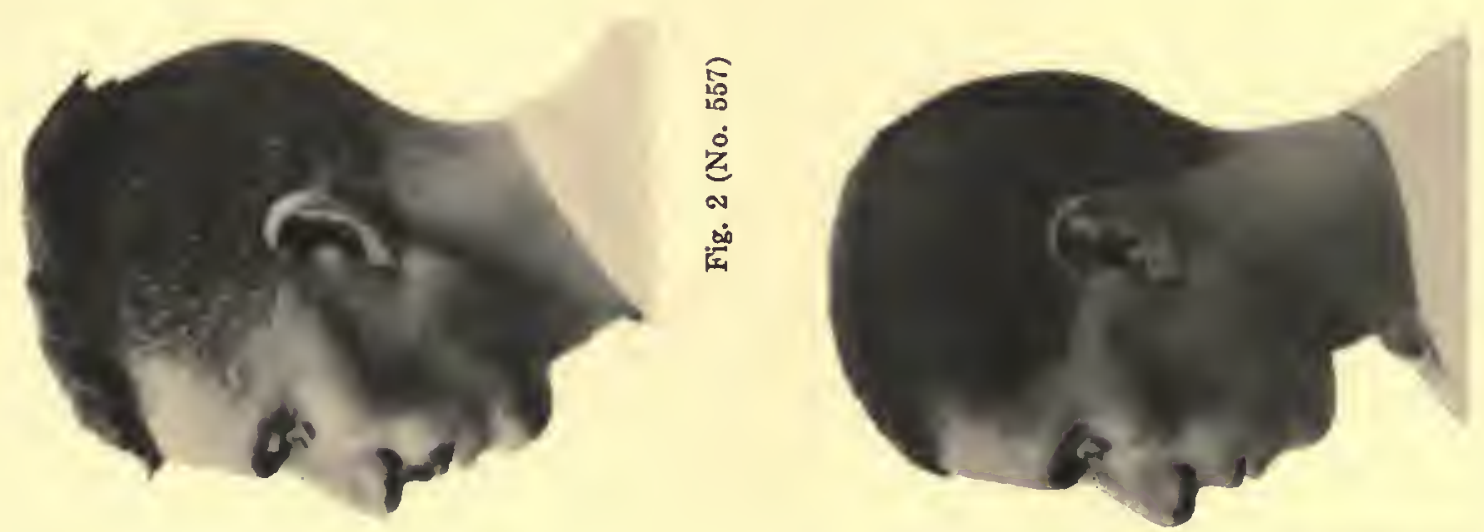

函 总

ì

递
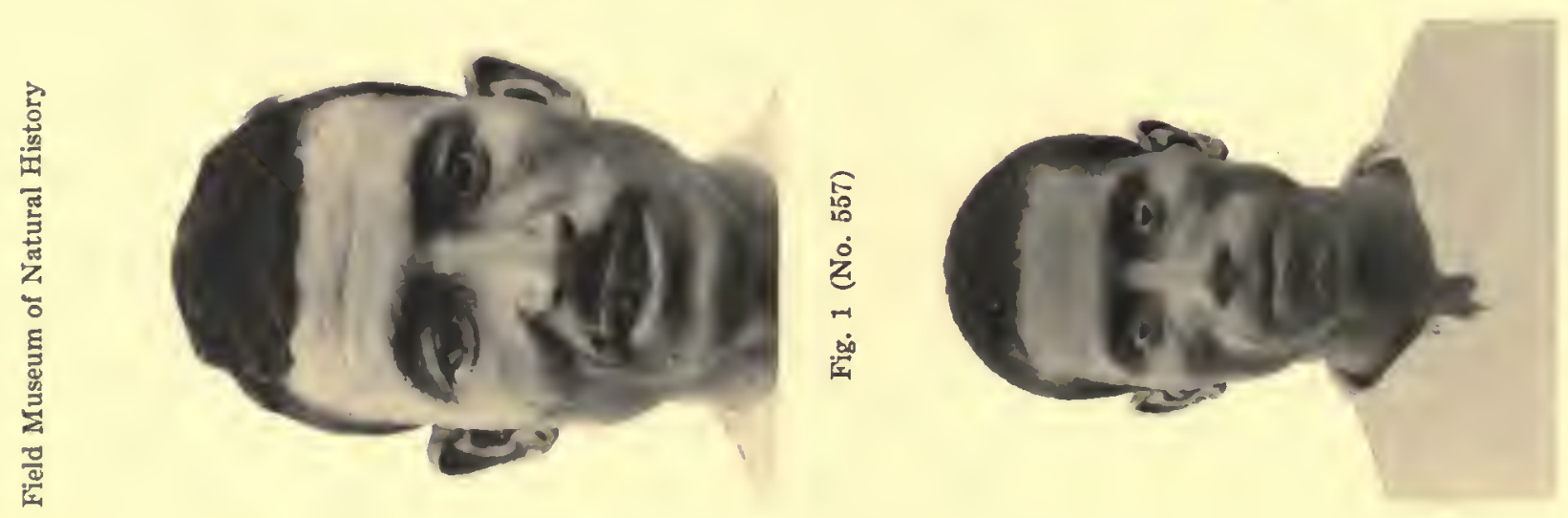

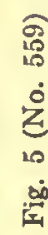

5 

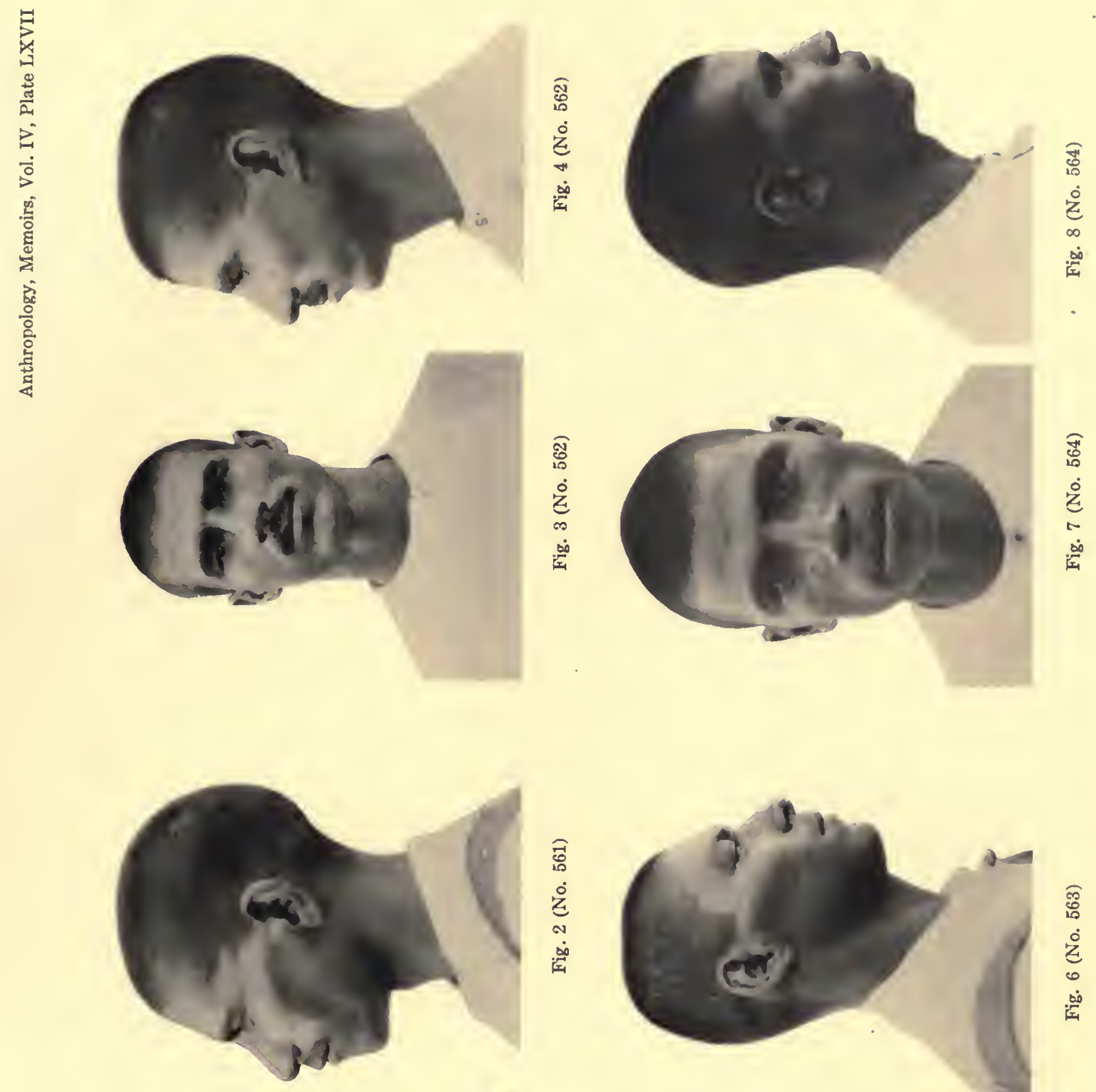

甭

章

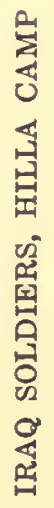

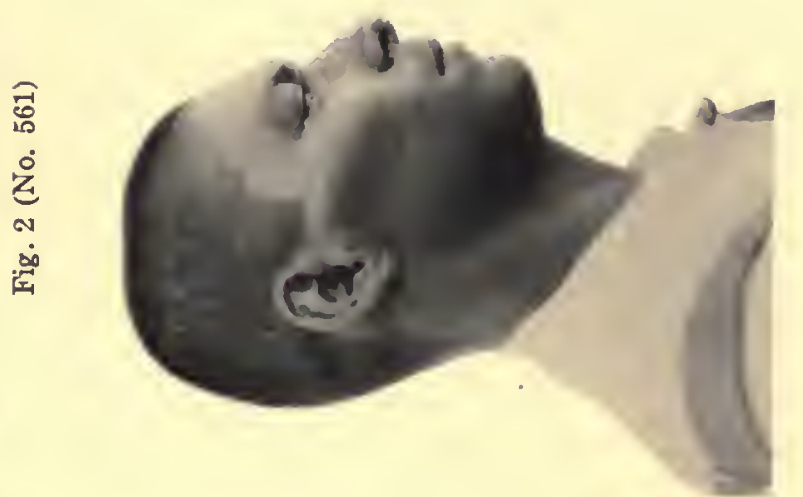

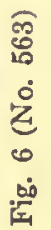

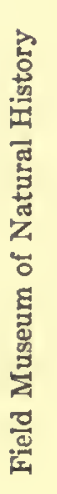
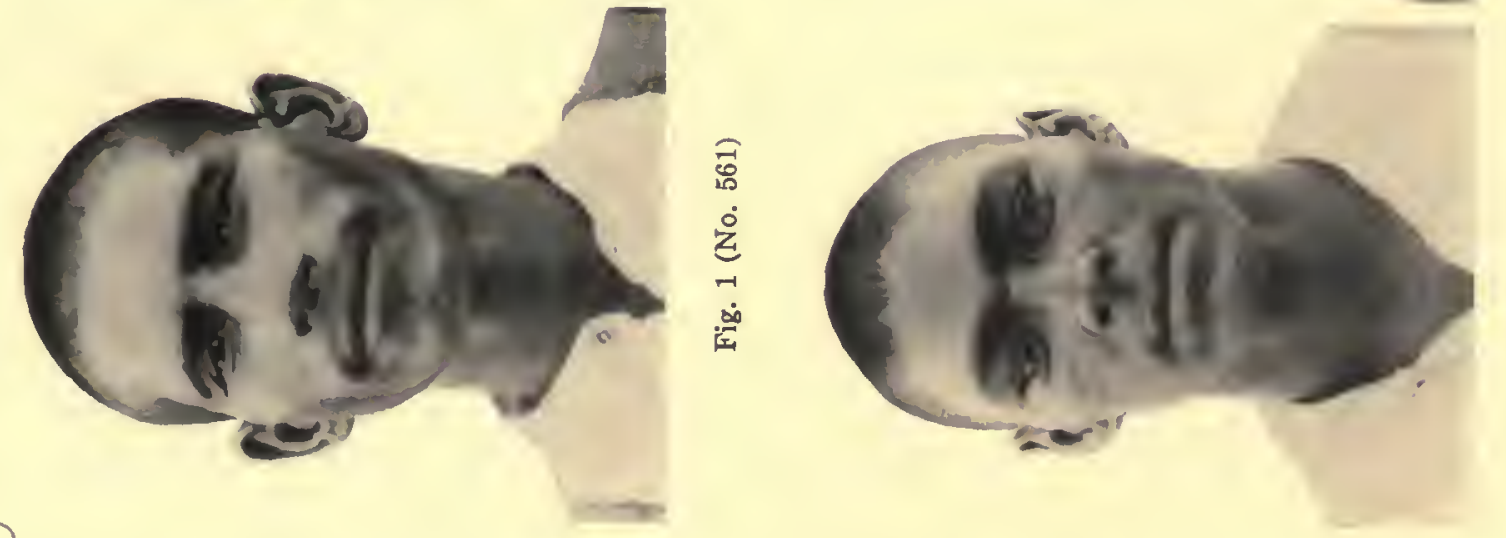

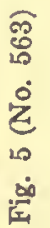

$x^{2}$

N 

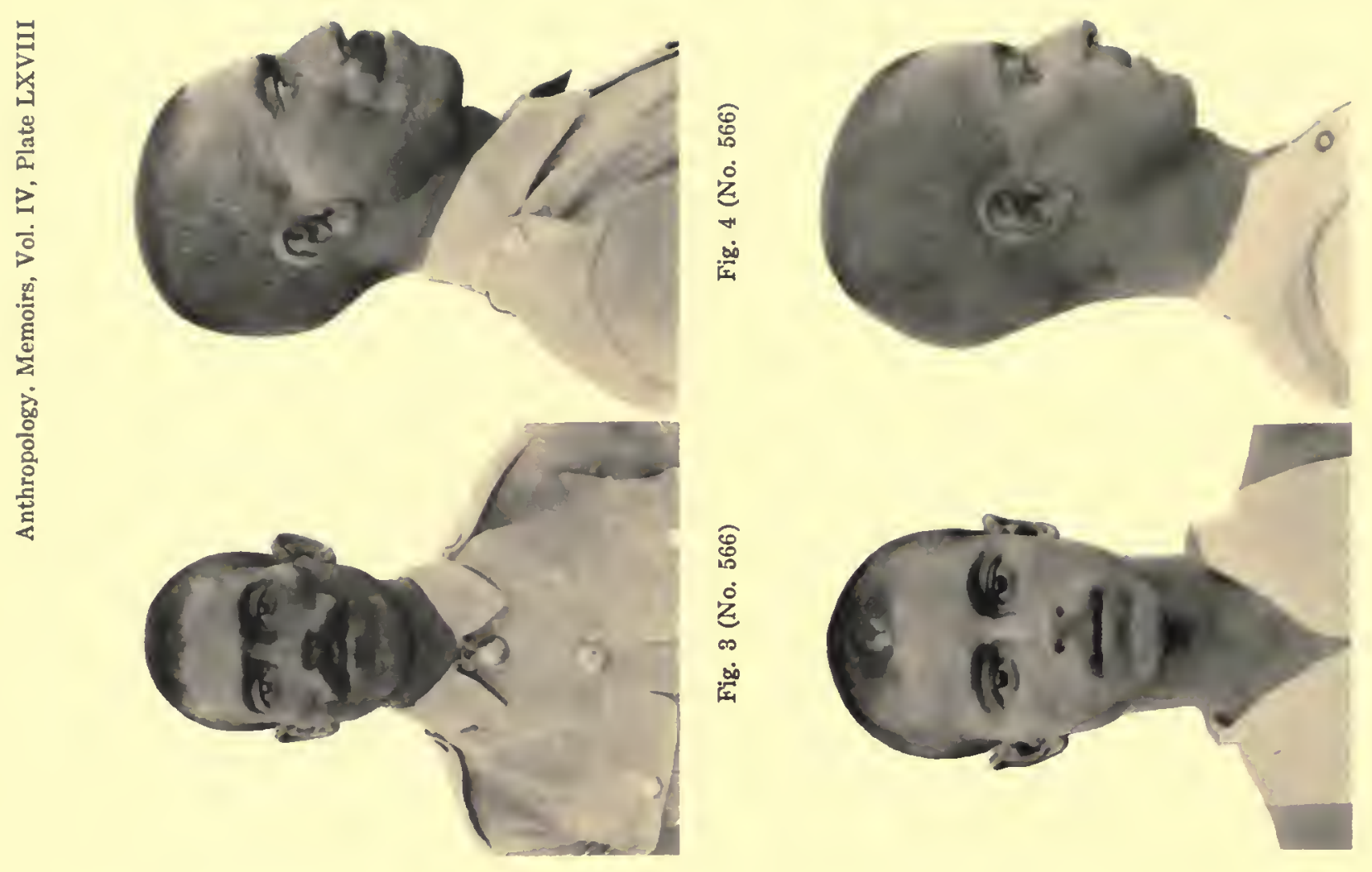

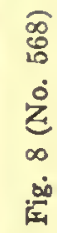

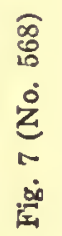
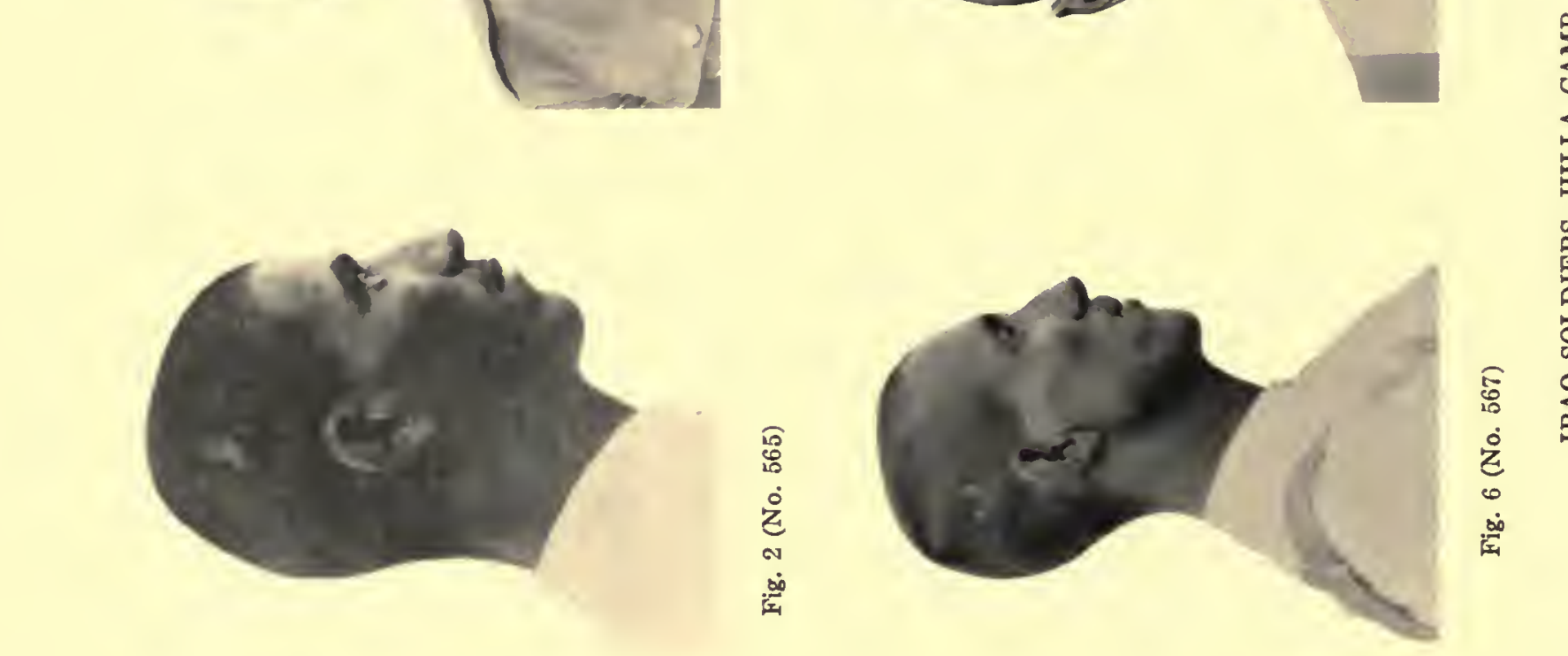

音
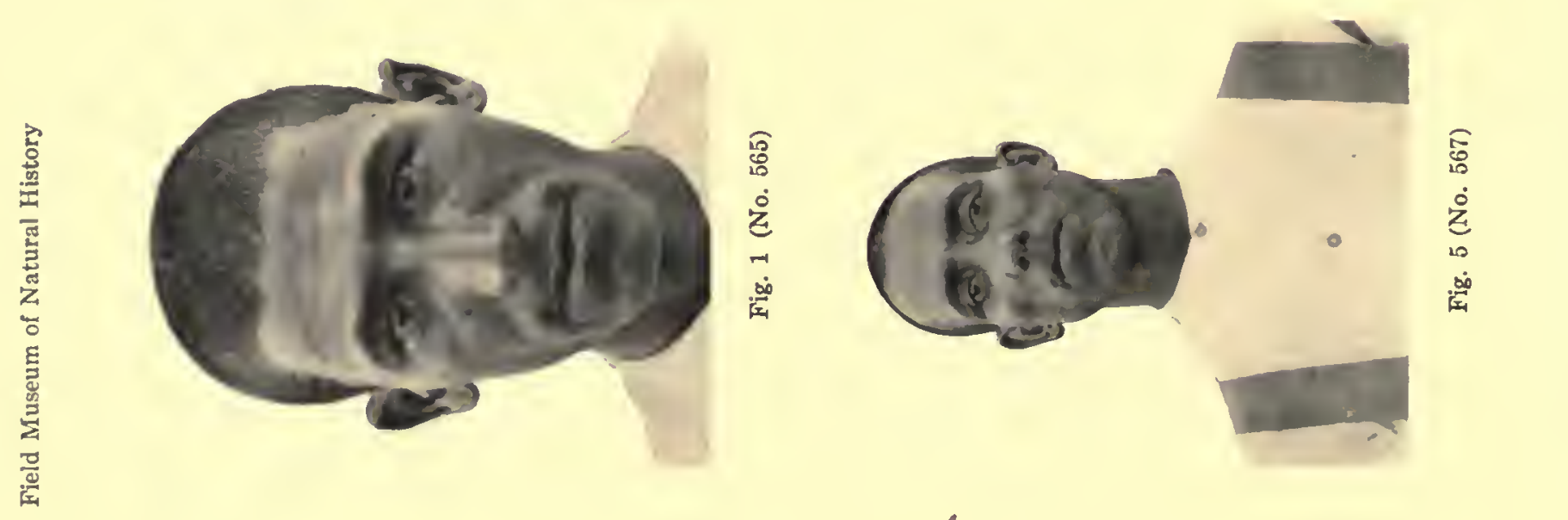


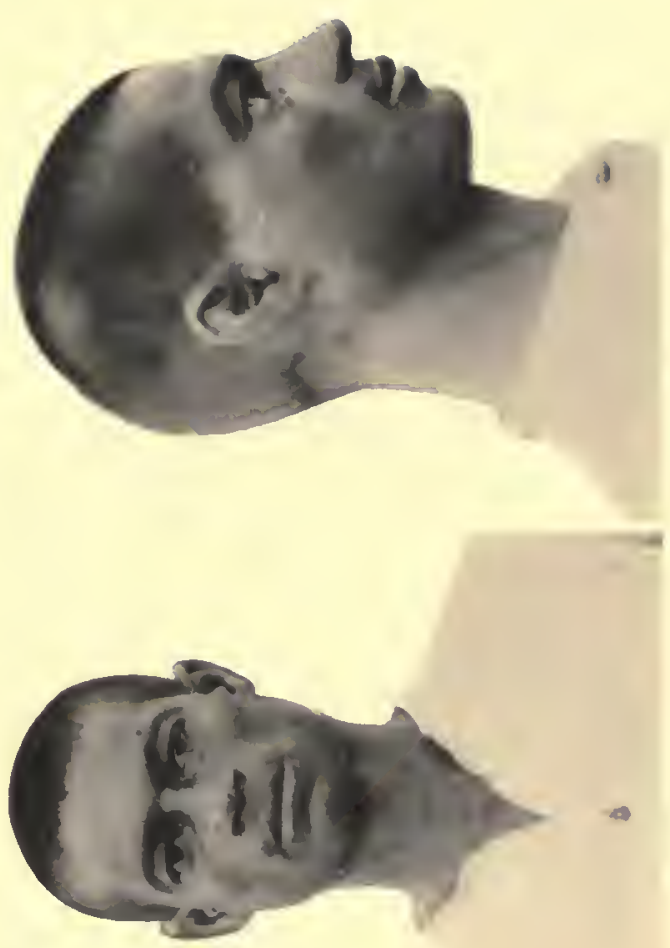

官

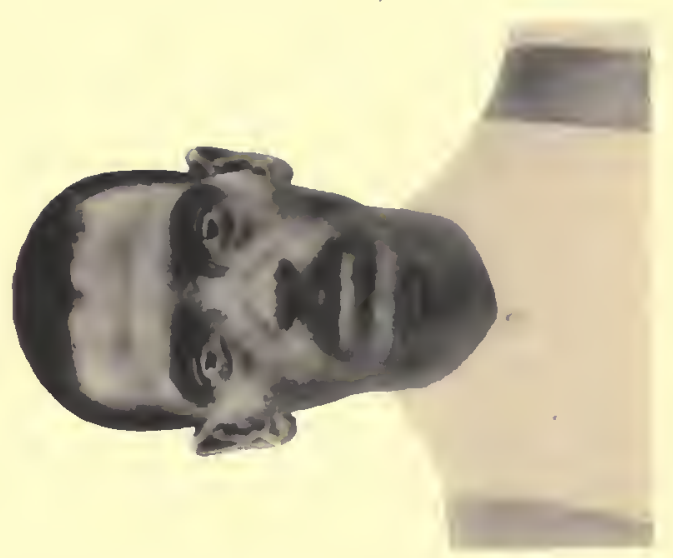

ลิธ

ż

옹

¿

近

菖
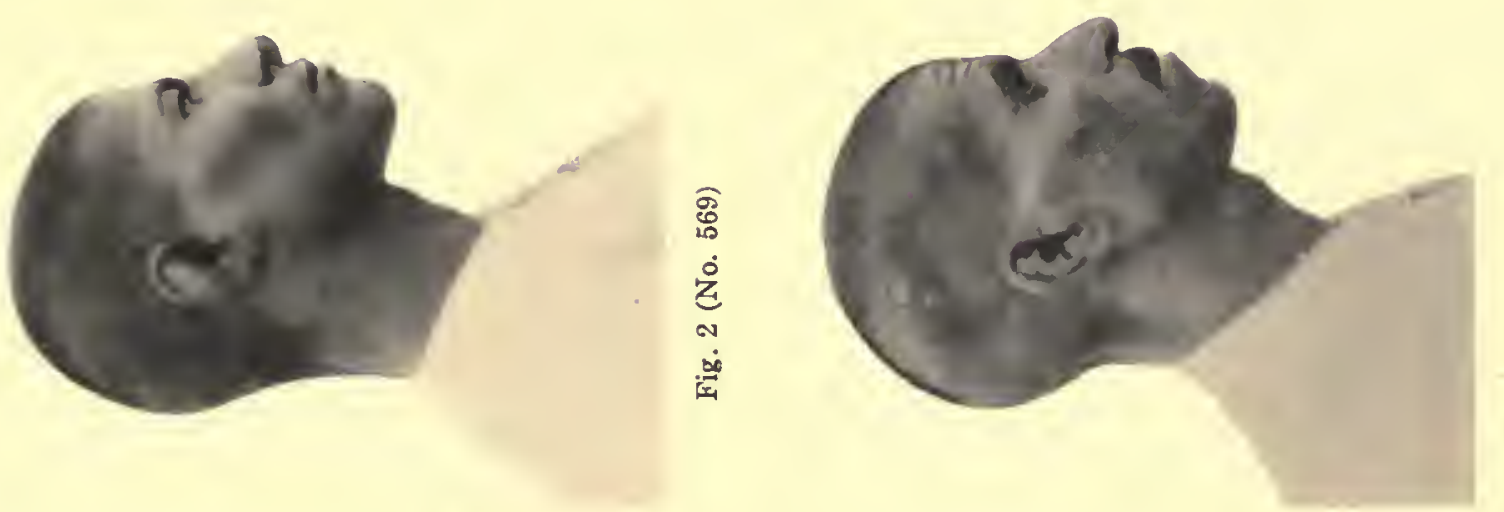

$E$
0
0
0
0
0
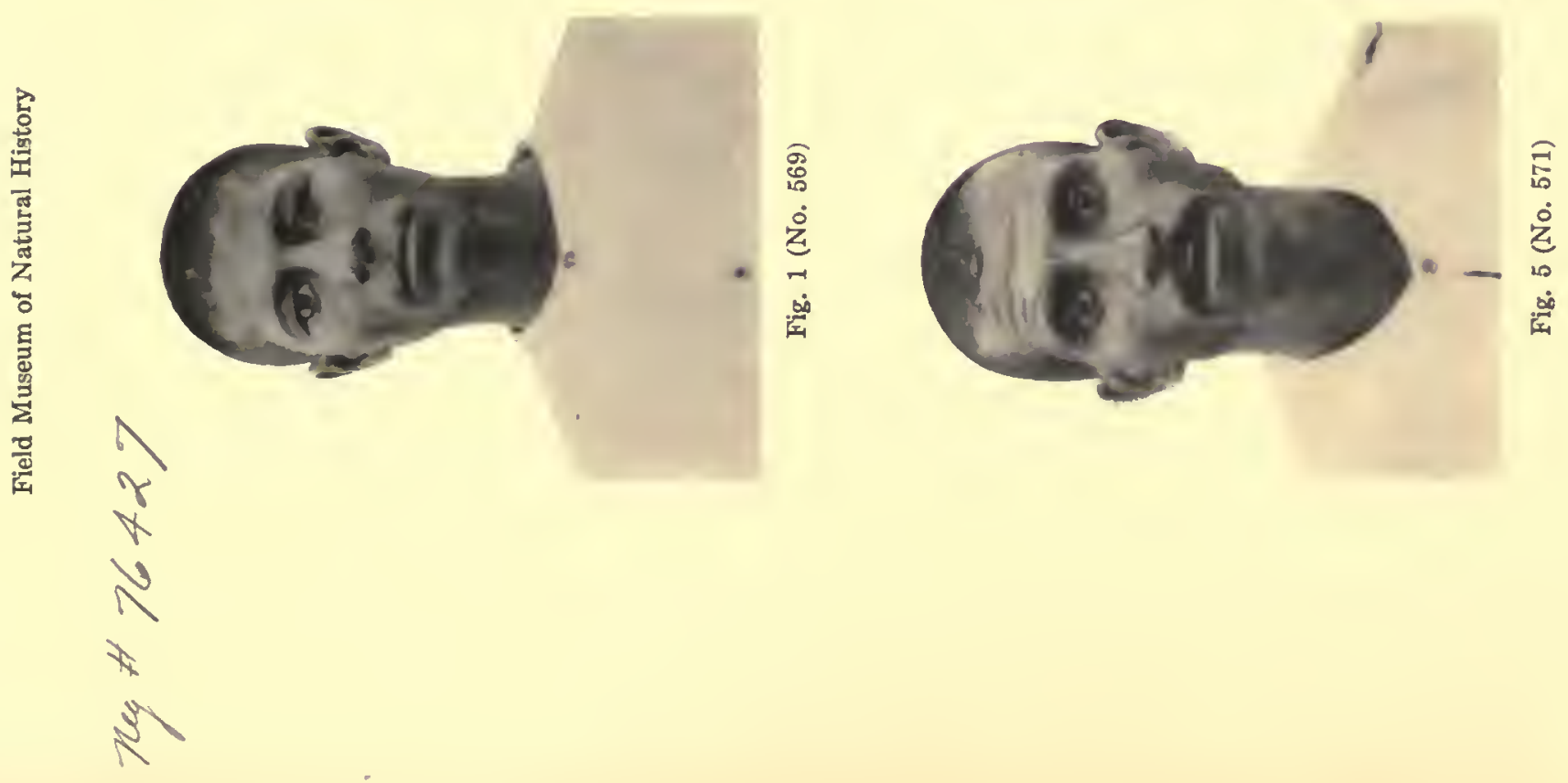

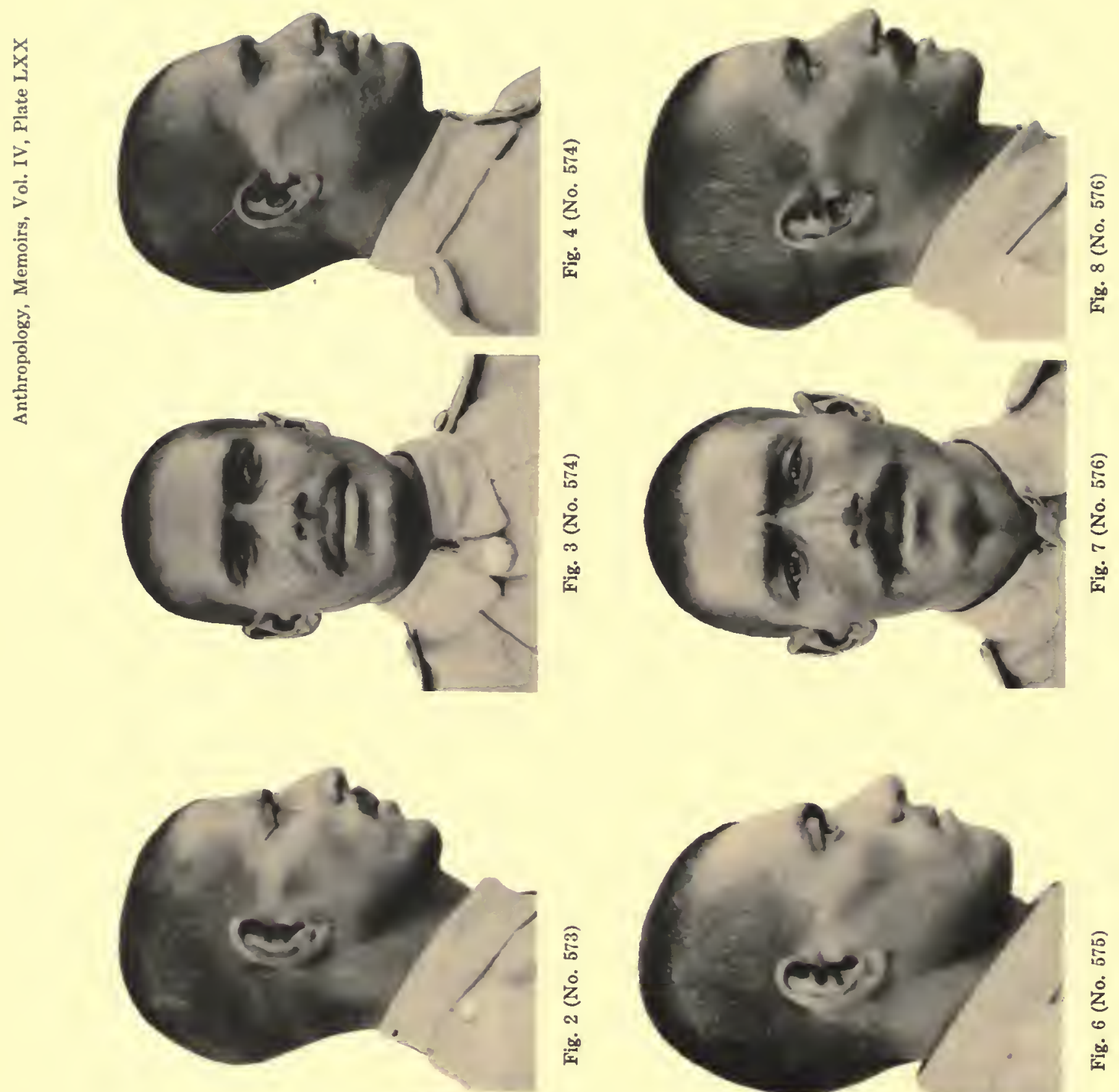

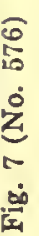

हो
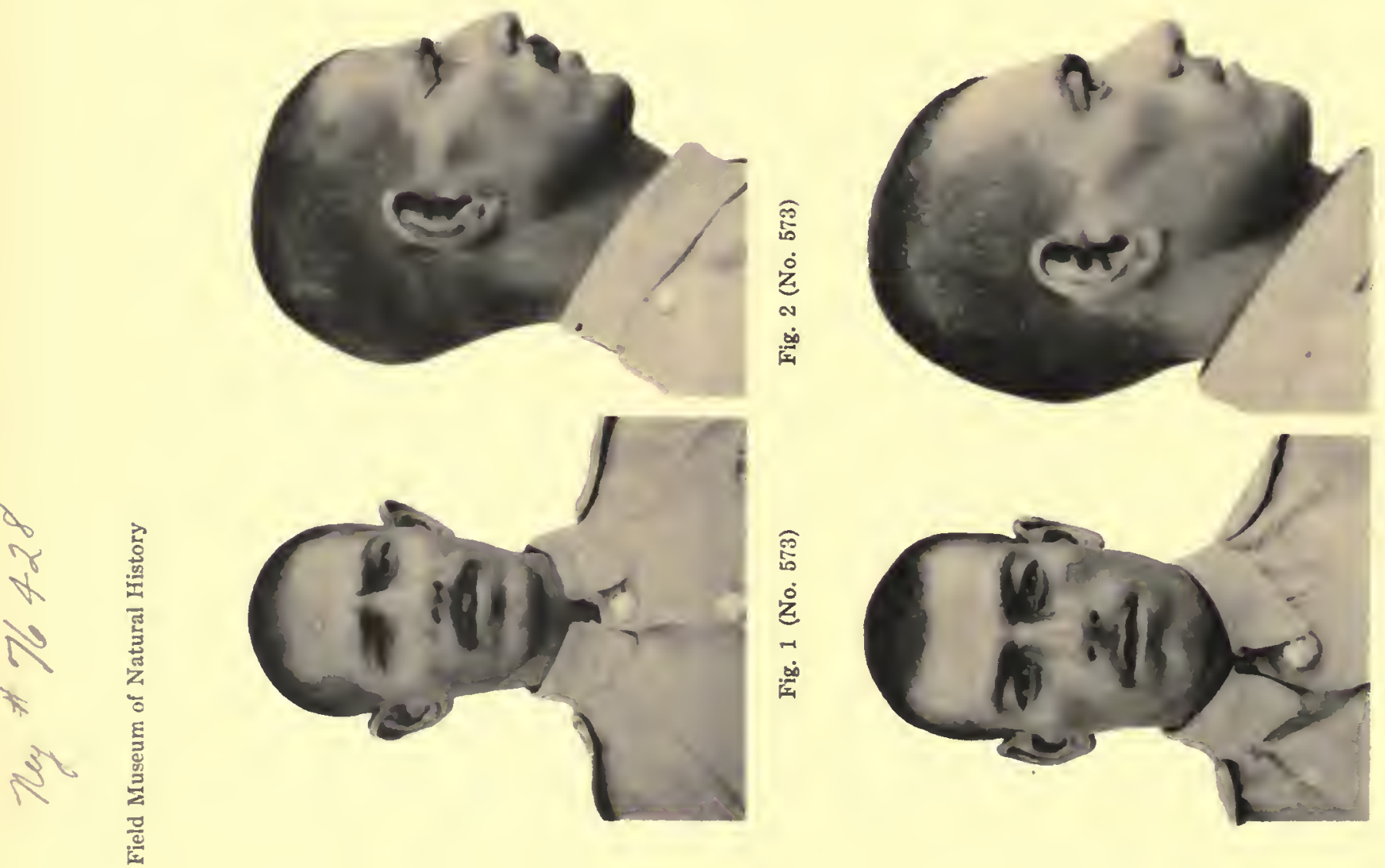

20
in
0
2
20
20 


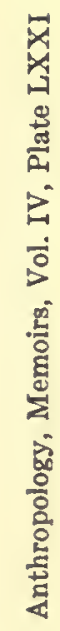
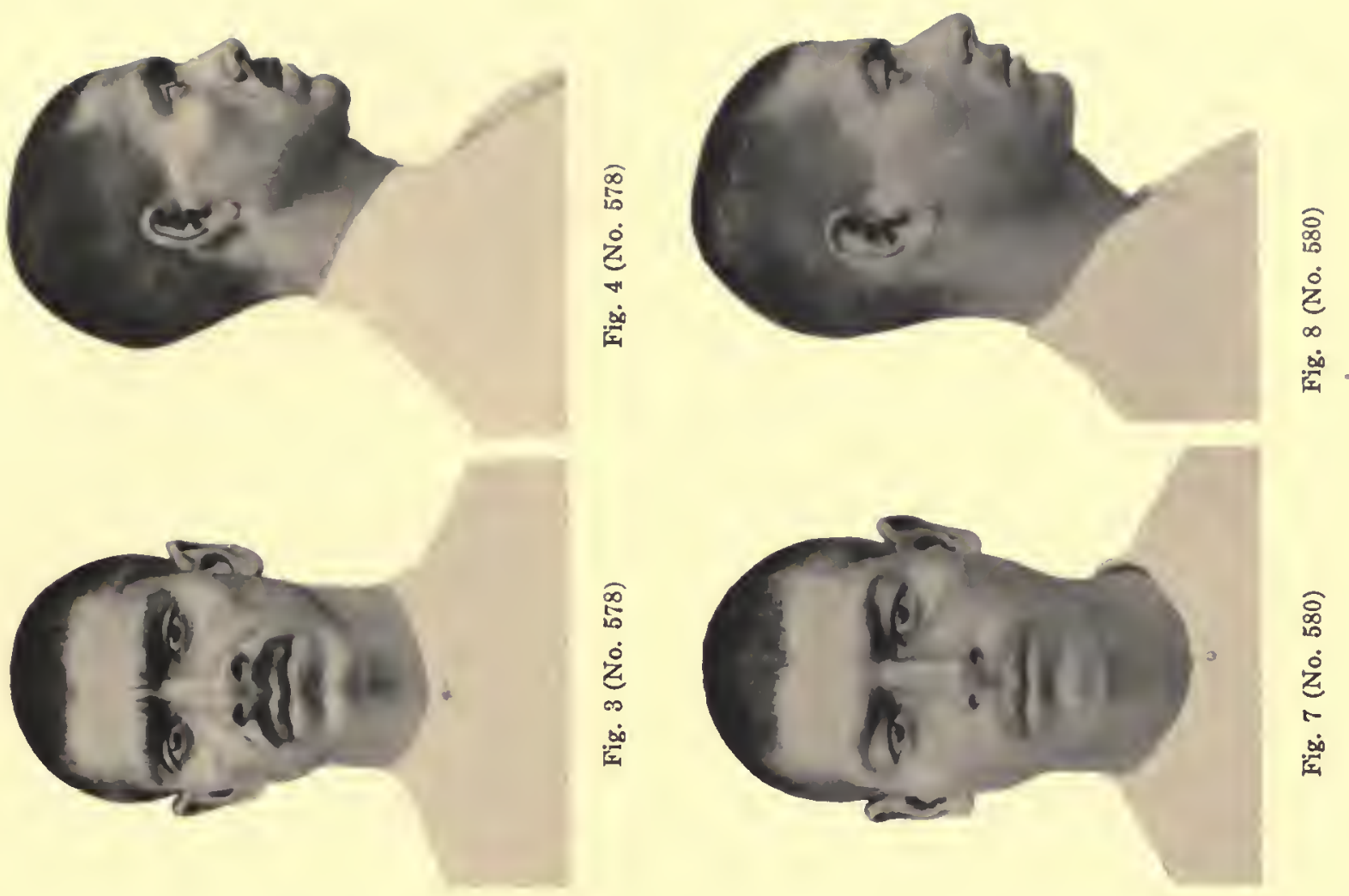

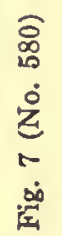

焉
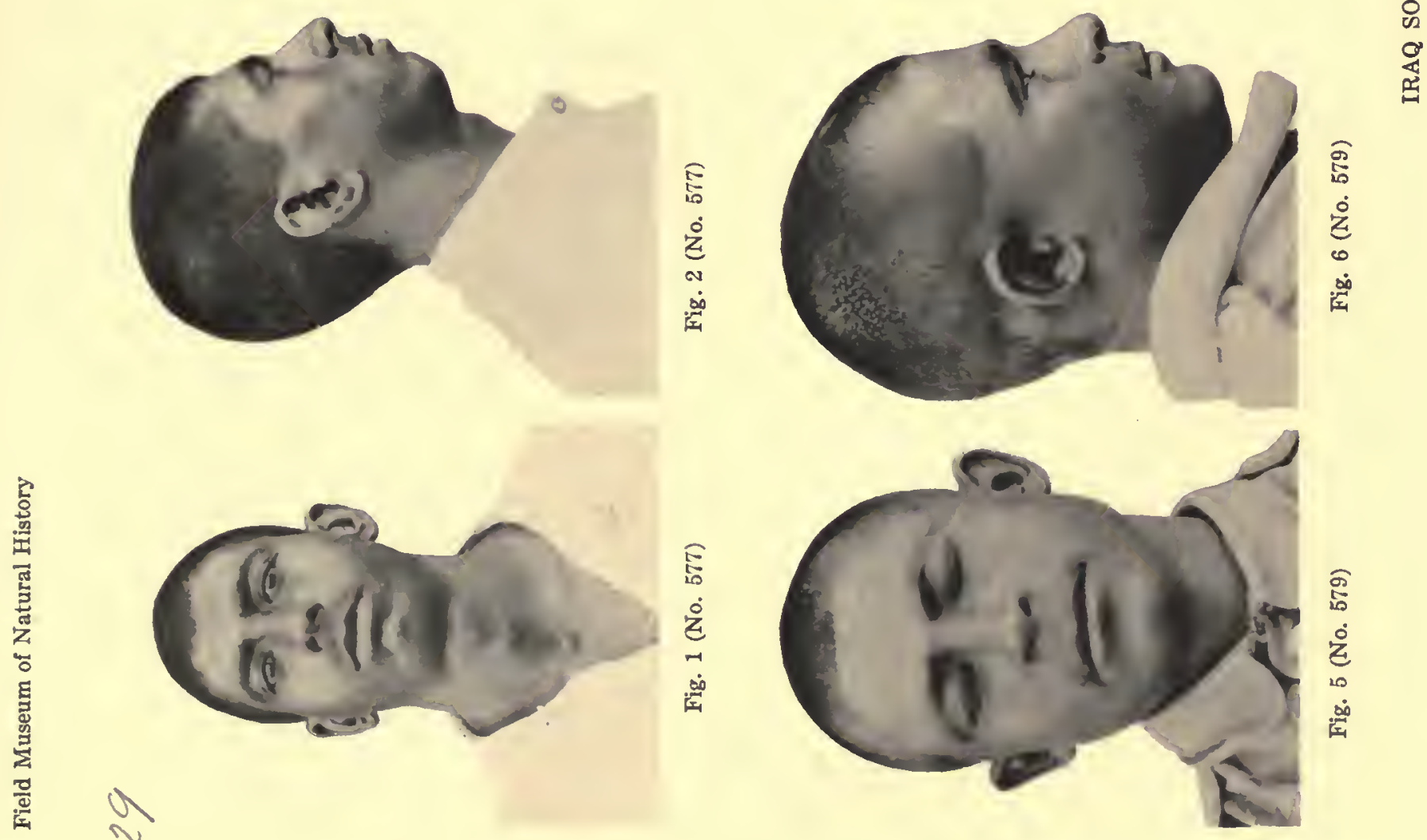

迹

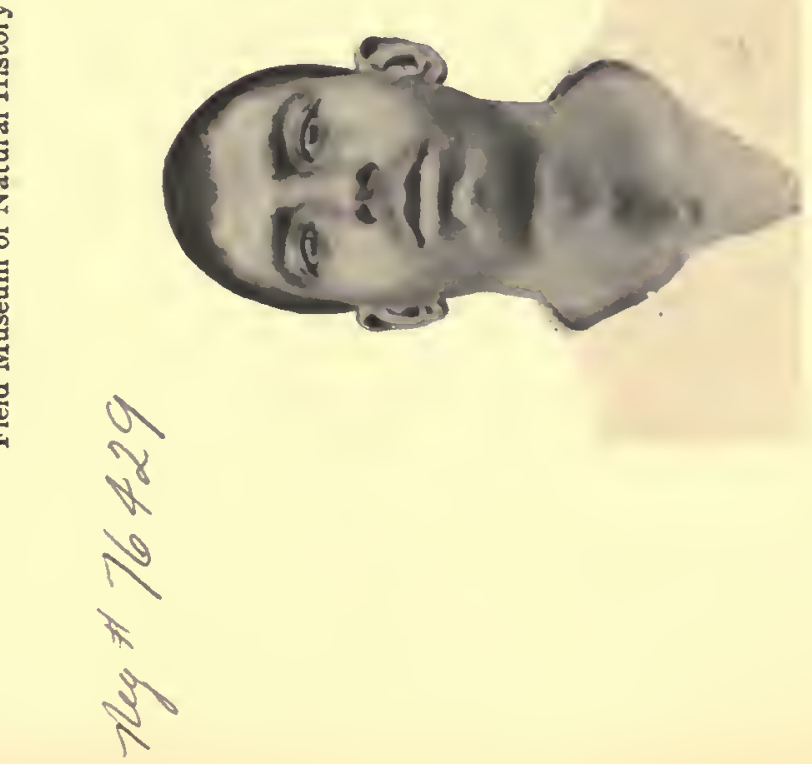

0
$y^{2}$
1

量
$\dot{0}$
$\dot{3}$
0
0
0 

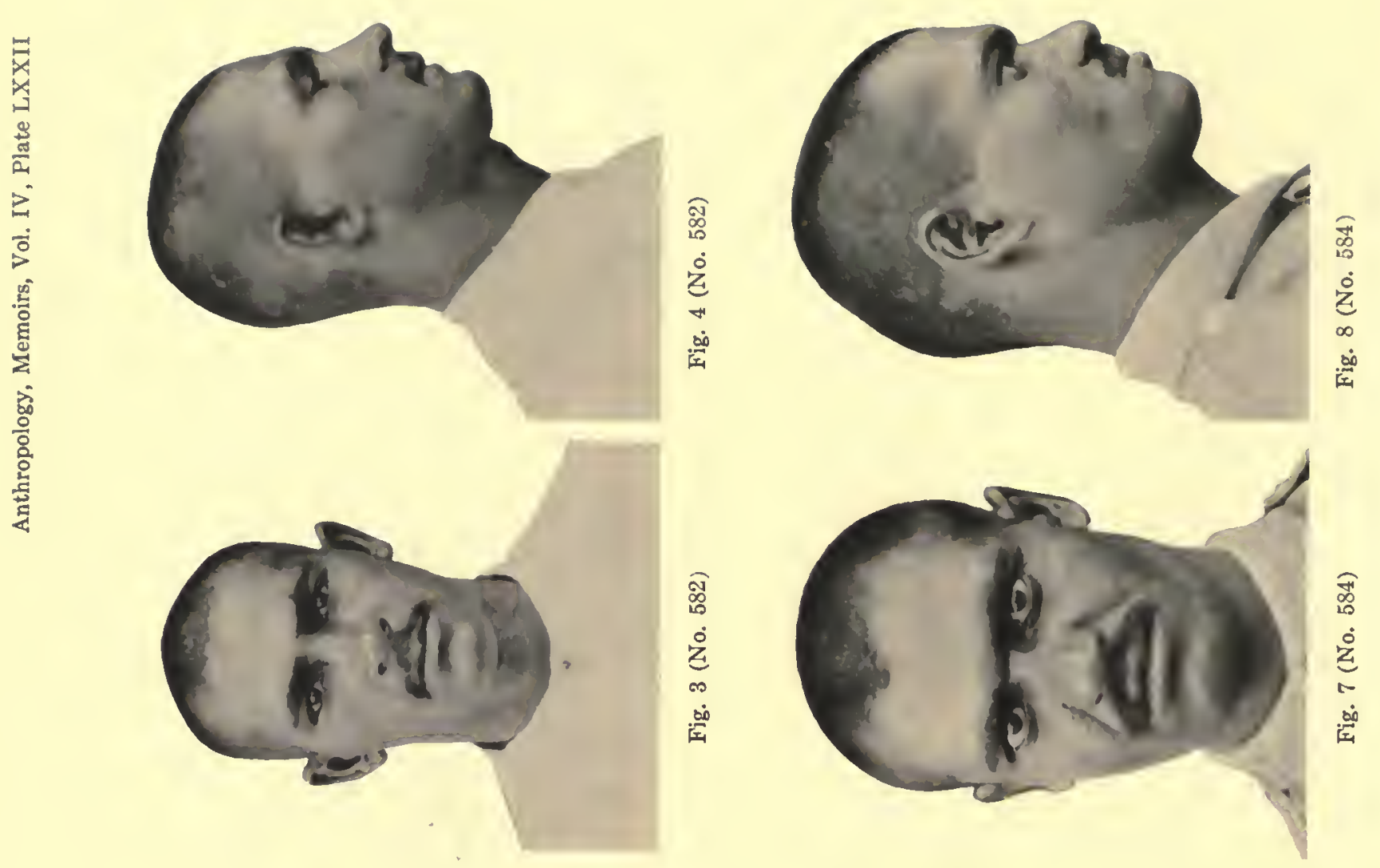

要
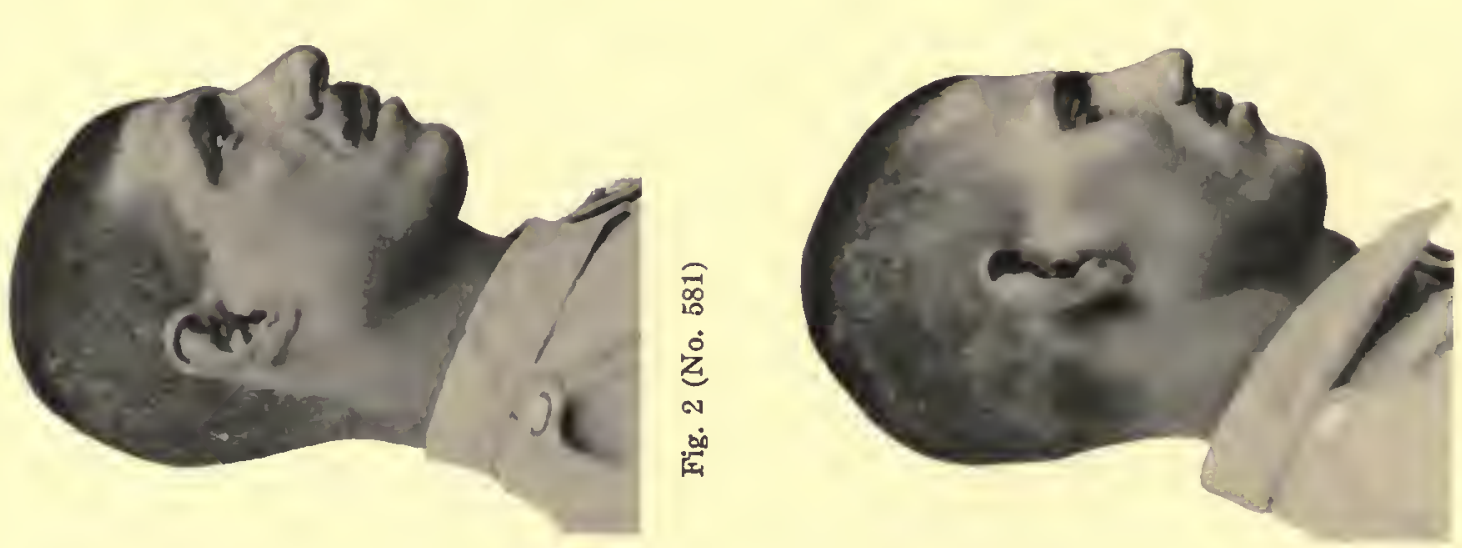

嵌

$x^{2}$
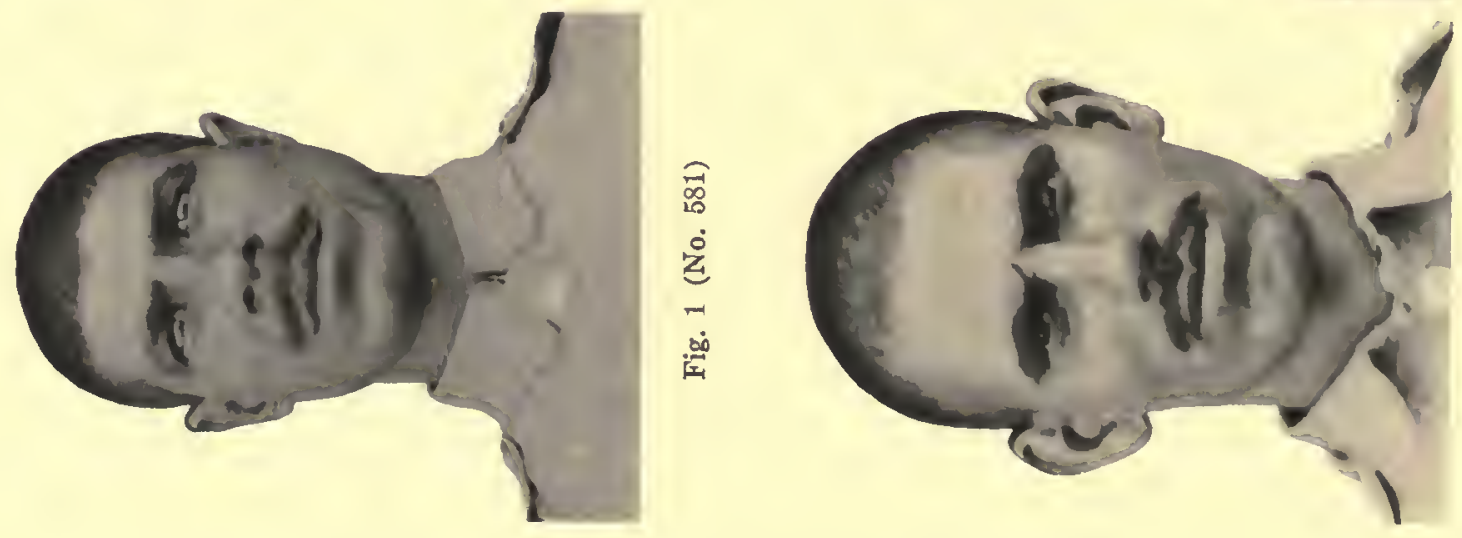

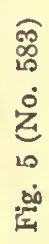

간 


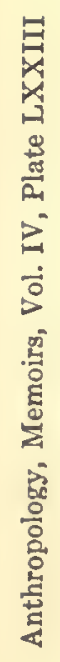
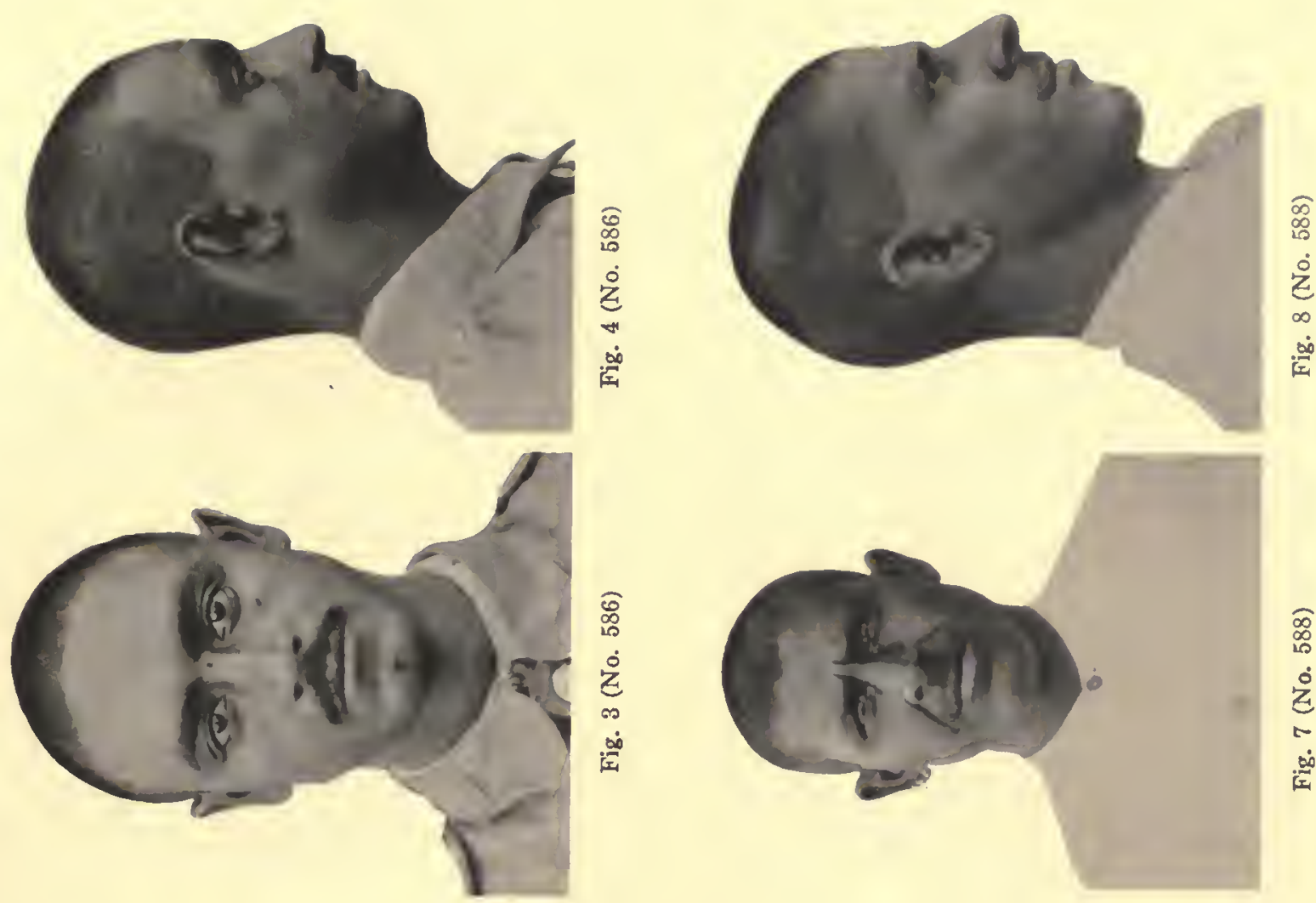

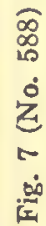

เ
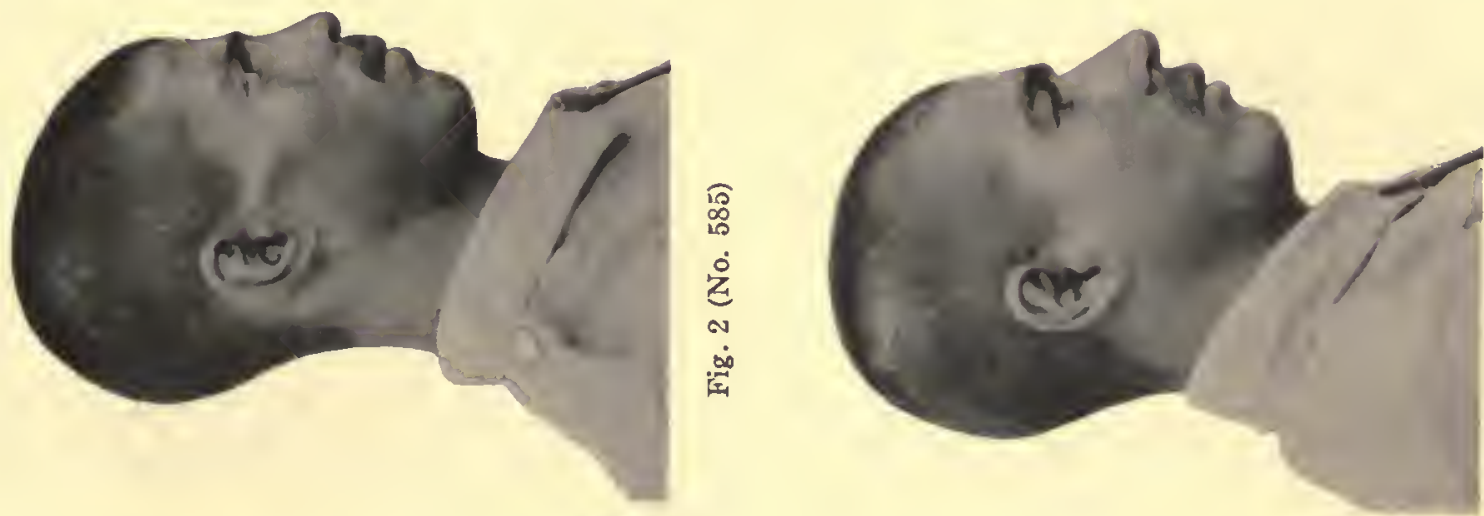

$\dot{z}$
$\infty$
$\infty$

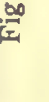

.
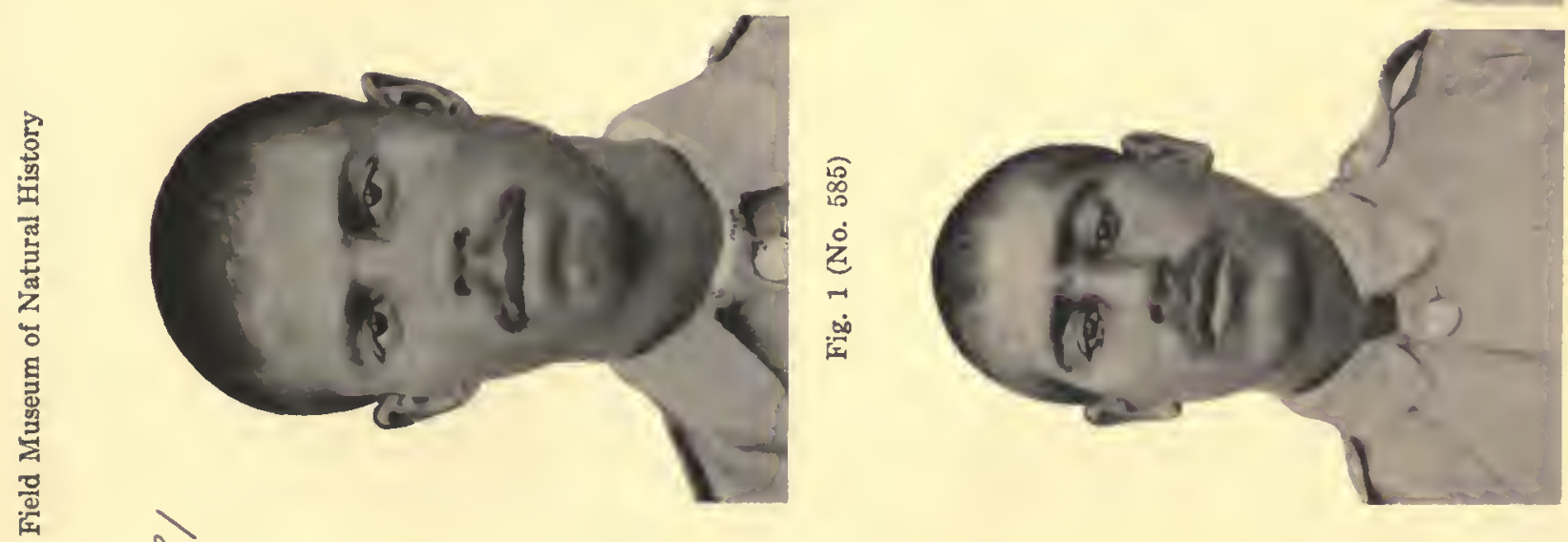

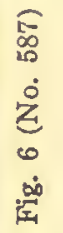

路

ż 离

$n$
$k$ 

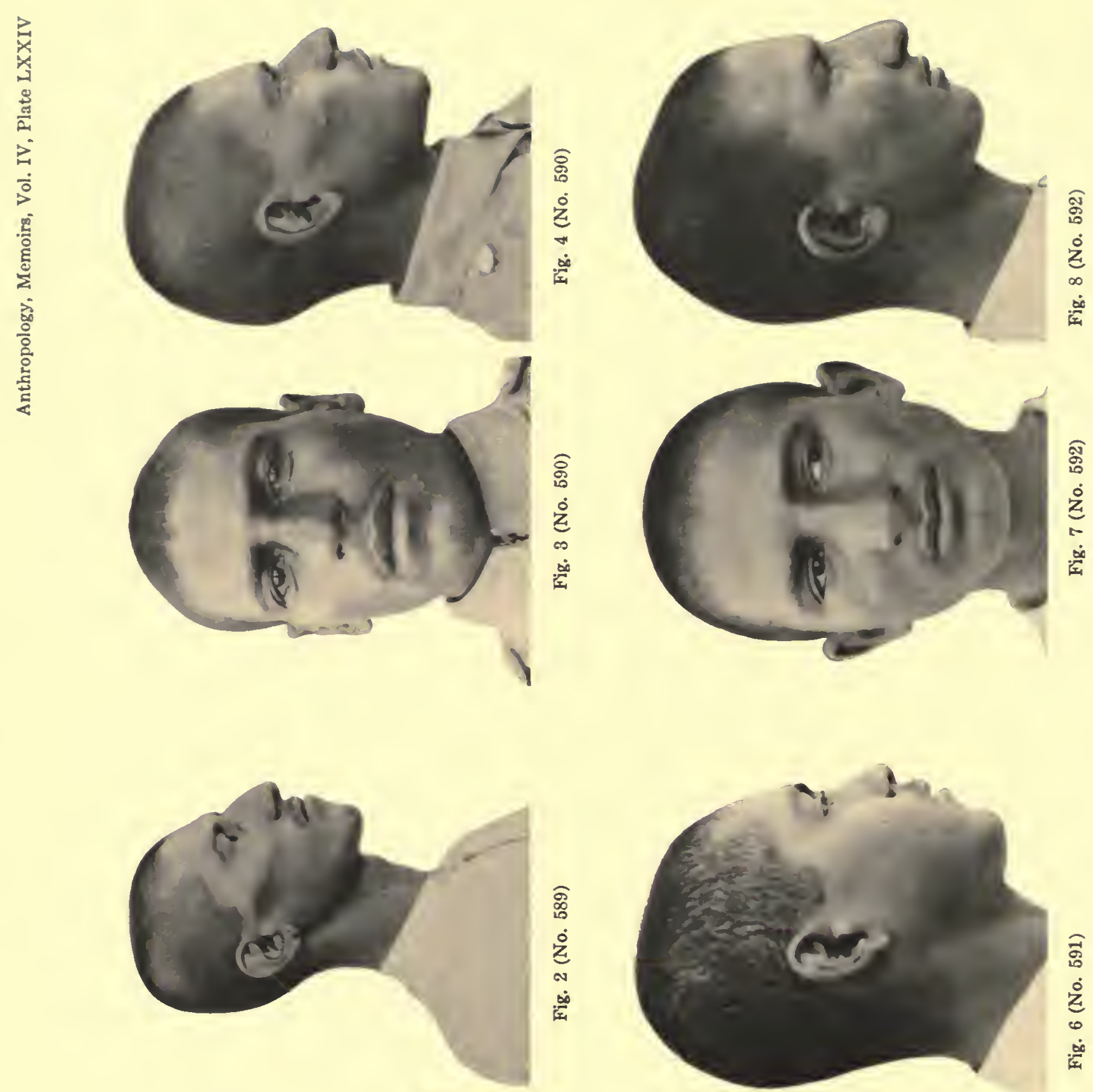

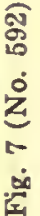

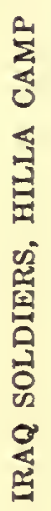

2
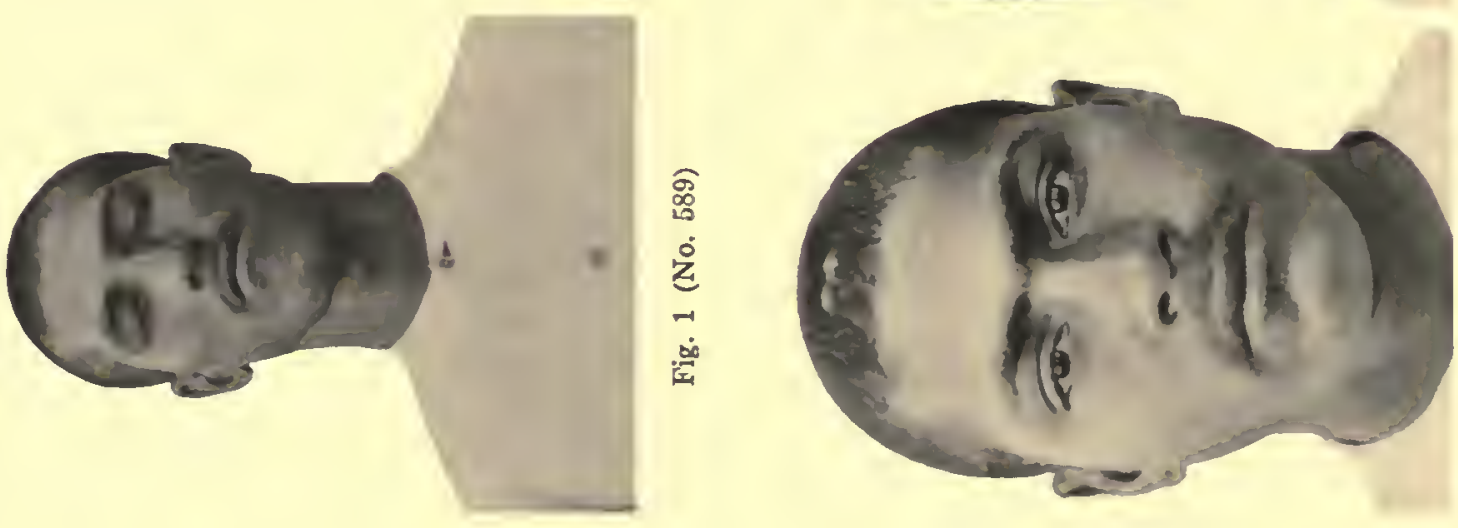

$\widehat{5}$
$\dot{5}$
$\dot{0}$
0
$\dot{0}$
$\dot{0}$

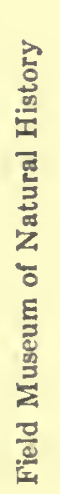

है 

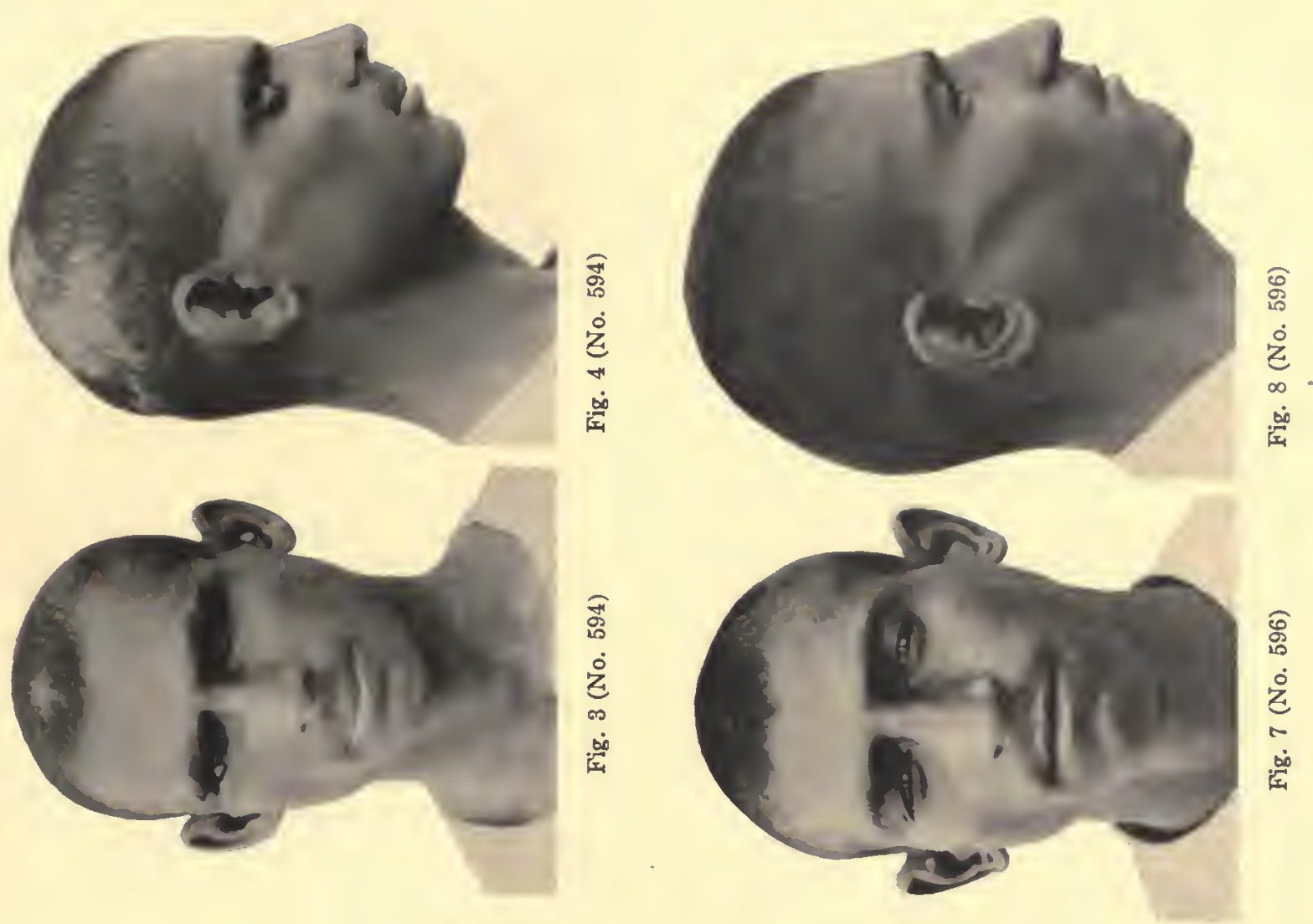

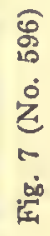

葛
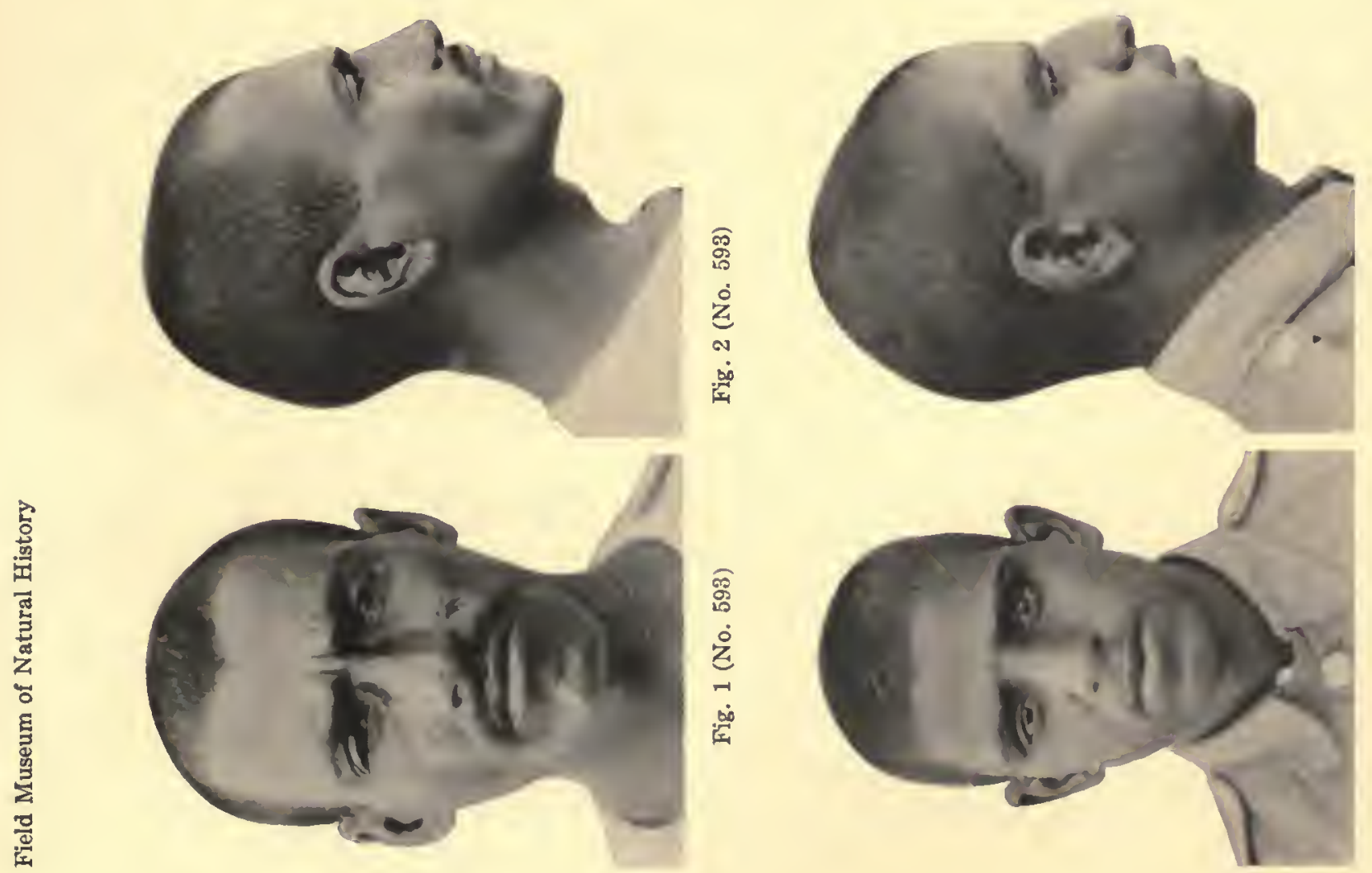

10
i⿱口口
$\dot{0}$
2
0
0
0

$n$
$x$

$k$

닌 

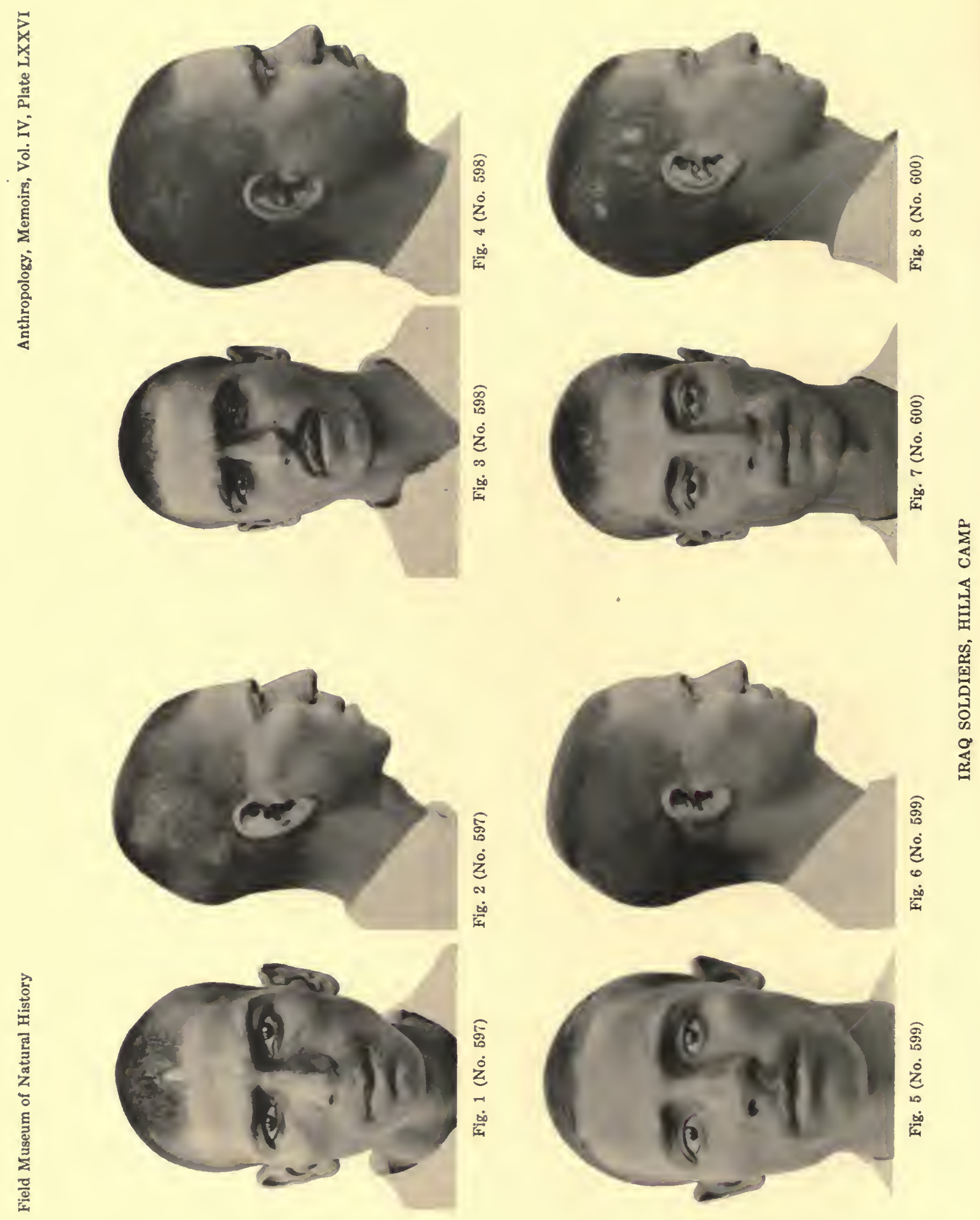
焉
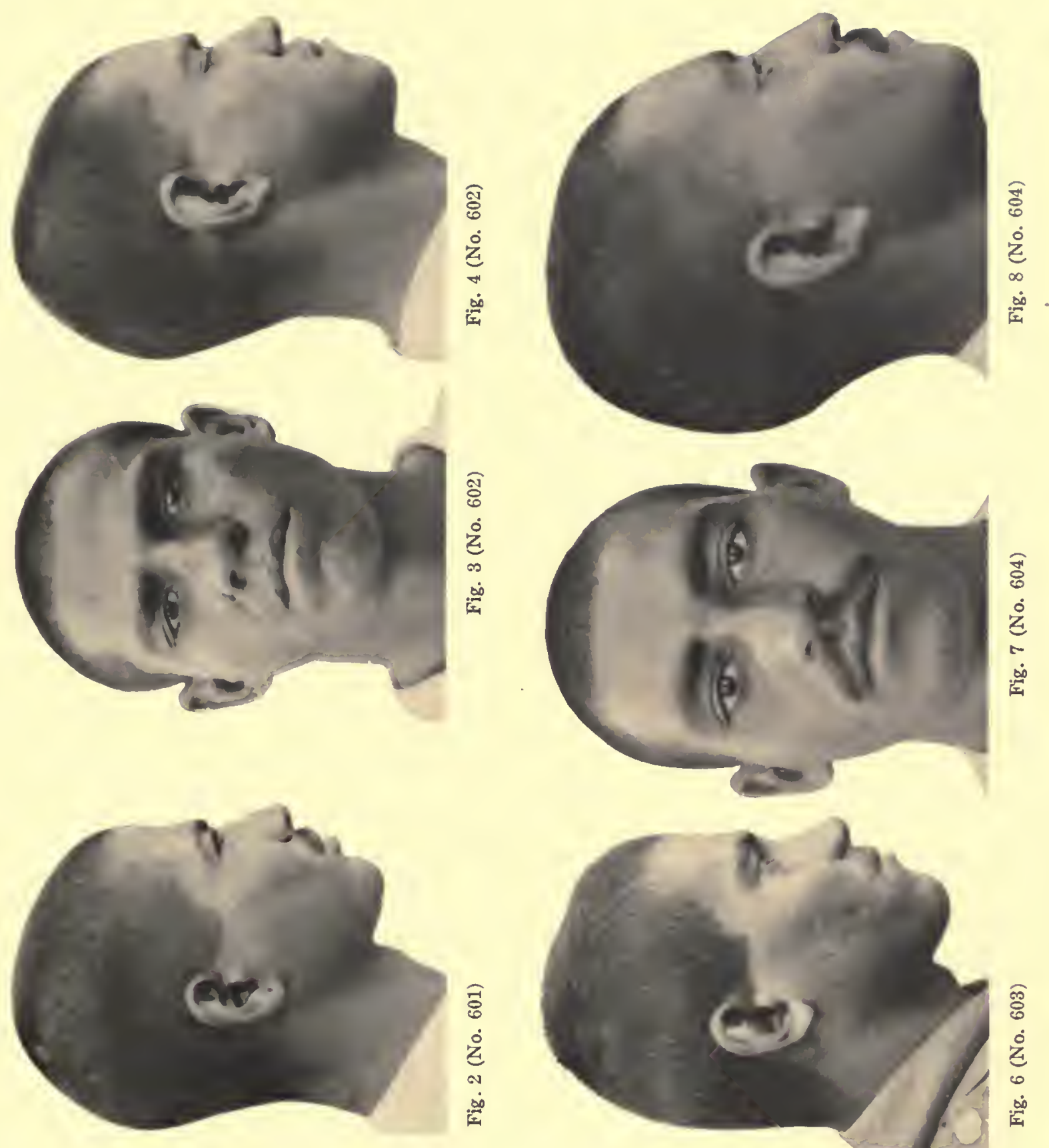

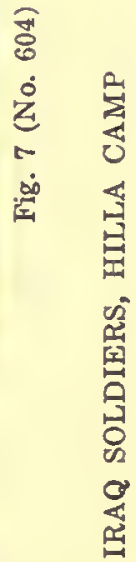
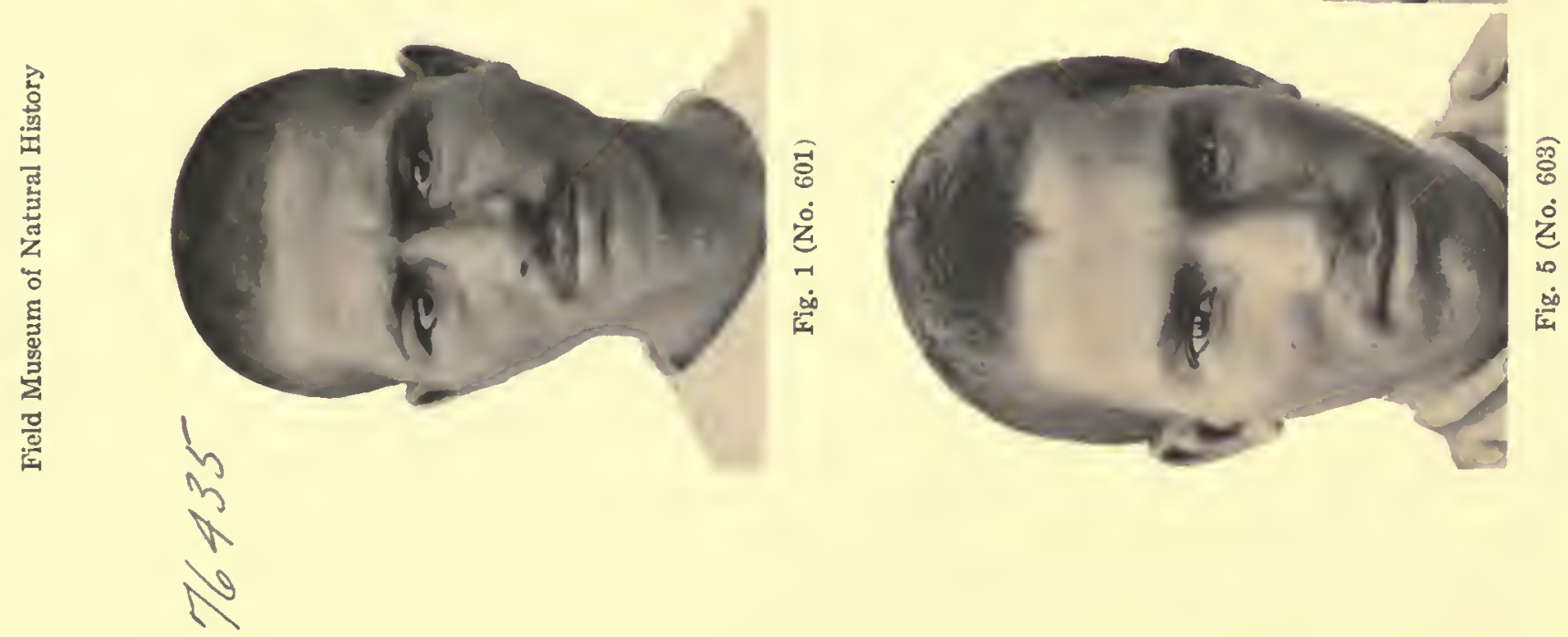

\

N 

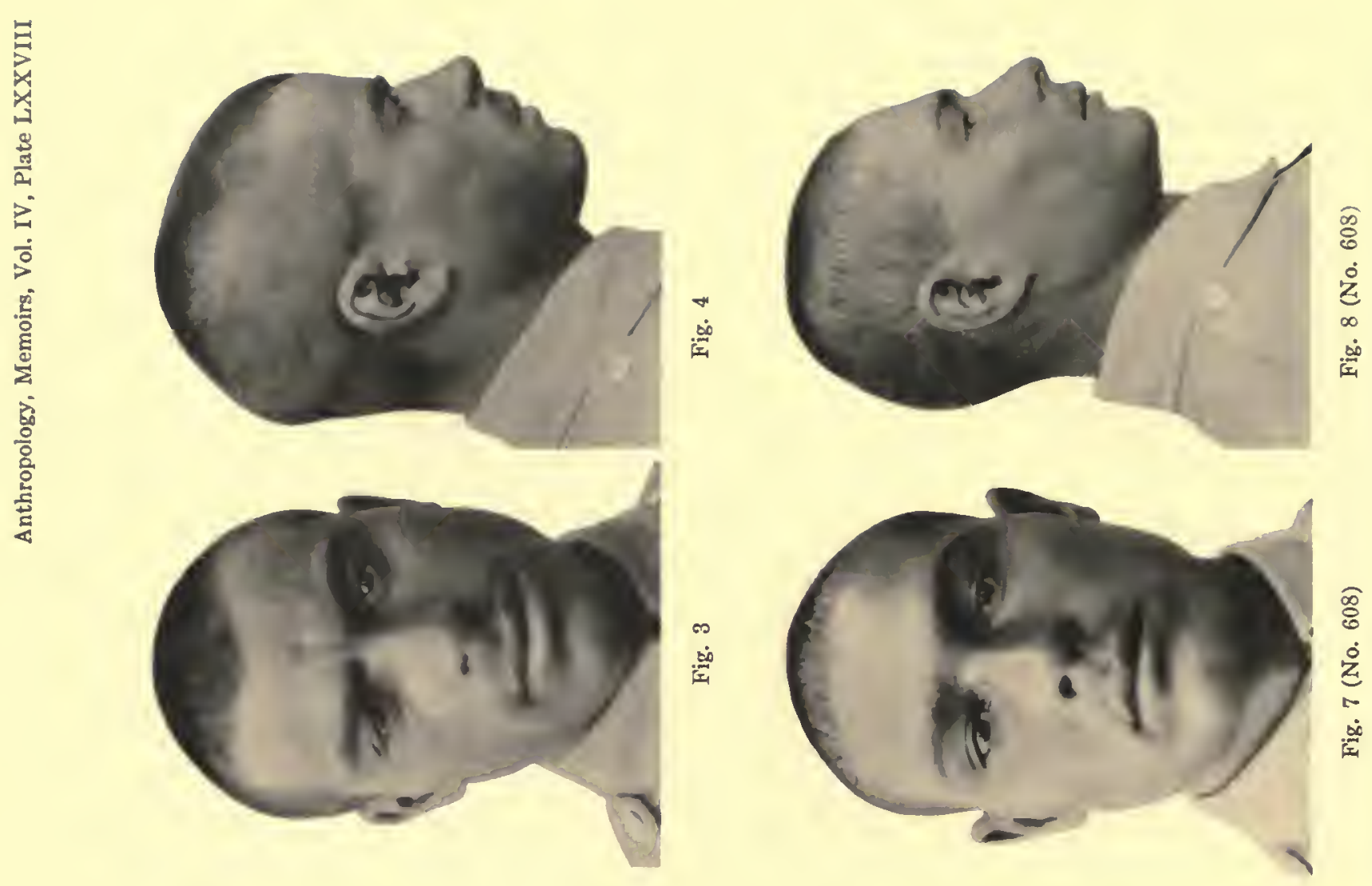

$b$
2
$x$
$n$
$x$
$n$
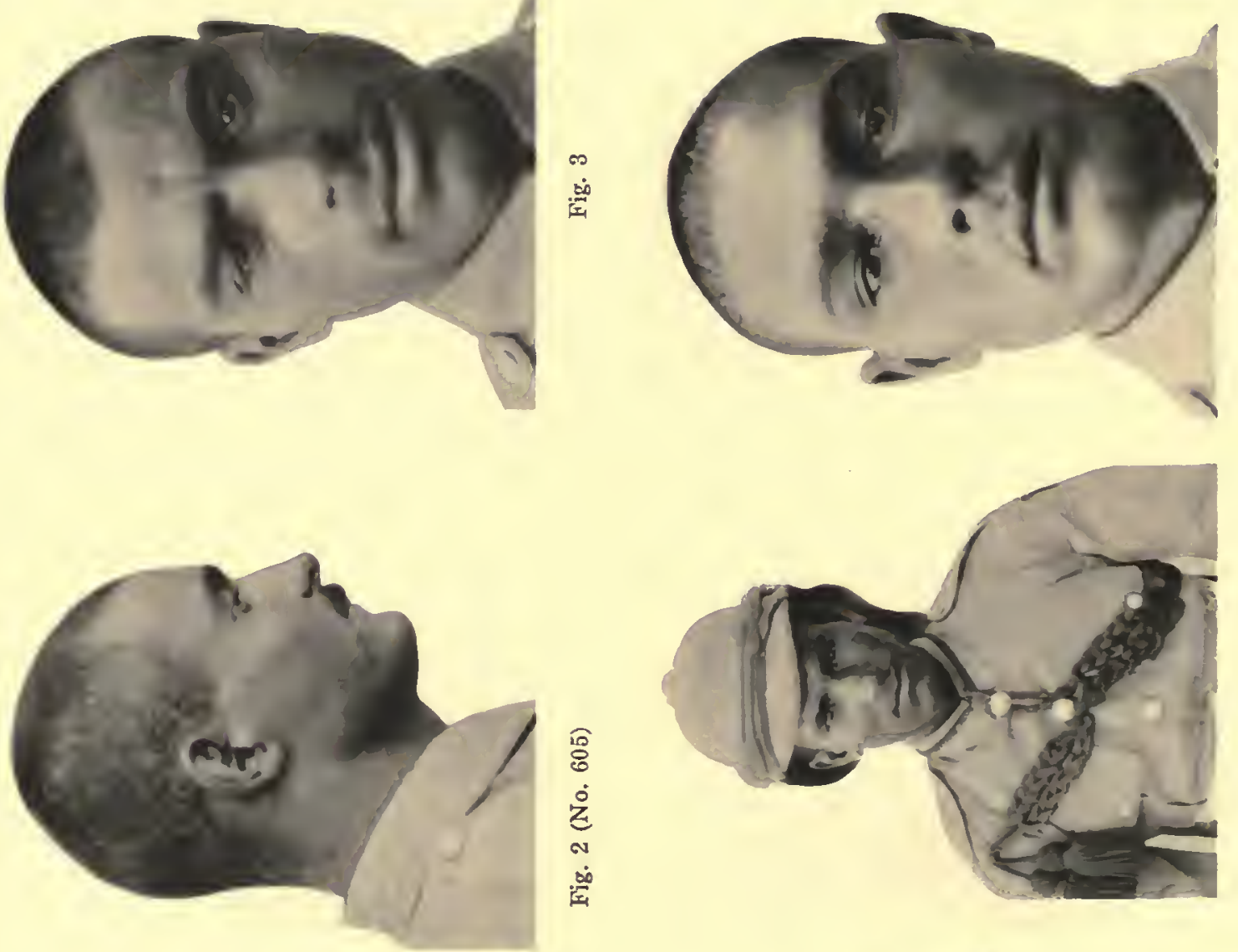

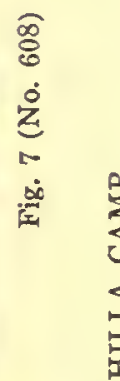

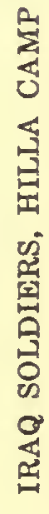

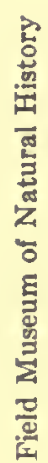
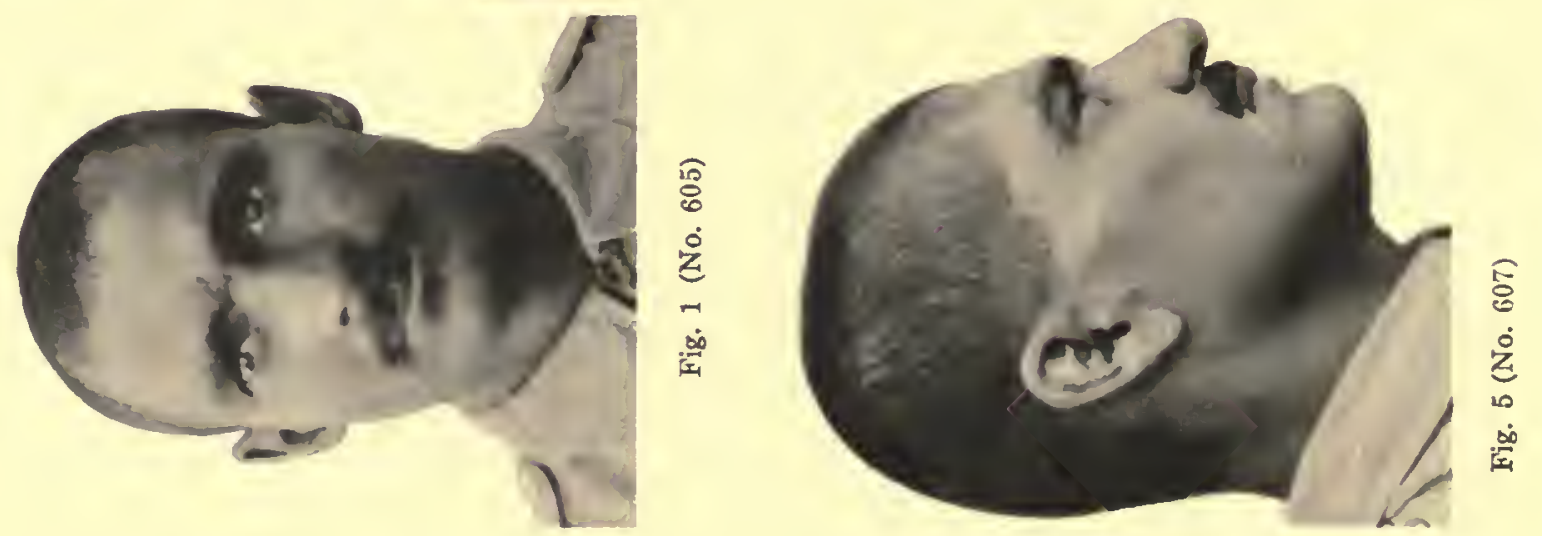

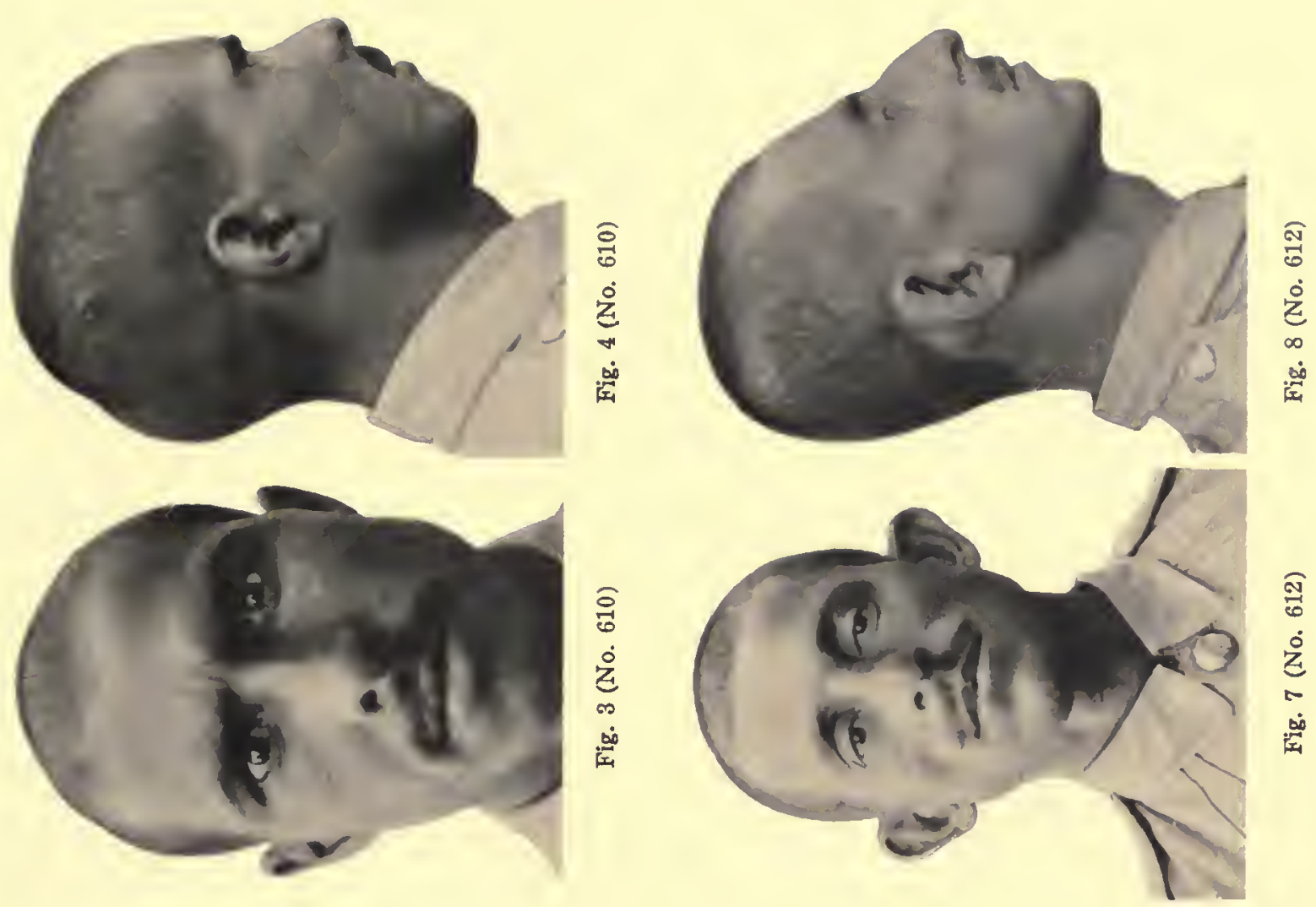

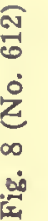
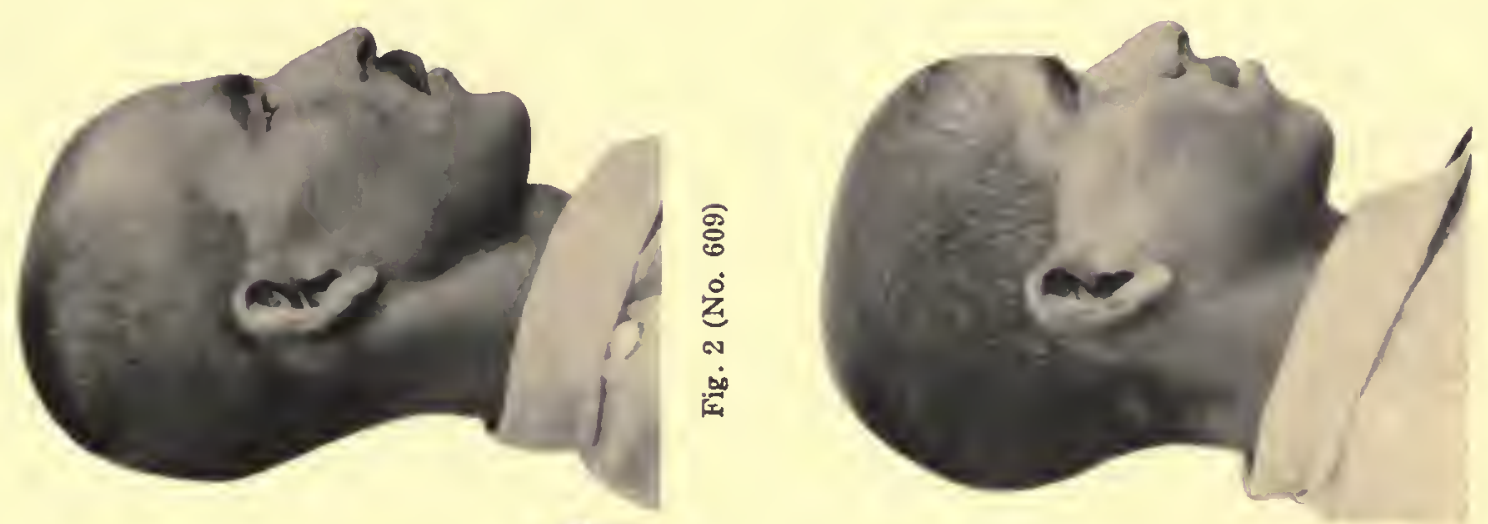

ลิ

\&

5

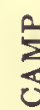

岁
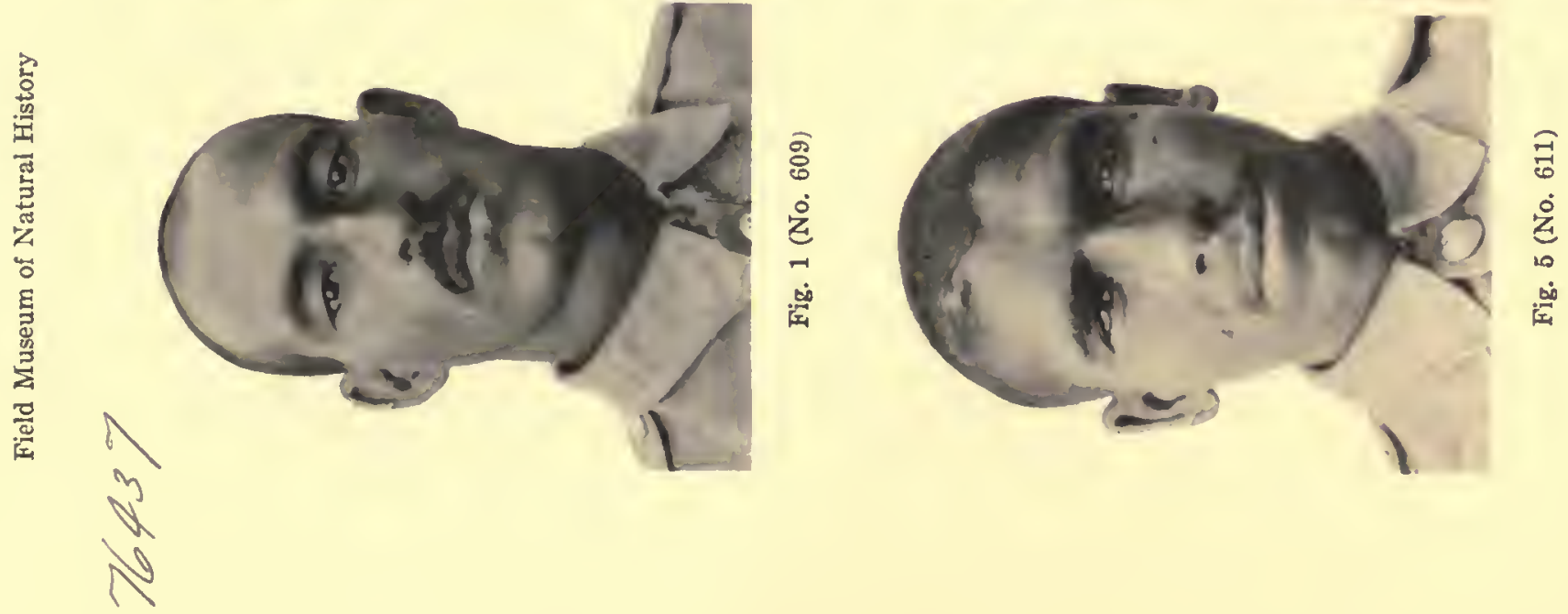

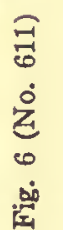

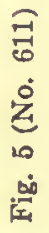




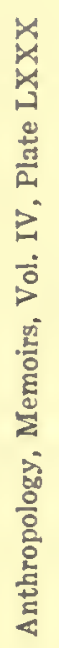
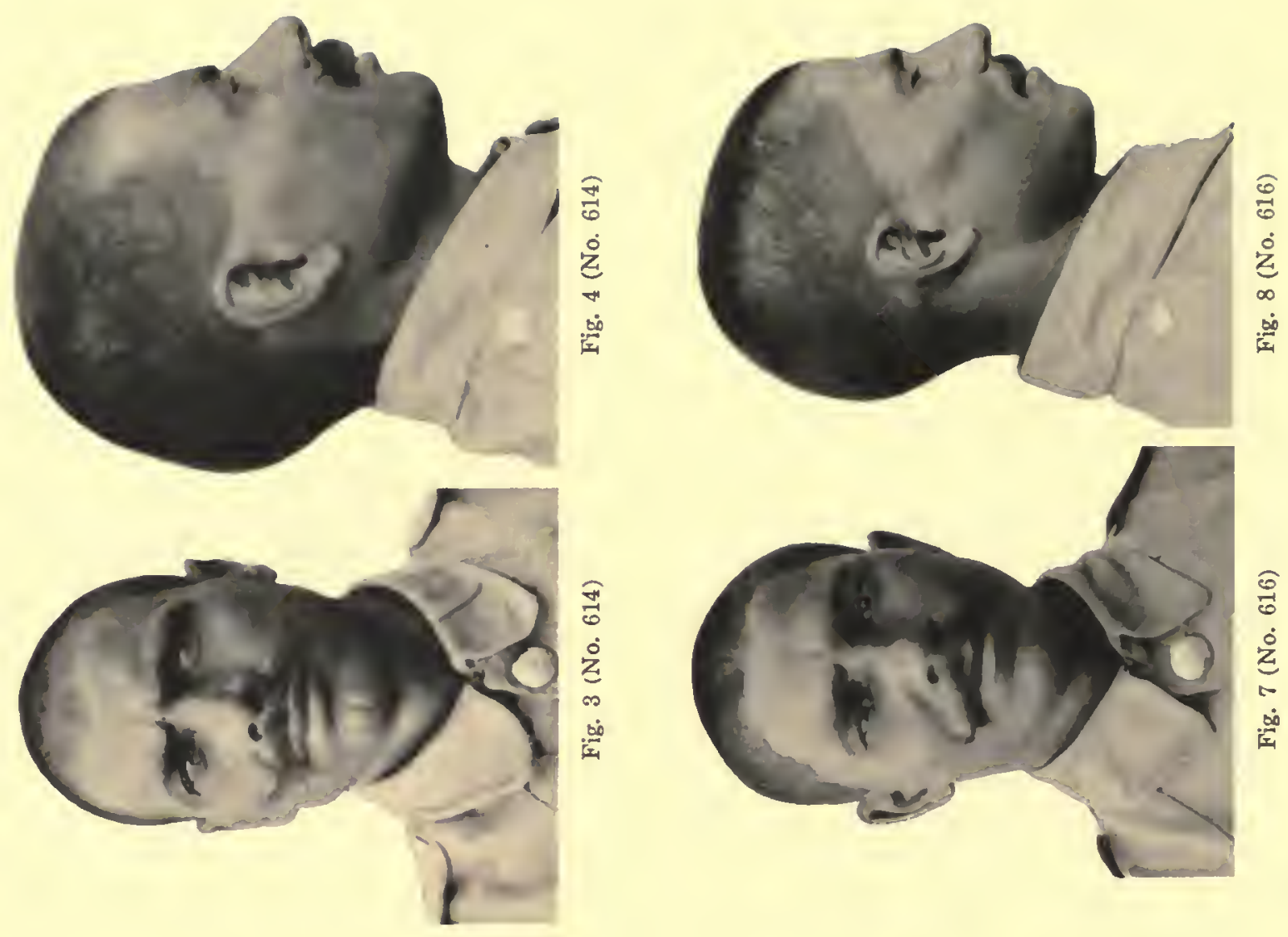

0
0
0
0
$\infty$
$\infty$
0
0
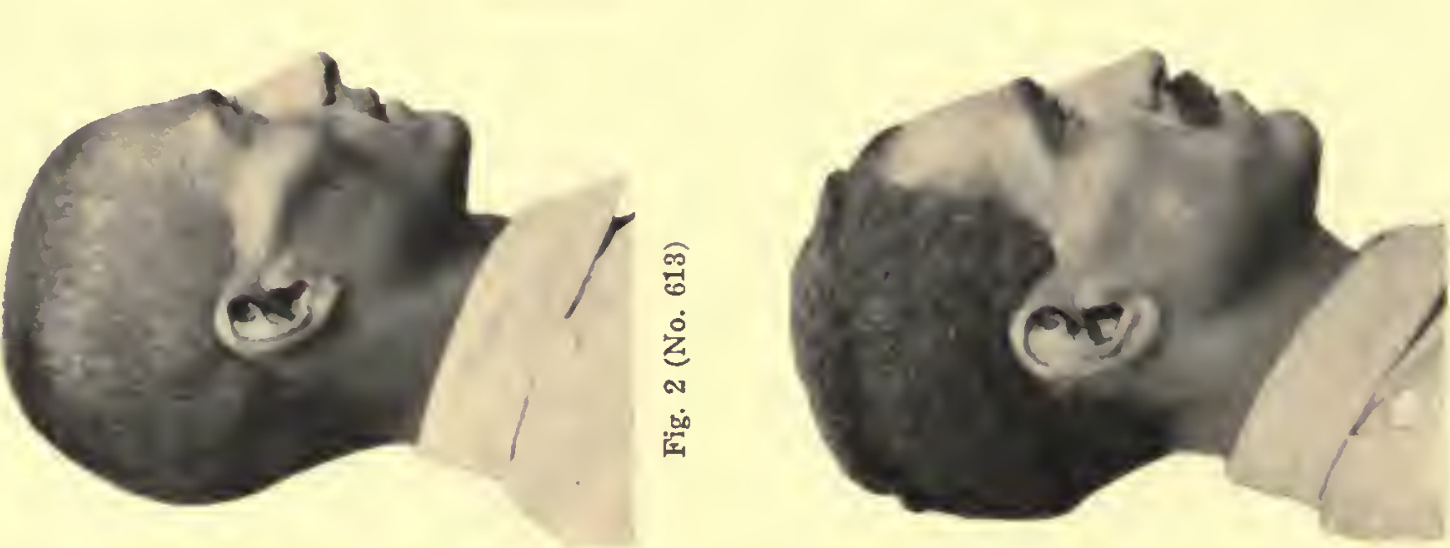

0
0
0
0
2
0
0
0
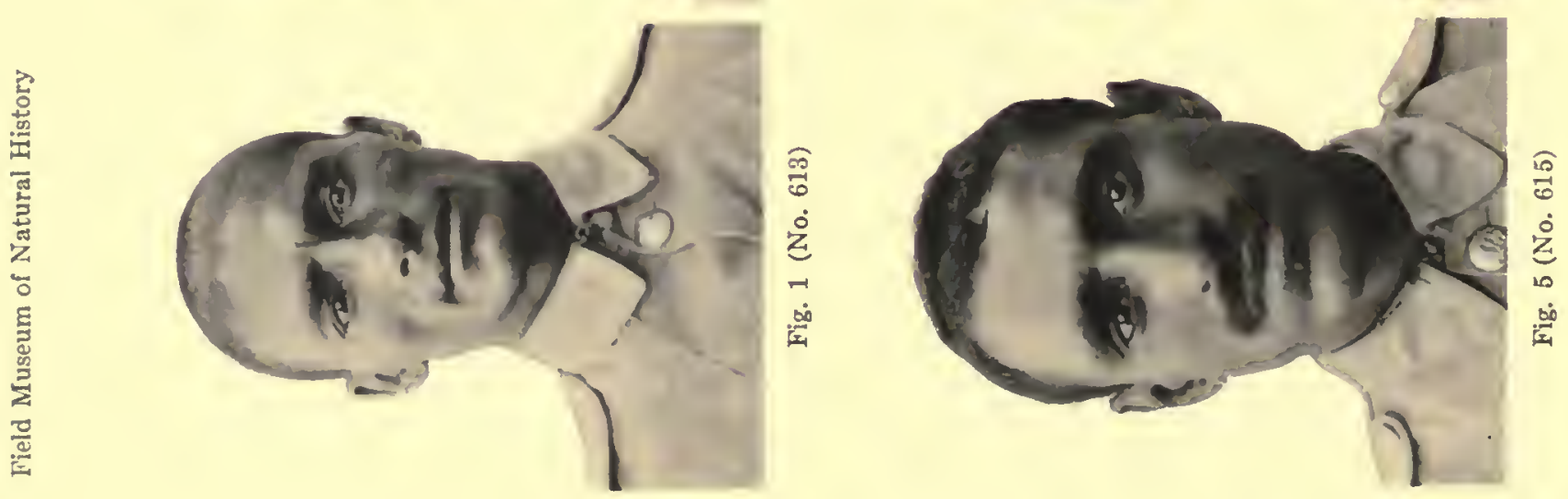

渵 s 

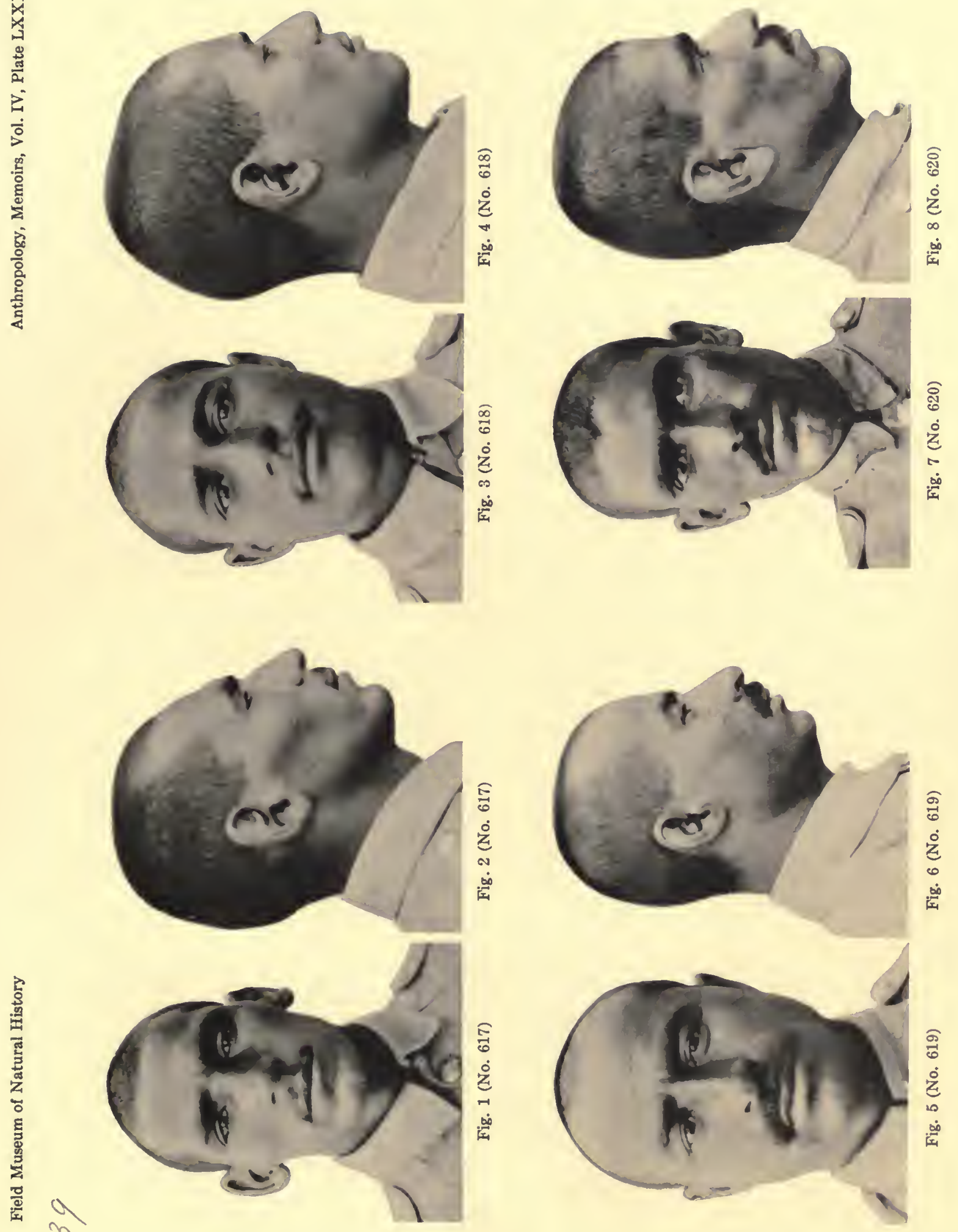

$n$

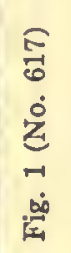

10 


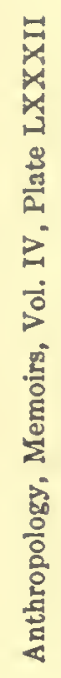
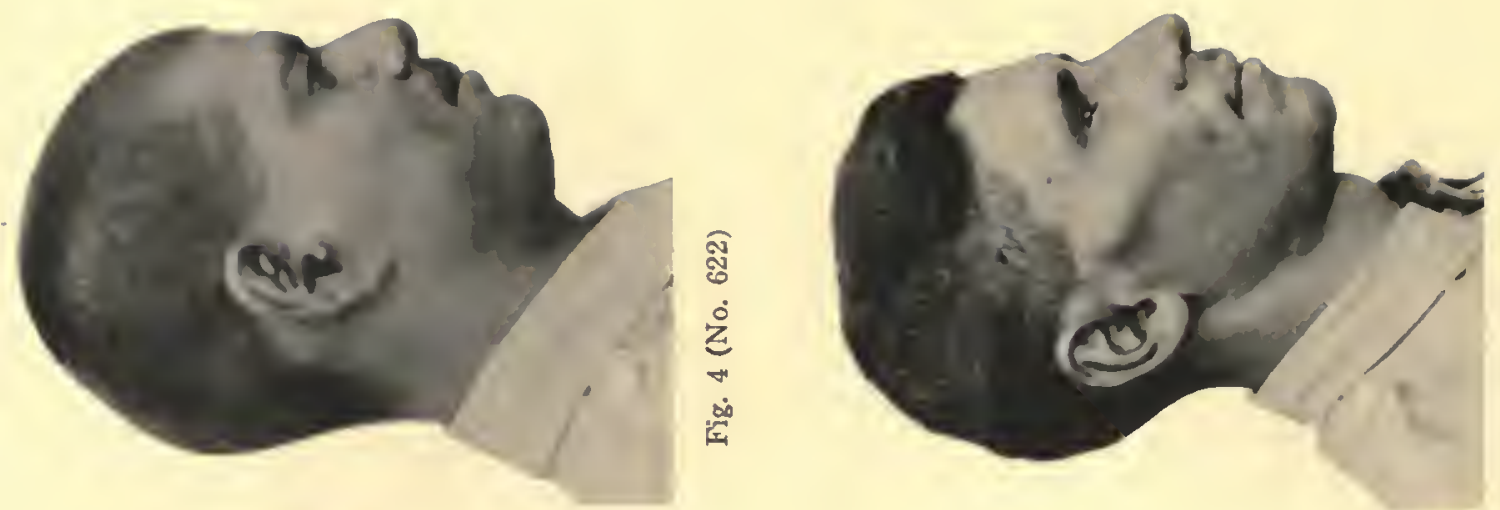

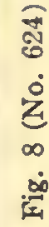
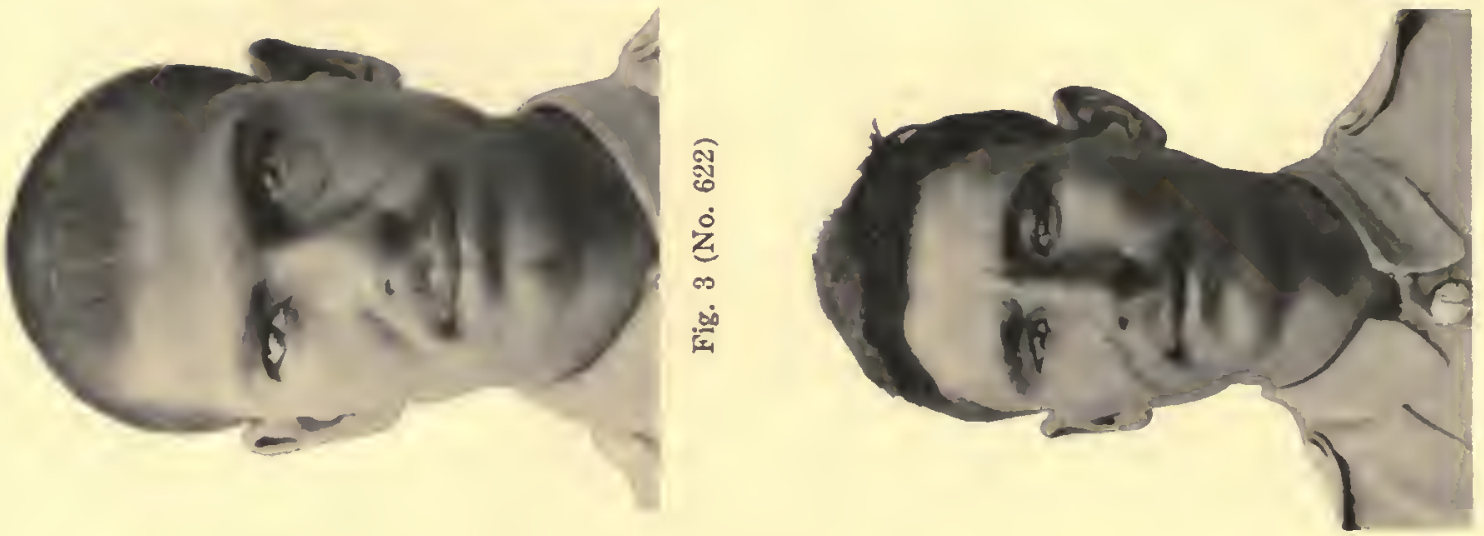

芯
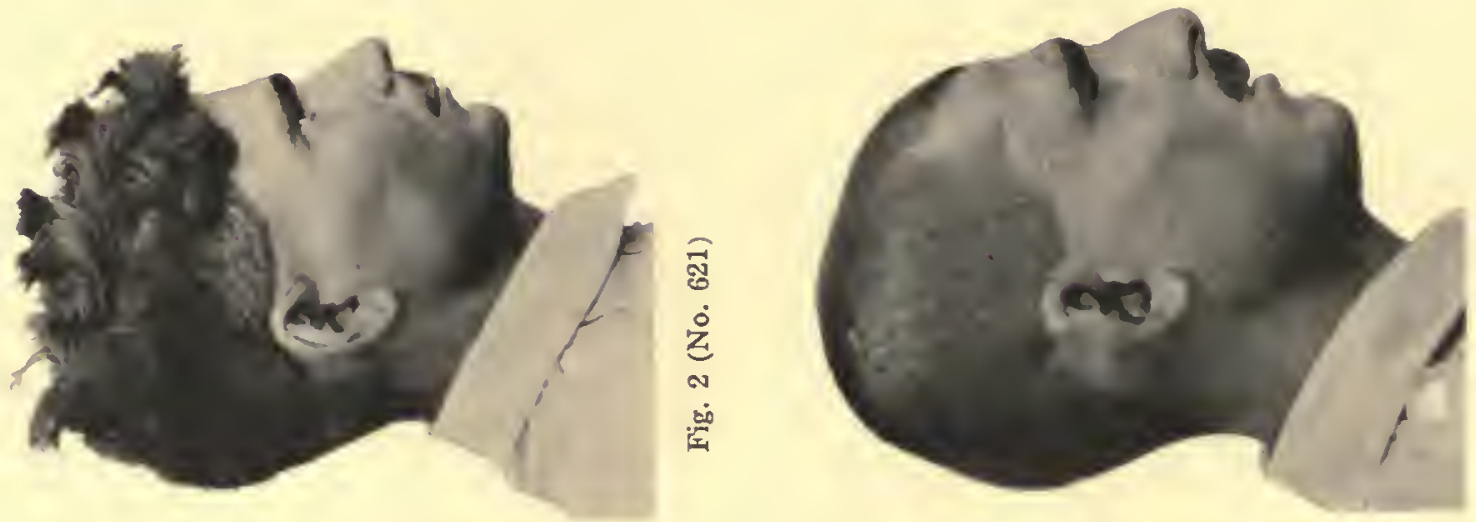

范

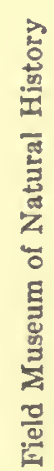
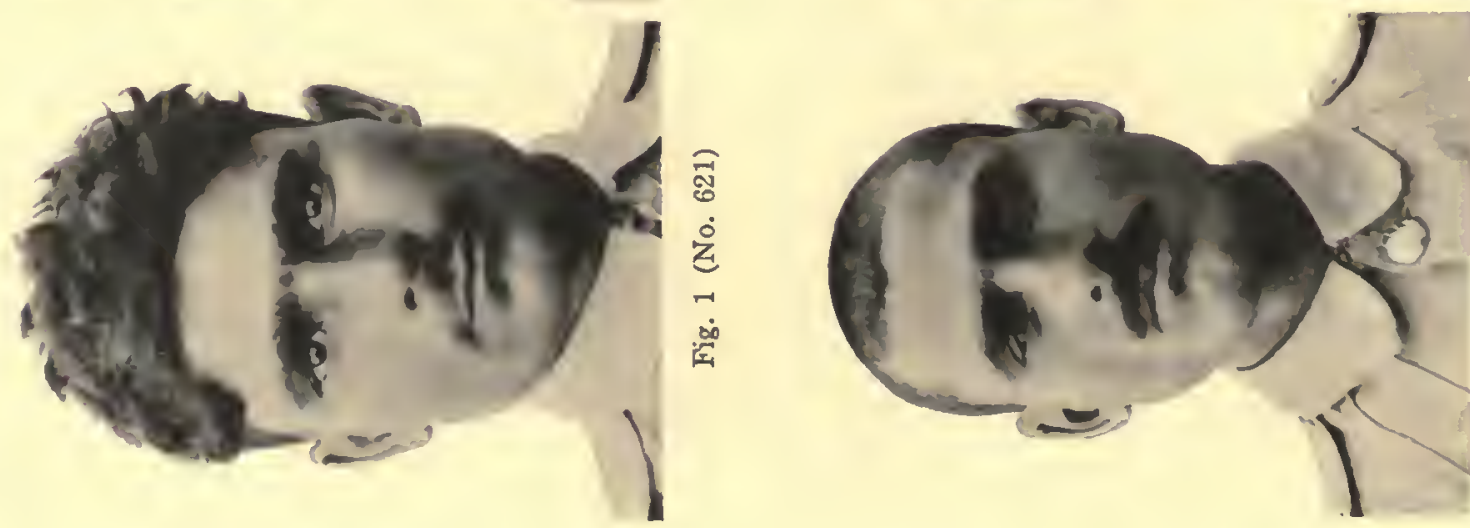

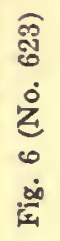

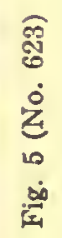




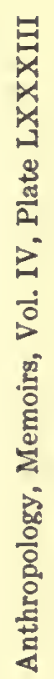
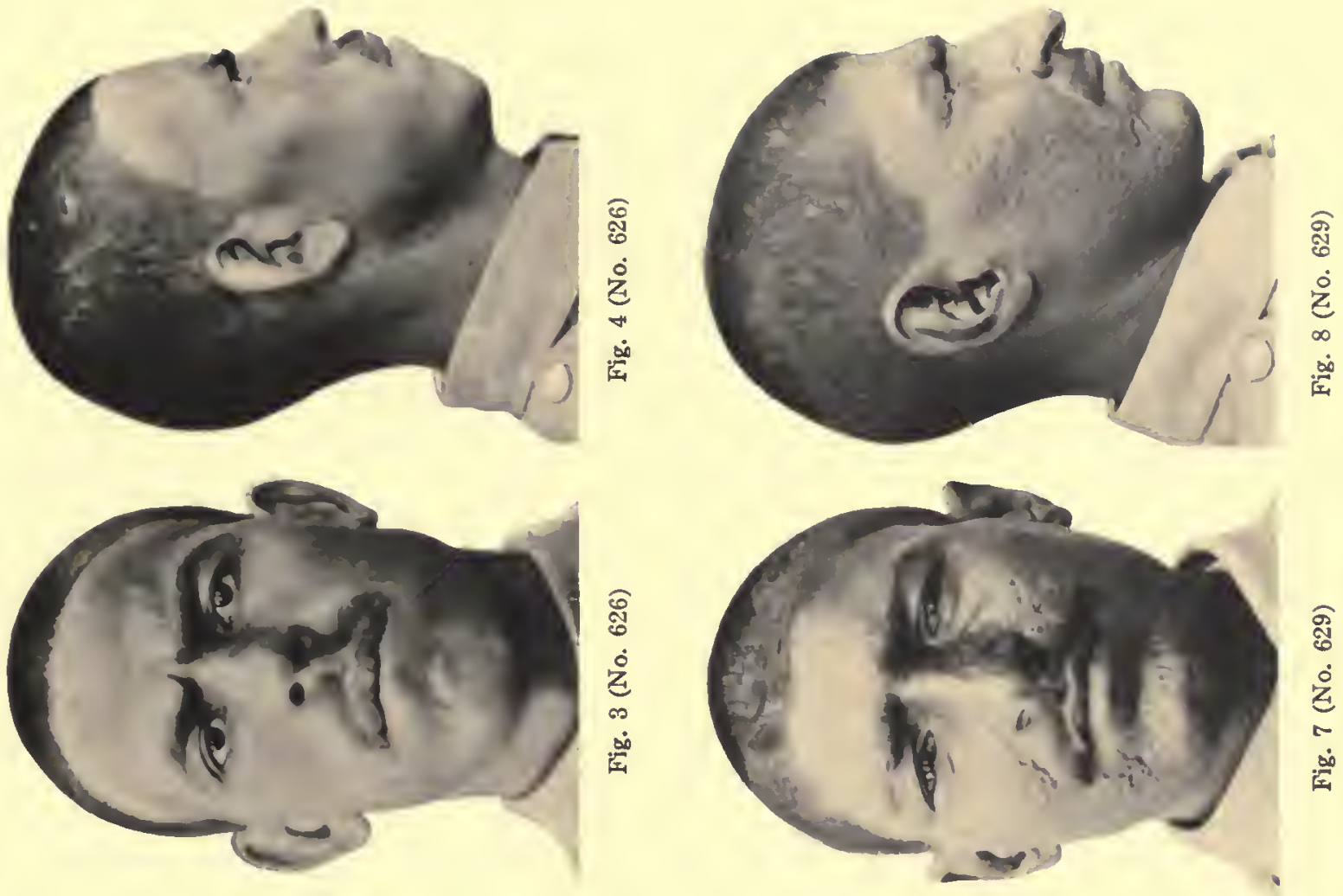

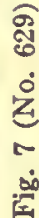

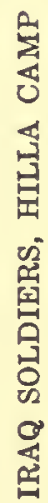
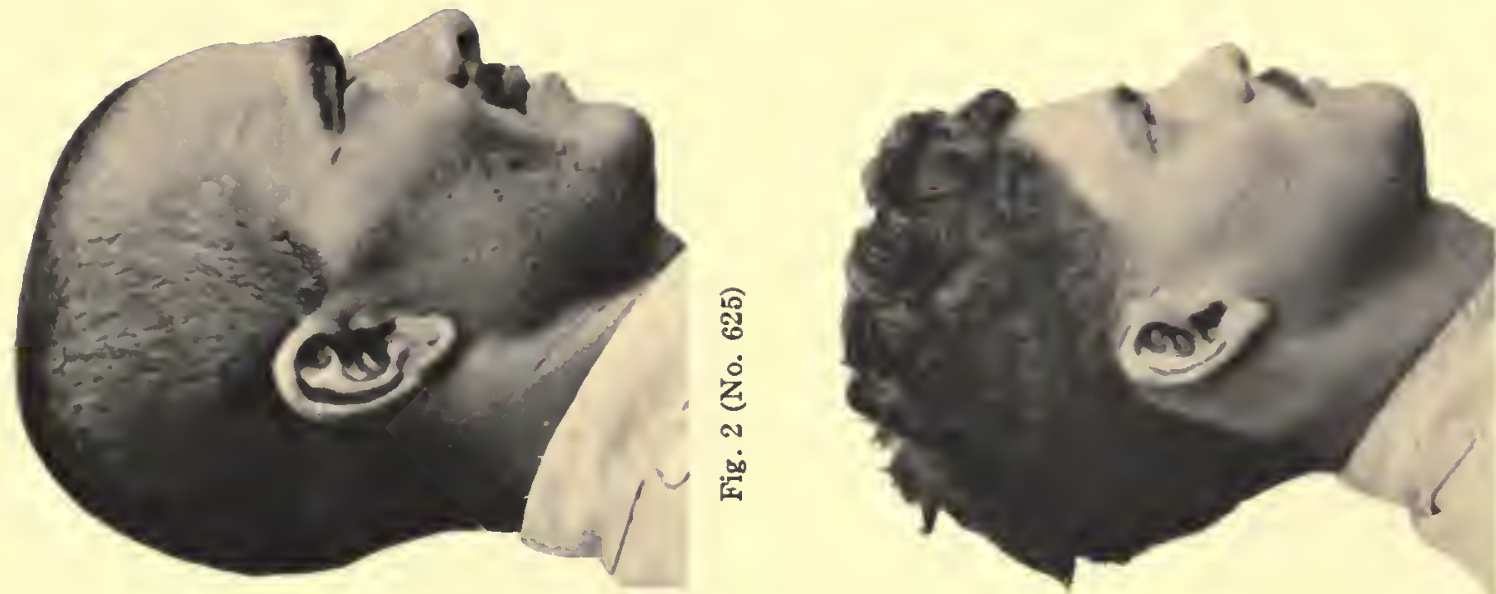

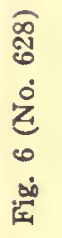
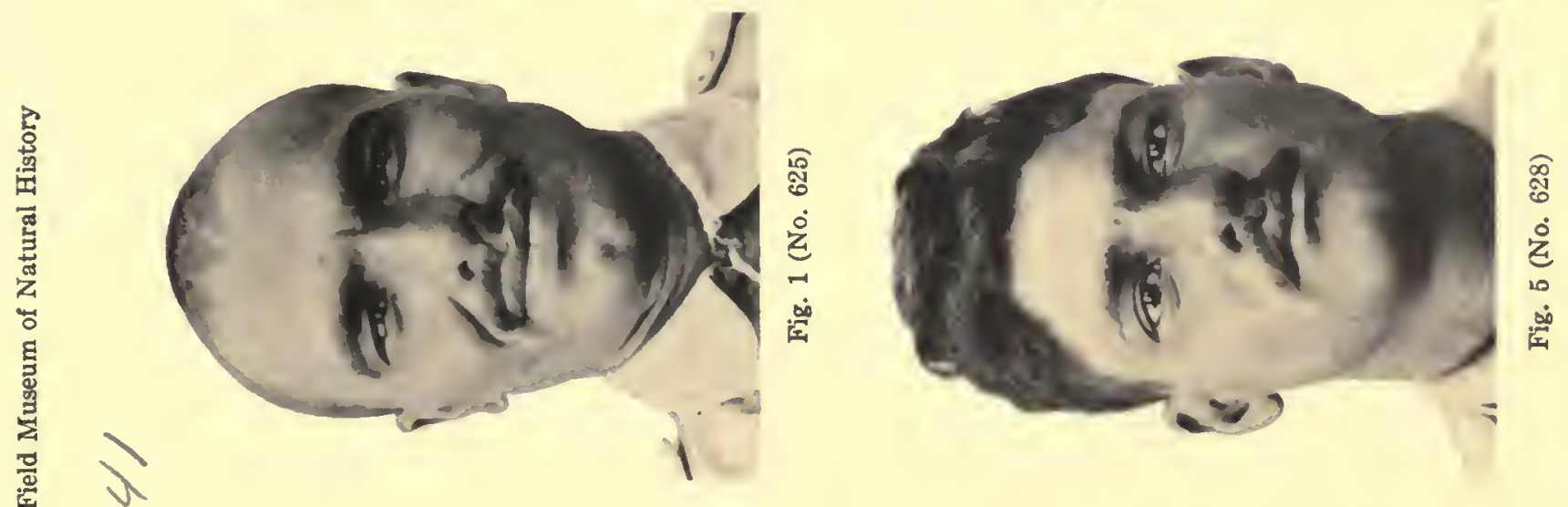
$\lambda$

1

$x$

s 

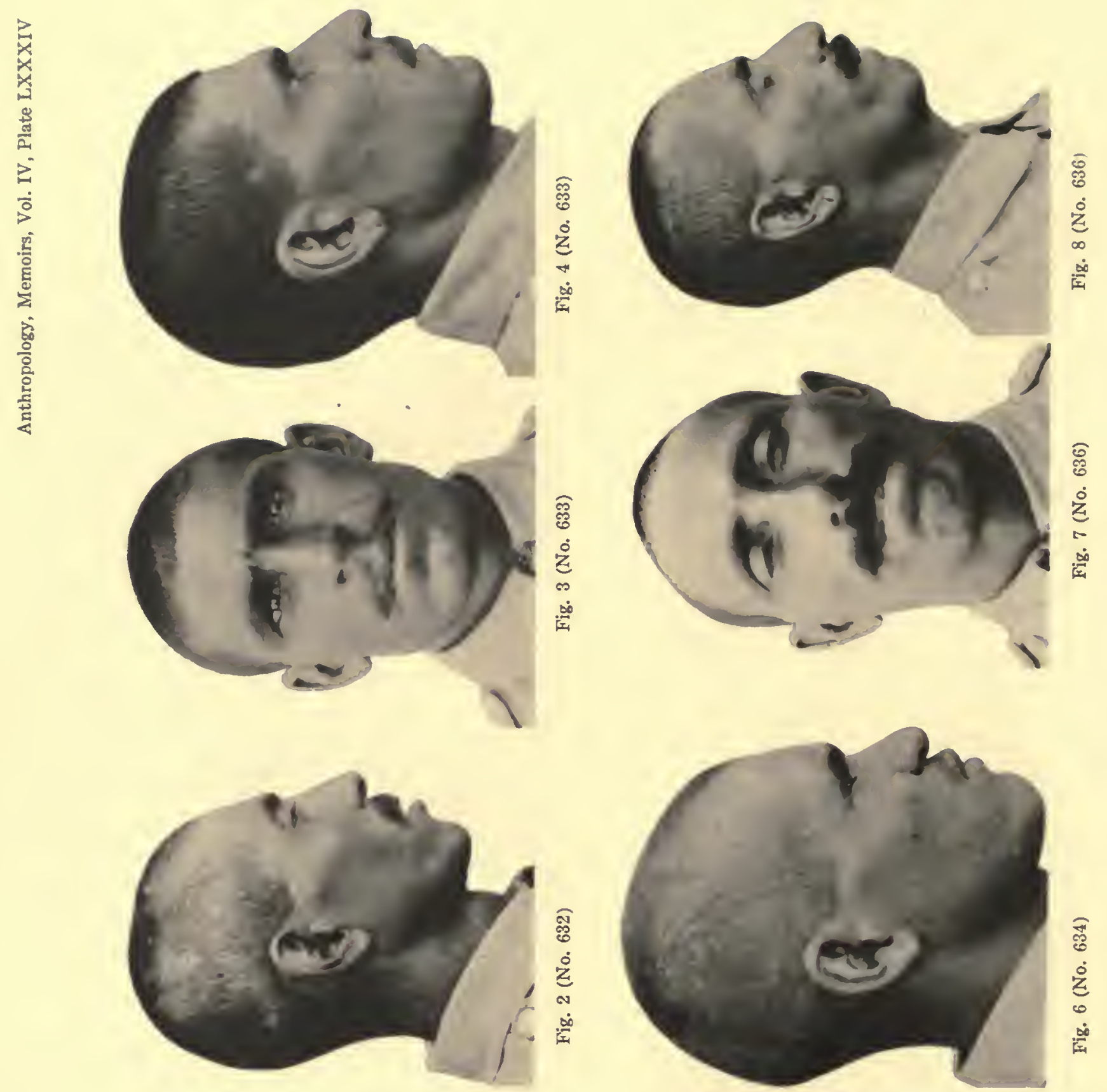

景

i
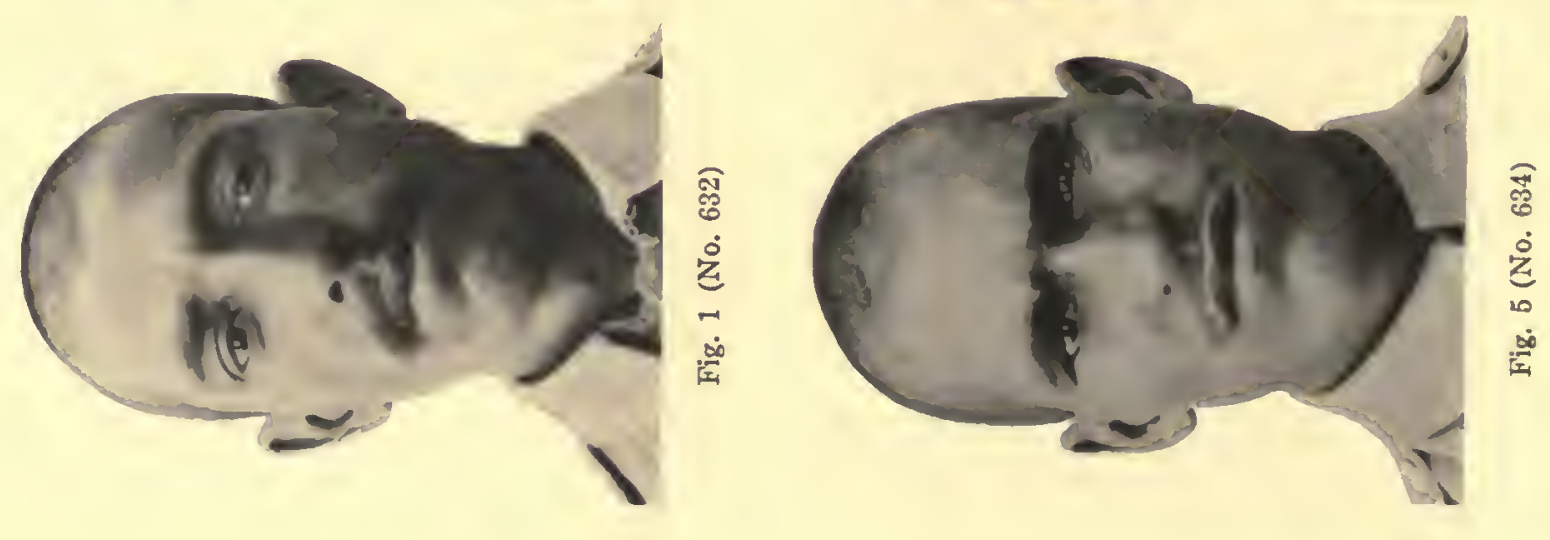


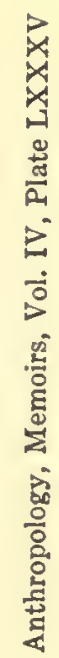
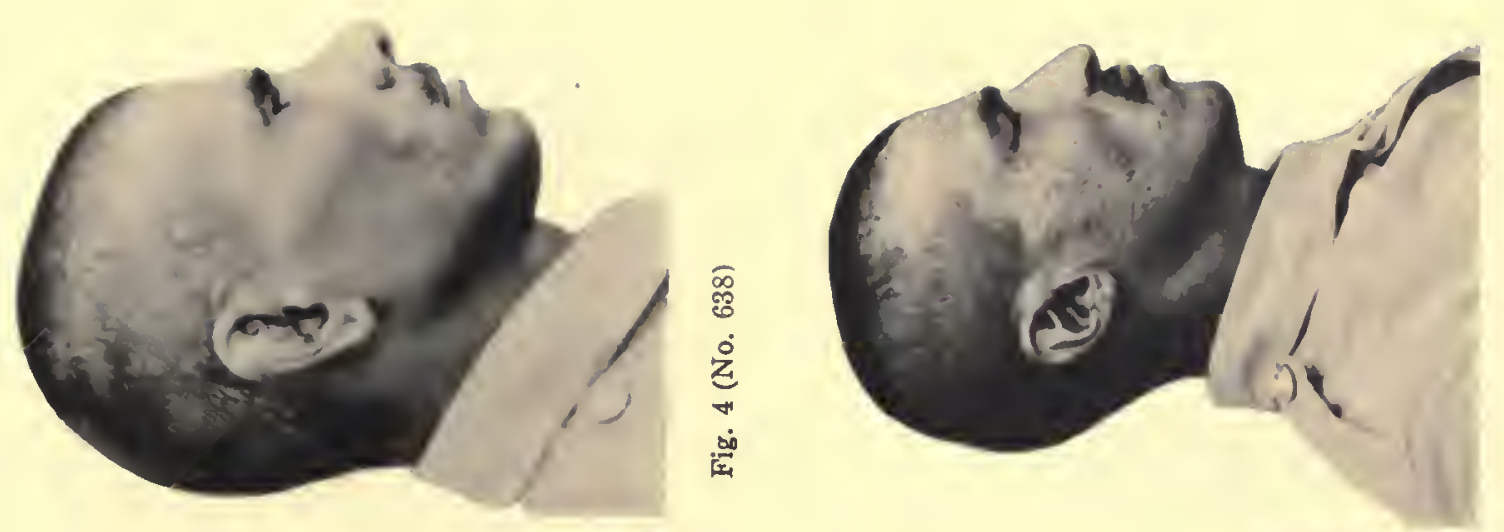

ज্
$\dot{0}$
$\dot{0}$
$\infty$
$\dot{0}$
$\dot{0}$
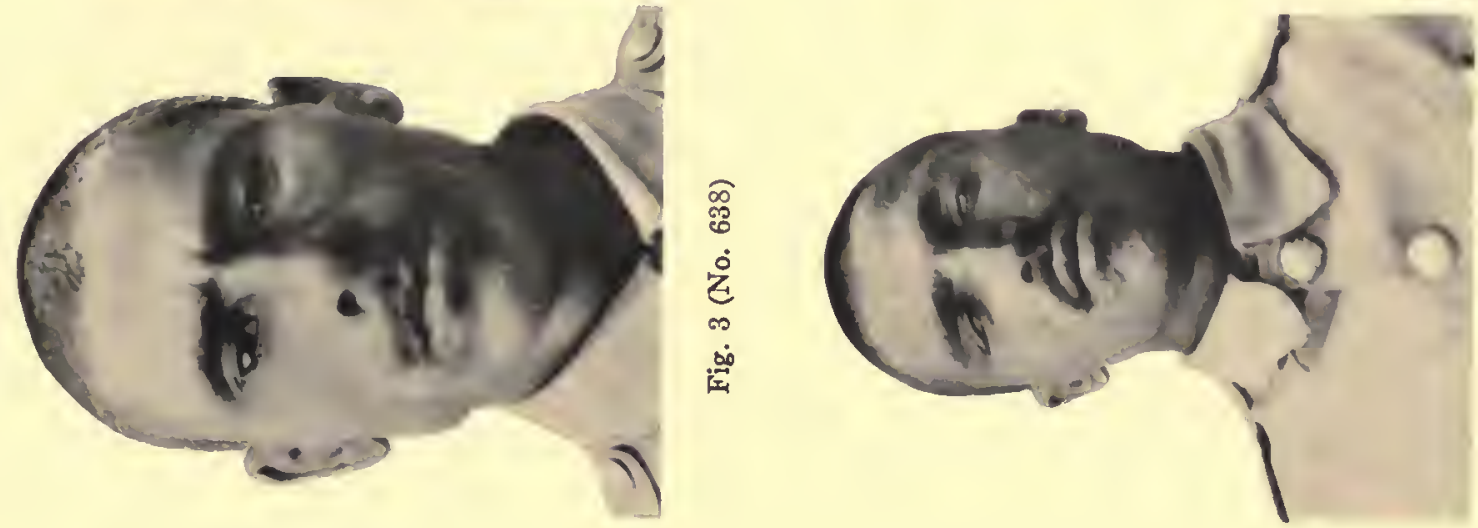

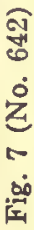

唯

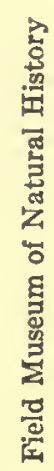
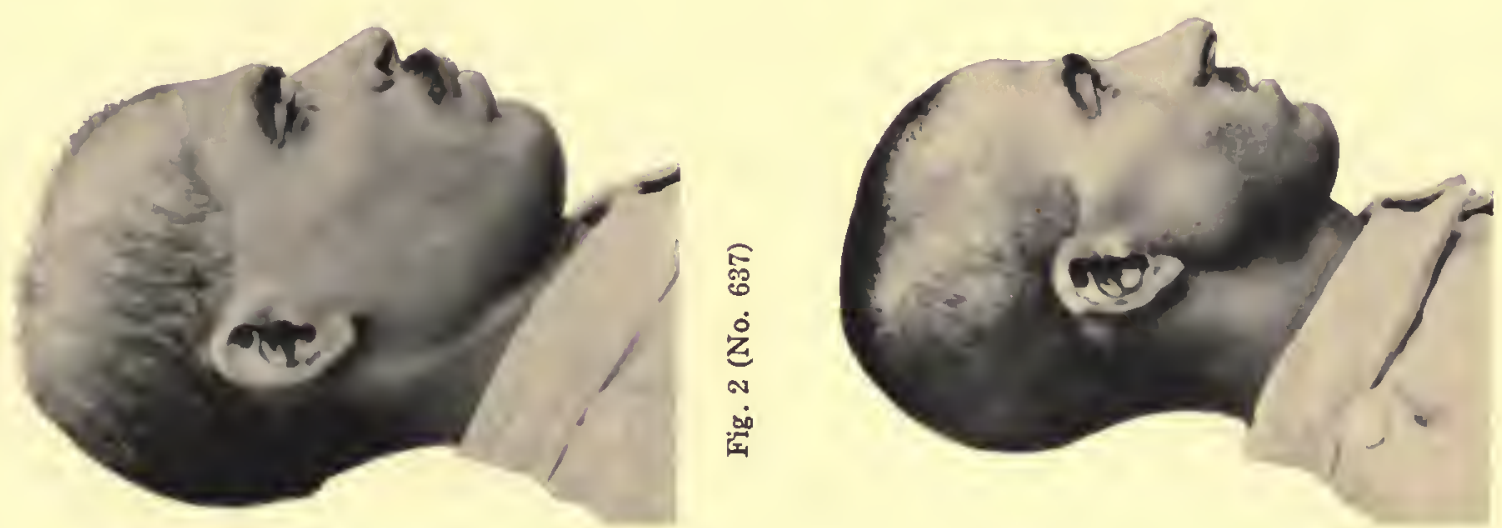

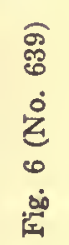
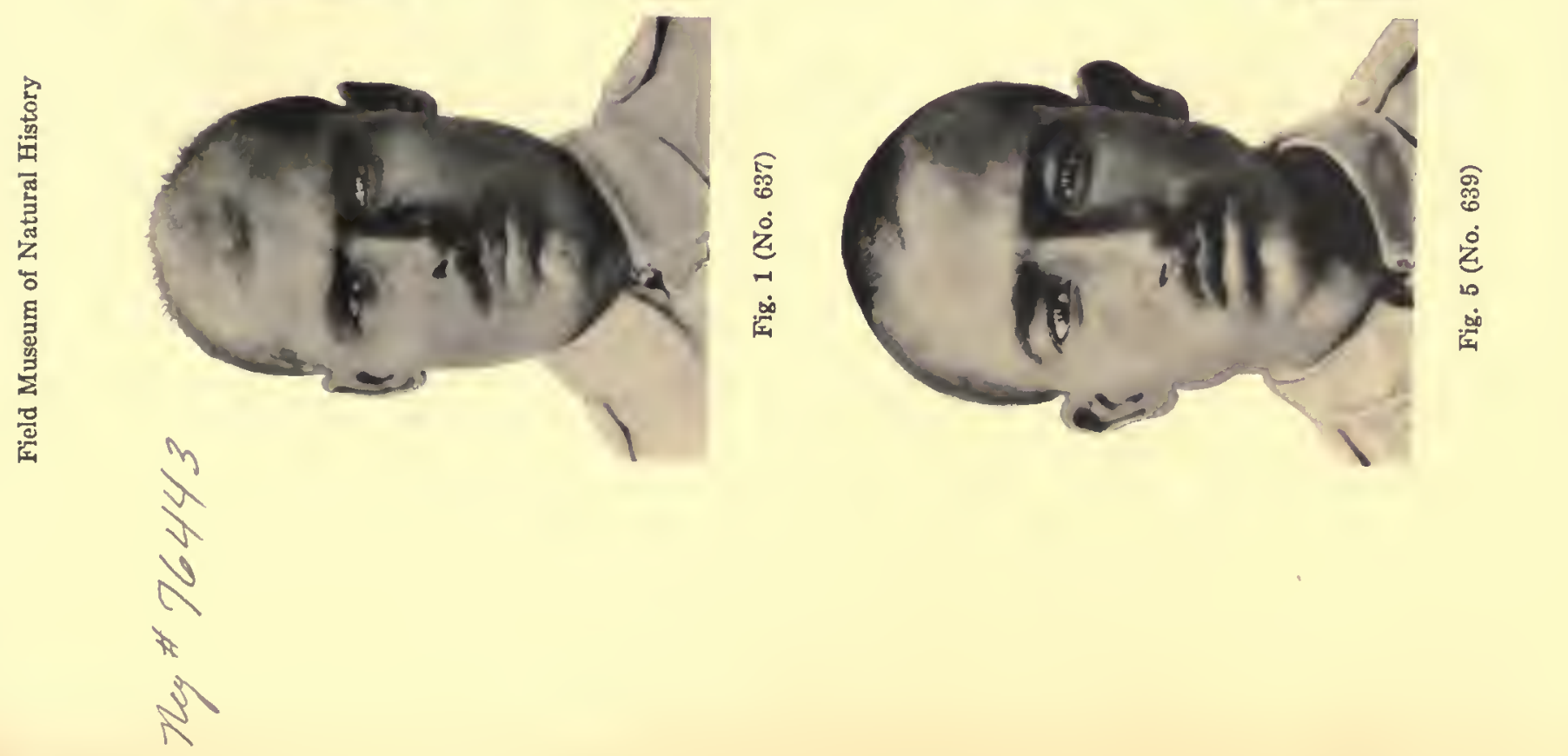

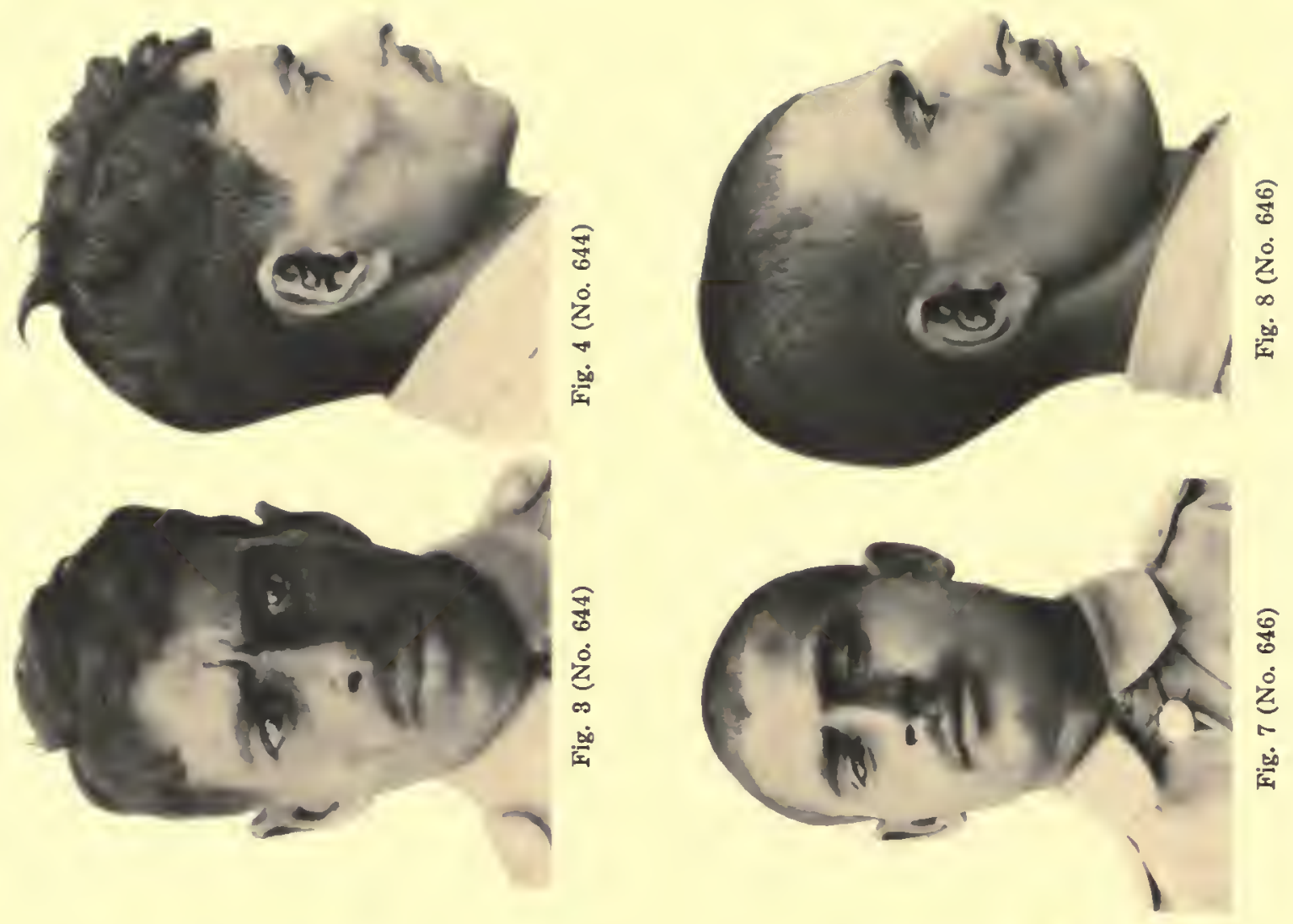

W
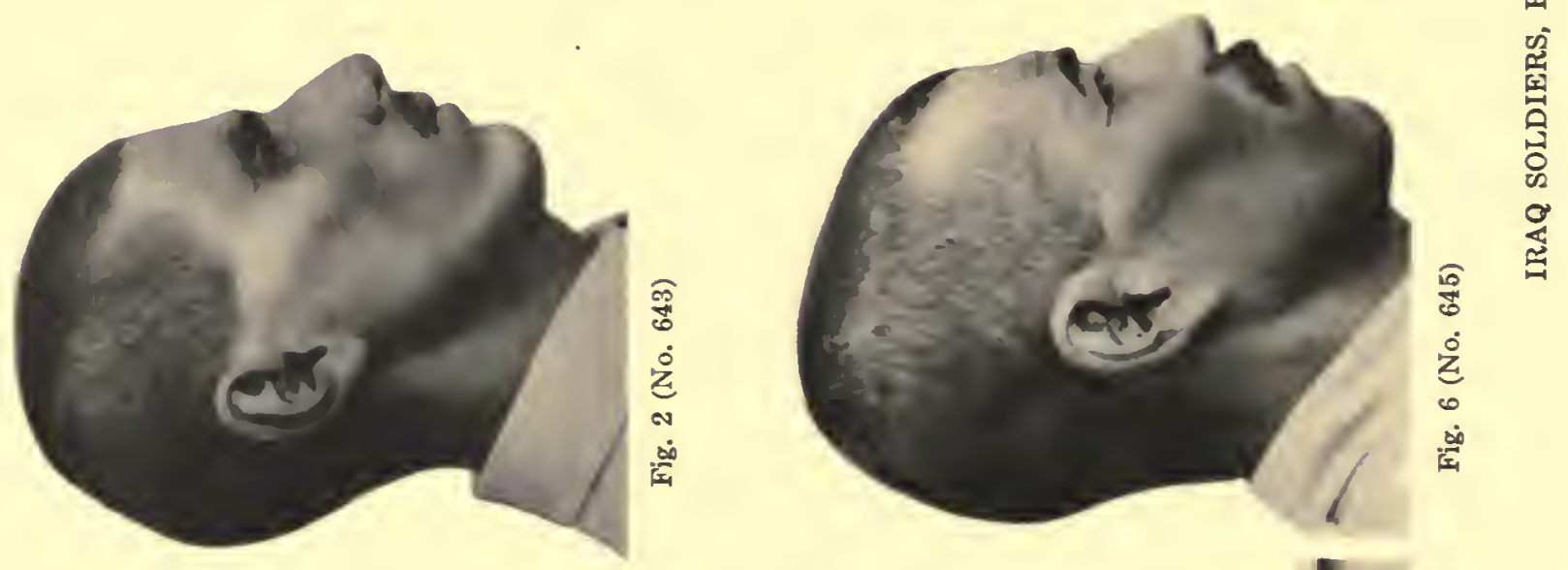

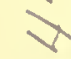

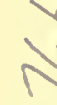

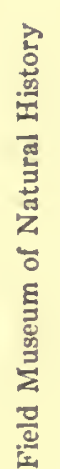
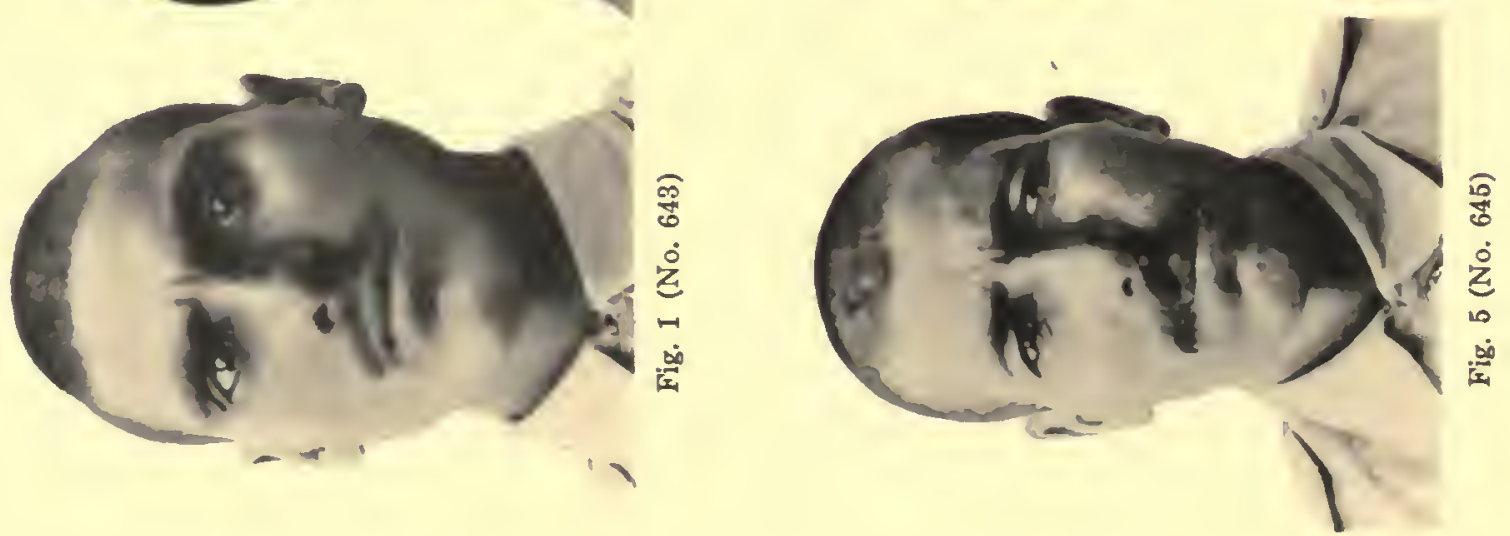

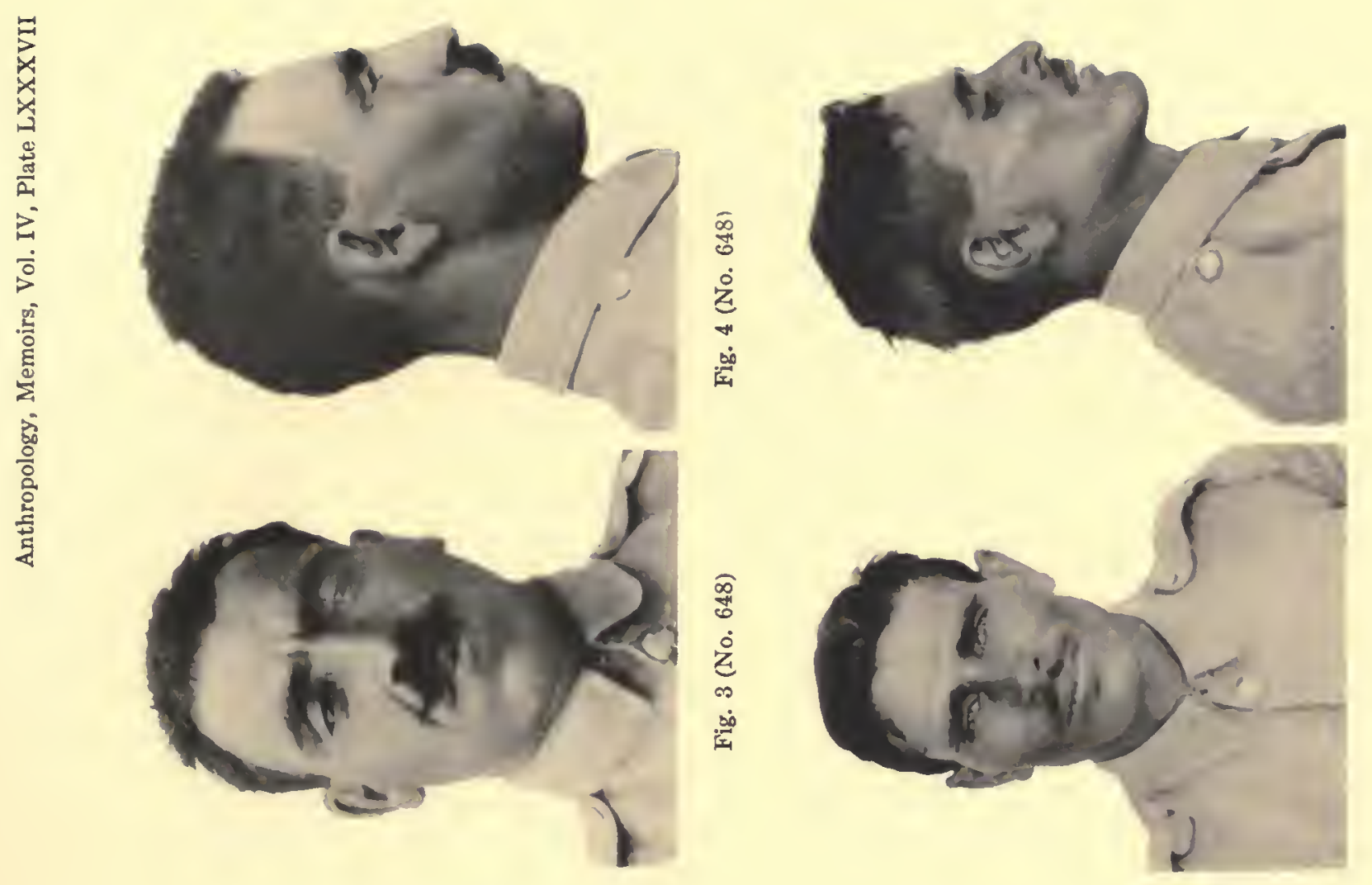

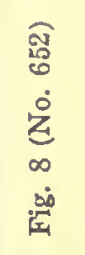

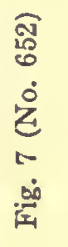

\begin{tabular}{l} 
量 \\
0 \\
哥 \\
\hline
\end{tabular}
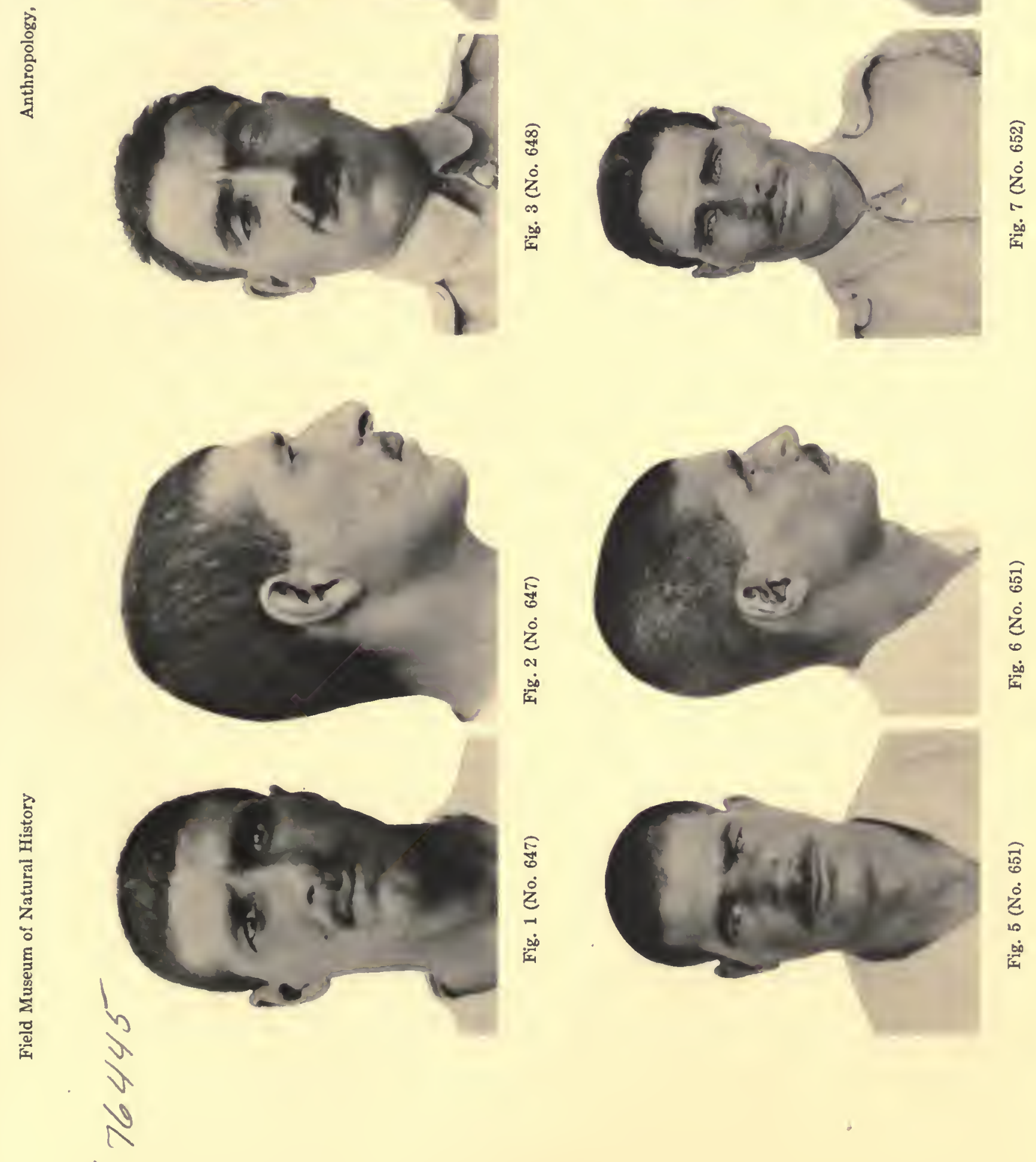

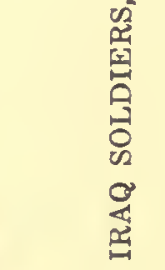

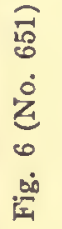

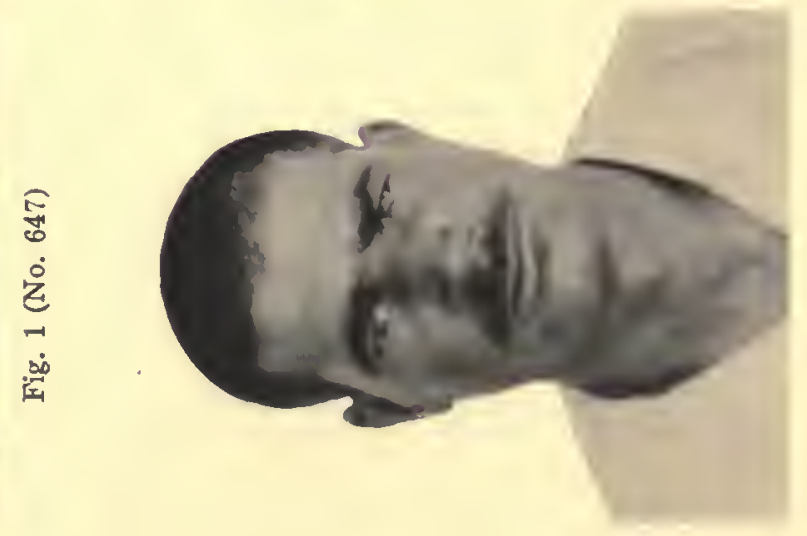

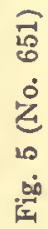
* 


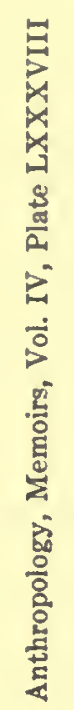
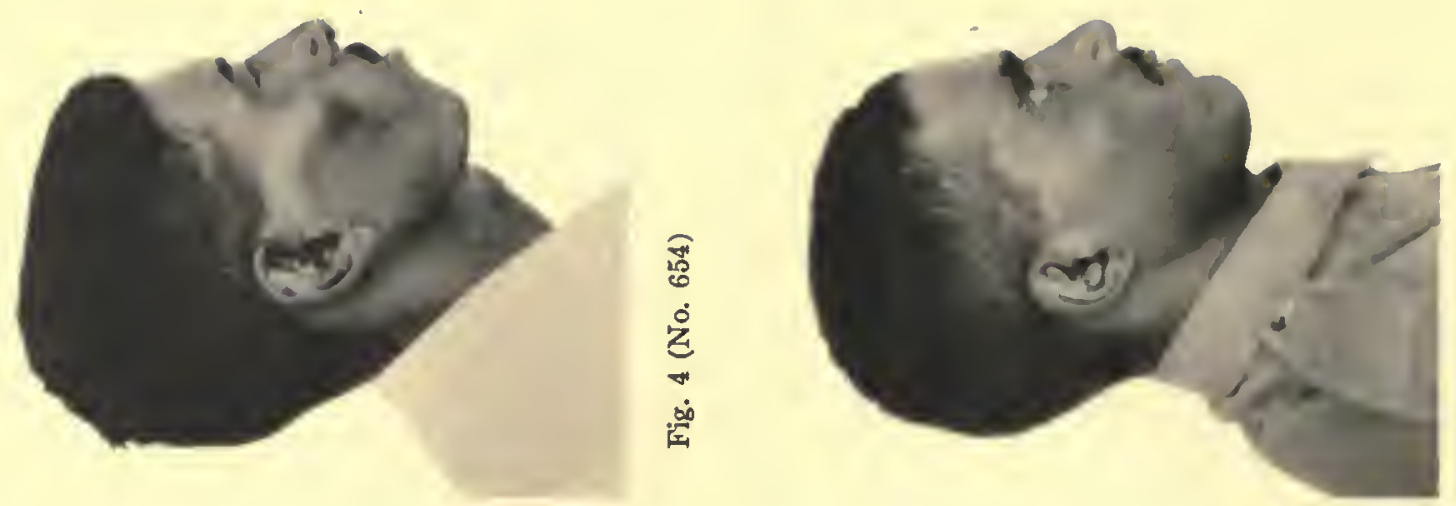

0
0
0
0
2
$\infty$
$\infty$
$\infty$
00
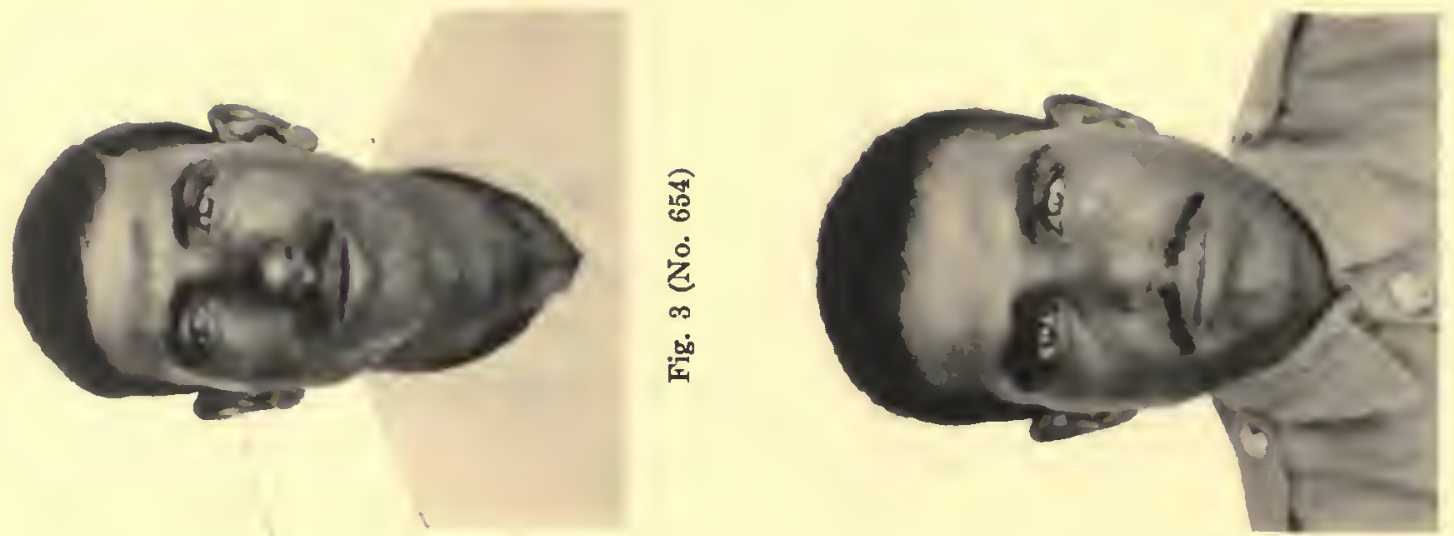

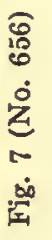
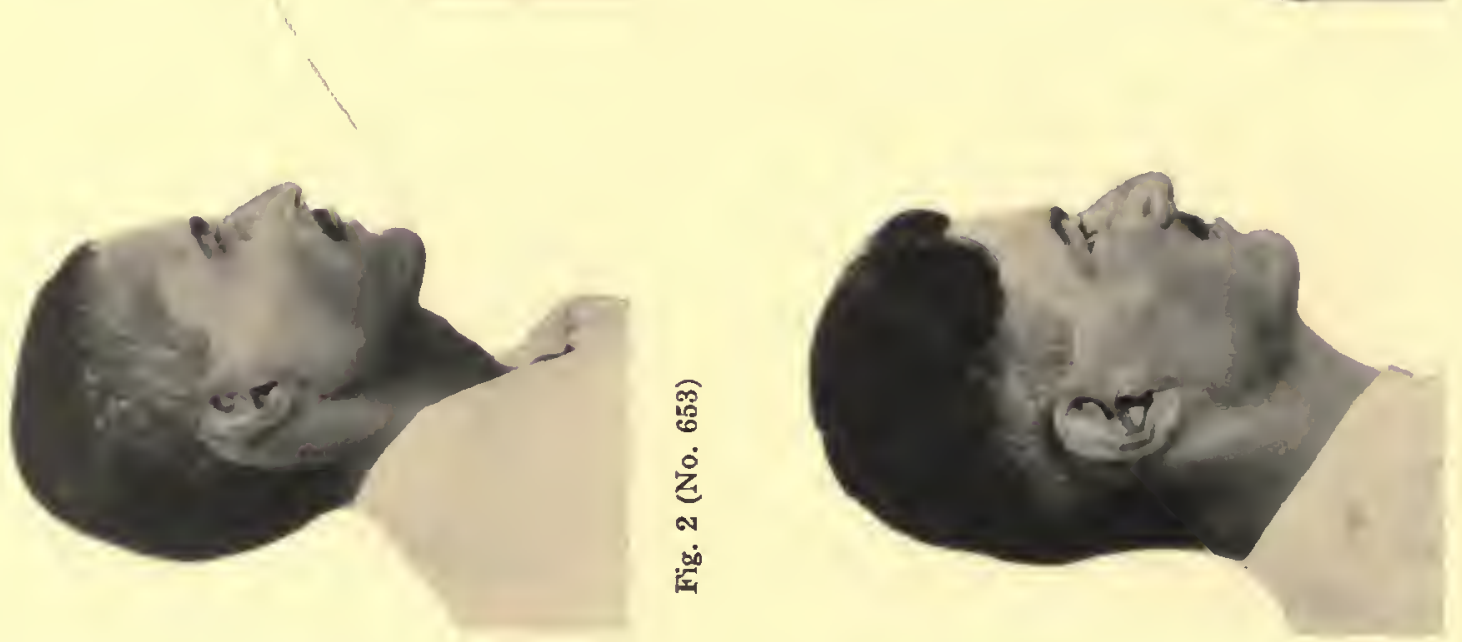

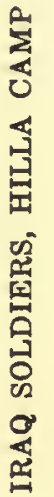

4
2
4
5
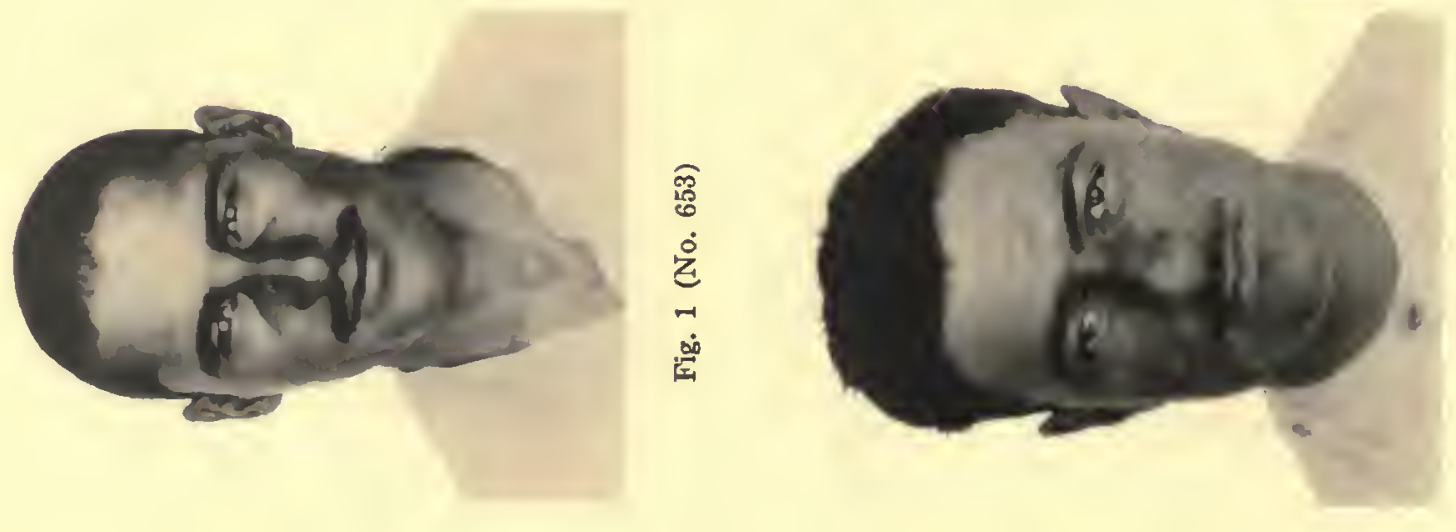

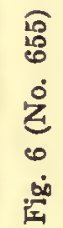

总

0
0
0
0
0
0
0
0 

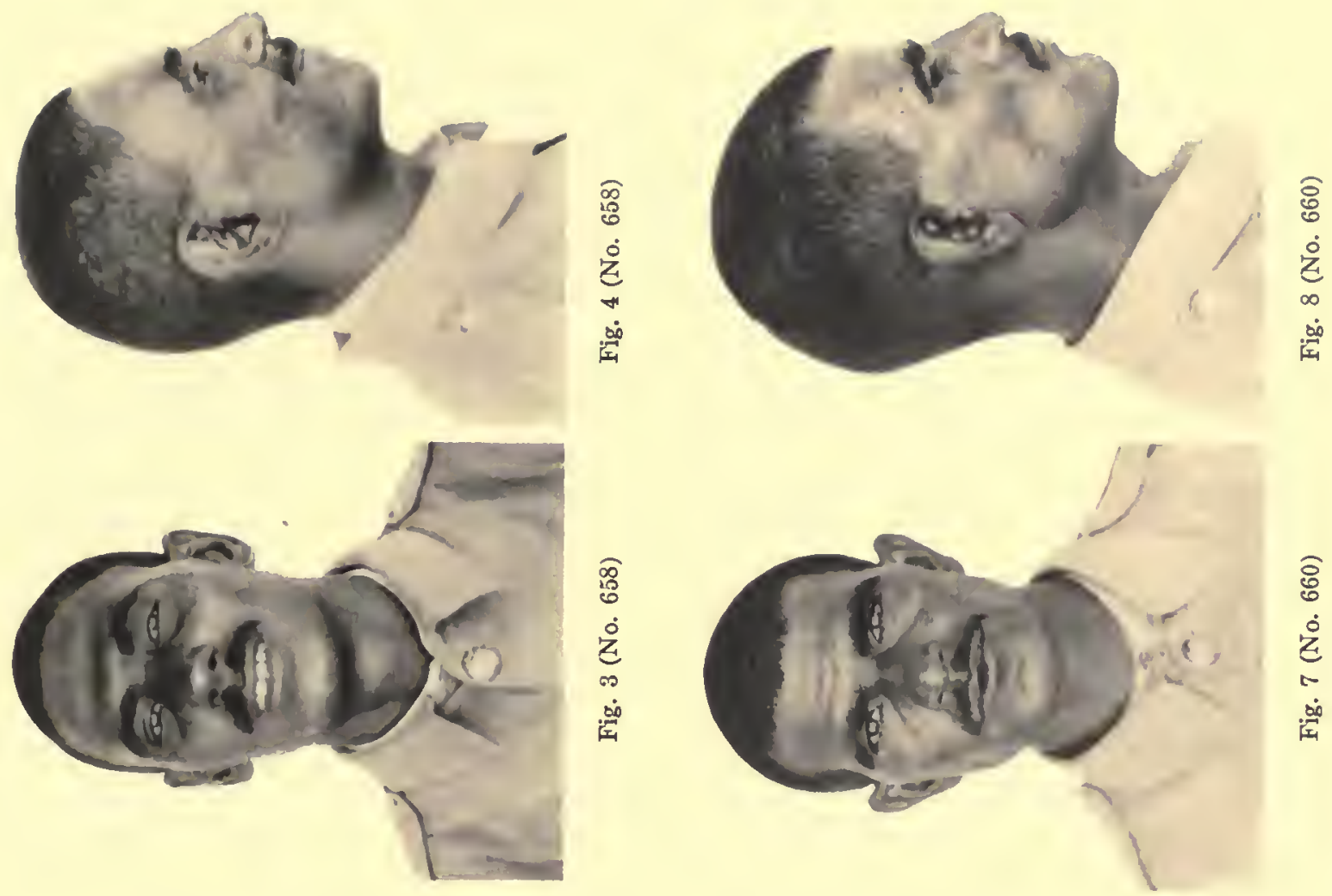

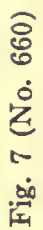
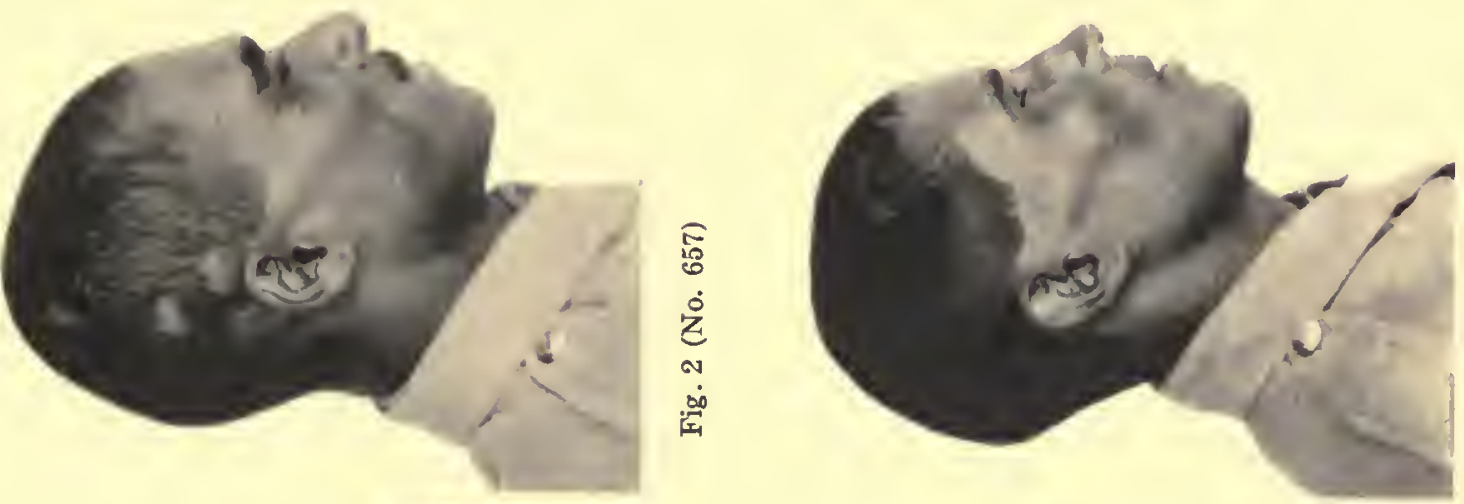

ठิ요
$\dot{0}$
$\infty$
$\infty$
$\dot{0}$
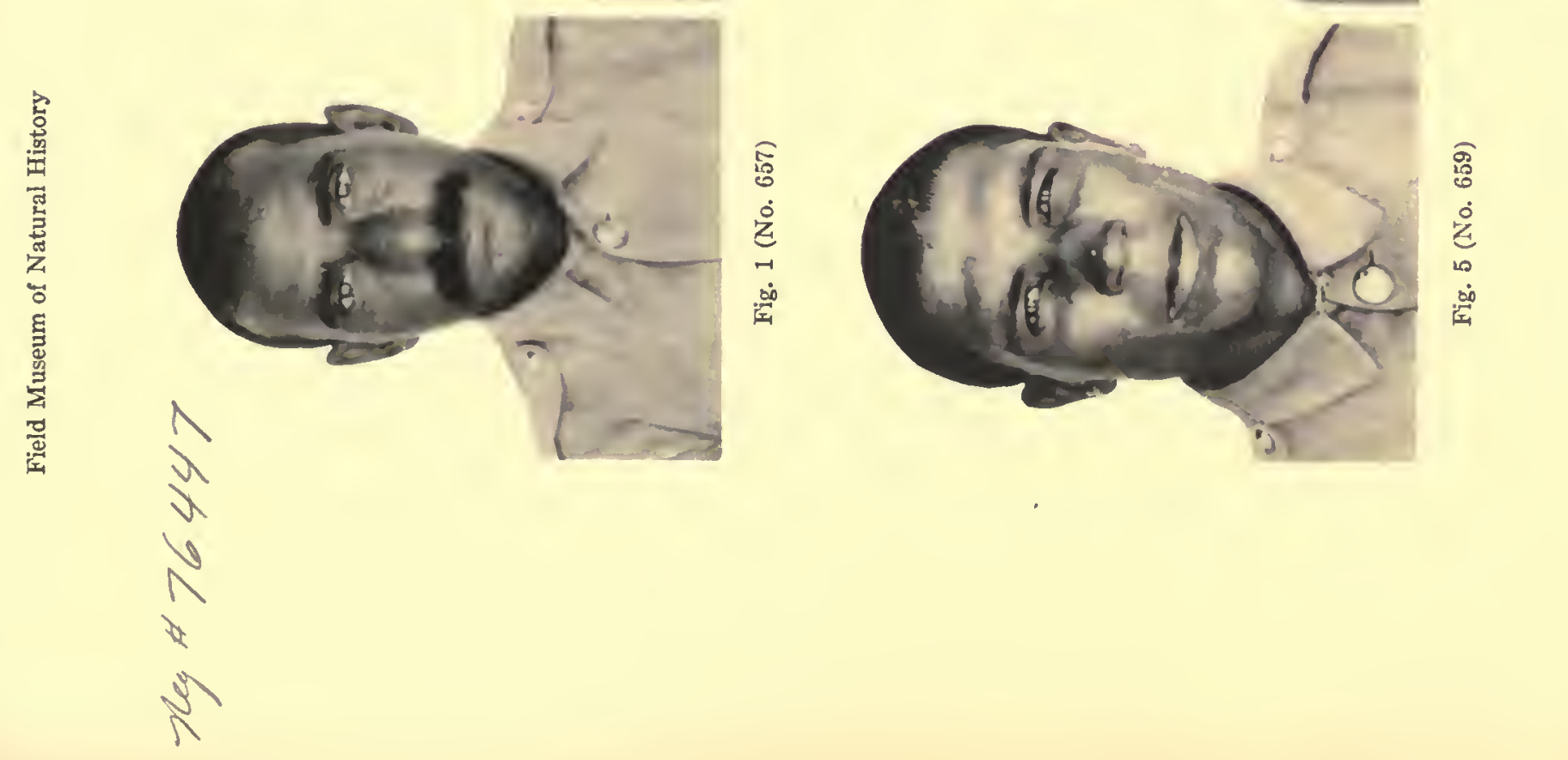

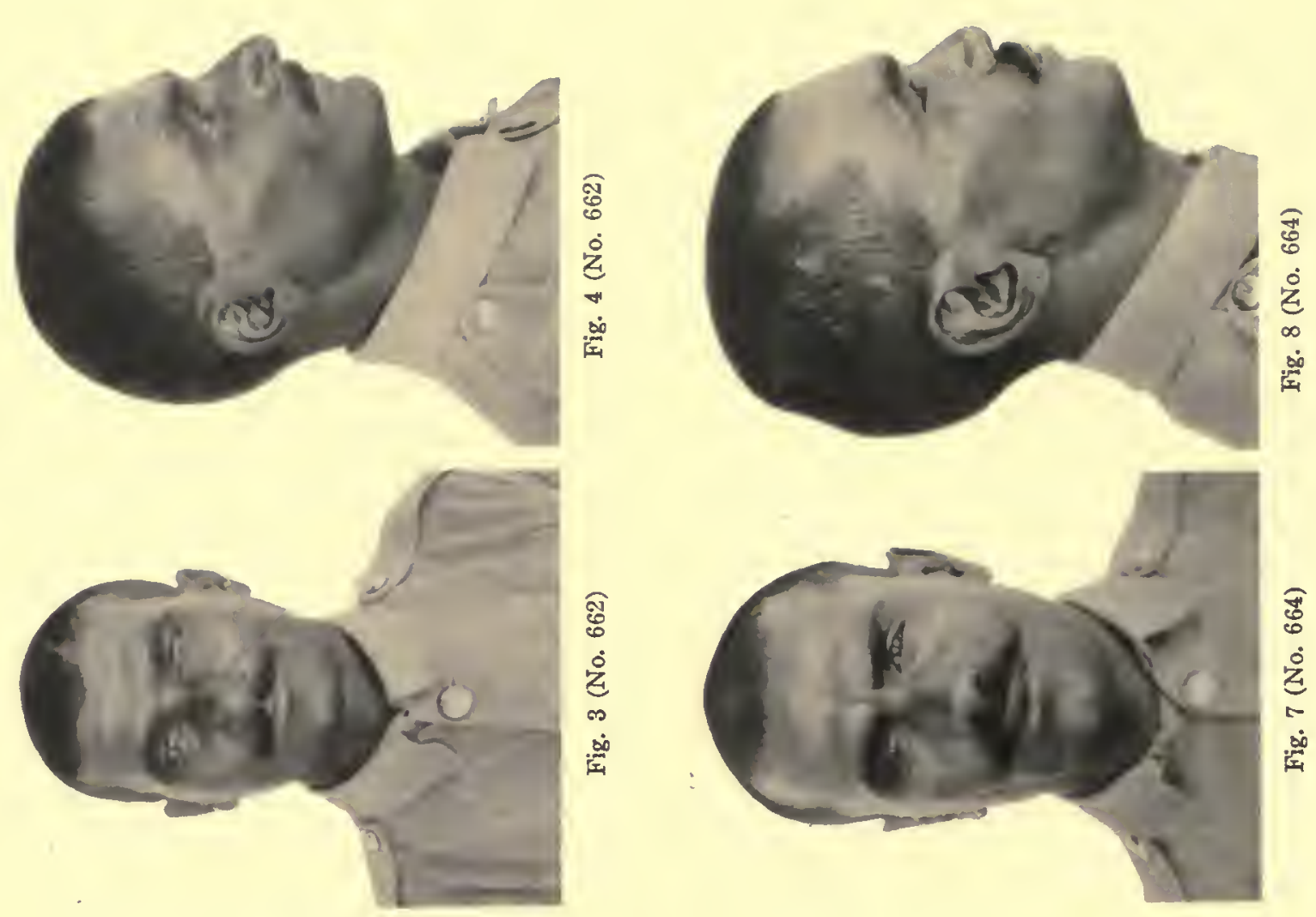

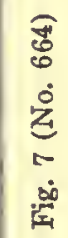

3
5
5
5
5
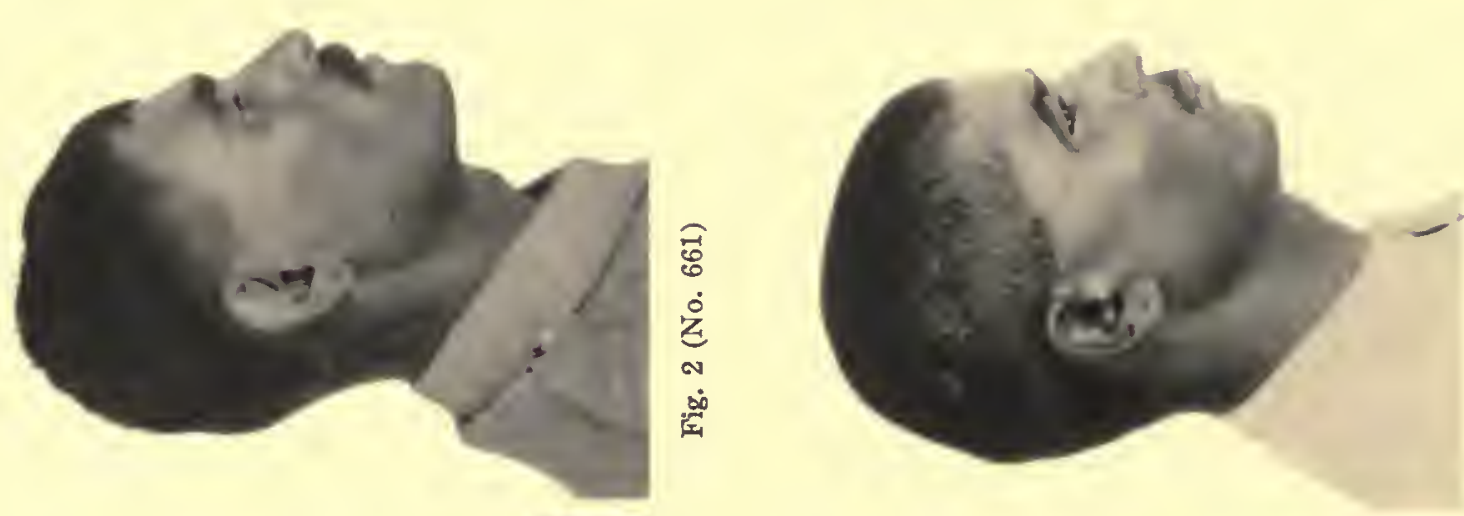

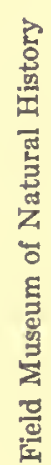
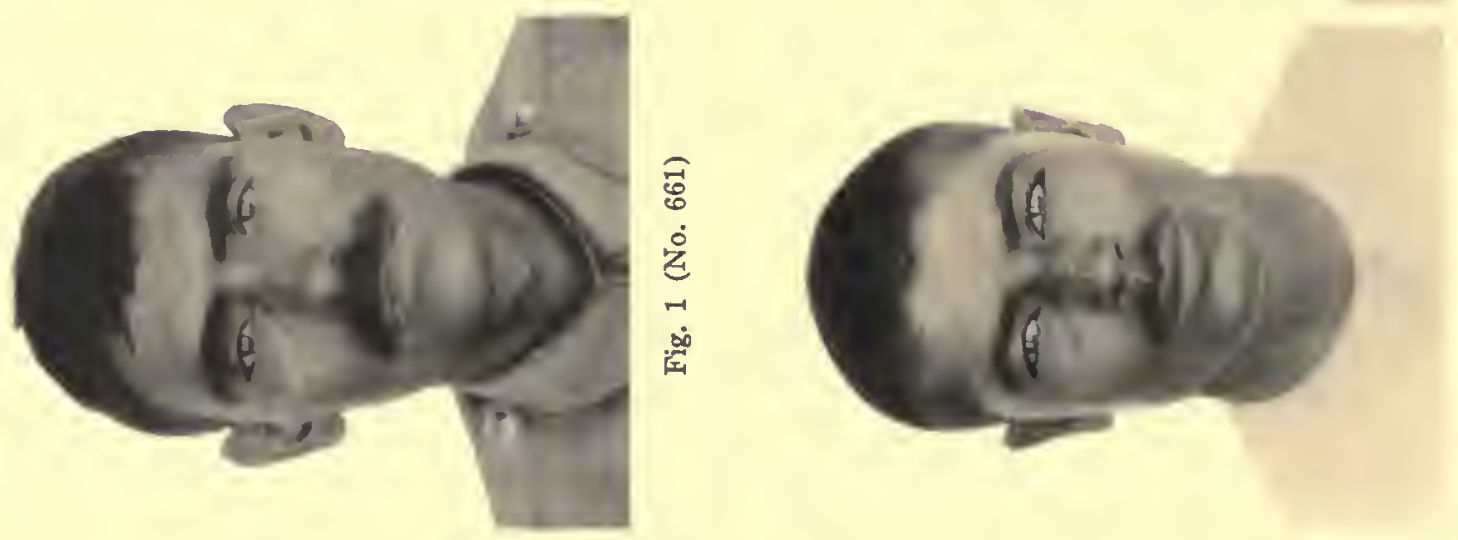

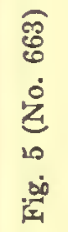




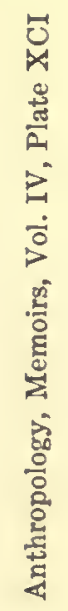
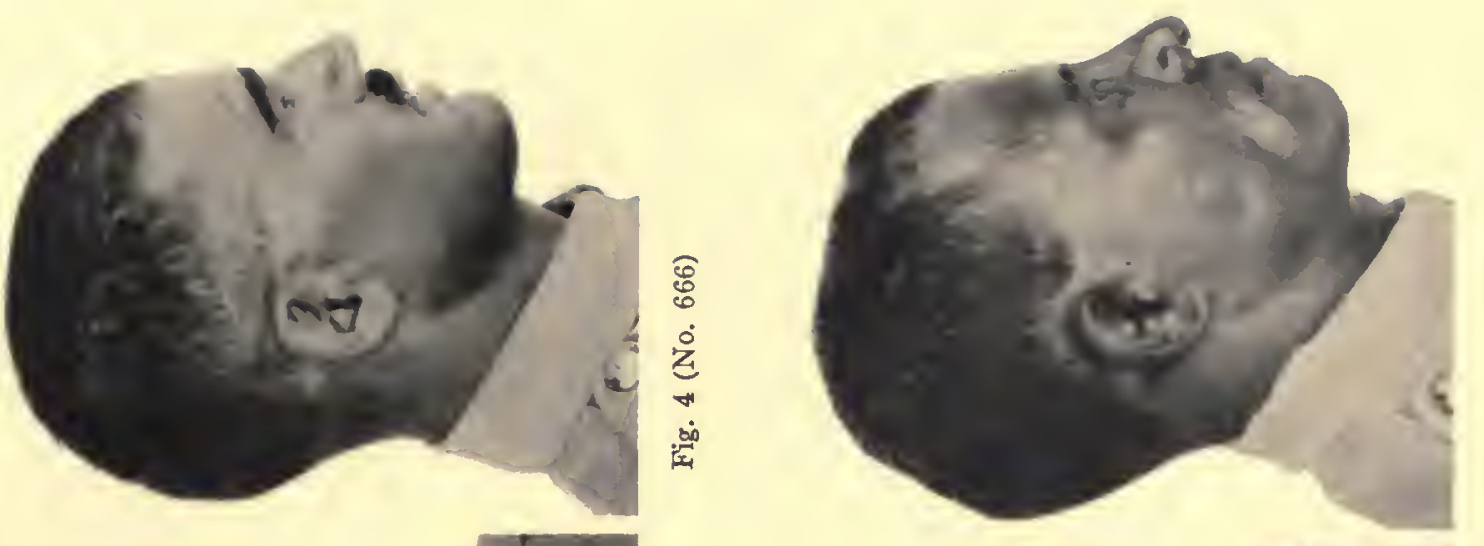

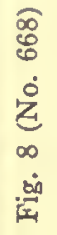
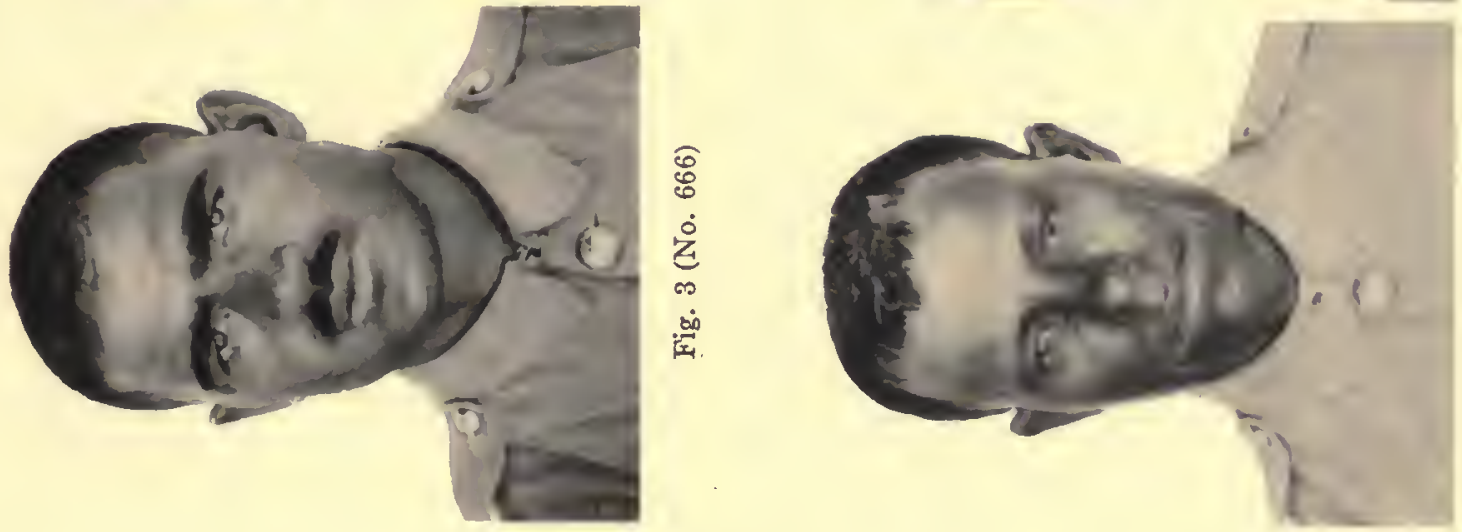

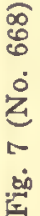

岂
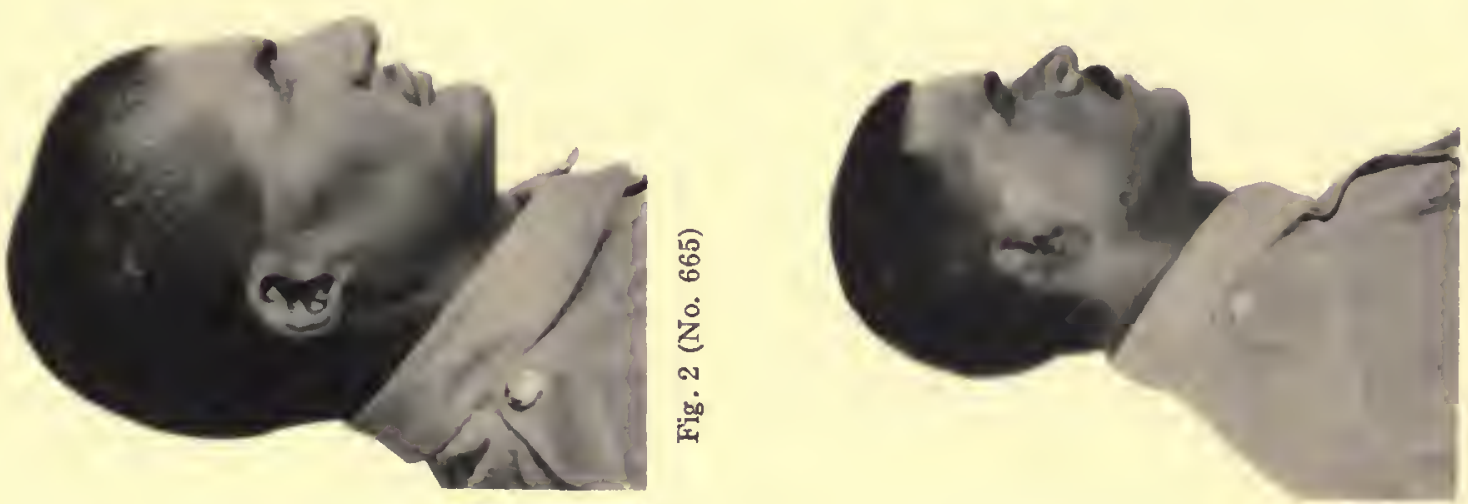

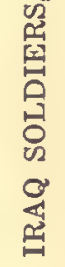

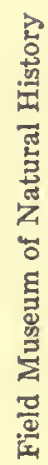
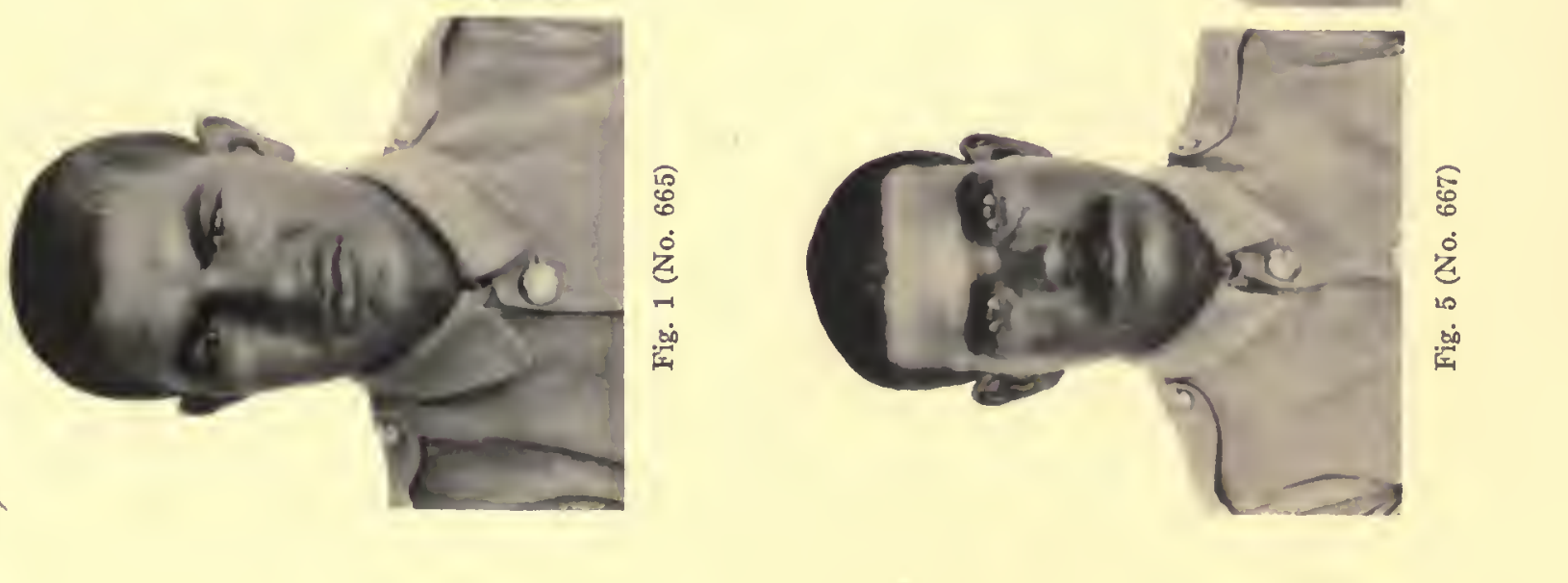

रे

k

र 


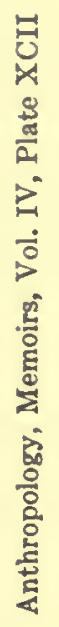
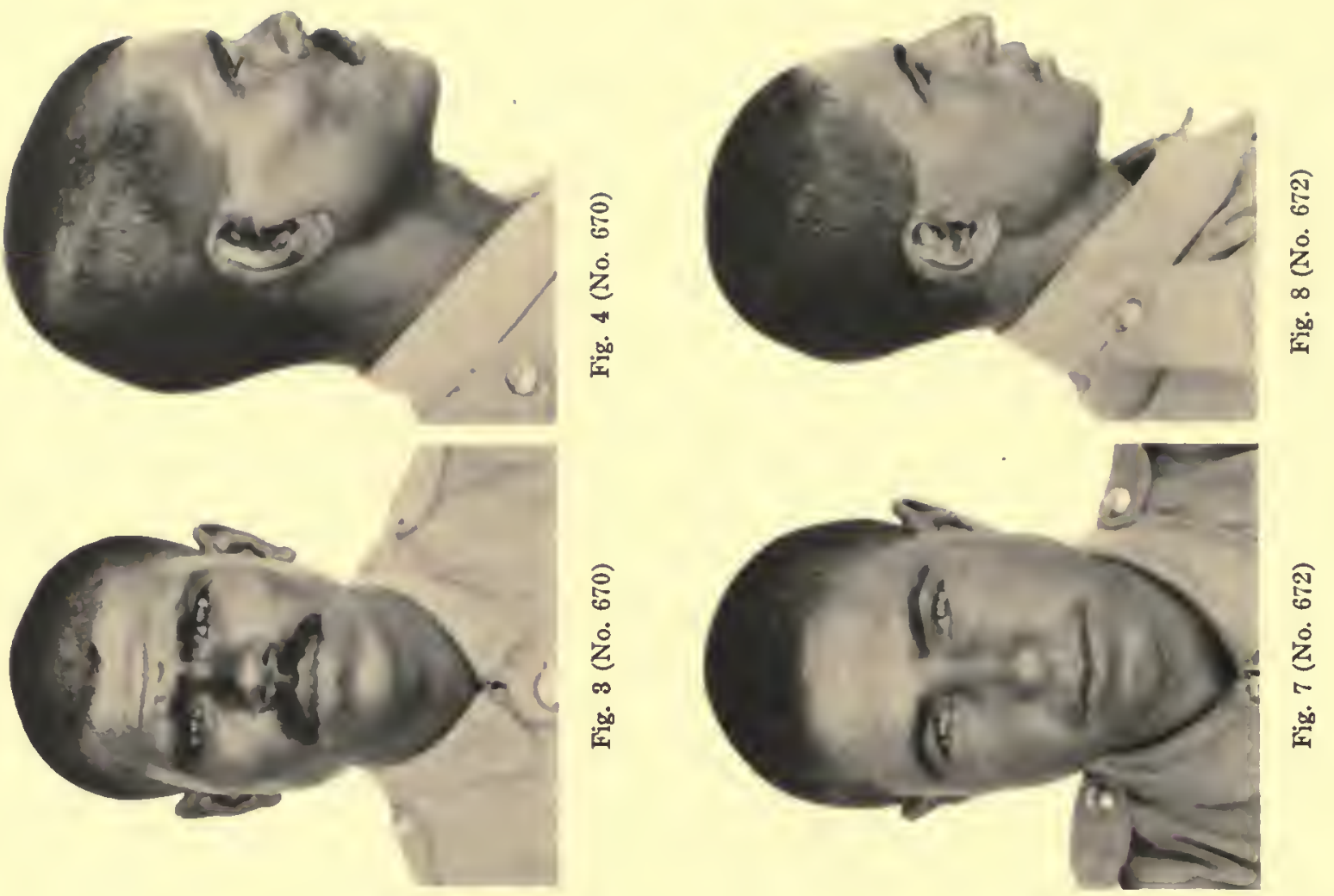

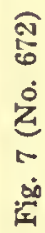
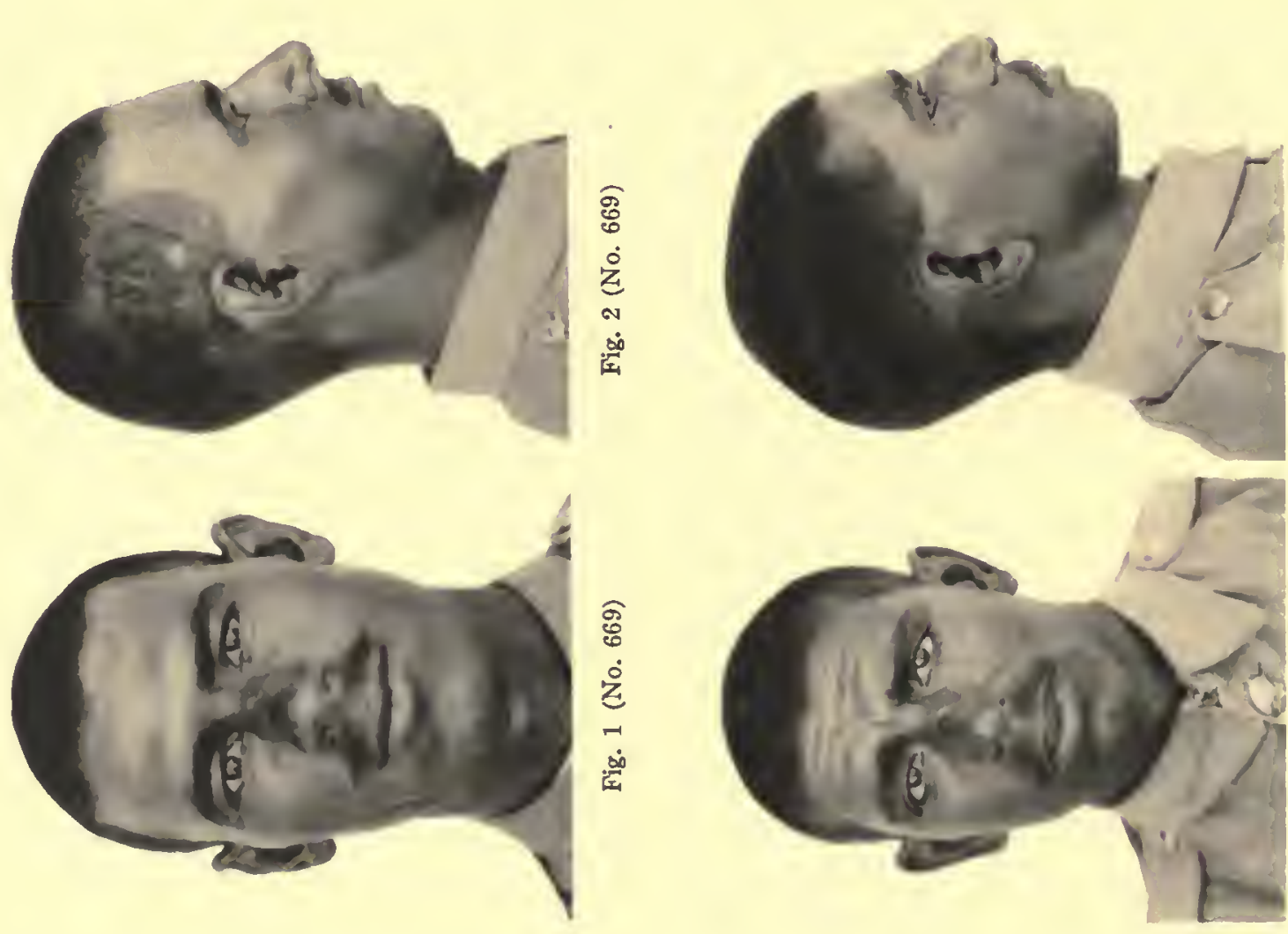

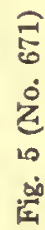

Ne
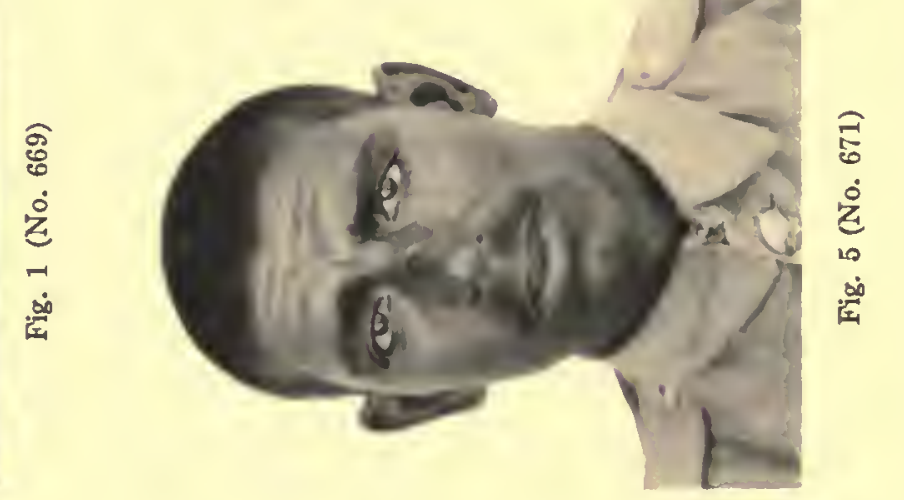


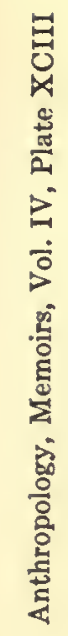
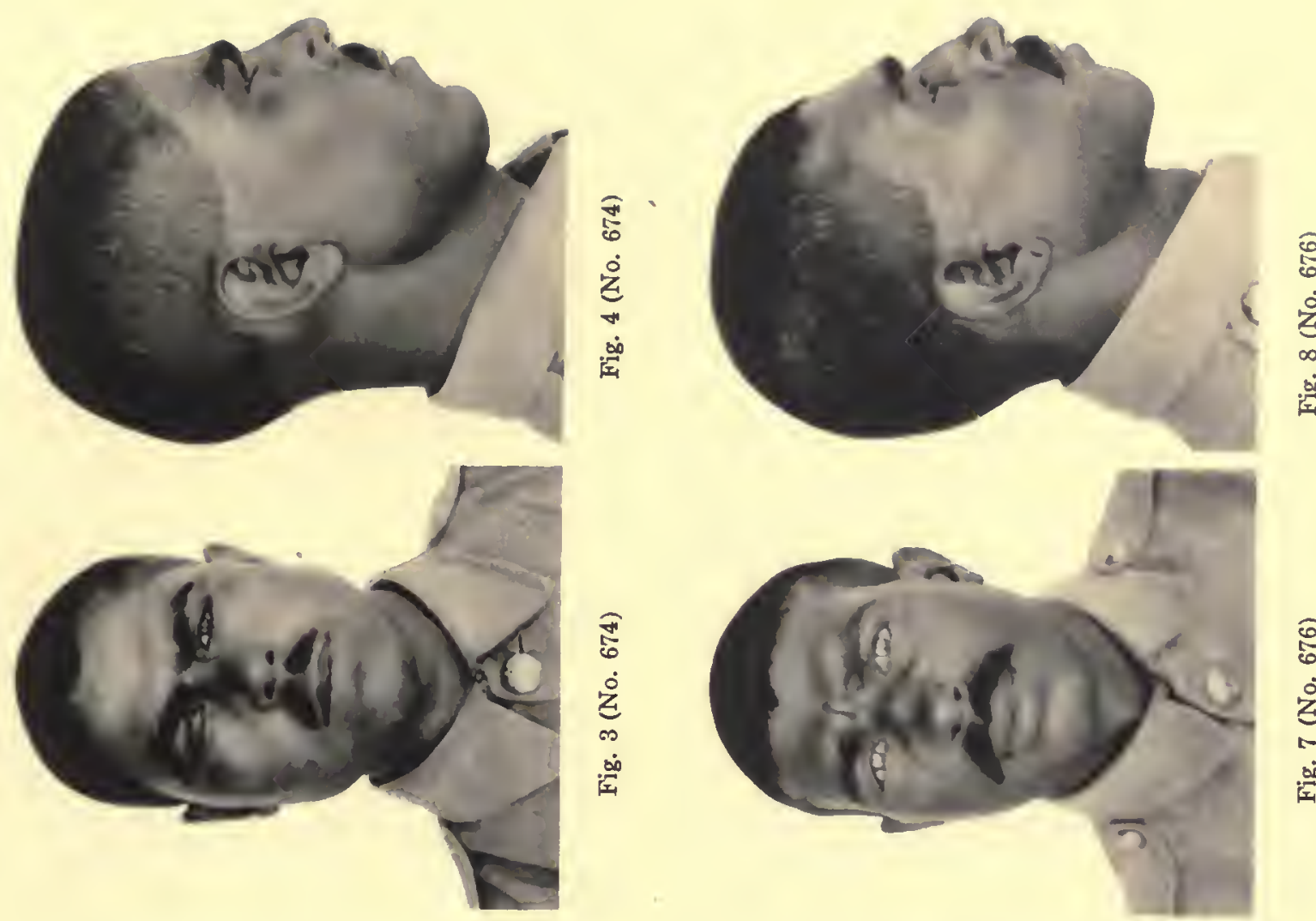

0
8
0
$\dot{0}$
$\infty$
$\infty$
0
0
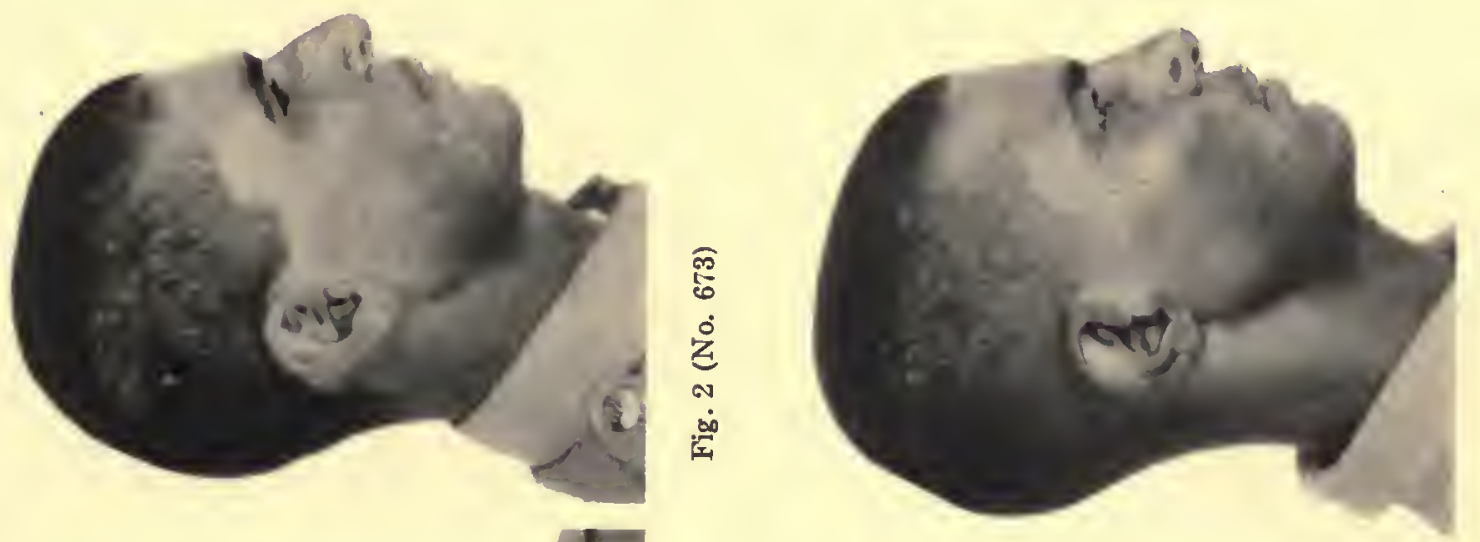

0
8
0
$\dot{0}$
3
5
0

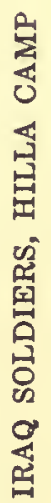
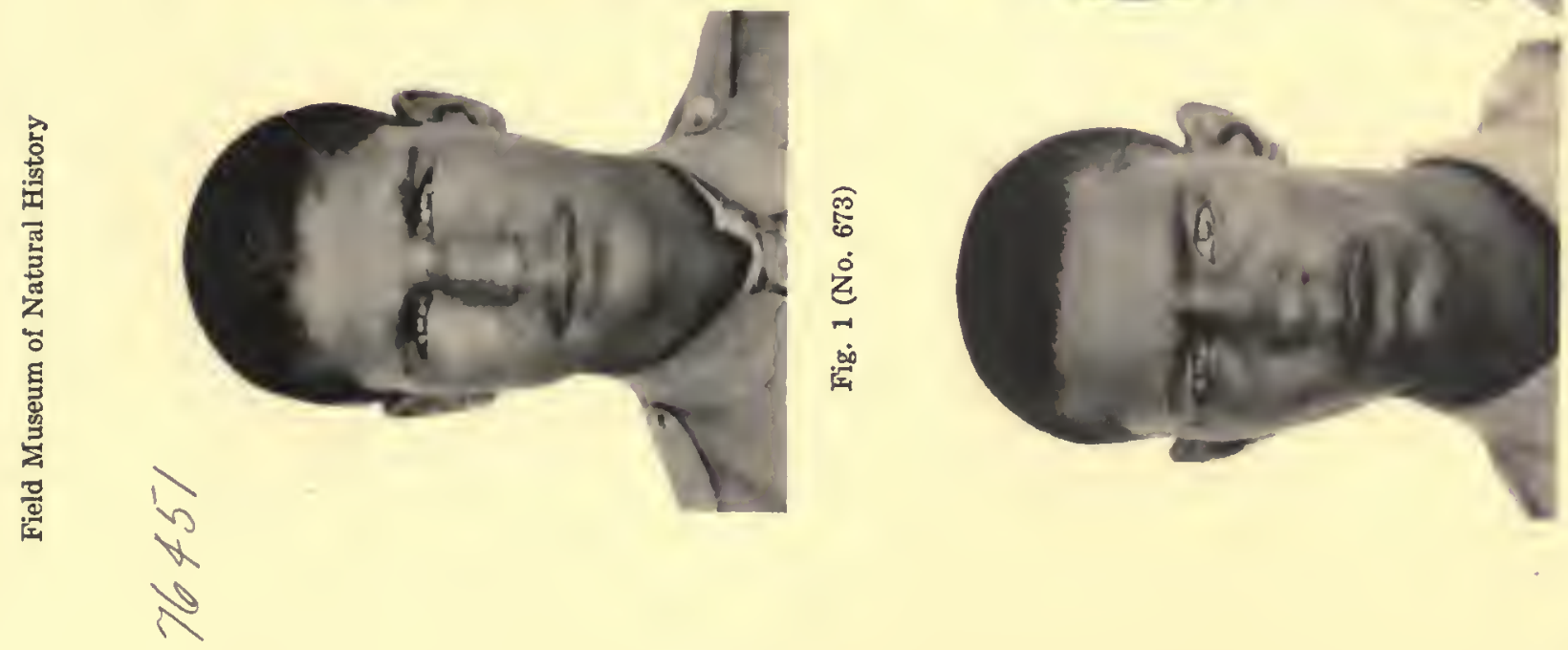

06
8
0
0
0
0
0
0

6
50
0
2
10
.00
10 
范
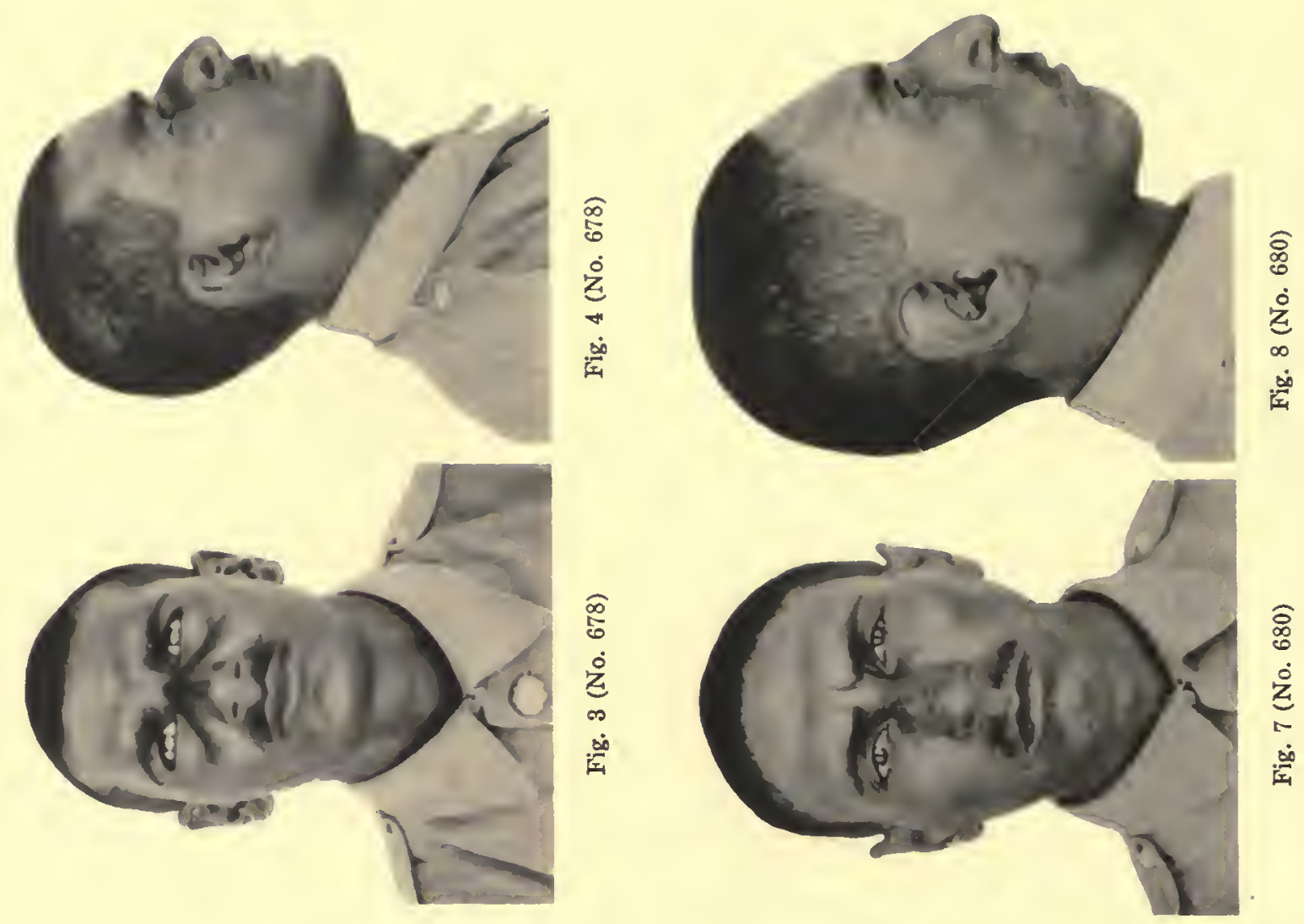

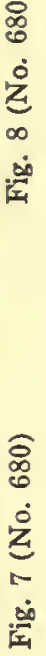
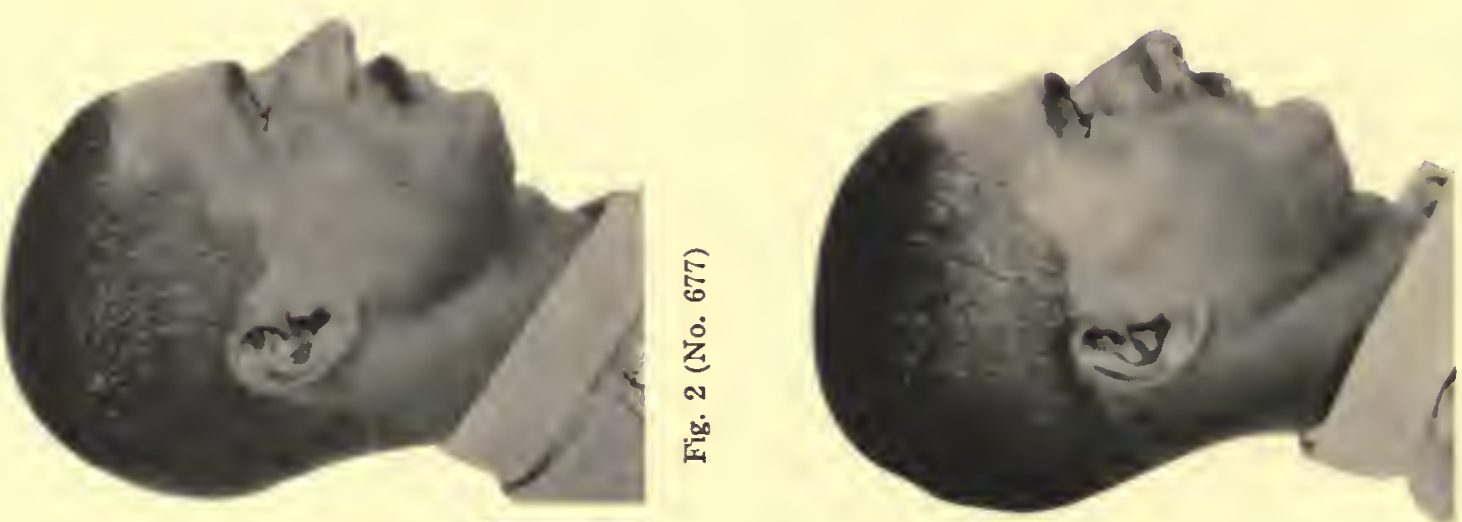

萣

v
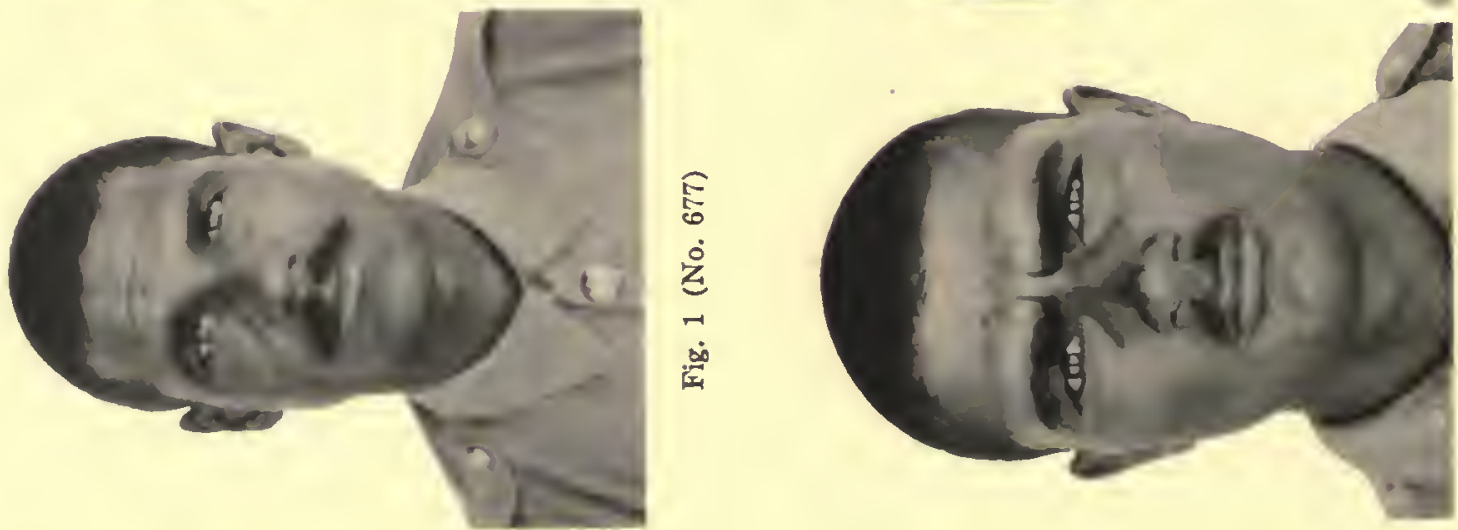

胥

No 혼 
ن
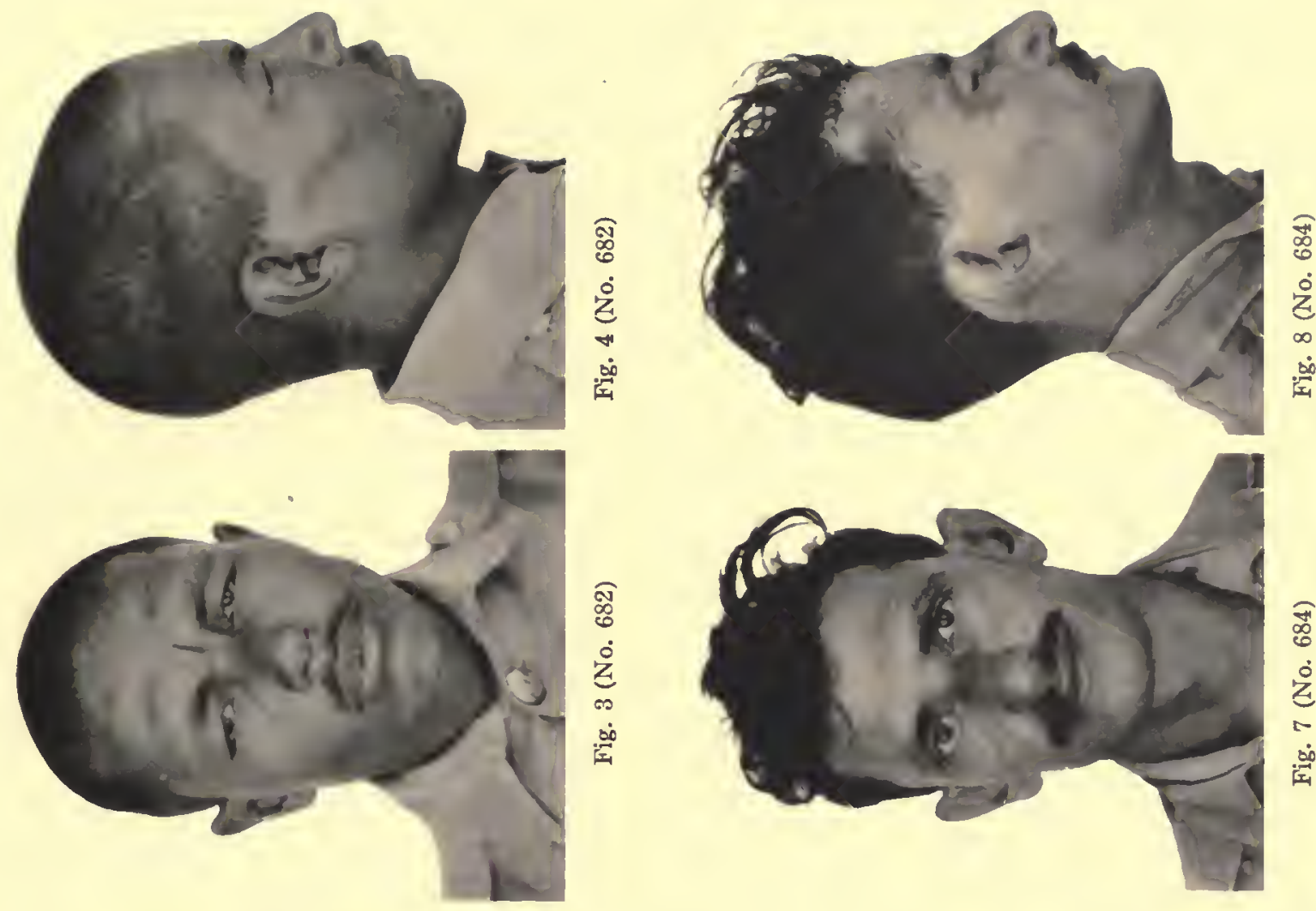

苟
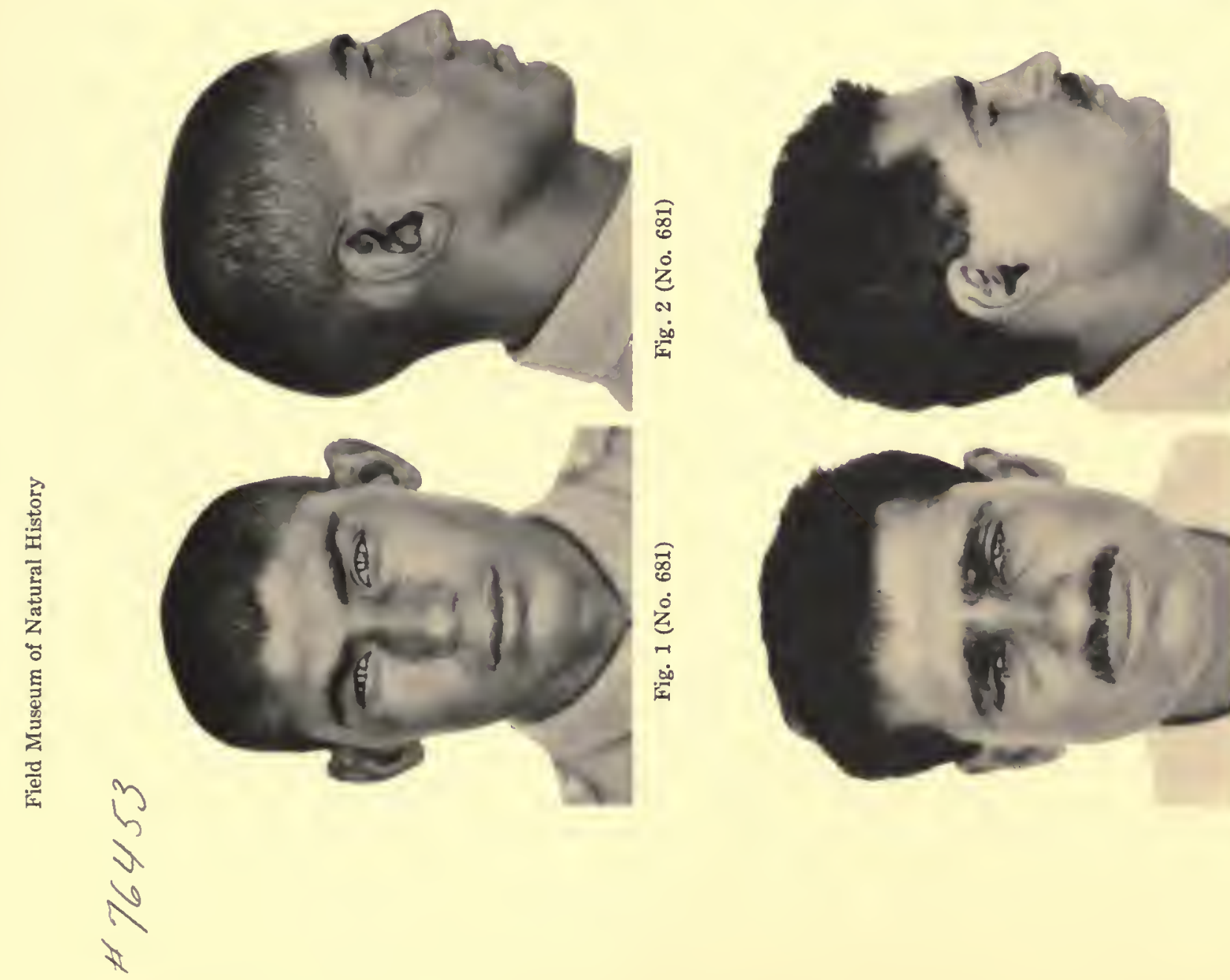

是

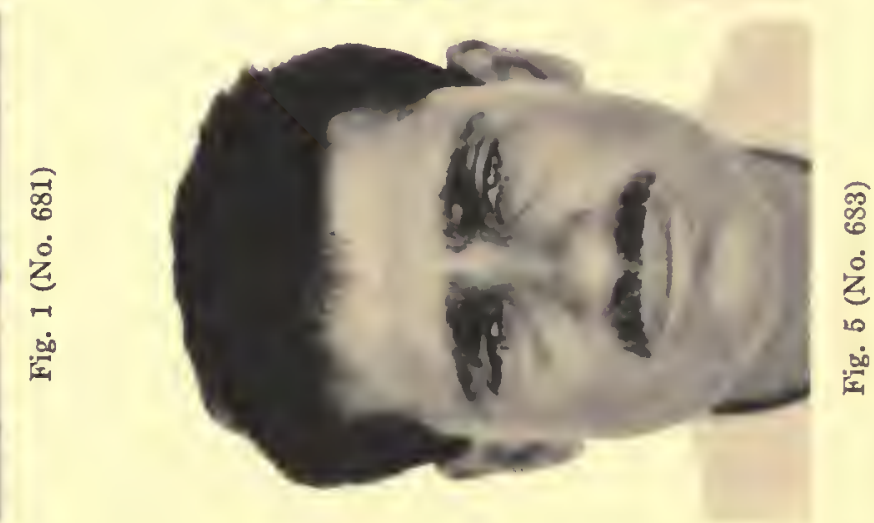

N 


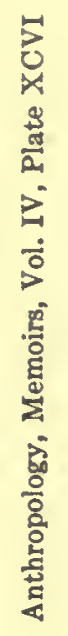
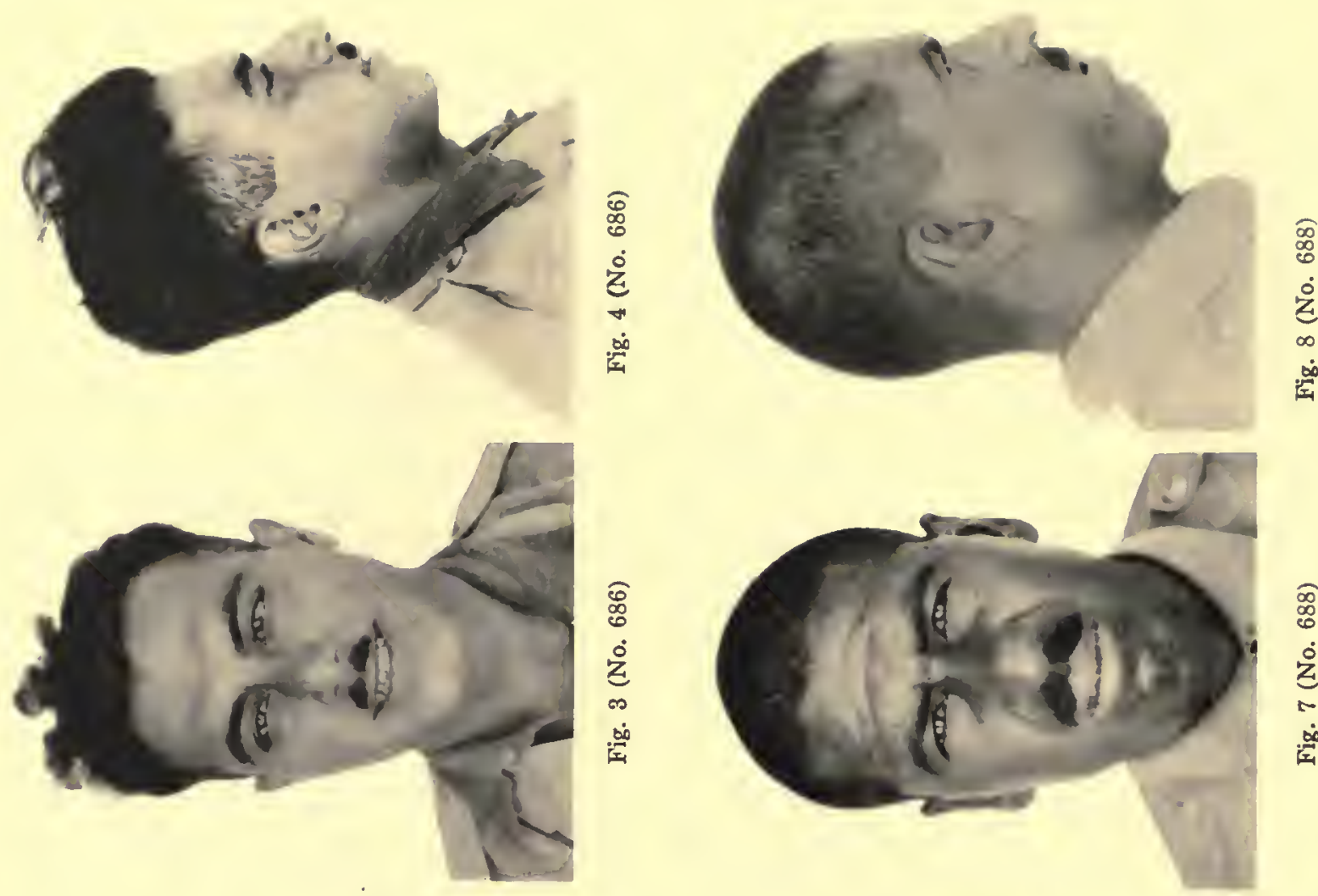

$\infty$
0
0
$\dot{0}$
$\infty$
$\infty$
0

$\infty$
0
0
$\dot{0}$
2
$\vdots$
0
0
0
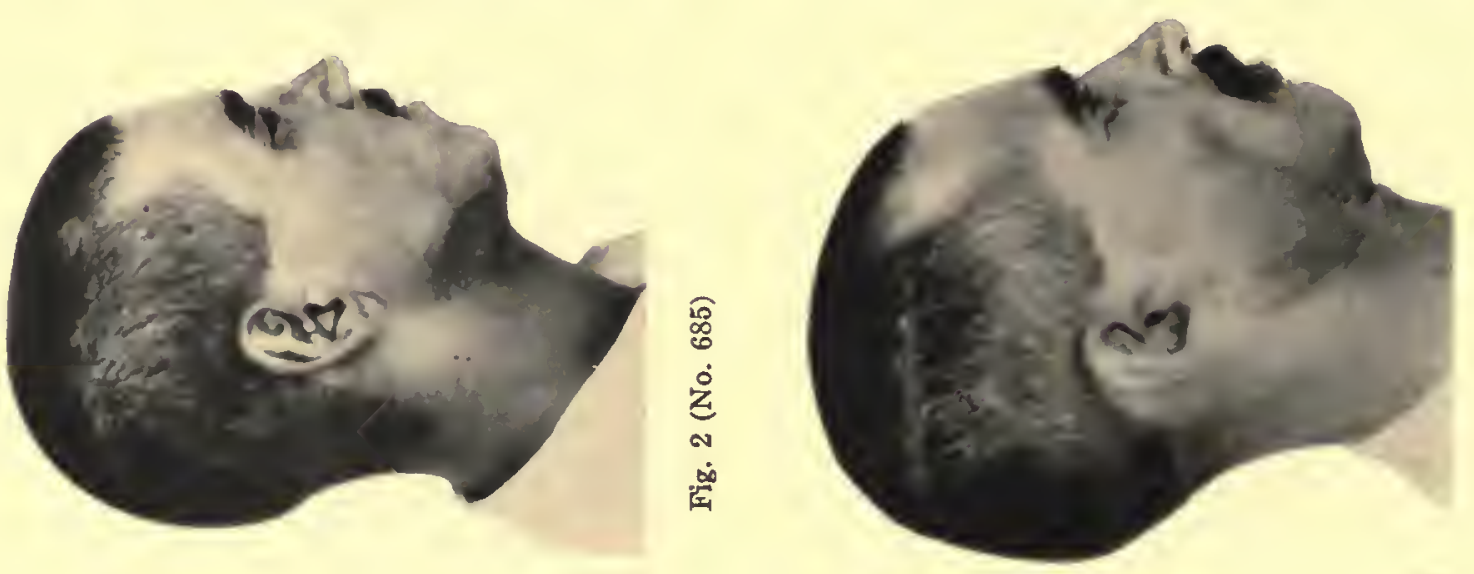

嵌

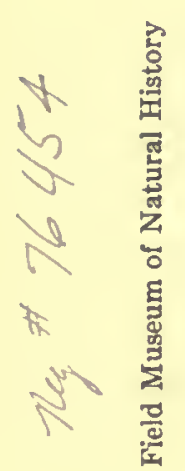

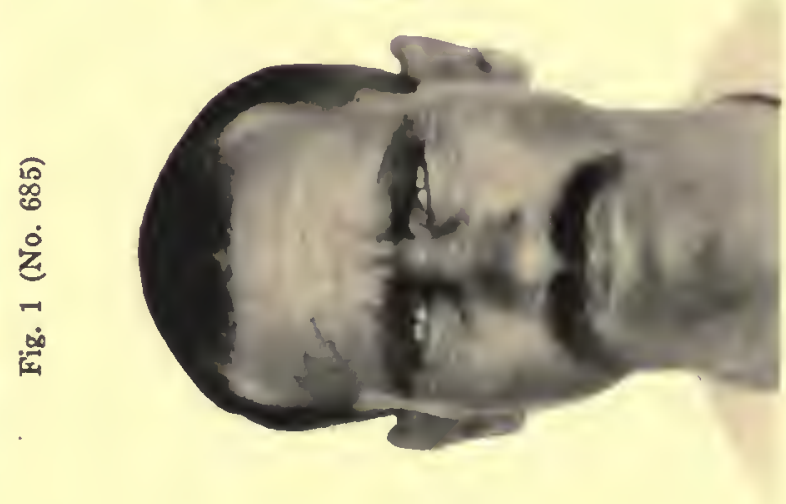

$E$
0
0
0
0
0
0
0

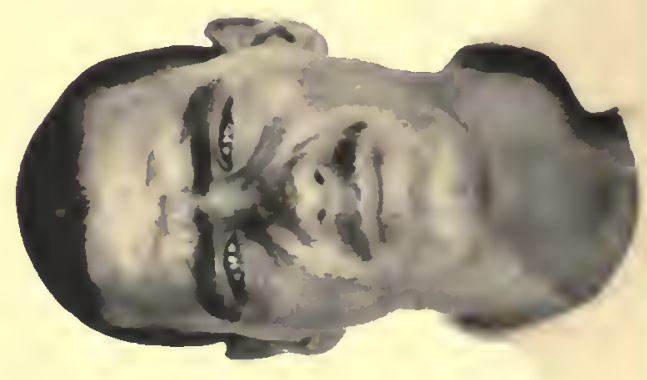


要
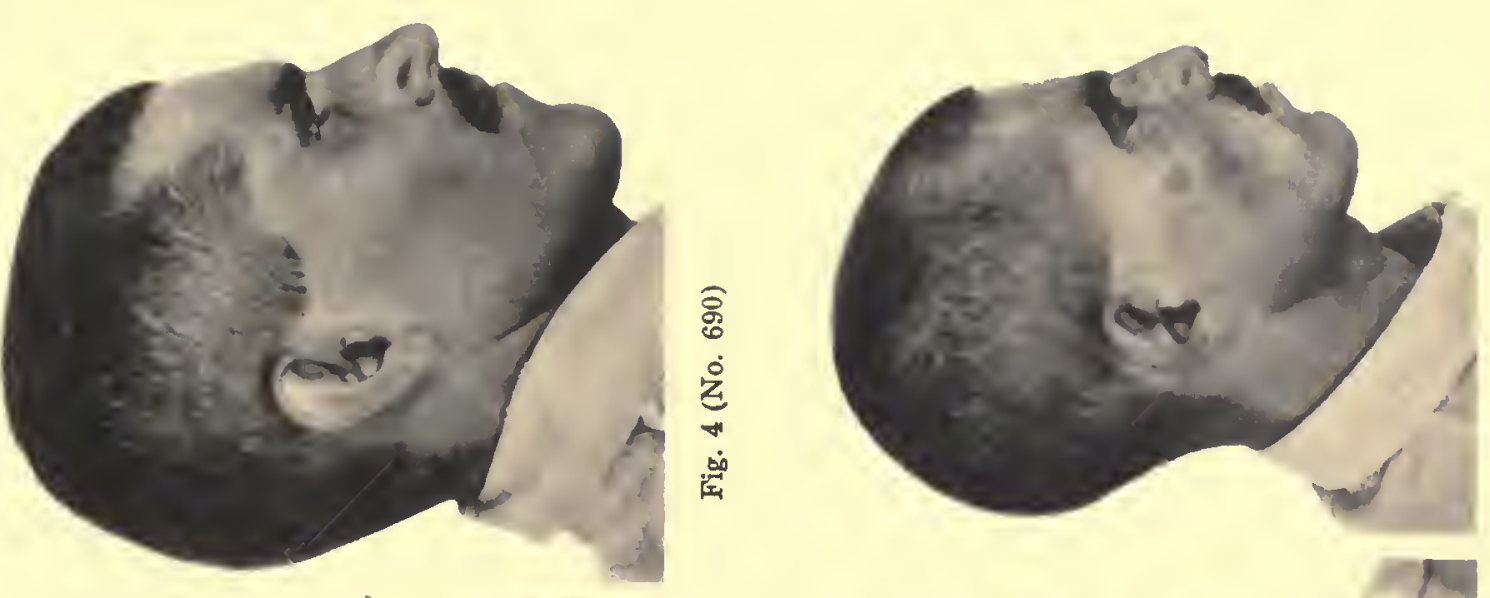

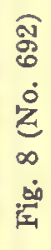
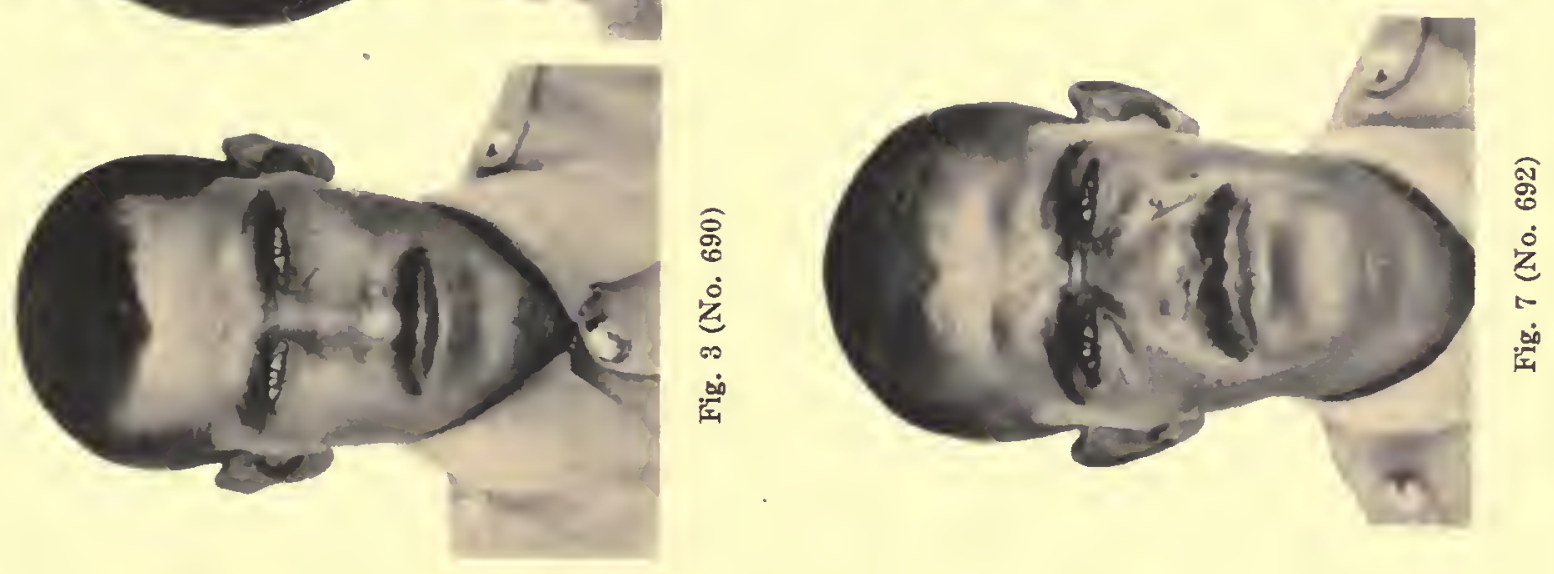

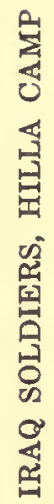
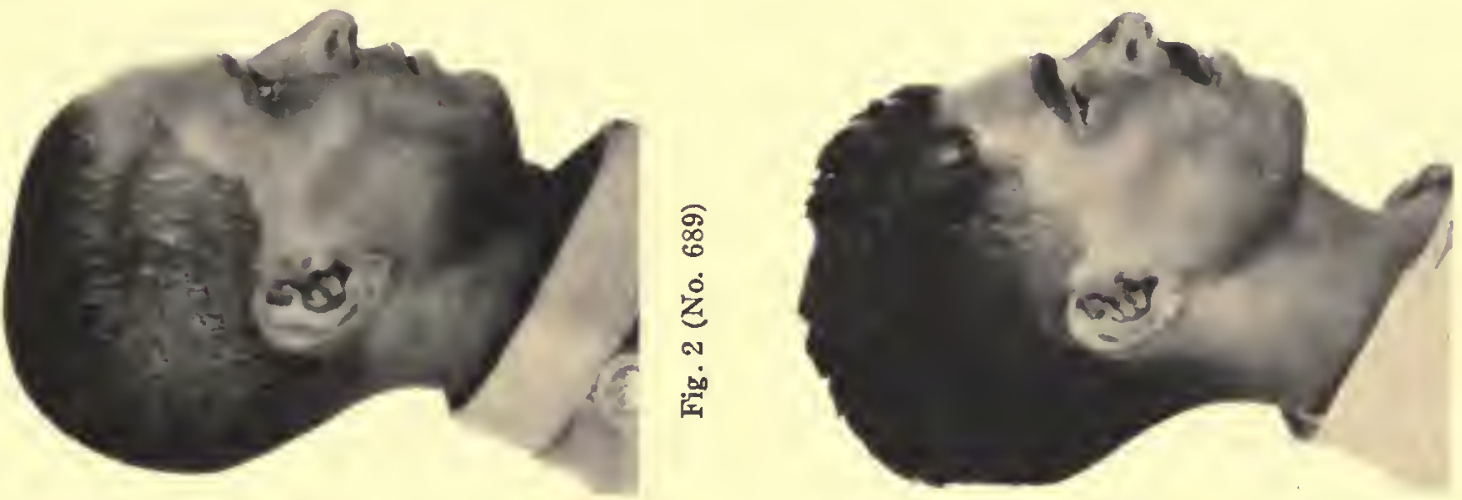

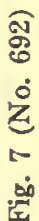

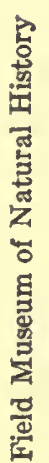
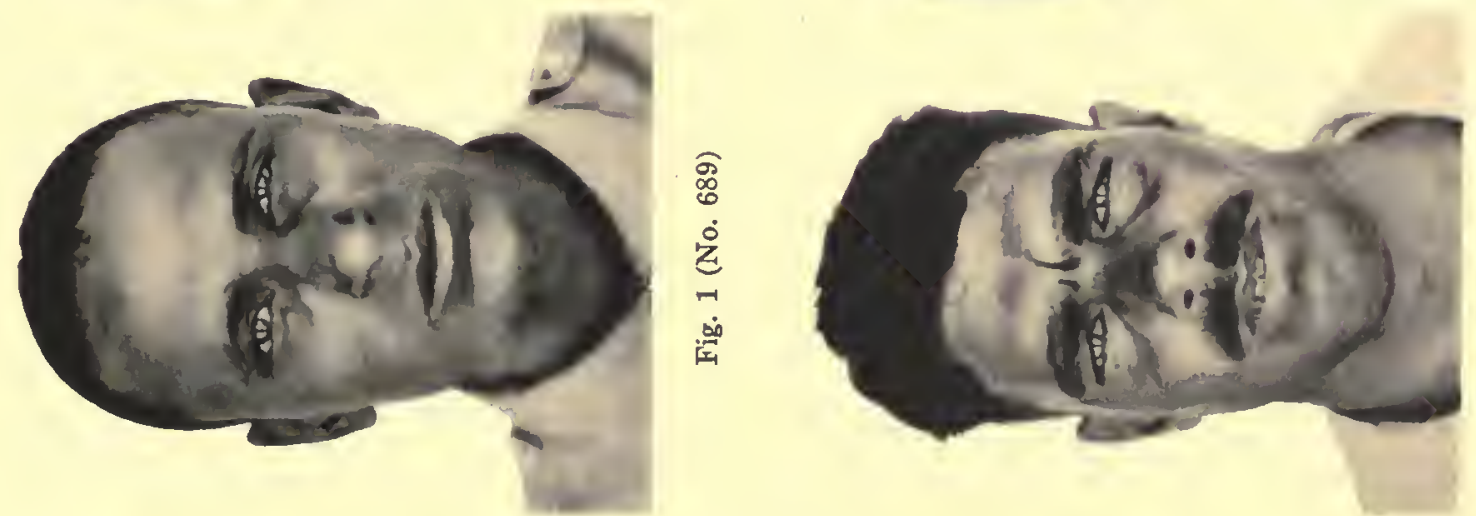

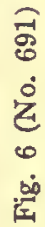

จำ

宅

我

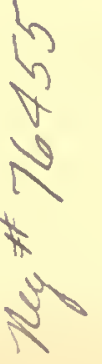



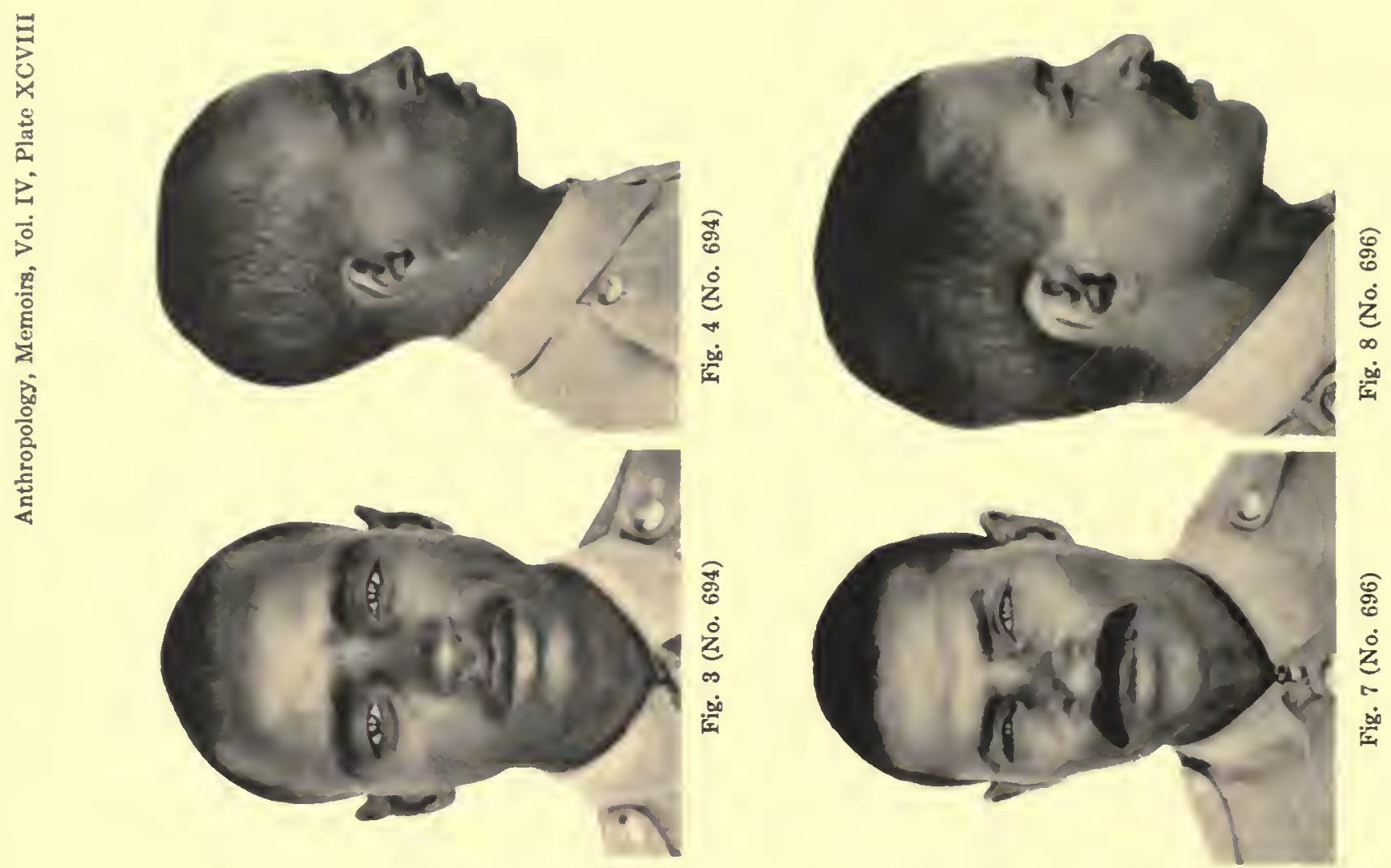

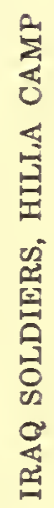
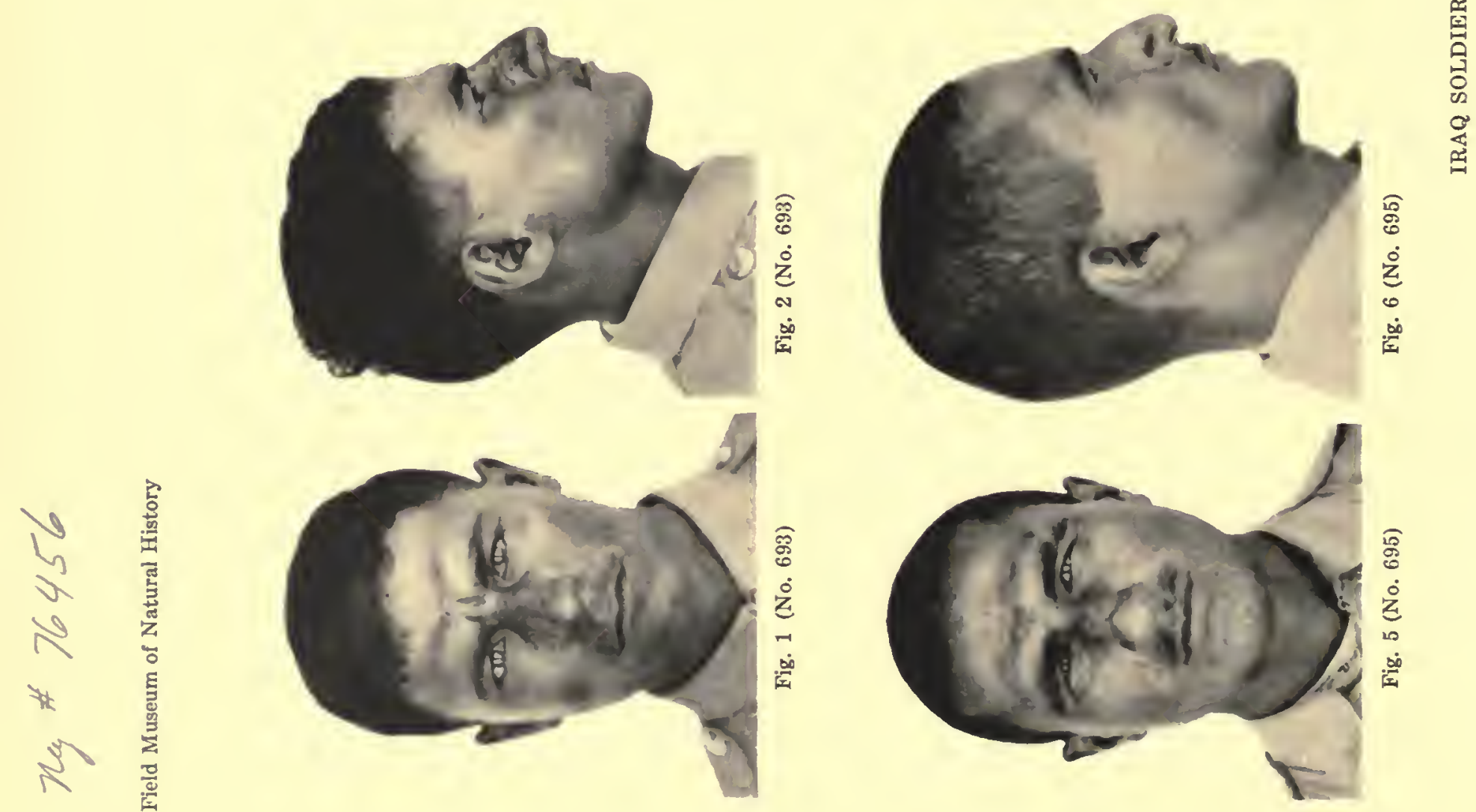
葆
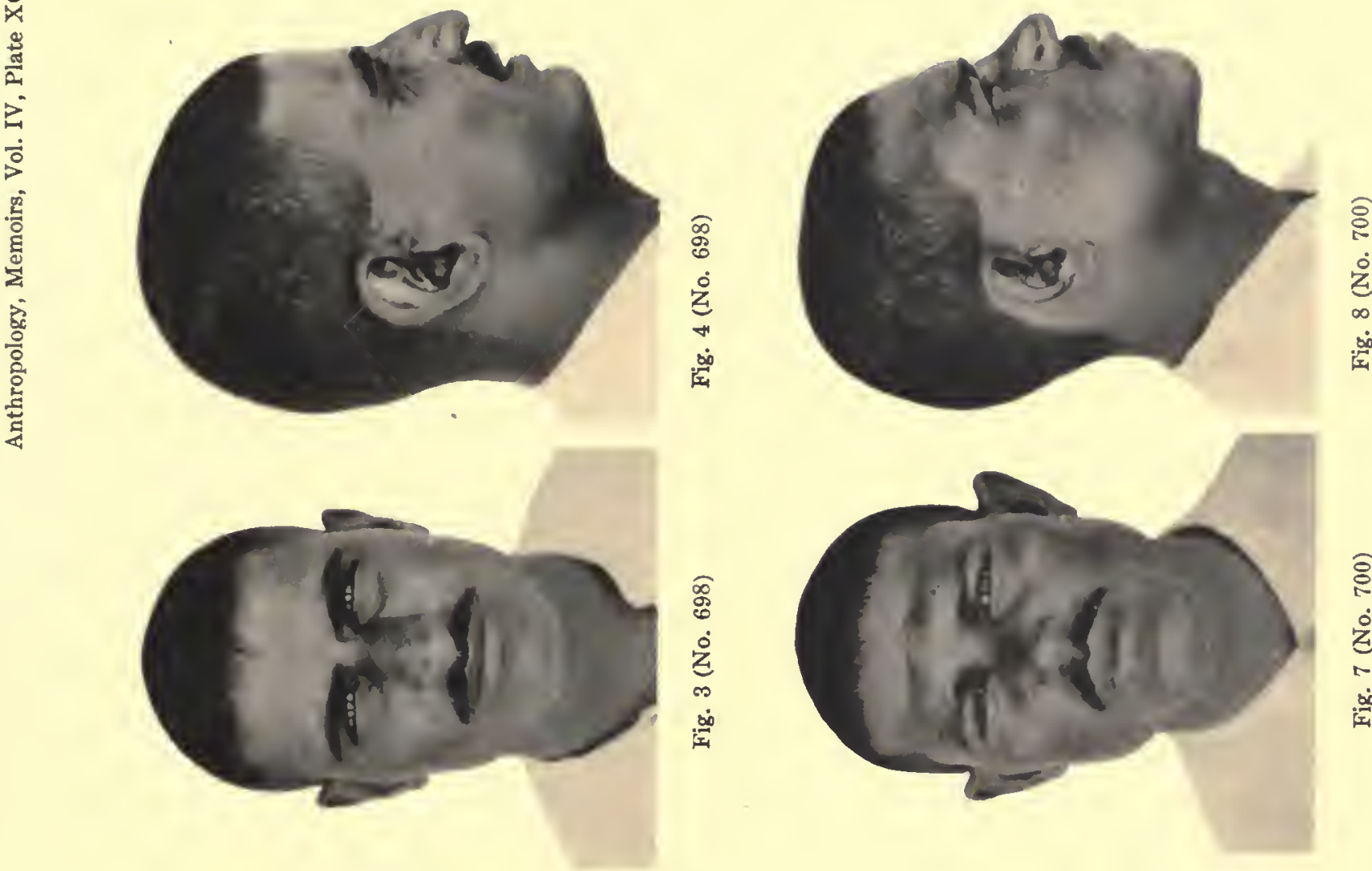

¿े
$\dot{0}$
$\infty$
$\infty$
$\dot{0}$
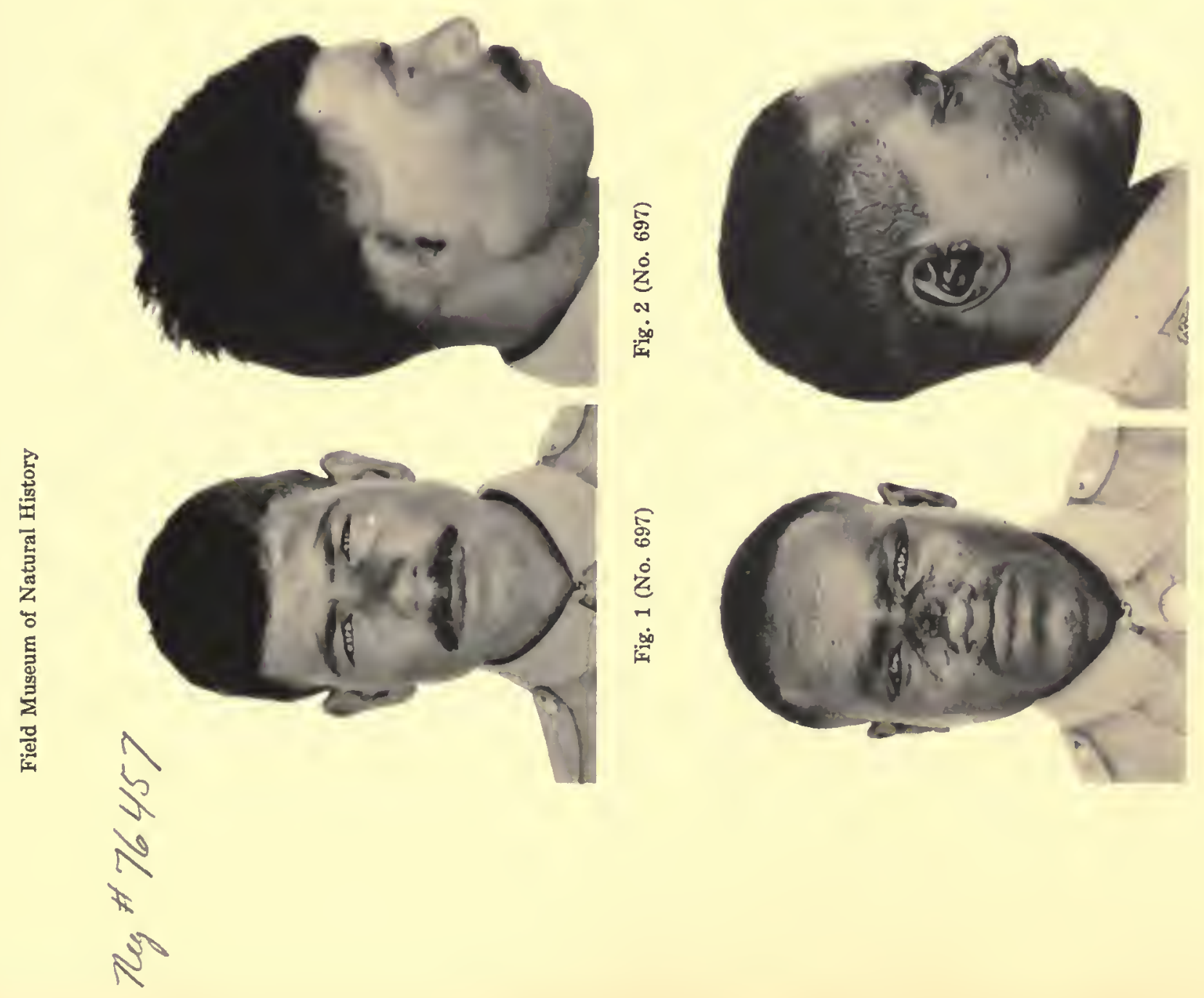

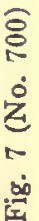

这

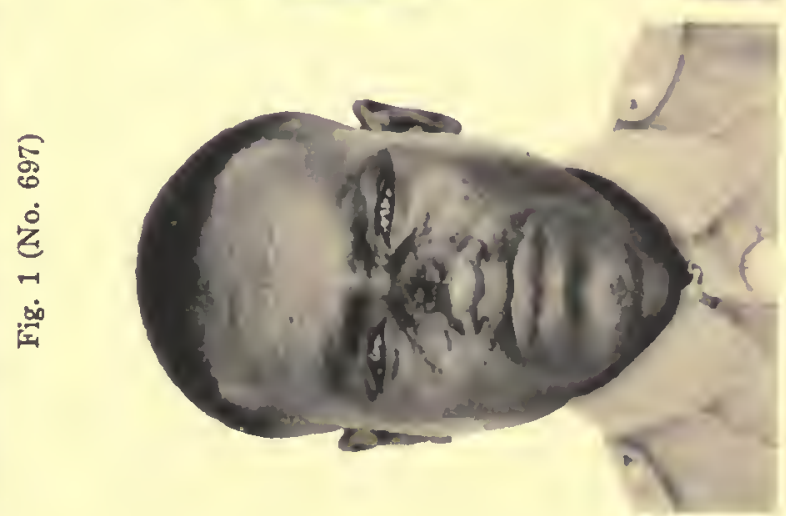

ब्
0
$\dot{8}$
$\dot{2}$
0
0
0

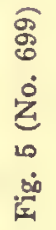


0
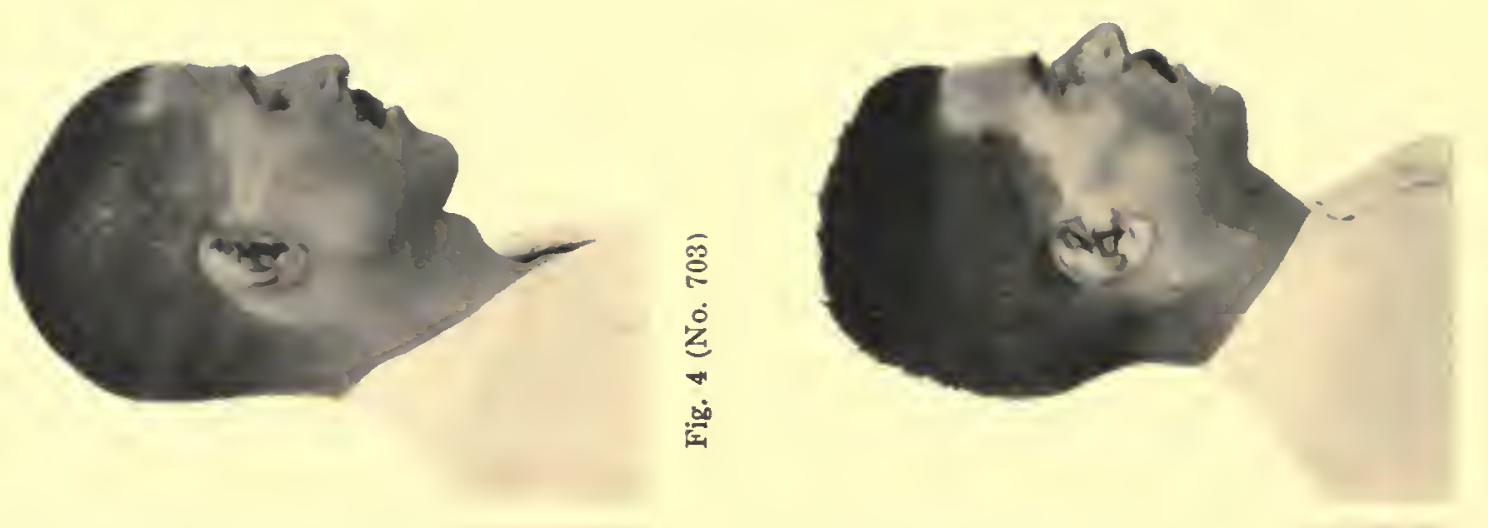

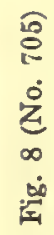
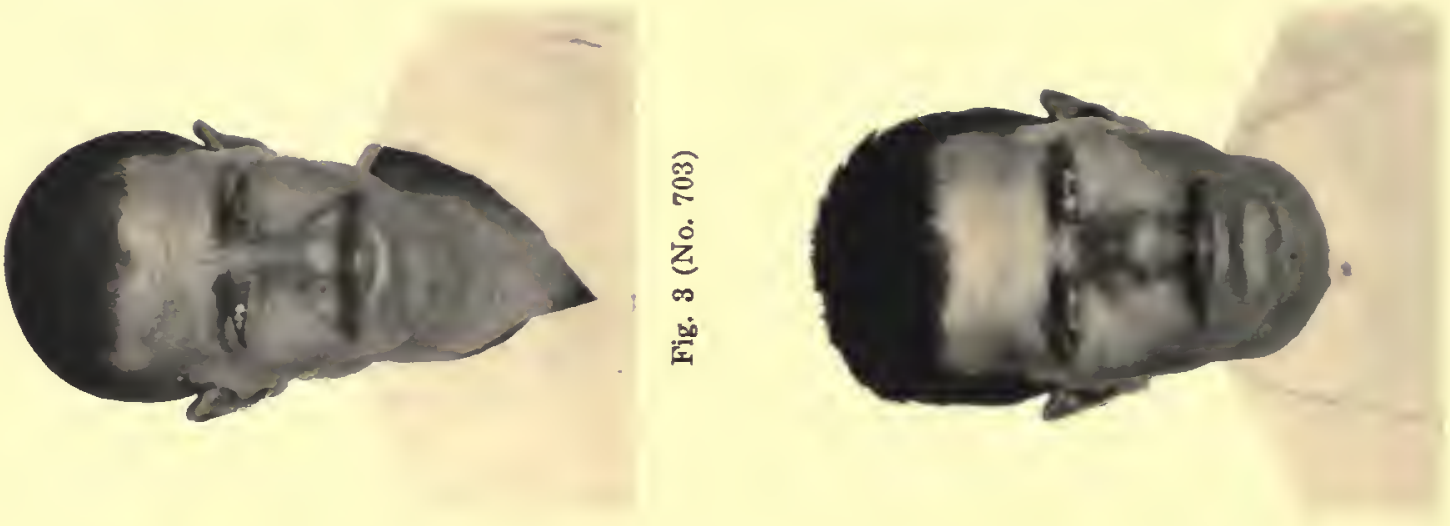

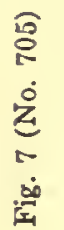
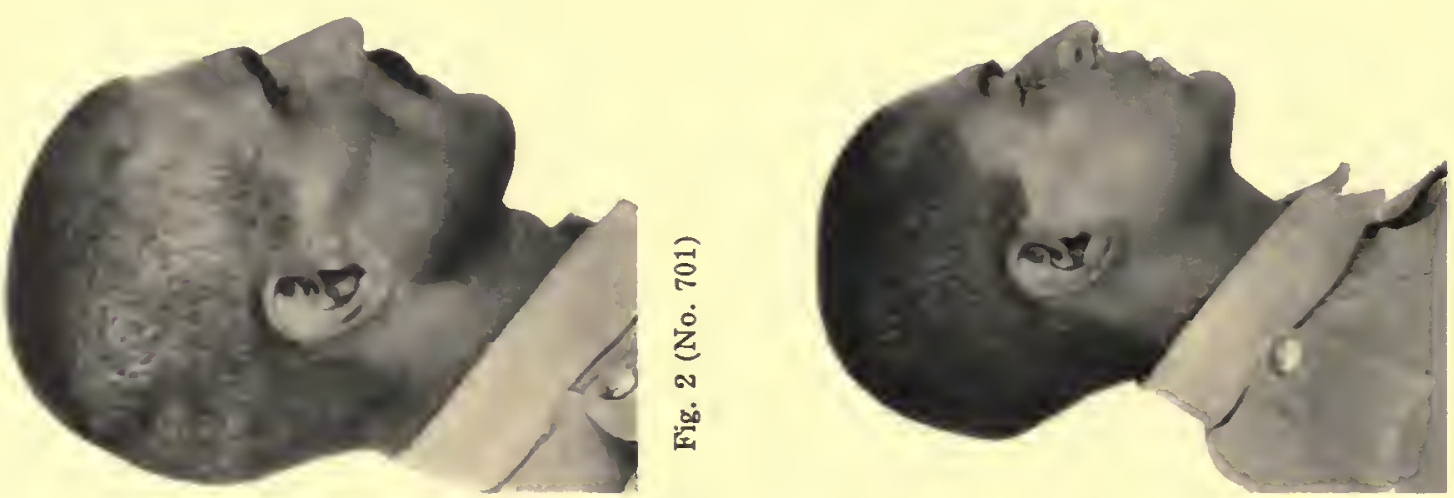

热

$b$
$x$
na
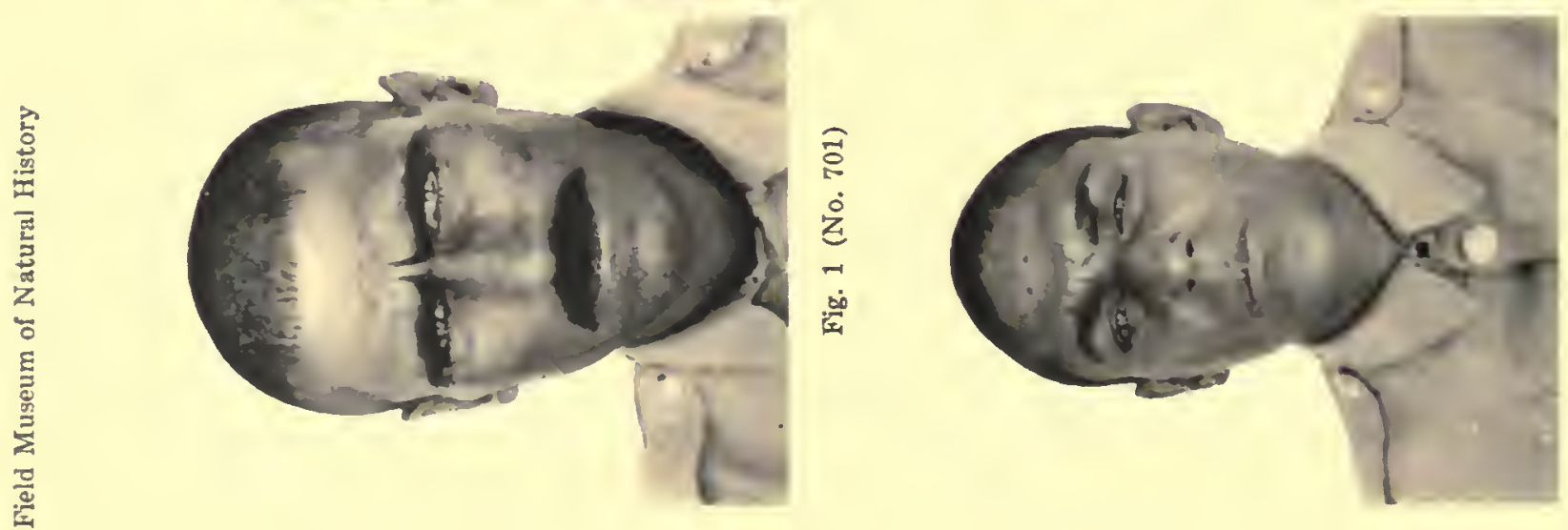

융
응
0
0
0

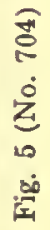

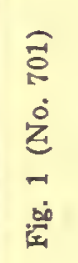


ज्ञ
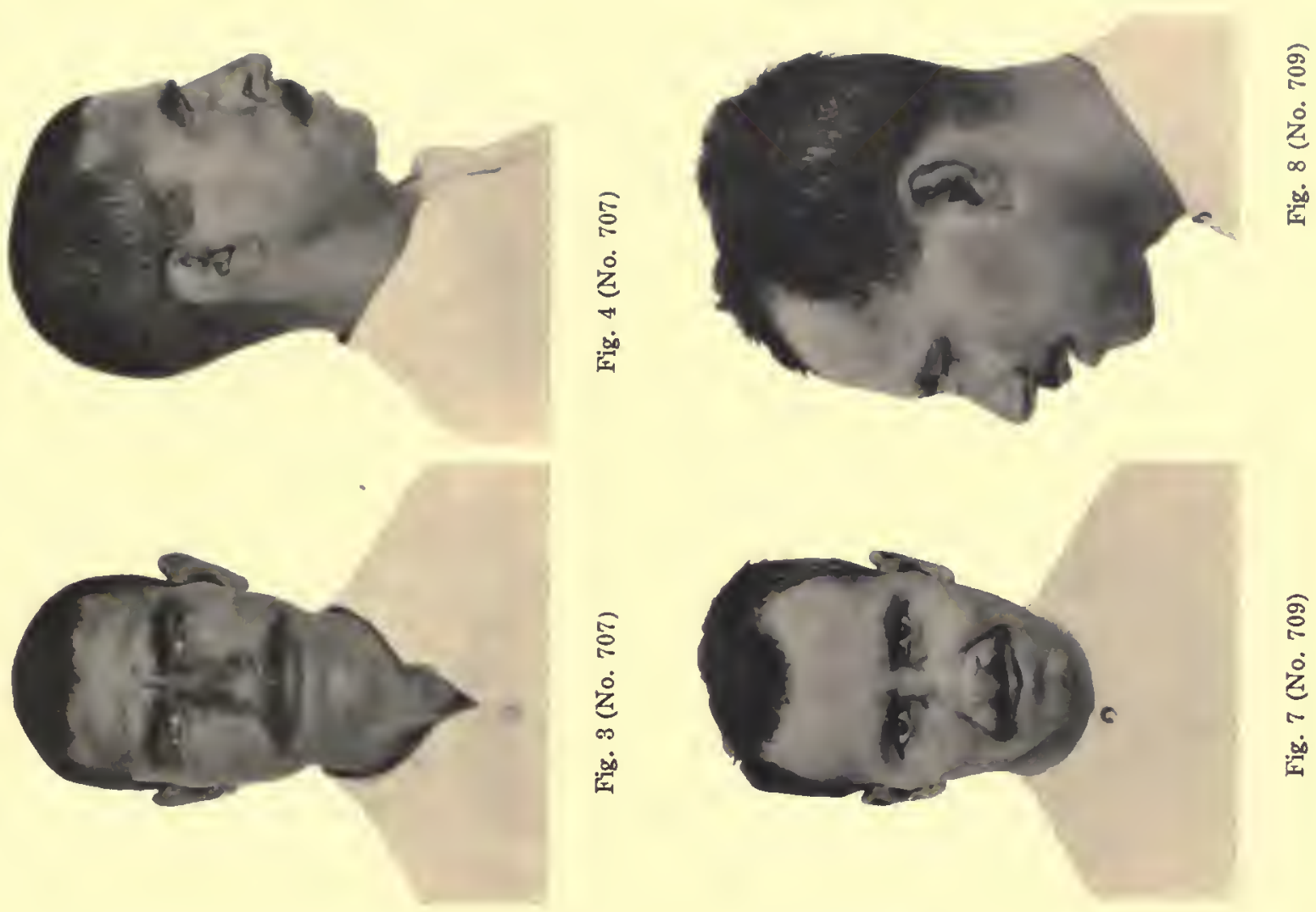

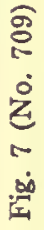
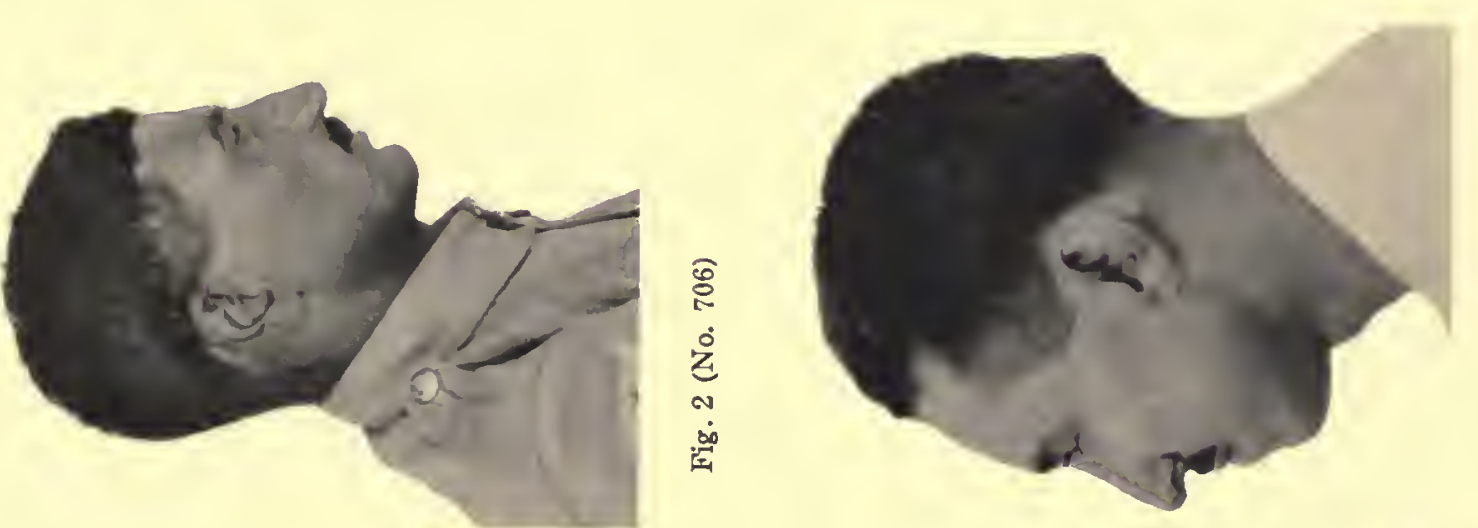

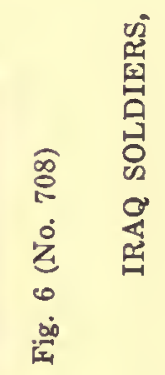
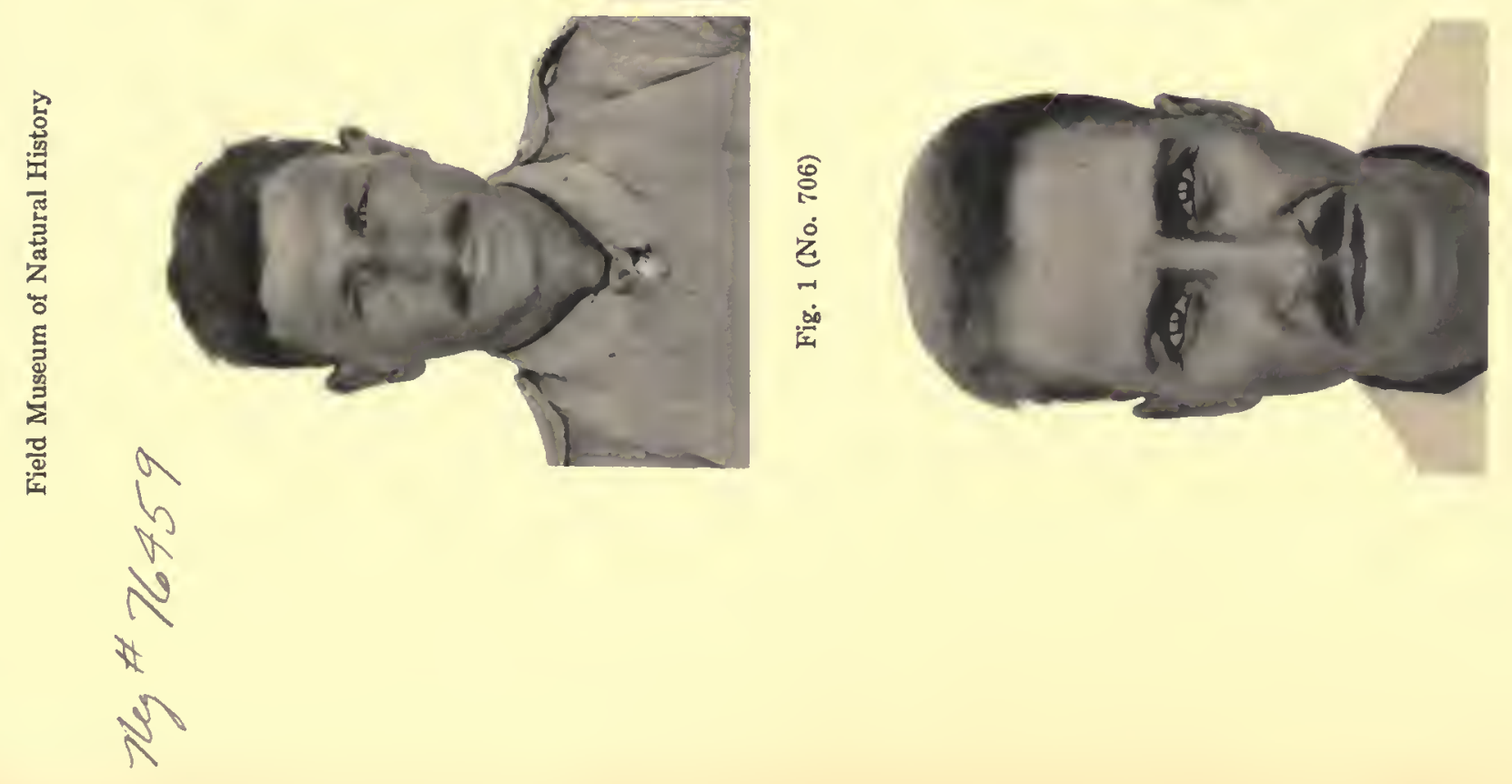

0
$\stackrel{0}{8}$
$\dot{0}$
20
20
$\dot{2}$

薄 

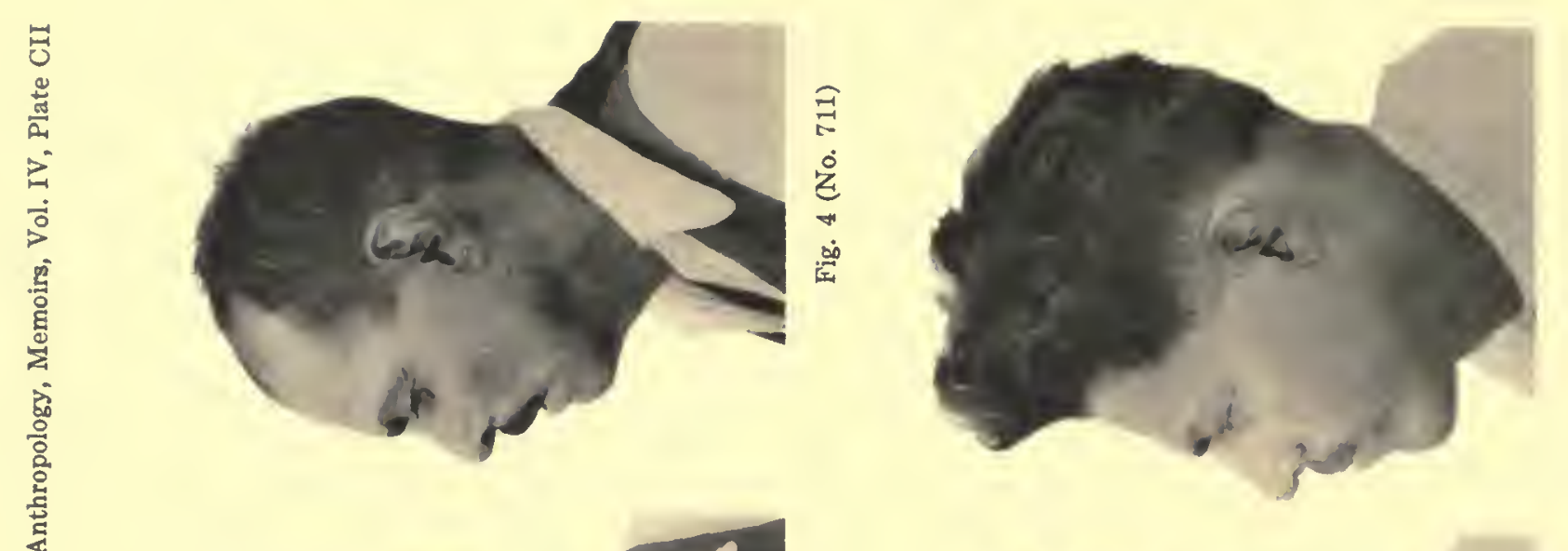

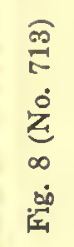
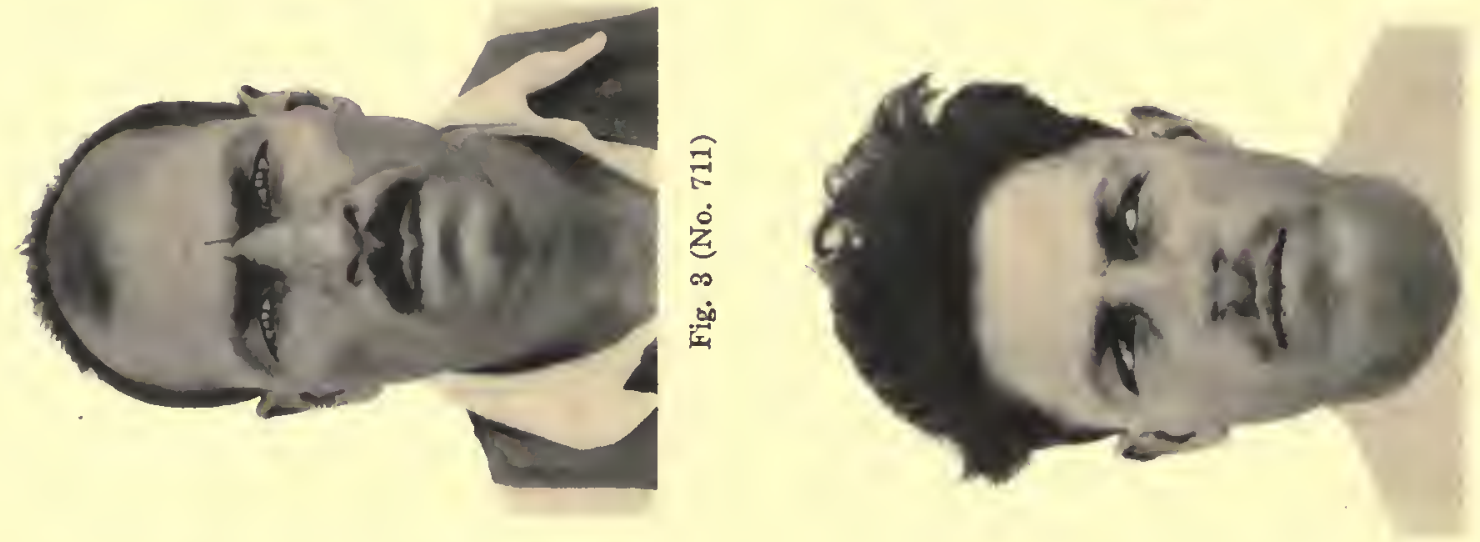

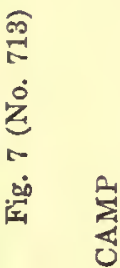
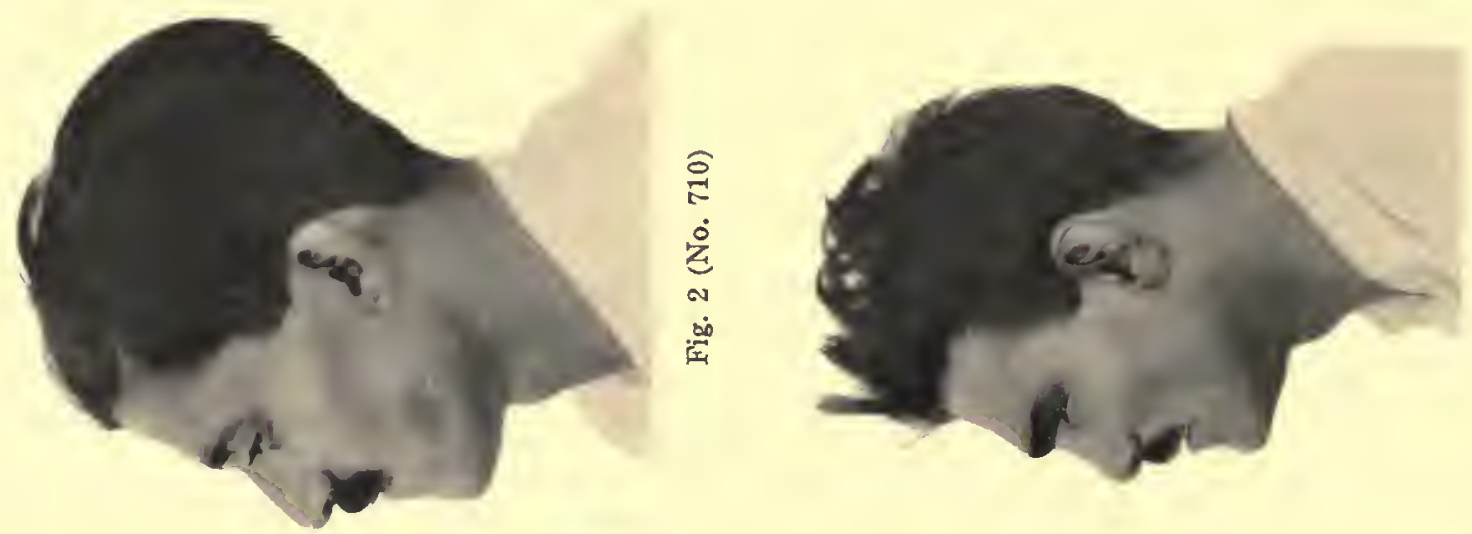

哥

0

$y$

$N$

k
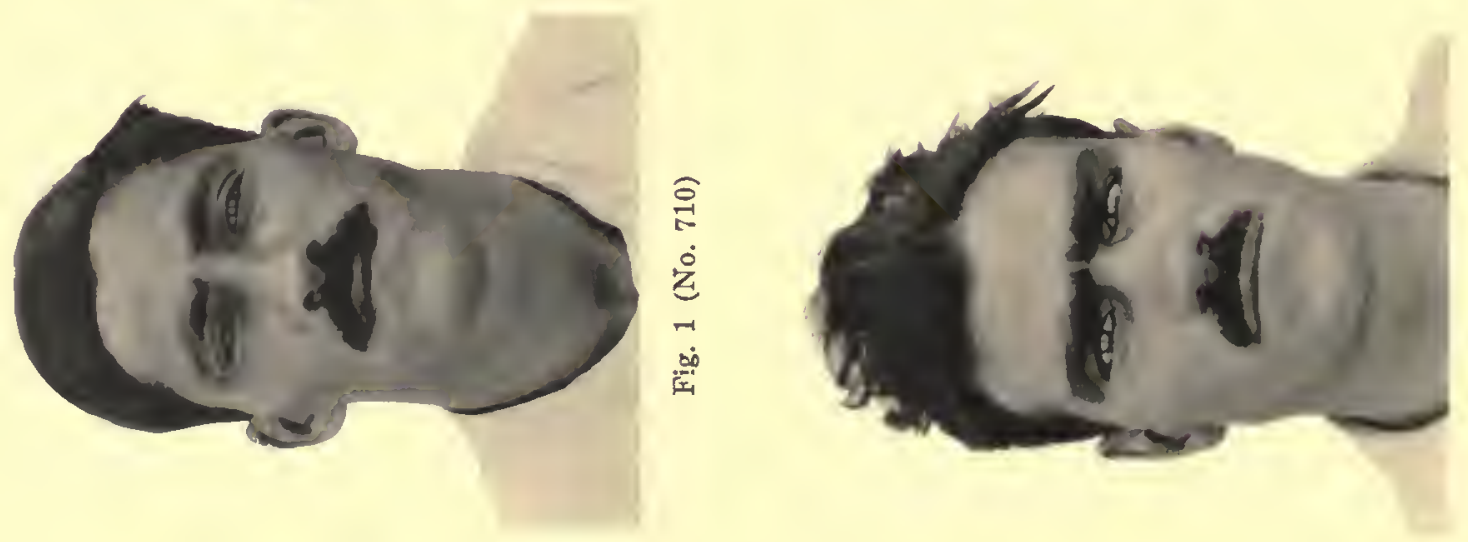

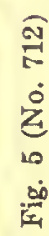

N)

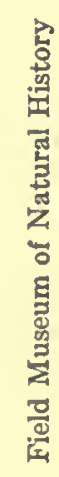




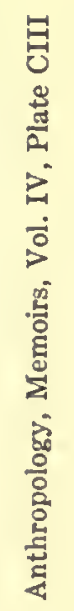
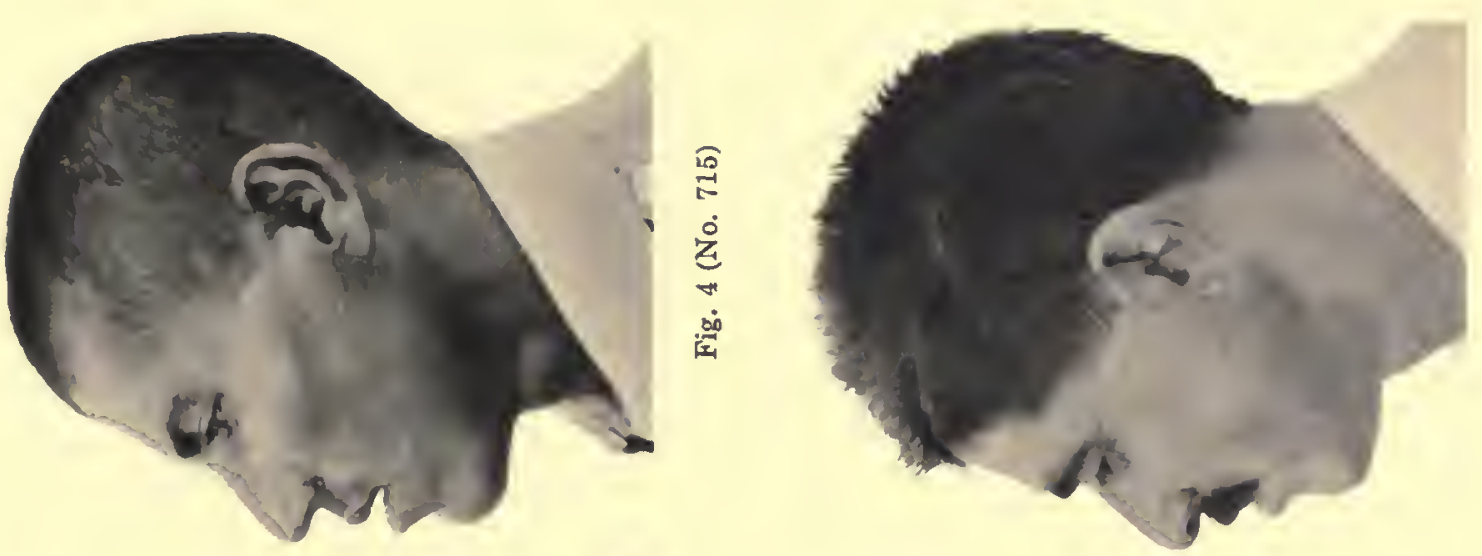

害
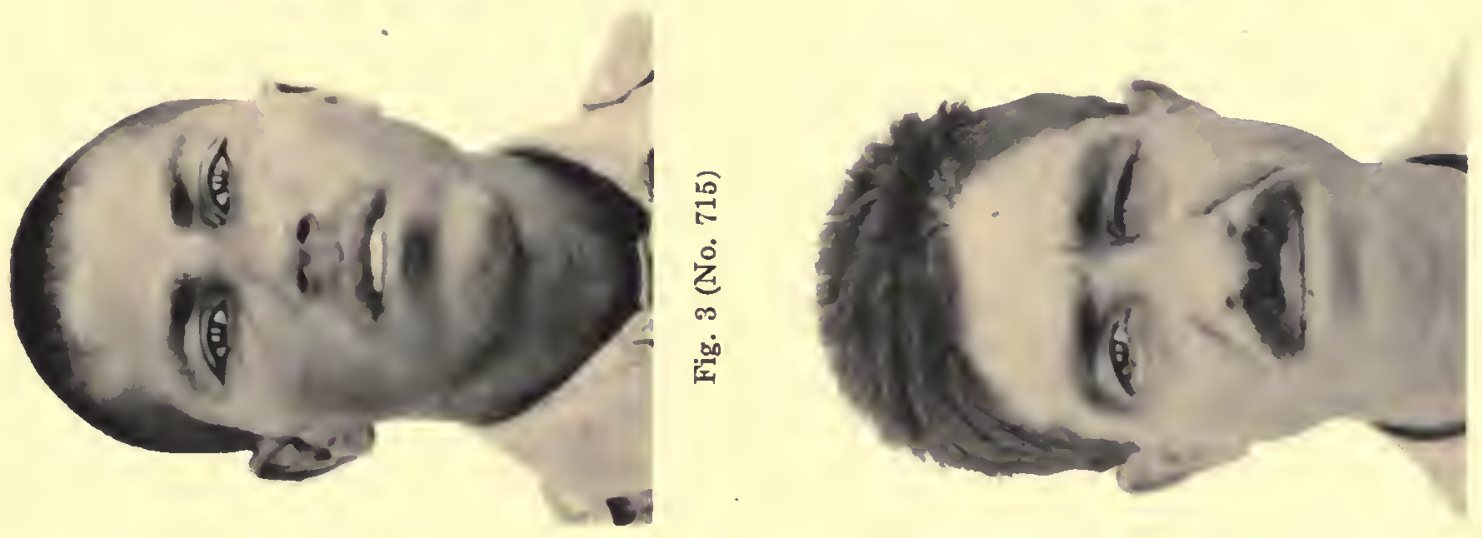

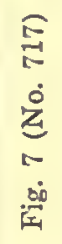

这

哥
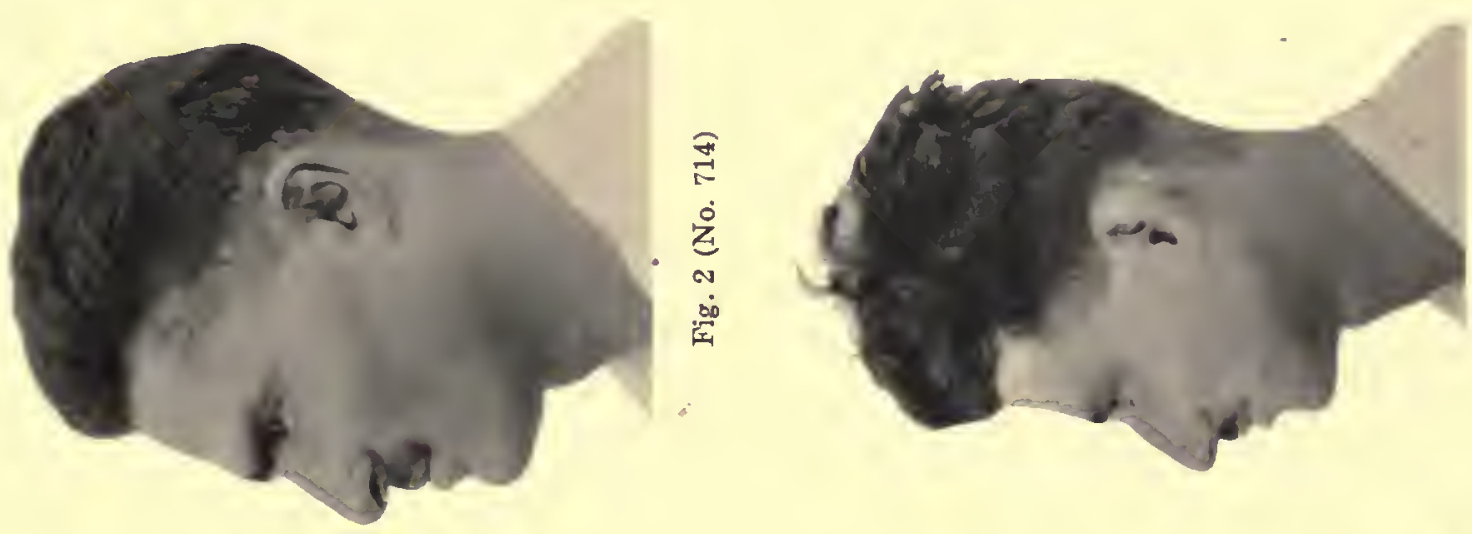

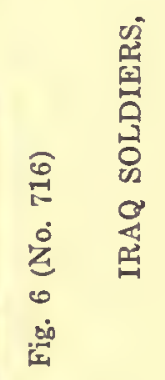
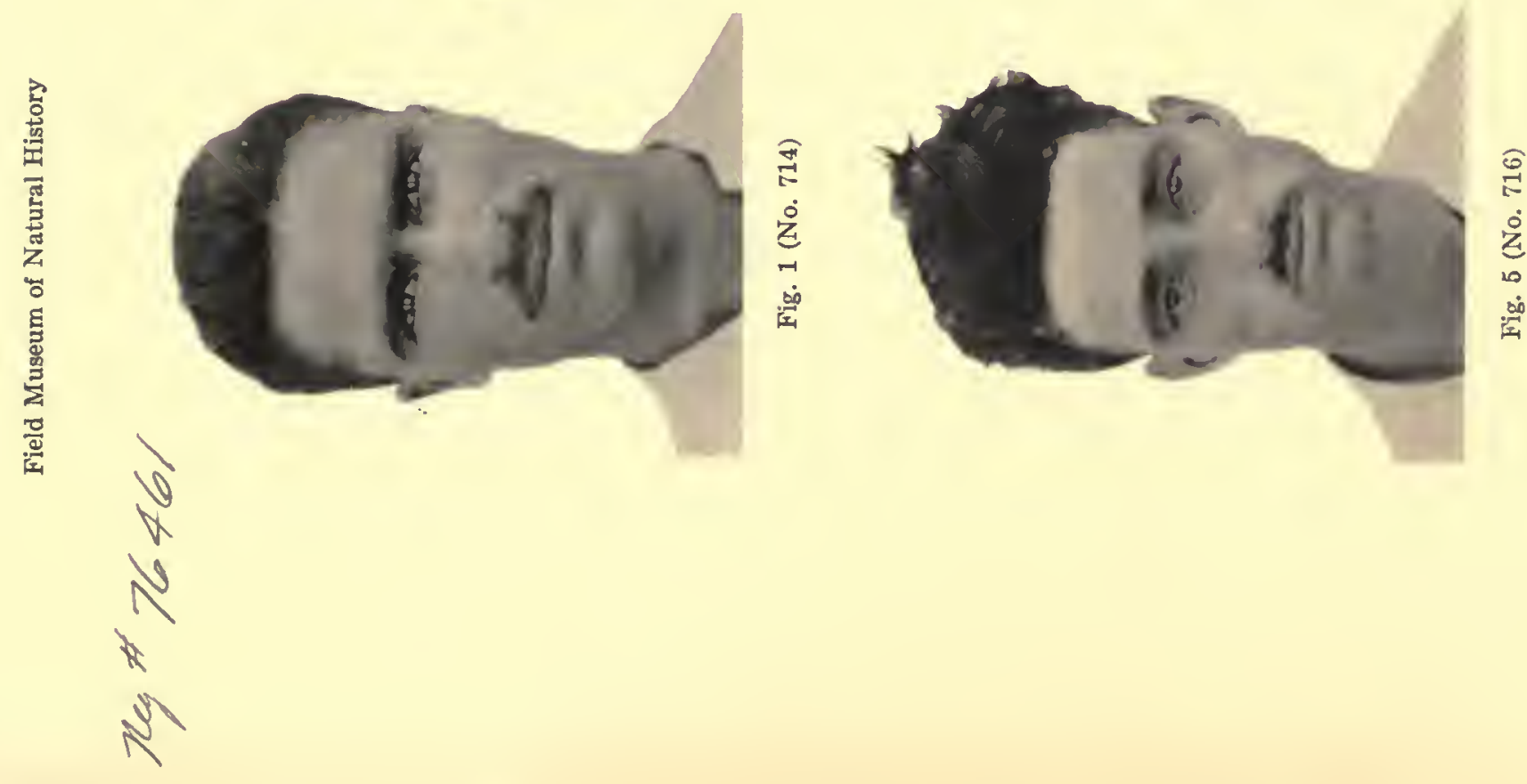

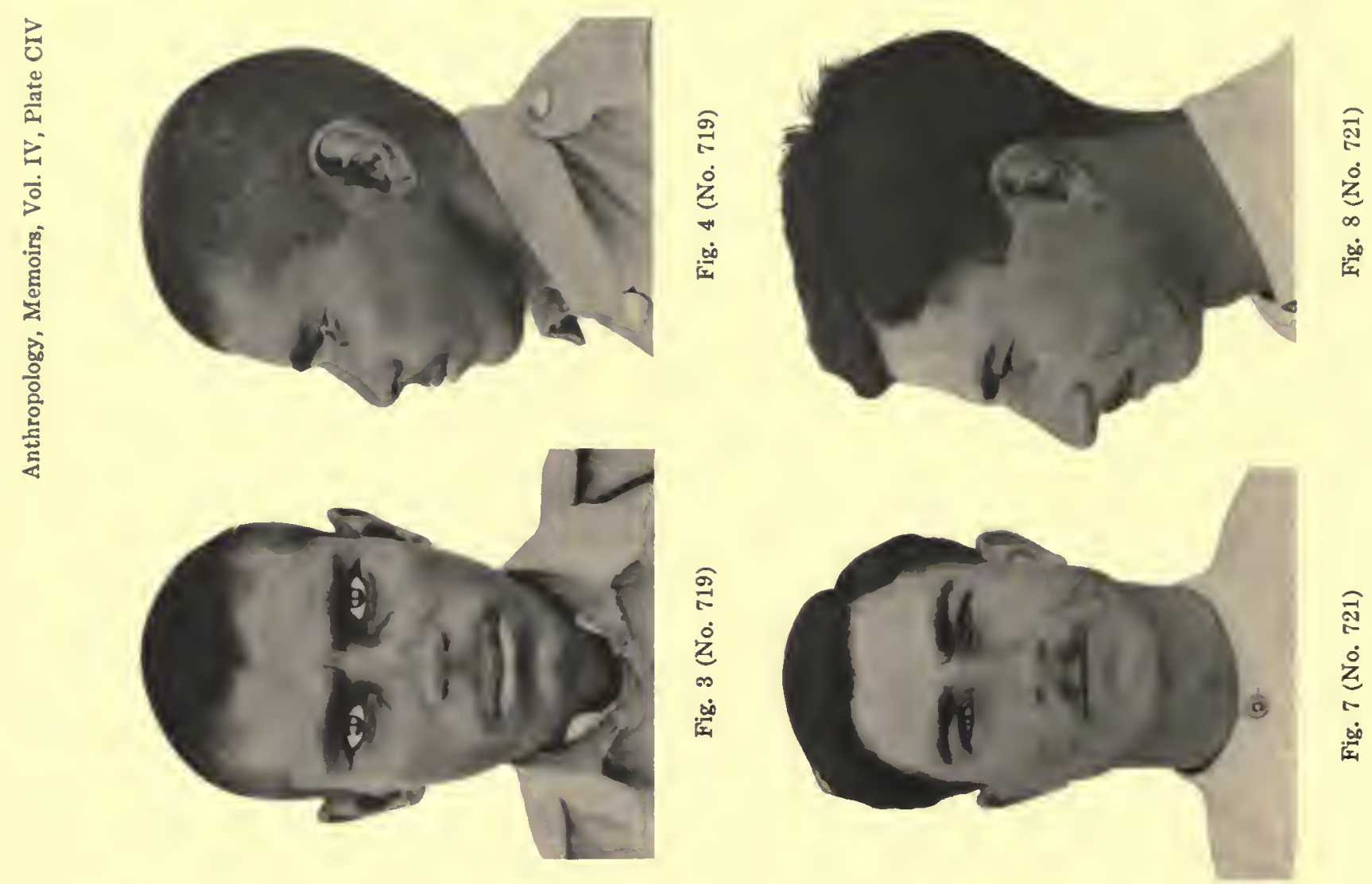

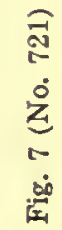
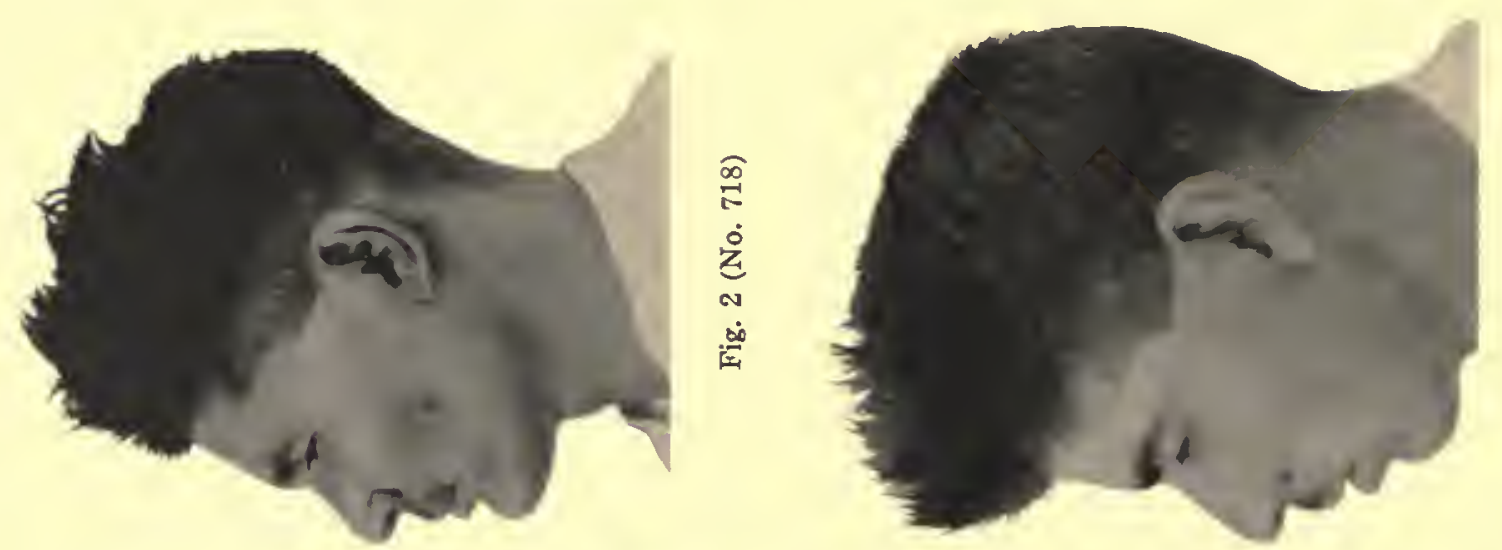

运

紊
哥

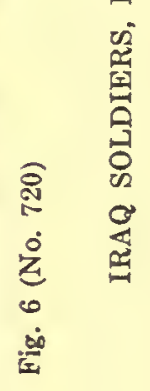

8
$y$
$y$
$y$
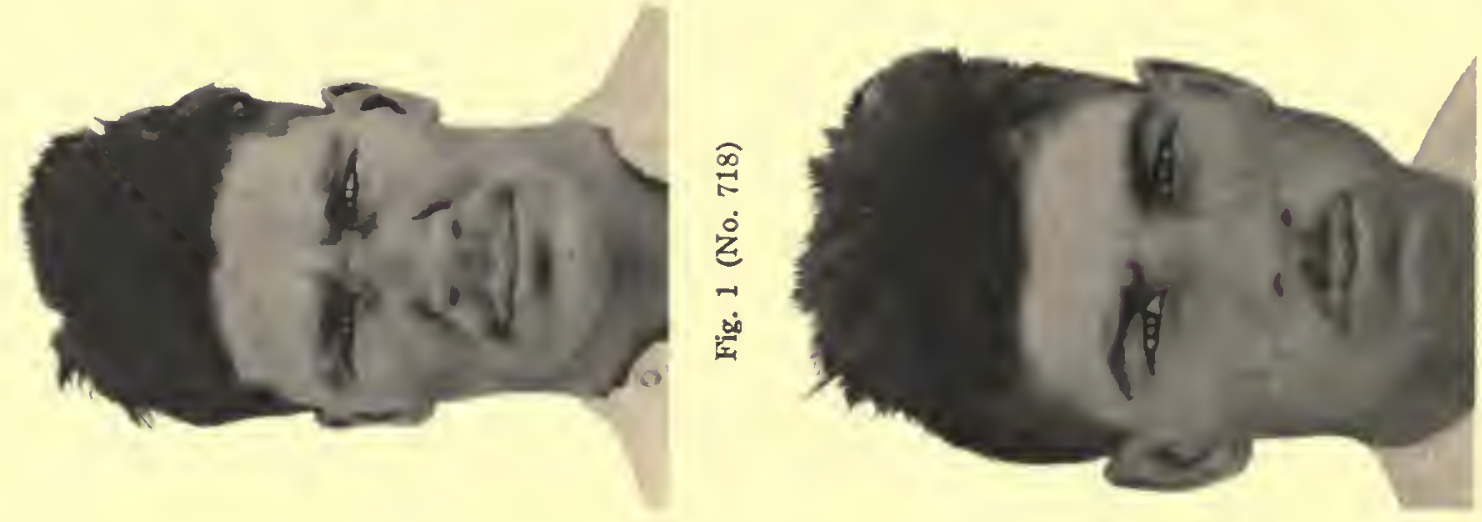

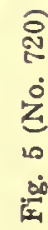



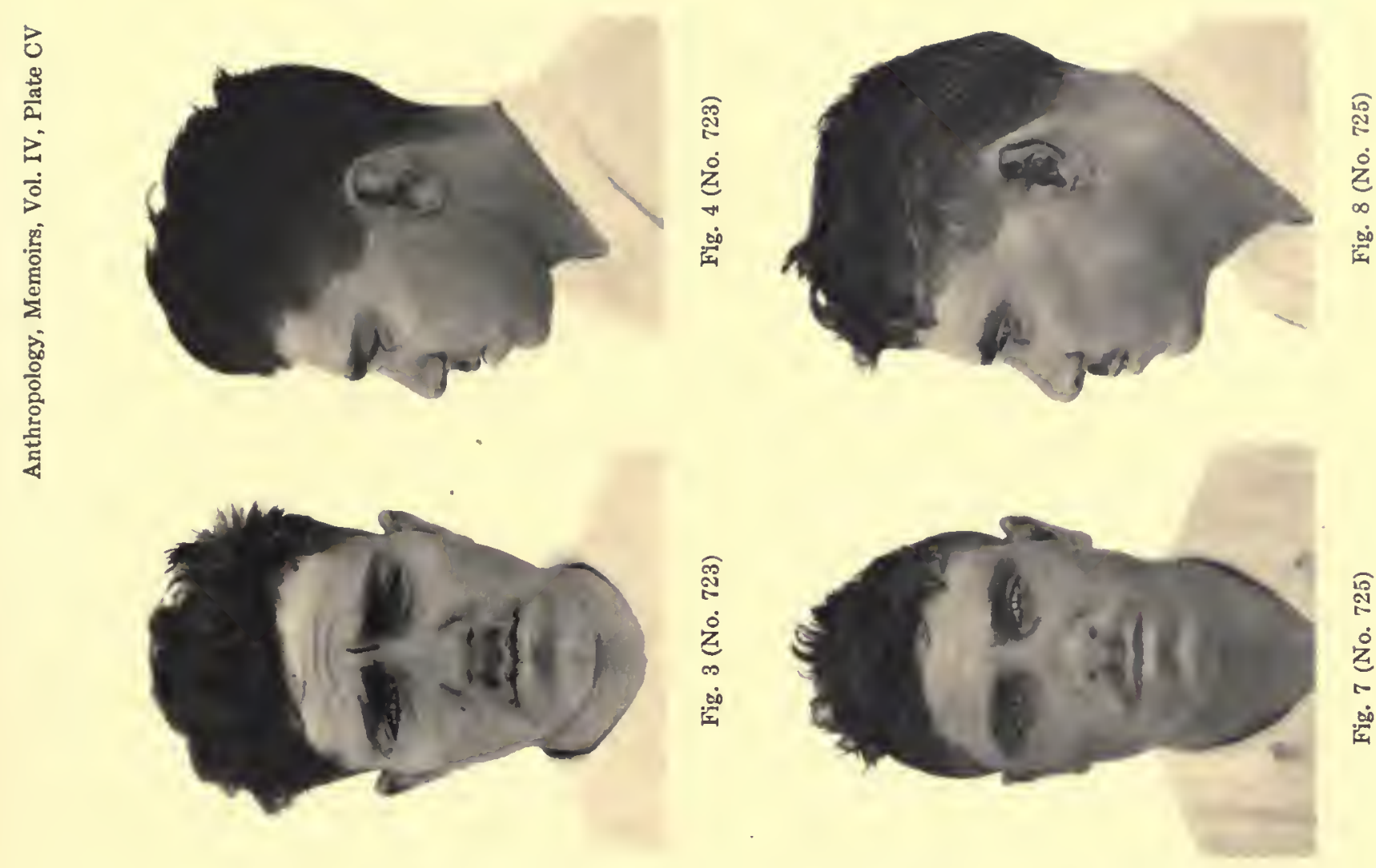

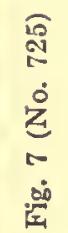
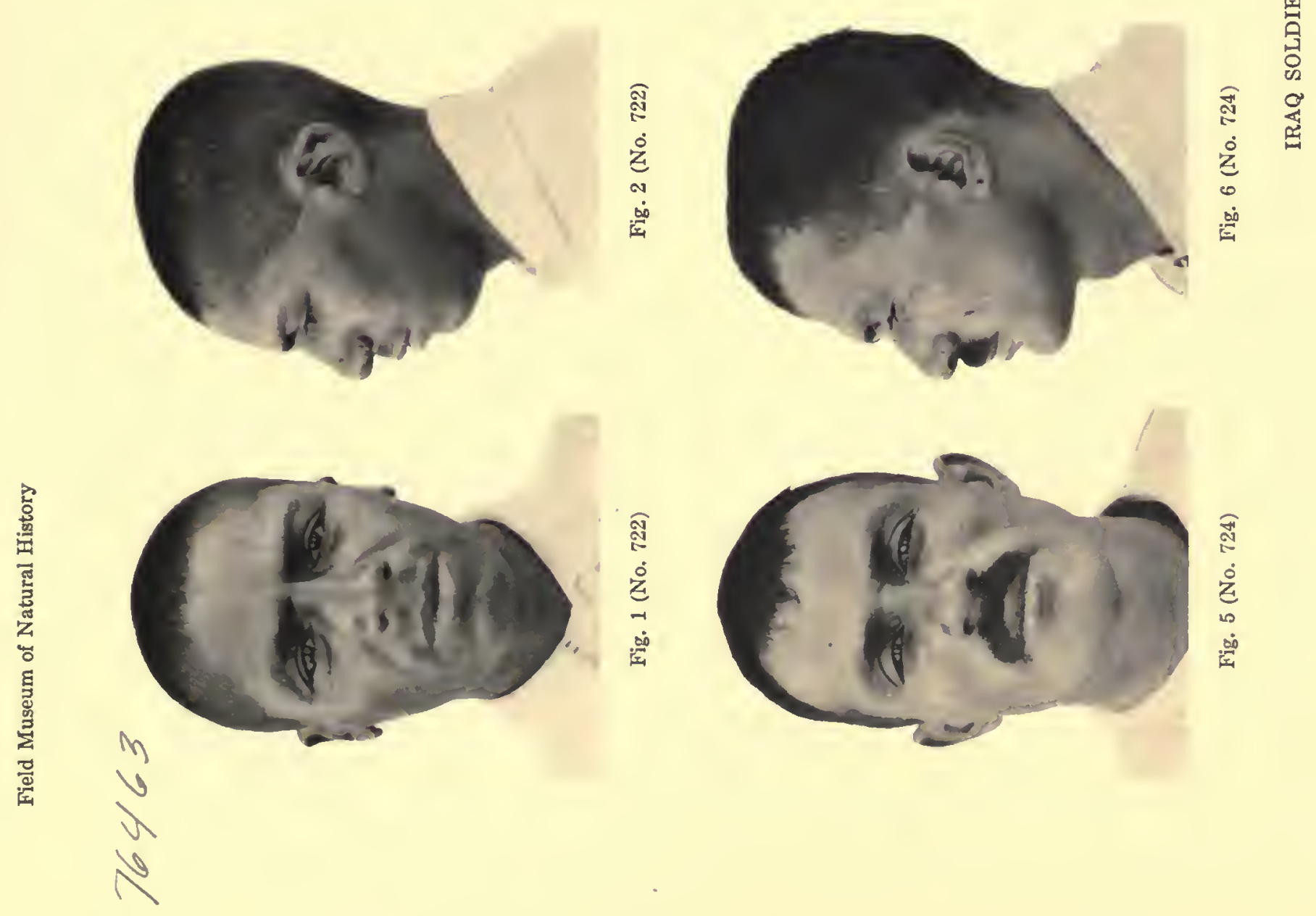


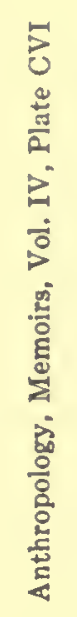
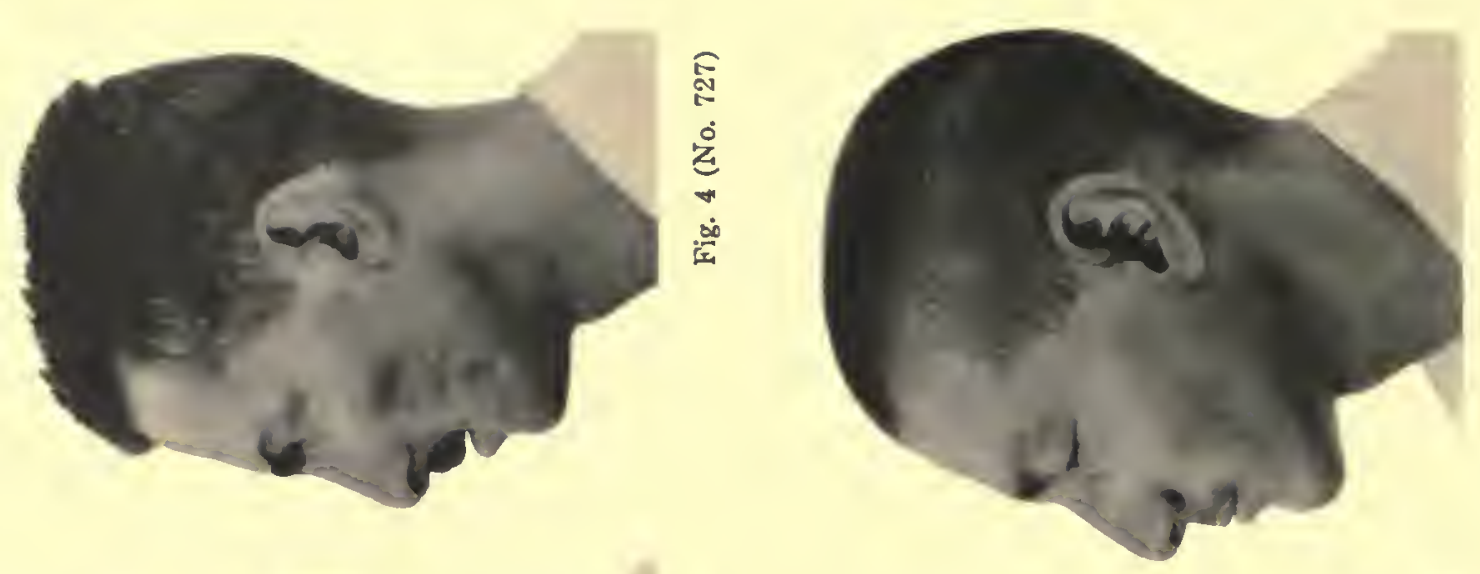

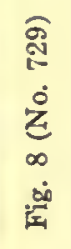
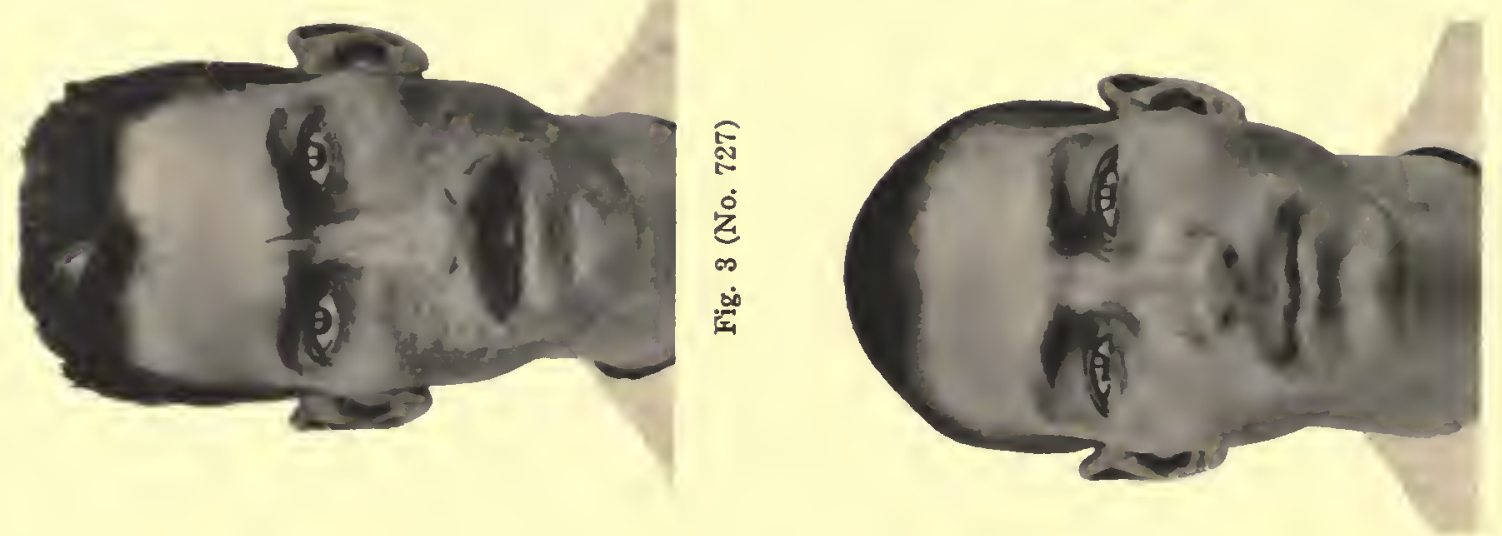

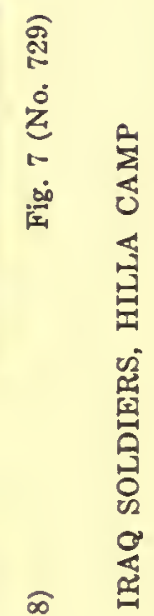
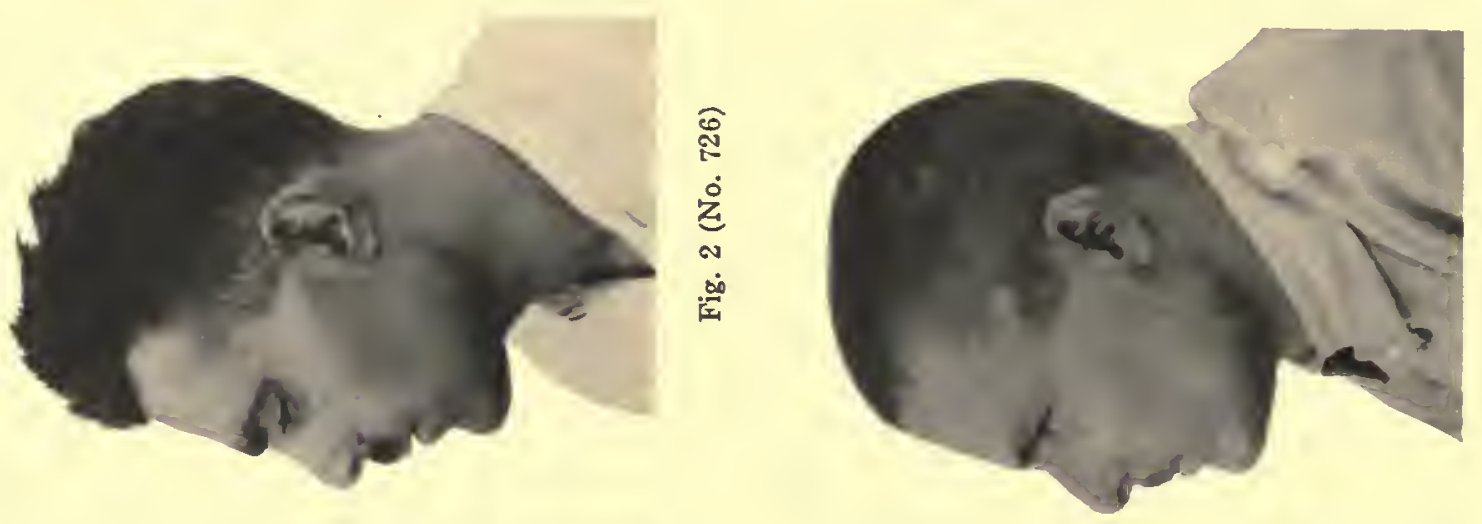

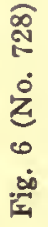
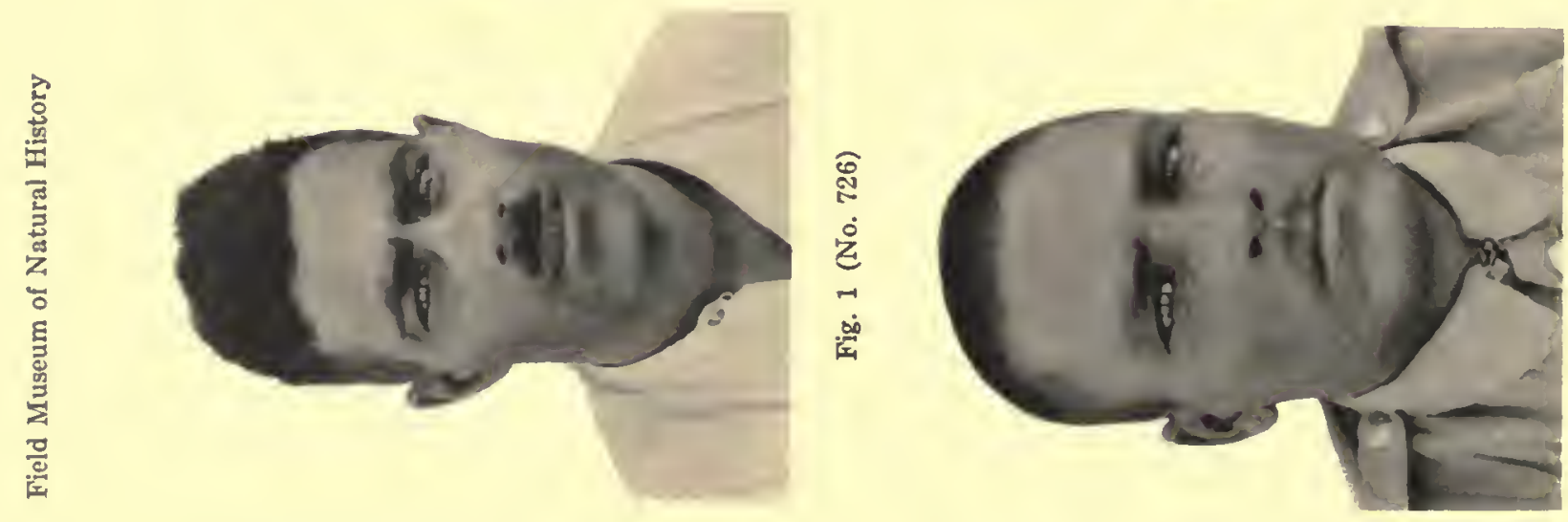

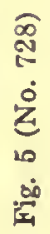

J 


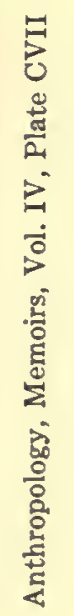
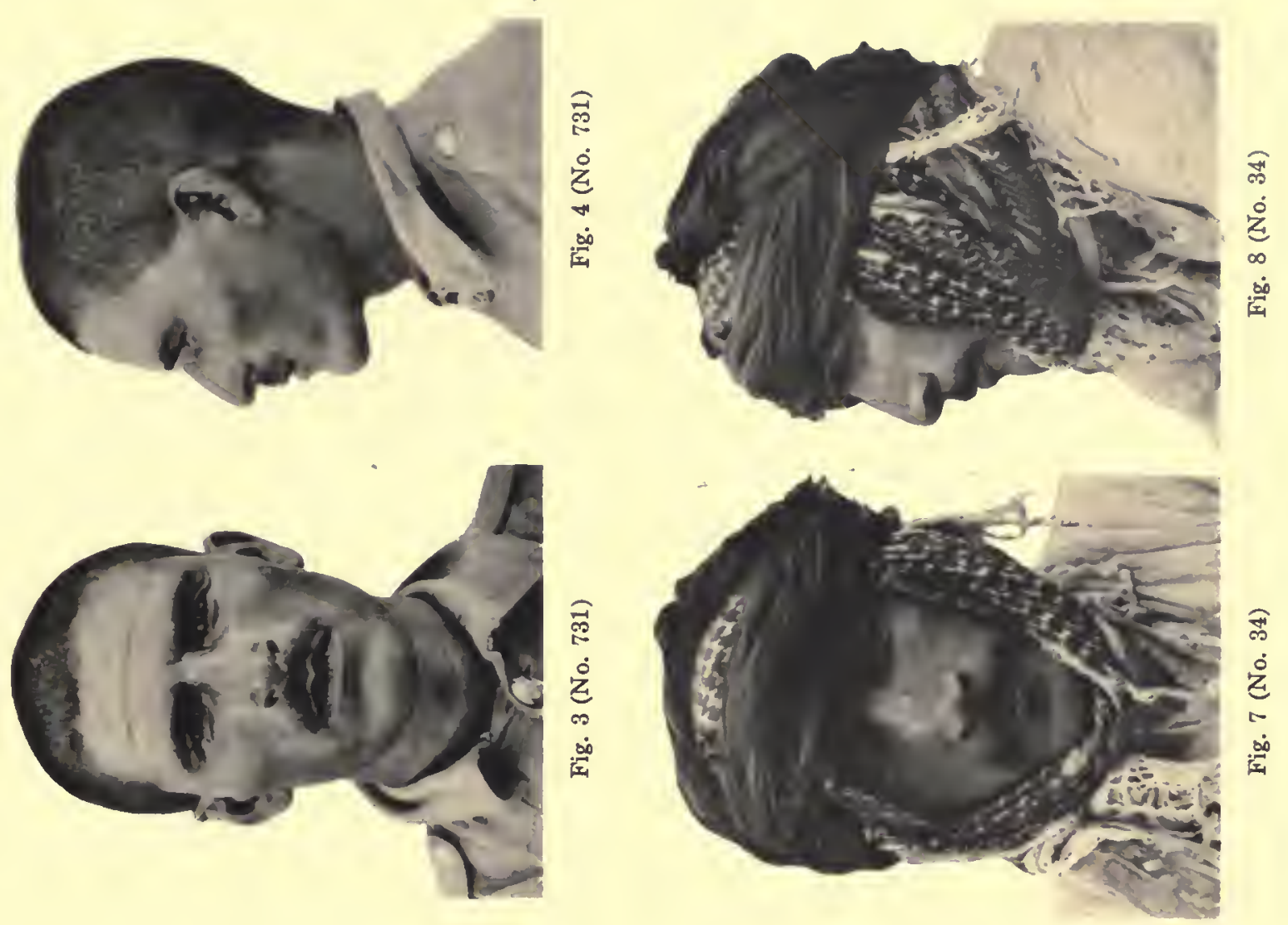

ก)

急

阏

¿ํ.

罚

$\infty$

然
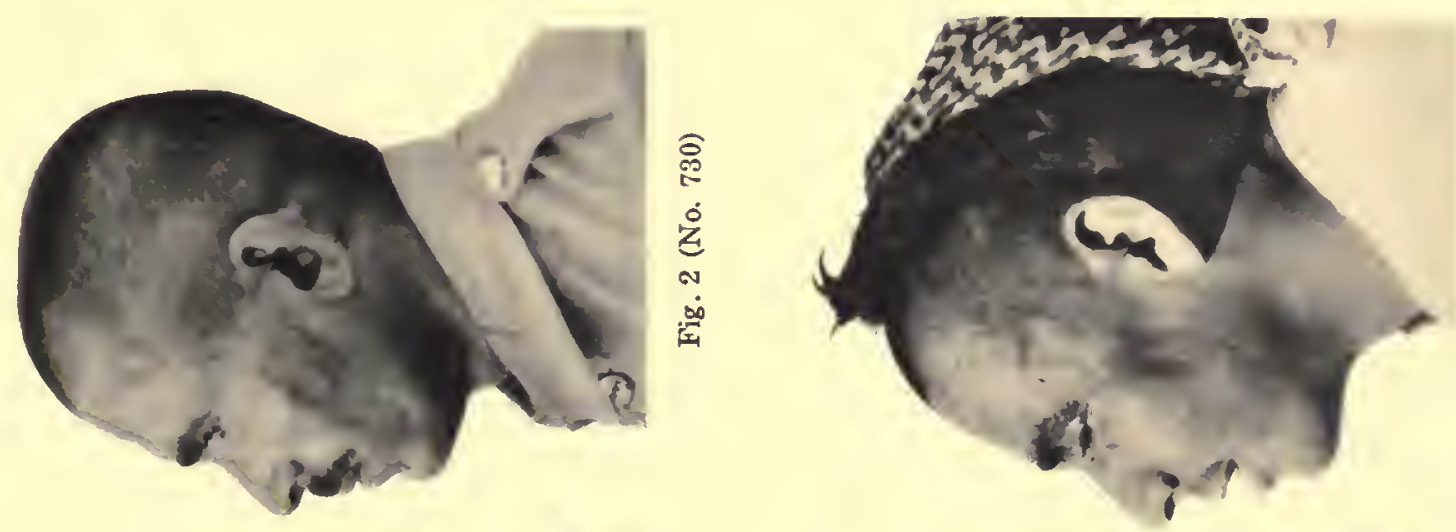

要
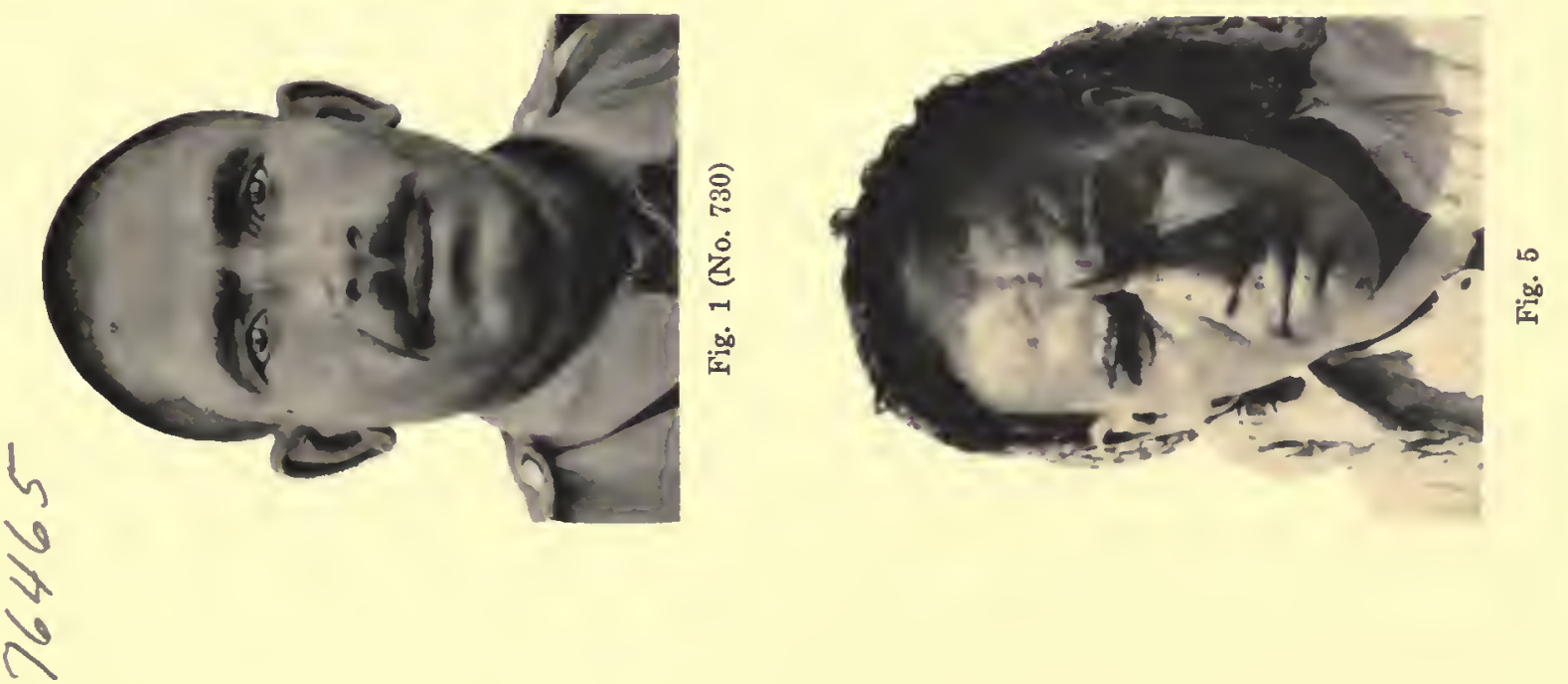

点悬

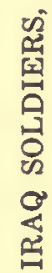

.

No 


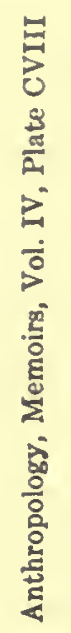
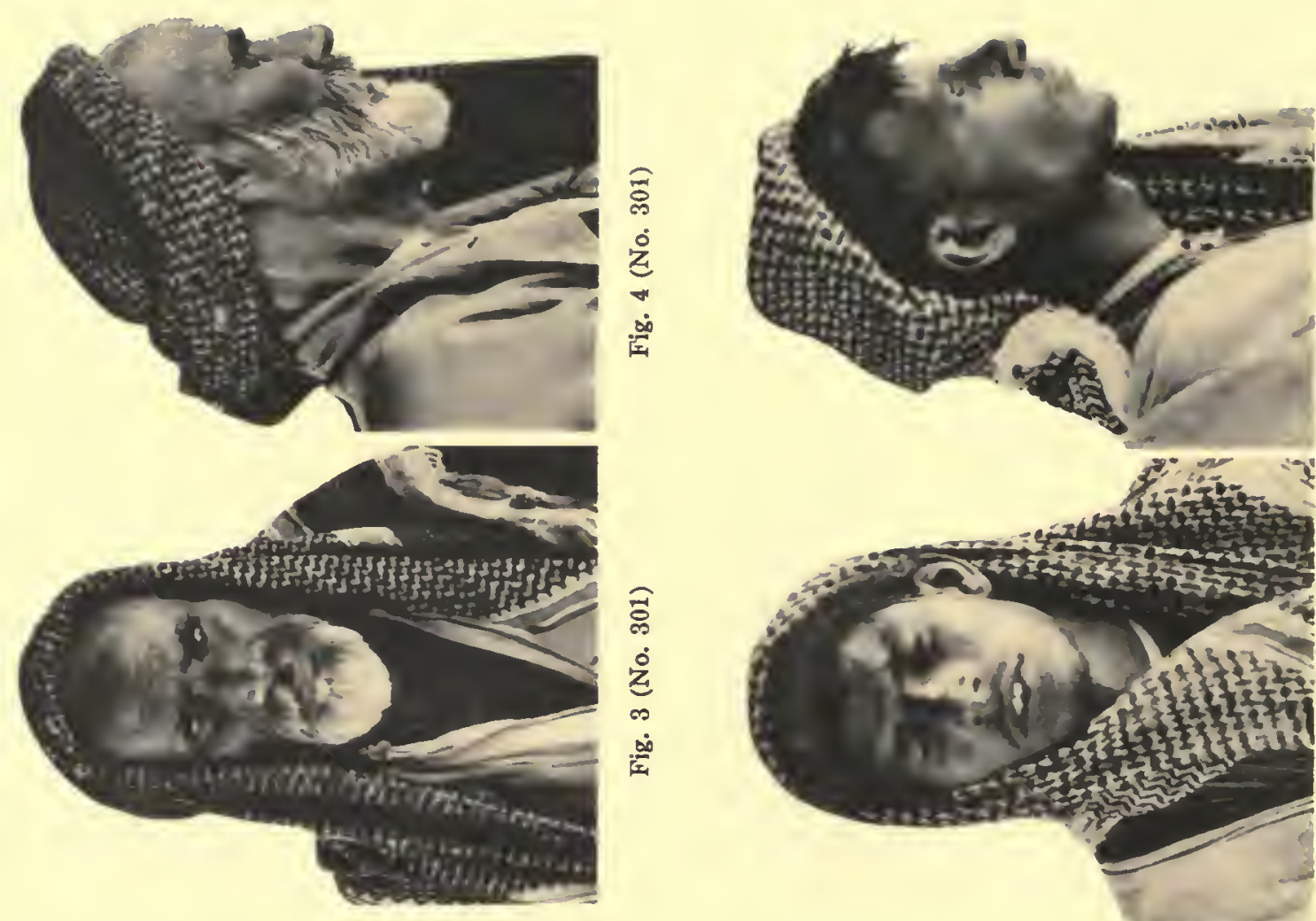

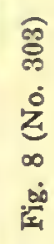

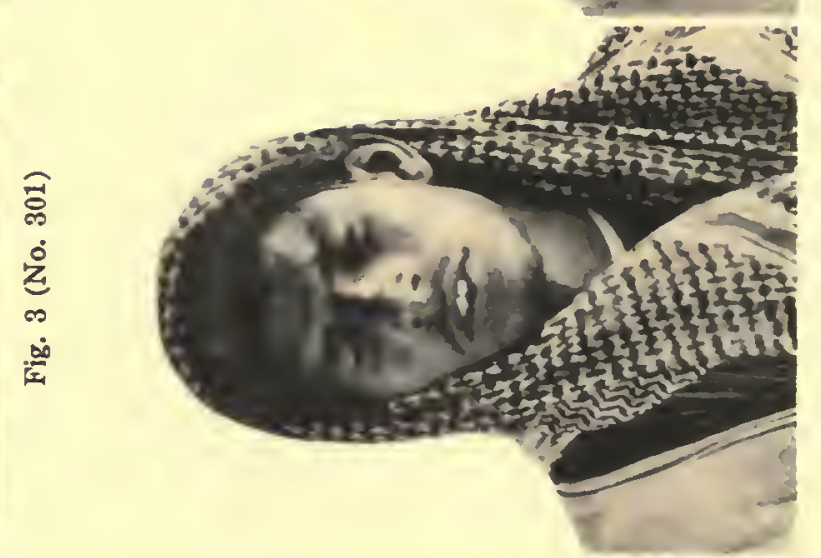

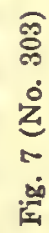
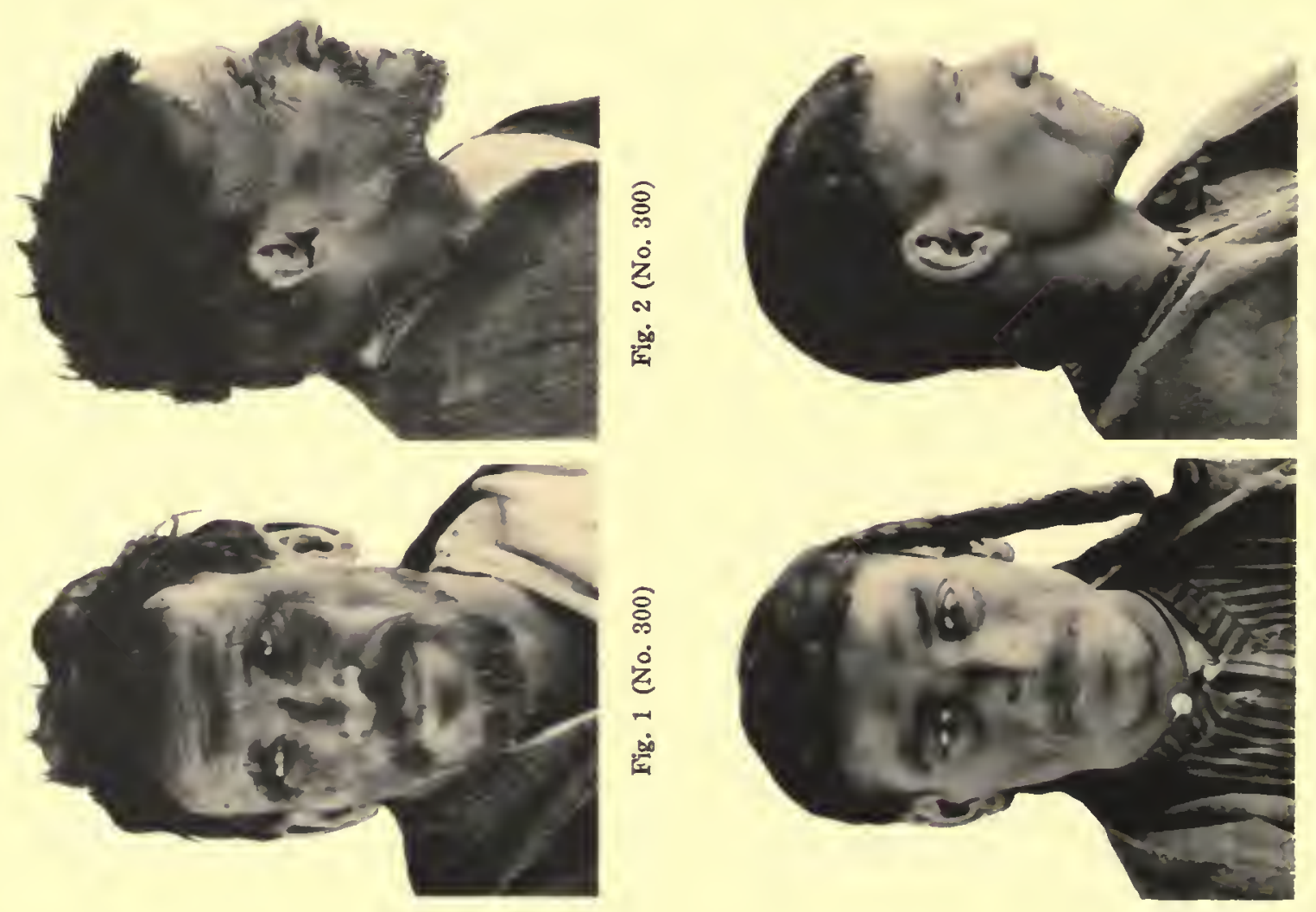

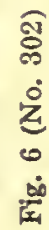



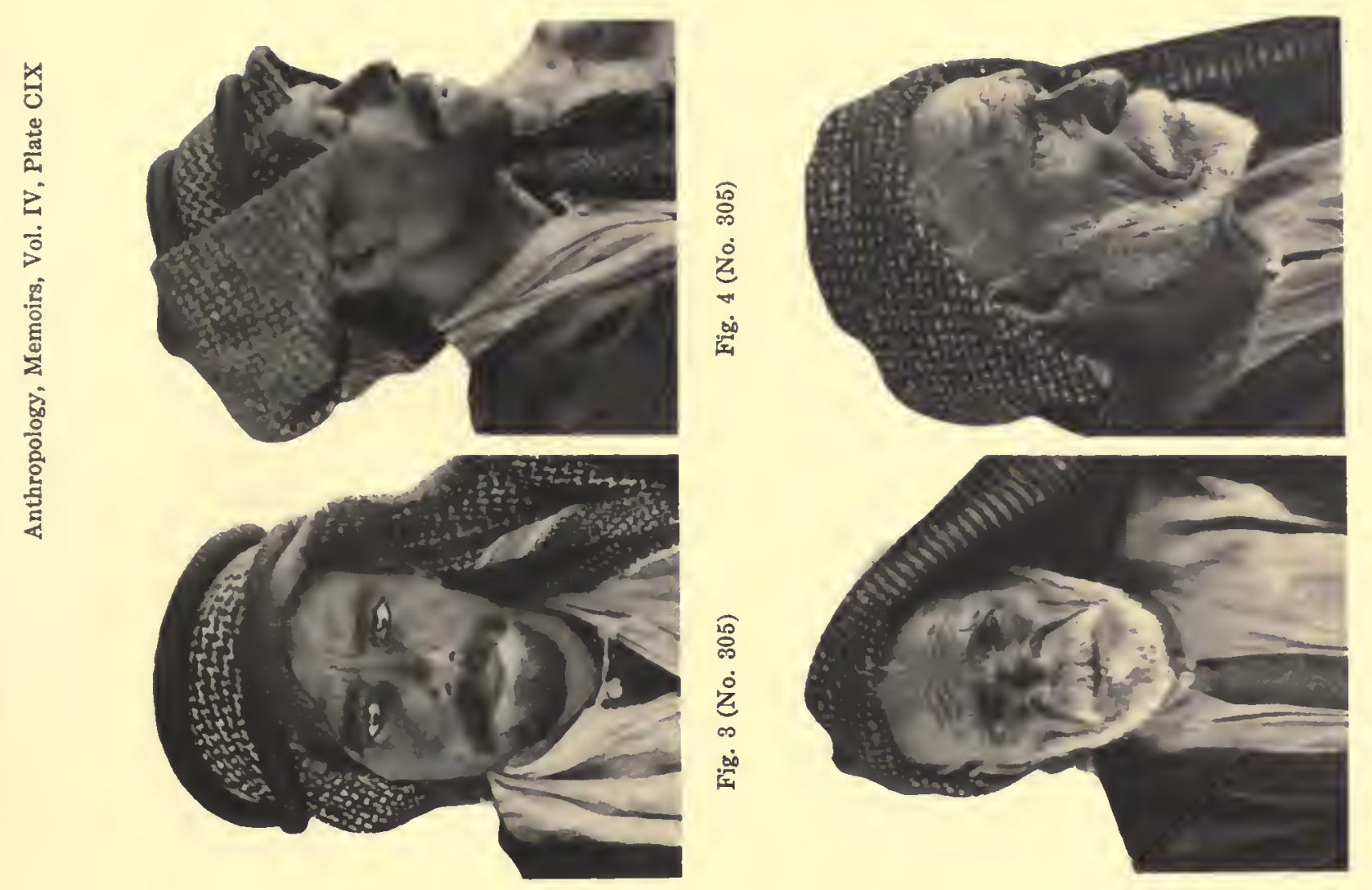

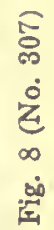

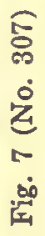
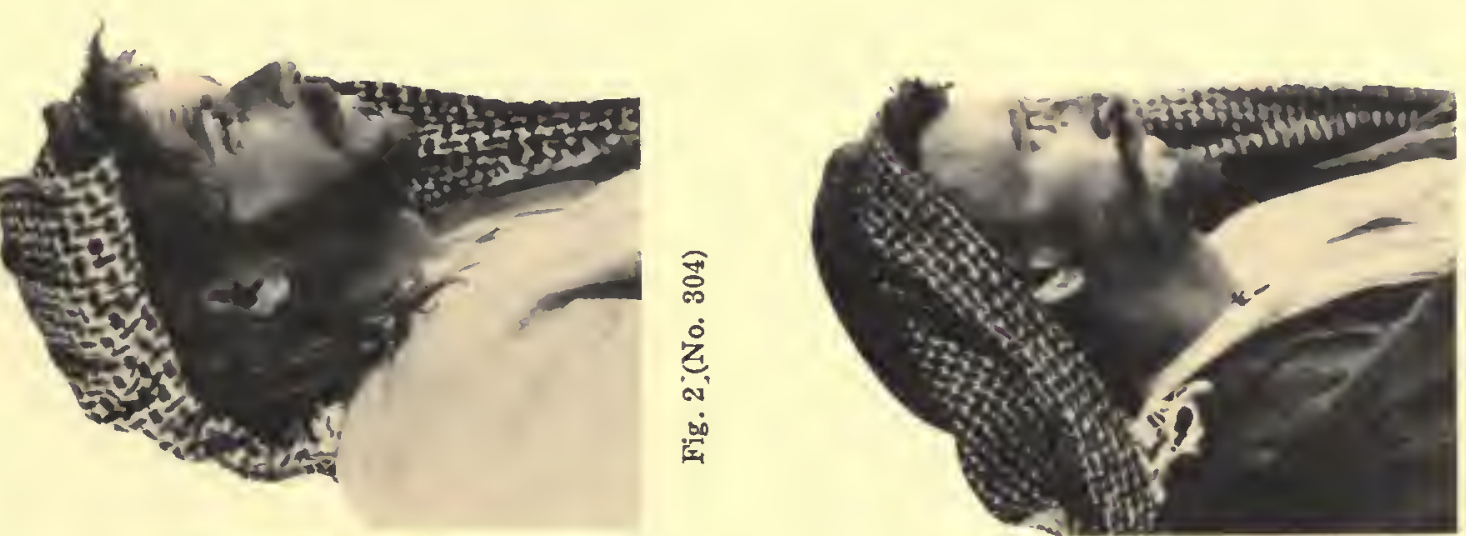

焉
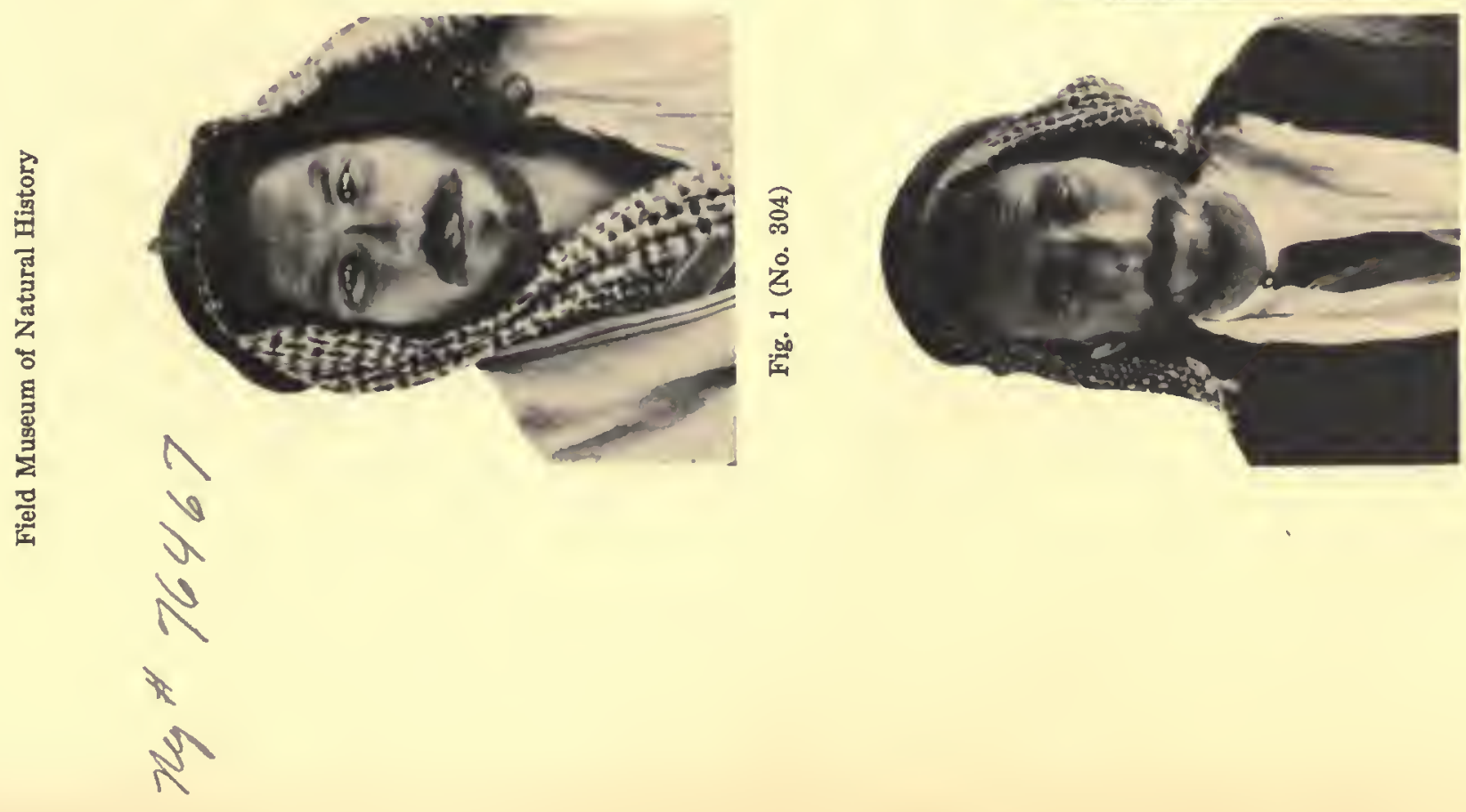

\&
\%
$\dot{8}$
0
0
0 

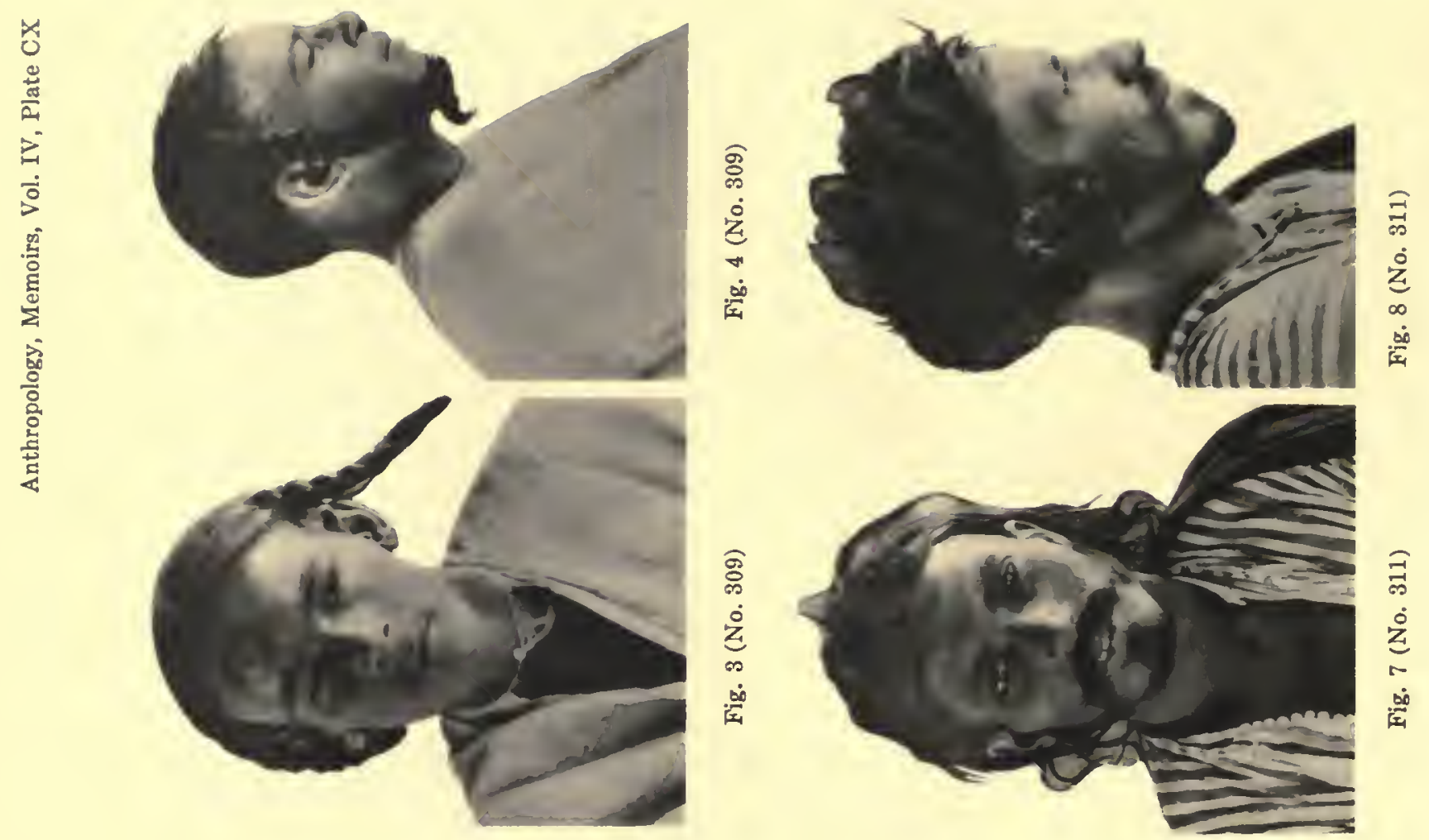

焉
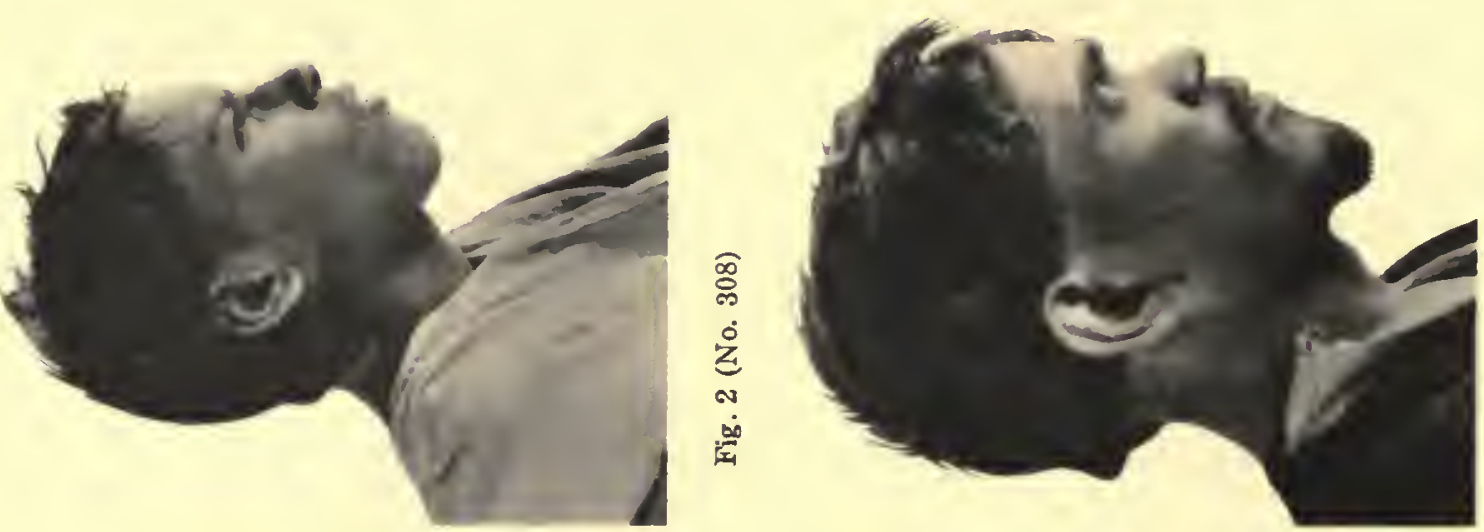

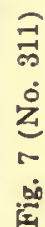
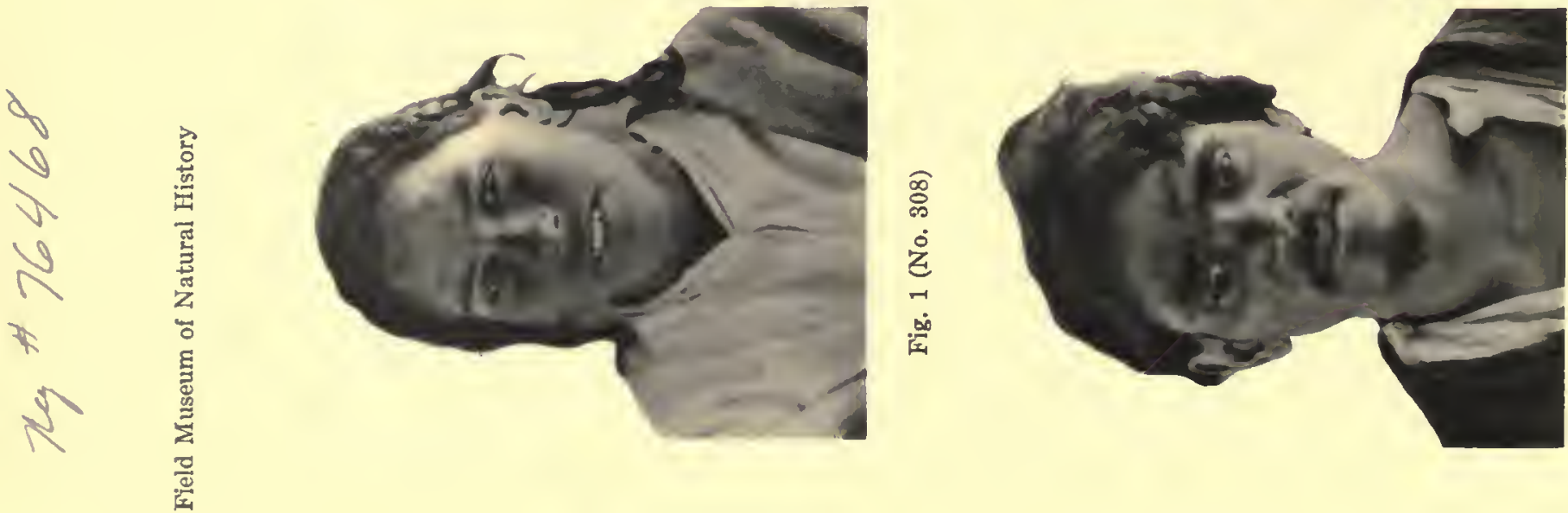

응 


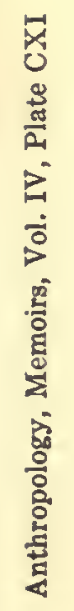
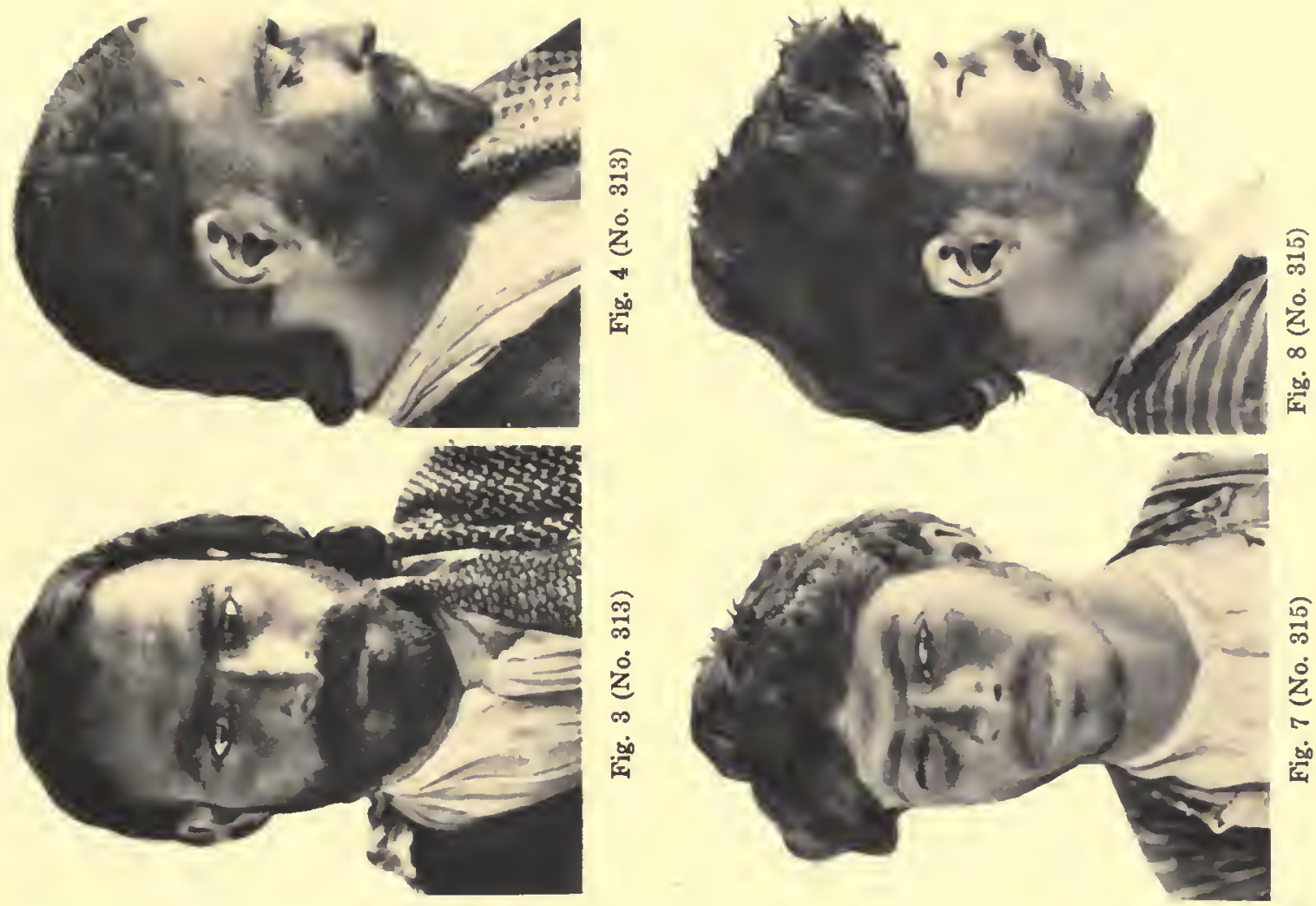

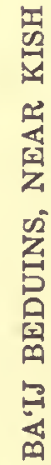
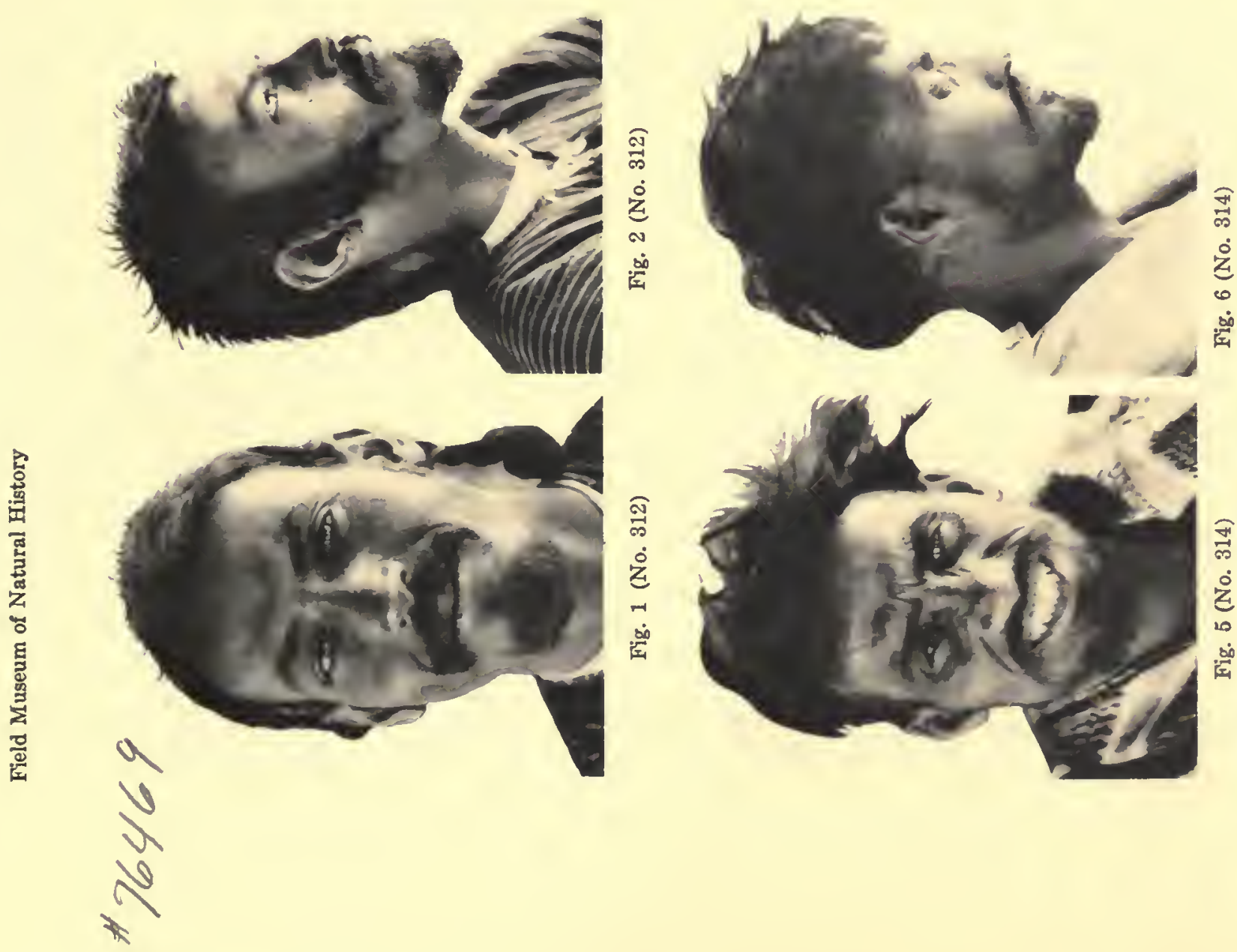

s 

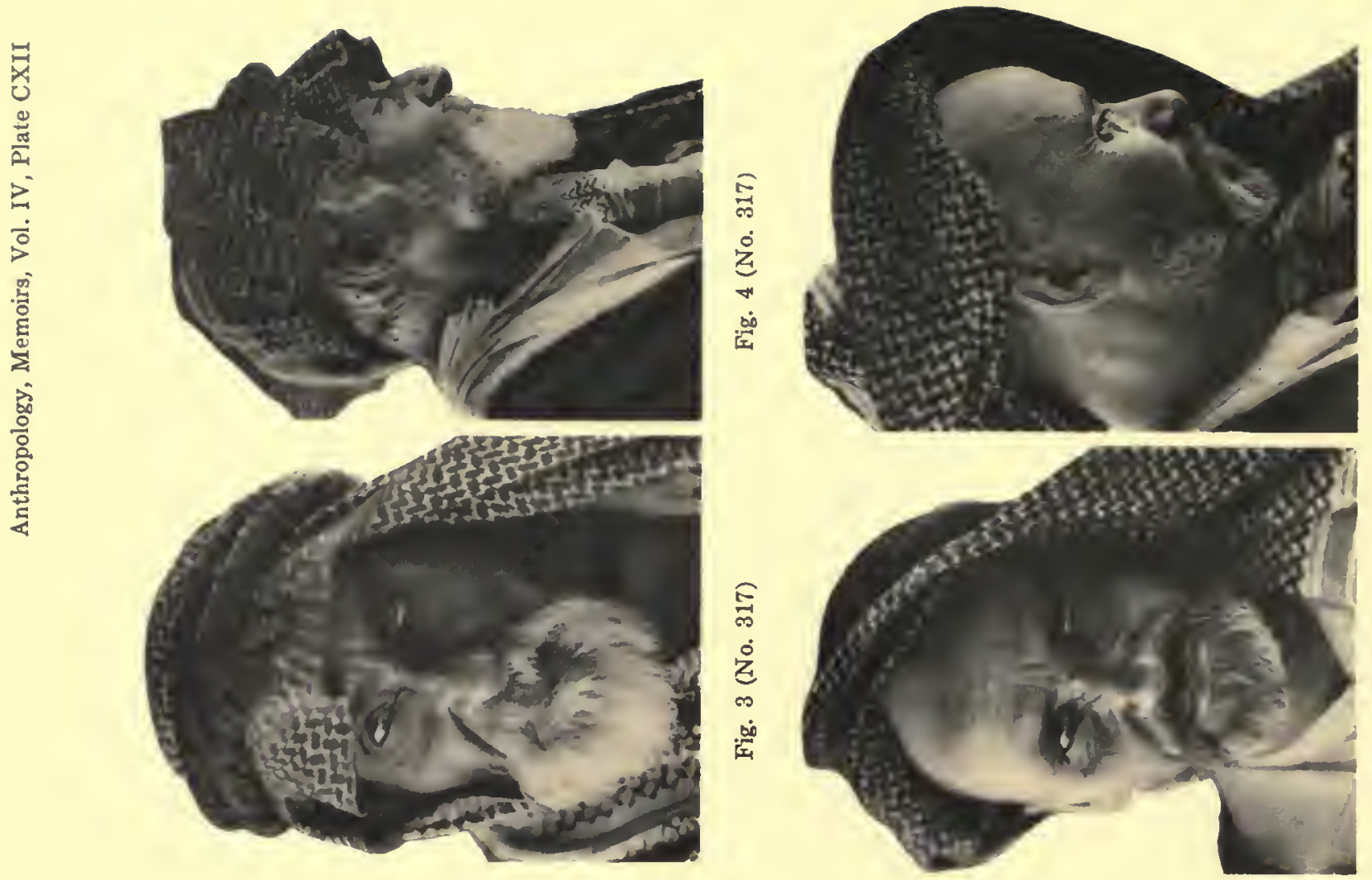

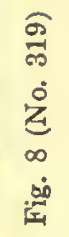
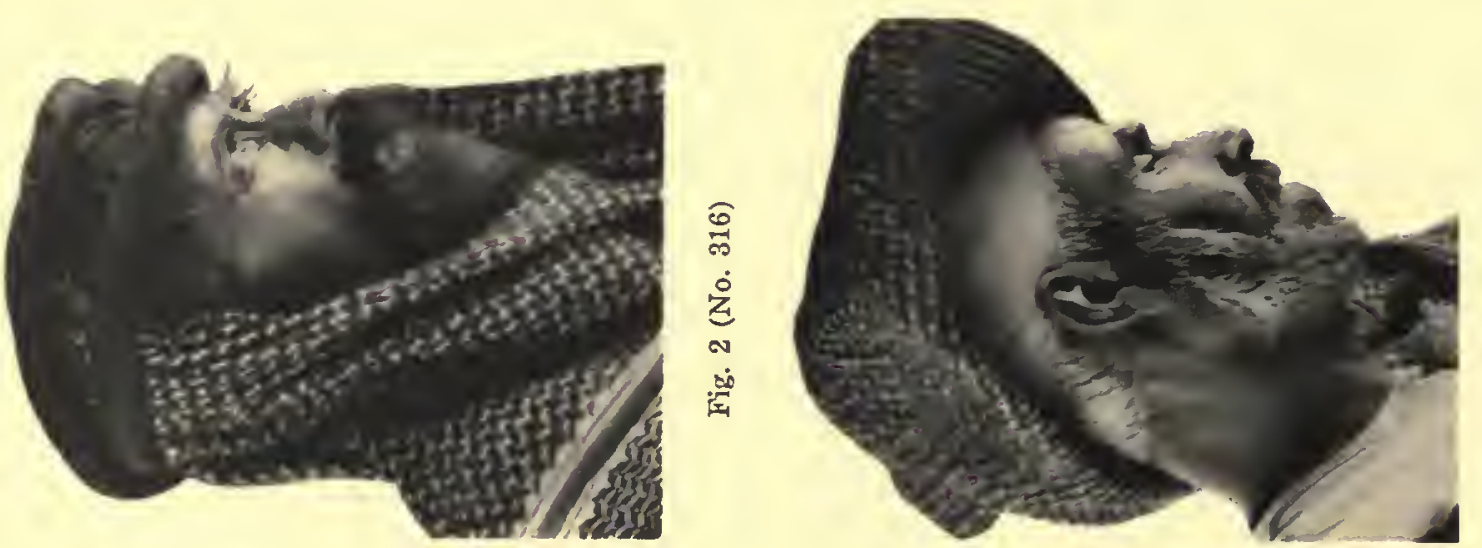

8
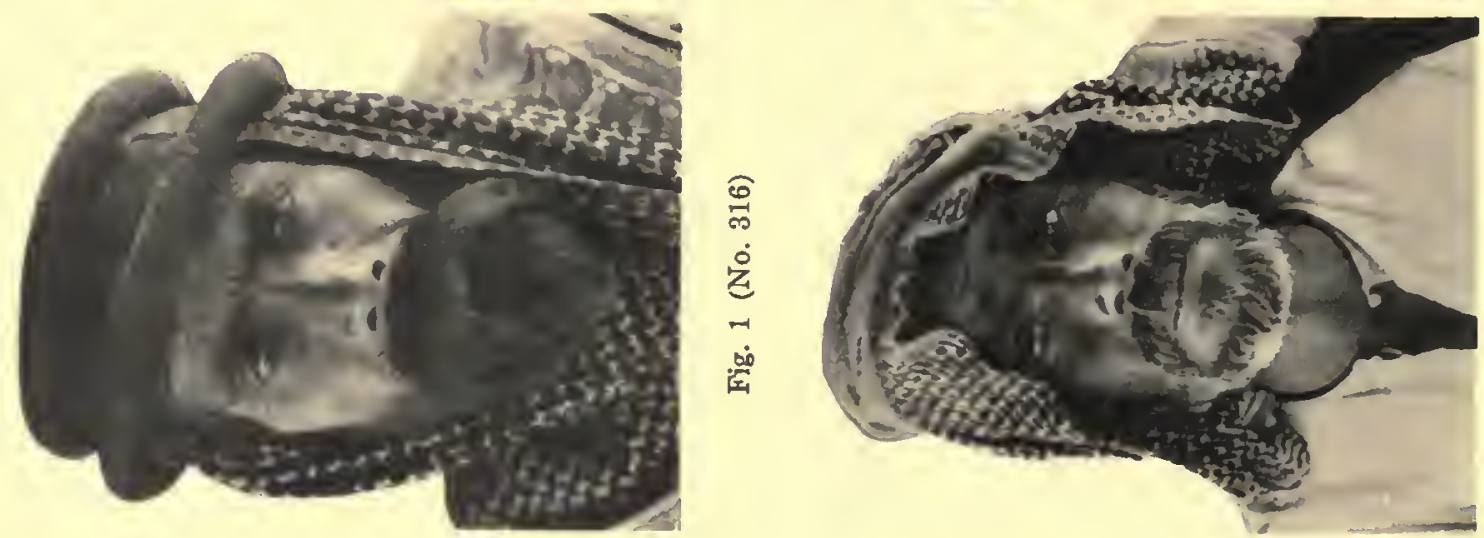

䙵 
当
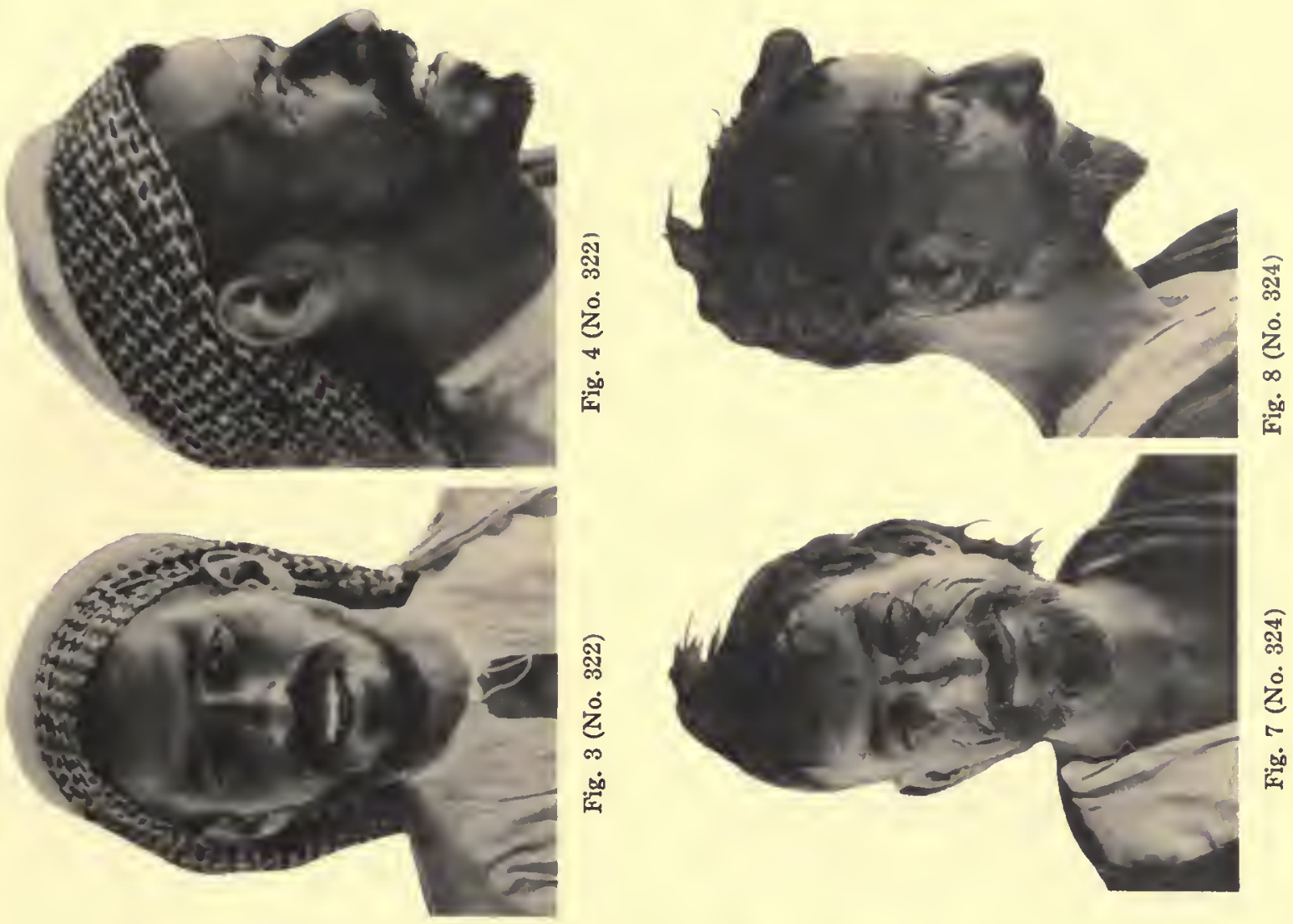

สิ

¿̊ำ

窂

舀
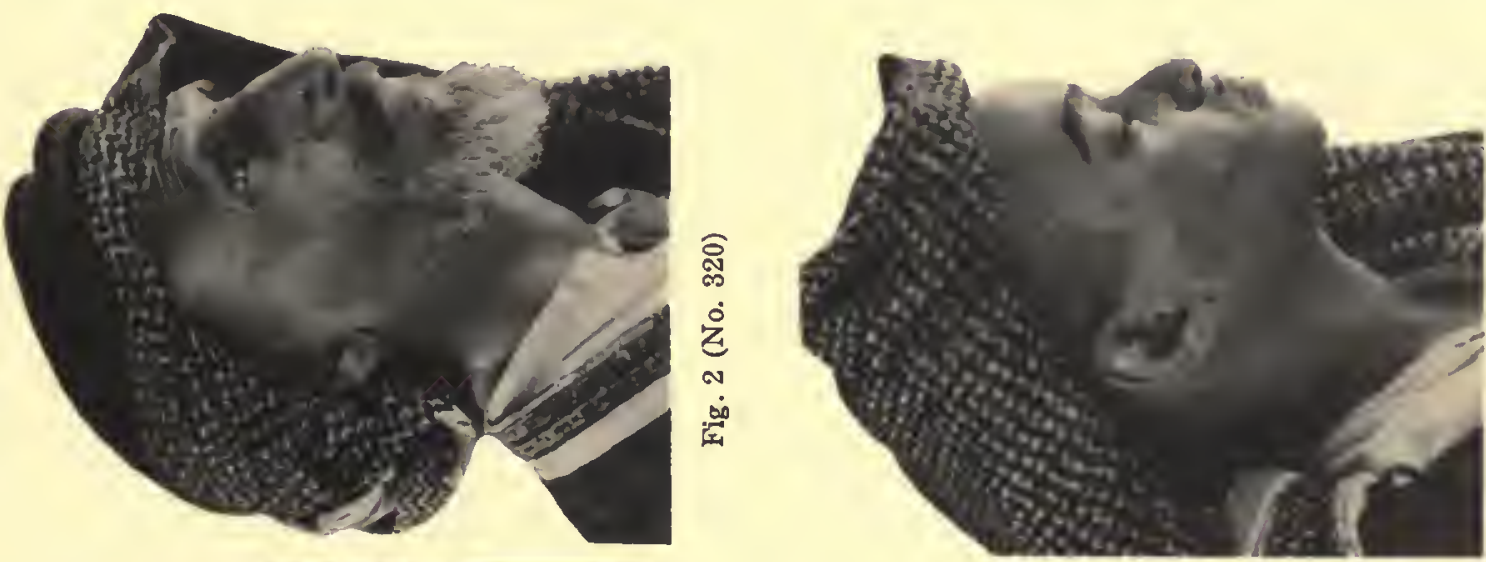

总

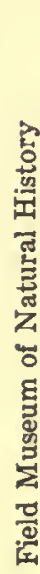
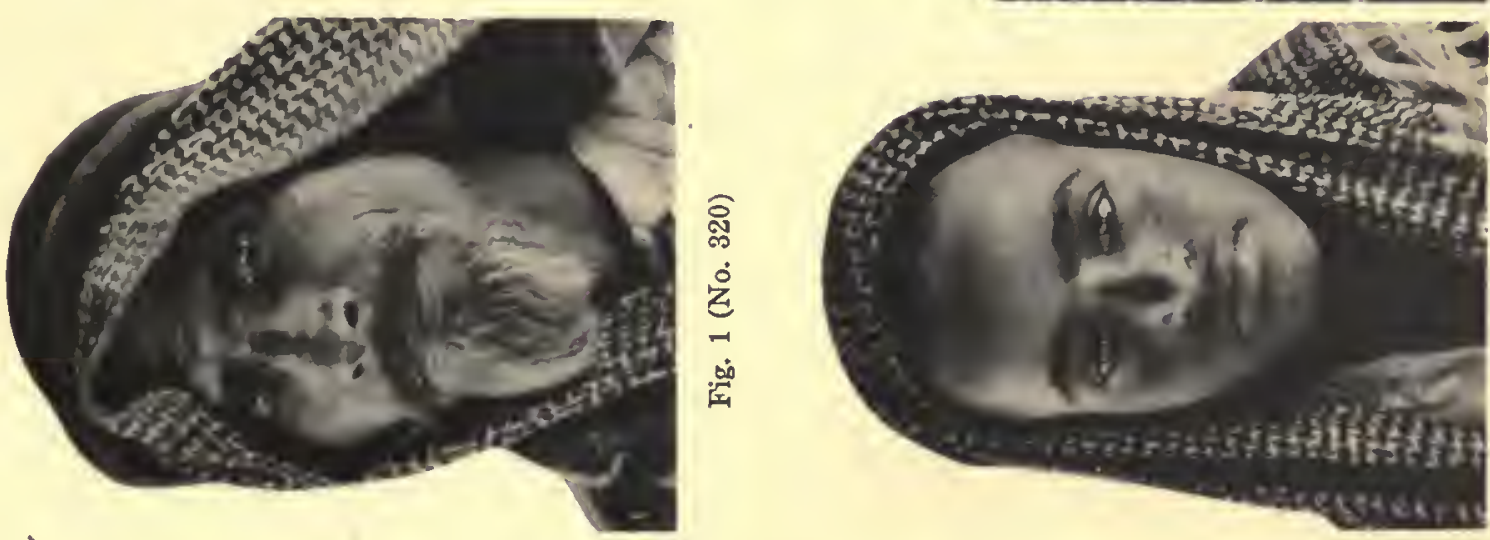

ळ్ల
$\dot{0}$
$\dot{3}$
0
0
0

ब్
$\dot{0}$
$\dot{3}$
10
10
00

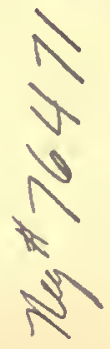




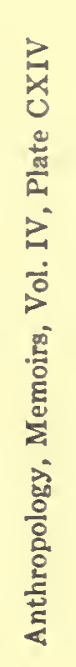
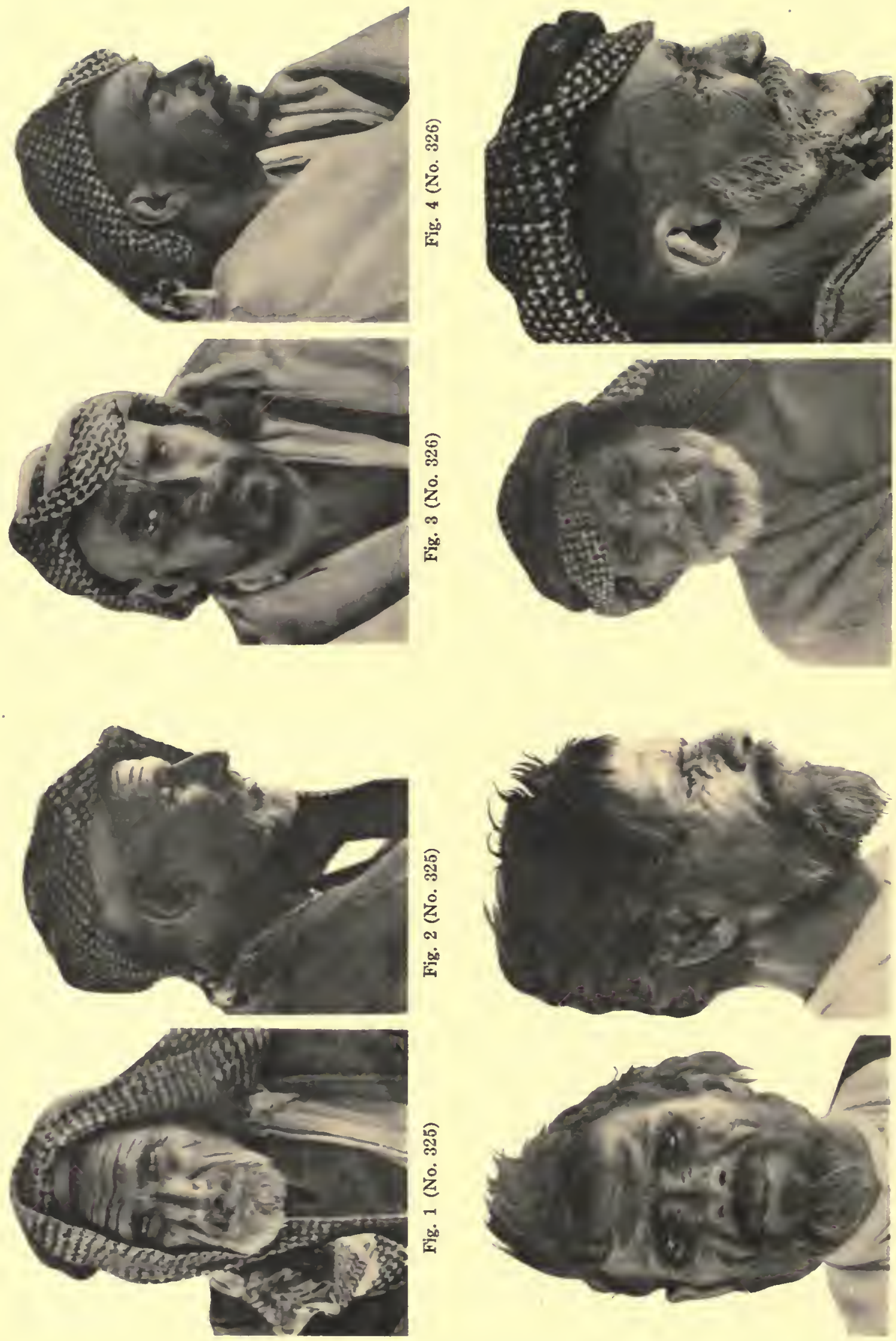
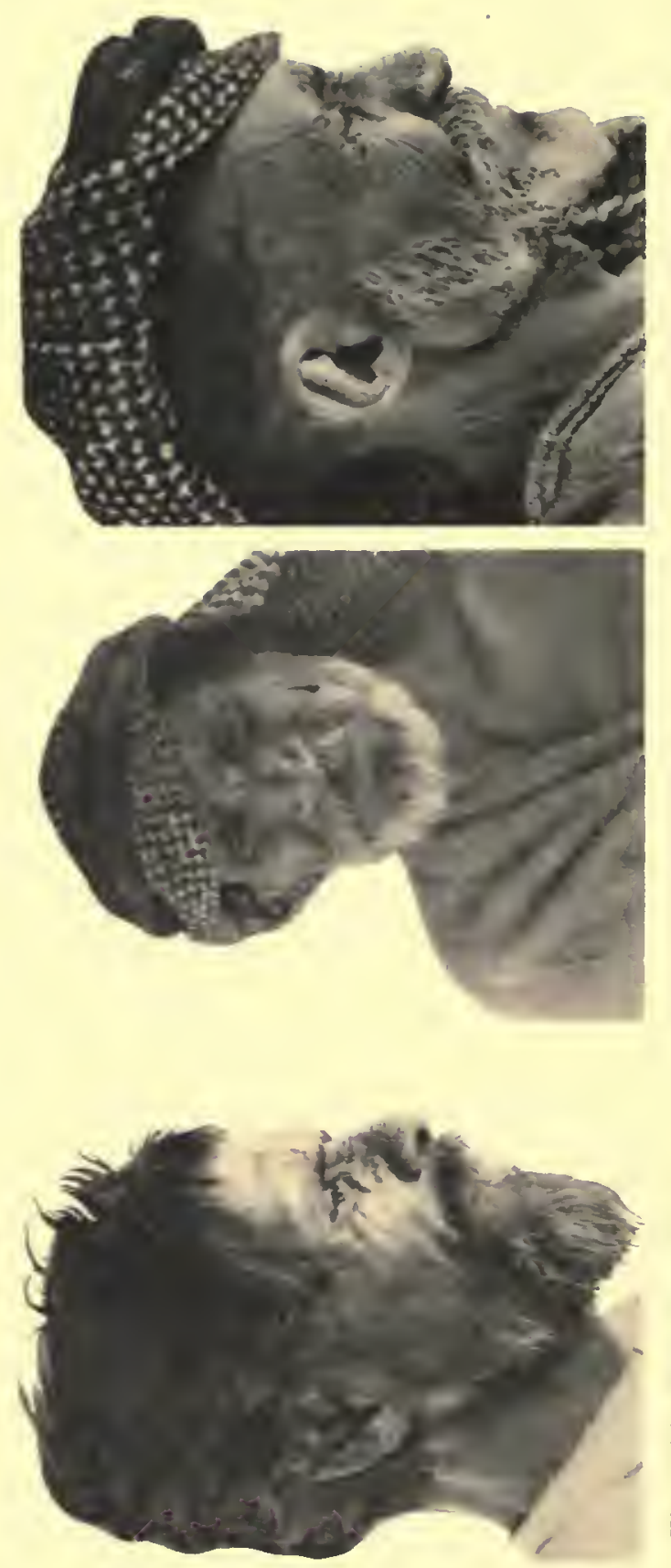

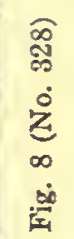

商

这

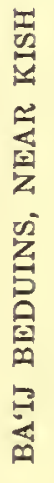

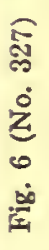

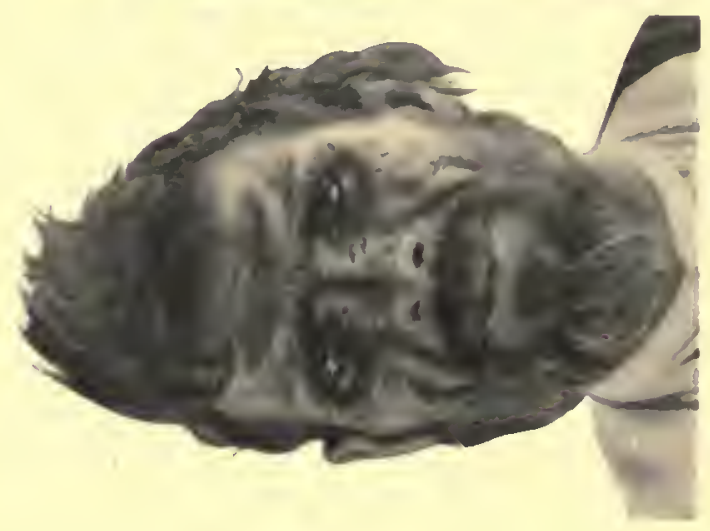


勿
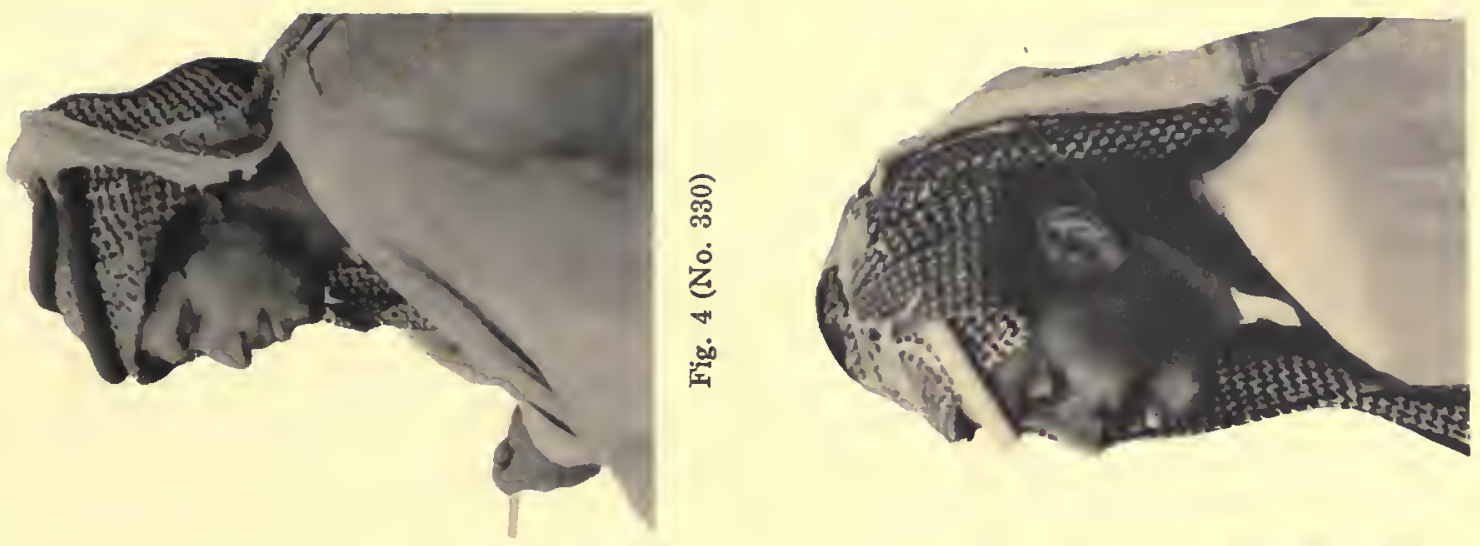

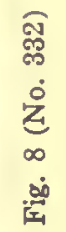
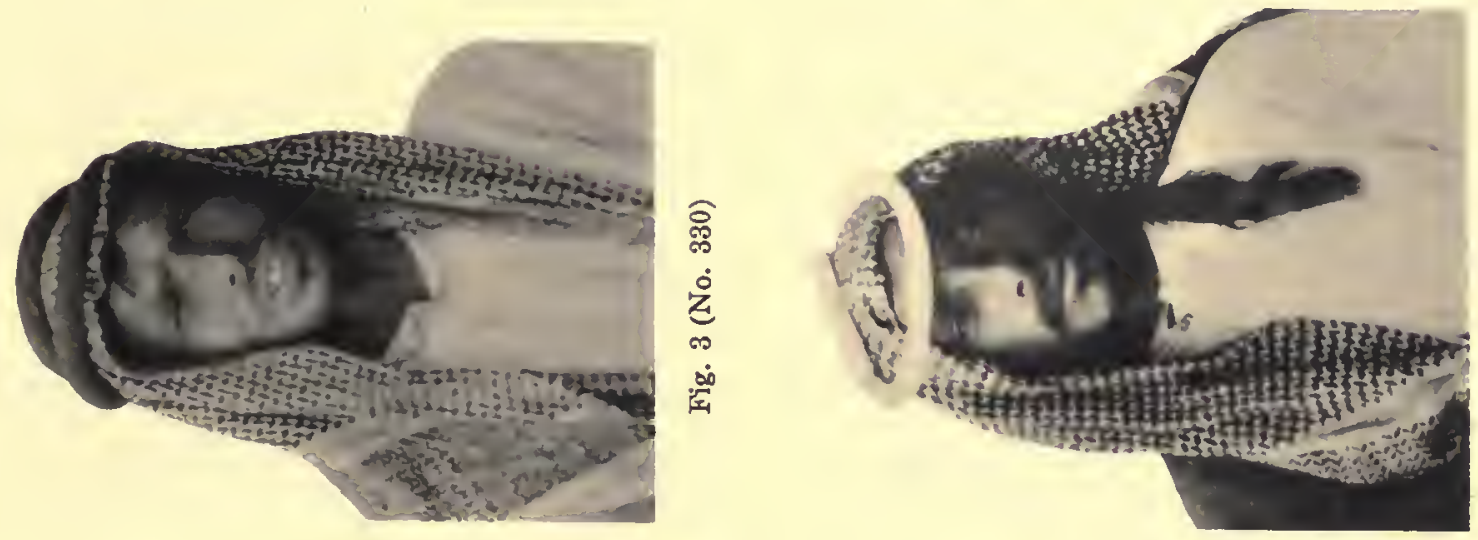

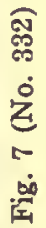

贾

悹
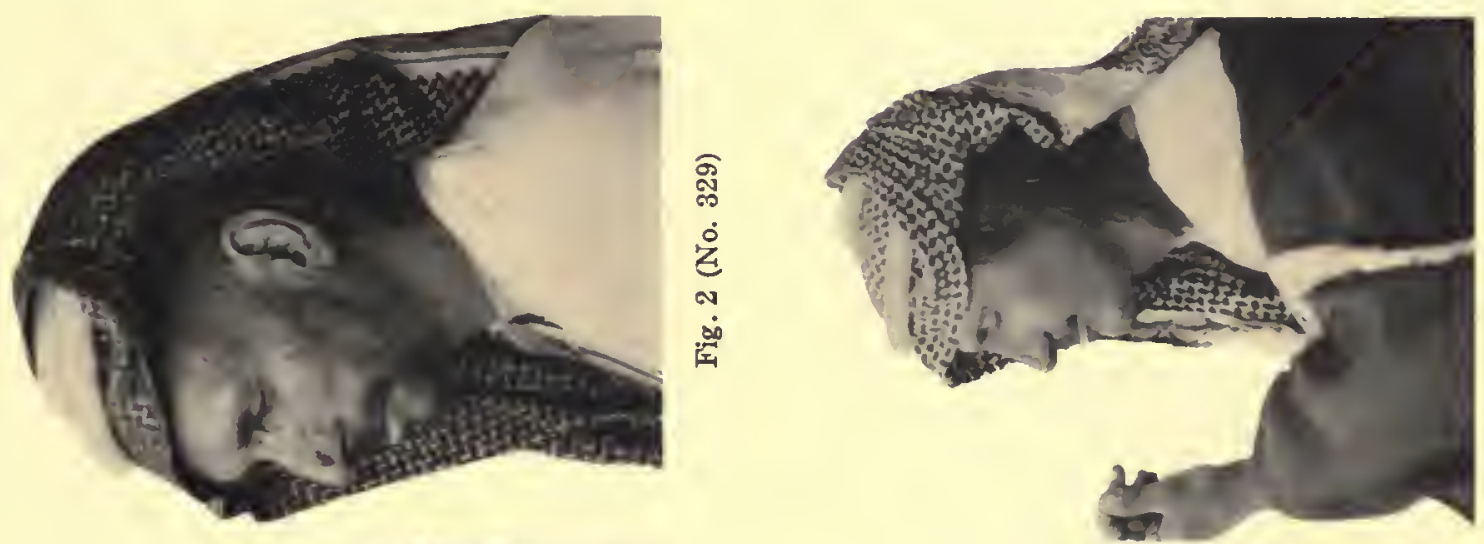

¿̊

禹
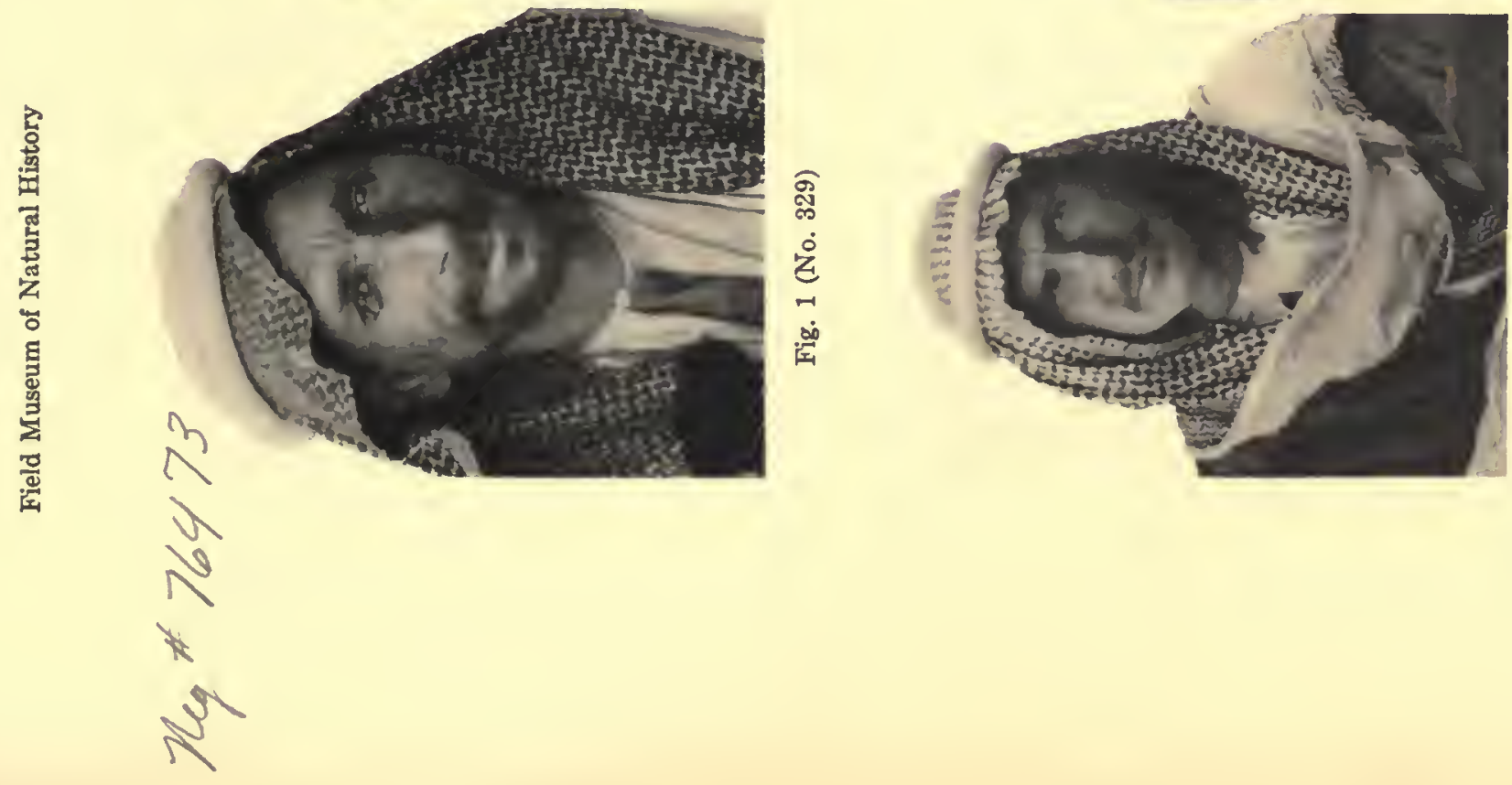

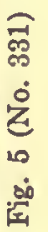




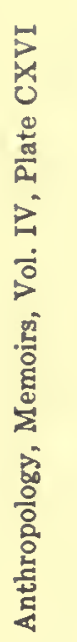
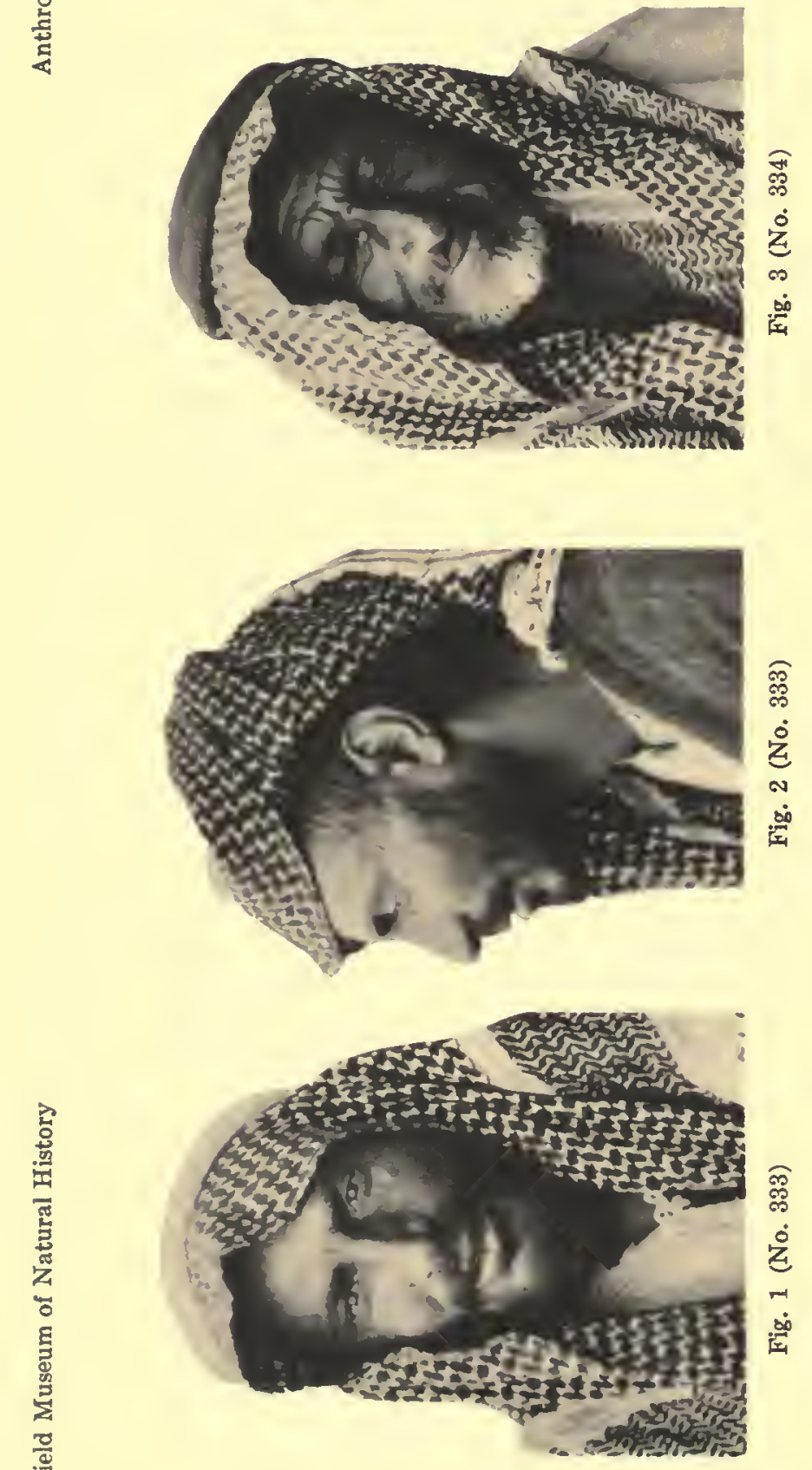
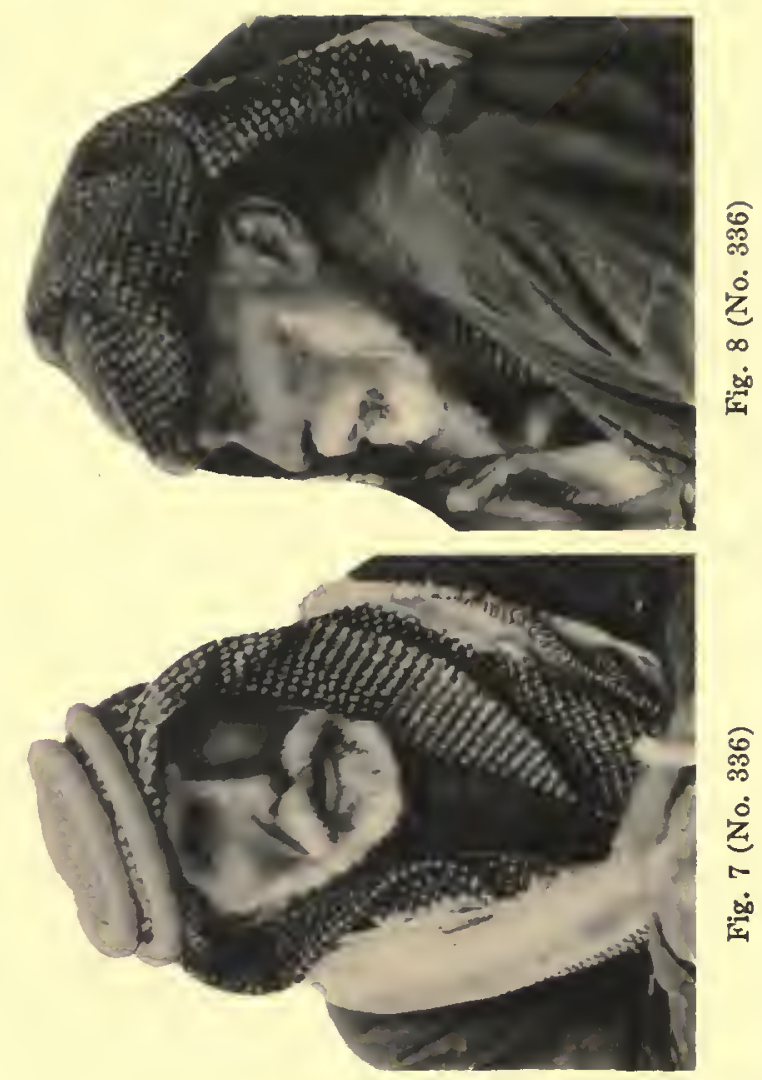

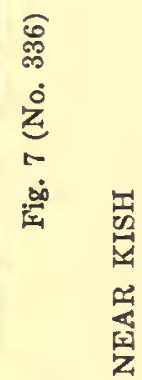

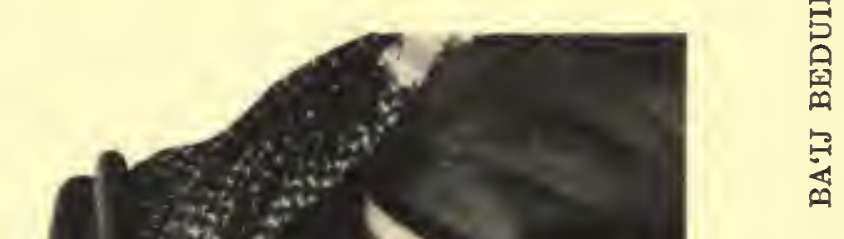

踏

它 它

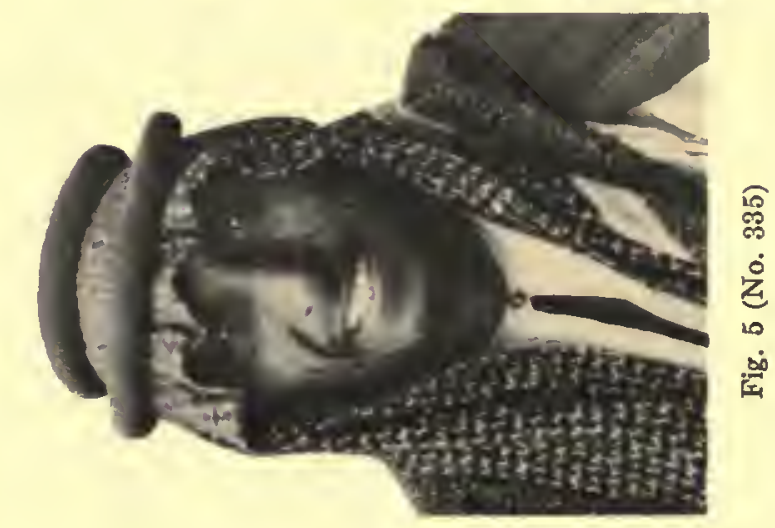




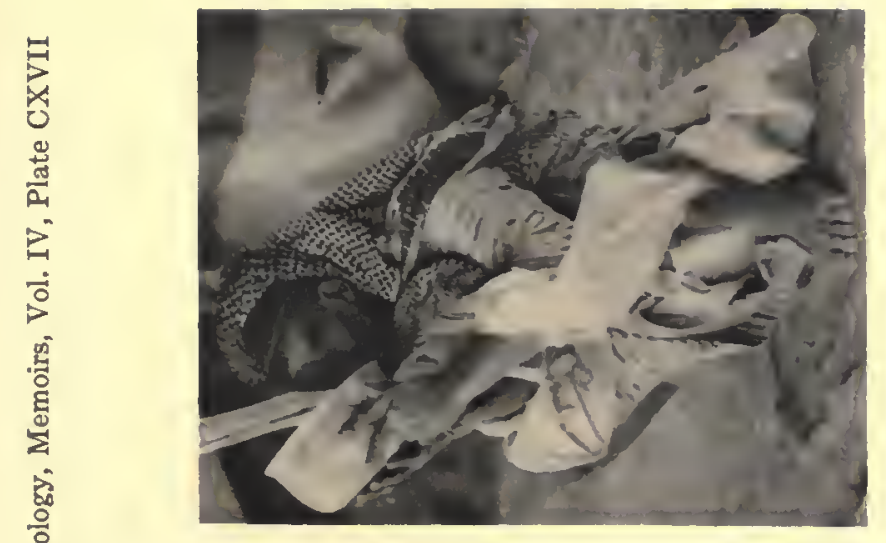

离
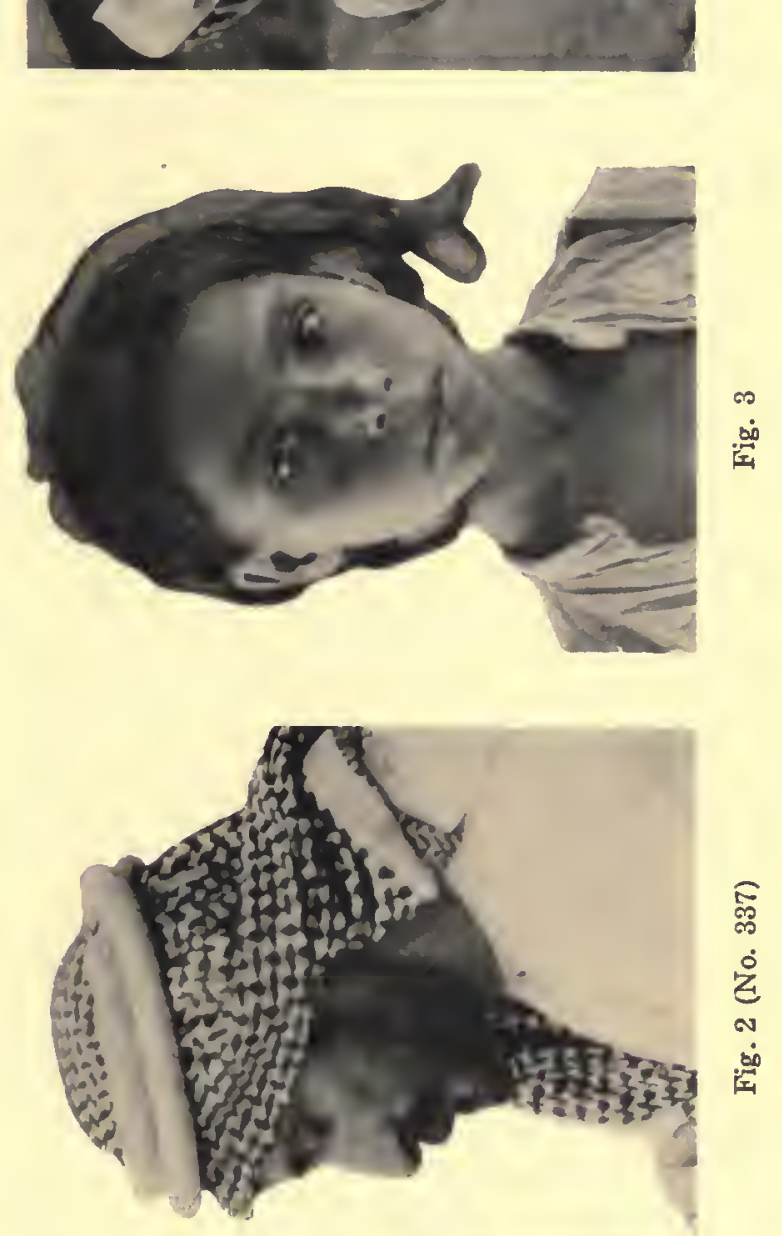

象

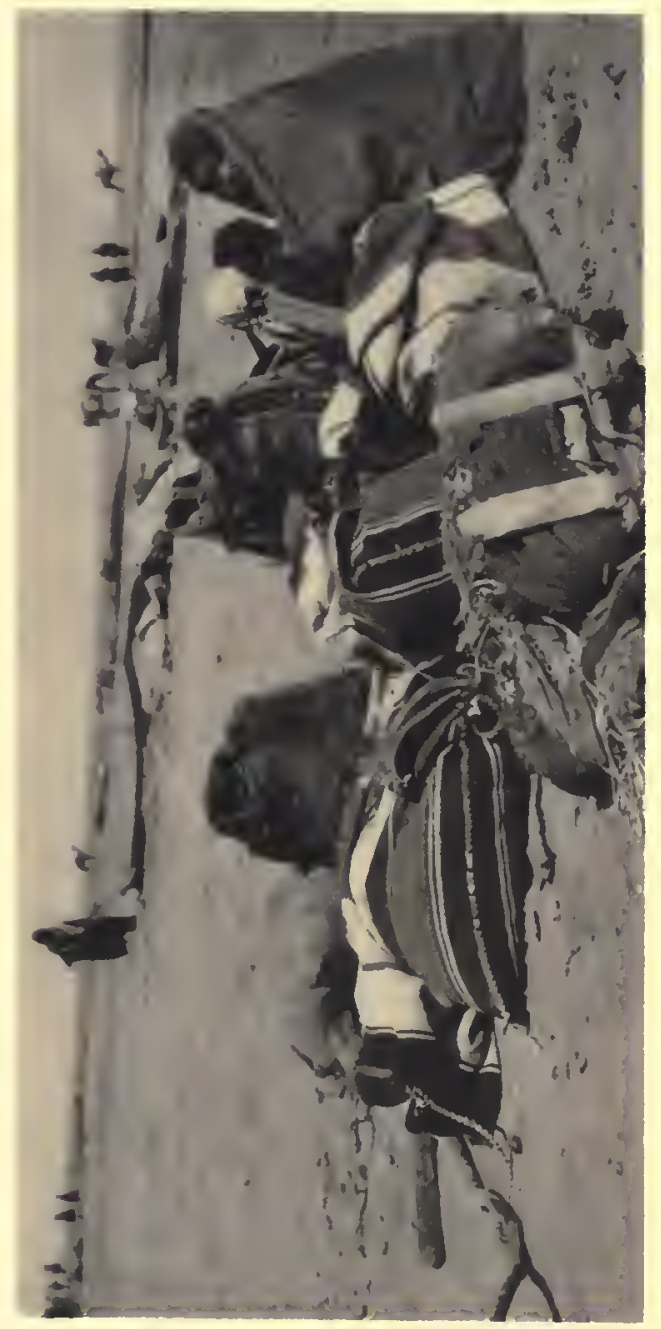

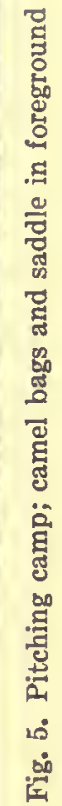

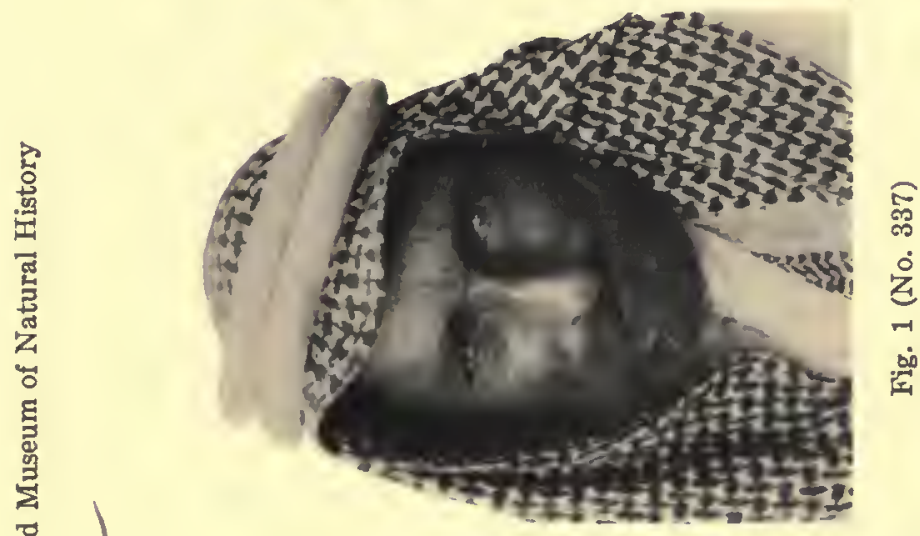

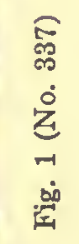

웠

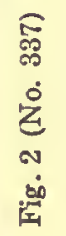

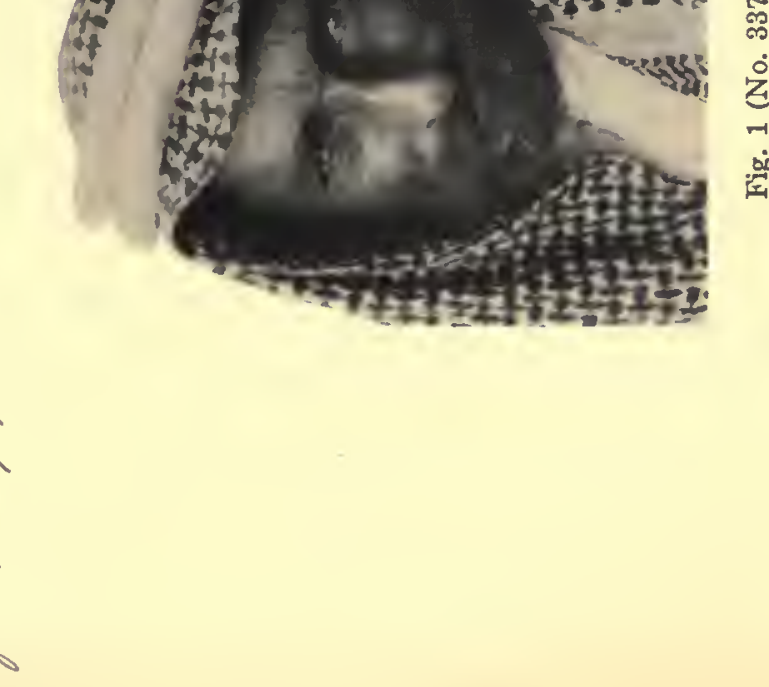

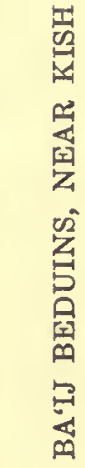




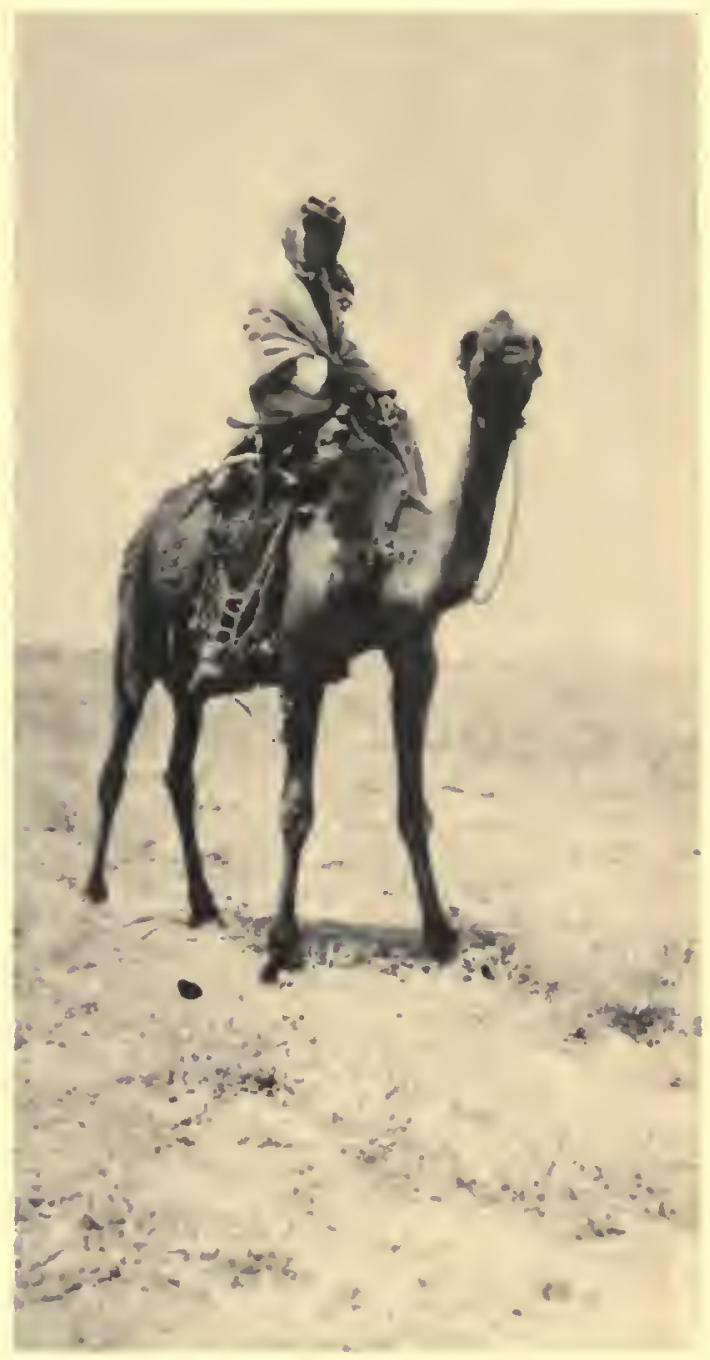

Fig. 1. Herdsman

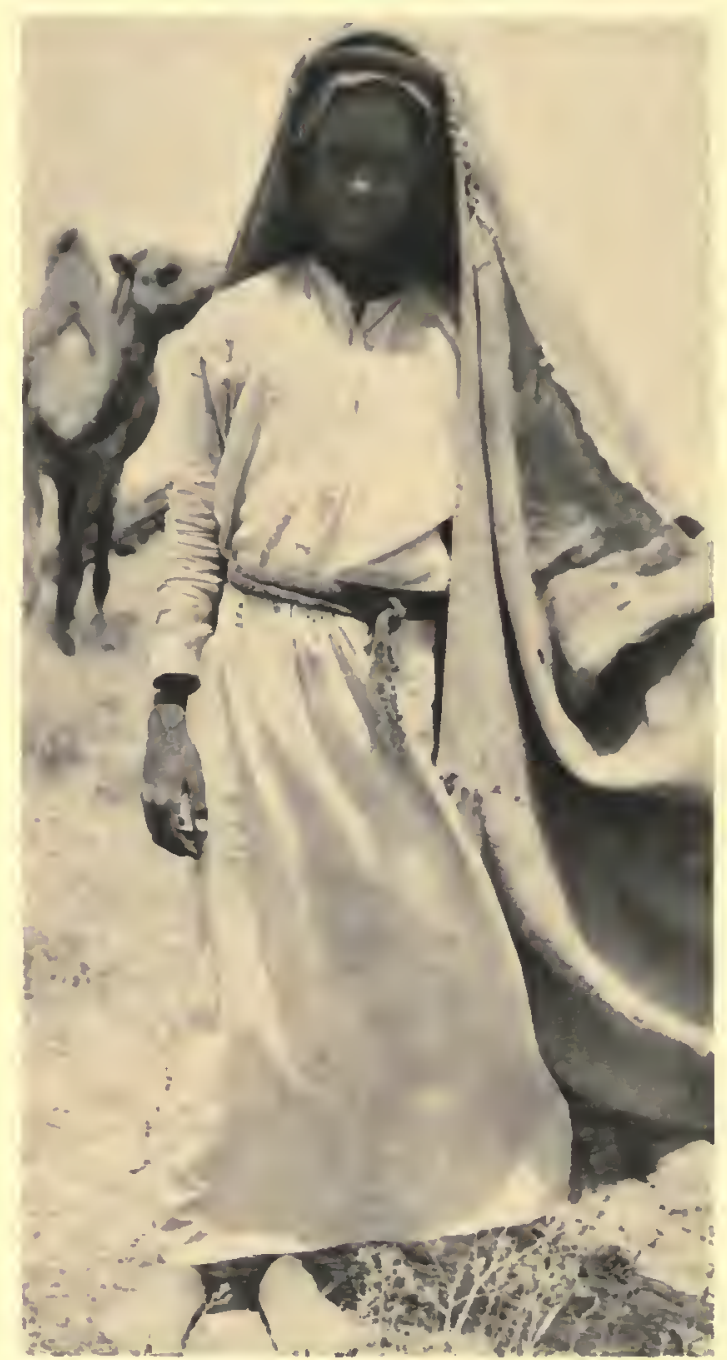

Fig. 2. Herdsman

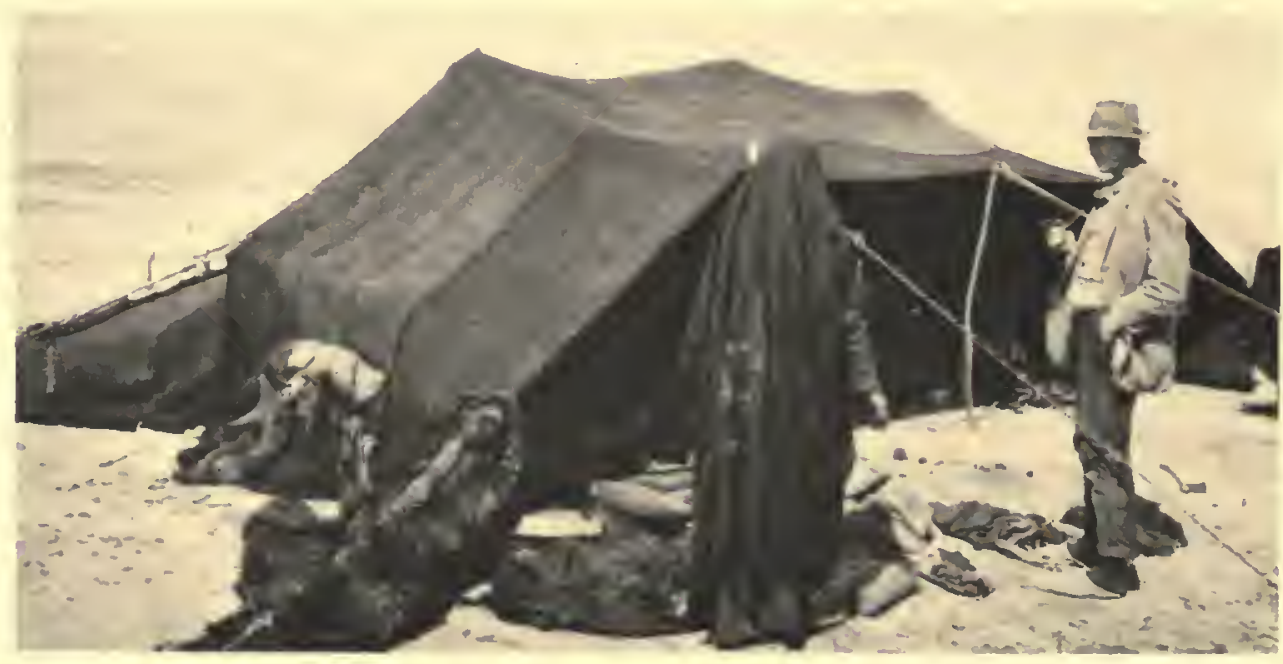

Fig. 3. Black tent made of camel's hair

BA'IJ BEDUINS, NEAR KISH 
Field Museum of Natural History

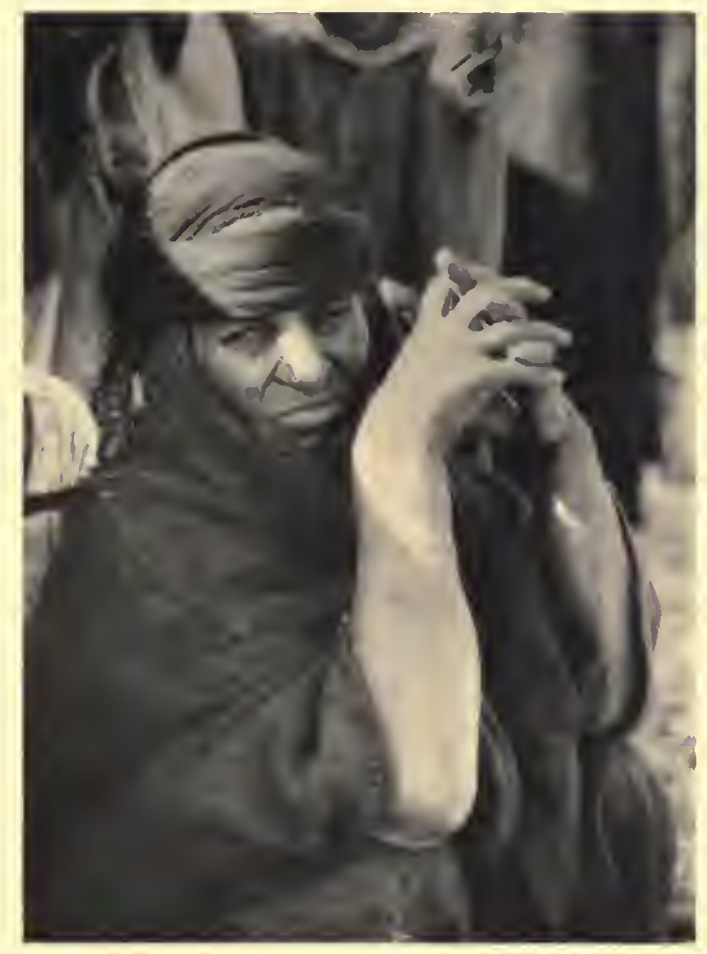

Fig. 1. Woman

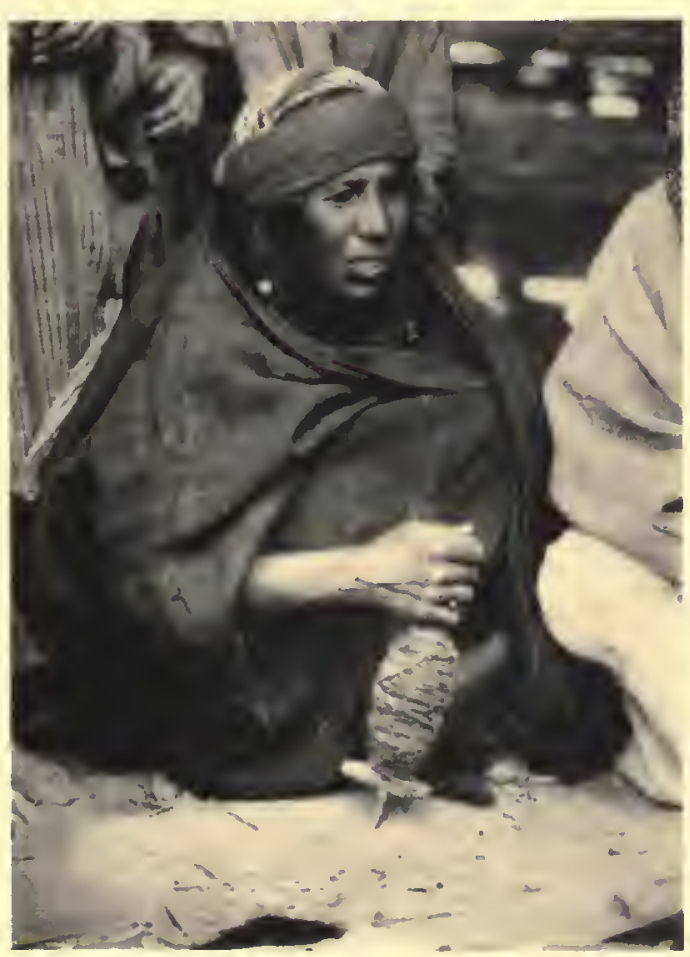

Fig. 3. Woman with spindle
Anthropology, Memoirs, Vol. IV, Plate CXIX

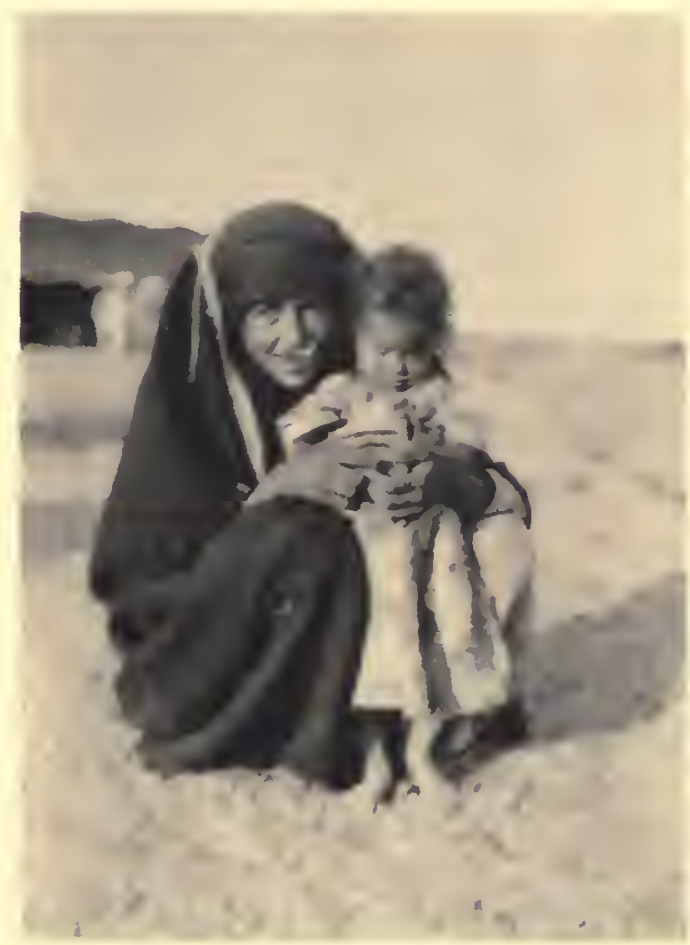

Fig. 2. Woman with child

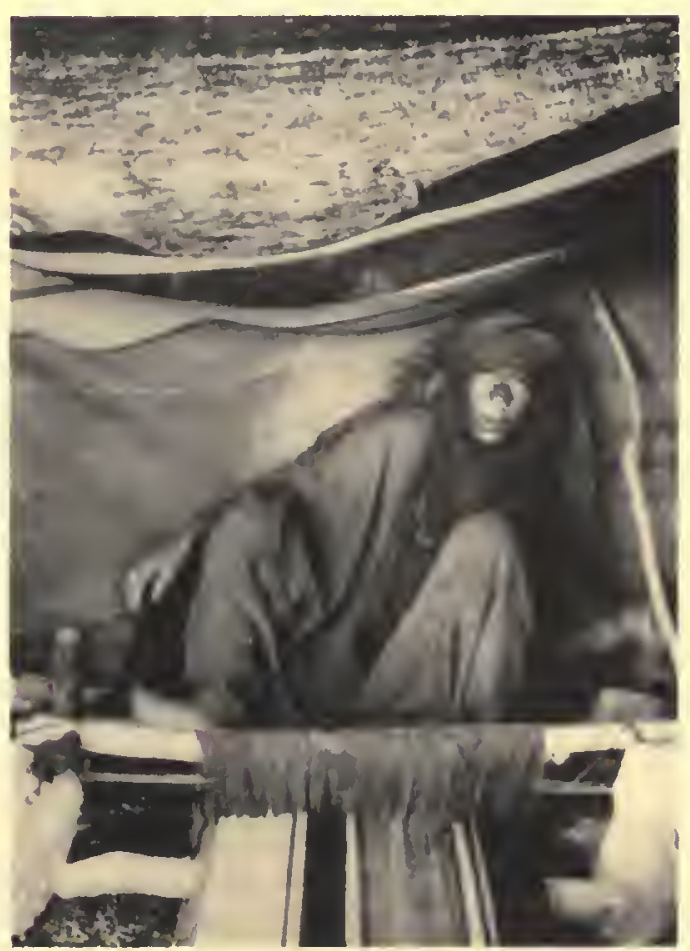

Fig. 4. Woman weaving 

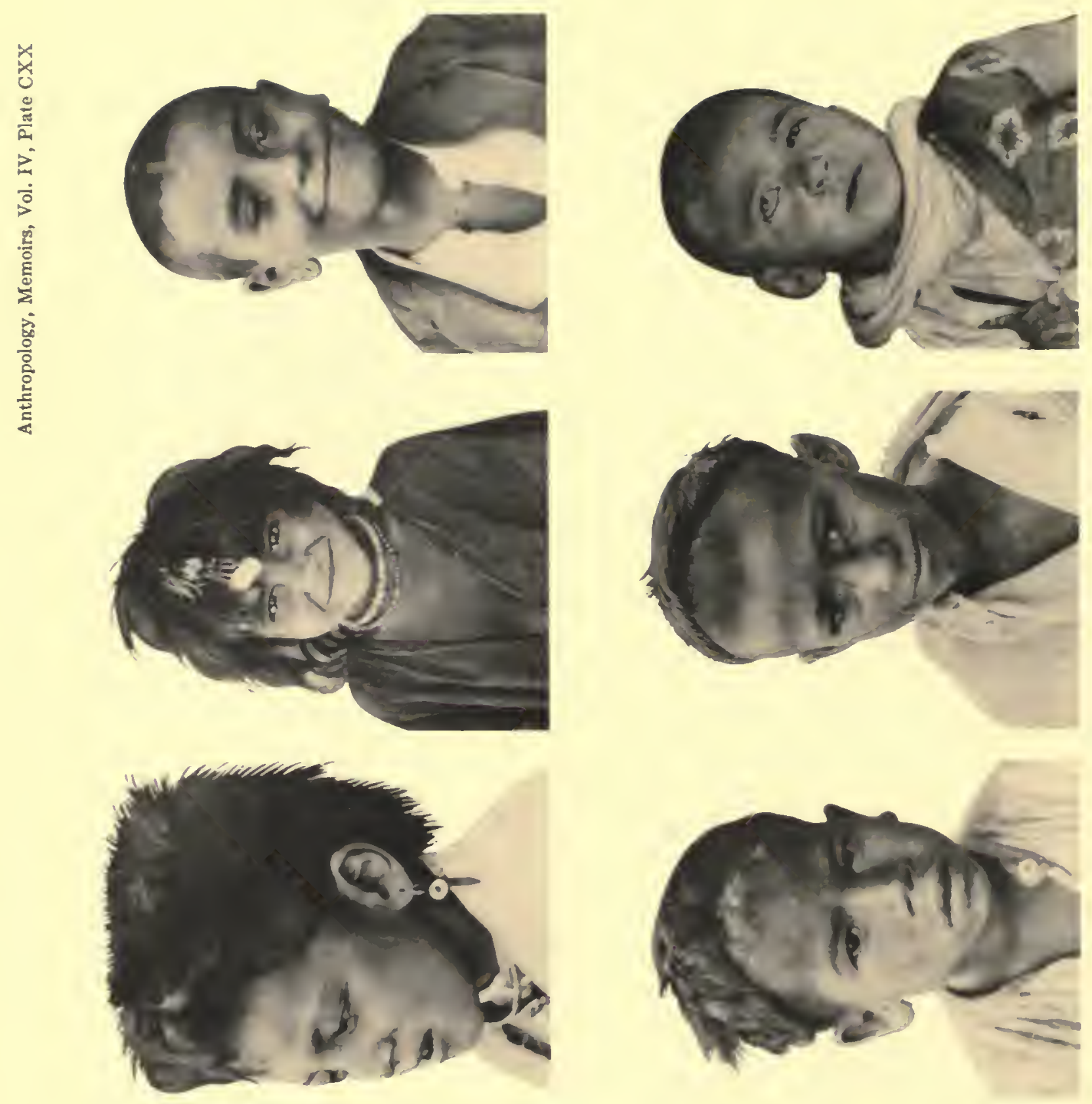

舀
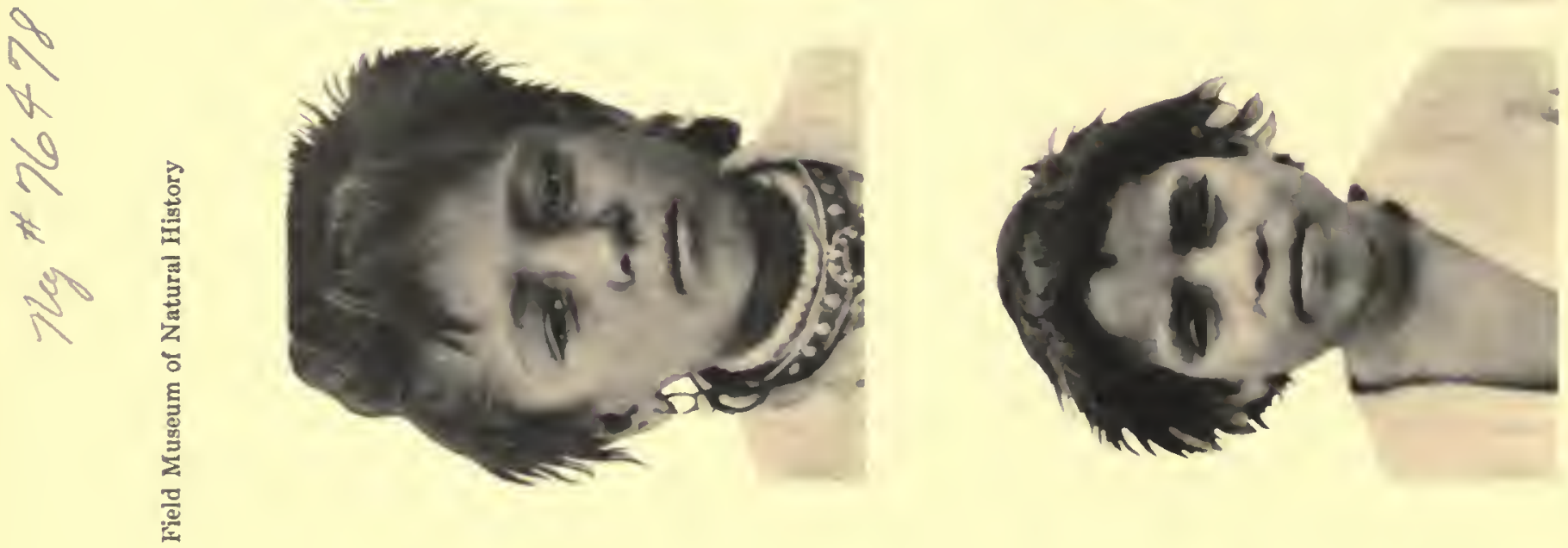

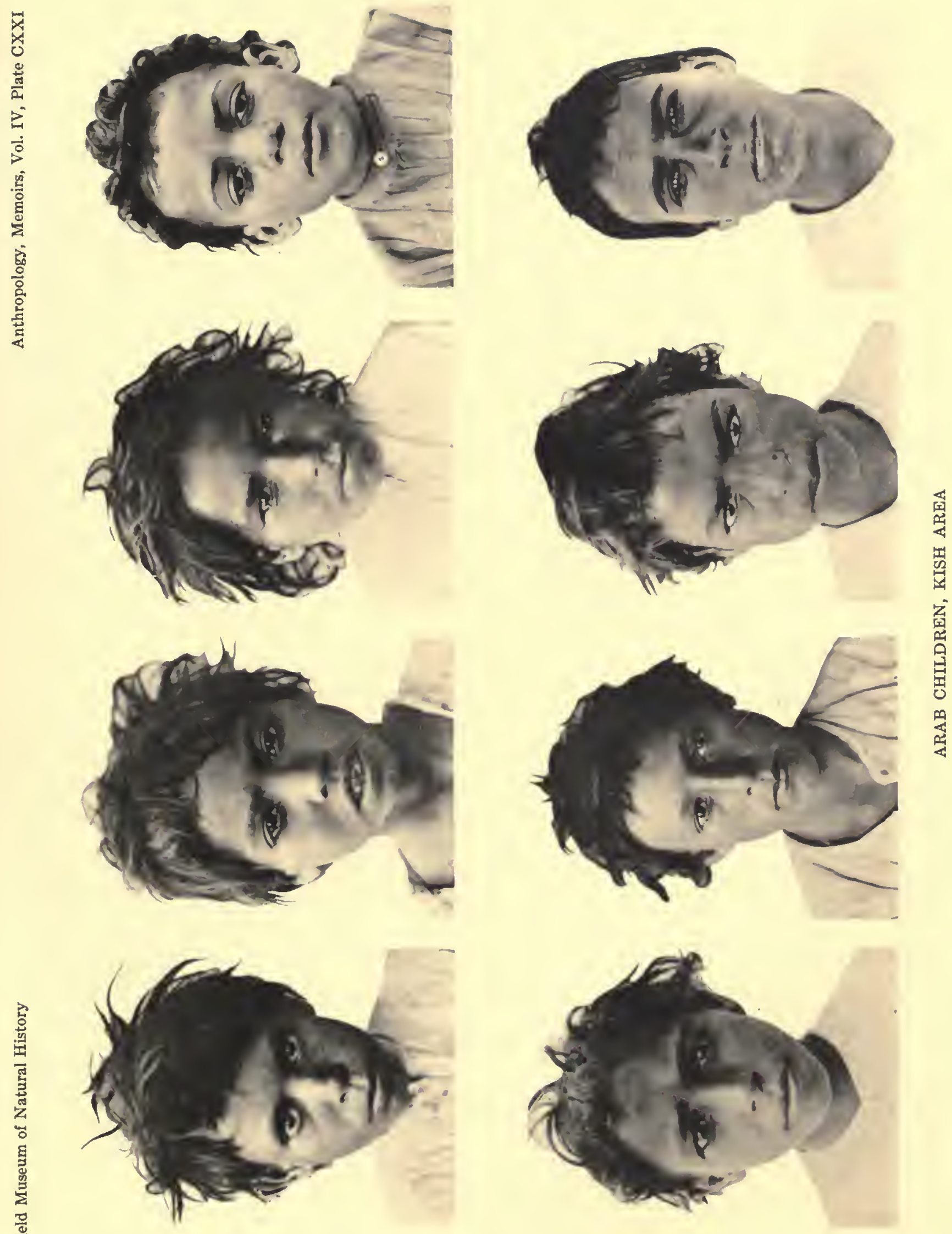

䍃

窑

N 

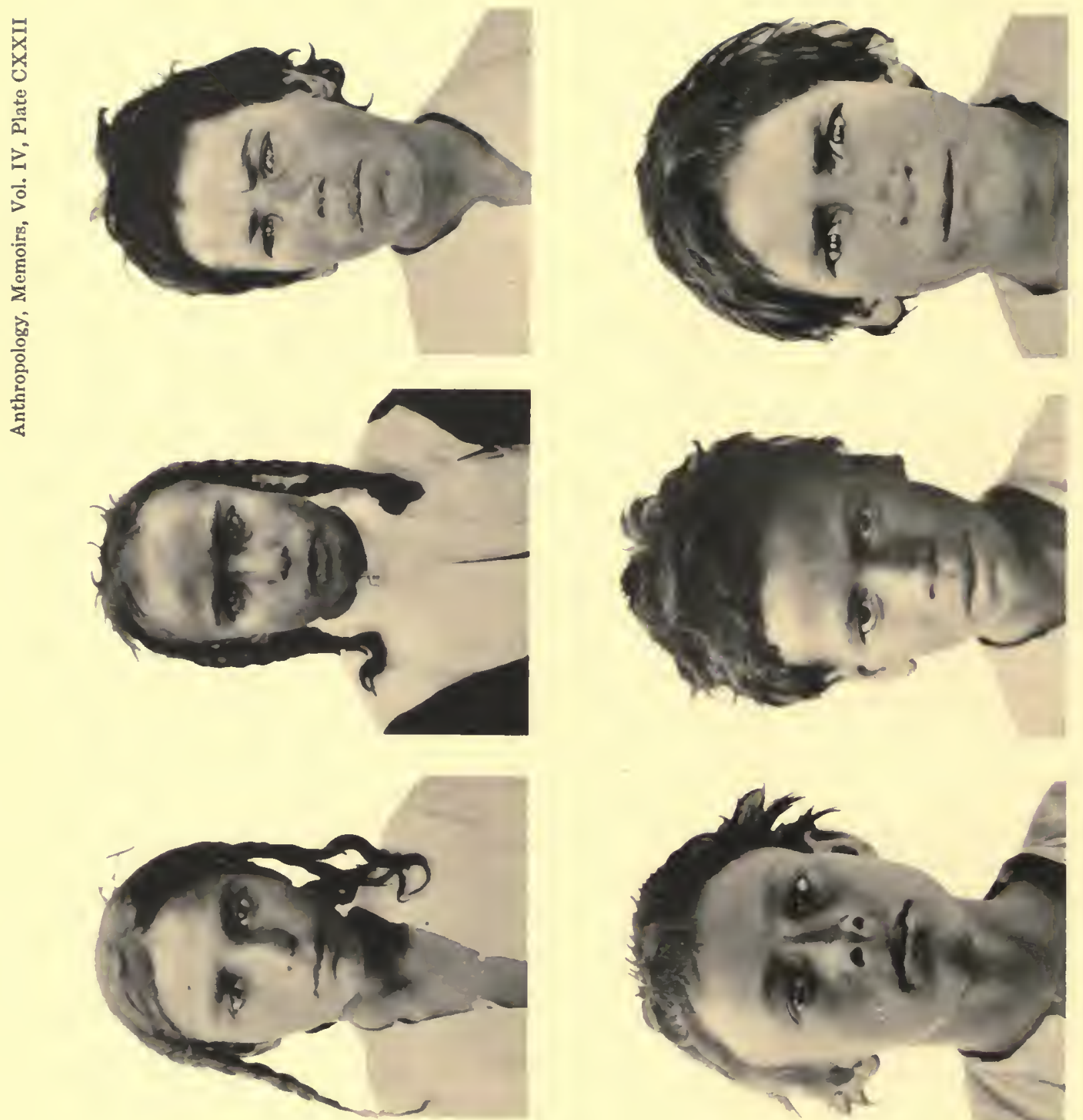

会
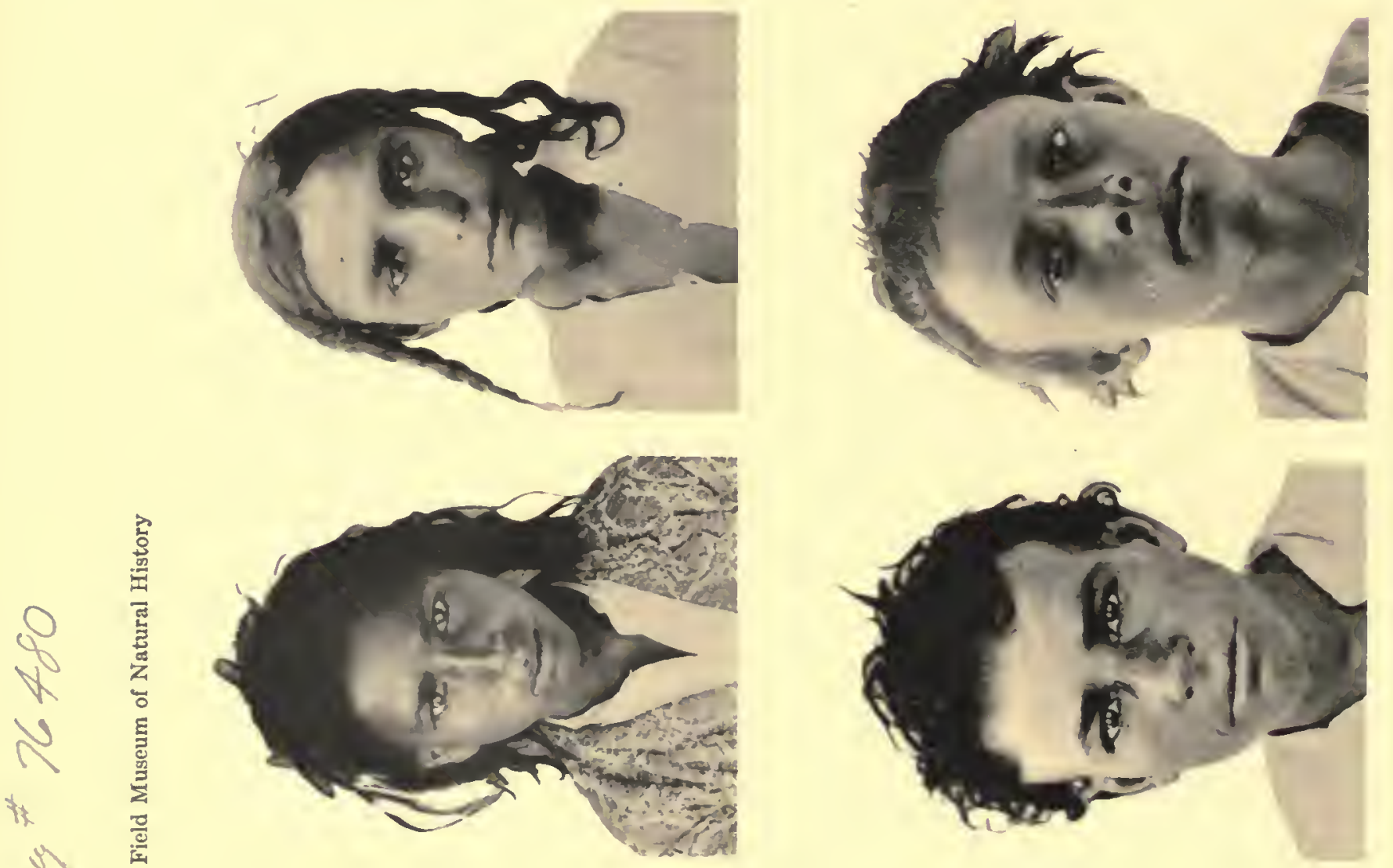

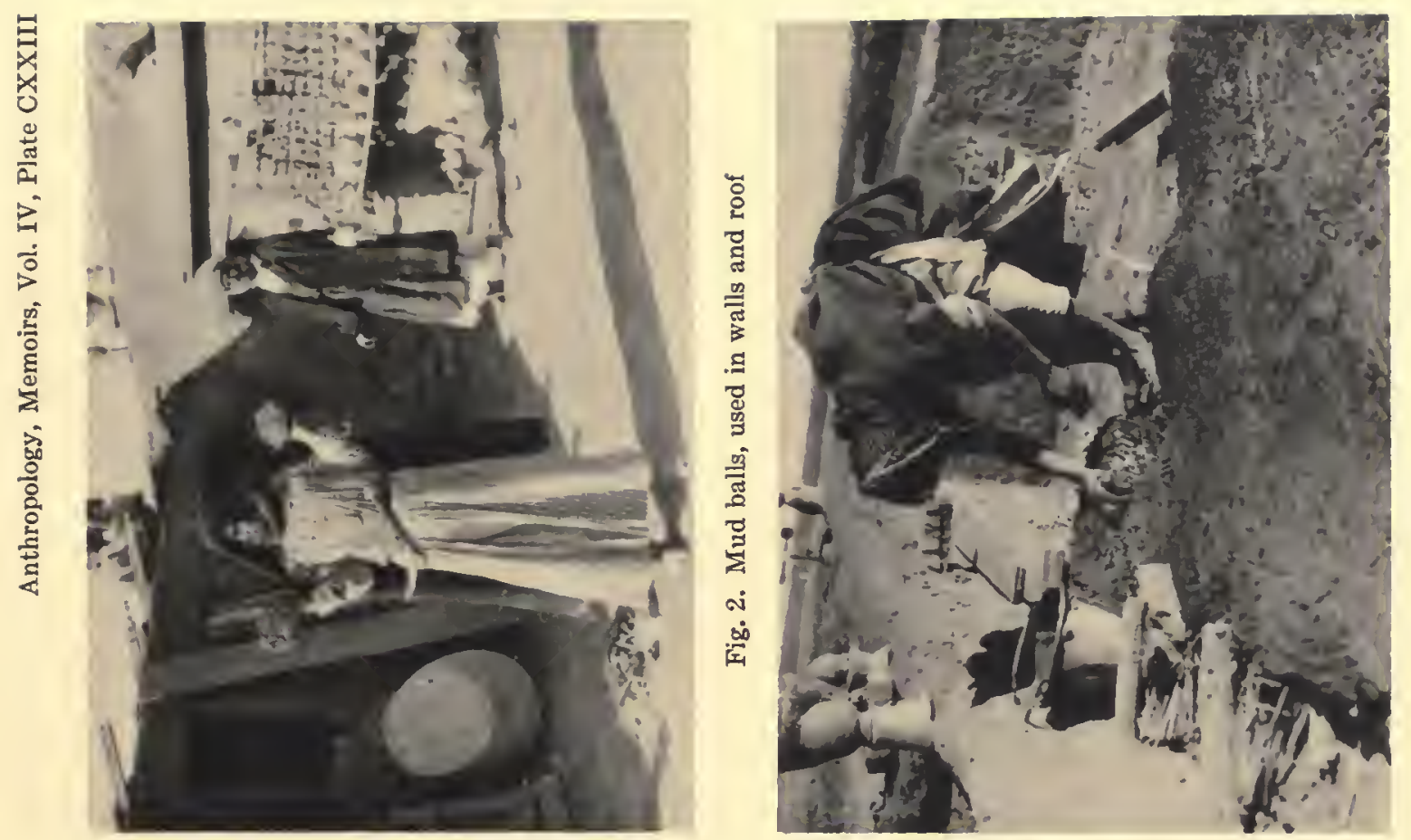

혼
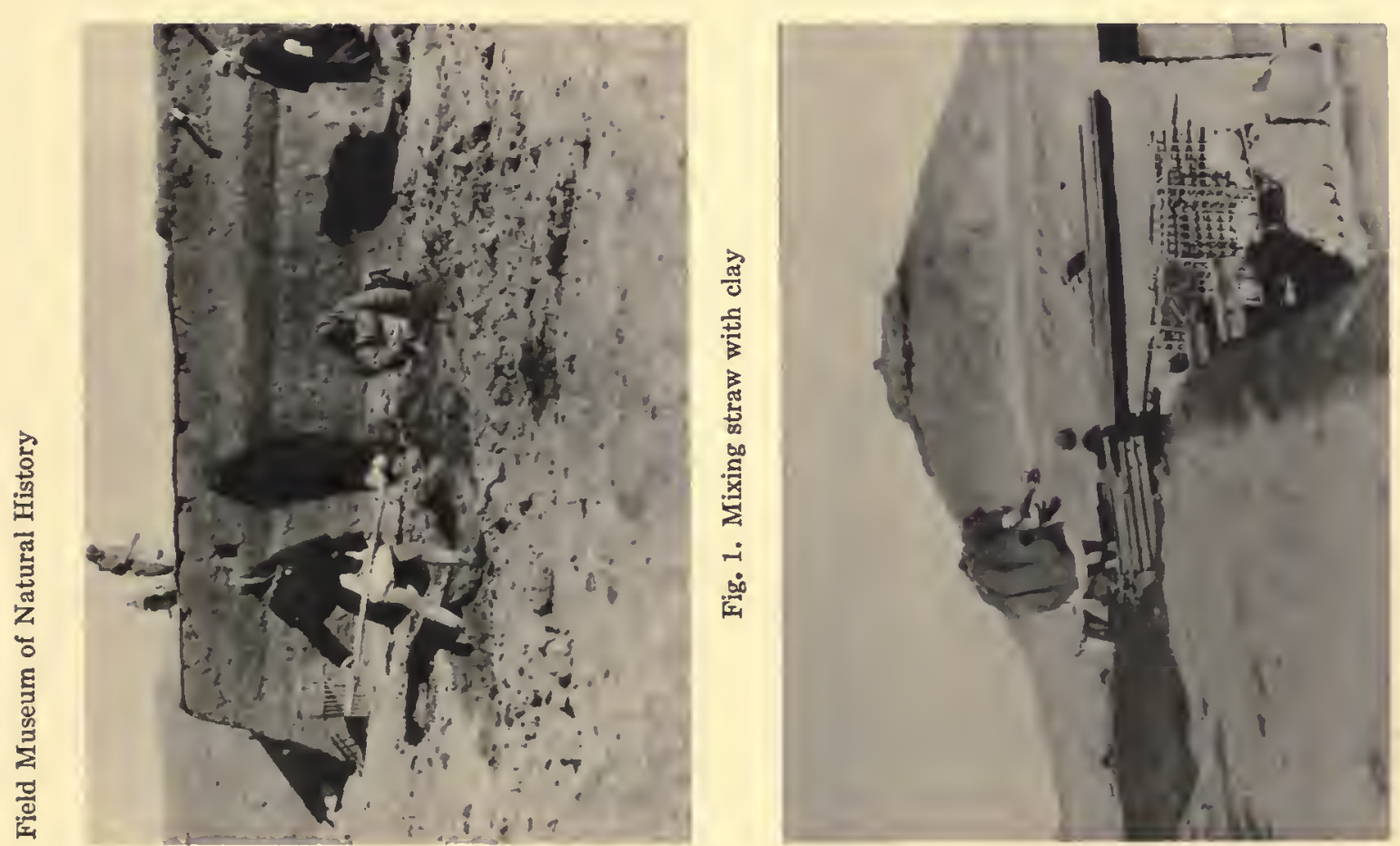

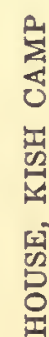

올

总

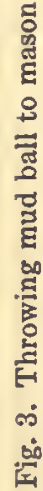

$b^{2}$

No 


\section{0,76482}

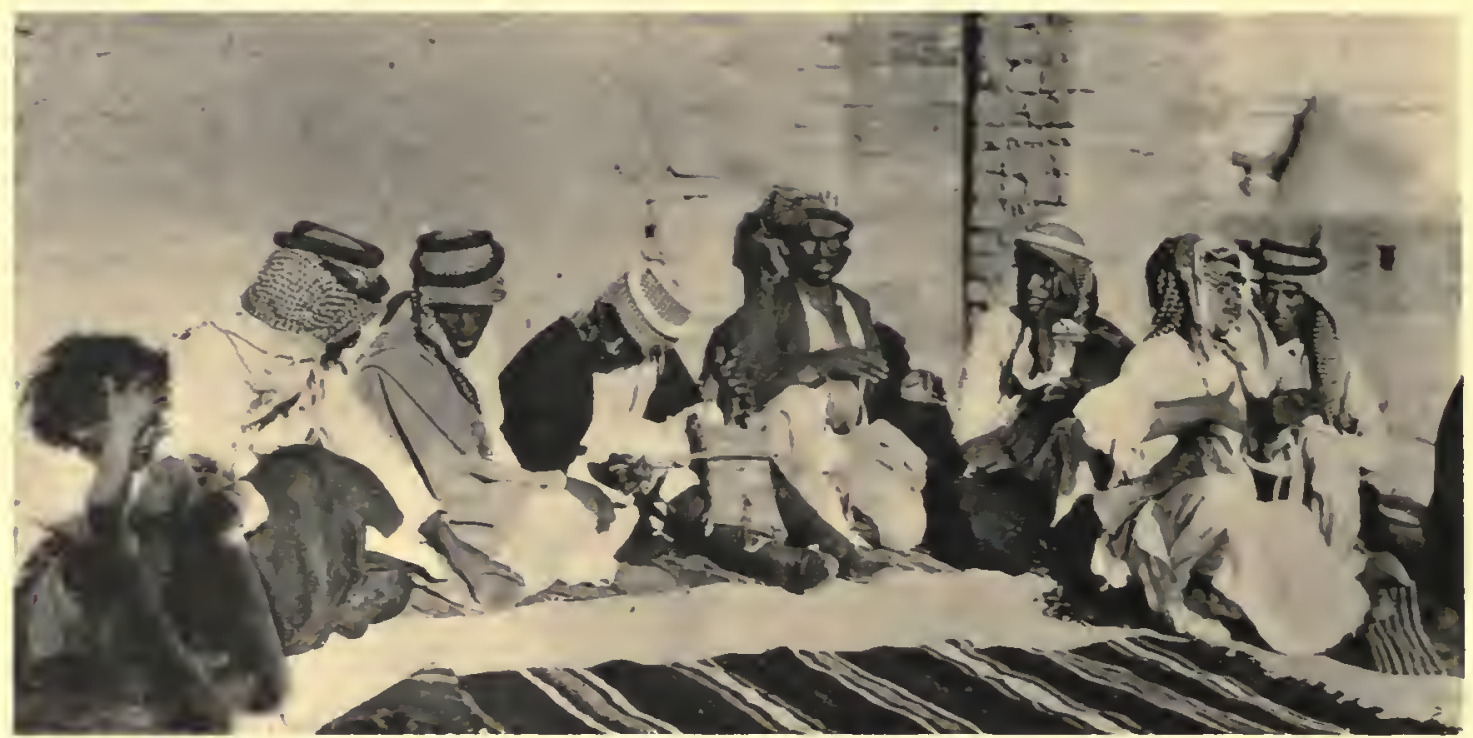

Fig. 1. Native orchestra

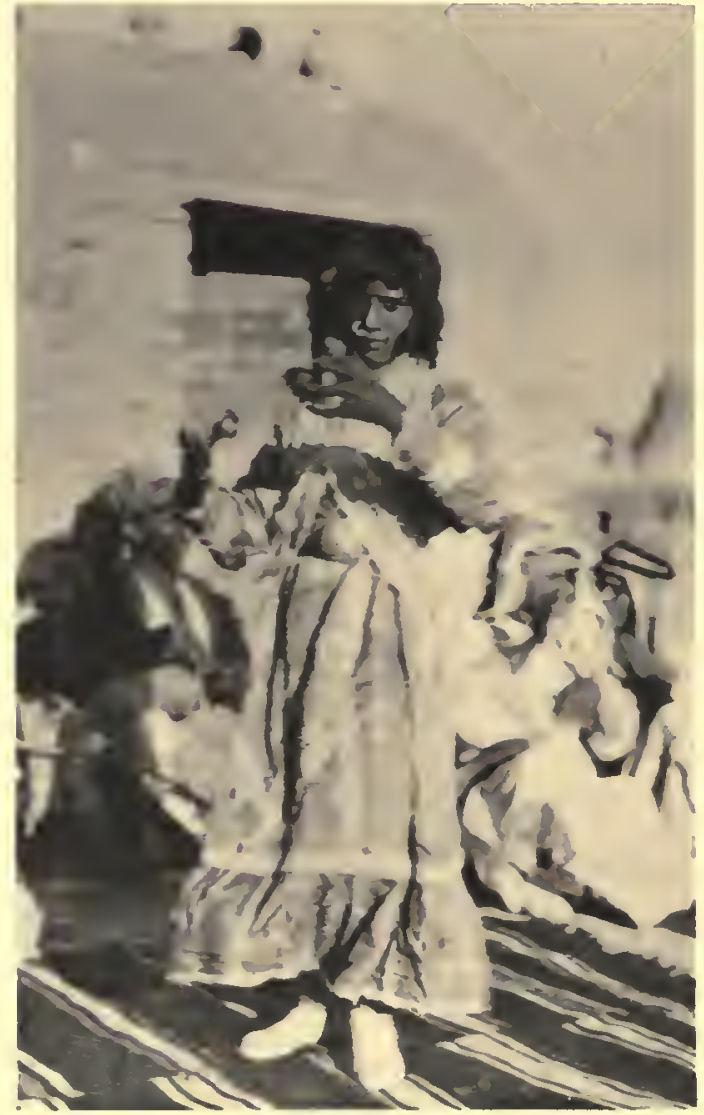

Fig. 2. Dancing girl

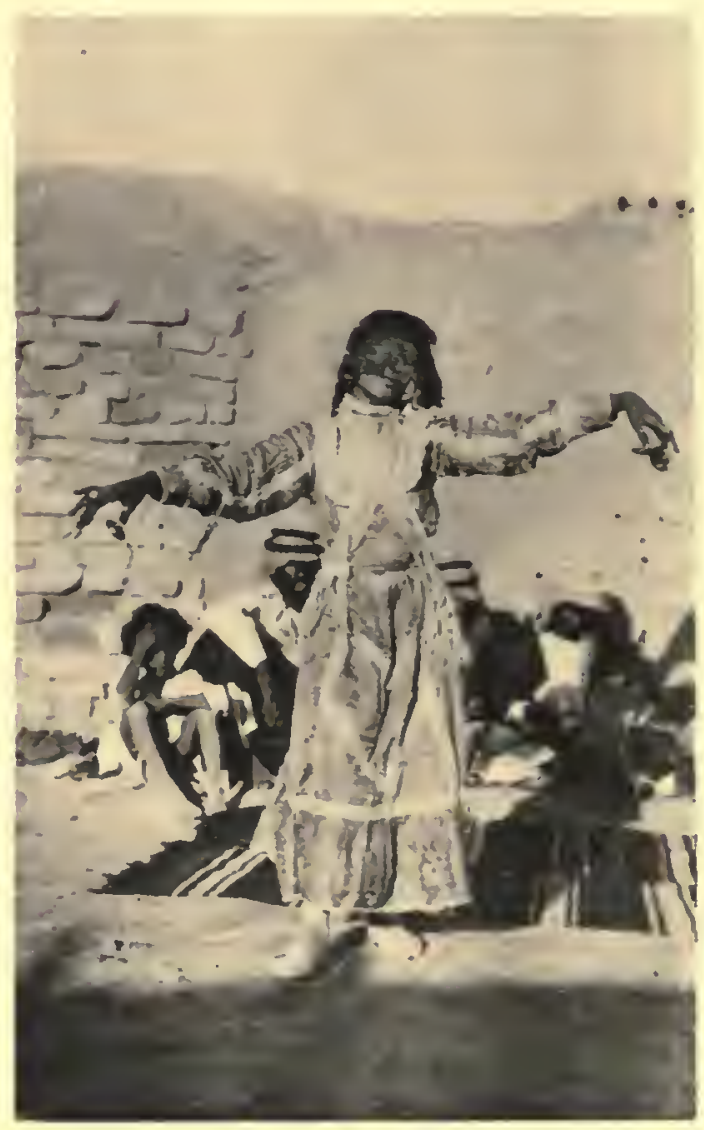

Fig. 3. Dancing girl ARAB DANCING GIRL AND MUSICIANS, KISH 

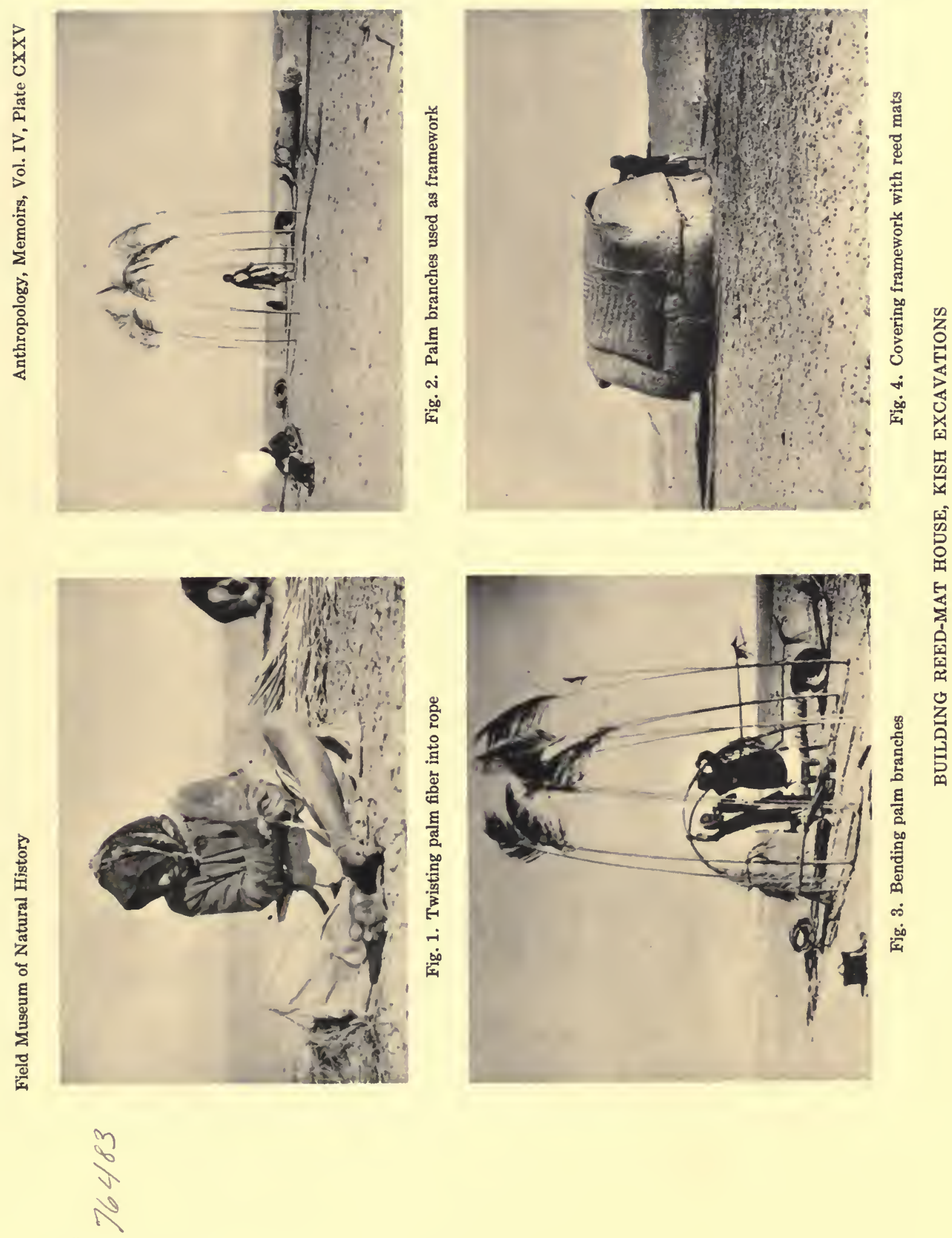

A

No 
Field Museum of Natural History

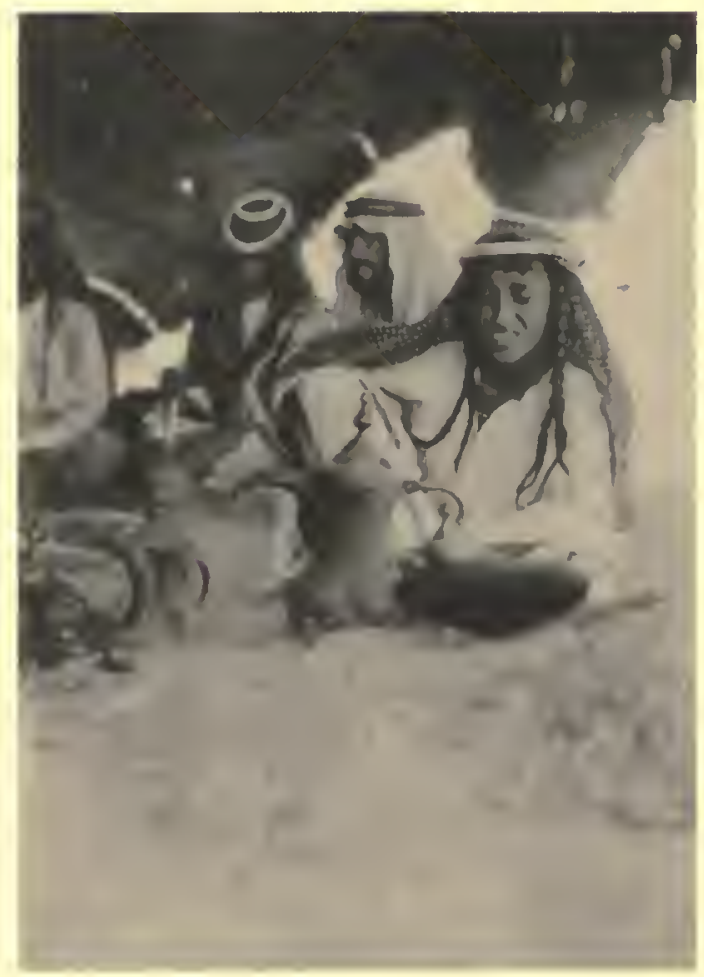

Fig. 1. Making coffee

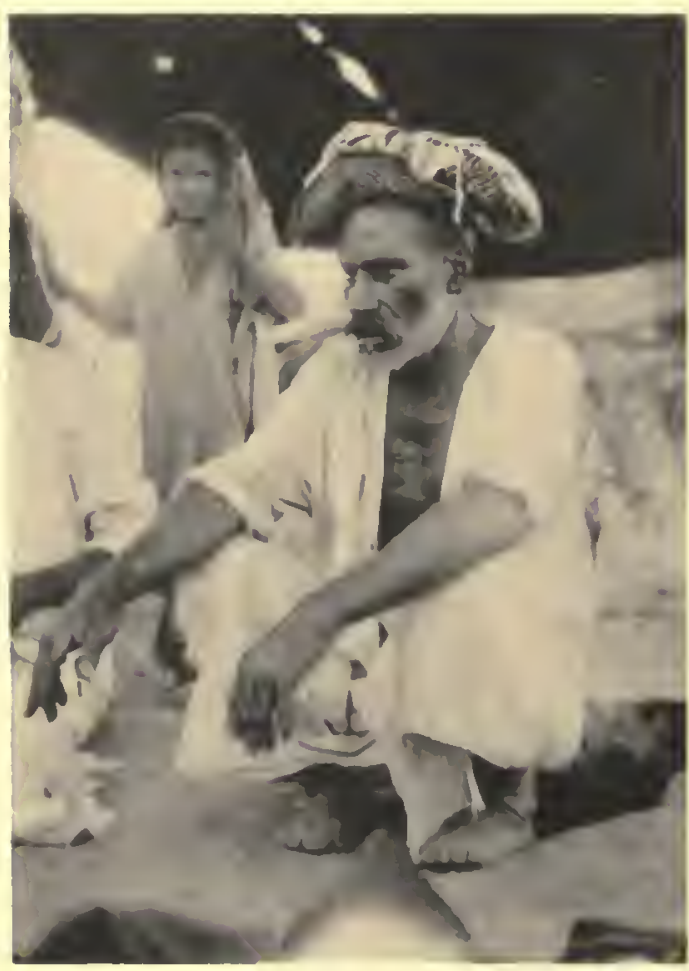

Fig. 3. Beside the hearth
Anthropology, Memoirs, Vol. IV, Plate CXXVI

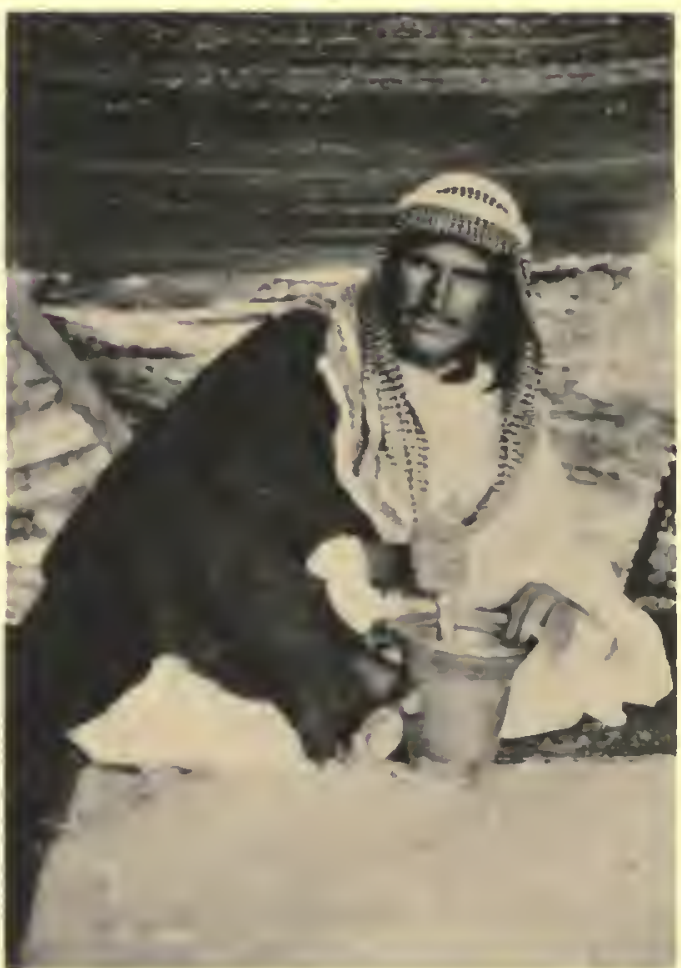

Fig. 2. Grinding coffee

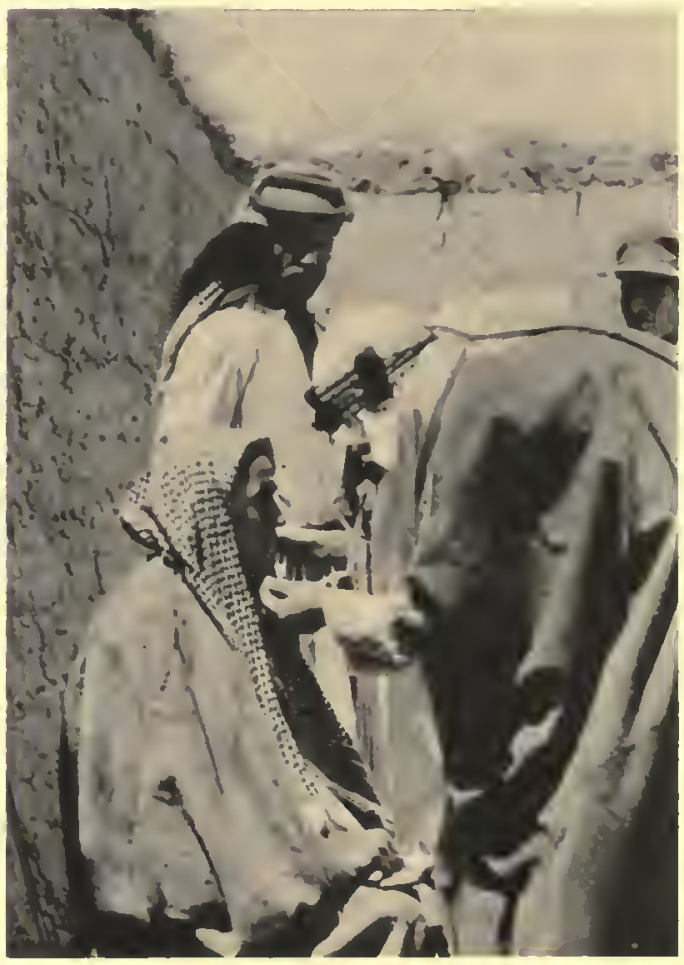

Fig. 4. Author, taking measurements 


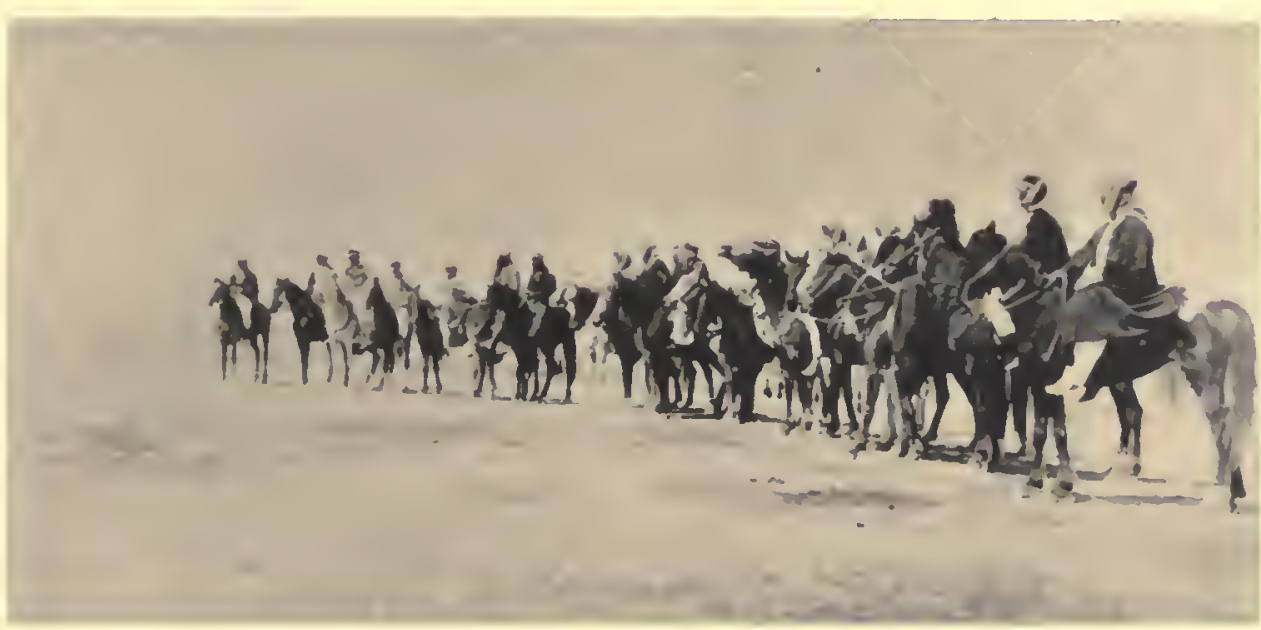

Fig. 1. Celebrating end of Ramadan

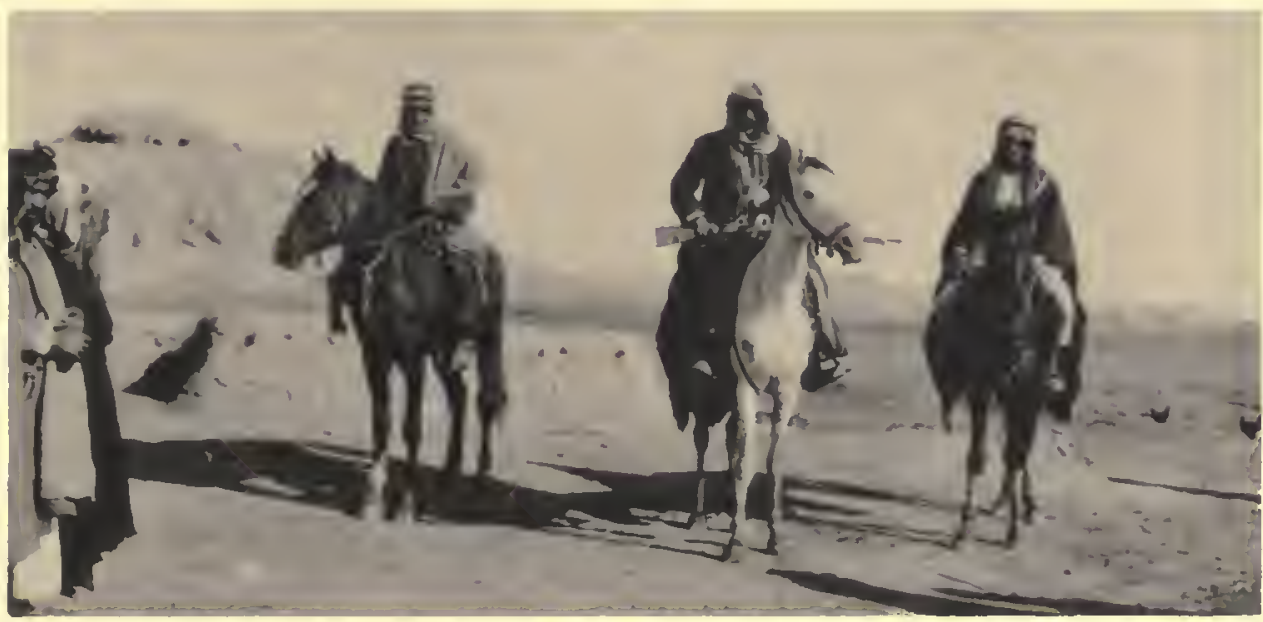

Fig. 2. Horsemen

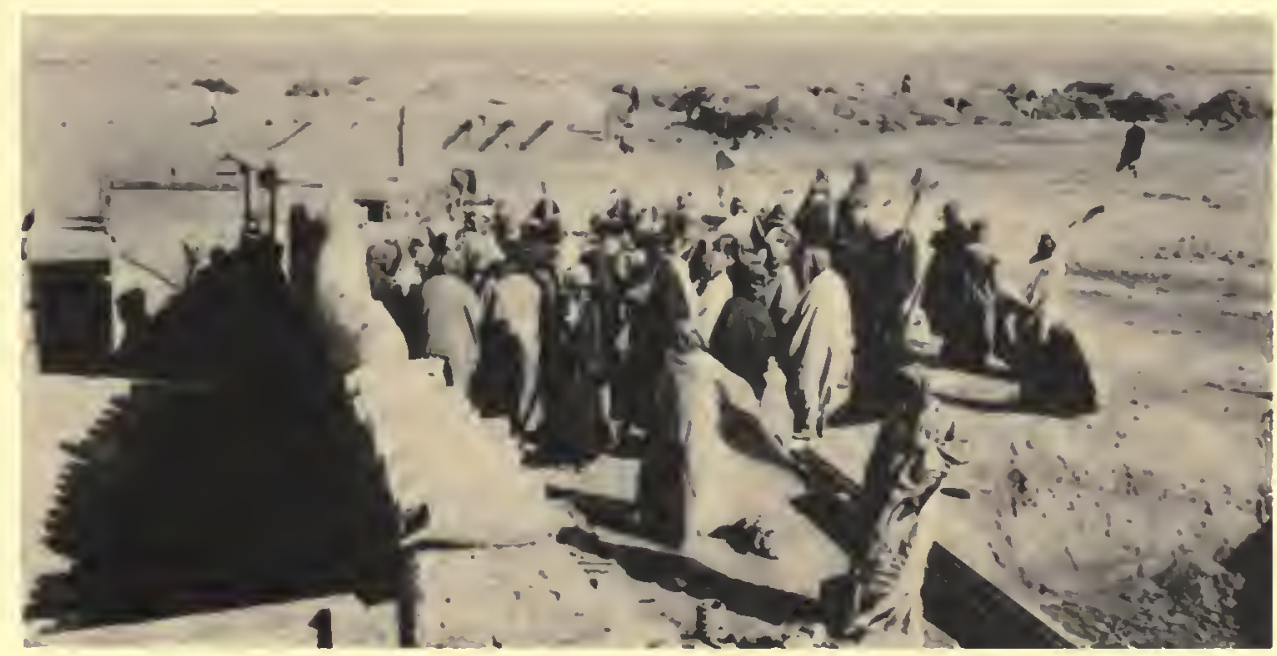

Fig. 3. Workmen outside camp, Kish ARABS, KISH AREA 


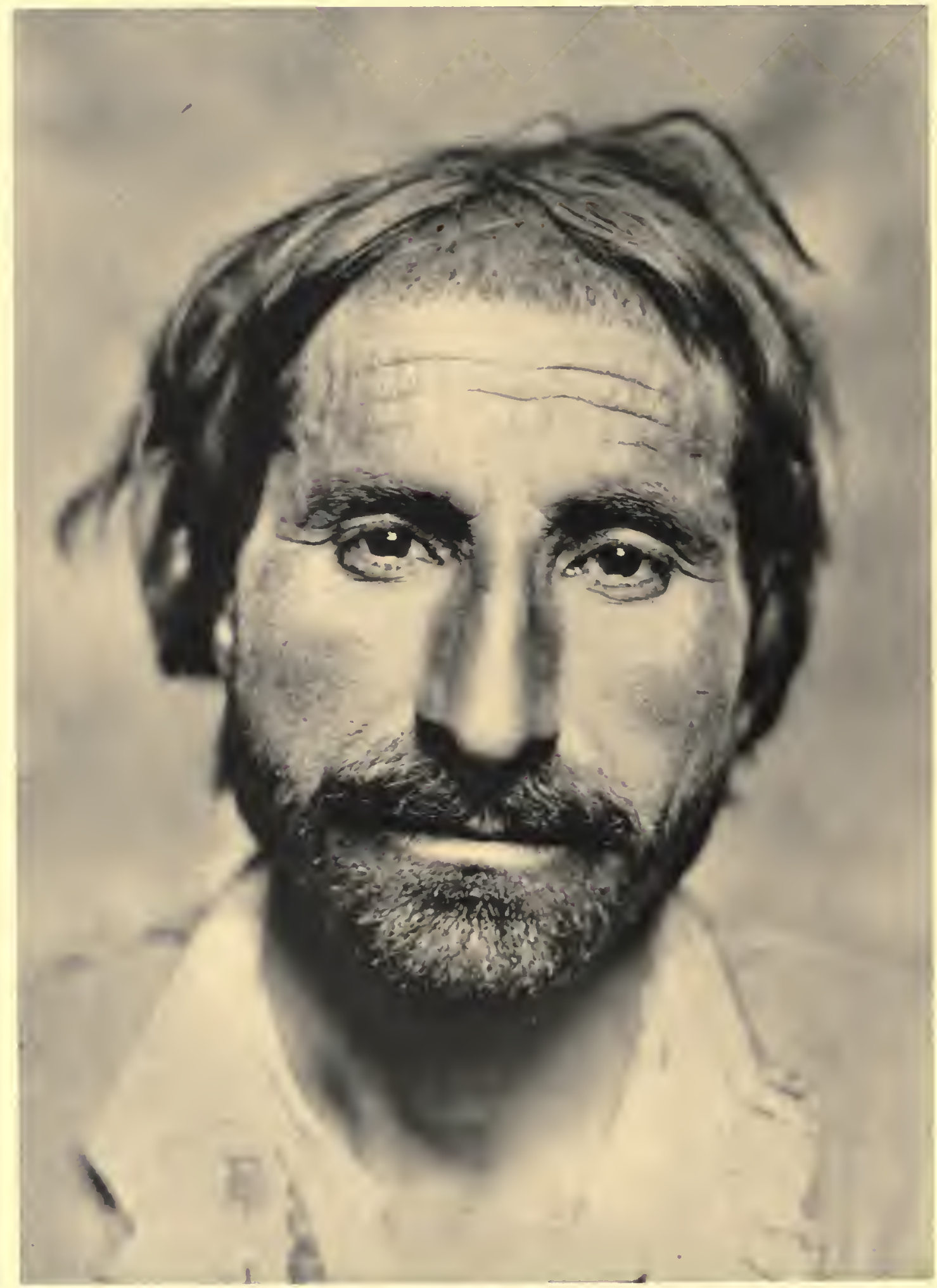

ARAB (No. 26), KISH AREA 


$$
\text { xy } \# 76487
$$

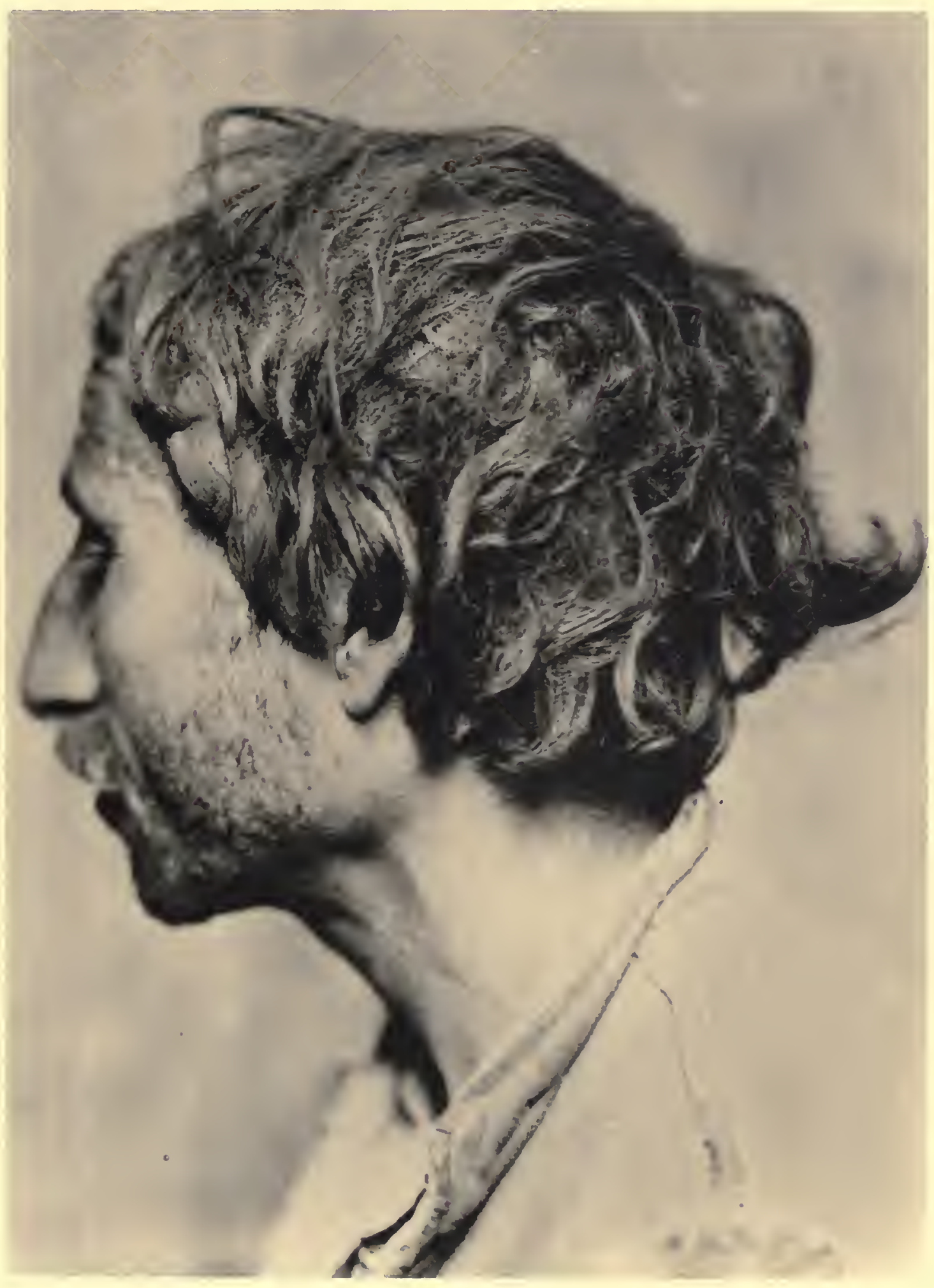

ARAB (No. 26), KISH AREA 


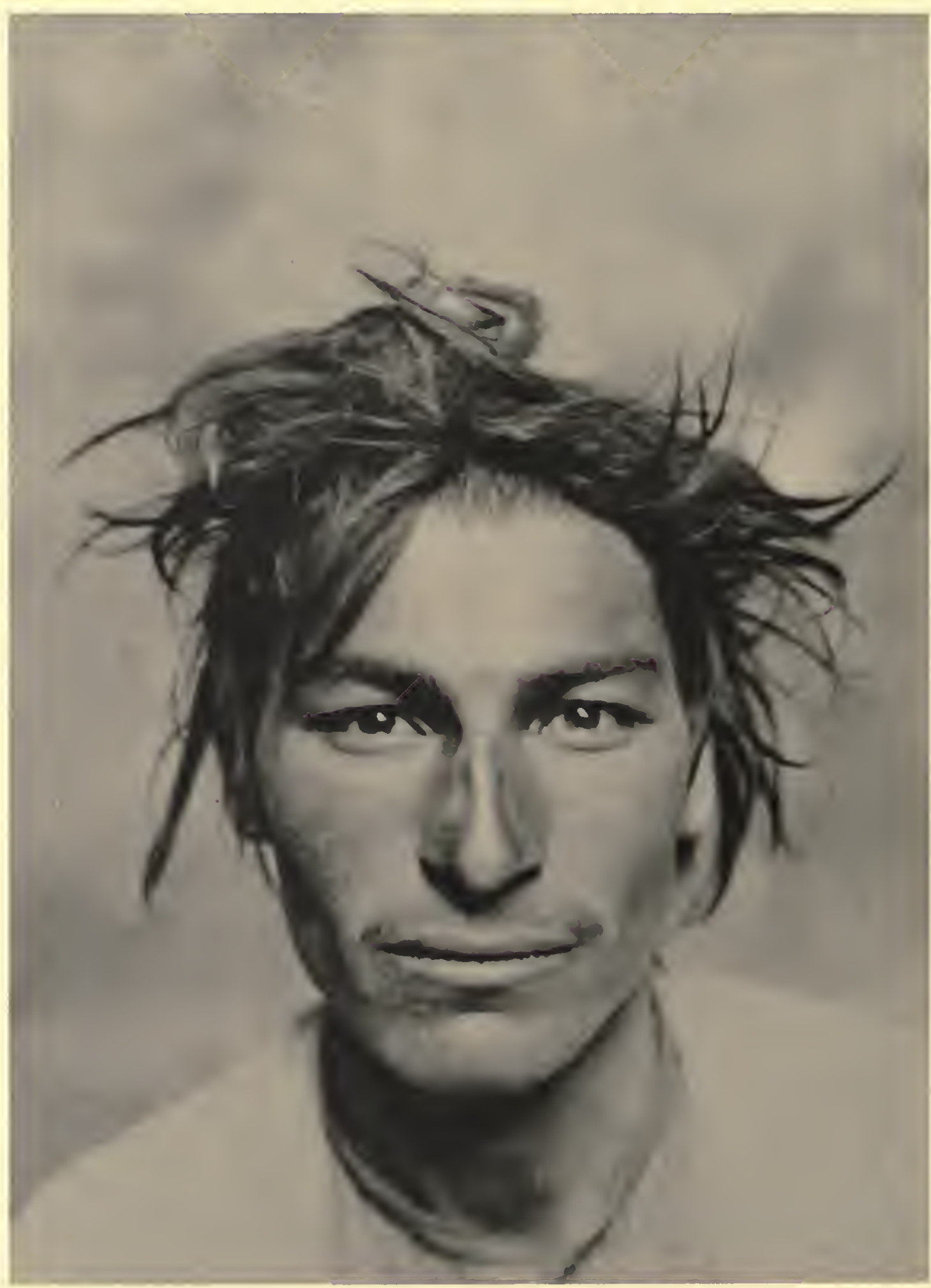

$\operatorname{ARAB}($ No. 2), KISH AREA 


$$
\begin{gathered}
\mathrm{Hey}^{*} 76489 \\
\left(6 \frac{1}{2} \times 8\right)
\end{gathered}
$$

Field Museum of Natural History Anthropology, Memoirs, Vol. IV, Plate CXXXI

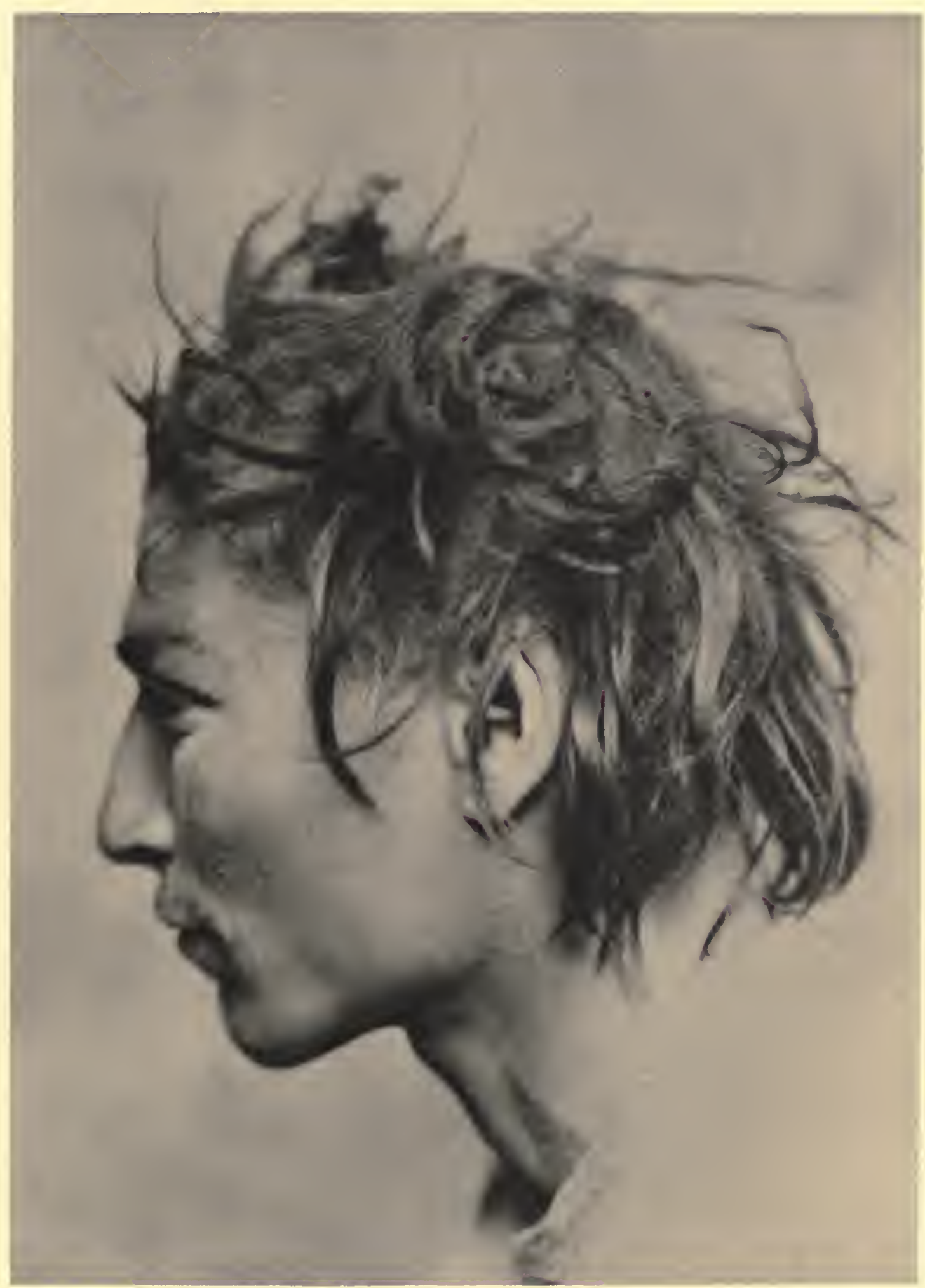

ARAB (No. 2), KISH AREA 


$$
7 l y \# 76489-A
$$

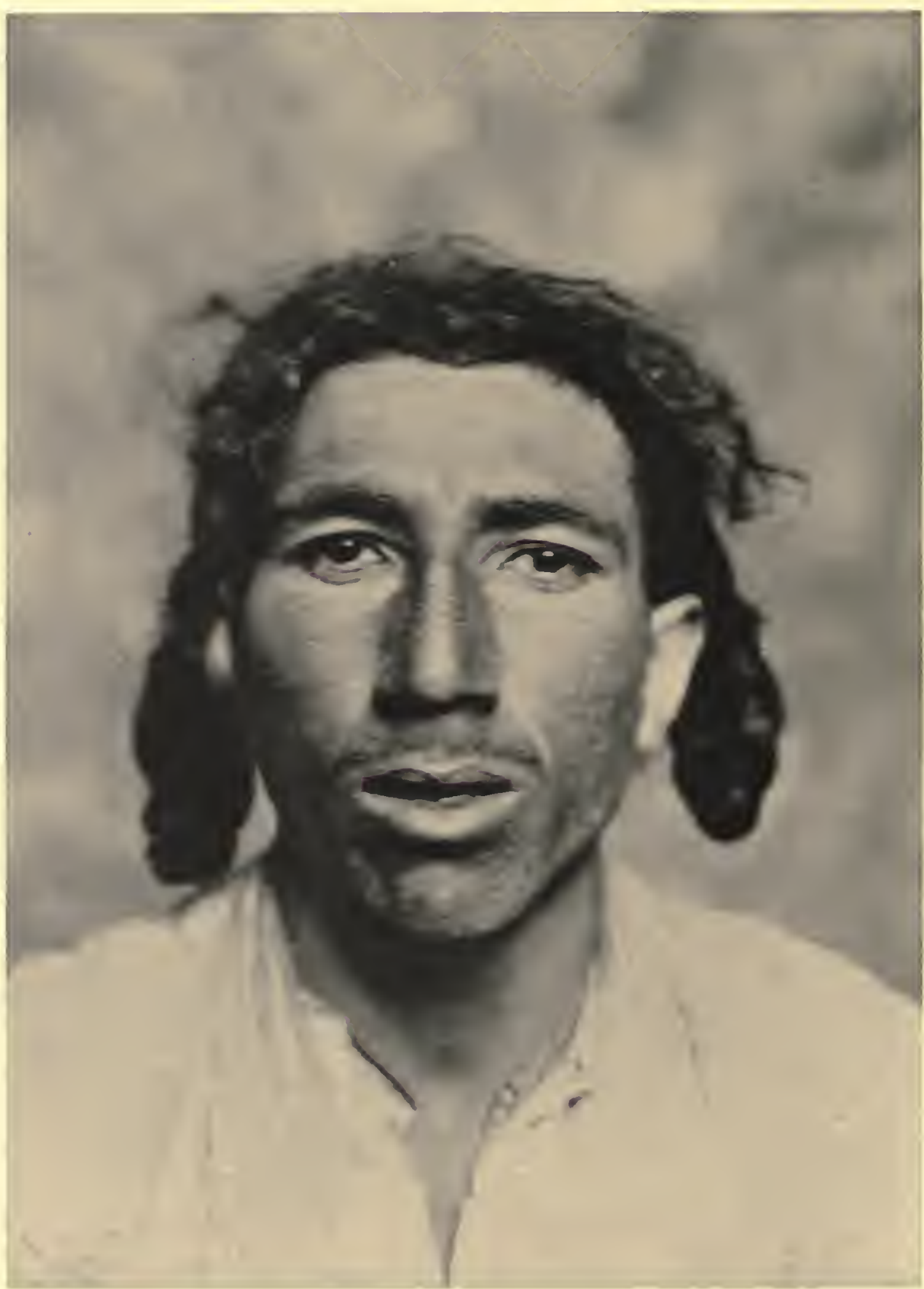

ARAB (No. 10), KISH AREA 


$$
10 \% * 76490
$$

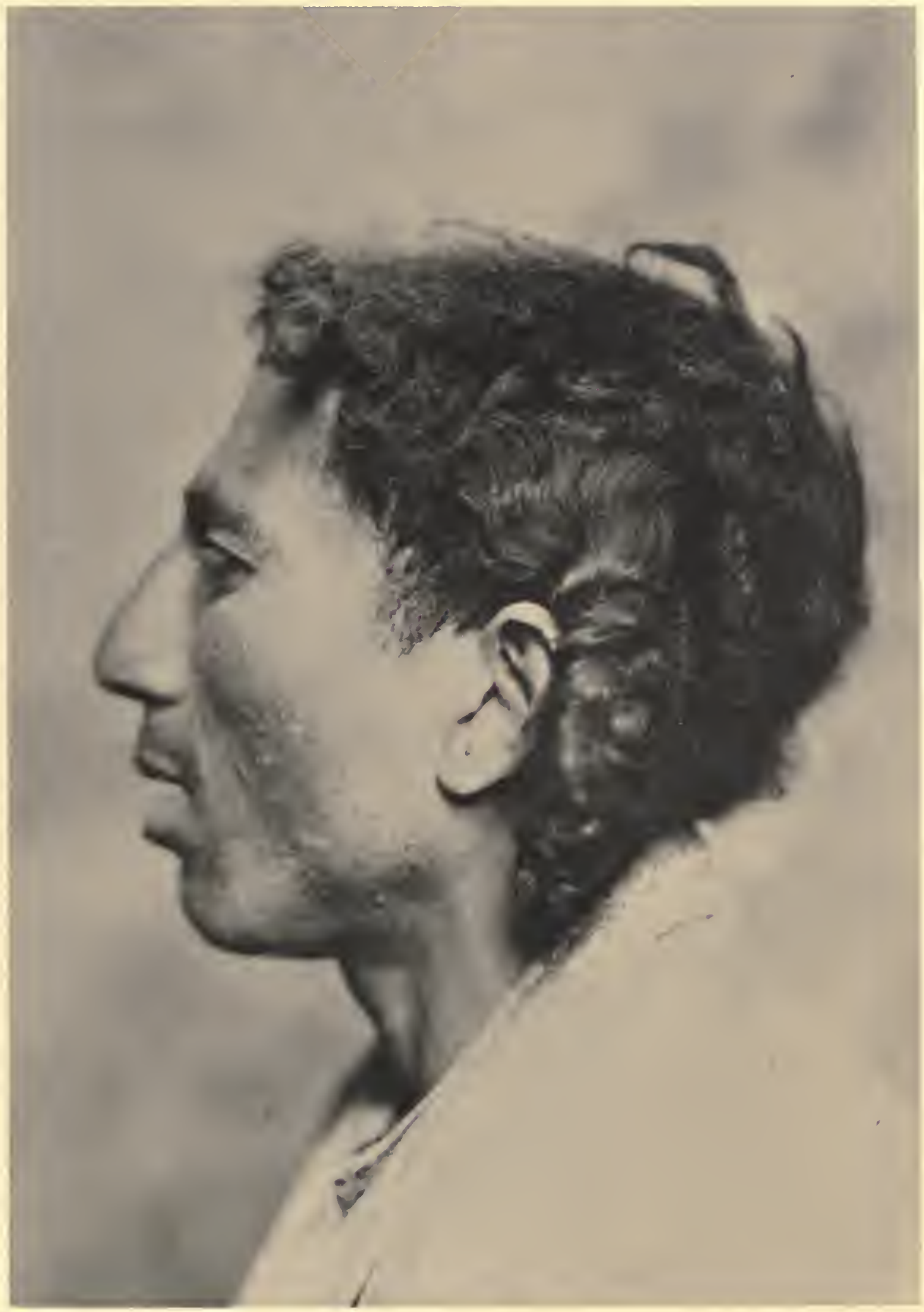

ARAB (No. 10), KISH AREA 


\section{$76 \% 76491$}

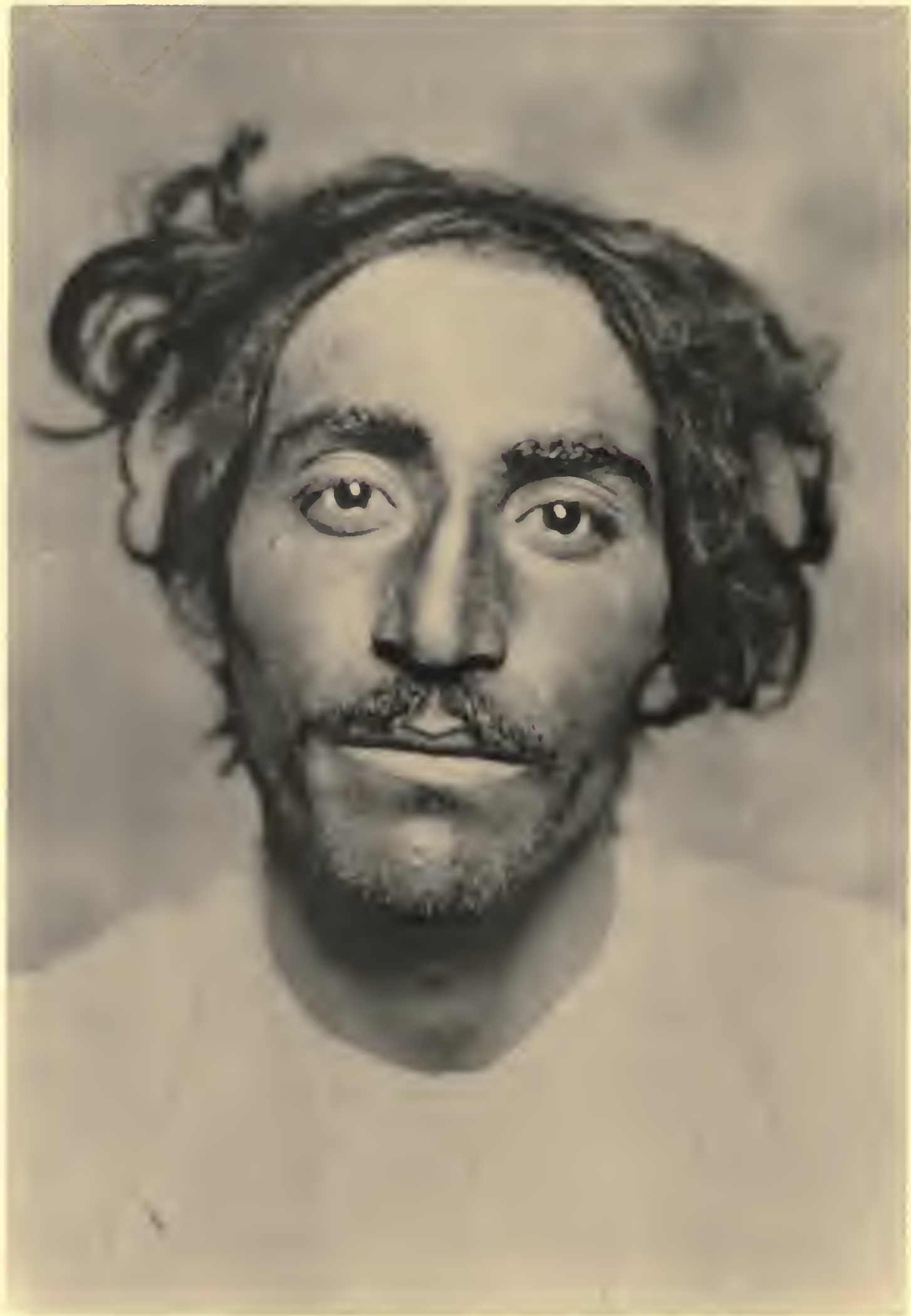

ARAB (No. 74), KISH AREA 


$$
\text { Hy \#.76492 }
$$

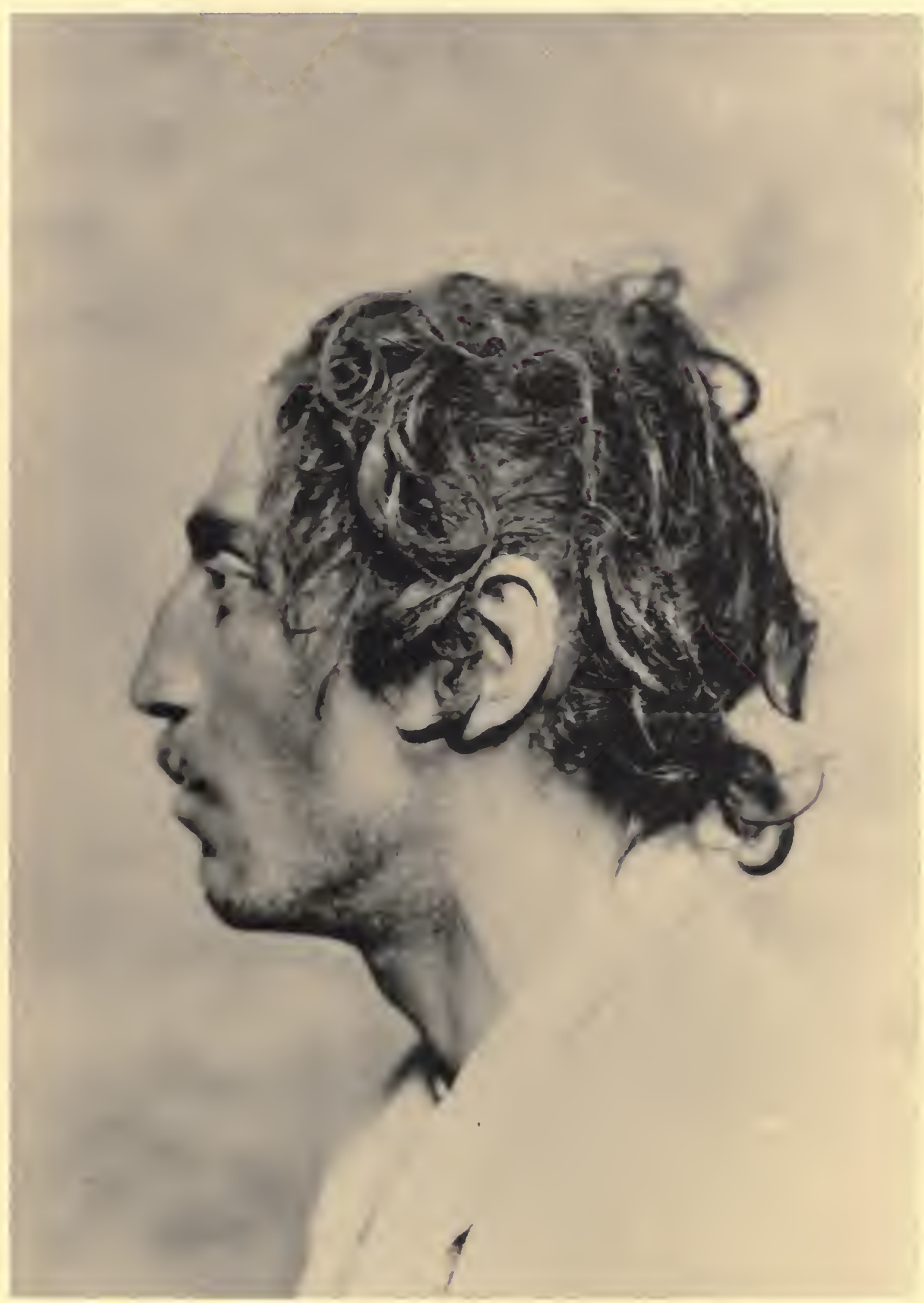

ARAB (No. 74), KISH AREA 


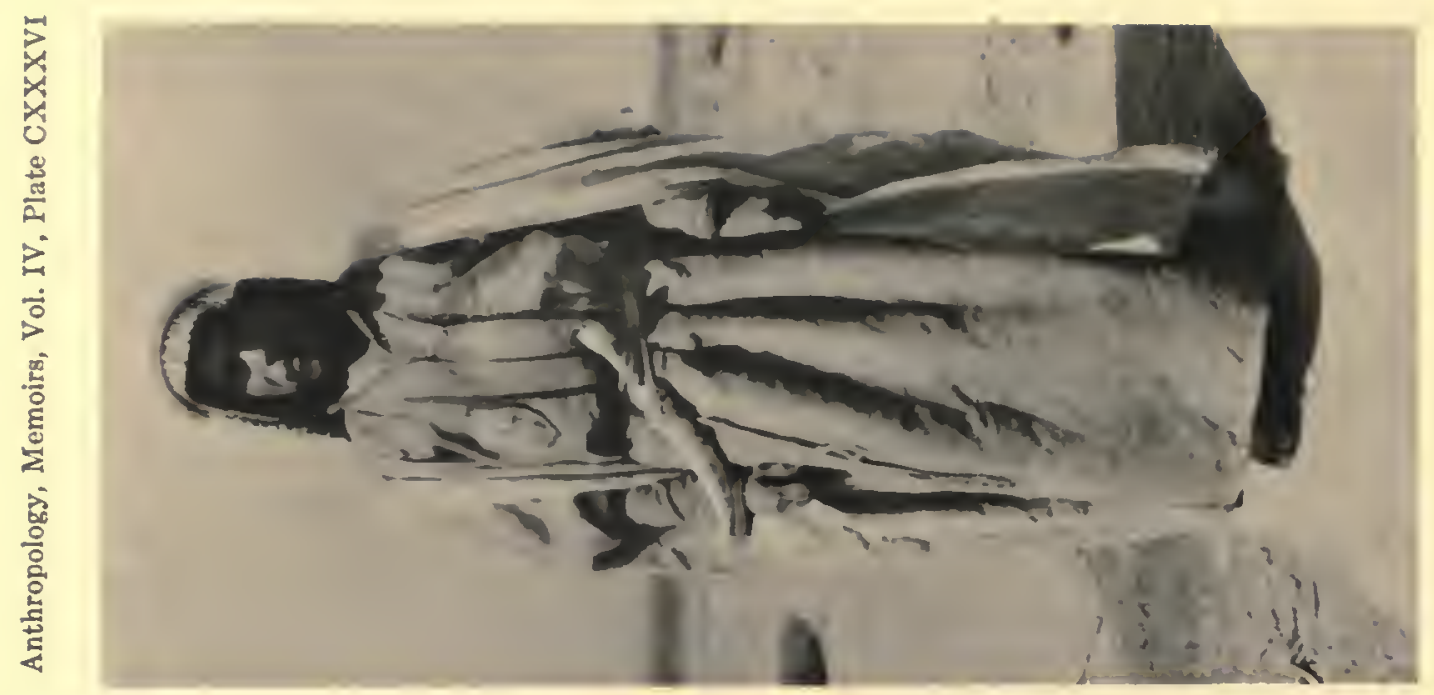

$y$
$x$

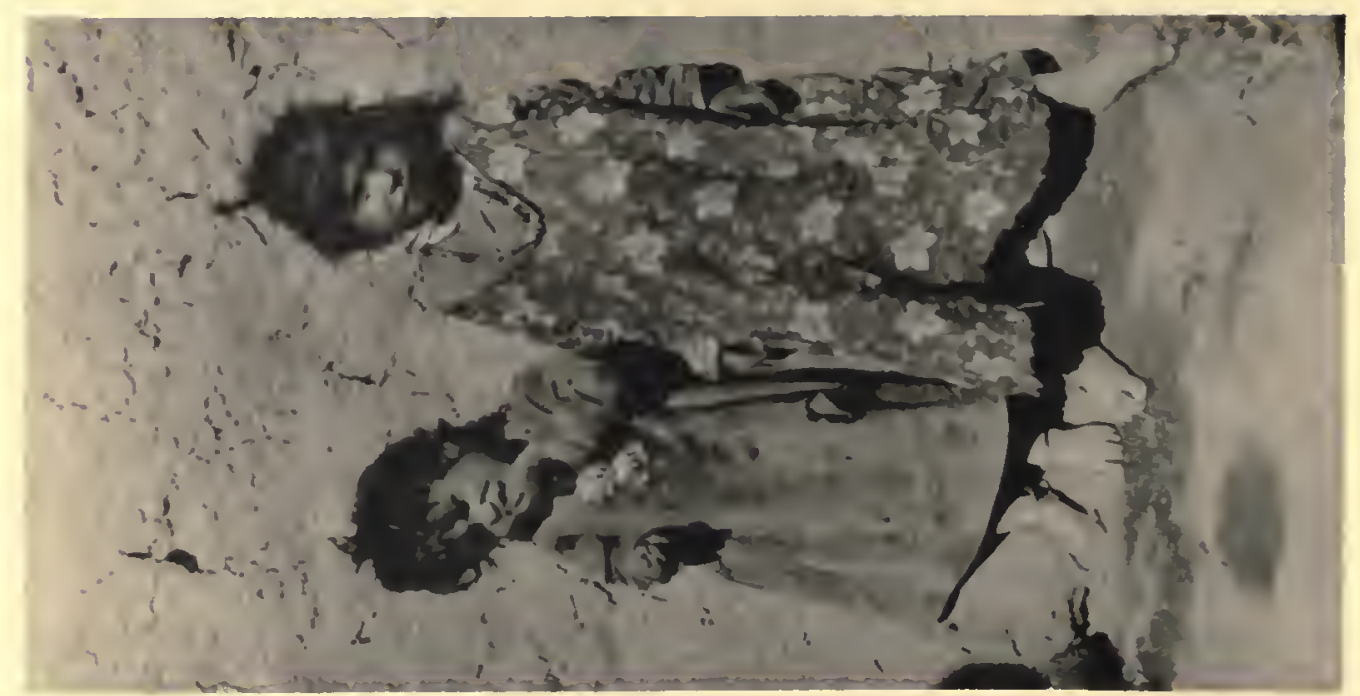

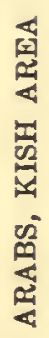

रू.

N

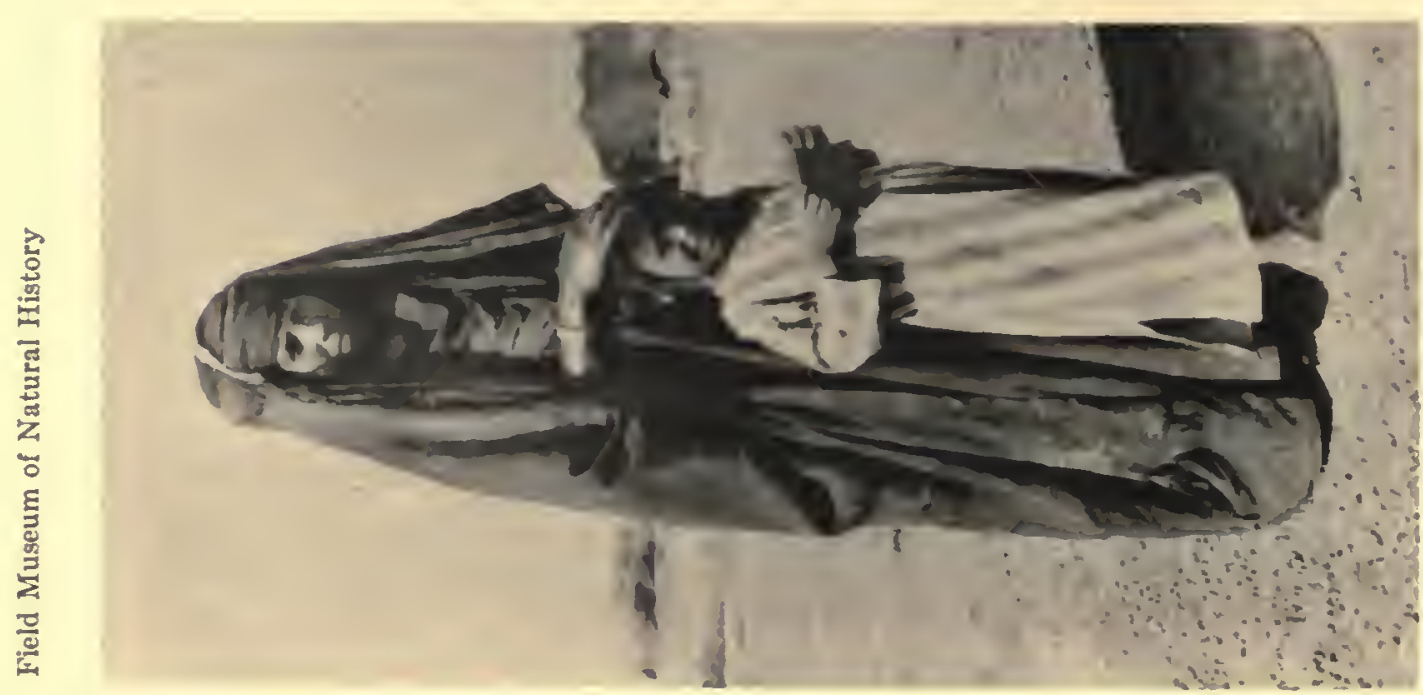




$$
7 y=76749
$$

Field Museum of Natural History Anthropology, Memoirs, Vol. IV, Plate CXXXVII

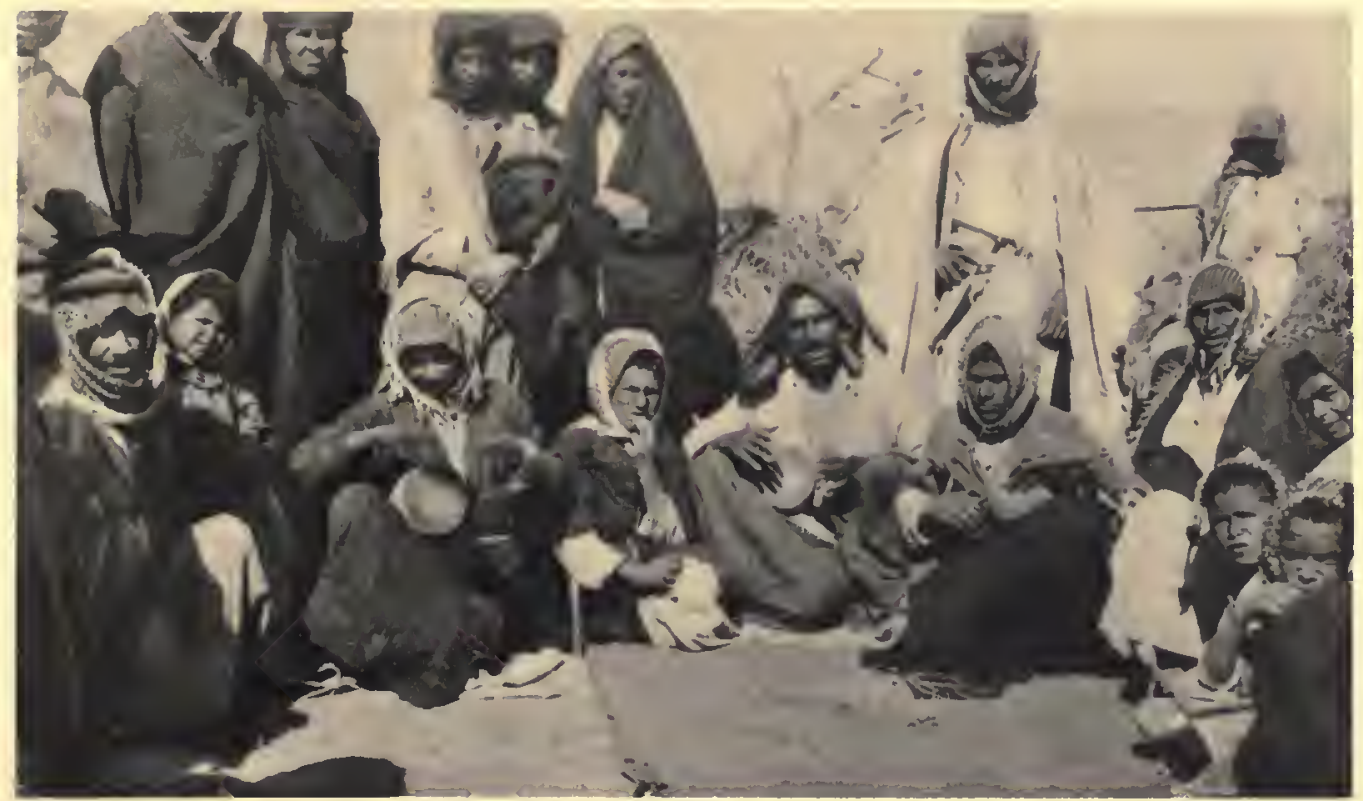

Fig. 1. Native orchestra

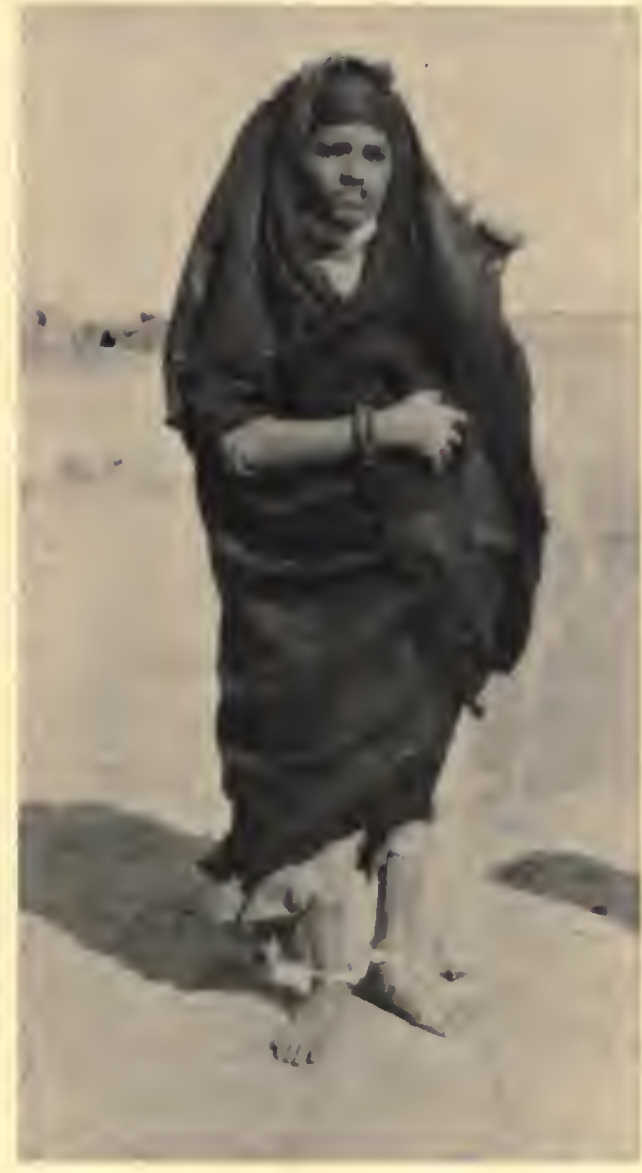

Fig. 2. Woman

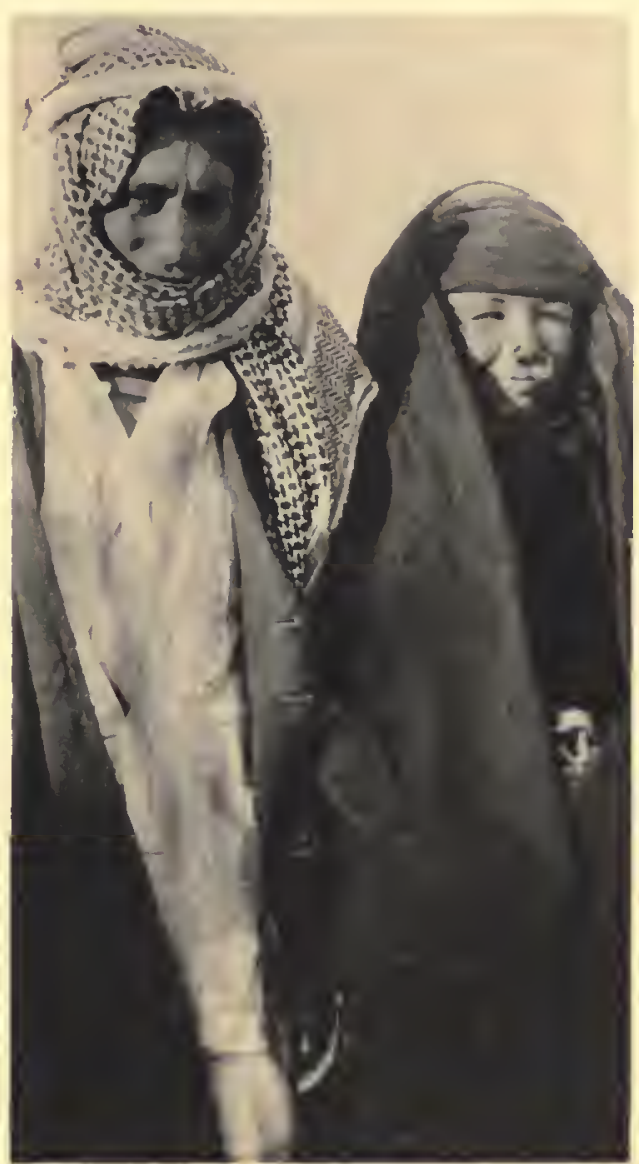

Fig. 3. Husband and wife 


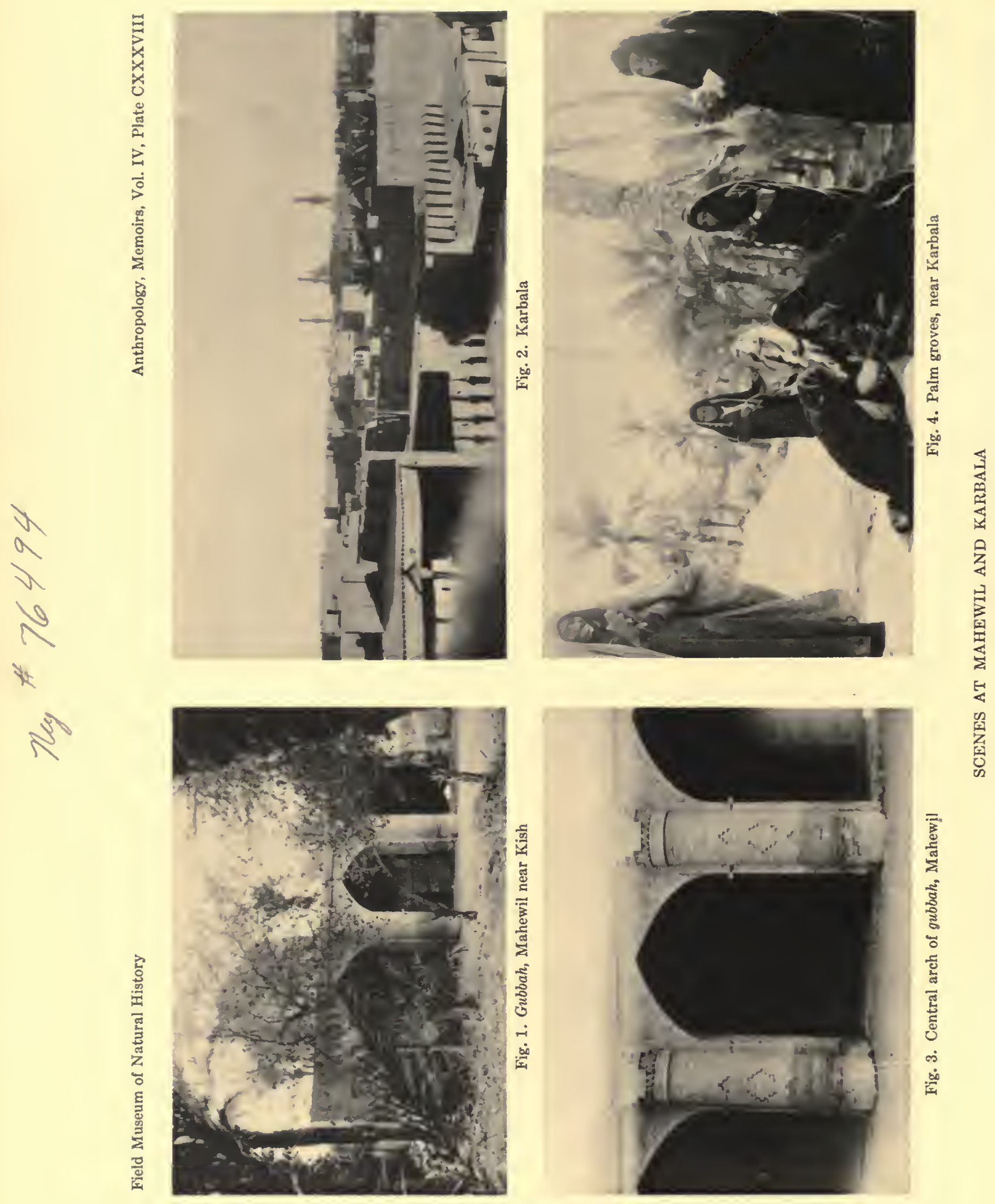


Field Museum of Natural History
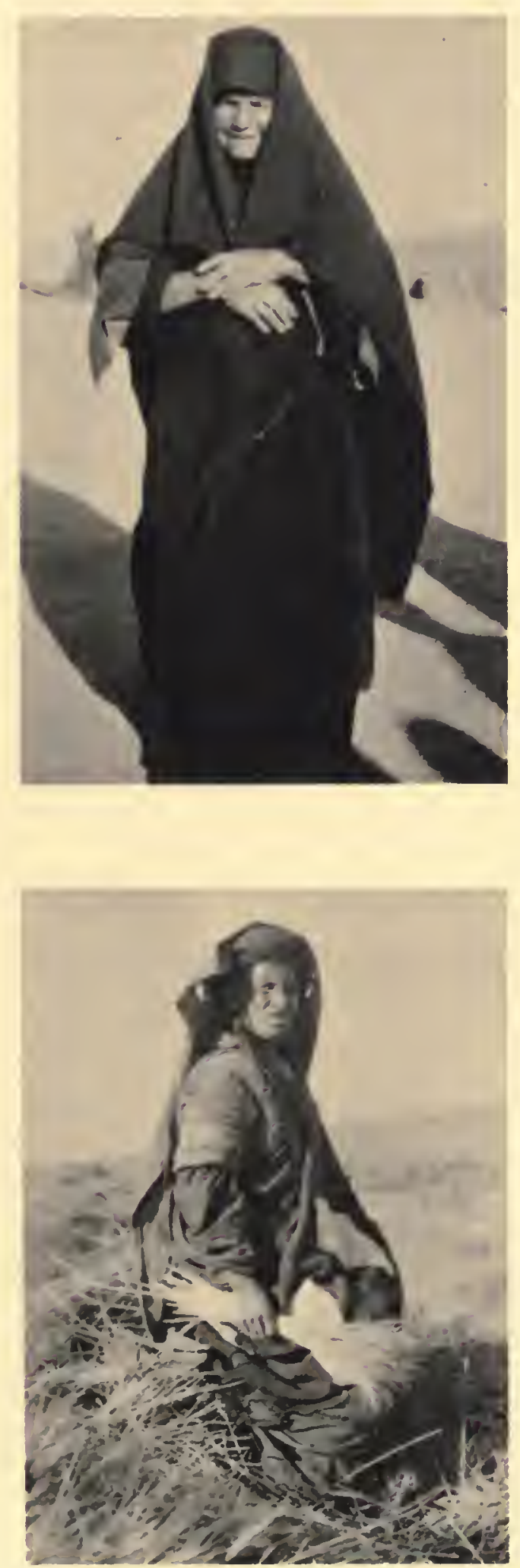

Anthropology, Memoirs, Vol. IV, Plate CXXXIX
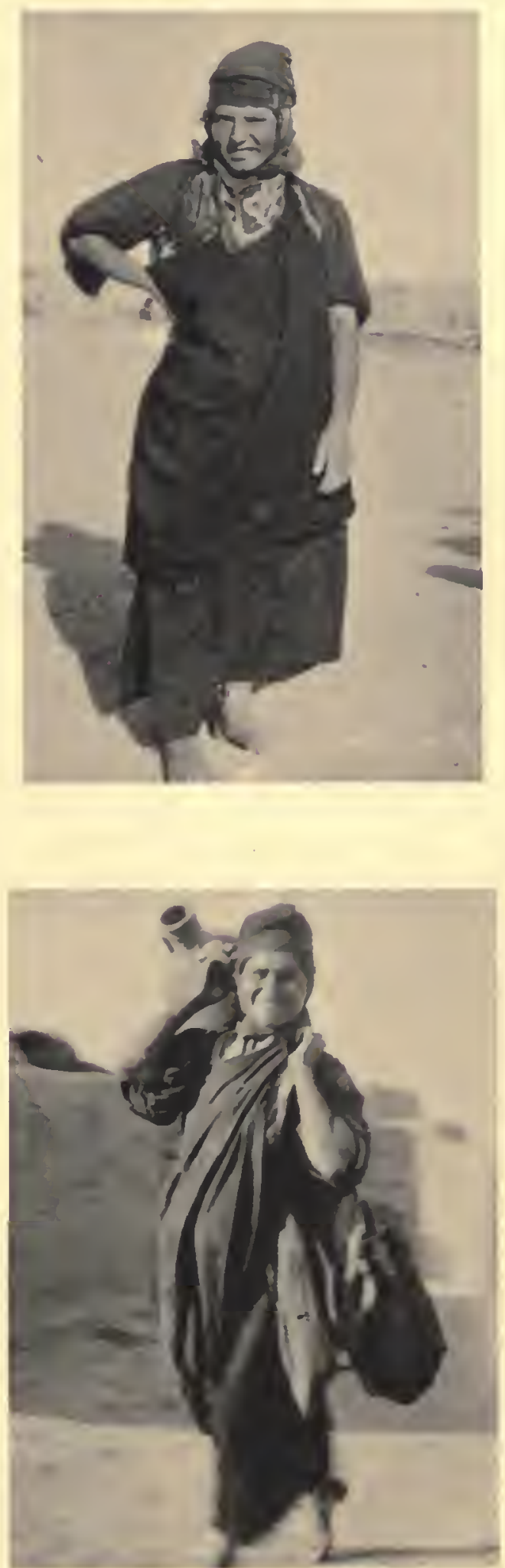

CHALDEAN WOMEN AND GIRLS, QARAQOSH 
$\frac{6}{15}$

$N$

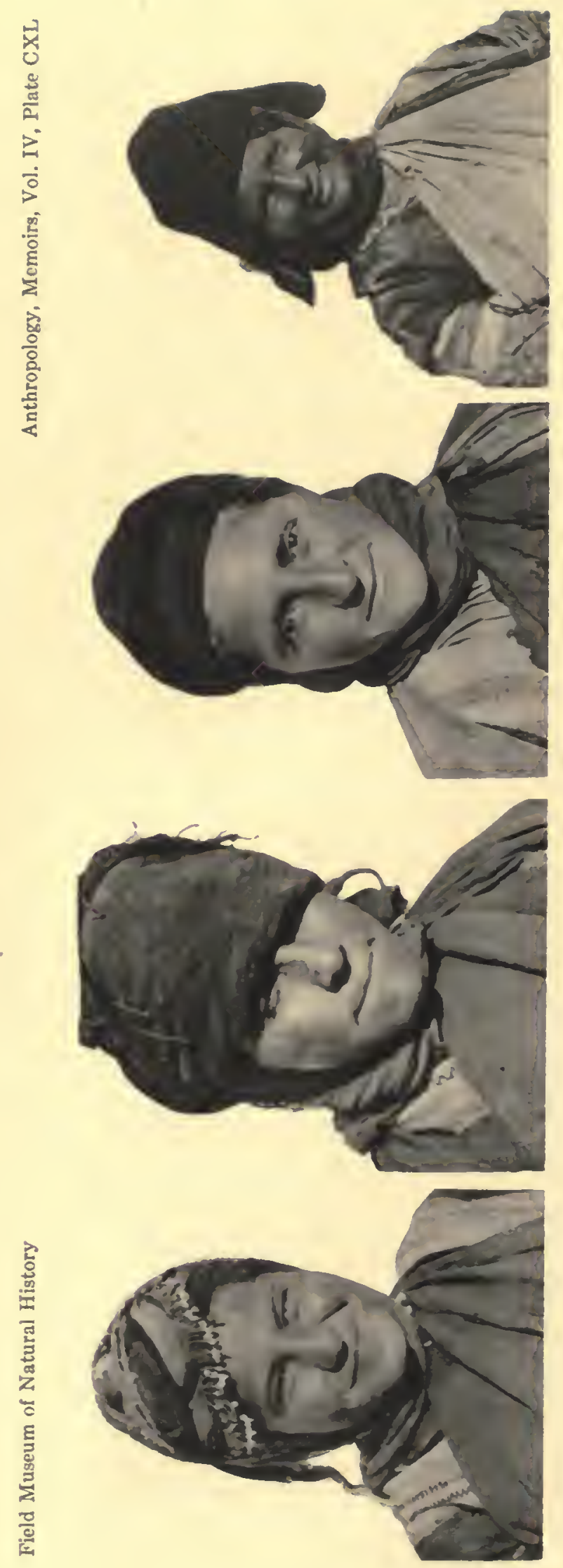
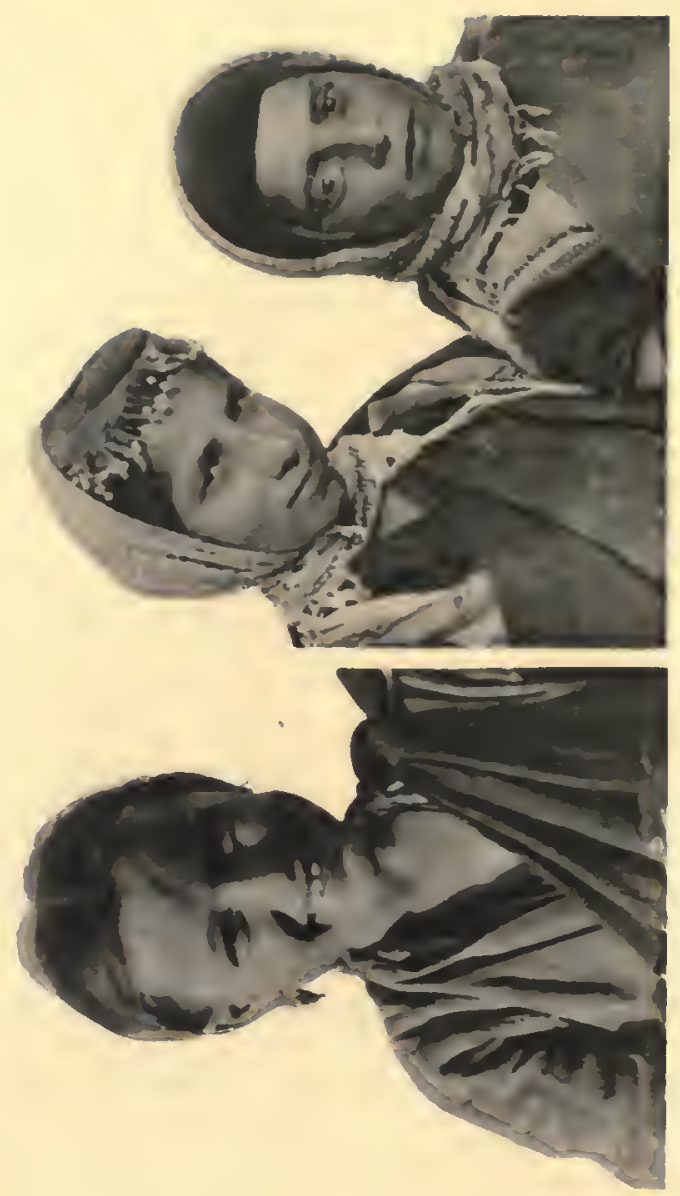

忨
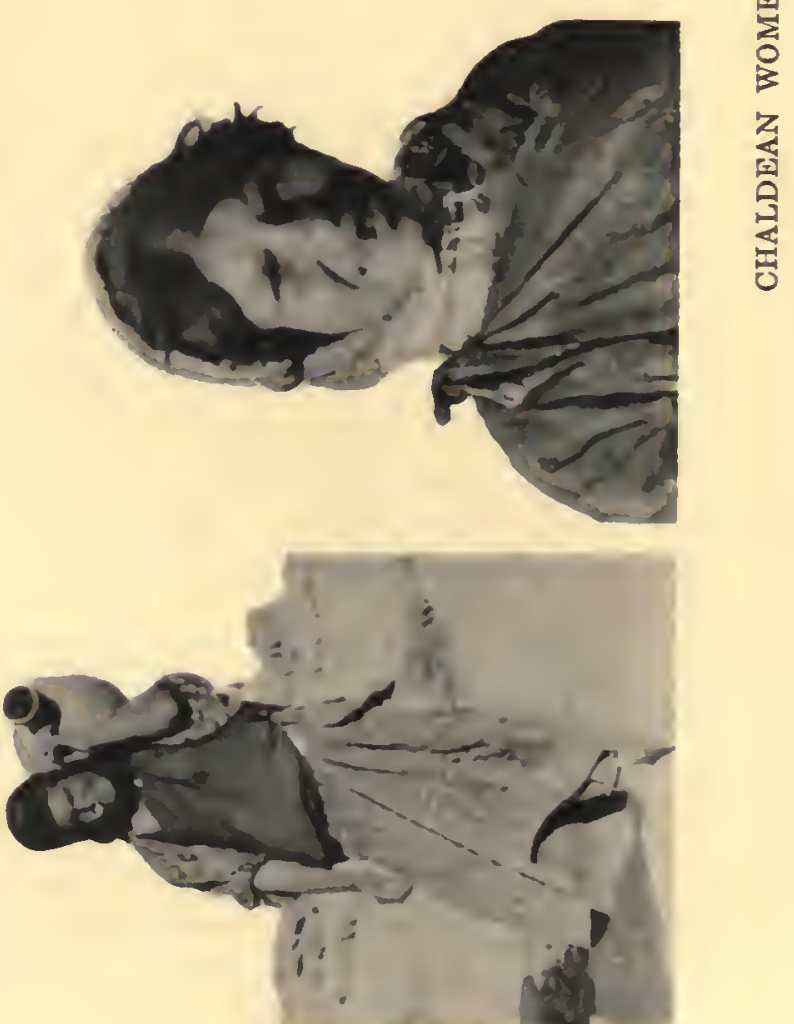
Field Museum of Natural History

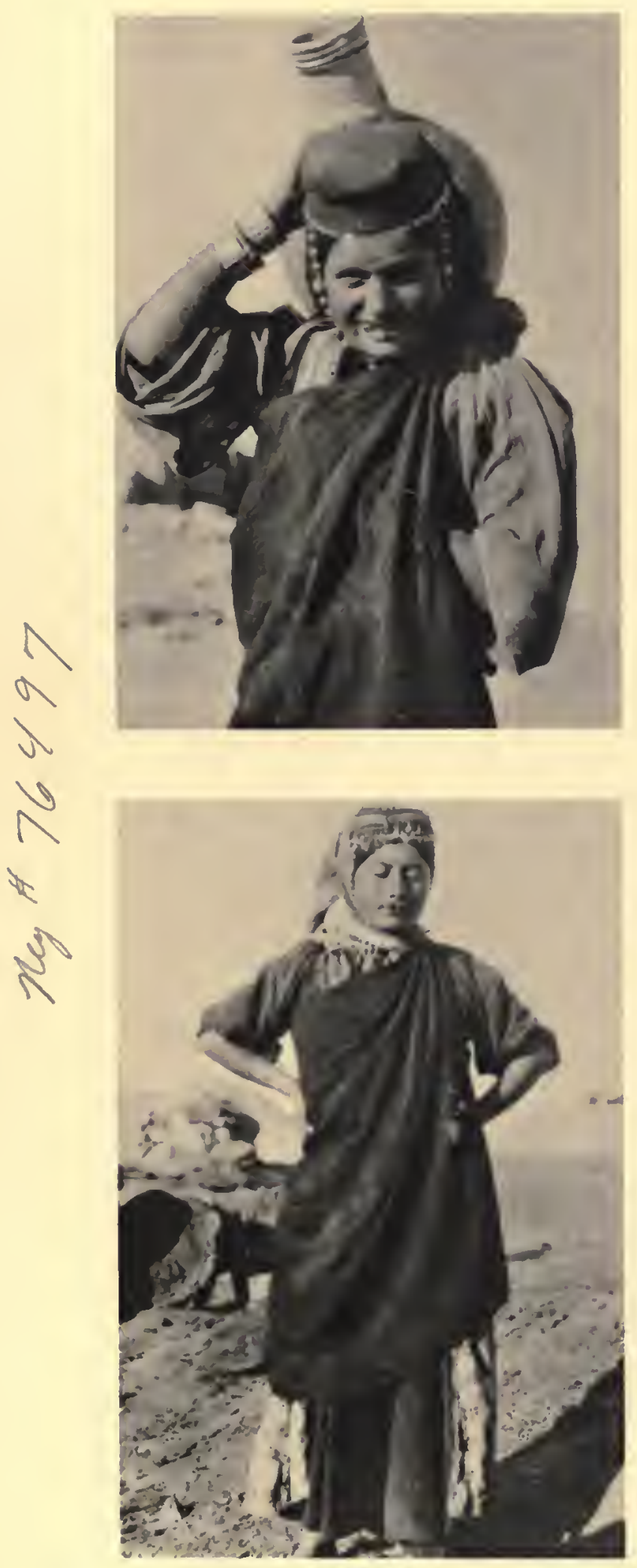

Anthropology, Memoirs, Vol. IV, Plate CXLI
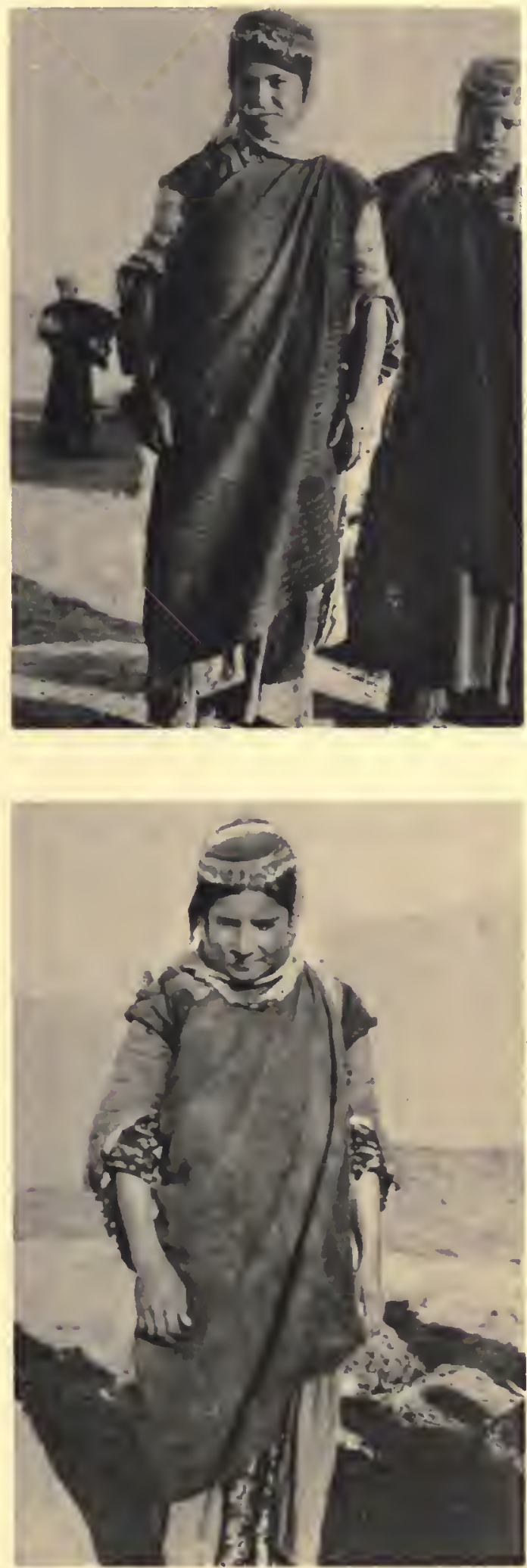

CHALDEAN WOMEN AND GIRLS, QARAQOSH 

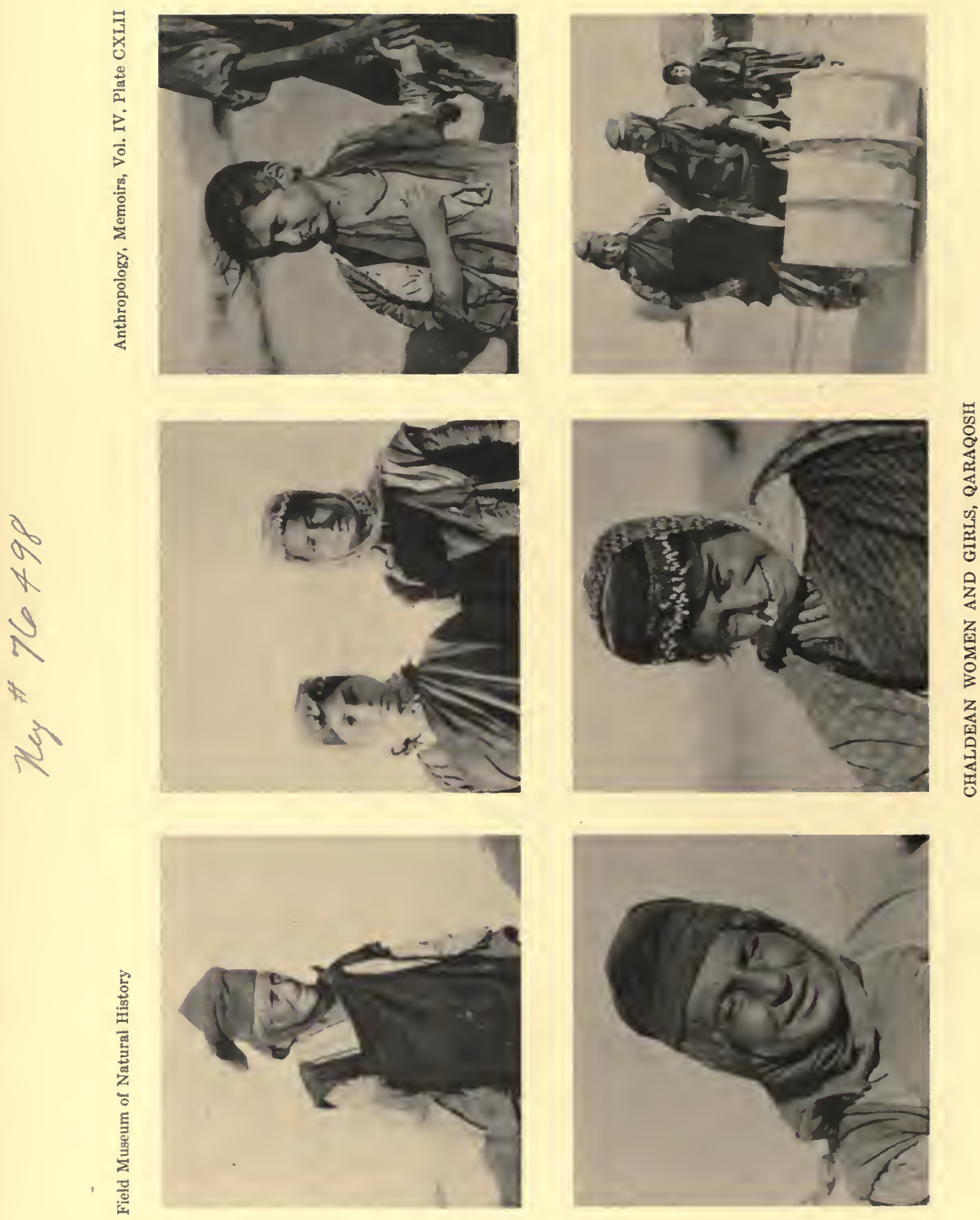
Field Museum of Natural History
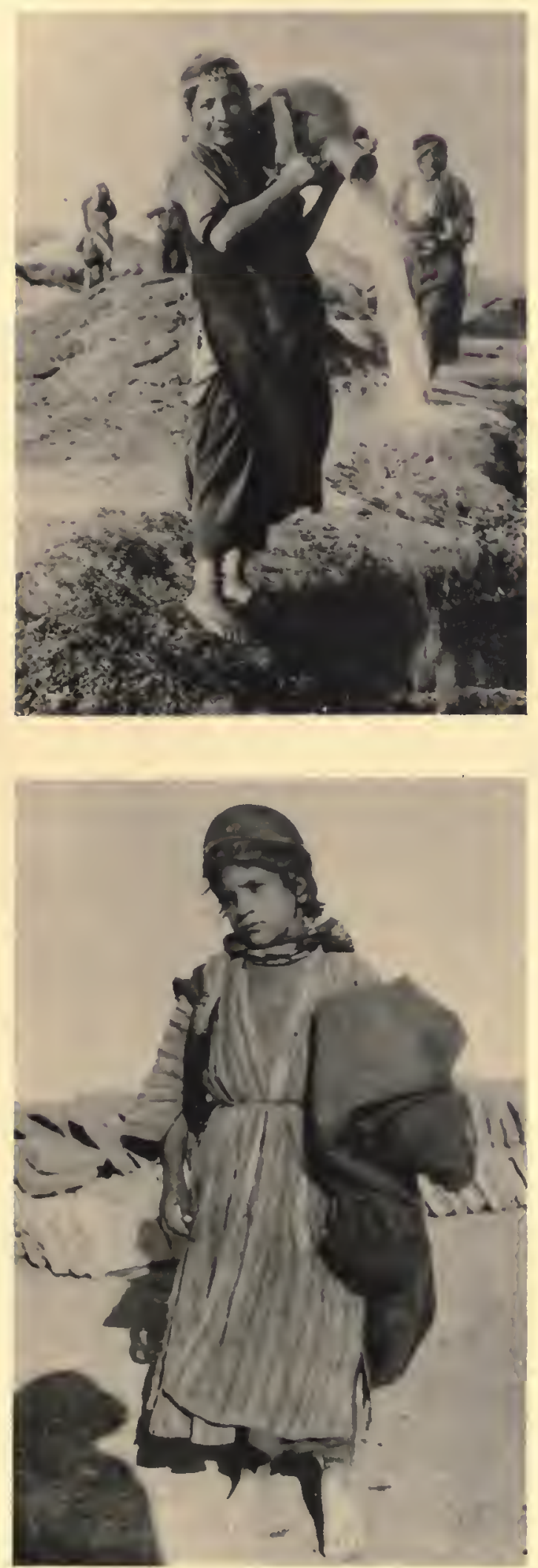

Anthropology, Memoirs, Vol. IV, Plate CXLIII
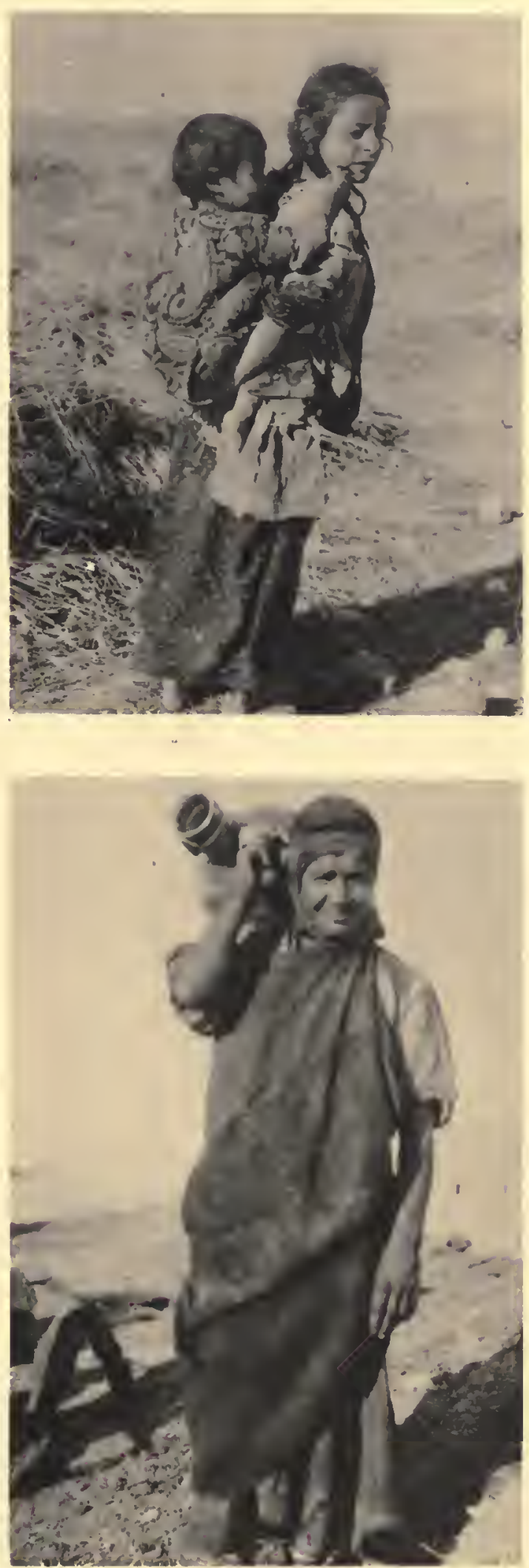

CHALDEAN WOMEN AND GIRLS, QARAQOSH 

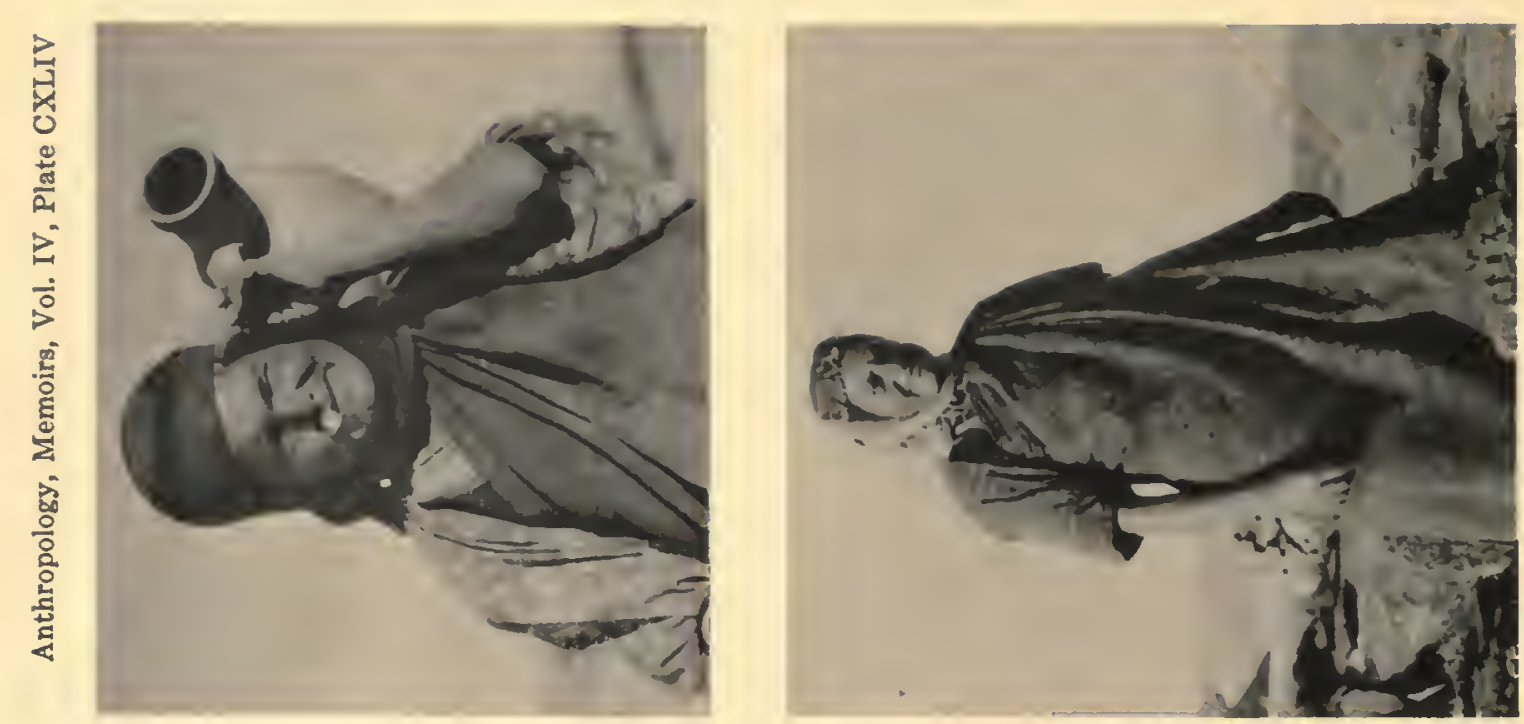

0
4 th N
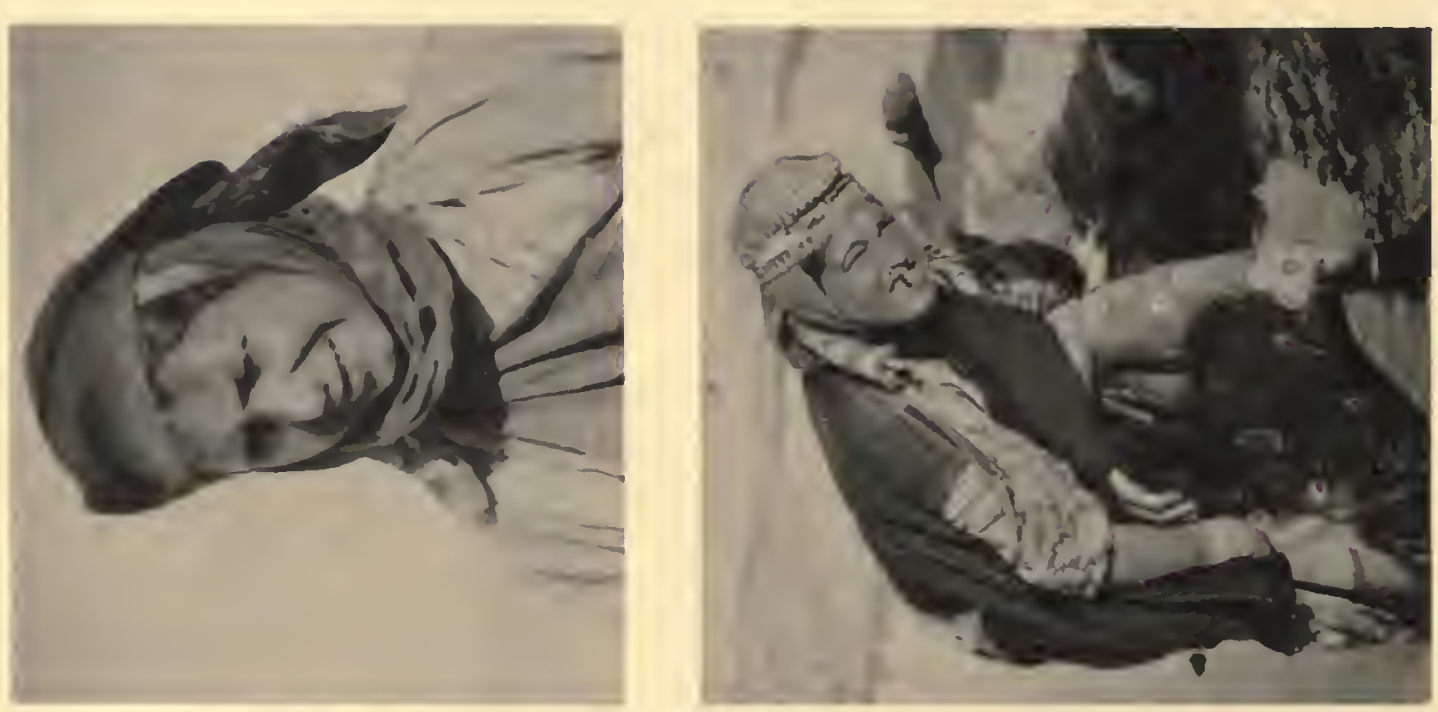

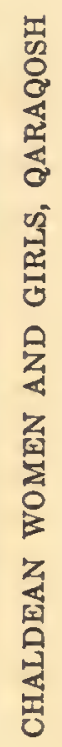
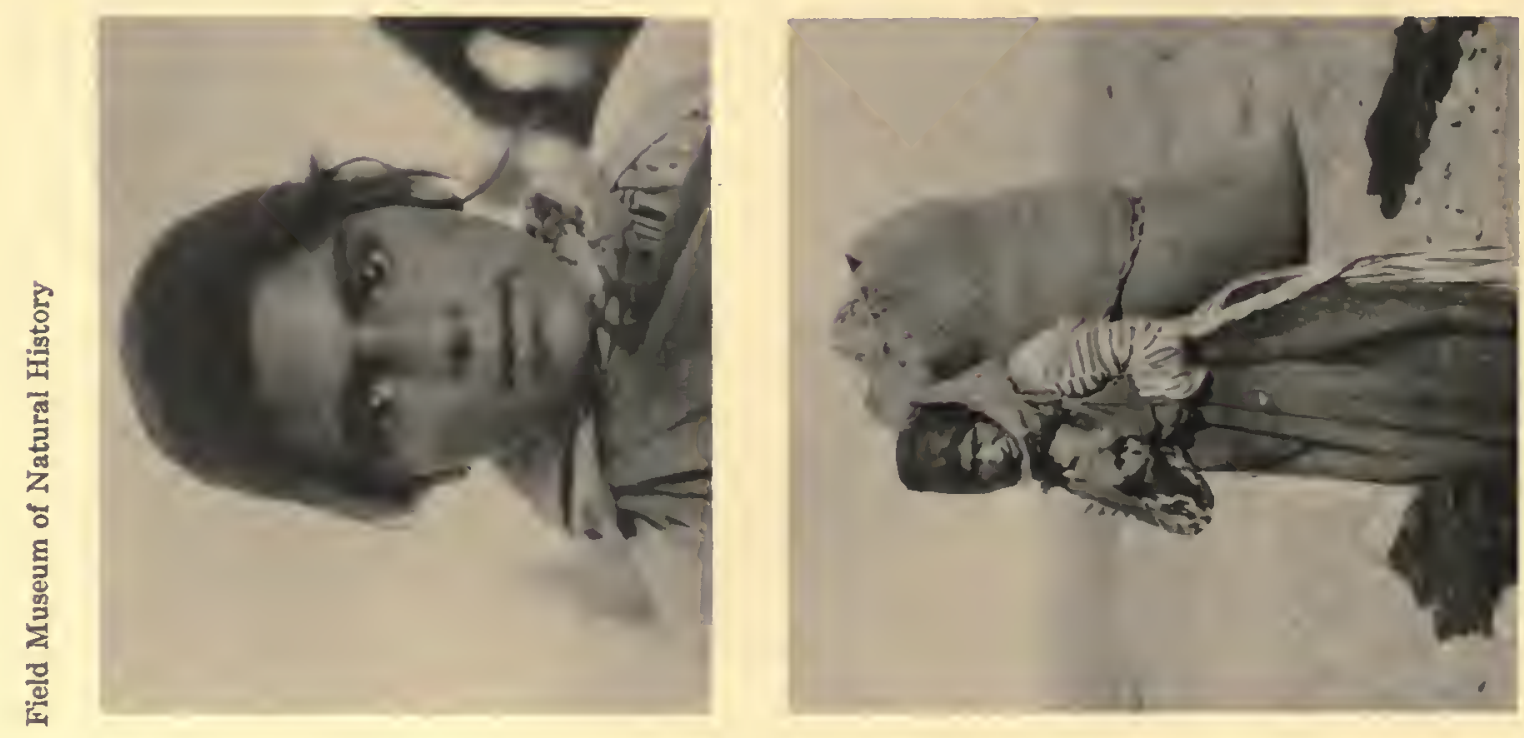


$$
\operatorname{Heg} \# 76501
$$

Field Museum of Natural History
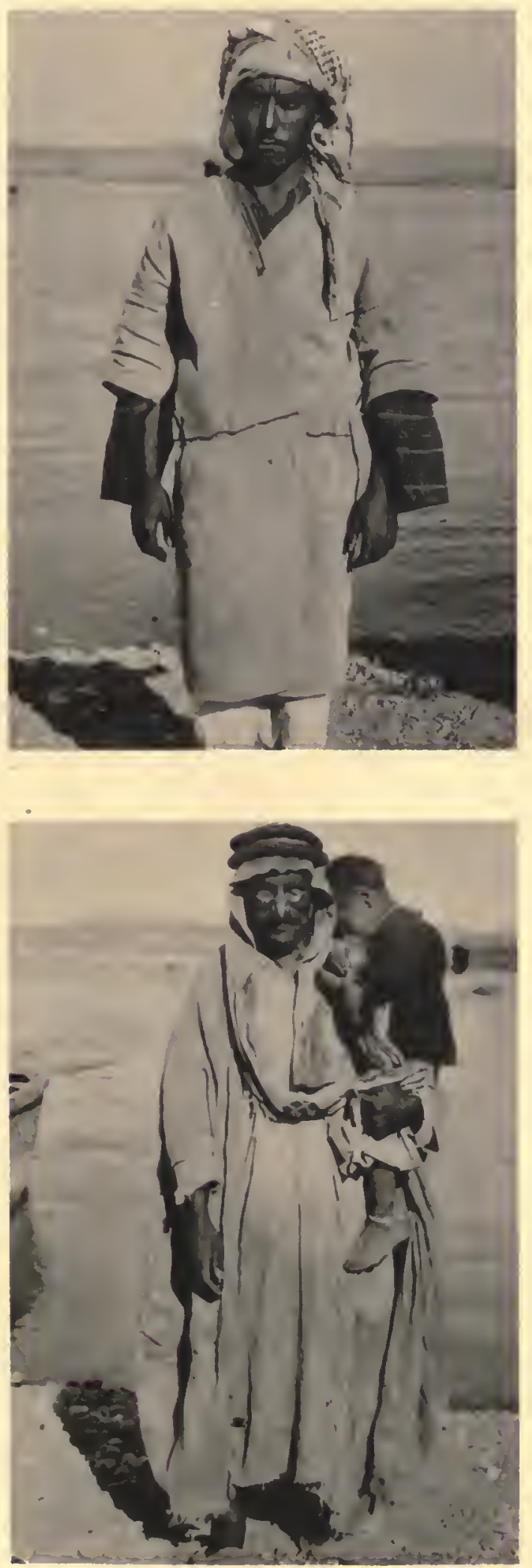

Anthropology, Memoirs, Vol. IV, Plate CXLV
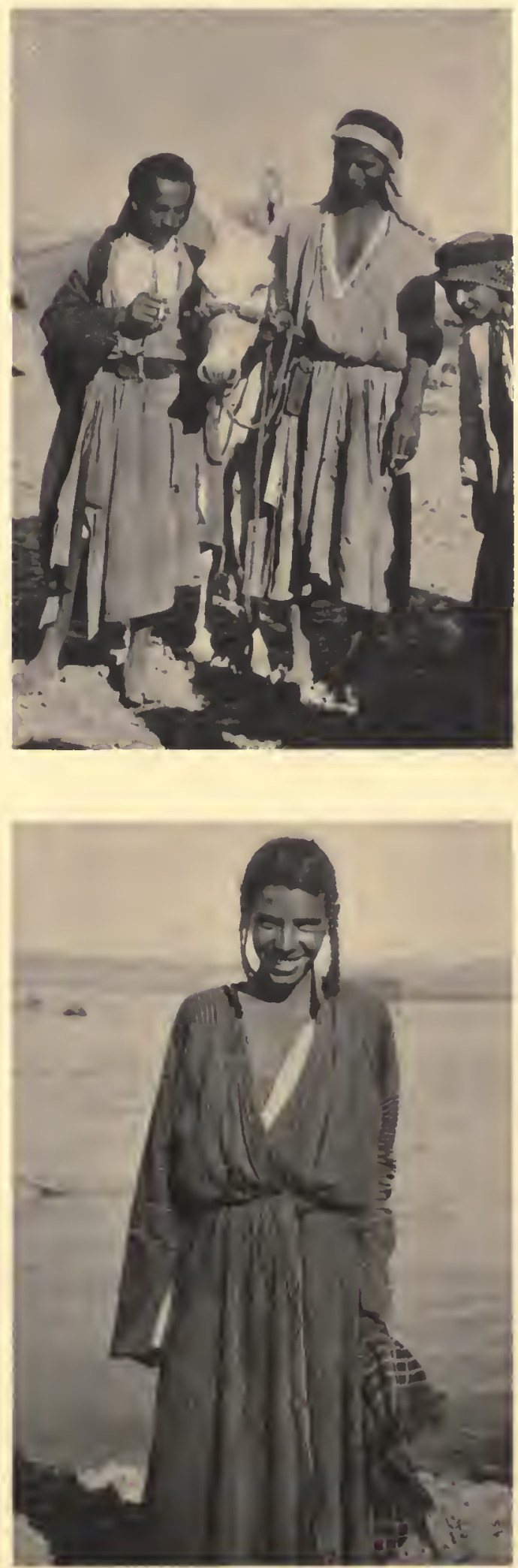

TYPES, NEAR ALTƯN KÖPRÜ 
$7 y \# 76502$

Field Museum of Natural History
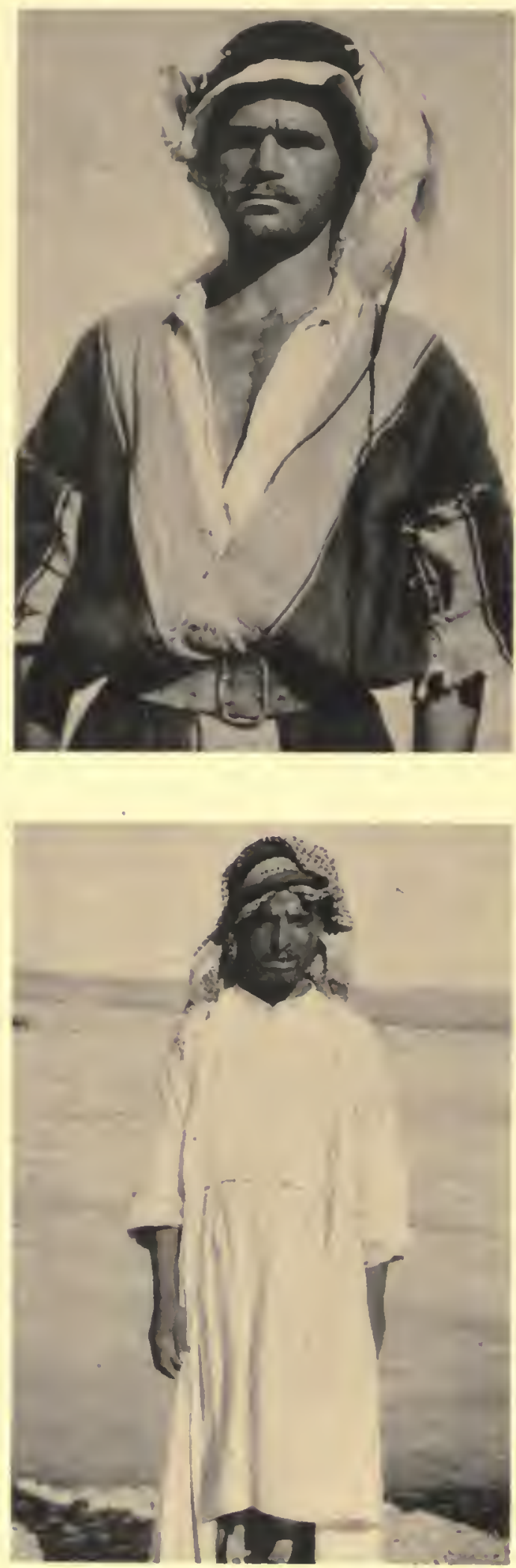

Anthropology, Memoirs, Vol. IV, Plate CXLVI
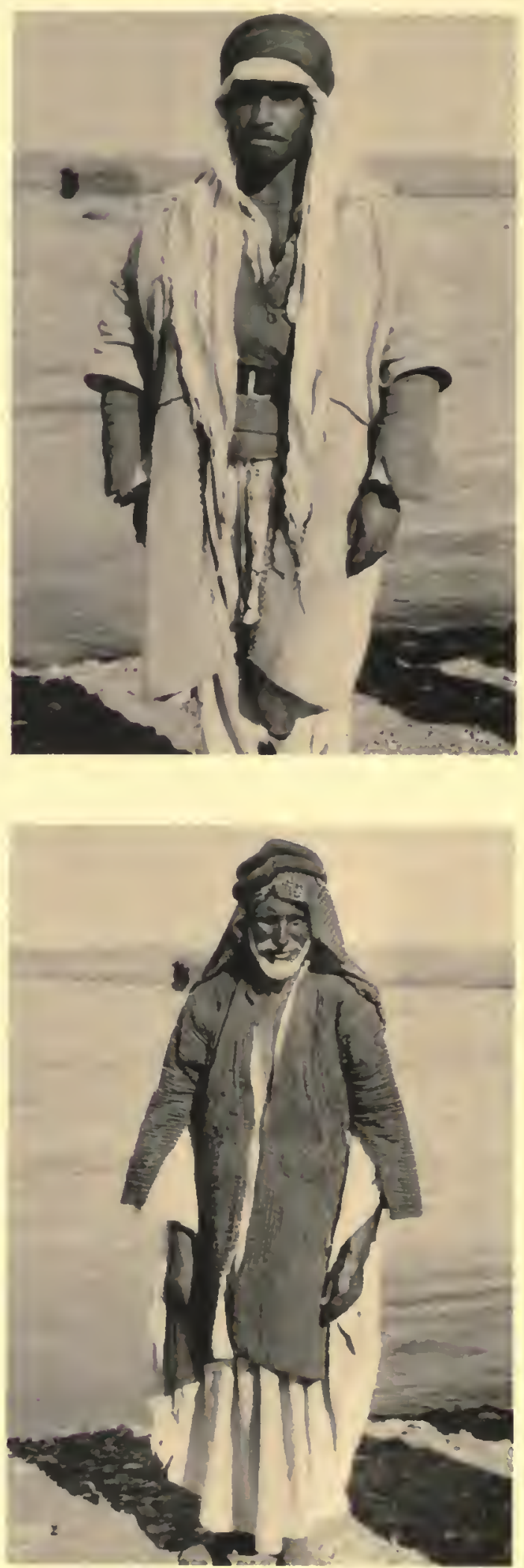

TYPES, NEAR ALTƯN KÖPRỦ 
ney 76503

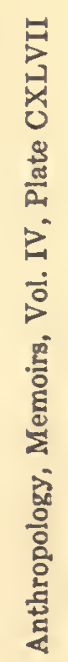
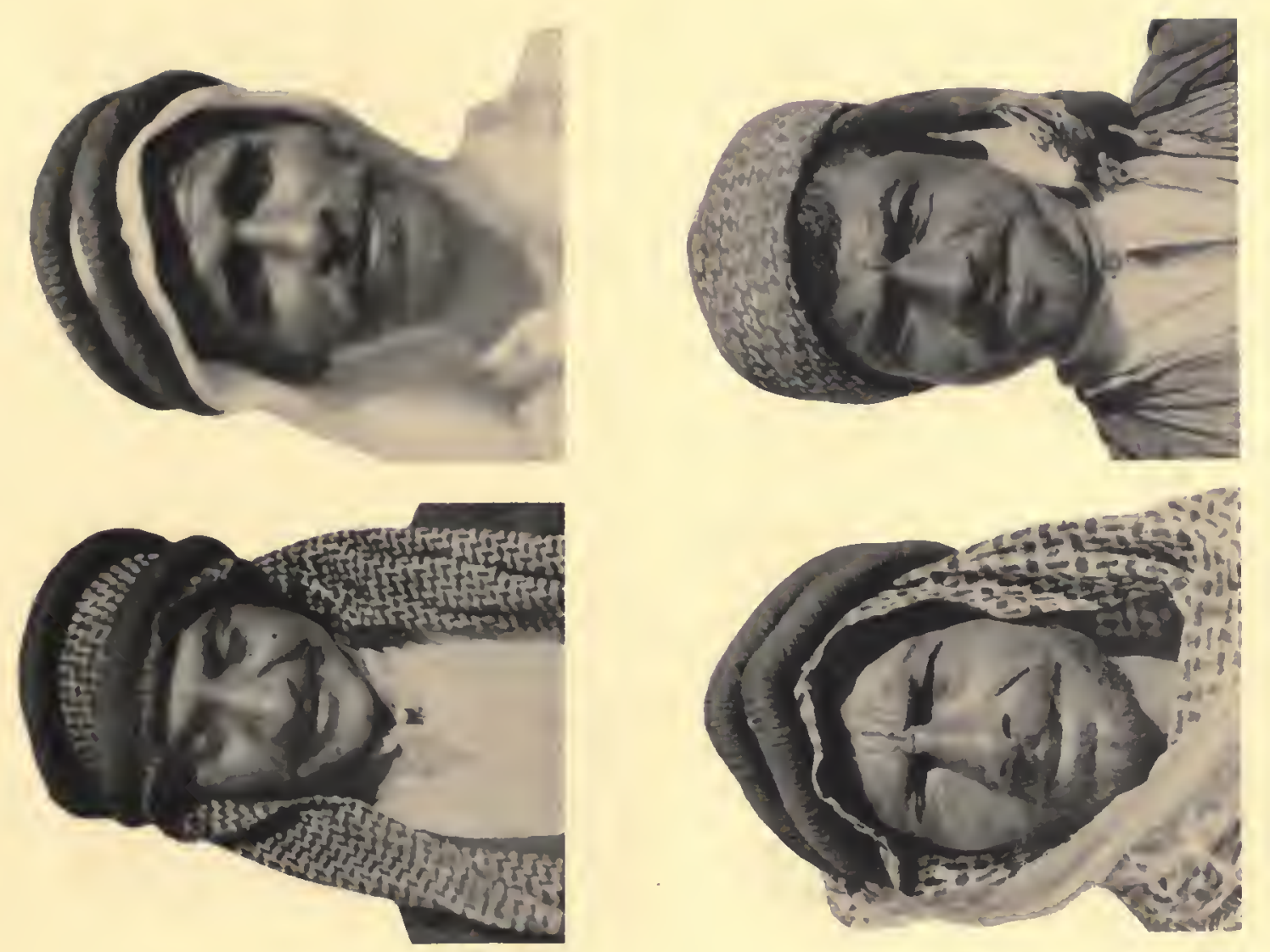

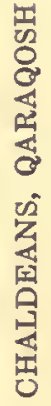
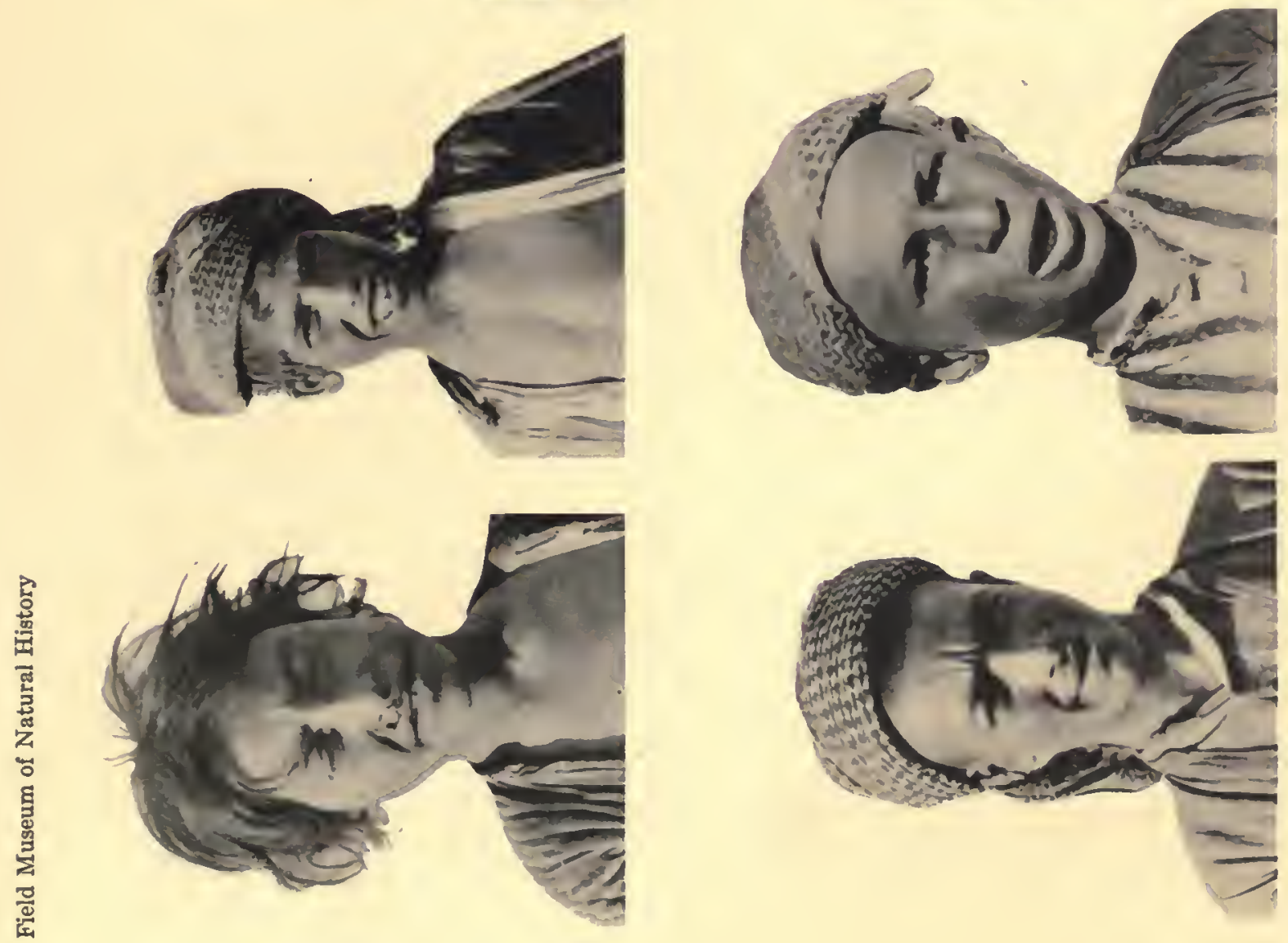
h

a

*

$\sqrt{ }$
ב
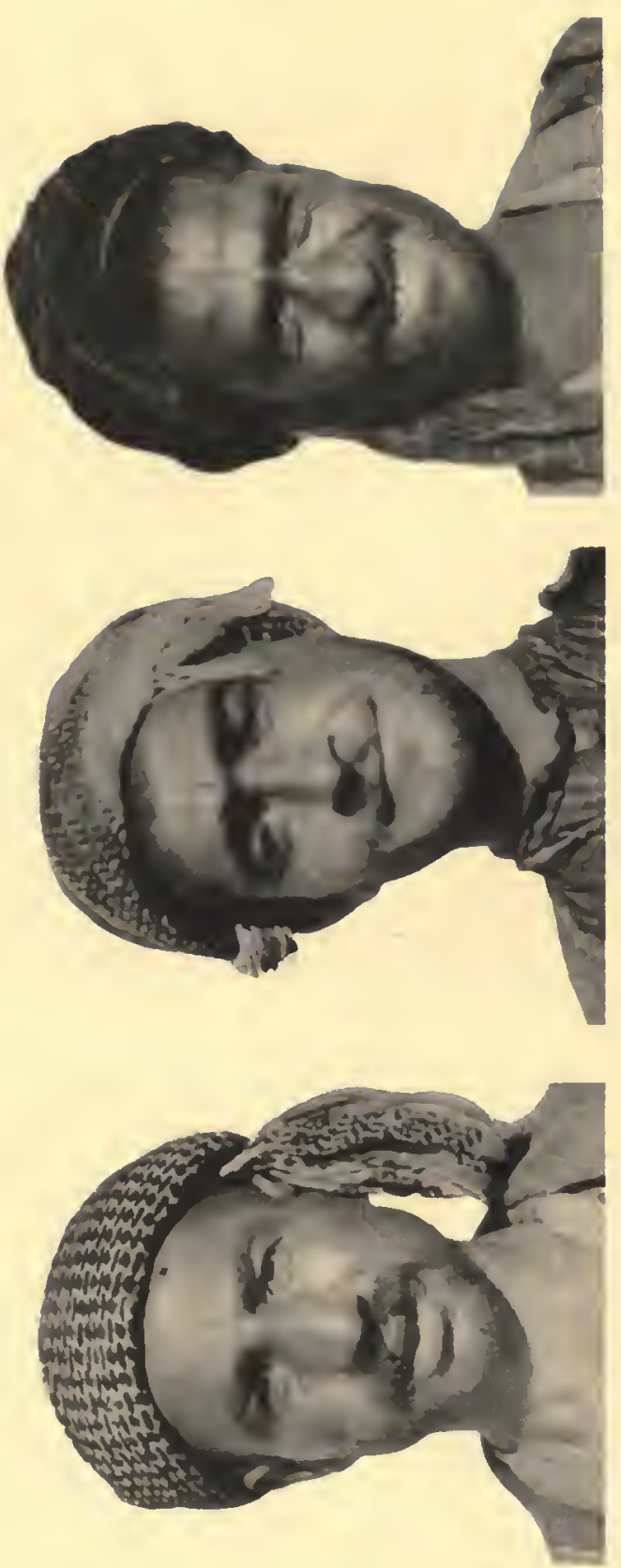

政

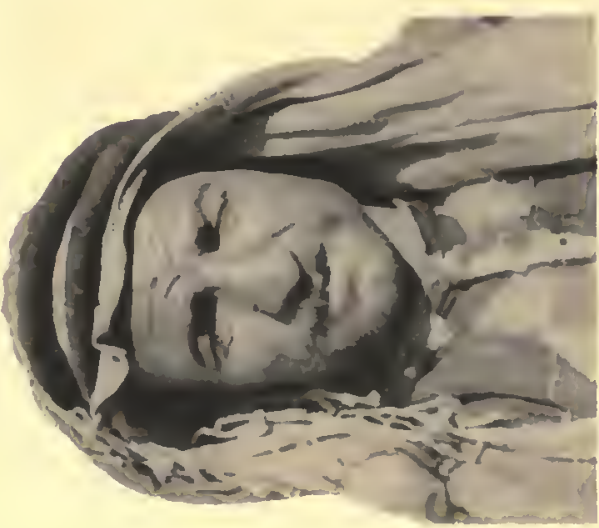

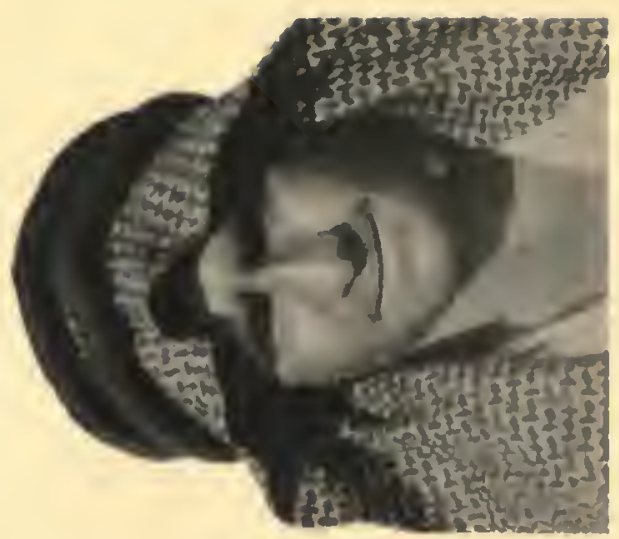

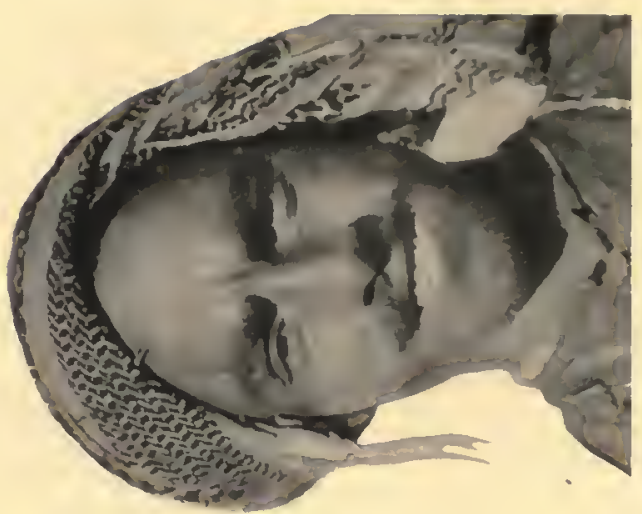

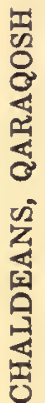
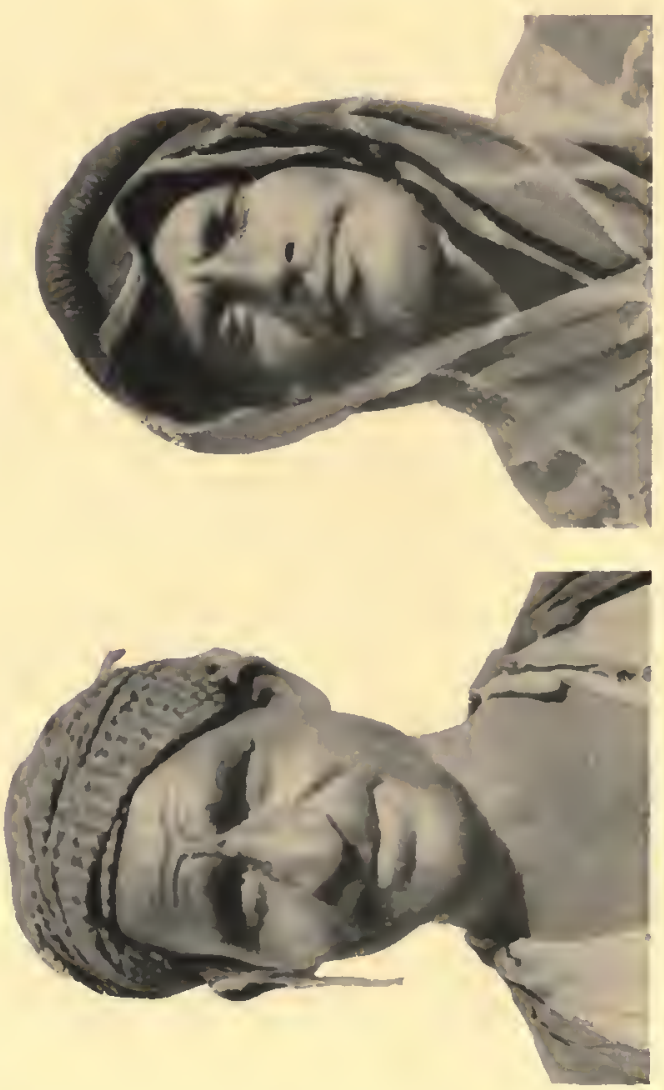


$$
\text { Hey } \# 76505
$$

Field Museum of Natural History
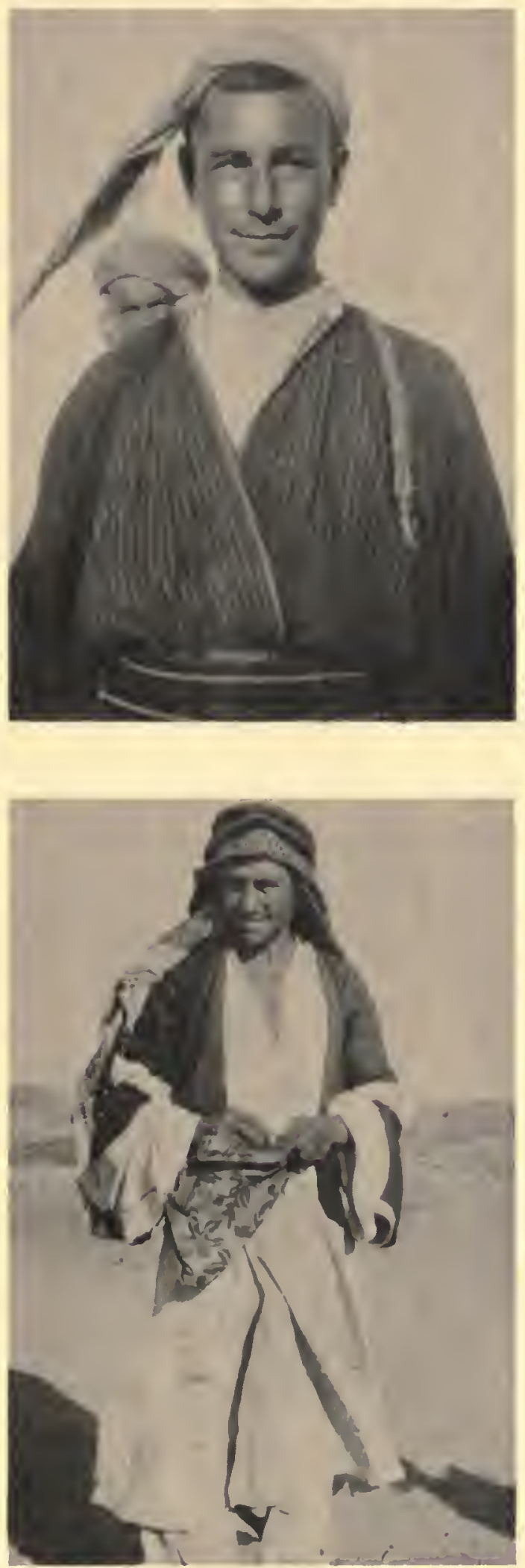

Anthropology, Memoirs, Vol. IV, Plate CXLIX
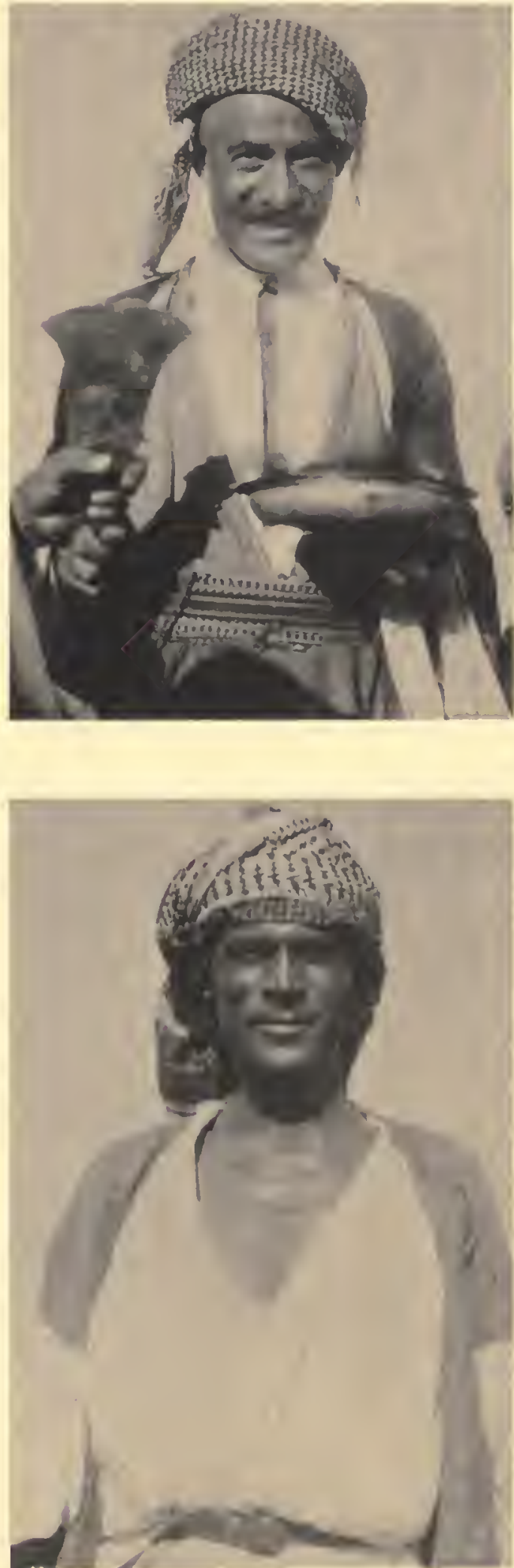

CHALDEANS, QARAQOSH 


$$
x_{y} * 76506
$$

Field Museum of Natural History

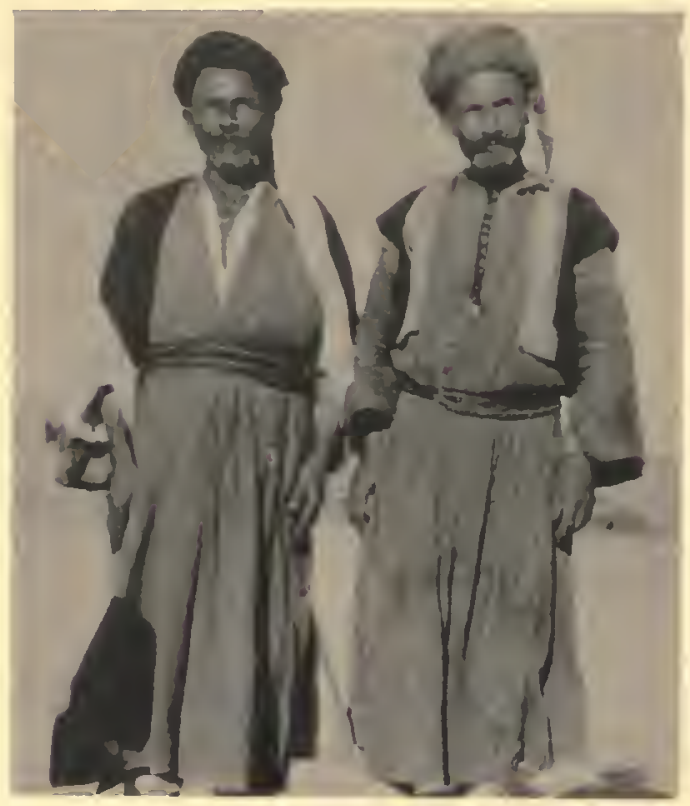

Fig. 1. Craftsmen

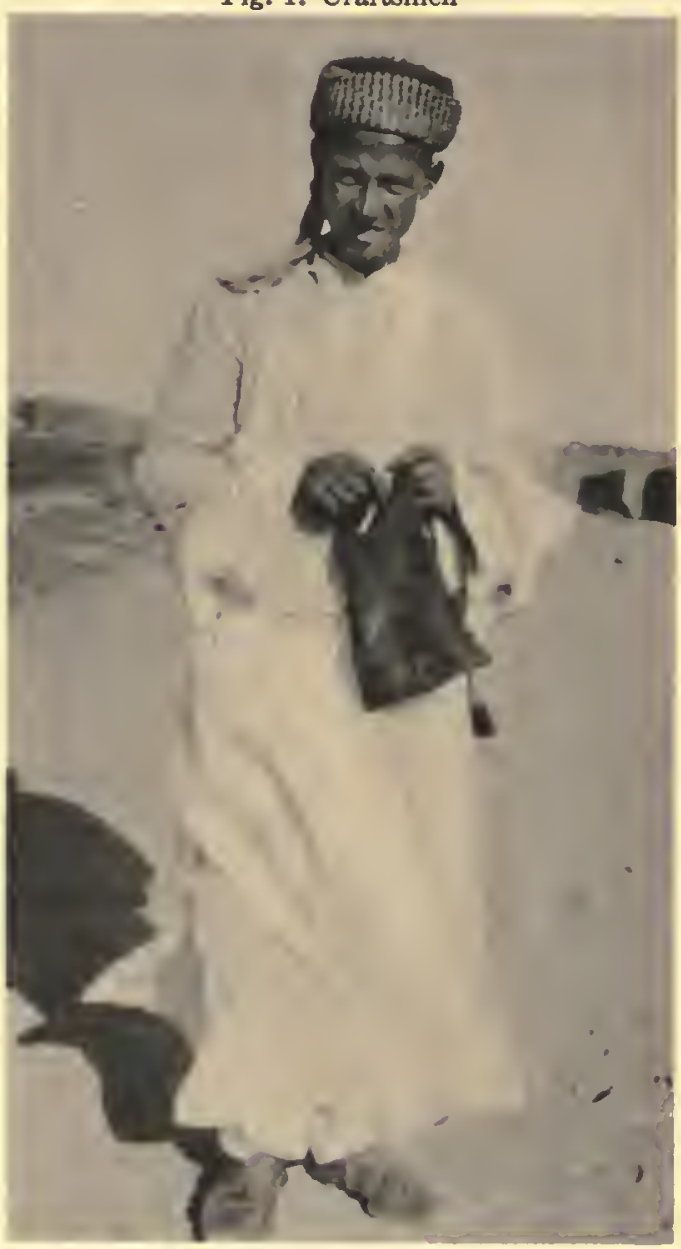

Fig. 3. Holding tobacco pouch
Anthropology, Memoirs, Vol. IV, Plate CL

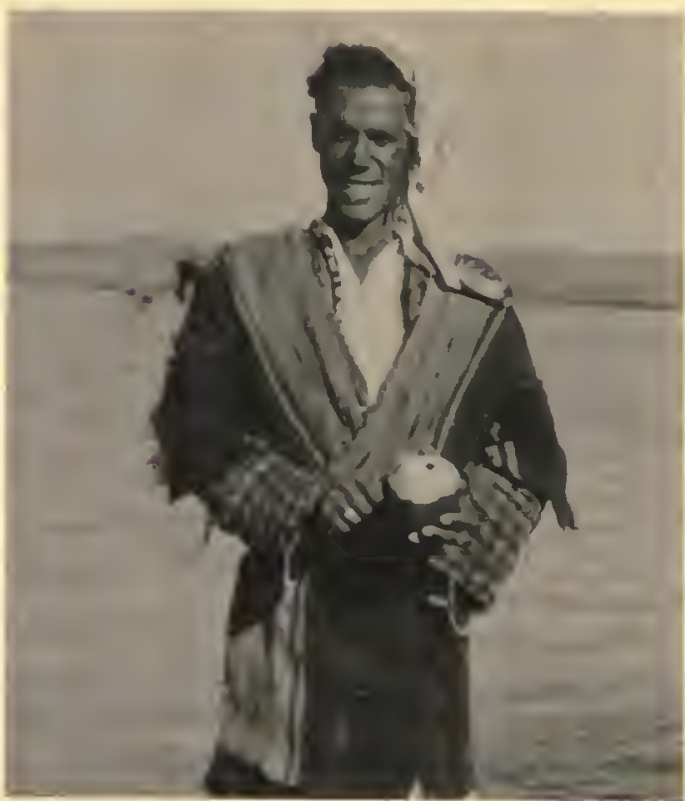

Fig. 2. Peeling melon

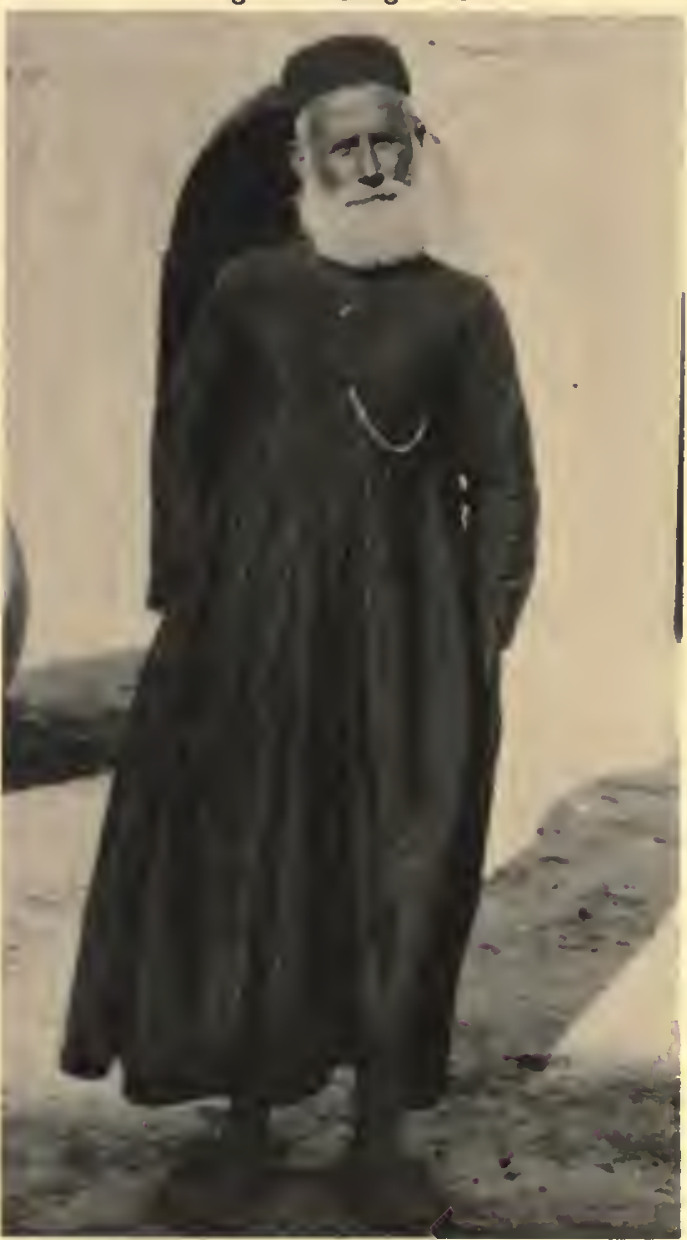

Fig. 4. Priest

CHALDEANS, QARAQOSH 


\section{$x_{y} \# 76507$}

ज्ञ
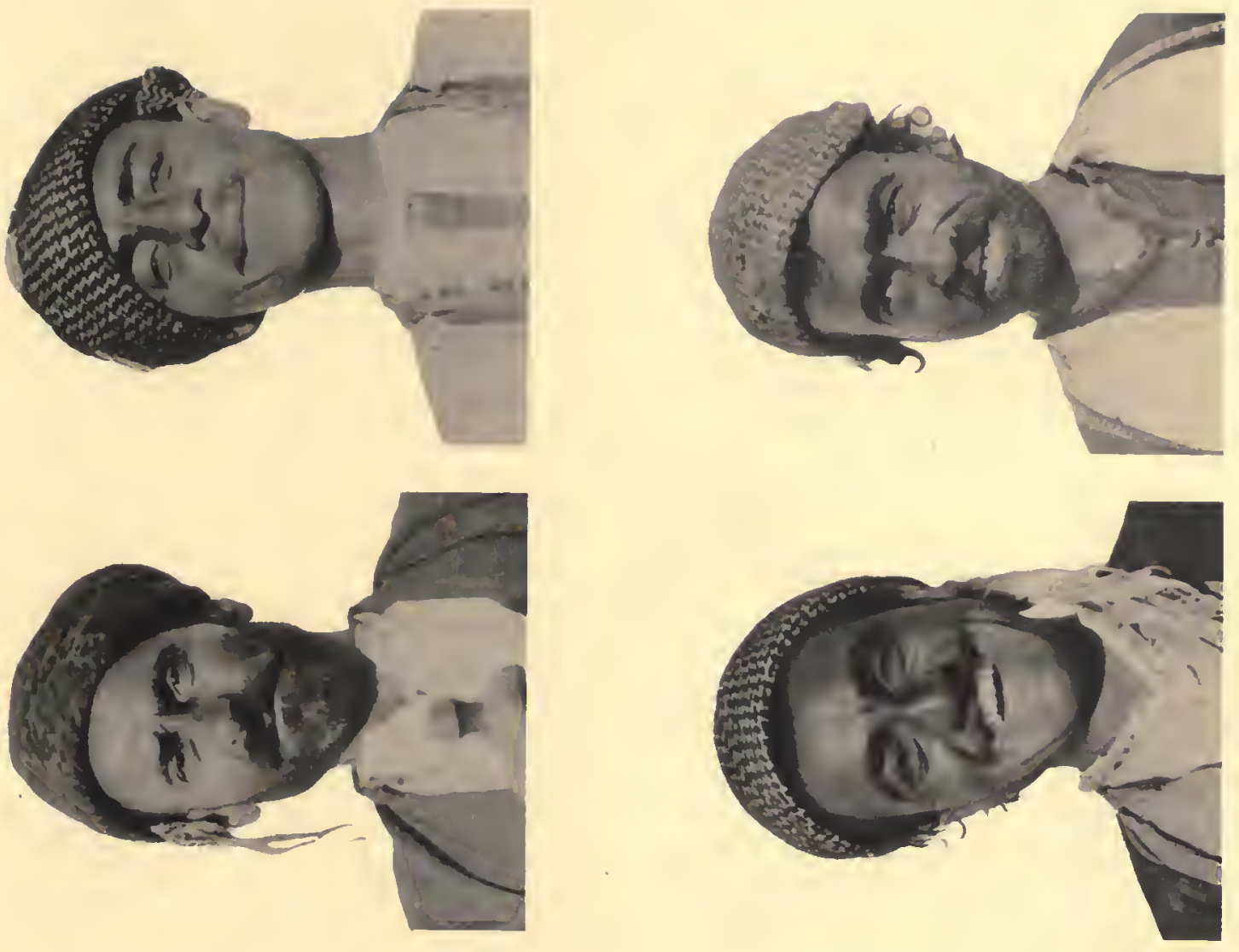

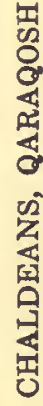
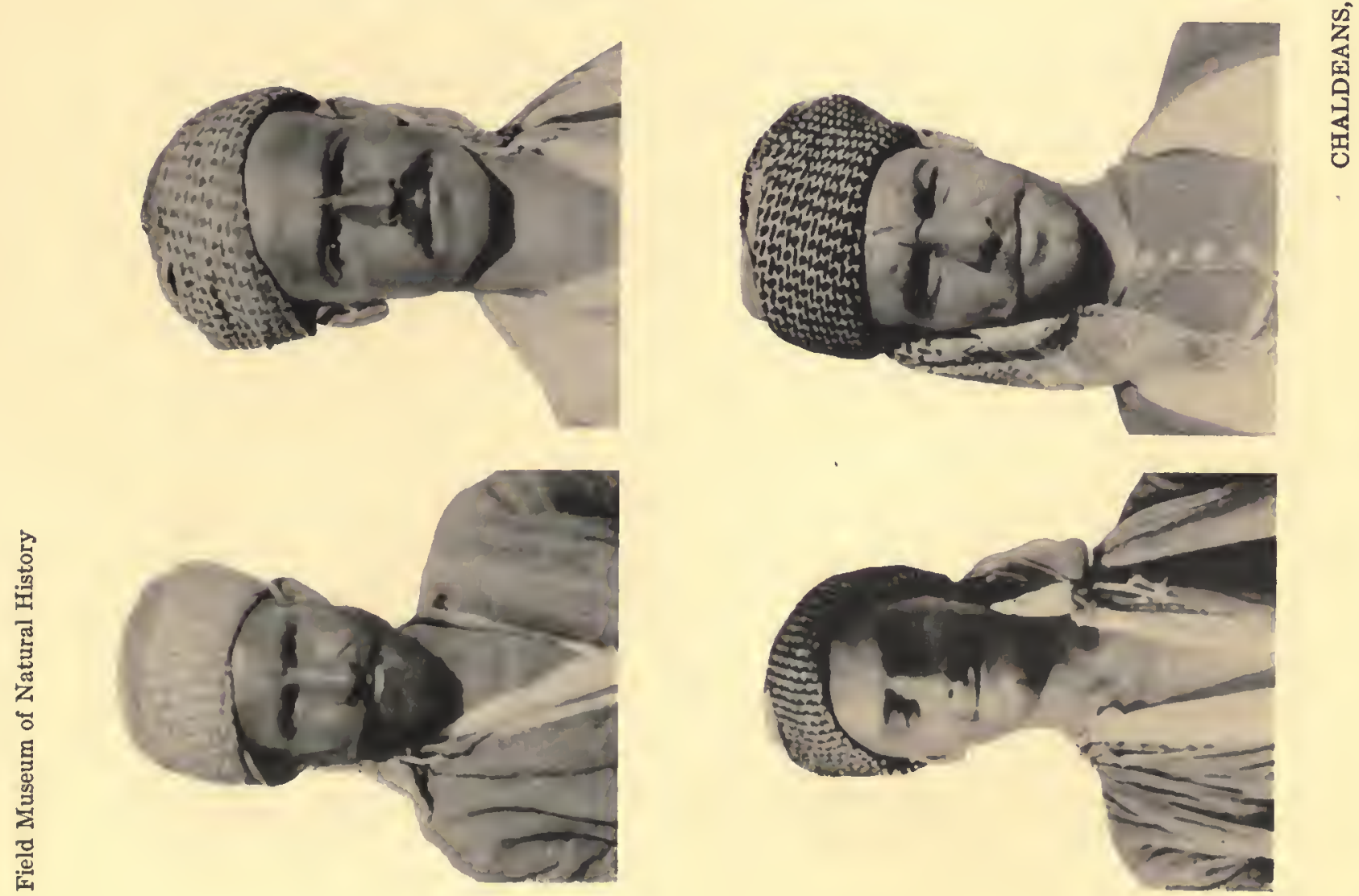

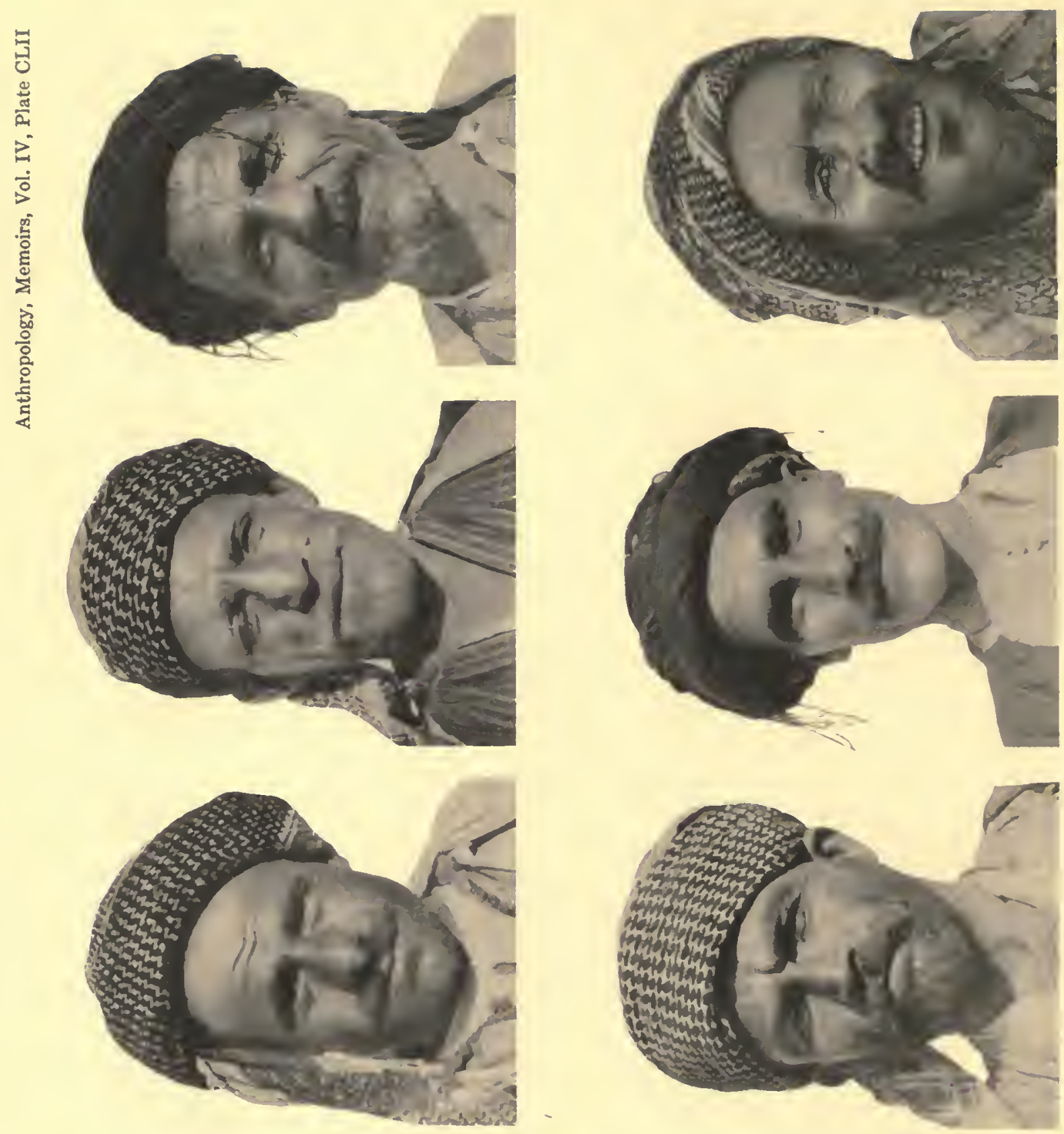

步
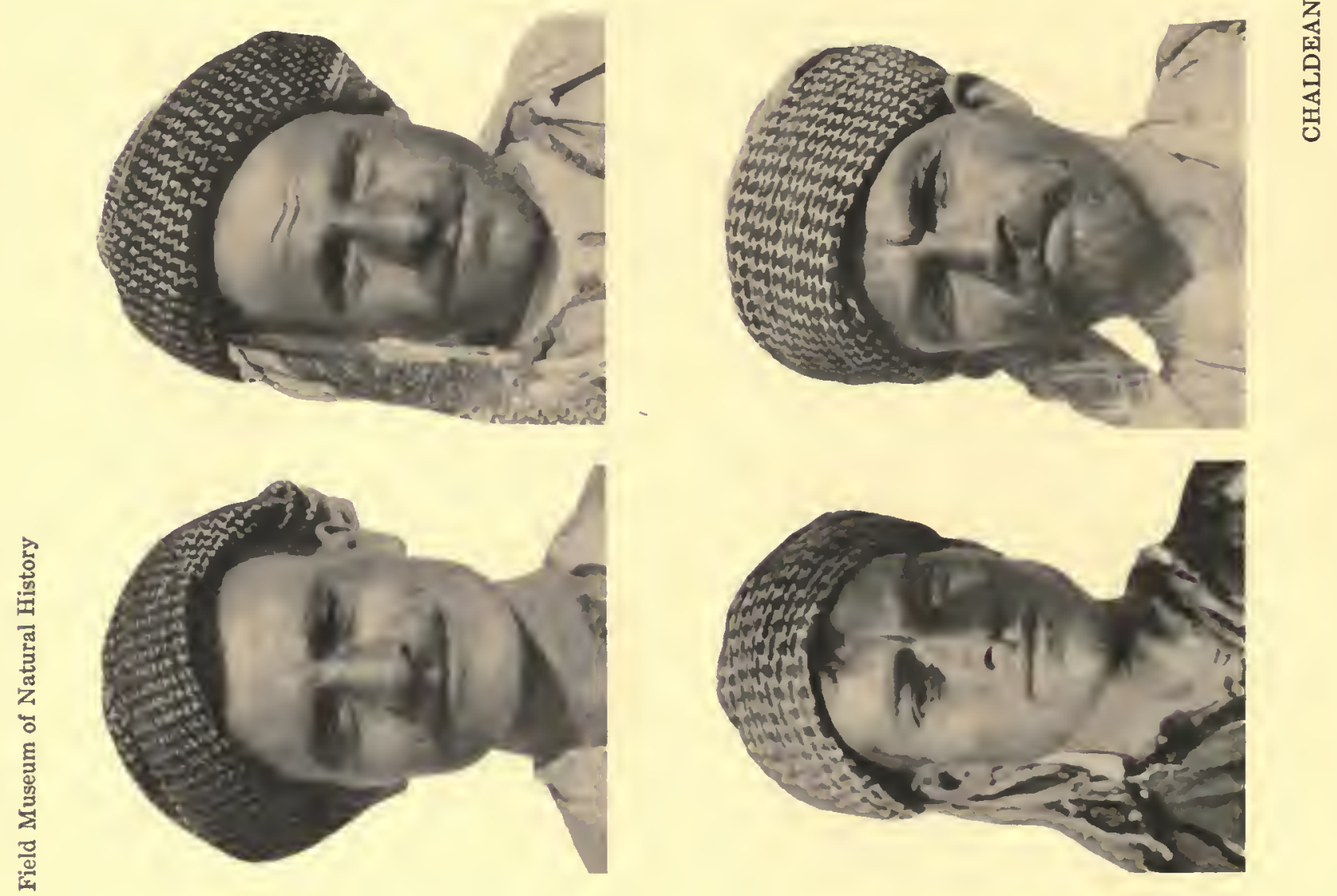

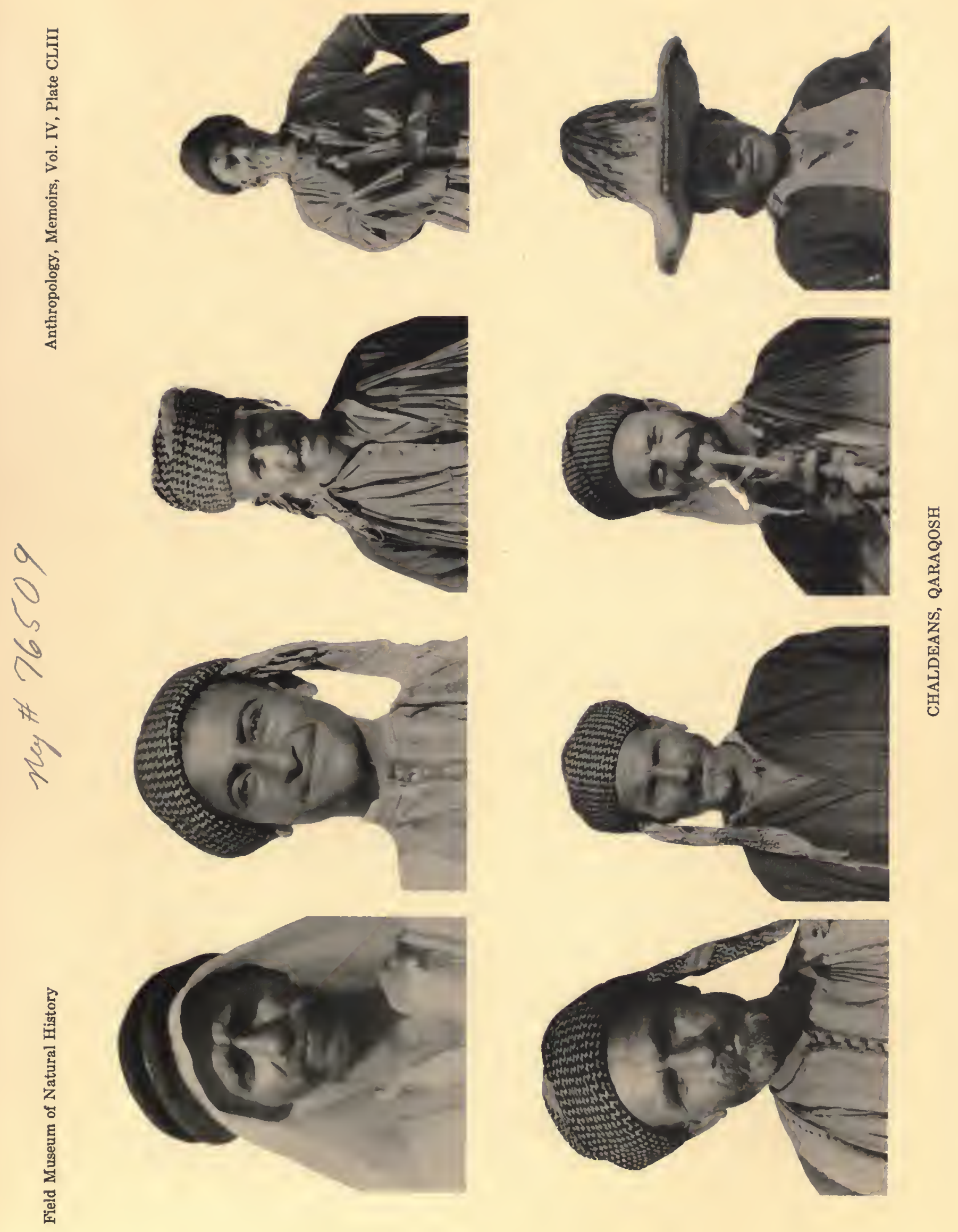


\section{$24 y^{\# ~} 76510$}

Field Museum of Natural History
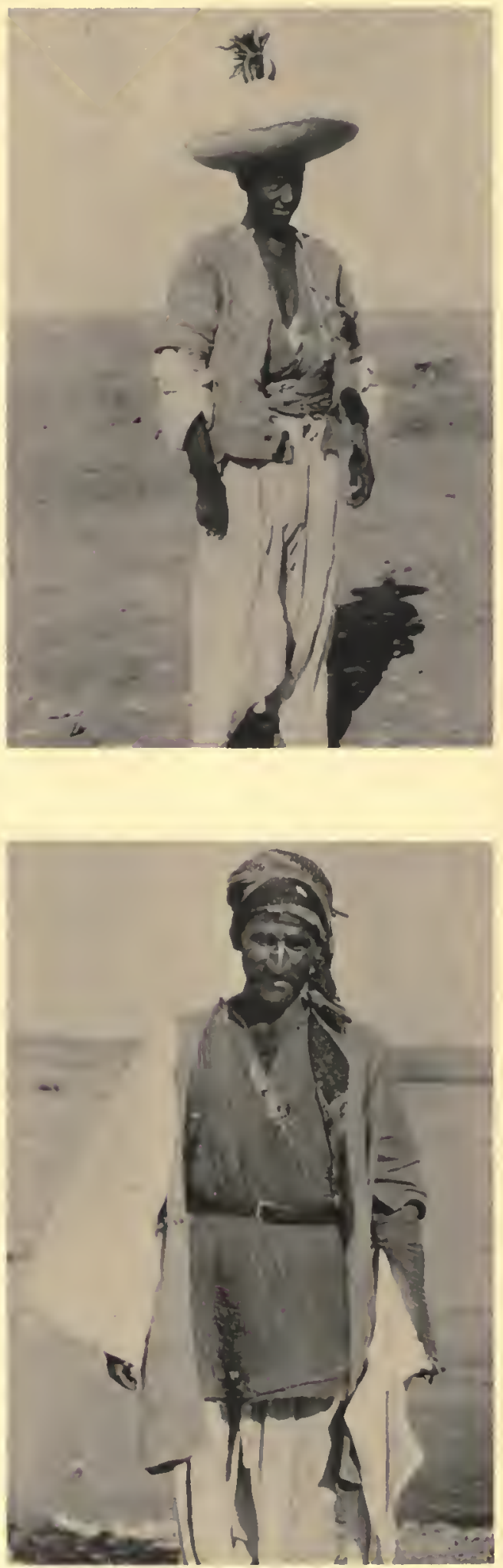

Anthropology, Memoirs, Vol. IV, Plate CLIV
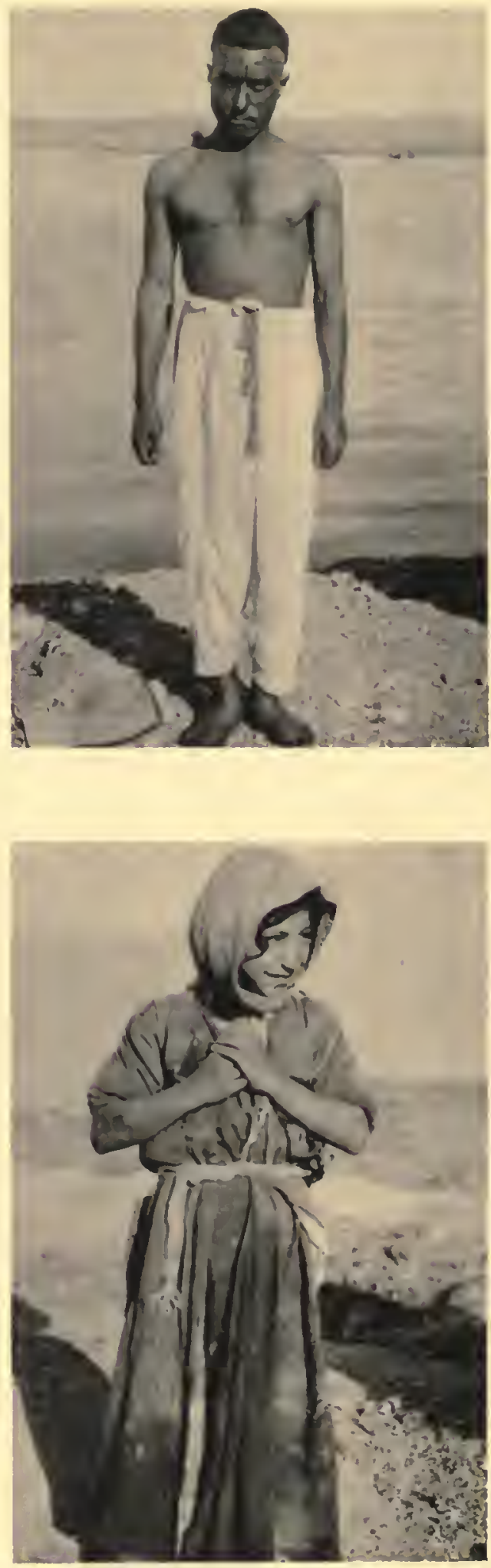

TYPES, NEAR ALTÜN KÖPRÜ 
Field Museum of Natural History
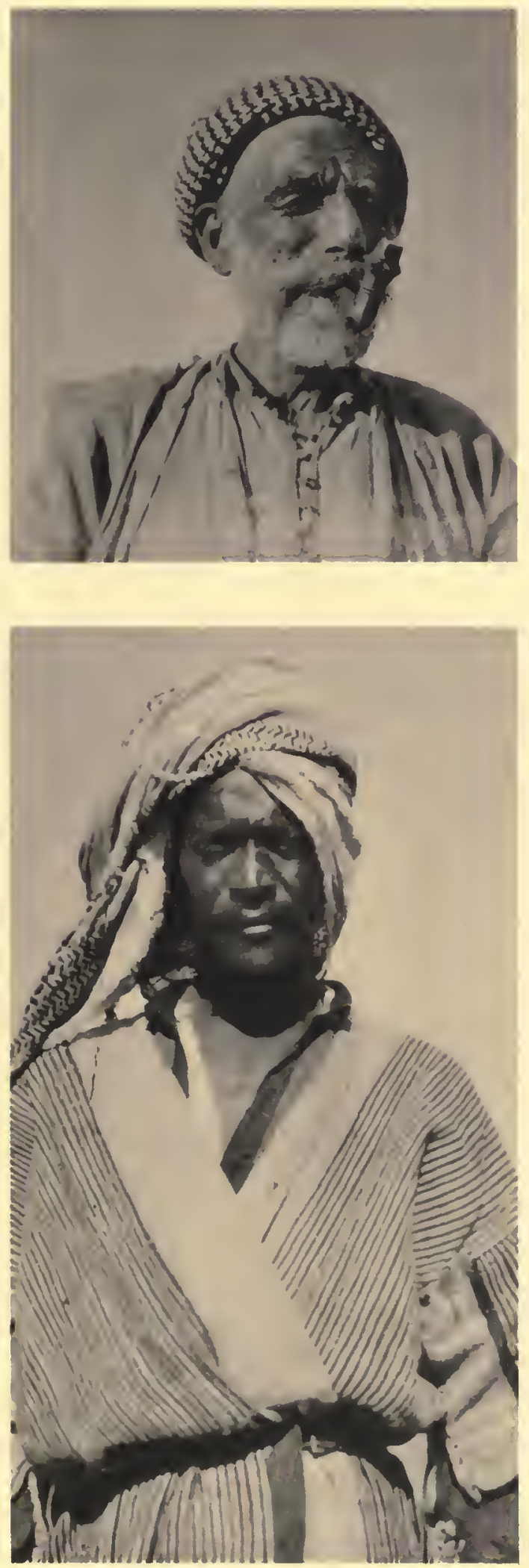

Anthropology, Memoirs, Vol. IV, Plate CLV
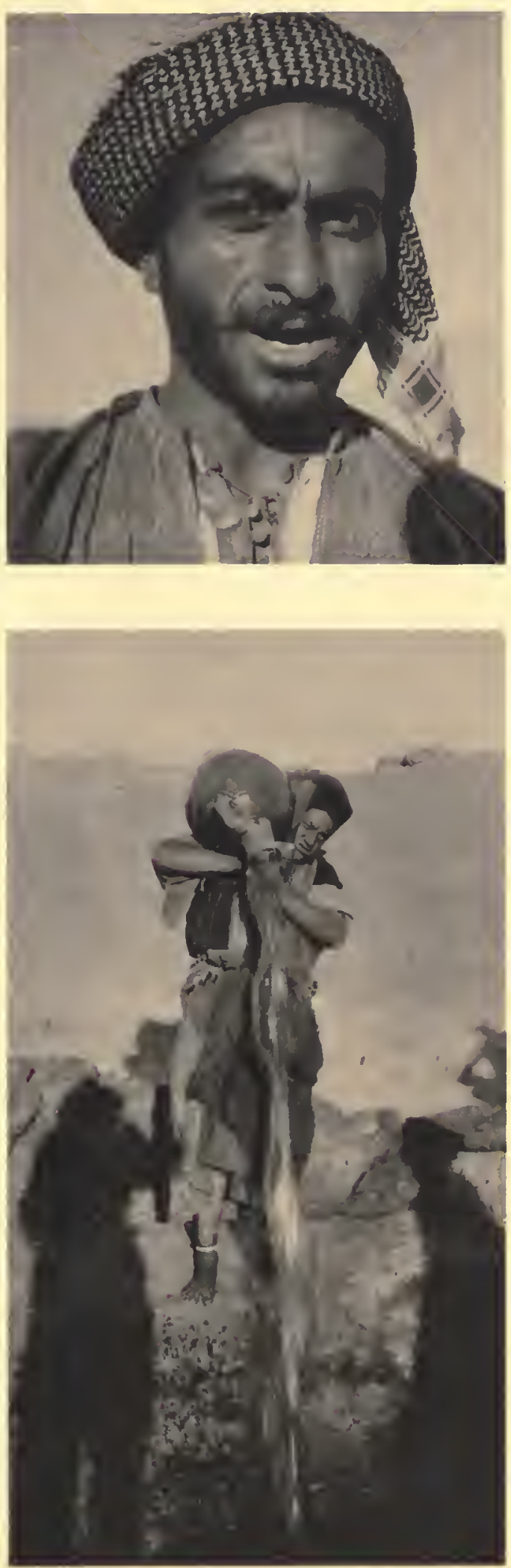

CHALDEANS, QARAQOSH 

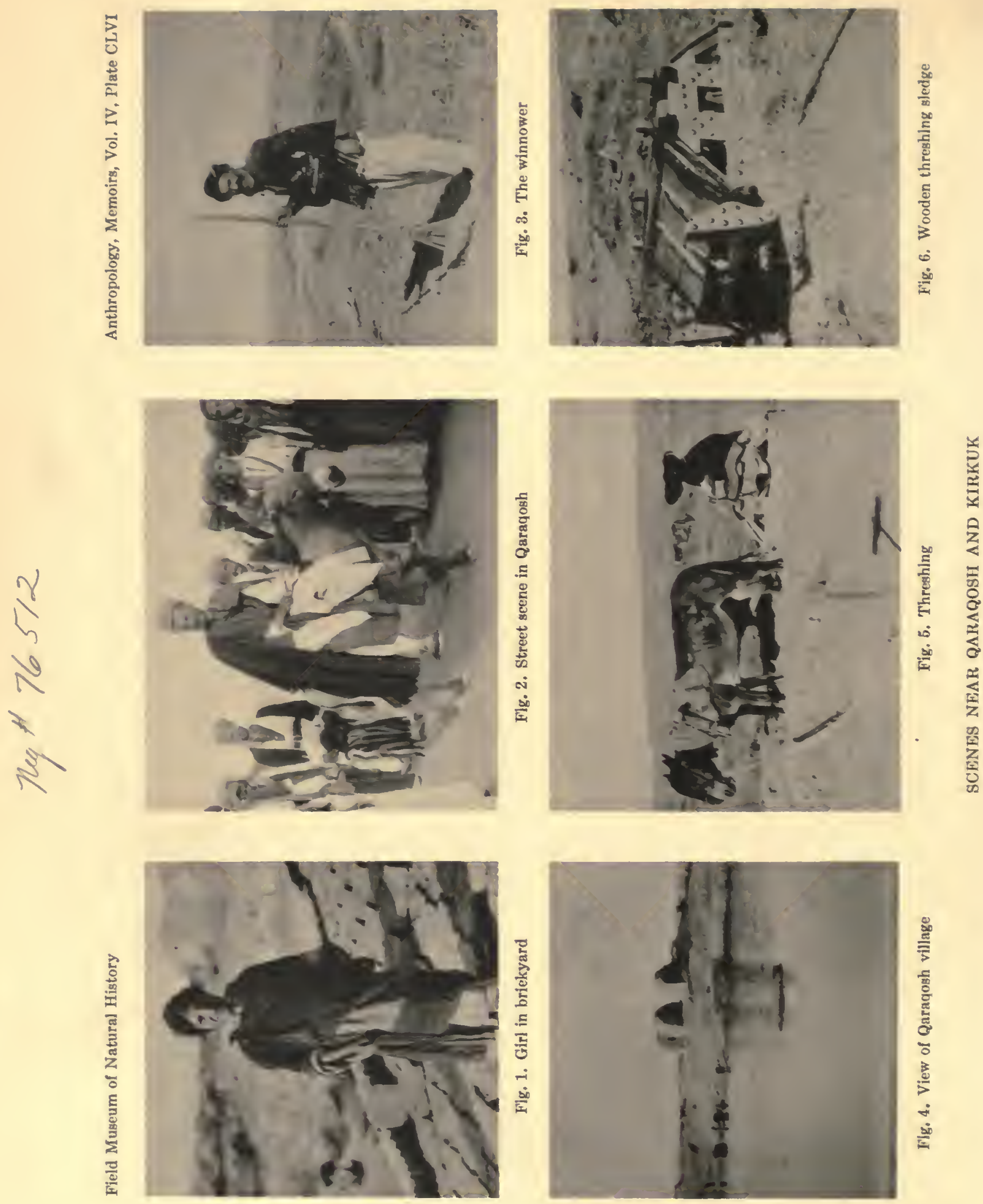


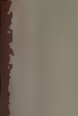



